Water Resources Data California

Water Year 1992

Volume 5. Ground-Water Data

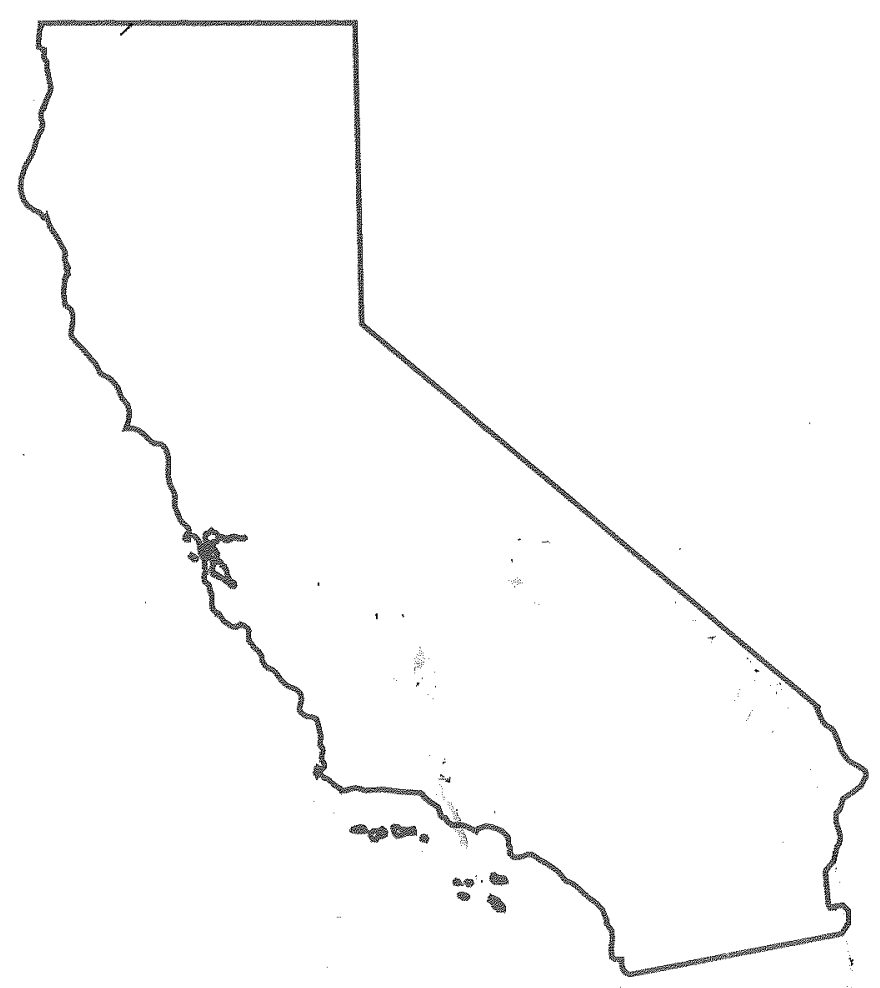

U.S. GEOLOGICAL SURVEY WATER-DATA REPORT CA $92-5$ Prepared in cooperation with other Federal, State, and local agencies 
1991

\begin{tabular}{|c|c|c|c|c|c|c|c|c|c|c|c|c|c|c|c|c|c|c|c|c|}
\hline \multicolumn{7}{|c|}{ OCTOBER } & \multicolumn{7}{|c|}{ NOVEMBER } & \multicolumn{7}{|c|}{ DECEMBER } \\
\hline S & M & $\mathrm{T}$ & W & $T$ & F & S & S & M & $\mathrm{T}$ & W & $\mathrm{T}$ & $\mathrm{E}$ & S & $S$ & M & $\mathrm{T}$ & W & $\mathrm{T}$ & $\mathrm{F}$ & S \\
\hline & & 1 & 2 & 3 & 4 & 5 & & & & & & 1 & 2 & & & & & & & \\
\hline 6 & 7 & 8 & 9 & 10 & 11 & 12 & 3 & 4 & 5 & 6 & 7 & 8 & 9 & 1 & 2 & 3 & 4 & 5 & 6 & 7 \\
\hline 13 & 14 & 15 & 16 & 17 & 18 & 19 & 10 & 11 & 12 & 13 & 14 & 15 & 16 & 8 & 9 & 10 & 11 & 12 & 13 & 14 \\
\hline 20 & 21 & 22 & 23 & 24 & 25 & 26 & 17 & 18 & 19 & 20 & 21 & 22 & 23 & 15 & 16 & 17 & 18 & 19 & 20 & 21 \\
\hline 27 & 28 & 29 & 30 & 31 & & & 24 & 25 & 26 & 27 & 28 & 29 & 30 & $\begin{array}{l}22 \\
29\end{array}$ & $\begin{array}{l}23 \\
30\end{array}$ & $\begin{array}{l}24 \\
31\end{array}$ & 25 & 26 & 27 & 28 \\
\hline
\end{tabular}

1992

\begin{tabular}{|c|c|c|c|c|c|c|c|c|c|c|c|c|c|c|c|c|c|c|c|c|}
\hline \multirow[b]{2}{*}{$S$} & \multicolumn{6}{|c|}{ JANUARY | } & \multicolumn{7}{|c|}{ EEBRUARY } & \multicolumn{7}{|c|}{ MARCH } \\
\hline & $M$ & $\mathrm{~T}$ & $W$ & $\mathrm{~T}$ & $F$ & $S$ & S & $M$ & $\mathrm{~T}$ & $W$ & $\mathrm{~T}$ & $\mathrm{~F}$ & $S$ & S & M & $T$ & $W$ & $\mathrm{~T}$ & $F$ & $\mathrm{~S}$ \\
\hline & & & 1 & 2 & 3 & 4 & & & & & & & 1 & 1 & 2 & 3 & 4 & 5 & 6 & 7 \\
\hline 5 & 6 & 7 & 8 & 9 & 10 & 11 & 2 & 3 & 4 & 5 & 6 & 7 & 8 & 8 & 9 & 10 & 11 & 12 & 13 & 14 \\
\hline 12 & 13 & 14 & 15 & 16 & 17 & 18 & 9 & 10 & 11 & 12 & 13 & 14 & 15 & 15 & 16 & 17 & 18 & 19 & 20 & 21 \\
\hline 19 & 20 & 21 & 22 & 23 & 24 & 25 & 16 & 17 & 18 & 19 & 20 & 21 & 22 & 22 & 23 & 24 & 25 & 26 & 27 & 28 \\
\hline 26 & 27 & 28 & 29 & 30 & 31 & & 23 & 24 & 25 & 26 & 27 & 28 & 29 & 29 & 30 & 31 & & & & \\
\hline
\end{tabular}

APRIL

MAY

JUNE

$\begin{array}{lllllll}S & M & T & W & T & F & S\end{array}$

$\begin{array}{llll}1 & 2 & 3 & 4\end{array}$

$\begin{array}{lllllll}5 & 6 & 7 & 8 & 9 & 10 & 11\end{array}$

$\begin{array}{lllllll}12 & 13 & 14 & 15 & 16 & 17 & 18\end{array}$

$\begin{array}{lllllll}19 & 20 & 21 & 22 & 23 & 24 & 25\end{array}$

$\begin{array}{lllll}26 & 27 & 28 & 29 & 30\end{array}$

JULY

$\begin{array}{lllllll}S & M & T & W & T & F & S\end{array}$

$\begin{array}{rrrrrrr}5 & 6 & 7 & 8 & 9 & 10 & 11\end{array}$

$\begin{array}{lllllll}12 & 13 & 14 & 15 & 16 & 17 & 18\end{array}$

$\begin{array}{lllllll}19 & 20 & 21 & 22 & 23 & 24 & 25\end{array}$

$\begin{array}{llllll}26 & 27 & 28 & 29 & 30 & 31\end{array}$

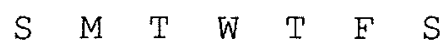

$\begin{array}{llll}1 & 1 & 2\end{array}$

$\begin{array}{lllllll}3 & 4 & 5 & 6 & 7 & 8 & 9\end{array}$

$\begin{array}{lllllll}10 & 11 & 12 & 13 & 14 & 15 & 16\end{array}$

$\begin{array}{lllllll}17 & 18 & 19 & 20 & 21 & 22 & 23\end{array}$

$\begin{array}{lllllll}24 & 25 & 26 & 27 & 28 & 29 & 30\end{array}$ 31

AUGUST

$S \quad M \quad T \quad W \quad T \quad F \quad S$

$\begin{array}{lllllll}2 & 3 & 4 & 5 & 6 & 7 & 8\end{array}$

$\begin{array}{lllllll}9 & 10 & 11 & 12 & 13 & 14 & 15\end{array}$

$\begin{array}{lllllll}16 & 17 & 18 & 19 & 20 & 21 & 22\end{array}$

$\begin{array}{lllllll}23 & 24 & 25 & 26 & 27 & 28 & 29\end{array}$

$30 \quad 31$
$\begin{array}{lllllll}S & M & T & W & T & F & S\end{array}$

$\begin{array}{llllll}1 & 2 & 3 & 4 & 5 & 6\end{array}$

$\begin{array}{lllllll}7 & 8 & 9 & 10 & 11 & 12 & 13\end{array}$

$\begin{array}{lllllll}14 & 15 & 16 & 17 & 18 & 19 & 20\end{array}$

$\begin{array}{lllllll}21 & 22 & 23 & 24 & 25 & 26 & 27\end{array}$

$\begin{array}{lll}28 & 29 & 30\end{array}$

\section{SEPTEMBER}

$\begin{array}{lllllll}S & M & T & W & T & F & S\end{array}$

$\begin{array}{llllll}6 & 1 & 2 & 3 & 4 & 5\end{array}$

$\begin{array}{lllllll}6 & 7 & 8 & 9 & 10 & 11 & 12\end{array}$

$\begin{array}{lllllll}13 & 14 & 15 & 16 & 17 & 18 & 19\end{array}$

$\begin{array}{lllllll}20 & 21 & 22 & 23 & 24 & 25 & 26\end{array}$

$\begin{array}{llll}27 & 28 & 29 & 30\end{array}$ 


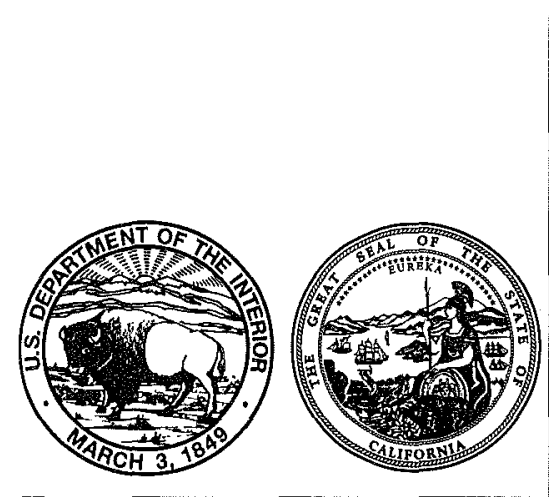

\section{Water Resources Data California}

Water Year 1992

Volume 5. Ground-Water Data

by J.A. Johnson and L.J. Fong-Frydendal

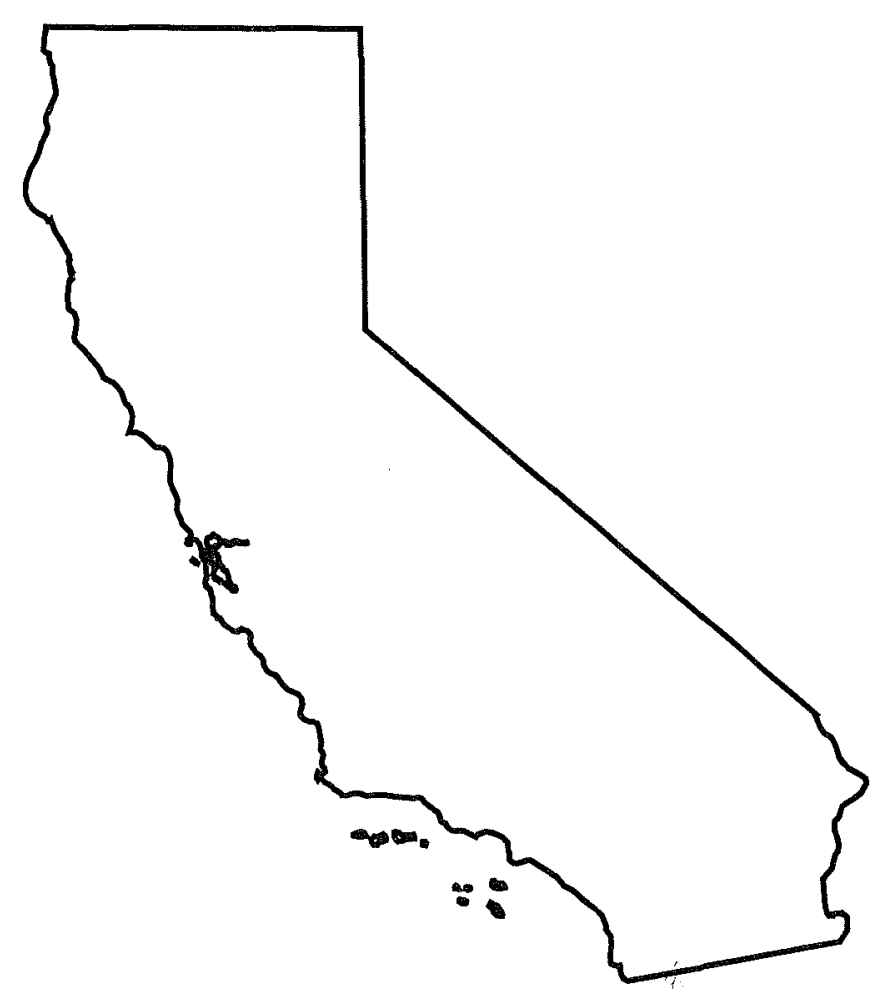

U.S. GEOLOGICAL SURVEY WATER-DATA REPORT CA-92-5

Prepared in cooperation with other Federal, State, and local agencies 


\section{U.S. DEPARTMENT OF THE INTERIOR}

BRUCE BABBITT, Secretary

\section{U.S. GEOLOGICAL SURVEY}

Dallas L. Peck, Director

For information on the water program in California write to

District Chief, Water Resources Division

U.S. Geological Survey

Federal Building, Room W-2233

2800 Cottage Way

Sacramento, CA 95825 


\section{PREFACE}

This volume of the annual hydrologic data report of California is one of a series of annual reports that document hydrologic data gathered from the U.S. Geological Survey's surface-and ground-water data-collection networks in each State, Puerto Rico, and the Trust Territories. These records of streamflow, ground-water levels, and quality of water provide the hydrologic information needed by State, local, and Federal agencies, and the private sector for developing and managing our Nation's land and water resources. Hydrologic data for California are contained in five volumes:

Volume 1. Southern Great Basin from Mexican Border to Mono Lake Basin and Pacific Slope Basins from the Tijuana River to Santa Maria River

Volume 2. Pacific Slope Basins from Arroyo Grande to Oregon State Line except Central Valley

Volume 3. Southern Central Valley Basins and The Great Basin from Walker River to Truckee River

Volume 4. Northern Central Valley Basins and The Great Basin from Honey Lake Basin to Oregon State Line

Volume 5. Ground-water data

This report is the culmination of a concerted effort by dedicated personnel of the U.S. Geological Survey who collected, compiled, analyzed, verified, and organized the data. In addition to the authors, who had primary responsibility for assuring that the information contained herein is accurate, complete, and adheres to U.S. Geological Survey policy and established guidelines, the individuals contributing significantly to the collection, processing, and tabulation of the data are given on page $V$.

This report was prepared in cooperation with the California Department of Water Resources and with other agencies, under the general supervision of John M. Klein, District Chief, California. 
4. THle and Subtitl.

Water Resources Data--California, Water Year 1992, Volume 5 Ground-Water Data

7. Author(s)

J.A. Johnson and L.J. Fong-Frydendal

9. Perfomine Oreanization Namo and Address

U.S. Geological Survey, Water Resources Division

California District

2800 Cottage Way, Room W-2233

Sacramento, CA 95825

12. Sponsoring Organization Name and Addross

U.S. Geological Survey, Water Resources Division

California District

2800 Cottage Way, Room W-2233

Sacramento, CA 95825

15. Supplementary Notes

Prepared in cooperation with other Federal, State, and 1ocal agencies.

\section{Abstract (Limit: 200 words)}

Water resources data for the 1992 water year for California consist of records of stage, discharge, and water quality of streams; stage and contents in lakes and reservoirs; and water levels and water quality in wells. This volume of the report series includes records on ground water in California. Additional ground-water information for California is contained in the files, data bases, and other published reports of the U.S. Geological Survey.

17. Document Analyzis a. Descriptors

*California, *Hydrologic data, $*$ Ground water, Chemical analyses, Water temperature, Sampling sites, Water levels, Water analyses

\section{b. Identifiers/Open.Ended Terms}

\section{c. CosAtI Fleld/Group}

12. Avellablity stateman: No restriction on distribution. This report may be purchased from National Technical Information Service Springfield, VA 22161

\begin{tabular}{|c|c|}
\hline $\begin{array}{l}\text { 86. Sacurty Cless (This Report) } \\
\text { Unclassified }\end{array}$ & $\begin{array}{l}\text { 21. No. of Pagers } \\
446\end{array}$ \\
\hline $\begin{array}{l}\text { 20. Socurty clags (Th/s Pagel } \\
\text { Unclassified }\end{array}$ & 22. Price \\
\hline
\end{tabular}


Anthony Buono, Assistant District Chief, South Steven J. Deverel, Assistant District Chief, North Kenneth W. Lee, Chief, Northern California Operations James C. Bowers, Chief, Southern California Operations

Jeffrey Agajanian, Hydrologic Technician

Steven W. Anderson, Supervisory Hydrologic Technician

Wende11 W. Ayers, Hydrologic Technician John S. Bader, Hydrologist

Brian S. Baharie, Hydrologic Technician

James B. Baker, Lead Editorial Assistant

Robin E. Bishop, Hydrologio Technician Daniel J, Bright, Hydrologist

Paul A. Buchanan, Hydrologic Technician

Louis A. Caldwell, Hydrologic Technician

Frank A. Carson, Hydrologio Technician Ann Thomas Chalmers, Chemist

Dennis A. Clark, Hydrologic Technician

Dwight D. Copeland, Hydrologic Technician

Michael J, De Grand, Hydrologic Technician Carol L. Donovan, Editor

Christopher D, Farrar, Hydrologist

Ronald G. Fay, Hydrologic Technician John R. Freckleton, Hydrologist

Lawrence A. Freeman, Hydrolog1c Technician

Michael F. Friebel, Lead Hydrologic Technician

Joseph R. Grant, Hydrologic Technician Scott N. Hamlin, Hydrologist

Paul D. Hayes, Supervisory Hydrologic Technician

Stuart A. Hill, Hydrologic Technician

Eugene B. Hoffman, Supervisory Hydrologic Technician Ernest. R, Houston, Hydrologic Technician

James F. Howle, Hydrologic Technician Francis A. Huber, Hydrologist Marti E. Ikehara, Hydrologist

Mark V. Johnson, Hydrologic Technician Charles A. Kaehler, Hydrologist

Willie B. Kinsey, Hydrologic Technician

Daniel S. Kogut, Hydrologic Technician

Michael L. Kuster, Hydrologic Technician Floyd E. Lee, Hydrologic Technician Peter Martin, Hydrologist

Gary A. Maxwell, Hydrologic Technician

Harold J. MeIntyre, Hydrologic Technician Gregory O. Mendez, Hydrologist

Lynn K. Millex, Hydrologic Technician

James R. Mullen, Supervisory Hydrologist

Clinton D. Nagel, Hydrolog 10 Technician

Nicole A. Norkoski, Hydrologic Technictan

Jessica L. Ollerton, Hydrologic Technician

Christine S, O'Neil, Hydrologic Technician

John R. Palmer, Supervisory Hydrologic Technician

Carlyle T, Peck, Hydrologic Technician

Loe A. Price, Hydrologic Technician Diane L. Rewis, Hydrologist

Gerald L. Rockwel1, Hydrologic Technician

Carol J. Simons, Office Automation Clerk

Michael Smith, Hydrologic Technician

David M. Sparks, Hydrologic Technician

Kathleen L, St.Clair, Hydrologic Technician

Kimball L, Stumpf, Hydrologic Technician

Gregory F. Susich, Hydrologic Technician Gloria Valdez, Clerk-Typist

Michael D. Webster, Lead Hydrologic Technician

Mark H. Woloszyk, Hydrologic Technician

George S, Yamamoto, Scientific Illustrator

Ferris F. Young, Hydrologic Technician

Ronald P. Fogelman, Supervisory Hydrologist Rick T. Iwatsubo, Biologist

Charles E, Lamb, Hydrologic Technician Robert W. Meyer, Hydrologist 



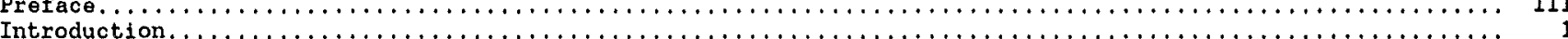

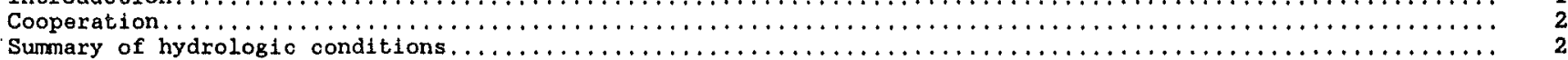

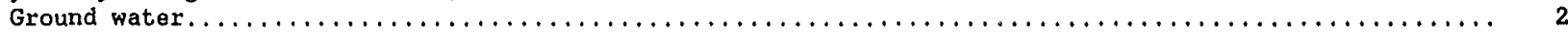

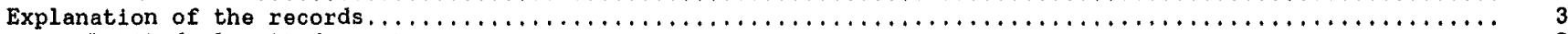

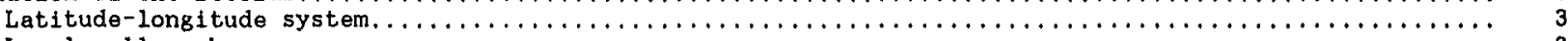

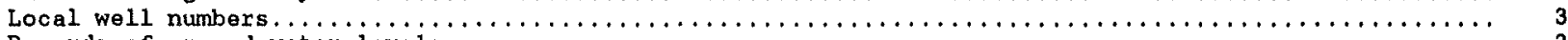

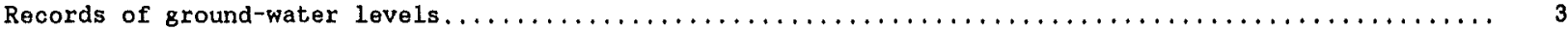

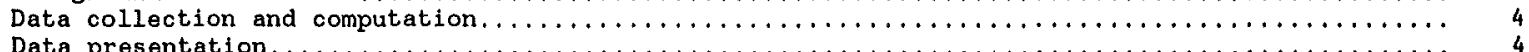

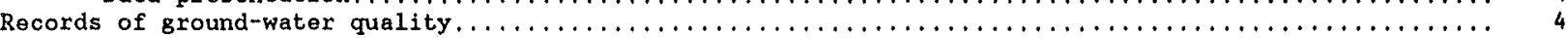

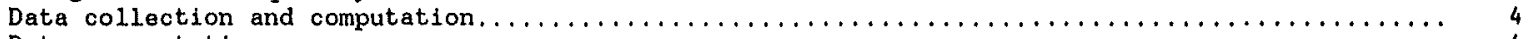

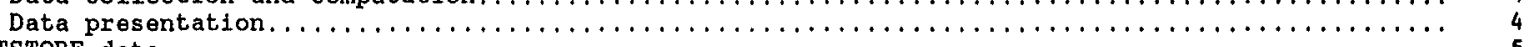

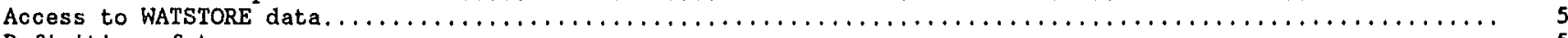

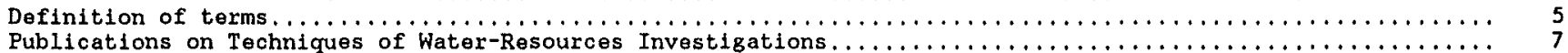

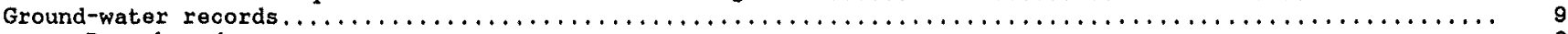

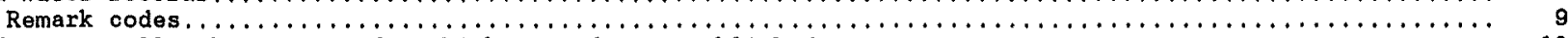

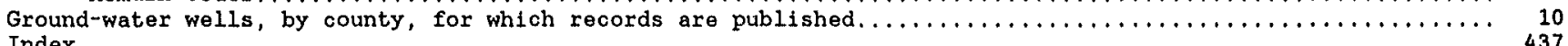

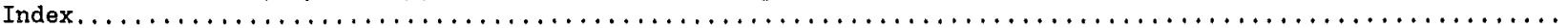

\section{ILLUSTRATIONS}

Figures 1,2. Diagrams showing:

Page

1. System for numbering wells and miscellaneous sites (latitude and longitude)....... 3

2. California well-numbering system $\ldots \ldots \ldots \ldots \ldots \ldots \ldots$

3-6. Index maps showing location of wells:

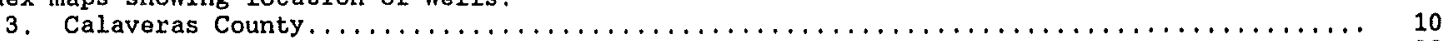

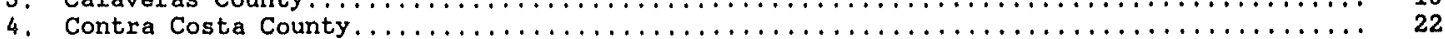

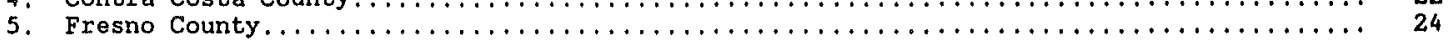

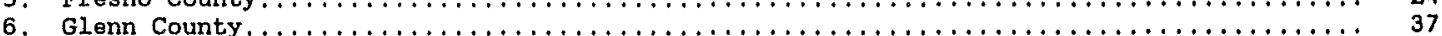

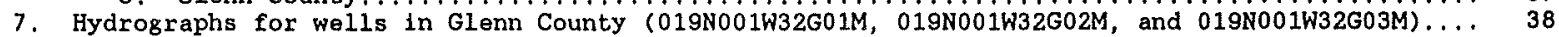

8-10. Index maps showing location of wells:

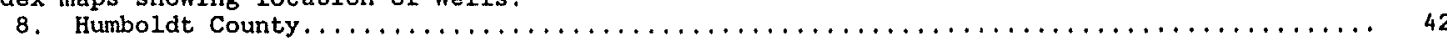

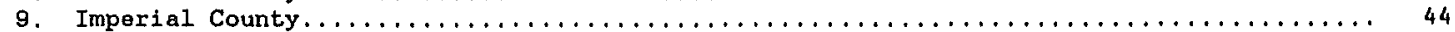

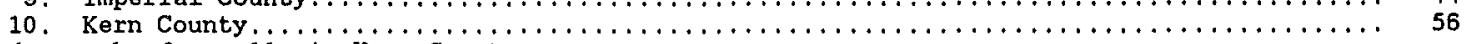

11, 12. Hydrographs for weils in Kern County:

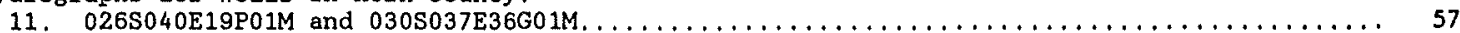

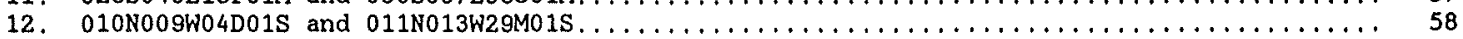

13,14. Index maps showing location of wells:

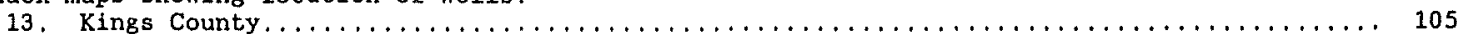

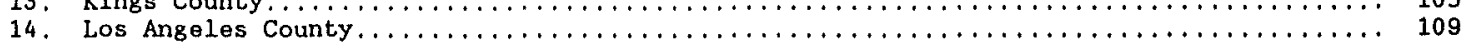

15, 16. Hydrographs for wells in Los Angeles County:

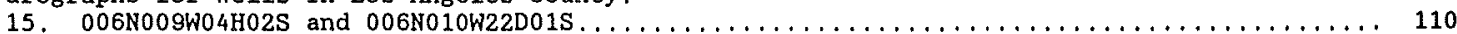

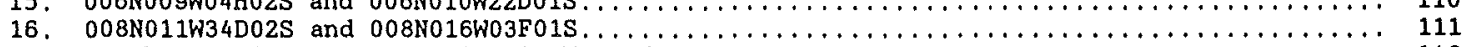

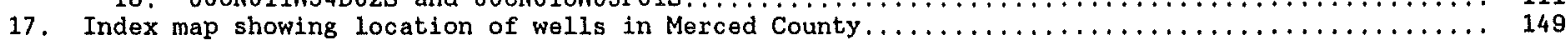

18. Hydrographs for wells in Merced County (006\$011E06L01M and 0095015E29F01M) ............. 150

19-22. Index maps showing location of wells:

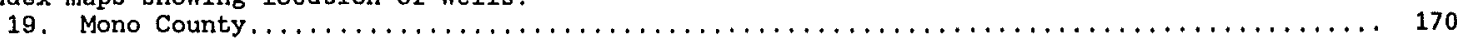

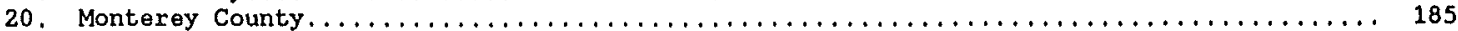

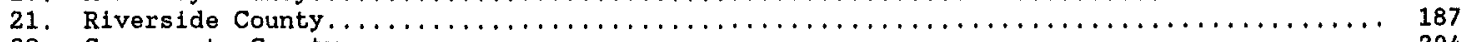

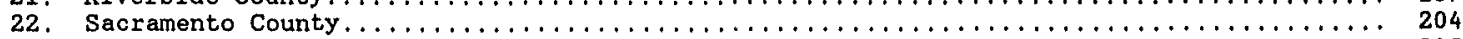

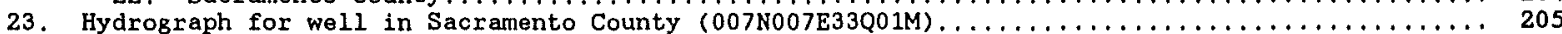

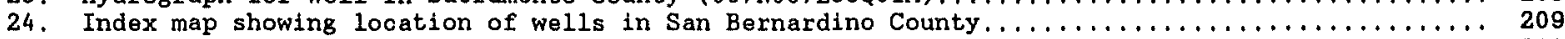

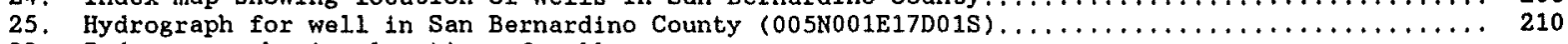

26-32. Index maps showing location of wells:

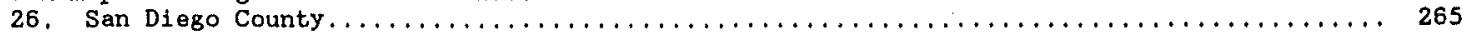

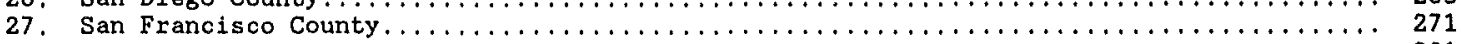

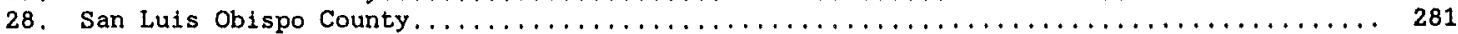

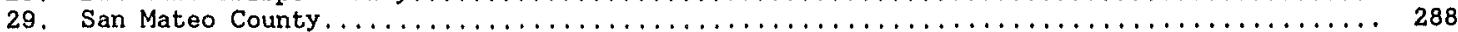

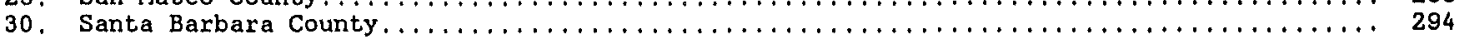

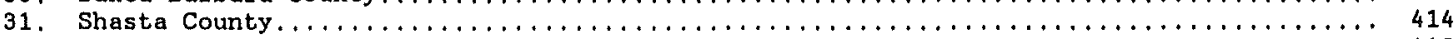

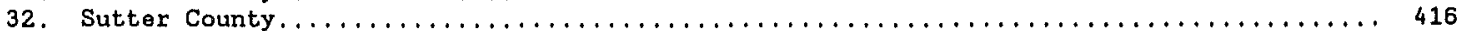

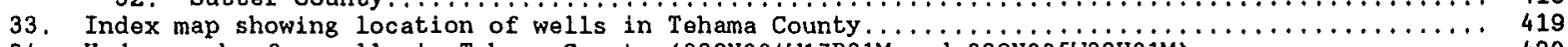

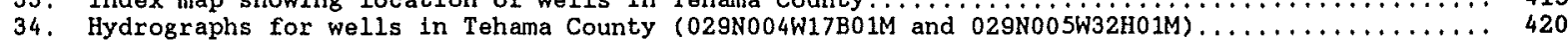

35-37. Index maps showing location of wells:

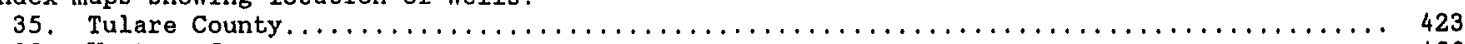

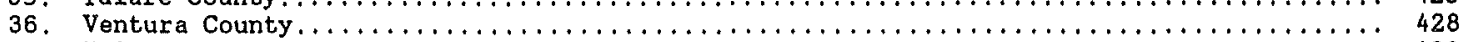

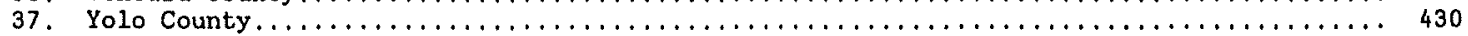

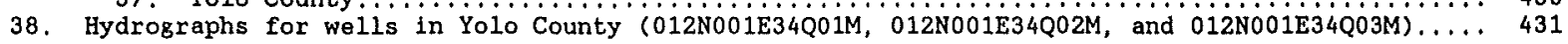


WATER RESOURCES DATA--CALIFORNIA, WATER YEAR 1992

VOLUME 5--GROUND-WATER DATA

By J.A. Johnson and L.J. Fong-Frydendal

\section{INTRODUCTION}

The Water Resources Division of the U.S. Geological Survey, in cooperation with State agencies, obtains a large amount of data pertaining to the water resources of California each water year. These data, accumulated during many water years, constitute a valuable data base for developing an improved understanding of the water resources of the State. To make these data readily available to interested parties outside the U.S. Geological Survey, the data are published annually in this report series entitled "Water Resources Data for California."

This volume of the report series includes records on ground water in California. Additional ground-water information for California is contained in the files, data bases, and other published reports of the U.S. Geological Survey.

The series of annual reports for California began with the 1961 water year with a report that contained only data relating to the quantities of surface water. For the 1964 water year, a similar report was introduced that contained only data relating to water quality. Beginning with the 1975 water year, the report format changed to include data on quantities of surface water, quality of surface and ground water, and ground-water levels. Beginning with the 1985 water year, a separate volume for ground-water levels and quality was published for California.

Prior to introduction of this series and for several water years concurrent with it, water-resources data for California were published in U.S. Geological Survey Water-Supply Papers. Data on stream discharge and stage and on lake or reservoir contents and stage, through September 1960, were published annually under the title "SurfaceWater Supply of the United States, Parts 10 and 11." For the 1961 through 1970 water years, the data were published in two 5-year reports. Data on chemical quality, temperature, and suspended sediment for the 1941 through 1970 water years were published annually under the title "Quality of Surface Waters of the United States," and water levels for the 1935 through 1974 water years were published under the title "Ground-Water Levels in the United States." These Water-Supply Papers may be consulted in public libraries of the principal cities of the United States and may be purchased from U.S. Geological Survey, Books and Open-File Reports Section, Federal Center, Box 25425, Denver, CO 80225 .

Publications similar to this report are published annually by the U.S. Geological Survey for all States. Each report has an identification number consisting of the two-letter state abbreviation, the last two digits of the weter year, and the volume number. For example, this volume is identified as "U.S. Geological Survey Water-Data Report CA-92-5." For archiving and general distribution, the reports for 1971-74 water years also are 1dentified as water-data reports. These water-data reports are for sale in paper copy or in microfiche by the National. Technical Information Service, U,S, Department of Commerce, Springfield, VA 22161. Beginning with the 1990 water year, all water-data reports also will be available on Compact Disc - Read Only Memory (CD-RoM). All data reports published for the current water year for the entire Nation, including Puerto Rico and the Trust Territories, will be reproduced on a single CD-ROM disc.

Additional information, including current prices, for ordering specific reports may be obtained from the District Office at the address given on the back of the title page or by telephone (916) $978-4668$. A limited number of CD-ROM discs will be available for purchase from U.S. Geological Survey, Books and Open-File Reports Section, Federal Center, Box 25425, Denver, Co 80225. 


\section{COOPERATION}

The U.S. Geological Survey and organizations of the State of California have had cooperative agreements for the systematic collection of water-resource records since 1903. Organizations that provided data are acknowledged in the well descriptions. Organizations that assisted through cooperative agreement with the Survey are:

Adelanto, City of, Jack E. Stonesifer, Superintendent, Water Department

Antelope Valley-East Kern Water Agency, Wallace G. Spinarski, General Manager

California Department of Water Resources, David N, Kennedy, Director

Calleguas Munlcipal Water District, Donald R. Kendall, General Manager

Carpinteria County Water District, Robert R, Lieberknecht, General Manager-Secretary

Channel Islands Beach Community Services District, Gerard W. Kapuscik, General Manager

Coachella Valley Water District, Thomas E. Levy, General Manager

Desert Water Agency, Jack H. Oberle, General Manager

East Bay Municipal Water District, Walter Bishop, Manager, Wastewater Division

Imperial County Department of Public Works, S. Harry Orfanos, Director

Indian Wells Valley Water District, Warren F. McGowan, General Manager

Merced, City of, Stevan M. Stroud, City Engineer

Mojave Water Agency, Larry W. Rowe, General Manager

Mono County Energy Management Department, Daniel L. Lyster, Director

Palmdale, City of, Steve Williams, Director, Public Works Department

Pechanga Indian Reservation, Jennie Miranda, Spokeswoman

San Bernardino Valley Municipal Water District, G. Louis Fletcher, General Manager

San Francisco Water Department, James D. Cooney, General Manager

Santa Barbara, City of, David H. Johnson, Director

Santa Barbara County Water Agency, Robert Almy, Manager

Santa Ynez River Water Conservation District, Joseph Foss, President

Assistance also was provided by the following Federal agencies:

U.S. Department of the Air Force, Edwards Air Force Base

U.S. Department of the Air Force, Vandenberg Air Force Base

U.S. Department of the Interior, Bureau of Indian Affairs

U.S. Department of the Interior, Bureau of Reclamation

U.S. Department of the Navy, Marine Corps Air Ground Combat Center, Twentynine Palms

U.S. Department of the Navy, Naval Weapons Center, China Lake

SUMMARY OF HYDROLOGIC CONDITIONS

\section{Ground Water}

The geography and geology of California are sufficiently complex that a single summary of ground-water conditions in the State is difficult. Descriptions of conditions in specific basins and valieys apply only to those areas and cannot be transferred to other areas.

Ground-water levels fluctuate in response to a variety of stresses and changes in stress. Short- and longterm climatic conditions can lead to changes in natural recharge and discharge. Superimposed on the natural fluctuations in water levels are changes caused by increasing or decreasing ground-water withdrawals and, in some areas, changes caused by irrigation and other sources of recharge.

Precipitation in California was below normal during the 1992 water year, extending drought conditions into the sixth consecutive year (1987-92). Five of the last six years were classified as "critically dry" by the Callfornia Department of Water Resources. Ground-water levels continued to decline in many areas of the State and historical lows were measured or expected as a result of the drought.

Hydrographs of two well clusters in the Sacramento Valley near the towns of Butte City in Glenn County (fig. 7) and Zamora in Yolo County (fig. 38) show typical seasonal fluctuations. Continuing drought conditions caused new historical low water levels in all three wells at the Butte City cluster-well site. The water level in the deep well reached a new low at the end of September 1992, exceeding the previous low in 1991 by $2.8 \mathrm{ft}$. Water: levels in the mid- and shallow-depth wells exceeded lows recorded in 1991 by 3.1 and $3.4 \mathrm{ft}$, respectively. The water level in the Zamora shallow well reached a new low of $20.08 \mathrm{ft}$, surpassing the 1979 historical low by $0.78 \mathrm{ft}$. The water level in the mid-depth well at this site declined 2.8 ft in the past year and was within $0.7 \mathrm{ft}$ of the historical low reached in 1979.

Water levels in well 007N007E33001M in Sacramento County (fig. 23) indicate seasonal fluctuations of about 5 to $6 \mathrm{ft} / \mathrm{yr}$; these fluctuations are caused by agricultural pumping and recharge. Since 1987, the average water level has declined about $10 \mathrm{ft}$ in response to the drought.

In Indiar Wells Valley in Kern County (fig. 11), the water level in well 026S040E19P01M declined 1.9 ft during water year 1992 reaching an historical low of 198.09 ft on September 29. Since 1946, the total water-level decline of this well was $62.2 \mathrm{ft}$.

The water level in well 005N001E17D01S in San Bernardino County (fig. 25) rose dramatically in response to the 7.6 magnitude Landers earthquake. This well is in Lucerne Valley, about 32 miles west northwest of the earthquake epicenter. On June 28,1992 , at $5 \mathrm{a}$. in., just minutes following the earthquake, the recorded water level was $10.26 \mathrm{ft}$ higher than the water level recorded 1 hour earlier. During the next 5 days, the water level recovered to within about $0.3 \mathrm{ft}$ of the pre-earthquake level. Since 1954, the water level in this well has declined nearly $100 \mathrm{ft}$ at an average rate of $2.5 \mathrm{ft} / \mathrm{yr}$. Continuous recording, begun in 1989, shows typical seasonal fluctuations and annual declines remain at $2.5 \mathrm{ft} / \mathrm{yr}$, despite the regional drought. 
EXPLANATION OF THE RECORDS

The ground-water records published in this report are for the 1992 water year that began October 1 , 1991, and ended September 30, 1992. A calendar of the water year is provided on the inside of the front cover. The records contain ground-water levels and quality of ground-water data. The following sections of the introductory text are presented to provide users with a more detailed explanation of how the hydrologic data published in this report were collected, analyzed, computed, and arranged for presentation.

\section{Latitude-Longitude System}

The identification numbers for wells are assigned according to the grid system of latitude and longitude (fig. 1). The number consists of 15 digits. The first six digits denote the degrees, minutes, and seconds of latitude, the next seven digits denote degrees, minutes, and seconds of longitude, and the last two digits (assigned sequentially) identify the wells or other sites within a 1-second grid. This site-identification number, once assigned, is a pure number, and has no locational significance. In the rare instance where the initial determination of latitude and longitude are in error, the station will retain its initial identification number.

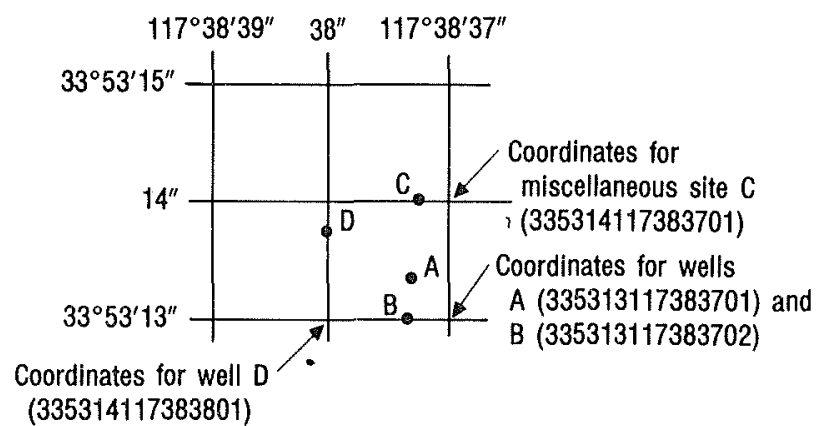

Figure 1. System for numbering wells and miscellaneous sites (latitude and longitude).

\section{Local Well Numbers}

Wells and springs in California are assigned numbers according to their location on the grid system for the subdivision of public land ( $\mathrm{fig}, 2$ ). For example, in the number 005S012E22P01M, the first four characters indicate the township (T.5 S,), and the next four characters indicate the range (R.12 E.); the digits following the range indicate the section (sec. 22); the letter following the section number indicates the 40-acre subdivision of the section. Within each 40-acre subdivision, the wells are numbered serially, as indicated by the last two digits. Springs are numbered similarly to wells except that an $S$ is placed between the 40-acre subdivision letter and the final digit (005S012E22AS1M). The final letter indicates the base line and meridian designations as follows: $\mathrm{H}$, Humboldt; M, Mount Diablo; S, San Bernardino.

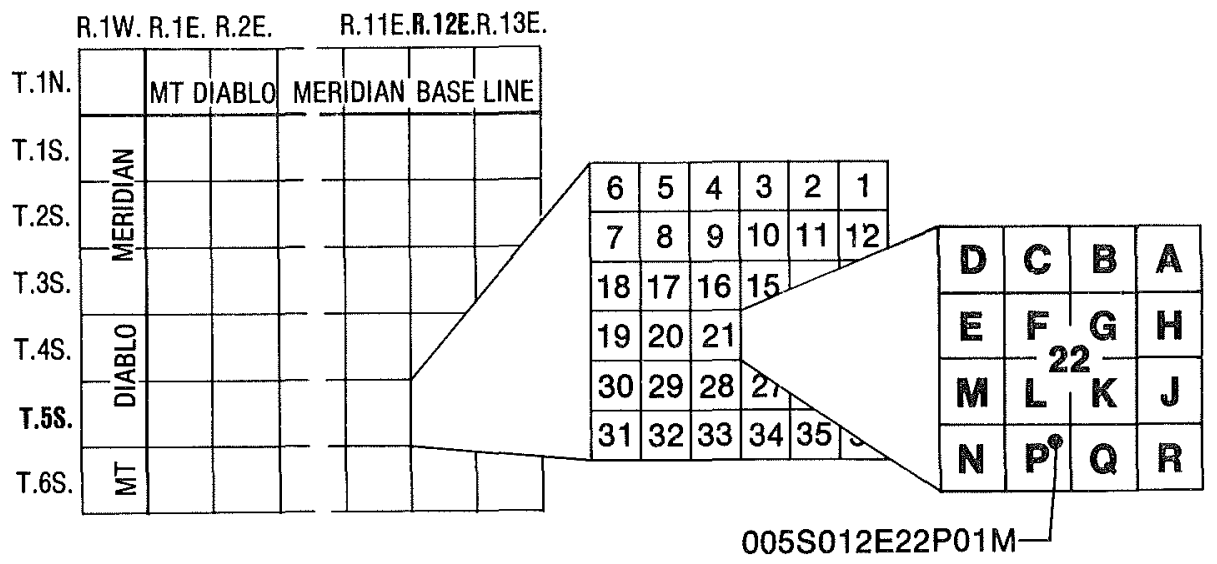

Figure 2. California woll-numbering system.

Records are obtained through cooperative efforts of many Federal, State, and local agencies for more than 1,000 observation wells throughout California and are placed in computer storage. Information about the availability of the data in the water-level file may be obtained from the District Chief, California District. (See address on back of title page.) 
Measurements of water levels are made in many types of wells under varying conditions, but the methods of measurement are standardized to the extent possible. The equipment and measuring techniques used at each observation well ensure that measurements at each well are of consistent accuracy and reliability.

Water-level records are obtained from direct measurements with a steel tape or electric sounder or from the graph, punched tape, or data logger of a water-stage recorder. The water-level measurements in this report are given in feet with reference to land-surface datum. Land-surface datum is a datum plane that is approximately at land surface at each well. The elevation of the land-surface datum is given in the well description.

Data Presentation

Water levels are reported to as many significant figures as can be justified by local conditions. For example, in a measurement of a depth to water of several hundred feet, the error may be a few tenths of a foot, whereas the error in determining the net change of water level between successive measurements may be only a hundredth or a few hundredths of a foot. For lesser depths to water, the accuracy is 8 reater. Accordingly, most measurements are reported to hundredth of a foot, but some are given to a tenth of a foot or a larger unt.

Tables of water-level data are presented by counties arranged in alphabetical order. A table of water levels follows the station description of each well. Water levels are reported in feet below land-surface datum, and all measurements of water level for the water year are listed. The highest and lowest static water levels of record and their dates of occurrence are shown below the data table. Missing records on daily tables are indicated by dashes in place of the water level.

Hydrographs are presented for selected wells in Glenn, Kern, Los Angeles, Merced, Sacramento, San Bernardino, Tehama, and Yolo Counties. Periods of missing record are shown as a straight line between measurements.

\section{Records of Ground-Water Quality}

Records of ground-water quality in this report differ from other types of records; for most sampling sites they consist of only one set of measurements for the water year. The quality of ground water ordinarily changes slowly; therefore, for most general purposes one annual sampling, or only a few samples taken at infrequent intervals during the year, is suffictent. Frequent measurement of the same constituents is not necessary unless there is concern with a particular problem, such as monitoring for trends in nitrate concentration. In special cases where the quality of ground water may change more rapidly, more frequent measurements are made to 1dentify the nature of the changes.

Traditionally, dissolved trace-element concentrations have been reported at the microgram per liter ( $\mu g / L$ ) level. Recent evidence, mostly from large rivers, indicates that actual dissolved-phase concentrations for a number of trace elements are within the range of 10's to 100's of nanograms per liter (ng/L). Present data above the $\mu \mathrm{g} / \mathrm{L}$ level should be viewed with caution. Such data may actually represent elevated environmental concentrations from natural or human causes; however, these data could reflect contamination introduced during sampling, processing, or analysis. T'o confidently produce dissolved trace-element data with insignificant contamination, the U.S. Geological Survey will begin using new trace-element protocols in the near future.

\section{Data Collection and Computation}

The records of ground-water quality in this report were obtained mostly as a part of special studies in specific areas. Consequently, a number of chemical analyses may be presented for some counties but none are presented for others. As a result, the records for a year, by themselves, do not provide a balanced view of ground-water quality statewide.

Most methods for collecting and analyzing water samples are described in the "U.S. Geological Survey Techniques of Water-Resources Investigations" manuals listed in this report. The values reported in this report represent water-quality conditions at the time of sampling as much as possible, consistent with available sampling techniques and methods of analysis. All samples were obtained by trained personnel. The wells sampled were pumped long enough to ensure that the water collected came directly from the aquifer.

\section{Data Presentation}

The records of ground-water quality are published in a section titled WATER QUALITY immediately following the ground-water-level records. Data for quality of ground water are listed alphabetically by county and are identified by well number. No descriptive statements are given for ground-water-quality records; however, the well number, date of sampling, and other pertinent data are given in the table containing the chemical analyses of the ground water.

Values for dissolved nitrite plus nitrate $\left(\mathrm{NO}_{2}+\mathrm{NO}_{3}\right.$, as $\mathrm{N}$ ) are not included in the calculation for dissolved solids, sum of constituents. 


\title{
ACCESS TO WATSTORE DATA
}

The U.S. Geological Survey is the principal Federal water-data agency and, as such, collects and disseminates about 70 percent of the water data currently being used by numerous State, local, private, and other Federal agencies to develop and manage our water resources. As part of the Geological Survey's program of releasing water data to the public, a large-scale computerized system has been developed for the storage and retrieval of water data collected through its activities. The National Water Data Storage and Retrieval System (WATSTORE) was established in 1972 to provide an effective and efficient means for the processing and maintenance of water data collected through the activities of the U.S. Geological Survey and to facilitate release of the data to the public. A variety of useful products ranging from data tables to complex statistical analyses, such as Log Pearson Type III, can be produced using WATSTORE. The system resides on the/central computer facilities of the U.S. Geological Survey at its National Center in Reston, Virginia, and consists of related files and data bases.

* Station Header File - Contains descriptive information on more than 440,000 sites throughout the United States and its territories where the U.S. Gological Survey collects or has collected data.

* Daily Values File - Contains more than 220 million daily values of streamflows, stages, reservoir contents, water temperatures, specific conductances, sediment concentrations, sediment discharges, and ground-water levels.

* Peak Flow File - Contains approximately 500,000 maximum (peak) streamflow and gage-height values at surface-water sites.

* Water Quality File - Contains approximately 2 million analyses of water samples that describe the chemical, physical, biological, and radio-chemical characteristics of both surface and ground water.

* Ground-Water Site Inventory Data Base - Contains inventory data for more than 900,000 wells, springs, and other sources of ground water. The data includes site location, geohydrologic characteristics, current well information and construction history.

In 1976, the U.S. Geological Survey opened WATSTORE to the public for direct access. The signing of a Memorandum of Agreement with the Survey is required to obtain direct access to WATSTORE. The system can be accessed either synchronously or asynchronously. The requestor will be expected to pay all computer costs he/she incurs. Direct access may be obtained by contacting:

\author{
U.S. Geological Survey \\ National Water Data Exchange \\ 421 USGS National Center \\ Reston, VA 22092
}

In addition to providing direct access to WATSTORE, data can be provided in various machine-readable formats on magnetic tape, $51 / 4$ or $31 / 2$-inch floppy disk and, as noted in the introduction, on CD-ROM discs. Beginning with the 1990 water year, all water-data reports will also be available on Compact Disc - Read Only Memory (CDROM). All data reports published for the current water year for the entire Nation, including Puerto Rico and the Trust Territories, will be reporduced on a single CD-ROM disc. Information about the availability of specific types of data or products, and user charges, can be obtained locally from each of the Water Resources Division's District offices. (See address on the back of the title page, A limited number of CD-ROM discs will be available for sale by the Books and Open-File Reports Section, U.S. Geological Survey, Federal Center, Box 25425, Denver, Colorado 80225 .

\section{DEFINITION OF TERMS}

Terms related to water-quality and other hydrologic data as used in this report are defined below. A table for converting inch-pound units to International System (SI) Units is printed on the inside of the back cover.

Aquifer is a geologic formation, group of formations, or part of a formation that contains sufficient saturated permeable material to yield significant quantities of water to wells and springs.

Artesian means confined and is used to describe a well in which the water level stands above the top of the aquifer tapped by a well. A flowing artesian well is one in which the water level is above the land surface.

Chemical oxygen demand (COD) is a measure of the chemically oxidizable material in the water and furnishes an approximation of the amount of organic and reducing material present. The determined value may correlate with natural water color or with carbonaceous organic pollution from sewage or industrial wastes.

Color unit is produced by 1 milligram per liter of platinum in the form of the chloroplatinate ion. Color is expressed in units of the platinum-cobalt scale.

Dissolved is that material in a representative water sample which passes through a 0.45 -micrometer membrane filter. This is a convenlent operational definition used by Federal agencies that collect water data. Determinations of "dissolved" constituents are made on subsamples of the filtrate. It is recognized that certain kinds of samples cannot be filtered; to provide for this, procedures that are considered equivalent to filtering through a 0.45 -micrometer membrane filter will be identified and announced at a later date.

Dissolved-solids concentration of water is determined either analytically by the "residue-on-evaporation" method, or mathematically by totaling the concentrations of individual constituents reported in a comprehensive chemical analysis. During the analytical determination of dissolved solids, the bicarbonate (generaliy a major dissolved component of water) is converted to carbonate. Therefore, in the mathematical calculation of dissolvedsolids concentration, the bicarbonate value, in milligrams per liter, is multiplied by 0.492 to reflect the change. 
Hardness of water is a physical-chemical characteristic that is commonly recognized by the increased quantity of soap that is required to produce lather. It is attributable to the presence of alkaline earths (principally calcium and magnesium) and is expressed as equivalent calcium carbonate $\left(\mathrm{CaCO}_{3}\right)$. well.

Land-surface datum is a datum plane that is approximately at land surface at each ground-water observation

Methylene blue active substance (MBAS) is a measure of apparent detergents. This determination depends on the formation of a blue color when methylene blue dye reacts with synthetic detergent compounds.

Micrograms per liter (UG/L, $\mu \mathrm{g} / \mathrm{L}$ ) is a unit expressing the concentration of chemical constituents in solution as mass (micrograms) of solute per unit volume (liter) of water. One thousand micrograms per liter is equivalent to 1 milligram pex liter.

Milligrams per liter (MG/L, $\mathrm{mg} / \mathrm{L}$ ) is a unit expressing the concentration of chemical constituents in solution as mass (milligrams) of solute per unit volume (liter) of water. Concentration of suspended sediment also is expressed in miliframs per liter and is based on the mass of sediment per liter of water-sediment mixture.

Nanograms per liter (NG/L, ng/L) is a unit expressing the concentration of chemical constituents in solution as mass (nanograms) of solute per unit volume (liter) of water. One million nanograms per liter is equivalent to 1 militgram per liter.

Pesticides are chemical compounds used to control undesirable organisms. Major categories of pesticides include insecticides, miticides, fungicides, herbicides, and rodenticides. Insecticides and herbicides, which control insects and plants respectively, are the two categories reported.

$\mathrm{pH}$ of water is the negative logarithm of the hydrogen-ion activity. Solutions with pH less than 7 are termed "acidic," and solutions with a pH greater than 7 are termed "basic." Solutions with a pH of 7 are neutral. The presence and concentration of many dissolved chemical constituents found in water are, in part, influenced by the hydrogen-ion activity of water. Biological processes including growth, distribution of organisms, and toxicity of the water to organisms also are influenced, in part, by the hydrogen-ion activity of water.

Polychlorinated biphenyls (PCBs) are industrial chemicals that are mixtures of chlorinated biphenyl compounds having various percentages of chlorine. They are similar in structure to organochlorine insecticides.

Sodium-adsorption-ratio (SAR) is the expression of relative activity of sodium lons in exchange reactions with soll and is an index of sodium or alkali hazard to the soil. Waters range in respect to sodium hazard from those which can be used for irrigation on almost all soils to those which are generally unsatisfactory for irrigation.

Specific conductance is a measure of the ability of water to conduct an electrical current. It is expressed in microsiemens per centimeter at $25^{\circ} \mathrm{C}$. Specific conductance is related to the type and concentration of ions in solution and can be used for approximating the dissolved-solids concentration in water. Commonly, the concentration of dissolved solids (in milligrams per 1iter) is about 65 percent of the specific conductance (in microsiemens). This relation is not constant from well to well, and it may vary with changes in the composition of the water.

Total, recoverable is the amount of a given constituent that is in solution after a representative watersuspended sediment sample has been digested by a method (usually using a dilute acid solution) that results in dissolution of only readily soluble substances. Complete dissolution of all particulate matter is not achieved by the digestion treatment; thus, the determination represents something less than the "total" amount (that is, less than 95 percent) of the constituent present in the dissolved and suspended phases of the sample. To achieve comparability of analytical data, equivalent digestion procedures would be required of all laboratories performing such analyses, because different digestion procedures are likely to produce different analytical results.

Total is the total amount of a given constituent in a representative water-suspended sediment sample, regardless of the constituent's physical or chemical form. This term is used only when the analytical procedure assures measurement of at least 95 percent of the constituent present in the dissolved and suspended phases of the sample. A knowledge of the expected form is required to judge when the results should be reported as "total." (Note that the word "total" does double duty here, indicating both that the sample consists of a water-suspended sediment mixture and that the analytical method determines all the constituent in the sample.)

Water year in U.S. Geological Survey reports dealing with water supply is the 12-month period, October 1 through September 30. The water year is designated by the calendar year in which it ends and which includes 9 of the 12 months. Thus, the year ending September 30, 1992, is called the "1992 water year."

WDR is used as an abbreviation for "Water-Data Reports" referring to previously published State annual basicdata reports.

WSP is used as an abbreviation for "Water-Supply Paper" in reference to previously published reports. 
PUBLICATIONS ON TECHNIQUES OF WATER-RESOURCES INVESTIGATIONS

The U.S. Geological Survey publishes a series of manuals describing procedures for planning and conducting specialized work in water-resources investigations. The material is grouped under major subject headings called books and is further divided into sections and chapters, For example, Section B of Book 3 (Applications of Hydraulics) pertains to ground water. The chapter, the unit of publication, is limited to a narrow field of subject matter. This format permits flexibility in revision and publication as the need arises. Complete lists of these publications are located in the other four volumes in this series.

The reports listed below are for sale by the U.S. Geological Survey, Books and Open-File Reports Section, Federal Center, Box 25425, Denver, CO 80225 (authorized agent of the Superintendent of Documents, Government Printing office). Prepayment is required. Remittance should be sent by check or money order payable to U.S. Geological Survey, Department of the Interior. Prices are not included because they are subject to change. Current prices can be obtained by writing to the above address. When ordering or inquiring about prices for any of these publications, please give the title, book number, chapter number, and "U.S. Geological Survey Techniques of Water-Resources Investigations."

1-D1. Water temperature--influential factors, field measurement, and data presentation, by H.H. Stevens, Jr., J.F. Ficke, and G.F. Smoot: USGS--TWRI Book 1, Chapter D1. 1975. 65 pages.

1-D2. Guidelines for collection and field analysis of ground-water samples for selected unstable constituents, by W.W. Wood: USGS--TWRI Book 1, Chapter D2. 1976. 24 pages.

2-D1. Application of surface geophysics to ground-water investigations, by A.A.R. Zohdy, G.P. Eaton, and D.R. Mabey: USGS--TWRI Book 2, Chapter D1, 1974, 116 pages.

2-D2. Application of seismic-refraction techniques to hydrologic studies, by F.P. Haeni: USGS--TWRI Book 2, Chapter D2. 1988. 86 pages.

2-E1. Application of borehole geophysics to water-resources investigations, by W.S. Keys, and L.M. MacCary: USGS--TWRI Book 2, Chapter E1. 1971. 126 pages.

2-E2, Borehole geophysics applied to ground-water investigations, by W. Scott Keys: USGS--TWRI Book 2, Chapter E2. 1990, 150 pages.

2-F1. Application of drilling, coring, and sampling techniques to test holes and wells, by Eugene Shuter and Warren E. Teasdale: USGS--TWRI Book 2, Chapter F1. 1989, 97 pages.

3-B1. Aquifer-test design, observation, and data analysis, by R.W. Stallman: USGS--TWRI Book 3, Chapter B1. 1971. 26 pages.

3-B2. Introduction to ground-water hydraulics, a programmed text for self-instruction, by G.D. Bennett: USGS-TWRI Book 3, Chapter B2. 1976. 172 pages.

3-B3. Type curves for selected problems of flow to wells in confined aquifers, by J.E. Reed: USGS--TWRI Book 3, Chapter B3. 1980.106 pages.

3-B4. Regression modeling of ground-water flow, by R,L. Cooley and R.L. Naff: USGS--TWRI Book 3, Chapter B4, 1990. 232 pages (5 1/4-inch DS/DD diskette included).

3-B5. Definition of boundary and initial conditions in the analysis of saturated ground-water flow systems--An introduction, by O.L. Franke, T.E. Reilly, and G.D. Bennett: USGS--TWRI Book 3, Chapter B5. 1987. 15 pages.

3-B6. The principle of superposition and its application in ground-water hydraulics, by T.E. Reilly, O.L. Franke, and G.D. Bennett: USGS--TWRI Book 3, Chapter B6. 1987. 28 pages.

3-B7. Analytical solutions for one-, two-, and three-dimensional solute transport in ground-water systems with uniform flow, by Eliezer J. Wexler: USGS--TWRI Book 3, Chapter B7. 1992. 190 pages.

4-A1. Some statistical tools in hydrology, by H.C. Riggs: USGS--TWRI Book 4, Chapter A1. 1968.39 pages.

4-D1. Computation of rate and volume of stream depletion by wells, by C.T. Jenkins: USGS--TWRI Book 4, Chapter D1. 1970. 17 pages.

5-A1. Methods for determination of inorganic substances in water and fluvial sediments, edited by $M . J$. Fishman and L.C. Friedman: USGS--TWRI Book 5, Chapter A1, 1989. 545 pages.

5-A2. Determination of minor elements in water by emission spectroscopy, by P.R. Barnett and E.C. Mallory, Jr.: USGS--TWRI Book 5, Chapter A2. 1971. 31 pages.

5-A3. Methods for the determination of organic substances in water and fluvial sediments, edited by R.L. Wershaw, M.J. Fishman, R.R. Grabbe, and L.E. Lowe: USGS--TWRI Book 5, Chapter A3. 1987.80 pages.

5-A4. Methods for collection and analysis of aquatic biological and microbiological samples, edited by L.J. Britton and P.E. Greeson: USGS--TWRI Book 5, Chapter A4, 1989, 363 pages.

5-A5. Methods for determination of radioactive substances in water and fluvial sediments, by L.L. Thatcher, V.J. Janzer, and K.W. Edwards: USGS--TWRI Book 5, Chapter A5. 1977.95 pages. 
5-A6, Quality assurance practices for the chemical and biological analyses of water and fluvial sediments, by L.C. Friedman, and D.E. Erdmann: USGS--TWRI Book 5, Chapter A6. 1982. 181 pages.

6-A1. A modular three-dimensional finite-difference ground-water flow model, by M.G. McDonald and A.W. Harbaugh: USGS-TWRI Book 6, Chapter A1. 1988. [varlously paged].

6-A2. Documentation of a computer program to sinulate aquifer-system compaction using the modular finitedifference ground-water flow model, by S.A. Leake and D.E. Prudic: USGS--TWRI Book 6, Chapter A2. 1991. 68 pages.

7-C1. Finite difference model for aquifer simulation in two dimensions with results of numerical experiments, by P.C. Trescott, G.F. Pinder, and S.P. Larson: USGS--TWRI Book 7, Chapter C1. 1976.116 pages.

7-C2, Computer model of two-dimensional solute transport and dispersion in ground water, by L.F. Konikow and J.D. Bredehoeft: USGS--TWRI Book 7, Chapter C2. 1978. 90 pages.

8-A1. Methods of measuring water levels in deep wells, by M.S. Garber and F.C. Koopman: USGS--TWRI Book 8, Chapter A1. 1968. 23 pages. 
GROUND-WATER RECORDS

\section{$\underline{\text { Remark Codes }}$}

Two remark codes may follow water-level data under the headings "M" (method) and "S" (status). In this report the following remark codes may appear with the data:

\section{PRINTED OUTPUT "M"}

A
$H$
$M$
R
S
V
Z

PRINTED OUTPUT "S"

D
E
F
G
H
N
O
P
R
S

T
V
W
X
Z

\section{REMARK}

Airline

Calibrated pressure gage

Manometer

Reported

Steel tape

Calibrated electric tape

Other

\section{REMARK}

Dry

Recently flowing

Flowing

Nearby flowing

Nearby recently flowing

Measurement discontinued

Obstruction

Pumping

Recently pumped

Nearby pumping

Nearby recently pumped

Foreign substance

Well destroyed

Surface-water effects

Other

Remark codes for water-quality data are shown in each data table. 


\section{CALAVERAS COUNTY}

Page

LOCAL NUMBER 004N010E04G01M A INTERVAL ......... 11, 19, 21

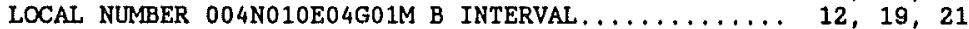

LOCAL NUMBER 004N010E04G02M.................. 12, 19, 21

LOCAL NUMBER 004 N010E04G03M. . . . . . . . . . . . . 13, 19, 21

LOCAI NUMBER 004N010E04G04M A INTERVAL.......... 13, 19, 21

LOCAL NUMBER 004N010E04G04M B INTERVAL . . . . . . 14, 19, 21

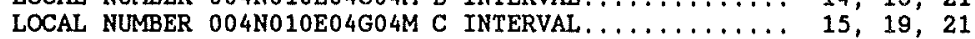

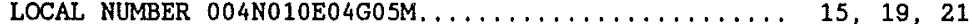

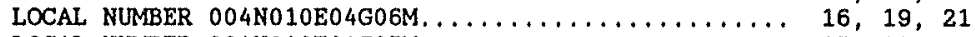

LOCAL NUMBER 004 N010E04G07M............... 17, 19, 21

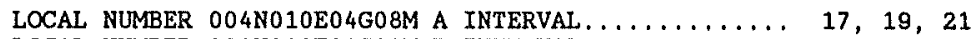

LOCAL NUMBER 004N010E04G08M B INTERVAL ............ 18, 19, 21

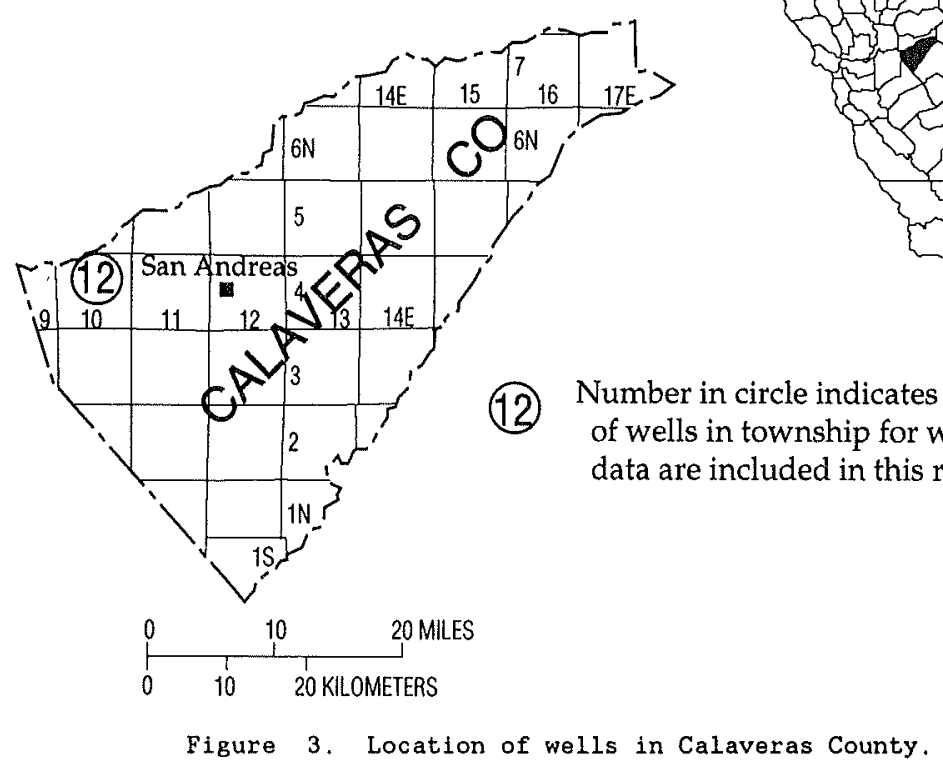


WATER LEVELS, CALAVERAS COUNTY

WELL DESCRIPTIONS AND WATER-LEVEL MEASUREMENTS

WATER YEAR OCTOBER 1991 TO SEPTEMBER 1992

SITE NUMBER 381355120524302

LOCAL NUMBER 004N010E04G01M A INTERVAL

About 1 mile northwest of Campo Seco. Observation well. Diameter 6 inches. Movable packer installed at 80 feet. Measurements represent water levels in A Interval, 0-80 feet. Altttude of land-surface datum 217 feet. Recorder installed May 1992. Water-level records available 1991 to current year.

WATER LEVELS IN FEET BELOW LAND-SURFACE DATUM (OBSERVATIONS AT 1200 HOURS)

\begin{tabular}{|c|c|c|c|c|c|c|c|c|c|c|c|c|}
\hline DAY & $\propto C T$ & NOV & DEC & JAN & FEB & MAR & APR & MAY & JUN & JUL & AUG & SEP \\
\hline 1 & -- & -- & -- & $\cdots$ & -- & -- & --- & -- & 24.28 & 24.34 & 24.42 & 24.76 \\
\hline 2 & -- & -- & -- & -- & $\cdots$ & --- & V22. 97 & --- & 24.29 & 24.22 & 24.43 & 24.77 \\
\hline 3 & -- & --- & -- & -- & -- & -- & -- & -- & 24.30 & 24.29 & 24.44 & 24.79 \\
\hline 4 & -- & -- & -- & $-\cdots$ & -- & V22.84 & --- & -- & 24.33 & 24,32 & 24.46 & 24.81 \\
\hline 5 & $\rightarrow-$ & --- & $-\infty$ & $\cdots$ & --- & -- & -- & --- & 24.34 & 24.32 & 24.48 & 24.82 \\
\hline 6 & --- & -- & -- & 522.70 & --- & -- & -- & --- & 24.33 & 24.32 & 24.48 & 24.84 \\
\hline 7 & --- & -- & -- & $\ldots$ & --- & -- & -- & V23.86 & 24.35 & 24.32 & 24.47 & 24.82 \\
\hline 8 & -- & -- & -- & $\cdots$ & --- & V21.79 & -- & 23.83 & 24.34 & 24.33 & 24.51 & 24.82 \\
\hline 9 & -- & -- & --- & -- & -- & $\mathrm{V} 22.00$ & $\cdots$ & 23.85 & 24.38 & 24.34 & 24.48 & 24.83 \\
\hline 10 & -- & -- & --- & $\cdots$ & -- & -- & $\cdots$ & 23.86 & 24.36 & 24.33 & 24.53 & 24.86 \\
\hline 11 & -- & -- & --- & --- & -- & --- & -- & 23.89 & 24.37 & 24.31 & 24.52 & 24.86 \\
\hline 12 & -- & --- & $\cdots$ & $\cdots$ & v22.19 & -- & -- & 23.96 & 24.35 & 24.36 & 24.57 & 24.88 \\
\hline 13 & -- & -- & -- & -- & $\cdots$ & -- & -- & 23.94 & 24.36 & 24.34 & 24.55 & 24.86 \\
\hline 14 & -- & -- & $\cdots$ & $-\cdots$ & -- & $\cdots$ & V23.29 & 23.96 & 24.33 & 24.33 & 24.59 & 24.86 \\
\hline 15 & --- & --- & -- & -- & -- & V22. 62 & V23.30 & 24.04 & 24.33 & 24.33 & 24.60 & 24.89 \\
\hline 16 & -- & -- & --- & -- & $\cdots$ & V22.72 & V23.40 & 24.06 & 24.37 & 24.34 & 24.60 & 24.89 \\
\hline 17 & $\cdots$ & $-\cdots$ & -- & -- & $\cdots$ & - & V24.36 & 24.11 & 24.32 & 24.34 & 24.62 & 24.90 \\
\hline 18 & --- & $\cdots$ & --- & -- & -. & V22.77 & -- & 24.11 & 24.32 & 24.34 & 24.61 & 24.94 \\
\hline 19 & $\cdots$ & -- & --- & -- & $\mathrm{V} 20.28$ & -- & $\cdots$ & 24.11 & 24.31 & 24.34 & 24.63 & 24.91 \\
\hline 20 & -- & -- & --- & --- & $-\cdot-$ & --- & -- & 24.13 & 24.31 & 24.35 & 24.65 & 24.92 \\
\hline 21 & -- & $\cdots$ & --- & --- &.- & --- & -- & 24.12 & 24.29 & 24.36 & 24.68 & 24.95 \\
\hline 22 & $\cdots$ & $\cdots$ & -- & \$23.69 & --- & -- & --- & 24.12 & 24.32 & 24.36 & 24.67 & 24.98 \\
\hline 23 & -- & -- & -- & - & -- & -- & -- & 24.14 & 24.31 & 24.33 & 24.69 & 24.97 \\
\hline 24 & --- & --- & $\mathrm{S} 24.15$ & -- & -- & V22. 86 & $\cdots$ & 24.18 & 24.28 & 24.36 & 24.71 & 25.00 \\
\hline 25 & --- & -- & -- & --- & $\cdots$ & --- & $\cdots$ & 24.17 & 24.29 & 24.39 & 24.71 & 25.00 \\
\hline 26 & $-\cdots$ & -- & -- & $=-$ & -- & -- & --- & 24.19 & 24.31 & 24.38 & 24.72 & 25.00 \\
\hline 27 & -- & $-\cdots$ & --- & --- & $\mathrm{V} 21.78$ & -- & -- & 24.19 & 24.26 & 24.39 & 24.75 & 24.99 \\
\hline 28 & -- & -- & -- & $-\cdots$ & --- & -- & $\cdots$ & 24.22 & 24.29 & 24,42 & 24.75 & 25.05 \\
\hline 29 & --- & $-m$ & --- & V23.90 & --- & -- & --- & 24.21 & 24.33 & 24.40 & 24.74 & 25.04 \\
\hline 30 & --- & -- & --- & -- & $\ldots$ & -- & -- & 24.25 & 24.37 & 24.42 & 24.75 & 25.02 \\
\hline 31 & -- & $\cdots$ & --- & -- & $\cdots$ & --- & --- & 24.26 & $\ldots$ & 24.42 & 24.77 & -- \\
\hline
\end{tabular}

PERIOD OF RECORD HIGHEST 20.28 FEB 19, 1992 LOWEST 25.05 SEP 28, 1992

S Manual steel tape measurement.

$\checkmark$ Manual calibrated electric tape measurement. 
WELL DESCRIPTIONS AND WATER-LEVEL MEASUREMENTS

WATER YEAR OCTOBER 1991 TO SEPTEMBER 1992

SITE NUMBER 381355120524303

LOCAL NUMBER 004N010E04G01M B INTERVAL

About 1 mile northwest of Campo Seco. Observation well. Diameter 2 inches. Movable packer installed at 80 feet. Measurements represent water levels in B Interval, 80-200 feet. Altitude of land-surface datum 217 feet. Recorder installed May 1992. Water-level records available 1991 to current year.

WATER LEVELS IN FEET BELOW LAND-SURFACE DATUM (OBSERVATIONS AT 1200 HOURS)

\begin{tabular}{|c|c|c|c|c|c|c|c|c|c|c|c|c|}
\hline DAY & $\mathrm{OCT}$ & NOV & $D E C$ & JAN & FEB & MAR & APR & MAY & JUN & JUL & AUG & SEP \\
\hline 1 & --- & --- & --- & --- & --- & --- & --- & --- & 9.94 & 8.34 & 7.74 & 6.85 \\
\hline 2 & --- & --- & --- & --- & --- & --- & v8. 35 & -- & 9.77 & 8.33 & 7.88 & 6.95 \\
\hline 3 & $-\infty$ & --- & --- & --- & $\mathrm{V} 10.24$ & --- & --- & --- & 9.68 & 8.21 & 7.89 & 6.98 \\
\hline 4 & $--\infty$ & --- & --- & --- & --- & $\mathrm{V} 10.14$ & --- & $--\infty$ & 9.55 & 8.19 & 7.89 & 7.02 \\
\hline 5 & -- & --- & -- & $m$ & $-\cdots$ & $-\cdots$ & --- & --- & 9.42 & 8.20 & 7.89 & 7.00 \\
\hline 6 & --- & --- & $-\cdots$ & $\mathrm{S} 15.30$ & $\cdots$ & $\cdots$ & --- & --- & 9.29 & 8.19 & 7.89 & 7.00 \\
\hline 7 & --- & $\cdots$ & $--m$ & -- & --- & --- & --- & $V_{1} 4.27$ & 9.24 & 8.11 & 7.94 & 7.08 \\
\hline 8 & $-\cdots$ & --- & --- & --- & --- & --- & --- & 13.91 & 9.19 & 8.12 & 7.88 & 7.04 \\
\hline 9 & $-\cdots$ & 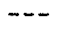 & $-\cdots$ & $-\cdots$ & $-\cdots$ & V9. 60 & --- & 13.52 & 9.20 & 8.14 & 7.91 & 6.99 \\
\hline 10 & --- & --- & --- & --- & --- & V9. 60 & --- & 13.21 & 9.13 & 8.10 & 7.89 & 7.07 \\
\hline 11 & --- & --- & --- & --- & --- & --- & --- & 13.06 & 9.12 & 8.09 & 7.82 & 7.10 \\
\hline 12 & --- & --- & --- & --- & V17.33 & --- & --- & 12.89 & 9.17 & 8.01 & 7.88 & 7.14 \\
\hline 13 & $--m$ & --- & --- & --- & --- & --- & --- & 12.70 & 9.15 & 7.98 & 8.01 & 7.08 \\
\hline 14 & $-\infty$ & $--m$ & $-m$ & $-\infty$ & --- & --- & V7. 85 & 12.48 & 9.04 & 7.99 & 8.85 & 7.03 \\
\hline 15 & $-\cdots$ & --- & -- & -- & --- & $\mathrm{V} 9.20$ & V7. 84 & 12.41 & 9.00 & 7.95 & 9.04 & 7.08 \\
\hline 16 & --- & --- & --- & --- & --- & v9. 15 & V7. 84 & 12.18 & 9.06 & 7.88 & 9.05 & 7.08 \\
\hline 17 & --- & --- & --- & --- & --- & -- & -- & 11.98 & 9.02 & 7.84 & 9.05 & 7.19 \\
\hline 18 & --- & --- & -- & $-\infty$ & $-\cdots$ & v9. 02 & V8. 01 & 11.84 & 8.98 & 7.86 & 9.03 & 7.13 \\
\hline 19 & $\cdots$ & $m-\infty$ & $-\cdots$ & $-m$ & V13.47 & --- & --- & 11.69 & 8.97 & 7.81 & 9.13 & 6.36 \\
\hline 20 & --- & --- & --- & --- & --- & --- & --- & 11.49 & 8.87 & 7.80 & 8.88 & 6.52 \\
\hline 21 & --- & --- & --- & -- & $\cdots$ & --- & -- & 11.27 & 8.80 & 7.77 & 7.59 & 6.58 \\
\hline 22 & --- & --- & --- & S11. 18 & --- & --- & --- & 11.15 & 8.76 & 7.72 & 7.24 & 6.72 \\
\hline 23 & $\cdots$ & --- & $\$ 16.62$ & --- & --- & V8. 75 & --- & 10.98 & 8.82 & 7.65 & 6.94 & 6.78 \\
\hline 24 & --- & $--\infty$ & --- & --- & --- & --- & --- & 10.81 & 8.74 & 7.60 & 6.84 & 6.83 \\
\hline 25 & --- & --- & --- & --- & --- & --- & $-\cdots$ & 10.69 & 8.65 & 7.60 & 6.94 & 6.84 \\
\hline 26 & -- & --- & --- & --- & $-\cdots$ & $\cdots$ & $\ldots$ & 10.60 & 8.64 & 7.64 & 7.00 & 6.86 \\
\hline 27 & --- & $m-m$ & --- & $--\infty$ & V11. 29 & $-\infty$ & --- & 10.41 & 8.57 & 7.63 & 6.95 & 6.92 \\
\hline 28 & --- & $-\cdots$ & --- & --- & $-m$ & $\cdots$ & $\cdots-$ & 10.28 & 8.50 & 7.67 & 6.92 & 6.88 \\
\hline 29 & --- & $-\infty$ & $-m$ & $-m$ & --- & --- & $-\cdots$ & 10.15 & 8.45 & 7.61 & 6.81 & 6.92 \\
\hline 30 & -- & --- & --- & --- & --- & $--\infty$ & $-\infty$ & 10.08 & 8.38 & 7.67 & 6.84 & 6.9 \\
\hline 31 & -- & $\cdots$ & $-\cdots-$ & $\cdots$ & $-\cdots$ & --- & --- & 9.97 & -- & 7.76 & 6.89 & \\
\hline
\end{tabular}

PERIOD OF RECORD HIGHEST 6.36 SEP $19,1992 \quad$ LOWEST 17.33 FEB 12, 1992

S Manual steel tape measurement.

$\checkmark$ Manual calibrated electric tape measurement.

SITE NUMBER 381354120524301

LOCAL, NUMBER O04N010E04G02M

About 1 mile northwest of Campo Seco. Observation well. Diameter 6 inches, depth 200 feet. Altitude of landsurface datum 222 feet. Water-level records avallable 1991 to current year.

WATER LEVELS IN FEET BELOW LAND-SURFACE DATUM

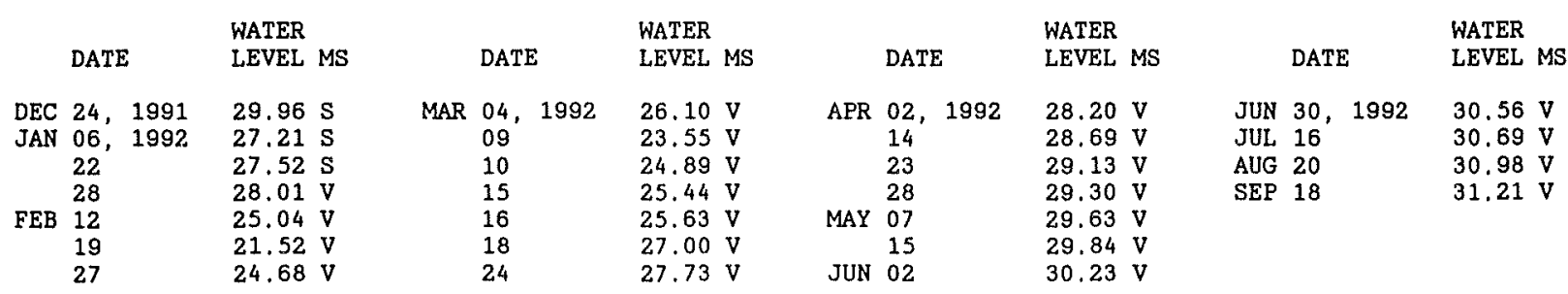

PERIOD OF RECORD HIGHEST 21.52 FEB $19,1992 \quad$ LOWEST $\quad 31.21 \quad$ SEP 18,1992 
WATER LEVELS, CALAVERAS COUNTY--Continued

WELL DESCRIPTIONS AND WATER-LEVEL MEASUREMENTS

WATER YEAR OCTOBER 1991 TO SEPTEMBER 1992

SITE NUMBER 381356120524301

LOCAL NUMBER 004N010E04G03M

About 1 mile northwest of Campo Seco. Observation well. Diameter 6 inches, depth 200 feet. Altitude of landsurface datum 226 feet. Water-level records available 1991 to current year.

WATER LEVELS IN FEET BELOW LAND-SURFACE DATUM

\begin{tabular}{|c|c|c|c|c|c|c|c|c|c|c|}
\hline DATE & $\begin{array}{l}\text { WATER } \\
\text { LEVEL MS }\end{array}$ & & DATE & $\begin{array}{l}\text { WATER } \\
\text { LEVEL MS }\end{array}$ & & DATE & $\begin{array}{l}\text { WATER } \\
\text { LEVEL MS }\end{array}$ & & DATE & $\begin{array}{l}\text { WATER } \\
\text { LEVEL MS }\end{array}$ \\
\hline $\begin{array}{ll}24, & 1991 \\
06, & 1992 \\
12 & \\
22 & \\
30 & \\
12 & \\
19 & \end{array}$ & $\begin{array}{r}10.38 \mathrm{~S} \\
8.90 \mathrm{~S} \\
8.97 \mathrm{~V} \\
9.85 \mathrm{~S} \\
9.90 \mathrm{~V} \\
8.74 \mathrm{~V} \\
7.63 \mathrm{~V}\end{array}$ & $\begin{array}{l}\text { FEB } \\
\text { MAR }\end{array}$ & $\begin{array}{ll}27, & 1992 \\
04 & \\
09 & \\
10 & \\
15 & \\
16 & \\
18 & \end{array}$ & $\begin{array}{r}8.62 \mathrm{~V} \\
9.09 \mathrm{~V} \\
8.61 \mathrm{~V} \\
8.67 \mathrm{~V} \\
9.70 \mathrm{~V} \\
8.95 \mathrm{~V} \\
10.05 \mathrm{~V}\end{array}$ & $\begin{array}{l}\text { MAR } \\
\text { APR }\end{array}$ & $\begin{array}{ll}24, & 1992 \\
02 & \\
15 & \\
16 & \\
23 & \\
28 & \\
07 & \end{array}$ & $\begin{array}{l}10.17 \mathrm{~V} \\
10.10 \mathrm{~V} \\
10.16 \mathrm{~V} \\
12.22 \mathrm{VR} \\
10.94 \mathrm{~V} \\
11.01 \mathrm{~V} \\
11.14 \mathrm{~V}\end{array}$ & $\begin{array}{l}\text { MAY } \\
\text { JUN } \\
\text { JUL } \\
\text { AUG } \\
\text { SEP }\end{array}$ & $\begin{array}{ll}15, & 1992 \\
02 & \\
30 & \\
16 & \\
20 & \\
18 & \end{array}$ & $\begin{array}{l}11.25 \mathrm{~V} \\
11.46 \mathrm{~V} \\
11.28 \mathrm{~V} \\
11.37 \mathrm{~V} \\
11.62 \mathrm{~V} \\
12.19 \mathrm{~V}\end{array}$ \\
\hline
\end{tabular}

PERIOD OF RECORD HIGHEST 7.63 FEB 19, 1992 LOWEST 12.19 SEP 18, 1992

SITE NUMBER 381357120524402

LOCAL NUMBER 004N010E04G04M A INTERVAL

About $1 \mathrm{mile}$ northwest of Campo Seco. Observation well. Diameter 6 inches. Movable packer installed at 55 feet. Measurements represent water levels in A Interval, 0-55 feet. Altitude of land-surface datum 221 feet. Recorder installed May 1992. Water-level records available 1992.

Missing record due to malfunction of recording instrument.

WATER LEVELS IN FEET BELOW LAND-SURFACE DATUM (OBSERVATIONS AT 1200 HOURS)

\begin{tabular}{|c|c|c|c|c|c|c|c|c|c|c|c|c|}
\hline DAY & OCT & NOV & DEC & JAN & FEB & MAR & APR & MAY & JUN & JUL & AUG & SEP \\
\hline 1 & -- & -- & $\cdots$ & -- & $\cdots$ & --- & --- & --- & 40.45 & 40.43 & 40.94 & -- \\
\hline 2 & --- & $\cdots$ & -- & $\cdots$ & -- & -- & V32.96 & $\cdots$ & 40.60 & 40.51 & 41.03 & -- \\
\hline 3 & -- & -- & --- & $\cdots$ & --- & --- & --- & $\cdots$ & 40.64 & 40.62 & 41.10 & -- \\
\hline 4 & -- & -- & -- & --- & -- & V37. 52 & -- & --- & 40.60 & 40.76 & 041.12 & --- \\
\hline 5 & -- & -- & --- & $\cdots$ & -- & --- & -- & --- & 40.59 & 40.54 & $e 41.12$ & $-\cdots$ \\
\hline 6 & $\cdots$ & -- & --- & S39.10 & -- & --- & $\cdots$ & --- & 40.67 & 40.43 & e 41.12 & $\cdots$ \\
\hline 7 & -- & $\cdots$ & -- & -- & -- & -- & -- & v36.73 & 40,75 & 40.56 & 841.13 & --- \\
\hline 8 & -- & -- & --- & -- & -- & --- & --- & -- & 40.76 & 40.68 & e41.13 & $\cdots$ \\
\hline 9 & -- & -- & -- & --- & $\cdots$ & V36.34 & -- & -- & 40.75 & 40.80 & 041.13 & $\cdots$ \\
\hline 10 & -- & -- & $\cdots$ & -- & $\cdots$ & V36.27 & -- & $\cdots$ & 40.75 & 40.28 & $e 41.13$ & -- \\
\hline 11 & -- & $\cdots$ & -- & $\cdots$ & -- & $-\infty$ & --- & -- & 40.73 & 39.58 & e 41.13 & --- \\
\hline 12 & -- & --- & --- & $\cdots$ & V39.20 & -- & --- & --- & 40.73 & 39.31 & 041.14 & -- \\
\hline 13 & -- & -- & --- & $\cdots$ & -- & -- & $\cdots$ & -- & 40.73 & 38.95 & e41.14 & -- \\
\hline 14 & --- & --- & -- & --- & $\cdots$ & --- & $\cdots$ & --- & 40.73 & 39.38 & 041.14 & $\cdots$ \\
\hline 15 & -- & --- & --- & --- & -- & V34. 35 & -- & v37.96 & 40.73 & 39.28 & $e 41.14$ & -- \\
\hline 16 & $-\cdots$ & -- & -- & --- & $\cdots$ & v34.20 & V34.42 & 38.11 & 40.74 & 40.87 & 041.14 & $\cdots$ \\
\hline 17 & -- & -- & $\ldots$ & -- & $\cdots$ & --- & $\ldots$ & 38.28 & 40.73 & 40.61 & 841.14 & $\ldots$ \\
\hline 18 & -- & -- & $\cdots$ & --- & ..- & V33. 83 & $\cdots$ & 38.42 & 40.73 & 40.50 & 041.14 & V41.59 \\
\hline 19 & $\cdots$ & -- & --- & -- & V38.16 & --- & --- & 38.56 & 40.73 & 39.98 & $\mathrm{e} 41.14$ & 41.58 \\
\hline 20 & $\cdots$ & $\cdots$ & --- & $\cdots$ & -- & $-\cdots$ & -- & 38.69 & 40.71 & 40.02 & V41.15 & 41.57 \\
\hline 21 & $\ldots$ & $-\cdots$ & $\cdots$ & --- & $\cdots$ & --- & $\cdots$ & 38.85 & 40.74 & 40.59 & -- & 41.54 \\
\hline 22 & -- & $\cdots$ & -- & S38, 92 & -- & -- & --- & 39.00 & 40.73 & 40.69 & -- & 41.54 \\
\hline 23 & $\cdots$ & --- & -- & --- & -- & V33.06 & V34.98 & 39.13 & 40.73 & 40.57 & --- & 41.54 \\
\hline 24 & $\cdots$ & -- & -- & -- & $\cdots$ & v33.00 & -- & 39.28 & 40.73 & 39.43 & --- & 41.54 \\
\hline 25 & $\cdots$ & --- & $\cdots$ & --- & $\cdots$ & --- & $\cdots$ & 39.43 & 40.68 & 39.42 & --- & 41.53 \\
\hline 26 & -- & --- & --- & $\cdots$ & --- & $\cdots$ & --- & 39.59 & 40.61 & 40.16 & $\cdots$ & 41.52 \\
\hline 27 & --- & $\cdots$ & -- & $\cdots$ & v37.64 & $\cdots$ & --- & 39.74 & 38.82 & 40.95 & $\cdots$ & 41.50 \\
\hline 28 & -- & --- & $\cdots$ & -- & --- & -- & V35.44 & 39.88 & 39.81 & 41.22 & -- & 41.51 \\
\hline 29 & $\cdots$ & --- & $\cdots$ & --- & $\cdots$ & --- & $\cdots$ & 40.03 & 41.31 & 40.23 & $-\cdots$ & 41.51 \\
\hline 30 & --- & -- & -- & $\cdots$ & -- & -- & --- & 40.17 & 40.68 & 40.39 & --- & 41.52 \\
\hline 31 & -- & $\cdots$ & -- & -- & $\cdots$ & --- & $-\infty$ & 40.32 & -.- & 40.71 & $\because-$ & --- \\
\hline
\end{tabular}

PERIOD OF RECORD HIGHEST 32.96 APR 02, 1992 LOWEST 41.59 SEP 18, 1992

e Estimated.

S Manual steel tape measurement.

V Manual calibrated electric tape measurement. 
SITE NUMBER 381357120524403

LOCAL NUMBER 004N010E04G04M B INTERVAL

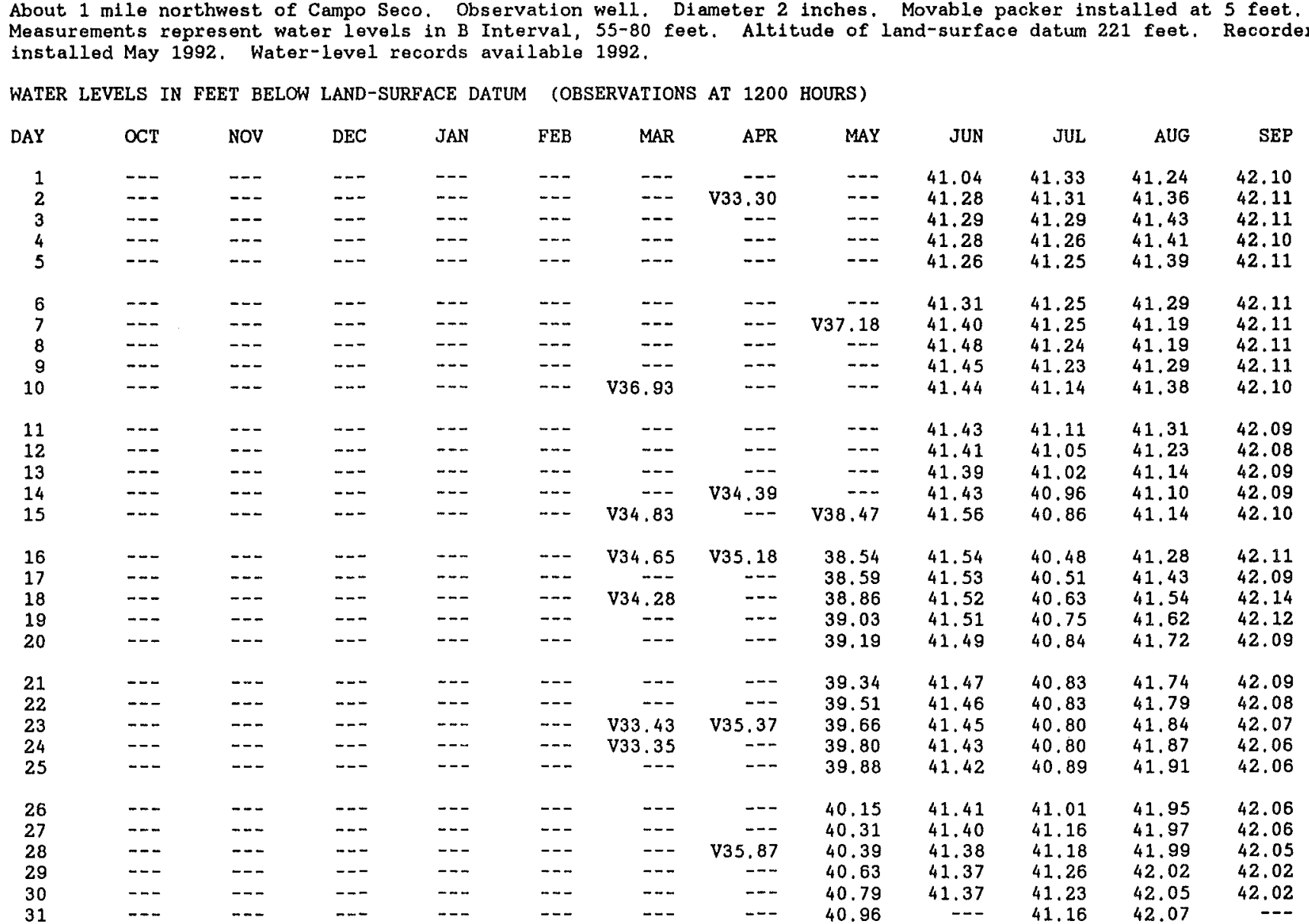


WELL DESCRIPTIONS AND WATER-LEVEL MEASUREMENTS

WATER YEAR OCTOBER 1991 TO SEPTEMBER 1992

SITE NUMBER 381357120524404

LOCAL NUMBER 004N010E04G04M C INTERVAL

About 1 mile northwest of Campo Seco. Observation well. Diameter 2 inches. Movable packer installed at 80 feet. Measurements represent water levels in C Interval, 80-200 feet. Altitude of land-surface datum 221 feet. Recorder installed May 1992. Water-level records available 1992.

WATER LEVELS IN FEET BELOW LAND-SURFACE DATUM (OBSERVATIONS AT 1200 HOURS)

\begin{tabular}{|c|c|c|c|c|c|c|c|c|c|c|c|c|}
\hline DAY & $\propto \mathrm{T}$ & NOV & DEC & JAN & FEB & MAR & APR & MAY & JUN & JUL & AUG & SEP \\
\hline 1 & -- & --- & -- & --- & --- & $-\cdots$ & --- & $m$ & 25.76 & 23.34 & 23.39 & 23.42 \\
\hline 2 & $\cdots$ & $\cdots$ & $\cdots$ & --- & -- & --- & v23.78 & $\cdots$ & 25.53 & 23.35 & 23,44 & 23.44 \\
\hline 3 & -- & -- & --- & $\cdots$ & -- & $\cdots$ & -- & -- & 25.22 & 23.33 & 23.45 & 23.44 \\
\hline 4 & -- & --- & --- & --- & -- & -- & $-\cdots$ & -- & 24.99 & 23.35 & 23.43 & 23.42 \\
\hline 5 & $\cdots$ & $\cdots$ & $-\cdots$ & $\cdots$ & $\cdots$ & $\cdots$ & -- & -- & 24.79 & 23.30 & 23.41 & 23.45 \\
\hline 6 & $-\cdots$ & $\cdots$ & $\cdots$ & -- & --- & --- & $\cdots$ & -.- & 24.63 & 23.26 & 23.45 & 23.43 \\
\hline 7 & --- & --- & --- & $\cdots$ & $\cdots$ & $\cdots$ & --- & V28.62 & 24.48 & 23.36 & 23.42 & 23.41 \\
\hline 8 & $\cdots$ & $\cdots$ & $\cdots$ & -- & --- & --- & $\cdots$ & -. & 24.39 & 23.33 & 23.42 & 23.45 \\
\hline 9 & -- & -- & --- & --- & -- & -- & $\cdots$ & --- & 24.26 & 23.37 & 23.39 & 23.43 \\
\hline 10 & $\cdots$ & $\cdots$ & -- & -- & --- & V33.08 & -- & $\cdots$ & 24.10 & 23.30 & 23.41 & 23.44 \\
\hline 11 & -- & --- & -- & -- & $-\cdots$ & $\cdots$ & --- & --- & 24.00 & 23,38 & 23.36 & 23.44 \\
\hline 12 & -- & -- & --- & -- & --- & $\cdots$ & -- & -- & 23.98 & 23,37 & 23.39 & 23.43 \\
\hline 13 & $\cdots$ & -- & -- & $\cdots$ & $\cdots$ & -- & $\cdots$ & $\cdots$ & 23.90 & 23.43 & 23.43 & 23.36 \\
\hline 14 & $\cdots$ & $-\cdots$ & -- & -- & -- & -- & v23.51 & -- & 23.76 & 23.39 & 23.42 & 23.47 \\
\hline 15 & -- & $\cdots$ & -- & --- & --- & v27.72 & $\ldots$ & V26.16 & 23.71 & 23.42 & 23,42 & 23.44 \\
\hline 16 & --- & -- & $\cdots$ & -- & $\cdots$ & v27. 24 & --- & 26.08 & 23.66 & 23.40 & 23,40 & 23.41 \\
\hline 17 & $\cdots$ & $\cdots$ & -- & --- & --- & --- & -- & 25.98 & 23.54 & 23.42 & 23,43 & 23.37 \\
\hline 18 & $\cdots$ & $\cdots$ & -.- & -- & --- & V26.55 & V23.70 & 25.97 & 23.49 & 23.37 & 23.42 & 23.45 \\
\hline 19 & $\cdots$ & $\cdots$ & -- & --- & --- & -- & --- & 25.95 & 23.45 & 23.36 & 23.38 & 23.43 \\
\hline 20 & --- & -- & $\cdots$ & $\cdots$ & $\cdots$ & --- & --- & 25.90 & 23.40 & 23.38 & 23.44 & 23.37 \\
\hline 21 & -- & $\cdots$ & -- & -- & --- & --- & $\cdots$ & 25.83 & 23.38 & 23.36 & 23.44 & 23.41 \\
\hline 22 & $\cdots$ & $\cdots$ & --- & -- & --- & --- & $\cdots$ & 25.87 & 23.39 & 23.32 & 23,44 & 23,34 \\
\hline 23 & $\cdots$ & $\cdots$ & -- & -- & --- & V25.41 & $\cdots$ & 25.92 & 23.42 & 23.37 & 23.44 & 23.46 \\
\hline 24 & -- & -- & -- & $\cdots$ & $\cdots$ & v25.33 & $\cdots$ & 25.99 & 23,40 & 23.37 & 23.41 & 23.43 \\
\hline 25 & --- & -- & $\cdots$ & $\cdots$ & $\cdots$ & $\cdots$ & --- & 26.02 & 23.42 & 23.39 & 23.43 & 23.43 \\
\hline 26 & -- & $\cdots$ & $-\infty$ & -- & --- & --- & -- & 26.14 & 23.36 & 23.47 & 23.44 & 23.43 \\
\hline 27 & $\ldots$ & $\cdots$ & -- & --- & --- & --- & $\cdots$ & 26.21 & 23.40 & 23.45 & 23.38 & 23.41 \\
\hline 28 & --- & $\cdots$ & --- & --- & --- & $\cdots$ & V34.64 & 26.24 & 23.37 & 23.38 & 23.42 & 23.38 \\
\hline 29 & $\cdots$ & $\cdots$ & --- & -- & --- & $\ldots$ & -.- & 26.29 & 23.35 & 23.40 & 23,43 & 23.42 \\
\hline 30 & -- & $\cdots$ & --- & -- & -- & $\cdots$ & --- & 26.27 & 23.36 & 23.43 & 23.42 & 23.43 \\
\hline 31 & $\cdots$ & $\cdots$ &.- & --- & --- & --- & $\cdots$ & 25.99 & -- & 23.41 & 23.42 & $\cdots$ \\
\hline
\end{tabular}

PERIOD OF RECORD HIGHEST 23.26 JUL 06, $1992 \quad$ LOWEST 34.64 APR 28, 1992

$\checkmark$ Manual calibrated electric tape measurement.

SITE NUMBER 381359120524501

LOCAL NUMBER 004N010E04G05M

About 1 mile northwest of Campo Seco. Observation well. Diameter 6 inches, depth 201.5 feet. Altitude of landsurface datum 218 feet. Water-level records available 1992.

WATER LEVELS IN FEET BELOW LAND-SURFACE DATUM

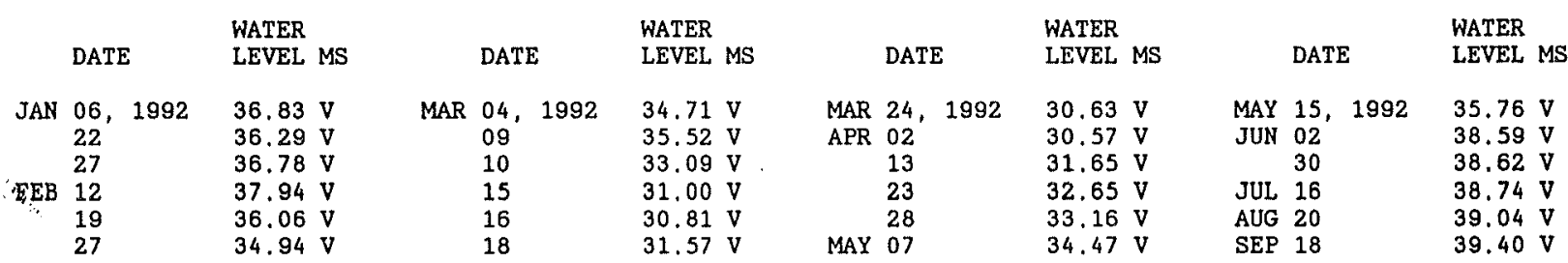

PERIOD OF RECORD HIGHEST 30.57 APR 02, 1992 LOWEST $39.40 \quad$ SEP 18,1992 
SITE NUMBER 381354120524401

LOCAL NUMBER 004N010E04G06M

About $1 \mathrm{mile}$ northwest of Campo Seco. Observation well. Diameter 6 inches, depth 401 feet. Altitude of landsurface datum 220 feet. Recorder installed May 1992. Water-level records avallable 1991 to current year.

WATER LEVELS IN FEET BELOW LAND-SURFACE DATUM (OBSERVATIONS AT 1200 HOURS)

\begin{tabular}{|c|c|c|c|c|c|c|c|c|c|c|c|c|}
\hline DAY & ○T & NOV & DEC & JAN & FEB & MAR & APR & MAY & JUN & JUL & AUG & SEP \\
\hline 1 &.-- & -- & -- & $\cdots$ & -- & -- & -- & --- & 36.30 & 36.48 & 36.58 & 36.91 \\
\hline 2 & -- & -- & -- & $\cdots$ & -- & -- & V31.71 & --- & 36.34 & 36.44 & 36,58 & 36.93 \\
\hline 3 & -- & -- & -- & $\cdots$ & $\cdots$ & $\cdots$ & $\ldots$ & --- & 36.38 & 36.41 & 36.61 & 36.93 \\
\hline 4 & --- & --- & --- & -- & -- & V33.55 & -- & -- & 36.39 & 36.45 & 36.61 & 36,92 \\
\hline 5 & --- & -- & -- & -- & --- & -- & -- & $\cdots$ & 36.40 & 36.46 & 36.62 & 36.93 \\
\hline 6 & --- & $-n$ & $\cdots$ & V34.37 & $\cdots$ & -- & $-\cdots$ & -- & 36.44 & 36.47 & 36.63 & 36.94 \\
\hline 7 & --- & --- & --- & --- & --- & --- & --- & v34.92 & 36.46 & 36.48 & 36.60 & 36.95 \\
\hline 8 & -- & --- & -- & --- & -- & -- & --- & 34.97 & 36.48 & 36.46 & 36.62 & 36.94 \\
\hline 9 & -- & $\cdots$ & $-\cdots$ & $\cdots$ & $\cdots$ & V32.97 & -- & 35.06 & 36.51 & 36.41 & 36.63 & 36.95 \\
\hline 10 & -- & -- & -- & -- & -- & V32. 85 & --- & 35.16 & 36.51 & 36.38 & 36.65 & 36.95 \\
\hline 11 & -- & --- & -- & -- & -- & -- & $-m$ & 35.25 & 36.54 & 36.35 & 36.65 & 36.97 \\
\hline 12 & -- & -- & --- & -- & V34.44 & -- & -- & 35.30 & 36.53 & 36.38 & 36.66 & 36.96 \\
\hline 13 & --- & -- & $-\cdots$ & -- & -- & -- & --- & 35.35 & 36.49 & 36.40 & 36.66 & 36.94 \\
\hline 14 & -- & -- & -- & --- & --- & --- & V32.62 & 35.40 & 36.50 & 36.37 & 36.65 & 36.95 \\
\hline 15 & -- & -- & --- & --- & --- & V32. 52 & v32.72 & 35.43 & 36.55 & 36.33 & 36.65 & 36.96 \\
\hline 16 & -- & -- & --- & --- & --- & V31. 34 & -- & 35.49 & 36.58 & 36.29 & 36.65 & 36.98 \\
\hline 17 & -- & -- & --- & -- & -- & - & --- & 35.55 & 36.54 & 36.29 & 36.69 & 36.97 \\
\hline 18 & -- & --- & -- & --- & -- & V32. 44 & -- & 35.60 & 36.54 & 36.32 & 36.71 & 36.97 \\
\hline 19 & --- & -- & -- & -- & v32. 56 & -- & --- & 35.63 & 36.51 & 36.32 & 36.72 & 36.95 \\
\hline 20 & -- & -- & --- & -- & -- & --- & -- & 35.68 & 36.48 & 36.33 & 36.74 & 36.96 \\
\hline 21 & --- & --- & $\cdots$ & -- & --- & --- & -- & 35.72 & 36.50 & 36.38 & 36.78 & 36.96 \\
\hline 22 & -- & -- & --- & V34.25 & -- & -- & -- & 35.78 & 36.54 & 36.37 & 36.80 & 36.98 \\
\hline 23 & $\cdots$ & $\cdots$ & -- & $-\cdots$ & -- & V31.81 & V34.37 & 35.83 & 36.52 & 36.35 & 36.81 & 36.97 \\
\hline 24 & --- & -- & 536.17 & -- & $-\cdots$ & V31.75 & -- & 35.86 & 36.51 & 36.37 & 36.81 & 36.97 \\
\hline 25 & --- & -- & -- & -- & --- & $\ldots$ & -- & 35.93 & 36.51 & 36.41 & 36.85 & 36.96 \\
\hline 26 & --- & --- & --- & -- & --- & -- & --- & 35.98 & 36.49 & 36.43 & 36.87 & 36.99 \\
\hline 27 & $\cdots$ & -- & --- & -- & V33.30 & $\cdots$ & -.- & 36.04 & 36.46 & 36.46 & 36.88 & 36.97 \\
\hline 28 & --- & --- & $\cdots$ & -- & -- & --- & V33. 88 & 36.10 & 36.50 & 36.50 & 36.88 & 36.97 \\
\hline 29 & $\cdots$ & $-\infty$ & $\cdots$ & V34.52 & --- & $-\cdots$ & $\ldots$ & 36.16 & 36.48 & 36.52 & 36.88 & 36.97 \\
\hline 30 & -- & -- & $\cdots$ & $-\cdots$ & --- & -- & $\cdots$ & 36.21 & 36.49 & 36.54 & 36.91 & 36.98 \\
\hline 31 & $\cdots$ & $\rightarrow-$ & -- & -- & -- & -- & -- & 36.25 & -- & 36.55 & 36.91 & --- \\
\hline
\end{tabular}


WELL DESCRIPTIONS AND WATER-LEVEL MEASUREMENTS

WATER YEAR OCTOBER 1991 TO SEPTEMBER 1992

SITE NUMBER 381354120524501

LOCAL NUMBER 004N010E04G07M

About 1 mile northwest of Campo Seco. Observation well. Diameter 6 inches. Movable packer installed at 76 feet. Measurements represent water levels in A Interval, 0-76 feet. Altitude of land-surface datum 220 feet. Water-1evel. records avallable 1991 to current year.

WATER LEVELS IN FEET BELON LAND-SURFACE DATUM

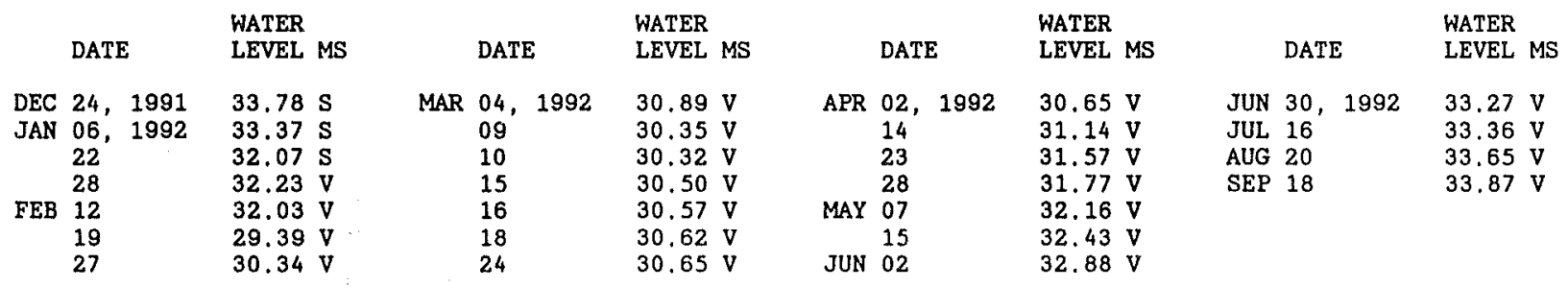

PERIOD OF RECORD HIGHEST 29.39 FEB 19, 1992 LOWEST 33.87 SEP 18,1992

SITE NUMBER 381356120524402

LOCAL NUMBER 004N010E04G08M A INTERVAL

About 1 mile northwest of Campo Seco. Observation well. Diameter 6 inches, depth 76 feet. Altitude of landsurface datum 219 feet. Recorder installed May 1992. Water-level records available 1992.

WATER LEVELS IN FEET BELOW LAND-SURFACE DATUM (OBSERVATIONS AT 1200 HOURS)

\begin{tabular}{|c|c|c|c|c|c|c|c|c|c|c|c|c|}
\hline DAY & $\propto \Gamma$ & NoV & DEC & JAN & FEB & MAR & APR & MAY & JUN & JUL & AUG & SEP \\
\hline 1 & -- & $\cdots$ & -- & -- & --- & $\cdots$ & -- & --- & 39.52 & 39.65 & 39.72 & 40.50 \\
\hline 2 & -- & --- & -- & $\cdots$ & --- & -- & V31.74 & -- & 39.69 & 39.56 & 39.83 & 40.51 \\
\hline 3 & --- & $\cdots$ & $\cdots$ & $\cdots$ & --- & -- & 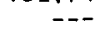 & -- & 39.70 & 39.47 & 39.88 & 40.51 \\
\hline 4 & -- & $\cdots$ & $\cdots$ & $\cdots$ & --- & -- & --- & $\cdots$ & 39.69 & 39.49 & 39.85 & 40.51 \\
\hline 5 & $-\cdots$ & $\cdots$ & -- & $\cdots$ & $-\cdots$ & --- & $\cdots$ & -- & 39.68 & 39.59 & 39.78 & 40.51 \\
\hline 6 & -- & -- & -- & $\cdots$ & --- & -- & --- & $\ldots$ & 39.75 & 39.63 & 39.73 & 40.51 \\
\hline 7 & -- & $\ldots$ & -- & -- & --- & $-\cdots$ & --- & V35.60 & 39.85 & 39.58 & 39.65 & 40.51 \\
\hline 8 & -- & $\cdots$ & -- & -- & $\ldots$ & $-n$ & $\ldots$ & 35.73 & 39.95 & 39.50 & 39.71 & 40.51 \\
\hline 9 & -- & $\cdots$ & $\cdots$ & -- & $\cdots$ & --- & --- & 35.87 & 39.94 & 39.30 & 39.80 & 40,51 \\
\hline 10 & -- & $\cdots$ & --- & -- & $\cdots$ & V35.38 & -- & 36.05 & 39.91 & 39.08 & 39.86 & 40.50 \\
\hline 11 & $\cdots$ & --- & -- & $\cdots$ & $\cdots$ & --- & $\cdots$ & 36.21 & 39.89 & 39.10 & 39.80 & 40.51 \\
\hline 12 & -- & $\cdots$ & --- & -- & -- & -- & $\cdots$ & 36.38 & 39.87 & 39.20 & 39.73 & 40.51 \\
\hline 13 & -- & $\cdots$ & --- & --- & -- & --- & -- & 36.54 & 39.88 & 39.27 & 39.67 & 40.50 \\
\hline 14 & -- & --- & -- & $\cdots$ & $\cdots$ & -- & V32.91 & 36.72 & 40.00 & 39.08 & 39.59 & 40.50 \\
\hline 15 & -- & --- & $-\cdots$ & $\cdots$ & -- & v33. 33 & -- & 36.89 & 40.13 & 38.85 & 39.67 & 40.51 \\
\hline 16 & -- & -- & --- & -- & -- & V33. 13 & $\cdots$ & 37.06 & 40.09 & 38.85 & 39.80 & 40.51 \\
\hline 17 & $\cdots$ & $\cdots$ & $\cdots$ & --- & $\cdots$ & -- & $\ldots$ & 37.20 & 40.00 & 38.93 & 39.94 & 40.50 \\
\hline 18 & 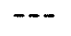 & --- & $\cdots$ & -- & $\cdots$ & v32.75 & --- & 37.38 & 39,92 & 39.06 & 40.00 & 40.50 \\
\hline 19 & -- & --- & -- & $\cdots$ & -- & -- & --- & 37.53 & 39.84 & 39.18 & 40.06 & 40.50 \\
\hline 20 & -- & --- & -- & --- & $\cdots$ & -- & --- & 37.67 & 39.80 & 39.28 & 40.12 & 40.48 \\
\hline 21 & --- & --- & $\cdots$ & --- & $\cdots$ & --- & -- & 37.78 & 39.89 & 39.23 & 40.17 & 40.47 \\
\hline 22 & -- & $-\cdots$ & $\cdots$ & --- & $\cdots$ & --- & $\cdots$ & 37.91 & 39.97 & 39.18 & 40.22 & 40.46 \\
\hline 23 & --- & $\cdots$ & $-\cdots$ & -- & $\cdots$ & -- & v33. 76 & 38.03 & 39.93 & 39.14 & 40.26 & 40.45 \\
\hline 24 & $\cdots$ & --- & $\cdots$ & -- & -- & V31. 81 & --- & 38.24 & 39.85 & 39.22 & 40.30 & 40.44 \\
\hline 25 & $-\cdots$ & --- & $\cdots$ & -- & $\cdots$ & -- & -- & 38.42 & 39.78 & 39.35 & 40.32 & 40.44 \\
\hline 26 & $\cdots$ & --- & $\cdots$ & -- & -- & -- & -- & 38.62 & 39.70 & 39.50 & 40.36 & 40.44 \\
\hline 27 & -- & --- & --- & $\cdots$ & -- & --- & $\cdots$ & 38.79 & 39.67 & 39.63 & 40.38 & 40.44 \\
\hline 28 & $\cdots$ & $\cdots$ & -- & $\cdots$ & $-\cdots$ & -- & v34.29 & 38.90 & 39.76 & 39.65 & 40.40 & 40.43 \\
\hline 29 & -- & -- & $\ldots$ & $\cdots$ & --- & $\cdots$ & -- & 39.06 & 39.82 & 39.73 & 40.44 & 40.42 \\
\hline 30 & --- & -- & $\cdots$ & --- & $\cdots$ & -- & $\cdots$ & 39.25 & 39.73 & 39.69 & 40.47 & 40.41 \\
\hline 31 & -- & --- & $-\cdots$ & 24.50 & -- & -- & --- & 39.32 & -- & 39.66 & 40.49 & 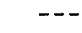 \\
\hline
\end{tabular}

PERIOD OF RECORD HIGHEST 24.50 JAN 31, 1992

LOWEST $\quad 40.51$ SEP $2-9,11,12,15,16,1992$

$\checkmark$ Manual calibrated electric tape measurement. 
WELL DESCRIPTIONS AND WATER-LEVEL MEASUREMENTS

WATER YEAR OCTOBER 1991 TO SEPTEMBER 1992

SITE NUMBER 381356120524403

LOCAL NUMBER 004N010E04G08M B INTERVAL

About 1 mile northwest of Campo Seco. Observation well. Diameter 2 inches, Movable packer installed at 76 feet. Measurements represent water levels in B Interval, 76-200 feet. Altitude of land-surface datum 219 feet. Recorder installed May 1992. Water-level records available 1992.

WATER LEVELS IN FEET BELOW LAND-SURFACE DATUM (OBSERVATIONS AT 1200 HOURS)

\begin{tabular}{|c|c|c|c|c|c|c|c|c|c|c|c|c|}
\hline DAY & OCT & NOV & DEC & JAN & FEB & MAR & APR & MAY & JUN & JUL & AUG & SEP \\
\hline 1 & -- & --- & --- & $\cdots$ & $\cdots$ & -.- & --- & -- & 23.83 & 24.16 & 24.09 & 24.25 \\
\hline 2 & --- & $\cdots$ & --- & $-\cdots$ & -- & $\cdots$ & V23.83 & --- & 23.79 & 24.10 & 24.08 & 24.26 \\
\hline 3 & -- & --- & --- & -- & $v 28.30$ & -- & -- & --- & 23.77 & 24.00 & 24.08 & 24.28 \\
\hline 4 & --- & --- & --- & $\cdots$ & - & V23.87 & --- & -- & 23.77 & 23.97 & 24.08 & 24.31 \\
\hline 5 & -- & -- & $\cdots$ & -- & --- & $\ldots$ & -- & -- & 23.76 & 23.98 & 24.07 & 24.29 \\
\hline 6 & $\ldots$ & -- & $\ldots-$ & $\$ 24.36$ & --- & -- & $\cdots$ & -- & 23.75 & 23.98 & 24.07 & 24.30 \\
\hline 7 & $\cdots$ & --- & $\cdots$ & $\cdots$ & --- & -- & $\cdots$ & V24.82 & 23.74 & 23.97 & 24.08 & 24.29 \\
\hline 8 & $\cdots$ & -- & $\cdots$ & --- & -- & -. & --- & 24.69 & 23.75 & 23.98 & 24.08 & 24.30 \\
\hline 9 & --- & -- & --- & --- & $-\infty$ & v23.76 & -- & 24.53 & 23.75 & 24.00 & 24.09 & 24.27 \\
\hline 10 & $\cdots$ & --- & $\cdots$ & $\cdots$ & --- & $\mathrm{V} 28.48$ & -- & 24.35 & 23.74 & 23.98 & 24.11 & 24.25 \\
\hline 11 & $\cdots$ & --- & -- & $\cdots$ & $\cdots$ & $-\cdots$ & --- & 24.39 & 23.78 & 23.95 & 24.09 & 24.28 \\
\hline 12 & --- & $\cdots$ & --- & --- & V26.07 & --- & $\cdots$ & 24.33 & 23.82 & 23.95 & 24.08 & 24.29 \\
\hline 13 & --- & -- & $\cdots$ & -- & -- & $\cdots$ & --- & 24.30 & 23.87 & 23.97 & 24.08 & 24.27 \\
\hline 14 & -- & -- & --- & -- & $\cdots$ & -- & V23. 53 & 24.27 & 23.82 & 23.99 & 24.11 & 24.25 \\
\hline 15 & $-\cdots$ & -- & --- & $\cdots$ & --- & $V 24.11$ & v23.78 & 24.20 & 23.82 & 24.00 & 24.12 & 24.25 \\
\hline 16 & $-\cdots$ & $\cdots$ & $\therefore$ & -- & $\cdots$ & V23.90 & $\ldots$ & 24.16 & 23.84 & 23.97 & 24.09 & 24.26 \\
\hline 17 & $\cdots$ & --- & --- & -- & -- & --- & V23.78 & 24.12 & 23.84 & 23.98 & 24.09 & 24.28 \\
\hline 18 & $-\infty$ & $\cdots$ & --- & --- & -. & V23.71 & $\ldots$ & 24.08 & 23.83 & 24.02 & 24.08 & 24.29 \\
\hline 19 & $\cdots$ & $\cdots$ & --- & -- & V 24,61 & -- & -- & 24.09 & 23.84 & 24.06 & 24.12 & 24.29 \\
\hline 20 & $\cdots$ & -- & --- & --- & -- & --- & -- & 24.08 & 23.79 & 24.03 & 24.10 & 24.28 \\
\hline 21 & -- & -- & $\cdots$ & -- & $\cdots$ & $\cdots$ & -- & 24.03 & 23.78 & 24.02 & 24.10 & 24.29 \\
\hline 22 & -- & $\cdots$ & --- & 524.29 & $\cdots$ & -- & -- & 23.99 & 23.79 & 24.02 & 24.09 & 24.31 \\
\hline 23 & $\ldots$ & -- & -- & $\ldots$ & $-\cdots$ & -- & $\ldots$ & 23.96 & 23.83 & 24.03 & 24.08 & 24.35 \\
\hline 24 & -- & -- & $\cdots$ & $-\cdot-$ & --- & V23.91 & $\cdots$ & 23.95 & 23.82 & 24.00 & 24.07 & 24.38 \\
\hline 25 & $\cdots$ & -- & $\cdots$ & --- & --- & $\ldots$ & $\cdots$ & 23.93 & 23.83 & 23.99 & 24.10 & 24.35 \\
\hline 26 & $\cdots$ & --- & -- & $\cdots$ & -- & --- & $\ldots$ & 23.91 & 23.85 & 24.06 & 24.16 & 24.36 \\
\hline 27 & $\cdots$ & --- & $\cdots$ & $\cdots$ & V24.24 & --- & 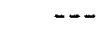 & 23.87 & 23.87 & 24.05 & 24.20 & 24.37 \\
\hline 28 & $\cdots$ & -- & $\cdots$ & $\cdots$ & -- & --- & V27.65 & 23.84 & 23.90 & 24.03 & 24.20 & 24.39 \\
\hline 29 & --- & $\cdots$ & --- & --- & -- & $\cdots$ & -- & 23,80 & 23.89 & 24.03 & 24.21 & 24.41 \\
\hline 30 & -- & -- & $\cdots$ & $\cdots$ & --- & --- & $\cdots$ & 23.80 & 23.92 & 24.01 & 24.24 & 24.42 \\
\hline 31 & $\cdots$ & -- & --- & --- & $\ldots$ & $\ldots$ & -- & 23.79 & - & 24.04 & 24.24 & -- \\
\hline
\end{tabular}

PERIOD OF RECORD HIGHEST 23.53 APR 14, 1992 LOWEST 28.48 MAR 10, 1992

S Manual steel tape measurement.

$\checkmark$ Manual calibrated electric tape measurement. 
INORGANIC DATA

WATER YEAR OCTOBER 1991 TO SEPTEMBER 1992

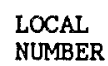

NUMBER

004N010E04G01M A INTERVAL 004N010E04G01M A INTERVAL 004N010E04G02M

004N010E04G03M

004N010E04G04M A INTERVAI 004N010E04G04M B INTERVAL. 004N010E04G04M C INTERVAI 004N010E04G05M

004 N010E04G06M

004N010E04G07M

004N010E04G08M A INTERVAL 004N010E04G08M B INTERVAL

DEPTH
BELOW
LAND
SURFACE
(WATER
LEVEL)
(FEET)
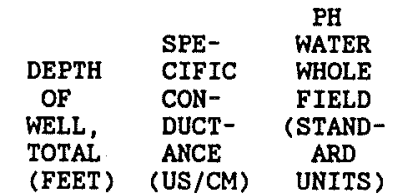

23.90

1250

1100

1345

1100

1740

1315

1210

1235

1630

1635

1405

1445

1520

1305

1615

\section{0}

1535
1625

1625
1525

1530

24.36

10.24

8.01200

28.01200

28.69200

$10.16 \quad 200$

$34.42 \quad 55$

$34.42 \quad 55$

35.18

$--$

$23.70 \quad 200$

$31.65 \quad 201.5$

$34.52 \quad 401$

$34.52 \quad 401$

$32.72 \quad 401$

$32.72 \quad 401$

$32.23 \quad 202$

$31.14 \quad 202$

$24.50 \quad 76$

$32.91 \quad 76$

$28.30 \quad 200$

$23.78 \quad 200$

1100
1225

5060
4950
5200
5980
1430
1810
5190
4600
2430
2430

3120
3440
4380
3730
3430
14000
14000
18000
18000
3150
2390
6900
5660
5540
6200

\section{DATE

DATE

\begin{abstract}
$01-29-92$
\end{abstract} 04-17-92 02-03-92 04-18-92 01-28-92

04-14-92 01-30-92 04-15-92 $04-16-92$
$04-16-92$

01-31-92 04-16-92 $02-03-92$ 04-18-92 01-27-92

04-13-92 01-29-92 01-29-92 04-15-92 $04-15-92$

\section{1-28-92}

04-14-92 01-31-92 04-14-92

$02-03-92$ 04-17-92
9.90200

- 80

$36.78 \quad 201.5$

MAGNE-

$\begin{array}{ccc} & \text { MAGNE- } \\ \text { CALCIUM } & \text { SIUM, } & \text { SODIUM, } \\ \text { DIS- } & \text { DIS- } & \text { DIS- } \\ \text { SOLVED } & \text { SOLVED } & \text { SOLVED }\end{array}$

LOCAL

NUMBER

DATE

(MG/L

VED SOLVE AS CA)

004N010E04G01M A

004N010E04G01M B

004N010E04G02M

01-29-92 $04-17-92 \quad 480$ 02-03-92 540 04-18-92 480 01-28-92 72

004N010E04G03M

04-14-92 72 01-30-92 480 04-15-92 440

004N010E04G04M A

210

200

004N010E04G04M B $01-31-92 \quad 380$

004N010E04G04M C

004N010E04G05M

$04-16-92 \quad 360$

02-03-92 360

04-18-92 380

01-27-92 440

$04-13-92 \quad 360$

01-29-92 520

01-29-92 570

04-15-92 560

04-15-92 600

$\begin{array}{llr}\text { (MG } / L & \text { (MG } / L & \text { SODIUM } \\ \text { AS MG) AS NA) PERCENT }\end{array}$

SODIUM
AD-
SORP-
TION
RATIO

$\begin{array}{rrr}350 & 370 & 22 \\ 350 & 280 & 19 \\ 320 & 410 & 25 \\ 260 & 810 & 43 \\ 38 & 180 & 53 \\ 34 & 320 & 68 \\ 600 & 57 & 3 \\ 550 & 57 & 4 \\ 260 & 29 & 4 \\ 240 & 31 & 4 \\ & & \\ 290 & 110 & 10 \\ 250 & 83 & 9 \\ 280 & 150 & 14 \\ 270 & 360 & 27 \\ 390 & 48 & 4 \\ & & \\ 320 & 49 & 5 \\ 430 & 1200 & 46 \\ 380 & 1700 & 55 \\ 290 & 3200 & 73 \\ 270 & 3100 & 72 \\ & & \end{array}$

22
19
25
43
53
68
3
4
4
4
10
9
14
27
4
5
46
55
73
72

250

170

04-14-92 300

01-31-92 510

04-14-92 480

$\begin{array}{ll}02-03-92 & 550 \\ 04-17-92 & 460\end{array}$

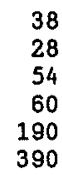

5.7

$5.1 \quad 460$

7.2263

$7.8 \quad 319$

$\begin{array}{rr}4.7 & =- \\ 4.8 & 451\end{array}$

$5.7 \quad 387$

$5.7 \quad 387$

$5.6=$

$\begin{array}{ll}6.6 & 400 \\ 6.7 & --\end{array}$

$7.3 \quad 293$

5.6 -

$5.4 \quad 438$

4.5
4.5

$4.2 \quad 484$

$4.1 \quad 484$

$4.6 \quad 565$

$\begin{array}{ll}4.9 & 565 \\ 3.7 & --\end{array}$

3.7500

$4.1 \quad 420$

XID-

HARD -

NESS

ATURE
DIS-

WATER SOLVED AS

(DEG C) (MG/L) CACO3)

$\begin{array}{llr}18.5 & -- & 2800 \\ 18.0 & -- & 2600 \\ 18.5 & -- & 2700 \\ 18.5 & -- & 2300 \\ 18.5 & -- & 340 \\ 18.5 & 0.9 & 320 \\ 19.5 & -- & 3700 \\ 19.5 & 1.7 & 3400 \\ 18.5 & -- & 1600 \\ 18.5 & -- & 1500 \\ 16.0 & -- & 2100 \\ 19.0 & -- & 1900 \\ 19.0 & -- & 2100 \\ 19.0 & -- & 2100 \\ 17.5 & -- & 2700 \\ 17.5 & 0.5 & 2200 \\ 17.5 & -- & 3100 \\ 17.5 & -- & 3000 \\ 18.5 & 0.5 & 2600 \\ 18.5 & -- & 2600 \\ 18.5 & -- & 2000 \\ 18.5 & 1.0 & 1400 \\ 18.0 & -- & 3500 \\ 18.5 & 0.2 & 3600 \\ 16.5 & -- & 2800 \\ 18.5 & -- & 3000\end{array}$

$\begin{array}{cccc} & \text { BICAR- } & \text { CAR- } & \text { ALKA- } \\ \text { POTAS- } & \text { BONATE } & \text { BONATE } & \text { LINITY } \\ \text { SIUM, } & \text { WATER } & \text { WATER } & \text { WAT WH } \\ \text { DIS- } & \text { WH IT } & \text { WH IT } & \text { TOT IT } \\ \text { SOLVED } & \text { FIELD } & \text { FIELD } & \text { FIELD } \\ \text { (MG } / L & \text { (MG /L AS } & \text { (MG/L AS } & \text { (MG } / L \text { AS }\end{array}$

(MG/L AS (MG/L AS (MG/L AS

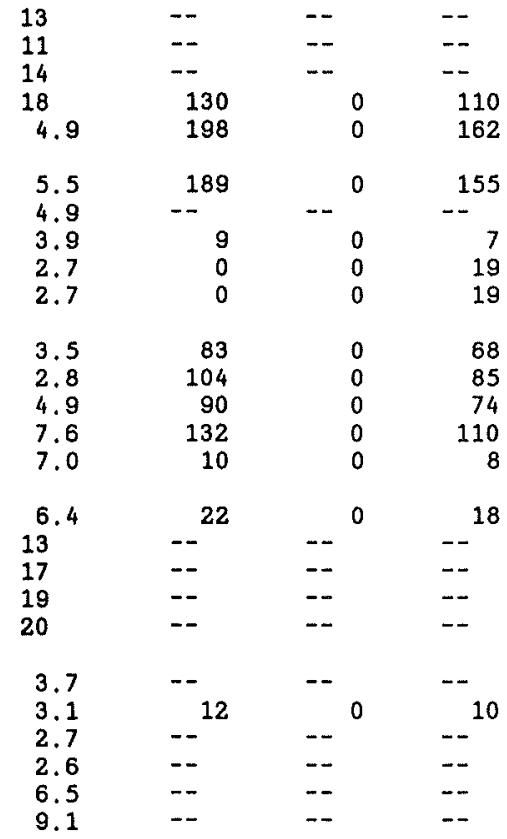




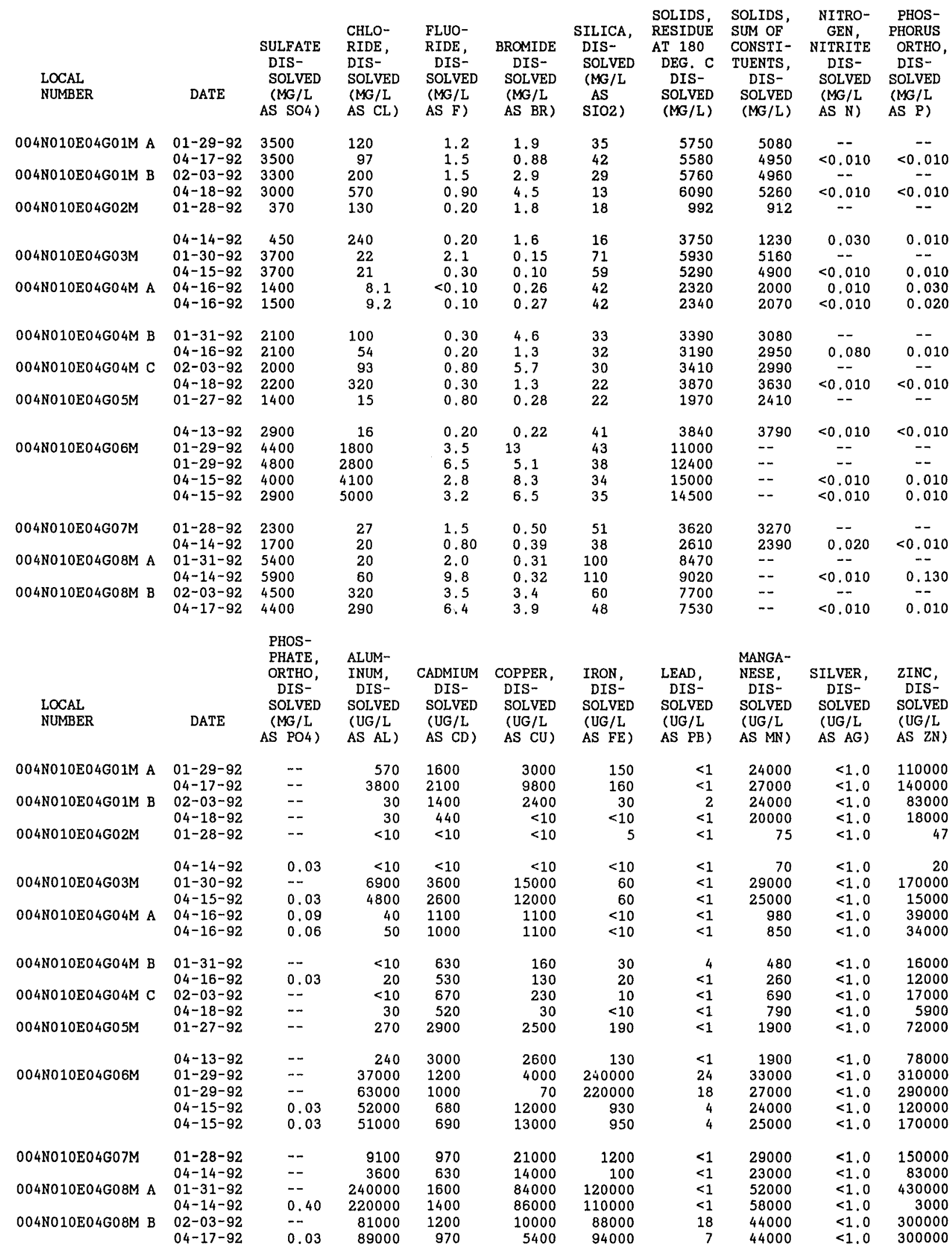


STABLE-ISOTORE DATA

WATER YEAR OCTOBER 1991 TO SEPTEMBER 1992

\begin{tabular}{|c|c|c|c|c|}
\hline $\begin{array}{l}\text { LOCAL } \\
\text { NUMBER }\end{array}$ & DATE & TIME & $\begin{array}{c}\text { H-2 / } \\
\text { H-1 } \\
\text { STABLE } \\
\text { ISOTOPE } \\
\text { RATIO } \\
\text { PER } \\
\text { MIL }\end{array}$ & $\begin{array}{c}0-18 \quad / \\
\text { O-16 } \\
\text { STABLE } \\
\text { ISOTOPE } \\
\text { RATIO } \\
\text { PER } \\
\text { MIL }\end{array}$ \\
\hline $\begin{array}{l}\text { 004N010E04G01M A INTERVAL } \\
\text { 004N010E04G01M B INTERVAL } \\
\text { 004N010E04G02M } \\
\text { 004N010E04G03M } \\
\text { 004N010E04G04M A INTERVAL }\end{array}$ & $\begin{array}{l}04-17-92 \\
04-18-92 \\
04-14-92 \\
04-15-92 \\
04-16-92\end{array}$ & $\begin{array}{l}1100 \\
1100 \\
1315 \\
1235 \\
1630\end{array}$ & $\begin{array}{l}-36.5 \\
-40.0 \\
-56.0 \\
-35.0 \\
-51.5\end{array}$ & $\begin{array}{l}-3.40 \\
-3.85 \\
-7.65 \\
-3.45 \\
-6.60\end{array}$ \\
\hline 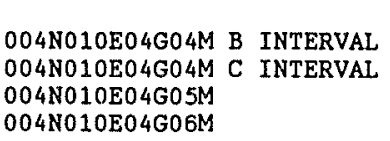 & $\begin{array}{l}04-16-92 \\
04-16-92 \\
04-18-92 \\
04-13-92 \\
04-15-92\end{array}$ & $\begin{array}{l}1635 \\
1445 \\
1305 \\
1640 \\
1525\end{array}$ & $\begin{array}{l}-50.0 \\
-48.5 \\
-48.5 \\
-46.5 \\
-35.0\end{array}$ & $\begin{array}{l}-6.65 \\
-6.30 \\
-6.25 \\
-5.40 \\
-2.90\end{array}$ \\
\hline $\begin{array}{l}\text { 004N010E04G07M } \\
\text { 004N010E04G08M A INTERVAL } \\
004 \text { N010E04G08M B INTERVAL }\end{array}$ & $\begin{array}{l}04-15-92 \\
04-14-92 \\
04-14-92 \\
04-17-92\end{array}$ & $\begin{array}{l}1530 \\
1250 \\
1830 \\
1225\end{array}$ & $\begin{array}{l}-33.0 \\
-58.0 \\
-32.5 \\
-38.0\end{array}$ & $\begin{array}{l}-2.90 \\
-8.20 \\
-2.65 \\
-3.85\end{array}$ \\
\hline
\end{tabular}


CONTRA COSTA COUNTY

Page
Page

LOCAL NUMBER 002N002W13P01M.............. 23

(1) Number in circle indicates number of wells in township for which data are included in this report
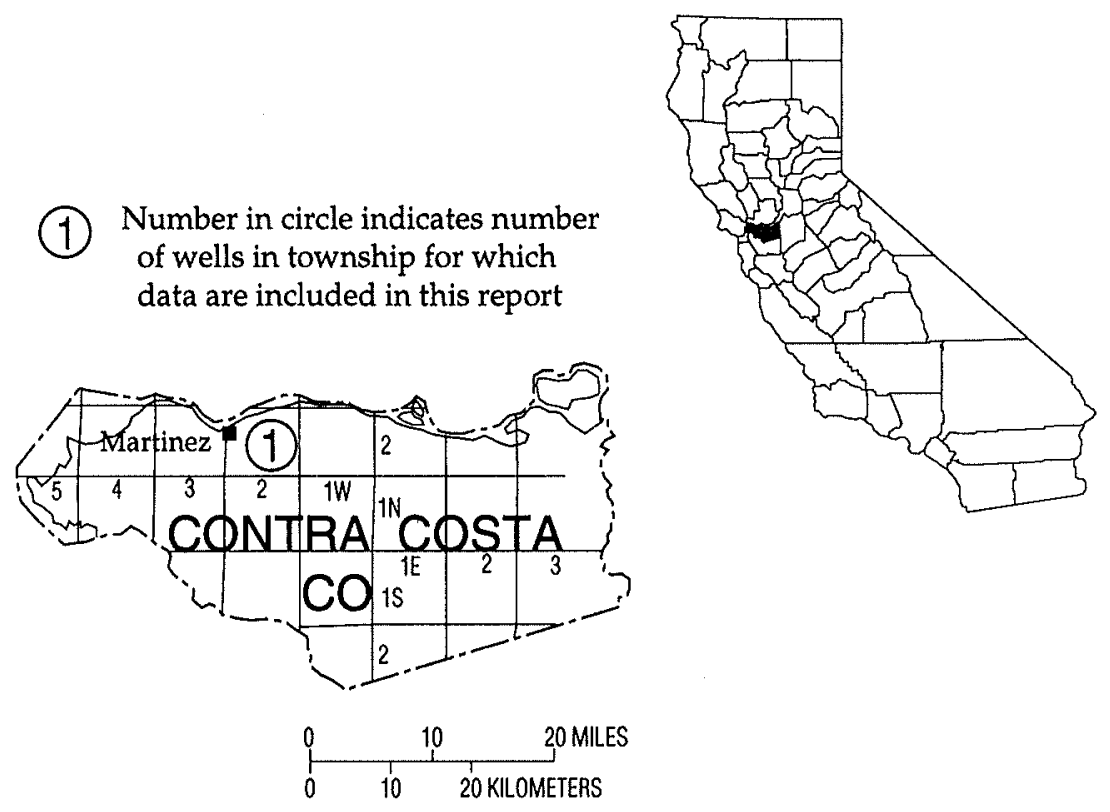

Figure 4. Location of well in Contra Costa County. 
WELL DESCRIPTIONS AND WATER-LEVEL MEASUREMENTS

WATER YEAR OCTOBER 1991 TO SEPTEMBER 1992

SITE NUMBER 380049122015301

LOCAL NUMBER 002N002W13P01M

Near Port Chicago. Drilled industrial water-table well in alluvium. Diameter unknown, depth 139 feet. Altitude of land-surface datum 45 feet. Water-level records available 1970 to current year.

WATER LEVELS IN FEET BELOW LAND-SURFACE DATUM

$$
\text { DATE WATER }
$$

MAY $22,1992 \quad 24.60 \mathrm{~s}$

PERIOD OF RECORD HIGHEST 19.61 APR 21, 1978 LOWEST 32.28 APR 18, 1979 


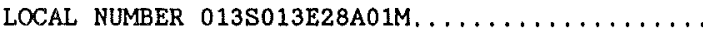

Page

25

LOCAL NLMBER 013S013E28A02M. ............

LOCAL NUMBER 013S013E28A03M...............

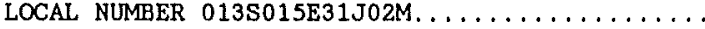

LOCAL NUMBER $0135015 E 31 J 03 M \ldots \ldots \ldots \ldots \ldots \ldots \ldots$

LOCAL NUMBER 013S015E31J04M..............

LOCAL NUMBER 013S015E31J05M............

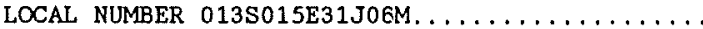

LOCAL NUMBER 013S015E31J07M. . . . . . . . . . . .

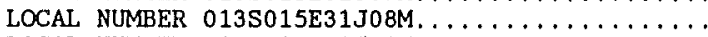

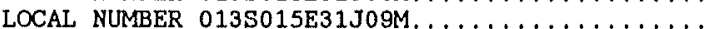

LOCAL NUMBER 013S015E31J10M.............

LOCAL NUMBER 013S015E31J11M............

LOCAL NUMBER 013S015E31J12M. . . . . . . . . . .

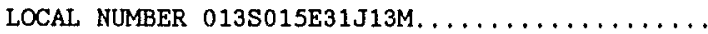

LOCAL NUMBER $0135015 E 31 \mathrm{~J} 14 \mathrm{M}, \ldots \ldots \ldots \ldots \ldots \ldots$

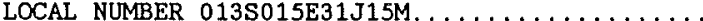

LOCAL NUMBER 014S013E24N02M. . . . . . . . .

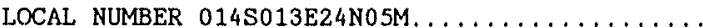

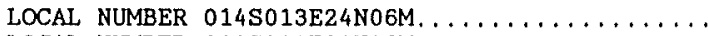

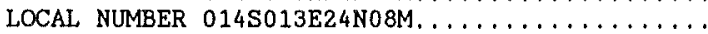

LOCAL NUMBER 014S013E24N09M . . . . . . . . . . .

LOCAL NUMBER 014 S013E24N10M. . . . . . . . . .

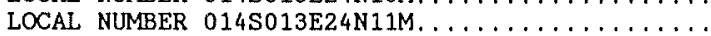

LOCAL NUMBER 014S013E24N12M. . . . . . . . .

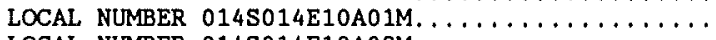

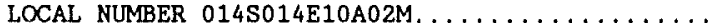

LOCAL NUMBER 014S014E10A03M. . . . . . . . . .

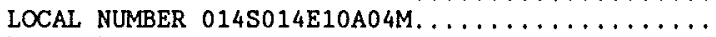

LOCAL NUMBER $015 \mathrm{~S} 012 \mathrm{E} 11 \mathrm{H} 01 \mathrm{M}, \ldots \ldots \ldots \ldots \ldots \ldots \ldots$

LOCAL NUMBER 015S012E11H02M. . . . . . . . .

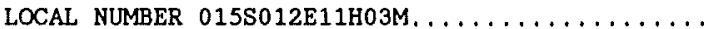

LOCAL NUMBER 015S013E05F03M . . . . . . . . .

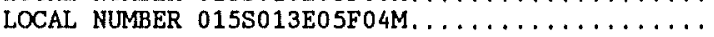

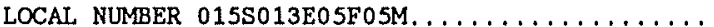

LOCAL NUMBER 015S013E11B03M. . . . . . . . . . .

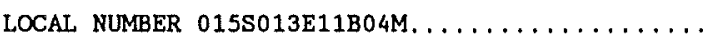

LOCAL NUMBER 015S013E11B05M...............

LOCAL NUMBER $015 S 013 E 11 B 06 M \ldots \ldots \ldots \ldots \ldots \ldots \ldots$

LOCAL NUMBER $0155013 E 11 B 07 M \ldots \ldots \ldots \ldots \ldots \ldots \ldots \ldots$

LOCAI NUMBER 015S013E22A01M..............

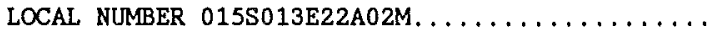

LOCAL NUMBER 015S015E08A01M . . . . . . . . . . .

LOCAL NUMBER 015S015E09D04M. . . . . . . . .

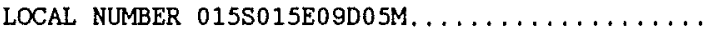

LOCAL NUMBER 015S015E09D06M . . . . . . . . . . .

LOCAL NUMBER $015 S 015 E 09 D 07 M \ldots \ldots \ldots \ldots \ldots$
Page

(3) Number in circle indicates number of wells in township for which

data are included in this report $N$
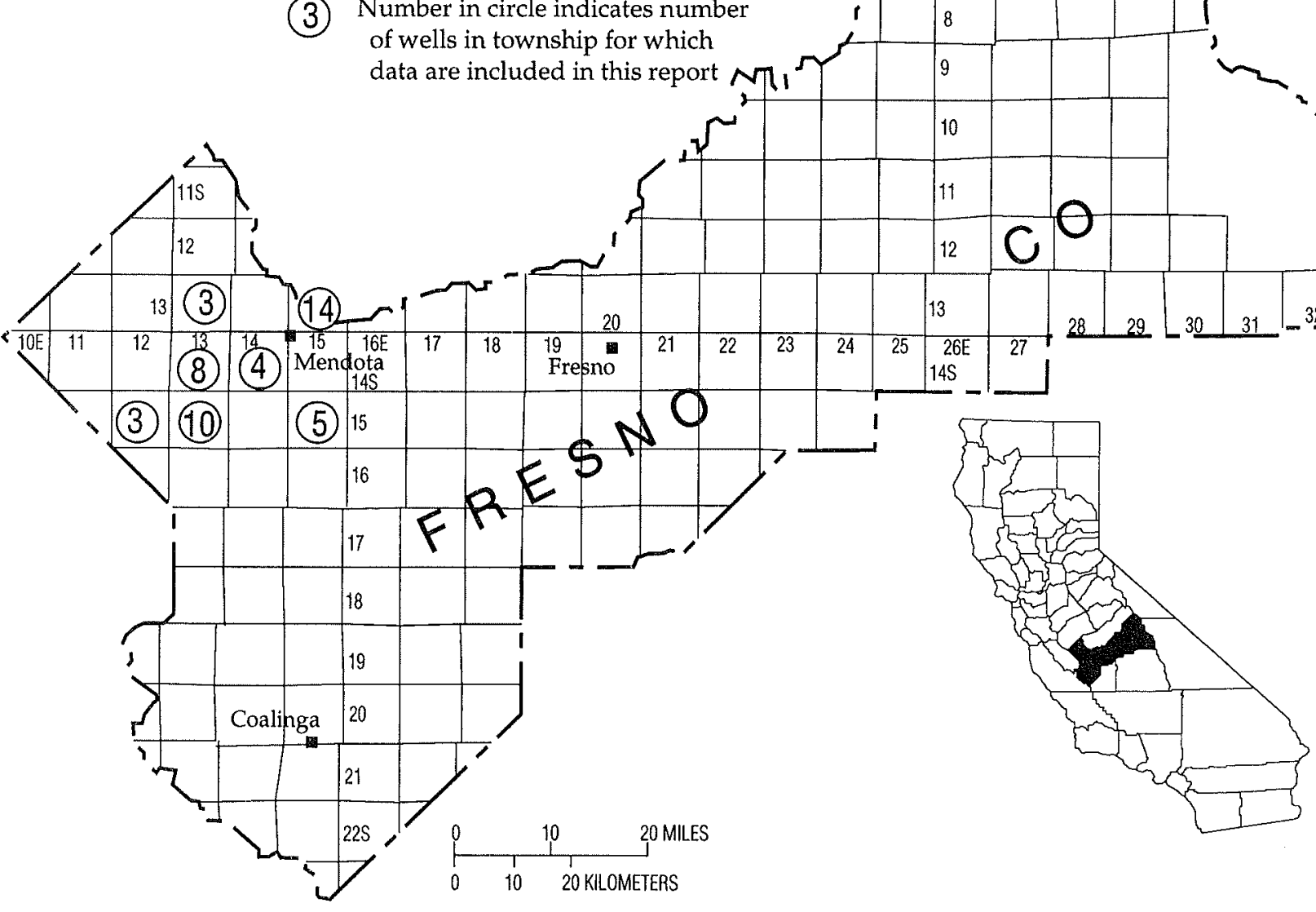

Figure 5. Location of wells in Fresno County. 
WELL DESCRIPTIONS AND WATER-LEVEL MEASUREMENTS

WATER YEAR OCTOBER 1991 TO SEPTEMBER 1992

SITE NUMBER 364640120325301

LOCAL NUMBER 013S013E28A01M

About 8 miles west of Mendota. Drilled observation well. Diameter 6 inches, depth 208 feet, perforated 193-203 feet. Altitude of land-surface datum 245 feet. Water-level records available 1987 to current year.

WATER LEVELS IN FEET BELOW LAND-SURFACE DATUM

$$
\text { DATE WATER }
$$

MAY 07, $1992 \quad 24.37 \mathrm{~s}$

$\begin{array}{llllllll}\text { PERIOD OF RECORD HIGHEST } 21,98 & \text { APR } 20,1990 & \text { LOWEST } & 27.29 & \text { NOV } & 1987\end{array}$

SITE NUMBER 364639120325301

LOCAL NUMBER 013S013E28A02M

About 8 miles west of Mendota. Drilled observation well. Diameter 6 inches, depth 103 feet, perforated 88-98 feet. Altitude of land-surface datum 245 feet. Water-level records available 1987 to current year.

WATER LEVELS IN FEET BELOW LAND-SURFACE DATUM

$$
\begin{array}{ll}
\text { WATER } \\
\text { DATE }
\end{array}
$$

MAY $07,199218.27 \mathrm{~S}$

PERIOD OF RECORD HIGHEST 17.31 SEP 14, 1990 LOWEST 24.59 NOV 1987

SITE NUMBER 364639120325303

LOCAL NUMBER 013S013E28A03M

About 8 miles west of Mendota. Drilled observation well. Diameter 5 inches, depth 437 feet, perforated 422-432 feet. Altitude of land-surface datum 245 feet. Water-level records available 1988 to current year.

WATER LEVELS IN FEET BELOW LAND-SURFACE DATUM

$$
\text { DATE WATER }
$$

MAY $07,199267.00 \mathrm{~S}$

PERIOD OF RECORD HIGHEST 62.82 JUN 30,1989 LOWEST 81.32 JUL 24,1991

SITE NUMBER 364522120222001

LOCAL NUMBER 013S015E31J02M

At Mendota Airport in Mendota. Drilled observation we11. Diameter 2 inches, depth 27 feet, perforated $22-27$ feet. Altitude of land-surface datum 152 feet. Water-level records available 1985 to current year.

WATER LEVELS IN FEET BELOW LAND-SURFACE DATUM

$$
\begin{array}{ll} 
& \text { WATER } \\
\text { DATE } & \text { LEVEL MS }
\end{array}
$$

MAY $07,1992 \quad$ SD

PERIOD OF RECORD HIGHEST 14.5 JAN 01, $1986 \quad$ LOWEST 23.22 APR 04,1988 
WATER LEVELS, FRESNO COUNTY--Continued

WELL DESCRIPTIONS AND WATER-LEVEL MEASUREMENTS

WATER YEAR OCTOBER 1991 TO SEPTEMBER 1992

SITE NUMBER 364521120222001

LOCAL NUMBER 013S015E31J03M

At Mendota Airport in Mendota. Drilled observation well. Diameter 6 inches, depth 415 feet, perforated 400-410 feet. Altitude of land-surface datum 152 feet. Water-level records available 1985 to current year.

WATER LEVELS IN FEET BELOW LAND-SURFACE DATUM

$$
\text { DATE WATER }
$$

MAY 07, $1992 \quad 98.73 \mathrm{~S}$

PERIOD OF RECORD HIGHEST 18.19 FEB 27, 1986 LOWEST 101.03 JUL 24, 1991

SITE NUMBER 364520120222001

LOCAL NUMBER 013S015E31J04M

At Mendota Airport in Mendota, Drilled observation well. Diameter 6 inches, depth 70 feet, perforated 55-65 feet. Altitude of land-surface datum 153 feet. Water-level records available 1985 to current year.

WATER LEVELS IN FEET BELOW LAND-SURFACE DATUM

$$
\begin{array}{ll} 
& \text { WATER } \\
\text { DATE } & \text { LEVEL MS }
\end{array}
$$

MAY 07, $199254.42 \mathrm{~s}$

PERIOD OF RECORD HIGHEST 15.87 MAY O6, 1986 LOWEST 54.42 MAY 07, 1992

SITE NUMBER 364519120222001

LOCAL NUMBER 013S015E31J05M

At Mendota Airport in Mendota. Drilled observation well. Diameter 6 inches, depth 255 feet, perforated 240-250 feet. Alt1tude of land-surface datum 153 feet. Water-level records available 1985 to current year.

WATER LEVELS IN FEET BELOW LAND-SURFACE DATUM

$$
\begin{array}{ll}
\text { WATER } & \text { WEVE MS }
\end{array}
$$

MAY $07,199284.84 \mathrm{~S}$

PERIOD OF RECORD HIGLEST 17.29 FEB 27, 1986 LOWEST 84.84 MAY 07, 1992

SITE NUMBER 364518120222001

LOCAL NUMBER O13S015E31J06M

At Mendota Airport in Mendota. Drilled observation well. Diameter 6 inches, depth 495 feet, perforated 480-490 feet. Altitude of land-surface datum 152 feet. Water-level records available 1985 to current year.

WATER LEVELS IN FEET BELOW LAND-SURFACE DATUM

$$
\text { DATE WATER }
$$

MAY $07,1992 \quad 94.38 \mathrm{~S}$

PERIOD OF RECORD HIGHEST 70.33 MAR 1988 LOWEST 125.97 JUL 24,1991 
WELL DESCRIPTIONS AND WATER-LEVEL MEASUREMENTS

WATER YEAR OCTOBER 1991 TO SEPTEMBER 1992

SITE NUMBER 364520120222006

LOCAL NUMBER 0135015E31J07M

At Mendota Airport in Mendota. Drilled observation well. Diameter 2 inches, depth 57 feet, perforated $54-56$ feet. Altitude of land-surface datum 152 feet. Water-level records available 1987 to current year.

WATER LEVELS IN FEET BELOW LAND-SURFACE DATUM

$$
\text { DATE NATER }
$$

MAY $07,1992 \quad 53.37 \mathrm{~s}$

PERIOD OF RECORD HIGHEST 18.72 FEB 01, 1988 LOWEST 53.37 MAY 07, 1992

SITE NUMBER 364520120222007

LOCAL NUMBER 013S015E31J08M

At Mendota Airport in Mendota. Drilled observation well. Diameter 2 inches, depth 51 feet, perforated 48-50 feet. Altitude of land-surface datum 152 feet. Water-level records avallable 1987 to current year.

WATER LEVELS IN FEET BELOW LAND-SURFACE DATUM

$$
\text { DATE WATER }
$$

MAY $07,199250.20 \mathrm{~s}$

PERIOD OF RECORD HIGHEST 19.01 FEB 01, 1988 LOWEST 50.97 JUL 24, 1991

SITE NUMBER 364520120222008

LOCAL NUMBER 013S015E31J09M

At Mendota Airport in Mendota. Drilled observation well. Diameter 2 inches, depth 41 feet, perforated $38-40$ feet. Altitude of land-surface datum 152 feet. Water-level records available 1987 to current year.

WATER LEVELS IN FEET BELOW LAND-SURFACE DATUM

$$
\text { DATE WATER }
$$

MAY $07,1992 \quad$ SD

PERIOD OF RECORD HIGHEST 18.30 APR 21, 1987 LOWEST 37.39 JUL 24, 1991

SITE NUMBER 364520120222009

LOCAL NUMBER 013S015E31J10M

At Mendota Airport in Mendota. Drilled observation well. Diameter 2 inches, depth 35 feet, perforated $32-34$ feet. Alt ltude of land-surface datum 152 feet. Water-level records available 1987 to current year.

WATER LEVELS IN FEET BELOW LAND-SURFACE DATUM

$$
\text { DATE WATER } \quad \text { LEVEL MS }
$$

MAY 07, $1992 \quad S D$

PERIOD OF RECORD HIGHEST 18.78 APR 21, 1987 LOWEST 31.39 AUG 15, 1990 
SITE NUMBER 364520120222010

LOCAL NUMBER 013S015E31J11M

At Mendota Airport in Mendota, Drilled observation well. Diameter 2 inches, depth 47 feet, perforated 44-46 feet. Altitude of land-surface datum 152 feet. Water-level records available 1987 to current year.

WATER LEVELS IN FEET BELOW LAND-SURFACE DATUM

$$
\begin{array}{ll} 
& \text { WATER } \\
\text { DATE } & \text { LEVEL MS }
\end{array}
$$

MAY 07, $199246.30 \mathrm{~s}$

PERIOD OF RECORD HIGHEST 18.69 APR 21, 1987 LOWEST 46.30 MAY 07, 1992

SITE NUMBER 364520120222011

LOCAL NUMBER 013S015E31J12M

At Mendota Airport in Mendota. Drilled observation well. Diameter 2 inches, depth 67 feet, perforated 59-64 feet. Altitude of land-surface datum 153 feet. Water-level records available 1988 to current year.

WATER LEVELS IN FEET BELOW LAND-SURFACE DATUM

$$
\begin{array}{ll} 
& \text { WATER } \\
\text { DATE } & \text { LEVEL MS }
\end{array}
$$

MAY 07, $199253.19 \mathrm{~S}$

PERIOD OF RECORD HIGHEST 26.86 FEB 28, 1990 LOWEST 53.19 MAY 07, 1992

SITE NUMBER 364520120222012

LOCAL NUMBER 013S015E31J13M

At Mendota Airport in Mendota. Drilled observation well. Diameter 2 inches, depth 38 feet, perforated $36-37$ feet. Altitude of land-surface datum 153 feet. Water-level records available 1988 to current year.

WATER LEVELS IN FEET BELOW LAND-SURFACE DATUM

$$
\text { DATE HATER }
$$

MAY 07, $199236.03 \mathrm{~S}$

PERIOD OF RECORD HIGHEST 26.26 JAN 24, 1989 LOWEST 36.03 MAY 07, 1992

SITE NUMBER 364520120222013

LOCAL NUMBER 013S015E31J14M

At Mendota Airport in Mendota. Drilled observation well. Diameter 2 inches, depth 27 feet, perforated 25-26 feet. Altitude of land-surface datum 153 feet. Water-level records available 1988 to current year.

WATER LEVELS IN FEET BELOW LAND-SURFACE DATUM

$$
\text { DATE WATER } \quad \text { LEVEL MS }
$$

MAY 07, $199226.10 \mathrm{~S}$

PERIOD OF RECORD HIGHEST 26.07 APR 28, 1989 LOWEST 26.60 MAY 25, 1989 
WELL DESCRIPTIONS AND WATER-LEVEL MEASUREMENTS

WATER YEAR OCTOBER 1991 TO SEPTEMBER 1992

SITE NUMBER 364520120222014

LOCAL NUMBER 013S015E31J15M

At Mendota Airport in Mendota. Drilled observation well. Diameter 2 inches, depth 24 feet, perforated $22-23$ feet. Altitude of land-surface datum 153 feet. Water-level records available 1988 to current year.

WATER LEVELS IN FEET BELOW LAND-SURFACE DATUM

$$
\begin{array}{ll}
\text { WATER } & \text { WEVEL MS }
\end{array}
$$

MAY $07,1992 \quad 22.40 \mathrm{~S}$

PERIOD OF RECORD HIGHEST 21.86 OCT 26,1988 LOWEST 23.63 MAY 25, 1989

SITE NUMBER 364129120304701

LOCAL NUMBER 014S013E24N02M

About 7 miles southwest of Mendota. Drilled observation well. Diameter 2 inches, depth 67 feet, perforated 62-67 feet. Altitude of land-surface datum 280 feet. Water-level records available 1985 to current year.

WATER LEVELS IN FEET BELOW LAND-SURFACE DATUM

DATE WATER

MAY $07,1992 \quad 53.68 \mathrm{~s}$

PERIOD OF RECORD HIGHEST 49.02 APR 28, 1989 LOWEST 60.5 AUG 23, 1985

SITE NUMBER 364127120304401

LOCAL NUMBER 014S013E24N05M

About 7 miles southwest of Mendota. Drilled observation well. Diameter 6 inches, depth 520 feet, perforated 505-515 feet. Altitude of land-surface datum 278 feet. Water-level records available 1985 to current year.

WATER LEVELS IN FEET BELOW LAND-SURFACE DATUM

$$
\text { DATE WATER }
$$

MAY $07,1992122.88 \mathrm{~S}$

PERIOD OF RECORD HIGHEST 85.18 APR 20, 1990 LOWEST 124.75 JUL 23, 1991

SITE NUMBER 364127120304301

LOCAL NUMBER 014S013E24N06M

About 7 miles southwest of Mendota. Drilled observation well. Diameter 6 inches, depth 114 feet, perforated 99-109 feet. Altitude of land-surface datum 278 feet. Water-level records available 1985 to current year.

WATER LEVELS IN FEET BELOW LAND-SURFACE DATUM

$$
\begin{array}{ll}
\text { WATE } & \text { WETER } \\
\text { LEVEL MS }
\end{array}
$$

MAY $07,1992 \quad 52.08 \mathrm{~S}$

PERIOD OF RECORD HIGHEST 46.51 APR 20, 1990 LOWEST 57.8 AUG 23, 1985 
WELL DESCRIPTIONS AND WATER-LEVEL MEASUREMENTS

WATER YEAR OCTOBER 1991 TO SEPTEMBER 1992

SITE NUMBER 364127120304402

LOCAL NUMBER 014S013E24N08M

About 7 miles southwest of Mendota. Drilled observation well. Diameter 2 inches, depth 90 feet, perforated 83-88 feet. Alttude of land-surface datum 278 feet. Water-1eve1 records available 1987 to current year.

WATER LEVELS IN FEET BELOW LAND-SURFACE DATUM

$$
\begin{array}{ll} 
& \text { WATER } \\
\text { DATE } & \text { LEVEL MS }
\end{array}
$$

MAY $07,1992 \quad 50.27 \mathrm{~S}$

PERIOD OF RECORD HIGHEST 47.29 FEB 28, $1990 \quad$ LOWEST 54.45 MAY 01,1987

SITE NUMBER 364127120304302

LOCAL NUMBER 014S013E24N09M

About $7 \mathrm{miles}$ southwest of Mendota. Drilled observation we11. Diameter 2 inches, depth 79 feet, perforated 72-77 feet. Altitude of land-surface datum 278 feet. Water-level records available 1987 to current year.

WATER LEVELS IN FEET BELOW LAND-SURFACE DATUM

$$
\begin{array}{ll}
\text { DATE } & \text { WATER } \\
\text { LEVEL MS }
\end{array}
$$

MAY $07,1992 \quad 51.48 \mathrm{~S}$

PERIOD OF RECORD HIGHEST 47.12 FEB 28,1990 LOWEST 54.44 MAY D1, 1987

SITE NUMBER 364127120304202

LOCAL NUMBER 014S013E24N10M

About 7 miles southwest of Mendota. Drilled observation well. Diameter 6 inches, depth 275 feet, perforated 260-270 feet. Altitude of land-surface datum 278 feet. Water-level records available 1987 to current year.

WATER LEVELS IN FEET BELOW LAND-SURFACE DATUM

$$
\begin{array}{ll} 
& \text { WATER } \\
\text { DATE } & \text { LEVEL MS }
\end{array}
$$

MAY $07,199280.48 \mathrm{~S}$

PERIOD OF RECORD HIGHEST 64.72 APR $20,1990 \quad$ LOWEST 83.35 JUL 23,1991

SITE NUMBER 364127120304502

LOCAL NUMBER 014S013E24N11M

About 7 miles southwest of Mendota. Drilled observation well. Diameter 4.5 inches, depth 879 feet, perforated 863-873 feet. Altitude of land-surface datum 279 feet. This well was 24 NO4 before deepening. Water-level records available 1988 to current year.

WATER LEVELS IN FEET BELOW LAND-SURFACE DATUM

$$
\begin{array}{ll} 
& \text { WATER } \\
\text { DATE } & \text { LEVEL MS }
\end{array}
$$

MAY $07,1992 \quad 385.97 \mathrm{~S}$

PERIOD OF RECORD HIGHEST 225.3 APR 20, $1990 \quad$ LOWEST 385.97 MAY 07,1992 
WELL DESCRIPTIONS AND WATER-LEVEL MEASUREMENTS

WATER YEAR OCTOBER 1991 TO SEPTEMBER 1992

SITE NUMBER 364127120304201

LOCAL NUMBER 014S013E24N12M

About 7 miles southwest of Mendota. Drilled observation well. Diameter 6 inches, depth 213 feet, perforated 198-208 feet. Altitude of land-surface datum 278 feet. Water-level records available 1985 to current year. Previously published in WDR CA-90-5 as well 014S013E24N07M.

WATER LEVELS IN FEET BELOW LAND-SURFACE DATUM

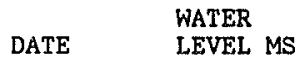

MAY 07, $199262.59 \mathrm{~s}$

PERIOD OF RECORD HIGHEST 54.07 FEB 28, $1990 \quad$ LOWEST 68.7 AUG 24, 1985

SITE NUMBER 364402120252201

LOCAL NUMBER 014S014E10A01M

About 2 miles southwest of Mendota. Drilled observation well. Diameter 2 inches, depth 18 feet, perforated 13-18 feet. Altitude of land-surface datum 188 feet. Water-level records available 1985 , 1987 to current year.

WATER LEVELS IN FEET BELOW LAND-SURFACE DATUM

$$
\text { DATE WATER }
$$

MAY $07,1992 \quad 4.28 \mathrm{~s}$

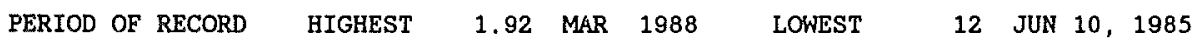

SITE NUMBER 364402120252101

LOCAL NUMBER 014S014E10A02M

About 2 miles southwest of Mendota. Drilled observation well. Diameter 2 inches, depth 88 feet, perforated 82-87 feet. Altitude of land-surface datum 188 feet. Water-level records available 1987 to current year.

WATER LEVELS IN FEET BELOW LAND-SURFACE DATUM

$$
\text { DATE WATER } \quad \text { LEVEL MS }
$$

MAY 07, $1992 \quad 9.24 \mathrm{~S}$

PERIOD OF RECORD HIGHEST 5.49 MAR 1988 LOWEST 10.22 JUL 23, 1991

SITE NUMBER 364403120252501

LOCAL NUMBER 014S014E10A03M

About 2 miles southwest of Mendota. Drilled observation well. Diameter 6 inches, depth 347 feet, perforated 332-342 feet. Altitude of land-surface datum 187 feet. Water-level records available 1987 to current year.

WATER LEVELS IN FEET BELOW LAND-SURFACE DATUM

$$
\text { DATE WATER } \quad \text { LEVEL MS }
$$

MAY 07, $1992120.77 \mathrm{~s}$

PERIOD OF RECORD HIGHEST 50.84 FEB 1988 LOWEST 123.66 JUL 23, 1991 
WATER LEVELS, FRESNO COUNTY--Continued

WELL DESCRIPTIONS AND WATER-LEVEL MEASUREMENTS

WATER YEAR OCTOBER 1991 TO SEPTEMBER 1992

SITE NUMBER 364402120252504

LOCAL NUMBER 014S014E10A04M

About 2 miles southwest of Mendota. Drilled observation well. Diameter 5 inches, depth 194 feet, perforated 180-190 feet. Altitude of land-surface datum 187 feet. Water-level records available 1988 to current year.

WATER LEVELS IN FEET BELOW LAND-SURFACE DATUM

$\begin{array}{ll} & \text { WATER } \\ \text { DATE LEVEL MS }\end{array}$

MAY 07, $199256.22 \mathrm{~S}$

PERIOD OF RECORD HIGHEST 28.37 FEB 23, 1989 LOWEST 57.64 JUL 23, 1991

SITE NUMBER 363830120372601

LOCAL NUMBER O15S012E11HO1M

About 13 miles southwest of Mendota. Drilled observation well. Diameter 6 inches, depth 570 feet, perforated 555-565 feet. Altitude of land-surface datum 475 feet. Water-level records available 1986 to current year.

WATER LEVELS IN FEET BELOW LAND-SURFACE DATUM

$$
\begin{array}{ll}
\text { DATE } & \text { WATER } \\
\text { LEVEL MS }
\end{array}
$$

MAY 07, $1992486.42 \mathrm{~S}$

PERIOD OF RECORD HIGHEST 439.6 APR 19, 1990 LOWEST 502.21 JUL 23, 1991

SITE NUMBER 363833120372801

LOCAL NUMBER 015S012E11H02M

About 13 miles southwest of Mendota. Drilled observation well. Diameter 6 inches, depth 350 feet, perforated 335-345 feet. Altitude of land-surface datum 475 feet. Water-level records available 1986 to current year.

WATER LEVELS IN FEET BELOW LAND-SURFACE DATUM

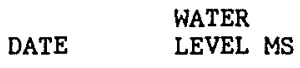

MAY 07, $1992266.52 \mathrm{~S}$

PERIOD OF RECORD HIGHEST 246.1 APR 19, 1990 LOWEST 270.27 JUN 11, 1986

SITE NUMBER 363831120372701

LOCAL NUMBER 015S012E11H03M

About 13 miles southwest of Mendota, Drilled observation well. Diameter 6 inches, depth 293 feet, perforated 278-288 feet. Altitude of land-surface datum 475 feet. Water-level records available 1986 to ourrent year.

WATER LEVELS IN FEET BELOW LAND-SURFACE DATUM

$$
\text { DATE WATER }
$$

MAY 07, $1992265.61 \mathrm{~s}$

PERIOD OF RECORD HIGHEST 246.0 APR 19, 1990 LOWEST 286.5 NOV 1986 
WELL DESCRIPTIONS AND WATER-LEVEL MEASUREMENTS

WATER YEAR OCTOBER 1991 TO SEPTEMBER 1992

SITE NUMBER 363924120343501

LOCAL NUMBER 015S013E05F03M

About 10 miles southwest of Mendota, Drilled observation well. Diameter 5 inches, depth 638 feet, perforated 623-633 feet. Altitude of land-surface datum 400 feet. Water-level records available 1988 to current year.

WATER LEVELS IN FEET BELOW LAND-SURFACE DATUM

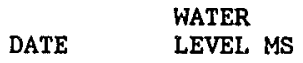

MAY 07, $1992323.07 \mathrm{~S}$

PERIOD OF RECORD HIGHEST 260.0 APR 19, 1990 LOWEST 323.07 MAY 07, 1992

SITE NUMBER 363925120343604

LOCAL NUMBER 015S013E05F04M

About 10 miles southwest of Mendota. Drilled observation well. Diameter 5 inches, depth 214 feet, perforated 198-209 feet. Altitude of land-surface datum 400 feet. Water-1evel records available 1988 to current year.

WATER LEVELS IN FEET BELOW LAND-SURFACE DATUM

$$
\text { DATE WATER }
$$

MAY 07, $1992193.60 \mathrm{~S}$

PERIOD OF RECORD HIGHEST 172.8 APR 19, 1990 LOWEST 193,60 MAY 07, 1992

SITE NUMBER 363925120343601

LOCAL NUMBER 015S013E05F05M

About 10 miles southwest of Mendota. Drilled observation well. Diameter 6 inches, depth 300 feet, perforated 285-295 feet. Altitude of land-surface datum 400 feet. Water-level records available 1987 to current year.

WATER LEVELS IN FEET BELOW LAND-SURFACE DATUM

$$
\begin{array}{ll} 
& \text { WATER } \\
\text { DATE } & \text { LEVEL MS }
\end{array}
$$

MAY $07,1992190.70 \mathrm{~S}$

PERIOD OF RECORD HIGHEST 172.8 APR 19, 1990 LOWEST 196.88 OCT 05, 1987

SITE NUMBER 363850120311901

LOCAL NUMBER 015S013E11B03M

About 7 miles southwest of Mendota. Drilled observation well. Diameter 6 inches, depth 50 feet, perforated 35-45 feet. Altitude of land-surface datum 335 feet. Water-level records available 1987 to current year.

WATER LEVELS IN FEET BELOW LAND-SURFACE DATUM

$$
\text { DATE WATER }
$$

MAY $07,1992 \quad 27.45 \mathrm{~S}$

PERIOD OF RECORD HIGHEST 24.52 SEP 14, $1990 \quad$ LOWEST 27.45 MAY 07, 1992 
SITE NUMBER 363850120311804

LOCAL NUMBER 015S013E11B04M

About 7 miles southwest of Mendota. Drilled observation well. Diameter 5 inches, depth 652 feet, perforated 637-647 feet. Altitude of land-surface datum 335 feet. Water-level records available 1988 to current year.

WATER LEVELS IN FEET BELOW LAND-SURFACE DATUM

$$
\text { DATE WATER }
$$

MAY 07, $1992168,15 \mathrm{~s}$

PERIOD OF RECORD HIGHEST 160.58 APR 20, 1990 LOWEST 182.64 JUL 23, 1991

SITE NUMBER 363850120311805

LOCAL NUMBER 015S013E11B05M

About 7 miles southwest of Mendota. Drilled observation well. Diameter 6 inches, depth 280 feet, perforated 265-275 feet. Altitude of land-surface datum 335 feet, Water-level records available 1988 to current year. WATER LEVELS IN FEET BELOW LAND-SURFACE DATUM

$$
\text { DATE WATER }
$$

MAY 07, $1992157.98 \mathrm{~S}$

PERIOD OF RECORD HIGHEST 110.61 FEB 28, 1990 LOWEST 157.98 MAY 07, 1992

SITE NUMBER 363849120311801

LOCAL NUMBER 015S013E11B06M

About 7 miles southwest of Mendota. Drilled observation well. Diameter 4.5 inches, depth 390 feet, perforated 375-385 feet. Altitude of land-surface datum 335 feet. Water-level records available 1987 to current year. Previously published in WDR CA-90-5 as well 015S013E11B01M.

WATER LEVELS IN FEET BELOW LAND-SURFACE DATUM

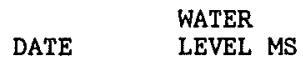

MAY $07,1992155.03 \mathrm{~S}$

PERIOD OF RECORD HIGHEST 123.03 APR 20, 1990 LOWEST 155.03 MAY 07, 1992

SITE NUMBER 363850120311801

LOCAL NUMBER 015S013E11B07M

About 7 miles southwest of Mendota. Drilled observation well. Diameter 6 inches, depth 199 feet, perforated 184-194 feet. Altitude of land-surface datum 335 feet. Water-level records available 1987 to current year. Previously published in WDR CA-90-5 as well 015S013E11B02M.

WATER LEVELS IN FEET BELOW LAND-SURFACE DATUM

$$
\begin{array}{ll} 
& \text { WATER } \\
\text { DATE } & \text { LEVEL MS }
\end{array}
$$

MAY 07, $1992118.16 \mathrm{~s}$

PERIOD OF RECORD HIGHEST 110.16 FEB 28, APR 20, 1990 LOWEST 121.23 JUN 1987 
WELL DESCRIPTIONS AND WATER-LEVEL MEASUREMENTS

WATER YEAR OCTOBER 1991 TO SEPTEMBER 1992

SITE NUMBER 363707120315401

LOCAL NUMBER 015S013E22A01M

About 9 miles southwest of Mendota. Drilled observation well. Diameter 5 inches, depth 380 feet, perforated 364-374 feet. Altitude of land-surface datum 430 feet. Water-level records available 1988 to current year.

WATER LEVELS IN FEET BELOW LAND-SURFACE DATUM

$$
\text { DATE WATER } \quad \text { LEVEL MS }
$$

MAY 07, $1992 \quad 287.94 \mathrm{~s}$

PERIOD OF RECORD HIGHEST 262.2 APR 20, 1990 LOWEST 287.94 MAY 07, 1992

SITE NUMBER 363707120315402

LOCAL NUMBER 015S013E22A02M

About 9 miles southwest of Mendota. Drilled observation well. Diameter 5 inches, depth 305 feet, perforated 290-300 feet. Altitude of land-surface datum 430 feet. Water-level records available 1989 to current year.

WATER LEVELS IN FEET BELOW LAND-SURFACE DATUM

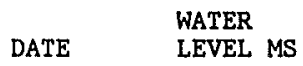

MAY 07, $1992263.78 \mathrm{~S}$

PERIOD OF RECORD HIGHEST 245.6 APR 20, 1990 LOWEST 267.00 JUN 05, 1989

SITE NUMBER 363848120210501

LOCAL NUMBER 015\$015E08A01M

About 7 miles south of Mendota. Drilled observation well. Diameter 6 inches, depth 487 feet, perforated 472-482 feet. Altitude of land-surface datum 172 feet. Water-level records available 1986 to current year. WATER LEVELS IN FEET BELOW LAND-SURFACE DATUM

$$
\text { DATE WATER }
$$

MAY $07,199272.56 \mathrm{~S}$

PERIOD OF RECORD HIGHEST 29.43 FEB 1988 LOWEST 91.85 JUL 23,1991

SITE NUMBER 363847120210201

LOCAL NUMBER 015S015E09D04M

About 7 miles south of Mendota. Drilled observation well. Diameter 6 inches, depth 130 feet, perforated 115-125 feet. Altitude of land-surface datum 172 feet. Water-level records available 1986 to current year. WATER LEVELS IN FEET BELOW LAND-SURFACE DATUM

$$
\text { DATE WATER }
$$

MAY 07, $199267.89 \mathrm{~S}$

PERIOD OF RECORD HIGHEST 28.22 MAR 1988 LOWEST 71.37 JUL 23,1991 
SITE NUMBER 363848120210301

LOCAL NUMBER 015S01.5E09D05M

About 7 miles south of Mendota. Drilled observation well. Diameter 6 inches, depth 70 feet, perforated 55-65 feet. Altitude of land-surface datum 172 feet. Water-level records available 1986 to current year.

WATER LEVELS IN FEET BELOW LAND-SURFACE DATUM

$$
\text { DATE WATER }
$$

MAY $07,1992 \quad 8.61 \mathrm{~S}$

PERIOD OF RECORD HIGHEST 5.87 JUN 1987 LOWEST 10.55 FEB 07,1991

SITE NUMBER 363846120210201

LOCAL NUMBER 015S015E09D06M

About 7 miles south of Mendota. Drilled observation well. Diameter 6 inches, depth 267 feet, perforated 252-262 feet. Altitude of land-surface datum 172 feet. Water-level records available 1986 to current year.

WATER LEVELS IN FEET BELOW LAND-SURFACE DATUM

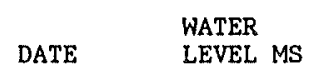

MAY 07, $199262.62 \mathrm{~S}$

PERIOD OF RECORD HIGHEST 28.64 MAY 24, 1989 LOWEST 72.58 JUL 03,1991

SITE NUMBER 363848120210302

LOCAL NUMBER 015S015E09D07M

About 7 miles south of Mendota. Drilled observation well. Diameter 2 inches, depth 28 feet, perforated 20-25 feet. Altitude of land-surface datum 172 feet. Water-level records available 1987 to current year. WATER LEVELS IN FEET BELOW LAND-SURFACE DATUM

$$
\begin{array}{ll} 
& \text { WATER } \\
\text { DATE } & \text { LEVEL MS }
\end{array}
$$

MAY $07,1992 \quad 5.73 \mathrm{~S}$

PERIOD OF RECORD HIGHEST 5.73 MAY 07, $1992 \quad$ LOWEST $8.92 \quad$ FEB 28,1990 
LOCAL NUMBER 019 N001W32G01M. . . . . . . . . . . . 39

LOCAL NUMBER 019 N001W32G02M................. 40

LOCAL NUMBER 019 N001W32G03M. .................. 41

(3) Number in circle indicates number of wells in township for which data are included in this report

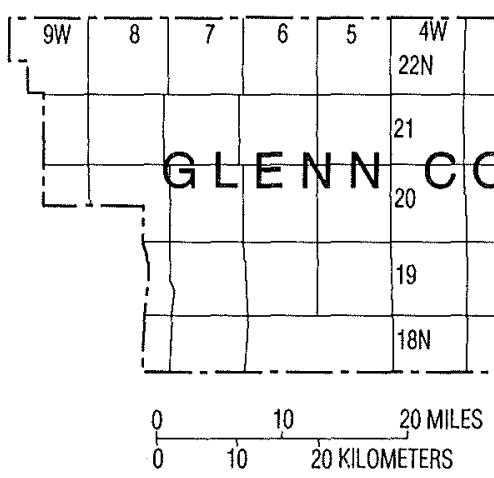

Figure 6. Location of wells in Glenn County. 


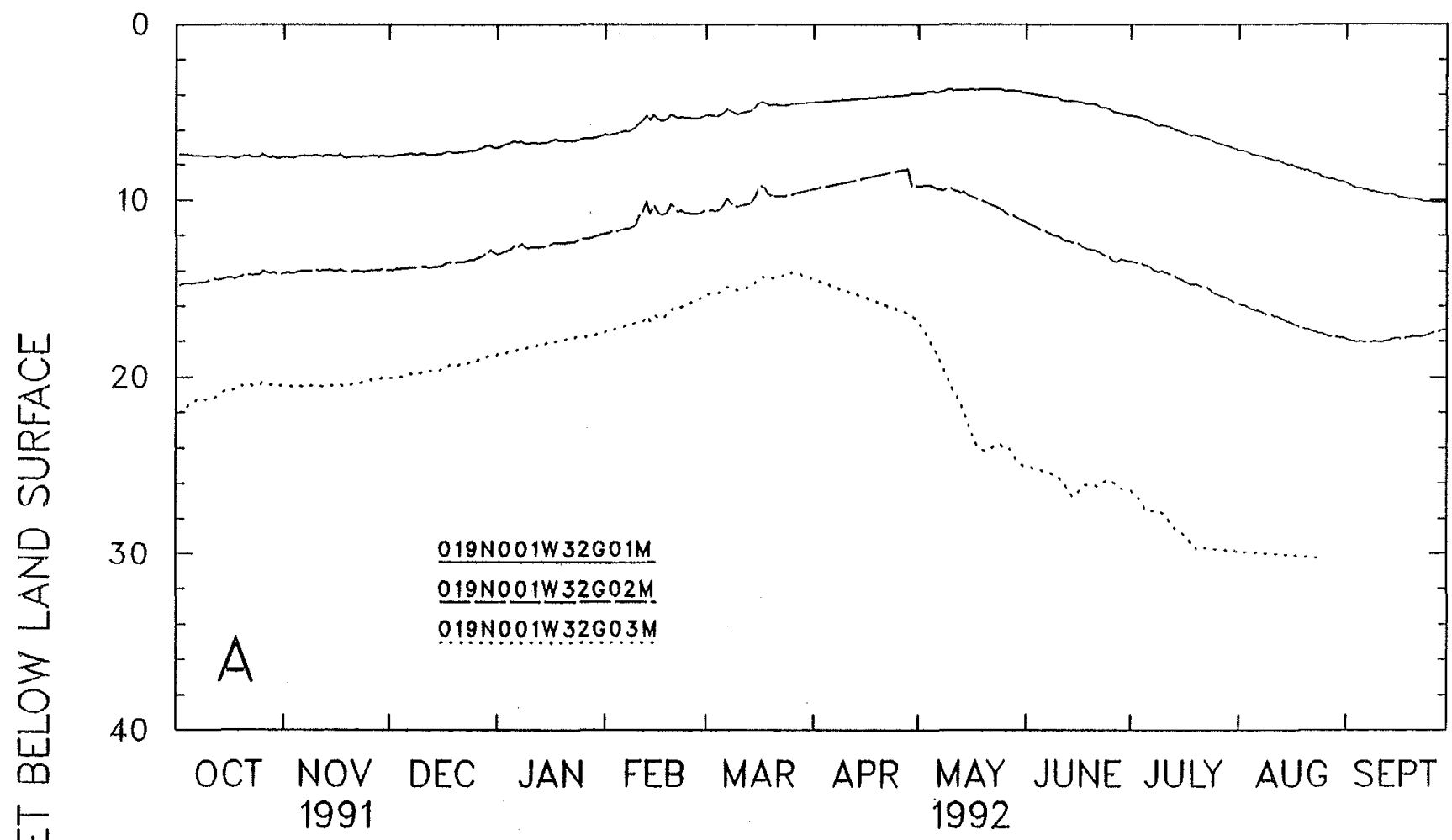

芘

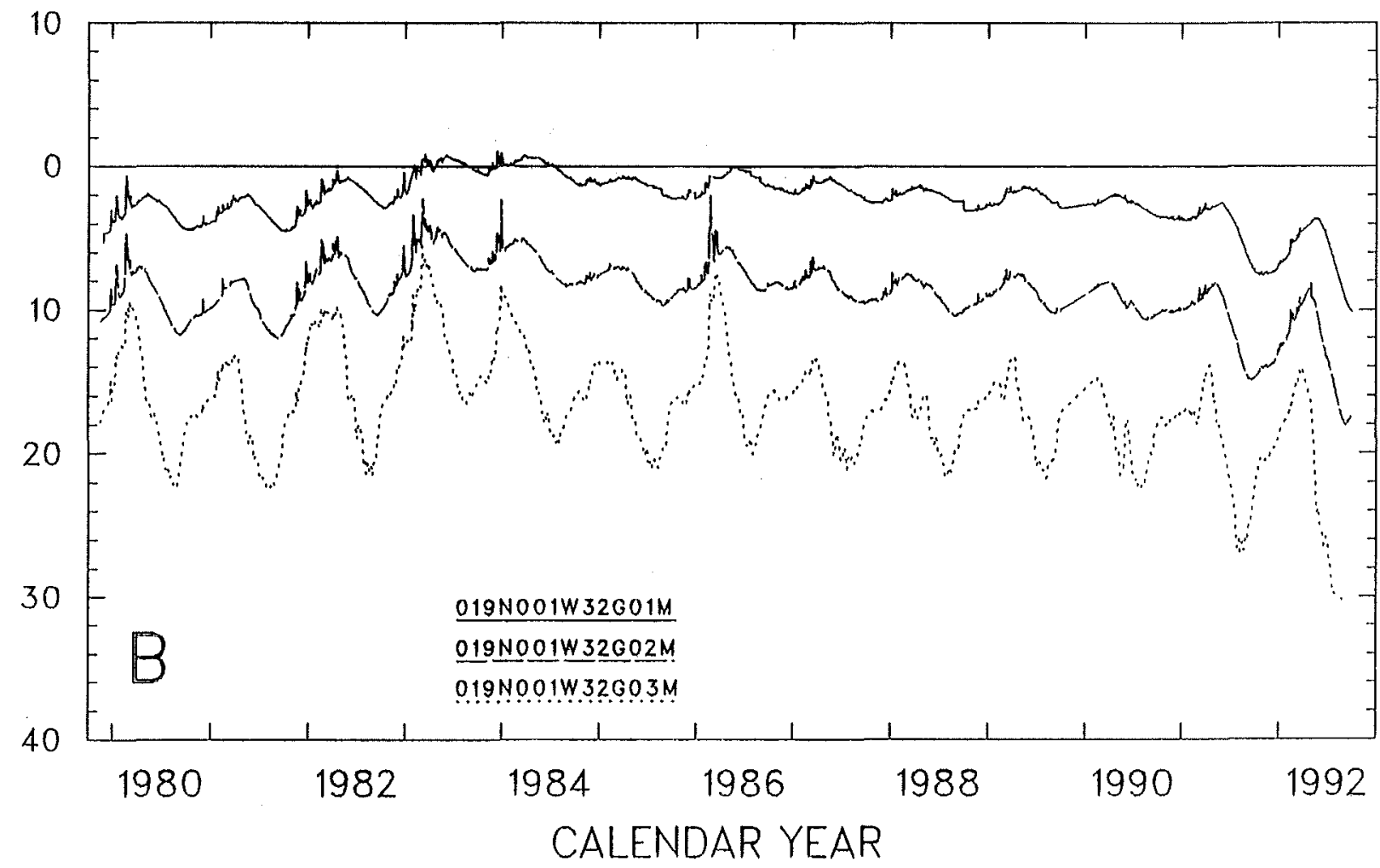

Figure 7. Hydrographs for wells in Glenn County. (O19N001W32G01M, 019N001W32G02M, and 019N001W32G03M)

A, 1992 water year. B, period of record. 
WATER LEVELS, GLENN COUNTY

WELI DESCRIPTIONS AND WATER-LEVEL MEASUREMENTS

WATER YEAR OCTOBER 1991 TO SEPTEMBER 1992

SITE NUMBER 392730121593001

LOCAL NUMBER 019N001W32G01M

About $0.5 \mathrm{mile}$ south of Butte City. Drilled observation water-table well in alluvium. Diameter 3 inches, depth 1,333 feet, screened 1,328-1,333 feet. Altitude of land-surface datum 87,40 feet. Recorder installed 1979 to current year. Water-level records available 1979 to current year.

Missing record due to malfunction of recording instrument.

WATER LEVELS IN FEET BELOW LAND-SURFACE DATUM (READINGS ABOVE LAND SURFACE INDICATED BY "+") (OBSERVATIONS AT 1200 HOURS)

\begin{tabular}{|c|c|c|c|c|c|c|c|c|c|c|c|c|}
\hline DAY & OCT & NOV & DEC & JAN & FEB & MAR & APR & MAY & JUN & JUL & AUG & SEP \\
\hline $\begin{array}{l}1 \\
2 \\
3 \\
4 \\
5\end{array}$ & $\begin{array}{r}57.41 \\
7.39 \\
7.40 \\
7.45\end{array}$ & $\begin{array}{l}7.57 \\
7.57 \\
7.60 \\
7.59 \\
7.53\end{array}$ & $\begin{array}{l}7.52 \\
7.51 \\
7.47 \\
7.43 \\
7.42\end{array}$ & $\begin{array}{l}6.99 \\
6.94 \\
6.85 \\
6.81 \\
6.70\end{array}$ & $\begin{array}{l}6.26 \\
6.28 \\
6.24 \\
6.21 \\
6.17\end{array}$ & $\begin{array}{l}5.18 \\
5.14 \\
5.18 \\
5.20 \\
5.12\end{array}$ & $\begin{array}{l}\cdots- \\
-- \\
\cdots- \\
\cdots-\end{array}$ & $\begin{array}{l}3.92 \\
3.89 \\
3.85 \\
3.80 \\
3.79\end{array}$ & $\begin{array}{l}--- \\
-\cdots \\
--- \\
---\end{array}$ & $\begin{array}{l}5.19 \\
5.23 \\
5.25 \\
5.35 \\
5.42\end{array}$ & $\begin{array}{l}7.17 \\
7.17 \\
7.21 \\
7.34 \\
7.38\end{array}$ & $\begin{array}{l}9.06 \\
9.16 \\
9.25 \\
9.32 \\
9.32\end{array}$ \\
\hline $\begin{array}{r}6 \\
7 \\
8 \\
9 \\
10\end{array}$ & $\begin{array}{l}7.47 \\
7.47 \\
7.48 \\
7.50 \\
7.53\end{array}$ & $\begin{array}{l}7.49 \\
7.48 \\
7.47 \\
7.46 \\
7.45\end{array}$ & $\begin{array}{l}7.39 \\
7.35 \\
7.41 \\
7.43 \\
7.35\end{array}$ & $\begin{array}{l}6.65 \\
6.70 \\
6.64 \\
6.74 \\
6.74\end{array}$ & $\begin{array}{l}6.10 \\
6.04 \\
6.08 \\
5.96 \\
5.86\end{array}$ & $\begin{array}{l}5.03 \\
4.81 \\
4.93 \\
5.02 \\
5.08\end{array}$ & $\begin{array}{l}--- \\
--- \\
--- \\
--- \\
---\end{array}$ & $\begin{array}{l}3.82 \\
3.84 \\
3.79 \\
3.71 \\
3.68\end{array}$ & $\begin{array}{r}-.- \\
0.14 \\
4.15\end{array}$ & $\begin{array}{l}5.47 \\
5.63 \\
5.71 \\
5.75 \\
5.74\end{array}$ & $\begin{array}{l}7.42 \\
7.48 \\
7.57 \\
7.65 \\
7.68\end{array}$ & $\begin{array}{l}9.38 \\
9.42 \\
9.48 \\
9.50 \\
9.58\end{array}$ \\
\hline $\begin{array}{l}11 \\
12 \\
13 \\
14 \\
15\end{array}$ & $\begin{array}{l}7.49 \\
7.49 \\
7.54 \\
7.57 \\
7.54\end{array}$ & $\begin{array}{l}7.49 \\
7.50 \\
7.45 \\
7.43 \\
7.47\end{array}$ & $\begin{array}{l}7.34 \\
7.42 \\
7.44 \\
7.43 \\
7.41\end{array}$ & $\begin{array}{l}6.71 \\
6.71 \\
6.75 \\
6.72 \\
6.71\end{array}$ & $\begin{array}{l}5.62 \\
5.47 \\
5.17 \\
5.47 \\
5.15\end{array}$ & $\begin{array}{l}5.03 \\
4.99 \\
4.95 \\
4.91 \\
4.75\end{array}$ & $\begin{array}{l}--- \\
--- \\
-\cdots \\
-\cdots\end{array}$ & $\begin{array}{l}3.73 \\
3.72 \\
3.72 \\
3.69 \\
3.72\end{array}$ & $\begin{array}{l}4.32 \\
4.36 \\
4.36 \\
4.33 \\
4.35\end{array}$ & $\begin{array}{l}5.76 \\
5.81 \\
5.91 \\
6.01 \\
6.04\end{array}$ & $\begin{array}{l}7.74 \\
7.76 \\
7.85 \\
7.92 \\
7.99\end{array}$ & $\begin{array}{l}9.62 \\
9.67 \\
9.66 \\
9.66 \\
9.72\end{array}$ \\
\hline $\begin{array}{l}16 \\
17 \\
18 \\
19 \\
20\end{array}$ & $\begin{array}{l}7.51 \\
7.54 \\
7.64 \\
7.61 \\
7.52\end{array}$ & $\begin{array}{l}7.50 \\
7.40 \\
7.54 \\
7.58 \\
7.55\end{array}$ & $\begin{array}{l}7.38 \\
7.29 \\
7.25 \\
7.33 \\
7.31\end{array}$ & $\begin{array}{l}6.69 \\
6.56 \\
6.54 \\
6.59 \\
6.59\end{array}$ & $\begin{array}{l}5.34 \\
5.46 \\
5.47 \\
5.38 \\
5.15\end{array}$ & $\begin{array}{l}4.49 \\
4.39 \\
4.43 \\
4.58 \\
4.55\end{array}$ & $\begin{array}{l}--- \\
--- \\
--- \\
--- \\
--\end{array}$ & $\begin{array}{l}3.68 \\
3.72 \\
3.72 \\
3.68 \\
3.68\end{array}$ & $\begin{array}{l}4.40 \\
4.44 \\
4.51 \\
4.49 \\
4.48\end{array}$ & $\begin{array}{l}6.13 \\
6.18 \\
6.31 \\
6.29 \\
6.32\end{array}$ & $\begin{array}{l}7.98 \\
8.08 \\
8.18 \\
8.25 \\
8.29\end{array}$ & $\begin{array}{l}9.81 \\
9.86 \\
9.89 \\
9.92 \\
9.93\end{array}$ \\
\hline $\begin{array}{l}21 \\
22 \\
23 \\
24 \\
25\end{array}$ & $\begin{array}{l}7.47 \\
7.48 \\
7.57 \\
7.57 \\
7.55\end{array}$ & $\begin{array}{l}7.53 \\
7.52 \\
7.56 \\
7.53 \\
7.50\end{array}$ & $\begin{array}{l}7.27 \\
7.26 \\
7.25 \\
7.21 \\
7.20\end{array}$ & $\begin{array}{l}6.59 \\
6.61 \\
6.60 \\
6.57 \\
6.53\end{array}$ & $\begin{array}{l}5.19 \\
5.32 \\
5.26 \\
5.31 \\
5.31\end{array}$ & $\begin{array}{l}4.56 \\
4.57 \\
4.58 \\
4.58 \\
4.54\end{array}$ & $\begin{array}{l}--- \\
--- \\
--- \\
--- \\
--\end{array}$ & $\begin{array}{l}3.68 \\
3.67 \\
3.67 \\
3.68 \\
3.71\end{array}$ & $\begin{array}{l}4.54 \\
4.68 \\
4.75 \\
4.75 \\
4.84\end{array}$ & $\begin{array}{l}6.38 \\
6.44 \\
6.48 \\
6.58 \\
6.70\end{array}$ & $\begin{array}{l}8.29 \\
8.48 \\
8.52 \\
8.56 \\
8.69\end{array}$ & $\begin{array}{r}9.99 \\
10.02 \\
10.08 \\
10.08 \\
10.10\end{array}$ \\
\hline $\begin{array}{l}26 \\
27 \\
28 \\
29 \\
30 \\
31\end{array}$ & $\begin{array}{l}7.38 \\
7.52 \\
7.58 \\
7.53 \\
7.62 \\
7.61\end{array}$ & $\begin{array}{r}7.48 \\
7.46 \\
7.50 \\
7.47 \\
7.50 \\
-\ldots\end{array}$ & $\begin{array}{l}7.17 \\
7.05 \\
6.99 \\
6.87 \\
6.86 \\
6.98\end{array}$ & $\begin{array}{l}6.47 \\
6.49 \\
6.48 \\
6.43 \\
6.39 \\
6.31\end{array}$ & $\begin{array}{r}5.32 \\
5.32 \\
5.32 \\
5.22 \\
-\ldots \\
-.-\end{array}$ & $\begin{array}{c}S 4.50 \\
\cdots \\
\cdots \\
\cdots- \\
\cdots\end{array}$ & $\begin{array}{r}--- \\
--.99 \\
\text { S3.99 } \\
3.90 \\
---\end{array}$ & $\begin{array}{l}3.77 \\
3.75 \\
3.74 \\
3.78 \\
3.79 \\
3.86\end{array}$ & $\begin{array}{r}4.98 \\
5.00 \\
5.04 \\
5.11 \\
5.17 \\
-.-\end{array}$ & $\begin{array}{l}6.76 \\
6.80 \\
6.88 \\
6.93 \\
7.00 \\
7.09\end{array}$ & $\begin{array}{l}8.75 \\
8.81 \\
8.80 \\
8.86 \\
8.94 \\
8.96\end{array}$ & $\begin{array}{r}10.12 \\
10.12 \\
10.12 \\
10.13 \\
10.15 \\
. .-\end{array}$ \\
\hline
\end{tabular}


WELL DESCRIPTIONS AND WATER-LEVEL MEASUREMENTS

WATER YEAR OCTOBER 1991 TO SEPTEMBER 1992

\section{SITE NUMBER 392730121593002}

LOCAL NUMBER 019N001W32G02M

About 0.5 mile south of Butte City. Drilled observation water-table well in alluvium. Diameter 3 inches, depth 968 feet, screened $963-968$ feet. Altitude of land-surface datum 87.40 feet. Recorder installed 1979 to current year. Water-level records available 1979 to current year.

Missing record due to malfunction of recording instrument.

WATER LEVELS IN FEET BELOW LAND-SURFACE DATUM (OBSERVATIONS AT 1200 HOURS)

\begin{tabular}{|c|c|c|c|c|c|c|c|c|c|c|c|c|}
\hline DAY & OCT & NOV & DEC & JAN & FEB & MAR & APR & MAY & JUN & JUL & AUG & SEP \\
\hline $\begin{array}{l}1 \\
2 \\
3 \\
4 \\
5\end{array}$ & $\begin{array}{r}514.81 \\
14.75 \\
14.73 \\
14.74\end{array}$ & $\begin{array}{l}14.12 \\
14.11 \\
14.14 \\
14.12 \\
14.07\end{array}$ & $\begin{array}{l}13.98 \\
13.97 \\
13.91 \\
13.89 \\
13.86\end{array}$ & $\begin{array}{l}13.05 \\
13.01 \\
12.91 \\
12.86 \\
12.76\end{array}$ & $\begin{array}{l}11.90 \\
11.86 \\
11.82 \\
11.80 \\
11.77\end{array}$ & $\begin{array}{l}10.65 \\
10.59 \\
10.62 \\
10.63 \\
10.52\end{array}$ & $\begin{array}{l}--- \\
--- \\
--- \\
---\end{array}$ & $\begin{array}{l}9.24 \\
9.23 \\
9.19 \\
9.18 \\
9.22\end{array}$ & $\begin{array}{l}-- \\
-- \\
-\cdots \\
---\end{array}$ & $\begin{array}{l}13.47 \\
13.53 \\
13.55 \\
13.63 \\
13.71\end{array}$ & $\begin{array}{l}15.90 \\
15.92 \\
15.98 \\
16.13 \\
16.19\end{array}$ & $\begin{array}{l}17.85 \\
17.96 \\
17.99 \\
18.03 \\
18.00\end{array}$ \\
\hline $\begin{array}{r}6 \\
7 \\
8 \\
9 \\
10\end{array}$ & $\begin{array}{l}14.73 \\
14.68 \\
14.66 \\
14.64 \\
14.62\end{array}$ & $\begin{array}{l}14.02 \\
14.02 \\
14.00 \\
14.01 \\
13.97\end{array}$ & $\begin{array}{l}13.85 \\
13.81 \\
13.82 \\
13.83 \\
13.79\end{array}$ & $\begin{array}{l}12.52 \\
12.65 \\
12.46 \\
12.67 \\
12.70\end{array}$ & $\begin{array}{l}11.71 \\
11.64 \\
11.65 \\
11.56 \\
11.47\end{array}$ & $\begin{array}{r}10.32 \\
9.94 \\
10.18 \\
10.33 \\
10.38\end{array}$ & $\begin{array}{l}--- \\
--- \\
--- \\
---\end{array}$ & $\begin{array}{l}9.32 \\
9.37 \\
9.41 \\
9.31 \\
9.33\end{array}$ & $\begin{array}{r}-.- \\
12.02 \\
12.04\end{array}$ & $\begin{array}{l}13.76 \\
13.89 \\
14.00 \\
14.07 \\
14.03\end{array}$ & $\begin{array}{l}16.24 \\
16.30 \\
16.39 \\
16.49 \\
16.51\end{array}$ & $\begin{array}{l}18.01 \\
18.00 \\
18.02 \\
17.99 \\
18.03\end{array}$ \\
\hline $\begin{array}{l}11 \\
12 \\
13 \\
14 \\
15\end{array}$ & $\begin{array}{l}14.53 \\
14.51 \\
14.52 \\
14.51 \\
14.44\end{array}$ & $\begin{array}{l}14.00 \\
13.99 \\
13.97 \\
13.95 \\
14.01\end{array}$ & $\begin{array}{l}13.78 \\
13.80 \\
13.80 \\
13.79 \\
13.77\end{array}$ & $\begin{array}{l}12.67 \\
12.66 \\
12.67 \\
12.62 \\
12.61\end{array}$ & $\begin{array}{l}10.93 \\
10.67 \\
10.14 \\
10.86 \\
10.31\end{array}$ & $\begin{array}{r}10.34 \\
10.30 \\
10.26 \\
10.18 \\
9.94\end{array}$ & $\begin{array}{l}--- \\
--- \\
--- \\
---\end{array}$ & $\begin{array}{l}9.45 \\
9.50 \\
9.59 \\
9.54 \\
9.74\end{array}$ & $\begin{array}{l}12.19 \\
12.31 \\
12.33 \\
12.41 \\
12.42\end{array}$ & $\begin{array}{l}14.10 \\
14.16 \\
14.24 \\
14.40 \\
14.46\end{array}$ & $\begin{array}{l}16.59 \\
16.60 \\
16.71 \\
16.81 \\
16.88\end{array}$ & $\begin{array}{l}18.01 \\
17.99 \\
17.94 \\
17.88 \\
17.81\end{array}$ \\
\hline $\begin{array}{l}16 \\
17 \\
18 \\
19 \\
20\end{array}$ & $\begin{array}{l}14.39 \\
14.39 \\
14.46 \\
14.38 \\
14.29\end{array}$ & $\begin{array}{l}14.02 \\
13.95 \\
14.05 \\
14.08 \\
14.07\end{array}$ & $\begin{array}{l}13.73 \\
13.60 \\
13.53 \\
13.58 \\
13.54\end{array}$ & $\begin{array}{l}12.57 \\
12.44 \\
12.42 \\
12.43 \\
12.42\end{array}$ & $\begin{array}{l}10.66 \\
10.84 \\
10.84 \\
10.74 \\
10.31\end{array}$ & $\begin{array}{l}9.38 \\
9.23 \\
9.35 \\
9.71 \\
9.74\end{array}$ & $\begin{array}{l}--- \\
--- \\
--- \\
---\end{array}$ & $\begin{array}{r}9.77 \\
9.89 \\
9.96 \\
10.02 \\
10.12\end{array}$ & $\begin{array}{l}12.47 \\
12.61 \\
12.75 \\
12.79 \\
12.81\end{array}$ & $\begin{array}{l}14.59 \\
14.65 \\
14.76 \\
14.76 \\
14.78\end{array}$ & $\begin{array}{l}16.91 \\
17.07 \\
17.13 \\
17.24 \\
17.31\end{array}$ & $\begin{array}{l}17.84 \\
17.86 \\
17.79 \\
17.77 \\
17.70\end{array}$ \\
\hline $\begin{array}{l}21 \\
22 \\
23 \\
24 \\
25\end{array}$ & $\begin{array}{l}14.22 \\
14.20 \\
14.26 \\
14.22 \\
14.20\end{array}$ & $\begin{array}{l}14.03 \\
14.03 \\
14.06 \\
14.04 \\
14.02\end{array}$ & $\begin{array}{l}13.52 \\
13.49 \\
13.48 \\
13.40 \\
13.37\end{array}$ & $\begin{array}{l}12.41 \\
12.41 \\
12.40 \\
12.28 \\
12.24\end{array}$ & $\begin{array}{l}10.36 \\
10.70 \\
10.60 \\
10.75 \\
10.77\end{array}$ & $\begin{array}{l}9.79 \\
9.79 \\
9.78 \\
9.76 \\
9.70\end{array}$ & $\begin{array}{l}--- \\
-- \\
-- \\
--\end{array}$ & $\begin{array}{l}10.21 \\
10.32 \\
10.38 \\
10.50 \\
10.58\end{array}$ & $\begin{array}{l}12.87 \\
12.98 \\
13.10 \\
13.21 \\
13.29\end{array}$ & $\begin{array}{l}14.87 \\
14.93 \\
14.98 \\
15.13 \\
15.28\end{array}$ & $\begin{array}{l}17.29 \\
17.44 \\
17.47 \\
17.53 \\
17.56\end{array}$ & $\begin{array}{l}17.75 \\
17.74 \\
17.71 \\
17.66 \\
17.57\end{array}$ \\
\hline $\begin{array}{l}26 \\
27 \\
28 \\
29 \\
30 \\
31\end{array}$ & $\begin{array}{l}14.02 \\
14.09 \\
14.13 \\
14.10 \\
14.19 \\
14.17\end{array}$ & $\begin{array}{r}13.99 \\
13.96 \\
13.98 \\
13.95 \\
13.98 \\
---\end{array}$ & $\begin{array}{l}13.32 \\
13.21 \\
13.14 \\
13.00 \\
12.82 \\
13.00\end{array}$ & $\begin{array}{l}12.18 \\
12.18 \\
12.15 \\
12.08 \\
12.04 \\
11.95\end{array}$ & $\begin{array}{r}10.80 \\
10.79 \\
10.79 \\
10.68 \\
- \\
-\end{array}$ & $\begin{array}{c}\text { S9. } 66 \\
\ldots \\
\ldots\end{array}$ & $\begin{array}{r}--- \\
\text { s8.25 } \\
9.21 \\
9.23 \\
-\end{array}$ & $\begin{array}{l}10.76 \\
10.84 \\
10.92 \\
11.02 \\
11.11 \\
11.22\end{array}$ & $\begin{array}{r}13.45 \\
13.51 \\
13.35 \\
13.38 \\
13.45 \\
.\end{array}$ & $\begin{array}{l}15.36 \\
15.43 \\
15.50 \\
15.59 \\
15.71 \\
15.80\end{array}$ & $\begin{array}{l}17.66 \\
17.71 \\
17.68 \\
17.73 \\
17.77 \\
17.79\end{array}$ & $\begin{array}{r}17.52 \\
17.46 \\
17.39 \\
17.36 \\
17.34 \\
-\end{array}$ \\
\hline
\end{tabular}

PERIOD OF RECORD HIGHEST 1.95 FEB 19, 1986 LOWEST 18.03 SEP 4, 10, 1992

S Manual steel tape measurement. 
WATER LEVELS, GLENN COUNTY--Continued

WELL DESCRIPTIONS AND WATER-LEVEL MEASUREMENTS

WATER YEAR OCTOBER 1991 TO SEPTEMBER 1992

SITE NUMBER 392730121593003

LOCAL NUMBER 019N001W32G03M

About 0.5 mile south of Butte City. Drilled observation water-table well in alluvium. Diameter 3 inches, depth 595 feet, screened 590-595 feet. Altitude of land-surface datum 87.40 feet. Recorder installed 1979 to ourrent year. Water-level records avallable 1979 to current year.

Missing record due to malfunction of recording instrument.

WATER LEVELS IN FEET BELOW LAND-SURFACE DATUM (OBSERVATIONS AT 1200 HOURS)

\begin{tabular}{|c|c|c|c|c|c|c|c|c|c|c|c|c|}
\hline DAY & $\infty \mathrm{C}$ & NOV & DEC & JAN & FEB & MAR & APR & MAY & JUN & JUL & AUG & SEP \\
\hline $\begin{array}{l}1 \\
2 \\
3 \\
4 \\
5\end{array}$ & $\begin{array}{r}\text { S22. } \\
21.92 \\
21.68 \\
21.53\end{array}$ & $\begin{array}{l}20.49 \\
20.50 \\
20.53 \\
20.55 \\
20.54\end{array}$ & $\begin{array}{l}20.06 \\
20.05 \\
19.99 \\
19.96 \\
19.95\end{array}$ & $\begin{array}{l}-\cdots \\
-\cdots \\
--- \\
-\cdots\end{array}$ & $\begin{array}{l}--- \\
--- \\
--- \\
---\end{array}$ & $\begin{array}{l}15.42 \\
15.34 \\
15.32 \\
15.31 \\
15.25\end{array}$ & $\begin{array}{l}--- \\
--- \\
--- \\
--- \\
---\end{array}$ & $\begin{array}{l}16.95 \\
17.21 \\
17.59 \\
17.97 \\
18.23\end{array}$ & $\begin{array}{l}--- \\
-- \\
-\cdots \\
-- \\
--\end{array}$ & $\begin{array}{l}26.44 \\
26.56 \\
26.80 \\
27.07 \\
27.43\end{array}$ & $\begin{array}{l}--- \\
--- \\
--- \\
--\end{array}$ & $\begin{array}{l}--- \\
--- \\
--- \\
--- \\
---\end{array}$ \\
\hline $\begin{array}{r}6 \\
7 \\
8 \\
9 \\
10\end{array}$ & $\begin{array}{l}21.40 \\
21.29 \\
21.27 \\
21.28 \\
21.29\end{array}$ & $\begin{array}{l}20.53 \\
20.50 \\
20.50 \\
20.49 \\
20.49\end{array}$ & $\begin{array}{l}19.82 \\
19.79 \\
19.79 \\
19.79 \\
19.75\end{array}$ & $\begin{array}{l}--- \\
-- \\
-\cdots \\
-\cdots\end{array}$ & $\begin{array}{l}--- \\
--- \\
--- \\
--- \\
-\cdots\end{array}$ & $\begin{array}{l}15.12 \\
14.95 \\
15.03 \\
15.07 \\
15.11\end{array}$ & $\begin{array}{l}-- \\
-- \\
-- \\
--\end{array}$ & $\begin{array}{l}18.57 \\
19.01 \\
19.48 \\
19.88 \\
20.42\end{array}$ & $\begin{array}{r}--- \\
--- \\
--- \\
25.49 \\
25.64\end{array}$ & $\begin{array}{l}27.58 \\
27.58 \\
27.58 \\
27.60 \\
27.68\end{array}$ & $\begin{array}{l}--- \\
--- \\
--- \\
--- \\
--\end{array}$ & $\begin{array}{l}--- \\
--- \\
-- \\
-- \\
--\end{array}$ \\
\hline $\begin{array}{l}11 \\
12 \\
13 \\
14 \\
15\end{array}$ & $\begin{array}{l}21.27 \\
21.16 \\
21.09 \\
20.95 \\
20.80\end{array}$ & $\begin{array}{l}20.51 \\
20.52 \\
20.51 \\
20.47 \\
20.50\end{array}$ & $\begin{array}{l}19.63 \\
19.64 \\
19.64 \\
19.63 \\
19.60\end{array}$ & $\begin{array}{l}--- \\
--- \\
-- \\
--\end{array}$ & $\begin{array}{l}--- \\
16.87 \\
16.59 \\
16.87 \\
16.56\end{array}$ & $\begin{array}{l}15.10 \\
15.06 \\
14.95 \\
14.90 \\
14.72\end{array}$ & $\begin{array}{l}--- \\
--- \\
--- \\
--- \\
---\end{array}$ & $\begin{array}{l}20.81 \\
21.11 \\
21.50 \\
22.00 \\
22.55\end{array}$ & $\begin{array}{l}25.86 \\
26.07 \\
26.37 \\
26.67 \\
26.75\end{array}$ & $\begin{array}{l}27.85 \\
28.17 \\
28.50 \\
28.61 \\
28.74\end{array}$ & $\begin{array}{l}--- \\
--- \\
--- \\
-\cdots\end{array}$ & $\begin{array}{l}-- \\
-- \\
-- \\
--\end{array}$ \\
\hline $\begin{array}{l}16 \\
17 \\
18 \\
19 \\
20\end{array}$ & $\begin{array}{l}20.74 \\
20.71 \\
20.71 \\
20.70 \\
20.49\end{array}$ & $\begin{array}{l}20.51 \\
20.43 \\
20.48 \\
20.48 \\
20.44\end{array}$ & $\begin{array}{l}19.58 \\
19.56 \\
19.34 \\
19.38 \\
19.35\end{array}$ & $\begin{array}{l}--- \\
--- \\
--- \\
--\end{array}$ & $\begin{array}{r}16.66 \\
16.71 \\
16.64 \\
-.- \\
-\end{array}$ & $\begin{array}{l}14.47 \\
14.36 \\
14.38 \\
14.46 \\
14.41\end{array}$ & $\begin{array}{l}-- \\
-\cdots \\
-\cdots \\
--\end{array}$ & $\begin{array}{l}23.12 \\
23.63 \\
23.98 \\
24.13 \\
24.16\end{array}$ & $\begin{array}{l}26.51 \\
26.24 \\
26.12 \\
26.12 \\
26.13\end{array}$ & $\begin{array}{l}28.90 \\
29.05 \\
29.35 \\
29.61 \\
29.77\end{array}$ & -- & $\begin{array}{l}-- \\
-- \\
-- \\
--\end{array}$ \\
\hline $\begin{array}{l}21 \\
22 \\
23 \\
24 \\
25\end{array}$ & $\begin{array}{l}20.42 \\
20.41 \\
20.47 \\
20.46 \\
20.44\end{array}$ & $\begin{array}{l}20.39 \\
20.34 \\
20.32 \\
20.27 \\
20.22\end{array}$ & $\begin{array}{l}19.30 \\
19.27 \\
1.9 .23 \\
19.15 \\
19.10\end{array}$ & $\begin{array}{r}--- \\
--- \\
\text { S17. } \\
17.76\end{array}$ & $\begin{array}{r}16.10 \\
16.16 \\
16.07 \\
15.98 \\
---\end{array}$ & $\begin{array}{l}14.35 \\
14.31 \\
14.25 \\
14.23 \\
14.15\end{array}$ & $\begin{array}{l}--- \\
-\cdots \\
--- \\
--- \\
---\end{array}$ & $\begin{array}{l}24.15 \\
23.99 \\
23.83 \\
23.83 \\
23.92\end{array}$ & $\begin{array}{l}26.17 \\
26.18 \\
26.00 \\
25.85 \\
25.87\end{array}$ & $\begin{array}{l}29.73 \\
29.67 \\
29.74 \\
29.76 \\
29.77\end{array}$ & $\begin{array}{r}--- \\
\cdots \\
--- \\
\\
\text { S30.- } \\
.26\end{array}$ & $\begin{array}{l}-- \\
-- \\
-- \\
-- \\
--\end{array}$ \\
\hline $\begin{array}{l}26 \\
27 \\
28 \\
29 \\
30 \\
31\end{array}$ & $\begin{array}{l}20.31 \\
20.41 \\
20.45 \\
20.44 \\
20.50 \\
20.50\end{array}$ & $\begin{array}{r}20.16 \\
20.10 \\
20.11 \\
20.05 \\
20.06 \\
.--\end{array}$ & $\begin{array}{r}19.05 \\
18.97 \\
18.87 \\
--- \\
--- \\
---\end{array}$ & $\begin{array}{r}17.74 \\
17.74 \\
17.73 \\
--- \\
--- \\
---\end{array}$ & $\begin{array}{r}15.81 \\
15.73 \\
15.66 \\
15.51 \\
\ldots .- \\
. .-\end{array}$ & $\begin{array}{r}\mathrm{S} 14.09 \\
\ldots \\
- \\
- \\
-\end{array}$ & $\begin{array}{r}\text { S16. } 41 \\
16.55 \\
16.74 \\
\end{array}$ & $\begin{array}{l}23.93 \\
24.02 \\
24.28 \\
24.66 \\
24.89 \\
24.99\end{array}$ & $\begin{array}{r}25.99 \\
26.13 \\
26.29 \\
26.39 \\
26.40 \\
---\end{array}$ & $\begin{array}{l}-- \\
-- \\
-- \\
-- \\
-- \\
--\end{array}$ & $\begin{array}{l}-- \\
-- \\
-- \\
-- \\
-- \\
-n\end{array}$ & $\begin{array}{l}-- \\
--- \\
--- \\
-- \\
-- \\
--\end{array}$ \\
\hline
\end{tabular}

S Manual steel tape measurement. 


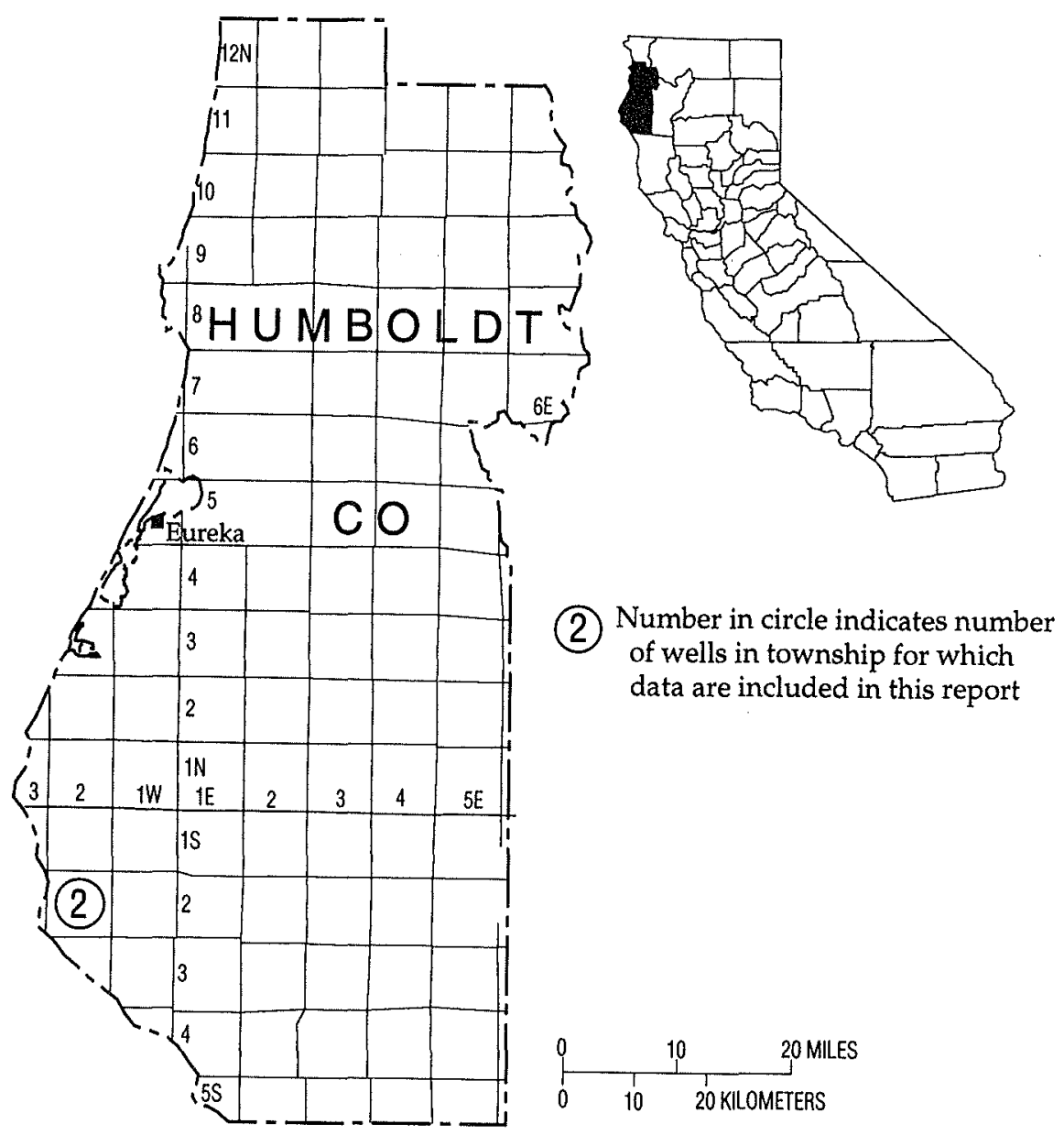

Figure 8. Location of wells in Humboldt County. 
WELL DESCRIPTIONS AND WATER-LEVEL MEASUREMENTS

WATER YEAR OCTOBER 1991 TO SEPTEMBER 1992

SITE NUMBER 401843124170301

LOCAL NUMBER OO2S002W03E01H

Near Petrolia. Drilled domestic water-table well in alluvium of the Coast Range of Pliocene-Holocene age.

Dlameter 8 inches, depth 50 feet. Altitude of land-surface datum 100 feet. Water-level records available 1978 to current year.

WATER LEVELS IN FEET BELOW LAND-SURFACE DATUM

$\begin{array}{lll} & \text { WATER } & \\ \text { DATE } & \text { WEVEL MS } & \text { DATE } \\ \text { LEVEL MS }\end{array}$

OCT 09, $1991 \quad 16.10 \mathrm{~S} \quad$ APR $13,1992 \quad 13.26 \mathrm{~S}$

PERIOD OF RECORD HIGHEST 13.26 APR 13, 1992 LOWEST 16.93 OCT 02,1984

SITE NUMBER 401928124171801

LOCAL NUMBER OO2SOO2WO9H01H

Near Petrolia. Drilled domestic water-table well in alluvium of the Coast Range of Pllocene-Holocene age.

Diameter 8 inches, depth 34 feet. Altitude of land-surface datum 76 feet. Water-level records available 1978 to current year.

WATER LEVELS IN FEET BELOW LAND-SURFACE DATUM

$\begin{array}{lll} & \text { WATER } & \\ \text { DATE } & \text { WEVEL MS } & \text { DATE } \\ \text { LEVEL MS }\end{array}$

OCT 09, $1991 \quad 15.30 \mathrm{SR} \quad$ APR 13, $1992 \quad 9.95 \mathrm{~S}$

PERIOD OF RECORD HIGHEST 5.04 MAR 04, 1980 LOWEST 14.53 OCT 24, 1990 


\section{IMPERIAL COUNTY}

Page

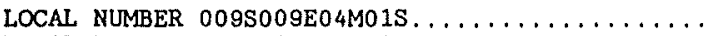
LOCAL NUMBER 010 S019E25R01S . . . . . . . . . . . . .

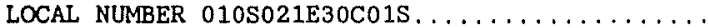

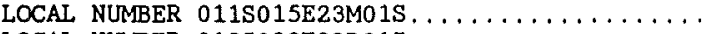

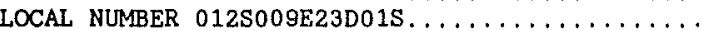

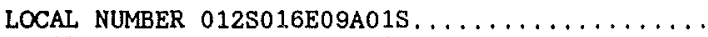
LOCAL NUMBER 013S018E33A01S. .

LOCAL NUMBER 015S011E32R01S.

LOCAL NUMBER $015 \mathrm{~S} 014 \mathrm{E} 18 \mathrm{C} 01 \mathrm{~s} \ldots \ldots \ldots \ldots \ldots \ldots \ldots$

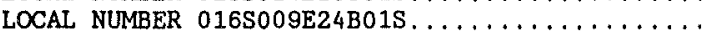

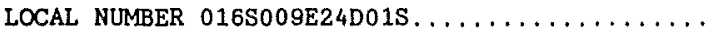
LOCAL NUMBER $016 \$ 009 E 25 K 025 \ldots \ldots \ldots \ldots \ldots \ldots \ldots \ldots$

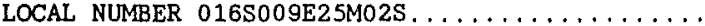

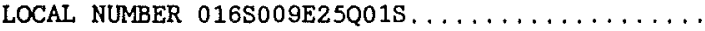

LOCAL NUMBER $0165009 E 36 D 025 \ldots \ldots \ldots \ldots \ldots \ldots \ldots$

LOCAL NUMBER 016S009E36D03S.

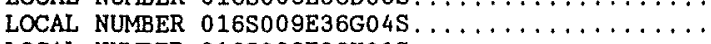

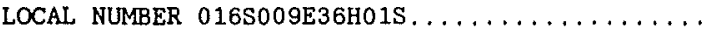

Page

LOCAL NUMBER 016S010E27R01S. . . . . . . . . .

LOCAL NUMBER $0165010 \mathrm{E} 29 \mathrm{H} 01 \mathrm{~S} \ldots \ldots \ldots \ldots \ldots \ldots \ldots \ldots$

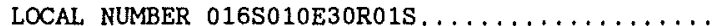

LOCAL NUMBER 016S010E42A05S...............

LOCAL NUMBER 016S011E23B01S . . . . . . . . . .

LOCAL NUMBER 016S011E27F01S . . . . . . . . .

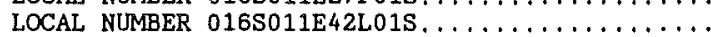

LOCAL NUMBER 016S011E42M05S...............

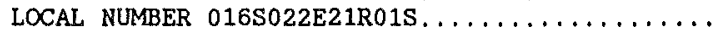

LOCAL NUMBER 016S023E09N01S...............

LOCAL NUMBER 017S010E11B01S. . . . . . . . .

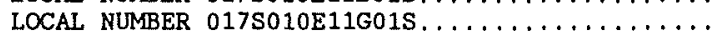

LOCAL NUMBER 017S010E11G04S..............

LOCAL NUMBER 017S010E11H01S..............

LOCAL NUMBER 017 SO10E11HO3S ................

LOCAL NUMBER 017S011E16J01S. . . . . . . . . .

LOCAL NUMBER 017 S011E22E02S . . . . . . . . . .

(1) Number in circle indicates number of wells in township for which data are included in this report
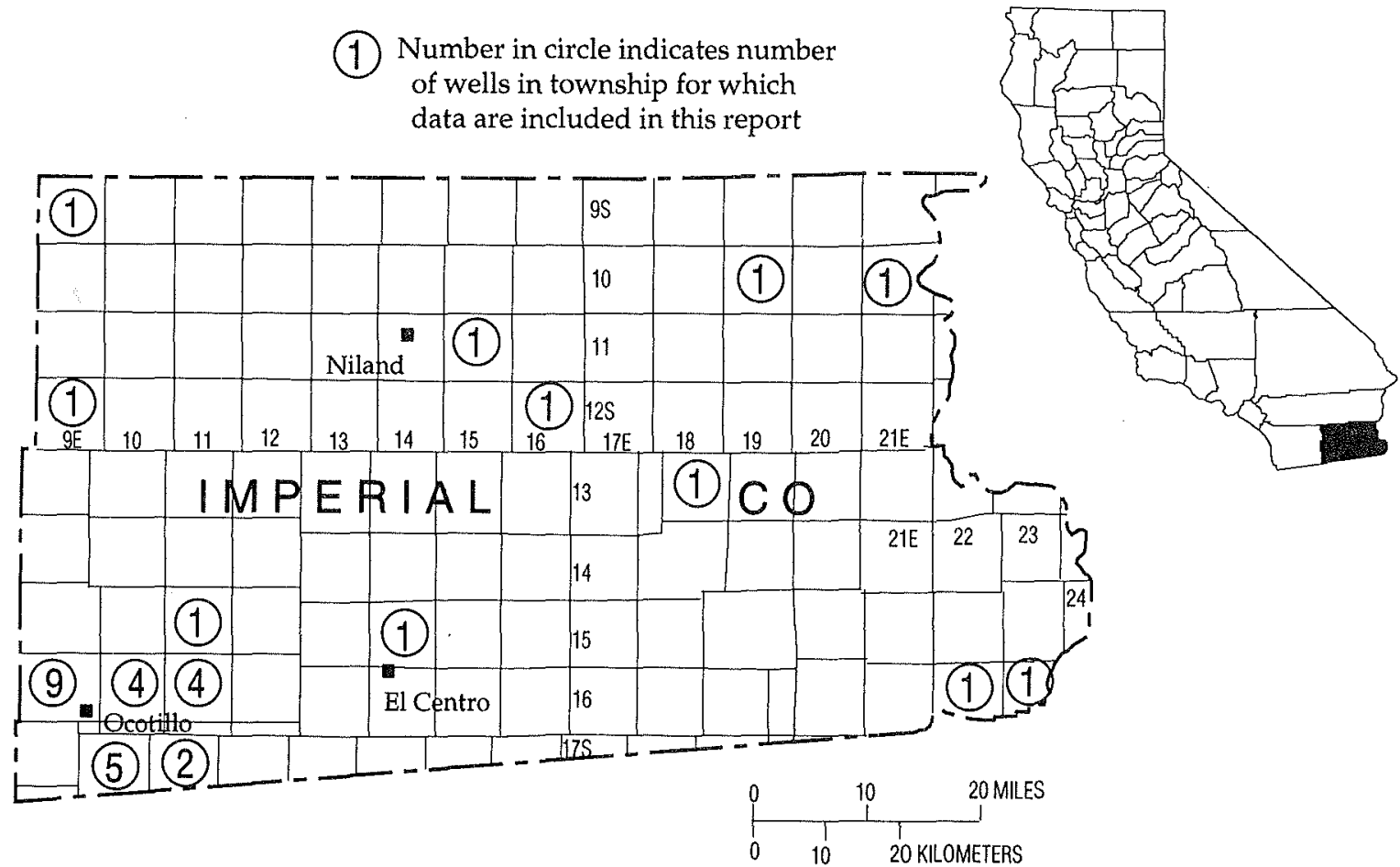

Figure 9. Location of wells in Imperial County. In Imperial County, half-townships are identified on quadrangle maps. For numbering purposes, the half-townships are not identified, but sections are numbered beyond 36 to accomodate the half-townships. 
WELL DESCRIPTIONS AND WATER-LEVEL MEASUREMENTS

WATER YEAR OCTOBER 1991 TO SEPTEMBER 1992

SITE NUMBER 332501116025701

LOCAL NUMBER OO9S009E04M01S

West side of the Salton Sea, north of Desert Shores. Drilled geothermal observation well. Diameter 2 inches, depth 489 feet, screened 486-489 feet. Altitude of land-surface datum -105 feet. Water-level records available 1979,1981 to current year.

WATER LEVELS IN FEET BELOW LAND-SURFACE DATUM

$\begin{array}{lllll} & \text { WATER } & \text { WATER } & & \text { WATER } \\ \text { DATE } & \text { DEVEL MS } & \text { DEVEL MS } & \text { DATE } & \text { LEVEL MS }\end{array}$

MAR 13, $1992114.47 \mathrm{~S} \quad$ SEP 03, $1992116.33 \mathrm{~S} \quad$ SEP 24, $1992116.28 \mathrm{~S}$

PERIOD OF RECORD HIGHEST 71.58 JUN 13,1979 LOWEST 116.33 SEP 03, 1992

SITE NUMBER 331603114550601

LCCAL NUMBER 010S019E25R01S

About 6 miles northwest of Highway 78 and west of Midway Road. Drilled water-table well. Diameter 8 inches, depth unknown. Altitude of land-surface datum 820 feet. Water-level records available 1979-85, 1989 to current year.

WATER LEVELS IN FEET BELOW LAND-SURFACE DATUM

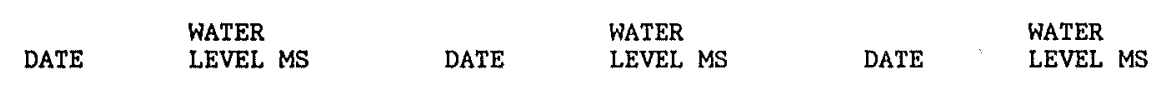

OCT 02, 1991 196.22 S MAR 17, $1992195.93 \mathrm{~S} \quad$ SEP 22, $1992196.34 \mathrm{~S}$

PERIOD OF RECORD HIGHEST 194.23 JAN 22, 1981 LOWEST 196.37 NOV 30,1989

SITE NUMBER 331659114481001

LOCAL NUMBER 010S021E30C01S

In Milpitas Wash, west of Ogilby Road. Drilled observation water-table well. Diameter 1.25 inches, depth 70.1 feet. Altitude of lend-surface datum 485 feet. Water-level records available 1972, 1979-85, 1989 to current year.

WATER LEVELS IN FEET BELOW LAND-SURFACE DATUM

$\begin{array}{llll} & & & \text { WATER } \\ \text { DATE } & \text { WEVEL MS } & \text { WATE } & \text { WEVEL MS } \\ \text { DATE } & \text { LEVEL MS }\end{array}$

OCT 02, $199140.03 \mathrm{~S} \quad$ MAR 17, $1992 \quad 39.77 \mathrm{~S} \quad \operatorname{SEP} 22,1992 \quad 36.28 \mathrm{~s}$

PERIOD OF RECORD HIGHEST 29.90 SEP 18, 1984 LOWEST 42.42 AUG 24,1972

SITE NUMBER 331144115231501

LOCAL NUMBER 011S015E23M01S

About 8 miles southeast of Niland in East Mesa area, near Siphon 3 on Coachella Canal. Drilled domestic well.

Diameter 12 inches, depth 550 feet in 1958. Altitude of land-surface datum 120 feet. Water-level records available $1953-58,1961-68,1978-85,1989$ to current year.

WATER LEVELS IN FEET BELOW LAND-SURFACE DATUM

\begin{tabular}{|c|c|c|c|c|c|}
\hline DATE & $\begin{array}{l}\text { WATER } \\
\text { LEVEL MS }\end{array}$ & DATE & $\begin{array}{l}\text { WATER } \\
\text { LEVEL MS }\end{array}$ & DATE & $\begin{array}{l}\text { WATER } \\
\text { LEVEL MS }\end{array}$ \\
\hline
\end{tabular}

OCT 08, $1991 \quad 42.46 \mathrm{~S} \quad \operatorname{MAR} 18,1992 \quad 42.70 \mathrm{~S} \quad \operatorname{SEP} 23,199243.07 \mathrm{~S}$

PERIOD OF RECORD HIGHEST 20.68 JAN 10, 1979 LOWEST 43.07 SEP 23, 1992 
SITE NUMBER 330701116003501

LOCAL NUMBER 012S009E23D01S

About 23 miles northwest of Westmorland, 0.5 mile south of Highway 78 , and about 0.75 mile north of San Felipe Creek. Drilled unused water-table well in alluvium. Diameter 14 inches, depth 580 feet. Altitude of land-surface datum -15 feet. Water-level records avallable 1953-58, 1961-68, 1978, 1980-85, 1989 to current year.

WATER LEVELS IN FEET BELOW LAND-SURFACE DATUM

$\begin{array}{llll} & \text { WATER } & \text { WATER } & \text { WATER } \\ \text { DATE } & \text { LEVEL MS } & \text { DATE } & \text { LEVEL MS }\end{array}$

OCT 02, $1991 \quad 171.66 \mathrm{~s} \quad$ MAR 16, $1992168.21 \mathrm{~S} \quad$ SEP 24, $1992165.74 \mathrm{~s}$

PERIOD OF RECORD HIGHEST 64.17 DEC 15, 1953 LOWEST 171.66 OCT 02, 1991

SITE NUMBER 330842115174701

LOCAL NUMBER 012S016E09A01S

About 14 miles east of Calipatria on Niland-Glamis Road. Drilled irrigation water-table well. Diameter 12 inches, depth 1,000 feet, perforated 150-1,000 feet. Altitude of land-surface datum 220 feet. Water-level records available 1979, 1981-85, 1990 to current year.

WATER LEVELS IN FEET BELOW LAND-SURFACE DATUM

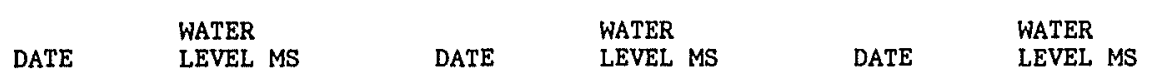

OCT 08, $1991 \quad 138.90 \mathrm{~V} \quad$ MAR 18, $1992139.11 \mathrm{~S} \quad$ SEP 23, $1992137.75 \mathrm{~S}$

PERIOD OF RECORD HIGHEST 126.33 AUG 02, 1979 LOWEST 139.20 APR 05, 1991

SITE NUMBER 325955115042601

LOCAL NUMBER 013S018E33A01S

In Glamis, 0.2 mile north of Highway 78. Drilled unused water-table well. Diameter unknown, depth 660 feet. Altitude of land-surface datum 335 feet. Water-level records available 1979-85, 1989 to current year.

WATER LEVELS IN FEET BELOW LAND-SURFACE DATUM

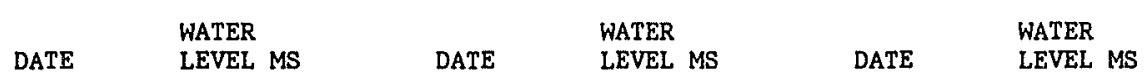

OCT 08, $1991227.33 \mathrm{~V} \quad$ MAR 17, $1992 \quad 227.22 \mathrm{~S} \quad \operatorname{SEP} 23,1992220.1 \mathrm{~V}$

PERIOD OF RECORD HIGHEST 193.45 AUG 26, 1981 LOWEST 230.87 FEB 28, 1985

SITE NUMBER 324851115505901

LOCAL NUMBER 015S011E32R01S

About 1.5 miles north of Plaster City. Augered observation water-table well. Diameter 1.25 inches, depth 152 feet, depth measured 145.8 feet in 1974, perforated 138-140 feet. Altitude of land-surface datum 65 feet. Water-level records available 1964, 1974, 1976 to current year.

WATER LEVELS IN FEET BELOW LAND-SURFACE DATUM

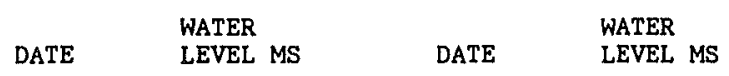

OCT 08, $199147.50 \mathrm{~S} \quad$ MAR $17,1992 \quad 47.39 \mathrm{~s}$

PERIOD OF RECORD HIGHEST 47.39 MAR 17, 1992 LOWEST 52.09 OCT 29, 1974 
WATER LEVELS, IMPERIAL COUNTY--Continued

WELL DESCRIPTIONS AND WATER-LEVEL MEASUREMENTS

WATER YEAR OCTOBER 1991 TO SEPTEMBER 1992

SITE NUMBER 325114115335201

LOCAL NUMBER 015S014E18C01S

In Imperial, east of Imperial Avenue, and about 0.5 mile north of Barioni Boulevard, Drilled unused water-table well. Diameter 8 inches, depth 500 feet in 1958, 379.02 feet in 1978, 378.5 feet in 1989, perforated 140-440 feet. Altitude of land-surface datum -64.97 feet. Water-level records available 1958, 1961, 1978-85, 1989 to current year.

WATER LEVELS IN FEET BELOW LAND-SURFACE DATUM

$\begin{array}{lllll}\text { WATER } & \text { WATER } & & \text { WATER } \\ \text { DATE } & \text { LEVEL MS } & \text { DATE } & \text { LEVEL MS } & \text { DATE }\end{array}$

OCT 02, $1991 \quad 7.69 \mathrm{~S} \quad$ MAR 16, $1992 \quad 8.14 \mathrm{~S} \quad$ SEP 22, $1992 \quad 8.15 \mathrm{~S}$

PERIOD OF RECORD HIGHEST 2.61 OCT 16, 1979 LOWEST 8.24 MAR 20, 1990

SITE NUMBER 324608115593501

LOCAL NUMBER 016S009E24B01S

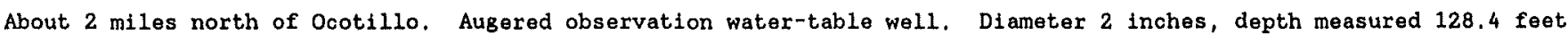

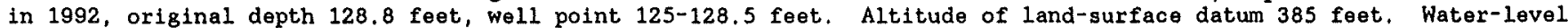
records avallable 1976 to current

year.

WATER LEVELS IN FEET BELOW LAND-SURFACE DATUM

$$
\begin{array}{lll} 
& \text { WATER } & \\
\text { DATE } & \text { LEVEL MS } & \text { WATER } \\
\text { LEVEL MS }
\end{array}
$$

OCT $07,1991 \quad 107.22 \mathrm{~S} \quad$ APR $06,1992107.32 \mathrm{~S}$

PERIOD OF RECORD HIGHEST 105.34 NOV 17,1976 LOWEST 107.32 APR 06,1992

SITE NUMBER 324558115595201

LOCAL NUMBER O16S009E24D01S

About 2 miles north of Ocotillo. Augered observation water-table well. Diameter 2 inches, depth 149 feet, well point 145.5-149 feet. Altitude of land-surface datum 382 feet. Water-level records available 1976 to current year.

WATER LEVELS IN FEET BELOW LAND-SURFACE DATUM

$$
\begin{array}{lll} 
& \text { WATER } & \\
\text { DATE } & \text { WEVEL MS } & \text { WATER } \\
\text { LEVEL MS }
\end{array}
$$

OCT 07, $1991 \quad 106.47 \mathrm{~S} \quad$ APR 06, $1992106.56 \mathrm{~s}$

PERIOD OF RECORD HIGHEST 103.86 APR 28, 1977 LOWEST 106.56 APR 06,1992

SITE NUMBER 324439115593401

LOCAL NUMBER 016SO09E25K02S

In Ocotillo, east of Imperial Highway. Drilled commercial water-table well. Diameter 10 inches, depth 372 feet, perforated 132-192, 242-372 feet. Altitude of land-surface datum 364 feet. Records available 1972 , 1975 to current year.

WATER LEVELS IN FEET BELOW LAND-SURFACE DATUM

$\begin{array}{lll} & \text { WATER } & \\ \text { DATE } & \text { LEVEL MS } & \text { WATER } \\ \text { LEVEL MS }\end{array}$

OCT $08,1991 \quad 94.57 \mathrm{~s} \quad$ APR $07,1992 \quad 94.46 \mathrm{~s}$

PERIOD OF RECORD HIGHEST 83.00 AUG 24,1972 LOWEST 101.47 MAR 14, 1979 
SITE NUMBER 324446115595901

LOCAL NUMBER 016S009E25M02S

In Ocot11lo, north of Interstate 8 and east of Imperial Highway. Drilled public-supply well. Diameter 8 inches, depth 336 feet, perforated 216-336 feet. Altitude of land-surface datum 410 feet. Water-level records available 1991 to current year.

WATER LEVELS IN FEET BELOW LAND-SURFACE DATUM

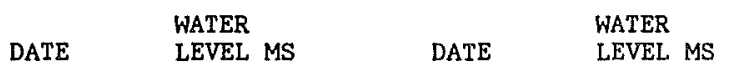

OCT 07, 1991 137.84 ST APR 06, 1992 136.75 S

PERIOD OF RECORD HIGHEST 136.69 APR 02, 1991 LOWEST 136.75 APR 06, 1992

SITE NUMBER 324426115593601

LOCAL NUMBER 0165009E25Q01S

In Ocotillo, north of Interstate 8, and east of Imperial Highway. Unused well. Diameter 8 inches, depth measured 128.5 feet in 1974. Altitude of land-surface datum 372 feet. Water-level records available 1974, 1976 , 1981 to current year.

WATER LEVELS IN FEET BELOW LAND-SURFACE DATUM

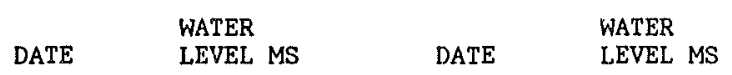

OCT 07, $1991 \quad 107.27 \mathrm{~S}$ MAR 17, $1992 \quad 0$

PERIOD OF RECORD HIGHEST 104.24 DEC 01, 1974 LOWEST 107.51 OCT 23, 1990

SITE NUMBER 324422116000301

LOCAL NUMBER 016S009E36D02S

In Ocotillo, about 300 feet east of Shell Canyon Road. Drilled domestic well. Diameter 8 inches, depth 200 feet, perforated $150-200$ feet. Altitude of land-surface datum 435 feet. Water-level records available 1975 to current year.

WATER LEVELS IN FEET BELOW LAND-SURFACE DATUM

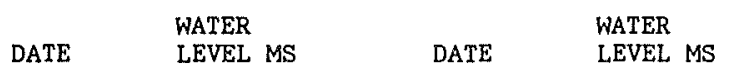

OCT 07, $1991161.36 \mathrm{~S} \quad$ MAR 17, $1992161.35 \mathrm{~S}$

PERIOD OF RECORD HIGHEST 157.90 JUN 26, 1975 LOWEST 161.36 OCT 07, 1991

SITE NUMBER 324401115593201

LOCAL NUMBER 016S009E36G04S

In Ocotillo, south of Interstate 8 , and east of Imperial Highway. Drilled industrial water-table well. Diameter 10.75 inches, depth 560 feet, perforated $340-560$ feet. Altitude of land-surface datum 382 feet. Water-level records available 1975 to current year.

WATER LEVELS IN FEET BELOW LAND-SURFACE DATUM

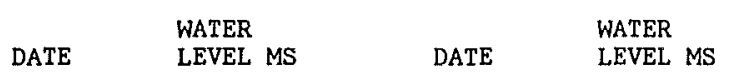

OCT $08,1991 \quad 131.26$ SR APR $07,1992127.85 \mathrm{~S}$

PERIOD OF RECORD HIGHEST 122.14 MAR 28, 1986 LOWEST 136.47 JUN 28, 1975 
WELL DESCRIPTIONS AND WATER-LEVEL MEASUREMENTS

WATER YEAR OCTOBER 1991 TO SEPTEMBER 1992

SITE NUMBER 324407115590901

LOCAL NUMBER O16SOO9E36H01S

About 0.5 mile east of Ocotillo, south of Interstate 8 . Drilled industrial water-table well in sand and clay. Diameter 10.75 inches, depth 410 feet, perforated $60-380$ feet. Altitude of land-surface datum 342 feet.

Water-level records available 1954, 1960, 1966, 1974, 1976 to current year.

WATER LEVELS IN FEET BELOW LAND-SURFACE DATUM

$$
\begin{array}{lll} 
& \text { WATER } & \\
\text { DATE } & \text { WEVEL MS } & \text { DATER } \\
\text { LEVEL MS }
\end{array}
$$

OCT 09, $199183.75 \mathrm{~S} \quad$ APR 07, $1992 \quad 83.96 \mathrm{~S}$

PERIOD OF RECORD HIGHEST 68.50 MAR 21, 1954 LOWEST 85.00 MAY 1960

SITE NUMBER 324430115555501

LOCAL NUMBER 016S010E27R01S

About 2 miles east of Coyote Wells. Augered observation water-table well. Diameter 2 inches, depth measured 103.76 feet in $1975,103.70$ feet in 1987, well point 102-104 feet. Altitude of land-surface datum 300 feat. Water-level records avaflable 1975 to current year.

WATER LEVELS IN FEET BELOW LAND-SURFACE DATUM

$$
\begin{array}{lll} 
& \text { WATER } & \\
\text { DATE } & \text { WETER } \\
\text { LEVE } & \text { LEVEL MS }
\end{array}
$$

OCT $09,199198.59 \mathrm{~S} \quad$ APR $07,1992 \quad 98.62 \mathrm{~S}$

PERIOD OF RECORD HIGHEST 98,48 MAR 15, 1988 LOWEST 98.97 MAY 12, 1975

SITE NUMBER 324458115570301

LOCAL NUMBER 016S010E29H01S

About 2.6 miles northeast of Coyote Wells on Highway S80. Augered observation water-table well. Diameter 2 inches, depth measured 38.7 feet in $1975,36.1$ feet in $1985,35.5$ feet in 1991 , wel1 point $36.7-38.7$ reet. Altitude of land-surface datum 250 feet. Water-level records available 1975 to current year.

WATER LEVELS IN FEET BELOW LAND-SURFACE DATUM

$$
\begin{array}{lll} 
& \text { WATER } & \\
\text { DATE } & \text { WEVEL MS } & \text { DATE } \\
\text { LEVEL MS }
\end{array}
$$

OCT 08, $199121.97 \mathrm{~s} \quad$ MAR 17, $1992 \quad 23.68 \mathrm{~s}$

PERIOD OF RECORD HIGHEST 21.53 APR 26, 1978 LOWEST 25.64 APR 04, 1991

SITE NUMBER 324329115580501

LOCAL NUMBER 016S010E42A05S

About 2 miles southeast of Ocotillo, north of Highway 98. Domestic well. Diameter and depth unknown. Altitude of land-surface datum 328 feet. Water-level records available 1974, 1976 to current year.

WATER LEVELS IN FEET BELOW LAND-SURFACE DATUM

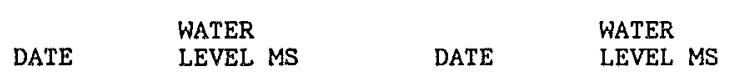

OCT $07,199179.78 \mathrm{~s} \quad \operatorname{MAR} 17,1992 \quad 79.63 \mathrm{~s}$

PERIOD OF RECORD HIGHEST 73.21 DEC $30,1974 \quad$ LOWEST $79.78 \quad$ OCT 07,1991 
About 3.5 miles southeast of Plaster City. Augered observation we11. Diameter 1.25 inches, depth measured 114.7 feet in 1974, perforated 121-123 feet. Altitude of land-surface datum 30 feet. Water-level records available $1964,1974,1976$ to current year.

WATER LEVELS IN FEET BELOW LAND-SURFACE DATUM

\begin{tabular}{|c|c|c|}
\hline DATE & WATER & DATE \\
\hline
\end{tabular}

OCT 09, $199140.83 \mathrm{~s}$ MAR 16, $199241.06 \mathrm{~s}$

PERIOD OF RECORD HIGHEST 39.34 APR 25, 1978 LOWEST 41.06 MAR 16, 1992

SITE NUMBER 324500115492101

LOCAL NUMBER 016S011E27F01S

About 3 miles southeast of Plaster City and $1.8 \mathrm{miles}$ southwest of Interstate 8 and Dunaway Road. Augered observation well. Diameter 2 inches, depth measured 134.5 feet in 1975 , well point 132.5-134.5 feet. Altitude of land-surface datum 100 feet. Water-level records avallable 1975 to current year.

WATER LEVELS IN FEET BELOW LAND-SURFACE DATUM

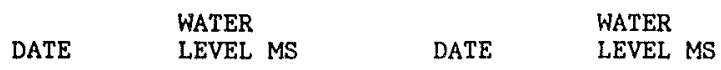

OCT $09,199199.68 \mathrm{~S} \quad$ MAR $16,1992 \quad 99.62 \mathrm{~S}$

PERIOD OF RECORD HIGHEST 92.95 MAY 07, 1975 LOWEST 99.68 OCT 09, 1991

SITE NUMBER 324251115522201

LOCAL NUMBER 016S011E42L01S

About 7.2 miles southeast of Ocotillo. Augered observation water-table well. Diameter 2 inches, depth measured 143.5 feet in $1975,130.4$ feet in 1987, well point 141.5-143.5 feet. Altitude of land-surface datum 195 feet. Water-level records avallable 1975-76, 1981 to current year.

WATER LEVELS IN FEET BELOW LAND-SURFACE DATUM

$\begin{array}{lll} & \text { WATER } & \\ \text { DATE } & \text { WETER MS }\end{array}$

OCT $09,199138.70 \mathrm{~S} \quad$ MAR $16,199238.65 \mathrm{~S}$

PERIOD OF RECORD HIGHEST 22.85 OCT 07, 1982 LOWEST 44.77 MAY 09, 1975

SITE NUMBER 324258115524101

LOCAL NUMBER O16S011E42M05S

About 7 miles southeast of Ocotillo. Unused water-table well. Diameter 5 inches, depth measured 6.5 feet in 1974 , 4.4 feet in 1980 , deepened to 9.3 feet in 1981 with 1 inch diameter casing, depth measured 8.0 feet in 1988 . Altitude of land-surface datum 220 feet. Water-level records available 1949, 1974, 1976 to current year.

WATER LEVELS IN FEET BELOW LAND-SURFACE DATUM

$\begin{array}{lll} & \text { WATER } & \\ \text { DATE } & \text { WEVEL MS }\end{array}$

OCT 09, $19915.14 \mathrm{~S} \quad$ MAR $16,19925.23 \mathrm{~s}$

PERIOD OF RECORD HIGHEST 3.33 APR 27, 1977 LOWEST 5.23 MAR 16, 1992 
WELL DESCRIPTIONS AND WATER-LEVEL MEASUREMENTS

WATER YEAR OCTOBER 1991 TO SEPTEMBER 1992

SITE NUMBER 324444114385901

LOCAL NUMBER 016S022E21R01S

About 1 mile northwest of Winterhaven, and 1 mile north of Colorado River, northwest of Yuma, Arizona. Drilled observation water-table well. Diameter 1.25 inches, depth measured 122.5 feet in 1989 . Altitude of land-surface datum 128 feet. Water-level records available 1964, 1967, 1975-85, 1989 to current year.

WATER LEVELS IN FEET BELOW LAND-SURFACE DATUM

\begin{tabular}{|c|c|c|c|c|c|}
\hline DATE & $\begin{array}{l}\text { WATER } \\
\text { LEVEL MS }\end{array}$ & DATE & $\begin{array}{l}\text { WATER } \\
\text { LEVEL MS }\end{array}$ & DATE & $\begin{array}{l}\text { WATER } \\
\text { LEVEL MS }\end{array}$ \\
\hline
\end{tabular}

$\begin{array}{lllllll}\text { OCT } 02,1991 & 10.76 \mathrm{~S} & \text { APR } 08,1992 & 10.35 \mathrm{~S} & \mathrm{SEP} 22,1992 & 10.09 \mathrm{~S}\end{array}$

PERIOD OF RECORD HIGHEST 4.66 SEP 20, 1983 LOWEST 12.67 JAN 05, 1978

SITE NUMBER 324641114333501

LOCAL NUMBER 016S023E09N01S

About 2 miles west of the Arizona boundary, $1 \mathrm{mile}$ south of Bard, east of Avenue $D$, and north of 5 th 5 treet.

Drilled irrigation water-table well. Diameter 20 inches, depth 235 feet, perforated 124-150, 154-225 feet.

Altitude of land-surface datum 137 feet. Water-level records available 1989 to current year.

WATER LEVELS IN FEET BELOW LAND-SURFACE DATUM

$\begin{array}{lllll} & \text { WATER } & \text { WATER } & \text { WATER } \\ \text { DATE } & \text { LEVEL MS } & \text { DATE } & \text { LEVEL MS } & \text { DATE } \\ \text { LEVEL MS }\end{array}$

$\begin{array}{llllll}\text { OCT 02, } 1991 & \mathrm{P} & \text { APR 08, } 1992 & 10.39 \mathrm{~S} & \text { AUG 26, } 1992 & 10.75 \mathrm{~S}\end{array}$

PERIOD OF RECORD HIGHEST 10.39 APR 08, 1992 LOWEST 11.76 MAR 14, 1991

SITE NUMBER 324138115552901

LOCAL NUMBER 017S010E11B01S

About 5 miles southeast of Ocotillo in Yuha Estates. Domestic well. Diameter 7 inches, depth reported 301 feet. Altitude of land-surface datum 376 feet. Water-level records avallable 1975-76, 1978 to current year.

WATER LEVELS IN FEET BELOW LAND-SURFACE DATUM

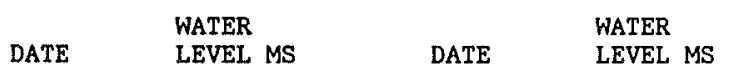

OCT 08, 1991 163.72 S APR 07, $1992163.13 \mathrm{~S}$

PERIOD OF RECORD HIGHEST 154.80 JAN 08, 1976 LOWEST 164.05 OCT 19, 1988

SITE NUMBER 324123115552901

LOCAL NUMBER 017S010E11G01S

About 5 miles southeast of Ocotillo in Yuha Estates. Drilled domestic well, Diameter 8 inches, depth 300 feet, perforated 160-300 feet. Altitude of land-surface datum 375 feet. Water-level records available 1967, $1971-72$, $1975-76,1978$ to current year.

WATER LEVELS IN FEET BELOW LAND-SURFACE DATUM

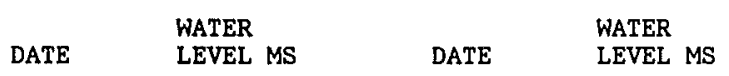

OCT 07, $1991 \quad 177.59 \mathrm{~S} \quad$ MAR 17, $1992 \quad 176.73 \mathrm{~S}$

PERIOD OF RECORD HIGHEST 164.94 JUN 27, 1975 LOWEST 232.80 NOV 06, 1981 
WATER LEVELS, IMPERIAL COUNTY--Continued

WELL DESCRIPTIONS AND WATER-LEVEL MEASUREMENTS

WATER YEAR OCTOBER 1991 TO SEPTEMBER 1992

SITE NUMBER 324119115553201

LOCAL NUMBER 017S010E11G04S

About 5 miles southeast of Ocotillo in Yuha Estates. Drilled unused well. Diameter 10.75 inches, depth measured 199.1 feet in 1978, deepened to a reported 500 feet in 1981. Altitude of land-surface datum 375 feet. Water-1evel records available 1978,1981 to current year.

WATER LEVELS IN FEET BELOW LAND-SURFACE DATUM

$$
\begin{array}{lll} 
& \text { WATER } & \\
\text { DATE } & \text { WEVEL MS } & \text { DATE } \\
\text { LEVEL MS }
\end{array}
$$

OCT $07,1991 \quad 180.70 \mathrm{~S} \quad$ MAR $17,1992179.76 \mathrm{~S}$

PERIOD OF RECORD HIGHEST 179.76 MAR 17, 1992 LOWEST 206.21 $\propto$ CT 07, 1982

SITE NUMBER 324114115552301

LOCAL NUMBER 017S010E11H01S

About 5 miles southeast of Ocotillo in Yuha Estates. Dr $\$ 1$ led domestic well. Diameter 8 inches, depth measured 329.9 feet in 1989. Altitude of land-surface datum 380 feet. Water-level records available 1964 , 1978 to current year.

WATER LEVELS IN FEET BELOW LAND-SURFACE DATUM

$$
\text { DATE WATER }
$$

OCT $07,1991 \quad 170.39 \mathrm{SR}$

PERIOD OF RECORD HIGHEST 158.27 JUN 15,1964 LOWEST 174.33 OCT 26,1983

SITE NUMBER 324117115552001

LOCAL NUMBER 017SO10E11HO3S

About 5 miles southeast of Ocotillo in Yuha Estates. Drilled domestic water-table well. Diameter 5 inches, depth 348 feet, perforated 202-217, 228-238,265-273,284-295, 304-318, 327-333 feet. Altitude of land-surface datum 380 feet. Water-level records available 1987 to current year.

WATER LEVELS IN FEET BELOW LAND-SURFACE DATUM

$$
\begin{array}{lll} 
& \text { WATER } & \\
\text { DATE } & \text { WEVEL MS } & \text { WATER } \\
\end{array}
$$

OCT 08, 1991 So APR 07, $1992175.57 \mathrm{~s}$

$\begin{array}{llllllll}\text { PERIOD OF RECORD HIGHEST } & 175.57 & \text { APR } & 07, & 1992 & \text { LOWEST } & 179.29 & \text { OCT } 23,1987\end{array}$

SITE NUMBER 324013115511101

LOCAL NUMBER 017S011E16J01S

About 15 miles west of Calexico south of Highway 98 . Drilled unused well. Diameter 4.5 inches, depth measured 336.5 feet in 1974, perforated 226-366 feet. Altitude of land-surface datum 298.7 feet. Water-1evel records available 1970,1974 to current year.

WATER LEVELS IN FEET BELOW LAND-SURFACE DATUM

$$
\begin{array}{lll}
\text { DATE } & \text { WATER } & \\
\text { LEVEL MS } & \text { WATER }
\end{array}
$$

OCT $07,1991 \quad 95.00 \mathrm{~s} \quad$ MAR $17,1992 \quad 95.10 \mathrm{~s}$

PERIOD OF RECORD HIGHEST 95.00 MAY 19, 1970 OCT 07, 1991 LOWEST 96.66 FEB 04, 1975 
WELL DESCRIPTIONS AND WATER-LEVEL MEASUREMENTS

WATER YEAR OCTOBER 1991 TO SEPTEMBER 1992

SITE NUMBER 323934115504701

LOCAL NUMBER 017SO11E22E02S

About 12 miles southeast of Ocotillo and $1 \mathrm{mile}$ south of Highway 98 and Sunrise Butte. Drilled observation well. Diameter 2 inches, depth measured 119,65 feet in 1975, well point 117,6-119.6 feet. Altitude of land-surface datum 303.9 feet. Water-level records avallable 1975 to current year.

WATER LEVELS IN FEET BELOW LAND-SURFACE DATUM

$\begin{array}{lll} & \text { WATER } & \\ \text { DATE } & \text { WEVEL MS } & \text { DATE } \\ \text { LEVEL MS }\end{array}$

OCT 07, $1991 \quad 100.61 \mathrm{~s} \quad$ MAR 17, $1992100.65 \mathrm{~s}$

PERIOD OF RECORD HIGHEST 100.61 OCT 07, 1991 LOWEST 102.48 MAY 08, 1975 


\begin{tabular}{|c|c|c|c|c|c|c|c|c|c|c|}
\hline $\begin{array}{l}\text { LOCAL } \\
\text { NUMBER }\end{array}$ & DATE & TIME & $\begin{array}{l}\text { DEPTH } \\
\text { BELOW } \\
\text { LAND } \\
\text { SURFACE } \\
\text { (WATER } \\
\text { LEVEL) } \\
\text { (FEET) }\end{array}$ & $\begin{array}{l}\text { DEPTH } \\
\text { OF } \\
\text { WELL, } \\
\text { TOTAL } \\
\text { (FEET) }\end{array}$ & $\begin{array}{l}\text { SPE- } \\
\text { CIFIC } \\
\text { CON- } \\
\text { DUCT- } \\
\text { ANCE } \\
\text { (US/CM) }\end{array}$ & $\begin{array}{c}\text { PH } \\
\text { WATER } \\
\text { WHOLE } \\
\text { FIELD } \\
\text { (STAND- } \\
\text { ARD } \\
\text { UNITS) }\end{array}$ & $\begin{array}{l}\text { TEMPER- } \\
\text { ATURE } \\
\text { WATER } \\
\text { (DEG C) }\end{array}$ & $\begin{array}{l}\text { CALCIUM } \\
\text { DIS- } \\
\text { SOLVED } \\
\text { (MG/L } \\
\text { AS CA) }\end{array}$ & $\begin{array}{l}\text { MAGNE- } \\
\text { SIUM, } \\
\text { DIS- } \\
\text { SOLVED } \\
\text { (MG/L } \\
\text { AS MG) }\end{array}$ & $\begin{array}{l}\text { SODIUM, } \\
\text { DIS- } \\
\text { SOLVED } \\
\text { (MG/L } \\
\text { AS NA) }\end{array}$ \\
\hline $\begin{array}{l}\text { 016S009E24B01S } \\
016 S 009 E 24 D 01 S \\
016 S 009 E 25 K 02 S \\
016 S 009 E 36 D 03 S \\
016 S 010 E 30 R 01 S\end{array}$ & $\begin{array}{l}04-06-92 \\
04-06-92 \\
04-07-92 \\
04-07-92 \\
04-06-92\end{array}$ & $\begin{array}{l}1115 \\
1415 \\
1000 \\
1500 \\
1700\end{array}$ & $\begin{array}{c}107.32 \\
106.56 \\
94.46 \\
-- \\
--\end{array}$ & $\begin{array}{l}128.4 \\
149 \\
372 \\
300 \\
75\end{array}$ & $\begin{array}{r}2160 \\
830 \\
535 \\
600 \\
1260\end{array}$ & $\begin{array}{l}8.6 \\
8.4 \\
8.1 \\
8.4 \\
7.7\end{array}$ & $\begin{array}{l}29.0 \\
29.5 \\
29.0 \\
31.0 \\
27.0\end{array}$ & $\begin{array}{l}5.2 \\
11 \\
22 \\
7.1 \\
--\end{array}$ & $\begin{array}{l}1.9 \\
3.3 \\
4.8 \\
1.3 \\
--\end{array}$ & $\begin{array}{r}440 \\
150 \\
77 \\
120 \\
150\end{array}$ \\
\hline $\begin{array}{l}\text { LOCAL } \\
\text { NUMBER }\end{array}$ & DATE & $\begin{array}{l}\text { SODIUM } \\
\text { PERCENT }\end{array}$ & $\begin{array}{l}\text { SODIUM } \\
\text { AD- } \\
\text { SORP- } \\
\text { TION } \\
\text { RATIO }\end{array}$ & $\begin{array}{l}\text { POTAS- } \\
\text { SIUM, } \\
\text { DIS- } \\
\text { SOLVED } \\
\text { (MG/L } \\
\text { AS K) }\end{array}$ & $\begin{array}{l}\text { ALKA- } \\
\text { LINITY } \\
\text { WAT WH } \\
\text { TOT FET } \\
\text { FIELD } \\
\text { MG/L AS } \\
\text { CACO3 }\end{array}$ & $\begin{array}{l}\text { SULFATE } \\
\text { DIS- } \\
\text { SOLVED } \\
\text { (MG/L } \\
\text { AS SO4) }\end{array}$ & $\begin{array}{l}\text { CHLO- } \\
\text { RIDE, } \\
\text { DIS- } \\
\text { SOLVED } \\
\text { (MG/L } \\
\text { AS CL) }\end{array}$ & $\begin{array}{l}\text { FLUO- } \\
\text { RIDE, } \\
\text { DIS- } \\
\text { SOLVED } \\
\text { (MG/L } \\
\text { AS F) }\end{array}$ & $\begin{array}{l}\text { SILICA, } \\
\text { DIS- } \\
\text { SOLVED } \\
\text { (MG/L } \\
\text { AS } \\
\text { SIO2) }\end{array}$ & \\
\hline $\begin{array}{l}016 \mathrm{~S} 009 \mathrm{E} 24 \mathrm{~B} 01 \mathrm{~S} \\
016 \mathrm{~S} 009 \mathrm{E} 24 \mathrm{D} 01 \mathrm{~S} \\
016 \mathrm{~S} 009 \mathrm{E} 25 \mathrm{~K} 02 \mathrm{~S} \\
016 \mathrm{~S} 009 \mathrm{E} 36 \mathrm{D} 03 \mathrm{~S} \\
016 \mathrm{~S} 010 \mathrm{E} 30 \mathrm{R} 01 \mathrm{~S}\end{array}$ & $\begin{array}{l}04-06-92 \\
04-06-92 \\
04-07-92 \\
04-07-92 \\
04-06-92\end{array}$ & $\begin{array}{l}98 \\
88 \\
68 \\
91 \\
61\end{array}$ & $\begin{array}{r}42 \\
10 \\
4 \\
11 \\
5\end{array}$ & $\begin{array}{l}2.8 \\
3.6 \\
4.4 \\
2.5 \\
--\end{array}$ & $\begin{array}{r}182 \\
84 \\
\mathrm{~L} 117 \\
150 \\
133\end{array}$ & $\begin{array}{r}270 \\
150 \\
39 \\
23 \\
--\end{array}$ & $\begin{array}{r}380 \\
96 \\
72 \\
86 \\
--\end{array}$ & $\begin{array}{l}1.6 \\
1.0 \\
0.80 \\
3.0 \\
0.80\end{array}$ & $\begin{array}{l}19 \\
16 \\
29 \\
36 \\
37\end{array}$ & \\
\hline
\end{tabular}

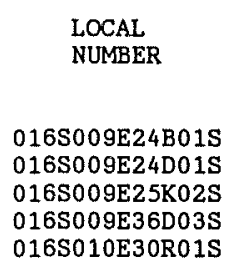

DATE
$04-06-92$
$04-06-92$
$04-07-92$
$04-07-92$
$04-06-92$

\begin{tabular}{|c|c|c|c|c|}
\hline $\begin{array}{l}\text { SOLIDS, } \\
\text { RESIDUE } \\
\text { AT } 180 \\
\text { DEG. C } \\
\text { DIS- } \\
\text { SOLVED } \\
\text { (MG/L) }\end{array}$ & $\begin{array}{c}\text { NITRO- } \\
\text { GEN, } \\
\text { NITRITE } \\
\text { DIS- } \\
\text { SOLVED } \\
\text { (MG /L } \\
\text { AS N) }\end{array}$ & $\begin{array}{c}\text { NITRO- } \\
\text { GEN, } \\
\text { NO2+NO3 } \\
\text { DIS- } \\
\text { SOLVED } \\
\text { (MG/L } \\
\text { AS N) }\end{array}$ & $\begin{array}{l}\text { NITRO- } \\
\text { GEN, } \\
\text { AMMONIA } \\
\text { DIS- } \\
\text { SOLVED } \\
\text { (MG/L } \\
\text { AS N) }\end{array}$ & $\begin{array}{c}\text { PHOS- } \\
\text { PHORUS } \\
\text { ORTHO, } \\
\text { DIS- } \\
\text { SOLVED } \\
\text { (MG/L } \\
\text { AS P) }\end{array}$ \\
\hline $\begin{array}{r}1230 \\
481 \\
315 \\
347 \\
700\end{array}$ & $\begin{array}{l}<0.010 \\
<0.010 \\
<0.010 \\
<0.010\end{array}$ & $\begin{array}{l}0.065 \\
1.40 \\
1.40 \\
0.450 \\
5.30\end{array}$ & $\begin{array}{r}0.020 \\
<0.010 \\
<0.010 \\
<0.010 \\
<0.010\end{array}$ & $\begin{array}{l}0.040 \\
0.030 \\
0.030 \\
0.030 \\
0.030\end{array}$ \\
\hline
\end{tabular}

\begin{tabular}{|c|c|c|}
\hline $\begin{array}{l}\text { BORON, } \\
\text { DIS- } \\
\text { SOLVED } \\
\text { (UG/L } \\
\text { AS B) }\end{array}$ & $\begin{array}{l}\text { IRON, } \\
\text { DIS- } \\
\text { SOLVED } \\
\text { (UG/L } \\
\text { AS FE) }\end{array}$ & $\begin{array}{l}\text { MANGA- } \\
\text { NESE, } \\
\text { DIS- } \\
\text { SOLVED } \\
\text { (UG/L } \\
\text { AS MN) }\end{array}$ \\
\hline $\begin{array}{l}770 \\
420 \\
220 \\
850 \\
530\end{array}$ & $\begin{array}{r}290 \\
<3 \\
5 \\
4 \\
7\end{array}$ & $\begin{array}{r}10 \\
<1 \\
1 \\
<1 \\
3\end{array}$ \\
\hline
\end{tabular}

< Actual value is less than value shown.

L Laboratory value. 
KERN COUNTY

Page

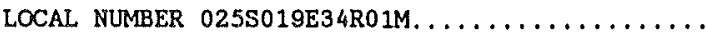

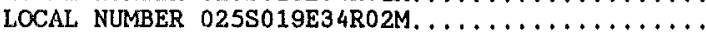

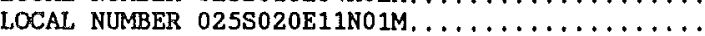

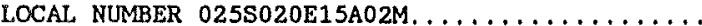

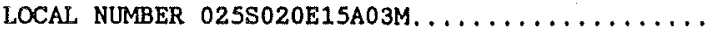

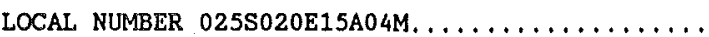

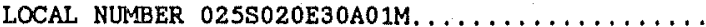

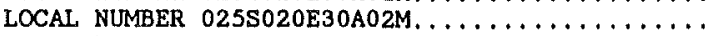

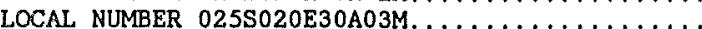

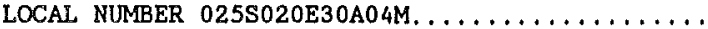

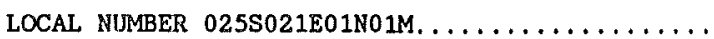

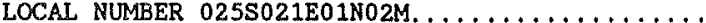

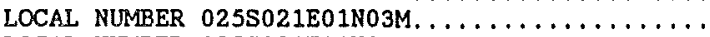
LOCAL NUMBER 025S021E01N04M. . . . . . . . . . LOCAL, NUMBER 025S021E07B01M.............

LOCAL NUMBER 025S021E07B02M. . . . . . . . . .

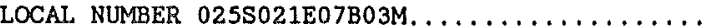

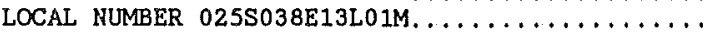

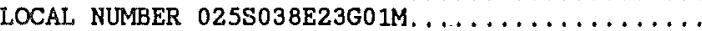

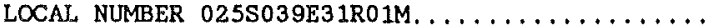

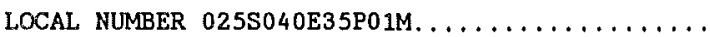
LOCAI, NUMBER 026S039E07N02M. . . . . . . . . .

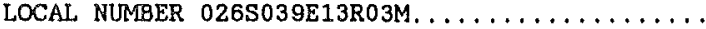

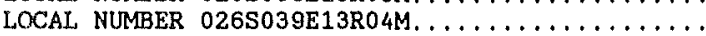

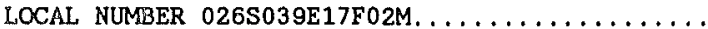

LOCAL, NUMBER 026S039E24K01M. . . . . . . . . . LOCAL NUMBER 026S040E19P01M. . . . . . . . . .

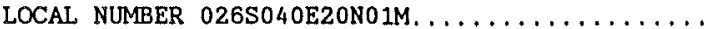

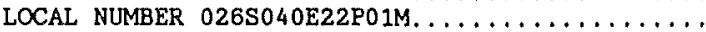

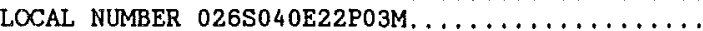

LOCAL NUMBER 026S040E22P04M..............

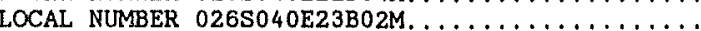

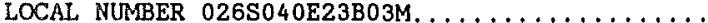

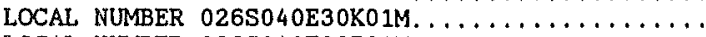

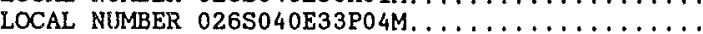

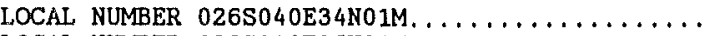

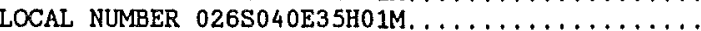

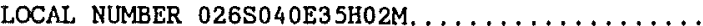

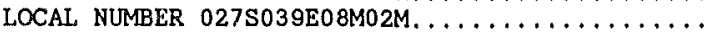

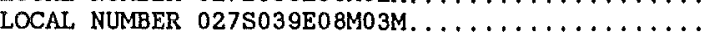

LOCAL NUMBER 027S039E08P01M.............

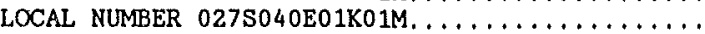

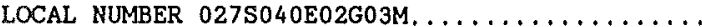

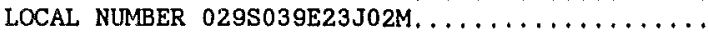

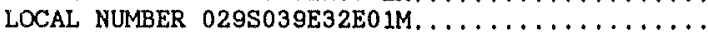

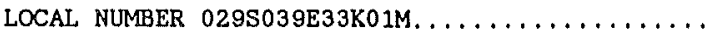

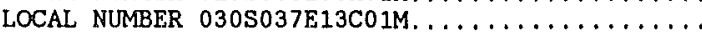

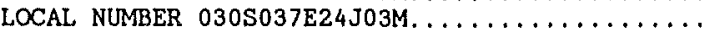

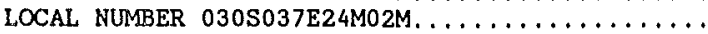

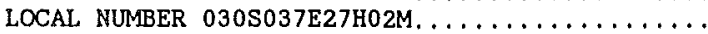

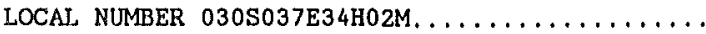

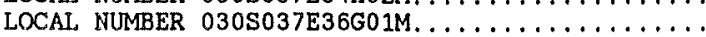
LOCAL NUMBER 030S038E03K02M. . . . . . . . . . LOCAL NUMBER 030S038E04D02M. . . . . . . . . .

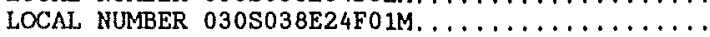

LOCAL NUMBER 030S038E30P01M. . . . . . . . . . .

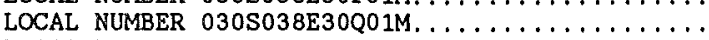

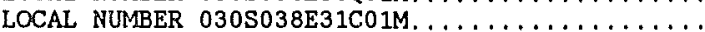

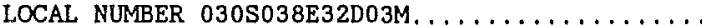

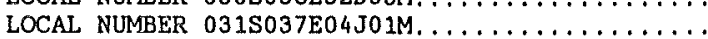

59

59

59

59

60

60

60

60

61

61

61

61

62

62

62

62

63

66

66,103

66,103

67

67,103

67,103

103

103

LOCAL NUMBER 031S037E04Q01M.

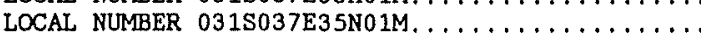

LOCAL NUMBER 031S038E18P01M. . . . . . . . . . .

LOCAL NUMBER 031S039E24P01M..............

LOCAL NUMBER 032S027E29P01M. . . . . . . . . . .

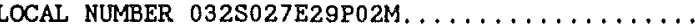

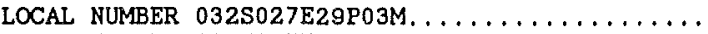

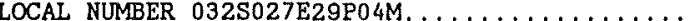

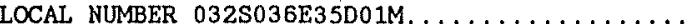

LOCAL NUMBER 032S037E11NO1M. . . . . . . . . .

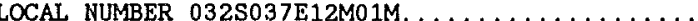
LOCAL NUMBER 032S037E22N01M. . . . . . . . . .

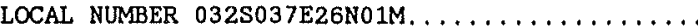

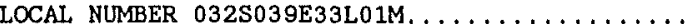

LOCAL NUMBER 009N008W06J01S. . . . . . . . . . .

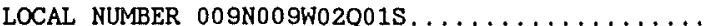

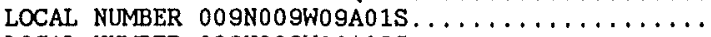

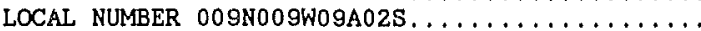
LOCAL NUMBER 009N009W10R01S, ..............

LOCAL NUMBER 009N009W13N01S.............. LOCAL NUMBER 009N009W15J01S. . . . . . . . . . . LOCAL NUMBER 009N009W18C01s..............

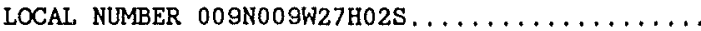
LOCAL NUMBER 009N009W28A01S..............

LOCAL NUMBER 009N009W28A02S. . . . . . . . .

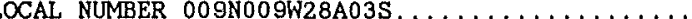

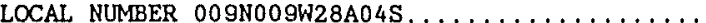

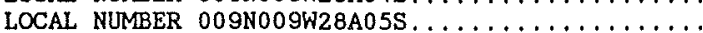

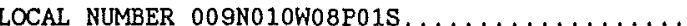

LOCAL NUMBER 009N010W16F01S.............

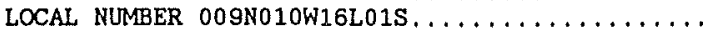

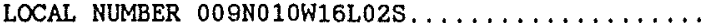

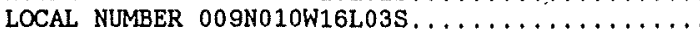

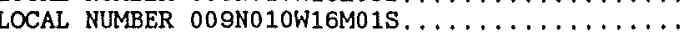

LOCAL NUMBER 009N010W16N01S..............

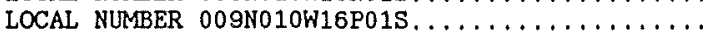

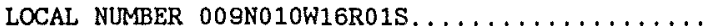

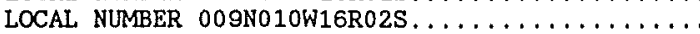

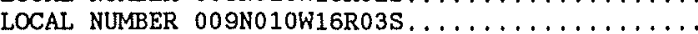

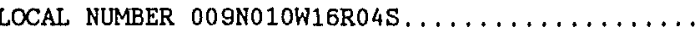

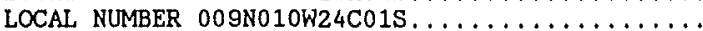

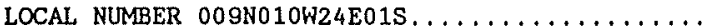

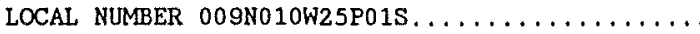

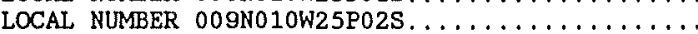

LOCAL NUMBER 009N010W28F02S............. LOCAL NUMBER 009N010W34R02S. . . . . . . . . LOCAL NUMBER OOSN010W34R03S. . . . . . . . . . LOCAL NUMBER OOSN010W34R04S, .............

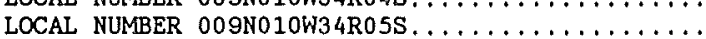

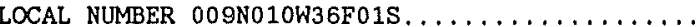

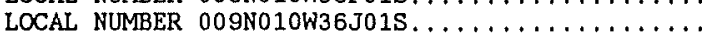
LOCAL NUMBER 009N010W36J02S. . . . . . . . . .

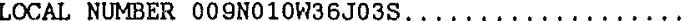

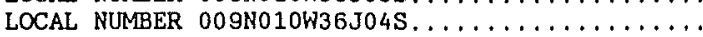

LOCAL NUMBER DOgN010W36P01S...............

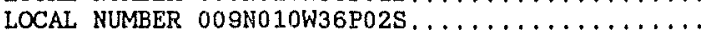
LOCAL NUMBER OOSN010W36P03S. . . . . . . . . .

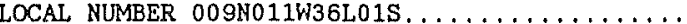

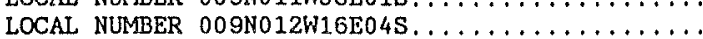


KERN COUNTY--Continued

Page

LOCAL NUMBER D09N012W23N01S ............

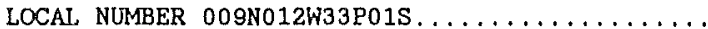

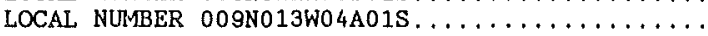

LOCAL NUMBER 009N013W07Q03S. . . . . . . . .

LOCAL NUMBER 009N013W14Q01S . . . . . . . . .

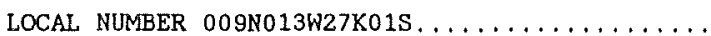

LOCAL NUMBER 009N014W01H01S . . . . . . . . . . .

LOCAL NUMBER $009 N 014$ W20B01S . . . . . . . . . .

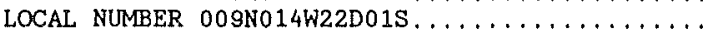

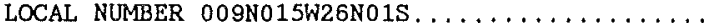

LOCAL NUMBER 009N015W30Q01S . . . . . . . . . .

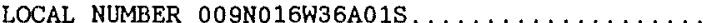

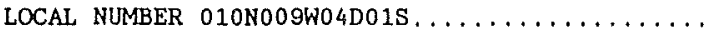

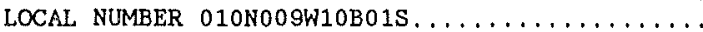

LOCAL NUMBER 010NOOSW10B02S..............

LOCAL NUMBER 010N009W24A02S ...........

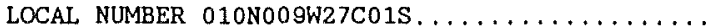

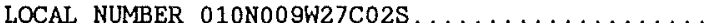

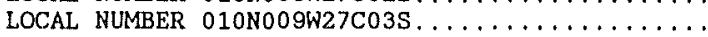

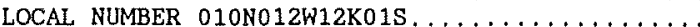

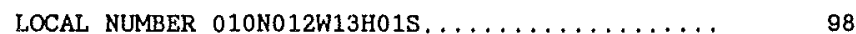

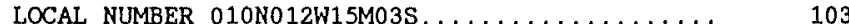

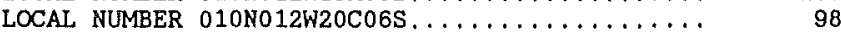

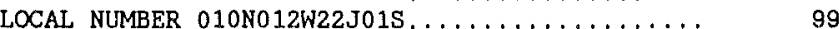

LOCAL NUMBER 010N013W32D01S, ............. 103

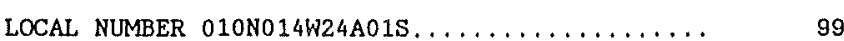

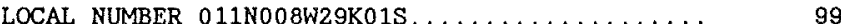

LOCAL NUMBER 011 N009W13D01S..............

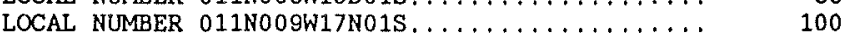

LOCAL NUMBER 011N009W24A01S................. 100

LOCAL NUMBER 011 N009W30H01S............... 100

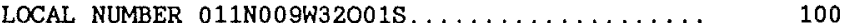

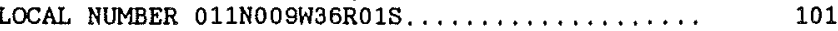

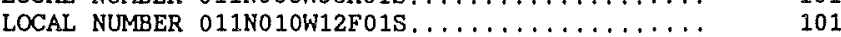

LOCAL NUMBER 011N011W07A01S............... 101

LOCAL NUMBER 011 N011W09A01S. . . . . . . . . . . 101

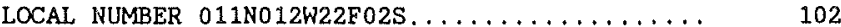

LOCAL NUMBER 011 N013W19C01S . . . . . . . . . . . . . 102

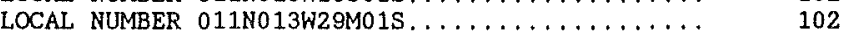

LOCAL NUMBER 012N012W35R01S . . . . . . . . . . . . . 102

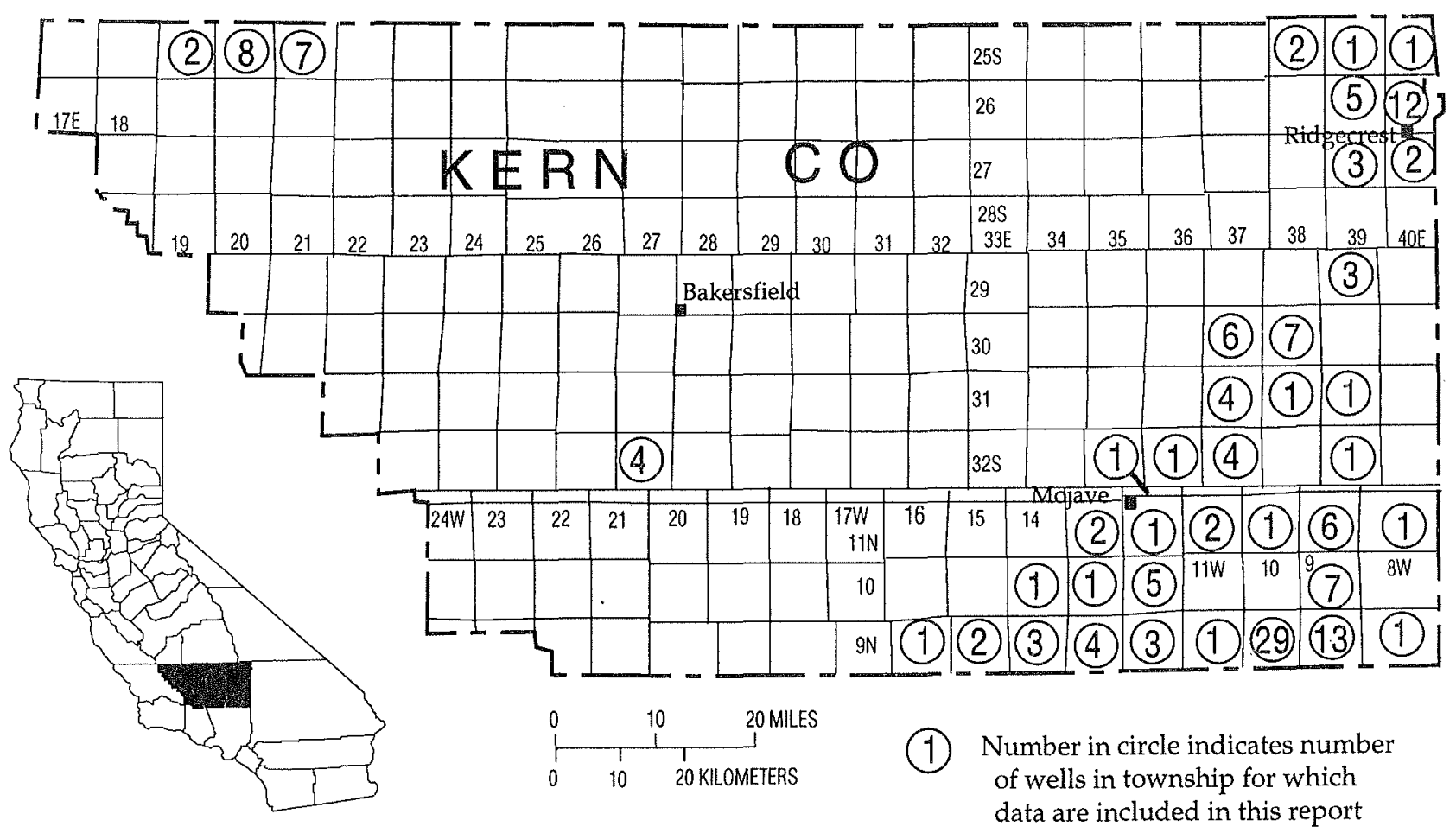

Figure 10. Location of wells in Kern County. 


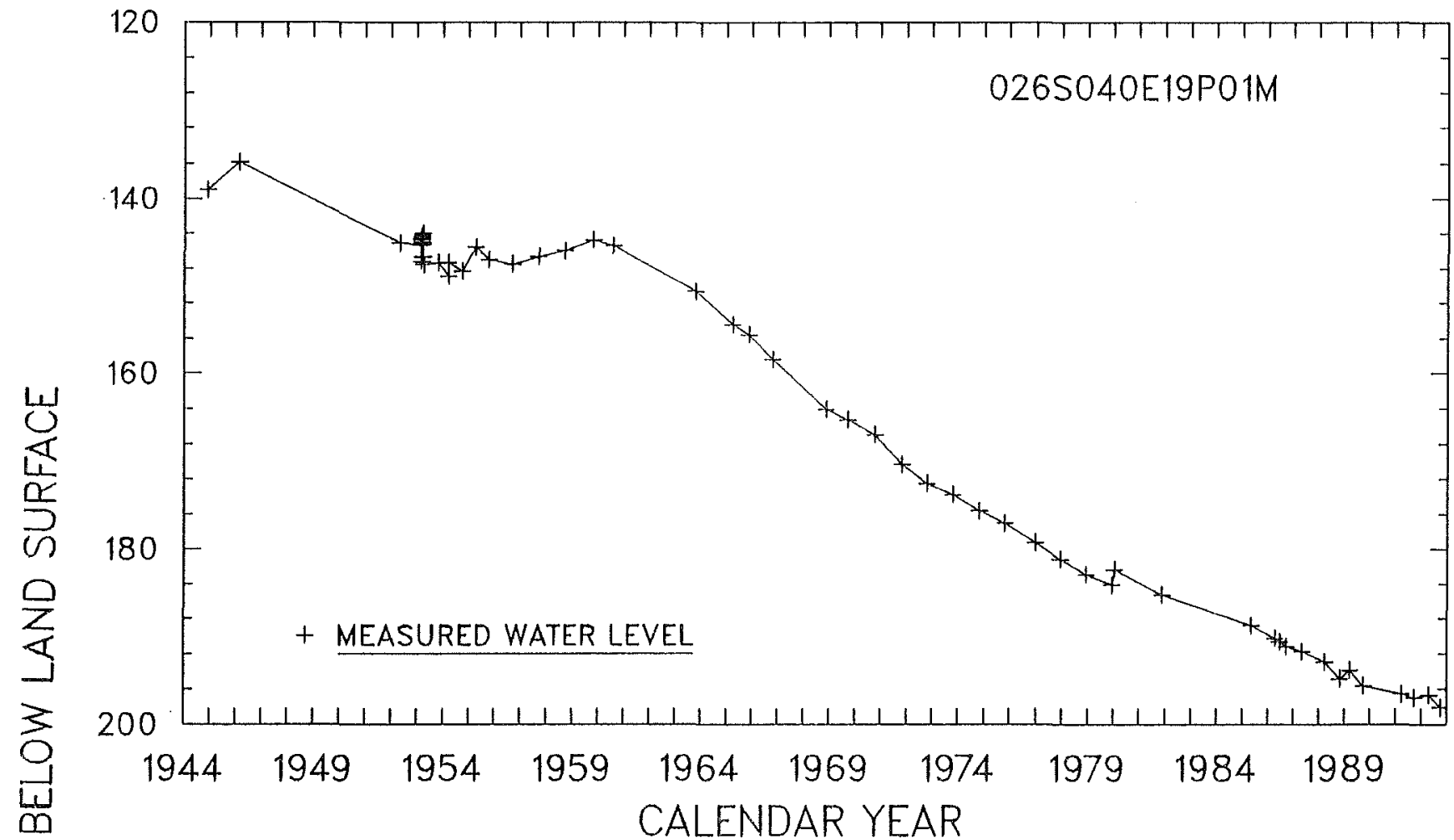

荘

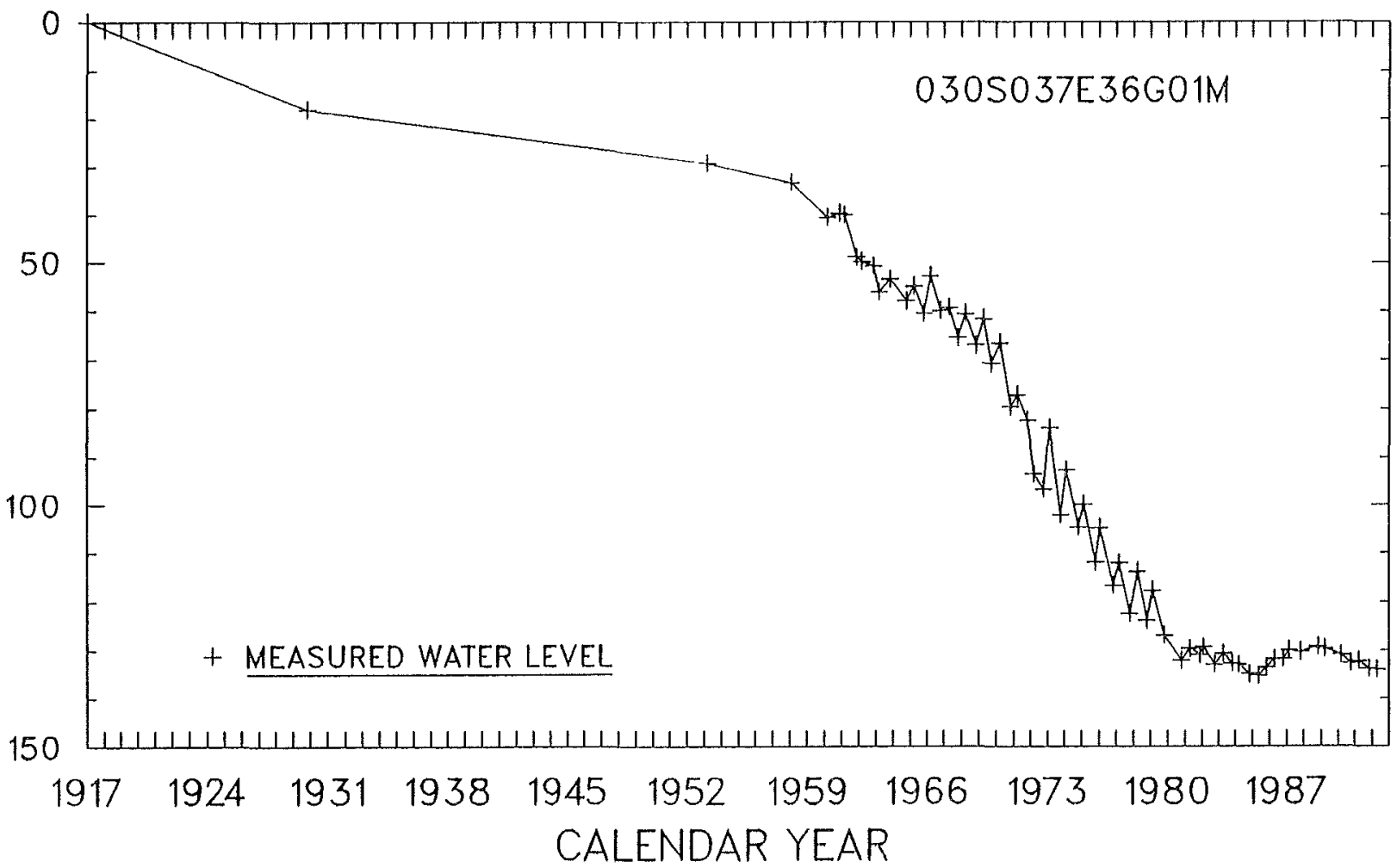

Figure 11. Hydrographs for wells in Kern County. (026S040E19P01M and 030S037E36G01M) 


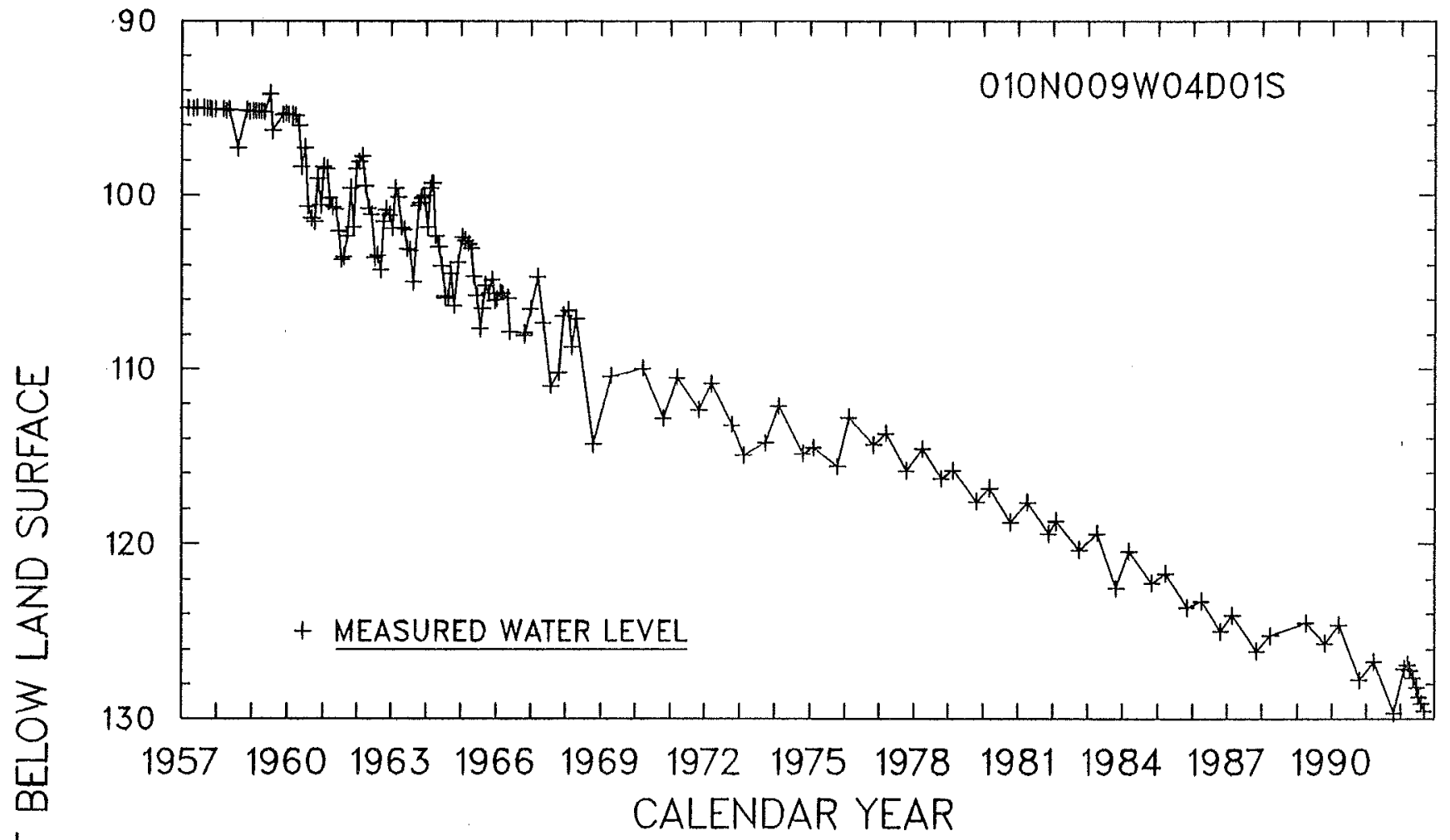

嵌

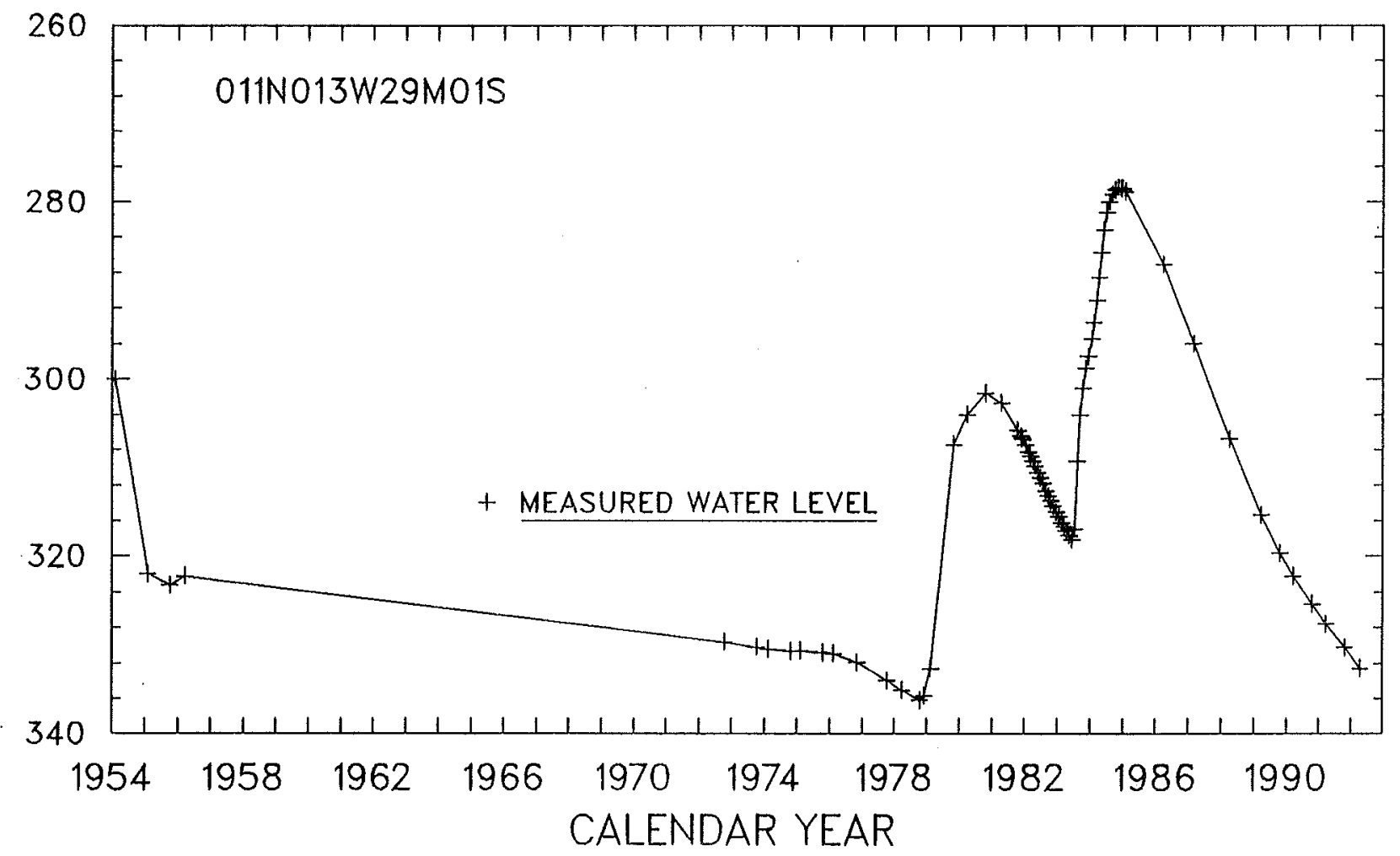

Figure 12. Hydrographs for wells in Kern County. (010N009W04DO1S and 011N013W29M01S) 
WELL DESCRIPTIONS AND WATER-LEVEL MEASUREMENTS

WATER YEAR OCTOBER 1991 TO SEPTEMBER 1992

SITE NUMBER 354212119540901

LOCAL NUMBER 025S019E34R01M

About 16 miles northwest of Lost Hills. Drilled observation well. Diameter 6 inches, depth 316 feet, perforated 301-311 feet. Altitude of land-surface datum 516 feet. Water-level records available 1988 to current year.

WATER LEVELS IN FEET BELOW LAND-SURFACE DATUM

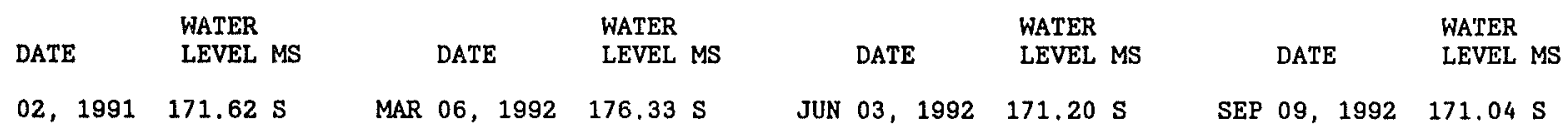

PERIOD OF RECORD HIGHEST 171.04 SEP 09, 1992 LOWEST 176.33 MAR 06, 1992

SITE NUMBER 354210119540901

LOCAL NUMBER 025S019E34R02M

About 16 miles northwest of Lost Hills, Drilled observation well. Diameter 6 inches, depth 225 feet, perforated 210-220 feet. Altitude of land-surface datum 516 feet. Water-level records available 1988 to current year.

WATER LEVELS IN FEET BELOW LAND-SURFACE DATUM

$\begin{array}{llllll} & \text { WATER } & & \text { WATER } & \text { WATER } & \text { WATER } \\ \text { DATE } & \text { LEVEL MS } & \text { DATE } & \text { LEVEL MS } & \text { DATE } & \text { LEVEL MS }\end{array}$

DEC 02, $1991 \quad 173.49 \mathrm{~S} \quad$ MAR 09, $1992 \quad 173.49 \mathrm{~S} \quad$ JUN 03, $1992 \quad 173.20 \mathrm{~S} \quad$ SEP $09,1992172.90 \mathrm{~S}$

PERIOD OF RECORD HIGHEST 172.90 SEP 09, 1992 LOWEST 177.58 NOV 21,1988

SITE NUMBER 354540119474101

LOCAL NUMBER 025SO2OE11N01M

About 13 miles northwest of Lost Hills. Drilled observation well. Diameter 3 inches, depth 10 feet. Altitude of land-surface datum 257 feet. Water-level records available 1988 to current year.

WATER LEVELS IN FEET BELOW LAND-SURFACE DATUM

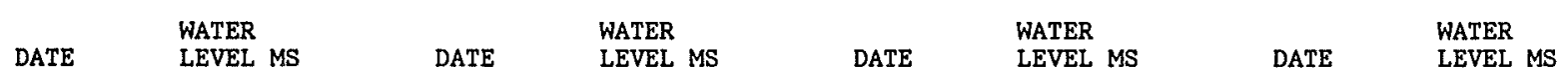

$\begin{array}{lllllllll}\text { DEC 02, } 1991 \quad 5.86 \mathrm{~S} & \text { MAR 09, } 1992 & 4.76 \mathrm{~S} & \text { JUN } 03,1992 & 4.07 \mathrm{~S} & \mathrm{SEP} & 09,1992 & 5.23 \mathrm{~S}\end{array}$

PERIOD OF RECORD HIGHEST 2.55 DEC $12,1990 \quad$ LOWEST 6.24 NOV 21, 1988

SITE NUMBER 354537119474401

LOCAL NUMBER O25S020E15A02M

About 13 miles northwest of Lost Hills. Drilled observation well. Diameter 6 inches, depth 105 feet, perforated 90-100 feet. Altitude of land-surface datum 260 feet. Water-level records available 1988 to current year.

WATER LEVELS IN FEET BELOW LAND-SURFACE DATUM

$\begin{array}{llllll}\text { DATE } & \text { WATER } & & \text { WATER } & \text { WATER } & \text { WATER } \\ \text { LEVEL MS } & \text { DATE } & \text { LEVEL MS } & \text { DATE } & \text { LEVEL MS } & \text { DATE }\end{array}$

$\begin{array}{llllllllll}\text { DEC } 02,1991 & 5.22 \mathrm{~s} & \text { MAR 09, } 1992 & 3.98 \mathrm{~s} & \text { JUN } 03,1992 & 2.87 \mathrm{~s} & \mathrm{SEP} 09,1992 & 3.70 \mathrm{~s}\end{array}$

PERIOD OF RECORD HIGHEST 1.42 APR $19,1990 \quad$ LOWEST 5.22 DEC 02, 1991 
WELL DESCRIPTIONS AND WATER-LEVEL MEASUREMENTS

WATER YEAR OCTOBER 1991 TO SERTEMBER 1992

SITE NUMBER 354535119474401

LOCAL NUMBER O25SO20E15A03M

About 13 miles northwest of Lost Hills. Drilled observation well. Diameter 6 inches, depth 55 feet, perforated 40-50 feet. Altitude of land-surface datum 260 feet. Water-level records available 1988 to current year.

WATER LEVELS IN FEET BELOW LAND-SURFACE DATUM

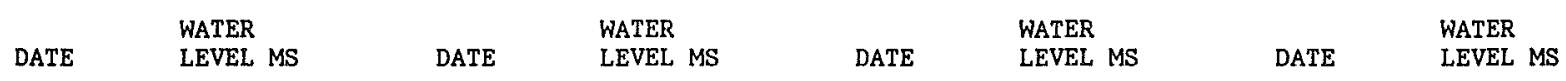

$\begin{array}{lllllllll}\text { DEC 02, } 1991 & 6.43 \mathrm{~S} & \text { MAR 09, } 1992 & 5.31 \mathrm{~S} & \text { JUN } 03,1992 & 4.29 \mathrm{~S} & \mathrm{SEP} & 09,1992 & 4.98 \mathrm{~S}\end{array}$

PERIOD OF RECORD HIGHEST 3.06 MAR 21, 1989 LOWEST 6.43 DEC 02, 1991

SITE NUMBER 354538119474401

LOCAL NUMBER 025S020E15A04M

About 13 miles northwest of Lost Hills, Drilled observation well. Diameter 6 inches, depth 224 feet, perforated 209-219 feet. Altitude of land-surface datum 260 feet. Water-level records available 1988 to current year.

WATER LEVELS IN FEET BELOW LAND-SURFACE DATUM (READINGS ABOVE LAND SURFACE INDICATED BY "+")

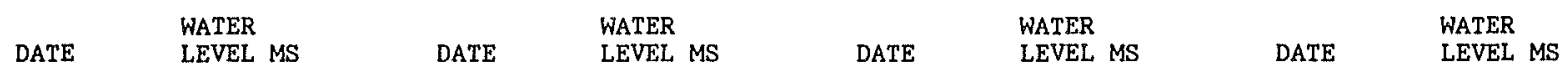

DEC 02, $1991+1.37 \mathrm{~F} \quad$ MAR 09, $1992+1.10 \mathrm{~F} \quad$ JUN 03, $1992+1.40 \mathrm{~F} \quad$ SEP $09,1992 \quad+1.40 \quad F$

PERIOD OF RECORD HIGHEST +1.40 JUN 03, SEPT 09, 1992 LOWEST 2.31 MAR 08, 1988

SITE NUMBER 354351119505701

LOCAL NUMBER 025S020E30A01M

About 11 miles northwest of Lost Hills. Drilled observation well. Diameter 6 inches, depth 152 feet, perforated 137-147 feet. Altitude of land-surface datum 380 feet. Water-level records available 1988 to current year.

WATER LEVELS IN FEET BELOW LAND-SURFACE DATUM

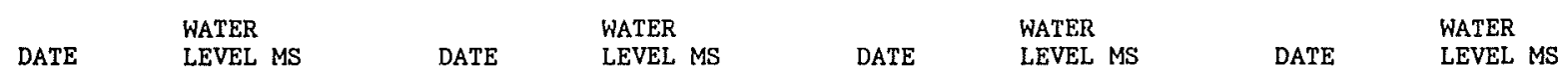

$\begin{array}{llllllllll}\text { DEC } 02,1991 & 37.90 \mathrm{~S} & \text { MAR 09, } 1992 & 39.05 \mathrm{~S} & \text { JUN } 03,1992 & 38.35 \mathrm{~S} & \text { SEP } 09,1992 & 38.15 \mathrm{~S}\end{array}$

PERIOD OF RECORD HIGHEST 36.61 SEP 28, 1989 LOWEST 42.87 MAR 10, 1988

SITE NUMBER 354350119505701

LOCAL NUMBER 025S020E30A02M

About 11 miles northwest of Lost Hills. Drilled observation well. Diameter 5 inches, depth 226 feet, perforated 211-221 feet. Altitude of land-surface datum 380 feet. Water-level records available 1988 to current year.

WATER LEVELS IN FEET BELOW LAND-SURFACE DATUM

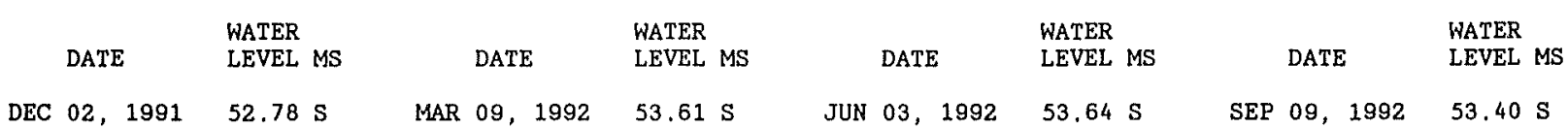

PERIOD OF RECORD HIGHEST 52.20 MAY $30,1991 \quad$ LOWEST 57.58 MAR 10,1988 
WELL DESCRIPTIONS AND WATER-LEVEL MEASUREMENTS

WATER YEAR OCTOBER 1991 TO SEPTEMBER 1992

SITE NUMBER 354349119505701

LOCAL NUMBER O25SO2OE30A03M

About 11 miles northwest of Lost Hills. Drilled observation well. Diameter 6 inches, depth 114 feet, perforated 99-109 feet. Altitude of land-surface datum 380 feet. Water-level records available 1988 to current year.

WATER LEVELS IN FEET BELOW LAND-SURFACE DATUM

\begin{tabular}{llllllll} 
& WATER & \multicolumn{3}{c}{ WATER } & & \multicolumn{3}{c}{ WATER } \\
DATE & LEVEL MS & DATE & LEVEL MS & DATE & LEVEL MS & DATE \\
02,1991 & $13.27 \mathrm{~S}$ & MAR 09,1992 & $14.26 \mathrm{~S}$ & JUN 03,1992 & $12.04 \mathrm{~S}$ & SEP 09,1992 & $12.82 \mathrm{~S}$
\end{tabular}

PERIOD OF RECORD HIGHEST 9.85 JUN 29, 1989 LOWEST $18.22 \quad$ MAR 10,1988

SITE NUMBER 354348119505701

LOCAL NUMBER 025S020E30A04M

About 11 miles northwest of Lost Hills. Drllled observation well. Diameter 6 inches, depth 71 feet, perforated 56-66 feet. Altitude of land-surface datum 380 feet. Water-level records available 1988 to current year.

WATER LEVELS IN FEET BELOW LAND-SURFACE DATUM

$\begin{array}{llllll} & \text { WATER } & \text { WATER } & & \text { WATER } \\ \text { DATE } & \text { LEVEL MS } & \text { DATE } & \text { LEVEL MS } & \text { DATE } & \text { LEVEL MS }\end{array}$

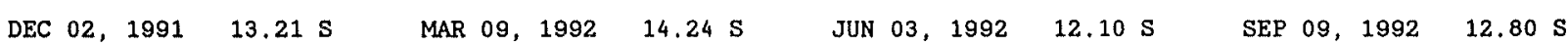

PERIOD OF RECORD HIGHEST 9.65 JUN 29,1989 LOWEST 17.79 MAR 10, 1988

SITE NUMBER 354634119401301

LOCAL NUMBER 025S021E01N01M

About 13 miles north of Lost Hills. Drilled observation well. Diameter 2 inches, depth 20 feet, screened 10-20 feet. Altitude of land-surface datum 290 feet. Water-level records available 1990 to current year.

WATER LEVELS IN FEET BELOW LAND-SURFACE DATUM

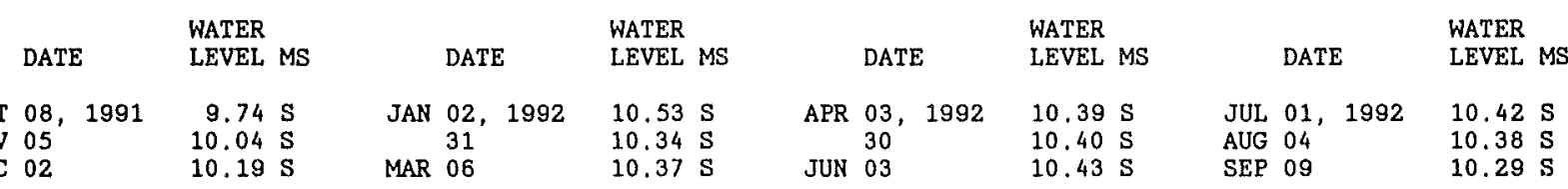

PERIOD OF RECORD HIGHEST 7.03 APR 08, 1991 LOWEST 10.53 JAN 02, 1992

SITE NUMBER 354634119401302

LOCAL NUMBER 025S021E01NO2M

About 13 miles north of Lost Hills. Drilled observation well. Diameter 2 inches, depth 67 feet, screened 52-62 feet. Altitude of land-surface datum 290 feet. Water-level records available 1990 to curront year.

WATER LEVELS IN FEET BELOW LAND-SURFACE DATUM

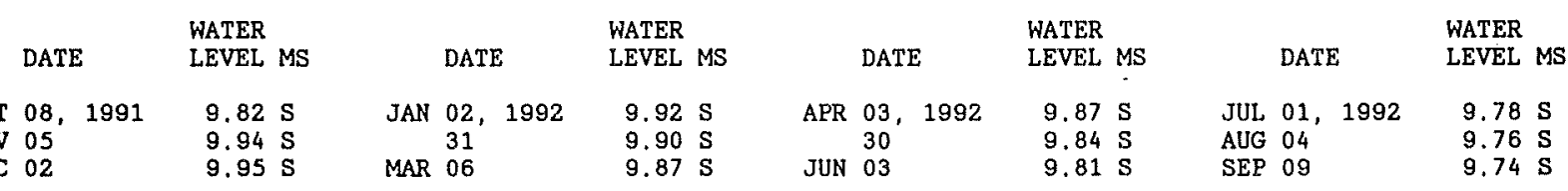

PERIOD OF RECORD HIGHEST 6.30 AUG 29,1990 LOWEST 9.95 DEC 02, 1991 
WELL DESCRIPTIONS AND WATER-LEVEL MEASUREMENTS

WATER YEAR OCTOBER 1991 TO SEPTEMBER 1992

SITE NUMBER 354634119401303

LOCAL NUMBER 025S021E01N03M

About 13 miles north of Lost Hills. Drilled observation well. Diameter 2 inches, depth 100 feet, screened 90-100 feet. Altitude of land-surface datum 290 feet. Water-level records avallable 1990 to current year. WATER LEVELS IN FEET BELOW LAND-SURFACE DATUM

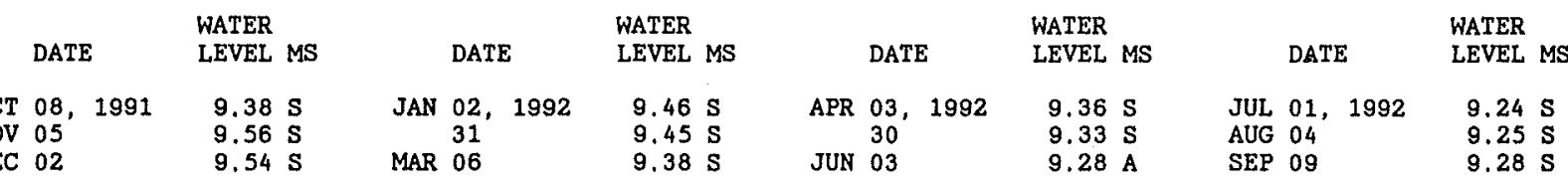

PERIOD OF RECORD HIGHEST 6.19 MAY 02, 1991 LOWEST 9.56 NOV 05, 1991

SITE NUMBER 354634119401304

LOCAL NUMBER 025S021E01NO4M

About 13 miles north of Lost Hills. Drilled observation well. Diameter 3 inches, depth 204 feet, screened 189-199 feet. Altitude of land-surface datum 290 feet. Water-level records available 1990 to current year. WATER LEVELS IN FEET BELOW LAND-SURFACE DATUM

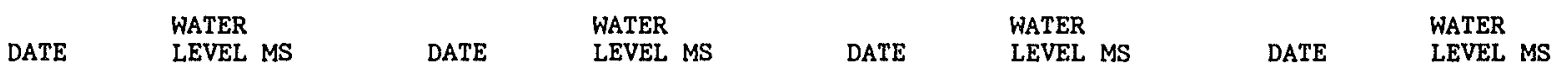

$\begin{array}{lllllllllll}\text { OCT } 08,1991 & 6.45 \mathrm{~S} & \text { JAN } 02,1992 & 6.78 \mathrm{~S} & \text { APR } 03,1992 & 6.66 \mathrm{~S} & \text { JUL } 01,1992 & 6.74 \mathrm{~S} \\ \text { NOV } 05 & 6.73 \mathrm{~S} & 31 & 6.79 \mathrm{~S} & 30 & 6.68 \mathrm{~S} & \text { AUG } 04 & 6.89 \mathrm{~S} \\ \text { DEC } 02 & 6.83 \mathrm{~S} & \text { MAR } 06 & 6.62 \mathrm{~S} & \text { JUN } 03 & 6.69 \mathrm{~S} & \text { SEP } 09 & 7.03 \mathrm{~S}\end{array}$

PERIOD OF RECORD HIGHEST 4.29 MAY 02, 1991 LOWEST 7.03 SEP 09, 1992

SITE NUMBER 354633119450401

LOCAL NUMBER 025S021E07B01M

About 12 miles north of Lost Hills. Drilled observation well. Diameter 2 inches, depth 83 feet, perforated

77-82 feet. Altitude of land-surface datum 217 feet, Water-level records available 1989 to current year.

WATER LEVELS IN FEET BELOW LAND-SURFACE DATUM

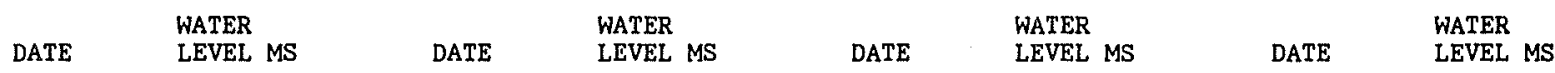
DEC 02,1991
$9.59 \mathrm{~S}$
MAR 06, 1992
$9.61 \mathrm{~S}$
JUN 03,1992
$9.33 \mathrm{~S}$
SEP 09,1992
$9.36 \mathrm{~s}$

PERIOD OF RECORD HIGHEST 5.24 SEP 28, 1989 LOWEST 9.61 MAR 06, 1992

SITE NUMBER 354633119450402

LOCAL NUMBER 025S021E07B02M

About 12 miles north of Lost Hills. Drilled observation well. Diameter 2 inches, depth 53 feet, perforated 47-52 feet. Altitude of land-surface datum 217 feet. Water-ievel records available 1989 to current year.

WATER LEVELS IN FEET BELOW LAND-SURFACE DATUM

\begin{tabular}{|c|c|c|c|c|c|c|c|}
\hline DATE & $\begin{array}{l}\text { WATER } \\
\text { LEVEL MS }\end{array}$ & DATE & $\begin{array}{l}\text { WATER } \\
\text { LEVEL MS }\end{array}$ & DATE & $\begin{array}{l}\text { WATER } \\
\text { LEVEL MS }\end{array}$ & DATE & $\begin{array}{l}\text { WATER } \\
\text { LEVEL MS }\end{array}$ \\
\hline
\end{tabular}

$\begin{array}{lllllllllll}\text { DEC 02, } 1991 & 8.35 \mathrm{~S} & \mathrm{MAR} & 06,1992 & 7.94 \mathrm{~S} & \text { JUN } 03,1992 & 7.67 \mathrm{~S} & \text { SEP } 09,1992 & 7.61 \mathrm{~S}\end{array}$

PERIOD OF RECORD HIGHEST 5.83 MAY 30, 1991 LOWEST 8.35 DEC 02, 1991 
WELL DESCRIPTIONS AND WATER-LEVEL MEASUREMENTS

WATER YEAR OCTOBER 1991 TO SEPTEMBER 1992

SITE NUMBER 354633119450403

LOCAL NUMBER 025S021E07B03M

About 12 miles north of lost Hills. Drilled observation well. Diameter 2 inches, depth 23 feet, perforated 17-22 feet. Altitude of land-surface datum 217 feet. Water-level records available 1989 to current year.

WATER LEVELS IN FEET BELOW LAND-SURFACE DATUM

\begin{tabular}{|c|c|c|c|c|c|c|c|}
\hline DATE & $\begin{array}{l}\text { WATER } \\
\text { LEVEL MS }\end{array}$ & DATE & $\begin{array}{l}\text { WATER } \\
\text { LEVEL MS }\end{array}$ & DATE & $\begin{array}{l}\text { WATER } \\
\text { LEVEL MS }\end{array}$ & DATE & $\begin{array}{l}\text { WATER } \\
\text { LEVEI MS }\end{array}$ \\
\hline
\end{tabular}

$\begin{array}{llllllllll}\text { DEC } 02,1991 & 8.36 \mathrm{~S} & \mathrm{MAR} 06,1992 & 7.79 \mathrm{~S} & \text { JUN } 03,1992 & 7.63 \mathrm{~S} & \text { SEP } 09,1992 & 7.59 \mathrm{~S}\end{array}$

PERIOD OF RECORD HIGHEST 5.94 MAY 30, 1991 LOWEST 8.36 DEC 02, 1991

SITE NUMBER 354541117501901

LOCAL NUMBER 025S038E13L01M

About 8 miles north of Inyokern, east of Brown Road. Drilled irrigation well. Diameter 16 inches, depth 444 feet, perforated 109-444 feet. Altitude of land-surface datum 2,320 feet. Water-level records available 1976 , 1978 to current year.

WATER LEVELS IN FEET BELOW LAND-SURFACE DATUM

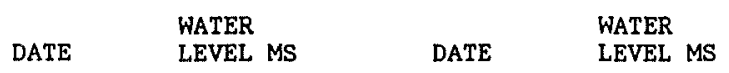

APR 17, $1992144.03 \mathrm{~S} \quad$ SEP 29, $1992140.82 \mathrm{SR}$

PERIOD OF RECORD HIGHEST 123.58 JAN 13, 1978 LOWEST 145.22 SEP 24, 1991

SITE NUMBER 354458117511801

LOCAL NUMBER 025S038E23G01M

About $7.5 \mathrm{miles}$ north of Inyokern, $0.8 \mathrm{mile}$ west of railroad tracks, and $2.6 \mathrm{miles}$ north of Leliter Road. Drilled unused water-table well. Diameter 12 inches, depth 259 feet in 1953 . Altitude of land-surface datum 2,412 feet. Water-level records available 1921, 1946, 1953-54, 1959, 1961 to current year.

WATER LEVELS IN FEET BELOW LAND-SURFACE DATUM

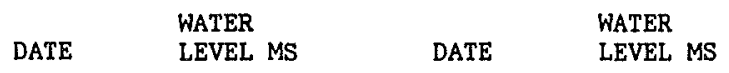

APR $17,1992224.55 \mathrm{~S} \quad$ SEP $29,1992 \quad 228.30 \mathrm{~S}$

PERIOD OF RECORD HIGHEST 202.22 APR 07, 1953 LOWEST 232.82 SEP 19,1987

SITE NUMBER 354240117485301

LOCAL NUMBER O25S039E31R01M

On China Lake Naval Weapons Center, about 4.5 miles north of Inyokern, and about 0.9 mile east of Leliter Road. Drilled observation well. Diameter 6 inches, depth drilled 603 feet, cased to 480 feet, perforated $120-180$ feet. Altitude of land-surface datum 2,260 feet. Water-level records avaliable 1986 to current year.

WATER LEVELS IN FEET BELOW LAND-SURFACE DATUM

$\begin{array}{lll} & \text { WATER } & \\ \text { DATE } & \text { WEVEL MS } & \text { DATE } \\ \text { LEVEL MS }\end{array}$

APR 16, $199274.38 \mathrm{~S} \quad \operatorname{SEP} 29,1992 \quad 75.09 \mathrm{~S}$

PERIOD OF RECORD HIGHEST 70.38 JAN 22, 1986 LOWEST 76.43 SEP 19, 1986 
WELL DESCRIPTIONS AND WATER-LEVEL MEASUREMENTS

WATER YEAR OCTOBER 1991 TO SEPTEMBER 1992

SITE NUMBER 354240117383501

LOCAL NUMBER 025SO40E35P01M

On China Lake Naval Weapons Center about 250 feet southwest of tower road. Bored observation water-table well. Diameter 1.25 inches, depth measured 15.4 feet, perforated 6.1-15.4 feet. Altitude of land-surface datum 2,158.8 feet. Water-level records available 1953-54, 1959, 1961 to current year.

WATER LEVELS IN FEET BELOW LAND-SURFACE DATUM

$$
\text { DATE WATER }
$$

APR 16, $1992 \quad 8.19 \mathrm{~S}$

PERIOD OF RECORD HIGHEST 7.77 APR 22, 1985 LOWEST 9.59 DEC 17, 1953

SITE NUMBER 354055117494402

LOCAL NUMBER 026S039E07NO2M

About 2.5 miles north of Inyokern, 0.45 mile east of Brown Road, and 50 feet north of Athel Street. Drilled domestic well. Diameter 6 inches, depth 368 feet. Altitude of land-surface datum 2,395.3 feet. Water-1evel records available 1986 to current year.

WATER LEVELS IN FEET BELOW LAND-SURFACE DATUM

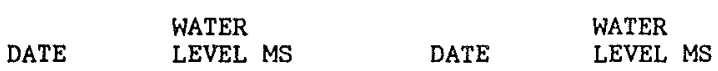

APR 17, $1992 \quad 215.43 \mathrm{~S} \quad \mathrm{SEP} 29,1992 \quad 214.50 \mathrm{~S}$

PERIOD OF RECORD HIGHEST 209.25 SEP 28,1986 LOWEST 216.81 OCT 29,1988

SITE NUMBER 353959117431903

LOCAL NUMBER 026S039E13R04M

On China Lake Naval Weapons Center about $0.5 \mathrm{mile}$ north of Snort access road and $1.2 \mathrm{miles}$ east of Snort tower. Drilled observation water-table weil. Diameter 6 inches, depth 800 feet, perforated $640-780$ feet. Altitude of land-surface datum 2,318 feet. Water-level records avallable 1987 to current year.

WATER LEVELS IN FEET BELOW LAND-SURFACE DATUM

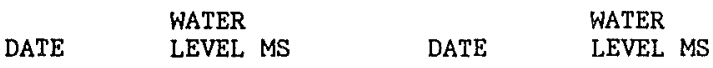

APR 16, $1992 \quad 197.53 \mathrm{~S} \quad$ SEP 29, $1992 \quad 212.08 \mathrm{~S}$

PERIOD OF RECORD HIGHEST 160.54 JUL 02, 1987 LOWEST 212.08 SEP 29, 1992

SITE NUMBER 354033117480901

LOCAL NUMBER 026S039E17F02M

On China Lake Naval Weapons Center, about 1.5 miles north of Inyokern, about 1 mile east of Brown Road, and $0.15 \mathrm{mile}$ north of Caddy Road. Drilled observation well. Diameter 14 inches, depth 881 feet, perforated 681-881 feet. Altitude of land-surface datum 2,340 feet. Water-level records available 1985 to current year.

WATER LEVELS IN FEET BELOW LAND-SURFACE DATUM

$$
\begin{array}{lll} 
& \text { WATER } & \\
\text { DATE } & \text { WATER } \\
\text { LEVEL MS } & \text { DEVEL MS }
\end{array}
$$

APR 17, $1992171.70 \mathrm{~S} \quad \operatorname{SEP} 29,1992179.60 \mathrm{~S}$

PERIOD OF RECORD HIGHEST 168.19 MAY 13, 1985 LOWEST 179.60 SEP 29, 1992 
WELL DESCRIPTIONS AND WATER-LEVEL MEASUREMENTS

WATER YEAR OCTOBER 1991 TO SEPTEMBER 1992

SITE NUMBER 353921117433901

LOCAL NUMBER 0265039E24K01M

On China Lake Naval Weapons Center, about 3.5 miles west of the town of China Lake, about 0.3 mile north of government railroad, and $3.2 \mathrm{miles}$ west of Sandquist Road, Drilled unused water-table well, Diameter 16 inches, depth 323.1 feet in 1953, perforated 190-197, 230-278, 287-301 feet. Altitude of land-surface datum 2,347.4 feet. Water-level records available $1952-79,1981$ to current year.

WATER LEVELS IN FEET BELOW LAND-SURFACE DATUM

$$
\begin{array}{lll} 
& \text { WATER } & \\
\text { DATE } & \text { WEVEL MS } & \text { WATER } \\
& \text { LEVEL MS }
\end{array}
$$

APR $17,1992 \quad 205,19 \mathrm{~S} \quad \operatorname{SEP} 28,1992 \quad 207,81 \mathrm{~S}$

PERIOD OF RECORD HIGHEST 153.54 MAR 16, 1953 LOWEST 207.81 SEP 28, 1992

SITE NUMBER 353910117430201

LOCAL NUMBER 026S040E19P01M

On China Lake Naval Weapons Center, about $2.5 \mathrm{miles}$ west of Ridgecrest, about $0.3 \mathrm{mile}$ east of extension of Jacks

Ranch Road. Drilled public-supply water-table well. Diameter 16 inches, depth 261 feet, perforated 192-220,

253-259 feet. Altitude of land-surface datum 2,336 feet. Water-level records available 1944, $1946,1952-60$, 1963, 1965 to current year.

WATER LEVELS IN FEET BELOW LAND-SURFACE DATUM

$$
\begin{array}{lll} 
& \text { WATER } & \\
\text { DATE } & \text { WEVEL MS } & \text { WATER } \\
& \text { LEVEL MS }
\end{array}
$$

APR $17,1992196.68 \mathrm{~S} \quad \operatorname{SEP} 29,1992198,09 \mathrm{~S}$

PERIOD OF RECORD HIGHEST 135.90 FEB 20,1946 LOWEST 198.09 SEP 29,1992

\section{SITE NUMBER 353910117420801}

LOCAL NUMBER 026S040E2ONO1M

On China Lake Naval Weapons Center, about 2 miles west of Ridgecrest, about 2.9 miles west of intersection of Inyokern Road, and north China Lake Boulevard. Drilled public-supply water-table well. Diameter 16 inches, depth 190.1 feet in 1952. Altitude of land-surface datum 2,311.8 feet. Water-level records available 1945 , 1952 to current year.

WATER LEVELS IN FEET BELOW LAND-SURFACE DATUM

$\begin{array}{lll} & \text { WATER } & \\ \text { DATE } & \text { LEVEL MS } & \text { WATER } \\ & \text { LEVEL MS }\end{array}$

APR 15, $1992164.98 \mathrm{~S} \quad$ SEP 29, $1992165.03 \mathrm{~S}$

PERIOD OF RFCORD HIGHEST $112.00 \quad$ SEP 07,1945 LOWEST $165.03 \quad$ SEP 29,1992

SITE NUMBER 353908117395201

LOCAL NUMBER 026S040E22P01M

In the town of China Lake about 100 feet west of Bullard Road and 0.25 mile north of Inyokern Road. Drilled unused water-table well. Diameter 8 inches, depth 830 feet, perforated $530-830$ feet. Altitude of land-surface datum $2,258.7$ feet. Water-level records available 1954 to current year.

WATER LEVELS IN FEET BELOW LAND-SURFACE DATUM

$$
\begin{array}{lll} 
& \text { WATER } & \\
\text { DATE } & \text { WEVEL MS } & \text { WATER } \\
\text { LEVEL MS }
\end{array}
$$

APR $15,1992112.88 \mathrm{~S} \quad \operatorname{SEP} 29,1992112.63 \mathrm{~s}$

PERIOD OF RECORD HIGHEST 64.00 MAR 01, 1954 LOWEST 116.41 SEP 24, 1991 
WELL DESCRIPTIONS AND WATER-LEVEL MEASUREMENTS

WATER YEAR OCTOBER 1991 TO SEPTEMBER 1992

SITE NUMBER 353912117394601

LOCAL NUMBER 026S040E22P03M

In the town of China Lake about $0.15 \mathrm{mile}$ north of East Inyokern Road. Drilled observation well. Diameter 6 inches, depth 415 feet in 1981, perforated 400-415 feet. Altitude of land-surface datum 2,273 feet. Water-level records available 1983 to current year.

WATER LEVELS IN FEET BELOW LAND-SURFACE DATUM

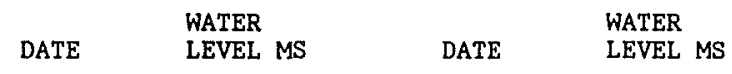

APR 16, $1992105.62 \mathrm{~S} \quad$ SEP 29, $1992105.12 \mathrm{~S}$

PERIOD OF RECORD HIGHEST 37.65 OCT 01,1983 LOWEST 105.62 APR 16, 1992

SITE NUMBER 353912117394602

LOCAL NUMBER 026S040E22PO4M

In the town of China Lake about 0.15 mile north of East Inyokern Road. Drilled observation well. Diameter 6 inches, depth 215 feet in 1981, perforated 200-215 feet. Altitude of land-surface datum 2,273 feet. Water-level records avaliable 1983 to current year.

WATER LEVELS IN FEET BELOW LAND-SURFACE DATUM

$$
\begin{array}{lll} 
& \text { WATER } & \\
\text { DATE } & \text { WEVEL MS } & \text { DATE }
\end{array}
$$

APR 16, $199270.32 \mathrm{~S}$ SEP 29, $1992 \quad 69.78 \mathrm{~S}$

PERIOD OF RECORD HIGHEST 36.73 JUN 02, 1985 LOWEST 74.68 MAR 26, 1991

SITE NUMBER 353955117381601

LOCAL NUMBER 026S040E23B02M

On China Lake Naval Weapons Centex about 1 mile north of the town of China Lake and 0.15 mile north of Knox Road. Drilled observation well. Diameter 6 inches, depth 360 feet, perforated 300-340 feet. Altitude of land-surface datum 2,210 feet. Water-level records available 1986 to current year.

WATER LEVELS IN FEET BELOW LAND-SURFACE DATUM

$$
\begin{array}{lll} 
& \text { WATER } & \text { WATER } \\
\text { DATE } & \text { DEVEL MS }
\end{array}
$$

APR 16, $1992 \quad 26.08 \mathrm{~S} \quad$ SEP $29,1992 \quad 27.22 \mathrm{~S}$

PERIOD OF RECORD HIGHEST 23.60 DEC 11, 1986 LOWEST 28.15 OCT 23, 1986

SITE NUMBER 353955117381602

LOCAL NUMBER 026S040E23B03M

On China Lake Naval Weapons Center about 1 mile north of the town of China Lake and 0.15 mile north of Knox Road. Drilled observation weli. Diameter 6 inches, depth 240 feet, perforated 180-220 feet. Altitude of land-surface datum 2,210 feet. Water-level records available 1986 to current year.

WATER LEVELS IN FEET BELOW LAND-SURFACE DATUM

$$
\begin{array}{lll} 
& \text { WATER } & \text { WATER } \\
\text { DATE } & \text { LEVEL MS } & \text { DATE } \\
\end{array}
$$

APR 16, $1992 \quad 22.82 \mathrm{~S} \quad$ SEP 29, $1992 \quad 23.64 \mathrm{~S}$

PERTOD OF RECORD HIGHEST 14.31 DEC 11, 1986 LOWEST 26.91 MAR 13, 1991 
WATER LEVELS, KERN COUNTY--Continued

WELL DESCRIPTIONS AND WATER-LEVEL MEASUREMENTS

WATER YEAR OCTOBER 1991 TO SEPTEMBER 1992

SITE NUMBER 353838117424401

LOCAL NUMBER 026S040E30KO1M

About 0.60 mile south of Ridgecrest Inyokern Road and 0.95 mile west of North Mahan Street. Drilled public-supply water-table well. Diameter 16 inches, depth 730 feet, cased to 730 feet, perforated 230-400, 610-730 feet.

Altitude of land-surface datum 2,340 feet. Water-level records available 1964-66, 1969, 1971-72, 1974-75, 1977 to current year.

WATER LEVELS IN FEET BELOW LAND-SURFACE DATUM

$$
\begin{array}{lll} 
& \text { WATER } & \text { WATER } \\
\text { DATE } & \text { LEVEL MS }
\end{array}
$$

APR 16, $1992 \quad 242.28 \mathrm{~S} \quad$ SEP 29, $1992 \quad 248.6 \quad \mathrm{MR}$

PERIOD OF RECORD HIGHEST 165.00 JAN $1,27,1966$ LONEST 242.28 APR 16,1992

SITE NUMBER 353723117404303

LOCAL NUMBER 026S040E33P04M

In Ridgecrest near the Intersection of West Ridgecrest Boulevard and Norma Road. Drilled public-supply

water-table well. Diameter 16 inches, depth 304 feet, perforated 169-182, 198-216, 233-252, 256-272, 278-290 feet. Altitude of land-surface datum 2,300 feet. Water-level records avallable $1966,1970-72,1974-78,1987$ to current year.

WATER LEVELS IN FEET BELOW LAND-SURFACE DATUM

$$
\begin{array}{lll} 
& \text { WATER } & \text { WATER } \\
\text { DATE } & \text { DEVEL MS }
\end{array}
$$

APR 17, $1992 \quad 176.30 \mathrm{~S} \quad$ SEP 29, $1992 \quad 184.22 \mathrm{SR}$

PERIOD OF RECORD HIGHEST 144.49 OCT 28, 1971 LOWEST 174.04 MAR 23, 1990

SITE NUMBER 353729117400301

LOCAL NUMBER 026S040E34N01M

About 0.15 mile north of East Ridgecrest Boulevard and $0.05 \mathrm{mile}$ east of China Lake Boulevard. Drilled publicsupply water-table well. Diameter 16 inches, depth 232 feet, cased to 12 inches, perforated 135-142, 146-155, 176-181 feet. Altitude of land-surface datum 2,290.4 feet. Water-leve1 records available 1945-50, 1953-76, 1978 to current year.

WATER LEVELS IN FEET BELOW LAND-SURFACE DATUM

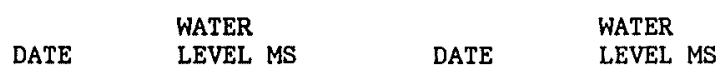

APR 17, $1992 \quad$ P $\quad$ SEP 29, $1992 \quad 147.77$ SR

PERIOD OF RECORD HIGHEST 67.40 JAN 13, 1946 LOWEST 136.08 OCT 05, 1989

SITE NUMBER 353601117483802

LOCAL NUMBER 027S039E0BMO2M

About $0.75 \mathrm{mile}$ west of Inyokern-Randsburg Road and $0.45 \mathrm{mile}$ south of Bowman Road. Drilled municipal supply well. Diameter 24 inches, depth 1,000 feet, perforated 400-1,000 feet. Altitude of land-surface datum 2,258 feet. Water-level records available 1989 to current year.

WATER LEVELS IN FEET BELOW LAND-SURFACE DATUM

DATE WATER

SEP $30,1992371.53 \mathrm{~S}$

PERIOD OF RECORD HIGHEST 367.55 MAR 22, 1990 LOWEST 371.53 SEP 30, 1992 
SITE NUMBER 353601117483301

LOCAL NUMBER 027S039E08M03M

About 3 miles south of Inyokern, 0.65 mile west of Inyokern-Randsburg Road, and 0.45 mile south of Bowman Road. Drilled observation water-table weil. Diameter 6 inches, depth 1,020 feet, perforated 600-1,000 feet. Altitude of land-surface datum 2,551.65 feet. Water-level records available 1989 to current year.

WATER LEVELS IN FEET BELOW LAND-SURFACE DATUM

$$
\text { DATE WATER }
$$

APR 17, 1992363.86 SS

PERIOD OF RECORD HIGHEST 362.15 MAR 24, 1989 LOWEST 363.72 SEP 24, 1991

SITE NUMBER 353537117480901

LOCAL NUMBER 027S039E08P01M

About 3 miles south of Inyokern, 0.5 mile west of Inyokern-Randsburg Road, and 1 mile south of Bowman Road. Drilled observation water-table well. Diameter 6 inches, depth 1,020 feet, perforated 600-1,000 feet. Altitude of land-surface datum 2,579.35 feet. Water-level records available 1989 to current year.

WATER LEVELS IN FEET BELOW LAND-SURFACE DATUM

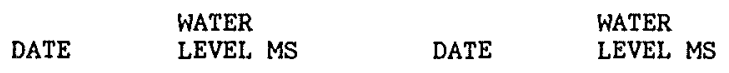

APR 17, $1992 \quad 391.63 \mathrm{~S} \quad \operatorname{SEP} 30,1992 \quad 393.55 \mathrm{~S}$

PERIOD OF RECORD HIGHEST 389.47 MAY 15, 1989 LOWEST 393.55 SEP 30,1992

SITE NUMBER 353652117372701

LOCAL NUMBER 027S040E01K01M

About $2.5 \mathrm{miles}$ east of Ridgecrest, $0.5 \mathrm{mile}$ south of Highway 178 , and $1.3 \mathrm{miles}$ east of south Richmond Street. Drilled unused well. Diameter 14 inches, depth unknown. Altitude of land-surface datum $2,318.1$ feet. Water-level records available 1952-58, 1960 to current year.

WATER LEVELS IN FEET BELOW LAND-SURFACE DATUM

$$
\begin{array}{ll} 
& \text { WATER } \\
\text { DATE } & \text { LEVEL MS }
\end{array}
$$

APR $17,1992 \quad 140.87 \mathrm{~S}$

PERIOD OF RECORD HIGHEST 127.65 MAY 21, 1952 LOWEST 142.92 NOV 14,1967

SITE NUMBER 352204117493001

LOCAL NUMBER 029S039E32E01M

Northeast of Koehn Lake, southwest of intersection of Redrock and Garlock Roads, Drilled domestic water-table well. Diameter 6 inches, depth reported 125 feet. Altitude of land-surface datum 1,965 feet. Water-level records available 1956, 1958, 1976, 1978-81, 1983 to current year.

WATER LEVELS IN FEET BELOW LAND-SURFACE DATUM

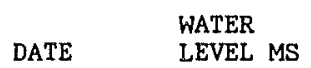

APR 16, $199278.15 \mathrm{~V}$

PERIOD OF RECORD HIGHEST 54.06 OCT 11,1956 LOWEST 78.15 APR 16, 1992 
WATER LEVELS, KERN COUNTY--Continued

WELL DESCRIPTIONS AND WATER-LEVEL MEASUREMENTS

WATER YEAR OCTOBER 1991 TO SEPTEMBER 1992

SITE NUMBER 352209117475201

LOCAL NUMBER 029S039E33K01M

About 3 miles northeast of Koehn Lake. Drilled unused water-table well in sand. Diameter 16 inches, original depth 402 feet, depth measured 403.4 feet in 1958, perforated 210-402 feet. Altitude of land-surface datum 2,050 feet. Water-level records avallable 1956, 1958, 1976, 1978-84, 1987 to current year.

WATER LEVELS IN FEET BELOW LAND-SURFACE DATUM

$$
\text { DATE } \quad \text { WATER }
$$

APR $16,1992 \quad 236.27 \mathrm{~s}$

PERIOD OF RECORD HIGHEST 131.16 FEB 13, 1958 LOWEST 244.46 MAR 06, 1984

SITE NUMBER 351948117573901

LOCAL NUMBER 030S037E13C01M

About 1.5 miles north of Cantil. Drilled unused water-table well in alluvium. Diameter 16 inches, original depth 336 feet, depth measured 333 feet in 1976, perforated 90-336 feet. Altitude of land-surface datum 2,150 feet. Water-level records available $1974,1976,1978$ to current year.

WATER LEVELS IN FEET BELOW LAND-SURFACE DATUM

DATE WATER

APR $16,1992141,46 \mathrm{~S}$

PERIOD OF RECORD HIGHEST 125.45 FEB 03,1981 LOWEST 144.84 JAN 14,1976

SITE NUMBER 351741117590901

LOCAL NUMBER 030S037E27H02M

About 1 mile southwest of Cantil along Neuralla Road. Unused well. Diameter 8 inches, depth measured 252.3 feet in 1992. Altitude of land-surface datum 2,040 feet. Water-level records available 1973-76, 1978 to current year.

WATER LEVELS IN FEET BELOW LAND-SURFACE DATUM

DATE WATER

APR 16, $1992 \quad 230.88 \mathrm{~S}$

PERIOD OF RECORD HIGHEST 170.79 FEB $15,1973 \quad$ LOWEST 251.70 MAR 07, 1984

SITE NUMBER 351659117591901

LOCAL NUMBER 030S037E34HO2M

About 2 miles southwest of Cantil, $0.3 \mathrm{mile}$ south of Munsey Road, and 0.1 mile west of Neuralia Road. Domestic water-table well. Diameter 12 inches, depth reported 456 feet. Altitude of land-surface datum 2,025 feet. Water-level records available 1978-79, 1982-84, 1986 to current year.

WATER LEVELS IN FEET BELOW LAND-SURFACE DATUM

$$
\begin{array}{ll} 
& \text { WATER } \\
\text { DATE } & \text { LEVEL MS }
\end{array}
$$

APR 16, $1992 \quad 225.80$ SR

PERIOD OF RECORD HIGHEST 232.56 MAR 26, 1986 LOWEST 247.37 MAR 07, 1984 
WELL DESCRIPTIONS AND WATER-LEVEL MEASUREMENTS

WATER YEAR OCTOBER 1991 TO SEPTEMBER 1992

SITE NUMBER 351659117571001

LOCAL NUMBER 030S037E36G01M

About 2 miles south of Cantil. Drilled domestic well in alluvium. Diameter 14 inches, depth 919 feet, perforated $12-52,106-110,138-144,171-180,238-250,293-309,418-424,436-440,446-450,457-463,767-787,816-824,832-838$, 902-907, 916-919 feet. Altitude of land-surface datum 1,968 feet. Water-level records available 1917, 1929, 1953, 1958 , 1960 to current year.

WATER LEVELS IN FEET BELOW LAND-SURFACE DATUM

$\begin{array}{lll} & \text { WATER } & \\ \text { DATE } & \text { WEVEL MS } & \text { DATE } \\ \text { LEVEL MS }\end{array}$

OCT $22,1991 \quad 133.82 \mathrm{~S} \quad$ APR $17,1992133.99 \mathrm{~S}$

PERIOD OF RECORD HIGHEST 18.00 OCT 29, 1929 LOWEST 135.30 MAY 16, 1985

SITE NUMBER 352101117530401

LOCAL NUMBER 030S038E03KO2M

About 1.5 miles south of Saltdale. Drilled unused well. Diameter 10 inches, depth measured 24.7 feet in 1990. Altitude of land-surface datum 1,895 feet. Water-level records avallable 1976 to current year.

WATER LEVELS IN FEET BELOW LAND-SURFACE DATUM

DATE WATER $\quad$ LEVEL MS

APR $17,199214.52 \mathrm{~S}$

PERIOD OF RECORD HIGHEST 14.39 MAR 22, 1991 LOWEST 52.91 MAR 08, 1977

SITE NUMBER 351838117511601

LOCAL NUMBER 030S038E24F01M

In Fremont Valley about $1 \mathrm{mile}$ east of Cantil. Drilled unused well. Diameter 5 inches, depth measured 445.9 feet in 1958. Altitude of land-surface datum 1,940 feet. Water-level records available 1953, 1958,1967 to current year.

WATER LEVELS IN FEET BELOW LAND-SURFACE DATUM

$$
\begin{array}{ll} 
& \text { WATER } \\
\text { DATE } & \text { LEVEL MS }
\end{array}
$$

APR $17,1992 \quad 41.69 \mathrm{~S}$

PERIOD OF RECORD HIGHEST 12.19 MAY 07, 1953 LOWEST 41.69 APR 17, 1992

SITE NUMBER 351714117563001

LOCAL NUMBER 030S038E30PO1M

About 2 miles southeast of Cantil, east of intersection of Munsey and Neuralia Roads. Drilled unused water-table well in alluvium. Diameter 20 inches $0-150$ feet, 12 inches 150-643 feet, original depth 643 feet, depth measured 330.5 feet in 1976, perforated 130-150, $153-643$ feet. Altitude of land-surface datum 1,957 feet. Water-1evel. records available $1953,1958-59,1974$ to current year.

WATER LEVELS IN FEET BELOW LAND-SURFACE DATUM

$$
\begin{array}{ll} 
& \text { WATER } \\
\text { DATE } & \text { LEVEL MS }
\end{array}
$$

APR $16,1992126.79 \mathrm{~S}$

PERIOD OF RECORD HIGHEST 23.88 JAN $31,1958 \quad$ LOWEST $133.12 \quad$ APR 17,1980 
WELL DESCRIPTIONS AND WATER-LEVEL MEASUREMENTS

WATER YEAR $\propto$ CTOBER 1991 TO SEPTEMBER 1992

SITE NUMBER 351714117562801

LOCAL NUMBER 030S038E30Q01M

About 2 miles southeast of Cantil near intersection of Pappas and Munsey Roads. Drilled unused water-table well. Diameter 12 inches, depth measured 93.8 feet in 1958,78.5 feet in 1976, 63.85 feet in 1990 . Altitude of land-surface datum 1,955 feet. Water-level records available 1958, 1976, 1978 to current year.

WATER LEVELS IN FEET BELOW LAND-SURFACE DATUM

$$
\text { DATE WATER }
$$

APR 16, $1992 \quad 45.09 \mathrm{~S}$

PERIOD OF RECORD HIGHEST 13.01 JAN 31, 1958 LOWEST 61.60 OCT 24, 1976

SITE NUMBER 351712117562801

LOCAL NUMBER 030S038E31C01M

About 2 miles southeast of Cantil near intersection of Pappas and Munsey Roads. Drilled irrigation well. Diameter 16 inches, depth unknown. Altitude of land-surface datum 1,957 feet. Water-level records available 1961-62, $1967-68,1976,1978$ to current year.

WATER LEVELS IN FEET BELOW LAND-SURFACE DATUM

$$
\text { DATE WATER }
$$

APR $17,1992120.19 \mathrm{~S}$

PERIOD OF RECORD HIGHEST 112.68 MAR 27, 1986 LOWEST 142.90 APR 23, 1980

SITE NUMBER 351548118001601

LOCAL NUMBER 031S037E04J01M

In Fremont Valley about 4 miles southeast of Cantil, near intersection of Neuralia and Munsey Roads. Drilled irrigation water-table well. Diameter 16 inches, depth 806 feet, depth of hole 864 feet, perforated $306-806$ feet. Altitude of land-surface datum 2,050 feet. Water-level records available 1974, 1976, 1979, 1982,1986 to current year.

WATER LEVELS IN FEET BELOW LAND-SURFACE DATUM

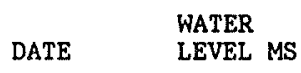

APR 16, $1992 \quad 307.81 \mathrm{~S}$

PERIOD OF RECORD HIGHEST 130.00 FEB 01, 1974 LOWEST 350.20 APR 23, 1986

SITE NUMBER 351528118003201

LOCAL NUMBER 031S037E04Q01M

In Fremont Valley about $4 \mathrm{miles}$ southwest of Cantil and 2 miles south of Munsey Road. Unused well. Diameter

16 inches, depth unknown. Altitude of land-surface datum 2,100 feet. Water-1evel records available 1974-76, 1978 to current year.

WATER LEVELS IN FEET BELOW LAND-SURFACE DATUM

$$
\text { DATE WATER }
$$

APR 16, $1992351.40 \mathrm{~S}$

PERIOD OF RECORD HIGHEST 152.00 FEB 1, 15, 1974 LOWEST 415.45 MAY 16, 1985 
WATER LEVELS, KERN COUNTY--Continued

WELL DESCRIPTIONS AND WATER-LEVEL MEASUREMENTS

WATER YEAR OCTOBER 1991 TO SEPTEMBER 1992

SITE NUMBER 351131118001201

LOCAL NUMBER 031S037E33H01M

About 3 miles north of California City and south of Phillips Road, Drilled unused well. Diameter 16 inches, depth reported 535 feet. Altitude of land-surface datum 2,340 feet. Water-level records available 1956 , $1958-59$, $1961,1964-65,1967$ to current year.

WATER LEVELS IN FEET BELOW LAND-SURFACE DATUM

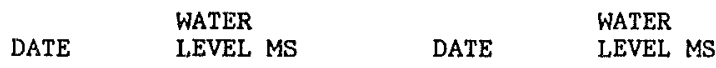

OCT $22,1991278.27 \mathrm{~S} \quad$ ARR $16,1992 \quad 277.09 \mathrm{~S}$

PERIOD OF RECORD HIGHEST 230.00 JUN 19, 1956 LOWEST 282.18 OCT 15,1990

SITE NUMBER 351104117590401

LOCAL NUMBER 031S037E35N01M

About 4 miles north of California City. Drilled unused water-table well. Diameter 16 inches, depth measured 429.2 feet in $1970,405.25$ feet in 1991. Altitude of land-surface datum 2,320 feet. Water-level records avallable 1953,1958 to current year.

WATER LEVELS IN FEET BELOW LAND-SURFACE DATUM

$$
\begin{array}{lll} 
& \text { WATER } & \\
\text { DATE } & \text { WEVEL MS } & \text { WATER } \\
\text { LEVEL MS }
\end{array}
$$

OCT 22, $1991257.39 \mathrm{~s} \quad$ APR $16,1992 \quad 256.53 \mathrm{~s}$

PERIOD OF RECORD HIGHEST 230.79 JAN 22, 1953 LOWEST 267.04 APR 15,1969

SITE NUMBER 351347117562601

LOCAL NUMBER 031S038E18P01M

About 6 miles north of California City near intersection of Phillips and Neuralia Roads. Drilled unused well in alluvium. Diameter 12 inches, depth measured 151.5 feet in 1974 . Altitude of 1 and-surface datum 2,225 feet. Water-level records available $1917,1953,1958,1964-65,1974-77,1979-81,1983$ to current year.

WATER LEVELS IN FEET BELOW LAND-SURFACE DATUM

$\begin{array}{ll} & \text { WATER } \\ \text { DATE } & \text { LEVEL MS }\end{array}$

APR $16,1992147.04 \mathrm{~S}$

PERIOD OF RECORD HIGHEST 132.39 FEB 23, 1987 LOWEST 149.17 ARR 14,1983

SITE NUMBER 351256117445401

LOCAL, NUMBER 031S039E24 P01M

East of Galileo Hill, northwest of intersection of Bushnell Road and Twenty Mule Team Parkway. Drilled unused water-table well. Diameter 14 inches, depth 793 feet. Altitude of land-surface datum 2,925 feet. Water-1evel. records available $1957,1961,1974$ to current year.

WATER LEVELS IN FEET BELOW LAND-SURFACE DATUM

$$
\begin{array}{ll}
\text { DATE } & \text { WATER } \\
\text { LEVEL MS }
\end{array}
$$

APR 15, $1992421.30 \mathrm{~s}$

PERIOD OF RECORD HIGHEST 384.00 NOV 1, 9, 1957 LOWEST 426.50 APR 30,1980 
WELL DESCRIPTIONS AND WATER-LEVEL MEASUREMENTS

WATER YEAR OCTOBER 1991 TO SEPTEMBER 1992

SITE NUMBER 350635119053801

LOCAL NUMBER 032S027E29P01M

About 25 miles southwest of Bakersfield near Lakeview. Drilled observation well. Diameter 2 inches, depth 20 feet, screened 10-20 feet. Altitude of land-surface datum 326 feet. Water-level records available 1990 to current year.

WATER LEVELS IN FEET BELOW LAND-SURFACE DATUM

$\begin{array}{llllll} & \text { WATER } & \text { WATER } & \text { WATER } \\ \text { DATE } & \text { LEVEL MS } & \text { DATE } & \text { LEVEL MS } & \text { DATE } & \text { LEVEL MS }\end{array}$

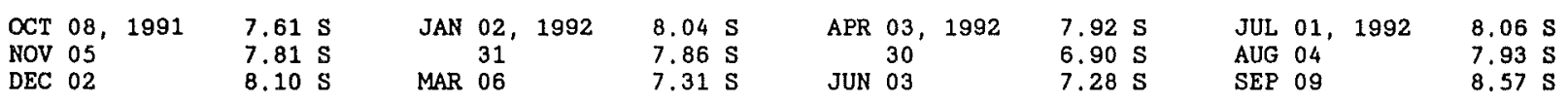

PERIOD OF RECORD HIGHEST 6.90 APR 30,1992 LOWEST 8.57 AUG 07,1990 SEP 09, 1992

SITE NUMBER 350635119053802

LOCAL, NUMBER 032S027E29P02M

About 25 miles southwest of Bakersfield near Lakeview. Drilled observation well. Diameter 2 inches, depth 57 feet, screened 42-52 feet. Altitude of land-surface datum 326 feet. Water-level records available 1990 to current year.

WATER LEVELS IN FEET BELOW LAND-SURFACE DATUM

$\begin{array}{llllll} & \text { WATER } & & & \text { WATER } \\ \text { DATE } & \text { LEVEL MS } & \text { DATE } & \text { LEVEL MS } & \text { DATE } & \text { LEVEL MS }\end{array}$

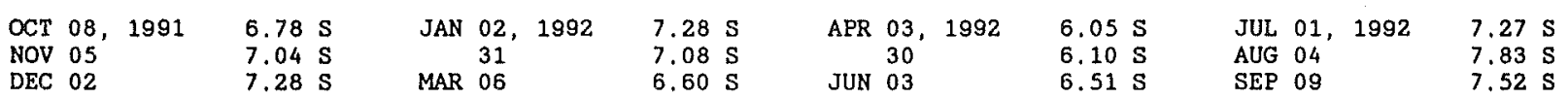

PERIOD OF RECORD HIGHEST 6.05 APR 03, $1992 \quad$ LOWEST $\quad 7.83 \quad$ AUG 04,1992

SITE NUMBER 350635119053803

LOCAL NUMBER 032S027E29P03M

About $25 \mathrm{miles}$ southwest of Bakersfield near Lakeview. Drilled observation well. Diameter 2 inches, depth 100 feet, screened 90-100 feet. Altitude of land-surface datum 326 feet. Water-level records available 1990 to current year.

WATER LEVELS IN FEET BELOW LAND-SURFACE DATUM

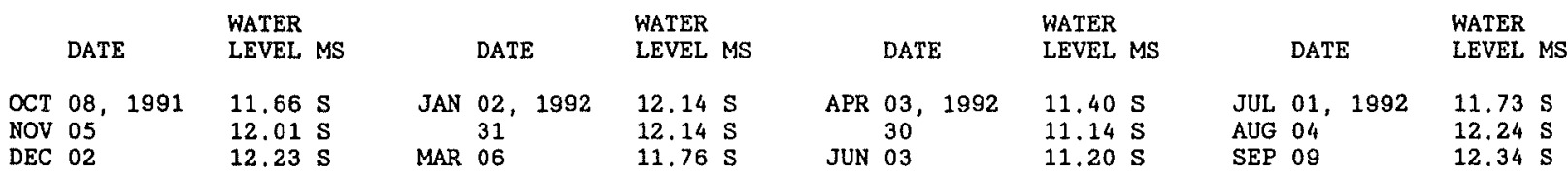

PERIOD OF RECORD HIGHEST 11.14 APR 30,1992 LOWEST $12.34 \quad$ SEP 09,1992

SITE NUMBER 350635119053804

LOCAL NUMBER 032S027E29P04M

About 25 miles southwest of Bakersfield near Lakeview. Drilled observation well. Diameter 3 inches, depth 214 feet, screened 199-209 feet. Altitude of land-surface datum 326 feet. Water-level records available 1990 to current year.

WATER LEVELS IN FEET BELOW LAND-SURFACE DATUM

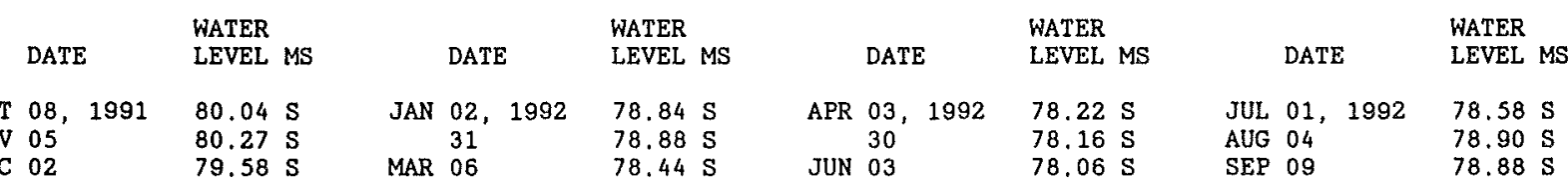

PERIOD OF RECORD HIGHEST 76.21 JAN 31,1991 LOWEST 80.27 NOV 05,1991 
WATER LEVELS, KERN COUNTY--Continued

WELL DESCRIPTIONS AND WATER-LEVEL MEASUREMENTS

WATER YEAR OCTOBER 1991 TO SEPTEMBER 1992

SITE NUMBER 350637118052901

LOCAL NUMBER 032S036E35D01M

About $6 \mathrm{miles}$ northeast of Mojave. Drilled unused well in alluvium. Diameter 14 inches, depth measured 800 feet in 1957, perforated 340-360, 395-411, 425-466, 482-494, 522-530, 542-550, 563-570, 577-606, 611-630, 717-725 feet. Altitude of land-surface datum 2,692 feet. Water-level records available 1957 to current year.

WATER LEVELS IN FEET BELOW LAND-SURFACE DATUM

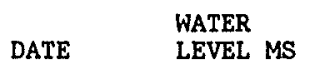

APR $15,1992 \quad 272.49 \mathrm{~S}$

PERIOD OF RECORD HIGHEST 263,93 JUL 17, AUG 14, 1957 LOWEST 272.80 FEB 23, 1987

SITE NUMBER 350919117590301

LOCAL NUMBER 032S037E11N01M

About 2 miles north of California City. Drilled unused well. Dianeter 16 inches, depth measured 600 feet in 1952 . Altitude of land-surface datum 2,375 feet. Water-level records available 1953, 1958-79, 1980 to current year.

WATER LEVELS IN FEET BELOW LAND-SURFACE DATUM

$$
\text { DATE WATER } \quad \text { LEVEL MS }
$$

APR 16, $1992 \quad 297.36 \mathrm{~S}$

PERIOD OF RECORD HIGHEST 268.09 JAN 22, 1953 LOWEST 297.36 APR 16,1992

SITE NUMBER 350933117575001

LOCAL NUMBER 032S037E12M01M

In California City. Drilled unused well. Diameter 16 inches, depth measured 430.82 feet in 1967 . Altitude of land-surface datum 2,350 feet. Water-level records available 1967 to current year.

WATER LEVELS IN FEET BELOW LAND-SURFACE DATUM

$$
\begin{array}{ll} 
& \text { WATER } \\
\text { DATE } & \text { LEVEL MS }
\end{array}
$$

APR 16, $1992 \quad 247.10 \mathrm{~S}$

PERIOD OF RECORD HIGHEST 242.00 MAR $17,1970 \quad$ LOWEST 247.10 APR 16,1992

SITE NUMBER 350737118000301

LOCAL NUMBER 032S037E22N01M

In California City, northeast of intersection of California City and Yorba Boulevards. Drilled irrigation well. Diameter 16 inches, depth reported 730 feet. Altitude of land-surface datum 2,460 feet. Water-level records available 1953-56, 1958-59, 1961-62, 1964 to current year.

WATER LEVELS IN FEET BELOW LAND-SURFACE DATUM

$$
\begin{array}{ll} 
& \text { WATER } \\
\text { DATE } & \text { LEVEL MS }
\end{array}
$$

APR $15,1992 \quad 379.10 \mathrm{~s}$

PERIOD OF RECORD HIGHEST 353 JAN 21, 1953 LOWEST 478,00 AUG 25, 1954 
WELL DESCRIPTIONS AND WATER-LEVEL MEASUREMENTS

WATER YEAR OCTOBER 1991 TO SEPTEMBER 1992

SITE NUMBER 350623117590401

LOCAL NUMBER 032S037E26NO1M

In California City, northeast of Intersection of Neuralia Road and Redwood Boulevard. Drilled unused well. Diameter 16 inches, depth reported 1,000 feet. Altitude of land-surface datum 2,420 feet. Water-1evel records available 1953-55, 1958, 1961-62, 1967 to current year.

WATER LEVELS IN FEET BELOW LAND-SURFACE DATUM

$$
\begin{array}{lll} 
& \text { WATER } & \text { WATER } \\
\text { DATE LEVEL MS } & \text { DATE LEVEL MS }
\end{array}
$$

OCT 22, $1991339.09 \mathrm{~S} \quad$ APR $15,1992345.35 \mathrm{~S}$

PERIOD OF RECORD HIGHEST 323.0 JUL 26,1961 LOWEST 441.0 JUL 15,1958

SITE NUMBER 350616117475501

LOCAL NUMBER 032S039E33LO1M

In Peerless Valley about 5 miles north of North Edwards. Drilled unused water-table well in alluvium. Diameter 16 inches, depth reported 1,400 feet. Altitude of land-surface datum 2,465 feet. Water-level records avallable 1956-57, 1978 to current year.

WATER LEVELS IN FEET BELOW LAND-SURFACE DATUM

$\begin{array}{lll} & \text { WATER } & \\ \text { DATE } & \text { WATER } \\ \text { LEVEL MS } & \text { LEVEL MS }\end{array}$

OCT 23, $1991479.35 \mathrm{~S} \quad$ APR $14,1992478.45 \mathrm{~S}$

PERIOD OF RECORD HIGHEST 267.5 DEC 12, 1956 LOWEST 518.60 SEP 14, 1978

SITE NUMBER 345609117452201

LOCAL NUMBER 009N008W06J01S

On Edwards AFB. Drilled public-supply well. Dlameter 14 inches, depth drilled 381 feet, perforated 147-363 feet. Altitude of land-surface datum 2,394 feet. Water-level records available 1966-68, 1991 to current year.

WATER LEVELS IN FEET BELOW LAND-SURFACE DATUM

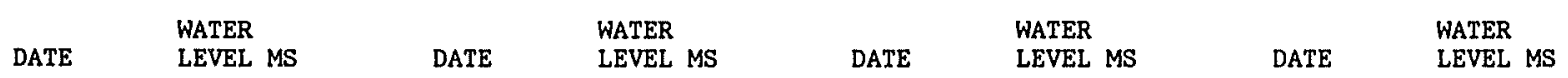

$\begin{array}{llllllllllll}\text { FEB } 06,1992 & 202.76 \mathrm{~S} & \text { MAY } 09,1992 & 203.30 \mathrm{~S} & \text { JUL } 09, & 1992 & 204.32 \mathrm{~S} & \text { SEP } 09,1992 & 204.39 \mathrm{~S} \\ \text { MAR } 13 & 204.06 \mathrm{~S} & \text { JUN } 17 & & 204.37 \mathrm{~S} & \text { AUG } 08 & & 203.91 \mathrm{~S} & \end{array}$

PERIOD OF RECORD HIGHEST 164.95 MAR 15, 1967 LOWEST 204.39 SEP 09, 1992

SITE NUMBER 345348117475801

LOCAL NUMBER OO9NOO9W02Q01S

On the east edge of Rogers Lake. Drilled unused well in dune sand. Diameter 8 inches, depth 122.8 feet. Altitude of land-surface datum $2,274.8$ feet. Water-level records available 1961-68, 1973-86, 1992 .

WATER LEVELS IN FEET BELOW LAND-SURFACE DATUM

$\begin{array}{lllll}\text { DATE } & \text { WATER } & \text { WATER } & & \text { WATER } \\ \text { LEVEL MS } & \text { DATE } & \text { LEVEL MS } & \text { DATE } & \text { LEVEL MS }\end{array}$

$\begin{array}{llllllll}\text { JAN } 08,1992 & 31.54 \mathrm{~S} & \text { APR 07, } 1992 & 31.61 \mathrm{~S} & \text { JUL 09, } 1992 & 31.21 \mathrm{~S} \\ \text { FEB 05 } & 32.16 \mathrm{~S} & \text { MAY 11 } & 32.06 \mathrm{~S} & \text { AUG 07 } & 32.25 \mathrm{~S} \\ \text { MAR 11 } & 31.87 \mathrm{~S} & \text { JUN } 17 & 32.17 \mathrm{~S} & \text { SEP 09 } & 32.34 \mathrm{~S}\end{array}$

PERIOD OF RECORD HIGHEST 25.35 MAR 01, 1961 LOWEST 66.77 MAR 25, 1980 
WATER LEVELS, KERN COUNTY--Continued

WELL DESCRIPTIONS AND WATER-LEVEL MEASUREMENTS

WATER YEAR OCTOBER 1991 TO SEPTEMBER 1992

SITE NUMBER 345344117493801

LOCAL NUMBER OO9N009W09A01S

On Edwards AFB. Drilled observation well. Diameter 2 inches, depth 345 feet, perforated $320-340$ feet. Deepest of three cluster wells. Altitude of land-surface datum 2,272 feet. Water-level records available 1991 to current year.

WATER LEVELS IN FEET BELOW LAND-SURFACE DATUM

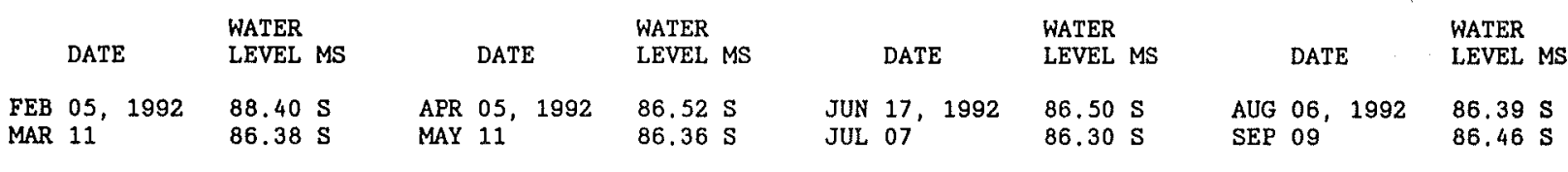

PERIOD OF RECORD HIGHEST 85.36 JUL 16,1991 LOWEST $88.40 \quad$ FEB 05,1992

SITE NUMBER 345344117493802

LOCAL NUMBER 009N009W09A02S

On Edwards AFB, Drilled observation well. Diameter 2 inches, depth 175 feet, perforated 160-170 feet. Mid-interval depth of three cluster wells. Altitude of land-surface datum 2,272 feet. Water-level records avallable 1991 to current year.

WATER LEVELS IN FEET BELOW LAND-SURFACE DATUM

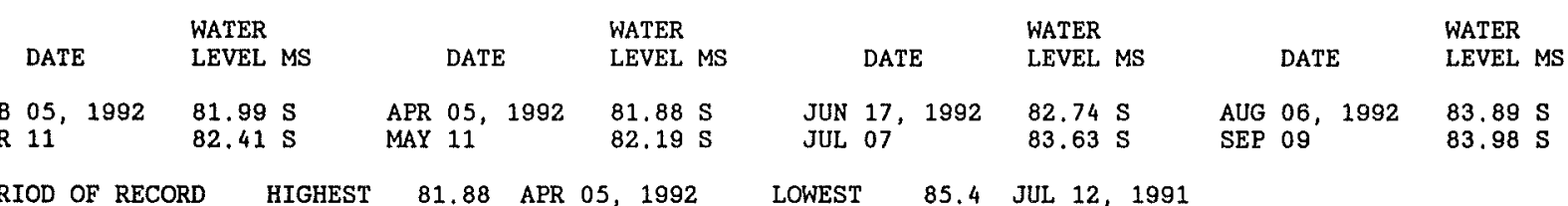

SITE NUMBER 345244117484201

LOCAL NUMBER 009N009W10R01S

On Edwards AFB at east edge of Rogers Lake. Drilled unused well in dune sand. Diameter 9.5 inches, depth measured 106 feet in 1951, 97.9 feet in 1990. Altitude of land-surface datum 2, 280 feet. Water-level records avallable $1951-53,1958-68,1970$ to current year.

WATER LEVELS IN FEET BELOW LAND-SURFACE DATUM

\begin{tabular}{|c|c|c|c|c|c|}
\hline & WATER & & WATER & & WATER \\
\hline DATE & LEVEL MS & DATE & LEVEL MS & DATE & LEVEL MS \\
\hline
\end{tabular}

$\begin{array}{llllllll}\text { NOV 08, } 1991 & 94.51 \mathrm{~S} & \text { APR 05, } 1992 & 94.76 \mathrm{~S} & \text { JUL 07, } 1992 & 95.15 \mathrm{~S} \\ \text { FEB 05, } 1992 & 94.58 \mathrm{~S} & \text { MAY 11. } & 94.98 \mathrm{~S} & \text { AUG 06 } & 95.28 \mathrm{~S} \\ \text { MAR 11 } & 94.75 \mathrm{~S} & \text { JUN } 17 & 95.04 \mathrm{~S} & \text { SEP 09 } & 95.52 \mathrm{~S}\end{array}$

PERIOD OF RECORD HIGHEST 17.59 MAY 06, $1952 \quad$ LOWEST $95.52 \quad$ SEP 09,1992

SITE NUMBER 345210117471601

LOCAL NUMBER OO9N0O9W13N01S

On Edwards AFB. Drilled public-supply well. Dianeter 14 inches, depth drilled 555 feet, perforated $178-533$ feet. Altitude of land-surface datum 2,350 feet. Water-level records available 1962-64, 1966, 1968 , 1991 to current year.

WATER LEVELS IN FEET BELOW LAND-SURFACE DATUM

\begin{tabular}{llllllll} 
& WATER & \multicolumn{3}{c}{ WATER } & & \multicolumn{2}{c}{ WATER } \\
DATE & LEVEL MS & DATE & LEVEL MS & DATE & LEVEL MS & DATE \\
& & & & & & & \\
LEVEL MS
\end{tabular}

PERIOD OF RECORD HIGHEST 104.41 JAN 14, 1963 LOWEST 162.35 SEP 10,1992 
WELL DESCRIPTIONS AND WATER-LEVEL MEASUREMENTS

WATER YEAR OCTOBER 1991 TO SEPTEMBER 1992

SITE NUMBER 345214117483701

LOCAL NUMBER OO9NOOOW15J01S

On Edwards AFB. Drilled public-supply well. Diameter 14 inches, depth drilled 534 feet, perforated 155-505 feet. Altitude of land-surface datum 2,297 feet. Water-level records available 1962-68, 1991 to current year.

WATER LEVELS IN FEET BELOW LAND-SURFACE DATUM

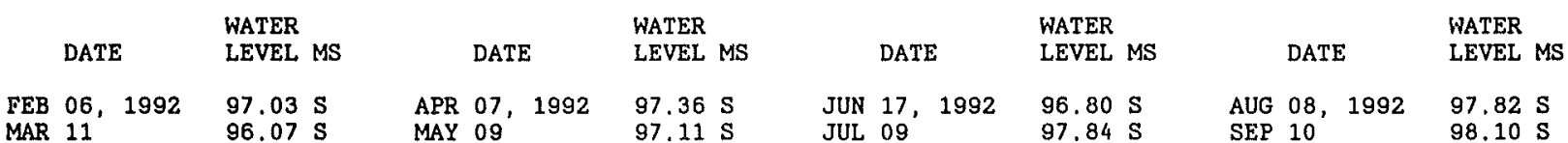

PERIOD OF RECORD HIGHEST 39.53 FEB 13, 1963 LOWEST 98.10 SEP 10, 1992

SITE NUMBER 345241117522301

LOCAL NUMBER OO9N009W18C01S

On Edwards AFB, Drilled unused well, Diameter 14 inches, depth drilled 360 feet, depth 221 feet in 1991 , perforated 25-340 feet. Altitude of land-surface datum 2,280 feet. Water-level records available 1948,1952 , $1957-58,1962-78,1992$.

WATER LEVELS IN FEET BELOW LAND-SURFACE DATUM

\begin{tabular}{|c|c|c|c|c|c|c|c|}
\hline DATE & $\begin{array}{l}\text { WATER } \\
\text { LEVEL MS }\end{array}$ & DATE & $\begin{array}{l}\text { WATER } \\
\text { LEVEL MS }\end{array}$ & DATE & $\begin{array}{l}\text { WATER } \\
\text { LEVEL MS }\end{array}$ & DATE & $\begin{array}{l}\text { WATER } \\
\text { LEVEL MS }\end{array}$ \\
\hline $\begin{array}{l}04,1992 \\
10\end{array}$ & $\begin{array}{l}101.42 \mathrm{~s} \\
101.28 \mathrm{~s}\end{array}$ & $\begin{array}{lll}\text { APR } & 05, & 1992 \\
\text { MAY } & 07 & \end{array}$ & $\begin{array}{l}101.11 \mathrm{~S} \\
101.29 \mathrm{~S}\end{array}$ & $\begin{array}{l}\text { JUN } 16,1992 \\
\text { JUL } 08\end{array}$ & $\begin{array}{l}101.66 \mathrm{~s} \\
101.75 \mathrm{~S}\end{array}$ & $\begin{array}{ll}\text { AUG } 10, & 1992 \\
\text { SEP } & 09\end{array}$ & $\begin{array}{l}102.05 \mathrm{~s} \\
102.22 \mathrm{~s}\end{array}$ \\
\hline
\end{tabular}

PERIOD OF RECORD HIGHEST 10.6 JAN 22, 1948 LOWEST 102.22 SEP 09, 1992

SITE NUMBER 345051117485001

LOCAL NUMBER 009N009W27H02S

On Edwards AFB at east edge of Rogers Lake. Drilled unused well in dune sand. Diameter 8 inches, depth measured 170.76 feet in 1991, original depth 200 feet, perforated 100-200 feet. Altitude of land-surface datum 2,280 feet. Water-level records available 1957 to current year.

WATER LEVELS IN FEET BELOW LAND-SURFACE DATUM

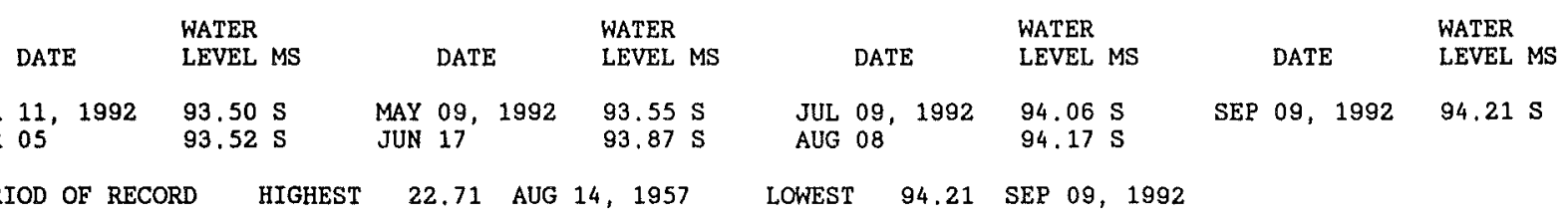


WELL DESCRIPTIONS AND WATER-LEVEL MEASUREMENTS

WATER YEAR OCTOBER 1991 TO SEPTEMBER 1992

SITE NUMBER 345056117501401

LOCAL NUMBER 009N009W28A01S

On Edwards AFB, on Rogers Lake playa. Drilled observation well. Diameter 2 inches, depth 755 feet, perforated 735-745 feet. Altitude of land-surface datum 2,271.08 feet. Recorder installed July 31, 1992. Water-1evel records aval lable 1991 to current year.

WATER LEVELS IN FEET BELOW LAND-SURFACE DATUM (OBSERVATIONS AT 1200 HOURS)

\begin{tabular}{|c|c|c|c|c|c|c|c|c|c|c|c|c|}
\hline DAY & $\mathrm{OCT}$ & Nov & DEC & JAN & FEB & MAR & APR & MAY & JUN & JUL & AUG & SEP \\
\hline 1 & -- & -- & -- & -- & -- & -- & --- & -- & -- & $\ldots$ & 94.97 & 95.37 \\
\hline 2 & -- & -- & -- & --- & -- & --- & --- & -- & $\ldots$ & $\ldots$ & 94.96 & 95.38 \\
\hline 3 & -- & -- & -- & $\cdots$ & -- & -- &.- & $\ldots$ & $\cdots$ & -- & 94.85 & 95.36 \\
\hline 4 & -- & -- & -- & -- & --- & --- & -. & -- & $\cdots$ & --- & 94.96 & 95.33 \\
\hline 5 & -- & --- & -- & -- & 592.24 & $\cdots$ & S92.18 & S92.36 & $\cdots$ & -- & 94.96 & 95,30 \\
\hline 6 & -- & $-\cdot-$ & -- & -- & -- & -- & -- & $-\cdots$ & -- & -. & 94.96 & 95.27 \\
\hline 7 & --- & $\ldots$ & -- & -- & $\ldots$ & $-\cdots$ & $\cdots$ & $\cdots$ & --- & 594.39 & 94.98 & 95.29 \\
\hline 8 & -- & --- & -- & --- & --- & --- & -- & --- & -- & $\ldots$ & 95.01 & 95.29 \\
\hline 9 & -.- & --- & --- & -- & -- & $\mathrm{s} 92.01$ & --- & --- & 594.44 & -- & 95.06 & 95.27 \\
\hline 10 & --- &.-- & $-\cdots$ & 592.49 & -- & - & $-\cdots$ & $\cdots$ & -. & -- & 95.09 & 95.24 \\
\hline 11 & --- & -- & -- & -- & -- & -- & --- & -- & --- & --- & 95.08 & 95.20 \\
\hline 12 & -- & -- & -- & -- & -- & $\mathrm{s} 92.11$ & --- & -- & -- & -- & 95.08 & 95.18 \\
\hline 13 & --- & -- & -- & --- & -- & - &.-- & $=-$ & -- & 594.57 & 95.09 & 95.15 \\
\hline 14 & -- & -- & -- & --- & -- & $\cdots$ & $\rightarrow$ & -- & -- & $\ldots$ & 95.13 & 95.08 \\
\hline 15 & --- & --- & --- & --- & -- & --- & -- & --- & --- & -- & 95.14 & 95.06 \\
\hline 16 & -- & -- & -- & S92.39 & -- & -- &.-- & $\ldots$ & $\ldots$ & $\ldots$ & 95.14 & 95.07 \\
\hline 17 & -- & $\ldots$ & $\ldots$ & - & --- & -- & -- & $\ldots$ & $\ldots$ &.- & 95.17 & 95.04 \\
\hline 18 & -- & -- & -- & -- & -- & -- & -- & -- & -- & -- & 95.21 & 94.98 \\
\hline 19 & $\ldots$ & -- & -- & -- & -.- & -.- & $\ldots$ & $\cdots$ & -- & --- & 95.24 & 94.98 \\
\hline 20 & --- & -- & -- & --- & -- & -- & -- & -- & -- & --- & 95.25 & 94.98 \\
\hline 21 & -- & --- & -- & --- & -.- & --- & -- & -- & -- & -- & 95.23 & 94.99 \\
\hline 22 & -- & -- & -- & -- & -- & -- & -- & $\cdots$ & -- & $\ldots$ & 95.21 & 95.05 \\
\hline 23 & -.- & -- & $-\cdots$ & -- & $\ldots$ & -- & -.- & $\cdots$ & $-\cdots$ & $\ldots$ & 95.22 & 95.09 \\
\hline 24 & -- & --- & --- & $\cdots$ & --- & -- & --- & $\cdots$ & -- & --- & 95.28 & 95.10 \\
\hline 25 & --- & -- & -- & -- & --- & --- & $\cdots$ &.- & -- & -- & 95.34 & 95.05 \\
\hline 26 & $\ldots$ & $\ldots$ & -- & -.. & $-\cdots$ & -- & $\ldots$ & $\ldots$ & -- & -- & 95.39 & 95.09 \\
\hline 27 &.-- & $\cdots$ & $\cdots$ & - - & $\ldots$ & $\cdots$ & -. & $\cdots$ & $-\cdots$ & $\cdots$ & 95.40 & 95.12 \\
\hline 28 & -- & -- & -- &.- & - & - & --- & $\ldots$ & -.. & -- & 95,37 & 95.15 \\
\hline 29 & -- & -- & -- & -- & $\ldots$ &.-- & $\cdots$ & $\cdots$ & $\cdots$ & 594.99 & 95.39 & 95.27 \\
\hline 30 & --- & -- & -- & -- & -- & --. & --- & $-\cdots$ & $-\ldots$ & - & 95,40 & 95.37 \\
\hline 31 & $\cdots$ & --- & -- & -- & --- & -- & -- & --- & -- & 94.95 & 95.38 & \\
\hline
\end{tabular}

PERIOD OF RECORD HIGHEST 92.01 MAR 09, 1992 LOWEST 95.40 AUG 27, 30, 1992

S Manual steel tape measurement.

SITE NUMBER 345056117501402

LOCAL NUMBER OOSNOOSW28A02S

On Edwards AFB, on Rogers Lake playa, north of runway 25. Drilled observation well. Diameter 2 inches, depth 524 feet, perforated 494-514 feet. Altitude of land-surface datum 2,271.08 feet. Water-level records available 1991 to current year.

WATER LEVELS IN FEET BELOW LAND-SURFACE DATUM

\begin{tabular}{|c|c|c|c|c|c|c|c|c|c|c|c|}
\hline & DATE & $\begin{array}{l}\text { WATER } \\
\text { LEVEL MS }\end{array}$ & & DATE & $\begin{array}{l}\text { WATER } \\
\text { LEVEL MS }\end{array}$ & & DATE & $\begin{array}{l}\text { WATER } \\
\text { LEVEL } \cdot \text { MS }\end{array}$ & & DATE & $\begin{array}{l}\text { WATER } \\
\text { LEVEL MS }\end{array}$ \\
\hline $\begin{array}{l}\text { JAN } \\
\text { FEB }\end{array}$ & $\begin{array}{ll}10, & 1992 \\
17 & \\
05 & \end{array}$ & $\begin{array}{l}91.32 \mathrm{~s} \\
91.22 \mathrm{~s} \\
91.08 \mathrm{~s}\end{array}$ & $\begin{array}{l}\text { MAR } \\
\text { APR } \\
\text { MAY }\end{array}$ & $\begin{array}{ll}11, & 1992 \\
05 & \\
05 & \end{array}$ & $\begin{array}{l}91.01 \mathrm{~s} \\
91.07 \mathrm{~s} \\
92.14 \mathrm{~s}\end{array}$ & JUL & $\begin{array}{ll}07, & 1992 \\
13 & \\
30 & \end{array}$ & $\begin{array}{l}93.02 \mathrm{~s} \\
93.20 \mathrm{~s} \\
93.49 \mathrm{~s}\end{array}$ & $\begin{array}{l}\text { AUG } \\
\text { SEP }\end{array}$ & $\begin{array}{l}11,1992 \\
09 \\
14\end{array}$ & $\begin{array}{l}93.54 \mathrm{~S} \\
93.81 \mathrm{~S} \\
93.65 \mathrm{~S}\end{array}$ \\
\hline
\end{tabular}

PERIOD OF RECORD HIGHEST 90.93 MAR 09, 1992 LOWEST 93.81 SEP 09, 1992 
WELL DESCRIPTIONS AND WATER-LEVEL MEASUREMENTS

WATER YEAR OCTOBER 1991 TO SEPTEMBER 1992

SITE NUMBER 345056117501403

LOCAL NUMBER OO9NOO9W28A03S

On Edwards AFB, on Rogers Lake playa, north of runway 25, Drilled observation well. Diameter 2 inches, depth 350 feet, perforated 320-340 feet. Altitude of land-surface datum 2,271.08 feet. Recorder installed July 31 , 1992. Water-level records available 1991 to current year.

WATER LEVELS IN FEET BELOW LAND-SURFACE DATUM (OBSERVATIONS AT 1200 HOURS)

\begin{tabular}{|c|c|c|c|c|c|c|c|c|c|c|c|c|}
\hline DAY & OCT & Nov & DEC & JAN & FEB & MAR & APR & MAY & JUN & JUL & AUG & SEP \\
\hline 1 & --- & -- & -- & -- & -- & --- & -- & --- & -- & -- & 89.91 & 90.12 \\
\hline 2 & -- & --- & $m-$ & $\cdots$ & $-\infty$ & -- & $-\infty$ & $\ldots$ & $\cdots$ & -- & 89.90 & 90.13 \\
\hline 3 & --- & --- & -- & -- & $\cdots$ & -- & -- & -- & -- & -- & 89.87 & 90.13 \\
\hline 4 & $-\infty$ & --- & --- & --- & --- & --- & --- & -- & -- & --- & 89.89 & 90.11 \\
\hline 5 & -- & $-\infty$ & --- & --- & 588.35 & -- & 588.34 & 588.86 & -- & $\cdots$ & 89.88 & 90.11 \\
\hline 6 & -- & --- & -- & -- & -- & -- & -- & $\cdots$ & -- & --- & 89.87 & 90,08 \\
\hline 7 & $-\cdots$ & -- & --- & --- & --- & $\cdots$ & $-\cdots$ & -- & --- & 589.50 & 89.90 & 90.09 \\
\hline 8 & --- & -- & -- & --- & -- & -- & $-\infty$ & -- & -- & - & 89.91 & 90.10 \\
\hline 9 & -- & $\cdots$ & $m$ & -- & $\cdots$ & 588.28 & $\cdots$ & -- & 589.46 & $\cdots$ & 89.95 & 90.08 \\
\hline 10 & $\cdots$ & $\cdots$ & -- & -- & -- & - & $-\cdots$ & -- & - & -- & 89.96 & 90.07 \\
\hline 11 & $\cdots$ & --- & -- & --- & -- & 588.32 & --- & --- & -- & --- & 89.92 & 90.06 \\
\hline 12 & -- & $\cdots$ & -- & -- & -- & - & -- & --- & -- & -- & 89.93 & 90.06 \\
\hline 13 & $\cdots$ & --- & -- & -- & -- & $-\cdots$ & $\cdots$ & -- & --- & $\$ 89.59$ & 89.94 & 90.05 \\
\hline 14 & --- & $m$ & --- & --- & --- & -- & --- & -- & $-\infty$ & -- & 89.99 & 90.02 \\
\hline 15 & --- & -- & -- & -- & $\cdots$ & --- & -- & --- & $\cdots$ & -- & 89.98 & 90.02 \\
\hline 16 & -- & -- & -- & -- & -- & -- & $\cdots$ & --- & -- & --- & 89.95 & 90.06 \\
\hline 17 & --- & -- & -- & S88.40 & -- & --- & -- & --- & $\cdots$ & -- & 89.97 & 90.07 \\
\hline 18 & -- & --- & -- & -- & $-\cdots$ & $-\cdot$ & -- & -- & $\cdots$ & -- & 90.00 & 90.04 \\
\hline 19 & $-\cdots$ & -- & --- & -- & $\cdots$ & -- & -- & --- & -- & -- & 90.03 & 90.02 \\
\hline 20 & $\cdots$ & -- & -- & $\cdots$ & -- & -- & $\cdots$ & $\cdots$ & $\cdots$ & -- & 90.04 & 90.02 \\
\hline 21 & --- & -- & $\cdots$ & --- & -- & -- & -- & -- & -- & -- & 90.01 & 90.03 \\
\hline 22 & $-\cdots$ & -- & --- & -- & $\cdots$ & -- & -- & -- & $\cdots$ & -- & 89.99 & 90.06 \\
\hline 23 & $\cdots$ & --- & -- & -- & $\cdots$ & $\cdots$ & -- & $\cdots$ & -- & -- & 89.98 & 90.07 \\
\hline 24 & --- & $\cdots$ & $\cdots$ & $\cdots$ & --- & $\cdots$ & $\cdots$ & $\cdots$ & $\cdots$ & $\cdots$ & 90.02 & 90.05 \\
\hline 25 & -- & -- & -- & -- & -- & -- & $\cdots$ & $m$ & --- & --- & 90.09 & 90.03 \\
\hline 26 & -- & -- & -- & -- & -- & --- & $\cdots$ & --- & --- & --- & 90.14 & 90.08 \\
\hline 27 & -- & -- & -- & -- & --- & -- & --- & --- & -- & --- & 90.17 & 90.10 \\
\hline 28 & --- & --- & $\ldots$ & $\cdots$ & -- & -- & $\cdots$ & $\cdots$ & -- & --- & 90.14 & 90.10 \\
\hline 29 & --- & $m$ & --- & --- & $-\infty$ & -- & --- & -+ & $\cdots$ & -- & 90.13 & 90.14 \\
\hline 30 & $\cdots$ & --- & --- & $\cdots$ & --- & -- & --- & --- & --- & S89.84 & 90.14 & 90.22 \\
\hline 31 & --- & --- & -- & $-\cdots$ & $\cdots$ & -- & -- & $\cdots$ & --- & 89.90 & 90.12 & - \\
\hline
\end{tabular}

PERIOD OF RECORD HIGHEST 88.28 MAR 09, 1992 LOWEST 90.22 SEP 30, 1992

S Manual steel tape measurement.

SITE NUMBER 345056117501404

LOCAL NUMBER 009N009W28A04S

On Edwards AFB, on Rogers Lake playa, north of runway 25. Drilled observation well. Diameter 2 inches, depth measured 218.6 feet in 1991, perforated 195-215 feet. Altitude of land-surface datum 2,271.08 feet. Water-1evel records available 1991 to current year.

WATER LEVELS IN FEET BELOW LAND-SURFACE DATUM

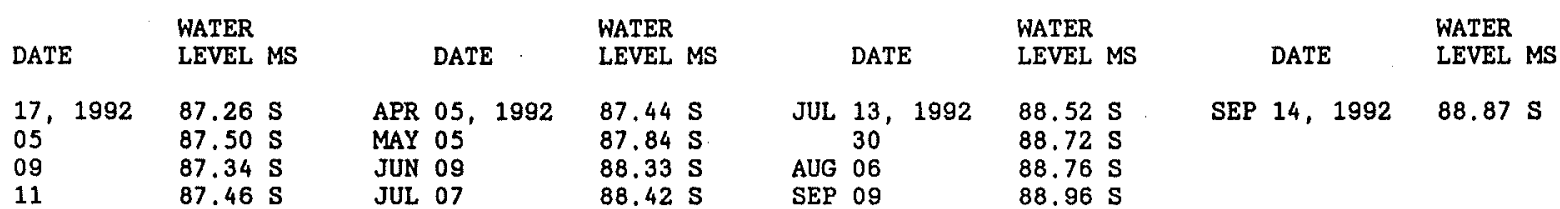

PERIOD OF RECORD HIGHEST 87.26 JAN 17, 1992 LOWEST 88.96 SEP 09,1992 
SITE NUMBER 345056117501405

LOCAL NUMBER O09N009W28A05S

On Edwards AFB, on Rogers Lake playa, north of runway 25. Drilled observation well. Diameter 3 inches, depth measured 54 feet in 1991, perforated 28-48 feet. Altitude of land-surface datum 2,271.08 feet. Recorder installed February 1992. Water-level records available 1991 to current year.

WATER LEVELS IN FEET BELOW LAND-SURFACE DATUM (OBSERVATIONS AT 1200 HOURS)

\begin{tabular}{|c|c|c|c|c|c|c|c|c|c|c|c|c|}
\hline DAY & $O C T$ & NOV & DEC & JAN & FEB & MAR & APR & MAY & JUN & JUL & AUG & SEP \\
\hline $\begin{array}{l}1 \\
2 \\
3 \\
4 \\
5\end{array}$ & $\begin{array}{l}--- \\
--- \\
--- \\
--- \\
---\end{array}$ & $\begin{array}{l}--- \\
--- \\
--- \\
--- \\
---\end{array}$ & $\begin{array}{l}--- \\
--- \\
--- \\
---\end{array}$ & $\begin{array}{l}--- \\
--- \\
--- \\
--- \\
---\end{array}$ & S39. 69 & $\begin{array}{l}40.09 \\
39.98 \\
40.11 \\
40.17 \\
40.18\end{array}$ & $\begin{array}{l}40.40 \\
40.46 \\
40.46 \\
40.40 \\
40.43\end{array}$ & $\begin{array}{l}40.52 \\
40.59 \\
40.58 \\
40.52 \\
40.43\end{array}$ & $\begin{array}{l}40.57 \\
40.52 \\
40.55 \\
40.51 \\
40.42\end{array}$ & $\begin{array}{l}40.52 \\
40.58 \\
40.53 \\
40.57 \\
40.59\end{array}$ & $\begin{array}{l}40.78 \\
40.76 \\
40.70 \\
40.70 \\
40.70\end{array}$ & $\begin{array}{l}40.73 \\
40.79 \\
40.79 \\
40.80 \\
40.80\end{array}$ \\
\hline $\begin{array}{r}6 \\
7 \\
8 \\
9 \\
10\end{array}$ & $\begin{array}{l}--- \\
--- \\
--- \\
---\end{array}$ & $\begin{array}{l}--- \\
--- \\
--- \\
-\cdots \\
---\end{array}$ & $\begin{array}{l}--- \\
--- \\
--- \\
--- \\
--\end{array}$ & $\begin{array}{l}--- \\
--- \\
--- \\
--- \\
---\end{array}$ & $\begin{array}{l}\cdots- \\
-- \\
-- \\
--\end{array}$ & $\begin{array}{l}40.17 \\
40.08 \\
40.09 \\
40.24 \\
40.39\end{array}$ & $\begin{array}{l}40.51 \\
40.57 \\
40.53 \\
40.50 \\
40.48\end{array}$ & $\begin{array}{l}40.52 \\
40.61 \\
40.49 \\
40.32 \\
40.44\end{array}$ & $\begin{array}{l}40.43 \\
40.52 \\
40.58 \\
40.60 \\
40.58\end{array}$ & $\begin{array}{l}40.62 \\
40.69 \\
40.76 \\
40.76 \\
40.67\end{array}$ & $\begin{array}{l}40.71 \\
40.74 \\
40.76 \\
40.78 \\
40.77\end{array}$ & $\begin{array}{l}40.75 \\
40.77 \\
40.77 \\
40.76 \\
40.75\end{array}$ \\
\hline $\begin{array}{l}11 \\
12 \\
13 \\
14 \\
15\end{array}$ & $\begin{array}{l}--- \\
--- \\
--- \\
---\end{array}$ & $\begin{array}{l}--- \\
--- \\
--- \\
--- \\
---\end{array}$ & $\begin{array}{l}--- \\
--- \\
--- \\
--- \\
-\cdots\end{array}$ & $\begin{array}{l}--- \\
--- \\
--- \\
--- \\
---\end{array}$ & $\begin{array}{l}\cdots- \\
\cdots- \\
\cdots- \\
\cdots-\end{array}$ & $\begin{array}{l}40.35 \\
40.34 \\
40.36 \\
40.35 \\
40.33\end{array}$ & $\begin{array}{l}40.47 \\
40.50 \\
40.51 \\
40.52 \\
40.51\end{array}$ & $\begin{array}{l}40.52 \\
40.55 \\
40.53 \\
40.49 \\
40.46\end{array}$ & $\begin{array}{l}40.62 \\
40.72 \\
40.67 \\
40.57 \\
40.61\end{array}$ & $\begin{array}{l}40.60 \\
40.62 \\
40.68 \\
40.71 \\
40.68\end{array}$ & $\begin{array}{l}40.71 \\
40.70 \\
40.75 \\
40.82 \\
40.79\end{array}$ & $\begin{array}{l}40.74 \\
40.74 \\
40.70 \\
40.66 \\
40.67\end{array}$ \\
\hline $\begin{array}{l}16 \\
17 \\
18 \\
19 \\
20\end{array}$ & $\begin{array}{l}--- \\
--- \\
--- \\
---\end{array}$ & $\begin{array}{l}--- \\
--- \\
--- \\
---\end{array}$ & $\begin{array}{l}--- \\
--- \\
--- \\
--- \\
---\end{array}$ & $\begin{array}{l}--- \\
--- \\
--- \\
--- \\
---\end{array}$ & $\begin{array}{l}--- \\
--- \\
--- \\
-\cdots \\
---\end{array}$ & $\begin{array}{l}40.29 \\
40.29 \\
40.35 \\
40.42 \\
40.30\end{array}$ & $\begin{array}{l}40.55 \\
40.57 \\
40.50 \\
40.62 \\
40.61\end{array}$ & $\begin{array}{l}40.47 \\
40.53 \\
40.60 \\
40.50 \\
40.46\end{array}$ & $\begin{array}{l}40.71 \\
40.67 \\
40.64 \\
40.61 \\
40.57\end{array}$ & $\begin{array}{l}40.65 \\
40.68 \\
40.74 \\
40.71 \\
40.63\end{array}$ & $\begin{array}{l}40.72 \\
40.71 \\
40.75 \\
40.75 \\
40.72\end{array}$ & $\begin{array}{l}40.76 \\
40.77 \\
40.72 \\
40.73 \\
40.70\end{array}$ \\
\hline $\begin{array}{l}21 \\
22 \\
23 \\
24 \\
25\end{array}$ & $\begin{array}{l}--- \\
--- \\
-\cdots \\
--- \\
---\end{array}$ & $\begin{array}{l}--- \\
--- \\
--- \\
--- \\
---\end{array}$ & $\begin{array}{l}--- \\
--- \\
-\cdots \\
--- \\
---\end{array}$ & $\begin{array}{l}-- \\
--- \\
\cdots- \\
\cdots-\end{array}$ & $\begin{array}{r}540.24 \\
40.22 \\
40.31 \\
40.37 \\
40.37\end{array}$ & $\begin{array}{l}40.24 \\
40.31 \\
40.35 \\
40.48 \\
40.40\end{array}$ & $\begin{array}{l}40.44 \\
40.50 \\
40.60 \\
40.62 \\
40.56\end{array}$ & $\begin{array}{l}40.48 \\
40.56 \\
40.61 \\
40.56 \\
40.52\end{array}$ & $\begin{array}{l}40.60 \\
40.70 \\
40.68 \\
40.63 \\
40.63\end{array}$ & $\begin{array}{l}40.62 \\
40.62 \\
40.63 \\
40.67 \\
40.76\end{array}$ & $\begin{array}{l}40.68 \\
40.66 \\
40.65 \\
40.69 \\
40.79\end{array}$ & $\begin{array}{l}40.75 \\
40.80 \\
40.78 \\
40.72 \\
40.71\end{array}$ \\
\hline $\begin{array}{l}26 \\
27 \\
28 \\
29 \\
30 \\
31\end{array}$ & $\begin{array}{l}--- \\
--- \\
--- \\
--- \\
--- \\
--\end{array}$ & $\begin{array}{l}--- \\
--- \\
--- \\
--- \\
--- \\
---\end{array}$ & $\begin{array}{l}--- \\
-- \\
--- \\
--- \\
-- \\
-\cdots\end{array}$ & $\begin{array}{l}--- \\
--- \\
--- \\
--- \\
--- \\
---\end{array}$ & $\begin{array}{r}40.40 \\
40.42 \\
40.38 \\
40.22 \\
\ldots . .\end{array}$ & $\begin{array}{l}40.31 \\
40.20 \\
40.34 \\
40.47 \\
40.46 \\
40.42\end{array}$ & $\begin{array}{r}40.56 \\
40.62 \\
40.62 \\
40.54 \\
40.47\end{array}$ & $\begin{array}{l}40.49 \\
40.48 \\
40.47 \\
40.48 \\
40.54 \\
40.62\end{array}$ & $\begin{array}{l}40.65 \\
40.65 \\
40.42 \\
40.50 \\
40.51\end{array}$ & $\begin{array}{l}40.79 \\
40.77 \\
40.75 \\
40.70 \\
40.69 \\
40.74\end{array}$ & $\begin{array}{l}40.87 \\
40.89 \\
40.83 \\
40.80 \\
40.80 \\
40.74\end{array}$ & $\begin{array}{r}40.83 \\
40.86 \\
40.85 \\
40.85 \\
40.88 \\
\ldots\end{array}$ \\
\hline
\end{tabular}

PERIOD OF RECORD HIGHEST 39.69 FEB 05, $1992 \quad$ LOWEST 40.89 AUG 27, 1992

S Manual steel tape measurement.

SITE NUMBER 345302117574001

LOCAL NUMBER 009N010W08P01S

On Edwards AFB about 0.2 mile south of Rosamond Boulevard and 2.5 miles west of Lancaster Boulevard.

Public-supply well. Diameter 6 inches, depth measured 136.65 feet in 1965, 132.6 feet in 1990 . Altitude of land-surface datum 2,370.54 feet, surveyed in May 1989. Water-level records available 1951-52, 1954, 1963-68, 1973 to current year.

WATER LEVELS IN FEET BELOW LAND-SURFACE DATUM

\begin{tabular}{|c|c|c|c|c|c|}
\hline DATE & $\begin{array}{l}\text { WATER } \\
\text { LEVEL MS }\end{array}$ & DATE & $\begin{array}{l}\text { WATER } \\
\text { LEVEL MS }\end{array}$ & DATE & $\begin{array}{l}\text { WATER } \\
\text { LEVEL MS }\end{array}$ \\
\hline $\begin{array}{l}09,1992 \\
04 \\
10\end{array}$ & $\begin{array}{l}81.58 \mathrm{~S} \\
81.53 \mathrm{~S} \\
81.56 \mathrm{~S}\end{array}$ & $\begin{array}{lll}\text { APR } & 05,1992 \\
\text { MAY } & 11 & \\
\text { JUN } & 17 & \end{array}$ & $\begin{array}{l}81.47 \mathrm{~S} \\
81.53 \mathrm{~S} \\
81.50 \mathrm{~S}\end{array}$ & $\begin{array}{lll}\text { JUL } & 08, & 1992 \\
\text { AUG } & 10 & \\
\text { SEP } & 08 & \end{array}$ & $\begin{array}{l}81.54 \mathrm{~S} \\
81.42 \mathrm{~S} \\
81.50 \mathrm{~S}\end{array}$ \\
\hline
\end{tabular}

PERIOD OF RECORD HIGHEST 80.89 MAR 19, 1991 LOWEST 88.04 MAR 14, 1963 
WELL DESCRIPTIONS AND WATER-LEVEL MEASUREMENTS

WATER YEAR OCTOBER 1991 TO SEPTEMBER 1992

SITE NUMBER 345230117564001

LOCAL NUMBER OOGNO10W16F01S

On Edwards AFB. Drilled observation well. Diameter 6 inches, depth 275 feet, open hole. Altitude of land-surface datum 2,320 feet. Water-level records available 1992.

WATER LEVELS IN FEET BELOW LAND-SURFACE DATUM

$\begin{array}{llll} & & & \\ \text { DATE } & \text { WATER } & \text { WATER } & \text { WATER } \\ \text { LEVEL MS } & \text { DATE } & \text { LEVEL MS } & \text { DATE }\end{array}$

$\begin{array}{lllllllll}\text { JAN 08, } 1992 & 111.53 \mathrm{~S} & \text { APR } 05,1992 & 111.33 \mathrm{~s} & \text { JUL } 07,1992 & 110.40 \mathrm{~S} \\ \text { FEB 04 } & 111.49 \mathrm{~S} & \text { MAY } 11 & 111.30 \mathrm{~S} & \text { AUG } 10 & 110.98 \mathrm{~S} \\ \text { MAR 10 } & 111.40 \mathrm{~S} & \text { JUN } 16 & 111.16 \mathrm{~S} & \text { SEP } 07 & 111.02 \mathrm{~S}\end{array}$

PERIOD OF RECORD HIGHEST 110.40 JUL 07, 1992 LOWEST 111.53 JAN 08, 1992

SITE NUMBER 345214117564601

LOCAL NUMBER OO9NO1OW16LO1S

On Edwards AFB. Drilled unused well. Diameter 14 inches, depth measured 465 feet in 1960. Altitude of land-surface datum 2,320 feet. Water-level records available 1951-52, 1960, 1991 to current year.

WATER LEVELS IN FEE'T BELOW LAND-SURFACE DATUM

\begin{tabular}{|c|c|c|c|c|c|c|c|}
\hline DATE & $\begin{array}{l}\text { WATER } \\
\text { LEVEL MS }\end{array}$ & DATE & $\begin{array}{l}\text { WATER } \\
\text { LEVEL MS }\end{array}$ & DATE & $\begin{array}{l}\text { WATER } \\
\text { LEVEL MS }\end{array}$ & DATE & $\begin{array}{l}\text { WATER } \\
\text { LEVEL MS }\end{array}$ \\
\hline
\end{tabular}

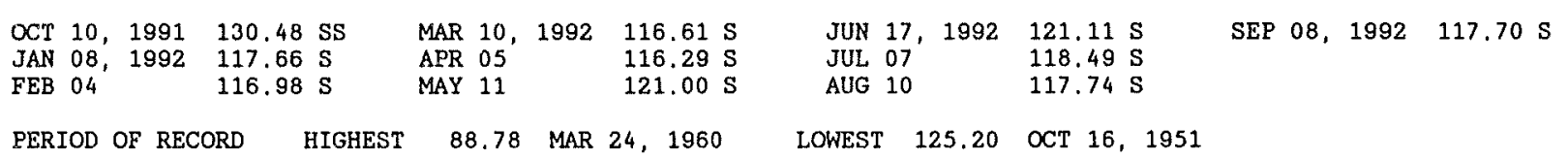

SITE NUMBER 345213117564001

LOCAL NUMBER OO9N010W16LO2S

On Edwards AFB. Drilled unused well. Diameter 14 inches, depth drilled 732 feet. Altitude of land-surface datum 2,320 feet. Water-level records available 1951-52, 1960, 1991 to current year.

WATER LEVELS IN FEET BELOW LAND-SURFACE DATUM

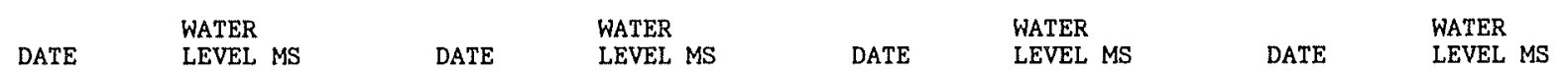

$\begin{array}{llllllllll}\text { OCT 09, } 1991 & 113.07 \mathrm{~S} & \text { MAR } 10,1992 & 112.42 \mathrm{~S} & \text { JUN } 16,1992 & 113.46 \mathrm{~S} & \text { SEP } 07,1992 & 113.06 \mathrm{~S} \\ \text { JAN 08, } 1992 & 113.40 \mathrm{~S} & \text { ARR } 05 & 112.16 \mathrm{~S} & \text { JUL } 07 & & 113.65 \mathrm{~S} & & & \\ \text { FEB } 04 & & 112.87 \mathrm{~S} & \text { MAY } 11 & 112.43 \mathrm{~S} & \text { AUG } 10 & 113.08 \mathrm{~S} & & \end{array}$

PERIOD OF RECORD HIGHEST 83.69 MAR 24, 1960 LOWEST 113.65 JUL 07, 1992

SITE NUMBER 345224117564701

LOCAL NUMBER OO9NO1OW16LO3S

On Edwards AFB, 1.5 miles west of Lancaster Boulevard. Drilled observation well in alluvium. Diameter 16 inches, depth 270 feet, perforated 130-260 feet. Altitude of land-surface datum 2,322 feet. Water-level records available 1990 to current year.

WATER LEVELS IN FEET BELOW LAND-SURFACE DATUM

\begin{tabular}{lllllll} 
& \multicolumn{1}{c}{ WATER } & \multicolumn{3}{c}{ WATER } \\
DATE & LEVEL MS & \multicolumn{2}{c}{ DATE } & LEVEL MS & DATE & \multicolumn{1}{c}{ WATER } \\
LEVEL MS
\end{tabular}

PERIOD OF RECORD HIGHEST 111.53 JUL 07,1992

LOWEST 113.06 JAN 07, FEB 04, 1992 
SITE NUMBER 345215117565801

LOCAL NUMBER 009N010W16M01S

On Edwards AFB. Drilled unused well. Diameter 16 inches, depth measured 140.5 feet in 1951 . Altitude of land-surface datum 2,325 feet. Water-level records available 1951-68, 1991 to current year.

WATER LEVELS IN FEET BELOW LAND-SURFACE DATUM

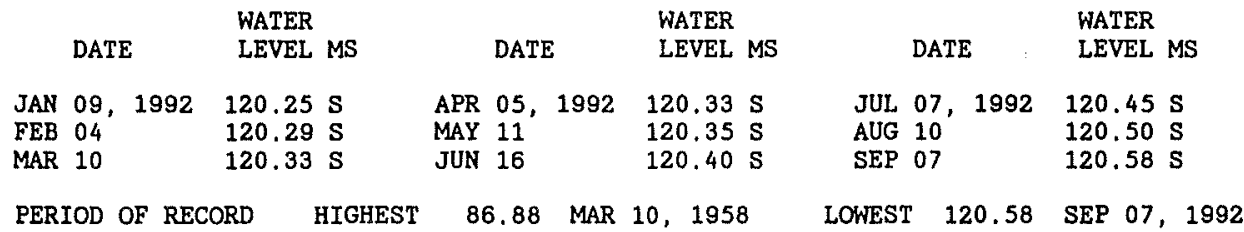

SITE NUMBER 345212117570301

LOCAL NUMBER OOSNO1OW16NO1S

On Edwards AFB. Drilled unused well. Diameter 14 inches, depth drilled 396 feet, depth measured 376 feet in 1961 , perforated $98-396$ feet. Altitude of land-surface datum 2,328 feet. Water-level records available 1954, $1956-61$, 1991 to current year.

WATER LEVELS IN FEET BELOW LAND-SURFACE DATUM

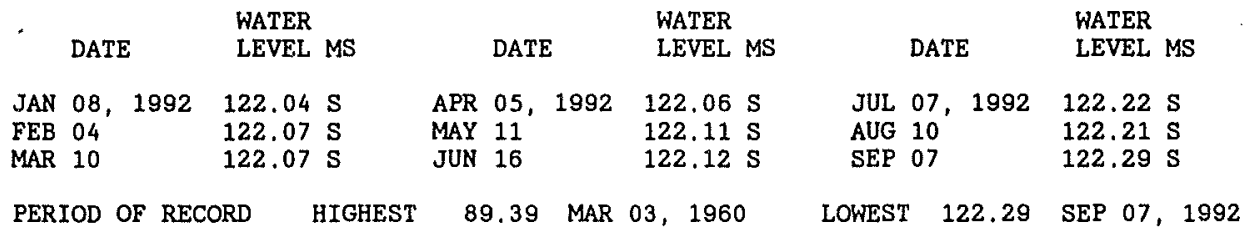

SITE-NUMBER, 345210117564501

LOCAL NUMBER OO9N010W16P01S

On Edwards AFB. Drilled irrigation well. Diameter 14 inches, depth drilled 532 feet, perforated 96-240, 264-288, 312-336, 408-432, 456-528 feet. Altitude of land-surface datum 2, 322 feet. Water-level records available 1952-54, $1956-68,1991$ to current year.

WATER LEVELS IN FEET BELOW LAND-SURFACE DATUM

\begin{tabular}{|c|c|c|c|c|c|}
\hline DATE & $\begin{array}{l}\text { WATER } \\
\text { LEVEL MS }\end{array}$ & DATE & $\begin{array}{l}\text { WATER } \\
\text { LEVEL MS }\end{array}$ & DATE & $\begin{array}{l}\text { WATER } \\
\text { LEVEL MS }\end{array}$ \\
\hline $\begin{array}{l}08,1992 \\
04 \\
10\end{array}$ & $\begin{array}{l}118.07 \mathrm{~S} \\
117.50 \mathrm{~S} \\
117.16 \mathrm{~S}\end{array}$ & $\begin{array}{lll}\text { APR } & 05, & 1992 \\
\text { MAY } & 11 & \\
\text { JUN } & 16 & \end{array}$ & $\begin{array}{l}116.88 \mathrm{~S} \\
121.33 \mathrm{~S} \\
121.00 \mathrm{~S}\end{array}$ & $\begin{array}{lll}\text { JUL } & 07, & 1992 \\
\text { AUG } & 10 & \\
\text { SEP } & 07 & \end{array}$ & $\begin{array}{l}118.75 \mathrm{~s} \\
118.17 \mathrm{~s} \\
118.11 \mathrm{~s}\end{array}$ \\
\hline
\end{tabular}

PERIOD OF RECORD HIGHEST 82.26 OCT 26, 1956 LOWEST 121.33 MAY 11, 1992 
WELL DESCRIPTIONS AND WATER-LEVEL MEASUREMENTS

WATER YEAR OCTOBER 1991 TO SEPTEMBER 1992

SITE NUMBER 345212117561801

LOCAL NUMBER OO9N010W16R01S

On Edwards AFB, west of Lancaster Boulevard. Drilled observation well in alluvium. Diameter 2 inches, depth 840 feet, perforated 800-830 feet. Altitude of land-surface datum 2,315 feet. Water-level records available 1991 to current year.

WATER LEVELS IN FEET BELOW LAND-SURFACE DATUM

\begin{tabular}{|c|c|c|c|c|c|c|c|c|c|c|}
\hline DATE & $\begin{array}{l}\text { WATER } \\
\text { LEVEL MS }\end{array}$ & & DATE & $\begin{array}{l}\text { WATER } \\
\text { LEVEL MS }\end{array}$ & & DATE & $\begin{array}{l}\text { WATER } \\
\text { LEVEL MS }\end{array}$ & & DATE & $\begin{array}{l}\text { WATER } \\
\text { LEVEL MS }\end{array}$ \\
\hline $\begin{array}{ll}10, & 1991 \\
21 & \\
19 & \\
08, & 1992\end{array}$ & $\begin{array}{l}101.65 \mathrm{~s} \\
100.32 \mathrm{~s} \\
100.39 \mathrm{~s} \\
100.45 \mathrm{~s}\end{array}$ & $\begin{array}{l}\text { JAN } \\
\text { FEB } \\
\text { MAR }\end{array}$ & $\begin{array}{ll}28, & 1992 \\
04 & \\
10 & \\
30 & \end{array}$ & $\begin{array}{l}100.46 \mathrm{~S} \\
100.48 \mathrm{~S} \\
100.47 \mathrm{~S} \\
100.40 \mathrm{~S}\end{array}$ & $\begin{array}{l}\text { MAY } \\
\text { JUN } \\
\text { JUL }\end{array}$ & $\begin{array}{ll}05, & 1992 \\
16 & \\
07 & \\
14 & \end{array}$ & $\begin{array}{r}100.66 \mathrm{~S} \\
100.69 \mathrm{~S} \\
99.71 \mathrm{~S} \\
100.00 \mathrm{~S}\end{array}$ & $\begin{array}{l}\text { AUG } \\
\text { SEP }\end{array}$ & $\begin{array}{ll}10, & 1992 \\
07 & \\
15 & \end{array}$ & $\begin{array}{l}101.79 \mathrm{~s} \\
103.55 \mathrm{~s} \\
103.53 \mathrm{~s}\end{array}$ \\
\hline
\end{tabular}

PERIOD OF RECORD HIGHEST 99.71 JUL 07, 1992 LOWEST 103.55 SEP 07, 1992

SITE NUMBER 345212117561802

LOCAL NUMBER O09NO10W16R02S

On Edwards AFB, west of Lancaster Boulevard. Drilled observation well in alluvium. Diameter 2 inches, depth 584 feet, perforated 494-564 feet. Deepest of two cluster wells. Altitude of land-surface datum 2,315 feet. Recorder installed June 1992. Water-level records available 1991 to current year.

WATER LEVELS IN FEET BELOW LAND-SURFACE DATUM (OBSERVATIONS AT 1200 HOURS)

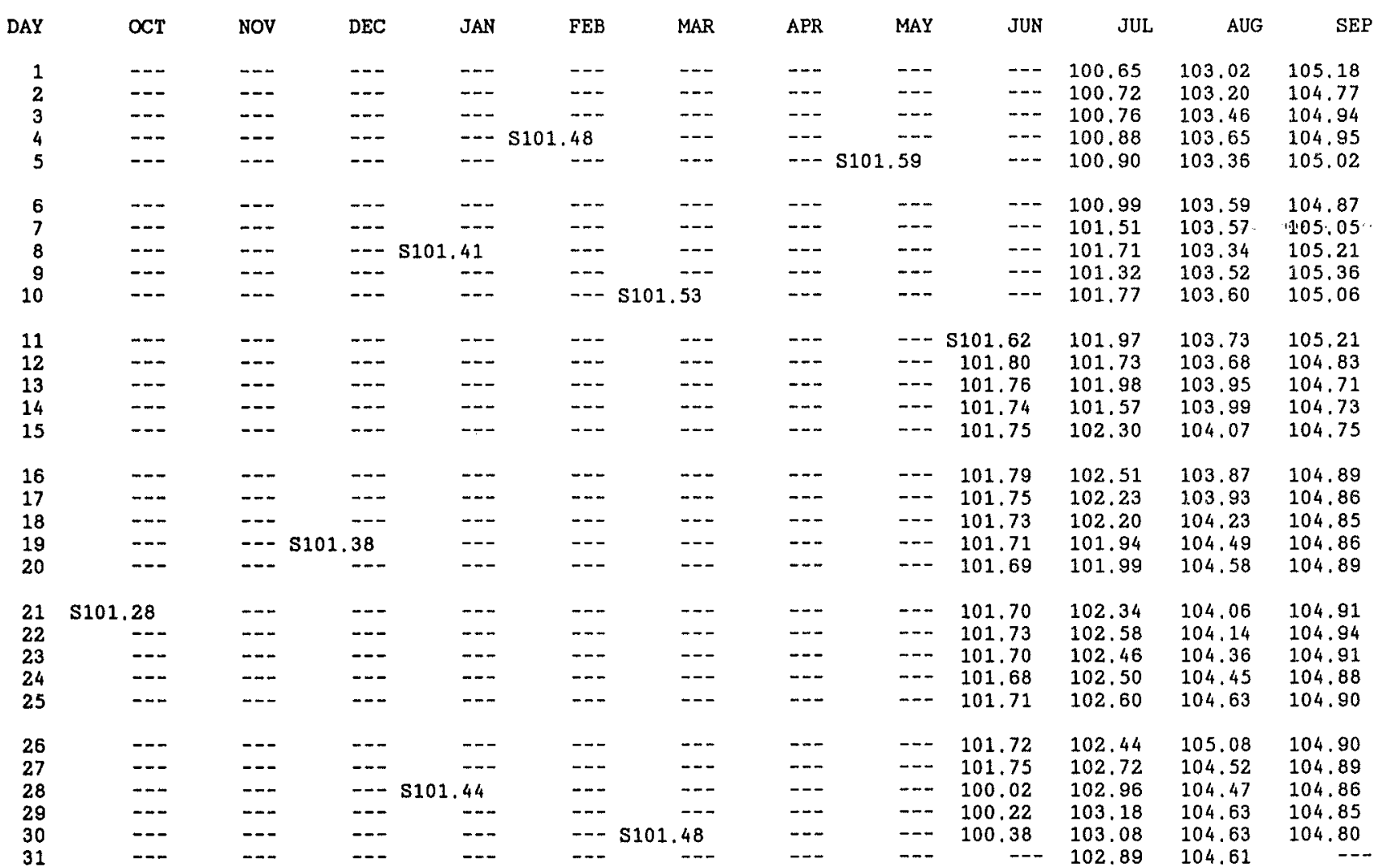

PERIOD OF RECORD HIGHEST 100.02 JUN 28,1992 LOWEST 105.36 SEP 09, 1992

S Manual steel tape measurement. 
WELL DESCRIPTIONS AND WATER-LEVEL MEASUREMENTS

WATER YEAR OCTOBER 1991 TO SEPTEMBER 1992

SITE NUMBER 345212117561803

LOCAL NUMBER OO9N010W16R03S

On Edwards AFB, west of Lancaster Boulevard. Drilled observation well in alluvium. Diameter 2 inches, depth 360 feet, perforated 300-340 feet. Shallowest of two cluster wells. Altitude of land-surface datum 2,315 feet. Recorder installed from June 1991 to February 1992. Water-level records available 1991 to current year.

WATER LEVELS IN FEET BELOW LAND-SURFACE DATUM, WATER YEAR OCTOBER 1990 TO SEPTEMBER 1991

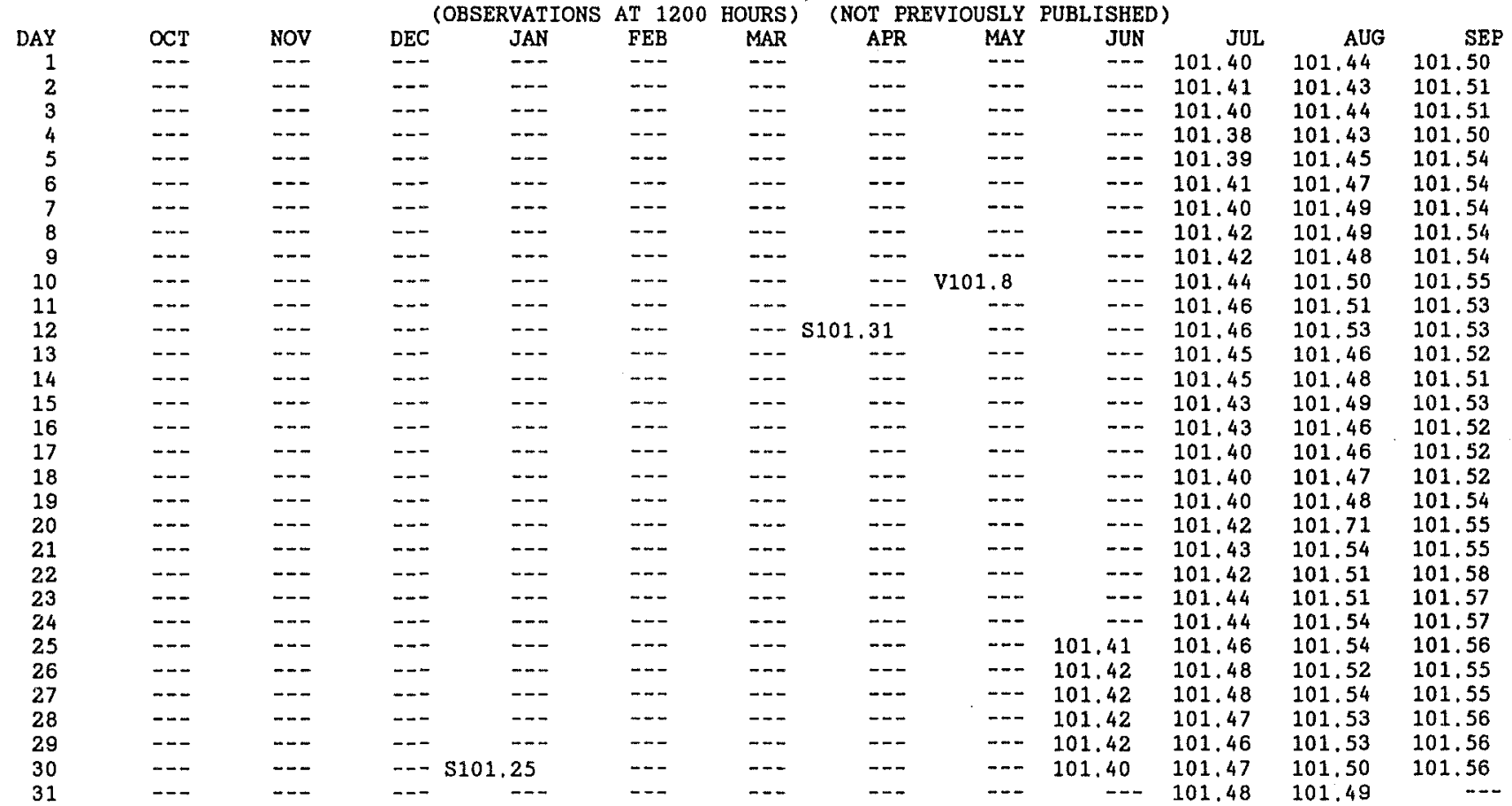

WATER LEVELS IN FEET BELOW LAND SURFACE DATUM, WATER YEAR OCTOBER 1991 TO SEPTEMBER 1992

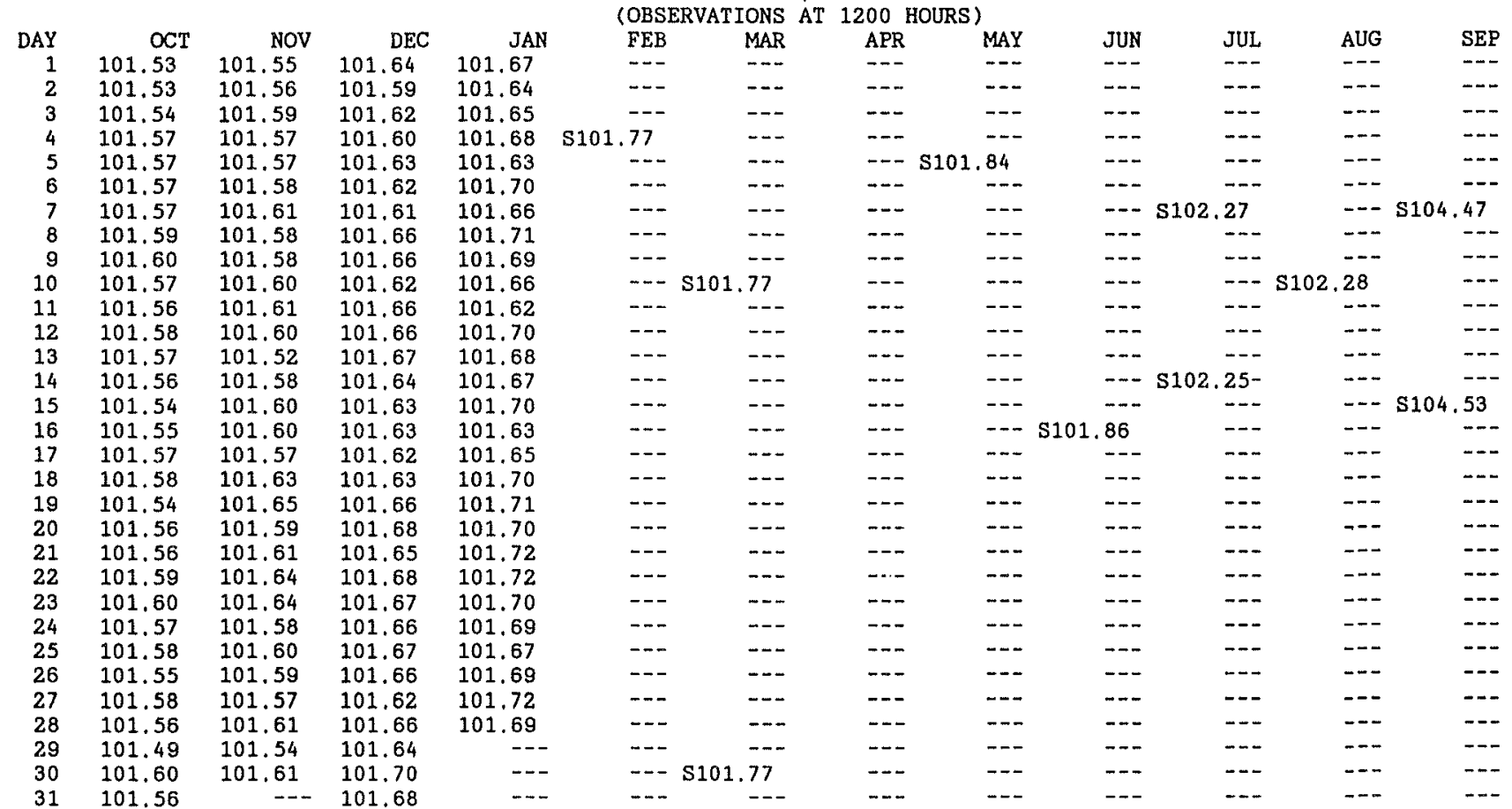

PERIOD OF RECORD HIGHEST 101.25 JAN 30, 1991 LOWEST 104.53 SEP 15, 1992

S Manual steel tape measurement. V Manual calibrated electric tape measurement. 
WATER LEVELS, KERN COUNTY--Continued

WELL DESCRIPTIONS AND WATER-LEVEL MEASUREMENTS

WATER YEAR OCTOBER 1991 TO SEPTEMBER 1992

\begin{abstract}
SITE NUMBER 345209117560801
\end{abstract}
LOCAL NUMBER OO9NO1OW16R04S

On Edwards AFB. Drilled public-supply well. Diameter 16 inches, depth 700 feet, screened 270-690 feet. Alt1tude of land-surface datum 2,318 feet. Water-level records available 1992.

WATER LEVELS IN FEET BELOW LAND-SURFACE DATUM

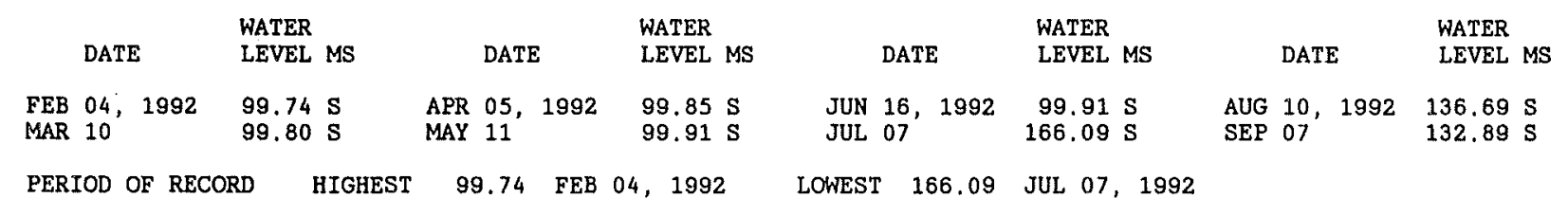

SITE NUMBER 345158117533201

LOCAL NUMBER 009N010W24C01S

On Edwards AFB about $1.4 \mathrm{miles}$ east of Lancaster Boulevard and $0.7 \mathrm{mile}$ north of Rogers Lake. Drilled unused water-table well. Diameter 14 inches, depth 750 feet, perforated 156-750 feet. Altitude of land-surface datum 2,280 feet. Water-level records available 1952-53, 1958, 1960, 1962-81, 1983 to current year.

WATER LEVELS IN FEET BELOW LAND-SURFACE DATUM

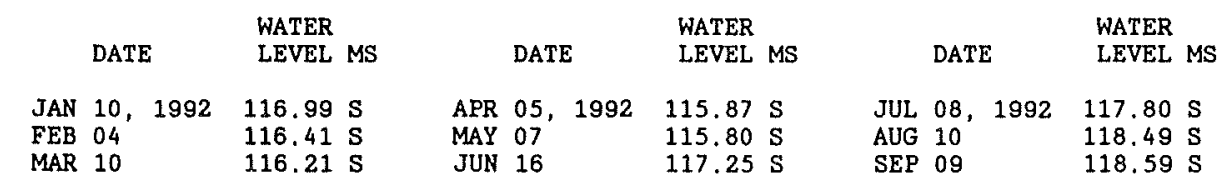

PERIOD OF RECORD HIGHEST 20.29 DEC 08, 1952 LOWEST 118.59 SEP 09, 1992

SITE NUMBER 345140117534201

LOCAL NUMBER OO9N010W24E01S

On Edwards AFB. Drilled unused well. Diameter 16 inches, depth 700 feet, perforated 280-650 feet. Altitude of land-surface datum 2,270,36 feet, surveyed in January 1990. Water-level records avallable 1958-68, 1991 to current year.

WATER LEVELS IN FEET BELOW LAND-SURFACE DATUM

\begin{tabular}{|c|c|c|c|c|c|c|c|}
\hline DATE & $\begin{array}{l}\text { WATER } \\
\text { LEVEL MS }\end{array}$ & DATE & $\begin{array}{l}\text { WATER } \\
\text { LEVEL MS }\end{array}$ & DATE & $\begin{array}{l}\text { WATER } \\
\text { LEVEL MS }\end{array}$ & DATE & $\begin{array}{l}\text { WATER } \\
\text { LEVEL MS }\end{array}$ \\
\hline $\begin{array}{l}04,1992 \\
10\end{array}$ & $\begin{array}{l}109.37 \mathrm{~s} \\
108.93 \mathrm{~s}\end{array}$ & $\begin{array}{ll}\text { APR } & 05,1992 \\
\text { MAY } & 07\end{array}$ & $\begin{array}{l}111.26 \mathrm{~S} \\
110.10 \mathrm{~S}\end{array}$ & $\begin{array}{ll}\text { JUN } 16,1992 \\
\text { JUL } 08\end{array}$ & $\begin{array}{l}133.60 \mathrm{~S} \\
130.14 \mathrm{~S}\end{array}$ & $\begin{array}{ll}\text { AUG } 10,1992 \\
\text { SEP } 09\end{array}$ & $\begin{array}{l}135.44 \mathrm{~S} \\
128.06 \mathrm{~S}\end{array}$ \\
\hline
\end{tabular}

PERIOD OF RECORD HIGHEST 29.38 MAR 12, 1958 LOWEST 135.44 AUG 10, 1932

SITE NUMBER 345016117532301

LOCAL NUMBER O09N010W25P01S

On Edwards AFB. Drilled observation well. Diameter 2 inches, depth 480 feet, perforated $450-470$ feet. Altitude of land-surface datum 2,269.49 feet, surveyed in July 1992. Water-level records available 1991 to current year. WATER LEVELS IN FEET BELOW LAND-SURFACE DATUM

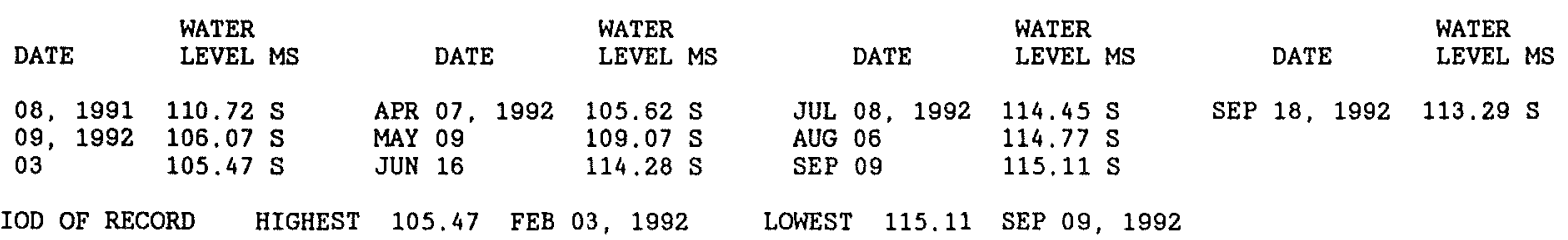


SITE NUMBER 345016117532302

LOCAL NUMBER 009N010W25P02S

On Edwards AFB, Drilled observation well. Diameter 2 inches, depth 130 feet, perforated 100-120 feet. Altitude of land-surface datum 2,269.49 feet, surveyed in July 1992. Water-level records avallable 1991 to current year.

WATER LEVELS IN FEET BELOW LAND-SURFACE DATUM

\begin{tabular}{|c|c|c|c|c|c|c|c|}
\hline DATE & $\begin{array}{l}\text { WATER } \\
\text { LEVEL MS }\end{array}$ & DATE & $\begin{array}{l}\text { WATER } \\
\text { LEVEL MS }\end{array}$ & DATE & $\begin{array}{l}\text { WATER } \\
\text { LEVEL MS }\end{array}$ & DATE & $\begin{array}{l}\text { WATER } \\
\text { LEVEL MS }\end{array}$ \\
\hline $\begin{array}{ll}08, & 1991 \\
09, & 1992 \\
03 & \end{array}$ & $\begin{array}{l}71.60 \mathrm{~S} \\
69.91 \mathrm{~S} \\
70.07 \mathrm{~S}\end{array}$ & $\begin{array}{lll}\text { APR } & 07, & 1992 \\
\text { MAY } & 09 & \\
\text { JUN } & 16\end{array}$ & $\begin{array}{l}70.06 \mathrm{~S} \\
70.79 \mathrm{~s} \\
71.97 \mathrm{~s}\end{array}$ & $\begin{array}{lll}\text { JUL } & 08,1992 \\
\text { AUG } & 06 & \\
\text { SEP } & 09 & \end{array}$ & $\begin{array}{l}71.32 \mathrm{~s} \\
71.77 \mathrm{~s} \\
72.11 \mathrm{~s}\end{array}$ & SEP 18, 1992 & $72.28 \mathrm{~s}$ \\
\hline
\end{tabular}

PERIOD OF RECORD HIGHEST 69.91 JAN 09, 1992 LOWEST 72.28 SEP 18, 1992

SITE NUMBER 345042117564301

LOCAL, NUMBER OO9N010W28F02S

On Edwards AFB about $2 \mathrm{miles}$ west of southwest area of Rogers Lake. Unused water-table well. Diameter 10 inches, depth measured 140.8 feet in 1958. Altitude of land-surface datum 2,290 feet. Water-level records available $1957-78,1980-82,1984$ to current year.

WATER LEVELS IN FEET BELOW LAND-SURFACE DATUM

\begin{tabular}{lllllll}
\multicolumn{1}{c}{ WATER } & \multicolumn{3}{c}{ WATER } \\
DATE & LEVEL MS & \multicolumn{2}{c}{ DATE } & LEVEL MS & DATE & WATER \\
LEVEL MS
\end{tabular}

SITE NUMBER 344923117550301

LOCAL NUMBER 009N010W34R02S

On Edwards AFB, north of Branch Park Road. Drilled observation well in alluvium. Diameter 2 inches, depth 838 feet, perforated 788-808, 818-828 feet. Altitude of land-surface datum 2,295 feet. Water-1evel records available 1991 to current year.

WATER LEVELS IN FEET BELOW LAND-SURFACE DATUM

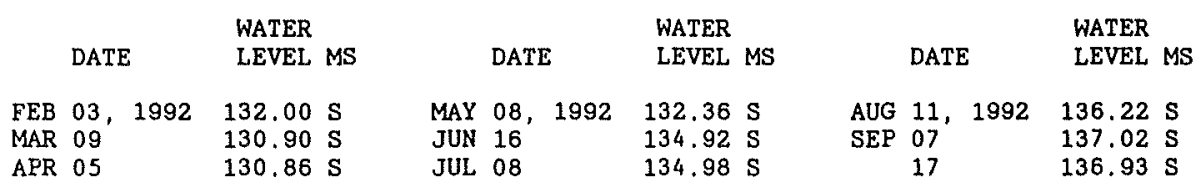

PERIOD OF RECORD HIGHEST 130.86 APR 05, 1992 LOWEST 137.02 SEP 07, 1992

SITE NUMBER 344923117550302

LOCAL NUMBER OOONO10W34RO3S

On Edwards AFB, north of Branch Park Road. Drilled observation well in alluvium. Diameter 2 inches, depth 520 feet, perforated 480-510 feet. Deepest of two cluster wells. Altitude of land-surface datum 2,295 feet. Water-level records avallable 1991 to current year.

WATER LEVELS IN FEET BELOW LAND-SURFACE DATUM

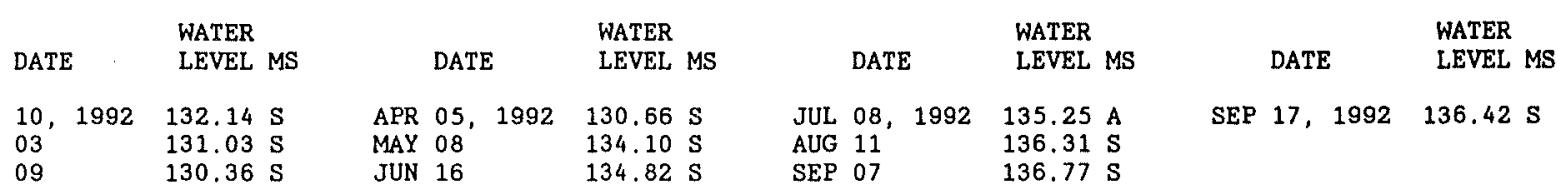

PERIOD OF RECORD HIGHEST 130.36 MAR 09, 1992 LOWEST 136,77 SEP 07, 1992 
WELL DESCRIPTIONS AND WATER-LEVEL MEASUREMENTS

WATER YEAR OCTOBER 1991 TO SEPTEMBER 1992

SITE NUMBER 344923117550303

LOCAL NUMBER 009N010W34R04S

On Edwards AFB, north of Branch Park Road. Drilled observation well in alluvium. Diameter 2 inches, depth 250 feet, perforated 210-240 feet. Shallowest of two cluster wells. Altitude of land-surface datum 2,295 feet. Water-level records avallable 1991 to curent year.

WATER LEVELS IN FEET BELOW LAND-SURFACE DATUM

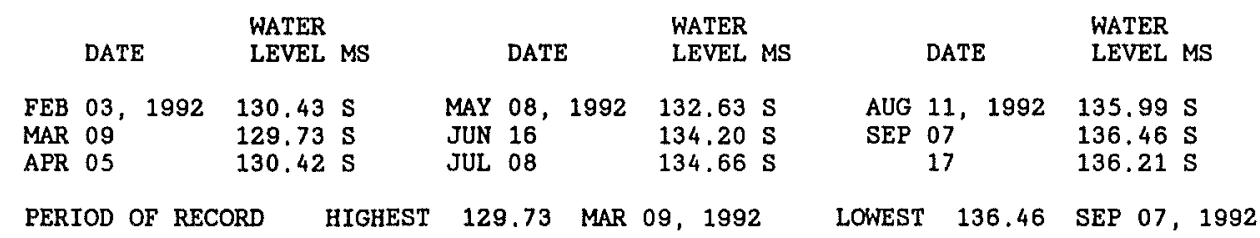

SITE NUMBER 344923117550305

LOCAL NUMBER O09N010W34R05S

On Edwards AFB. Drilled observation well. Diameter 2 inches, depth 90 feet, perforated 60-80 feet. Altitude of land-surface datum 2,295 feet. Water-level records available 1991 to current year.

WATER LEVELS IN FEET BELOW LAND-SURFACE DATUM

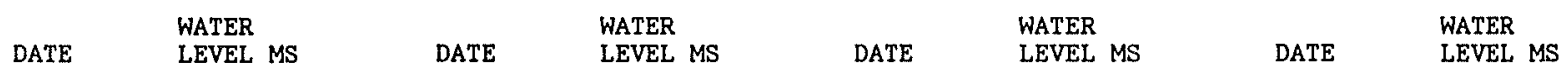

$\begin{array}{llllllllllll}\text { OCT } 09,1991 & 17.90 \mathrm{~S} & \text { MAR 09, } 1992 & 17.49 \mathrm{~S} & \text { JUN } 16,1992 & 17.85 \mathrm{~S} & \text { SEP } 07,1992 & 18.33 \mathrm{~S} \\ \text { JAN 10, } 1992 & 17.45 \mathrm{~S} & \text { APR } 05 & & 17.70 \mathrm{~S} & \text { JUL } 08 & & 17.99 \mathrm{~S} & & 17 & 18.26 \mathrm{~S} \\ \text { FEB 03 } & 17.41 \mathrm{~S} & \text { MAY } 08 & & 17.92 \mathrm{~S} & \text { AUG } 11 & 18.21 \mathrm{~S} & & & \end{array}$

FEB 03

MAY 08

AUG 11

LOWEST 18,33 SEP 07,1992

SITE NUMBER 344948117533601

LOCAL NUMBER OOONO1OW36F01S

On Edwards AFB. Drilled public-supply well. Diameter 14 inches, depth 672 feet, perforated 216-288, 324-540, 564-662 feet. Altitude of land-surface datum 2,285.55 feet, surveyed in July 1992. Water-level records available 1992.

WATER LEVELS IN FEET BELOW LAND-SURFACE DATUM

\begin{tabular}{|c|c|c|c|c|c|c|c|}
\hline DATE & $\begin{array}{l}\text { WATER } \\
\text { LEVEL MS }\end{array}$ & DATE & $\begin{array}{l}\text { WATER } \\
\text { LEVEL MS }\end{array}$ & DATE & $\begin{array}{l}\text { WATER } \\
\text { LEVEL MS }\end{array}$ & DATE & $\begin{array}{l}\text { WATER } \\
\text { LEVEL MS }\end{array}$ \\
\hline $\begin{array}{l}09,1992 \\
03\end{array}$ & $\begin{array}{l}122.36 \mathrm{~S} \\
141.85 \mathrm{~S}\end{array}$ & $\begin{array}{ll}\text { MAR } & 10,1992 \\
\text { APR } & 07\end{array}$ & $\begin{array}{l}124.22 \mathrm{~S} \\
122.27 \mathrm{~S}\end{array}$ & $\begin{array}{lll}\text { MAY } & 09, & 1992 \\
\text { JUN } & 16 & \end{array}$ & $\begin{array}{l}126.15 \mathrm{~S} \\
152.5 \mathrm{~V}\end{array}$ & $\begin{array}{lll}\text { AUG } 06,1992 \\
\text { SEP } 09\end{array}$ & $\begin{array}{l}151.68 \mathrm{~S} \\
133.50 \mathrm{~S}\end{array}$ \\
\hline
\end{tabular}

PERIOD OF RECORD HIGHEST 122.27 APR 07, 1992 LOWEST 152.5 JUN 16, 1992 
WELL DESCRIPTIONS AND WATER-LEVEL MEASUREMENTS

WATER YEAR OCTOBER 1991 TO SEPTEMBER 1992

SITE NUMBER 344932117525401

LOCAL NUMBER 009N010W36J01S

On Edwards AFB, at Survival School. Drilled observation well. Diameter 2 inches, depth 900 feet, perforated 870-890 feet. Altitude of land-surface datum 2,283.00 feet, surveyed in July 1992 . Recorder installed July 1992. Water-level records available 1991 to current year.

WATER LEVELS IN FEET BELOW LAND-SURFACE DATUM (OBSERVATIONS AT 1200 HOURS)

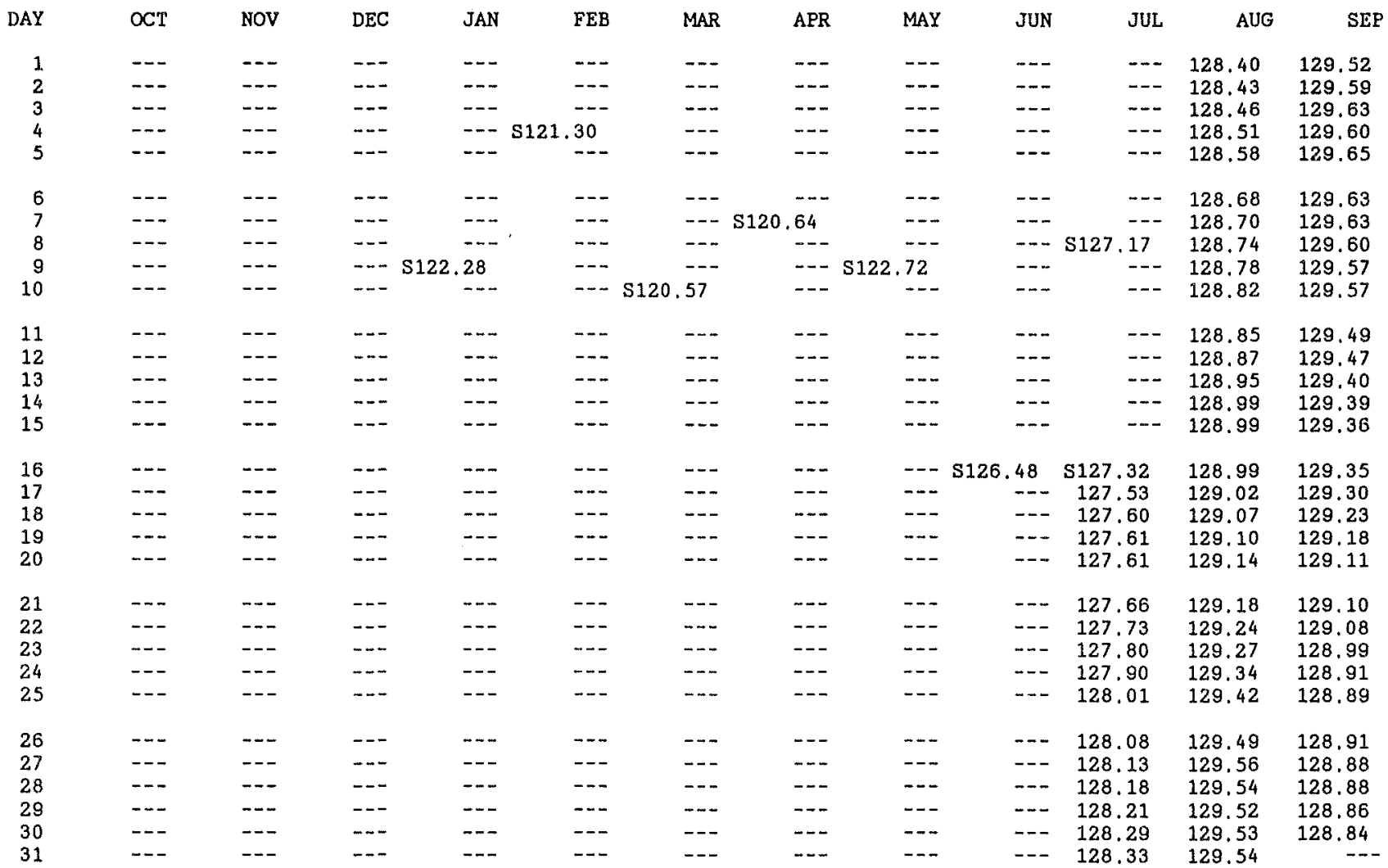


WELL DESCRIPTIONS AND WATER-LEVEL MEASUREMENTS

WATER YEAR OCTOBER 1991 TO SEPTEMBER 1992

SITE NUMBER 344932117525402

LOCAL NUMBER OO9N010W36J02S

On Edwards AFB, at Survival School. Drilled observation well, Diameter 2 inches, depth 529 feet, perforated 503-523 feet. Altitude of land-surface datum 2,283.00 feet, surveyed in July 1992. Recorder installed July 1992. Water-level records available 1991 to current year.

WATER LEVELS IN FEET BELOW LAND-SURFACE DATUM (OBSERVATIONS AT 1200 HOURS)

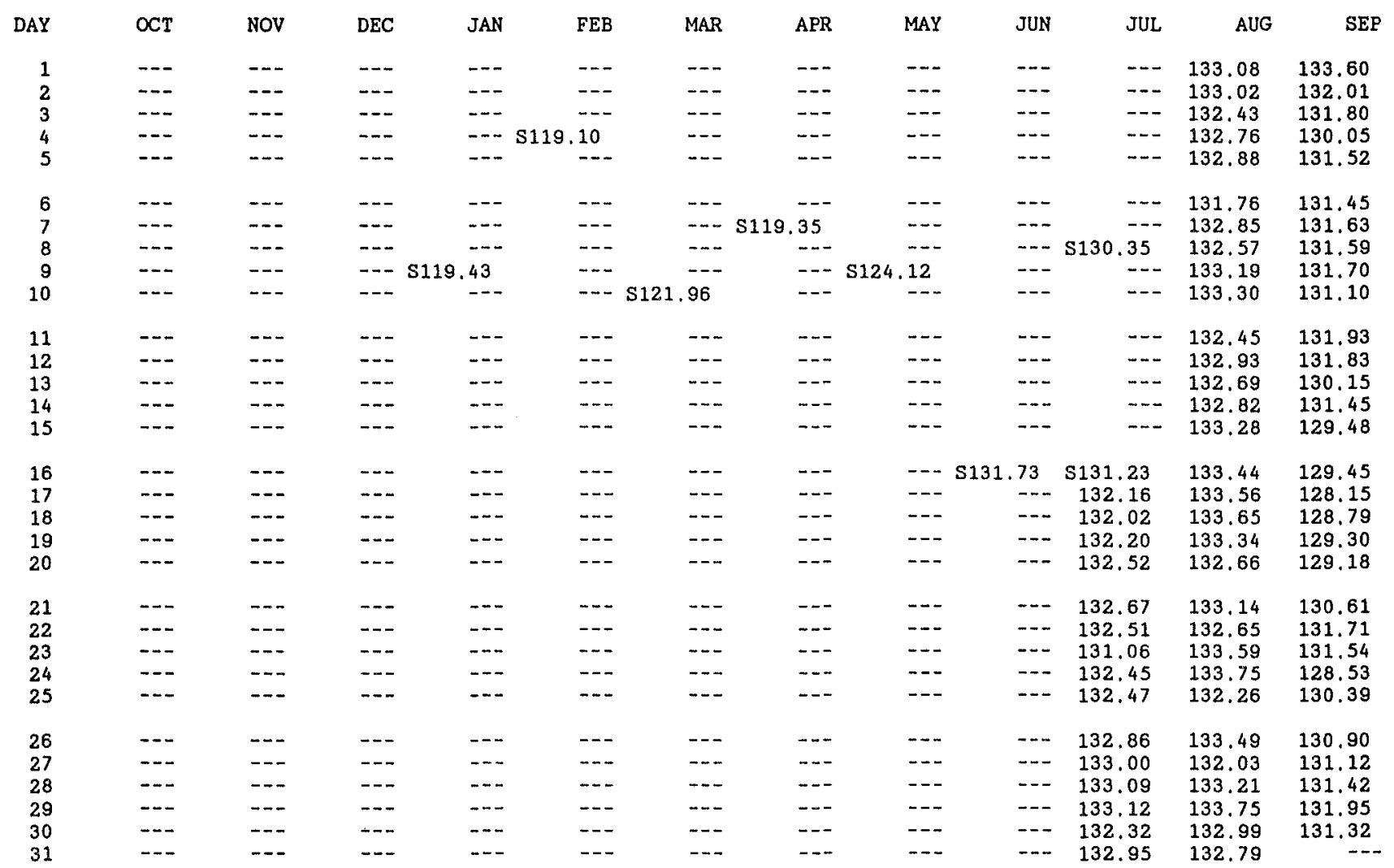


WELL DESCRIPTIONS AND WATER-LEVEL MEASUREMENTS

WATER YEAR OCTOBER 1991 TO SEPTEMBER 1992

SITE NUMBER 344932117525403

LOCAL NUMBER 009N010W36J03S

On Edwards AFB, at Survival Sohool. Drilled observation well. Diameter 2 inches, depth 237 feet, perforated 212-232 feet. Altitude of land-surface datum 2,283.00 feet, surveyed in July 1992 . Water-level records available 1991 to current year.

WATER LEVELS IN FEET BELOW LAND-SURFACE DATUM

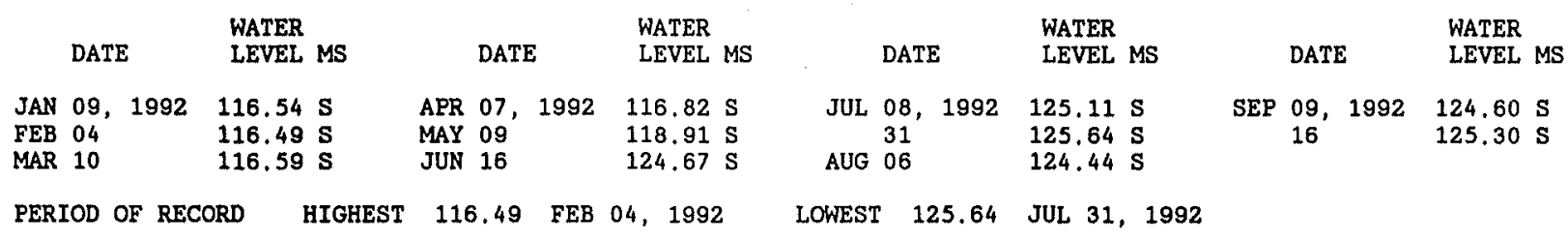

SITE NUMBER 344932117525404

LOCAL NUMBER OO9N010W36J04S

On Edwards AFB, at Survival School. Drilled observation well. Diameter 2 inches, depth 95 feet, perforated 70-90 feet. Altitude of land-surface datum 2,283.00 feet, surveyed in July 1992, Water-level records available 1991 to current year.

WATER LEVELS IN FEET BELOW LAND-SURFACE DATUM

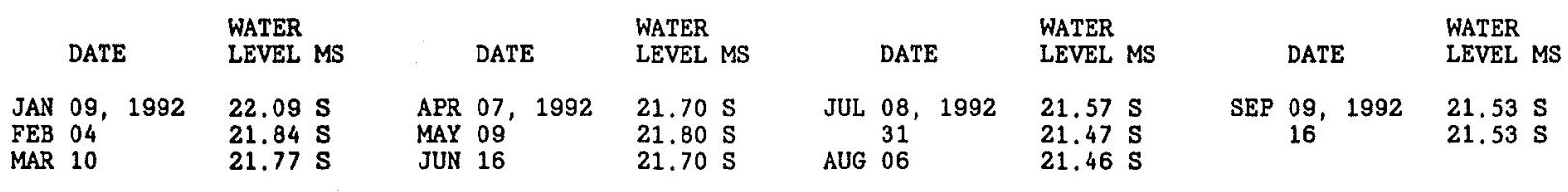

PERIOD OF RECORD HIGHEST 21.46 AUG 06, 1992 LOWEST 22.09 JAN 09, 1992

\section{SITE NUMBER 344927117533401}

LOCAL NUMBER O09N010W36PO1S

On Edwards AFB. Drilled public-supply well. Diameter 16 inches, depth 667 feet, perforated 223-655 feet. Altitude of land-surface datum 2,288.27 feet, surveyed in July 1992, Water-level records available 1992.

WATER LEVELS IN FEET BELOW LAND-SURFACE DATUM

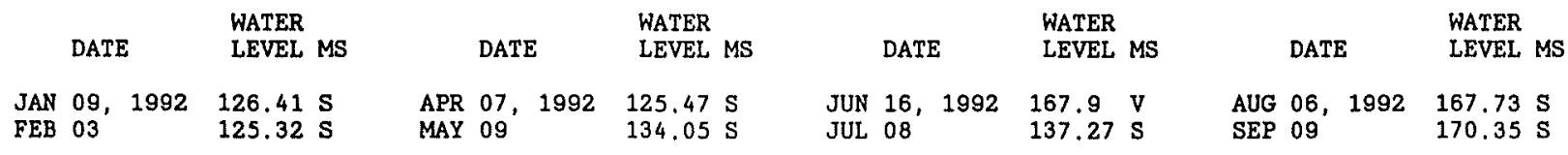

PERIOD OF RECORD HIGHEST 125.32 FEB 03, 1992 LOWEST 170.35 SEP 09, 1992 
WELL DESCRIPTIONS AND WATER-LEVEL MEASUREMENTS

WATER YEAR OCTOBER 1991 TO SEPTEMBER 1992

SITE NUMBER 344922117543301

LOCAL NUMBER OOSNO10W36P02S

On Edwards AFB. Drilled observation well. Diameter 2 inches, depth 465 feet, perforated 435-455 feet. Altitude of land-surface datum 2,290.09 feet, surveyed in July 1992. Recorder installed July 1992. Water-level records available 1991 to current year.

WATER LEVELS IN FEET BELOW LAND-SURFACE DATUM (OBSERVATIONS AT 1200 HOURS)

\begin{tabular}{|c|c|c|c|c|c|c|c|c|c|c|c|c|}
\hline DAY & OCT & NOV & DEC & JAN & FEB & MAR & APR & MAY & JUN & JUL & AUG & SEP \\
\hline 1 & --- & -- & -- & --- & $\cdots$ & -- & --- & --- & -- & $\ldots$ & 143.48 & 144.16 \\
\hline 2 & --- & -- & --- & -- & -- & --- & -- & -- & -- & -- & 143.34 & 142.03 \\
\hline 3 & --- & --- & -- & -- & S127.22 & -- & --- & --- & -- & -- & 142.17 & 142.27 \\
\hline 4 & --- & $-\cdots$ & --- & -- & -- & --- & $-\cdots$ & -- & $-\cdots$ & --- & 141.88 & 138.33 \\
\hline 5 & $\cdots$ & -- & --- & -- & --- & --- & --- & $\cdots$ & --- & $\cdots$ & 143.20 & 141.63 \\
\hline 6 & --- & $\cdots$ & --- & $\cdots$ & --- & --- & -- & -- & --- & -- & 141.86 & 141.42 \\
\hline 7 & $\cdots$ & -- & $\cdots$ & -- & -- & -- & S127.48 & $\cdots$ & --- & -- & 143.12 & 141.58 \\
\hline 8 & --- & S135.35 & -- & -- & -- & --- & -- & --- & --- & $\mathrm{S} 138.10$ & 142.98 & 141.50 \\
\hline 9 & $\cdots$ & $\cdots$ & --- & S128.34 & -- & -- & $-\cdots$ & $\mathrm{S} 136.92$ & -- & -- & 143.48 & 141.62 \\
\hline 10 & $-\cdot$ & $\ldots$ &.- & - & -- & -- & -- & $\ldots$ & -- & -- & 143.59 & 141.00 \\
\hline 11 & $\cdots$ & --- & -- & --- & -- & $\cdots$ & $=-$ & --- & -- & -- & 142.92 & 141.95 \\
\hline 12 & -- & --- & -- & $-\cdots$ & $-\cdots$ & $\cdots$ & --- & --- & $\cdots$ & $\cdots$ & 143.23 & 141.78 \\
\hline 13 & -- & -- & $\cdots$ & --- & $\cdots$ & -- & $\cdots$ & -- & --- & -- & 142.72 & 138.55 \\
\hline 14 & --- & -- & $-\cdots$ & --- & $-\cdots$ & --- & -- & --- & -- & -- & 143.33 & 142.30 \\
\hline 15 & --- & $m$ & --- & --- & --- & $-\cdots$ & -- & -- & -- & $\mathrm{S} 138,63$ & 143.74 & 140.63 \\
\hline 16 & --- & --- & --- & -- & -- & -- & -- & --- & $\mathrm{S} 142.81$ & 142.28 & 143.89 & 138.17 \\
\hline 17 & --- & 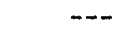 & -- & --- & $\cdots$ & $\cdots$ & --- & -- & -- & 142.87 & 144.02 & 137.27 \\
\hline 18 & -- & --- & -- & --- & --- & -- & --- & --- & --- & 142.82 & 144.11 & 137.72 \\
\hline 19 & -- & --- & -- & $\cdots$ & -- & $\cdots$ & --- & $-\cdots$ & --- & 142.96 & 143.45 & 138.01 \\
\hline 20 & --- & $\cdots$ & --- & --- & -- & --- & $-\infty$ & --- & --- & 143.11 & 143.49 & 137.96 \\
\hline 21 & --- & --- & -- & -- & -- & -- & -- & --- & $\rightarrow$ & 143.21 & 143.79 & 141.29 \\
\hline 22 & -- & -- & $\cdots$ & $-\infty$ & --- & $\cdots$ & --- & -- & -- & 143.20 & 143.13 & 142.33 \\
\hline 23 & --- & -- & --- & --- & -- & --- & $-\cdots$ & --- & $-\cdots$ & 142.23 & 144.18 & 141.71 \\
\hline 24 & --- & $m$ & -- & $-\cdots$ & $\cdots$ & --- & $\cdots$ & --- & --- & 143.10 & 144.27 & 137.11 \\
\hline 25 & --- & -- & $\cdots$ & -- & -- & -- & -- & --- & $\cdots$ & 143.14 & 143.24 & 140.88 \\
\hline 26 & --- & --- & $\cdots$ & -- & -- & --- & $\cdots$ & $\cdots$ & -- & 143.38 & 144.20 & 141.10 \\
\hline 27 & $\cdots$ & $\cdots$ & --- & $\rightarrow$ & --- & $\cdots$ & --- & -- & $\cdots$ & 143.47 & 142.16 & 141.25 \\
\hline 28 & --- & $\cdots$ & $\cdots$ & -- & -- & -- & $\cdots$ & $\cdots$ & -- & 143.53 & 143.86 & 145.52 \\
\hline 29 & --- & -- & -- & -- & -- & -- & -- & -- & --- & 143.55 & 144.38 & 146.11 \\
\hline 30 & --- & --- & $\cdots$ & -- & $\cdots$ & --- & $\cdots$ & --- & -- & 142.67 & 143.00 & 142.18 \\
\hline 31 & --- & -- & --- & -- & -- & --- & -- & --- & --- & 143.33 & 143.32 & -- \\
\hline
\end{tabular}

PERIOD OF RECORD HIGHEST 127.22 FEB 03, 1992 LOWEST 146.11 SEP 29, 1992

S Manual steel tape measurement. 
WELL DESCRIPTIONS AND WATER-LEVEL MEASUREMENTS

WATER YEAR OCTOBER 1991 TO SEPTEMBER 1992

SITE NUMBER 344922117543302

LOCAL NUMBER OOSNO1OW36P03S

On Edwards AFB. Drilled observation well. Diameter 2 inches, depth 120 feet, perforated 90-110 feet. Altitude of land-surface datum 2,291.21 feet, surveyed in July 1992. Recorder installed July 1992. Water-level records available 1991 to current year.

WATER LEVELS IN FEET BELOW LAND-SURFACE DATUM (OBSERVATIONS AT 1200 HOURS)

\begin{tabular}{|c|c|c|c|c|c|c|c|c|c|c|c|c|}
\hline DAY & $\propto C T$ & NOV & DEC & JAN & FEB & MAR & APR & MAY & JUN & JUL & AUG & SEP \\
\hline 1 & -- & $m$ & $\cdots$ & --- & --- & $\cdots$ & $\cdots$ & -- & -- & $\cdots$ & 27.38 & 27.31 \\
\hline 2 & -- & -- & --- & $-\cdots$ & $\ldots$ & -- & -- & $\ldots$ & -- & -- & 27.37 & 27.32 \\
\hline 3 & -- & --- & --- & -- & S27. 55 & $\cdots$ & --- & -- & $\cdots$ & --- & 27.38 & 27.32 \\
\hline 4 & -- & -- & --- & --- & $\ldots$ & -- & -- & $\cdots$ & -- & --- & 27.37 & 27.36 \\
\hline 5 & -- & --- & -- & -- & $\cdots$ & $\cdots$ & --- & $\cdots$ & -- & --- & 27.37 & 27.32 \\
\hline 6 & -- & -- & -- & $\cdots$ & --- & --- & -- & $\cdots$ & --- & --- & 27.38 & 27.32 \\
\hline 7 & --- & -- & $\cdots$ & -- & --- & $-\cdots$ & S27.00 & --- & --- & $\cdots$ & 27.37 & 27.32 \\
\hline 8 & --- & S27.88 & $\cdots$ & --- & --- & $\cdots$ & -- & --- & -- & \$27. 36 & 27.37 & 27.32 \\
\hline 9 & $\cdots$ & $\ldots$ & --- & s27. 53 & $\cdots$ & -- & --- & S27.01 & $\cdots$ & $\cdots$ & 27.36 & 27.31 \\
\hline 10 & --- & -- & -- & -- & $-\cdots$ & 527.06 & --- & - & $-\cdots$ & $\cdots$ & 27.36 & 27.31 \\
\hline 11 & --- & $-\cdots$ & $\cdots$ & --- & --- & $-\cdots$ & $\ldots$ & $\cdots$ & --- & -- & 27.36 & 27.30 \\
\hline 12 & $\cdots$ & --- & -- & $\cdots$ & --- & --- & $\cdots$ & $\cdots$ & $\cdots$ & $-\cdots$ & 27.35 & 27.30 \\
\hline 13 & -- & -- & --- & -- & $\cdots$ & $\cdots$ & -- & --- & -- & $\cdots$ & 27.35 & 27.34 \\
\hline 14 & --- & $\cdots$ & -- & --- & $\cdots$ & $\cdots$ & -- & --- & --- & $\cdots$ & 27.35 & 27.30 \\
\hline 15 & $\cdots$ & -- & $\cdots$ & $-\cdots$ & --- & -- & $\cdots$ & $-\cdots$ & -- & $\mathrm{S} 27.35$ & 27.34 & 27.33 \\
\hline 16 & --- & $\cdots$ & $\cdots$ & --- & $\cdots$ & $\cdots$ & -- & --- & --- & 27.43 & 27.33 & 27.35 \\
\hline 17 & $\cdots$ & --- & -- & $\cdots$ & --- & --- & --- & --- & $\cdots$ & 27.43 & 27.33 & 27.36 \\
\hline 18 & -- & $\cdots$ & $\cdots$ & --- & -- & $\cdots$ & $\cdots$ & $\cdots$ & --- & 27.42 & 27.33 & 27.35 \\
\hline 19 & -- & -- & -- & -- & -- & $\cdots$ & -- & $\ldots$ & -- & 27.42 & 27.33 & 27.34 \\
\hline 20 & $\cdots$ & -- & --- & $-\cdots$ & --- & --- & $\cdots$ & $\cdots$ & --- & 27.42 & 27.34 & 27.34 \\
\hline 21 & --- & -- & --- & --- & --- & -- & -- & $-\cdots$ & $\cdots$ & 27.41 & 27.33 & 27.30 \\
\hline 22 & -- & --- & --- & -- & --- & -- & --- & -- & --- & 27.43 & 27.33 & 27.30 \\
\hline 23 & -- & --- & --- & --- & --- & --- & --- & $\cdots$ & -- & 27.43 & 27.32 & 27.30 \\
\hline 24 & $\cdots$ & $\cdots$ & --- & --- & --- & -- & -- & $\cdots$ & --- & 27.42 & 27.32 & 27.35 \\
\hline 25 & -- & --- & --- & $\cdots$ & $\cdots$ & $\cdots$ & --- & --- & $\cdots$ & 27.41 & 27.35 & 27.30 \\
\hline 26 & -- & --- & --- & --- & --- & -- & $\cdots$ & $\cdots$ & --- & 27.41 & 27.33 & 27.30 \\
\hline 27 & $\cdots$ & -- & --- & -- & -- & $-\cdots$ & $\cdots$ & -- & --- & 27.40 & 27.33 & 27.30 \\
\hline 28 & -- & $\cdots$ & $\cdots$ & $\cdots$ & $\cdots$ & -- & --- & $\cdots$ & $\cdots$ & 27.40 & 27.32 & 27.23 \\
\hline 29 & -- & $\cdots$ & $\cdots$ & $\cdots$ & -- & --- & -- & $\cdots$ & $\cdots$ & 27.39 & 27.31 & 27.22 \\
\hline 30 & -- & $\cdots$ & $\cdots$ & $\cdots$ & -- & --- & --- & $\cdots$ & $\cdots$ & 27.39 & 27.31 & 27.28 \\
\hline 31 & $\cdots$ & $\cdots$ & $\cdots$ & $\cdots$ & --- & --- & -- & $\cdots$ & $\cdots$ & 27.38 & 27.32 & $\ldots$ \\
\hline
\end{tabular}

PERIOD OF RECORD HIGHEST 27.00 APR 07, 1992 LOWEST 27.88 NOV 08, 1991

s Manual steel tape measurement. 
WELL DESCRIPTIONS AND WATER-LEVEL MEASUREMENTS

WATER YEAR OCTOBER 1991 TO SEPTEMBER 1992

SITE NUMBER 344947117594801

LOCAL NUMBER OOSN011W36L01S

On Edwards AFB about 1 mile east of Rosamond Lake. Drilled unused well. Diameter 12 inches, depth measured 134.7 feet in 1970, 127.1 feet in 1991. Altitude of land-surface datum 2,289.54 feet, surveyed in May 1989 . Water-level records available 1951-54,1956-71, 1974-78, 1981-82, 1984 to current year.

WATER LEVELS IN FEET BELOW LAND-SURFACE DATUM

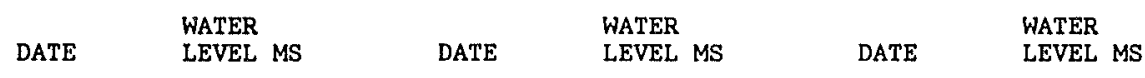

$\begin{array}{lrlllll}\text { APR } 06,1992 & 92.19 \mathrm{~S} & \text { JUN } 17,1992 & 100.11 \mathrm{~s} & \text { AUG } 05,1992 & 94.97 \mathrm{~s}\end{array}$

$\begin{array}{llllll}\text { MAY } 11 & 101.47 \mathrm{~S} & \text { JUL } 08 & 100.23 \mathrm{~S} & \text { SEP } 07 & 99.99 \mathrm{~s}\end{array}$

PERIOD OF RECORD HIGHEST 25.22 APR 10, 1951 LOWEST 106.78 APR 14, 1981

SITE NUMBER 345237118095101

LOCAL NUMBER OO9N012W16E04S

In Rosamond about $0.7 \mathrm{mile}$ north of Rosamond Boulevard and 50 feet west of Slerra Highway. Unused we11. Diameter 8 inches, depth unknown. Altitude of land-surface datum 2,380 feet. Water-level records available 1973 to current year.

WATER LEVELS IN FEET BELON LAND-SURFACE DATUM

$$
\text { DATE WATER }
$$

APR 16, $1992 \quad 23.62 \mathrm{~S}$

PERIOD OF RECORD HIGHEST 21.38 MAR 26, 1985 LOWEST 29.18 FEB 15,1974

SITE NUMBER 345112118075101

LOCAL NUMBER OOON012W23N01S

On Edwards AFB, east of Rosamond, and about 0.8 mile south of Rosamond Boulevard on Division Street. Drilled unused well. Diameter 12 inches, depth measured 266.7 feet in $1948,263.65$ feet in $1973,263.95$ feet in 1991. Altitude of land-surface datum 2,294 feet. Water-level records available 1951-54, 1956-68, 1973 to current year.

WATER LEVELS IN FEET BELOW LAND-SURFACE DATUM

\begin{tabular}{|c|c|c|c|c|c|c|c|}
\hline DATE & $\begin{array}{l}\text { WATER } \\
\text { LEVEL MS }\end{array}$ & DATE & $\begin{array}{l}\text { WATER } \\
\text { LEVEL MS }\end{array}$ & DATE & $\begin{array}{l}\text { WATER } \\
\text { LEVEL MS }\end{array}$ & DATE & $\begin{array}{l}\text { WATER } \\
\text { LEVEL MS }\end{array}$ \\
\hline $\begin{array}{l}12, \quad 1992 \\
06\end{array}$ & $\begin{array}{l}72.69 \mathrm{~S} \\
72.64 \mathrm{~S}\end{array}$ & $\begin{array}{ll}\text { APR } & 16, \\
\text { MAY } & 1992\end{array}$ & $\begin{array}{l}72.61 \mathrm{~s} \\
72.56 \mathrm{~s}\end{array}$ & $\begin{array}{l}\text { JUN } 17,1992 \\
\text { JUL } 08\end{array}$ & $\begin{array}{l}72.65 \mathrm{~S} \\
72.65 \mathrm{~S}\end{array}$ & $\begin{array}{lll}\text { AUG } 05, & 1992 \\
\text { SEP } 09 & \end{array}$ & $\begin{array}{l}72.77 \mathrm{~S} \\
72.94 \mathrm{~S}\end{array}$ \\
\hline
\end{tabular}

SITE NUMBER 344917118093501

LOCAL NUMBER 009N012W33P01S

About 2 miles south of Rosamond, 200 feet north of Avenue A, and 0.25 mile west of Sierra Highway. Unused well. Diameter 12 inches, depth reported 146 feet in 1990 . Altitude of land-surface datum 2,310 feet. Water-level records available 1978 to current year.

WATER LEVELS IN FEET BELOW LAND-SURFACE DATUM

$$
\text { DATE WATER }
$$

APR 16, $1992 \quad 74.59 \mathrm{~S}$

PERIOD OF RECORD HIGHEST 69.74 FEB 28, 1979 LOWEST 74.74 MAR 22, 1991 
SITE NUMBER 345423118152101

LOCAL NUMBER OO9N013W04A01S

About 7 miles northwest of Rosamond. Drilled domestic well. Diameter 12 inches, depth reported 282 feet. Altitude of land-surface datum $2,636.8$ feet. Water-level records avallable 1929, $1954-78,1980$ to current year.

WATER LEVELS IN FEET BELOW LAND-SURFACE DATUM

$$
\text { DATE WATER }
$$

APR 13, $1992192.30 \mathrm{SR}$

PERIOD OF RECORD HIGHEST 69.70 NOV 15, 1929 LOWEST 154.82 FEB 20, 1975

SITE NUMBER 345252118174601

LOCAL NUMBER 009N013W07Q03S

In Willow Springs, west of Manley Road. Unused well. Diameter 8 inches, depth reported 185 feet. Altitude of land-surface datum 2,605 feet. Water-level records available 1929, 1956, 1961 to current year.

WATER LEVELS IN FEET BELOW LAND-SURFACE DATUM

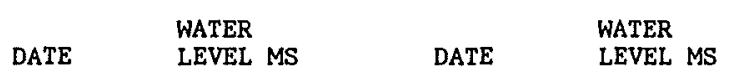

OCT 22, $1991 \quad 96.57 \mathrm{~s} \quad$ APR $13,1992 \quad 82.07 \mathrm{~s}$

PERIOD OF RECORD HIGHEST 0.31 NOV 14, $1929 \quad$ LOWEST 96.57 OCT 22, 1991

SITE NUMBER 345202118133501

LOCAL NUMBER 009N013W14Q01S

About 3 miles west of Rosamond at the Tropico School. Institutional water-table well. Diameter 10 inches, depth 400 feet, perforated 250-400 feet. Altitude of land-surface datum 2,442 feet. Water-level records available 1957 , $1967-76,1978-80,1982$ to current year.

WATER LEVELS IN FEET BELOW LAND-SURFACE DATUM

$$
\begin{array}{ll} 
& \text { WATER } \\
\text { DATE } & \text { LEVEL MS }
\end{array}
$$

APR 13, $1992189.07 \mathrm{~S}$

PERIOD OF RECORD HIGHEST 176 NOV 1957 LOWEST 202.88 MAR 30, 1978

SITE NUMBER 345021118144601

LOCAL NUMBER O09N013W27K01S

About 4 miles southwest of Rosamond, 0.3 mile north of Gaskill Road, and 30 feet east of 65th Street West. Drilled irrigation water-table well. Diameter 14 inches, depth reported 550 feet. Altitude of land-surface datum 2,390 feet. Water-level records available 1964, 1971, 1976, 1979 to current year.

WATER LEVELS IN FEET BELOW LAND-SURFACE DATUM

$$
\begin{array}{ll} 
& \text { WATER } \\
\text { DATE } & \text { LEVEL MS }
\end{array}
$$

APR $13,1992184.47 \mathrm{~S}$

PERIOD OF RECORD HIGHEST 169.02 MAR 29, 1988 LOWEST 272.00 MAY 11, 1971 
WELL DESCRIPTIONS AND WATER-LEVEL MEASUREMENTS

WATER YEAR OCTOBER 1991 TO SEPTEMBER 1992

SITE NUMBER 344400118184501

LOCAL NUMBER O09N014W01H01S

About 1.5 miles north of Willow Springs, and 1.25 miles west of Willow Springs Road. Drilled unused water-table we11. Diameter 18 inches, depth measured 760.5 feet. Altitude of land-surface datum 2,700 feet. Water-1eve1 records available 1956, 1967 to current year.

WATER LEVELS IN FEET BELOW LAND-SURFACE DATUM

DATE WATER $\quad$ LEVEL MS

APR $13,1992162.83 \mathrm{~S}$

PERIOD OF RECORD HIGHEST 115.00 MAY 23, 1956 LOWEST 168,87 OCT 19, 1970

SITE NUMBER 345143118231401

LOCAL NUMBER 009N014W20B01S

About 13 miles west of Rosamond, southeast of intersection of Rosamond Boulevard and 145th Street West. Drilled unused water-table well. Diameter 14 inches, depth 540 feet, perforated 290-540 feet. Altitude of land-surface datum 2,656.4 feet. Water-level records available 1955, 1960-63, 1967 to current year.

WATER LEVELS IN FEET BELOW LAND-SURFACE DATUM

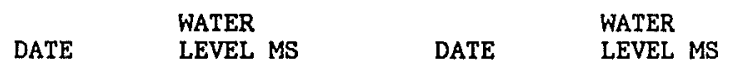

OCT 21, $1991338.65 \mathrm{~S} \quad$ APR 13, $1992 \quad 338.49 \mathrm{~S}$

PERIOD OF RECORD HIGHEST 231.60 MAY 24, 1955 LOWEST 339.59 OCT 27, 1983

SITE NUMBER 345144118213201

LOCAL NUMBER 009N014W22D01S

About 11 miles west of Rosamond, 0.2 mile east of 130 th Street West and 150 feet south of Rosamond Boulevard, Domestic water-table well. Diameter 6 inches, depth measured 415 feet in 1978 . Altitude of land-surface datum 2,565 feet. Water-level records available 1978-84, 1988 to current year.

WATER LEVELS IN FEET BELOW LAND-SURFACE DATUM

$$
\text { DATE WATER }
$$

APR 13, $1992 \quad 327.20 \mathrm{~S}$

PERIOD OF RECORD HIGHEST 294.39 APR 17, 1991 LOWEST 394.74 MAR 14, 1980

SITE NUMBER 345001118264501

LOCAL NUMBER OO9N015W26N01S

About 16 miles west of Rosamond near intersection of 170 th Street West and Gaskill Road. Drilled domestic water-table well in alluvium. Diameter 8 inches, depth 600 feet, perforated $280-600$ feet. Altitude of land-surface datum 2,642.4 feet. Water-level records available 1962-63, 1978-81, 1983 to current year.

WATER LEVELS IN FEET BELOW LAND-SURFACE DATUM

$$
\text { DATE WATER }
$$

APR 14, $1992 \quad 274.48 \mathrm{~S}$

PERIOD OF RECORD HIGHEST 182.35 FEB 19, 1963 LOWEST 300.62 APR 15, 1981 
WELL DESCRIPTIONS AND WATER-LEVEL MEASUREMENTS

WATER YEAR OCTOBER 1991 TO SEPTEMBER 1992

SITE NUMBER 345856117485502

LOCAL NUMBER 010N009W10B02S

On Edwards AFB. Drilled observation well. Diameter 2 inches, depth 150 feet, perforated 117-137 feet. Altitude of land-surface datum 2,280 feet. Water-level records available 1991 to current year.

WATER LEVELS IN FEET BELOW LAND-SURFACE DATUM

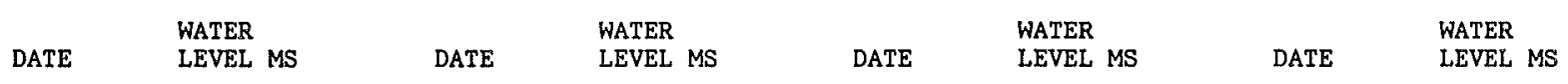

OCT 10,1991

JAN 08, 1992

SEP 10,1992

$95.88 \mathrm{~s}$

FEB 05

$95.52 \mathrm{~s}$

MAR 11, 1992

$95.51 \mathrm{~S}$

JUN 17, 1992

$\begin{array}{ll}\text { APR } & 05 \\ \text { MAY } 09\end{array}$

$95.61 \mathrm{~S}$
$95.60 \mathrm{~S}$

JUL 09

$95.67 \mathrm{~s}$

$95.72 \mathrm{~S}$

AUG 07

$95.77 \mathrm{~S}$

PERIOD OF RECORD HIGHEST 95.10 OCT 10,1991 LOWEST 95.88 SEP 10, 1992

SITE NUMBER 345712117463601

LOCAL NUMBER 010N009W24A02S

On Edwards AFB at east edge of Rogers Lake. Drilled unused well. Diameter 14 inches, depth measured 281 feet in $1968,278,7$ feet in 1991. Altitude of land-surface datum 2,290.60 feet. Water-level records available 1954, 1956 to current year.

WATER LEVELS IN FEET BELOW LAND-SURFACE DATUM

\begin{tabular}{lllllll} 
DATE & \multicolumn{1}{c}{ WATER } & \multicolumn{3}{c}{ WATER } & & WATER \\
& LEVEL MS & DATE & LEVEL MS & DATE & LEVEL MS \\
08,1992 & $94.51 \mathrm{~S}$ & APR 05, 1992 & $94.83 \mathrm{~S}$ & JUL 09, 1992 & $94.78 \mathrm{~S}$ \\
05 & $94.49 \mathrm{~S}$ & MAY 09 & $94.65 \mathrm{~S}$ & AUG 07 & $94.85 \mathrm{~S}$ \\
11 & $94.54 \mathrm{~S}$ & JUN 17 & $94.73 \mathrm{~S}$ & SEP 10 & $94.99 \mathrm{~S}$
\end{tabular}

PERIOD OF RECORD HIGHEST 72.25 NOV 29, 1956 MAR 07, JUN 05, 1957 LOWEST 94.99 SEP 10,1992

SITE NUMBER 345616117491001

LOCAL NUMBER 010N009W27C01S

On Edwards AFB. Drilled observation well. Diameter 2 inches, depth 225 feet, perforated 207-217 feet. Deepest of three cluster wells. Altitude of land-surface datum 2,273 feet. Water-level records avallable 1991 to current year.

WATER LEVELS IN FEET BELOW LAND-SURFACE DATUM

\begin{tabular}{|c|c|c|c|c|c|c|c|}
\hline & WATER & & WATER & & WATER & & WATER \\
\hline DATE & LEVEL MS & DATE & LEVEL MS & DATE & LEVEL MS & DATE & LEVEL, MS \\
\hline $\begin{array}{l}05,1992 \\
11\end{array}$ & $\begin{array}{l}79.03 \mathrm{~S} \\
80.18 \mathrm{~S}\end{array}$ & 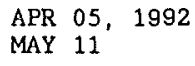 & $\begin{array}{l}79.14 \mathrm{~S} \\
79.30 \mathrm{~S}\end{array}$ & $\begin{array}{ll}\text { JUN } 17, & 1992 \\
\text { JUL } 07 & \end{array}$ & $\begin{array}{l}79.42 \mathrm{~S} \\
79.35 \mathrm{~S}\end{array}$ & $\begin{array}{lll}\text { AUG } & 07,1992 \\
\text { SEP } & 09\end{array}$ & $\begin{array}{l}79.50 \mathrm{~S} \\
79.60 \mathrm{~S}\end{array}$ \\
\hline
\end{tabular}

PERIOD OF RECORD HIGKEST 79.03 FEB 05, 1992 LOWEST 80.18 MAR 11, 1992

SITE NUMBER 345616117491002

LOCAL NUMBER 010N009W27C02S

On Edwards AFB. Drilled observation well. Diameter 2 inches, depth 160 feet, perforated 130-150 feet. Altitude of land-surface datum 2,273 feet. Mid-interval depth of three cluster wells. Water-level records available 1991 to current year.

WATER LEVELS IN FEET BELOW LAND-SURFACE DATUM

\begin{tabular}{|c|c|c|c|c|c|c|c|}
\hline DATE & $\begin{array}{l}\text { WATER } \\
\text { LEVEL MS }\end{array}$ & DATE & $\begin{array}{l}\text { WATER } \\
\text { LEVEL MS }\end{array}$ & DATE & $\begin{array}{l}\text { WATER } \\
\text { LEVEL MS }\end{array}$ & DATE & $\begin{array}{l}\text { WATER } \\
\text { LEVEL MS }\end{array}$ \\
\hline $\begin{array}{l}05,1992 \\
11\end{array}$ & $\begin{array}{l}78.90 \mathrm{~S} \\
79.07 \mathrm{~S}\end{array}$ & $\begin{array}{l}\text { APR 05, } 1992 \\
\text { MAY 11 }\end{array}$ & $\begin{array}{l}79.11 \mathrm{~S} \\
79.18 \mathrm{~S}\end{array}$ & $\begin{array}{l}\text { JUN } 17,1992 \\
\text { JUL } 07\end{array}$ & $\begin{array}{l}79.28 \mathrm{~S} \\
79.23 \mathrm{~s}\end{array}$ & $\begin{array}{l}\text { AUG } 06,1992 \\
\text { SEP 09 }\end{array}$ & $\begin{array}{l}79.36 \mathrm{~S} \\
79.45 \mathrm{~S}\end{array}$ \\
\hline
\end{tabular}

PERIOD OF RECORD HIGHEST 78.39 JUL 16, 1991 LOWEST 79.45 SEP 09, 1992 
WELL DESCRIPTIONS AND WATER-LEVEL MEASUREMENTS

WATER YEAR OCTOBER 1991 TO SEPTEMBER 1992

SITE NUMBER 345616117491003

LOCAL NUMBER 010N009W27C03S

On Edwards AFB. Drilled observation well. Diameter 2 inches, depth 80 feet, perforated 55-75 feet. Altitude of land-surface datum 2,273 feet. Shallowest of three cluster wells. Water-level records avallable 1991 to current year.

WATER LEVELS IN FEET BELOW LAND-SURFACE DATUM

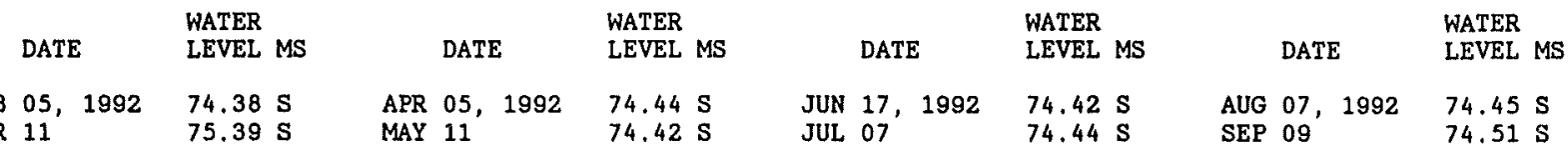

PERIOD OF RECORD HIGHEST 70.29 JUL 16, 1991 LOWEST 75.39 MAR 11, 1992

SITE NUMBER 345829118061301

LOCAL NUMBER O1ONO12W12KO1S

About 7 miles north of Rosamond Lake, north of Edwards AFB. Unused water-table well. Diameter 14 inches, depth measured 224 feet in 1978. Altitude of land-surface datum 2,520 feet. Water-level records available 1978 to current year.

WATER LEVELS IN FEET BELOW LAND-SURFACE DATUM

$$
\text { DATE WATER } \quad \text { LEVEL MS }
$$

APR 16, $1992 \quad 85.65 \mathrm{~S}$

PERIOD OF RECORD HIGHEST 81.43 MAR 25, 1985 LOWEST 85.65 APR 16, 1992

SITE NUMBER 345747118054301

LOCAL NUMBER 010N012W13H01S

On Edwards AFB about 6 miles north of Rosamond Lake. Drilled observation well. D1ameter 12 inches, depth 156 feet, 167.9 feet in 1992, perforated 75-147 feet. Altitude of land-surface datum 2,505 feet. Water-1evel records available 1956, 1967 to current year.

WATER LEVELS IN FEET BELOW LAND-SURFACE DATUM

$$
\text { DATE WATER } \quad \text { LEVEL MS }
$$

APR $16,199262.25 \mathrm{~s}$

PERIOD OF RECORD HIGHEST 51.76 NOV 08, 1956 LOWEST 63.24 FEB 04, 1975

SITE NUMBER 345701118103601

LOCAL NUMBER 010N012W20C06S

About 8 miles south of Mojave and 200 feet south of Backus Road. Domestic well. Diameter and depth unknown. Altitude of land-surface datum 2,655 feet. Water-level records available 1973-78, 1980 to current year.

WATER LEVELS IN FEET BELOW LAND-SURFACE DATUM

$$
\text { DATE WATER }
$$

APR 16, $1992108.70 \mathrm{~V}$

PERIOD OF RECORD HIGHEST 101.60 MAR 27, 1978 LOWEST 109.20 MAR 22, 1991 
WELL DESCRIPTIONS AND WATER-LEVEL MEASUREMENTS

WATER YEAR OCTOBER 1991 TO SEPTEMBER 1992

SITE NUMBER 345640118080301

LOCAL NUMBER 010N012W22J01S

On Edwards AFB about 8 miles south of Mojave. Drilled observation well. Diameter 12 inches, depth 240 feet, 230.55 feet in 1992, perforated 130-230 feet. Altitude of land-surface datum 2,530 feet. Water-level records available 1956, 1967 to current year.

WATER LEVELS IN FEET BELOW LAND-SURFACE DATUM

$$
\text { DATE WATER MS }
$$

APR 16, $1992 \quad 44.77 \mathrm{~S}$

PERIOD OF RECORD HIGHEST 33.00 MAY 18, 1956 LOWEST 44.77 APR 16, 1992

SITE NUMBER 345651118183201

LOCAL NUMBER 010N014W24A01S

About 5 miles northwest of Willow Springs, 1 mile west of Willow Springs Road, and 0.24 mile south of Backus Road. Unused well. Diameter 8 inches, depth unknown. Altitude of land-surface datum 2,945 feet. Water-level records available $1978-83,1985$ to current year.

WATER LEVELS IN FEET BELOW LAND-SURFACE DATUM

$$
\text { DATE NATER } \quad \text { LEVEL MS }
$$

APR 16, $1992345.50 \mathrm{~V}$

PERIOD OF RECORD HIGHEST 337.92 FEB 22, 1979 LOWEST 346.50 MAR 22, 1991

SITE NUMBER 350113117444801

LOCAL NUMBER 011N008W29K01S

About 5.5 miles west of Boron. Drilled unused water-table well. Diameter 12 inches, depth measured 485.37 feet in 1973, original depth 495 feet, perforated 96-495 feet. Altitude of land-surface datum 2,351,82 feet. Some measurements provided by U.S. Borax and Chemical Corporation 1955-73. Water-level records available 1955 to current year.

WATER LEVELS IN FEET BELOW LAND-SURFACE DATUM

$$
\begin{array}{ll} 
& \text { WATER } \\
\text { DATE } & \text { LEVEL MS }
\end{array}
$$

APR 14, $1992184.55 \mathrm{~S}$

PERIOD OF RECORD HIGHEST 138.96 JAN 24, 1957 LOWEST 184.55 APR 14, 1992

SITE NUMBER 350323117471901

LOCAL NUMBER 011N009W13D01S

About 4 miles northeast of North Edwards. Drilled unused water-table well. Diameter 12 inches, depth 312 feet, perforated 96-312 feet. Altitude of land-surface datum 2,375 feet. Measurements provided by U.S. Borax and Chemical Corporation 1956-73. Water-level records available 1956 to current year.

WATER LEVELS IN FEET BELOW LAND-SURFACE DATUM

$$
\text { DATE WATER } \quad \text { LEVEL MS }
$$

APR 14, $1992201.75 \mathrm{~S}$

PERIOD OF RECORD HIGHEST 157.47 DEC 26, 1956 LOWEST 202.65 MAR 19, 1991 
SITE NUMBER 350249117514301

LOCAL NUMBER 011N009W17N01S

About 3 miles northwest of North Edwards. Unused water-table well. Diameter 12 inches, depth measured 181.9 feet in 1991. Altitude of land-surface datum 2,319.93 feet. Some measurements provided by U.S. Borax and Chemical Corporation 1960-73. Water-level records avallable 1951-58, 1960 to current year.

WATER LEVELS IN FEET BELOW LAND-SURFACE DATUM

$$
\begin{array}{ll} 
& \text { WATER } \\
\text { DATE } & \text { LEVEL MS }
\end{array}
$$

APR 14, $1992156.57 \mathrm{~S}$

PERIOD OF RECORD HIGHEST 129.53 NOV 03, 1952 LOWEST 156.57 APR 14,1992

\section{SITE NUMBER 350232117463201}

LOCAL NUMBER O11N009W24A01S

About 2 miles northeast of North Edwards, 2.1 miles west of Gephardt Road, and 2.5 miles north of Highway 58 . Drilled unused water-table well. Diameter 16 inches, depth 888 feet, perforated 200-870 feet. Altitude of land-surface datum 2,348,77 feet. Water-level records available 1958, 1966-68, 1973 to current year.

WATER LEVELS IN FEET BELOW LAND-SURFACE DATUM

$$
\begin{array}{ll} 
& \text { WATER } \\
\text { DATE } & \text { LEVEL MS }
\end{array}
$$

APR 14, $1992174.62 \mathrm{~S}$

PERIOD OF RECORD HIGHEST 136.28 JAN 16, 1958 LOWEST 174.62 APR 14, 1992

SITE NUMBER 350114117514901

LOCAL NUMBER 011N009W30H01S

About 2 miles west of North Edwards, 0.85 mile north of Highway 58 , and 200 feet west of Castle Butte Road. Drilled unused water-table well. Diameter 10 inches, original depth 270 feet, depth measured 248.6 feet in 1973. Altitude of land-surface datum 2,298.31 feet. Some measurements provided by U.S. Borax and Chemical Corporation 1960-73. Water-level records available 1958 to current year.

WATER LEVELS IN FEET BELOW LAND-SURFACE DATUM

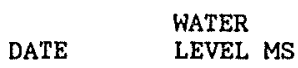

APR $14,1992118,74 \mathrm{~S}$

PERIOD OF RECORD HIGHEST 93.20 JAN 28, 1958 LOWEST 118.74 APR 14, 1992

\section{SITE NUMBER 350002117510301}

LOCAL NUMBER 011N009W32Q01S

On Edwards AFB. Drilled unused well. Diameter 16 inches, depth drilled 450 feet, perforated 234-450 feet. Altitude of land-surface datum 2,302.94 feet, surveyed in May 1989. Water-level records available 1957, 1959-60, $1966-71,1992$

WATER LEVELS IN FEET BELOW LAND-SURFACE DATUM

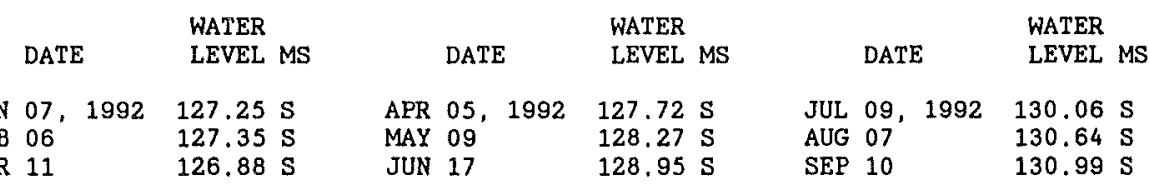

PERIOD OF RECORD HIGHEST 93.61 OCT 16,1957 LOWEST 130.99 SEP 10, 1992 
WELL DESCRIPTIONS AND WATER-LEVEL MEASUREMENTS

WATER YEAR OCTOBER 1991 TO SEPTEMBER 1992

SITE NUMBER 350002117463301

LOCAL NUMBER 011NOO9W36R01S

About 3 miles east of North Edwards, 1.5 miles southwest of intersection of Twenty Mule Team Road and Highway 58. Drilled unused water-table well. Diameter 10 inches, original depth 298 feet, depth measured 254.1 feet in 1991 , perforated 100-132 feet. Altitude of land-surface datum 2,311.95 feet. Some measurements provided by U.S. Borax and Chemical Corporation 1960-73. Water-level records available 1953-54, 1956, 1958 to current year.

WATER LEVELS IN FEET BELOW LAND-SURFACE DATUM

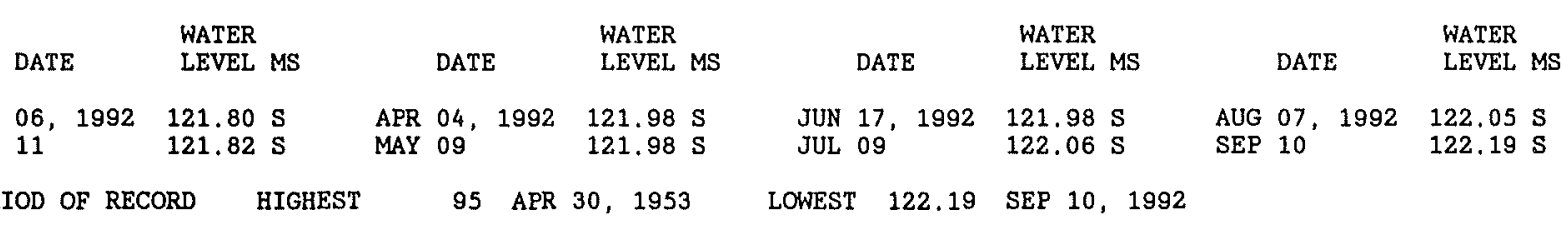

SITE NUMBER 350405117532201

LOCAL NUMBER 011N010W12F01S

About. 3 miles southeast of California City, 0.7 mile north of Twin Buttes Avenue, and 100 feet west of Jacinto Street. Drilled unused well. Diameter 8 inches, depth unknown. Altitude of land-surface datum 2,350 feet. Water-level records available 1967, 1973 to current year.

WATER LEVELS IN FEET BELOW LAND-SURFACE DATUM

$$
\begin{array}{ll} 
& \text { WATER } \\
\text { DATE } & \text { LEVEL MS }
\end{array}
$$

APR 14, $1992 \quad 187.91 \mathrm{~s}$

PERIOD OF RECORD HIGHEST 170.90 APR $25,1967 \quad$ LOWEST 187.91 APR 14,1992

SITE NUMBER 350411118044101

LOCAL NUMBER 011N011W07A01S

About 5 miles southwest of California City. Drilled test well. Diameter 14 inches, depth 414 feet, perforated 305-356 feet. Altitude of land-surface datum 2,627.9 feet. Water-level records available $1957-58$, 1967 to current year.

WATER LEVELS IN FEET BELOW LAND-SURFACE DATUM

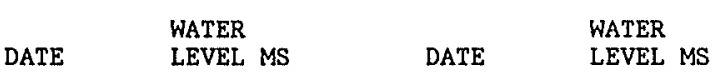

NOV $01,1991209.07 \mathrm{~S} \quad$ APR $14,1992 \quad 209.01 \mathrm{~s}$

PERIOD OF RECORD HIGHEST 201.63 FEB 03, 1958 LOWEST 209.09 MAR 20, 1991

SITE NUMBER 350411118023601

LOCAL NUMBER 011N011W09A01S

About 3 miles southwest of California City. Drilled unused water-table well. Diameter 14 inches, depth 422 feet, perforated 262-295, 352-362 feet. Altitude of land-surface datum 2,549.6 feet. Water-level records available $1956-58,1967$ to current year.

WATER LEVELS IN FEET BELOW LAND-SURFACE DATUM

$\begin{array}{lll} & \text { WATER } & \text { WATER } \\ \text { DATE } & \text { LEVEL MS } & \text { DATE } \\ \text { LEVEL MS }\end{array}$

NOV $01,1991 \quad 131.83 \mathrm{~S} \quad$ APR $14,1992131.82 \mathrm{~S}$

PERIOD OF RECORD HIGHEST 124.59 OCT 17,1956 LOWEST 131.83 NOV 01,1991 
SITE NUMBER 350212118083701

LOCAL NUMBER 011N012W22FO2S

About 2.3 miles southeast of Mojave, $0.45 \mathrm{mile}$ south of Highway 58 . Drilled unused water-table well. Diameter 12 inches, depth 346 feet, perforated 244-346 feet. Altitude of land-surface datum 2,663 feet. Water-level records available 1968, 1974, 1976 to current year.

WATER LEVELS IN FEET BELOW LAND-SURFACE DATUM

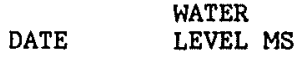

APR 16, $1992 \quad 244.84 \mathrm{~S}$

PERIOD OF RECORD HIGHEST 244.84 APR 16,1992 LOWEST 260.50 JUN 03,1974

SITE NUMBER 350214118180701

LOCAL NUMBER 011N013W19C01S

About 7 miles west of Mojave near Oak Creek. Unused water-table well. Diameter 16 inches, depth 430 feet, perforated 230-358, 376-430 feet. Altitude of land-suface datum 3,610 feet. Water-level records available 1953 , 1955-56, 1972 to current year.

WATER LEVELS IN FEET BELOW LAND-SURFACE DATUM

$$
\begin{array}{lll} 
& \text { WATER } & \\
\text { DATE } & \text { WEVEL MS }
\end{array}
$$

OCT $21,1991354.18 \mathrm{~S} \quad$ APR $16,1992356.53 \mathrm{~s}$

PERIOD OF RECORD HIGHEST 180.88 OCT 27,1983 LOWEST 356.53 APR 16, 1992

SITE NUMBER 350055118172601

LOCAL NUMBER 011N013W29M01S

About 7 miles west of Mojave. Drilled unused water-table well in alluvium. Diameter 16 inches, depth 744 feet, perforated 520-724 feet. Altitude of land-surface datum 3,350 feet. Water-level records available $1954-56$, 1972 to current year.

WATER LEVELS IN FEET BELOW LAND-SURFACE DATUM

$\begin{array}{lll} & \text { WATER } & \\ \text { DATE } & \text { WETER } \\ & \text { LEVE } & \text { LEVEL MS }\end{array}$

OCT $21,1991330.24 \mathrm{~S} \quad$ APR $16,1992 \quad 332.57 \mathrm{~S}$

PERIOD OF RECORD HIGHEST 278,40 NOV 01, 1984 LOWEST 336.19 OCT 17,1978

SITE NUMBER 350505118065001

LOCAL NUMBER 012N012W35R01S

About 4 miles northeast of Mojave. Drilled unused well. Diameter 14 inches, depth 590 feet, perforated 350-590 feet. Altitude of land-surface datum 2,743.3 feet. Water-level records available $1957-58$, 1967 to current year.

WATER LEVELS IN FEET BELOW LAND-SURFACE DATUM

$$
\begin{array}{ll} 
& \text { WATER } \\
\text { DATE } & \text { LEVEL MS }
\end{array}
$$

APR $14,1992 \quad 323.93 \mathrm{~S}$

PERIOD OF RECORD HIGHEST 316.11 JUL 17, AUG 14, 1957 LOWEST 324.08 MAR 13, 1990 
INORGANIC DATA

WATER YEAR OCTOBER 1991 TO SEPTEMBER 1992

LOCAL
NUMBER
026S039E13R03M
026S040E23B02M
026S040E23B03M
026S040E33P04M
026S040E34N01M
026S040E35H01M
026S040E35HO2M
027S040E02G03M
029S039E23J02M
030S037E24J03M
030S037E24M02M
030S037E34H02M
030S038E04D02M
030S038E32D03M
009N015W26N01S
010N012W15M03S
010N013W32D01S

\begin{tabular}{|c|c|c|c|c|c|c|c|c|c|}
\hline DATE & TIME & $\begin{array}{l}\text { DEPTH } \\
\text { BELOW } \\
\text { LAND } \\
\text { SURFACE } \\
\text { (WATER } \\
\text { LEVEL) } \\
\text { (FEET) }\end{array}$ & $\begin{array}{l}\text { DEPTH } \\
\text { OF } \\
\text { WELL, } \\
\text { TOTAL } \\
\text { (FEET) }\end{array}$ & $\begin{array}{l}\text { SPE- } \\
\text { CIFIC } \\
\text { CON- } \\
\text { DUCT- } \\
\text { ANCE } \\
\text { (US/CM) }\end{array}$ & $\begin{array}{c}\text { PH } \\
\text { WATER } \\
\text { WHOLE } \\
\text { FIELD } \\
\text { (STAND- } \\
\text { ARD } \\
\text { UNITS) }\end{array}$ & $\begin{array}{l}\text { TEMPER- } \\
\text { ATURE } \\
\text { AIR } \\
\text { (DEG C) }\end{array}$ & $\begin{array}{c}\text { TEMPER- } \\
\text { ATURE } \\
\text { WATER } \\
\text { (DEG C) }\end{array}$ & $\begin{array}{l}\text { HARD- } \\
\text { NESS } \\
\text { TOTAL } \\
\text { (MG /L } \\
\text { AS } \\
\text { CACO3) }\end{array}$ & $\begin{array}{l}\text { CALCIUM } \\
\text { DIS- } \\
\text { SOLVED } \\
\text { (MG/L } \\
\text { AS CA) }\end{array}$ \\
\hline $\begin{array}{l}04-16-92 \\
04-16-92 \\
04-16-92 \\
04-17-92 \\
04-17-92\end{array}$ & $\begin{array}{l}1250 \\
0830 \\
0945 \\
1445 \\
1330\end{array}$ & $\begin{array}{r}136.41 \\
26.08 \\
22.82 \\
176.30 \\
\ldots\end{array}$ & $\begin{array}{l}300 \\
360 \\
240 \\
304 \\
232\end{array}$ & $\begin{array}{r}343 \\
2230 \\
2330 \\
1608 \\
1786\end{array}$ & $\begin{array}{r}7.9 \\
8.3 \\
8.7 \\
17.9 \\
18.2\end{array}$ & $\begin{array}{l}26.0 \\
18.0 \\
20.0 \\
-- \\
--\end{array}$ & $\begin{array}{l}24.5 \\
22.0 \\
22.0 \\
-- \\
--\end{array}$ & $\begin{array}{r}96 \\
47 \\
49 \\
39 \\
140\end{array}$ & $\begin{array}{c}30 \\
9.8 \\
9.3 \\
12 \\
36\end{array}$ \\
\hline $\begin{array}{l}04-17-92 \\
04-17-92 \\
04-16-92 \\
08-06-92 \\
08-06-92\end{array}$ & $\begin{array}{l}0845 \\
1100 \\
1810 \\
0920 \\
1000\end{array}$ & $\begin{array}{c}72.54 \\
82.98 \\
88.02 \\
-- \\
--\end{array}$ & $\begin{array}{l}150 \\
500 \\
-- \\
600 \\
250\end{array}$ & $\begin{array}{r}1450 \\
498 \\
2020 \\
673 \\
874\end{array}$ & $\begin{array}{r}7.5 \\
8.3 \\
8.1 \\
-- \\
--\end{array}$ & $\begin{array}{l}22.0 \\
25.0 \\
31.0 \\
-- \\
--\end{array}$ & $\begin{array}{l}29.0 \\
29.0 \\
23.0 \\
26.5 \\
21.0\end{array}$ & $\begin{array}{r}380 \\
49 \\
150 \\
-- \\
--\end{array}$ & $\begin{array}{r}110 \\
14 \\
42 \\
-- \\
--\end{array}$ \\
\hline $\begin{array}{l}08-06-92 \\
08-06-92 \\
08-06-92 \\
08-06-92 \\
08-05-92\end{array}$ & $\begin{array}{l}1040 \\
1115 \\
1245 \\
1335 \\
0835\end{array}$ & $\begin{array}{l}-- \\
-- \\
-- \\
--\end{array}$ & $\begin{array}{l}615 \\
456 \\
300 \\
250 \\
600\end{array}$ & $\begin{array}{r}770 \\
758 \\
4560 \\
1880 \\
368\end{array}$ & $\begin{array}{l}-- \\
-- \\
-- \\
- \\
8.0\end{array}$ & $\begin{array}{l}-- \\
-- \\
-- \\
--\end{array}$ & $\begin{array}{l}28.0 \\
24.0 \\
35.0 \\
23.0 \\
24.5\end{array}$ & $\begin{array}{l}-- \\
-- \\
-- \\
-- \\
130\end{array}$ & $\begin{array}{l}-- \\
-- \\
-- \\
45\end{array}$ \\
\hline $\begin{array}{l}08-05-92 \\
08-05-92\end{array}$ & $\begin{array}{l}1505 \\
1055\end{array}$ & -- & $\begin{array}{l}175 \\
900\end{array}$ & $\begin{array}{l}365 \\
662\end{array}$ & $\begin{array}{l}8.0 \\
7.7\end{array}$ & -- & $\begin{array}{l}23.0 \\
23.5\end{array}$ & $\begin{array}{r}90 \\
210\end{array}$ & $\begin{array}{l}26 \\
62\end{array}$ \\
\hline
\end{tabular}

\section{LOCAL \\ NUMBER}

026SO39E13R03M 026SO 40E23BO2M 026S0 40E23B03M 026S040E33P0 4M 026S040E34NO1M

026S040E35HO1M 026SO $40 \mathrm{E} 35 \mathrm{HO} \mathrm{2M}$ $027 \mathrm{~S} 040 \mathrm{E} 02 \mathrm{GO} \mathrm{M}$ 029S039E23JO2M 030S037E24 J03M

030S037E24M02M 030S037E34H02M 030S038E04D02M 030 S038E32D03M 009N015W26N01S

$010 \mathrm{~N} 012 \mathrm{~W} 15 \mathrm{M} 03 \mathrm{~S}$ 010N013W32D01S
MAGNE-

SIUM, SODIUM,

DIS- DIS-

SOLVED SOLVED

(MG/L (MG/L

DATE

AS MG)

AS NA)

$\begin{array}{lcc}04-16-92 & 5.2 & 32 \\ 04-16-92 & 5.5 & 460 \\ 04-16-92 & 6.2 & 490 \\ 04-17-92 & 2.1 & 130 \\ 04-17-92 & 11 & 100 \\ 04-17-92 & 26 & 150 \\ 04-17-92 & 3.3 & 81 \\ 04-16-92 & 12 & 340 \\ 08-06-92 & -- & -- \\ 08-06-92 & -- & -- \\ 08-06-92 & -- & -- \\ 08-06-92 & -- & -- \\ 08-06-92 & -- & -- \\ 08-06-92 & -- & -- \\ 08-05-92 & 4.6 & 28 \\ 08-05-92 & 6.0 & 41 \\ 08-05-92 & 13 & 54\end{array}$

$\begin{array}{cccccc} & & \text { BICAR- } & \text { ALKA- } & & \\ \text { SODIUM } & \text { POTAS- } & \text { BONATE } & \text { LINITY } & & \text { CHLO- } \\ \text { AD- } & \text { SIUM, } & \text { WATER } & \text { WAT WH } & \text { SULFATE } & \text { RIDE, } \\ \text { SORP- } & \text { DIS- } & \text { WH IT } & \text { TOT FET } & \text { DIS- } & \text { DIS- } \\ \text { TION } & \text { SOLVED } & \text { FIELD } & \text { FIELD } & \text { SOLVED } & \text { SOLVED } \\ \text { RATIO } & (M G / L & \text { MG/L AS } & \text { MG/L AS } & \text { (MG/L } & \text { (MG/L } \\ & \text { AS K) } & \text { HCO3 } & \text { CACO3 } & \text { AS SO4) } & \text { AS CL) }\end{array}$

$\begin{array}{rrclrrr}41 & 1 & 3.0 & -- & 116 & 18 & 29 \\ 94 & 29 & 12 & -- & 458 & 97 & 410 \\ 94 & 31 & 14 & -- & 566 & 150 & 320 \\ 87 & 9 & 3.7 & -- & 177 & 36 & 140 \\ 61 & 4 & 5.4 & -- & 189 & 63 & 150 \\ & & & & & & \\ 45 & 3 & 9.0 & -- & 187 & 210 & 220 \\ 74 & 5 & 11 & -- & 162 & 6.3 & 64 \\ 81 & 12 & 11 & -- & 126 & 100 & 480 \\ -- & -- & -- & -- & -- & -- & - \\ -- & -- & -- & -- & -- & -- & -- \\ -- & -- & -- & -- & -- & -- & -- \\ -- & -- & -- & -- & -- & -- & - \\ -- & -- & -- & -- & -- & -- & -- \\ -- & -- & -- & - & -- & -- & - \\ 31 & 1 & 2.3 & 189 & -- & 14 & 11 \\ 49 & 2 & 2.2 & 127 & -- & 61 & 18 \\ 36 & 2 & 2.2 & 228 & -- & 82 & 45\end{array}$

L Laboratory value. 


LOCAL
NUMBER
026S039E13R03M
026S040E23B02M
026S040E23B03M
026S040E33PO4M
026S040E34N01M
026S040E35H01M
026S040E35H02M
027S040E02G03M
029S039E23J02M
030S037E24J03M
030S037E24MO2M
030S037E34HO2M
030S038E04DO2M
030S038E32D03M
009N015W26N01S
010N012W15M03S
010N013W32D01S

010N013W32D01S

LOCAL
NUMBER
026S039E13R03M
026S040E23B02M
026S040E23B03M
026S040E33P04M
026S040E34N01M
026S040E35H01M
026S040E35H02M
027S040E02G03M
029S039E23J02M
030S037E24J03M
030S037E24M02M
030S037E34H02M
030S038E04D02M
030S038E32D03M
009N015W26N01S
010N012W15M03S
010N013W32D01S

\begin{tabular}{|c|c|c|c|c|c|c|c|c|}
\hline DATE & $\begin{array}{l}\text { FLUO- } \\
\text { RIDE, } \\
\text { DIS- } \\
\text { SOLVED } \\
\text { (MG/L } \\
\text { AS F) }\end{array}$ & $\begin{array}{l}\text { SILICA, } \\
\text { DIS- } \\
\text { SOLVED } \\
\text { (MG/L } \\
\text { AS } \\
\text { SIO2) }\end{array}$ & $\begin{array}{l}\text { SOLIDS, } \\
\text { RESIDUE } \\
\text { AT } 180 \\
\text { DEG. C } \\
\text { DIS- } \\
\text { SOLVED } \\
\text { (MG/L) }\end{array}$ & $\begin{array}{c}\text { SOLIDS, } \\
\text { SUM OF } \\
\text { CONSTI- } \\
\text { TUENTS, } \\
\text { DIS- } \\
\text { SOLVED } \\
\text { (MG/L) }\end{array}$ & $\begin{array}{c}\text { NITRO- } \\
\text { GEN, } \\
\text { NITRITE } \\
\text { DIS- } \\
\text { SOLVED } \\
\text { (MG/L } \\
\text { AS N) }\end{array}$ & $\begin{array}{l}\text { NITRO- } \\
\text { GEN } \\
\text { NO2+NO3 } \\
\text { DIS- } \\
\text { SOLVED } \\
\text { (MG/L } \\
\text { AS N) }\end{array}$ & $\begin{array}{l}\text { NITRO- } \\
\text { GEN, } \\
\text { AMMONIA } \\
\text { DIS- } \\
\text { SOLVED } \\
\text { (MG/L } \\
\text { AS N) }\end{array}$ & $\begin{array}{c}\text { NITRO- } \\
\text { GEN, } \\
\text { AMMONIA } \\
\text { DIS- } \\
\text { SOLVED } \\
\text { (MG/L } \\
\text { AS NH4) }\end{array}$ \\
\hline $\begin{array}{l}04-16-92 \\
04-16-92 \\
04-16-92 \\
04-17-92 \\
04-17-92\end{array}$ & $\begin{array}{l}0.60 \\
1.9 \\
2.4 \\
0.90 \\
0.60\end{array}$ & $\begin{array}{l}46 \\
60 \\
53 \\
39 \\
38\end{array}$ & $\begin{array}{l}-- \\
-- \\
-- \\
--\end{array}$ & $\begin{array}{r}234 \\
1340 \\
1390 \\
420 \\
463\end{array}$ & $\begin{array}{l}<0.010 \\
<0.010 \\
<0.010 \\
<0.010 \\
<0.010\end{array}$ & $\begin{array}{c}<0.050 \\
<0.050 \\
<0.050 \\
2.00 \\
1.20\end{array}$ & $\begin{array}{l}0.020 \\
0.930 \\
0.860 \\
0.020 \\
0.020\end{array}$ & $\begin{array}{l}0.03 \\
1.2 \\
1.1 \\
0.03 \\
0.03\end{array}$ \\
\hline $\begin{array}{l}04-17-92 \\
04-17-92 \\
04-16-92 \\
08-06-92 \\
08-06-92\end{array}$ & $\begin{array}{l}0.40 \\
1.3 \\
1.3 \\
-- \\
-.\end{array}$ & $\begin{array}{l}58 \\
60 \\
60 \\
-- \\
--\end{array}$ & $\begin{array}{l}-- \\
-- \\
-- \\
--\end{array}$ & $\begin{array}{l}906 \\
339 \\
1120 \\
-- \\
--\end{array}$ & $\begin{array}{c}<0.010 \\
<0.010 \\
<0.010 \\
-- \\
--\end{array}$ & $\begin{array}{c}1.90 \\
<0.050 \\
<0.050 \\
-- \\
--\end{array}$ & $\begin{array}{l}0.020 \\
0.380 \\
0.040 \\
-- \\
--\end{array}$ & $\begin{array}{l}0.03 \\
0.49 \\
0.05 \\
-- \\
--\end{array}$ \\
\hline $\begin{array}{l}08-06-92 \\
08-06-92 \\
08-06-92 \\
08-06-92 \\
08-05-92\end{array}$ & $\begin{array}{l}-- \\
-- \\
-- \\
-- \\
0.20\end{array}$ & $\begin{array}{l}-- \\
-- \\
-- \\
-- \\
27\end{array}$ & $\begin{array}{l}-- \\
-- \\
-- \\
-- \\
220\end{array}$ & $\begin{array}{l}-- \\
-- \\
-- \\
-- \\
234\end{array}$ & $\begin{array}{c}-- \\
- \\
-- \\
-- \\
<0.010\end{array}$ & $\begin{array}{l}-- \\
-- \\
-- \\
-- \\
2.10\end{array}$ & $\begin{array}{l}-- \\
-- \\
-- \\
-- \\
0.010\end{array}$ & $\begin{array}{l}-- \\
-- \\
-- \\
-- \\
0.01\end{array}$ \\
\hline $\begin{array}{l}08-05-92 \\
08-05-92\end{array}$ & $\begin{array}{l}0.30 \\
0.20\end{array}$ & $\begin{array}{l}65 \\
24\end{array}$ & $\begin{array}{l}273 \\
386\end{array}$ & $\begin{array}{l}285 \\
408\end{array}$ & $\begin{array}{l}<0.010 \\
<0.010\end{array}$ & $\begin{array}{l}0.670 \\
3.10\end{array}$ & $\begin{array}{l}0.020 \\
0.010\end{array}$ & $\begin{array}{l}0.03 \\
0.01\end{array}$ \\
\hline
\end{tabular}

< Actual value is less than value shown. 

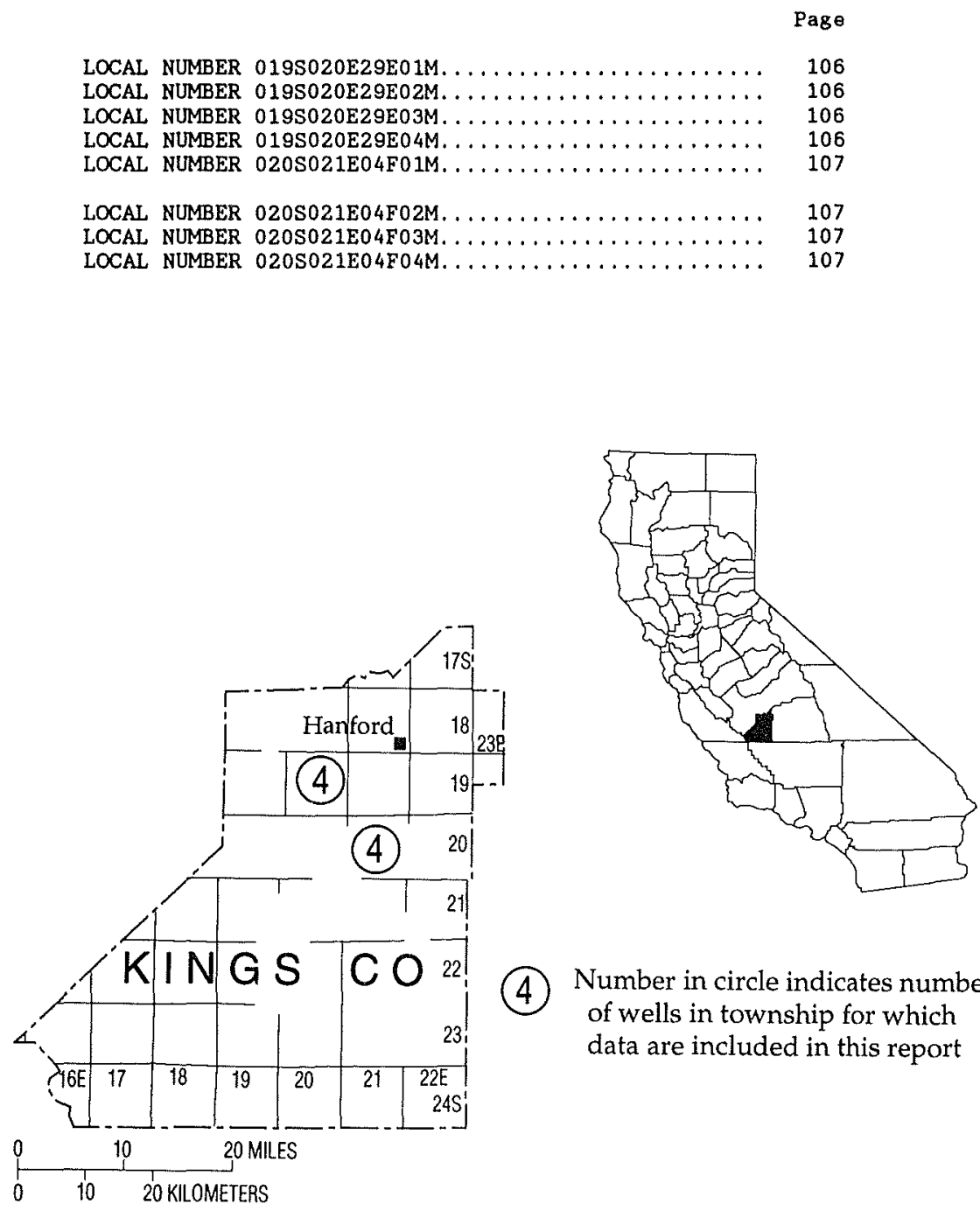

(4) Number in circle indicates number of wells in township for which data are included in this report

Figure 13. Location of wells in Kings County. 
SITE NUMBER 361455119495701

LOCAL NUMBER O19S020E29E01M

About 4 miles southwest of Lemoore. Drilled observation well. Diameter 2 inches, depth 20 feet, screened 10-20 feet. Altitude of land-surface datum 208 feet. Water-level records available 1990 to current year.

WATER LEVELS IN FEET BELOW LAND-SURFACE DATUM

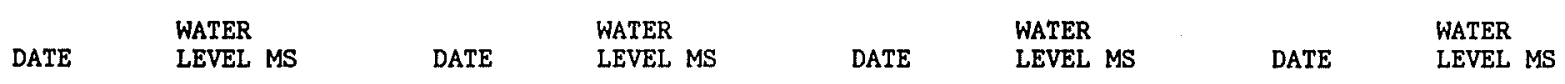

$\begin{array}{lllllll}\text { OCT } & 08,1991 & 6.88 \mathrm{~S} & \text { JAN } 02,1992 & 6.97 \mathrm{~S} \\ \text { NOV } & 05 & & 7.28 \mathrm{~S} & & 31 & 6.25 \mathrm{~S} \\ \text { DEC } 02 & & 7.37 \mathrm{~S} & \text { MAR } 06 & & 5.28 \mathrm{M}\end{array}$

DEC $02-7.37 \mathrm{~s}$

MAR $06 \quad 5.28 \mathrm{M}$

HIGHEST

4.52 JUL 01, 1992

APR 03,1992
30
JUN 03

$\begin{array}{ll}5.38 & \mathrm{~s} \\ 5.21 & \mathrm{~S} \\ 5.87 & \mathrm{~S}\end{array}$

JUL 01, 1992

$4.52 \mathrm{~S}$

SITE NUMBER 361455119495702

LOCAL NUMBER 019S020E29E02M

About 4 miles southwest of Lemoore. Drilled observation well. Diameter 2 inches, depth 60 feet, screened 45-55 feet. Altitude of land-surface datum 208 feet. Water-level records available 1990 to current year.

WATER LEVELS IN FEET BELOW LAND-SURFACE DATUM

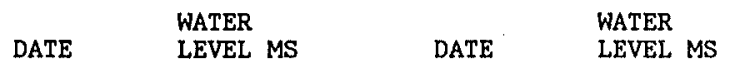

OCT 08, 1991

NOV 05

DEC 02

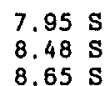

$8.48 \mathrm{~S}$

JAN 02, 1992

MAR 06
DATE

$8.33 \mathrm{~s}$

$7.63 \mathrm{~S}$

$6.32 \mathrm{~s}$

LOWEST

9.25 AUG 09,1990

$5.99 \mathrm{~s}$

PERIOD OF RECORD HIGHEST 5.14 JUL 02, 1991 LOWEST 8.65 DEC 02, 1991

SITE NUMBER 361455119495703

LOCAL NUMBER 019S020E29E03M

About 4 miles southwest of Lemoore. Drilled observation well. Diameter 2 inches, depth 90 feet, screened 75-85 feet. Altitude of land-surface datum 208 feet. Water-ievel records available 1990 to current year.

WATER LEVELS IN FEET BELOW LAND-SURFACE DATUM

\begin{tabular}{|c|c|c|c|c|c|c|c|}
\hline DATE & $\begin{array}{l}\text { WATER } \\
\text { LEVEL MS }\end{array}$ & DATE & $\begin{array}{l}\text { WATER } \\
\text { LEVEL MS }\end{array}$ & DATE & $\begin{array}{l}\text { WATER } \\
\text { LEVEL MS }\end{array}$ & DATE & $\begin{array}{l}\text { WATER } \\
\text { LEVEL MS }\end{array}$ \\
\hline $\begin{array}{ll}08,1991 \\
05 & \\
02 & \end{array}$ & $\begin{array}{l}17.73 \mathrm{~S} \\
18.19 \mathrm{~S} \\
18.45 \mathrm{~S}\end{array}$ & $\begin{array}{lll}\text { JAN } 02, & 1992 \\
& 31 & \\
\text { MAR } & 06 & \end{array}$ & $\begin{array}{l}18.35 \mathrm{~S} \\
18.05 \mathrm{~S} \\
17.24 \mathrm{~S}\end{array}$ & $\begin{array}{lll}\text { APR } & 03,1992 \\
30 & \\
\text { JUN } 03 & \end{array}$ & $\begin{array}{l}17.26 \mathrm{~s} \\
17.23 \mathrm{~s} \\
17.60 \mathrm{~s}\end{array}$ & $\begin{array}{lll}\text { JUL } & 01,1992 \\
\text { AUG } & 04 & \\
\text { SEP } & 09\end{array}$ & $\begin{array}{l}17.06 \mathrm{~S} \\
17.19 \mathrm{~S} \\
17.69 \mathrm{~s}\end{array}$ \\
\hline
\end{tabular}

PERIOD OF RECORD HIGHEST 16.91 JUL 24,1991 LOWEST 18.45 DEC 02, 1991

SITE NUMBER 361455119495704

LOCAL NUMBER 019S020E29E04M

About 4 miles southwest of Lemoore. Drilled observation well. Diameter 3 inches, depth 200 feet, screened 185-195 feet. Altitude of land-surface datum 208 feet. Water-level records available 1990 to current year.

WATER LEVELS IN FEET BELOW LAND-SURFACE DATUM

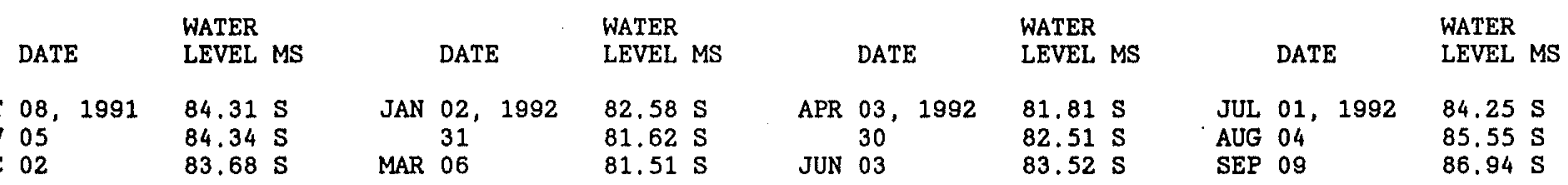

\footnotetext{
PERIOD OF RECORD HIGHEST 78.35 JAN 31, $1991 \quad$ LOWEST 86.94 SEP 09, 1992
} 
WELL DESCRIPTIONS AND WATER-LEVEL MEASUREMENTS

WATER YEAR OCTOBER 1991 TO SEPTEMBER 1992

SITE NUMBER 361307119422001

LOCAL NUMBER 020S021E04F01M

About 8 miles southwest of Hanford. Drilled observation well. Diameter 2 inches, depth 20 feet, screened 10-20 feet. Altitude of land-surface datum 326 feet. Water-level records available 1990 to current year. WATER LEVELS IN FEET BELOW LAND-SURFACE DATUM

$\begin{array}{lll} & \text { WATER } & \\ \text { DATE } & \text { WEVEL MS } & \text { DATE } \\ \text { LEVEL MS }\end{array}$

OCT $08,1991 \quad 10.17 \mathrm{~s}$

NOV $05 \quad 10.44 \mathrm{~S}$

DEC $02 \quad 10.63 \mathrm{~s}$

PERIOD OF RECORD HIGHEST
10.76

$10.71 \mathrm{~s}$

$\begin{array}{ll}02,1992 & 10.76 \mathrm{~S} \\ 31 & 10.83 \mathrm{~S} \\ 06 & 10.71 \mathrm{~S}\end{array}$

8.47 APR 08, 1991 $\begin{array}{ll} & \text { WATER } \\ \text { DATE } & \text { LEVEL MS }\end{array}$

APR 03, 1992

JUN 03
$10.84 \mathrm{~S}$

$11.04 \mathrm{~S}$
WATER

DATE LEVEL MS

JUL 01, $199211.19 \mathrm{~s}$

AUG $04 \quad 11.25 \mathrm{~S}$

SEP $09 \quad 11.38 \mathrm{~S}$

SITE NUMBER 361307119422002

LOCAL NUMBER 020S021E04F02M

About 8 miles southwest of Hanford. Drilled observation well. Diameter 3 inches, depth 205 feet, screened 190-200 feet. Altitude of land-surface datum 326 feet. Water-level records avaliable 1990 to current year.

WATER LEVELS IN FEET BELOW LAND-SURFACE DATUM

$\begin{array}{llllll} & \text { WATER } & & \text { WATER } & \text { WATER } & \text { WATER } \\ \text { DATE } & \text { LEVEL MS } & \text { DATE } & \text { LEVEL MS } & \text { DATE } & \text { LEVEL MS }\end{array}$

OCT $08,1991 \quad 55.71 \mathrm{~S}$

NOV $05,1991 \quad 54.43$

DEC $02 \quad 52.63 \mathrm{~S}$

$\begin{array}{lll}\text { JAN } 02,1992 & 51.40 \mathrm{~S} \\ & 31 & 50.33 \mathrm{~S} \\ \text { MAR } 06 & & 49.60 \mathrm{~S}\end{array}$

APR 03, 1992

$51.04 \mathrm{~S}$

JUN 03

$51.82 \mathrm{~S}$

JUL 01,1992

$55.47 \mathrm{~S}$

PERIOD OF RECORD HIGHEST

47.05 JAN 31, 1991

LOWEST 58.64 SEP 09, 1992

SITE NUMBER 361307119422003

LOCAL NUMBER 020S021E04F03M

About 8 miles southwest of Hanford. Drilled observation well. Diameter 2 inches, depth 108 feet, perforated 93-103 feet. Altitude of land-surface datum 326 feet. Water-level records available 1991 to'current year.

WATER LEVELS IN FEET BELOW LAND-SURFACE DATUM

\begin{tabular}{|c|c|c|c|c|c|c|c|}
\hline DATE & $\begin{array}{l}\text { WATER } \\
\text { LEVEL MS }\end{array}$ & DATE & $\begin{array}{l}\text { WATER } \\
\text { LEVEL MS }\end{array}$ & DATE & $\begin{array}{l}\text { WATER } \\
\text { LEVEL MS }\end{array}$ & DATE & $\begin{array}{l}\text { WATER } \\
\text { LEVEL MS }\end{array}$ \\
\hline
\end{tabular}

OCT $08,1991 \quad 27.72$

NOV 05 , 29.22

DEC $02 \quad 28.46 \mathrm{~S}$

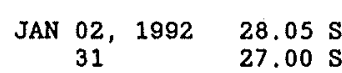

APR 03, $1992 \quad 27.36 \mathrm{~S}$

$\begin{array}{ll}30 & 28.14 \mathrm{~S} \\ \text { JUN } 03 & 29.70 \mathrm{~S}\end{array}$

JUL 01, 1992

$30.28 \mathrm{~S}$

MAR $06 \quad 26.77 \mathrm{~S}$

PERIOD OF RECORD HIGHEST 26.77 MAR 06, 1992 LOWEST 31.89 AUG 04, 1992

SITE NUMBER 361307119422004

LOCAL NUMBER 020S021E04F04M

About 8 miles southwest of Hanford. Drilled observation well. Diameter 2 inches, depth 63 feet, perforated 45-50 feet. Altitude of land-surface datum 326 feet. Water-level records available 1991 to current year.

WATER LEVELS IN FEET BELOW LAND-SURFACE DATUM

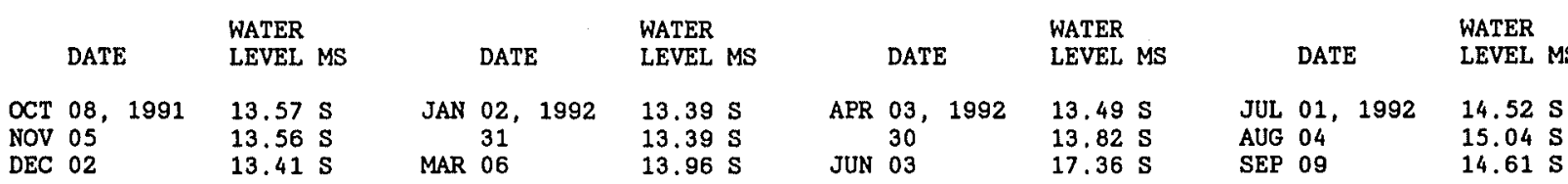

PERIOD OF RECORD HIGHEST 13.39 JAN 02, 31, 1992 LOWEST 17.36 JUN 03, 1992 
LOS ANGELES COUNTY

Page

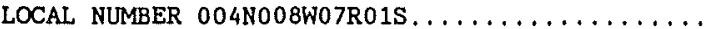

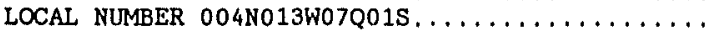

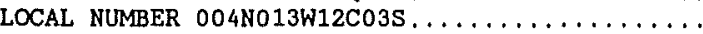

LOCAL NUMBER 004N013W13L01S . . . . . . . . . .

LOCAL NUMBER 004N013W15A01S............

LOCAL NUMBER 005N009W05E02S.............

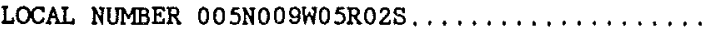

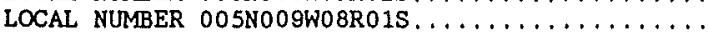

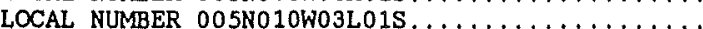

LOCAL NUMBER 005NO10W06N01S..............

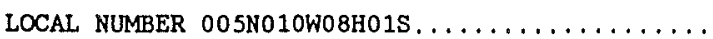

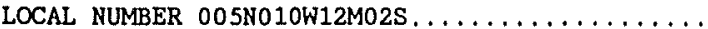
LOCAL NUMBER O05NO10W17LO1S, ..............

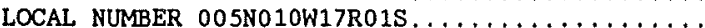
LOCAL NUMBER 005NO11W01M01S

LOCAL NUMBER 005N011W12R01S. . . . . . . . . . . . LOCAL NUMBER 005N012W32M01S . . . . . . . . . .

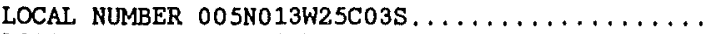
LOCAL NUMBER 006N008W09Q01S, ............ LOCAL NUMBER 006N008W19E01S.

LOCAL NUMBER 006N009W04H02S.............. LOCAL NUMBER 006N009W07J01S.............. LOCAL NUMBER O06N009W11N01S..............

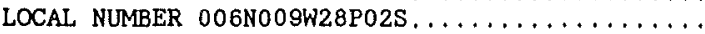
LOCAL NUMBER O06NO09W30F01S.............

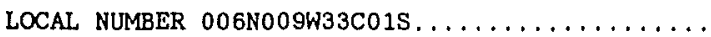

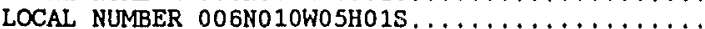
LOCAL NUMBER 006N010W17N01S . . . . . . . . . .

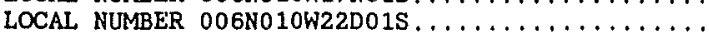

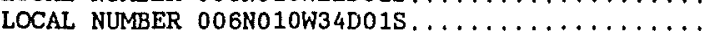

LOCAL NUMBER 006N010W35A01S.............

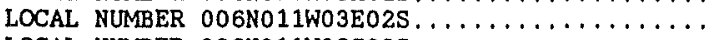

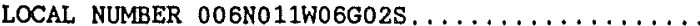
LOCAL NUMBER 006N011W09P01S..............

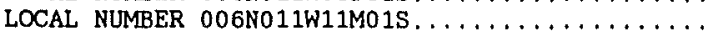

LOCAL NUMBER 006N011W16J01S. . . . . . . . . .

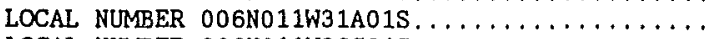

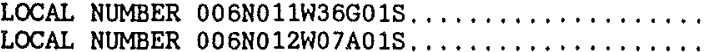
LOCAL NUMBER 006N012W07A01S $\ldots \ldots \ldots \ldots \ldots \ldots \ldots$

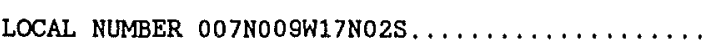

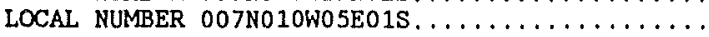

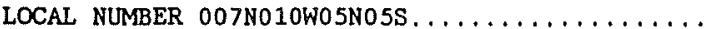
LOCAL NUMBER 007N010W19D01S,.............

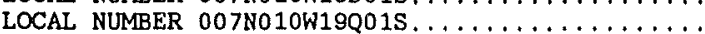

LOCAL NUMBER 007N010W22P01S..............

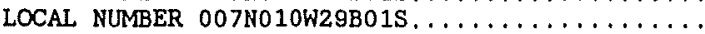
LOCAL NUMBER 007N010W29P01S.............. LOCAL NUMBER 007N010W32B01S..............

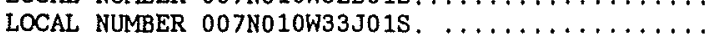

LOCAL NUMBER D07N011W01Q01S.............

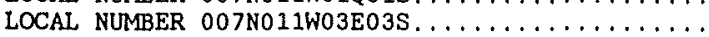

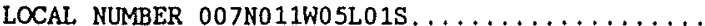

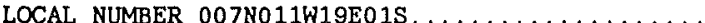
LOCAL NUMBER $007 N 011 W 21 E 01 S \ldots \ldots \ldots \ldots \ldots \ldots \ldots$

LOCAL NUMBER 007N011W27Q01S LOCAL NUMBER $007 N 011 W 28001 \mathrm{~S} \ldots \ldots \ldots \ldots \ldots \ldots \ldots$

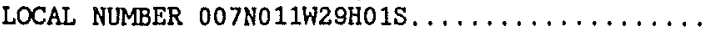

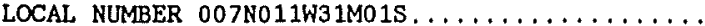
LOCAL NUMBER 007 N011W33N01S..............

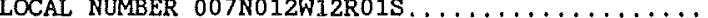

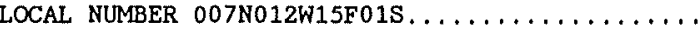

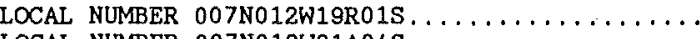

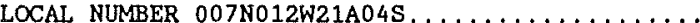

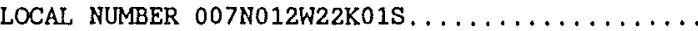

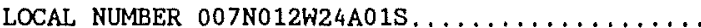

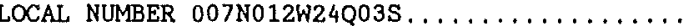

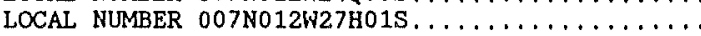

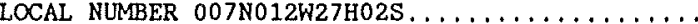

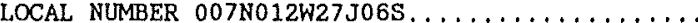

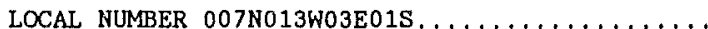

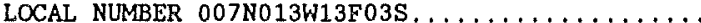

LOCAL NUMBER $007 N 013 W 13 N 015 \ldots \ldots \ldots \ldots \ldots \ldots \ldots$

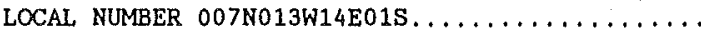

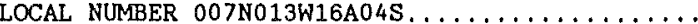

LOCAL NUMBER 007N013W19D01S. . . . . . . . . .

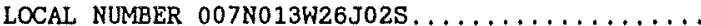

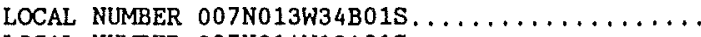

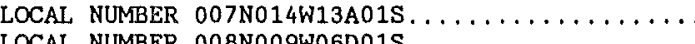

1s....................

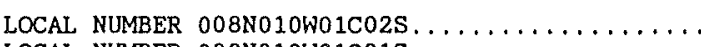

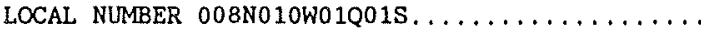

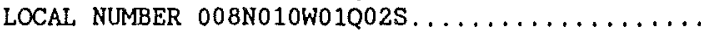

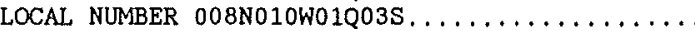

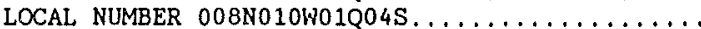

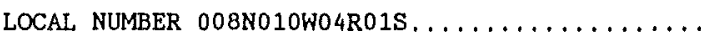

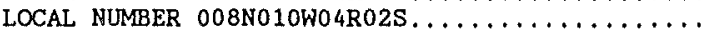

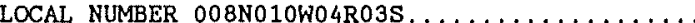

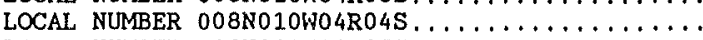

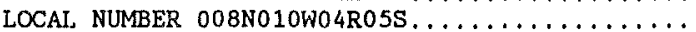

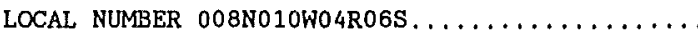

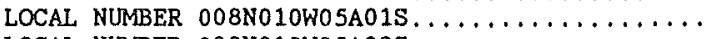

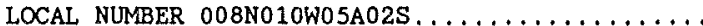

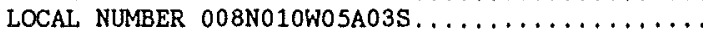

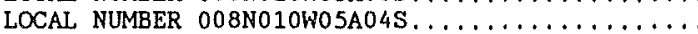

LOCAL NUMBER 008N010W05A06S..............

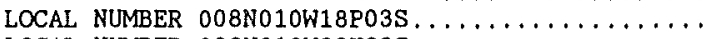
LOCAL NUMBER 008N010W22H03S............. LOCAL NUMBER 008N010W22P03S..............

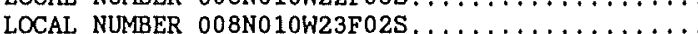

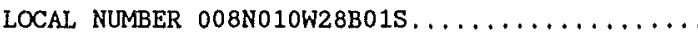

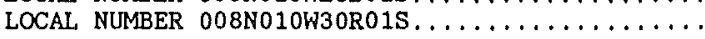

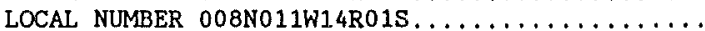

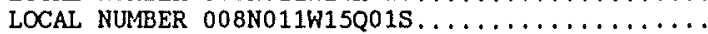

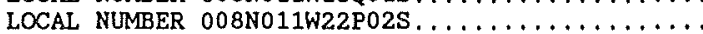

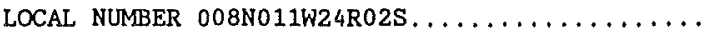

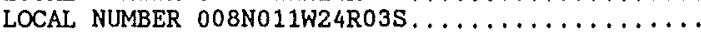

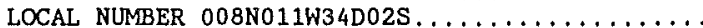

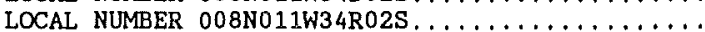

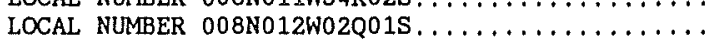

LOCAL NUMBER 008N012W05D01S . . . . . . . . . . .

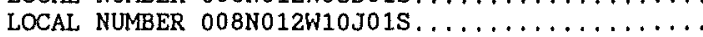

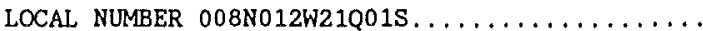

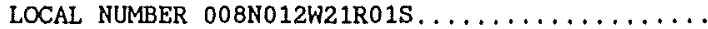

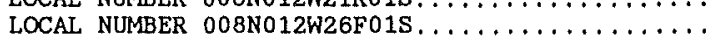

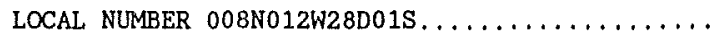

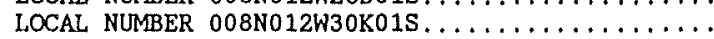
LOCAL NUMBER 008N012W31002S ...............

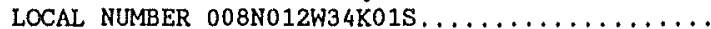

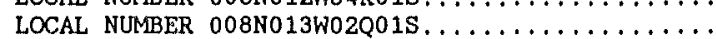


LOS ANGELES COUNTY--Continued

\begin{tabular}{|c|c|c|c|}
\hline Page & & & Page \\
\hline 139 & LOCAL NUMBER & 008N014W17M01S... & 143 \\
\hline 139 & LOCAL NUMBER & $008 \mathrm{~N} 014 \mathrm{~W} 18 \mathrm{~N} 01 \mathrm{~S} \ldots \ldots \ldots \ldots$ & 143 \\
\hline 140 & LOCAL NUMBER & $008 \mathrm{~N} 014 \mathrm{~W} 23 \mathrm{G} 01 \mathrm{~s} \ldots \ldots \ldots \ldots \ldots \ldots \ldots$ & 143 \\
\hline 140 & LOCAL NUMBER & $008 \mathrm{~N} 014 \mathrm{~W} 24 \mathrm{C} 01 \mathrm{~S}$. & 144 \\
\hline 140 & LOCAL NUMBER & 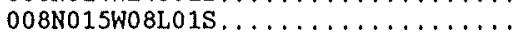 & 144 \\
\hline 140 & LOCAL, NUMBER & 008N015W10P02S. & 144 \\
\hline 141 & LOCAL NUMBER & 008 N015W33G01S. . & \\
\hline 141 & LOCAL NUMBER & 008N016W02R01S... & \\
\hline 141 & LOCAL NUMBER & 008N016W03F01S, $\ldots \ldots \ldots \ldots \ldots \ldots$ & \\
\hline 141 & LOCAL NUMBER & 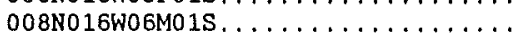 & 145 \\
\hline 142 & LOCAL NUMBER & 008N016W09G02S. & \\
\hline 142 & LOCAL NUMBER & 008N016W18H02S.. & \\
\hline 142 & LOCAL NUMBER & $008 N 016 \mathrm{~W} 22 \mathrm{Q} 04 \mathrm{~S}$. & 14 \\
\hline 142 & LOCAL NUMBER & 008N017W01N01S. & \\
\hline 143 & LOCAL NUMBER & 008 N017W04D01s........... & 14 \\
\hline
\end{tabular}
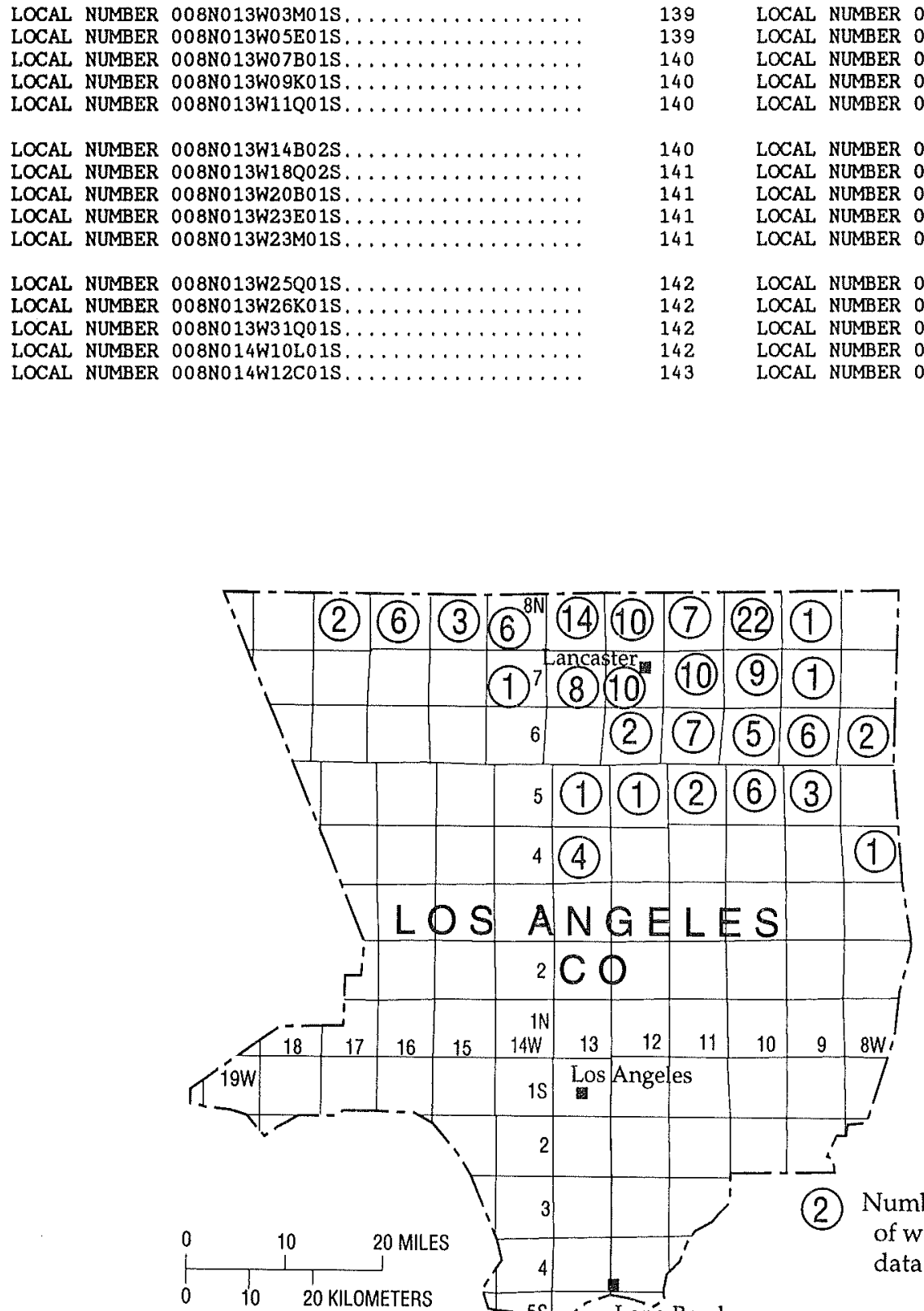

LOCAL NUMBER 008N017W04D01S............. 


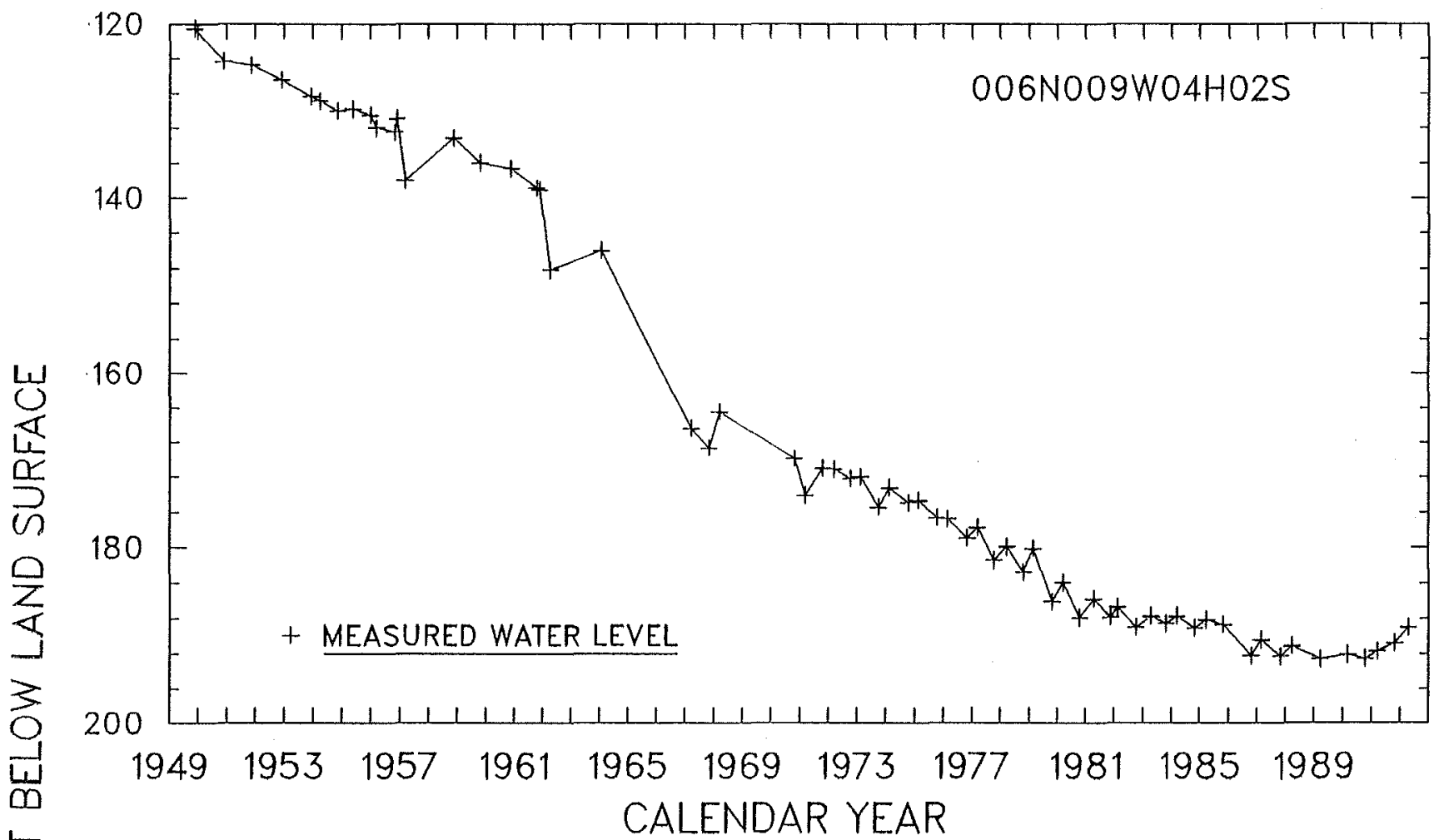

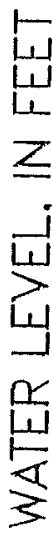

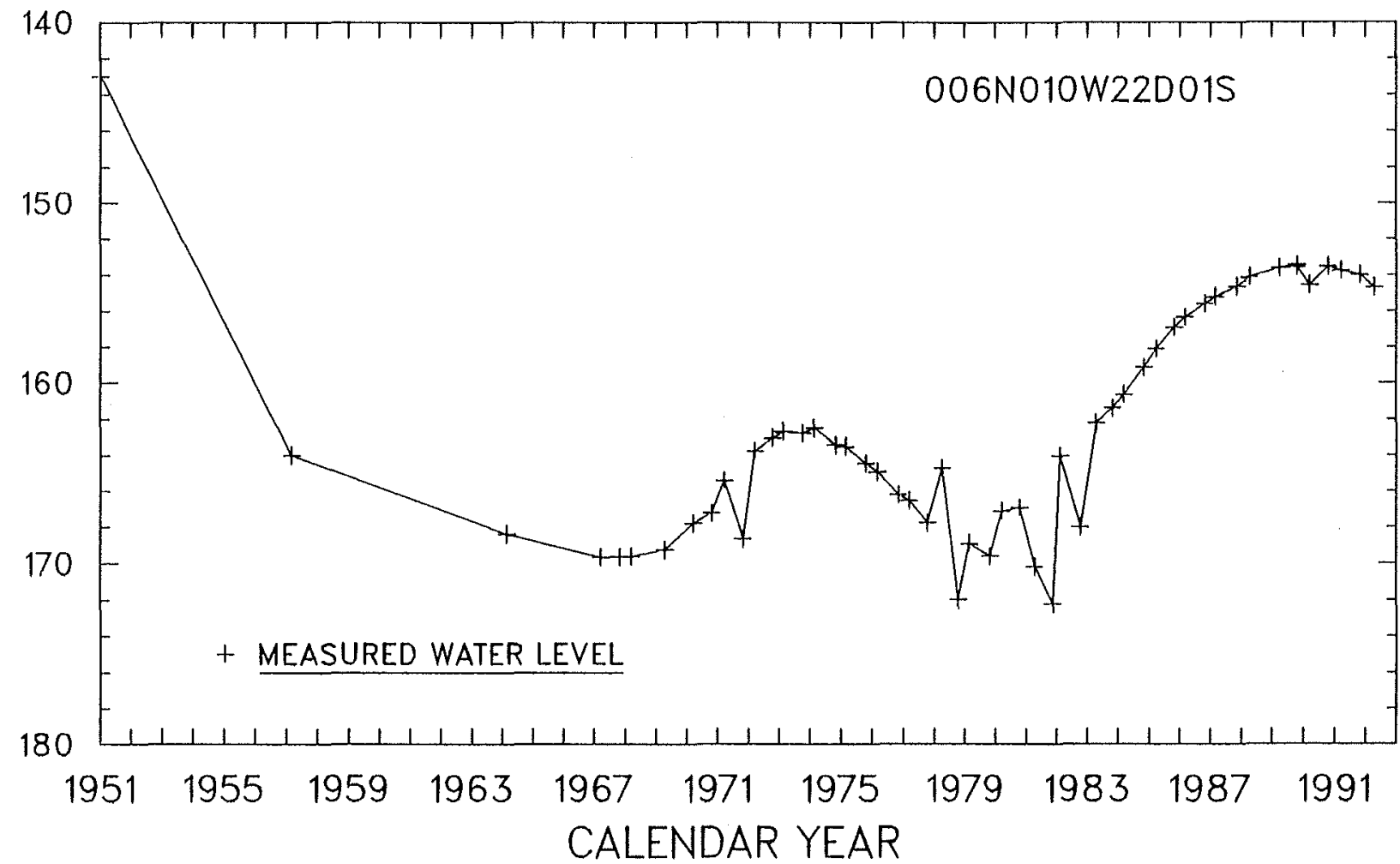

Figure 15. Hydrographs for wells in Los Angeles County. (006N009W04H02S and 006N010W22D01S) 


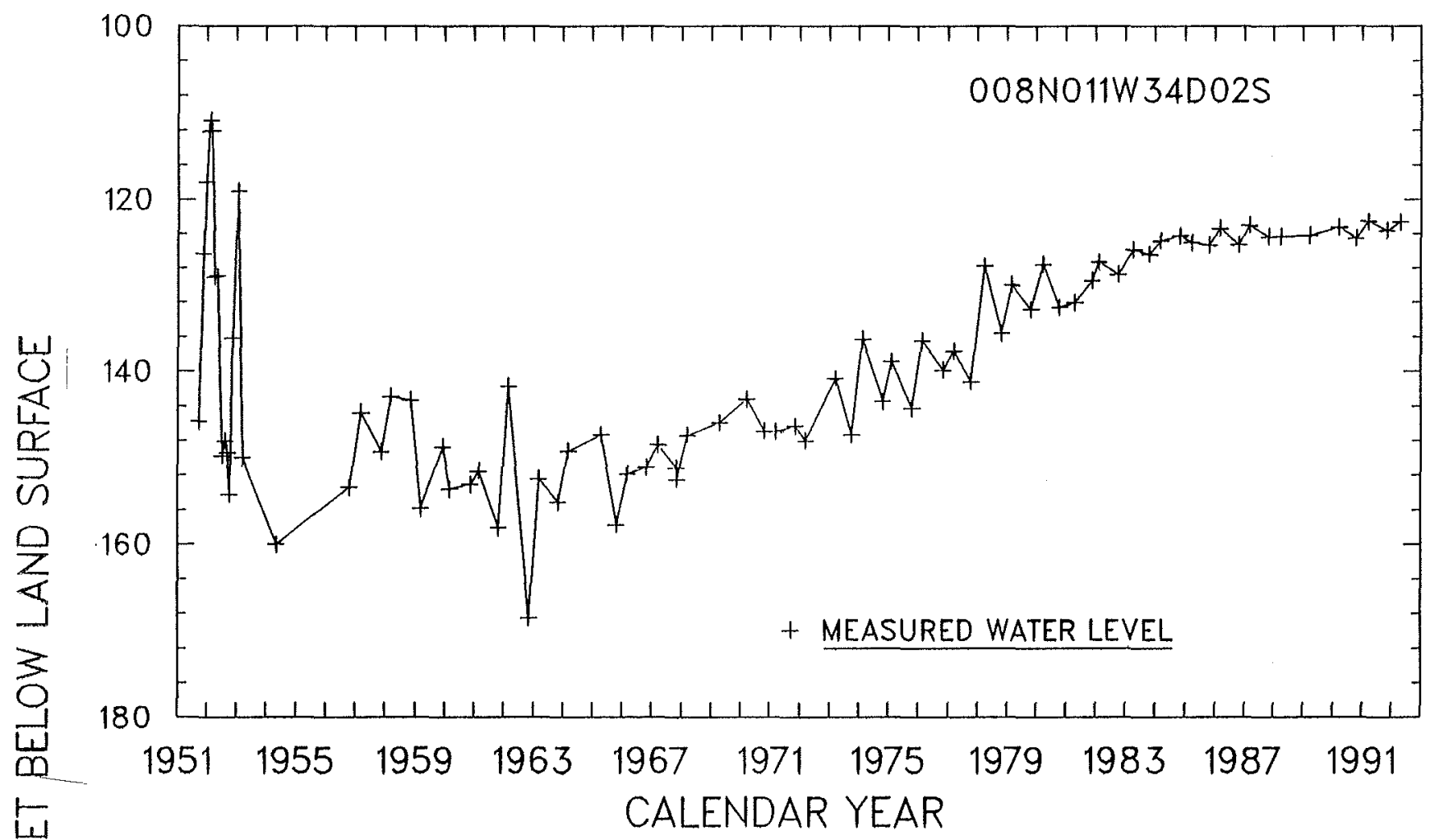

崖

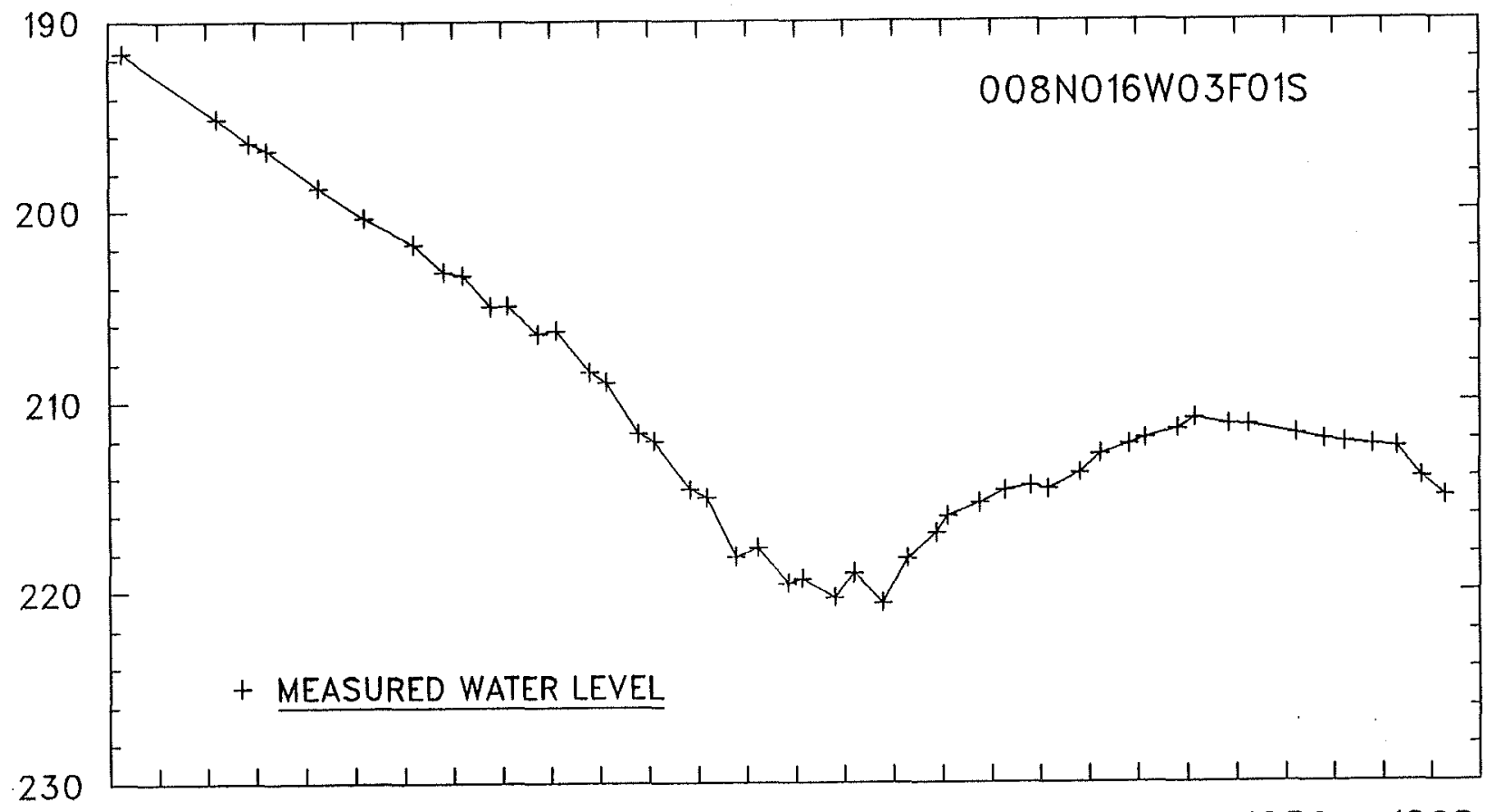

$\begin{array}{llllllllll}1965 & 1968 & 1971 & 1974 & 1977 & 1980 & 1983 & 1986 & 1989 & 1992\end{array}$ CALENDAR YEAR

Figure 16. Hydrographs for wells in Los Angeles County. (008N011W34D02S and 008N016W03F01S) 
SITE NUMBER 342631117445101

LOCAL NUMBER 004N008W07R01S

About 6 miles west of Pinon Hills and about 4.4 miles south of intersection of Pearblossom Highway and 213 th Street East. Drilled unused well. Diameter 12 inches, depth reported 185 feet. Altitude of land-surface datum 4,307 feet. Water-level records available 1964, 1974 to current year.

WATER LEVELS IN FEET BELOW LAND-SURFACE DATUM

$$
\begin{array}{ll}
\text { WATER } \\
\text { DATE } & \text { LEVEL MS }
\end{array}
$$

APR $14,1992112.43 \mathrm{~S}$

PERIOD OF RECORD HIGHEST 94.68 APR 15, 1983 LOWEST 130.75 MAR 28, 1978

SITE NUMBER 342612118163401

LOCAL NUMBER 004N013W07Q01S

About 7 miles southwest of Acton, and 300 feet north of Soledad Canyon Road, and 0.1 mile east of Indian Canyon Road to Santa Clara divide. Domestic well in alluvium. Diameter 8 inches, depth reported 40 feet. Altitude of land-surface datum 2,200 feet. Water-level records available 1965, 1978-82, 1984 to current year.

WATER LEVELS IN FEET BELOW LAND-SURFACE DATUM

$$
\text { DATE WATER }
$$

APR 15, $1992 \quad 7.21 \mathrm{~S}$

PERIOD OF RECORD HIGHEST 3.56 MAR 13, $1980 \quad$ LOWEST 7,95 FEB 26, 1987

SITE NUMBER 342706118114501

LOCAL NUMBER 004N013W12C03S

About $0.1 \mathrm{mile}$ northeast of Acton Camp. Institutional water-table well. Diameter 12 inches, depth reported 115 feet. Altitude of land-surface datum 2,635 feet. Reported measurements provided by Los Angeles County Flood Control District. Water-level records available 1950-51, 1953, 1955-58, 1963, 1965, 1973 to current year.

WATER LEVELS IN FEET BELOW LAND-SURFACE DATUM

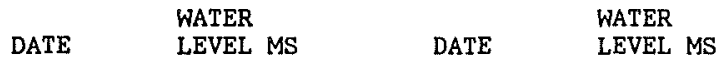

OCT $31,1991 \quad 38.49 \mathrm{~S} \quad$ APR $15,1992 \quad 32.00 \mathrm{~S}$

PERIOD OF RECORD HIGHEST 3.07 MAR 06, $1984 \quad$ LOWEST 50.11 SEP 21,1965

SITE NUMBER 342539118113601

LOCAL NUMBER 004N013W13L01S

South of Acton, east of Moody Canyon Road. Drilled irrigation well. Diameter 12 inches, depth unknown. Altitude of land-surface datum 2,960 feet. Water-level records available 1965, 1974-76, 1978 to current year.

WATER LEVELS IN FEET BELOW LAND-SURFACE DATUM

$$
\text { DATE WATER } \quad \text { LEVEL MS }
$$

APR 15, 1992

$$
\mathbf{P}
$$

PERIOD OF RECORD HIGHEST 7.68 MAR 13, 1980 LOWEST 57.00 SEP 01, 1965 
WELL DESCRIPTIONS AND WATER-LEVEL MEASUREMENTS

WATER YEAR OCTOBER 1991 TO SEPTEMBER 1992

SITE NUMBER 342609118131101

LOCAL NUMBER 004N013W15A01S

About 2 miles southwest of Acton. Domestic well. Diameter 14 inches, depth reported 60 feet. Altitude of land-surface datum 2,475 feet. Water-level records available 1965, 1978-80, 1982 to current year.

WATER LEVELS IN FEET BELOW LAND-SURFACE DATUM

$$
\begin{array}{ll} 
& \text { WATER } \\
\text { DATE } & \text { LEVEL MS }
\end{array}
$$

APR 15, $1992 \quad 7.21 \mathrm{~S}$

PERIOD OF RECORD HIGHEST 6.64 MAR 13, 1980 LOWEST 29.55 SEP 21, 1965

SITE NUMBER 343312117510101

LOCAL NUMBER 005N009W05E02S

About 15 miles east of Palmdale, $0.3 \mathrm{mile}$ south of Avenue $\mathrm{S}$, and 0.9 mile west of $165 \mathrm{th}$ Street East. Drilled unused well. Diameter 16 inches, depth measured greater than 500 feet in 1990 . Altitude of land-surface datum 2,866 feet. Water-level records available 1981 to current year.

WATER LEVELS IN FEET BELOW LAND-SURFACE DATUM

$$
\text { DATE NATER } \quad \text { LEVEL MS }
$$

APR 14, $1992 \quad 72.64 \mathrm{~S}$

PERIOD OF RECORD HIGHEST 53,50 MAR 08, 1984 LOWEST 72.64 APR 14, 1992

SITE NUMBER 343242117500601

LOCAL NUMBER 005N009W05R02S

About 15 miles east of Palmdale, 0.9 mile south of Avenue $\mathrm{S}$, and 100 feet west of 165 th Street East. Drilled unused well. Diameter 8 inches, depth measured 143.7 feet in $1964,137.3$ feet in 1983 . Altitude of land-surface datum 2,908 feet. Water-level records available 1964, 1981 to current year.

WATER LEVELS IN FEET BELOW LAND-SURFACE DATUM

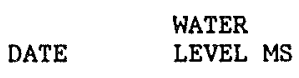

APR 14, $1992116.43 \mathrm{~S}$

PERIOD OF RECORD HIGHEST 97.18 MAR 05, 1984 LOWEST 138,34 MAY 05, 1964

SITE NUMBER 343141117501201

LOCAL NUMBER O05NOO9W08R01S

About 16 miles east of Palmdale and about 1.5 miles north of the intersection of Pearblossom Highway and 165th Street East. Drilled domestic well in alluvium. Diameter 10 inches, depth measured 230 feet in 1986 . Altitude of land-surface datum 3,000 feet. Water-level records available 1964, 1978 to current year.

WATER LEVELS IN FEET BELOW LAND-SURFACE DATUM

$$
\text { DATE WATER }
$$

APR $14,1992163.74 \mathrm{~S}$

PERIOD OF RECORD HIGHEST 128.83 MAR 28, 1985 LOWEST 188.30 MAR 31,1978 
SITE NUMBER 343251117545901

LOCAL NUMBER O05N010W03LO1S

About 2.5 miles north of Pearblossom, 0.3 mile east of 116 th Street East, and 0.3 mile north of Avenue $T$, Domestio well. Diameter 8 inches, depth measured 148.5 feet in 1990 . Altitude of land-surface datum 2,802 feet.

Water-level records available 1961, 1963, 1968-70, 1973 to current year.

WATER LEVELS IN FEET BELOW LAND-SURFACE DATUM

$$
\text { DATE WATER }
$$

APR $14,1992 \quad 91.05 \mathrm{~S}$

PERIOD OF RECORD HIGHEST 83.33 MAR 21, 1989 LOWEST 110.55 FEB 23, 1979

\section{SITE NUMBER 343237117582601}

LOCAL NUMBER DOSN010W06N01S

In Littlerock near intersection of Avenue $T$ and 87 th Street East. Unused well. Diameter 14 inches, depth measured 303.8 feet in 1982. Altitude of land-surface datum 2,777 feet. Reported measurements provided by Los Angeles County Flood Control District. Water-level records available 1926, 1928, 1930, 1938-78, 1982 to current year.

WATER LEVELS IN FEET BELOW LAND-SURFACE DATUM

$$
\begin{array}{ll}
\text { WATER } \\
\text { DATE }
\end{array}
$$

APR $14,1992114.62 \mathrm{~S}$

PERIOD OF RECORD HIGHEST 85.05 MAR 02, 1945 LOWEST 147.30 JUL 17,1956

SITE NUMBER 343205117525801

LOCAL NUMBER 005N010W12M02S

About 2 miles northwest of Pearblossom and 0.24 mile east of 136 th Street East. Unused well. Diameter 8 inches, depth measured 115.9 feet in 1982. Altitude of land-surface datum 2,912 feet. Water-level records available 1982 to current year.

WATER LEVELS IN FEET BELOW LAND-SURFACE DATUM

$$
\text { DATE WATER } \quad \text { LEVEL MS }
$$

APR $14,199275.08 \mathrm{~S}$

PERIOD OF RECORD HIGHEST 55.43 MAR 28, 1985 LOWEST 75.08 APR 14, 1992

SITE NUMBER 343115117565601

LOCAL NUMBER 005N010W17LO1S

About $1 \mathrm{mile}$ east of Littlerock, $0.5 \mathrm{mile}$ east of $96 \mathrm{th}$ Street East, and 100 feet south of Pearblossom Highway. Unused well. Diameter 8 inches, depth 370 feet, perforated 250-370 feet. Altitude of land-surface datum 2,955 feet. Measurements provided by Los Angeles County Flood Control District 1960-81. Water-level records avallable $1960,1964,1976-78,1981$ to current year.

WATER LEVELS IN FEET BELOW LAND-SURFACE DATUM

$$
\text { DATE WATER }
$$

APR 14, $1992192.61 \mathrm{~S}$

PERIOD OF RECORD HIGHEST 166.60 APR 19, 1960 LOWEST 217.50 NOV 04,1977 
WELL DESCRIPTIONS AND WATER-LEVEL MEASUREMENTS

WATER YEAR OCTOBER 1991 TO SEPTEMBER 1992

SITE NUMBER 343051117563001

LOCAL NUMBER 005N010W17R01S

About 2 miles east of Littlerock, 0.1 mile west of 106th Street East, and 50 feet north of Avenue V. Unused well. Diameter 14 inches, depth measured 452 feet in 1982. Altitude of land-surface datum 3,032 feet. Water-level records available 1964, 1982 to current year.

WATER LEVELS IN FEET BELOW LAND-SURFACE DATUM

$$
\text { DATE WATER }
$$

APR 14, $1992 \quad 272.84 \mathrm{~S}$

PERIOD OF RECORD HIGHEST 249.02 JAN 09, 1964 LOWEST 273.69 MAR 21, 1989

SITE NUMBER 343259117593101

LOCAL NUMBER 005N011W01M01S

North of Littlerock, about $0.5 \mathrm{mile}$ north of Avenue T. Drilled commercial water-table well in alluvium.

Diameter 14 inches, original depth 414 feet, depth measured 396,29 feet in 1967, perforated 100-364 feet. Altitude of land-surface datum 2,739 feet. Water-level records available 1955, 1963, 1967-68, 1970 to current year.

WATER LEVELS IN FEET BELOW LAND-SURFACE DATUM

$\begin{array}{lll} & \text { WATER } & \\ \text { DATE } & \text { WEVEL MS } & \text { WATER } \\ \text { LEVEL MS }\end{array}$

OCT 31, 1991 111.03 SR APR 15, $1992100.27 \mathrm{~s}$

PERIOD OF RECORD HIGHEST 64.48 APR 10, 1980 LOWEST 119.99 MAR 21, 1991

SITE NUMBER 343148117582901

LOCAL, NUMBER 005NO11W12R01S

In Littlerock, west of $87 \mathrm{th}$ Street East, and $0.1 \mathrm{mile}$ north of Avenue U. Public-supply water-table well. Diameter 14 inches, depth reported 602 feet. Altitude of land-surface datum 2,841 feet. Measurements provided by Los Angeles County Flood Control District 1937-45, 1949-52. Water-level records ava1lable 1927, 1937-45, 1949-52, $1961,1963-64,1968,1972$ to current year.

WATER LEVELS IN FEET BELOW LAND-SURFACE DATUM

$$
\text { DATE WATER }
$$

APR 14, 1992188.62 SR

PERIOD OF RECORD HIGHEST 110.00 NOV 1927 LOWEST 204.40 AUG 09, 1972

SITE NUMBER 342824118094301

LOCAL NUMBER 005N012W32M01S

About 1.5 miles east of Acton, 25 feet north of Carson Mesa Road. Unused well. Diameter unknown, depth measured 131.3 feet in 1992. Altitude of land-surface datum 2,835 feet. Water-level records available 1978 to current year.

WATER LEVELS IN FEET BELOW LAND-SURFACE DATUM

$$
\begin{array}{ll} 
& \text { WATER } \\
\text { DATE } & \text { LEVEL MS }
\end{array}
$$

APR 15, $1992 \quad 82.96 \mathrm{~S}$

PERIOD OF RECORD HIGHEST 40.25 APR 12, 1983 LOWEST 127.39 MAR 19, 1991 
SITE NUMBER 342934118114901

LOCAL NUMBER 005N013W25C03S

About 1.5 miles north of Acton, southeast of intersection of Sierra Highway and Crown Valley Road. Domestic well. Diameter 6 inches, depth unknown, Altitude of land-surface datum 2,961 feet. Water-1evel records available 1965 , 1974 to current year.

WATER LEVELS IN FEET BELOW LAND-SURFACE DATUM

$$
\begin{array}{ll} 
& \text { WATER } \\
\text { DATE } & \text { LEVEL MS }
\end{array}
$$

APR $15,1992 \quad 193.90 \mathrm{~S}$

PERIOD OF RECORD HIGHEST 188.87 FEB 26, 1987 LOWEST 215.78 MAR 07, 1977

SITE NUMBER 343701117433001

LOCAL NUMBER 006N008W09Q01S

About $30 \mathrm{miles}$ east of Lancaster, 200 feet north of Avenue 0 , and $0.6 \mathrm{mile}$ east of 220 th Street East. Unused well. Diameter 6 inches, depth measured 59.2 feet in 1985, 52 feet in 1992 . Altitude of 1 and-surface datum 2,791 feet. Water-level records available 1974 to current year.

WATER LEVELS IN FEET BELOW LAND-SURFACE DATUM

$$
\text { DATE WATER }
$$

APR $15,1992 \quad$ D

PERIOD OF RECORD HIGHEST 52.17 FEB $28,1974 \quad$ LOWEST $53.76 \quad$ FEB 27,1979

SITE NUMBER 343541117461801

LOCAL NUMBER 006N008W19E01S

About 29 miles east of Lancaster near intersection of 200 th Street East and Avenue P-8. Drilled unused well. Diameter 14 inches, depth measured 303,6 feet in 1992. Altitude of land-surface datum 2,774 feet. Water-1evel records available 1964,1978 to current year.

WATER LEVELS IN FEET BELOW LAND-SURFACE DATUM

$$
\begin{array}{ll} 
& \text { WATER } \\
\text { DATE } & \text { LEVEL MS }
\end{array}
$$

APR $15,1992155.10 \mathrm{~S}$

PERIOD OF RECORD HIGHEST 147.44 MAR 26, 1964 LOWEST 156.33 APR 14,1981

SITE NUMBER 343824117493801

LOCAL NUMBER 006N009W04H02S

At Wilsona School about $0.6 \mathrm{mile}$ north of Avenue $N$ and 100 feet west of 170 th Street East. Drilled institutional well. Dianeter 10 inches, depth reported 336 feet. Altitude of land-surface datum 2,595 feet. Water-1evel. records avallable 1949-62, 1964, 1967-68, 1970 to current year.

WATER LEVELS IN FEET BELOW LAND-SURFACE DATUM

$$
\begin{array}{lll} 
& \text { WATER } & \\
\text { DATE } & \text { WEVEL MS } & \text { WATER }
\end{array}
$$

OCT $24,1991190.83 \mathrm{~s} \quad$ APR $14,1992189.00 \mathrm{~s}$

PERIOD OF RECORD HIGHEST 120.56 NOV 23, 1949 LOWEST 192.56 OCT 17,1990 
WELL DESCRIPTIONS AND WATER-LEVEL MEASUREMENTS

WATER YEAR OCTOBER 1991 TO SEPTEMBER 1992

\section{SITE NUMBER 343726117514501}

LOCAL NUMBER OO6NOO9W07J01S

About 20 miles east of Lancaster, 140 feet west of 150 th Street East, and 0.5 mile south of Avenue N. Drilled unused water-table well. Diameter 14 inches, depth 243 feet, perforated 163-243 feot. Altitude of land-surface datum 2,618 feet. Water-level records available 1964, 1974 to current year.

WATER LEVELS IN FEET BELOW LAND-SURFACE DATUM

$$
\text { DATE WATER } \quad \text { LEVEL MS }
$$

APR $14,1992146.43 \mathrm{~S}$

PERIOD OF RECORD HIGHEST 128.02 JAN 27, 1964 LOWEST 150.59 FEB 27, 1979

\section{SITE NUMBER 343703117482801}

LOCAL NUMBER OO6N009W11NO1S

About 24 miles east of Lancaster, 550 feet north of Avenue 0 , and 300 feet east of 180 th Street East. Unused well. Diameter 8 inches, depth 295 feet. Altitude of land-surface datum 2,666 feet. Water-level records available $1951-62,1964,1967$ to current year.

WATER LEVELS IN FEET BELOW LAND-SURFACE DATUM

$$
\text { DATE WATER } \quad \text { LEVEL MS }
$$

APR $14,1992177.21 \mathrm{~S}$

PERIOD OF RECORD HIGHEST 136.88 DEC 27, 1951 LOWEST 179.09 MAR 24, 1989

\section{SITE NUMBER 343450117522501}

LOCAL NUMBER 006N009W30F01S

About 14 miles east of Palmdale, 130 feet north of Palmdale Boulevard, and 0.7 mile west of 150 th Street East. Unused water-table well. Diameter 12 inches, depth reported 312 feet in 1922, depth measured 92.3 feet in 1964. Altitude of land-surface datum 2,758 feet. Water-level records available 1951, 1954-62, 1964,1966 to current year.

WATER LEVELS IN FEET BELOW LAND-SURFACE DATUM

$$
\text { DATE WATER }
$$

APR $14,1992 \quad 46.39 \mathrm{~S}$

PERIOD OF RECORD HIGHEST 15.05 APR 14, 1969 LOWEST 59.63 MAR 08, 1977

SITE NUMBER 343408117500701

LOCAL NUMBER O06N009W33C01S

About 16 miles east of Palmdale, 0.25 mile south of Avenue R, and 140 feet west of 165 th Street East. Drilled irrigation water-table well. Diameter 14 inches, depth reported 738 feet, perforated 180-738 feet. Altitude of land-surface datum 2,823 feet. Water-level records available 1964, 1967, 1973-80, 1982, 1986 to current year.

WATER LEVELS IN FEET BELOW LAND-SURFACE DATUM

$$
\text { DATE WATER }
$$

APR 14, $1992118.57 \mathrm{SR}$

PERIOD OF RECORD HIGHEST 99.04 MAR 20, 1991 LOWEST 196.53 FEB 26, 1976 
SITE NUMBER 343820117570501

LOCAL NUMBER 006N010W05H01S

About 13 miles east of Palmdale, 20 feet north of Avenue M-8 and 40 feet west of 100th Street East. Public-supply well. Diameter 14 inches, depth 425 feet. Altitude of land-surface datum 2,552 feet. Records available 1952-53, $1956,1963,1974-78,1980$ to current year.

WATER LEVELS IN FEET BELOW LAND-SURFACE DATUM

$$
\text { DATE WATER }
$$

APR 13, $1992 \quad 260.75$ VR

PERIOD OF RECORD HIGHEST 230.00 JUL 1952. AUG 07, 1953 LOWEST 266.2 APR 15, 1981

SITE NUMBER 343608117580501

LOCAL NUMBER 006N010W17N01S

About 9 miles east of Palmdale, 58 feet east of 90 th Street East, and 45 feet north of Avenue P. Unused well. Diameter 14 inches, depth measured 336.2 feet in 1991. Altitude of land-surface datum 2,605 feet. Water-level records available $1945,1964,1973$ to current year.

WATER LEVELS IN FEET BELOW LAND-SURFACE DATUM

$$
\text { DATE WATER }
$$

APR $13,1992179.86 \mathrm{~S}$

PERIOD OF RECORD HIGHEST 132.64 MAR 08, 1984 LOWEST 202.85 JAN 20, 1964

SITE NUMBER 343557117554801

LOCAL NUMBER O06N010W22D01S

About 11 miles east of Palmdale, 0.25 mile south of Avenue P, and 0.16 mile east of 110 th Street East. Domestic water-table well. Diameter 12 inches, depth 200 feet, perforated 100-200 feet. Altitude of land-surface datum 2,645 feet. Water-level records available 1951, 1957, 1964, 1967 to current year.

WATER LEVELS IN FEET BELOW LAND-SURFACE DATUM

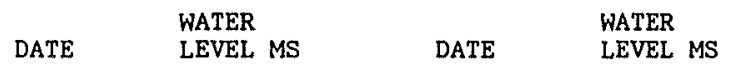

OCT 24, $1991154.00 \mathrm{~S} \quad$ APR 13, $1992154.68 \mathrm{~S}$

PERIOD OF RECORD HIGHEST 143.00 JAN 1951 LOWEST 172.22 NOV 17,1981

SITE NUMBER 343422117555801

LOCAL NUMBER D06N010W34D01S

About 10.5 miles east of Palmdale, 100 feet east of 110th Street East, and 90 feet south of Avenue R. Drilled public-supply water-table well. Diameter 8 inches, depth reported 300 feet. Altitude of land-surface datum 2,706 feet. Water-level records available 1957-60, 1964, 1967 to current year.

WATER LEVELS IN FEET BELOW LAND-SURFACE DATUM

$$
\text { DATE WATER } \quad \text { LEVEL MS }
$$

APR $13,1992126.03 \mathrm{~S}$

PERIOD OF RECORD HIGHEST 124.50 OCT 09, 1957 LOWEST 141.87 FEB 14, 1973 
WELL DESCRIPTIONS AND WATER-LEVEL MEASUREMENTS

WATER YEAR OCTOBER 1991 TO SEPTEMBER 1992

SITE NUMBER 343420117535501

LOCAL NUMBER 006N010W35A01S

About 12 miles east of Palmdale, 2.0 miles east of 110 th Street East, and 160 feet south of Avenue R. Unused well, Diameter 10 inches, depth measured 80.5 feet in 1982 . Altitude of land-surface datum 2,750 feet. Water-1evel records avallable 1964,1982 to current year.

WATER LEVELS IN FEET BELOW LAND-SURFACE DATUM

$$
\text { DATE WATER } \quad \text { LEVEL MS }
$$

APR $13,199253.28 \mathrm{~s}$

PERIOD OF RECORD HIGHEST 48.50 FEB 25, 1987 LOWES' $\quad 68.70$ MAR 09, 1964

SITE NUMBER 343820118022101

LOCAL. NUMBER O06N011W03E02S

Southeast of Lancaster, about 0.25 mile east of 50 th Street East, and south of Avenue M. Irrigation well. Diameter 16 inches, depth 700 feet, perforated 325-700 feet. Altitude of land-surface datum 2,493 feet. Water-level records available $1960,1962-63$, 1981 to current year.

WATER LEVELS IN FEET BELOW LAND-SURFACE DATUM

$$
\text { DATE WATER }
$$

APR 13, $1992 \quad 298.27 \mathrm{~s}$

PERIOD OF RECORD HIGHEST 202.00 FEB 12, 1962 LOWEST 352.35 SEP 03, 1981

SITE NUMBER 343829118050301

LOCAL NUMBER 006N011W06G02S

On Edwards AFB (Plant 42) about 0.5 mile east of 20 th Street East and 0.3 mile south of Avenue M. Drilled water-table well. Diameter unknown, depth reported 1,200 feet. Altitude of land-surface datum 2,480 feet. Water-level records avallable 1967, 1969-73, 1975-83, 1985 to current year.

WATER LEVELS IN FEET BELOW LAND-SURFACE DATUM

$$
\begin{array}{ll} 
& \text { WATER } \\
\text { DATE } & \text { LEVEL MS }
\end{array}
$$

APR $15,1992 \quad 339.76 \mathrm{~S}$

PERIOD OF RECORD HIGHEST 282.40 MAR 15, 1967 LOWEST 350.02 MAR 29, 1988

SITE NUMBER 343700118031101

LOCAL NUMBER OOGN011W09P01S

About 4 miles northeast of Palmdale, 0.3 mile east of intersection of Avenue 0 and 40 th Street East. Drilled irrigation water-table well. Diameter 16 inches, depth reported 652 feet, perforated $537-652$ feet. Altitude of land-surface datum 2,523 feet. Water-level records available 1956, 1963, 1981 to current year.

WATER LEVELS IN FEET BELOW LAND-SURFACE DATUM

DATE WATER $\quad$ LEVEL MS

APR 15, $1992 \quad$ P

PERIOD OF RECORD HIGHEST 257 AUG 1956 LOWEST 341.30 SEP 03, 1981 
SITE NUMBER 343726118012001

LOCAL NUMBER O06N011W11M01S

About 6 miles northeast of Palmdale along 60th Street East and 0.5 mile south of Avenue N. Drilled unused water-table well. Diameter 14 inches, depth 431 feet, perforated 311-431 feet. Altitude of land surface datum 2,523 feet. Water-level records available $1960,1963,1974$ to current year.

WATER LEVELS IN FEET BELOW LAND-SURFACE DATUM

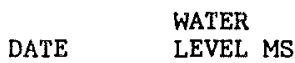

APR $13,1992304.64 \mathrm{~S}$

PERIOD OF RECORD HIGHEST 300.00 APR 1963 LOWEST 341.48 APR 10,1980

SITE NUMBER 343628118022701

LOCAL NUMBER 006N011W16J01S

About 4 miles northeast of Palmdale, $0.4 \mathrm{mile}$ north of intersection of Avenue $P$ and 50 th Street East. Drilled unused water-table well in alluvium. Diameter 14 inches, depth 630 feet, perforated $322-630$ feet, Altitude of land-surface datum 2,547 feet. Water-level records available 1964, 1970, 1972-75, 1977-78, 1980 to current year.

WATER LEVELS IN FEET BELOW LAND-SURFACE DATUM

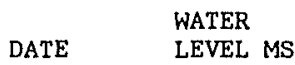

APR $13,1992 \quad 317,62 \mathrm{~s}$

PERIOD OF RECORD HIGHEST 310.28 MAR 29, 1988 LOWEST 370.65 OCT 22, 1970

SITE NUMBER 343419118044401

LOCAL NUMBER O0GN011W31A01S

In Palmdale, $0.2 \mathrm{mile}$ west of 30 th Street East and $0.06 \mathrm{mile}$ south of Avenue R. Drilled domestic water-table well. Diameter 8 inches, depth 444 feet. Altitude of land-surface datum 2,633 feet. Water-level records available 1963, $1974-76,1978$ to current year.

WATER LEVELS IN FEET BELOW LAND-SURFACE DATUM

$$
\text { DATE WATER }
$$

APR 13, $1992268.35 \mathrm{~V}$

PERIOD OF RECORD HIGHEST 220.10 NOV 13, 1963 LOWEST 268.35 APR 13, 1992

SITE NUMBER 343404117593101

LOCAL NUMBER O06N011W36G01S

About 3 miles east of Pearland, 0.3 mile south of Avenue $R$ and 0.3 mile west of 80 th Street East. Commercial wel1. Diameter 12 inches, depth 572 feet, perforated 235-572 feet. Altitude of land-surface datum 2,679 feet. Water-level records available 1956, $1974-78,1980$ to current year.

WATER LEVELS IN FEET BELOW LAND-SURFACE DATUM

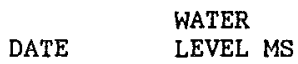

APR $15,1992114.87 \mathrm{~s}$

PERIOD OF RECORD HIGHEST 73.51 MAR 09, 1984 LOWEST 192 JAN 12, 1956 
WELL DESCRIPTIONS AND WATER-LEVEL MEASUREMENTS

WATER YEAR OCTOBER 1991 TO SEPTEMBER 1992

SITE NUMBER 343741118110301

LOCAL NUMBER O06N012W07A01S

About 1 mile southeast of Quartz Hill, 500 feet west of 30 th Street West and 40 feet south of Avenue N-3. Drilled public-supply well. Diameter 14 inches, depth 432 feet, perforated 276-432 feet. Altitude of land-surface datum 2,597 feet. Water-level records available 1955, 1957, 1961, 1974 to current year.

WATER LEVELS IN FEET BELOW LAND-SURFACE DATUM

DATE HATER MS

APR 13, $1992 \quad 346.16 \mathrm{~S}$

PERIOD OF RECORD HIGHEST 284.10 MAR 12, 1957 LOWEST 349.43 FEB 26, 1986

SITE NUMBER 343716118063501

LOCAL NUMBER 006N012W12M01S

On Edwards AFB (Plant 42), 1.4 miles northwest of intersection of Avenue $P$ and 15 th Street East. Drilled unused water-table well. Diameter 10.5 inches, depth measured 430 feet in 1982. Altitude of land-surface datum 2,560 feet. Water-level records avallable 1963, 1982 to current year.

WATER LEVELS IN FEET BELOW LAND-SURFACE DATUM

$\begin{array}{ll} & \text { WATER } \\ \text { DATE } & \text { LEVEL MS }\end{array}$

APR 15, $1992 \quad$ VD

PERIOD OF RECORD HIGHEST 308.40 MAY 13, 1963 LOWEST 418.89 MAR 21, 1989

SITE NUMBER 344124117514301

LOCAL NUMBER 007N009W17N02S

About 17 miles east of Lancaster, 250 feet east of 150 th Street East, and 100 feet north of Avenue J. Drilled unused mell in alluvium. Diameter 12 inches, depth measured 303 feet in 1960, 274.15 feet in 1990 . Altitude of land-surface datum 2,492 feet. Water-level records available 1959 to current year.

WATER LEVELS IN FEET BELOW LAND-SURFACE DATUM

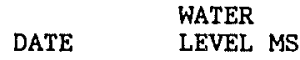

APR $15,1992 \quad 241.70 \mathrm{~S}$

PERIOD OF RECORD HIGHEST 192.35 MAR 01, 1960 LOWEST 255.70 NOV 03, 1967

SITE NUMBER 344337117575501

LOCAL NUMBER O07N010W05E01S

About 10 miles east of Lancaster, 0.2 mile east of 90th Street East. Drilled domestic well. Diameter 14 inches, 0-120 feet, 10 inches 120-400 feet, depth 400 feet, perforated 120-400 feet. Altitude of land-surface datum 2,391 feet. Water-level records available 1951-53, 1967-75, 1977 to current year.

WATER LEVELS IN FEET BELOW LAND-SURFACE DATUM

DATE WATER

APR 14, $1992 \quad 170.69 \mathrm{~S}$

PERIOD OF RECORD HIGHEST 112.04 FEB 14, 1952 LOWEST 213.95 OCT 21, 1970 


\section{SITE NUMBER 344320117575601}

LOCAL NUMBER OO7N010W05NOSS

About 10 miles east of Lancaster, 500 feet north of Avenue $\mathrm{H}$, and $0.2 \mathrm{mile}$ east of 90 th Street East. Unused well. Diameter 12 inches, depth reported 400 feet. Altitude of land-surface datum 2, 398 feet. Water-level records aveilable 1973-74, 1976 to current year.

WATER LEVELS IN FEET BELOW LAND-SURFACE DATUM

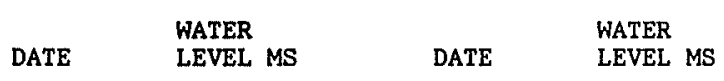

OCT 23, $1991215.04 \mathrm{~S} \quad$ APR 14, $1992211.14 \mathrm{~S}$

PERIOD OF RECORD HIGHEST 211.14 APR 14, 1992 LOWEST 264.75 MAR 09, 1976

SITE NUMBER 344119117591201

LOCAL NUMBER OOTNO1OW19DO1S

About 7.5 miles east of Lancaster, 0.1 mile south of Avenue J, and 50 feet east of 80th Street East. Domestic water-table we11. Diameter 8 inches, depth measured 320 feet in 1954. Altitude of land-surface datum 2,446 feet. Measurements provided by Los Angeles County Flood Control District 1954-61. Water-leve1 records available 1951, $1954-61,1964-68,1970$ to current year.

WATER LEVELS IN FEET BELOW LAND-SURFACE DATUM

$$
\text { DATE WATER }
$$

APR 14, $1992 \quad 266.67$ SS

PERIOD OF RECORD HIGHEST 183.83 MAR 27, 1951 LOWEST 293.05 MAR 07, 1977

SITE NUMBER 344033117582401

LOCAL NUMBER 007N010W19Q01S

About 8 miles east of Lancaster, 60 feet north of Avenue K, and 0.3 mile west of 90th Street East. Drilled unused well. Diameter 16 inches, depth reported 600 feet. Altitude of land-surface datum 2,473 feet. Water-level records available 1981 to current year.

WATER LEVELS IN FEET BELOW LAND-SURFACE DATUM

$$
\text { DATE WATER } \quad \text { LEVEL MS }
$$

APR 14, $1992291.20 \mathrm{~S}$

PERIOD OF RECORD HIGHEST 291.20 APR 14,1992 LOWEST 344,60 SEP 03, 1981

SITE NUMBER 344044117552901

LOCAL NUMBER 007NO1OW22PO1S

About $10.5 \mathrm{miles}$ east of Lancaster, $0.5 \mathrm{mile}$ east of $110 \mathrm{th}$ Street East, and $0.5 \mathrm{mile}$ south of Avenue J. Drilled unused water-table well. Diameter 12 inches, depth measured 474 feet in 1974 . Altitude of land-surface datum 2,481 feet. Water-level records available 1967,1974 to current year.

WATER LEVELS IN FEET BELOW LAND-SURFACE DATUM

$$
\text { DATE WATER }
$$

APR 15, $1992 \quad 309.93 \mathrm{~S}$

PERIOD OF RECORD HIGHEST 309.93 APR 15, 1992 LOWEST 345.80 MAR 07, 1977 
WELL DESCRIPTIONS AND WATER-LEVEL MEASUREMENTS

WATER YEAR OCTOBER 1991 TO SEPTEMBER 1992

SITE NUMBER 344029117573601

LOCAL NUMBER 007N010W29B01S

About 10 miles east of Lancaster, east of intersection of Avenue $K$ and 90th Street East. Drilled unused well. Diameter 16 inches, depth 585 feet. Altitude of land-surface datum 2,487 feet, Water-level records available 1950,1981 to current year.

WATER LEVELS IN FEET BELOW LAND-SURFACE DATUM

$$
\text { DATE NATER } \quad \text { LEVEL MS }
$$

APR 14, $1992303.08 \mathrm{~S}$

PERIOD OF RECORD HIGHEST 201.52 DEC 19, 1950 LOWEST 348.70 SEP 03, 1981

SITE NUMBER 343946117574701

LOCAL NUMBER 007N010W29P01S

About 10 miles east of Lancaster, east of intersection of Avenue $\mathbf{L}$ and 90 th Street East, Drilled unused well. Diameter 16 inches, original depth 410 feet, depth measured 378.7 feet in 1990 , perforated $200-408$ feet. Altitude of land-surface datum 2,505 feet. Water-level records available 1981 to current year.

WATER LEVELS IN FEET BELOW LAND-SURFACE DATUM

$$
\text { DATE WATER }
$$

APR 15, $1992314.20 \mathrm{~s}$

PERIOD OF RECORD HIGHEST 314.20 ARR 15, 1992 LOWEST 355.02 SEP 02, 1981

SITE NUMBER 343926117573701

LOCAL NUMBER 007N010W32B01S

About 13 miles east of Palmdale, $0.5 \mathrm{mile}$ east of $90 \mathrm{th}$ Street East, and $0.3 \mathrm{mile}$ south of Avenue L. Drilled unused well. Diameter 16 inches, depth reported 504 feet. Altitude of land-surface datum 2,517 feet. Water-1evel records available 1981 to current year.

WATER LEVELS IN FEET BELOW LAND-SURFACE DATUM

DATE WATER

APR 15, $1992 \quad$ So

PERIOD OF RECORD HIGHEST 320.45 MAR 14, 1990 LOWEST 343.50 SEP 02, 1981

SITE NUMBER 343925117561301

LOCAL NUMBER 007N010W33J01S

About 14 miles east of Palmdale, 0.75 mile north of Avenue M, and 0.2 mile west of 110th Street East. Drilled unused water-table well. Diameter 14 inches, depth reported 415 feet. Altitude of land-surface datum 2,538 feet. Water-level records available 1950-51, 1957, 1973-77, 1979 to current year.

WATER LEVELS IN FEET BELOW LAND-SURFACE DATUM

DATE WATER $\quad$ LEVEL MS

APR $15,1992 \quad 317.63 \mathrm{~S}$

PERIOD OF RECORD HIGHEST 241.32 DEC 21, 1950 LOWEST 341.60 MAR 21, 1973 
SITE NUMBER 344310117593001

LOCAL NUMBER 007N011W01Q01S

About 7 miles east of Lancaster, 30 feet north of Avenue $H$, and 0.25 mile west of 80 th Street East. Drilled unused well in alluvium. Diameter 10 inches, depth measured 390 feet in 1992 . Altitude of land-surface datum 2,385 feet. Water-level records available 1958-63, 1965-68, 1970-71, 1973, 1975 to current year.

WATER LEVELS IN FEET BELOW LAND-SURFACE DATUM

$$
\text { DATE WATER }
$$

APR $15,1992 \quad 185.84 \mathrm{~S}$

PERIOD OF RECORD HIGHEST 179,49 MAR 12, 1958 LOWEST $218.72 \quad$ OCT 21,1970

\section{SITE NUMBER 344323118041001}

LOCAL NUMBER OOTNO11W05LO1S

Northeast of Lancaster, $0.4 \mathrm{mile}$ east of $30 \mathrm{th}$ Street East, and $0.25 \mathrm{mile}$ north of Avenue M. Domestic well,

Diameter 8 inches, depth unknown. Altitude of land-surface datum 2,363 feet. Water-level records available 1963, $1974-83,1986$ to current year.

WATER LEVELS IN FEET BELOW LAND-SURFACE DATUM

$$
\text { DATE WATER } \quad \text { LEVEL MS }
$$

APR $14,1992130.88 \mathrm{~S}$

PERIOD OF RECORD HIGHEST 104.00 OCT 01, 1963 LOWEST 131.35 MAR 15, 1990

SITE NUMBER 344058118032401

LOCAL NUMBER OOTNO11W21E01S

About 4 miles east of Lancaster, $0.5 \mathrm{mile}$ north of Avenue $\mathrm{K}$, and $0.1 \mathrm{mile}$ east of 40 th Street East. Unused well. Diameter 8 inches, depth measured 162.95 feet in 1990 . Altitude of land-surface datum 2,422 feet. Water-level records available 1963, 1967 to current year.

WATER LEVELS IN FEET BELOW LAND-SURFACE DATUM

$$
\text { DATE WATER } \quad \text { LEVEL MS }
$$

APR 15, $1992153.10 \mathrm{~S}$

PERIOD OF RECORD HIGHEST 102.76 MAR 20, 1967 LOWEST 161.80 MAR 21, 1989

SITE NUMBER 343939118013701

LOCAL NUMBER 007N011W27Q01S

About 6 miles southeast of Lancaster, 0.3 mile west of 60 th Street East. Unused well. Diameter 14 inches, depth reported 650 feet. Altitude of land-surface datum 2,467 feet. Water-level records available 1953-54, 1963, 1974, to current year.

WATER LEVELS IN FEET BELOW LAND-SURFACE DATUM

$$
\text { DATE WATER } \quad \text { LEVEL MS }
$$

APR $15,1992 \quad 284.26 \mathrm{~S}$

PERIOD OF RECORD HIGHEST 200.00 1953 LOWEST 343.41 MAR 07, 1977 
WELL DESCRIPTIONS AND WATER-LEVEL MEASUREMENTS

WATER YEAR OCTOBER 1991 TO SEPTEMBER 1992

SITE NUMBER 343939118025201

LOCAL NUMBER 007N011W28Q01S

About 4.5 miles southeast of Lancaster, $0.45 \mathrm{mile}$ west of 50 th Street East on Avenue L. Unused well. Diameter

17 inches, depth unknown. Altitude of land-surface datum 2,453 feet. Water-level records available 1963,1982 to current year.

WATER LEVELS IN FEET BELOW LAND-SURFACE DATUM

$$
\begin{array}{ll} 
& \text { WATER } \\
\text { DATE } & \text { LEVEL MS }
\end{array}
$$

APR 15, $1992 \quad 276.82 \mathrm{~S}$

PERIOD OF RECORD HIGHEST 267.84 DEC 31, $1963 \quad$ LOWEST 315.85 OCT 08, 1982

SITE NUMBER 344006118033001

LOCAL NUMBER 007N011W29H01S

About 4 miles southeast of Lancaster, 35 feet west of 40 th Street East along Avenue K-8. Drilled irrigation water-table well. Diameter 14 inches, depth 679 feet, perforated 319-679 feet. Altitude of land-surface datum 2,442 feet. Water-level records available 1959-60, 1963, 1974 to current year.

WATER LEVELS IN FEET BELOW LAND-SURFACE DATUM

$$
\text { DATE WATER }
$$

APR 16, $1992269.15 \mathrm{~S}$

PERIOD OF RECORD HIGHEST 249.00 DEC 10, 1963 LOWEST 313.02 APR 15, 1981

SITE NUMBER 343909118053601

LOCAL NUMBER 007N011W31M01S

North of Edwards AFB (Plant 42), $0.5 \mathrm{mlle}$ north of Avenue M. Unused water-table well. Diameter 14 inches, depth measured 385.6 feet in 1974. Aititude of land-surface datum 2,468 feet. Water-leve1 records avallable $1951-52$, $1964,1974-75,1977$ to current year.

WATER LEVELS IN FEET BELOW LAND-SURFACE DATUM

$\begin{array}{ll} & \text { WATER } \\ \text { DATE } & \text { LEVEL MS }\end{array}$

APR 15, $1992314.69 \mathrm{~s}$

PERIOD OF RECORD HIGHEST 184.57 MAR 04, 1952 LOWEST 314.69 ARR 15, 1992

SITE NUMBER 343847118032001

LOCAL NUMBER 007N011W33N01S

North of Edwards AFB (Plant 42), east of intersection of 40 th Street East and Avenue M. Irrigation water-table well. Diameter 20 inches, depth measured 351.22 feet in 1992 . Altitude of land-surface datum 2,473 feet. Waterlevel records available 1951, 1954, 1956-65, 1967-68, 1970 to current year.

WATER LEVELS IN FEET BELOW LAND-SURFACE DATUM

$$
\text { DATE WATER } \quad \text { LEVEL MS }
$$

APR 15, $1992 \quad 293.96 \mathrm{~S}$

PERIOD OF RECORD HIGHEST 215.39 NOV 17, 1951 LOWEST 327.80 MAR 09, 1976 
SITE NUMBER 344217118054101

LOCAL NUMBER 007N012W12R01S

In Lancaster about 225 feet west of 20 th Street East and 250 feet north of Avenue 1 . Unused well. Diameter 6 inches, depth unknown. Altitude of land-surface datum 2,375 feet. Water-level records available 1963, 1978-81, 1983 to current year.

WATER LEVELS IN FEET BELOW LAND-SURFACE DATUM

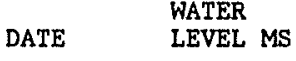

APR 14, $1992 \quad 133.44 \mathrm{~S}$

PERIOD OF RECORD HIGHEST 94.90 SEP $25,1963 \quad$ LOWEST $142.00 \quad$ SEP 20,1978

SITE NUMBER 344159118083101

LOCAL NUMBER 007N012W15F01S

In Lancaster about 50 feet north of Kittering Street and 130 feet west of Elm Street. Domestic well. Diameter and depth unknown. Altitude of land-surface datum 2,348 feet. Water-1evel records available 1924, 1934, 1942-63, 1965-66, 1968-1992. We11 destroyed.

WATER LEVELS IN FEET BELOW LAND-SURFACE DATUM

$$
\begin{array}{ll} 
& \text { WATER } \\
\text { DATE } & \text { LEVEL MS }
\end{array}
$$

APR 13, 1992

$$
\text { W }
$$

PERIOD OF RECORD HIGHEST 26.70 OCT 28, 1942 LOWEST 184.07 MAR 28, 1988

SITE NUMBER 344030118110001

LOCAL NUMBER 007N012W19R01S

West of Lancaster about 100 feet north of Avenue $K$ and 200 feet west of 30 th Street West. Unused well. Diameter 12 inches, depth 400 feet, perforated $100-400$ feet. Altitude of land-surface datum 2,386 feet. Some measurements provided by Los Angeles County Flood Control District, Los Angeles County Engineers, and California Water Rights Board 1951-67. Water-level records available 1951, 1953, 1961 to current year.

WATER LEVELS IN FEET BELOW LAND-SURFACE DATUM

$$
\begin{array}{ll} 
& \text { WATER } \\
\text { DATE } & \text { LEVEL MS }
\end{array}
$$

APR $14,1992 \quad 228.45 \mathrm{~S}$

PERIOD OF RECORD HIGHEST 96.00 NOV 07, 1951 LOWEST 229.05 MAR 18, 1991

SITE NUMBER 344109118090601

LOCAL NUMBER 007N012W21A04S

In Lancaster, $0.25 \mathrm{mile}$ west of 10 th Street West, and $0.25 \mathrm{mile}$ south of Avenue J. Unused well. Diameter 8 inches, depth unknown. Altitude of land-surface datum 2,365 feet. Water-level records available 1963 , 1974 to current year.

WATER LEVELS IN FEET BELOW LAND-SURFACE DATUM

$$
\begin{array}{ll} 
& \text { WATER } \\
\text { DATE } & \text { LEVEL MS }
\end{array}
$$

APR 13, $1992203.90 \mathrm{~S}$

PERIOD OF RECORD HIGHEST 124.70 DEC 13, 1963 LOWEST $203.90 \quad$ APR 13,1992 
WELL DESCRIPTIONS AND WATER-LEVEL MEASUREMENTS

WATER YEAR OCTOBER 1991 TO SEPTEMBER 1992

SITE NUMBER 344043118080301

LOCAL NUMBER O07N012W22K01S

In Lancaster, 360 feet west of Sierra Highway, and $0.3 \mathrm{mile}$ north of Avenue K. Unused water-table well. Diameter 8 inches, depth reported 400 feet. Altitude of land-surface datum 2,407 feet. Some measurements provided by Los Angeles County Flood Control District. Water-level records available 1961,1963 to current year.

WATER LEVELS IN FEET BELOW LAND-SURFACE DATUM

$\begin{array}{ll} & \text { WATER } \\ \text { DATE } & \text { LEVEL MS }\end{array}$

APR 13, $1992 \quad 256.68 \mathrm{~V}$

PERIOD OF RECORD HIGHEST 169.60 MAY 01, 1961 LOWEST 257.68 MAR 13, 1990

SITE NUMBER 344120118055301

LOCAL NUMBER 007N012W24A01S

In Lancaster about $0.8 \mathrm{mile}$ east of $10 \mathrm{th}$ Street East and 250 feet south of Avenue $\mathrm{J}$. Drilled unused well.

Diameter 8 inches, depth measured 230.5 feet in 1982 , perforated 180-400 feet. Altitude of land-surface datum

2,401 feet. Water-level records available 1963, 1982 to current year.

WATER LEVELS IN FEET BELOW LAND-SURFACE DATUM

$$
\text { DATE WATER }
$$

APR $14,1992 \quad 222.28 \mathrm{~V}$

PERIOD OF RECORD HIGHEST 177.74 OCT 03,1963 LOWEST 223.50 MAR 31,1988

SITE NUMBER 344032118060601

LOCAL NUMBER 007N012W24Q03S

In Lancaster, $0.5 \mathrm{mile}$ west of $20 \mathrm{th}$ Street East, and 200 feet north of Avenue K. Dri1led domestic well. Diameter 8 inches, depth reported 630 feet. Altitude of land-surface datum 2,435 feet. Water-level records available 1982 to current year.

WATER LEVELS IN FEET BELOW LAND-SURFACE DATUM

$$
\text { DATE WATER }
$$

APR $14,1992 \quad 270.19 \mathrm{~V}$

PERIOD OF RECORD HIGHEST 263.07 MAY 09, 1983 LOWEST 270.19 APR 14,1992

SITE NUMBER 344004118075901

LOCAL NUMBER 007N012W27H01S

In Lancaster, $0.14 \mathrm{mile}$ west of Sierra Highway, and $0.5 \mathrm{mile}$ south of Avenue $\mathrm{K}$. Unused water-table well.

Diameter 14 inches, depth reported 500 feet, perforated 189-500 feet. Altitude of land-surface datum 2,449 feet. Water-level records avallable 1949, 1954, 1959, 1964, 1974 to current year.

WATER LEVELS IN FEET BELOW LAND-SURFACE DATUM

$$
\text { DATE WATER }
$$

APR 13, $1992311.02 \mathrm{~S}$

PERIOD OF RECORD HIGHEST 151 DEC 1949 LOWEST 328,20 MAR 22, 1989 
SITE NUMBER 344338118151301

LOCAL NUMBER 007N013W03E01S

About 7 miles west of Lancaster, $0.4 \mathrm{mile}$ south of Avenue G. Unused water-table well. Diameter 12 inches 0-75 feet, 10 inches 75-400 feet, depth 400 feet, perforated 69-400 feet. Altitude of land-surface datum 2,381 feet. Water-level records available 1920, 1963, 1967 to current year.

WATER LEVELS IN FEET BELOW LAND-SURFACE DATUM

$$
\begin{array}{lll} 
& \text { WATER } & \text { WATER } \\
\text { DATE } & \text { LEVEL MS } & \text { DATE }
\end{array}
$$

OCT 24, $1991 \quad 159.69 \mathrm{~S} \quad$ APR 14, $1992155.60 \mathrm{~S}$

PERIOD OF RECORD HIGHEST 32.00 JAN 16, 1920 LOWEST 219.10 MAY 29, 1963

SITE NUMBER 344122118125701

LOCAL NUMBER 007N013W13N01S

About 5 miles west of Lancaster, 55 feet north of Avenue J, and $0.1 \mathrm{mile}$ east of 50 th Street West. Unused well. Diameter unknown, depth measured 458.08 feet in 1992. Altitude of land-surface datum 2,350 feet. Water-1evel records avallable 1978 to current year.

WATER LEVELS IN FEET BELOW LAND-SURFACE DATUM

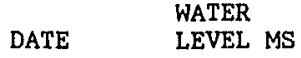

APR $14,1992143.00 \mathrm{~S}$

PERIOD OF RECORD HIGHEST 142.72 MAR 24,1980 LOWEST 157.55 DEC 08, 1978

SITE NUMBER 344213118151301

LOCAL NUMBER 007N013W16A04S

About 7 miles west of Lancaster, southwest of the intersection of Avenue I and 70 th Street West. Domestic well. Diameter 10 inches, depth unknown. Altitude of land-surface datum 2,367 feet. Water-level records available 1978 to current year.

WATER LEVELS IN FEET BELOW LAND-SURFACE DATUM

$$
\text { DATE WATER } \quad \text { LEVEL MS }
$$

APR 14, 1992108.89 SR

PERIOD OF RECORD HIGHEST 98.58 SEP 20, 1978 LOWEST 107.57 MAR 18, 1991

SITE NUMBER 344000118130601

LOCAL NUMBER 007N013W26J02S

In Quartz Hill near Avenue $\mathrm{K}-10$ and 50 th Street West. Drilled public-supply water-table well. Diameter 14 inches, depth 606 feet, perforated 288-606 feet. Altitude of land-surface datum 2, 417 feet. Water-level records avallable $1957-61,1963-64,1967,1969$ to current year.

WATER LEVELS IN FEET BELOW LAND-SURFACE DATUM

$$
\begin{array}{ll} 
& \text { WATER } \\
\text { DATE } & \text { LEVEL MS }
\end{array}
$$

APR $14,1992 \quad 236.82$ SS

PERIOD OF RECORD HIGHEST 216.00 APR 05, 1957 LOWEST 329.60 AUG 24,1972 
WELL DESCRIPTIONS AND WATER-LEVEL MEASUREMENTS

WATER YEAR OCTOBER 1991 TO SEPTEMBER 1992

SITE NUMBER 343932118144001

LOCAL NUMBER 007N013W34B01S

West of Quartz Hill, west of intersection of Avenue L and 60 th Street West. Drilled irrigation water-table well. Diameter 14 inches, depth 475 feet, perforated 250-475 feet. Alt1tude of land-surface datum 2,433 feet. Water-level records available 1963, 1967 to current year.

WATER LEVELS IN FEET BELOW LAND-SURFACE DATUM

$$
\begin{array}{ll} 
& \text { WATER } \\
\text { DATE } & \text { LEVEL MS }
\end{array}
$$

APR 14, $1992220.50 \mathrm{~V}$

PERIOD OF RECORD HIGHEST 219.25 MAR 19, 1991 LOWEST 371.14 OCT 27, 1971

SITE NUMBER 344209118183801

LOCAL NUMBER 007N014W13A01S

About 10 miles west of Lancaster, 200 feet south of Avenue I, and 0.2 mile west of $100 t h$ Street West. Drilled irrigation water-table well. Diameter 12 inches, depth 519 feet, perforated 249-519 feet. Altitude of land-surface datum 2,467 feet. Records 1953-62 provided by Southern California Edison. Water-level records available 1953-63, 1967 to current year.

WATER LEVELS IN FEET BELOW LAND-SURFACE DATUM

$$
\begin{array}{ll} 
& \text { WATER } \\
\text { DATE } & \text { LEVEL MS }
\end{array}
$$

APR 14, $1992 \quad 234.41 \mathrm{~S}$

PERIOD OF RECORD HIGHEST 219.00 OCT 14,1953 LOWEST 307.23 OCT 22,1970

SITE NUMBER 344911117524001

LOCAL NUMBER 008N009W06D01S

South of Rogers Lake, about $0.8 \mathrm{mile}$ north of Avenue B. Drilled unused well. Diameter 8 inches, original depth 200 feet, depth measured 135.6 feet in 1991. Altitude of land-surface datum 2,293 feet. Water-level records available 1959 to current year.

WATER LEVELS IN FEET BELOW LAND-SURFACE DATUM

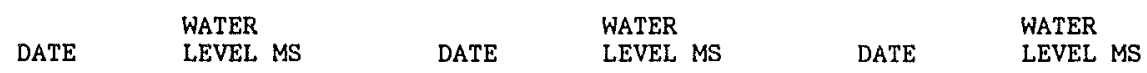

$\begin{array}{llllllllll}\text { JAN 09, 1992 } & 40.73 \mathrm{~S} & \text { APR 07, } 1992 & 39.82 \mathrm{~S} & \text { JUL 08, } 1992 & 42.61 \mathrm{~S} \\ \text { FEB 04 } & 40.83 \mathrm{~S} & \text { MAY 09 } & & 40.23 \mathrm{~S} & \text { AUG 06 } & 42.44 \mathrm{~S} \\ \text { MAR } 10 & & 39.96 \mathrm{~S} & \text { JUN } 16 & & 40.61 \mathrm{~S} & \text { SEP } 09 & 42.05 \mathrm{~S}\end{array}$

PERIOD OF RECORD HIGHEST 26.90 MAR 12, 1959 LOWEST 47,30 APR 13, 1983

SITE NUMBER 344910117532402

LOCAL NUMBER O08N010W01C02S

On Edwards AFB. Drilled public-supply well. Diameter 16 inches, depth 700 feet, perforated $300-690$ feet. Altitude of land-surface datum 2,296.36 feet. Water-level records available 1984, 1992.

WATER LEVELS IN FEET BELOW LAND-SURFACE DATUM

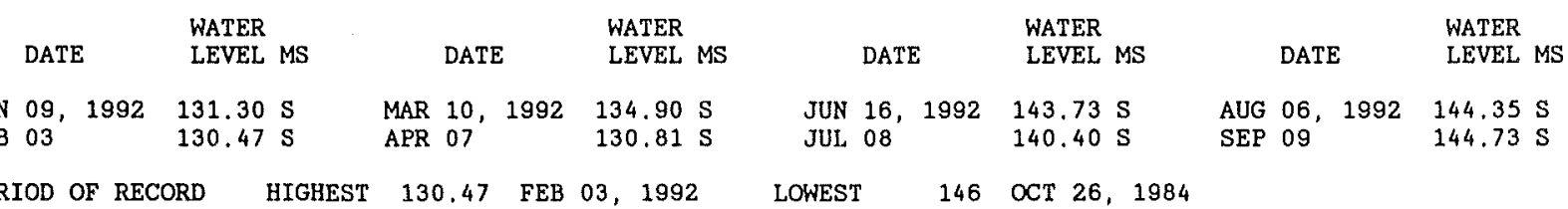


SITE NUMBER 344835117531301

LOCAL NUMBER 008N010W01Q01S

On Edwards AFB, about 2 miles south of Rogers Lake. Drilled observation well in alluvium. Diameter 2 inches, depth 1,020 feet, perforated 980-1,010 feet. Altitude of land-surface datum 2,301.85 feet. Water-level records avallable 1990 to current year.

WATER LEVELS IN FEET BELOW LAND-SURFACE DATUM

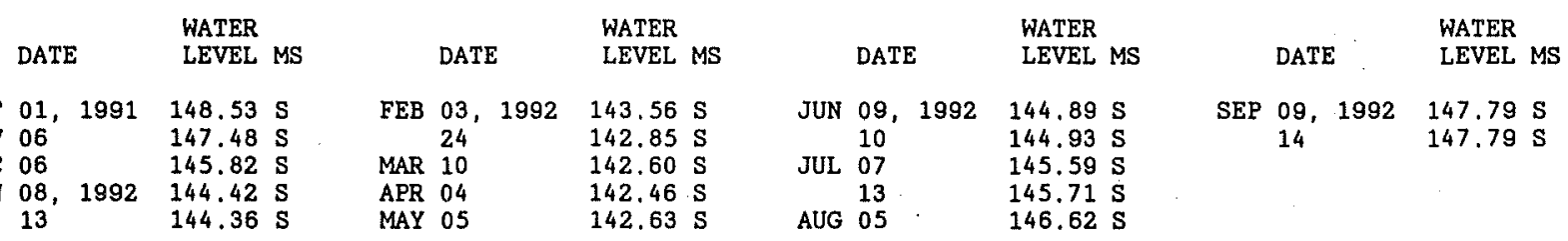

PERIOD OF RECORD HIGHEST 142.46 APR 04, 1992

LOWEST 149.17 AUG 03,1990

SITE NUMBER 344835117531302

LOCAL NUMBER 008N010W01Q02S

On Edwards AFB, about 2 miles south of Rogers Lake. Drilled observation well in alluvium. Diameter 3 inches, depth 643 feet. Deepest of three nested wells. Altitude of land-surface datum 2301.72 feet. Water-level records available 1990 to current year.

WATER LEVELS IN FEET BELOW LAND-SURFACE DATUM

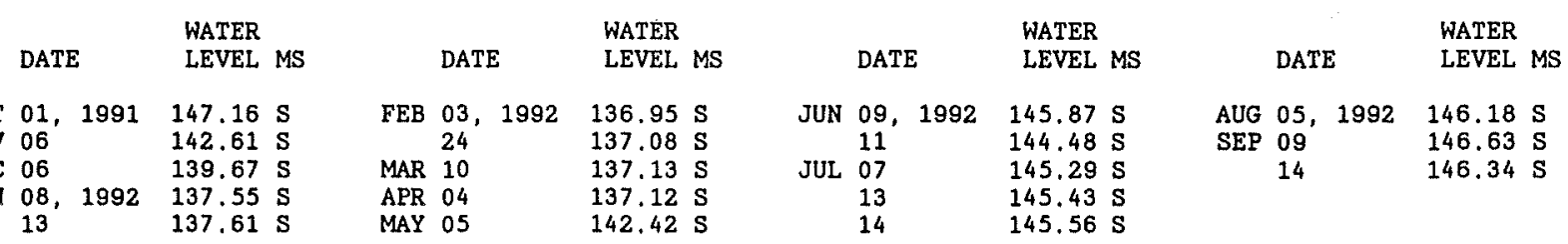

PERIOD OF RECORD HIGHEST 136.95 FEB 03, 1992 LOWEST 149.64 AUG 03, 1990

SITE NUMBER 344835117531303

LOCAL NUMBER OOBNO10W01Q03S

On Edwards AFB, about 2 miles south of Rogers Lake. Drilled observation well in alluvium. Diameter 2 inches, depth 475 feet. Middle of three nested wells. Altitude of land-surface datum 2,301.72 feet. Water-level records avallable 1990 to current year.

WATER LEVELS IN FEET BELOW LAND-SURFACE DATUM

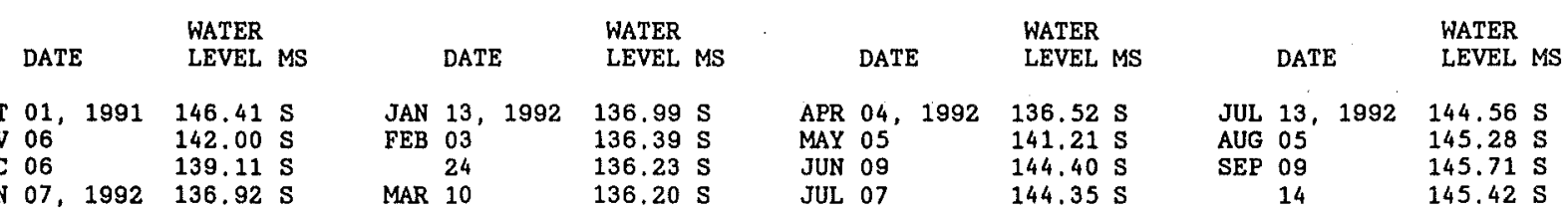

PERIOD OF RECORD HIGHEST 136.20 MAR 10, 1992 LOWEST 148.57 AUG 03, 1990 
WELL DESCRIPTIONS AND WATER-LEVEL MEASUREMENTS

WATER YEAR OCTOBER 1991 TO SEPTEMBER 1992

SITE NUMBER 344835117531304

LOCAL NUMBER 008N010W01Q04S

On Edwards AFB, about 2 miles south of Rogers Lake. Drilled observation well in alluvium. Diameter 2 inches, depth 110 feet, perforated 80-110 feet. Shallowest of three nested wells. Altitude of land-surface datum $2,301.72$ feet, Water-level records available 1990 to current year.

WATER LEVELS IN FEET BELOW LAND-SURFACE DATUM

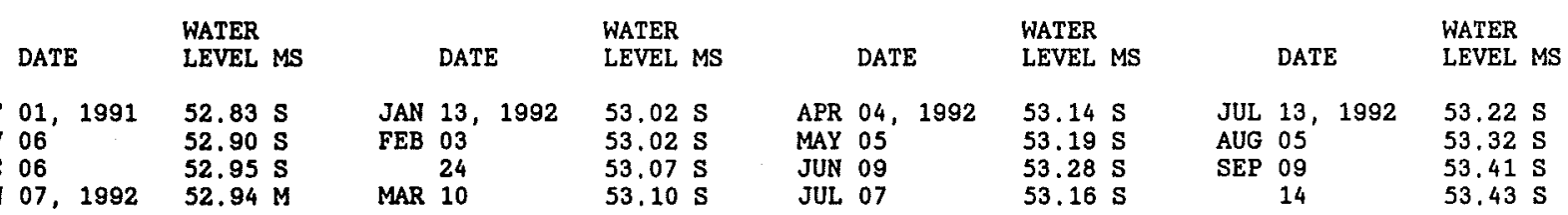

PERIOD OF RECORD HIGHEST 51.98 MAY 10, 1990 LOWEST 54.43 SEP 14, 1992

SITE NUMBER 344833117561301

LOCAL NUMBER O08N010W04R01S

On Edwards AFB. Drilled observation well. Diameter 2 inches, depth 980 feet, perforated 920-960 feet. Altitude of land-surface datum 2,305 feet. Water-level records available 1991 to current year.

WATER LEVELS IN FEET BELOW LAND-SURFACE DATUM

\begin{tabular}{lllllll} 
DATE & $\begin{array}{l}\text { WATER } \\
\text { LEVEL MS }\end{array}$ & \multicolumn{2}{c}{ DATE } & $\begin{array}{l}\text { WATER } \\
\text { LEVEL MS }\end{array}$ & DATE & $\begin{array}{l}\text { WATER } \\
\text { LEVEL MS }\end{array}$ \\
09,1992 & $146.71 \mathrm{~S}$ & APR 05, 1992 & $143.66 \mathrm{~S}$ & JUL 08, 1992 & $144.39 \mathrm{~S}$ \\
03 & $145.83 \mathrm{~S}$ & MAY 07 & $143.44 \mathrm{~S}$ & AUG 11 & $145.36 \mathrm{~S}$ \\
09 & $144.43 \mathrm{~S}$ & JUN 16 & $144.31 \mathrm{~S}$ & SEP 09 & $146.28 \mathrm{~S}$
\end{tabular}

PERIOD OF RECORD HIGHEST 143.44 MAY 07, 1992 LOWEST 146.71 JAN 09, 1992

SITE NUMBER 344833117561302

LOCAL NUMBER 008NO1OWO4RO2S

On Edwards AFB. Drilled observation well. Diameter 2 inches, depth 750 feet, perforated $700-740$ feet. Altitude of land-surface datum 2,305 feet. Water-level records available 1991 to current year.

WATER LEVELS IN FEET BELOW LAND-SURFACE DATUM

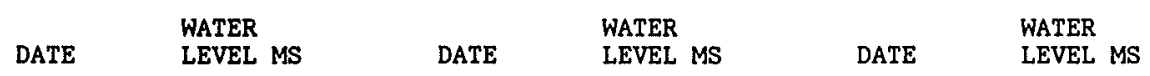

$\begin{array}{lllllllll}\text { JAN 09, } 1992 & 143.00 \mathrm{~S} & \text { APR 05, } 1992 & 140.27 \mathrm{~S} & \text { JUL 08, } 1992 & 143.36 \mathrm{~S} \\ \text { FEB 03 } & 142.00 \mathrm{~S} & \text { MAY 07 } & 140.89 \mathrm{~S} & \text { AUG } 11 & 144.60 \mathrm{~S} \\ \text { MAR 10 } & 140.59 \mathrm{~S} & \text { JUN } 16 & 143.14 \mathrm{~S} & \text { SEP 09 } & 145.70 \mathrm{~S} \\ \text { PERIOD OF RECORD } & \text { HIGHEST } & 140.27 & \text { APR 05, } 1992 & \text { LOWEST } 145.70 & \text { SEP 09, } 1992\end{array}$


SITE NUMBER 344833117561303

LOCAL NUMBER 008N010W04R03S

On Edwards AFB. Drilled observation well. Diameter 2 inches, depth 546 feet, perforated 516-536 feet. Altitude of land-surface datum 2,305 feet. Water-level records available 1991 to current year.

WATER LEVELS IN FEET BELOW LAND-SURFACE DATUM

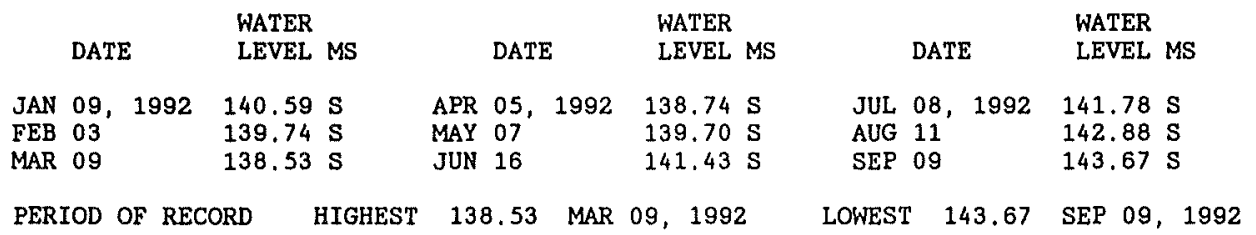

SITE NUMBER 344833117561304

LOCAL NUMBER 008N010W04R04S

On Edwards AFB. Drilled observation well. Diameter 2 inches, depth 250 feet, perforated 220-240 feet. Altitude of land-surface datum 2,305 feet. Water-level records available 1991 to current year.

WATER LEVELS IN FEET BELOW LAND-SURFACE DATUM

\begin{tabular}{llllll}
\multicolumn{1}{c}{ WATER } & \multicolumn{3}{c}{ WATER } \\
DATE & LEVEL MS & DATE & LEVEL MS & DATE & WATER \\
LEVEL MS
\end{tabular}

PERIOD OF RECORD HIGHEST 132.00 MAR 09, 1992 LOWEST 135.28 SEP 09, 1992

SITE NUMBER 344833117561305

LOCAL NUMBER 008N010W04R05S

On Edwards AFB. Drilled observation well. Diameter 2 inches, depth 150 feet, perforated 135-150 feet. Altitude of land-surface datum 2,305 feet. Water-level records available 1991 to current year.

WATER LEVELS IN FEET BELOW LAND-SURFACE DATUM

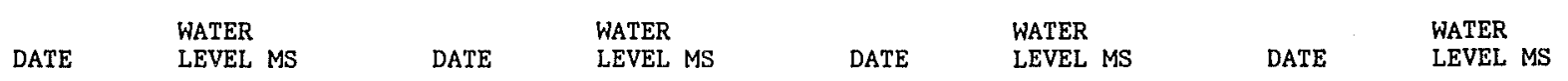

$\begin{array}{llllllllll}\text { OCT 30, 1991 } & 89.54 \mathrm{~S} & \text { MAR 09, } 1992 & 88.93 \mathrm{~S} & \text { JUN } 16,1992 & 89.14 \mathrm{~S} & \text { SEP } 09,1992 & 89.42 \mathrm{~S} \\ \text { JAN 09, 1992 } & 89.17 \mathrm{~S} & \text { APR 05 } & 88.87 \mathrm{~S} & \text { JUL 08 } & 89.11 \mathrm{~S} & & \\ \text { FEB 03 } & 89.11 \mathrm{~S} & \text { MAY 07 } & 89.01 \mathrm{~S} & \text { AUG } 11 & 89.25 \mathrm{~S} & & \end{array}$

PERIOD OF RECORD HIGHEST 88.87 APR 05, 1992 LOWEST 89.54 OCT 30,1991

SITE NUMBER 344833117561306

LOCAL NUMBER 008N010W04R06S

On Edwards AFB. Drilled observation well. Diameter 2 inches, depth 100 feet, perforated 80-100 feet. Altitude of land-surface datum 2,305 feet. Water-level records available 1991 to current year.

WATER LEVELS IN FEET BELOW LAND-SURFACE DATUM

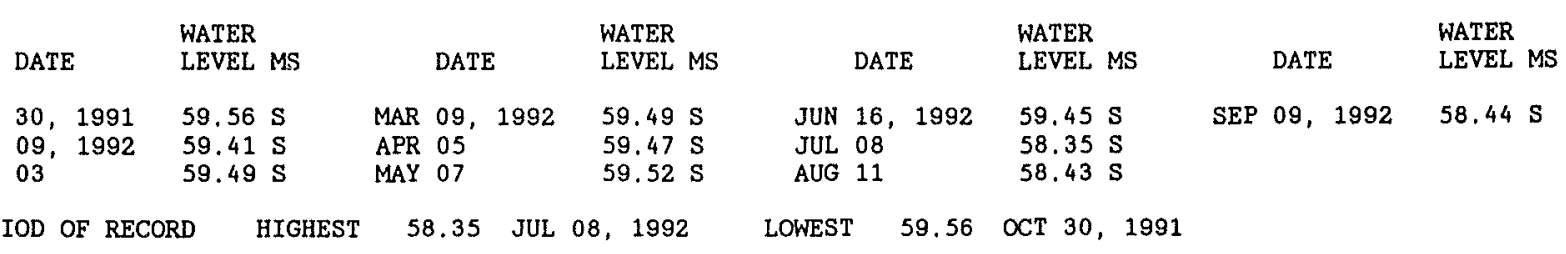


WELL DESCRIPTIONS AND WATER-LEVEL MEASUREMENTS

WATER YEAR OCTOBER 1991 TO SEPTEMBER 1992

SITE NUMBER 344921117570601

LOCAL NUMBER OOBNO1OWOSA01S

On Edwards AFB, 2 miles west of Lancaster Boulevard on Branch Park Road. Drilled observation well in alluvium. Diameter 2 inches, depth 947 feet, perforated 897-927 feet. Altitude of land-surface datum 2,287 feet. Water-level records available 1990 to current year.

WATER LEVELS IN FEET BELOW LAND-SURFACE DATUM

$\begin{array}{lllll} & \text { WATER } & \text { WATER } & \text { WATER } \\ \text { DATE } & \text { LEVEL MS } & \text { DATE } & \text { LEVEL MS } & \text { DATE } \\ \text { LEVEL MS }\end{array}$

$\begin{array}{lllllllllll}\text { JAN 09, } 1992 & 127.23 \mathrm{~S} & \text { MAR } 31,1992 & 125.72 \mathrm{~S} & \text { JUL } 08,1992 & 125.21 \mathrm{~S} \\ \text { FEB } 03 & 126.79 \mathrm{~S} & \text { MAY } 08 & & 125.48 \mathrm{~S} & \text { AUG } 11 & 125.73 \mathrm{~S} \\ \text { MAR 09 } & 126.18 \mathrm{~S} & \text { JUN } 16 & 125.72 \mathrm{~S} & \text { SEP } 09 & 126.16 \mathrm{~S}\end{array}$

PERIOD OF RECORD HIGHEST 125.21 JUL 08, 1992 LOWEST 128.03 JAN 30,1991

SITE NUMBER 344921117570602

LOCAL NUMBER 008N010W05A02S

On Edwards AFB. Drilled observation well. Diameter 2 inches, depth 560 feet, perforated 530-550 feet. Altitude of land-surface datum 2,287 feet. Water-ievel records available 1991 to current year.

WATER LEVELS IN FEET BELOW LAND-SURFACE DATUM

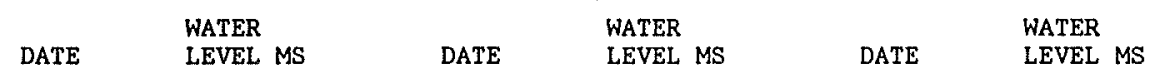

$\begin{array}{llllllllll}\text { JAN 09, } 1992 & 126.44 \mathrm{~S} & \text { MAR } 31,1992 & 124.60 \mathrm{~S} & \text { JUL 08, } 1992 & 125.56 \mathrm{~S} \\ \text { FEB 03 } & 125.80 \mathrm{~S} & \text { MAY } 08 & 124.75 \mathrm{~S} & \text { AUG } 11 & & 126.18 \mathrm{~S} \\ \text { MAR 09 } & 124.93 \mathrm{~S} & \text { JUN } 16 & 125.52 \mathrm{~S} & \text { SEP } 09 & 126.74 \mathrm{~S}\end{array}$

PERIOD OF RECORD HIGHEST 124.60 MAR 31, 1992 LOWEST 127.02 JUL 15, 1991

SITE NUMBER 344921117570603

LOCAL NUMBER 008N010W05A03S

On Edwards AFB. Drilled observation well. Diameter 2 inches, depth 390 feet, perforated 360-380 feet. Altitude of land-surface datum 2,287 feet. Water-level records available 1991 to current year,

WATER LEVELS IN FEET BELOW LAND-SURFACE DATUM

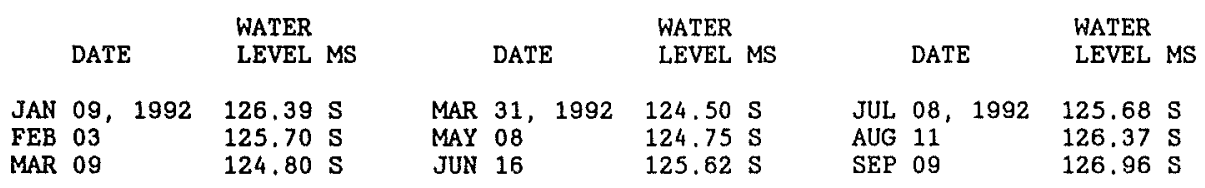

PERIOD OF RECORD HIGHEST 124,50 MAR 31, 1992 LOWEST 126.96 SEP 09, 1992

SITE NUMBER 344921117570604

LOCAL NUMBER OO8N010W05A04S

On Edwards AFB. Drilled observation well. Diameter 2 inches, depth 274 feet, perforated 246-266 feet. Altitude of land-surface datum 2,287 feet. Water-level records avadlable 1991 to current year.

WATER LEVELS IN FEET BELOW LAND-SURFACE DATUM

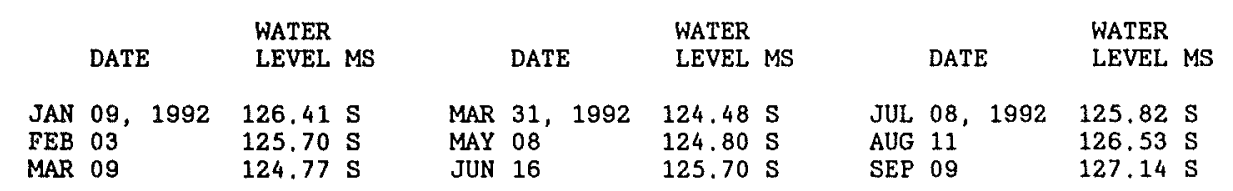

PERIOD OF RECORD HIGHEST 121.99 JUL 15,1991 LOWEST 127.14 SEP 09, 1992 
WELL DESCRIPTIONS AND WATER-LEVEL MEASUREMENTS

WATER YEAR OCTOBER 1991 TO SEPTEMBER 1992

SITE NUMBER 344921117570606

LOCAL NUMBER O08N010W05A06S

On Edwards AFB. Drilled observation well. Diameter 2 inches, depth 55 feet, perforated 30-50 feet. Altitude of land-surface datum 2,287 feet. Water-level records available 1991 to current year.

WATER LEVELS IN FEET BELOW LAND-SURFACE DATUM

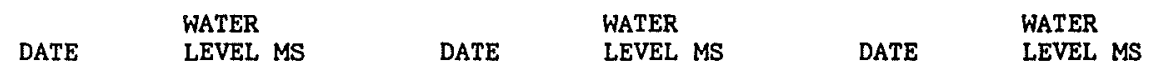

$\begin{array}{lllllllll}\text { JAN } 09,1992 & 28.36 \mathrm{~S} & \text { MAR } 31,1992 & 28.38 \mathrm{~S} & \text { JUL } 08,1992 & 28.49 \mathrm{~S} \\ \text { FEB } 03 & 28.36 \mathrm{~S} & \text { MAY } 08 & & 28.39 \mathrm{~S} & \text { AUG } 11 & 28.56 \mathrm{~S} \\ \text { MAR } 09 & 28.36 \mathrm{~S} & \text { JUN } 16 & & 28.46 \mathrm{~S} & \text { SEP } 09 & 28.62 \mathrm{~S}\end{array}$

PERIOD OF RECORD HIGHEST 28.02 JUL 15,1991 LOWEST 28.62 SEP 09, 1992

SITE NUMBER 344643117581703

LOCAL NUMBER O08N010W18P03S

On Edwards AFB. Unused well. Diameter 14 inches, depth unknown. Altitude of land-surface datum 2,325 feet. Water-level records available 1951, 1992.

WATER LEVELS IN FEET BELOW LAND-SURFACE DATUM

\begin{tabular}{|c|c|c|c|c|c|c|c|}
\hline DATE & $\begin{array}{l}\text { WATER } \\
\text { LEVEL MS }\end{array}$ & DATE & $\begin{array}{l}\text { WATER } \\
\text { LEVEL MS }\end{array}$ & DATE & $\begin{array}{l}\text { WATER } \\
\text { LEVEL MS }\end{array}$ & DATE & $\begin{array}{l}\text { WATER } \\
\text { LEVEL MS }\end{array}$ \\
\hline $\begin{array}{l}07,1992 \\
12\end{array}$ & $\begin{array}{l}93.28 \mathrm{~S} \\
93.39 \mathrm{~s}\end{array}$ & $\begin{array}{ll}\text { MAR } 31, & 1992 \\
\text { MAY } 06 & \end{array}$ & $\begin{array}{l}93.42 \mathrm{~S} \\
93.46 \mathrm{~S}\end{array}$ & $\begin{array}{l}\text { JUN } 17,1992 \\
\text { JUL } 08\end{array}$ & $\begin{array}{l}93.54 \mathrm{~s} \\
93.57 \mathrm{~s}\end{array}$ & $\begin{array}{l}\text { AUG } 05,1992 \\
\text { SEP 09 }\end{array}$ & $\begin{array}{l}93.57 \mathrm{~s} \\
93.62 \mathrm{~s}\end{array}$ \\
\hline
\end{tabular}

PERIOD OF RECORD HIGHEST 78.52 MAY 24, 1951 LOWEST 93.62 SEP 09, 1992

SITE NUMBER 344627117543001

LOCAL NUMBER 008N010W23F02S

South of Edwards AFB, about 0.25 mile east of 120 th Street East, and 0.25 mile south of Avenue D. Drilled domestic well. Diameter 12 inches, depth 226 feet, perforated 120-226 feet. Altitude of land-surface datum 2,350 feet. Water-level records available 1968, 1970-72, 1974 to current year.

WATER LEVELS IN FEET BELOW LAND-SURFACE DATUM

$$
\text { DATE WATER }
$$

APR $15,1992138.43 \mathrm{~S}$

PERIOD OF RECORD HIGHEST 108.10 MAR 06, 1968 LOWEST 138.43 AER 15,1992

SITE NUMBER 344542117562301

LOCAL NUMBER 008N010W28B01S

South of Edwards AFB, about $0.8 \mathrm{mile}$ east of $100 \mathrm{th}$ Street East, and $0.2 \mathrm{mile}$ south of Avenue E. Drilled unused well in alluvium. Diameter 16 inches, depth 215 feet. Altitude of land-surface datum 2,358 feet. Water-1evel records available 1951-54, 1956-78, 1982 to current year.

WATER LEVELS IN FEET BELOW LAND-SURFACE DATUM

$$
\text { DATE WATER }
$$

APR $15,1992 \quad 151.12 \mathrm{~S}$

PERIOD OF RECORD HIGHEST 66.75 JAN 23, 1951 LOWEST 151.40 MAR 18, 1991 
WELL DESCRIPTIONS AND WATER-LEVEL MEASUREMENTS

WATER YEAR OCTOBER 1991 TO SEPTEMBER 1992

SITE NUMBER 344457117581001

LOCAL NUMBER 008N010W30R01S

South of Edwards AFB, northwest of intersection of Avenue F and 90th Street East. Drilled irrigation well. Diameter 16 inches, depth 1,064 feet, perforated 650-1,064 feet. Altitude of land-surface datum 2,361 feet. Water-level records available 1973, 1975, 1978 to current year.

WATER LEVELS IN FEET BELOW LAND-SURFACE DATUM

$$
\text { DATE WATER }
$$

APR $14,1992143.65 \mathrm{~S}$

PERIOD OF RECORD HIGHEST 131.84 MAR 30,1978 LOWEST 158.2 APR 16, 1981

SITE NUMBER 344643118002001

LOCAL NUMBER 008N011W14R01S

Southeast of Rosamond Lake, northwest of intersection of Avenue $D$ and 70 th Street East. Drilled unused well. Diameter 12 inches, depth measured 167.5 feet in 1960, 164.9 feet in 1991 . Altitude of land-surface datum $2,314.03$ feet. Water-level records available 1960,1966 to current year.

WATER LEVELS IN FEET BELOW LAND-SURFACE DATUM

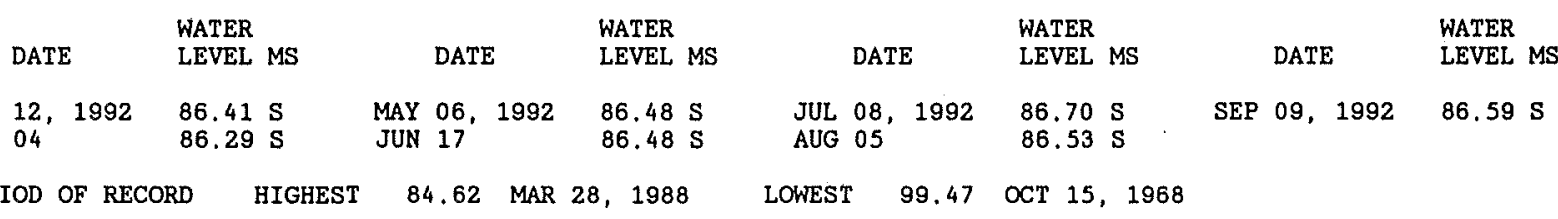

SITE NUMBER 344641118015301

LOCAL NUMBER 008N011W15Q01S

Southeast of Rosamond Lake, 0.5 mile east of 50 th Street East, and 40 feet north of Avenue D. Drilled unused well. Diameter 12 inches, depth measured 179.2 feet in 1958, 177.8 feet in 1991. Altitude of land-surface datum 2,303.80 feet. Water-level records available 1952, 1954, 1958-72, 1974 to current year.

WATER LEVELS IN FEET BELOW LAND-SURFACE DATUM

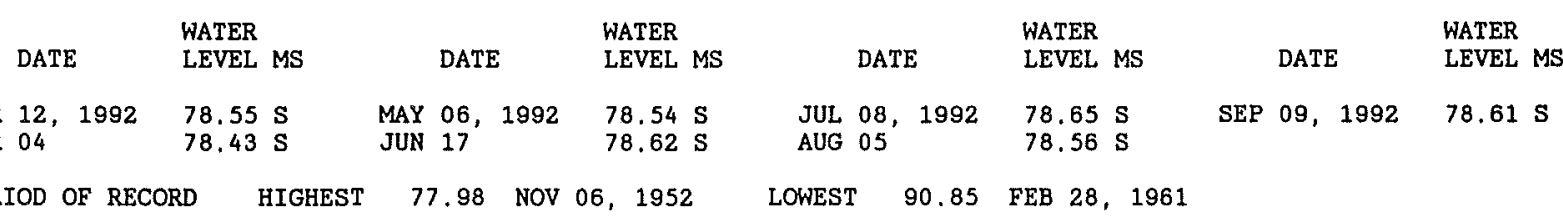

SITE NUMBER 344548118005401

LOCAL NUMBER 008N011W22P02S

Southeast of Rosamond Lake, northeast of intersection of Avenue E and 50th Street East. Drilled unused well. Diameter 12 inches, depth measured 202.3 feet in 1990 . Altitude of land-surface datum 2,323 feet. Water-1evel records available 1978 to current year.

WATER LEVELS IN FEET BELOW LAND-SURFACE DATUM

$$
\begin{array}{ll}
\text { WATER } & \text { WATER MS }
\end{array}
$$

APR $14,199299.82 \mathrm{~s}$

PERIOD OF RECORD HIGHEST 99.67 MAR 18, 1991 LOWEST 109.75 DEC 08, 1978 
SITE NUMBER 344551117591401

LOCAL NUMBER 008N011W24R02S

Southeast of Rosamond Lake, northwest of intersection of Avenue $E$ and 80 th Street East. Drilled unused water-table well. Diameter 12 inches, original depth 270 feet, depth measured 132.2 feet in 1991, perforated $120-270$ feet. Altitude of land-surface datum 2,337 feet. Water-level records available 1946, 1951, 1973 to current year.

WATER LEVELS IN FEET BELOW LAND-SURFACE DATUM

$$
\text { DATE WATER }
$$

APR 14, $1992 \quad 109.57 \mathrm{~S}$

PERIOD OF RECORD HIGHEST 75 AUG 1946 LOWEST 124.86 MAY 25, 1951

SITE NUMBER 344550117591901

LOCAL NUMBER O08N011W24R03S

Southeast of Rosamond Lake about 0.1 mile west of 80 th Street East. Drilled unused water-table well. Diameter 12 inches, depth 302 feet, perforated 158-302 feet. Altitude of land-surface datum 2,337 feet. Water-level records available 1973 to current year.

WATER LEVELS IN FEET BELOW LAND-SURFACE DATUM

$$
\begin{array}{ll}
\text { WATER } \\
\text { DATE } & \text { LEVEL MS }
\end{array}
$$

APR 14, 1992 So

PERIOD OF RECORD HIGHEST 108.00 APR 13, 1983 LOWEST 121.03 MAR 07, 1977

SITE NUMBER 344443118021301

LOCAL NUMBER O08N011W34D02S

About 5 miles northeast of Lancaster, $0.25 \mathrm{mile}$ south of Avenue F, and 0.2 mile east of 50 th Street East. Drilled domestic well. Diameter 12 inches, depth measured 250.5 feet in 1951. Altitude of land-surface datum 2,340 feet. Water-level records available 1951-54, 1956 to current year.

WATER LEVELS IN FEET BELOW LAND-SURFACE DATUM

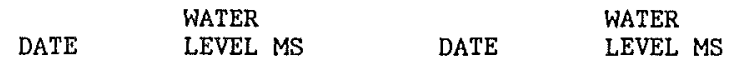

OCT 31, $1991 \quad 123.64 \mathrm{~S} \quad$ APR 15, $1992122.64 \mathrm{SP}$

PERIOD OF RECORD HIGHEST 110.89 FEB 15, 1952 LOWEST 158.09 OCT 26,1961

SITE NUMBER 344403118012101

LOCAL NUMBER 008N011W34RO2S

About 5 miles northeast of Lancaster, northwest of intersection of Avenue $G$ and $60 t h$ Street East. Drilled unused water-table we11. Diameter 12 inches, depth measured 260.1 feet in 1991 . Altitude of land-surface datum 2,358 feet. Water-level records available 1951-52, 1956 to current year.

WATER LEVELS IN FEET BELOW LAND-SURFACE DATUM

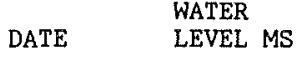

APR 15, $1992131.22 \mathrm{~S}$

PERIOD OF RECORD HIGHEST 126.36 MAR 18, 1991 LOWEST 215.30 SEP 19, 1964 
WELL DESCRIPTIONS AND WATER-LEVEL MEASUREMENTS

WATER YEAR OCTOBER 1991 TO SEPTEMBER 1992

SITE NUMBER 344825118071801

LOCAL NUMBER OOBN012W02Q01S

At west edge of Rosamond Lake. Drilled unused well. Diameter 6 inches, original depth 260 feet, depth measured 226.4 feet in 1965,72.7 feet in 1990. Altitude of land-surface datum 2,283 feet. Water-level records available 1951-54, 1956-77, 1979 to current year.

WATER LEVELS IN FEET BELOW LAND-SURFACE DATUM

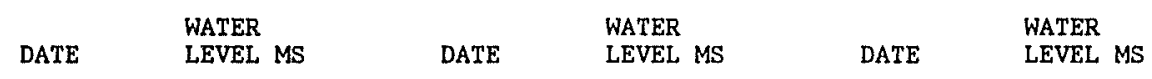

APR 16, $1992 \quad 49.00 \mathrm{~S}$ JUN $17,1992 \quad 49.26 \mathrm{~s} \quad$ AUG $05,1992 \quad 50.30 \mathrm{~s}$

$\begin{array}{llllll}\text { MAY } 06 & 48.94 \mathrm{~S} & \text { JUL } 08 & 49 & & \end{array}$

PERIOD OF RECORD HIGHEST 0.66 APR 14, 1953 LOWEST 50.84 FEB 10, 1982

SITE NUMBER 344913118110001

LOCAL, NUMBER 008N012W05D01S

About 2 miles south of Rosamond, 200 feet east of 30 th Street West, and 130 feet south of Avenue A, Drilled unused water-table well. Diameter 14 inches, depth measured 465.3 feet in 1974 . Altitude of land-surface datum 2,329 feet. Water-level records available 1951, 1974 to current year.

WATER LEVELS IN FEET BELOW LAND-SURFACE DATUM

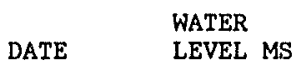

APR 13, $1992112.06 \mathrm{~S}$

PERIOD OF RECORD HIGHEST 41.70 APR 19, 1951 LOWEST 154.30 FEB 12, 1974

SITE NUMBER 344747118075001

LOCAL NUMBER 008N012W10J01S

On Edwards AFB, about $0,3 \mathrm{mile}$ north of Avenue $C$, and 100 feet west of Division Street. Unused well. Diameter 6 inches, depth measured 91 feet in $1974,90.5$ feet in 1992. Altitude of land-surface datum 2,285 feet. Waterlevel records available $1960,1965-67,1974$ to current year.

WATER LEVELS IN FEET BELOW LAND-SURFACE DATUM

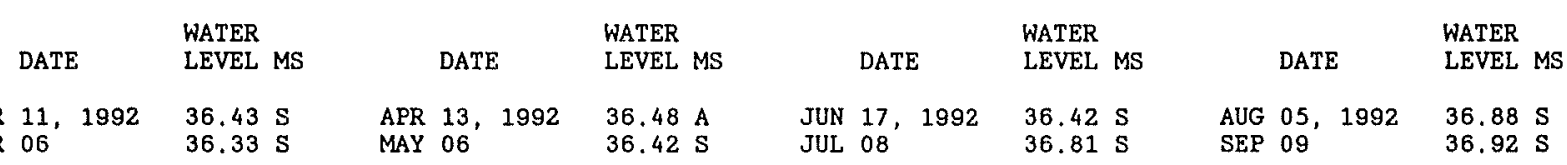

PERIOD OF RECORD HIGHEST 13.67 MAR 23, 1960 LOWEST 37.60 MAR 29, 1988

SITE NUMBER 344547118090601

LOCAL NUMBER 008N012W21R01S

About $6 \mathrm{miles}$ south of Rosamond, $0.3 \mathrm{mile}$ west of Sierra Highway, and 200 feet north of Avenue E. Unused well. Diameter 8 inches, depth measured 138.5 feet in 1982, Altitude of land-surface datum 2,303 feet. Water-1evel records available 1982 to current year.

WATER LEVELS IN FEET BELOW LAND-SURFACE DATUM

$$
\text { DATE WATER }
$$

APR $13,199271.05 \mathrm{~S}$

PERIOD OF RECORD HIGHEST 71.05 APR 13, 1992 LOWEST 83.65 OCT 08,1982 
SITE NUMBER 344523118073301

LOCAL NUMBER OOBNO12W26F01S

About 7 miles southeast of Rosamond, 0.3 mile east of Division Street, and 0.4 mile south of Avenue E, Drilled unused well. Diameter 6 inches, depth measured 123.1 feet in 1990 . Altitude of land-surface datum 2,303 feet. Water-level records available 1951, 1974 to current year.

WATER LEVELS IN FEET BELOW LAND-SURFACE DATUM

$$
\text { DATE WATER }
$$

APR $13,1992 \quad 23.50 \mathrm{~S}$

PERIOD OF RECORD HIGHEST 14.27 JAN 14, 24, 1951 LOWEST 23.50 APR 13, 1992

SITE NUMBER 344534118094301

LOCAL NUMBER O08N012W28D01S

About 6 miles south of Rosamond, 0.2 mile south of Avenue $E$, and 0.8 mile west of 10th Street West. Drilled irrigation well. Diameter 12 inches, depth 316 feet, perforated 48-316 feet. Altitude of land-surface datum 2,308 feet. Water-level records available 1951, 1974-75, 1978-80, 1982 to current year.

WATER LEVELS IN FEET BELOW LAND-SURFACE DATUM

$$
\text { DATE WATER } \quad \text { LEVEL MS }
$$

APR 13, $1992 \quad 59.14 \mathrm{~S}$

PERIOD OF RECORD HIGHEST 11.89 APR 26, 1951 LOWEST 64.39 MAR 19, 1986

SITE NUMBER 344517118112801

LOCAL NUMBER 008N012W30K01S

About 7 miles south of Rosamond, 0.5 mile south of Avenue $E$, and 0.4 mile west of 30 th Street West. Drilled unused well in alluvium. Diameter 6 inches, depth measured 170.9 feet in $1965,164.4$ feet in 1990 . Altitude of land-surface datum 2,324 feet. Water-level records available 1951, 1956 to current year.

WATER LEVELS IN FEET BELOW LAND-SURFACE DATUM

$$
\begin{array}{ll} 
& \text { WATER } \\
\text { DATE } & \text { LEVEL MS }
\end{array}
$$

APR 13, $1992 \quad 91.28 \mathrm{~S}$

PERIOD OF RECORD HIGHEST 33.68 APR 26, 1951 LOWEST 105.85 OCT 20,1970

SITE NUMBER 344405118111901

LOCAL NUMBER O08N012W31Q02S

About 3 miles northwest of Lancaster, 0.1 mile north of Avenue G, and 0.3 mile west of 30 th Street West. Drilled unused well. Diameter 6 inches, depth measured 83.72 feet in 1992. Altitude of land-surface datum 2,322 feet. Water-level records available 1963, 1967 to current year.

WATER LEVELS IN FEET BELOW LAND-SURFACE DATUM

$$
\begin{array}{ll} 
& \text { WATER } \\
\text { DATE } & \text { LEVEL MS }
\end{array}
$$

APR 13, $1992 \quad 71.31 \mathrm{~s}$

PERIOD OF RECORD HIGHEST 43.69 AUG 15,1963 LOWEST 71.38 MAR 18,1991 
WELL DESCRIPTIONS AND WATER-LEVEL MEASUREMENTS

WATER YEAR OCTOBER 1991 TO SEPTEMBER 1992

SITE NUMBER 344418118081401

LOCAL NUMBER OOBN012W34KO1S

About 2 miles north of Lancaster, $0.6 \mathrm{mile}$ south of Avenue $F$, and $0.1 \mathrm{mile}$ east of 5 th Street West. Domestic well. Diameter unknown, depth measured 143.9 feet in 1990. Altitude of land-surface datum 2,316 feet. Water-1evel records avallable 1978 to current year.

WATER LEVELS IN FEET BELOW LAND-SURFACE DATUM

DATE WATER

APR $13,199256.49 \mathrm{~S}$

PERIOD OF RECORD HIGHEST 48.75 FEB 13, 1979. LOWEST 56.65 MAR 16, 1991

SITE NUMBER 344822118134001

LOCAL NUMBER 008N013W02Q01S

About 4 miles southwest of Rosamond, $0.6 \mathrm{mile}$ east of $60 \mathrm{th}$ Street West, and 100 feet north of Avenue B. Unused well. Diameter and depth unknown. Altitude of land-surface datum 2,373 feet. Water-level records available 1963 , 1973 to current year.

WATER LEVELS IN FEET BELOW LAND-SURFACE DATUM

$$
\text { DATE WATER }
$$

APR 14, $1992162.89 \mathrm{~S}$

PERIOD OF RECORD HIGHEST 162.89 APR 14, 1992 LOWEST 216.90 MAR 09, 1977

SITE NUMBER 344834118151401

LOCAL NUMBER O08N013W03M01S

About 5 miles southwest of Rosamond at intersection of Avenue A-12 and 70th Street West. Drilled unused water-table well. Diameter 14 inches, depth 765 feet, perforated $350-765$ feet. Altitude of land-surface datum 2,400 feet. Water-level records available 1955-56, 1974 to current year.

WATER LEVELS IN FEET BELOW LAND-SURFACE DATUM

$$
\text { DATE WATER }
$$

APR $14,1992188.52 \mathrm{~S}$

PERIOD OF RECORD HIGHEST 188.52 ARR 14, 1992 LOWEST 265.90 MAR 09, 1977

SITE NUMBER 344848118172301

LOCAL NUMBER 008N013W05E01S

About 7 miles southwest of Rosamond, northeast of intersection of Avenue A-8 and 90th Street West. Unused water-table well. Diameter 18 inches, depth reported 552 feet. Altitude of land-surface datum 2,440 feet. Water-level records available 1945, 1957-63, 1967 to current year.

WATER LEVELS IN FEET BELOW LAND-SURFACE DATUM

$$
\text { DATE WATER }
$$

APR 14, $1992 \quad 234,20 \mathrm{~S}$

PERIOD OF RECORD HIGHEST 104.0 DEC 03, 1945 LOWEST 306.28 FEB 19, 1975 
SITE NUMBER 344819118175201

LOCAL NUMBER 008N013W07B01S

About $8 \mathrm{miles}$ southwest of Rosamond, $0.5 \mathrm{mile}$ west of intersection of Avenue B and 90th Street West. Drilled unused well. Diameter 14 inches, depth 496 feet, perforated $320-496$ feet. Altitude of land-surface datum 2,453 feet. Water-level records available 1961, 1983 to current year.

WATER LEVELS IN FEET BELOW LAND-SURFACE DATUM

$$
\text { DATE WATER } \quad \text { LEVEL MS }
$$

APR 14, $1992250.26 \mathrm{~S}$

PERIOD OF RECORD HIGHEST 250.26 APR 14, 1992 LOWEST 290.83 MAY 18, 1983

SITE NUMBER 344758118154401

LOCAL NUMBER 008N013W09K01S

About 6 miles west of Rosamond at southeast corner of intersection of 75 th Street West and Avenue B-6. Unused well. Diameter 14 inches, depth reported 411 feet. Altitude of land-surface datum 2,412 feet. Water-level records available $1957-63,1967$ to current year.

WATER LEVELS IN FEET BELOW LAND-SURFACE DATUM

$$
\text { DATE WATER }
$$

APR 14, $1992 \quad 204.86 \mathrm{~S}$

PERIOD OF RECORD HIGHEST 169.50 MAR 12,1958 LOWEST 228.35 OCT 13,1970

SITE NUMBER 344733118133901

LOCAL NUMBER OOBNO13W11Q01S

About 4 miles southwest of Rosamond, 0.5 mile east of 60 th Street West, and 0.1 mile north of Avenue L. Irrigation water-table well. Diameter 16 inches, depth 575 feet, perforated 200-575 feet. Altitude of land-surface datum 2,374 feet. Water-level records available 1947, 1951, 1956-63, 1974-76, 1979-80, 1982-83, 1986 to current year.

WATER LEVELS IN FEET BELOW LAND-SURFACE DATUM

$$
\text { DATE WATER }
$$

APR $16,1992 \quad 164.18 \mathrm{~S}$

PERIOD OF RECORD HIGHEST 110.00 JAN 26, 1947 LOWEST 209.96 FEB 10,1976

SITE NUMBER 344725118132701

LOCAL NUMBER OO8N013W14B02S

About 5 miles southwest of Rosamond, 250 feet south of Avenue C, and 200 feet west of 52 nd Street West. Drilled unused water-table well in alluvium. Diameter 6.5 inches, depth measured 159.2 feet in 1978 . Altitude of land-surface datum 2,370 feet. Water-level records available 1978 to current year.

WATER LEVELS IN FEET BELOW LAND-SURFACE DATUM

$$
\text { DATE WATER }
$$

APR 16, $1992137.91 \mathrm{~S}$

PERIOD OF RECORD HIGHEST 134.80 MAR 28, 1978 LOWEST 148.46 APR 08, 1980 
WELL DESCRIPTIONS AND WATER-LEVEL MEASUREMENTS

WATER YEAR OCTOBER 1991 TO SEPTEMBER 1992

SITE NUMBER 344647118175401

LOCAL NUMBER 008N013W18Q02S

About 10 miles southwest of Rosamond, 0.5 mile west of 90 th Street West, and 0.2 mile north of Avenue D. Unused water-table well. Diameter 14 inches, depth 533 feet, perforated 255-533 feet. Altitude of land-surface datum 2,453 feet. Water-level records available 1963,1973 to current year.

WATER LEVELS IN FEET BELOW LAND-SURFACE DATUM

DATE WATER

APR 16, $1992 \quad 235.53 \mathrm{~S}$

PERIOD OF RECORD HIGHEST 235.53 APR 16, 1992 LOWEST 295.00 FEB 01, 1973

SITE NUMBER 344623118164901

LOCAL NUMBER 008N013W20B01S

About 7 miles southwest of Rosamond, 0.5 mile east of 90 th Street West, and 0.2 mile south of Avenue D. Unused water-table well. Diameter 16 inches, depth 618 feet, perforated 214-614 feet. Altitude of land-surface datum 2,430 feet. Water-level records available 1952-59, 1961-63, 1967, 1970, 1973 to current year.

WATER LEVELS IN FEET BELOW LAND-SURFACE DATUM

DATE WATER

APR 16, $1992219.48 \mathrm{~S}$

PERIOD OF RECORD HIGHEST 165.00 DEC 1952 LOWEST 298.50 OCT 23, 1970

SITE NUMBER 344620118140901

LOCAL NUMBER O08N013W23E01S

About 6 miles southwest of Rosamond, 250 feet east of 60 th Street West, and 0.2 mile north of Avenue D-8. Unused water-table well. Diameter 14 inches, depth 529 feet, depth of hole 2,063 feet. Altitude of land-surface datum 2,382 feet. Water-level records available 1963,1974 to current year.

WATER LEVELS IN FEET BELOW LAND-SURFACE DATUM

DATE WATER

APR 16, $1992169.72 \mathrm{~S}$

PERIOD OF RECORD HIGHEST 169.72 APR 16, 1992 LOWEST 208.67 FEB 13, 1974

SITE NUMBER 344607118140801

LOCAL NUMBER 008N013W23MO1S

About 6 miles southwest of Rosamond, 125 feet east of 60 th Street West, and 100 feet south of Avenue D-8. Unused well. Diameter 8 inches, depth 450 feet, perforated $50-450$ feet. Altitude of land-surface datum 2,376 feet. Measurements prior to 1983 provided by Los Angeles County Flood Control District, Southern Cailfornia Electric and California Department of Water Resources. Water-level records available 1942-43, 1945-56, 1983 to current year.

WATER LEVELS IN FEET BELOW LAND-SURFACE DATUM

$$
\text { DATE WATER }
$$

APR 16, $1992158.23 \mathrm{~S}$

PERIOD OF RECORD HIGHEST 50.62 NOV 28, 1942 LOWEST 186.95 APR 26, 1983 
SITE NUMBER 344500118122201

LOCAL NUMBER 008N013W25Q01S

About 4 miles northwest of Lancaster, 0.2 mile north of Avenue F, and 200 feet west of 42nd Street West. Unused well. Diameter 6 inches, depth measured 62.4 feet in 1991. Altitude of land-surface datum 2,333 feet.

Water-level records available 1963, 1974 to current year.

WATER LEVELS IN FEET BELOW LAND-SURFACE DATUM

$$
\text { DATE WATER }
$$

APR 16, $1992 \quad 43,88 \mathrm{~S}$

PERIOD OF RECORD HIGHEST 43.88 APR 16, 1992 LOWEST 86.25 MAR 13, 1990

SITE NUMBER 344509118133801

LOCAL NUMBER 008N013W26K01S

About $5 \mathrm{miles}$ northwest of Lancaster, $0.3 \mathrm{mile}$ north of Avenue $\mathrm{F}$, and east of 55 th Street West. Drilled domestic water-table well. Diameter 6 inches, depth reported 238 feet. Altitude of land-surface datum 2,348 feet. Water-level records available 1978 to current year.

WATER LEVELS IN FEET BELOW LAND-SURFACE DATUM

$$
\text { DATE NATER }
$$

APR 16, $1992115.40 \mathrm{SR}$

PERIOD OF RECORD HIGHEST 115.70 APR 15, 1991 LOWEST 120.58 FEB 08, 1982

SITE NUMBER 344403118175001

LOCAL NUMBER 008N013W31Q01S

About $10 \mathrm{miles}$ west of Lancaster, $0.1 \mathrm{mile}$ north of Avenue G, and $0.5 \mathrm{mile}$ west of 90th Street West. Drilled unused water-table well. Diameter 14 inches, depth 655 feet, perforated 300-655 feet. Altitude of land-surface datum 2,440 feet. Water-level records available 1948, 1956, 1962, 1974 to current year.

WATER LEVELS IN FEET BELOW LAND-SURFACE DATUM

$$
\begin{array}{ll} 
& \text { WATER } \\
\text { DATE } & \text { LEVEL MS }
\end{array}
$$

APR $16,1992 \quad 212.09 \mathrm{~S}$

PERIOD OF RECORD HIGHEST 212.09 APR 16,1992 LOWEST 293.10 OCT 02, 1962

SITE NUMBER 344751118210701

LOCAL NUMBER O08N014W10LO1S

About $18 \mathrm{miles}$ west of Lancaster, $0.5 \mathrm{mile}$ south of intersection of Avenue B and 130 th Street West. Unused well. Diameter 12 inches, depth measured greater than 400 feet. Altitude of land-surface datum 2,525 feet. Water-level records available 1967, 1983 to current year.

WATER LEVELS IN FEET BELOW LAND-SURFACE DATUM

$$
\text { DATE WATER }
$$

APR 14, $1992250.03 \mathrm{~S}$

PERIOD OF RECORD HIGHEST 248.38 MAR 18, 1991 LOWEST 276.05 MAY 18, 1983 
WELL DESCRIPTIONS AND WATER-LEVEL MEASUREMENTS

WATER YEAR OCTOBER 1991 TO SEPTEMBER 1992

SITE NUMBER 344819118191301

LOCAL NUMBER OOBNO14W12C01S

About 17 miles west of Lancaster, 50 feet south of Avenue B, and 0.25 mile east of 110th Street West. Drilled unused water-table well. Diameter 14 inches, depth reported 653 feet. Altitude of land-surface datum 2,480 feet. Water-level records available 1963, 1978 to current year.

WATER LEVELS IN FEET BELOW LAND-SURFACE DATUM

$$
\begin{array}{ll} 
& \text { WATER } \\
\text { DATE } & \text { LEVEL MS }
\end{array}
$$

APR 14, 1992 So

PERIOD OF RECORD HIGHEST 278.59 MAR 18, 1991 LOWEST 354.40 SEP 21, 1978

\section{SITE NUMBER 344658118234201}

LOCAL NUMBER 008N014W17MOIS

About 19 miles west of Lancaster, 0.45 mile north of Avenue D, and 150 feet east of 150 th Street West. Drilled unused water-table well. Diameter 14 inches, depth 670 feet, depth of hole 828 feet, perforated $270-670$ feet. Altitude of land-surface datum 2,592 feet. Water-level records available 1958, 1962, 1974 to current year.

WATER LEVELS IN FEET BELOW LAND-SURFACE DATUM

$$
\begin{array}{ll}
\text { WATE } & \text { WATER } \\
\text { LEVEL MS }
\end{array}
$$

APR 14, $1992173.59 \mathrm{~S}$

PERIOD OF RECORD HIGHEST 172.50 MAY 08, 1962 LOWEST 190.00 NOV 20, 1958

SITE NUMBER 344635118244301

LOCAL NUMBER 008N014W18N01S

About 20 miles west of Lancaster, 350 feet north of Avenue $D$, and 50 feet east of 160 th Street West. Drilled unused water-table well. Diameter 16 inches $0-450$ feet, 14 inches $450-865$ feet, depth 865 feet, perforated 250-865 feet. Altitude of land-surface datum 2,642 feet. Water-level records available 1954-55, 1958, 1960, $1962,1967-68,1970$ to current year.

WATER LEVELS IN FEET BELOW LAND-SURFACE DATUM

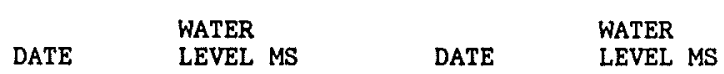

OCT 23, $1991111.73 \mathrm{~S} \quad$ APR $14,1992112.49 \mathrm{~S}$

PERIOD OF RECORD HIGHEST 110.21 MAR 30, 1988 LOWEST 220 FEB 23, 1954

\section{SITE NUMBER 344607118195901}

LOCAL NUMBER OOBN014W23G01S

About 16 miles west of Lancaster, 100 feet east of 115 th Street West, and 0.5 mile south of Avenue D. Unused water-table well. Diameter 14 inches, depth reported 420 feet. Altitude of land-surface datium 2,500 feet. Water-level records available 1952, 1954, 1956-60, 1974-79, 1981 to current year.

WATER LEVELS IN FEET BELOW LAND-SURFACE DATUM

$$
\text { DATE HATER }
$$

APR 16, $1992260.10 \mathrm{~S}$

PERIOD OF RECORD HIGHEST 182.40 JUL 22, 1952 LOWEST 293.69 FEB 22, 1979 
SITE NUMBER 344652118185701

LOCAL NUMBER 008N014W24CO1S

About 15 miles west of Lancaster, 0.5 mile east of 110 th Street West, and 100 feet south of Avenue D. Drilled unused water-table well. Diameter 14 inches, depth reported 618 feet, perforated $500-618$ feet. Altitude of land-surface datum 2,475 feet. Water-level records avallable 1955, 1967, 1978 to current year.

WATER LEVELS IN FEET BELOW LAND-SURFACE DATUM

$$
\text { DATE WATER }
$$

APR $16,1992 \quad 246.95 \mathrm{~S}$

PERIOD OF RECORD HIGHEST 230.5 JUN 15, 1955 LOWEST 289.02 DEC 26, 1967

SITE NUMBER 344745118293301

LOCAL NUMBER OOBN015W08L01S

About 25 miles northwest of Lancaster near intersection of 205 th Street West and Avenue B-8. Unused well.

Diameter and depth unknown. Altitude of land-surface datum 2,720 feet. Water-level records available 1981 to current year.

WATER LEVELS IN FEET BELOW LAND-SURFACE DATUM

$$
\text { DATE WATER }
$$

APR 14, $1992147.72 \mathrm{~S}$

PERIOD OF RECORD HIGHEST 147.72 APR 14, 1992 LOWEST 165.07 AUG 19, 1981

SITE NUMBER 344727118273101

LOCAL NUMBER OO8NO15W1OP02S

About 23 miles northwest of Lancaster, northeast of intersection of 190 th Street West and Avenue C. Drilled unused water-table well. Diameter 12 inches, depth 621 feet, perforated 361-621 feet. Altitude of land-surface datum 2,710 feet. Water-level records available 1965, 1981 to current year.

WATER LEVELS IN FEET BELOW LAND-SURFACE DATUM

$$
\begin{array}{ll}
\text { DATE } & \text { WATER } \\
\text { LEVEL MS }
\end{array}
$$

APR 14, $1992150.77 \mathrm{~S}$

PERIOD OF RECORD HIGHEST 150.77 APR 14, 1992 LOWEST 200.00 MAR 23, 1965

SITE NUMBER 344421118282201

LOCAL NUMBER 008N015W33G01S

About 19 miles west of Lancaster, 100 feet north of Avenue F-8, and 0.4 mile west of 190th Street West. Domestic water-table well. Diameter 12 inches, depth 281.5 feet. Altitude of land-surface datum 2,930 feet. Measurements prior to 1968 provided by California Department of Water Resources. Water-level records available 1946-50, $1953-63,1967$ to current year.

WATER LEVELS IN FEET BELOW LAND-SURFACE DATUM

$$
\text { DATE WATER }
$$

APR $16,1992 \quad 237.34 \mathrm{~S}$

PERIOD OF RECORD HIGHEST 194.00 JAN 23, 1946 LOWEST 284.00 NOV 12, 1959 
WELL DESCRIPTIONS AND WATER-LEVEL MEASUREMENTS

WATER YEAR OCTOBER 1991 TO SEPTEMBER 1992

SITE NUMBER 344814118322401

LOCAL NUMBER 008N016W02R01S

About 27 miles west of Lancaster, $0.2 \mathrm{mile}$ west of 230 th Street West, and 60 feet north of Avenue B. Drilled observation well. Diameter 1.5 inches, depth measured 347 feet in 1965, perforated 343.5-347 feet. Altitude of land-surface datum 2,795 feet. Water-level records avallable 1965, 1973-78, 1980 to current year.

WATER LEVELS IN FEET BELOW LAND-SURFACE DATUM

$$
\text { DATE WATER }
$$

APR 15, $1992181.68 \mathrm{~S}$

PERIOD OF RECORD HIGHEST 165.03 APR 06, 1965 LOWEST 190.67 MAR 29, 1978

SITE NUMBER 344841118335001

LOCAL NUMBER 008N016W03F01S

About $28 \mathrm{miles}$ west of Lanoaster, $0.5 \mathrm{mile}$ north of Avenue B, and $0.5 \mathrm{mile}$ west of 240 th Street West. Drilled observation well in alluvium. Diameter 1.5 inches 0-295.5 feet, 2 inches 295.5-326 feet, depth 326 feet,

perforated 317-326 feet. Altitude of land-surface datum 2,835 feet. Water-level records available 1965 , 1967 to current year.

WATER LEVELS IN FEET BELOW LAND-SURFACE DATUM

\begin{tabular}{|c|c|c|c|}
\hline DATE & $\begin{array}{l}\text { WATER } \\
\text { LEVEL MS }\end{array}$ & DATE & $\begin{array}{l}\text { WATER } \\
\text { LEVEL MS }\end{array}$ \\
\hline
\end{tabular}

OCT 23, $1991214.03 \mathrm{~S} \quad$ APR $15,1992215.04 \mathrm{~S}$

PERIOD OF RECORD HIGHEST 191.64 APR 13, 1965 LOWEST 220.57 OCT 14,1980

SITE NUMBER 344828118372601

LOCAL NUMBER 008N016W06M01S

About 30 miles west of Lancaster, and 1.7 miles north of Highway 138 on 280 th Street West. Drilled domestic well. Diameter 12 inches, original depth 845 feet, cased to 600 feet, depth 546 feet after well rehab in 1988 , perforated 230-546 feet. Altitude of land-surface datum 2,927 feet. Water-level records available 1956, $1959-62,1992$.

WATER LEVELS IN FEET BELOW LAND-SURFACE DATUM

DATE WATER $\quad$ LEVEL MS

AUG 20, $1992 \quad 262.23 \mathrm{~S}$

PERIOD OF RECORD HIGHEST 228.5 OCT 02, 1956 LOWEST 262.23 AUG 20, 1992

SITE NUMBER 344806118343801

LOCAL NUMBER 008N016W09G02S

About 30 miles west of Lancaster, 300 feet west-southwest of Holiday Lake and 0.5 mile east of California Aqueduct. Domestic water-table well. Diameter 6 inches, depth reported 300 feet. Altitude of land-surface datum 2,890 feet. Water-level records available 1965, 1974-77, 1980-84, 1986 to current year.

WATER LEVELS IN FEET BELOW LAND-SURFACE DATUM

$$
\text { DATE WATER }
$$

APR 15, $1992265.38 \mathrm{~S}$

PERIOD OF RECORD HIGHEST 240.29 FEB 26, 1986 LOWEST 265.38 APR 15, 1992 
SITE NUMBER 344701118363401

LOCAL NUMBER OOBNO16W18HO2S

About 31 miles west of Lancaster, 0.2 mile west of 270 th Street West, and 150 feet north of Highway 138 . Drilled domestic water-table well. Diameter 10 inches $0-183$ feet, 8 inches $183-400$ feet, original depth 193 feet, deepened to 400 feet in 1961. Altitude of land-surface datum 2,987 feet. Water-leve1 records available $1956-62$, 1978 to current year.

WATER LEVELS IN FEET BELOW LAND-SURFACE DATUM

$$
\begin{array}{ll} 
& \text { WATER } \\
\text { DATE } & \text { LEVEL MS }
\end{array}
$$

APR 15, 1992182.45 SR

PERIOD OF RECORD HIGHEST 120.4 OCT 07,1958 LOWEST 191.56 MAR 25, 1980

SITE NUMBER 344541118333901

LOCAL NUMBER OOBN016W22Q04S

About $28 \mathrm{miles}$ west of Lancaster, 1 mile south of intersection of Avenue D and 245 th Street West. Drilled domestic well. Dianeter 9 inches, depth 151.75 feet. Altitude of land-surface datum 3,049 feet. Water-1evel records available 1956-63, 1983 to current year.

WATER LEVELS IN FEET BELOW LAND-SURFACE DATUM

$$
\begin{array}{ll} 
& \text { HATER } \\
\text { DATE } & \text { LEVEL MS }
\end{array}
$$

APR 15, $1992 \quad 28.11 \mathrm{~S}$

PERIOD OF RECORD HIGHEST 28.11 APR 15, 1992 LOWEST 76.2 OCT 26, 1960

SITE NUMBER 344812118383401

LOCAL NUMBER 008N017W01N01S

About 34 miles northwest of Lancaster near intersection of Avenue $B$ and 290 th Street West. Drilled unused water-table well. Diameter 14 inches $0-489$ feet, 12 inches $489-603$ feet, 10 inches $603-787$ feet, depth 787 feet. Altitude of land-surface datum 2,955 feet. Water-level records available 1945, 1953-54, 1962, 1964, 1967-68, $1970-72,1974$ to current year.

WATER LEVELS IN FEET BELOW LAND-SURFACE DATUM

$$
\begin{array}{ll} 
& \text { WATER } \\
\text { DATE } & \text { LEVEL MS }
\end{array}
$$

APR $15,1992266.03 \mathrm{~S}$

PERIOD OF RECORD HIGHEST 199.60 NOV 13,1945 LOWEST 315.91 MAR 21,1967

SITE NUMBER 344858118414101

LOCAL NUMBER 008N017W04D01S

About $35 \mathrm{miles}$ northwest of Lancaster, about $4 \mathrm{miles}$ north of San Andreas Fault, $2.5 \mathrm{miles}$ north of Highway 138 , and 2 miles west of 300 th Street West. Unused water-table well. Diameter 12 inohes, depth 530 feet. Altitude of land-surface datum 3,036 feet. Water-level records available 1948, 1951, 1962 , 1972 to current year.

WATER LEVELS IN FEET BELOW LAND-SURFACE DATUM

$$
\begin{array}{lll} 
& \text { WATER } & \\
\text { DATE } & \text { WEVEL MS } & \text { WATER } \\
\end{array}
$$

OCT 23, $1991137.96 \mathrm{~S} \quad$ APR $15,1992128.28 \mathrm{~s}$

PERIOD OF RECORD HIGHEST 105.56 OCT 23,1979 LOWEST 161.33 OCT 11,1972 
INORGANIC DATA

WATER YEAR OCTOBER 1991 TO SEPTEMBER 1992

LOCAL
NUMBER

005N010W08H01S OOSNO11W01MO1S 006NOO9W28P02S 006N011W06G02S 006N011W36G01S

007N011W03E03S 007N011W19E01S $007 \mathrm{~N} 012 \mathrm{~W} 27 \mathrm{H} 02 \mathrm{~S}$ $007 \mathrm{~N} 012 \mathrm{~W} 27 \mathrm{~J} 06 \mathrm{~S}$ 007N013W13F03S

$007 N 013 W 14 E 01 S$ 007N013W19D01S 007N014W13A01S 008N010W22H03S 008N010W22P03S

$008 \mathrm{~N} 012 \mathrm{~W} 21 \mathrm{Q} 01 \mathrm{~S}$

\section{LOCAL \\ NUMBER}

005N010W08H01S $005 \mathrm{~N} 011 \mathrm{~W} 01 \mathrm{M} 01 \mathrm{~S}$ 006N009W28P02S 006N011W06G02S 006N011W36G01S

007N011W03E03S 007N011W19E01S $007 N 012 W 27 \mathrm{H} 02 \mathrm{~S}$ 007 N012W27J06S 007N013W13F03S

007N013W14E01S 007N013W19D01S $007 \mathrm{~N} 014 \mathrm{~W} 13 \mathrm{AO} 1 \mathrm{~S}$ $008 \mathrm{~N} 010 \mathrm{~W} 22 \mathrm{H} 03 \mathrm{~S}$ 008N010W22P03S

008N012W21Q01S

\begin{tabular}{|c|c|c|c|c|c|c|c|c|c|}
\hline DATE & TIME & $\begin{array}{l}\text { DEPTH } \\
\text { OF } \\
\text { WELL, } \\
\text { TOTAL } \\
\text { (FEET) }\end{array}$ & $\begin{array}{l}\text { SPE- } \\
\text { CIFIC } \\
\text { CON- } \\
\text { DUCT- } \\
\text { ANCE } \\
\text { (US/CM) }\end{array}$ & $\begin{array}{c}\text { PH } \\
\text { WATER } \\
\text { WHOLE } \\
\text { FIELD } \\
\text { (STAND- } \\
\text { ARD } \\
\text { UNITS) }\end{array}$ & $\begin{array}{c}\text { TEMPER- } \\
\text { ATURE } \\
\text { WATER } \\
\text { (DEG C) }\end{array}$ & $\begin{array}{l}\text { HARD- } \\
\text { NESS } \\
\text { TOTAL } \\
\text { (MG/L } \\
\text { AS } \\
\text { CACO3) }\end{array}$ & $\begin{array}{l}\text { CALCIUM } \\
\text { DIS- } \\
\text { SOLVED } \\
\text { (MG/L } \\
\text { AS CA) }\end{array}$ & $\begin{array}{l}\text { MAGNE- } \\
\text { SIUM, } \\
\text { DIS- } \\
\text { SOLVED } \\
\text { (MG/L } \\
\text { AS MG) }\end{array}$ & $\begin{array}{l}\text { SODIUM, } \\
\text { DIS- } \\
\text { SOLVED } \\
\text { (MG/L } \\
\text { AS NA) }\end{array}$ \\
\hline $\begin{array}{l}08-19-92 \\
08-21-92 \\
08-19-92 \\
08-19-92 \\
08-17-92\end{array}$ & $\begin{array}{l}0825 \\
0815 \\
0950 \\
1135 \\
1510\end{array}$ & $\begin{array}{r}630 \\
396 \\
731 \\
1200 \\
572\end{array}$ & $\begin{array}{l}321 \\
322 \\
754 \\
217 \\
287\end{array}$ & $\begin{array}{l}7.9 \\
-- \\
-- \\
8.2 \\
7.8\end{array}$ & $\begin{array}{l}24.5 \\
16.5 \\
21.5 \\
22.5 \\
18.0\end{array}$ & $\begin{array}{l}95 \\
-- \\
-- \\
61 \\
100\end{array}$ & $\begin{array}{c}34 \\
-- \\
23 \\
32\end{array}$ & $\begin{array}{l}2.5 \\
-- \\
-- \\
0.91 \\
6.0\end{array}$ & $\begin{array}{c}29 \\
-- \\
-- \\
22 \\
17\end{array}$ \\
\hline $\begin{array}{l}08-03-92 \\
08-03-92 \\
08-20-92 \\
08-19-92 \\
05-04-92\end{array}$ & $\begin{array}{l}1210 \\
1305 \\
0815 \\
1410 \\
0930\end{array}$ & $\begin{array}{r}370 \\
508 \\
690 \\
1150 \\
100\end{array}$ & $\begin{array}{l}444 \\
205 \\
240 \\
400 \\
815\end{array}$ & $\begin{array}{l}-- \\
8.1 \\
8.5 \\
--\end{array}$ & $\begin{array}{l}20.5 \\
22.5 \\
22.0 \\
27.0 \\
24.5\end{array}$ & $\begin{array}{l}-- \\
-- \\
59 \\
--\end{array}$ & $\begin{array}{c}-- \\
-- \\
20 \\
9.9 \\
--\end{array}$ & $\begin{array}{l}-- \\
-- \\
2.1 \\
0.72 \\
--\end{array}$ & $\begin{array}{c}-- \\
-- \\
28 \\
76 \\
--\end{array}$ \\
\hline $\begin{array}{l}08-04-92 \\
08-04-92 \\
08-20-92 \\
08-03-92 \\
08-03-92\end{array}$ & $\begin{array}{l}1000 \\
0905 \\
1600 \\
1130 \\
1150\end{array}$ & $\begin{array}{l}930 \\
500 \\
519 \\
150 \\
200\end{array}$ & $\begin{array}{l}506 \\
555 \\
295 \\
380 \\
356\end{array}$ & $\begin{array}{l}-- \\
-- \\
7.4 \\
--\end{array}$ & $\begin{array}{l}30.0 \\
25.5 \\
23.0 \\
21.0 \\
21.5\end{array}$ & $\begin{array}{l}-- \\
-- \\
-- \\
--\end{array}$ & $\begin{array}{c}-- \\
25 \\
-- \\
--\end{array}$ & $\begin{array}{l}-- \\
-- \\
5.7 \\
-- \\
--\end{array}$ & $\begin{array}{c}-- \\
26 \\
--\end{array}$ \\
\hline $08-18-92$ & 1600 & 207 & 460 & 8.0 & 19.0 & 99 & 29 & 6.4 & 54 \\
\hline
\end{tabular}

DATE $\begin{array}{r}\text { SORP- } \\ \text { TION } \\ \text { SODIUM } \\ \text { RERTENT }\end{array}$

$08-19-92$
$08-21-92$
$08-19-92$
$08-19-92$
$08-17-92$
$08-03-92$
$08-03-92$
$08-20-92$
$08-19-92$
$05-04-92$
$08-04-92$
$08-04-92$
$08-20-92$
$08-03-92$
$08-03-92$

(MG/L
AS K)
1

$\begin{array}{cccccc}39 & 1 & 1.9 & 154 & 126 & 40 \\ -- & -- & -- & -- & -- & -- \\ -- & -- & -- & - & - & -- \\ 43 & 1 & 1.1 & 122 & 100 & 14 \\ 26 & 0.7 & 2.1 & 139 & 114 & 26\end{array}$

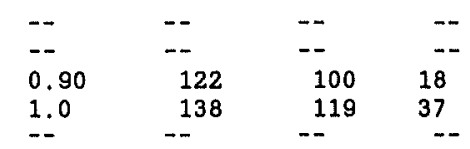

$$
\begin{array}{lll}
\text { CHLO- } & \text { FLUO- } & \text { SILICA, } \\
\text { RIDE, } & \text { RIDE, } & \text { DIS- } \\
\text { DIS- } & \text { DIS- } & \text { SOLVED } \\
\text { SOLVED } & \text { SOLVED } & \text { (MG/L } \\
\text { (MG/L } & \text { (MG/L } & \text { AS } \\
\text { AS CL) } & \text { AS F) } & \text { SIO2) }
\end{array}
$$

$$
\begin{array}{lll}
4.7 & 0.20 & 26 \\
-- & -- & - \\
-- & -- & -
\end{array}
$$$$
4.1<0.10 \quad 22
$$$$
\begin{array}{rrr}
4.1 & <0.10 & 22 \\
8.7 & 0.20 & 24
\end{array}
$$$$
\begin{array}{lllllllll}
-- & -- & -- & -- & -- & -- & -- & -- & -- \\
-- & -- & -- & -- & -- & -- & -- & -- & - \\
51 & 2 & 0.90 & 122 & 100 & 18 & 7.4 & 0.30 & 25 \\
85 & 6 & 1.0 & 138 & 119 & 37 & 32 & 0.80 & 25 \\
-- & -- & -- & -- & -- & -- & -- & --
\end{array}
$$$$
\text { -- }
$$$$
\begin{array}{lllllllll}
-- & -- & -- & -- & -- & -- & -- & -- & - \\
-- & -- & -- & - & -- & -- & -- & -- & - \\
39 & 1 & 1.5 & 116 & 95 & 8.6 & 12 & 0.30 & 44 \\
-- & -- & -- & -- & -- & -- & -- & -- & -- \\
-- & -- & -- & -- & -- & -- & -- & -- & -
\end{array}
$$

08-18-92

$\begin{array}{llll}1.8 & 143 & 117 & 33\end{array}$

52

$0.60 \quad 29$

< Actual value is less than value shown. 
WATER YEAR OCTOBER 1991 TO SEPTEMBER 1992

\section{LOCAL}

NUMBER

\begin{abstract}
005N010W08H01S
$005 N 011 W 01 M 01 S$

$006 N 009 W 28 P 02 S$

006N011W06G02S

006N011W36G01S
\end{abstract}

007N011W03E03S

$007 N 011 W 19 E 01 \mathrm{~S}$

$007 \mathrm{~N} 012 \mathrm{~W} 27 \mathrm{H} 02 \mathrm{~S}$

$007 \mathrm{~N} 012 \mathrm{~W} 27 \mathrm{~J} 06 \mathrm{~S}$

007 N013W13F03S

007N013W14E01S

007N013W19D01S

$007 N 014 W 13 A 01 S$

008N010W22H03S

008 N010W22P03S

008N012W21Q01S

$\begin{array}{ccccccc} & \text { SOLIDS, } & & \text { NITRO- } & \text { NITRO- } & \text { NITRO- } & \text { PHOS- } \\ & \text { RESIDUE } & \text { RESIDUE } & \text { GEN, } & \text { GEN, } & \text { GEN, } & \text { PHORUS } \\ & \text { AT } 180 & \text { VOLA- } & \text { NITRITE } & \text { NO2+NO3 } & \text { AMPONIA } & \text { ORTHO, } \\ & \text { DEG. C } & \text { TILE, } & \text { DIS- } & \text { DIS- } & \text { DIS- } & \text { DIS- } \\ & \text { DIS- } & \text { DIS- } & \text { SOLVED } & \text { SOLVED } & \text { SOLVED } & \text { SOLVED } \\ \text { DATE } & \text { SOLVED } & \text { SOLVED } & (M G / L & \text { (MG/L } & \text { (MG/L } & \text { (MG/L } \\ & (M G / L) & (M G / L) & \text { AS N) } & \text { AS N) } & \text { AS N) } & \text { AS P) }\end{array}$

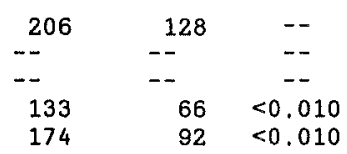
08-21-92 $08-19-92$ $08-19-92$ 08-17-92

$$
\begin{aligned}
& 08-03-92 \\
& 08-03-92 \\
& 08-20-92 \\
& 08-19-92 \\
& 05-04-92
\end{aligned}
$$
$--$ -

247<smiles>C1CCCCC1</smiles>

62

08-04-92 $08-04-92$ $08-20-92$ 08-03-92 08-03-92

08-18-92
-- $\quad 172$

$\begin{array}{ll}-- & -- \\ -- & --\end{array}$

$-$

200

$--$

260
--
--
-
0.020

0.010

-

--
--
--
--

$-$

$-\infty$

$-$

$--$

$--$

0.020

$--$

$-$
--
--
$-\overline{0}$
0.010

$--$

--

$--$

$-$

$-$

0.050

$--$

$--$

$--$

$\begin{array}{ccl} & & \text { MANGA- } \\ \text { BORON, } & \text { IRON, } & \text { NESE, } \\ \text { DIS- } & \text { DIS- } & \text { DIS- } \\ \text { SOLVED } & \text { SOLVED } & \text { SOLVED } \\ \text { (UG/L } & \text { (UG/L } & \text { (UG/L } \\ \text { AS B) } & \text { AS FE) } & \text { AS MN) }\end{array}$

$\begin{array}{rrr}50 & 5 & <1 \\ -- & -- & -- \\ <10 & -- & -- \\ 30 & <3 & <1 \\ 4 & <1\end{array}$

--

20

220

$--$

$-$

30

$--$

230
$-$

$<3$

$<1$

--

$-$

$<3$

$\ldots$

(5)
$<1$

$<1$

$<1$

$<1$

$--$

$--$

$<1$

$-\cdots$

< Actual value is less than value shown. 
MERCED COUNTY

Page

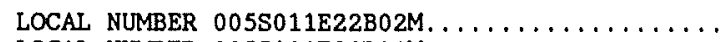

LOCAL NUMBER $005 S 011 E 29 R 01 M \ldots \ldots \ldots \ldots \ldots \ldots \ldots$

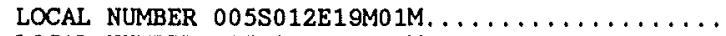

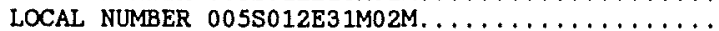

LOCAL NUMBER 005S013E07C01M..............

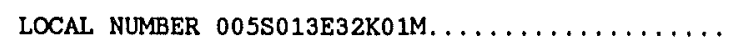

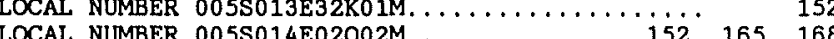

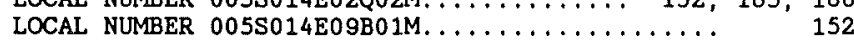

LOCAL NUMBER 005S014E09B02M................. 165, 168

LOCAL NUMBER 005S014E18K01M.......... 153, 165, 168

LOCAL NUMBER 006S011E01M01M. . . . . . . . . . .

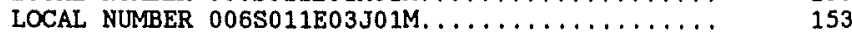

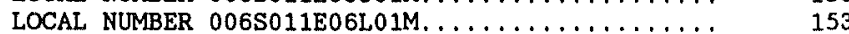

LOCAL NUMBER OO6S011E19J01M............... 154

LOCAL NUMBER 006S011E34F01M............. 154

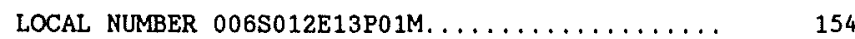

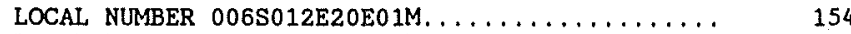

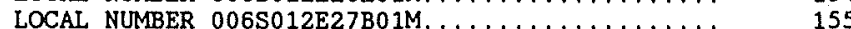

LOCAL NUMBER O06S012E34D01M.............. 155

LOCAL NUMBER 006S013E04Q01M................. 165, 168

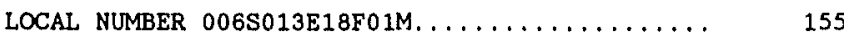

LOCAL NUMBER O06S013E25M01M.................. 165, 168

LOCAL NUMBER 006S013E28A01M. ............. 155

LOCAL NUMBER 006S014E17H01M............... 156

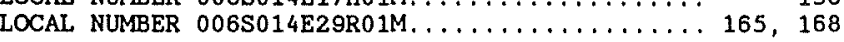

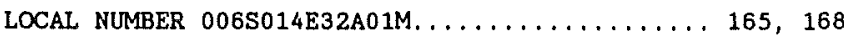

LOCAL NUMBER $006 \$ 014 E 32 B 01 M \ldots \ldots \ldots \ldots \ldots \ldots$ 156, 165, 168

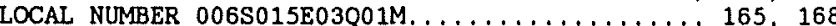

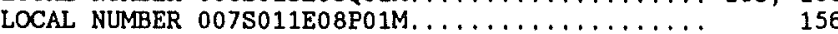

LOCAL NUMBER O07S011E13R01M.............. 156

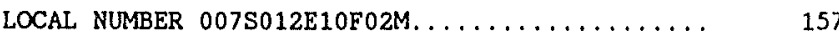

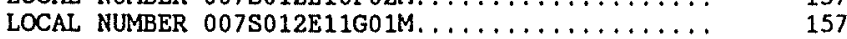

LOCAL NUMBER 007S013E09B03M. . . . . . . . . . . .
LOCAL NUMBER 007S013E21K01M.............

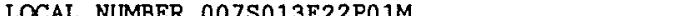

LOCAL NUMBER 007S013E33K01M. ............... 158

LOCAL NUMBER 007 S014E05A01M................... 165, 168

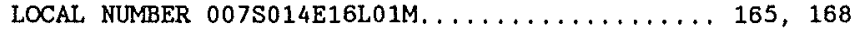

LOCAL NUMBER 007S014E29R01M. . . . . . . . . . 165, 168

LOCAL NUMBER O07S014E31M01M. ............. 158

LOCAL NUMBER 007 S014E35B01M............... 158

LOCAL NUMBER 007 S015E18K01M. ............... 158

LOCAL NUMBER 007S015E28K01M. . . . . . . 159, 165, 168

LOCAL NUMBER 007S015E28M01M............. 159

LOCAL NUMBER O07S015E30E01M, .............. 159

LOCAL NUMBER 007S015E33F01M. ............... 159

LOCAL NUMBER 007 S015E33H01M................ 160

LOCAL NUMBER 007S015E35A02M, ............. 165, 168

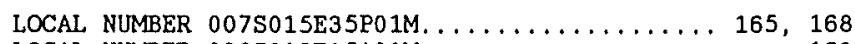

LOCAL NUMBER 008 S012E $15 A 01 M \ldots \ldots \ldots \ldots \ldots \ldots \ldots, 160$

LOCAL NUMBER 008S013E19H01M.............. 160

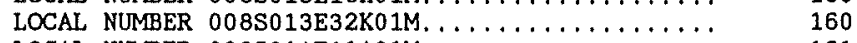

LOCAL NUMBER 008S014E11A01M............... 161

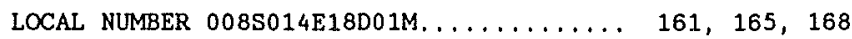

LOCAL NUMBER DO8SO14E19E01M.............. 161, 165, 168

LOCAL NUMBER 008S014E31A01M. . . . . . . . . .

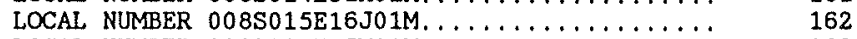

LOCAL NUMBER OOSS013E05M01M. .............. 162

LOCAL NUMBER 009S013E11K01M............... 162

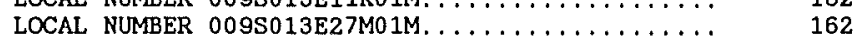

LOCAL NUMBER 009S014E13J01M............... 163

LOCAL NUMBER O09S015E15A01M............. 163

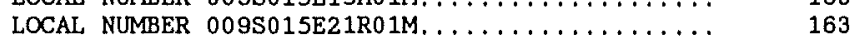

LOCAL NUMBER OO9S015E29F01M. . . . . . . . . . . . 163

LOCAL NUMBER OOSS016E18D01M..............

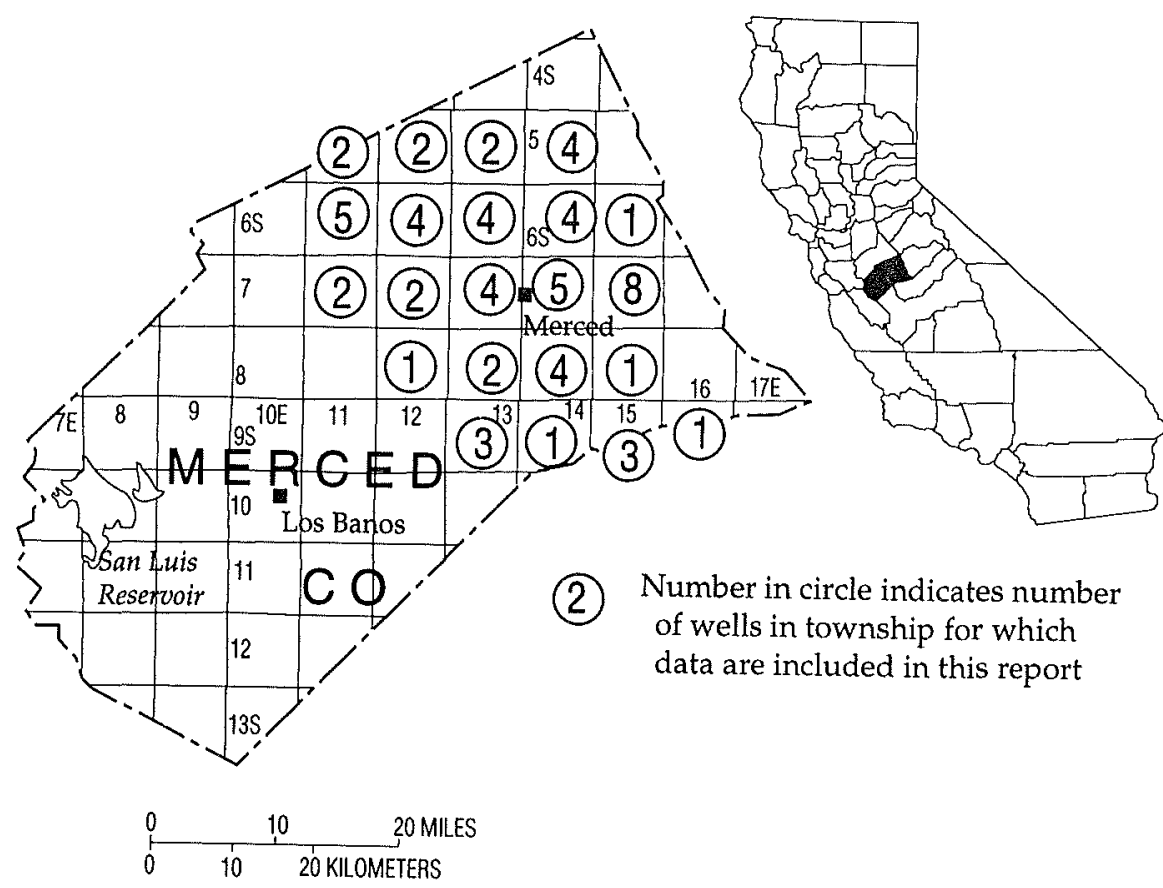

Figure 17. Location of wells in Merced County. 


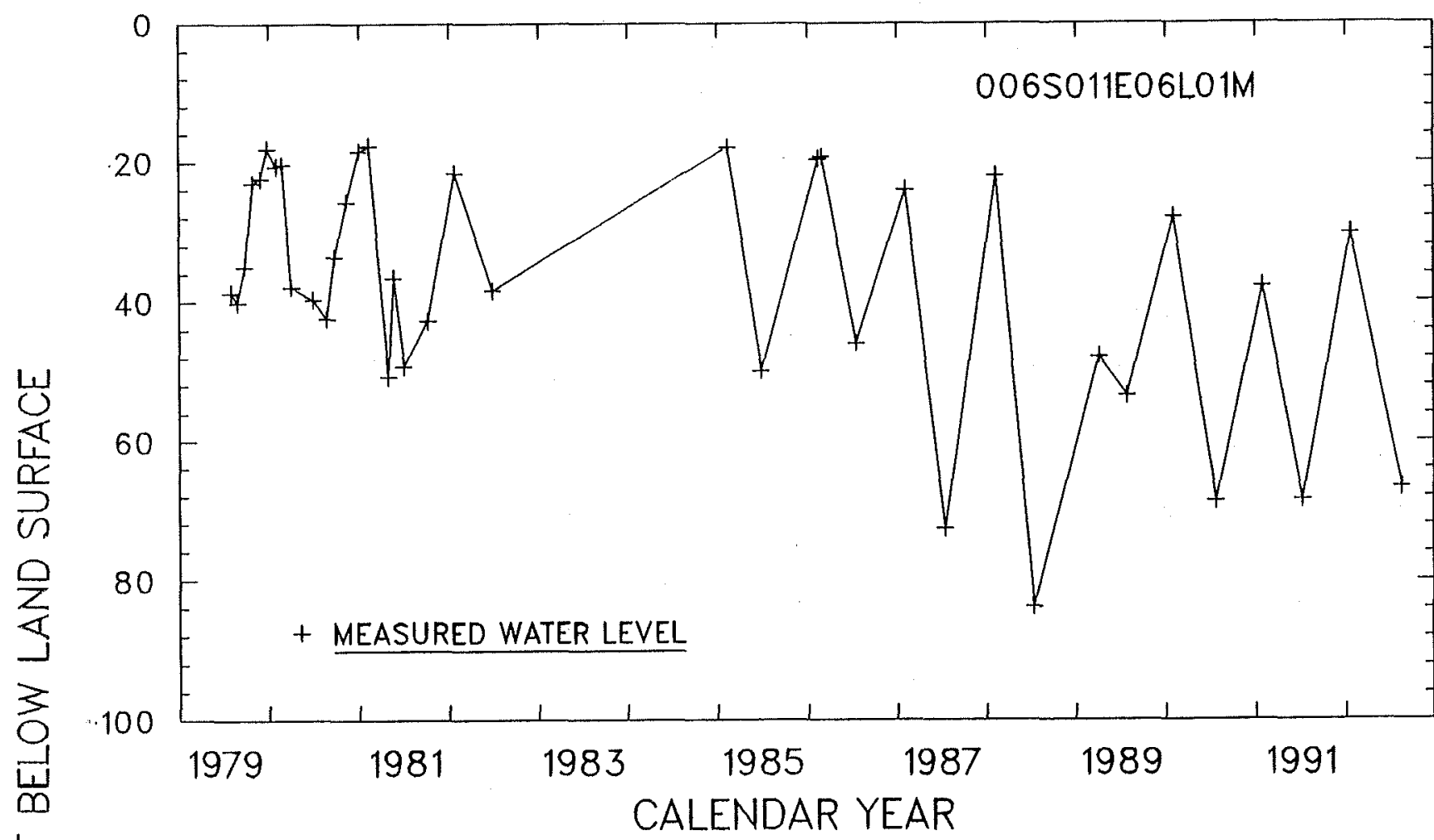

壵

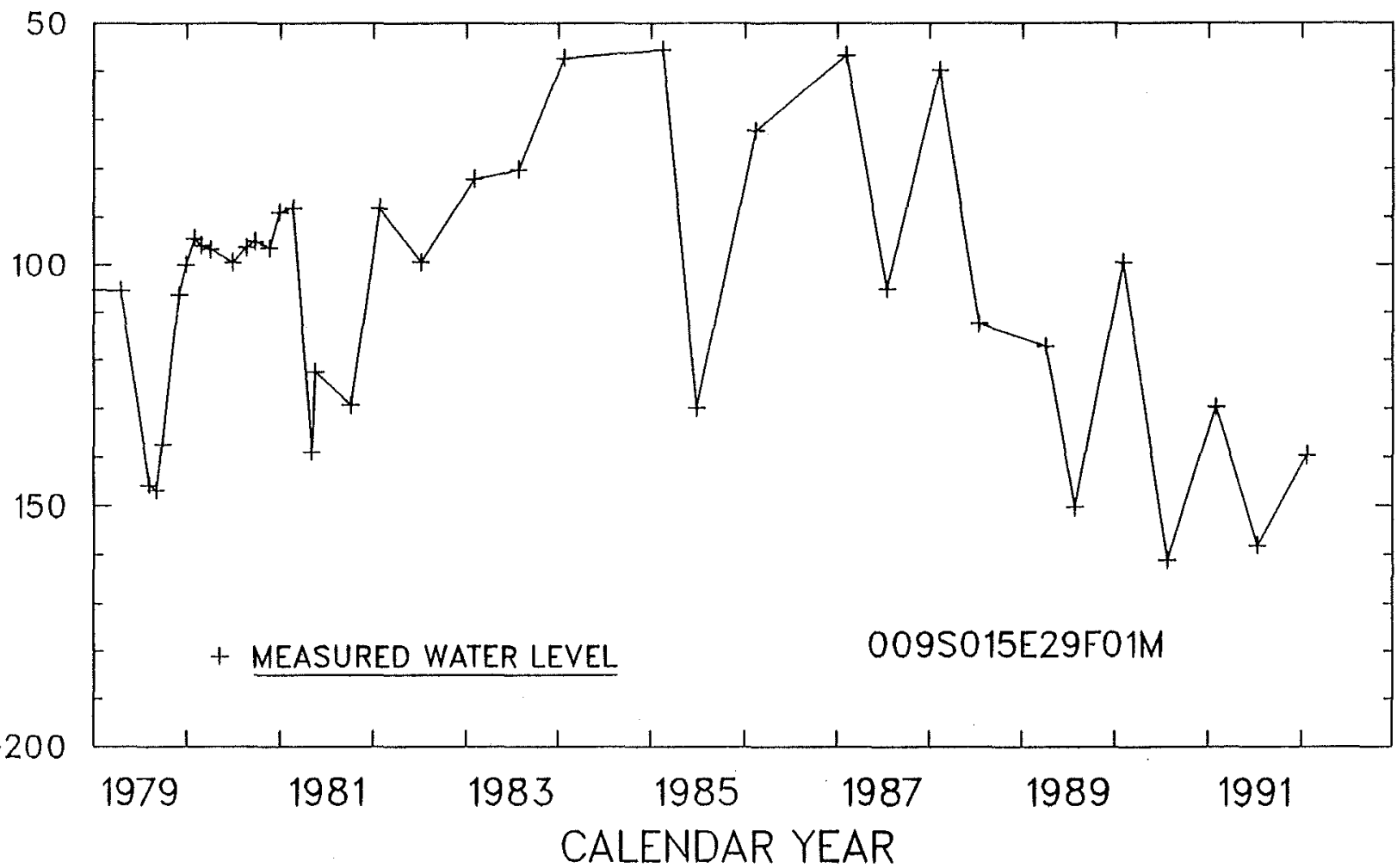

Figure 18. Hydrographs for wells in Merced County. (006S011E06L01M and 009S015E29F01M) 
WATER LEVELS, MERCED COUNTY

WELL DESCRIPTIONS AND WATER-LEVEL MEASUREMENTS

WATER YEAR OCTOBER 1991 TO SEPTEMBER 1992

SITE NUMBER 372919120444401

LOCAL NUMBER 005S011E22B02M

About 2.3 miles east of Turlock. Drilled irrigation well. Diameter 16 inches 0-228 feet, 14 inches 222-402 feet, perforated 226-398 feet. Altitude of land-surface datum 189 feet. Water-level records available 1976 , 1991 to current year.

WATER LEVELS IN FEET BELOW LAND-SURFACE DATUM

$$
\text { DATE WATER }
$$

FEB 06, $1992121.41 \mathrm{~s}$

PERIOD OF RECORD HIGHEST 121.41 FEB 06, 1992 LOWEST 130.07 JAN 28, 1991

SITE NUMBER 372753120464101

LOCAL NUMBER O0SS011E29R01M

About 3 miles southeast of Turlock. Drilled irrigation well. Diameter 16 inches, depth 410 fet, perforated 140-410 feet. Altitude of land-surface datum 121 feet. Water-level records available 1977, 1991 to current year. WATER LEVELS IN FEET BELOW LAND-SURFACE DATUM

$\begin{array}{lll} & \text { WATER } & \\ \text { DATE } & \text { WATER } \\ \text { LEVEL MS } & \text { DATE }\end{array}$

JAN $21,1992 \quad 45.49 \mathrm{~S} \quad$ SEP $01,1992 \quad 74.85 \mathrm{~S}$

PERIOD OF RECORD HIGHEST 45.49 JAN 21, 1992 LOWEST 79.16 JUL 09, 1991

SITE NUMBER 372904120415501

LOCAL NUMBER O05S012E19M01M

About 8 miles west of Turlock. Observation we11. Diameter 6 inches, depth 194 feet, perforated 155-165, 184-194 feet. Altitude of land-surface datum 156 feet. Water-level records available 1974,1984 to current year. WATER LEVELS IN FEET BELOW LAND-SURFACE DATUM

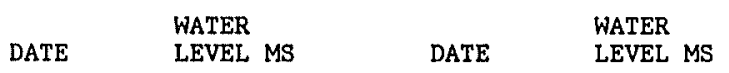

JAN 25, $1992102.38 \mathrm{~s}$ AUG $18,1992109.10 \mathrm{~s}$

PERIOD OF RECORD HIGHEST 74.68 FEB 13, 1985 LOWEST 109.10 AUG 18, 1992

SITE NUMBER 372709120415901

LOCAL NUMBER 005S012E31M02M

In Ballico. Drilled domestic well. Diameter 6 inches, depth 158 feet, perforated 138-158 feet. Altitude of land-surface datum 155 feet. Water-level records available 1974, 1978, 1982-83, 1992.

WATER LEVELS IN FEET BELOW LAND-SURFACE DATUM

$\begin{array}{lll} & \text { WATER } & \text { WATER } \\ \text { DATE } & \text { LEVEL MS }\end{array}$

FEB $04,199287.24 \mathrm{~S}$ AUG $18,1992117.26 \mathrm{~S}$

PERIOD OF RECORD HIGHEST 67.90 MAR 28, 1978 LOWEST 117.26 AUG 18, 1992 
SITE NUMBER 373107120351401

LOCAL NUMBER 005S013E07C01M

About 11 miles east of Turlock. Drilled irrigation well. Diameter 14 inches, depth 600 feet, perforated 312-600 feet. Altitude of land-surface datum 190 feet. Water-level records available 1992 .

WATER LEVELS IN FEET BELOW LAND-SURFACE DATUM

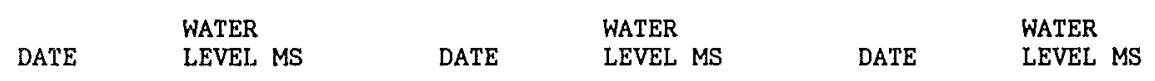

FEB 04, $1992114.95 \mathrm{~S}$ AUG 17, $1992 \quad \mathrm{P} \quad$ AUG 20, $1992 \quad$ P

PERIOD OF RECORD HIGHEST 114.95 FEB 04, 1992 LOWEST 114.95 FEB 04, 1992

SITE NUMBER 372708120334001

LOCAL NUMBER 005S013E32K01M

About 6 miles northeast of Winton. Drilled irrigation well. Diameter 18 inches $0-112$ feet, 16 inches 111-268 feet, 14 inches 268-359 feet, 12 inches 359-488 feet, perforated 112-488 feet. Altitude of land-surface datum 211 feet. Water-level records available 1971, 1991 to current year.

WATER LEVELS IN FEET BELOW LAND-SURFACE DATUM

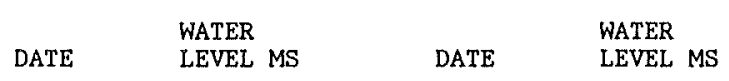

JAN 23, $1992 \quad 82.95 \mathrm{~S}$ AUG $18,1992100.06 \mathrm{~S}$

PERIOD OF RECORD HIGHEST 60.17 APR 19, 1971 LOWEST 100.06 AUG 18, 1992

SITE NUMBER 373116120235701

LOCAL NUMBER 005S014E02Q02M

About 3 miles east of Snelling. Domestic well. Diameter 6 inches, depth 50 feet. Altitude of land-surface datum 276 feet. Water-level records available 1986 to current year.

WATER LEVELS IN FEET BELOW LAND-SURFACE DATUM

$\begin{array}{ll} & \text { WATER } \\ \text { DATE } & \text { LEVEL MS }\end{array}$

SEP 02, $1992 \quad 21.77$ SR

PERIOD OF RECORD HIGHEST 13.33 FEB 02, $1987 \quad$ LOWEST 20.37 JUL 26,1990

SITE NUMBER 373109120261401

LOCAL NUMBER 005S014E09B01M

In Snelling. Drilled domestio well. Diameter 8 inches, depth 60 feet, depth of hole 165 feet. Altitude of land-surface datum 250 feet. Water-level records available $1978,1992$.

WATER LEVELS IN FEET BELOW LAND-SURFACE DATUM

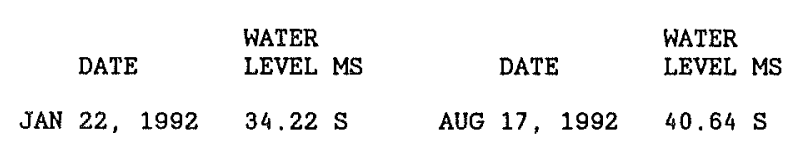

PERIOD OF RECORD HIGHEST 34.22 JAN 22, 1992 LOWEST 42.90 APR 18,1978 
WELL DESCRIPTIONS AND WATER-LEVEL MEASUREMENTS

WATER YEAR OCTOBER 1991 TO SEPTEMBER 1992

SITE NUMBER 372956120282201

LOCAL NUMBER 005S014E18K01M

About 3 miles southwest of Snelling. Drilled domestic well. Diameter 8 inches, depth reported 100 feet. Altitude of land-surface datum 221 feet. Water-level records avallable 1971, 1992.

WATER LEVELS IN FEET BELOW LAND-SURFACE DATUM

DATE WATER

AUG $31,1992 \quad 9.10 \mathrm{SR}$

PERIOD OF RECORD HIGHEST 9.00 APR 01, 1971 LOWEST 9.00 APR 01, 1971

SITE NUMBER 372617120431201

LOCAL NUMBER 006S011E01M01M

About 3.5 miles north of Livingston. Drilled irrigation well. Diameter 14 inches, depth 206 feet, perforated 155-163 feet. Altitude of land-surface datum 132 feet. Water-level records available 1973, 1991 to current year.

WATER LEVELS IN FEET BELOW LAND-SURFACE DATUM

$\begin{array}{lll} & \text { WATER } & \\ \text { DATE } & \text { WEVEL MS } & \text { DATE } \\ \text { LEVEL MS }\end{array}$

JAN $21,1992 \quad 59.61 \mathrm{~S} \quad$ AUG $21,1992 \quad \mathrm{P}$

PERIOD OF RECORD HIGHEST 41 DEC 05, 1973 LOWEST 101.38 JUL 12, 1991

SITE NUMBER 372626120442301

LOCAL NUMBER O06S011E03J01M

About 2 miles east of Delhi. Drilled domestic well. Diameter 6 inches, depth 145 feet, perforated $125-145$ feet. Altitude of land-surface datum 127 feet. Water-level records available 1977, 1992.

WATER LEVELS IN FEET BELOW LAND-SURFACE DATUM

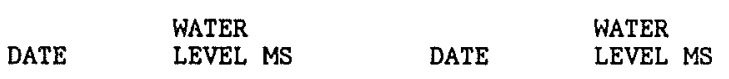

FEB 04, $1992 \quad 47.25$ SR SEP 01, $1992 \quad 0$

PERIOD OF RECORD HIGHEST 51 SEP 22, 1977 LOWEST 51 SEP 22, 1977

SITE NUMBER 372627120482301

LOCAL NUMBER 006S011E06L01M

About 1.5 miles northwest of Delhi. Irrigation well. Diameter 14 inches, depth 286 feet, perforated 202-286 feet. Altitude of land-surface datum 105 feet. Water-level records avallable 1979 to current year.

WATER LEVELS IN FEET BELOW LAND-SURFACE DATUM

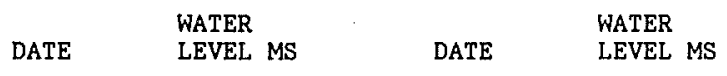

JAN $21,1992 \quad 30.32 \mathrm{~S} \quad$ AUG $18,199266.76 \mathrm{~s}$

PERIOD OF RECORD HIGHEST 17.67 FEB 09, 1981 LOWEST 83.82 JUL 14, 1988 
SITE NUMBER 372338120474401

LOCAL NUMBER 006S011E19J01M

About $2.8 \mathrm{mlles}$ southwest of Delhi. Domestic well. Diameter 6 inches, depth 182 feet, perforated $170-182$ feet. Alt1tude of land-surface datum 110 feet. Water-level records available 1981 to current year.

WATER LEVELS IN FEET BELOW LAND-SURFACE DATUM

$\begin{array}{lll} & \text { WATER } & \\ \text { DATE } & \text { WATER } \\ \text { LEVEL MS } & \text { DEVEL MS }\end{array}$

FEB $04,1992 \quad 35.62 \mathrm{~S} \quad$ AUG $19,1992 \quad 51.52 \mathrm{~s}$

PERIOD OF RECORD HIGHEST 14 FEB 25, $1981 \quad$ LOWEST 51.52 AUG 19, 1992

SITE NUMBER 372209120451201

LOCAL NUMBER 006S011E34F01M

About 2.2 miles southwest of Livingston. Irrigation well. Diameter 16 inches, depth 148 feet, perforated 10-25, 101-106, 119-124, 127-131 feet. Altitude of land-surface datum 117 feet, Water-level records available 1970 , 1980,1982 to current year.

WATER LEVELS IN FEET BELOW LAND-SURFACE DATUM

$$
\begin{array}{lll} 
& \text { WATER } & \text { WATER } \\
\text { DATE } & \text { DEVEL MS } & \text { DATE }
\end{array}
$$

FEB 05, $1992 \quad 41.95 \mathrm{~S} \quad$ AUG 19, $1992 \quad \mathrm{P}$

PERIOD OF RECORD HIGHEST 20 NOV 20, 1970 LOWEST 41.95 FEB 05, 1992

SITE NUMBER 372427120362601

LOCAL NUMBER 006S012E13P01M

About 1 mile north of Winton. Drilled irrigation well. Diameter 12 inches, depth 160 feet, perforated

120-160 feet. Altitude of land-surface datum 184 feet. Water-level records available 1977, 1991 to current year.

WATER LEVELS IN FEET BELOW LAND-SURFACE DATUM

$$
\begin{array}{lll} 
& \text { WATER } & \text { WATER } \\
\text { DATE } & \text { DEVEL MS }
\end{array}
$$

JAN $22,1992 \quad 73.68 \mathrm{~s}$ AUG $18,199281.50 \mathrm{~s}$

PERIOD OF RECORD HIGHEST 51 MAR 08, 1977 LOWEST 81.50 AUG 18, 1992

SITE NUMBER 372350120410201

LOCAL NUMBER 006S012E20E01M

About 2 miles northeast of Livingston. Drilled domestic well. Diameter 6 inches, depth 200 feet, perforated 120-140, 180-200 feet. Altitude of land-durface datum 150 feet. Water-level records available 1977 , 1991 to current year.

WATER LEVELS IN FEET BELOW LAND-SURFACE DATUM

$\begin{array}{lll} & \text { WATER } & \text { WATER } \\ \text { DATE } & \text { LEVEL MS } & \\ & & \text { DATE }\end{array}$
JAN 21, 1992
$60.70 \mathrm{~s}$
SEP 01, 1992
$69.60 \mathrm{~S}$

PERIOD OF RECORD HIGHEST 52 NOV 02, 1977 LOWEST 69.60 SEP 01,1992 
WELL DESCRIPTIONS AND WATER-LEVEL MEASUREMENTS

WATER YEAR OCTOBER 1991 TO SEPTEMBER 1992

SITE NUMBER 372318120381601

LOCAL NUMBER O06S012E27B01M

About 1.4 miles west of Winton. Irrigation well. Diameter 20 inches, depth 214 feet, depth of hole 284 feet. Altitude of land-surface datum 158 feet. Water-level records available 1923, 1975 to current year.

WATER LEVELS IN FEET BELOW LAND SURFACE DATUM

\begin{tabular}{|c|c|c|c|c|c|}
\hline DATE & $\begin{array}{l}\text { WATER } \\
\text { LEVEL MS }\end{array}$ & DATE & $\begin{array}{l}\text { WATER } \\
\text { LEVEL MS }\end{array}$ & DATE & WATER \\
\hline
\end{tabular}

FEB 05, $1992 \quad 66.67 \mathrm{~S} \quad$ AUG 19, $1992 \quad$ P $\quad$ AUG 20, $1992 \quad$ P

PERIOD OF RECORD HIGHEST 39.68 JAN 25, 1984 LOWEST 72.18 MAY 30,1978

SITE NUMBER 372217120384501

LOCAL NUMBER 006S012E34D01M

About 2.3 miles southwest of Winton. Irrigation well. Diameter 16 inches, depth 44 feet, depth of hole 82 feet. Altitude of land-surface datum 155 feet. Water-level records available 1924, 1970, 1975 to current year.

WATER LEVELS IN FEET BELOW LAND-SURFACE DATUM

$$
\begin{array}{lll} 
& \text { WATER } & \\
\text { DATE } & \text { WEVEL MS } & \text { DATE } \\
\text { LEVEL MS }
\end{array}
$$

FEB 05, $1992 \quad 61.30 \mathrm{~S} \quad$ AUG $19,1992 \quad 59.97 \mathrm{~s}$

$\begin{array}{llllllll}\text { PERIOD OF RECORD HIGHEST } & 2 & \text { JUN } & 1924 & \text { LOWEST } & 61,30 & \text { FEB } & 05,1992\end{array}$

SITE NUMBER 372440120350401

LOCAL NUMBER 006S013E18F01M

About 2 miles northeast of Winton. Drilled irrigation well. Diameter 12 inches, depth 172 feet, depth of hole 204 feet, perforated 123-126, 139-147, 156-159, 163-168 feet. Altitude of land-surface datum 201 feet. Water-level records available 1973, 1975, 1991 to current year.

WATER LEVELS IN FEET BELOW LAND-SURFACE DATUM

$$
\begin{array}{lll} 
& \text { WATER } & \\
\text { DATE } & \text { WEVEL MS } & \text { DATE } \\
\text { LEVEL MS }
\end{array}
$$

JAN $21,199276.15 \mathrm{~S} \quad$ AUG $18,1992 \quad 87.51 \mathrm{~S}$

PERIOD OF RECORD HIGHEST 53.6 SEP 10, 1975 LOWEST 87.51 AUG 18, 1992

SITE NUMBER 372323120323401

LOCAL NUMBER O06S013E28A01M

About 3.8 miles west of Winton. Drilled irrigation well. Diameter 18 inches $0-156$ feet, 16 inches $144-312$ feet, depth 312 feet, depth of hole 386 feet, perforated 168-312 feet. Altitude of land-surface datum 195 feet.

Water-level records available 1969-70, 1975, 1977 to current year.

WATER LEVELS IN FEET BELOW LAND-SURFACE DATUM

$$
\begin{array}{lll} 
& \text { WATER } & \\
\text { DATE } & \text { WATER } \\
\text { LEVEL MS } & \text { LEVEL MS }
\end{array}
$$

JAN 22, $199269.54 \mathrm{~s} \quad$ AUG $19,1992 \quad 82.02 \mathrm{~s}$

PERIOD OF RECORD HIGHEST 49.20 FEB 01, 1977 LOWEST 96.48 AUG 29, 1977 
SITE NUMBER 372444120265701

LOCAL NUMBER 006S014E17H01M

About 4.0 miles north of Merced. Observation well. Diameter 3 inches, depth 150 feet, perforated $130-140$ feet. Altitude of land-surface datum 273 feet. Water-level records available 1988 to current year.

WATER LEVELS IN FEET BELOW LAND-SURFACE DATUM

$$
\begin{array}{lll} 
& \text { WATER } & \text { WATER } \\
\text { DATE } & \text { LEVEL MS } & \text { DATE }
\end{array}
$$

JAN 22, $1992 \quad 108.10 \mathrm{~s} \quad$ AUG $17,1992110.49 \mathrm{~s}$

PERIOD OF RECORD HIGHEST 101.05 FEB 09, 1988 LOWEST 120.35 JUL 11,1991

SITE NUMBER 372225120272101

LOCAL NUMBER 006S014E32B01M

About 3 miles northeast of Merced. Drilled domestic well. Diameter 8 inches, depth 88 cased feet, depth drilled 126 feet. Altitude of land-surface datum 227 feet. Water-level records available 1979-83, 1992.

WATER LEVELS IN FEET BELOW LAND-SURFACE DATUM

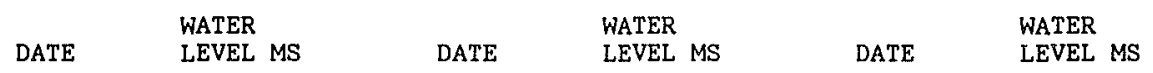

JAN 23, $1992 \quad 78.72 \mathrm{~s} \quad$ AUG $18,1992 \quad 82.15 \mathrm{SR} \quad$ AUG 19, $1992 \quad 81.36$ SR

PERIOD OF RECORD HIGHEST 28.65 SEP $18,1980 \quad$ LOWEST $\quad 78,72 \quad$ JAN 23, 1992

SITE NUMBER 372006120472201

LOCAL NUMBER 007S011E08P01M

About 4 miles southwest of Livingston. Drilled irrigation well. Diameter 16 inches, depth 168 feet, perforated 72-168 feet. Altitude of land-surface datum 96 feet. Water-level records available 1976, $1978-80,1992$.

WATER LEVELS IN FEET BELOW LAND-SURFACE DATUM

$\begin{array}{lllll} & \text { WATER } & \text { WATER } & \text { WATER } \\ \text { DATE } & \text { LEVEL MS } & \text { DATE } & \text { LEVEL MS } & \text { DATE }\end{array}$

JAN 22, $1992 \quad 21.77 \mathrm{~S} \quad$ AUG $19,1992 \quad$ P $\quad$ AUG $21,1992 \quad$ P

PERIOD OF RECORD HIGHEST 8 MAR 30,1976 LOWEST 22.55 AUG 28, 1979

SITE NUMBER 371914120421701

LOCAL NUMBER 007S011E13R01M

About 4 miles south of Livingston. Drilled domestic well. Diameter 6 inches, depth 180 feet, depth of hole 231 feet, perforated 160-180 feet. Altitude of land-surface datum 115 feet. Water-1evel records avallable 1991 to current year.

WATER LEVELS IN FEET BELOW LAND-SURFACE DATUM

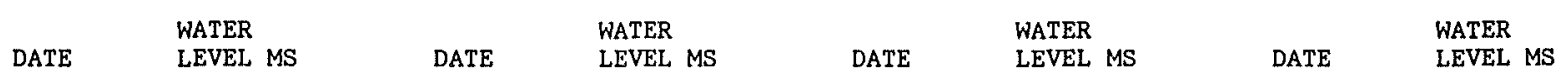

$\begin{array}{lllllllll}\text { JAN 22, } 1992 & P & \text { JAN } 23,1992 & \mathrm{P} & \text { FEB } 04,1992 & 28,18 \text { SP } & \text { AUG } 19,1992 & 31.20 \text { S }\end{array}$

PERIOD OF RECORD HIGHEST 31.20 AUG 19, 1992 LOWEST $38.93 \quad$ JUL 12,1991 
WELL DESCRIPTIONS AND WATER-LEVEL MEASUREMENTS

WATER YEAR OCTOBER 1991 TO SEPTEMBER 1992

SITE NUMBER 372020120383501

LOCAL NUMBER O07S012E10F02M

About $0.1 \mathrm{mile}$ southwest of Atwater. Drilled irrigation well in alluvium. Diameter unknown, depth 55 feet. Altitude of land-surface datum 145 feet. Water-level records avallable 1975 to current year.

WATER LEVELS IN FEET BELOW LAND-SURFACE DATUM

$$
\text { DATE WATER }
$$

AUG $19,199259.17 \mathrm{~s}$

PERIOD OF RECORD HIGHEST 23.36 JAN 23, 1984 LOWEST 59.17 AUG 19, 1992

SITE NUMBER 372030120371301

LOCAL NUMBER OO7S012E11G01M

About 0.4 mile southwest of Atwater. Drilled irrigation water-table well. Diameter 16 inches $0-100,14$ inches 100-220 feet, depth 220 feet, perforated 177-182, 186-190, 198-203, 213-217 feet. Altitude of land-surface datum 145 feet. Water-level records available 1971, 1977-81, 1991 to current year.

WATER LEVELS IN FEET BELOW LAND-SURFACE DATUM

$$
\begin{array}{lll} 
& \text { WATER } & \text { WATER } \\
\text { DATE } & \text { DEVEL MS } & \text { DETE }
\end{array}
$$

JAN 21, $1992 \quad 48.12 \mathrm{~S} \quad$ AUG $19,199269.17 \mathrm{~s}$

PERIOD OF RECORD HIGHEST 20.40 JAN 08, 1971 LOWEST 99.3 AUG 29, 1977

SITE NUMBER 372045120325301

LOCAL NUMBER OO7S013E09B03M

About 3 miles northeast of Merced. Drilled irrigation we11. Diameter 16 inches, depth 250 feet, perforated 172-250 feet. Altitude of land-surface datum 165 feet. Water-level records available 1984,1991 to current year. WATER LEVELS IN FEET BELOW LAND-SURFACE DATUM

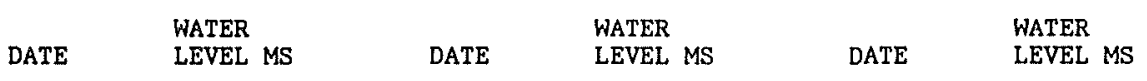

JAN 21, $1992 \quad 50.76 \mathrm{~S} \quad$ AUG 19, $1992 \quad \mathrm{P} \quad$ AUG 20, 1992 P

PERIOD OF RECORD HIGHEST 36 OCT 1984 LOWEST 54.72 JUL 10,1991

SITE NUMBER 371830120324601

LOCAL NUMBER 007S013E21K01M

About 3 miles west of Merced. Drilled irrigation well. Diameter 16 inches, depth 192 feet, perforated $108-114$, 134-142, 180-188 feet. Altitude of land-surface datum 150 feet. Water-level records avallable 1970 , 1975 , 1991 to current year.

WATER LEVELS IN FEET BELOW LAND-SURFACE DATUM

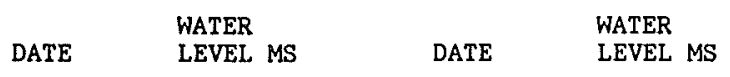

JAN 21, $1992 \quad 27.48 \mathrm{~S} \quad$ AUG $18,1992 \quad 38.90 \mathrm{~s}$

PERTOD OF RECORD HIGHEST 11.42 DEC $11,1970 \quad$ LOWEST $38.90 \quad$ AUG 18,1992 
SITE NUMBER 371650120325901

LOCAL NUMBER 007S013E33K01M

About 3 miles west of Merced. Drilled irrigation well. Diameter 16 inches, depth 388 feet, perforated 165-177, 302-311, 354-378 feet. Altitude of land-surface datum 140 feet. Water-level records available 1975,1991 to current year.

WATER LEVELS IN FEET BELOW LAND-SURFACE DATUM

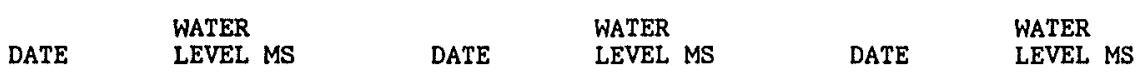

JAN 22, $1992 \quad 24.06 \mathrm{~S} \quad$ AUG $18,1992 \quad P \quad$ AUG 19, $1992 \quad$ P

PERIOD OF RECORD HIGHEST 12.45 SEP 12, $1975 \quad$ LOWEST 45.92 JUL 10,1991

SITE NUMBER 371650120285701

LOCAL NUMBER O07S014E31M01M

In south Merced. Drilled irrigation well. Diameter 16 inches, depth 204 feet, perforated 72-75, 122-125,

174-178 feet. Altitude of land-surface datum 160 feet. Water-level records available 1970, 1975-82, 1991 to current year.

WATER LEVELS IN FEET BELOW LAND-SURFACE DATUM

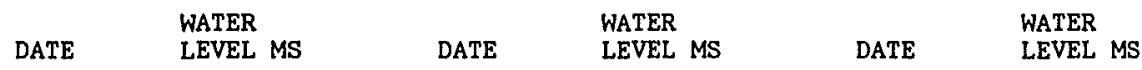

$\begin{array}{llllll}\text { JAN 22, } 1992 & 40.67 \mathrm{~s} & \text { AUG } 18,1992 & \text { P } & \text { AUG 20, } 1992 & \text { P }\end{array}$

PERIOD OF RECORD HIGHEST 18.47 NOV 10,1970 LOWEST 79.10 AUG 30,1977

SITE NUMBER 371709120240801

LOCAL NUMBER 007S014E35B01M

About 2 miles east of Merced. Drilled irrigation well. Diameter 16 inches, depth 272 feet, depth of hole 536 feet, perforated 155-161, 236-242, 263-268 feet. Altitude of land-surface datum 192 feet. Water-level records available 1969, 1975, 1991 to current year.

WATER LEVELS IN FEET BELOW LAND-SURFACE DATUM

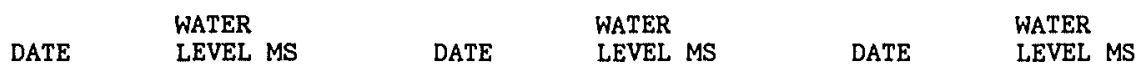

JAN 22, $1992 \quad 45.20 \mathrm{~S}$ AUG 18, $1992 \quad \mathrm{P} \quad$ AUG 21, $1992 \quad$ P

PERIOD OF RECORD HIGHEST 18.00 DEC 06, 1969 LOWEST 48.98 FEB 06, 1991

SITE NUMBER 371919120215801

LOCAL NUMBER 007S015E18K01M

About 6.5 miles east of Merced. Unused well. Diameter 18 inches, depth 84 feet, depth of hole 550 feet. Altitude of land-surface datum 212 feet. Water-level records available 1970, 1981 to current year.

WATER LEVELS IN FEET BELOW LAND-SURFACE DATUM

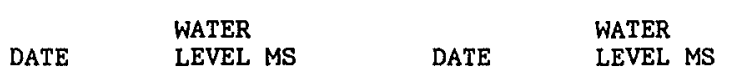

FEB $05,1992 \quad 46.15 \mathrm{~S} \quad$ AUG $17,1992 \quad 54.17 \mathrm{~S}$

PERIOD OF RECORD HIGHEST 25.8 JUL 26,1983 LOWEST 54.17 AUG 17,1992 
WELL DESCRIPTIONS AND WATER-LEVEL MEASUREMENTS

WATER YEAR OCTOBER 1991 TO SEPTEMBER 1992

SITE NUMBER 371743120194301

LOCAL NUMBER 007S015E28K01M

About 7 miles east of Merced. Drilled domestic well. Diameter 10 inches, depth 56 feet, perforated $44-52$ feet. Altitude of land-surface datum 225 feet. Water-level records available 1971 to current year.

WATER LEVELS IN FEET BELOW LAND-SURFACE DATUM

$$
\text { DATE WATER }
$$

AUG 19, $1992 \quad 22.01 \mathrm{~s}$

PERIOD OF RECORD HIGHEST 16.44 FEB 08, $1979 \quad$ LOWEST 22.01 AUG 19,1992

SITE NUMBER 371733120201801

LOCAL NUMBER 007S015E28M01M

West of Planada. Drilled irrigation we11. Diameter 16 inches, depth 200 feet, depth of hole 642 feet, perforated 84-196 feet. Altitude of land-surface datum 216 feet. Water-level records available 1967 , 1979 , 1991 to current year.

WATER LEVELS IN FEET BELOW LAND-SURFACE DATUM

$$
\begin{array}{lll} 
& \text { WATER } & \\
\text { DATE } & \text { LEVEL MS } & \text { WATER } \\
\text { LEVEL MS }
\end{array}
$$

FEB $05,1992 \quad 31.45 \mathrm{~S} \quad$ AUG $17,1992 \quad 38.15 \mathrm{~s}$

PERIOD OF RECORD HIGHEST 19.34 FEB $08,1979 \quad$ LOWEST $38.15 \quad$ AUG 17,1992

SITE NUMBER 371747120222701

LOCAL NUMBER 007S015E30E01M

About 3 miles west of Planada. Drilled irrigation well. Diameter 16 inches, depth 192 feet, depth of hole

642 feet, periorated 80-188 feet. Altitude of land-surface datum 140 feet. Water-1evel records available 1967 , 1979,1991 to current year.

WATER LEVELS IN FEET BELOW LAND-SURFACE DATUM

$$
\begin{array}{lll} 
& \text { WATER } & \\
\text { DATE } & \text { WEVEL MS } & \text { WATER } \\
& & \text { LEVEL MS }
\end{array}
$$

FEB $05,1992 \quad 38.23 \mathrm{~s} \quad$ AUG $17,1992 \quad 51.65 \mathrm{~s}$

PERIOD OF RECORD HIGHEST 17 NOV $16,1967 \quad$ LOWEST 55.32 JUL 11,1991

SITE NUMBER 371652120195901

LOCAL NUMBER 007S015E33F01M

About 7 miles east of Merced, Drilled domestic well. Diameter 14 inches, depth 360 feet, depth of hole 560 feet, perforated 164-207, 270-296, 304-318, 323-354 feet. Altitude of land-surface datum 217 feet. Water-1evel records available 1977 to current year.

WATER LEVELS IN FEET BELOW LAND-SURFACE DATUM

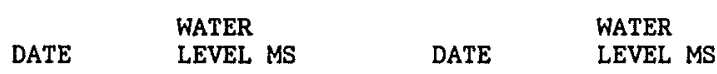

FEB 05, $1992 \quad 37.05 \mathrm{~s} \quad$ AUG $17,1992 \quad 41.40 \mathrm{~s}$

PERIOD OF RECORD HIGHEST 20.38 APR 22, 1987 LOWEST 41.40 AUG 17,1992 
SITE NUMBER 371653120193901

LOCAL NUMBER 007S015E33H01M

About 7 miles east of Merced. Drilled domestic well. Diameter 12 inches, depth 205 feet, perforated 80-100, 120-200 feet. Altitude of land-surface datum 221 feet. Water-level records available 1968, 1975, 1992.

WATER LEVELS IN FEET BELOW LAND-SURFACE DATUM

\begin{tabular}{|c|c|c|c|c|c|}
\hline & WATER & & WATER & & WATER \\
\hline DATE & LEVEL MS & DATE & LEVEL MS & DATE & LEVEL MS \\
\hline
\end{tabular}

$\begin{array}{llllll}\text { FEB } 05,1992 & 26.81 \mathrm{~S} & \text { AUG } 18,1992 & \mathrm{P} & \text { AUG 20, } 1992 & \mathrm{P}\end{array}$

PERIOD OF RECORD HIGHEST 17,85 FEB 08, 1975 LOWEST 60 JUL 03, 1968

SITE NUMBER 371438120375901

LOCAL NUMBER O08S012E15A01M

About 8 miles southwest of Merced. Drilled irrigation well. Diameter 16 inches, depth 254 feet, perforated 125-240 feet. Altitude of land-surface datum 108 feet. Water-level records available 1975, 1979-81, 1987,1991 to current year.

WATER LEVELS IN FEET BELOW LAND-SURFACE DATUM

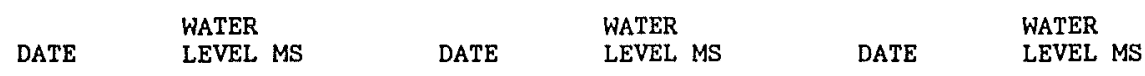

JAN 22, $1992 \quad 40.42 \mathrm{~S}$ AUG 18, $1992 \quad$ P $\quad$ AUG 19, $1992 \quad$ P

PERIOD OF RECORD HIGHEST 19.41 APR 01, 1980 LOWEST 53.30 JUL 10, 1991

SITE NUMBER 371320120344301

LOCAL NUMBER DO8S013E19H01M

About 7.6 miles southwest of Merced. Domestic well. Diameter 8 inches, depth 241 feet, perforated 207-239 feet. Altitude of land-surface datum 121 feet. Water-level records available 1982 to current year.

WATER LEVELS IN FEET BELOW LAND-SURFACE DATUM

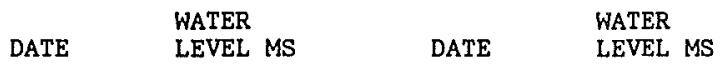

AUG 18, 1992 P AUG 20, 1992 P

PERIOD OF RECORD HIGHEST 17,70 FEB 14, 1985 LOWEST 64.59 JUL 24, 1990

SITE NUMBER 371134120341001

LOCAL NUMBER 008S013E32K01M

About $8 \mathrm{miles}$ southwest of Merced. Drilled irrigation well. Diameter 14 inches, depth 244 feet, perforated 107-117, 220-240 feet. Altitude of land-surface datum 120 feet. Water-level records available 1976, 1979, 1991. to current year.

WATER LEVELS IN FEET BELOW LAND-SURFACE DATUM

$$
\begin{array}{lll} 
& \text { WATER } & \text { WATER } \\
\text { DATE } & \text { LEVEL MS } & \text { DATE }
\end{array}
$$

JAN $23,1992 \quad 53.01 \mathrm{~s} \quad$ AUG $18,1992 \quad 65.65 \mathrm{~s}$

PERIOD OF RECORD HIGHEST 34 MAR 23, 1976 LOWEST 65.65 AUG 18,1992 
WELL DESCRIPTIONS AND WATER-LEVEL MEASUREMENTS

WATER YEAR OCTOBER 1991 TO SEPTEMBER 1992

SITE NUMBER 371524120234901

LOCAL NUMBER 008S014E11A01M

About 5.8 miles southwest of Merced. Irrigation well. Diameter 16 inches, depth 380 feet, depth of hole 765 feet, perforated 83-90, 147-154. 262-269 feet. Altitude of land-surface datum 188 feet. Water-level records avallable $1967,1970,1975,1985$ to current year.

WATER LEVELS IN FEET BELOW LAND-SURFACE DATUM

$\begin{array}{lll} & \text { WATER } & \\ \text { DATE } & \text { WEVEL MS } & \text { DATE } \\ \text { LEVEL MS }\end{array}$

JAN 22, $199265.80 \mathrm{~s}$ AUG $17,199275.10 \mathrm{~s}$

PERIOD OF RECORD HIGHEST 21.73 FEB 03, 1987 LOWEST 82.13 JUL 10, 1991

SITE NUMBER 371430120291001

LOCAL NUMBER 008S014E18D01M

About 4 miles south of Merced. Drilled stock well. Diameter 6 inches, depth 86 feet, perforated $76-86$ feet. Altitude of land-surface datum 153 feet. Water-level records available 1992.

WATER LEVELS IN FEET BELOW LAND-SURFACE DATUM

$$
\text { DATE WATER }
$$

SEP $03,1992 \quad 4.99 \mathrm{~S}$

PERIOD OF RECORD HIGHEST 4.99 SEP 03, 1992 LOWEST 4.99 SEP 03, 1992

SITE NUMBER 371326120290601

LOCAL NUMBER 008S014E19E01M

About 4.5 miles south of Merced. Drilled domestic well. Diameter 6 inches, depth 94 feet, perforated 84-94 feet. Altitude of land-surface datum 147 feet. Water-level records available 1981-82, 1992.

WATER LEVELS IN FEET BELOW LAND-SURFACE DATUM

$\begin{array}{ll} & \text { WATER } \\ \text { DATE } & \text { LEVEL MS }\end{array}$

SEP 03, $199290.82 \mathrm{SR}$

PERIOD OF RECORD HIGHEST 32.39 JAN 28, 1982 LOWEST 47.09 DEC 09, 1981

SITE NUMBER 371156120280901

LOCAL NUMBER 008S014E31A01M

About 7 miles south of Merced. Drilled irrigation well. Diameter 16 inches, depth 496 feet, perforated 180-485 feet. Altitude of land-surface datum 151 feet. Water-level records available 1976, 1979,1991 to current year.

WATER LEVELS IN FEET BELOW LAND-SURFACE DATUM

$\begin{array}{llll} & \text { WATER } & \text { WATER } & \text { WATER } \\ \text { DATE } & \text { LEVEL MS } & \text { DATE } & \text { LEVEL MS }\end{array}$

FEB 22, $199262.85 \mathrm{~S}$ AUG 18, $1992 \quad \mathrm{P} \quad$ AUG $21,1992 \quad$ P

PERIOD OF RECORD HIGHEST 45.15 JAN 31, 1979 LOWEST 67.48 FEB 06, 1991 
SITE NUMBER 371411120194001

LOCAL NUMBER 008S015E16J01M

About 0.3 mile northwest of Plainsburg. Irrigation well. Diameter 14 inches, depth 284 feet, depth of hole 420 feet, perforated 200-280 feet. Altitude of land-surface datum 216 feet. Water-level records avallable 1972 , 1979,1982 to current year.

WATER LEVELS IN FEET BELOW LAND-SURFACE DATUM

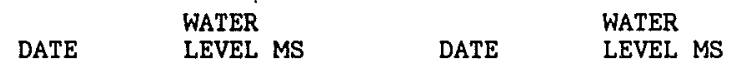

JAN 23, $1992123.35 \mathrm{~S}$ AUG $17,1992139.50 \mathrm{~S}$

PERIOD OF RECORD HIGHEST 75.15 FEB 14, 1985 LOWEST 139.50 AUG 17, 1992

SITE NUMBER 371043120344701

LOCAL NUMBER OO9S013E05MO1M

About 8 miles southwest of Merced. Drilled irrigation well. Diameter 18 inches, depth 290 feet, perforated 110-290 feet. Altitude of land-surface datum 114 feet. Water-level records available 1979-81, 1991 to current year.

WATER LEVELS IN FEET BELOW LAND-SURFACE DATUM

$\begin{array}{lll} & \text { WATER } & \\ \text { DATE } & \text { WEVEL MS } & \text { WATER } \\ \text { LEVEL MS }\end{array}$

JAN 22, $199252.20 \mathrm{~S}$ AUG 18, $199262.34 \mathrm{SR}$

PERIOD OF RECORD HIGHEST 42.90 APR 01, 1980 LOWEST 112.19 AUG 02, 1979

SITE NUMBER 370939120304901

LOCAL NUMBER OOSS013E11KO1M

About 3 miles north of El Nido. Drilled irrigation well. Diameter 16 inches, depth 321 feet, perforated 80-320 feet. Altitude of land-surface datum 131 feet. Water-level records available 1992.

WATER LEVELS IN FEET BELOW LAND-SURFACE DATUM

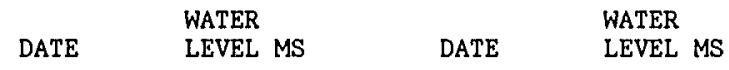

JAN $22,1992 \quad 54.50 \mathrm{~S} \quad$ AUG $18,1992 \quad \mathrm{P}$

PERIOD OF RECORD HIGHEST 54.50 JAN 22, 1992 LOWEST $54.50 \quad$ JAN 22, 1992

SITE NUMBER 370713120322801

LOCAL NUMBER 009S013E27M01M

About 3 miles west of El Nido. Drilled irrigation well. Altitude of land-surface datum 120 feet. Water-level records available 1970 to current year.

WATER LEVELS IN FEET BELOW LAND-SURFACE DATUM

\begin{tabular}{|c|c|c|c|c|c|}
\hline & WATER & & WATER & & WATER \\
\hline DATE & LEVEL MS & DATE & LEVEL MS & DATE & LEVEL MS \\
\hline JAN 22, 1992 & $61.60 \mathrm{~s}$ & AUG 18,1992 & $\mathbf{P}$ & AUG 20,1392 & $\mathbf{P}$ \\
\hline
\end{tabular}


WELL DESCRIPTIONS AND WATER-LEVEL MEASUREMENTS

WATER YEAR OCTOBER 1991 TO SEPTEMBER 1992

SITE NUMBER 370855120225601

LOCAL NUMBER OO9S014E13J01M

About 6 miles east of El Nido. Drilled irrigation well. Diameter 14 inches, depth 304 feet, perforated 161-300 feet. Altitude of land-surface datum 190 feet. Water-level records available 1991 to current year.

WATER LEVELS IN FEET BELOW LAND-SURFACE DATUM

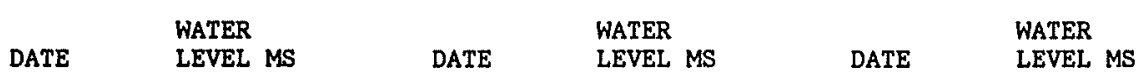

JAN 23, $1992126.42 \mathrm{~S}$ AUG 17, $1992 \quad \mathrm{P} \quad$ AUG 18, $1992 \quad$ P

PERIOD OF RECORD HIGHEST 122.51 JAN 30, 1991 LOWEST 126.42 JAN 23, 1992

SITE NUMBER 370923120183601

LOCAL NUMBER OO9S015E15A01M

About 3 miles northwest of Chowchilla. Drilled irrigation well. Diameter 16 inches, depth 449 feet, perforated 200-226, 270-283, 334-358, 400-440 feet. Altitude of land-surface datum 219 feet. Water-level records available $1979,1982,1991$ to current year.

WATER LEVELS IN FEET BELOW LAND-SURFACE DATUM

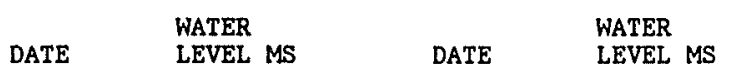

JAN 23, $1992187.70 \mathrm{~S}$ AUG $31,1992 \quad \mathrm{P}$

PERIOD OF RECORD HIGHEST 128.46 FEB 11, 1982 LOWEST 191.55 JUL 10, 1991

SITE NUMBER 370748120194101

LOCAL NUMBER O09S015E21R01M

About 4 miles west of Chowchilla. Drilled irrigation well. Diameter 14 inches, depth 450 feet, perforated 150-214, 242-265, 286-310, 320-360, 394-418, 426-450 feet. Altitude of land-surface datum 211 feet. Water-1evel records available 1964, 1992.

WATER LEVELS IN FEET BELOW LAND-SURFACE DATUM

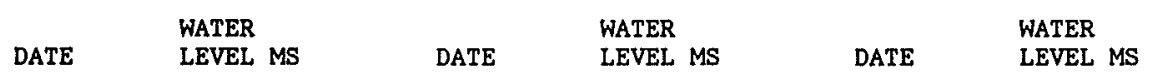

FEB 05, $1992104.40 \mathrm{~S}$ AUG 17, $1992 \quad \mathrm{P} \quad$ AUG 18, $1992 \quad$ P

PERIOD OF RECORD HIGHEST 102.00 NOV 01, 1964 LOWEST 104.40 FEB 05, 1992

SITE NUMBER 370713120212401

LOCAL NUMBER 009S015E29F01M

About 5.2 miles west of Chowchilla. Irrigation well. Diameter 14 inches, depth 342 feet, perforated 150-186, 210-232, 243-292, 308-339 feet. Altitude of land-surface datum 196 feet. Water-level records available 1964 , 1979 to current year.

WATER LEVELS IN FEET BELOW LAND-SURFACE DATUM

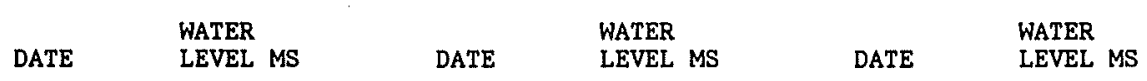

JAN 23, $1992139.70 \mathrm{~S}$ AUG 17, $1992 \quad \mathrm{P} \quad$ AUG 18, $1992 \quad$ P

PERIOD OF RECORD HIGHEST 55.55 FEB 14, 1985 LOWEST 161.09 JUL 24, 1990 
WELL DESCRIPTIONS AND WATER-LEVEL MEASUREMENTS

WATER YEAR OCTOBER 1991 TO SEPTEMBER 1992

SITE NUMBER 370915120162301

LOCAL NUMBER O09S016E18D01M

About 2.5 miles north of Chowchilla. Domestic well. Diameter 10 inches, depth 320 feet, depth of hole 500 feet, perforated 306-314 feet. Altitude of land-surface datum 239 feet. Water-1evel records available 1972 , 1981 to current yoar.

WATER LEVELS IN FEET BELOW LAND-SURFACE DATUM

$$
\begin{array}{lll} 
& \text { WATER } & \\
\text { DATE } & \text { WEVEL MS } & \text { WATER } \\
\text { LEVEL MS }
\end{array}
$$

FEB $05,1992 \quad 171.87 \mathrm{~S} \quad$ AUG $17,1992188.60 \mathrm{~S}$

PERIOD OF RECORD HIGHEST 121.48 FEB 03, 1987 LOWEST 188.60 AUG 17, 1992 
INORGANIC DATA

WATER YEAR OCTOBER 1991 TO SEPTEMBER 1992

LOCAL
NUMBER
005S014E02Q02M
005S014E09B02M
$005 \mathrm{~S} 014 \mathrm{E} 18 \mathrm{~K} 01 \mathrm{M}$
006S013E04Q01M
006S013E25M01M
006S014E29R01M
006S014E32A01M
006S014E32B01M
006S015E03Q01M
007S013E22P01M

007S014E05A01M
007S014E16L01M
007S014E29R01M
007S015E28K01M
$007 \mathrm{~S} 015 \mathrm{E} 35 \mathrm{~A} 02 \mathrm{M}$
007S015E35P01M
008S014E18D01M
008S014E19E01M

$008 \mathrm{~S} 014 \mathrm{E} 19 \mathrm{E} 01 \mathrm{M}$

\begin{tabular}{|c|c|c|c|c|c|c|c|c|c|}
\hline DATE & TIME & $\begin{array}{l}\text { DEPTH } \\
\text { BELOW } \\
\text { LAND } \\
\text { SURFACE } \\
\text { (WATER } \\
\text { LEVEL) } \\
\text { (FEET) }\end{array}$ & $\begin{array}{l}\text { DEPTH } \\
\text { OF } \\
\text { WELL, } \\
\text { TOTAL } \\
\text { (FEET) }\end{array}$ & $\begin{array}{l}\text { SPE- } \\
\text { CIFIC } \\
\text { CON- } \\
\text { DUCT- } \\
\text { ANCE } \\
\text { (US/CM) }\end{array}$ & $\begin{array}{c}\text { PH } \\
\text { WATER } \\
\text { WHOLE } \\
\text { FIELD } \\
\text { (STAND- } \\
\text { ARD } \\
\text { UNITS) }\end{array}$ & $\begin{array}{c}\text { TEMPER- } \\
\text { ATURE } \\
\text { AIR } \\
\text { (DEG C) }\end{array}$ & $\begin{array}{c}\text { TEMPER- } \\
\text { ATURE } \\
\text { WATER } \\
\text { (DEG C) }\end{array}$ & $\begin{array}{l}\text { HARD- } \\
\text { NESS } \\
\text { TOTAL } \\
\text { (MG/L } \\
\text { AS } \\
\text { CACO3) }\end{array}$ & $\begin{array}{c}\text { CALC } \\
\text { DIS } \\
\text { SOL } \\
\text { (MG } \\
\text { AS }\end{array}$ \\
\hline $\begin{array}{l}09-02-92 \\
09-01-92 \\
08-31-92 \\
09-01-92 \\
09-02-92\end{array}$ & $\begin{array}{l}1115 \\
1140 \\
1720 \\
0920 \\
1230\end{array}$ & $\begin{array}{l}21.77 \\
-. \\
9.10 \\
-- \\
--\end{array}$ & $\begin{array}{r}50 \\
64 \\
100 \\
128 \\
202\end{array}$ & $\begin{array}{l}260 \\
147 \\
190 \\
332 \\
264\end{array}$ & $\begin{array}{l}7.2 \\
7.2 \\
7.1 \\
7.1 \\
7.3\end{array}$ & $\begin{array}{l}28.5 \\
27.0 \\
29.0 \\
22.0 \\
28.5\end{array}$ & $\begin{array}{l}21.5 \\
20.0 \\
20.0 \\
18.0 \\
22.0\end{array}$ & $\begin{array}{r}95 \\
57 \\
79 \\
130 \\
67\end{array}$ & $\begin{array}{l}20 \\
14 \\
20 \\
32 \\
16\end{array}$ \\
\hline $\begin{array}{l}09-01-92 \\
08-20-92 \\
08-19-92 \\
08-31-92 \\
08-20-92\end{array}$ & $\begin{array}{l}1712 \\
1120 \\
1745 \\
1930 \\
0930\end{array}$ & $\begin{array}{c}-- \\
\overline{--} \\
81.36 \\
-- \\
--\end{array}$ & $\begin{array}{r}152 \\
165 \\
88 \\
- \\
100\end{array}$ & $\begin{array}{l}213 \\
255 \\
287 \\
169 \\
739\end{array}$ & $\begin{array}{l}7.6 \\
7.2 \\
7.2 \\
7.5 \\
7.2\end{array}$ & $\begin{array}{l}31.0 \\
32.5 \\
38.5 \\
20.0 \\
27.0\end{array}$ & $\begin{array}{l}23.0 \\
20.0 \\
22.5 \\
22.0 \\
20.0\end{array}$ & $\begin{array}{r}65 \\
70 \\
84 \\
40 \\
320\end{array}$ & $\begin{array}{c}16 \\
17 \\
21 \\
9.0 \\
73\end{array}$ \\
\hline $\begin{array}{l}09-03-92 \\
09-02-92 \\
09-02-92 \\
08-19-92 \\
09-01-92\end{array}$ & $\begin{array}{l}1400 \\
0835 \\
0740 \\
1021 \\
1400\end{array}$ & $\begin{array}{c}-- \\
-- \\
22.01 \\
--\end{array}$ & $\begin{array}{r}268 \\
184 \\
56 \\
120\end{array}$ & $\begin{array}{l}167 \\
290 \\
470 \\
597 \\
534\end{array}$ & $\begin{array}{l}7.6 \\
7.5 \\
7.8 \\
7.4 \\
7.8\end{array}$ & $\begin{array}{l}25.5 \\
20.5 \\
19.0 \\
33.0 \\
29.5\end{array}$ & $\begin{array}{l}21.5 \\
20.5 \\
20.5 \\
21.0 \\
21.0\end{array}$ & $\begin{array}{r}37 \\
110 \\
170 \\
230 \\
210\end{array}$ & $\begin{array}{l}8.9 \\
24 \\
43 \\
53 \\
50\end{array}$ \\
\hline $\begin{array}{l}08-19-92 \\
09-03-92 \\
09-03-92\end{array}$ & $\begin{array}{l}2000 \\
1030 \\
0930\end{array}$ & $\begin{array}{r}-- \\
4.99 \\
90.82\end{array}$ & $\begin{array}{l}76 \\
86 \\
94\end{array}$ & $\begin{array}{l}572 \\
580 \\
491\end{array}$ & $\begin{array}{l}7.6 \\
7.8 \\
7.8\end{array}$ & $\begin{array}{l}30.5 \\
26.0 \\
25.0\end{array}$ & $\begin{array}{l}19.5 \\
20.0 \\
20.5\end{array}$ & $\begin{array}{l}230 \\
210 \\
190\end{array}$ & $\begin{array}{l}53 \\
48 \\
45\end{array}$ \\
\hline
\end{tabular}

MAGNE-

SIUM, SODIUM,

DIS- DIS-

SOLVED SOLVED

(MG/L (MG/L SODIUM

AS MG) AS NA) PERCENT

\begin{tabular}{|c|c|c|c|c|c|}
\hline SODIU & & $\begin{array}{l}\text { BICAR- } \\
\text { BONATE }\end{array}$ & $\begin{array}{l}\text { ALKA- } \\
\text { LINITY }\end{array}$ & & CHLC \\
\hline$A D-$ & IU & WATER & WAT WH & SULFATE & RIDE, \\
\hline SORP- & DIS- & WH IT & TOT IT & DIS & DIS- \\
\hline TTON & SOLVED & FIELD & FIELD & SOLVED & SOLVED \\
\hline RATIO & $\begin{array}{l}\text { (MG/L } \\
\text { AS K) }\end{array}$ & $\underset{\mathrm{HCO} / \mathrm{LS}}{\mathrm{MCS}}$ & $\begin{array}{c}\text { MG } / L \text { AS } \\
\text { CACO3 }\end{array}$ & $\begin{array}{c}(M G / L \\
\text { AS SO 4) }\end{array}$ & $\begin{array}{l}(M G / L \\
\text { AS CL) }\end{array}$ \\
\hline
\end{tabular}

$09-02-92$

09-01-92

$08-31-92$

$09-01-92$

09-02-92

11

$\begin{array}{rc}11 & 15 \\ 5.2 & 6.1 \\ 7.1 & 5.4 \\ 12 & 19 \\ 6.4 & 24\end{array}$

25

$\begin{array}{ll}0.7 & 2.8\end{array}$

153

125
60

$0.3 \quad 1.6$

0.7

2.5

73
123
201

09-01-92

08-20-92

08-19-92

08-31-92

08-20-92

$6.0 \quad 19$

$6.6 \quad 26$

$7.6 \quad 25$

$\begin{array}{rr}4.3 & 17 \\ 32 & 28\end{array}$

$09-03-92$

$09-02-92$

09-02-92

$3.7 \quad 21$

12

16

$09-01-92$

24
21

2.7

76

51
165

4.0
4.7

16

16
4.9

7.0

62

5.8

4.4
6.0

12.2

1114

2. 8

3.0

2.1

94
110

110
115

2.7

3.2

8.1

3.9

290

38

$\begin{array}{ll}1 & 2.6 \\ 0.7 & 4.3 \\ 1 & 5.9\end{array}$

$\begin{array}{ll}1 & 5.9 \\ 1 & 2.1\end{array}$

77
122

4.2

93
149
1268

317
294

122

260

11.

11
18

17

08-19-92 23

$09-03-92 \quad 22$

8.0

2.6
2.5

$1_{284}^{326}$

$1_{233}^{267}$

17
47

26
17

6.9

8.

15

14
32

6.6
8.2
13
13
22
19
31
20

$L$ Laboratory value. 


\section{LOCAL \\ NUMBER \\ $005 \mathrm{S014E02Q02M}$ 005S014E09B02M 005S014E18K01M 006S013E04Q01M 006S013E25M01M \\ 006S014E29R01M 006S014E32A01M 006S014E32B01M 006S015E03Q01M 007S013E22P01M \\ 007S014E0SA01M 007S014E16L01M 007S014E29R01M 007S015E28K01M 007 S015E35A02M \\ 007S015E35P01M 008S014E18D01M 008S014E19E01M}

DATE

09-02-92

09-01-92

$08-31-92$

09-01-92

09-02-92

09-01-92

08-20-92

08-19-92

08-31-92

08-20-92

09-03-92

09-02-92

09-02-92

08-19-92

09-01-92

08-19-92

09-03-92

09-03-92
FLUO- SILICA,

RIDE, DIS-

DIS-

SOLVED

$\begin{array}{lc}\text { (MG/L } & \text { AS } \\ \text { AS F) } & \text { SIO2) }\end{array}$

0.20

$<0.10$

$<0.10$

0.10

0.20

0.10

0.30

0.20

0.40

0.10

0.20

0.20

0.10

0.20

0.20

0.20

0.20
0.20

82
29

29

24

60
50

45

46

46

60
60

55

44

71

47
57

61

61
57
41
SOLIDS,

NITRO- NITRO- NITRO- PHOS-

GEN, GEN, GEN, PHORUS

AT 180 NITRITE NO2+NO3 AMMONIA ORTHO, INUM,

DIS- SOLVED SOLVED SOLVED SOLVED SOLVED

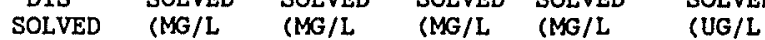

$(M G / L)$ AS $N)$ AS $N)$ AS N) AS P) AS AL)

$\begin{aligned} 196 & <0.010 \\ 95 & <0.010 \\ 127 & <0.010 \\ 240 & <0.010 \\ 193 & <0.010 \\ & \\ 137 & <0.010 \\ 169 & <0.010 \\ 176 & <0.010 \\ 152 & <0.010 \\ 487 & <0.010 \\ & \\ 150 & <0.010 \\ 216 & <0.010 \\ 308 & <0.010 \\ 364 & <0.010 \\ 337 & <0.010 \\ 376 & <0.010 \\ 378 & <0.010 \\ 303 & <0.010\end{aligned}$

0.400

0.460

$<0.010$

0.040

0.050

0.040

0.070

0.140

$2.30<0.010$

8.30

0.030

0.090

2.90

3.20

$<0.010$

2.90

$<0.010$

$<0.010$

$<0.010$

$<0.010$

0.110

0.070

0.070

0.020

12.0

0.020

1.40

2.70

$<0.010$

0.020

0.030

0.020

$3.50<0.010$

$9.10<0.010$

$5.20<0.010$

0.050

0.050

$3.80<0.010$

3. 00

$<0.010$
0.010

0.020

0.070

0.040

0.020
20

30

30

$<10$

10

10

10

$<10$

$<10$

$<10$

$<10$

$<10$

$<10$

$<10$

$<10$

$<10$
LOCAL.

NUMBER

005S014E02Q02M $005 S 014 \mathrm{E} 09 \mathrm{~B} 02 \mathrm{M}$ 005S014E18K01M 006S013E04Q01M 006S013E25M01M

006S014E29R01M 006S014E32A01M 006 S014E32B01M $006 \mathrm{~S} 015 \mathrm{E} 03001 \mathrm{M}$ 007S013E22P01M

007S014E05A01M $007 S 014 \mathrm{E} 16 \mathrm{LO} 1 \mathrm{M}$ 007S014E29R01M $007 \mathrm{~S} 015 \mathrm{E} 28 \mathrm{~K} 01 \mathrm{M}$ 007S015E35A02M

$0075015 E 35 P 01 M$ $008 \mathrm{~S} 014 \mathrm{E} 18 \mathrm{D} 01 \mathrm{M}$ 008S014E19E01M
DATE

$\begin{array}{cc}\text { ARSENIC } & \text { CADMIUM } \\ \text { DIS- } & \text { DIS- } \\ \text { SOLVED } & \text { SOLVED } \\ \text { (UG/L } & \text { (UG/L } \\ \text { AS AS) } & \text { AS CD) }\end{array}$

CHRO-

MIUM, COBALT, COPPER, IRON, LEAD, LITHIUM DIS- DIS- DIS- DIS- DIS- DISSOLVED SOLVED SOLVED SOLVED SOLVED SOLVED (UG/L (UG/L (UG/L (UG/L AS CR) AS CO) AS CU) AS FE)

AS PB) AS LI)

AS CU) AS FE)

$09-02-92$

$09-01-92$

08-31-92

09-01-92

09-02-92

09-01-92

08-20-92

08-19-92

08-31-92

08-20-92

09-03-92

$09-02-92$

09-02-92

$08-19-92$

09-01-92

08-19-92

09-03-92

09-03-92

$\begin{array}{rlc}1 & -- & -- \\ <1 & <1.0 & <1 \\ <1 & -- & -- \\ 1 & -- & -- \\ 2 & <1.0 & 3 \\ 2 & -- & - \\ 1 & <1.0 & <1 \\ 1 & <1.0 & <1 \\ 3 & <1.0 & <1 \\ 1 & <1,0 & <1\end{array}$

$--$

${ }_{--}^{<1}$

$-2$

$-$

$-$

$-$

$<1$
$<1$
$<1$
$<1$

$--$

5
$<1$
$<1$

$<1$

-- $\quad-$

$\begin{array}{lll}-- & -1 & - \\ -- & - & -2\end{array}$

$--$

$<1.0$

$<1.0$

$--$

$<1$
2

$--$

$--$

$--$

$<1$

1
4

38
33
3
$<3$
$<3$

$\begin{array}{ll}-- & <1 \\ -- & -- \\ -- & -- \\ <1 & <4\end{array}$

$--$

$\begin{array}{lr}<1 & -- \\ <1 & 4 \\ <1 & 4 \\ <1 & 4\end{array}$

$\begin{aligned}-- & -- \\ -- & -- \\ <1 & <4 \\ <1 & <4\end{aligned}$

5

$<1.0$

$n^{<1}$

$--<$

$-1$

< Actual value is less than value shown. 
WATER QUALITY, MERCED COUNTY--Continued

INORGANIC DATA

WATER YEAR OCTOBER 1991 TO SEPTEMBER 1992

LOCAL

NUMBER

$0055014 \mathrm{E} 02 \mathrm{Q} 02 \mathrm{M}$ $005 S 014 \mathrm{E} 09 \mathrm{~B} 02 \mathrm{M}$ 005S014E18KO1M 006S013E04Q01M 006S013E25M01M

006S014E29R01M O06S014E32A01M 006S014E32B01M 006S015E03Q01M 007S013E22P01M

007S014E05A01M 007S014E16L01M 007S014E29R01M 007SO15E28K01M DOTS015E35A02M

007S015E35P01M 008S014E18D01M 008S014E19E01M

\begin{tabular}{|c|c|c|c|c|c|c|c|c|}
\hline DATE & $\begin{array}{l}\text { MANGA- } \\
\text { NESE, } \\
\text { DIS- } \\
\text { SOLVED } \\
\text { (UG/L } \\
\text { AS MN) }\end{array}$ & $\begin{array}{c}\text { MERCURY } \\
\text { DIS- } \\
\text { SOLVED } \\
\text { (UG/L } \\
\text { AS HG) }\end{array}$ & $\begin{array}{l}\text { MOLYB- } \\
\text { DENUM, } \\
\text { DIS- } \\
\text { SOLVED } \\
\text { (UG/L } \\
\text { AS MO) }\end{array}$ & $\begin{array}{l}\text { NICKEL, } \\
\text { DIS- } \\
\text { SOLVED } \\
\text { (UG/L } \\
\text { AS NI) }\end{array}$ & $\begin{array}{l}\text { SELE- } \\
\text { NIUM, } \\
\text { DIS- } \\
\text { SOLVED } \\
\text { (UG/L } \\
\text { AS SE) }\end{array}$ & $\begin{array}{l}\text { STRON- } \\
\text { TIUM, } \\
\text { DIS- } \\
\text { SOLVED } \\
\text { (UG/L } \\
\text { AS SR) }\end{array}$ & $\begin{array}{l}\text { VANA- } \\
\text { DIUM, } \\
\text { DIS- } \\
\text { SOLVED } \\
\text { (UG/L } \\
\text { AS V) }\end{array}$ & $\begin{array}{l}\text { ZINC, } \\
\text { DIS- } \\
\text { SOLVED } \\
\text { (UG/L } \\
\text { AS ZN) }\end{array}$ \\
\hline $09-02-92$ & 21 & -- & -- & -- & - & -- & - & -- \\
\hline $09-01-92$ & 24 & $<0.1$ & $<1$ & $<1$ & $<1$ & 170 & 1 & 330 \\
\hline $08-31-92$ & $<1$ & -- & -- & -- & - & - & -- & -- \\
\hline $09-01-92$ & $<1$ & -- & -- & -- & -- & - & -- & -- \\
\hline $09-02-92$ & $<1$ & $<0.1$ & $<1$ & $<1$ & $<1$ & 190 & 13 & 60 \\
\hline $09-01-92$ & $<1$ & -- & -- & - & - & -- & -- & -- \\
\hline $08-20-92$ & $<1$ & $<0.1$ & $<1$ & $<1$ & $<1$ & 210 & $\mathbf{g}$ & 67 \\
\hline $08-19-92$ & 1 & $<0.1$ & $<1$ & $<1$ & $<1$ & 280 & 9 & 19 \\
\hline $08-31-92$ & 3 & $<0.1$ & $<1$ & $<1$ & $<1$ & 120 & 15 & 52 \\
\hline $08-20-92$ & $<1$ & $<0.1$ & $<1$ & $<1$ & $<1$ & 780 & 16 & 21 \\
\hline $09-03-92$ & $<1$ & - & - & -- & - & - & -- & -- \\
\hline $09-02-92$ & $<1$ & -- & -- & -- & -- & -- & -- & -- \\
\hline $09-02-92$ & $<1$ & -- & -- & -- & $\cdots$ & -- & -- & -- \\
\hline $08-19-92$ & $<1$ & 0.1 & $<1$ & $<1$ & $<1$ & 550 & 19 & 9 \\
\hline $09-01-92$ & $<\overline{1}$ & $<0.1$ & 1 & $<1$ & $<1$ & 380 & 20 & 110 \\
\hline $3-19-92$ & $<1$ & $<0.1$ & 1 & $<1$ & $<1$ & 450 & 21 & 5 \\
\hline $09-03-92$ & $<1$ & -- & -- & $\ldots$ & -- & $\ldots$ & $-\infty$ & $\cdots$ \\
\hline $09-03-92$ & $<1$ & -- & -- & -- & -- & -- & -- & -- \\
\hline
\end{tabular}

< Actual value is less than value shown. 
WATER YEAR OCTOBER 1991 TO SEPTEMBER 1992

\section{LOCAL \\ NUMBER \\ 005S014E02Q02M $005 \mathrm{~S} 014 \mathrm{E} 09 \mathrm{~B} 02 \mathrm{M}$ $005 S 014 \mathrm{E} 18 \mathrm{~K} 01 \mathrm{M}$ 006S013E04001M 006S013E25M01M \\ 006S014E29R01M 0065014E32A01M 0065014E32B01M 006S015E03001M 007S013E22P0 1M \\ 007S014E05A01M 007S014E16L01M OOTSO14E29R01M 007S015E28K01M 007S015E35A02M \\ 007S015E35P01M $0085014 \mathrm{E} 18 \mathrm{D} 01 \mathrm{M}$ 008S014E19E01M}

$\begin{array}{cc} & \\ & \\ \text { DATE } & \text { TIME } \\ & \\ 09-02-92 & 1115 \\ 09-01-92 & 1140 \\ 08-31-92 & 1720 \\ 09-01-92 & 0920 \\ 09-02-92 & 1230 \\ 09-01-92 & 1712 \\ 08-20-92 & 1120 \\ 08-19-92 & 1745 \\ 08-31-92 & 1930 \\ 08-20-92 & 0930 \\ & \\ 09-03-92 & 1400 \\ 09-02-92 & 0835 \\ 09-02-92 & 0740 \\ 08-19-92 & 1021 \\ 09-01-92 & 1400 \\ & \\ 08-19-92 & 2000 \\ 09-03-92 & 1030 \\ 09-03-92 & 0930 \\ & \end{array}$

\section{DEPTH \\ BELOW}

LAND
SURFACE

(WATER

LEVEL)

(FEET)

$\begin{array}{lr}21.77 & 50 \\ -- & 64 \\ 9.10 & 100 \\ -- & 128 \\ -- & 202\end{array}$

152

$\begin{array}{rr}-- & 165 \\ 81.36 & 88\end{array}$

$\cdots \quad 100$

$-\infty$

$\begin{array}{cr}-- & - \\ -- & 268 \\ -- & 184 \\ 22.01 & 56 \\ -- & 120\end{array}$

-- 76

4.99

90.82

DEPTH
OF
WELL, PCB,
TOTAL, TOTAL
(FEET) (UG/L)

NAPH-

THA-

LENES,

POLY-

CHLOR. ALDRIN,

TOTAL TOTAL

(UG/L)

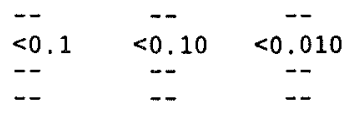

$<0.1$

$<0.10$

$<0.010$

$<0$.

$<0.1$

$--$

$<0.1$

$<0.10$

$--$

$<0.010$

$<0.010$

$<0.1$

$<0.10$

$<0.010$

$<0.010$

$\begin{array}{ll}-- & -- \\ -- & --\end{array}$

$<0.1<0.10$

$<0.1<0.10$

--

$--$

$<0.010$

$<0.010$

$<0.1<0.10<0.010$

$<0.1$

$--$
$<0.010$
$-$
LOCAL

NUMBER

$0055014 \mathrm{E} 02 \mathrm{Q} 02 \mathrm{M}$ $005 \mathrm{~S} 014 \mathrm{E} 09 \mathrm{~B} 02 \mathrm{M}$ 005S014E18K01M 006S013E04Q01M 006S013E25M01M

006S014E29R01M 0065014E32A01M 006S014E32B01M 006S015E03Q01M 007S013E22P01M

007S014E05A01M 007S014E16L01M 007S014E29R01M 007S015E28K01M 007S015E35A02M

007S015E35P01M 008S014E18D01M 008S014E19E01M

< Actual value is less than value shown.

DIBROMO

CHLORO-

CHLOR- PROPANE

CHLOR- PYRIFOS WATER

DANE, TOTAL WHOLE

$\begin{array}{lll}\text { (UG/L) } & \text { RECOVER } & \text { TOT.REC } \\ \text { (UG } / \mathrm{L}) & (\mathrm{UG} / \mathrm{L})\end{array}$

$<0.1<0.01<0.0$

$--\quad--\quad<0.0$

$\begin{array}{lll}-- & -- & <0.0\end{array}$

$<0.1<0.01<0.0$

$-\infty \quad--<00$

$<0.1<0.01<0.0$

$<0.1<0.01<0.0$

$<0.1<0.01<0.0$

$<0.1<0.01<0.0$

$--\quad--\quad<<0$

$-. \quad--\quad<\quad<0.0$

$--\quad \cdots \quad<0.0$

$<0.1<0.01 \quad 0.0$

$<0.1<0.01<0.0$

$<0.1<0.01<0.0$

$\begin{array}{lll}-- & \ldots & <0.0\end{array}$

$<0.0$

\section{DATE}

09-02-92 09-01-92

$08-31-92$

$09-01-92$

$09-02-92$

09-01-92

$08-20-92$

08-19-92

$08-31-92$

08-20-92

09-03-92

$09-02-92$

09-02-92

08-19-92

09-01-92

08-19-92

$09-03-92$

$09-03-92$
DDD, DDE, DDT, DEF

TOTAL TOTAL TOTAL TOTAL

(UG/L)
(UG/L) (UG/L)

DI-

AZINON,

TOTAL

(UG/L)

$<0.010$

$<0.010$

$<0$

$<0.01$

-.

$--$

0.010

$\ldots$

--

$<0.01$

$<0.01$

$--$
$<0.01$

$<0.01$

$<0.01$

$<0.010<0.010<0.010$

$<0.010$

$<0.010$

$<0.010$

$<0.010$

$<0.010$

$<0.01$

$<0.01$

$<0.01$

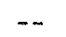

--

$<0.0$

$<0.01$

$<0.01$

$<0.010$

$<0.01$
1,2-

DIBROMO

ETHANE

WATER

WHOLE.

TOTAL

(UG/L) 
ORGANIC DATA

WATER YEAR OCTOBER 1991 TO SEPTEMBER 1992

\begin{abstract}
LOCAL
NUMBER

$005 \mathrm{~S} 014 \mathrm{E} 02 \mathrm{Q} 02 \mathrm{M}$ $005 S 014$ E09B02M O05S014E18K01M 006S013E04Q01M 006S013E25M01M

006S014E29R01M 006S014E32A01M 006S014E32B01M 006S015E03Q01M 007S013E22P01M

007S014E05A01M 007S014E16L01M 007S014E29R01M 007S015E28K01M 007S015E35A02M

007S015E35P01M $008 \mathrm{~S} 014 \mathrm{E} 18 \mathrm{DO} 1 \mathrm{M}$ 008S014E19E01M
\end{abstract}

LOCAL

NUMBER

$005 S 014 \mathrm{E} 02 \mathrm{Q} 02 \mathrm{M}$ $005 S 014 \mathrm{E} 09 \mathrm{~B} 02 \mathrm{M}$ $0055014 \mathrm{E} 18 \mathrm{KO} 1 \mathrm{M}$ 006S013E04Q01M 006S013E25MO1M

006S014E29R01M $006 \mathrm{~S} 014 \mathrm{E} 32 \mathrm{A0} \mathrm{M}$ 006S014E32B01M 006S015E03Q01M 007S013E22P01M

007S014E05A01M $007 \mathrm{~S} 014 \mathrm{E} 16 \mathrm{~L} 01 \mathrm{M}$ 007S014E29R01M 007S015E28K01M $007 \mathrm{S015E3} 5 \mathrm{A02M}$

007S015E35P01M $008 \mathrm{~S} 014 \mathrm{E} 18 \mathrm{D} 01 \mathrm{M}$ 008S014E19E01M
DATE

\section{9-02-92}

09-01-92

08-31-92

09-01-92

09-02-92

09-01-92

08-20-92

08-19-92

$08-31-92$

08-20-92

$09-03-92$

09-02-92

09-02-92

$08-19-92$

09-01-92

08-19-92 09-03-92 09-03-92

\begin{tabular}{|c|c|c|c|c|c|c|c|}
\hline $\begin{array}{l}\text { ENDO- } \\
\text { SULFAN, } \\
\text { TOTAL } \\
\text { (UG/L) }\end{array}$ & $\begin{array}{c}\text { ENDRIN } \\
\text { WATER } \\
\text { UNFLTRD } \\
\text { REC } \\
\text { (UG/L) }\end{array}$ & $\begin{array}{l}\text { ETHION, } \\
\text { TOTAL } \\
\text { (UG/L) }\end{array}$ & $\begin{array}{c}\text { FONOFOS } \\
\text { (DY- } \\
\text { FONATE) } \\
\text { WATER } \\
\text { WHOLE } \\
\text { TOT .REC } \\
\text { (UG/L) }\end{array}$ & $\begin{array}{l}\text { HEPTA- } \\
\text { CHLOR, } \\
\text { TOTAL } \\
\text { (UG/L) }\end{array}$ & $\begin{array}{l}\text { HEPTA- } \\
\text { CHLOR } \\
\text { EFOXIDE } \\
\text { TOTAL } \\
\text { (UG/L) }\end{array}$ & $\begin{array}{l}\text { LINDANE } \\
\text { TOTAL } \\
\text { (UG/L) }\end{array}$ & $\begin{array}{l}\text { MALA- } \\
\text { THION } \\
\text { TOTAL } \\
\text { (UG/L }\end{array}$ \\
\hline-- & - & -- & -- & -- & -- & -- & -- \\
\hline$<0.010$ & $<0.010$ & $<0,01$ & $<0.01$ & $<0.010$ & $<0.010$ & $<0.010$ & $<0.01$ \\
\hline $\begin{array}{l}-- \\
--\end{array}$ & $\begin{array}{l}-- \\
--\end{array}$ & -- & $\begin{array}{l}-- \\
--\end{array}$ & -- & $\begin{array}{l}-- \\
--\end{array}$ & -- & -- \\
\hline$<0.010$ & $<0.010$ & $<0,01$ & $<0.01$ & $<0.010$ & $<0.010$ & $<0.010$ & $<0.01$ \\
\hline $\begin{array}{l}<-- \\
<0.010 \\
<0.010 \\
<0.010 \\
<0.010\end{array}$ & $\begin{array}{l}<-- \\
<0.010 \\
<0.010 \\
<0.010 \\
<0.010\end{array}$ & $\begin{array}{l}-- \\
<0.01 \\
<0.01 \\
<0.01 \\
<0.01\end{array}$ & $\begin{array}{l}<- \\
<0.01 \\
<0.01 \\
<0.01 \\
<0.01\end{array}$ & $\begin{array}{l}<0.010 \\
<0.010 \\
<0.010 \\
<0.010\end{array}$ & $\begin{array}{c}-- \\
<0.010 \\
<0.010 \\
<0.010 \\
<0.010\end{array}$ & $\begin{array}{c}-- \\
<0.010 \\
<0.010 \\
<0.010 \\
<0.010\end{array}$ & $\begin{array}{l}<- \\
<0.01 \\
<0.01 \\
<0.01 \\
<0.01\end{array}$ \\
\hline
\end{tabular}

\section{- $\quad-$}

$--\quad \quad-$

$<0.010$

$<0.010$

$<0.010$

$<0.010$
--

$<0.01$

$<0.01$

$<0.01$

$\ldots$ $\overline{--}$

$<0.01$

$<0.01$

$<0.01$

$+-$
$<0,010$

$<0.010$

$<0.010$
$--$

$-$

$<0.010$

$<0.010$

$<0.010$

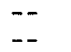

$-$

$-$

$<0.01$

$\begin{array}{ll}<0.010 & <0.01 \\ <0.010 & <0.01\end{array}$

$<0.010<0.01$

--

< Actual value is less than value shown.

$\begin{array}{lcccccccc}09-02-92 & -- & -- & -- & -- & - & -- & -- & -- \\ 09-01-92 & <0.01 & <0.01 & <0.01 & <0.01 & <0.1 & <0.01 & <1 & <0.01 \\ 08-31-92 & -- & -- & -- & -- & -- & -- & -- & -- \\ 09-01-92 & -- & -- & -- & -- & -- & -- & -- & -- \\ 09-02-92 & <0.01 & <0.01 & <0.01 & <0.01 & <0.1 & <0.01 & <1 & <0.01 \\ 09-01-92 & -- & -- & -- & -- & -- & -- & -- & -- \\ 08-20-92 & <0.01 & <0.01 & <0.01 & <0.01 & <0.1 & <0.01 & <1 & <0.01 \\ 08-19-92 & <0.01 & <0.01 & <0.01 & <0.01 & <0.1 & <0.01 & <1 & <0.01 \\ 08-31-92 & <0.01 & <0.01 & <0.01 & <0.01 & <0.1 & <0.01 & <1 & <0.01 \\ 08-20-92 & <0.01 & <0.01 & <0.01 & <0.01 & <0.1 & <0.01 & <1 & <0.01 \\ 09-03-92 & -- & -- & -- & -- & -- & -- & -- & -- \\ 09-02-92 & -- & -- & -- & -- & -- & -- & -- & -- \\ 09-02-92 & -- & -- & -- & -- & -- & -- & -- & -- \\ 08-19-92 & <0.01 & <0.01 & <0.01 & <0.01 & <0.1 & <0.01 & <1 & <0.01 \\ 09-01-92 & <0.01 & <0.01 & <0.01 & <0.01 & <0.1 & <0.01 & <1 & <0.01 \\ 08-19-92 & <0.01 & <0.01 & <0.01 & <0.01 & <0.1 & <0.01 & <1 & <0.01 \\ 09-03-92 & -- & -- & -- & -- & -- & -- & -- & -- \\ 09-03-92 & -- & -- & -- & -- & -- & -- & -- & --\end{array}$




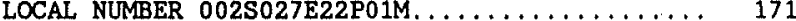

LOCAL NUMBER 002S027E34A01M............. 171

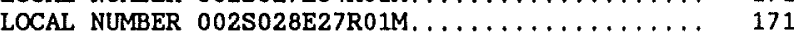

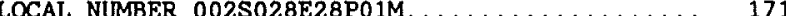

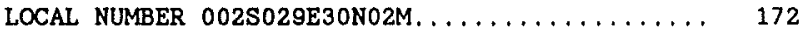

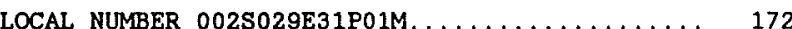

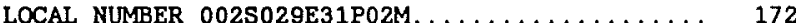

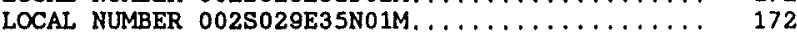

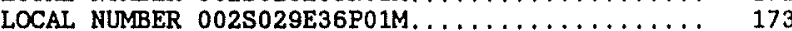

LOCAL NUMBER 003S027E03K01M............., 173

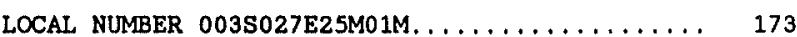

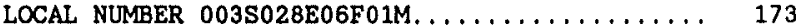

LOCAL NUMBER O03S028E15P01M.............. 174

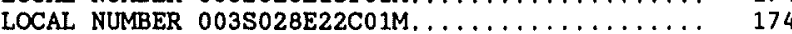

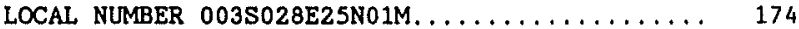

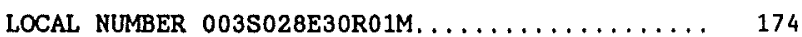

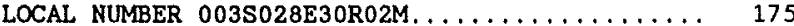

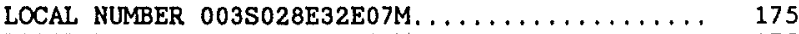

LOCAL NUMBER O03SO28E32E08M. ................. 175

LOCAL NUMBER O03S028E33P01M............. 175

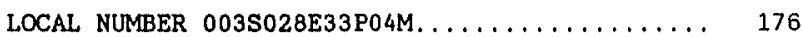

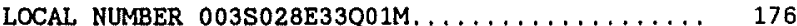

LOCAL NUMBER 003S028E34C01M............ 176

LOCAL NUMBER O03S028E34R02M................ 176

LOCAL NUMBER 003S028E35E02M. . . . . . . . . . . 177

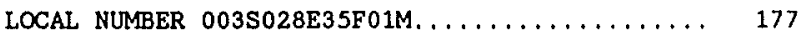

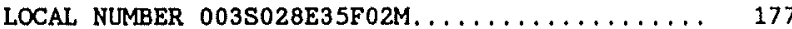

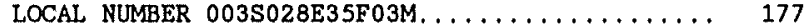

LOCAL NUMBER O03S029E02A01M. ............ 178

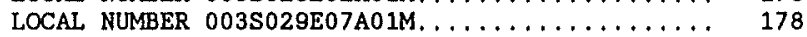

LOCAL NUMBER 003S029E18J01M............. 178

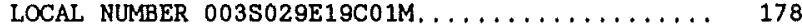

LOCAL NUMBER 003S029E19R01M . . . . . . . . . . 179

LOCAL NUMBER 003S029E27L05M $\ldots \ldots \ldots \ldots \ldots \ldots$

LOCAL NUMBER 003S029E29N01M................ 179

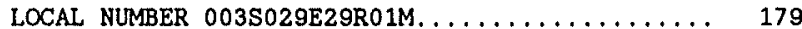

LOCAL NUMBER 003S029E30E01M. ............. 180

LOCAL NUMBER 003SO29E30E02M. . ........... 180

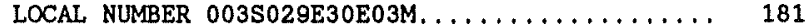

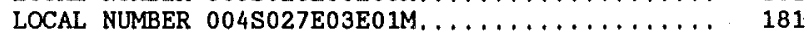

LOCAL NUMBER 004 S027E03J03M. ................. 181

LOCAL NUMBER 004S027E10B01M. ............. 181

LOCAL NUMBER 004S027E10C01M.............. 182

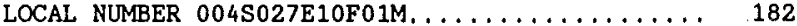

LOCAL NUMBER 004S028E01F01M.............. 182

LOCAL NUMBER 004S028E01L01M.................. 182

LOCAL NUMBER 004S028E03J03M. .............. 183

LOCAL NUMBER 004S028E04P02M............. 183

LOCAL NUMBER 004S028E06L01M............... 183

LOCAL NUMBER 004S028E06L02M.............. 183

LOCAL NUMBER 004 S028E12K01M.................. 184

LOCAL NUMBER 004S029E05B01M.............. 184

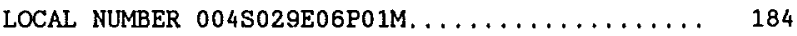

LOCAL NUMBER 004S029E07E01M............... 184

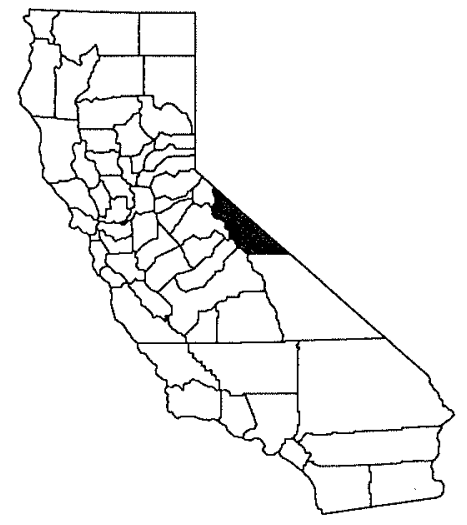

(2) Number in circle indicates number of wells in township for which data are included in this report
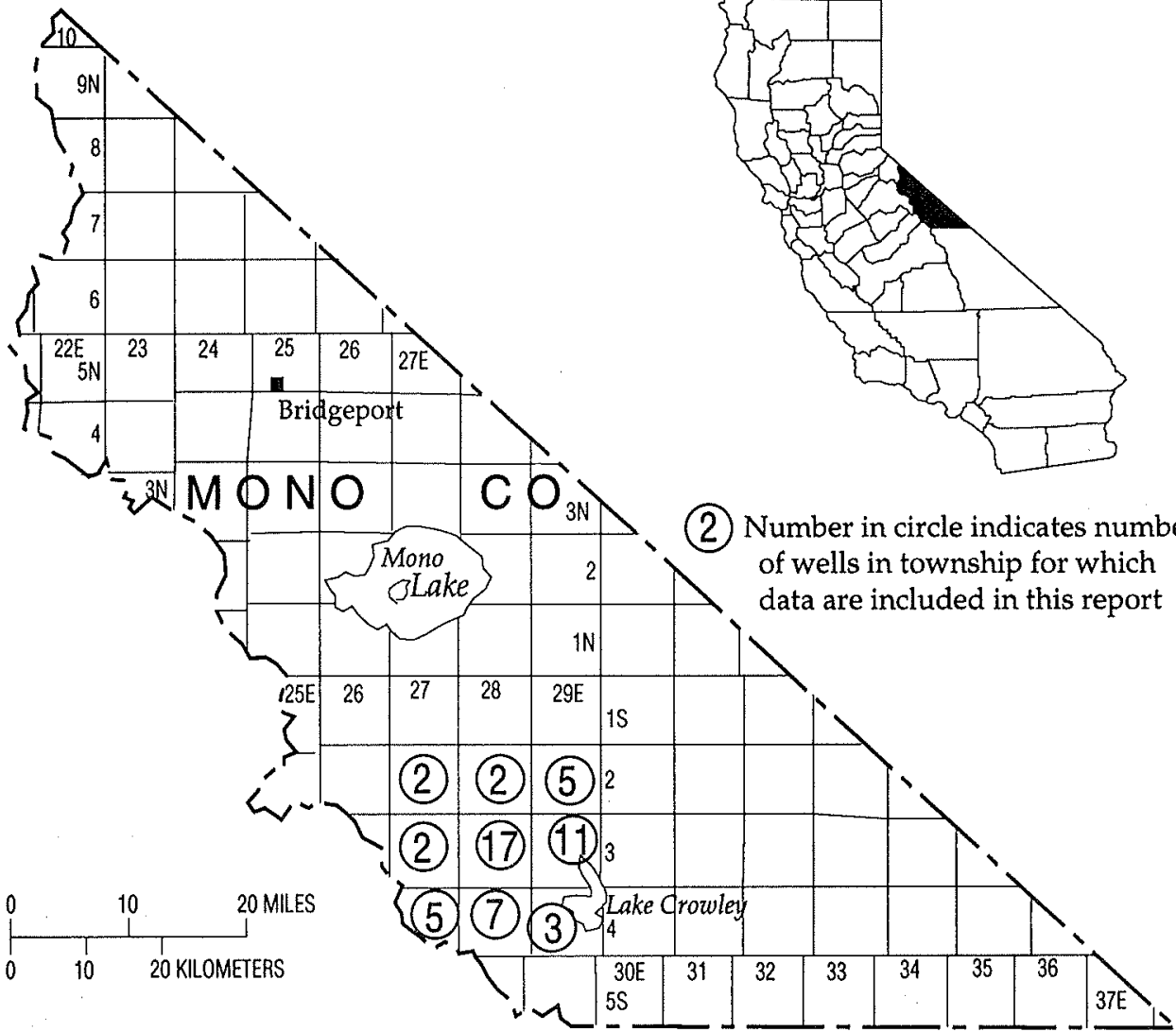

Figure 19. Location of wells in Mono County. 
WELL DESCRIPTIONS AND WATER-LEVEL MEASUREMENTS

WATER YEAR OCTOBER 1991 TO SEPTEMBER 1992

SITE NUMBER 374511118585801

LOCAL NUMBER 002S027E22P01M

About 7.5 miles north of Mammoth Lakes at the Caltrans Crestview Maintenance Station. Drilled commercial well. Diameter 10 inches, depth 490 feet. Altitude of land-surface datum 7,520 feet. Water-level records available 1990 to current year.

WATER LEVELS IN FEET BELOW LAND-SURFACE DATUM

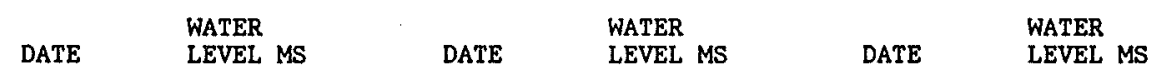

NOV $20,1991133.81 \mathrm{~V}$ APR 07, $1992133.03 \mathrm{~V} \quad$ JUL $20,1992129.33 \mathrm{~s}$

PERIOD OF RECORD HIGHEST 123.72 AUG 02, 1990 LCWEST 133.81 NOV 20, 1991

SITE NUMBER 374410118583401

LOCAL NUMBER 002S027E34A01M

About 6.3 miles north of Mammoth Lakes and $0.4 \mathrm{mile}$ west of Highway 395 . Drilled observation well. Diameter 1.25 inches, depth 633 feet. Altitude of land-surface datum 7,515.27 feet. Water-level records available 1983 to current year.

WATER LEVELS IN FEET BELOW LAND-SURFACE DATUM

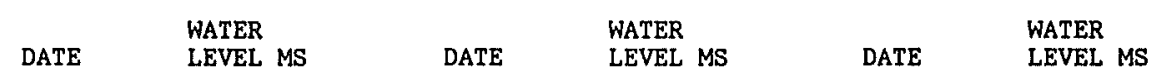
NoV 19,1991
$212.98 \mathrm{~V}$
APR 07, $1992 \quad 213.85 \mathrm{~V}$
APR $07,1992 \quad 213.70 \mathrm{~s}$

PERIOD OF RECORD HIGHEST 171.30 JUL 20, 1983 LOWEST 213.85 APR 07, 1992

SITE NUMBER 374420118515501

LOCAL NUMBER 002S028E27RO1M

About 8.3 miles northeast of Mammoth Lakes and $2.6 \mathrm{miles}$ southeast of Arcularius Ranch. Drilled 1ivestock well. Diameter 6 inches, depth 44.3 feet. Altitude of land-surface datum $7,039.18$ feet. Water-1evel records available 1982 to current year.

WATER LEVELS IN FEET BELOW LAND-SURFACE DATUM

$\begin{array}{lllll}\text { WATE } & \text { WATER } & \text { WATER } & \text { WATER } \\ \text { LEVEL MS } & \text { DATE } & \text { LEVEL MS } & \text { DATE } & \text { LEVEL MS }\end{array}$

NOV 19, $1991 \quad 36.26 \mathrm{~S} \quad$ APR 07, $1992 \quad 36.44 \mathrm{~S} \quad$ JUL $21,1992 \quad 37.44 \mathrm{~S}$

PERIOD OF RECORD HIGHEST 33.2 JUL 16, 1985 LOWEST 37.5 NOV 18, 1982 NOV 04,1983

SITE NUMBER 374420118532501

LOCAL NUMBER 002S028E28P01M

About 7.5 miles northeast of Mamnoth Lakes and 1.4 miles southeast of Arcularius Ranch. Drilled 1ivestock well. Diameter 6 inches, depth 125 feet. Altitude of land-surface datum 7,109.02 feet. Water-level records available 1983 to current year.

WATER LEVELS IN FEET BELOW LAND-SURFACE DATUM

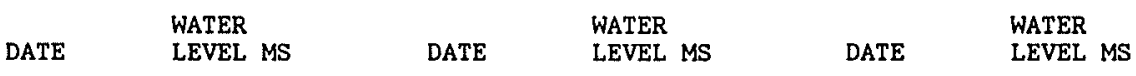

NOV 19, $199186.76 \mathrm{~V} \quad$ APR $07,1992 \quad 87.20 \mathrm{~s} \quad$ JUL $21,1992 \quad 87.63 \mathrm{~V}$

PERIOD OF RECORD HIGHEST 75.28 JUL 25, 1984 LOWEST 116.55 JUN 03, 1983 
WELL DESCRIPTIONS AND WATER-LEVEL MEASUREMENTS

WATER YEAR OCTOBER 1991 TO SEPTEMBER 1992

SITE NUMBER 374420118493301

LOCAL NUMBER O02SO29E3ONO2M

About 7.8 miles northeast of Mammoth Lakes and $4.8 \mathrm{miles}$ southeast of Arcularius Ranch. Drilled observation well. Diameter 8 inches, depth measured 31.8 feet in 1992. Altitude of land-surface datum 6,913.21 feet. Water-level records available 1972,1982 to current year.

WATER LEVELS IN FEET BELOW LAND-SURFACE DATUM

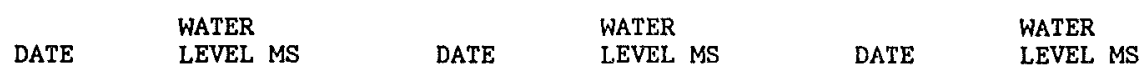
Nov 19, 1991
$9.04 \mathrm{~S}$
APR 07, 1992
$8.77 \mathrm{~S}$
JUL 21,1992
$9.09 \mathrm{~s}$

PERIOD OF RECORD HIGHEST

2.95 MAY 10, 1986

LOWEST

9.44 NOV 14, 1990

SITE NUMBER 374332118491001

LOCAL NUMBER O02S029E31P01M

About 9.4 miles northeast of Mammoth Lakes and 5.4 miles southeast of Arcularius Ranch. Unused water-table well in alluvium of Quaternary age. Diameter 30 inches, depth 7.6 feet. Altitude of land-surface datum 6,914.01 feet. Water-level records available 1966, 1972-73, 1978 to current year.

WATER LEVELS IN FEET BELOW LAND-SURFACE DATUM

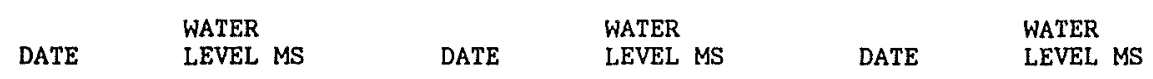

NOV 19, $1991 \quad 4.96 \mathrm{~S} \quad$ APR 07, $1992 \quad 4.41 \mathrm{~S} \quad$ JUL 21, $1992 \quad 5.18 \mathrm{~S}$

PERIOD OF RECORD HIGHEST 0.00 APR 02, $1984 \quad$ LOWEST $6.00 \quad$ JUN 13, 1966

SITE NUMBER 374332118491002

LOCAL NUMBER O02S029E31P02M

About 9.4 miles northeast of Mammoth Lakes and $5.4 \mathrm{miles}$ southeast of Arcularius Ranch. Livestock well. Diameter 4 inches, depth unknown. Altitude of land-surface datum 6,915.7 feet. Water-level records available 1988 to current year.

WATER LEVELS IN FEET BELOW LAND-SURFACE DATUM

$\begin{array}{lllll} & \text { WATER } & \text { WATER } & \text { WATER } \\ \text { DATE } & \text { LEVEL MS } & \text { DATE } & \text { LEVEL MS } & \text { DATE }\end{array}$

NOV 19, $1991 \quad 4.83 \mathrm{~S} \quad$ APR $07,1992 \quad 4.30 \mathrm{~S} \quad$ JUL $21,1992 \quad 5.05 \mathrm{~S}$

PERIOD OF RECORD HIGHEST 2.09 APR 28, 1988 LOWEST 5.05 JUL 21, 1992

SITE NUMBER 374327118445901

LOCAL NUMBER 002S029E35N01M

About 13 miles northeast of Mammoth Lakes. Drilled livestock well. Diameter unknown, depth 75 feet. Altitude of land-surface datum 6,914 feet. Water-level records available 1972, 1984 to current year.

WATER LEVELS IN FEET BELOW LAND-SURFACE DATUM

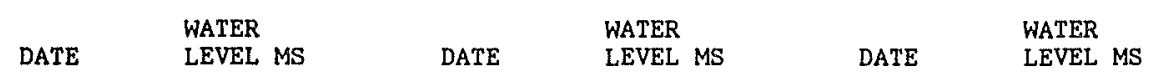

NOV 20, $1991 \quad 3.22 \mathrm{~S} \quad$ APR 08, $1992 \quad 1.80 \mathrm{~s} \quad$ JUL $23,1992 \quad 3.70 \mathrm{~s}$

PERIOD OF RECORD HIGHEST 1.05 JUL 25,1984 LOWEST 4.39 JUL 17,1985 
WATER LEVELS, MONO COUNTY--Continued

WELL DESCRIPTIONS AND WATER-LEVEL MEASUREMENTS

WATER YEAR OCTOBER 1991 TO SEPTEMBER 1992

SITE NUMBER 374335118434101

LOCAL NUMBER 002S029E36P01M

About 14.2 miles northeast of Marmoth Lakes. Unused livestock well. Diameter unknown, depth measured 72 feet in 1992. Altitude of land-surface datum 6,995 feet. Water-level records available 1986 to current year.

WATER LEVELS IN FEET BELOW LAND-SURFACE DATUM

$\begin{array}{lllll} & \text { WATER } & \text { WATER } & & \text { WATER } \\ \text { DATE } & \text { LEVEL MS } & \text { DATE } & \text { LEVEL MS } & \text { DATE }\end{array}$

NOV 20, $199169.11 \mathrm{~S} \quad$ APR 08, $199269.16 \mathrm{~S} \quad$ JUL 23, $1992 \quad 69.17 \mathrm{~S}$

PERIOD OF RECORD HIGHEST 68.70 MAY 30, 1987 LOWEST 71.51 NOV 29, 1986

SITE NUMBER 374249118584501

LOCAL NUMBER 003S027E03K01M

About 4.7 miles north of Mamnoth Lakes and 1.3 miles west of Highway 395. Drilled observation well. Diameter 1.75 inches, depth 2,330 feet, perforated 1,845-1,855 feet. Altitude of land-surface datum 7,734 feet. Water-1evel records available 1984 to current year.

WATER LEVELS IN FEET BELOW LAND-SURFACE DATUM

$\begin{array}{lllll} & \text { WATER } & & & \text { WATER } \\ \text { DATE } & \text { LEVEL MS } & \text { DATE } & \text { WEVEL MS } & \text { DATE }\end{array}$

NOV 19, $1991440.80 \mathrm{~V}$ APR 06, $1992444.63 \mathrm{~V}$ JUL $20,1992444.06 \mathrm{~V}$

PERIOD OF RECORD HIGHEST 398 AUG 30,1984 LOWEST 444.63 APR 06, 1992

SITE NUMBER 373924118571201

LOCAL NUMBER 003S027E25M01M

About $0.8 \mathrm{mile}$ northeast of Mammoth Lakes and $0.7 \mathrm{mile}$ north of U.S Fish and Wildlife ranger station. Drilled observation well. Diameter 2 inches, depth 1,304 feet, depth of hole 2,346 feet. Altitude of land-surface datum $7,788.4$ feet. Water-level records avallable 1986 to current year.

WATER LEVELS IN FEET BELOW LAND-SURFACE DATUM

\begin{tabular}{|c|c|c|c|c|c|c|c|}
\hline DATE & $\begin{array}{l}\text { WATER } \\
\text { LEVEL MS }\end{array}$ & DATE & $\begin{array}{l}\text { WATER } \\
\text { LEVEL MS }\end{array}$ & DATE & $\begin{array}{l}\text { WATER } \\
\text { LEVEL MS }\end{array}$ & DATE & $\begin{array}{l}\text { WATER } \\
\text { LEVEL MS }\end{array}$ \\
\hline $\begin{array}{l}15,19 \\
17\end{array}$ & $\begin{array}{l}430.22 \mathrm{~V} \\
430.87 \mathrm{~V}\end{array}$ & $\begin{array}{ll}\text { JAN } & 18, \\
\text { FEB } & 1992\end{array}$ & $\begin{array}{l}431.70 \mathrm{~V} \\
432.3 \mathrm{~V}\end{array}$ & $\begin{array}{l}\text { MAR } 13,1992 \\
\text { APR } 10\end{array}$ & $\begin{array}{l}432.13 \mathrm{~V} \\
433.05 \mathrm{~V}\end{array}$ & $\begin{array}{l}\text { JUN } 11,1992 \\
\text { JUL } 20\end{array}$ & $\begin{array}{l}432.65 \mathrm{~V} \\
434.28 \mathrm{~V}\end{array}$ \\
\hline
\end{tabular}

PERIOD OF RECORD HIGHEST 430.22 NOV 15, 1991 LOWEST 448.40 JUN 07, 1989

SITE NUMBER 374300118554401

LOCAL NUMBER 003S028E06F01M

About 5.2 miles northeast of Mammoth Lakes and 1.3 miles east of Highway 395 . Drilled observation well. Diameter 6 inches, depth 3,500 feet, perforated 500-3,500 feet. Altitude of land-surface datum 7,350 feet. Water-level records available 1984 to current year.

WATER LEVELS IN FEET BELOW LAND-SURFACE DATUM

$$
\begin{array}{lllll} 
& \text { WATER } & \text { WATER } & \text { WATER } \\
\text { DATE } & \text { LEVEL MS } & \text { DATE } & \text { LEVEL MS } & \text { DATE }
\end{array}
$$

$\begin{array}{llllllll}\text { NOV 19, } 1991 & 68.46 \mathrm{~S} & \text { MAR 13, } 1992 & 69.00 \mathrm{~V} & \text { JUN } 11,1992 & 69.56 \mathrm{~S} \\ \text { DEC } 17 & & 68.48 \mathrm{~S} & \text { APR } 07 & 69.17 \mathrm{~S} & \text { JUL } 09 & & 70.86 \mathrm{~S}\end{array}$

$\begin{array}{lrrrrr}\text { JAN } 18,1992 & 68.48 \mathrm{~S} & \text { APR } 07 & 69.17 \mathrm{~S} & \text { JUL } 09 & 70.86 \mathrm{~S} \\ & & 10 & 69.15 \mathrm{~S} & 21 & 70.73 \mathrm{~S}\end{array}$

PERIOD OF RECORD HIGHEST 57.61 JAN 05, 1987 LOWEST 70.86 JUL 09, 1992 
WELL DESCRIPTIONS AND WATER-LEVEL MEASUREMENTS

WATER YEAR OCTOBER 1991 TO SEPTEMBER 1992

SITE NUMBER 374051118523301

LOCAL NUMBER 003S028E15P01M

About 5.3 miles northeast of Mammoth Lakes in Little Antelope Valley, Observation we1l. Diameter 30 inches, depth 14 feet. Altitude of land-surface datum 7,200 feet. Water-level records available 1972-73, 1982 to current year.

WATER LEVELS IN FEET BELOW LAND-SURFACE DATUM (READINGS ABOVE LAND SURFACE INDICATED BY "+")

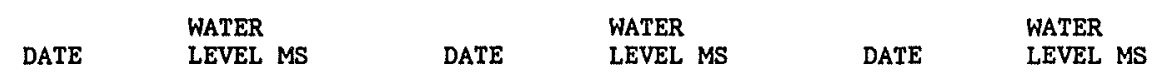

NOV 19, $1991 \quad 7.90 \mathrm{~S} \quad$ APR 07, $1992 \quad 2.81 \mathrm{~S} \quad$ JUL $21,1992 \quad 7.40 \mathrm{~S}$

PERIOD OF RECORD HIGHEST +1.35 MAY 10, AUG 27, 1986 LOWEST 8.62 NOV 15, 1990

SITE NUMBER 374040118522501

LOCAL NUMBER 003S028E22C01M

About 5.3 miles northeast of Marmoth Lakes in Little Antelope Valley. Drilled observation well. Diameter 1.25 inches, depth 677 feet, perforated 535-539 feet. Altitude of land-surface datum 7,248.23 feet. Water-1evel records available 1982 to current year.

WATER LEVELS IN FEET BELOW LAND-SURFACE DATUM

$$
\begin{array}{lllll} 
& \text { WATER } & \text { WATER } & \text { WATER } \\
\text { DATE } & \text { DEVEL MS } & \text { DATE } & \text { LEVEL MS }
\end{array}
$$

NOV 19, $1991209.25 \mathrm{~V} \quad$ APR 07, $1992208.41 \mathrm{~V}$ JUL 21, $1992207.11 \mathrm{~V}$

PERIOD OF RECORD HIGHEST 202.55 AUG 27, 1986 LOWEST 209.25 NOV 19, 1991

SITE NUMBER 373907118503801

LOCAL NUMBER 003S028E25NO1M

About 6.5 miles east of Mammoth Lakes near Hot Creek Fish Hatchery, Drilled observation well. Diameter 6 inches 0-80 feet, 2 inches 80-340 feet, depth 340 feet, perforated 320-340 feet. Altitude of land-surface datum

$7,081.26$ feet. Water-level records available 1986 to current year.

WATER LEVELS IN FEET BELOW LAND-SURFACE DATUM

$\begin{array}{lllll} & \text { WATER } & & \text { WATER } & \text { WATER } \\ \text { DATE } & \text { LEVEL MS } & \text { DATE } & \text { LEVEL MS } & \text { DATE }\end{array}$

NOV 19, $199148.64 \mathrm{~S}$ APR 07, $199249.26 \mathrm{~S} \quad$ JUL $23,199248.2 \mathrm{~s}$

PERIOD OF RECORD HIGHEST 43.60 AUG 12, 1986 LOWEST 49.26 APR 07, 1992

SITE NUMBER 373913118551401

LOCAL NUMBER OO3S028E30R01M

About $2.4 \mathrm{miles}$ northeast of Mammoth Lakes and $0.9 \mathrm{mile}$ north of junction of Highways 395 and 203 . Drilled observation wel1. Diameter 2 inches, depth 57 feet, depth measured 14 feet in 1992 , perforated $55-57$ feet. Altitude of land-surface datum $7,340^{\circ}$ feet. Water-level records available 1982 , 1987 to current year.

WATER LEVELS IN FEET BELOW LAND-SURFACE DATUM

$\begin{array}{lllll} & \text { WATER } & \text { WATER } & & \text { WATER } \\ \text { DATE } & \text { LEVEL MS } & \text { DATE } & \text { LEVEL MS } & \text { DATE }\end{array}$

NOV 19, $1991 \quad 13.05 \mathrm{~S} \quad$ APR 07, $1992 \quad$ D $\quad$ JUL 21, $1992 \quad$ SD

PERIOD OF RECORD HIGHEST 0.19 AUG 26, 1987 LOWEST 13.05 NOV 19, 1991 
WELL DESCRIPTIONS AND WATER-LEVEL MEASUREMENTS

WATER YEAR OCTOBER 1991 TO SEPTEMBER 1992

SITE NUMBER 373917118551402

LOCAL NUMBER O03S028E30R02M

About 2.4 miles northeast of Mammoth Lakes and $1 \mathrm{mile}$ north of junction of Highways 395 and 203. Drilled observation well. Diameter 2 inches, depth 79.7 feet, depth measured 55.2 feet in 1992, well point $77.7-79.7$ feet. Altitude of land-surface datum 7,340 feet. Water-level records avallable 1973 to current year.

WATER LEVELS IN FEET BELOW LAND-SURFACE DATUM (READINGS ABOVE LAND SURFACE INDICATED BY "+")

$\begin{array}{lllll} & \text { WATER } & \text { WATER } & \text { WATER } \\ \text { DATE } & \text { LEVEL MS } & \text { DATE } & \text { LEVEL MS } & \text { DATE } \\ \text { LEVEL MS }\end{array}$

NOV 19, $1991 \quad 18.45 \mathrm{~S} \quad$ APR 07, $1992 \quad 22.21 \mathrm{~S} \quad$ JUL $21,1992 \quad 24.75 \mathrm{~S}$

PERIOD OF RECORD HIGHEST +1.00 MAY 21, 1986 LOWEST 24.75 JUL 21,1992

SITE NUMBER 373847118545801

LOCAL NUMBER 003S028E32E07M

About 2.5 miles east of Mammoth Lakes and 0.1 mile northwest of Casa Diablo Hot Springs. Drilled unused watertable well. Diameter 6 inches, depth 22 feet, depth measured 19.4 feet in 1992 . Altitude of land-surface datum 7,316 feet. Water-level records available 1989 to current year.

WATER LEVELS IN FEET BELOW LAND-SURFACE DATUM

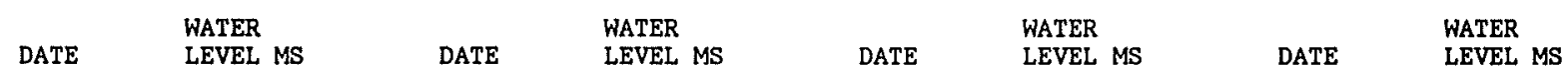

$\begin{array}{llllllllll}\text { OCT 22, } 1991 & \text { SD } & \text { DEC } 12,1991 & \text { SD } & \text { MAY } 15,1992 & \text { D } & \text { JUL 22, } 1992\end{array}$

\begin{tabular}{|c|}
\hline VV 20 \\
\hline
\end{tabular}

PERIOD OF RECORD HIGHEST 8.58 DEC $20,1989 \quad$ LOWEST 17.95 FEB 15, 1991

SITE NUMBER 373847118545802

LOCAL NUMBER O03S028E32E08M

About 2.5 miles east of Mammoth Lakes and 0.1 mile northwest of Casa Diablo Hot Springs. Drilled irrigation well. Diameter 6 inches, depth 75 feet. Altitude of land-surface datum 7,316 feet. Water-level records available 1990 to current year.

WATER LEVELS IN FEET BELOW LAND-SURFACE DATUM

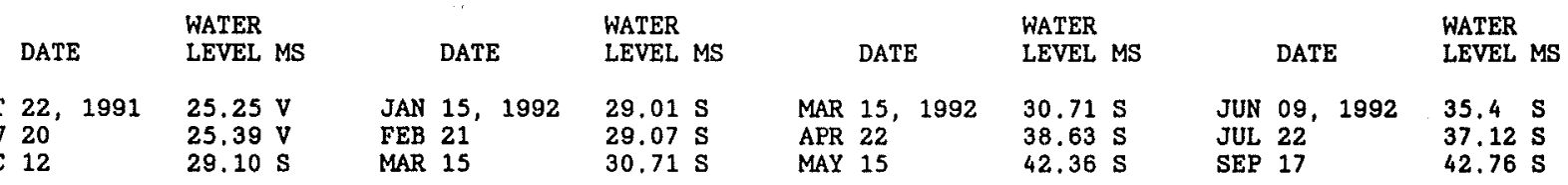

PERIOD OF RECORD HIGHEST 13.94 AUG 29, 1990 LOWEST 42.76 SEP 17, 1992

SITE NUMBER 373818118533201

LOCAL NUMBER 003S028E33P01M

About 3.9 miles east of Mammoth Lakes at the Mono County Sheriff Substation. Drilled public-supply well. Diameter 8 inches, depth 75 feet, perforated 51-75 feet. Altitude of land-surface datum 7,180,61 feet. Water-level records available $1971-72,1982$ to current year.

WATER LEVELS IN FEET BELOW LAND-SURFACE DATUM

$\begin{array}{lllll} & \text { WATER } & \text { WATER } & & \text { WATER } \\ \text { DATE } & \text { LEVEL MS } & \text { DATE } & \text { LEVEL MS } & \text { DATE } \\ \text { LEVEL MS }\end{array}$

NOV 20, $1991 \quad 36.48 \mathrm{~V} \quad$ APR 08, $1992 \quad 34.44 \mathrm{~S} \quad$ JUL $21,1992 \quad 34.08 \mathrm{VR}$

PERIOD OF RECORD HIGHEST 16.53 MAY 21, 1986 LOWEST 36.48 NOV 20, 1991 
WELL DESCRIPTIONS AND WATER-LEVEL MEASUREMENTS

WATER YEAR OCTOBER 1991 TO SEPTEMBER 1992

SITE NUMBER 373818118533202

LOCAL NUMBER 003S028E33P04M

About 3.9 miles east of Mammoth Lakes at the Mono County Sheriff Substation. Drilled public-supply well. Diameter 6 inches, depth 100 feet, perforated 34-60, 80-100 feet. Altitude of land-surface datum 7,179.13 feet. Water-

level records available 1978, 1984 to current year.

WATER LEVELS IN FEET BELOW LAND-SURFACE DATUM

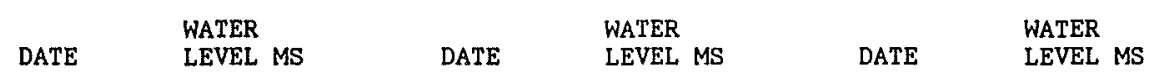

NOV 20, $199120.00 \mathrm{~V} \quad$ APR 08, $1992 \quad 21.28 \mathrm{SP} \quad$ JUL $21,1992 \quad 17.95 \mathrm{VT}$

PERIOD OF RECORD HIGHEST 6.35 JUL 19, 1984 LOWEST 20.00 NOV 20, 1991

SITE NUMBER 373816118532001

LOCAL NUMBER 003S028E33001M

About 4 miles east of Mammoth Lakes. Drilled domestic well. Diameter 6 inches, depth 80 feet. Altitude of landsurface datum 7,161,76 feet. Water-level records available 1986 to current year.

WATER LEVELS IN FEET BELOW LAND-SURFACE DATUM

DATE WATER $\quad$ LEVEL MS

JUL $21,1992 \quad 22.71 \mathrm{SR}$

PERIOD OF RECORD HIGHEST 10.40 MAY 21, 1986 LOWEST 26.60 JUL 29, 1988

SITE NUMBER 373906118522301

LOCAL, NUMBER O03S028E34C01M

About 4.9 miles east of Mammoth Lakes near Hot Creek Fish Hatchery. Drilled observation well. Diameter 4 inches, depth 152 feet. Altitude of land-surface datum 7,077,0 feet. Water-level records available 1985 to current year.

WATER LEVELS IN FEET BELOW LAND-SURFACE DATUM

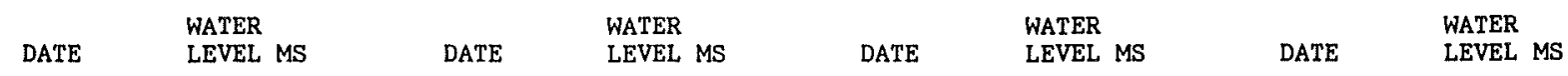

$\begin{array}{llllllllll}\text { NOV 19, } 1991 & 6.84 \mathrm{~S} & \text { APR } 07,1992 & 7.25 \mathrm{~S} & \text { JUL } 07,1992 & 6.65 \mathrm{~S} & \text { SEP } 16,1992 & 7.01 \mathrm{~S} \\ \text { DEC 17 } & 6.80 \mathrm{~S} & \text { JUN } 10 & 6.62 \mathrm{~S} & & 23 & & 6.08 \mathrm{~S} & & \\ \text { MAR 14, 1992 } & 7.08 \mathrm{~S} & 24 & 6.85 \mathrm{~S} & \text { AUG } 03 & 6.11 \mathrm{~S} & & \end{array}$

PERIOD OF RECORD HIGHEST 0.25 AUG 27, 1986 LOWEST 7.25 APR 07, 1992

SITE NUMBER 373818118514502

LOCAL NUMBER 003S028E34RO2M

About 5.5 miles east of Mammoth Lakes at elementary school. Drilled institutional well. Diameter 6 inches, depth 44.3 feet. Altitude of land-surface datum 7,090 feet. Water-level records available 1985 to current year.

WATER LEVELS IN FEET BELOW LAND-SURFACE DATUM

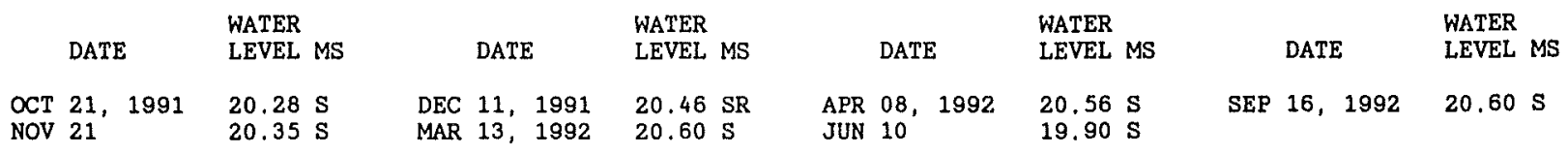

PERIOD OF RECORD HIGHEST 12.55 MAY 12, 1986 LOWEST 22.30 DEC 19, 1989 
WELL DESCRIPTIONS AND WATER-LEVEL MEASUREMENTS

WATER YEAR OCTOBER 1991 TO SEPTEMBER 1992

SITE NUMBER 373850118513601

LOCAL NUMBER 003S028E35E02M

About 5.6 miles east of Mammoth Lakes at elementary school. Drilled observation well. Diameter 18 inches, depth 223 feet, depth of hole 805 feet. Altitude of land-surface datum 7,084,99 feet. Water-level records available 1973,1982 to current year.

WATER LEVELS IN FEET BELOW LAND-SIJRFACE DATUM

\begin{tabular}{|c|c|c|c|c|c|}
\hline DATE & $\begin{array}{l}\text { WATER } \\
\text { LEVEL MS }\end{array}$ & DATE & $\begin{array}{l}\text { WATER } \\
\text { LEVEL MS }\end{array}$ & DATE & $\begin{array}{l}\text { WATER } \\
\text { LEVEL MS }\end{array}$ \\
\hline
\end{tabular}

$\begin{array}{lllllll}\text { NOV } 19,1991 & 14.68 \mathrm{~V} & \text { MAR } 11,1992 & 15.02 \mathrm{~V} & \text { APR 23, } 1992 & 15.15 \mathrm{~V} \\ \text { DEC } 17 & & 14.63 \mathrm{~V} & \text { APR } 09 & 14.93 \mathrm{~V} & \text { JUL 23 } & 13.83 \mathrm{~V} \\ \text { JAN } 16,1992 & 14.45 \mathrm{~V} & 17 & 14.97 \mathrm{~V} & & \end{array}$

PERIOD OF RECORD HIGHEST 6.77 AUG 13, 1983 LOWEST 19.01 MAR 11, 1992

SITE NUMBER 373849118513001

LOCAL NUMBER 003S028E35F01M

About 5.7 miles east of Mammoth Lakes near Hot Creek Fish Hatchery. Drilled observation well. Diameter 13 1nches, depth 420 feet, depth of hole 917 feet. Altitude of land-surface datum $7,062.20$ feet. Water-1evel records available 1986 to current year.

WATER LEVELS IN FEET BELOW LAND-SURFACE DATUM

$\begin{array}{llllll} & \text { WATER } & \text { WATER } & \text { WATER } & \text { WATER } \\ \text { DATE } & \text { LEVEL MS } & \text { DATE } & \text { LEVEL MS } & \text { DATE } & \text { LEVEL MS }\end{array}$

$\begin{array}{llllrlllll}\text { NOV 19, } 1991 & 9.85 \mathrm{~S} & \text { MAR } 11,1992 & 9.95 \mathrm{~S} & \text { JUN } 24,1992 & 9.84 \mathrm{~S} & \text { SEP } 16,1992 & 9.58 \mathrm{~S} \\ \text { DEC } 17 & & 9.52 \mathrm{~S} & \text { APR } 07 & 9.98 \mathrm{~S} & \text { JUL } 07 & 9.26 \mathrm{~S} & & \\ \text { JAN } 16,1992 & 9.85 \mathrm{~S} & & 23 & 10.05 \mathrm{~S} & & 23 & 8.68 \mathrm{~S} & & \\ & 18 & 9.85 \mathrm{~S} & \text { JUN } 10 & 9.80 \mathrm{~S} & \text { AUG } 03 & 8.67 \mathrm{~S} & \end{array}$

PERIOD OF RECORD HIGHEST 1.82 SEP 01, $1986 \quad$ LOWEST 10.05 APR 23, 1992

SITE NUMBER 373841118512901

LOCAL NUMBER 003S028E35F02M

About 5.6 miles east of Mammoth Lakes near Hot Creek Fish Hatchery. Drilled observation well. Diameter 6 inches 0-50 feet, 2 inches 50-496 feet, depth 496 feet, perforated 50-496 feet. Altitude of land-surface datum $7,054.12$ feet. Water-level records available 1986 to current year.

WATER LEVELS IN FEET BELOW LAND-SURFACE DATUM

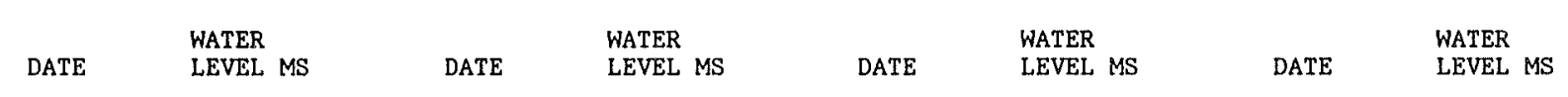

$\begin{array}{llllllllll}\text { NOV 19, } 1991 & 5.92 \mathrm{~S} & \text { JUN } 24,1992 & 6.63 \mathrm{~S} & \text { JUL } 23,1992 & 6.96 \mathrm{~S} & \text { SEP } 16,1992 & 7.08 \mathrm{~S} \\ \text { APR 07, } 1992 & 6.69 \mathrm{~S} & \text { JUL 07 } & 6.97 \mathrm{~S} & \text { AUG 03 } & 6.98 \mathrm{~S} & & \end{array}$
PERIOD OF RECORD HIGHEST 0.21 SEP 01, 1986 LOWEST 7.08 SEP 16, 1992

SITE NUMBER 373841118512902

LOCAL NUMBER 003S028E35F03M

About 5.6 miles east of Mammoth Lakes near Hot Creek Fish Hatchery. Drilled observation well. Diameter 5 inches 0-40 feet, 2 inches 40-255 feet, depth 255 feet, perforated 230-255 feet. Altitude of land-surface daturn $7,057.08$ feet. Water-level records available 1987 to current year.

WATER LEVELS IN FEET BELOW LAND-SURFACE DATUM (READINGS ABOVE LAND SURFACE INDICATED BY " $+"$ )
$\begin{array}{ll} & \text { WATER } \\ \text { DATE } & \text { LEVEL MS }\end{array}$
$\begin{array}{ll} & \text { WATER } \\ \text { DATE } & \text { LEVEL MS }\end{array}$
DATE WATER
NoV 19,1991
$1.41 \mathrm{~s}$
APR 07, 1992
$1.64 \mathrm{~S}$
JUL 23, 1992
$1.97 \mathrm{~S}$

PERIOD OF' RECORD

HIGHEST $\quad+0.35$ APR 19,1987

LOWEST

1.97 JUL 23,1992 
WELL DESCRIPTIONS AND WATER-LEVEL MEASUREMENTS

WATER YEAR OCTOBER 1991 TO SEPTEMBER 1992

SITE NUMBER 374315118441201

LOCAL NUMBER O03S029E02A01M

About 13.7 miles northeast of Mammoth Lakes. Drilled livestock well. Diameter 8 inches, depth 32 feet. Altitude of land-surface datum 6,922 feet. Water-level records available 1982 to current year.

WATER LEVELS IN FEET BELOW LAND-SURFACE DATUM

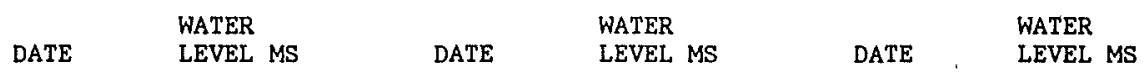

NOV 20, $1991 \quad 4.13 \mathrm{~S} \quad$ APR 08, $1992 \quad 4.27 \mathrm{~S} \quad$ JUL 23, $1992 \quad 4.36 \mathrm{~S}$

PERIOD OF RECORD HIGHEST 3.08 MAY 20, 1986 LOWEST 5.59 MAY 30, 1987

SITE NUMBER 374233118482701

LOCAL NUMBER 003S029E07A01M

About 8.9 miles northeast of Mammoth Lakes and $0.8 \mathrm{mile}$ north of Cashbaugh Ranch. Drilled observation well. Diameter 2 inches, depth 18 feet, depth measured 5.45 feet in 1992 , perforated 16-18 feet. Altitude of landsurface datum $6,873.81$ feet. Water-level records available 1972-73, 1982 to current year.

WATER LEVELS IN FEET BELOW LAND-SURFACE DATUM

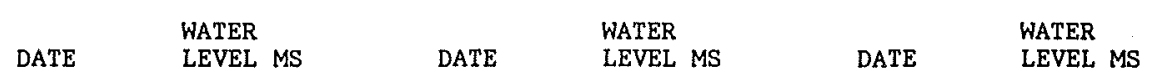

NOV 19, $1991 \quad 3.75 \mathrm{~S} \quad$ APR 07, $1992 \quad 1.96 \mathrm{~S} \quad$ JUL $21,1992 \quad 2.65 \mathrm{~S}$

PERIOD OF RECORD HIGHEST 0.62 JUN 03, 1983 LOWEST 10.44 JUL 25, 1972

SITE NUMBER 374109118484101

LOCAL NUMBER OO3SO29E18J01M

About 8.9 miles northeast of Mammoth Lakes and $0.1 \mathrm{mile}$ north of Cashbaugh Ranch. Drilled observation well. Diameter 2 inches, depth 9.1 feet, depth measured 5.9 feet in 1991, perforated 7.1-9.1 feet. Altitude of landsurface datum 6,895.62 feet. Water-level records available 1982 to current year.

WATER LEVELS IN FEET BELOW LAND-SURFACE DATUM

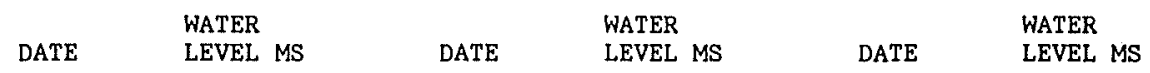

NOV 19, $1991 \quad 4.94 \mathrm{~S} \quad$ APR 07, $1992 \quad 1.05 \mathrm{~s} \quad$ JUL $21,1992 \quad 1.94 \mathrm{~s}$

PERIOD OF RECORD HIGHEST 0.05 JUN 03, 1983 LOWEST 5.50 NOV 29, 1986

SITE NUMBER 374045118491001

LOCAL NUMBER 003S029E19C01M

About 8.1 miles northeast of Mammoth Lakes and 0.7 mile southwest of Cashbaugh Ranch. Drilled observation well. Diameter 1.25 inches, depth 973 feet, perforated $470-475$ feet. Altitude of land-surface datum $6,969.93$ feet. Water-level records available 1982 to current year.

WATER LEVELS IN FEET BELOW LAND-SURFACE DATUM

$\begin{array}{llllll} & \text { WATER } & \text { WATER } & & \text { WATER } & \text { WATER } \\ \text { DATE } & \text { LEVEL MS } & \text { DATE } & \text { LEVEL MS } & \text { DATE } & \text { LEVEL MS }\end{array}$

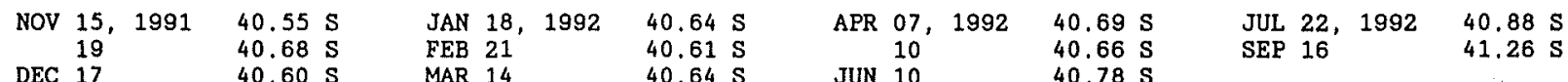

PERIOD OF RECORD HIGHEST 33.67 AUG 03, 1982 LOWEST 41.26 SEP 16, 1992 
WELL DESCRIPTIONS AND WATER-LEVEL MEASUREMENTS

WATER YEAR OCTOBER 1991 TO SEPTEMBER 1992

SITE NUMBER 374000118483101

LOCAL NUMBER O03S029E19RO1M

About 8.6 miles northeast of Mammoth Lakes and 1.2 miles south of Cashbaugh Ranch. Drilled observation well. Dlameter 2 inches, depth 22.3 feet, perforated $20.3-22.3$ feet. Altitude of land-surface datum 6,960 feet. Water-level records avallable 1989 to current year.

WATER LEVELS IN FEET BELOW LAND-SURFACE DATUM (READINGS ABOVE LAND SURFACE INDICATED BY "+")

$\begin{array}{lllll} & & & & \text { WATER } \\ \text { DATE } & \text { WEVEL MS } & \text { WATER } & \text { WATER } \\ \text { LATE } & \text { LEVEL MS } & \text { DATE }\end{array}$

$\begin{array}{lllllll}\text { NOV 20, } 1991 & 2.02 \mathrm{~S} & \text { APR } 23,1992 & 0.67 \mathrm{~S} & \text { JUN } 24,1992 & +0.38 \mathrm{SE} \\ \text { APR 08, } 1992 & 1.07 \mathrm{~S} & \text { JUN } 11 & & +0.42 \mathrm{SE} & \text { JUL 23 } & +0.37 \mathrm{~S}\end{array}$

PERIOD OF RECORD HIGHEST +0.37 JUL 23, 1992 LOWEST 5.26 AUG 08, 1989

SITE NUMBER 373929118455405

LOCAL NUMBER 003S029E27LOSM

About 11.2 miles east of Marmoth Lakes and 3.2 miles northeast of Whitmore Hot Springs. Drilled observation well. Diameter 1.25 inches, depth 160 feet. Altitude of land-surface datum 6,870 feet. Water-level records available 1982 to current year.

WATER LEVELS IN FEET BELOW LAND-SURFACE DATUM

$\begin{array}{lllll} & \text { WATER } & \text { WATER } & \text { WATER } \\ \text { DATE } & \text { LEVEL MS } & \text { DATE } & \text { LEVEL MS } & \text { DATE }\end{array}$

NOV 20, $1991 \quad 5.85 \mathrm{~s} \quad$ APR $08,1992 \quad 5.69 \mathrm{~s} \quad$ JUL $23,1992 \quad 6.71 \mathrm{~s}$

PERIOD OF RECORD HIGHEST 5.55 MAY 20, 1986 LOWEST 7.73 JUN 02, 1983

SITE NUMBER 373918118481901

LOCAL NUMBER 003S029E29NO1M

About 8.8 miles east of Mammoth Lakes and $1.9 \mathrm{miles}$ northeast of Whitmore Hot Springs. Drilled observation well. Diameter 2 inches, depth 71 feet, perforated $69-71$ feet. Altitude of land-surface datum $6,966.0$ feet. Water-level records available 1972-73, 1982 to current year.

WATER LEVELS IN FEET BELOW LAND-SURFACE DATUM

\begin{tabular}{|c|c|c|c|c|c|}
\hline DATE & $\begin{array}{l}\text { WATER } \\
\text { LEVEL MS }\end{array}$ & DATE & $\begin{array}{l}\text { WATER } \\
\text { LEVEL MS }\end{array}$ & DATE & $\begin{array}{l}\text { WATER } \\
\text { LEVEL MS }\end{array}$ \\
\hline
\end{tabular}

NOV 20, $1991 \quad 19.73 \mathrm{~s} \quad$ APR 08, $1992 \quad 19.77 \mathrm{~s} \quad$ JUL 23, $1992 \quad 19.98 \mathrm{~s}$

PERIOD OF RECORD HIGHEST 12.44 JUN 02, $1983 \quad$ LOWEST 21 JUN 02, 1972

SITE NUMBER 373914118472201

LOCAL NUMBER 003S029E29R01M

About 9.6 miles east of Marmoth Lakes and 2 miles northeast of Whitmore Hot Springs. Drilled observation well. Diameter 2 inches, depth 96 feet. Altitude of land-surface datum 6,985.38 feet. Water-level records available 1982 to current year.

WATER LEVELS IN FEET BELOW LAND-SURFACE DATUM
DATE WATER
$\begin{array}{ll} & \text { WATER } \\ \text { DATE } & \text { LEVEL MS }\end{array}$
DATE WATER

Nov 20,1991

$39.07 \mathrm{~s}$

APR 08, $1992 \quad 39.45 \mathrm{~S}$

JUL 23, 1992

$39.59 \mathrm{~S}$

PERIOD OF RECORD HIGHEST 26.63 OCT 19, 1985 LOWEST 39.59 JUL 23, 1992 
WELL DESCRIPTIONS AND WATER-LEVEL MEASUREMENTS

WATER YEAR OCTOBER 1991 TO SEPTEMBER 1992

SITE NUMBER 373930118491601

LOCAL NUMBER 003S029E30E01M

About $7.8 \mathrm{miles}$ east of Mammoth Lakes and 2 miles north of Whitmore Hot Springs. Drilled observation well. Diameter 6 inches, depth 110 feet, perforated $90-110$ feet. Altitude of land-surface datum $7,078,80$ feet. Waterlevel records available 1983 to current year.

WATER LEVELS IN FEET BELOW LAND-SURFACE DATUM

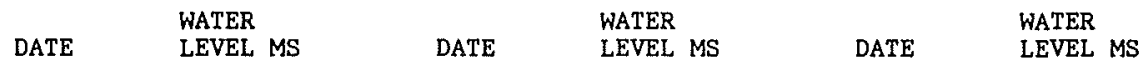

APR $08,1992 \quad 74.10 \mathrm{~V} \quad$ APR $10,1992 \quad 74.28 \mathrm{~V} \quad$ JUL $22,1992 \quad 74.22 \mathrm{~V}$

PERIOD OF RECORD HIGHEST 69.8 SEP $28,1983 \quad$ LOWEST 74.96 AUG 30,1987

SITE NIMBER 373930118491602

LOCAL NUMBER 003S029E30E02M

About $7.8 \mathrm{miles}$ east of Mammoth Lakes and 2 miles north of Whitmore Hot Springs, Drilled observation well. Dianeter 6 inches, depth 300 feet, depth of hole 315 feet. Altitude of land-surface datum $7,078.99$ feet. Waterlevel records available 1983 to current year.

Missing record due to malfunction of recording instrument.

WATER LEVELS IN FEET BELOW LAND-SURFACE DATUM (DAILY MEAN VALUES)

\begin{tabular}{|c|c|c|c|c|c|c|c|c|c|c|c|c|}
\hline DAY & OCT & NOV & DEC & JAN & FEB & MAR & APR & MAY & JUN & JUL & AUG & SEP \\
\hline $\begin{array}{l}1 \\
2 \\
3 \\
4 \\
5\end{array}$ & $\begin{array}{l}74.16 \\
74.13 \\
74.13 \\
74.15 \\
74.17\end{array}$ & $\begin{array}{l}74.36 \\
74.37 \\
74.41 \\
74.41 \\
74.39\end{array}$ & $\begin{array}{l}74.55 \\
74.56 \\
74.57 \\
74.53 \\
74.52\end{array}$ & $\begin{array}{l}74.60 \\
74.55 \\
74.46 \\
74.46 \\
74.39\end{array}$ & $\begin{array}{l}74.33 \\
74.40 \\
74.40 \\
74.40 \\
74.39\end{array}$ & $\begin{array}{l}74.51 \\
74.50 \\
74.53 \\
74.56 \\
74.53\end{array}$ & $\begin{array}{l}74.61 \\
74.63 \\
74.61 \\
74.59 \\
74.60\end{array}$ & $\begin{array}{l}74.59 \\
74.62 \\
74.62 \\
74.59 \\
74.56\end{array}$ & $\begin{array}{r}74.55 \\
--- \\
74.52 \\
74.49 \\
74.46\end{array}$ & $\begin{array}{l}74.53 \\
74.56 \\
74.54 \\
74.54 \\
74.56\end{array}$ & $\begin{array}{l}74.51 \\
74.50 \\
74.48 \\
74.47 \\
74.46\end{array}$ & $\begin{array}{r}74.45 \\
74.46 \\
74.44 \\
--= \\
74.46\end{array}$ \\
\hline $\begin{array}{r}6 \\
7 \\
8 \\
9 \\
10\end{array}$ & $\begin{array}{r}74.18 \\
--- \\
--- \\
74.22 \\
74.23\end{array}$ & $\begin{array}{l}74.38 \\
74.40 \\
74.38 \\
74.37 \\
74.40\end{array}$ & $\begin{array}{l}74.49 \\
74.45 \\
74.54 \\
74.56 \\
74.48\end{array}$ & $\begin{array}{l}74.44 \\
74.48 \\
74.56 \\
74.57 \\
74.52\end{array}$ & $\begin{array}{l}74.36 \\
74.37 \\
74.42 \\
74.38 \\
74.40\end{array}$ & $\begin{array}{r}74.52 \\
74.52 \\
74.56 \\
74.63 \\
---\end{array}$ & $\begin{array}{l}74.62 \\
74.66 \\
74.64 \\
74.64 \\
74.63\end{array}$ & $\begin{array}{r}74.57 \\
-.- \\
74.55 \\
74.52 \\
74.54\end{array}$ & $\begin{array}{r}--- \\
74.47 \\
74.48 \\
74.45\end{array}$ & $\begin{array}{l}74.57 \\
74.59 \\
74.61 \\
74.59 \\
74.55\end{array}$ & $\begin{array}{l}74.47 \\
74.47 \\
74.47 \\
74.50 \\
74.52\end{array}$ & $\begin{array}{r}74.45 \\
74.47 \\
74.46 \\
-9 \\
74.49\end{array}$ \\
\hline $\begin{array}{l}11 \\
12 \\
13 \\
14 \\
15\end{array}$ & $\begin{array}{r}74.20 \\
74.21 \\
--- \\
74.26 \\
74.22\end{array}$ & $\begin{array}{r}74.43 \\
74.43 \\
74.37 \\
74.34 \\
---\end{array}$ & $\begin{array}{l}74.47 \\
74.52 \\
74.57 \\
74.57 \\
74.56\end{array}$ & $\begin{array}{l}74.44 \\
74.49 \\
74.51 \\
74.50 \\
74.51\end{array}$ & $\begin{array}{l}74.46 \\
74.42 \\
74.43 \\
74.46 \\
74.43\end{array}$ & $\begin{array}{l}74.64 \\
74.63 \\
74.61 \\
74.58 \\
74.56\end{array}$ & $\begin{array}{l}74.61 \\
74.61 \\
74.61 \\
74.61 \\
74.61\end{array}$ & $\begin{array}{r}7.5 \\
74.54 \\
74.56 \\
74.64\end{array}$ & $\begin{array}{l}74.45 \\
74.45 \\
74.43 \\
74.40 \\
74.41\end{array}$ & $\begin{array}{l}74.51 \\
74.52 \\
74.53 \\
74.54 \\
74.53\end{array}$ & $\begin{array}{l}74.52 \\
74.52 \\
74.61 \\
74.62 \\
74.61\end{array}$ & $\begin{array}{l}74.52 \\
74.54 \\
74.55 \\
74.54 \\
74.57\end{array}$ \\
\hline $\begin{array}{l}16 \\
17 \\
18 \\
19 \\
20\end{array}$ & $\begin{array}{l}--- \\
--- \\
--- \\
--- \\
---\end{array}$ & $\begin{array}{r}74.48 \\
74.47 \\
74.52 \\
74.56\end{array}$ & $\begin{array}{l}74.53 \\
74.49 \\
74.47 \\
74.53 \\
74.58\end{array}$ & $\begin{array}{l}74.43 \\
74.36 \\
74.38 \\
74.39 \\
74.59\end{array}$ & $\begin{array}{l}74.50 \\
74.55 \\
74.58 \\
74.59 \\
74.58\end{array}$ & $\begin{array}{l}74.55 \\
74.57 \\
74.59 \\
74.61 \\
74.55\end{array}$ & $\begin{array}{l}74.63 \\
74.64 \\
74.64 \\
74.66 \\
74.63\end{array}$ & $\begin{array}{r}74.65 \\
-.- \\
74.57 \\
74.51\end{array}$ & $\begin{array}{l}74.45 \\
74.44 \\
74.43 \\
74.42 \\
74.42\end{array}$ & $\begin{array}{r}74.53 \\
74.53 \\
- \\
74.48\end{array}$ & $\begin{array}{l}74.56 \\
74.51 \\
74.52 \\
74.51 \\
74.50\end{array}$ & $\begin{array}{l}74.60 \\
74.61 \\
74.60 \\
74.59 \\
74.59\end{array}$ \\
\hline $\begin{array}{l}21 \\
22 \\
23 \\
24 \\
25\end{array}$ & $\begin{array}{l}74.20 \\
74.19 \\
74.20 \\
74.25 \\
74.25\end{array}$ & $\begin{array}{r}74.51 \\
-5 \\
74.58 \\
-=- \\
74.53\end{array}$ & $\begin{array}{l}74.54 \\
74.55 \\
74.55 \\
74.54 \\
74.55\end{array}$ & $\begin{array}{l}74.37 \\
74.40 \\
74.42 \\
74.40 \\
74.36\end{array}$ & $\begin{array}{l}74.62 \\
74.62 \\
74.64 \\
74.64 \\
74.65\end{array}$ & $\begin{array}{l}74.54 \\
74.54 \\
74.57 \\
74.62 \\
74.58\end{array}$ & $\begin{array}{l}74.59 \\
74.61 \\
74.66 \\
74.65 \\
74.64\end{array}$ & $\begin{array}{l}74.54 \\
74.56 \\
74.59 \\
74.66 \\
74.66\end{array}$ & $\begin{array}{r}74.43 \\
74.45 \\
-. \\
74.40 \\
74.39\end{array}$ & $\begin{array}{r}74.46 \\
\text { S74. } \\
74.44 \\
74.46 \\
74.50\end{array}$ & $\begin{array}{l}74.47 \\
74.43 \\
74.41 \\
74.42 \\
74.45\end{array}$ & $\begin{array}{r}74.61 \\
74.61 \\
74.60 \\
74.56 \\
-.-\end{array}$ \\
\hline $\begin{array}{l}26 \\
27 \\
28 \\
29 \\
30 \\
31\end{array}$ & $\begin{array}{l}74.21 \\
74.27 \\
74.30 \\
74.27 \\
74.37 \\
74.36\end{array}$ & $\begin{array}{r}74.52 \\
74.48 \\
74.51 \\
74.35 \\
74.53 \\
-\end{array}$ & $\begin{array}{l}74.57 \\
74.52 \\
74.50 \\
74.49 \\
74.55 \\
74.60\end{array}$ & $\begin{array}{l}74.36 \\
74.41 \\
74.40 \\
74.43 \\
74.42 \\
74.37\end{array}$ & $\begin{array}{r}74.65 \\
74.65 \\
74.62 \\
74.54 \\
-.- \\
---\end{array}$ & $\begin{array}{l}74.55 \\
74.53 \\
74.59 \\
74.61 \\
74.59 \\
74.61\end{array}$ & $\begin{array}{r}74.63 \\
74.64 \\
74.63 \\
74.58 \\
74.55 \\
.\end{array}$ & $\begin{array}{r}74.59 \\
74.52 \\
74.52 \\
74.56 \\
74.61 \\
\end{array}$ & $\begin{array}{r}74.39 \\
- \\
74.49 \\
-\end{array}$ & $\begin{array}{l}74.51 \\
74.51 \\
74.50 \\
74.48 \\
74.50 \\
74.52\end{array}$ & $\begin{array}{l}74.48 \\
74.51 \\
74.50 \\
74.47 \\
74.46 \\
74.45\end{array}$ & $\begin{array}{r}74.62 \\
74.60 \\
74.59 \\
74.57 \\
-\end{array}$ \\
\hline
\end{tabular}

PERIOD OF RECORD HIGHEST 71.72 SEP 28, 1983 LOWEST 75.10 AUG 25, 1987

S Manual steel tape measurement. 
WELL DESCRIPTIONS AND WATER-LEVEL MEASUREMENTS

WATER YEAR OCTOBER 1991 TO SEPTEMBER 1992

SITE NUMBER 373932118491501

LOCAL NUMBER 003S029E30E03M

About 7.8 miles east of Marmoth Lakes and 2 miles north of Whitmore Hot Springs. Drilled observation well. Diameter 1.25 inches, depth 175 feet, perforated 115-120 feet. Altitude of land-surface datum 7,074.88 feet. Water-level records avaliable 1982 to current year.

WATER LEVELS IN FEET BELOW LAND-SURFACE DATUM

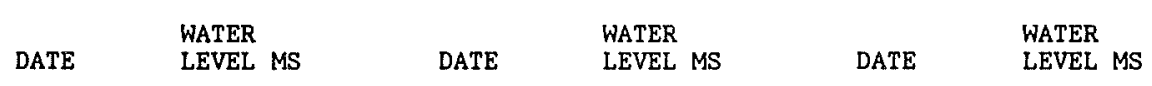

APR 08, $1992 \quad 70.35 \mathrm{~V} \quad$ APR $10,1992 \quad 70.37 \mathrm{~V} \quad$ JUL $22,1992 \quad 70.24 \mathrm{~V}$

PERIOD OF RECORD HIGHEST 67.70 NOV 03, 1983 LOWEST 70.98 AUG 30,1987

SITE NUMBER 373757118591801

LOCAL NUMBER O04S027E03E01M

In Old Mammoth near Mammoth Creek. Drilled unused well. Diameter 8 inches, depth 357.5 feet, perforated 59.8-112,7, 132.7-357,5 feet. Altitude of land-surface datum 7,985.28 feet. Water-level records available 1988 to current year.

WATER LEVELS IN FEET BELOW LAND-SURFACE DATUM

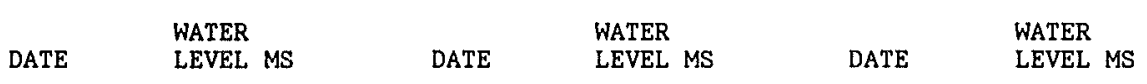

$\begin{array}{llrrrrrrr}\text { NOV } 18,1991 \quad 4.54 \mathrm{~S} & \text { APR } 09,1992 & 3.77 \mathrm{~S} & \text { JUL } 21, & 1992 & 4.28 \mathrm{~S} \\ \text { PERIOD OF RECORD } & \text { HIGHEST } & 0.22 & \text { APR } 21,1988 & \text { LOWEST } & 4.54 & \text { NOV } 18,1991\end{array}$

SITE NUMBER 373739118582101

LOCAL NUMBER 004S027E03J03M

About $0.3 \mathrm{mile}$ south of Mammoth Lakes in the Snow Creek development. Drilled observation well. Diameter 4 inches, depth 501 feet, perforated 100-501 feet, Altitude of land-surface datum $7,877.79$ feet. Water-level records available 1988 current year.

WATER LEVELS IN FEET BELOW LAND-SURFACE DATUM

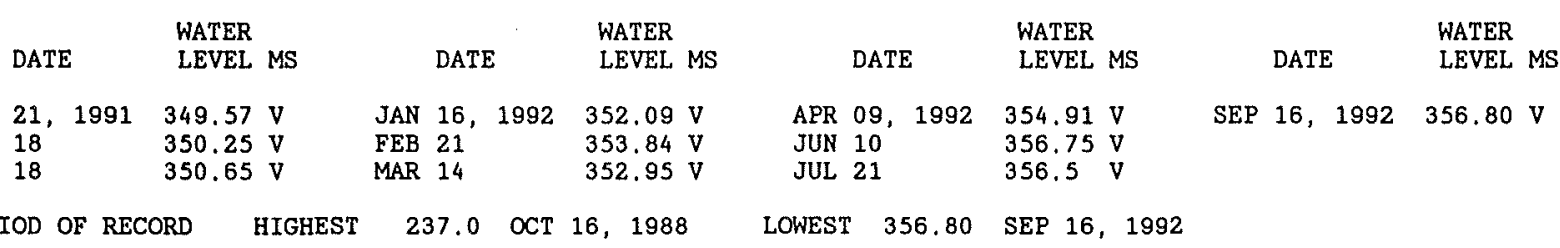

SITE NUMBER 373719118585301

LOCAL NUMBER 004S027E10B01M

About $0.8 \mathrm{mile}$ south of Mammoth Lakes near the Snow Creek development. Drilled observation well. Diameter

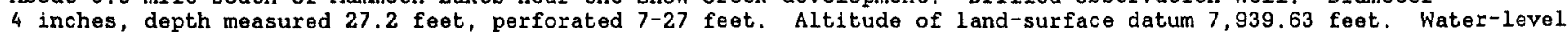
records available 1988 to current year.

WATER LEVELS IN FEET BELOW LAND-SURFACE DATUM

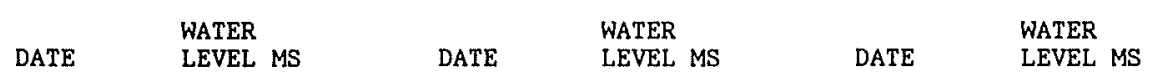

NOV 18, 1991 SD APR 09, $1992 \quad$ D $\quad$ JUL 21, $1992 \quad$ SD

PERIOD OF RECORD HIGHEST 13.27 JUL 28,1988 LOWEST 27.14 MAY 24,1990 
LOCAL NUMBER 004S027E10C01M

About $0.9 \mathrm{mile}$ south of Mammoth Lakes near the Snow Creek development. Drilled observation well. Diameter

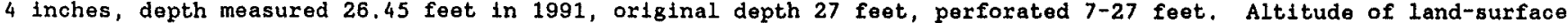
datum 7,952.92 feet. Water-level records available 1988 to current year.

WATER LEVELS IN FEET BELOW LAND-SURFACE DATUM

$\begin{array}{lllll} & \text { WATER } & \text { WATER } & \text { WATER } \\ \text { DATE } & \text { LEVEL MS } & \text { DATE } & \text { LEVEL MS } & \text { DATE }\end{array}$

NOV 18, 1991 SD APR 09, $1992 \quad$ D $\quad$ JUL 21, $1992 \quad$ SD

PERIOD OF RECORD HIGHEST 18.83 AUG 08, 1989 LOWEST 26.00 MAR 19, 1990

SITE NUMBER 373705118585601

LOCAL NUMBER 004S027E10F01M

About $0.8 \mathrm{mile}$ south of Mammoth Lakes near the Snow Creek development. Drilled observation well. Diameter 4 inches, depth 43 feet, perforated 5-43 feet. Altitude of land-surface datum $7,968.56$ feet. Water-level records available 1988 to current year.

WATER LEVELS IN FEET BELOW LAND-SURFACE DATUM

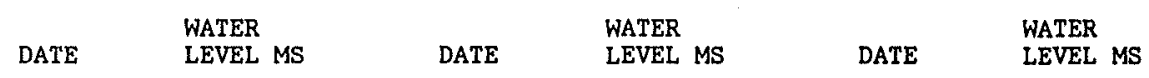

NOV 18, $199130.46 \mathrm{~S} \quad$ APR 09, $1992 \quad 33.67 \mathrm{~S} \quad$ JUL $21,1992 \quad 22.75$

PERIOD OF RECORD HIGHEST 12.14 JUL 28,1988 LOWEST 33.67 APR 09,1992

SITE NUMBER 373754118501701

LOCAL NUMBER 004S028E01F01M

About 7 miles southeast of Mammoth Lakes and 1.4 miles west of Whitmore Hot Springs. Drilled observation well. Diameter 2 inches, depth 98.6 feet, perforated 96.6-98.6 feet. Altitude of land-surface datum 7,090.40 feet. Water-level records available 1972-73, 1986 to current year.

WATER LEVELS IN FEET BELOW LAND-SURFACE DATUM

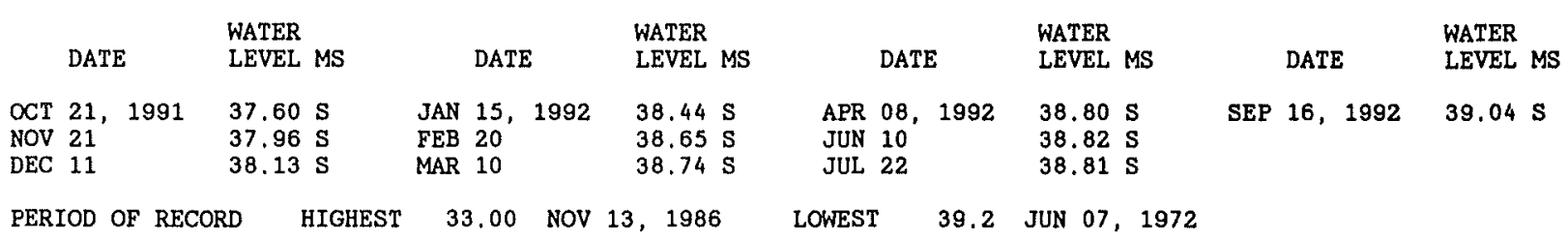

SITE NUMBER 373737118501701

LOCAL NUMBER 004S028E01L01M

About 7 miles southeast of Mammoth Lakes at the airport. Drilled public-supply well. Diameter 10 inches, depth 70 feet, perforated 52-66 feet. Altitude of land-surface datum 7,120 feet. Water-level records avallable 1984 to current year.

WATER LEVELS IN FEET BELOW LAND-SURFACE DATUM

\begin{tabular}{|c|c|c|c|c|c|}
\hline DATE & WATER & DATE & WATER & DATE & WATER \\
\hline
\end{tabular}
NOV 21,1991
$29.02 \mathrm{~S}$
APR 08, 1992
$29.91 \mathrm{SR}$
JUL 22, 1992
$29.51 \mathrm{sP}$

PERIOD OF RECORD * HIGHEST 19.24 AUG 30,1986 LOWEST 29.24 JUL 16,1990 
WELL DESCRIPTIONS AND WATER-LEVEL MEASUREMENTS

WATER YEAR OCTOBER 1991 TO SEPTEMBER 1992

SITE NUMBER 373742118515703

LOCAL NUMBER 004S028E03J03M

About $5.6 \mathrm{mlles}$ southeast of Mammoth Lakes. Drilled unused well, Diameter 8 inches, depth 125 feet, perforated 27-125 feet. Altitude of land-surface datum 7,102.22 feet. Water-level records available 1984 to current year. WATER LEVELS IN FEET BELOW LAND-SURFACE DATUM

$\begin{array}{llllll} & \text { WATER } & & \text { WATER } & & \text { WATER } \\ \text { DATE } & \text { LEVEL MS } & \text { DATE } & \text { LEVEL MS } & \text { DATE } & \text { WEVEL MS }\end{array}$

$\begin{array}{lllllllll}\text { OCT } 21,1991 & 20,48 \mathrm{~S} & \text { JAN } 15,1992 & 21.45 \mathrm{~S} & \text { APR } 08,1992 & 21.65 \mathrm{~S} & \mathrm{SEP} 16,1992 & 21.72 \mathrm{~S} \\ \text { NOV } 20 & 20.95 \mathrm{~S} & \text { FEB } 21 & & 21.58 \mathrm{~S} & \text { JUN } 08 & 19.96 \mathrm{~S} & \\ \text { DEC } 11 & 21.18 \mathrm{~S} & \text { MAR } 10 & & 21.79 \mathrm{~S} & \text { JUL } 21 & 20.76 \mathrm{~S}\end{array}$

DEC 11

LOWEST $\quad 21.79$ MAR 10,1992

SITE NUMBER 373729118532802

LOCAL NUMBER 004S028E04P02M

About $4.5 \mathrm{miles}$ southeast of Mammoth Lakes. Drilled observation well. Diameter 2 inches, depth 44 feet, depth measured 41.5 feet in 1992, perforated 40-42 feet. Altitude of land-surface datum 7 , 167.39 feet. Water-level records available $1972-73,1982$ to current year.

WATER LEVELS IN FEET BELOW LAND-SURFACE DATUM

\begin{tabular}{|c|c|c|c|c|c|}
\hline DATE & $\begin{array}{l}\text { WATER } \\
\text { LEVEL MS }\end{array}$ & DATE & $\begin{array}{l}\text { WATER } \\
\text { LEVEL MS }\end{array}$ & DATE & WATER \\
\hline
\end{tabular}

$\begin{array}{llllllll}\text { NOV } 20,1991 & \text { SD } & \text { APR } 08,1992 & \text { D } & \text { JUL } 21, & 1992 & \text { SD }\end{array}$

PERIOD OF RECORD HIGHEST 24.07 MAY 26, 1982 LOWEST 42.65 MAY 17,1972

SITE NUMBER 373745118554001

LOCAL NUMBER 004S028E06L01M

About 2.3 miles southeast of Mammoth Lakes. Drilled observation well. Diameter 2.5 inches, depth 40 feet, depth of hole 132 feet. Altitude of land-surface datum 7,469.84 feet. Water-level records available 1982 to current year.

WATER LEVELS IN FEET BELOW LAND-SURFACE DATUM

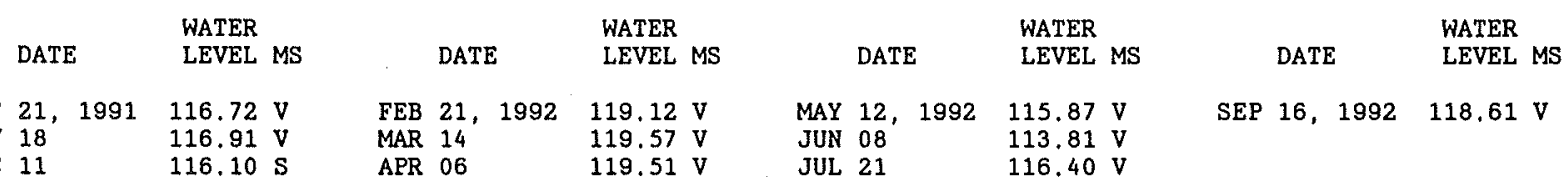

PERIOD OF RECORD HIGHEST 69.95 JUL $06,1983 \quad$ LOWEST 119.57 MAR 14,1992

SITE NUMBER 373745118554002

LOCAL NUMBER 004S028E06L02M

About 2.3 miles southeast of Mammoth Lakes. Drilled observation well. Diameter 4 inches, depth 215 feet, depth of hole 230 feet. Altitude of land surface datum $7,470,65$ feet. Water-level records available 1983 to current year.

WATER LEVELS IN FEET BELOW LAND-SURFACE DATUM

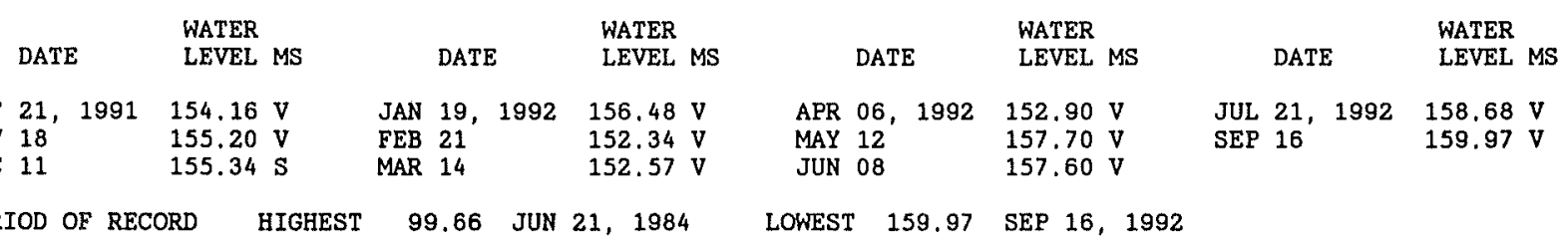


SITE NUMBER 373654118495401

LOCAL NUMBER 004S028E12K01M

About 7.6 miles southeast of Mammoth Lakes at the Sierra Nevada Aquatic Research Laboratory. Drilled commercial well. Diameter 6 inches, depth 70 feet, perforated 34-70 feet. Altitude of land-surface datum 7,095 feet. Waterlevel records available 1959 to current year.

WATER LEVELS IN FEET BELOW LAND-SURFACE DATUM

\begin{tabular}{|c|c|c|c|c|}
\hline & WATER & & WATER & \\
\hline DATE & LEVEL MS & DATE & LEVEL MS & DATE \\
\hline
\end{tabular}

NOV 20, $199129.57 \mathrm{~V} \quad$ APR 08, $1992 \quad 32.55 \mathrm{~S} \quad$ JUL $22,1992 \quad 30.44 \mathrm{~V}$

PERIOD OF RECORD HIGHEST 22.67 JUL 17,1991 LOWEST 33.24 APR 24, 1990

SITE NUMBER 373759118474101

LOCAL NUMBER 0045029E05B01M

About 9.9 miles east of Mammoth Lakes and 1.6 miles northeast of Whitmore Hot Springs. Drilled observation well. Diameter 2 inches, depth 98 feet, perforated 96-98 feet. Altitude of land-surface daturn 6,918 feet. Water-level records avallable 1972-73, 1982-85, 1988 to current year.

WATER LEVELS IN FEET BELOW LAND-SURFACE DATUM

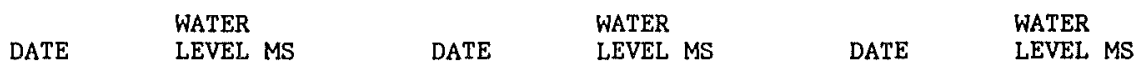

NOV 20, $199145.17 \mathrm{~S} \quad$ APR $08,1992 \quad 45.37 \mathrm{~s} \quad$ JUL $23,1992 \quad 45.48 \mathrm{~S}$

PERIOD OF RECORD HIGHEST 42.95 NOV 12,1984 LOWEST 46.14 NOV 28,1988

SITE NUMBER 373732118490001

LOCAL NUMBER 004S029E06PO1M

About 8.2 miles southeast of Mammoth Lakes at Mono County Probation Department Camp. Drilled institutional well. Diameter 6 inches, depth 150 feet. Altitude of land-surface datum 7,014 feet. Water-level records available 1986 to current year.

WATER LEVELS IN FEET BELOW LAND-SURFACE DATUM

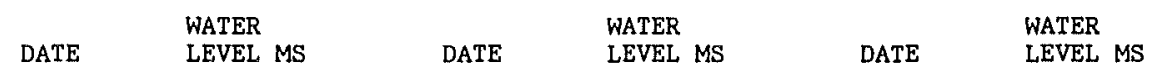

NOV 20, $199125.69 \mathrm{~V} \quad$ APR 08, $1992 \quad 25.60$ VR JUL 22, $1992 \quad 24.78 \mathrm{~V}$

PERIOD OF RECORD HIGHEST 21.69 AUG 30, 1986 LOWEST 29.45 APR 25, 1989

SITE NUMBER 373707118491701

LOCAL NUMBER 004S029E07E01M

About 8 miles southeast of Mammoth Lakes and 1 mile southwest of Whitmore Hot Springs. Drilled domestic well. Diameter 6 inches, depth 45.5 feet. Altitude of land-surface datum 7,035 feet. Water-level records available 1989 to current year.

WATER LEVELS IN FEET BELOW LAND-SURFACE DATUM

$\begin{array}{lllll}\text { DATE } & \text { WATER } & \text { WATER } & & \text { WATER } \\ \text { LEVEL MS } & \text { DATE } & \text { LEVEL MS } & \text { DATE } & \text { LEVEL MS }\end{array}$

NOV 20, $1991 \quad 7.61 \mathrm{~S} \quad$ APR 08, $1992 \quad 8.02 \mathrm{~S} \quad$ JUL $22,1992 \quad 8.54 \mathrm{~S}$

PERIOD OF RECORD HIGHEST 4.15 JUL 17, 1991 LOWEST 8.54 JUL 22, 1992 


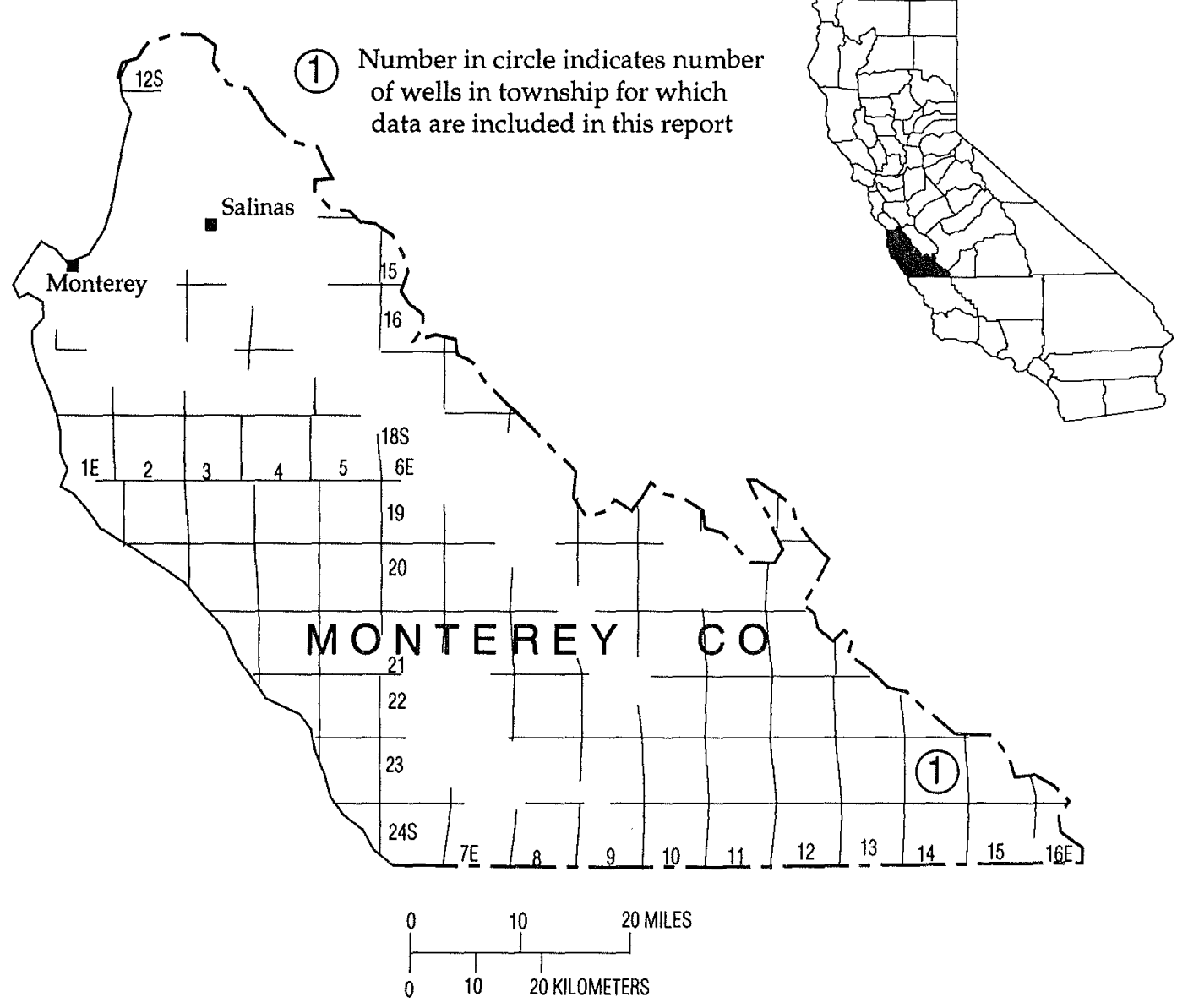

Figure 20. Location of well in Monterey County. 
WELL DESCRIPTIONS AND WATER-LEVEL MEASUREMENTS

WATER YEAR OCTOBER 1991 TO SEPTEMBER 1992

SITE NUMAER 355405120263301

LOCAL NUMBER 023S014E27H01M

About 0.6 mile west of Parkfield. Drilled domestic water-table well in alluvium of Quaternary age. Diameter 10 inches, depth unknown. Altitude of land-surface datum 1,533 feet. Water-level records available 1979 to current year.

WATER LEVELS IN FEET BELOW LAND-SURFACE DATUM

$\begin{array}{llll}\text { DATE } & \begin{array}{l}\text { WATER } \\ \text { LEVEL MS }\end{array} & \text { DATE } & \begin{array}{l}\text { WATER } \\ \text { LEVEL MS }\end{array} \\ 17,1991 & 37.61 \mathrm{SR} & \text { APR 17, 1992 } & 34.47 \mathrm{~S}\end{array}$

PERIOD OF RECORD HIGHEST 16.18 APR 19, 1983 LOWEST 41.9 SEP 21,1982 


\section{RIVERSIDE COUNTY}

Page

LOCAL NUMBER 003S004F20D01S $\ldots \ldots \ldots 18,203$

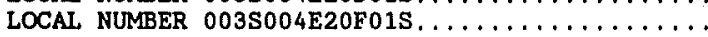

LOCAL NUMBER $0035004 E 20 F 025 \ldots \ldots \ldots \ldots \ldots \ldots \ldots \ldots \ldots 18,203$

LOCAL NUMBER 003S004E20F03S . . . . . . . . . . 188, 203

LOCAL NUMBER 003 S004E20J01S. . . . . . . . . . 189, 203

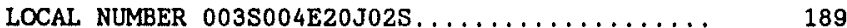

LOCAL NUMBER $0035004 E 20 J 035 \ldots \ldots \ldots \ldots \ldots \ldots \ldots \ldots \ldots$ 189, 203

LOCAL NUMBER 003\$004E29F01S . . . . . . . . . . . 189

LOCAL NUMBER 003S004E29R01S . . . . . . . . . . 190, 203

LOCAL NUMBER $0035004 E 30 C 01 S \ldots \ldots \ldots \ldots \ldots \ldots \ldots \ldots$. 203

LOCAL NUMBER $005 S 016 E 07 M 015 \ldots \ldots \ldots \ldots \ldots \ldots \ldots \ldots \ldots$

LOCAL NUMBER 006S011E16E01S............... 190

LOCAL NUMBER 007S003E15P01S . . . . . . . . . . . . . 190

LOCAL NUMBER 007S003E21L03S................ 191

LOCAL NUMBER 007 S003E34E01S................ 191

LOCAL NUMBER 008S003E02K01S. . . . . . . . . .

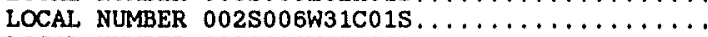

LOCAL NUMBER $0035001 W 05 Q 015 \ldots \ldots \ldots \ldots \ldots \ldots \ldots$

LOCAL NUMBER $004 \$ 003 W 290015 \ldots \ldots \ldots \ldots \ldots \ldots$

LOCAL NUMBER 005S002W19N01S..............

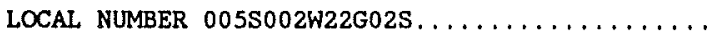

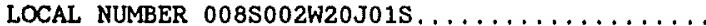

LOCAL NUMBER $008 S 002 W 20 J 02 S \ldots \ldots \ldots \ldots \ldots \ldots \ldots \ldots$

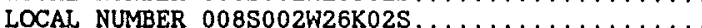

LOCAL NUMBER 008S002W28M01S ..............

LOCAL NUMBER 008S002W28M02S.............

LOCAL NUMBER 008S002W28M03S.............
LOCAL NUMBER 008S002W28P01S . . . . . . . .

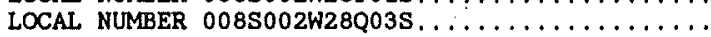

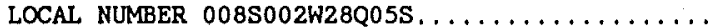

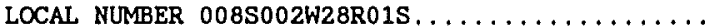

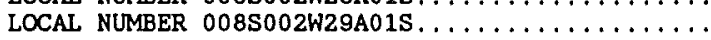

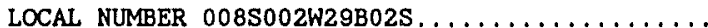

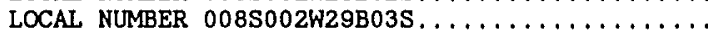

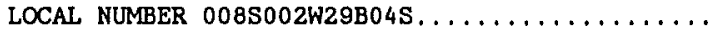

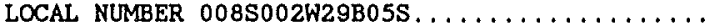

LOCAL NUMBER 008S002W29B06S . . . . . . . . .

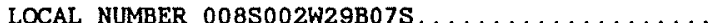

LOCAL NUMBER 008S002W29B08S. . . . . . . . . .

LOCAL NUMBER 008S002W29B09S. . . . . . . . . .

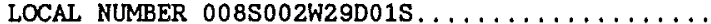

LOCAL NUMBER 008S002W29D02S. . . . . . . . . .

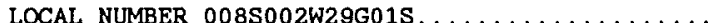

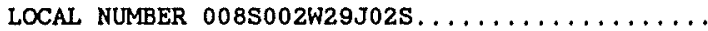

LOCAL NUMBER $0085002 W 29 K 015 \ldots \ldots \ldots \ldots \ldots \ldots$

LOCAL NUMBER 008S002W33H02S . . . . . . . . . . .

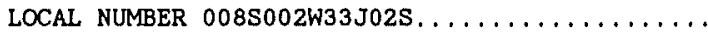

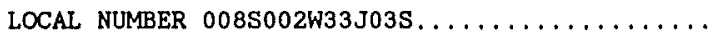

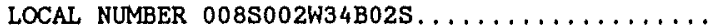

LOCAL NUMBER $0085002 W 34 B 04$ S . . . . . . . . . .

LOCAL NUMBER 008\$002W34E01S . . . . . . . . . . .

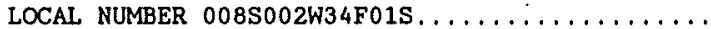

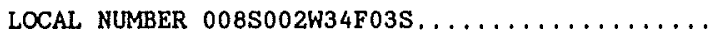

LOCAL NUMBER 008S002W35D01S . . . . . . . . .
Page

194

195

195

195

195

196

196

196

197

197

197

198

198

198

199

199

199

199

200

200

200

200

201

201

201

201

202
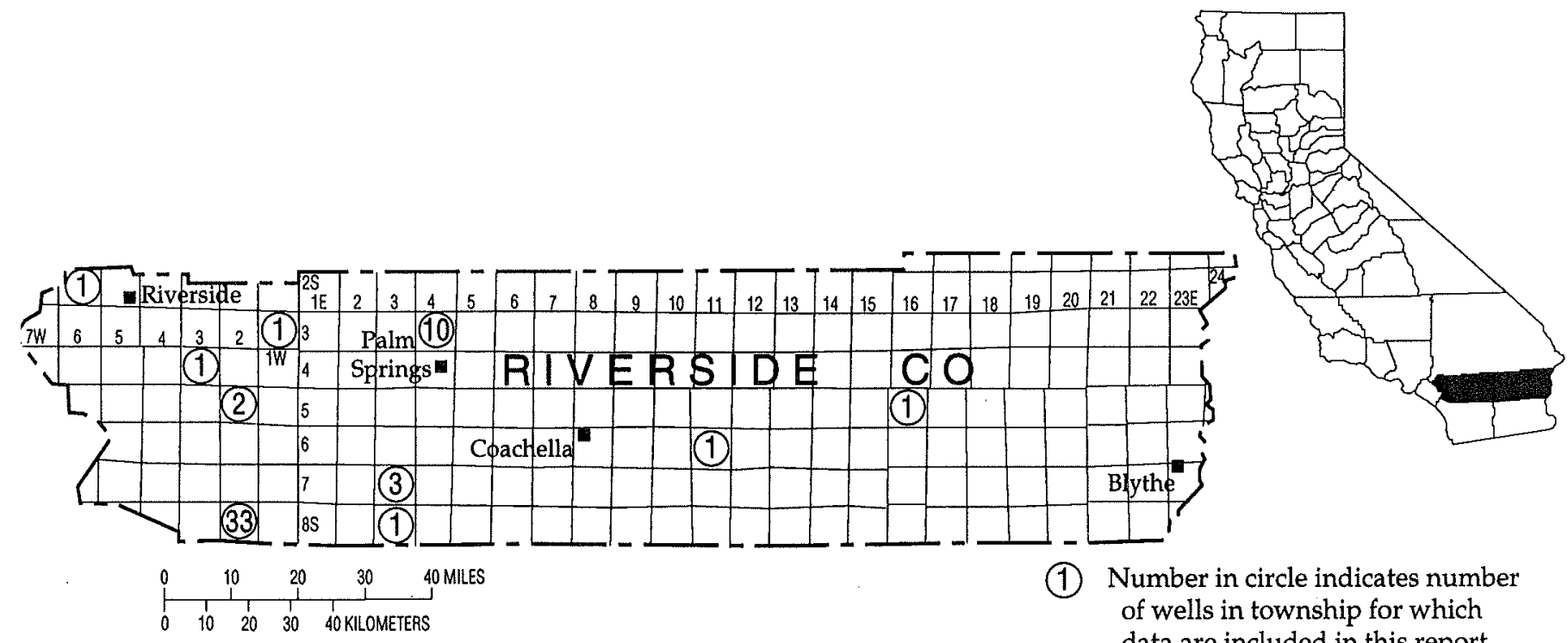

Figure 21, Location of wells in Riverside County. 
SITE NUMBER 335407116353401

LOCAL NUMBER 003S004E20D01S

About $7 \mathrm{miles}$ northwest of Palm Springs, $0.75 \mathrm{mile}$ south of Interstate 10 . Drilled observation water-table well in alluvium. Diameter 3 inches, depth measured 566 feet in 1991, perforated 560-580 feet. Altitude of land-surface datum 910 feet. Water-level records available 1972 to current year.

WATER LEVELS IN FEET BELOW LAND-SURFACE DATUM

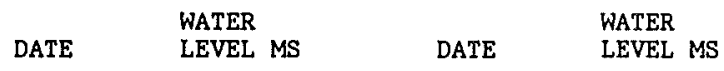

DEC 02, 1991396.94 V APR 20, $1992407.66 \mathrm{~V}$

PERIOD OF RECORD HIGHEST 150.98 APR 28, 1987 LOWEST 554.50 MAR 14, 1978

SITE NUMBER 335348116352701

LOCAL NUMBER 003S004E20F01S

About 7 miles northwest of Palm Springs, 0.2 mile south of Southern Pacific Railroad tracks and 2.7 miles west of Indian Avenue. Drilled observation water-table well. Diameter 2 inches, depth measured 624 feet in 1991 , original depth 640 feet, perforated 600-640 feet. Altitude of land-surface datum 890 feet. Water-level records available 1979 to current year.

WATER LEVELS IN FEET BELOW LAND-SURFACE DATUM

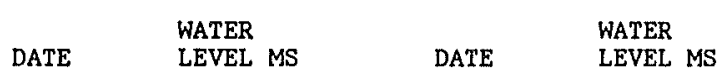

DEC $04,1991378.87 \mathrm{~V} \quad$ APR $21,1992388.54 \mathrm{~V}$

PERIOD OF RECORD HIGHEST 132.57 NOV 12, 1986 LOWEST 486.40 SEP 25, 1979

SITE NUMBER 335348116352702

LOCAL NUMBER 003S004E2OFO2S

About 7 miles northwest of Palm Springs, 0.2 mile south of Southern Pacific Railroad tracks, and 2.7 miles west of Indian Avenue. Drilled observation water-table well. Diameter 2 inches, depth 890 feet, depth measured 864 feet in 1991, perforated 850-890 feet. Altitude of land-surface datum 890 feet. Water-level records available 1979 to current year.

WATER LEVELS IN FEET BELOW LAND-SURFACE DATUM

$$
\begin{array}{lll} 
& \text { WATER } & \\
\text { DATE } & \text { WEVEL MS } & \text { DATER } \\
\text { LEVEL MS }
\end{array}
$$

DEC $04,1991381.62 \mathrm{~V} \quad$ APR 21, $1992390.95 \mathrm{~V}$

PERIOD OF RECORD HIGHEST 144.78 APR 29, 1987 LOWEST 490.60 SEP 25, 1979

SITE NUMBER 335348116352703

LOCAL NUMBER 003S004E20F03S

About 7 miles northwest of Palm Springs, $0.2 \mathrm{mile}$ south of Southern Pacific Rallroad tracks, and 2.7 miles west of

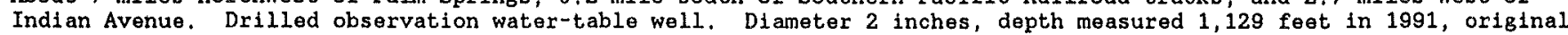
depth 1,140 feet, perforated 1,100-1,140 feet. Altitude of land-surface datum 890 feet. Water-level records available 1979 to current year.

WATER LEVELS IN FEET BELOW LAND-SURFACE DATUM

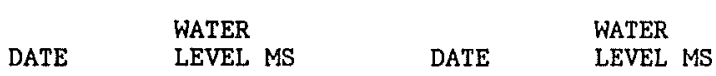

DEC $04,1991384.58 \mathrm{~V} \quad$ APR 21, $1992393.84 \mathrm{~V}$

PERTOD OF RECORD HIGHEST 153.70 APR 29, 1987 LOWEST $494.20 \quad$ SEP 25,1979 
WELL DESCRIPTIONS AND WATER-LEVEL MEASUREMENTS

WATER YEAR OCTOBER 1991 TO SEPTEMBER 1992

SITE NUMBER 335339116345301

LOCAL NUMBER 003S004E20J01S

About 7 miles northwest of Palm Springs, $0.45 \mathrm{mile}$ south of Southern Pacific Railroad tracks, and $2.1 \mathrm{miles}$ west of Indian Avenue. Drilled observation water-table well. Diameter 2 inches, depth measured 565 feet in 1991 , original depth 590 feet, perforated 550-590 feet. Altitude of land-surface datum 840 feet. Water-level records available 1980 to current year.

WATER LEVELS IN FEET BELOW LAND-SURFACE DATUM

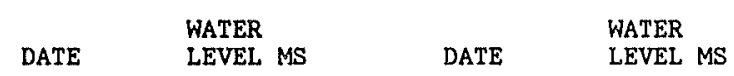

DEC 04, $1991343.32 \mathrm{~V} \quad$ APR 21, $1992352.88 \mathrm{~V}$

PERIOD OF RECORD HIGHEST 57.90 NOV 12,1986 LOWEST 417.20 OCT 02, 1980

\section{SITE NUMBER 335339116345302}

LOCAL NUMBER O03S004E20J02S

About 7 miles northwest of Palm Springs, $0.45 \mathrm{mile}$ south of Southern Pacific Railroad tracks and $2.1 \mathrm{miles}$ west of Indian Avenue. Drilled observation water-table well. Diameter 2 inches, depth measured 705 feet in 1991 , original depth 730 feet, perforated 690-730 feet. Altitude of land-surface datum 840 feet. Water-level records available 1980 to current year.

WATER LEVELS IN FEET BELOW LAND-SURFACE DATUM

$\begin{array}{lll} & \text { WATER } & \text { WATER } \\ \text { DATE } & \text { LEVEL MS } & \text { DATE }\end{array}$

DEC 04, $1991343.64 \mathrm{~V} \quad$ APR 21, $1992353.18 \mathrm{~V}$

PERIOD OF RECORD HIGHEST 103.89 NOV 12, 1986 LOWEST 419.00 JUL 16,1980

SITE NUMBER 335339116345303

LOCAL NUMBER 003S004E20J03S

About 7 miles northwest of Palm Springs, $0.45 \mathrm{mile}$ south of Southern Pacific Railroad tracks, and $2.1 \mathrm{miles}$ west of Indian Avenue. Drilled observation water-table well. Diameter 2 inches, depth measured 869 feet in 1991 , original depth 890 feet, perforated 850-890 feet. Altitude of land-surface datum 840 feet. Water-level records available 1980 to current year.

WATER LEVELS IN FEET BELOW LAND-SURFACE DATUM

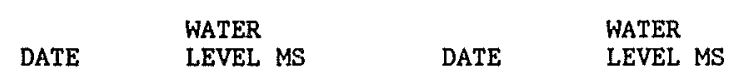

DEC $04,1991345.1 \mathrm{~V} \quad$ APR 21, $1992354.19 \mathrm{~V}$

PERIOD OF RECORD HIGHEST 112.64 APR 28, 1987 LOWEST 423.40 JUL 16,1980

\section{SITE NUMBER 335304116353001}

LOCAL NUMBER O03S004E29F01S

About 6 miles northwest of Palm Springs near Highway 111. Drilled unused water-table well in alluvium. Diameter 3 inches, depth 575 feet, perforated 555-575 feet. Altitude of land-surface datum 865 feet. Water-level records available 1972 to current year.

WATER LEVELS IN FEET BELOW LAND-SURFACE DATUM

$$
\begin{array}{lll} 
& \text { WATER } & \\
\text { DATE } & \text { WEVER MS }
\end{array}
$$

DEC 03, $1991373.89 \mathrm{~V} \quad$ APR 22, $1992381.35 \mathrm{~V}$

PERIOD OF RECORD HIGHEST 116.97 NOV 11, 1986 LOWEST 547.00 DEC 21, 1977 
SITE NUMBER 335231116345401

LOCAL NUMBER 003S004E29R01S

About 6 miles northwest of Palm Springs near Highway 111. Drilled unused water-table well in alluvium. Diameter 8 inches, depth measured 494 feet in 1991, perforated 431-551 feet. Altitude of land-surface datum 777 feet. Waterlevel records available 1971 to current year.

WATER LEVELS IN FEET BELOW LAND-SURFACE DATUM

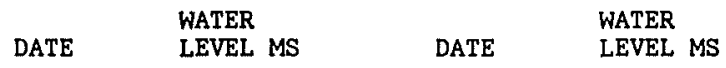

DEC 02, $1991365.03 \mathrm{~V} \quad$ APR 20, $1992375.65 \mathrm{~V}$

PERIOD OF RECORD HIGHEST 127.84 APR 27, 1987 LOWEST 516.39 MAR 14, 1978

SITE NUMBER 334452115212801

LOCAL NUMBER 005S016E07M01S

About 3.5 miles northeast of Desert Center. Drilled unused well. Diameter 14 inches $0-350$ feet, 12-3/4 inches 350-648 feet, depth 648 feet, perforated 250-648 feet. Altitude of land-surface datum 614 feet. Water-1evel records available October 1991 to current year.

WATER LEVELS IN FEET BELOW LAND-SURFACE DATUM

$$
\begin{array}{lllll} 
& \text { WATER } & \text { WATER } & \text { WATER } \\
\text { DATE } & \text { DEVEL MS } & \text { DATE } & \text { LEVEL MS } & \text { DATE } \\
\text { LEVEL MS }
\end{array}
$$

OCT 03, $1991194.37 \mathrm{~S} \quad$ MAR 18, $1992189.85 \mathrm{~S} \quad$ SEP 23, $1992188.42 \mathrm{~S}$

PERIOD OF RECORD HIGHEST 188.42 SEP 23, 1992 LOWEST 194.37 OCT 03, 1991

SITE NUMBER 333911115505701

LOCAL NUMBER 006\$011E16E01S

In Shavers Valley, west of Chiriaco Sumit about $0.6 \mathrm{mile}$ south of Interstate 10 and 2.5 miles west of Cottonwood Spring Road. Drilled unused water-table well. Diameter 16 inches, depth 515.4 feet, perforated $320-460$ feet. Altitude of land-surface datum 1,320 feet. Water-level records available 1933-34, 1940, 1961, 1979, 1981-85, 1989 to current year.

WATER LEVELS IN FEET BELOW LAND-SURFACE DATUM

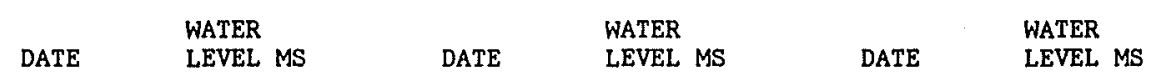

OCT 03, $1991246.01 \mathrm{~S} \quad$ MAR 18, $1992246.20 \mathrm{~S}$

SEP 23, $1992246.04 \mathrm{~S}$

PERIOD OF RECORD HIGHEST 243 MAR 20, 1934 LOWEST 274 JUN 10, 1934

SITE NUMBER 333321116392401

LOCAL NUMBER 007S003E15P01S

About 1.1 miles east of Anza. Domestic well. Diameter 7 inches, depth reported 70 feet. Altitude of land-surface datum 3,943 feet. Water-level records available 1953-54, 1960, 1969, 1973, 1986, 1989 to current year.

WATER LEVELS IN FEET BELOW LAND-SURFACE DATUM (READINGS ABOVE LAND SURFACE INDICATED BY "+")

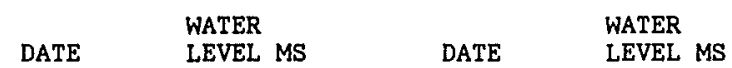

DEC 19, 199133.50 SR MAR 17, $199217.52 \mathrm{~S}$

PERIOD OF RECORD HIGHEST +2.80 NOV 24, 1953 LOWEST 30.0 MAY 22, 1953 
WELL DESCRIPTIONS AND WATER-LEVEL MEASUREMENTS

WATER YEAR OCTOBER 1991 TO SEPTEMBER 1992

SITE NUMBER 333240116403201

LOCAL NUMBER 007S003E21L03S

About 1.5 miles south of Anza. Drilled unused water-table well. Diameter 12 inches, depth 117 feet, depth measured 51.5 feet in 1989. Altitude of land-surface datum 3,847 feet. Water-level records available 1960 , 1989 to current year.

WATER LEVELS IN FEET BELOW LAND-SURFACE DATUM

$\begin{array}{lll} & \text { WATER } & \\ \text { DATE } & \text { WEVEL MS } & \text { DATER } \\ \text { LEVEL MS }\end{array}$

DEC $19,1991 \quad 18.74 \mathrm{~s} \quad$ MAR $17,199216.79 \mathrm{~s}$

PERIOD OF RECORD HIGHEST 15.05 MAR 09, 1989 LOWEST 51.00 AUG 01, 1960

SITE NUMBER 333122116394001

LOCAL NUMBER 007S003E34E01S

On Cahuilla Indian Reservation, south of Anza, 0.35 mile north of reservation road. Drilled unused water-table well. Diameter 9 inches, depth 249 feet, perforated 40-182 feet. Altitude of land-surface datum 3,870 feet. Water-level records available 1946, 1951-54, 1960, 1969, 1972 to current year.

WATER LEVELS IN FEET BELOW LAND-SURFACE DATUM

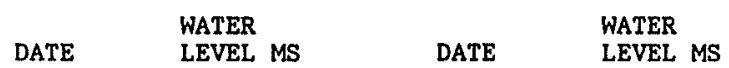

DEC $19,199162.3 \quad \mathrm{~V} \quad$ MAR $17,199260.64 \mathrm{~s}$

PERIOD OF RECORD HIGHEST 34.50 JAN 31,1951 LOWEST 76.75 JUL 24,1973

SITE NUMBER 333012116380801

LOCAL NUMBER 008S003E02K01S

About 3 miles southeast of Anza, 0.2 mile west of intersection of Bailey and Terwilliger Roads. Drilled unused water-table well. Diameter 8 inches, depth 296 feet, Altitude of land-surface datum 3,870 feet. Water-level records avallable 1972 to current year.

WATER LEVELS IN FEET BELOW LAND-SURFACE DATUM

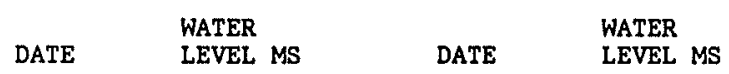

DEC $19,199150.26 \mathrm{~S} \quad \operatorname{MAR} 17,199248.92 \mathrm{~S}$

PERIOD OF RECORD HIGHEST 36.20 MAR 12, 1973 LOWEST 60.45 SEP 15, 1981

SITE NUMBER 335731117330601

LOCAL NUMBER 002S006W31C01S

North of Norco and the Santa Ana River, about $0.35 \mathrm{mile}$ southeast of intersection of Adams Avenue and Schleisman Road. Drilled domestic water-table well. Diameter and depth unknown. Altitude of land-surface datum 601 feet. Most measurements provided by Riverside County Flood Contro1 and Water Conservation District prior to 1982; measured periodically by U.S. Geological Survey. Water-level records available 1962 to current year.

WATER LEVELS IN FEET BELOW LAND-SURFACE DATUM

$\begin{array}{lll} & \text { WATER } & \text { WATER } \\ \text { DATE } & \text { DEVEL MS }\end{array}$

OCT 23, $199133.33 \mathrm{~S} \quad$ JUN $15,1992 \quad 29.94 \mathrm{~S}$

PERIOD OF RECORD HIGHEST 22.50 JUL 02, 1970 LOWEST 41.20 JUN 06, 1974 
WATER YEAR OCTOBER 1991 TO SEPTEMBER 1992

SITE NUMBER 335603117000601

LOCAL NUMBER 003S001W05Q01S

About 1.5 miles west of Beaumont, north of Highway 60, and west of Interstate 10. Drilled domestic well. Diameter 16 inches, depth 1,300 feet, perforated 384-388, 454-494 feet. Altitude of land-surface datum 2,525 feet. Waterlevel records available 1926-29, 1937-41, 1953-57, 1960-81, 1986 to current year.

WATER LEVELS IN FEET BELOW LAND-SURFACE DATUM

$$
\begin{array}{lll} 
& \text { WATER } & \\
\text { DATE } & \text { WEVEL MS } & \text { DATE } \\
\text { LEVEL MS }
\end{array}
$$

NOV $26,1991131.77 \mathrm{~S}$ JUN $12,1992134.08 \mathrm{SR}$

PERIOD OF RECORD HIGHEST 90.6 JUN 25, 1941 LOWEST 132.3 OCT 26,1955

SITE NUMBER 334717117124401

LOCAL NUMBER 004S003W29Q01S

In Perris, north of intersection of Wilson and San Jacinto Roads. Drilled unused water-table well. Diameter 14 inches, depth 1,624 feet. Altitude of land-surface datum 1,417 feet. Most measurements provided by Riverside County Flood Control and Water Conservation District; measured periodically by U.S. Geological Survey. Water-level records available $1974-77,1979$ to current year.

WATER LEVELS IN FEET BELOW LAND-SURFACE DATUM

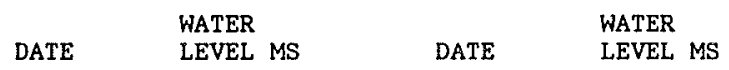

$\operatorname{OCT} 23,1991123.40 \mathrm{~S} \quad$ JUN $15,1992118.64 \mathrm{~S}$

PERIOD OF RECORD HIGHEST 118.64 JUN 15, 1992 LOWEST 209.60 ○CT 16,1977

SITE NUMBER 333712117080901

LOCAL NUMBER 005S002W19N01S

About 3 miles east of Sun City, east of Briggs Road, and north of Grand Avenue. Drilled domestic water-table well. Diameter 10 inches, depth 358 feet, perforated $96-312$ feet. Altitude of land-surface datum 1,459 feet. Water-level records available 1987 to current year.

WATER LEVELS IN FEET BELOW LAND-SURFACE DATUM

$\begin{array}{lll} & \text { WATER } & \\ \text { DATE } & \text { WEVEL MS } & \text { DATE } \\ \text { LEVEL MS }\end{array}$

MAR 18, $1992 \quad 37.79$ S $\quad$ SEP 03, $199242.47 \quad \mathrm{~S}$

PERIOD OF RECORD HIGHEST 29.40 SEP 18,1987 LOWEST 42.47 SEP 03, 1992

SITE NUMBER 334320117042102

LOCAL NUMBER 005SO02W22G02S

North of Winchester, east of intersection of Winchester and Stowe Roads. Drilled domestic water-table well. Diameter 14 inches, depth 118.6 feet, perforated 48-104 feet. Altitude of land-surface datum 1,506 feet. Water-level records available 1951, 1987 to current year.

WATER LEVELS IN FEET BELOW LAND-SURFACE DATUM

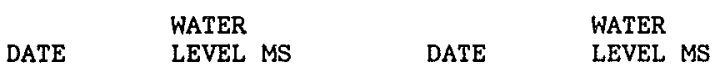

MAR 18, $199243.77 \mathrm{~S} \quad$ SEP 03, $199258.34 \mathrm{~S}$

PERIOD OF RECORD HIGHEST 31.25 SEP 18, 1987 LOWEST 58.34 SEP 03, 1992 
WELL DESCRIPTIONS AND WATER-LEVEL MEASUREMENTS

WATER YEAR OCTOBER 1991 TO SEPTEMBER 1992

SITE NUMBER 332747117061101

LOCAL NUMBER 008S002W20J01S

Southeast of Temecula in Wolf Valley, adjacent to Wolf Valley Road, about 1,670 feet east of Pala-Temecula Highway. Drilled observation well (piezometer). Diameter 2 inches, depth 575 feet. Altitude of land-surface datum $1,076.23$ feet. Water-level records avallable 1990 to current year.

WATER LEVELS IN FEET BELOW LAND-SURFACE DATUM

\begin{tabular}{|c|c|c|c|c|c|c|c|c|c|c|c|c|c|c|c|}
\hline DATE & $\begin{array}{l}\text { WATER } \\
\text { LEVEL }\end{array}$ & MS & & DATE & $\begin{array}{l}\text { WATER } \\
\text { LEVEL }\end{array}$ & MS & & DATE & $\begin{array}{l}\text { WATER } \\
\text { LEVEL }\end{array}$ & MS & & DATE & & $\begin{array}{l}\text { WATER } \\
\text { LEVEL }\end{array}$ & $\mathrm{MS}$ \\
\hline $\begin{array}{ll}08, & 1991 \\
26 & \\
12 & \\
10, & 1992\end{array}$ & $\begin{array}{l}70.15 \\
70.17 \\
70.28 \\
70.03\end{array}$ & $\begin{array}{l}\mathrm{V} \\
\mathrm{V} \\
\mathrm{V} \\
\mathrm{V}\end{array}$ & $\begin{array}{l}\text { JAN } \\
\text { FEB } \\
\text { MAR }\end{array}$ & $\begin{array}{l}27 \\
07 \\
28 \\
13\end{array}$ & $\begin{array}{l}70.01 \\
69.81 \\
68.56 \\
69.30\end{array}$ & $\begin{array}{l}V \\
V \\
V \\
V\end{array}$ & $\begin{array}{l}\text { APR } \\
\text { MAY } \\
\text { JUN }\end{array}$ & $\begin{array}{ll}10, & 1992 \\
01 & \\
28 & \\
19 & \end{array}$ & $\begin{array}{l}68.90 \\
68.87 \\
68.84 \\
69.05\end{array}$ & $\begin{array}{l}\mathrm{V} \\
\mathrm{V} \\
\mathrm{V} \\
\mathrm{V}\end{array}$ & $\begin{array}{l}\text { JUL } \\
\text { SEP }\end{array}$ & $\begin{array}{l}15 \\
23 \\
01 \\
17\end{array}$ & 1992 & $\begin{array}{l}69.44 \\
69.41 \\
69.77 \\
69.86\end{array}$ & $\begin{array}{l}\mathrm{V} \\
\mathrm{V} \\
\mathrm{V} \\
\mathrm{V}\end{array}$ \\
\hline
\end{tabular}

PERIOD OF RECORD HIGHEST 68.56 FEB 28, 1992 LOWEST 73.93 MAR 15, 1990

SITE NUMBER 332747117061102

LOCAL NUMBER O08S002W20J02S

Southeast of Temecula in Wolf Valley, adjacent to Wolf Valley Road, about 1,670 feet east of Pala-Temecula Highway. Drilled observation well (piezometer). Diameter 2 inches, depth 180 feet. Altitude of land-surface datum 1,076.23 feet. Water-level records available 1990 to current year.

WATER LEVELS IN FEET BELOW LAND-SURFACE DATUM

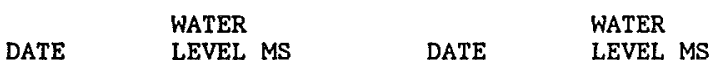

NoV $08,199160.49$

DEC $12 \quad 60.67 \quad \mathrm{~V}$

JAN $10,199260.68 \mathrm{~V}$

PERIOD OF RECORD

HIGHEST
DATE WATER

APR 10, 1992

MAY 01

28

JUN 19
$60.47 \mathrm{~V}$

$60.39 \mathrm{~V}$

$60.33 \mathrm{~V}$

$60.33 \mathrm{~V}$

LOWEST 62.47 MAR 05, 1990

\begin{tabular}{|c|c|c|c|}
\hline & & WATER & \\
\hline & DATE & & \\
\hline JUL & $\begin{array}{l}15,1992 \\
23\end{array}$ & $\begin{array}{l}60.42 \\
60.46\end{array}$ & \\
\hline SEP & 01 & 60.61 & \\
\hline & 17 & 60.67 & \\
\hline
\end{tabular}

WATER

$60.46 \mathrm{~V}$ $60.67 \mathrm{~V}$

SITE NUMBER 332659117032102

LOCAL NUMBER 008S002W26K02S

On Pechanga Indian Reservation, southeast of Temecula, about 2 miles east of Pala-Temecula Highway. Drilled unused well. Diameter 8.6 inches, depth 120 feet. Altitude of land-surface datum 1,330 feet. Water-1evel records available 1989 to current year.

WATER LEVELS IN FEET BELOW LAND-SURFACE DATUM

$$
\begin{array}{lll} 
& \text { WATER } & \\
\text { DATE } & \text { WATER } \\
\text { LEVEL MS } & \text { LEVEL MS }
\end{array}
$$

MAY $04,199267.92 \mathrm{~S}$ JUN $12,199269,89 \mathrm{~S}$

PERIOD OF RECORD HIGHEST 67.64 JAN 23, 1989 LOWEST 70.80 NOV 14, 1990 
SITE NUMBER 332657117054601

LOCAL NUMBER 008S002W28M01S

Southeast of Temecula in Wolf Valley, about 0.1 mile southwest of Pala-Temecula Highway. Drilled irrigation water-table well. Diameter unknown, depth measured 269.5 feet. Altitude of land-surface datum 1,126 feet. Records provided by California Department of Water Resources in 1953-54. Water-1evel records available 1953-54, $1958-68,1971-73,1975$ to current year.

WATER LEVELS IN FEET BELOW LAND-SURFACE DATUM

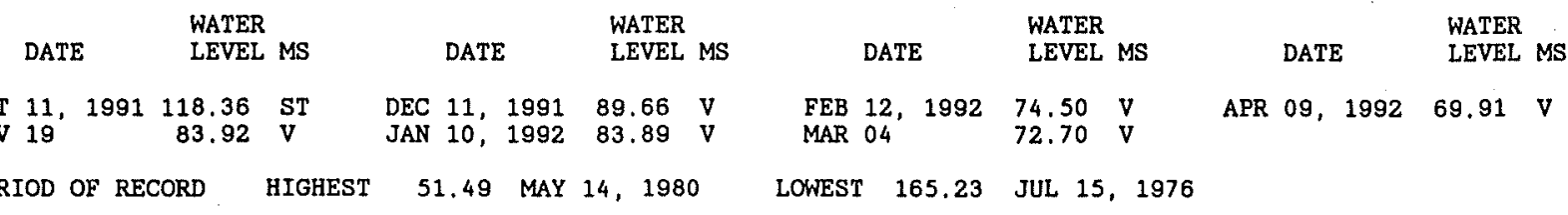

SITE NUMBER 332657117055101

LOCAL NUMBER 008S002W28M02S

Southeast of Temecula and west of Pala-Temecula Highway. Drilled unused water-table well. Diameter 8 inches, depth 98.5 feet. Altitude of land-surface datum 1,120 feet. Water-level records available 1958-61, 1967, 1975 to current year.

WATER LEVELS IN FEET BELOW LAND-SURFACE DATUM

$$
\begin{array}{llllll} 
& \text { WATER } & & & \text { WATER } & \text { WATER } \\
\text { DATE } & \text { LEVEL MS } & \text { DATE } & \text { WATER MS } & \text { DATE } & \text { LEVEL MS }
\end{array}
$$

$\begin{array}{llllllllllllllllll}\text { OCT } 11,1991 & 50.14 & \mathrm{~S} & \text { DEC } 11,1991 & 50.29 & \mathrm{~V} & \text { FEB } 12, & 1992 & 50.39 & \mathrm{~V} & & \text { APR } & 09, & 1992 & 49.49 & \mathrm{~V} \\ \text { NOV } & 19 & & 49.99 & \mathrm{~S} & \text { JAN } 10,1992 & 50.60 & \mathrm{~V} & \text { MAR } & 04 & & 50.13 & \mathrm{~V} & & & & \end{array}$

PERIOD OF RECORD HIGHEST 27.06 APR 08, 1980 LOWEST 57.00 NOV 08, 1990

SITE NUMBER 332705117054901

LOCAL NUMBER 008S002W28M03S

Southeast of Temecula and west of Pala-Temecula Highway. Drilled irrigation water-table well. Diameter 16 inches, depth 600 feet, perforated 200-600 feet. Altitude of land-surface datum 1,130 feet. Historically this well recovers very slowly to the regional static water level after pumping. Water levels with a ' $Z$ ' site status represent this condition. Water-level records available 1976 to current year.

WATER LEVELS IN FEET BELOW LAND-SURFACE DATUM

$$
\begin{array}{lllll} 
& & \text { WATER } & \text { WATER } \\
\text { DATE } & \text { WATER } & \text { DATE } & \text { LEVEL MS } & \text { DATE }
\end{array}
$$

OCT 11, $1991 \quad 99.82$ SR $\quad$ DEC $11,1991 \quad 76.00 \quad \mathrm{~V} \quad$ MAR 04, $1992 \quad 67.12 \quad \mathrm{~V}$

$\begin{array}{lllllllllll} & \text { NOV } 19 & 71.62 & \mathrm{~V} & \text { FEB } 12,1992 & 68.28 & \mathrm{~V} & \text { APR } 09 & 65.78 \mathrm{~V}\end{array}$

PERIOD OF RECORD HIGHEST 49.33 MAY 14, 1980 LOWEST 135.96 NOV 08, 1990

SITE NUMBER 332649117053701

LOCAL NUMBER OOBS002W28P01S

Southeast of Temecula in Wolf Valley, about 0.1 mile west of Pala-Temecula Highway. Drilled domestic water-table well. Diameter 6 inches, depth 138 feet. Altitude of land-surface datum 1,150 feet. Water-level records available 1972 to current year.

WATER LEVELS IN FEET BELOW LAND-SURFACE DATUM

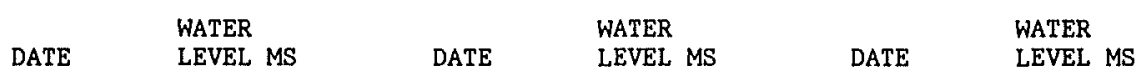

OCT 11, $1991 \quad 27.92 \quad \mathrm{~S} \quad$ DEC 11, $199127.98 \quad \mathrm{~V} \quad$ MAR 04, $1992 \quad 25.91 \mathrm{~V}$

$\begin{array}{lllllllllll}\text { NOV } 19 & 27.83 & \text { SR } & \text { FEB } 12,1992 & 27.48 & \text { V } & \text { APR } 09 & & & & \end{array}$

PERIOD OF RECORD HIGHEST 6.77 FEB 29, 1980 LOWEST 32.40 NOV 07, 1975 
WELL DESCRIPTIONS AND WATER-LEVEL MEASUREMENTS

WATER YEAR OCTOBER 1991 TO SEPTEMBER 1992

SITE NUMBER 332655117051801

LOCAL NUMBER 008SO02W28Q03S

On Pechanga Indian Reservation, southeast of Temecula, about 0.3 mile east of Pala-Temecula Highway. Drilled unused well. Diameter 8.5 inches, depth measured 94.78 feet in 1991. Alt1tude of land-surface datum 1,170 feet. Water-lovel records available 1972 to current year.

WATER LEVELS IN FEET BELOW LAND-SURFACE DATUM

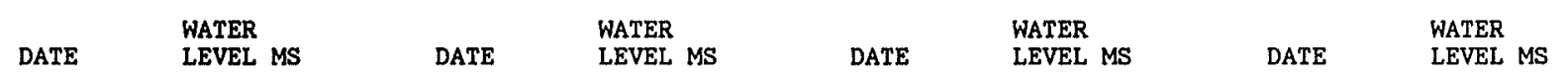

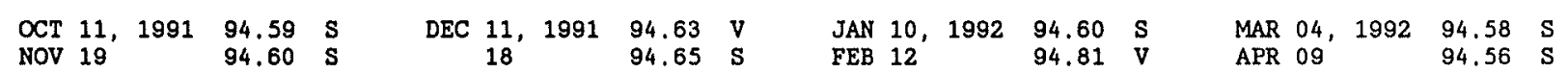

PERIOD OF RECORD HIGHEST 54.47 MAY 14, 1980 LOWEST 95.56 MAR 30, 1973

SITE NUMBER 332654117051701

LOCAL NUMBER 008S002W28Q05S

On Pechanga Indian Reservation, southeast of Temecula. Drilled unused we11. Diameter 8 inches, depth 390 feet, perforated 249-256, 276-285, 292-304, 308-344, 349-360, 386-370 feet. Water-level records available 1975 to current year.

WATER LEVELS IN FEET BELOW LAND-SURFACE DATUM

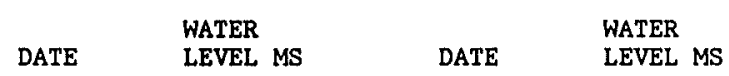

MAY $04,199284.79 \mathrm{~S} \quad$ SEP 22, $199283.93 \mathrm{~S}$

PERIOD OF RECORD HIGHEST 0.00 OCT 19, 1976 LOWEST 123.83 MAR 27, 1975

SITE NUMBER 332653117050301

LOCAL NUMBER 008S002W28R01S

On Pechanga Indian Reservation, southeast of Temecula. Drilled unused water-table well in sand and gravel. Diameter 12 inches, depth 1,000 feet, perforated 130-220, 250-350, 400-710, 750-780, 830-870, 930-940, 975-1,000 feet. Aititude of land-surface datum 1,190 feet. "Z" site status is water level affected by falling water. Water-level records available 1973 to current year.

WATER LEVELS IN FEET BELOW LAND-SURFACE DATUM

\begin{tabular}{|c|c|c|c|c|c|c|}
\hline DATE & $\begin{array}{l}\text { WATER } \\
\text { LEVEL MS }\end{array}$ & DATE & $\begin{array}{l}\text { WATER } \\
\text { LEVEL MS }\end{array}$ & DATE & $\begin{array}{l}\text { WATER } \\
\text { LEVEL MS }\end{array}$ & $\begin{array}{l}\text { WATER } \\
\text { LEVEL MS }\end{array}$ \\
\hline
\end{tabular}

$\begin{array}{lrrrrrrrrr}\text { OCT } 11,1991 & 145.85 & \mathrm{~S} & \text { DEC } 11,1991 & 109.55 & \mathrm{~V} & \text { FEB } 12,1992 & 100.25 & \mathrm{~V} \\ \text { NOV 19 } & 66.76 & \mathrm{~V} & \text { JAN } 10,1992 & 107.58 & \mathrm{~V} & \text { MAR } 04 & 155.38 & \text { VZ }\end{array}$

PERIOD OF RECORD HIGHEST 46.86 APR 08, 1980 LOWEST 168.55 AUG 07, 1991

SITE NUMBER 332724117061101

LOCAL NUMBER 008S002W29A01S

On Pechanga Indian Reservation, southeast of Temecula, southwest of Pala-Temecula Highway. Domestic well. Diameter 8 inches, depth 348 feet. Altitude of land-surface datum 1,090 feet. Water-level records avallable 1980-81, 1983 to current year.

WATER LEVELS IN FEET BELOW LAND-SURFACE DATUM

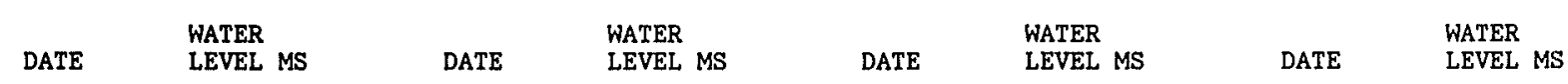

$\begin{array}{lllllllllllllllllll}\text { OCT } 11,1991 & & \text { P } & \text { DEC 11, } 1991 & 64.27 & \text { V } & \text { FEB } 12, & 1992 & 63.75 & \text { V } & \text { APR } & 09, & 1992 & 60.79 & \text { S } \\ \text { NOV } 19 & & 64.63 & \text { SR } & \text { JAN } 10, & 1992 & 63.31 & \text { V } & \text { MAR } 04 & & 62.77 & \text { V } & & & & \end{array}$

PERIOD OF RECORD HIGHEST 21.21 FEB 29, 1980 LOWEST 65.90 SEP 06, NOV 08, 1990 
WELL DESCRIPTIONS AND WATER-LEVEL MEASUREMENTS

WATER YEAR OCTOBER 1991 TO SEPTEMBER 1992

SITE NUMBER 332732117062301

LOCAL NUMBER 008S002W29B02S

On Kelsey Tract of Pechanga Indian Reservation, about 250 feet southwest of intersection of Pala-Temecula Highway and Wolf Valley Road. Drilled observation well (piezometer). Diameter 2 inches, depth 785 feet. Altitude of land-surface daturn 1,074.08 feet. Water-level records available 1990 to current year.

WATER LEVELS IN FEET BELOW LAND-SURFACE DATUM

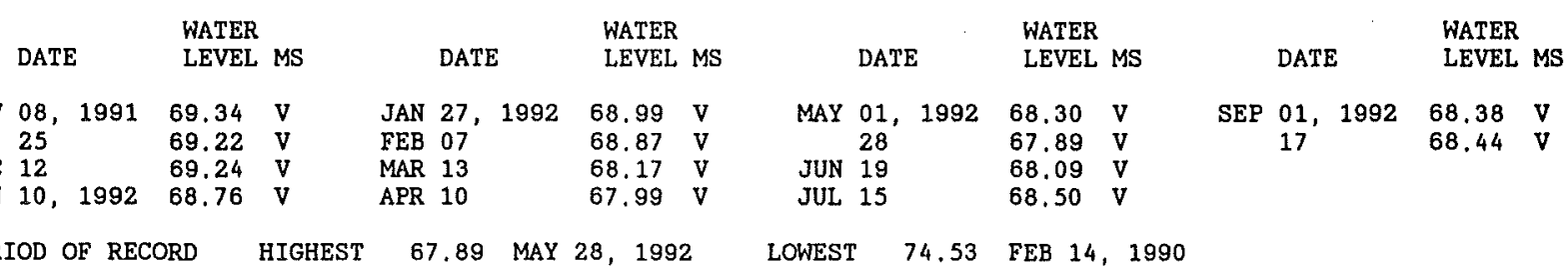

SITE NUMBER 332732117062302

LOCAL NUMBER 008S002W29B03S

On Kelsey Tract of Pechanga Indian Reservation, about 250 feet soutbwest of intersection of Pala-Temecula Highway and Wolf Valley Road. Drilled observation well (piezometer). Diameter 2 inches, depth 493 feet. Altitude of land-surface datum $1,074.08$ feet. Water-level records available 1990 to current year.

WATER LEVELS IN FEET BELOW LAND-SURFACE DATUM

\begin{tabular}{|c|c|c|c|c|c|c|c|c|c|c|c|c|c|c|c|}
\hline & DATE & $\begin{array}{l}\text { WATER } \\
\text { LEVEL }\end{array}$ & MS & & DATE & $\begin{array}{l}\text { WATER } \\
\text { LEVEL }\end{array}$ & MS & & DATE & $\begin{array}{l}\text { WATER } \\
\text { LEVEL }\end{array}$ & MS & & DATE & $\begin{array}{l}\text { WATER } \\
\text { LEVEL }\end{array}$ & MS \\
\hline NOV & $\begin{array}{ll}08,1991 \\
25 & \\
26 & \end{array}$ & $\begin{array}{l}70.13 \\
69.99 \\
70.04\end{array}$ & $\begin{array}{l}\mathrm{V} \\
\mathrm{V} \\
\mathrm{V}\end{array}$ & $\begin{array}{l}\text { JAN } \\
\text { FEB }\end{array}$ & $\begin{array}{ll}10,1992 \\
27 & \\
07 & \end{array}$ & $\begin{array}{l}69.62 \\
69.79 \\
69.66\end{array}$ & $\begin{array}{l}\text { V } \\
\text { v } \\
\text { v }\end{array}$ & $\begin{array}{l}\text { MAR } \\
\text { APR } \\
\text { MAY }\end{array}$ & $\begin{array}{ll}13, & 1992 \\
10 & \\
01 & \end{array}$ & $\begin{array}{l}69.01 \\
68.82 \\
68.93\end{array}$ & $\begin{array}{l}\mathrm{V} \\
\mathrm{V} \\
\mathrm{V}\end{array}$ & $\begin{array}{l}\text { JUN } \\
\text { JUL } \\
\text { SEP }\end{array}$ & $\begin{array}{ll}19, & 1992 \\
15 & \\
01 & \end{array}$ & $\begin{array}{l}68.71 \\
69.02 \\
69.27\end{array}$ & $\begin{array}{l}\mathrm{V} \\
\mathrm{v} \\
\mathrm{v}\end{array}$ \\
\hline DEC & 12 & 70.00 & v & & 28 & 69.43 & V & & 28 & 68.54 & V & & 17 & 69.11 & V \\
\hline
\end{tabular}

PERIOD OF RECORD HIGHEST 68.54 MAY 28, 1992 LOWEST 75.62 FEB 14, 1990

SITE NUMBER 332732117062303

LOCAL NUMBER 008S002W29B04S

On Kelsey Tract of Pechanga Indian Reservation, about 250 feet southwest of intersection of Pala-Temecula Highway and Wolf Valley Road. Drilled observation well (plezometer). Diameter 2 inches, depth 80 feet. Altitude of land-surface datum $1,074.08$ feet. Water-level records available 1990 to current year.

WATER LEVELS IN FEET BELOW LAND-SURFACE DATUM

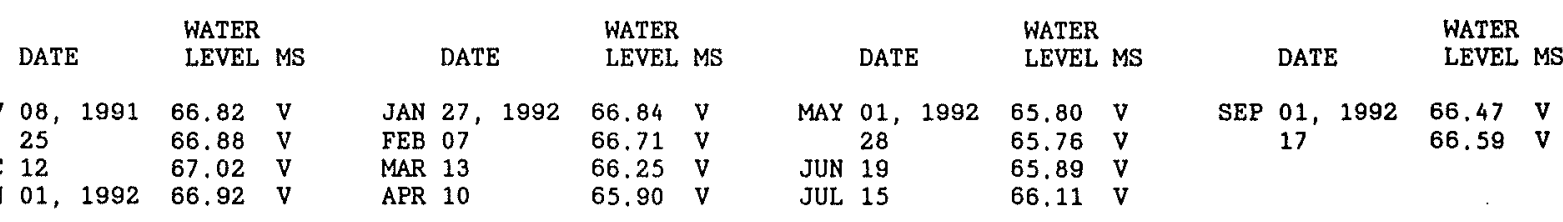

PERIOD OF RECORD HIGHEST 65.76 MAY 28, 1992 LOWEST 70.11 MAR 05, 1990 
WELL DESCRIPTIONS AND WATER-LEVEL MEASUREMENTS

WATER YEAR OCTOBER 1991 TO SEPTEMBER 1992

SITE NUMBER 332732117062304

LOCAL NUMBER 008S002W29B05S

On Kelsey Tract of Pechanga Indian Reservation, about 250 feet southwest of intersection of Pala-Temeoula Highway and Wolf Valley Road. Drilled observation well (piezometer). Diameter 2 inches, depth 400 feet. Altitude of land-surface datum 1,074.00 feet. Water-level records available 1990 to current year.

WATER LEVELS IN FEET BELOW LAND-SURFACE DATUM

\begin{tabular}{|c|c|c|c|c|c|c|c|c|c|c|c|c|c|c|}
\hline DATE & $\begin{array}{l}\text { WATER } \\
\text { LEVEL }\end{array}$ & MS & & DATE & $\begin{array}{l}\text { WATER } \\
\text { LEVEL }\end{array}$ & MS & & DATE & $\begin{array}{l}\text { WATER } \\
\text { LEVEL }\end{array}$ & MS & & DATE & $\begin{array}{l}\text { WATER } \\
\text { LEVEL }\end{array}$ & MS \\
\hline $\begin{array}{ll}08, & 1991 \\
25 & \\
26 & \\
12 & \end{array}$ & $\begin{array}{l}68.99 \\
68.90 \\
68.92 \\
68.99\end{array}$ & $\begin{array}{l}\mathrm{v} \\
\mathrm{v} \\
\mathrm{v} \\
\mathrm{v}\end{array}$ & $\begin{array}{l}\text { JAN } \\
\text { FEB }\end{array}$ & $\begin{array}{ll}10, & 1992 \\
27 & \\
07 & \\
28 & \end{array}$ & $\begin{array}{l}68.68 \\
68.78 \\
68.63 \\
68.38\end{array}$ & $\begin{array}{l}V \\
V \\
v \\
V\end{array}$ & $\begin{array}{l}\text { MAR } \\
\text { APR } \\
\text { MAY } \\
\text { JUN }\end{array}$ & $\begin{array}{ll}13, & 1992 \\
10 & \\
01 & \\
19 & \end{array}$ & $\begin{array}{l}68.02 \\
67.74 \\
67.50 \\
67.64\end{array}$ & $\begin{array}{l}\text { V } \\
\text { V } \\
\text { V } \\
\text { V }\end{array}$ & $\begin{array}{l}\text { JUL } \\
\text { SEP }\end{array}$ & $\begin{array}{l}15,1992 \\
01 \\
17\end{array}$ & $\begin{array}{l}68.13 \\
68.21 \\
68.28\end{array}$ & $\begin{array}{l}\text { V } \\
\text { V } \\
\text { V }\end{array}$ \\
\hline
\end{tabular}

PERIOD OF RECORD HIGHEST 67.50 MAY 01, 1992 LOWEST 74.22 MAR 01, 1990

SITE NUMBER 332732117062305

LOCAL NUMBER D08S002W29B06S

On Kelsey Tract of Pechanga Indian Reservation, about 250 feet southwest of intersection of Pala-Temecula Highway and Wolf Valley Road. Drilled observation well (piezometer). Diameter 2 inches, depth 174 feet. Altitude of land-surface datum 1,074.00 feet. Water-level records available 1990 to current year.

WATER LEVELS IN FEET BELOW LAND-SURFACE DATUM

\begin{tabular}{|c|c|c|c|c|c|c|c|c|c|c|c|c|c|c|}
\hline DATE & $\begin{array}{l}\text { WATER } \\
\text { LEVEL }\end{array}$ & MS & & DATE & $\begin{array}{l}\text { WATER } \\
\text { LEVEL }\end{array}$ & MS & & DATE & $\begin{array}{l}\text { WATER } \\
\text { LEVEL }\end{array}$ & MS & & DATE & $\begin{array}{l}\text { WATER } \\
\text { LEVEL }\end{array}$ & MS \\
\hline $\begin{array}{ll}08, & 1991 \\
25 & \\
26 & \\
12 & \end{array}$ & $\begin{array}{l}67.69 \\
67.72 \\
67.70 \\
67.85\end{array}$ & $\begin{array}{l}V \\
V \\
V \\
V\end{array}$ & $\begin{array}{l}\text { JAN } \\
\text { FEB }\end{array}$ & $\begin{array}{ll}10, & 1992 \\
27 & \\
07 & \\
28 & \end{array}$ & $\begin{array}{l}67.59 \\
67.56 \\
67.47 \\
67.13\end{array}$ & $\begin{array}{l}\text { V } \\
v \\
v \\
v\end{array}$ & $\begin{array}{l}\text { MAR } \\
\text { APR } \\
\text { MAY }\end{array}$ & $\begin{array}{ll}13, & 1992 \\
10 & \\
01 & \\
28 & \end{array}$ & $\begin{array}{l}66.88 \\
66.52 \\
66.48 \\
66.40\end{array}$ & $\begin{array}{l}\text { V } \\
V \\
V \\
V\end{array}$ & $\begin{array}{l}\text { JUN } \\
\text { SEP }\end{array}$ & $\begin{array}{ll}19, & 1992 \\
01 & \\
17 & \end{array}$ & $\begin{array}{l}66.58 \\
67.27 \\
67.37\end{array}$ & $\begin{array}{l}\mathrm{V} \\
\mathrm{V} \\
\mathrm{V}\end{array}$ \\
\hline
\end{tabular}

PERIOD OF RECORD HIGHEST 66.40 MAY 28, 1992 LOWEST 71.40 MAR 01, 1990

SITE NUMBER 332728117062701

LOCAL NUMBER 008S002W29B07S

On Kelsey Tract of Pechanga Indian Reservation, about 250 feet southwest of intersection of Pala-Temecula Highway and Wolf Valley Road. Drilled observation well (piezometer). Diameter 2 inches, depth 510 feet. Altitude of land-surface datum 1,075.98 feet. Water-level records available 1990 to current year.

WATER LEVELS IN FEET BELOW LAND-SURFACE DATUM

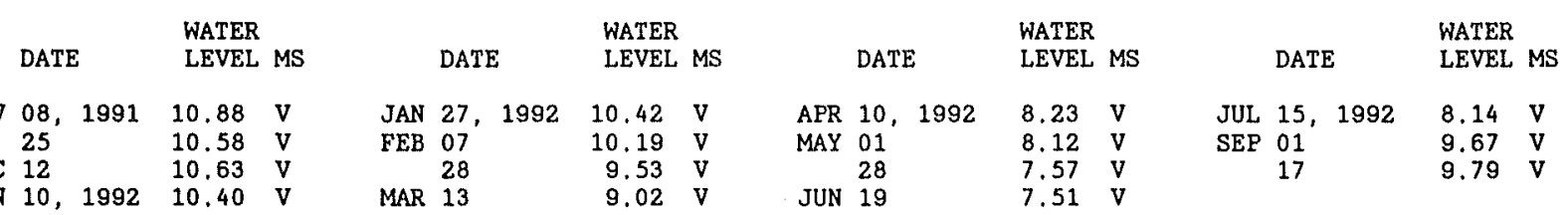

PERIOD OF RECORD HIGHEST 7.51 JUN 19, 1992 LOWEST 20.74 DEC 10, 1990 
WELL DESCRIPTIONS AND WATER-LEVEL MEASUREMENTS

WATER YEAR OCTOBER 1991 TO SEPTEMBER 1992

SITE NUMBER 332728117062702

LOCAL NUMBER 008S002W29B08S

On Kelsey Tract of Pechanga Indian Reservation, about 250 feet southwest of intersection of Pala-Temecula Highway and Wolf Valley Road. Drilled observation well (piezometer). Diameter 2 inches, depth 332 feet. Altitude of land-surface datum 1,075.98 feet. Water-level records available 1990 to current year.

WATER LEVELS IN FEET BELOW LAND-SURFACE DATUM

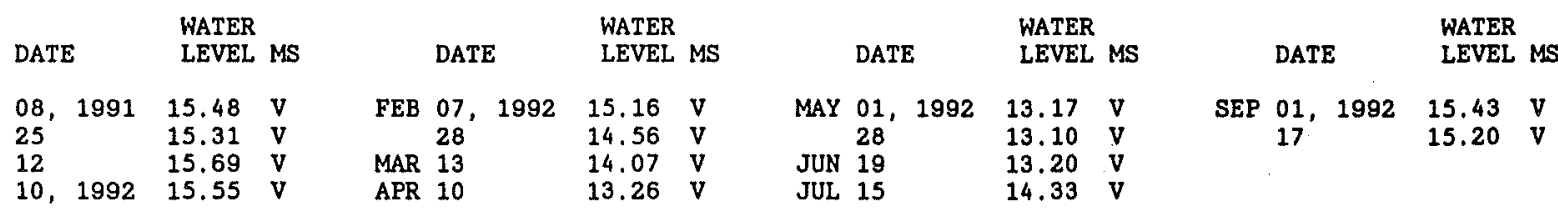

PERIOD OF RECORD HIGHEST 13.10 MAY 28, 1992 LOWEST 21.85 DEC 10, 1990

SITE NUMBER 332728117062703

LOCAL NUMBER 008S002W29B09S

On Kelsey Tract of Pechanga Indian Reservation, about 250 feet southwest of intersection of Pala-Temecula Highway and Wolf Valley Road. Drilled observation well (piezometer). Diameter 2 inches, depth 113 feet. Altitude of land-surface datum 1,075.93 feet. Water-level records available 1990 to current year.

WATER LEVELS IN FEET BELOW LAND-SURFACE DATUM

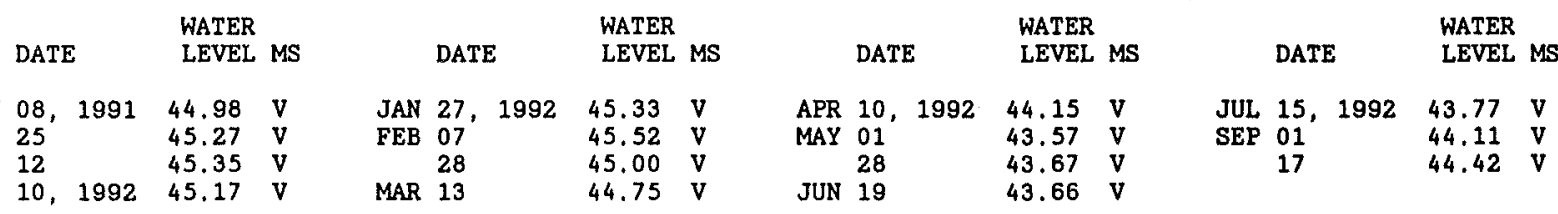

PERIOD OF RECORD HIGHEST 43.57 MAY 01, 1992 LOWEST 46.45 DEC 14,1990

SITE NUMBER 332729117065101

LOCAL NUMBER 008S002W29D01S

Southeast of Temecula in Wolf Valley, about 0.4 mile west of Pala-Temecula Highway and about $0.3 \mathrm{mile}$ north of Via Eduardo. Drilled unused well. Diameter 8 inches, depth 90 feet. Altitude of land-surface datum 1,065 feet. Water-level records avallable 1967, 1989 to current year.

WATER LEVELS IN FEET BELOW LAND-SURFACE DATUM

$\begin{array}{ll} & \text { WATER } \\ \text { DATE } & \text { LEVEL MS }\end{array}$

MAY $04,199214.65 \mathrm{~s}$

PERIOD OF RECORD HIGHEST 13.00 SEP 01, $1967 \quad$ LOWEST 29.24 NOV 29, 1990 
SITE NUMBER 332722117064701 LOCAL NUMBER O08S002W29D02S

$$
85 / 2 w-29 D 2
$$

Southeast of Temecula in Wolf Valley. Drilled domestic woll. Diameter 5 inches, depth 265 feet. Altitude of land-surface datum 1,090 feet. Water-level records avallable 1987 to current year.

WATER LEVELS IN FEET BELOW LAND-SURFACE DATUM

$\begin{array}{lllllll} & \text { WATER } & & \text { WATER } & & \text { WATER } & \text { WATER } \\ \text { DATE } & \text { LEVEL MS } & \text { DATE } & \text { LEVEL MS } & \text { DATE } & \text { LEVEL MS } & \text { DATE }\end{array}$

$\begin{array}{lllllllllllllll}\text { OCT } 11,1991 & 12.83 & \mathrm{~S} & \text { DEC } 11,1991 & 13.45 & \mathrm{~V} & \text { FEB } 12,1992 & 13.30 & \mathrm{~V} & \text { APR } 09,1992 & 9.18 & \mathrm{~S} \\ \text { NOV } 19 & & 13.38 & \mathrm{~S} & \text { JAN } 10,1992 & 13.37 & \mathrm{~S} & \text { MAR } & 04 & & 11.65 & \mathrm{~V} & & \end{array}$

PERIOD OF RECORD HIGHEST 9.18 APR 09, 1992 LOWEST 16.84 NOV 08, 1990

\section{SITE NUMBER 332719117061501}

LOCAL NUMBER 008S002W29G01S

On Pechanga Indian Reservation, southeast of Temecula. Drilled unused water-table well. Diameter 12 inches, depth measured 176 feet in 1951, 159.1 feet in 1972. Altitude of land-surface datum 1,091.1 feet. Recorder instailed October 1987. Water-level records available 1925-28, 1934-37, 1940, 1951-54, 1956, 1958-68, 1971 to current year.

WATER LEVELS IN FEET BELOW LAND-SURFACE DATUM

\begin{tabular}{|c|c|c|c|c|c|c|}
\hline DATE & $\begin{array}{l}\text { WATER } \\
\text { LEVEL MS }\end{array}$ & DATE & $\begin{array}{l}\text { WATER } \\
\text { LEVEL MS }\end{array}$ & DATE & $\begin{array}{l}\text { WATER } \\
\text { LEVEL MS }\end{array}$ & $\begin{array}{l}\text { WATER } \\
\text { LEVEL MS }\end{array}$ \\
\hline
\end{tabular}

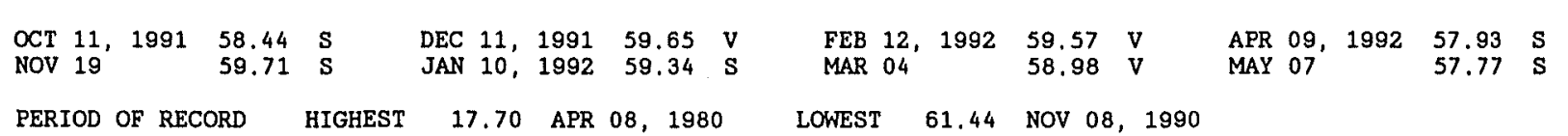

SITE NUMBER 332703117060401

LOCAL NUMBER 008S002W29J02S

On Pechanga Indian Reservation, southeast of Temecula. Drilled domestic water-table well. Diameter 8 inches, depth 150 feet. Altitude of land-surface datum 1,110 feet. Water-level records available 1967,1972 to current year.

WATER LEVELS IN FEET BELOW LAND-SURFACE DATUM

\begin{tabular}{|c|c|c|c|c|c|c|c|}
\hline DATE & $\begin{array}{l}\text { WATER } \\
\text { LEVEL MS }\end{array}$ & DATE & $\begin{array}{l}\text { WATER } \\
\text { LEVEL MS }\end{array}$ & DATE & $\begin{array}{l}\text { WATER } \\
\text { LEVEL MS }\end{array}$ & DATE & $\begin{array}{l}\text { WATER } \\
\text { LEVEL MS }\end{array}$ \\
\hline
\end{tabular}

$\begin{array}{llllllllllllllll}\text { OCT } 11,1991 & 34.37 & \text { SR } & \text { DEC 11, } 1991 & 32.36 & \mathrm{~V} & \text { FEB } 12,1992 & 32.33 & \mathrm{~V} & \text { APR } 09,1992 & 27.44 & \mathrm{~S} \\ \text { NOV } 19 & & 32.46 & \mathrm{~S} & \text { JAN } 10,1992 & 32.40 & \mathrm{~S} & \text { MAR } & 04 & & 30.80 & \mathrm{~V} & & \end{array}$

PERIOD OF RECORD HIGHEST 12.33 APR 08, 1980 LOWEST 39.52 OCT 05, 1990

SITE NUMBER 332703117061701

\section{LOCAL NUMBER 008S002W29K01S}

On Pechanga Indian Reservation, southeast of Temecula. Unused well. Diameter 8 inches, depth 39 feet. Altitude of land-surface datum 1,110 feet. Recorder installed October 1987-91. Water-level records available 1987 to current year.

WATER LEVELS IN FEET BELOW LAND-SURFACE DATUM

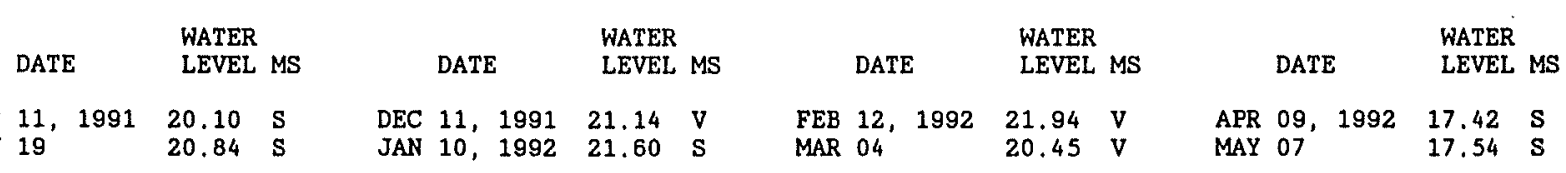

PERIOD OF RECORD HIGHEST 16.60 MAY 08, 1991 LOWEST 30.40 FEB 08, 1991 
WELL DESCRIPTIONS AND WATER-LEVEL MEASUREMENTS

WATER YEAR OCTOBER 1991 TO SEPTEMBER 1992

SITE NUMBER 332623117050801

LOCAL NUMBER 008SO02W33H02S

Southeast of Temecula at southern end of Wolf Valley, about 0.3 mile east of Pala-Temecula Highway and about 80 feet north of Los Encinos Drive. Drilled domestic well. Diameter 8 inches, depth 200 feet. Altitude of land-surface datum 1,190 feet. Water-level records avallable 1989 to current year.

WATER LEVELS IN FEET BELOW LAND-SURFACE DATUM

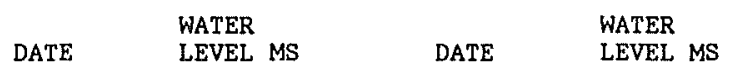

MAY 13, 199270.86 SR JUN 22, $199268.98 \mathrm{~S}$

PERIOD OF RECORD HIGHEST 64.93 OCT 12,1989 LOWEST 68.98 JUN 22, 1992

SITE NUMBER 332604117051001

LOCAL NUMBER 008S002W33J02S

Southeast of Temecula, south of Wolf Valley, about 0.1 mile east of Pala-Temecula Highway. Drilled unused well. Diameter 5 inches, depth 290 feet. Altitude of land-surface datum 1,220 feet. Water-level records 1989 to current year.

WATER LEVELS IN FEET BELOW LAND-SURFACE DATUM

$$
\text { DATE WATER }
$$

MAY $04,199226.38 \mathrm{~S}$

PERTOD OF RECORD HIGHEST 26.38 MAY 04, 1992 LOWEST 32.38 DEC 14, 1990

SITE NUMBER 332605117051601

LOCAL NUMBER 008S002W33J03S

Southeast of Temecula, south of Wolf Valley, about 120 feet east of Pala-Temecula Highway, Drilled well, used occasionally to fill pond. Diameter 6 inches, depth 250 feet. Altitude of land-surface datum 1,190 feet. Waterlevel. records available 1989 to current year.

WATER LEVELS IN FEET BELOW LAND-SURFACE DATUM

$$
\text { DATE WATER }
$$

MAY $04,199212.72 \mathrm{~S}$

PERIOD OF RECORD HIGHEST 12.72 MAY 04, 1992 LOWEST 26.05 DEC 14, 1990

SITE NUMBER 332639117042301

LOCAL NUMBER 008S002W34B02S

On Pechanga Indian Reservation, southeast of Temecula. Drilled unused water-table well. Diameter 8 inches, depth 221 feet. Altitude of land-surface datum 1,240 feet. Recorder installed October 1987-91. Water-1evel records available 1972,1979 to current year.

WATER LEVELS IN FEET BELOW LAND-SURFACE DATUM

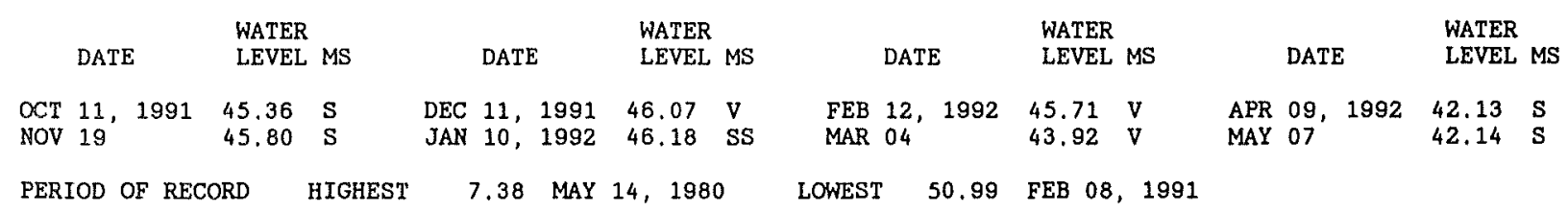


WELL DESCRIPTIONS AND WATER-LEVEL MEASUREMENTS

WATER YEAR $\propto$ CTOBER 1991 TO SEPTEMBER 1992

SITE NUMBER 332632117042001

LOCAL NUMBER 008S002W34B04S

On Pechanga Indian Reservation. Drilled unused water-table well. Diameter 6 Inches, depth reported 140 feet. Altitude of land-surface datum 1,245 feet. Water-level records available 1973, 1989 to current year.

WATER LEVELS IN FEET BELOW LAND-SURFACE DATUM

DATE WATER

MAY 04, $1992 \quad 78.14 \mathrm{~S}$

PERIOD OF RECORD HIGHEST 74.00 APR 01, 1973 LOWEST 84.39 NOV 30, 1990

\section{SITE NUMBER 332625117044101}

LOCAL NUMBER O08S002W34E01S

Southeast of Temecula on private land within Pechanga Indian Reservation boundaries, about 1 mile east of PalaTemecula Highway. Drilled unused well. Diameter 6 inches, depth about 200 feet. Altitude of land-surface datum 1,235 feet. Water-level records available 1967, 1989 to current year.

WATER LEVELS IN FEET BELOW LAND-SURFACE DATUM

$$
\text { DATE WATER }
$$

MAY 04, $199245,64 \mathrm{~S}$

PERIOD OF RECORD HIGHEST 45.18 JAN 23, 1989 LOWEST 143.00 SEP 01, 1967

SITE NUMBER 332620117043101

LOCAL NUMBER 008S002W34F01S

Southeast of Temecula on private land within Pechanga Indian Reservation boundaries, about 1 mile east of PalaTemecula Highway. Drilled unused well. Diameter 8 inches, depth unknown. Altitude of land-surface datum

1,235 feet. Water-level records available 1953, 1958-59, 1967,1989 to current year.

WATER LEVELS IN FEET BELOW LAND-SURFACE DATUM

DATE WATER

MAY 04, $199235.60 \mathrm{~S}$

PERIOD OF RECORD HIGHEST 34.62 OCT 12,1989 LOWEST 73.72 NOV 05, 1959

SITE NUMBER 332622117043801

LOCAL NUMBER 008S002W34F03S

Southeast of Temecula on private land within Pechanga Indian Reservation boundaries, about 1 mile east of PalaTemecula Highway. Drilled unused well. Diameter 8 inches, depth greater than 260 feet. Altitude of land-surface datum 1,235 feet. Water-level records available 1967, 1989 to current year.

WATER LEVELS IN FEET BELOW LAND-SURFACE DATUM

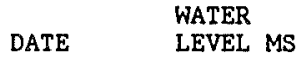

MAY $04,199240.72 \mathrm{~S}$

PERIOD OF RECORD HIGHEST 40.65 JUN 13, 1991 LOWEST 189.00 SEP 01, 1967 
WELL DESCRIPTIONS AND WATER-LEVEL MEASUREMENTS

WATER YEAR OCTOBER 1991 TO SEPTEMBER 1992

SITE NUMBER 332638117034601

LOCAL NUMBER 008S002W35D01S

On Pechanga Indian Reservation. Drilled unused water-table well. Diameter 9 inches, depth 405 feet. Altitude of land-surface datum 1,285 feet. Water-level records available 1979, 1989 to current year.

WATER LEVELS IN FEET BELOW LAND-SURFACE DATUM

$$
\text { DATE WATER }
$$

MAY 04, $199259.20 \quad$ SR

PERIOD OF RECORD HIGHEST 28.41 AUG 14, 1979 LOWEST 86.15 MAY 03, 1990 
INORGANIC DATA

WATER YEAR OCTOBER 1991 TO SEPTEMBER 1992

\begin{tabular}{|c|c|c|c|c|c|c|c|c|c|c|}
\hline $\begin{array}{l}\text { LOCAL } \\
\text { NUMBER }\end{array}$ & DATE & TIME & $\begin{array}{l}\text { DEPTH } \\
\text { BELOW } \\
\text { LAND } \\
\text { SURFACE } \\
\text { (WATER } \\
\text { LEVEL) } \\
\text { (FEET) }\end{array}$ & $\begin{array}{l}\text { DEPTH } \\
\text { OF } \\
\text { WELL, } \\
\text { TOTAL } \\
\text { (FEET) }\end{array}$ & $\begin{array}{l}\text { SPE- } \\
\text { CIFIC } \\
\text { CON- } \\
\text { DUCT- } \\
\text { ANCE } \\
\text { (US/CM) }\end{array}$ & $\begin{array}{c}\text { PH } \\
\text { WATER } \\
\text { WHOLE } \\
\text { FIELD } \\
\text { (STAND- } \\
\text { ARD } \\
\text { UNITS) }\end{array}$ & $\begin{array}{l}\text { TEMPER- } \\
\text { ATURE } \\
\text { WATER } \\
\text { (DEG C) }\end{array}$ & $\begin{array}{l}\text { CALCIUM } \\
\text { DIS- } \\
\text { SOLVED } \\
\text { (MG/L } \\
\text { AS CA) }\end{array}$ & $\begin{array}{l}\text { MAGNE- } \\
\text { SIUM, } \\
\text { DIS- } \\
\text { SOLVED } \\
\text { (MG/L } \\
\text { AS MG) }\end{array}$ & $\begin{array}{l}\text { SODIUM, } \\
\text { DIS- } \\
\text { SOLVED } \\
\text { (MG/L } \\
\text { AS NA) }\end{array}$ \\
\hline $\begin{array}{l}\text { 003S004E20D01S } \\
\text { 003S004E20F02S } \\
\text { 003S004E20F03S }\end{array}$ & $\begin{array}{l}12-02-91 \\
04-21-92 \\
12-05-91 \\
04-23-92 \\
12-05-91\end{array}$ & $\begin{array}{l}1430 \\
1245 \\
1015 \\
1045 \\
1230\end{array}$ & $\begin{array}{c}396.94 \\
-- \\
-- \\
-- \\
--\end{array}$ & $\begin{array}{r}566 \\
566 \\
864 \\
864 \\
1129\end{array}$ & $\begin{array}{l}800 \\
790 \\
385 \\
360 \\
280\end{array}$ & $\begin{array}{l}7.8 \\
7.8 \\
7.9 \\
8.0 \\
8.2\end{array}$ & $\begin{array}{l}18.0 \\
22.5 \\
19.0 \\
19.5 \\
20.5\end{array}$ & $\begin{array}{r}110 \\
110 \\
45 \\
45 \\
26\end{array}$ & $\begin{array}{l}19 \\
20 \\
9.6 \\
9.6 \\
5.4\end{array}$ & $\begin{array}{l}28 \\
24 \\
20 \\
18 \\
21\end{array}$ \\
\hline $\begin{array}{l}\text { 003S004E20J01S } \\
\text { 003S004E20J03S }\end{array}$ & $\begin{array}{l}04-23-92 \\
12-04-91 \\
04-22-92 \\
12-04-91 \\
04-22-92\end{array}$ & $\begin{array}{l}1330 \\
0845 \\
0845 \\
1345 \\
1415\end{array}$ & $\begin{array}{c}343.32 \\
-- \\
345.1 \\
--\end{array}$ & $\begin{array}{r}1129 \\
565 \\
565 \\
869 \\
869\end{array}$ & $\begin{array}{l}280 \\
790 \\
800 \\
350 \\
390\end{array}$ & $\begin{array}{l}8.0 \\
7.8 \\
7.9 \\
7.8 \\
8.1\end{array}$ & $\begin{array}{l}20.5 \\
17.5 \\
20.0 \\
19.5 \\
20.0\end{array}$ & $\begin{array}{l}27 \\
83 \\
84 \\
41 \\
46\end{array}$ & $\begin{array}{l}5.7 \\
15 \\
17 \\
8.7 \\
9.8\end{array}$ & $\begin{array}{l}24 \\
57 \\
53 \\
19 \\
21\end{array}$ \\
\hline $003 \mathrm{~S} 004 \mathrm{E} 29 \mathrm{R} 01 \mathrm{~S}$ & $\begin{array}{l}12-03-91 \\
04-21-92 \\
12-02-91 \\
04-20-92\end{array}$ & $\begin{array}{l}0915 \\
0915 \\
1715 \\
1745\end{array}$ & $\begin{array}{l}-- \\
-- \\
--\end{array}$ & $\begin{array}{l}494 \\
494 \\
726 \\
726\end{array}$ & $\begin{array}{l}710 \\
650 \\
635 \\
610\end{array}$ & $\begin{array}{l}8.0 \\
7.8 \\
7.8 \\
7.7\end{array}$ & $\begin{array}{l}17.0 \\
21.0 \\
17.5 \\
20.5\end{array}$ & $\begin{array}{l}74 \\
63 \\
80 \\
75\end{array}$ & $\begin{array}{l}13 \\
12 \\
14 \\
14\end{array}$ & $\begin{array}{l}61 \\
51 \\
26 \\
24\end{array}$ \\
\hline $\begin{array}{l}\text { LOCAL } \\
\text { NUMBER }\end{array}$ & DATE & $\begin{array}{l}\text { SODIUM } \\
\text { PERCENT }\end{array}$ & $\begin{array}{l}\text { SODIUM } \\
\text { AD- } \\
\text { SORP- } \\
\text { TION } \\
\text { RATIO }\end{array}$ & $\begin{array}{l}\text { POTAS- } \\
\text { SIUM, } \\
\text { DIS- } \\
\text { SOLVED } \\
\text { (MG/L } \\
\text { AS K) }\end{array}$ & $\begin{array}{l}\text { ALKA- } \\
\text { LINITY } \\
\text { WAT WH } \\
\text { TOT FET } \\
\text { FIELD } \\
\text { MG/L AS } \\
\text { CACO3 }\end{array}$ & $\begin{array}{l}\text { ALKA- } \\
\text { LINITY } \\
\text { WAT DIS } \\
\text { FIX END } \\
\text { FIELD } \\
\text { CACO3 } \\
(M G / L)\end{array}$ & $\begin{array}{l}\text { SULFATE } \\
\text { DIS- } \\
\text { SOLVED } \\
\text { (MG/L } \\
\text { AS SO4) }\end{array}$ & $\begin{array}{l}\text { CHLO- } \\
\text { RIDE, } \\
\text { DIS- } \\
\text { SOLVED } \\
\text { (MG / } \\
\text { AS CL) }\end{array}$ & $\begin{array}{l}\text { FLUO- } \\
\text { RIDE, } \\
\text { DIS- } \\
\text { SOLVED } \\
\text { (MG/L } \\
\text { AS F) }\end{array}$ & $\begin{array}{l}\text { SILICA, } \\
\text { DIS- } \\
\text { SOLVED } \\
\text { (MG/L } \\
\text { AS } \\
\text { SIO2) }\end{array}$ \\
\hline $\begin{array}{l}\text { 003S004E20D01S } \\
\text { 003S004E20F02S } \\
\text { 003S004E20F03S }\end{array}$ & $\begin{array}{l}12-02-91 \\
04-21-92 \\
12-05-91 \\
04-23-92 \\
12-05-91\end{array}$ & $\begin{array}{l}14 \\
12 \\
22 \\
20 \\
34\end{array}$ & $\begin{array}{l}0.6 \\
0.5 \\
0.7 \\
0.6 \\
1\end{array}$ & $\begin{array}{l}5.9 \\
5.6 \\
2.8 \\
2.8 \\
1.9\end{array}$ & $\begin{array}{c}135 \\
135 \\
-- \\
162 \\
114\end{array}$ & $\begin{array}{c}-- \\
-- \\
160 \\
--\end{array}$ & $\begin{array}{r}220 \\
210 \\
17 \\
18 \\
13\end{array}$ & $\begin{array}{l}56 \\
57 \\
13 \\
14 \\
10\end{array}$ & $\begin{array}{l}0.50 \\
0.50 \\
0.70 \\
0.80 \\
0.80\end{array}$ & $\begin{array}{l}20 \\
17 \\
23 \\
23 \\
23\end{array}$ \\
\hline $\begin{array}{l}\text { 003S004E20J01S } \\
\text { 003S004E20J03S }\end{array}$ & $\begin{array}{l}04-23-92 \\
12-04-91 \\
04-22-92 \\
12-04-91 \\
04-22-92\end{array}$ & $\begin{array}{l}36 \\
31 \\
29 \\
23 \\
22\end{array}$ & $\begin{array}{l}1 \\
2 \\
1 \\
0.7 \\
0.7\end{array}$ & $\begin{array}{l}2.5 \\
5.4 \\
5.1 \\
2.1 \\
2.9\end{array}$ & $\begin{array}{l}124 \\
-- \\
143 \\
133 \\
152\end{array}$ & $\begin{array}{r}-- \\
130 \\
-- \\
--\end{array}$ & $\begin{array}{r}14 \\
210 \\
210 \\
28 \\
35\end{array}$ & $\begin{array}{l}12 \\
54 \\
56 \\
15 \\
18\end{array}$ & $\begin{array}{l}1.0 \\
0.70 \\
0.70 \\
0.70 \\
1.0\end{array}$ & $\begin{array}{l}24 \\
17 \\
18 \\
23 \\
23\end{array}$ \\
\hline $003 \mathrm{~S} 004 \mathrm{E} 30 \mathrm{C} 01 \mathrm{~S}$ & $\begin{array}{l}12-03-91 \\
04-21-92 \\
12-02-91 \\
04-20-92\end{array}$ & $\begin{array}{l}35 \\
34 \\
18 \\
17\end{array}$ & $\begin{array}{l}2 \\
2 \\
0.7 \\
0.7\end{array}$ & $\begin{array}{l}6.6 \\
6.2 \\
5.7 \\
5.2\end{array}$ & $\begin{array}{l}-- \\
-- \\
-- \\
119\end{array}$ & $\begin{array}{r}120 \\
120 \\
1130 \\
--\end{array}$ & $\begin{array}{l}180 \\
150 \\
150 \\
140\end{array}$ & $\begin{array}{l}50 \\
44 \\
38 \\
38\end{array}$ & $\begin{array}{l}0.60 \\
0.70 \\
0.60 \\
0.70\end{array}$ & $\begin{array}{l}16 \\
14 \\
20 \\
21\end{array}$ \\
\hline $\begin{array}{l}\text { LOCAL } \\
\text { NUMBER }\end{array}$ & DATE & $\begin{array}{c}\text { SOLIDS, } \\
\text { RESIDUE } \\
\text { AT } 180 \\
\text { DEG. C } \\
\text { DIS- } \\
\text { SOLVED } \\
(M G / L)\end{array}$ & $\begin{array}{c}\text { NITRO- } \\
\text { GEN, } \\
\text { NITRITE } \\
\text { DIS- } \\
\text { SOLVED } \\
\text { (MG/L } \\
\text { AS N) }\end{array}$ & $\begin{array}{c}\text { NITRO- } \\
\text { GEN, } \\
\text { NO2+NO3 } \\
\text { DIS- } \\
\text { SOLVED } \\
\text { (MG/L } \\
\text { AS N) }\end{array}$ & $\begin{array}{l}\text { NITRO- } \\
\text { GEN, } \\
\text { AMMONIA } \\
\text { DIS- } \\
\text { SOLVED } \\
\text { (MG/L } \\
\text { AS N) }\end{array}$ & $\begin{array}{l}\text { PHOS- } \\
\text { PHORUS } \\
\text { ORTHO, } \\
\text { DIS- } \\
\text { SOLVED } \\
\text { (MG/L } \\
\text { AS P) }\end{array}$ & $\begin{array}{l}\text { BORON, } \\
\text { DIS- } \\
\text { SOLVED } \\
\text { (UG/L } \\
\text { AS B) }\end{array}$ & $\begin{array}{l}\text { IRON, } \\
\text { DIS- } \\
\text { SOLVED } \\
\text { (UG/L } \\
\text { AS FE) }\end{array}$ & $\begin{array}{l}\text { MANGA- } \\
\text { NESE, } \\
\text { DIS- } \\
\text { SOLVED } \\
\text { (UG/L } \\
\text { AS MN) }\end{array}$ & \\
\hline $\begin{array}{l}\text { 003S004E20D01S } \\
\text { 003S004E2OF02S } \\
\text { 003S004E20F03S }\end{array}$ & $\begin{array}{l}12-02-91 \\
04-21-92 \\
12-05-91 \\
04-23-92 \\
12-05-91\end{array}$ & $\begin{array}{l}535 \\
514 \\
209 \\
238 \\
154\end{array}$ & $\begin{array}{l}<0.010 \\
<0.010 \\
<0.010 \\
<0.010 \\
<0.010\end{array}$ & $\begin{array}{l}0.610 \\
0.370 \\
1.30 \\
1.20 \\
0.660\end{array}$ & $\begin{array}{r}<0.010 \\
0.010 \\
<0.010 \\
0.010 \\
<0.010\end{array}$ & $\begin{array}{r}0.020 \\
<0.010 \\
0.020 \\
0.010 \\
0.020\end{array}$ & $\begin{array}{l}10 \\
20 \\
20 \\
10 \\
30\end{array}$ & $\begin{array}{l}5 \\
<3 \\
14 \\
<3 \\
<3\end{array}$ & $\begin{array}{r}2 \\
4 \\
2 \\
<1 \\
<1\end{array}$ & \\
\hline $003 \mathrm{~S} 004 \mathrm{E} 20 \mathrm{J03S}$ & $\begin{array}{l}04-23-92 \\
12-04-91 \\
04-22-92 \\
12-04-91 \\
04-22-92\end{array}$ & $\begin{array}{l}170 \\
533 \\
505 \\
201 \\
238\end{array}$ & $\begin{array}{r}<0.010 \\
0.010 \\
<0.010 \\
<0.010 \\
<0.010\end{array}$ & $\begin{array}{l}0.700 \\
0.500 \\
0.400 \\
0.970 \\
1.10\end{array}$ & $\begin{array}{r}0.040 \\
<0.010 \\
0.020 \\
<0.010 \\
0.020\end{array}$ & $\begin{array}{r}0.020 \\
0.020 \\
<0.010 \\
0.020 \\
0.020\end{array}$ & $\begin{array}{l}30 \\
60 \\
60 \\
20 \\
20\end{array}$ & $\begin{array}{r}<3 \\
5 \\
<3 \\
<3 \\
<3\end{array}$ & $\begin{array}{r}<1 \\
1 \\
<1 \\
<1 \\
2\end{array}$ & \\
\hline $003 \mathrm{~S} 004 \mathrm{E} 30 \mathrm{CO} 1 \mathrm{~S}$ & $\begin{array}{l}12-03-91 \\
04-21-92 \\
12-02-91 \\
04-20-92\end{array}$ & $\begin{array}{l}456 \\
398 \\
425 \\
383\end{array}$ & $\begin{array}{r}<0.010 \\
0.010 \\
<0.010 \\
<0.010\end{array}$ & $\begin{array}{r}<0.050 \\
0.790 \\
0.380 \\
.0 .620\end{array}$ & $\begin{array}{r}<0.010 \\
0.030 \\
<0.010 \\
0.010\end{array}$ & $\begin{array}{r}<0.010 \\
<0.010 \\
<0.010 \\
0.010\end{array}$ & $\begin{array}{l}60 \\
60 \\
20 \\
20\end{array}$ & $\begin{array}{r}5 \\
<3 \\
5 \\
<3\end{array}$ & $\begin{array}{l}20 \\
11 \\
<1 \\
<1\end{array}$ & \\
\hline
\end{tabular}

< Actual value is less than value shown.

L Laboratory value. 
LOCAL NUMBER 007N007E33Q01M............... 206

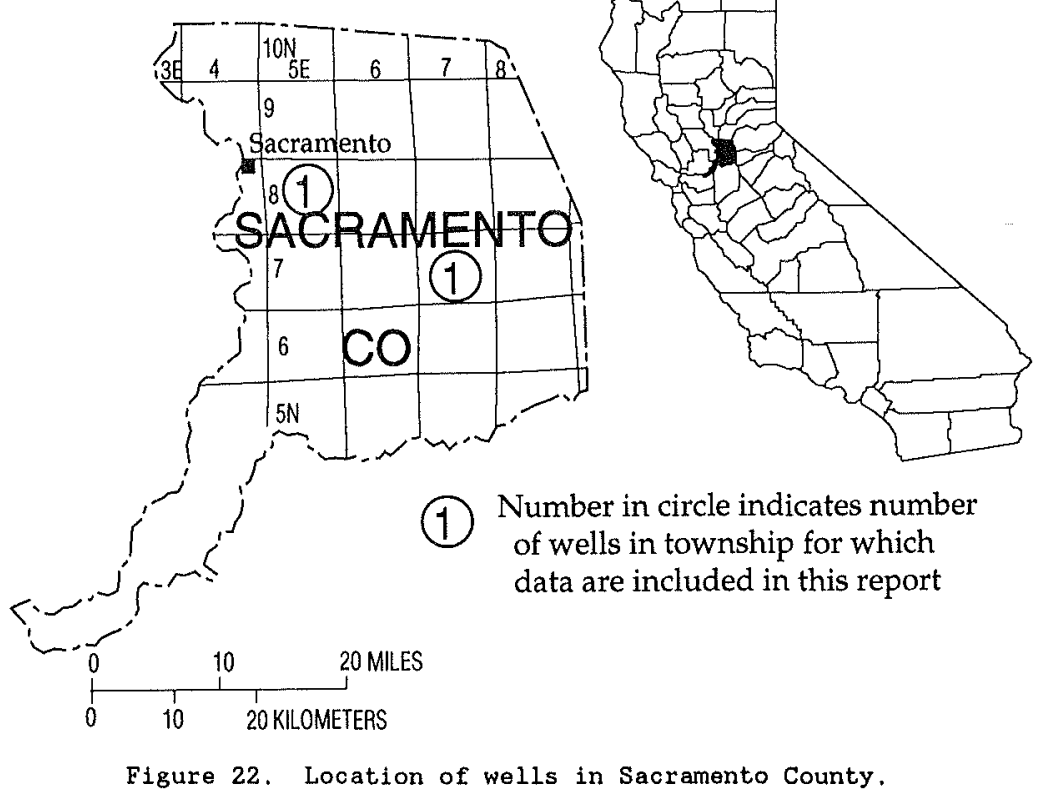




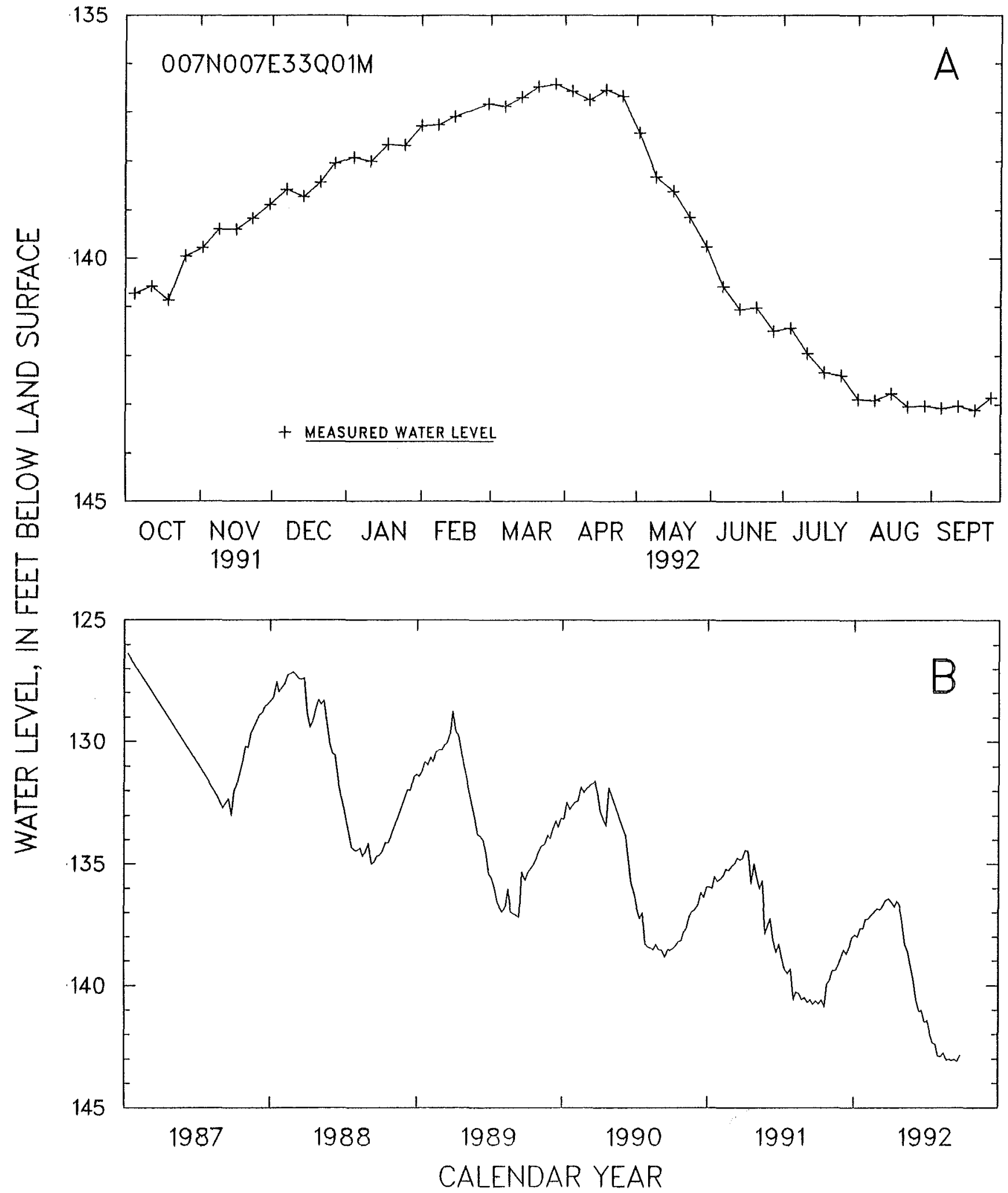

Figure 23. Hydrographs for well in Sacramento County (007N007E33Q01M).

A, 1992 water year. B, period of record. 
WATER LEVELS, SACRAMENTO COUNTY

WELL DESCRIPTIONS AND WATER-LEVEL MEASUREMENTS

WATER YEAR OCTOBER 1991 TO SEPTEMBER 1992

SITE NUMBER 382444121123301

LOCAL NUMBER 007N007E33Q01M

About 9 miles east of Elk Grove. Drilled domestic well in Mehrten Formation. Diameter 8 inches, depth cased 250 feet, open hole 250-300 feet. Altitude of land-surface datum 125 feet. Water-level records availeble 1987 to current year.

WATER LEVELS IN FEET BELOW LAND-SURFACE DATUM

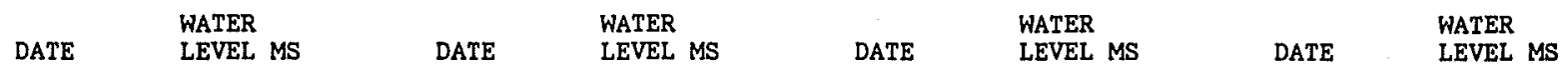

$\begin{array}{rrrr}\text { OCT } 05,1991 & 140.74 \mathrm{~S} & \text { JAN } 04,1992 & 137.92 \mathrm{~S} \\ 12 & 140.59 \mathrm{~S} & 11 & 138.00 \mathrm{~S} \\ 19 & 140.87 \mathrm{~S} & 18 & 137.65 \mathrm{~S} \\ 26 & 139.96 \mathrm{~S} & 25 & 137.67 \mathrm{~S} \\ \text { NOV } 02 & 139.78 \mathrm{~S} & \text { FEB } 01 & 137.27 \mathrm{~S} \\ 09 & 139.40 \mathrm{~S} & 08 & 137.26 \mathrm{~S} \\ 16 & 139.40 \mathrm{~S} & 15 & 137.09 \mathrm{~S} \\ 23 & 139.17 \mathrm{~S} & 29 & 136.83 \mathrm{~S} \\ 30 & 138.88 \mathrm{~S} & \text { MAR } 07 & 136.88 \mathrm{~S} \\ \text { DEC } 07 & 138.57 \mathrm{~S} & 14 & 136.70 \mathrm{~S} \\ 14 & 138.72 \mathrm{~S} & 21 & 136.48 \mathrm{~S} \\ 21 & 138.42 \mathrm{~S} & 28 & 136.43 \mathrm{~S} \\ 27 & 138.04 \mathrm{~S} & \text { APR } 04 & 136.57 \mathrm{~S}\end{array}$

PERIOD OF RECORD HIGHEST 126,39 JAN 10,1987

$\begin{array}{rll}\text { APR } 11,1992 & 136.75 \mathrm{~S} \\ 18 & & 136.54 \mathrm{~S} \\ 25 & 136.67 \mathrm{~S} \\ \text { MAY } 02 & 137.42 \mathrm{~S} \\ 09 & 138.32 \mathrm{~S} \\ 16 & 138.61 \mathrm{~S} \\ 23 & 139.15 \mathrm{~S} \\ 30 & 139.76 \mathrm{~S} \\ \text { JUN } 06 & 140.59 \mathrm{~S} \\ 13 & 141.06 \mathrm{~S} \\ 20 & 141.02 \mathrm{~S} \\ 27 & 141.50 \mathrm{~S} \\ \text { JUL } 04 & 141.44 \mathrm{~S}\end{array}$

$\begin{array}{cll}\text { JUL } 11,1992 & 141.96 \mathrm{~S} \\ 18 & & 142.35 \mathrm{~S} \\ 25 & 142.41 \mathrm{~S} \\ \text { AUG } 01 & 142.89 \mathrm{~S} \\ 08 & 142.91 \mathrm{~S} \\ 15 & 142.77 \mathrm{~S} \\ 22 & 143.04 \mathrm{~S} \\ 29 & 143.03 \mathrm{~S} \\ \text { SEP } 05 & 143.07 \mathrm{~S} \\ 12 & 143.03 \mathrm{~S} \\ 19 & 143.11 \mathrm{~S} \\ 26 & 142.86 \mathrm{~S}\end{array}$

LOWEST 143.11 SEP 19,1992

SITE NUMBER 383350121254301

LOCAL NUMBER O08N005E09H01M

In Sacramento, on California State University campus. Drilled observation well. Diameter 4 inches, depth measured 47.9 feet in 1991, perforated 27-47 feet. Altitude of land-surface datum 35 feet. Water-level records available 1991 to current year.

WATER LEVELS IN FEET BELOW LAND-SURFACE DATUM

$$
\text { DATE WATER } \quad \text { LEVEL MS }
$$

JUL $20,1992 \quad 28.89 \mathrm{~S}$

PERIOD OF RECORD HIGHEST 28.37 APR 24, 1991 LOWEST 29.07 MAY 23, 1991 
SAN BERNARDINO COUNTY

Page

LOCAL NUMBER 001N005E02N01S.

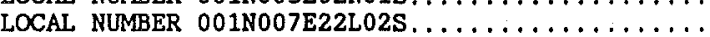

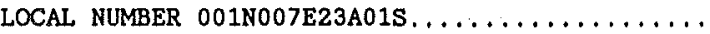

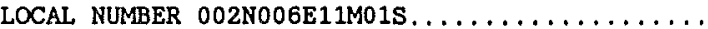

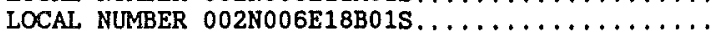

LOCAL NUMBER 002N007E02C01S.

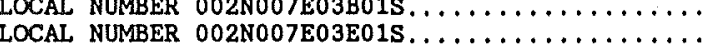

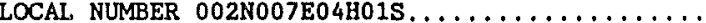

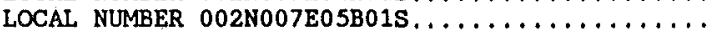

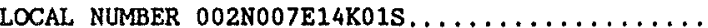

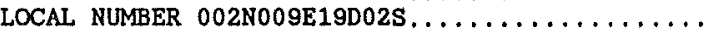

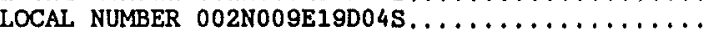
LOCAL NUMBER 003N004E04K02S. . . . . . . . . . . .

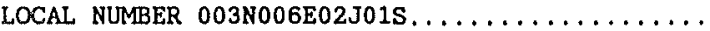

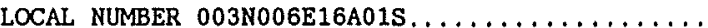

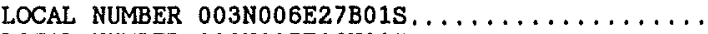

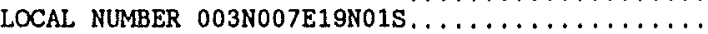

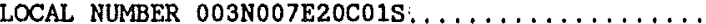
LOCAL NUMBER 003NO07E2OMO1S.............

LOCAL NUMBER 003NO07E27H01S.............

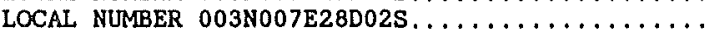
LOCAL NUMBER 003 N007E29F01S. . . . . . . . . . .

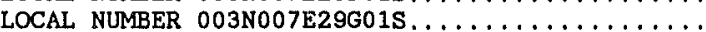
LOCAL NUMBER 003N007E29R01S..............

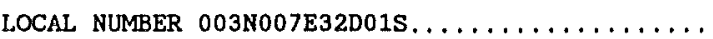

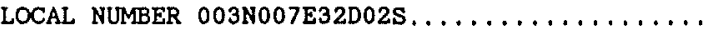

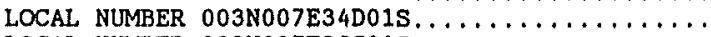
LOCAL NUMBER O03N007E36G01S. . . . . . . . . . .

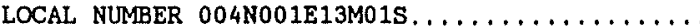

LOCAL NUMBER 004N001E17L01S............... LOCAL NUMBER 004N003E35J01S . . . . . . . . . . .

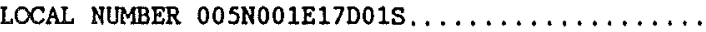

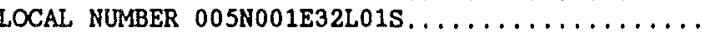

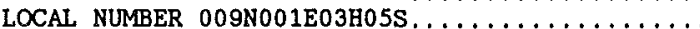

LOCAL NUMBER OOSNO01E06H02S. . . . . . . . . . .

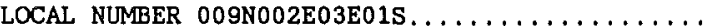

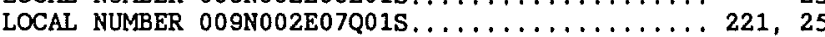
LOCAL NUMBER OOSN002E11C03S............... 222, 257

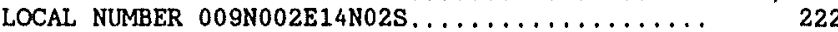

LOCAL NUMBER OOSNO03E18N01S.............. 222

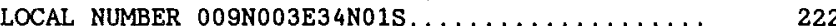
LOCAL NUMBER O09N004E05R02S............... LOCAL NUMBER OOSN004E21B02S ................. LOCAL NUMBER 010N001E28G03S................ 223

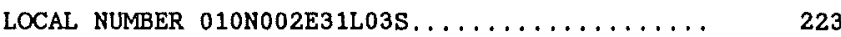

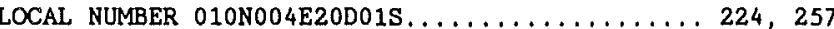

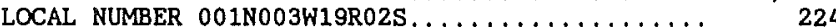
LOCAL NUMBER 001 N003W29M02S................. 224 LOCAL NUMBER 001 N003W31Q02S, ............. 224

LOCAL NUMBER 001N003W33C01s.............

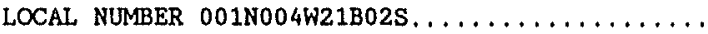

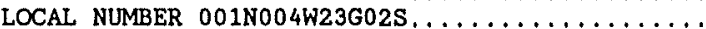

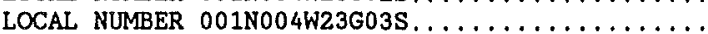

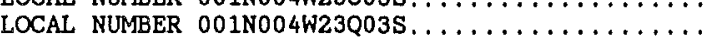

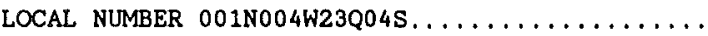

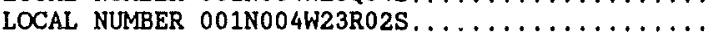
LOCAL NUMBER 001N004W23R03S..............

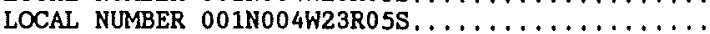
LOCAL NUMBER O01N004W26E02S. .

3

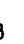
24

LOCAL NUMBER 001N004W27B01S............. 227

LOCAL NUMBER 001 N004W34G03S................. 257, 262

LOCAL NUMBER 001 N004W35L01S.................. 227

LOCAL NUMBER 001N005W06F01S.............. 227

LOCAL NUMBER 001 N005W15Q01S................... 228

LOCAL NUMBER 001 N005W34M01S.............. 228

LOCAL NUMBER 002N005W33J04S................ 228

LOCAL NUMBER O03N004W02C01S................. 228

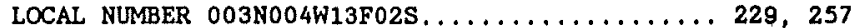

LOCAL NUMBER 003 N004W33H05S............... 229, 257

LOCAL NUMBER 004N001W14C02S............... 229

LOCAL NUMBER 004 N001W21G01S................. 229

LOCAL NUMBER 004 N002W16E01S..................

LOCAL NUMBER 004 N003W15C01S............... 230

LOCAL NUMBER 004 N003W21M03S.............. 230

LOCAL NUMBER 004N003W30A06S............. 230, 257

LOCAL NUMBER 004 N004W01A01S................ 231, 257

LOCAL NUMBER 004 N004W03Q01S................. 231

LOCAL NUMBER 004 N004W08N03S................ 231

LOCAL NUMBER 004N004W24P03S.............. 231

LOCAL NUMBER 004 N005W01K04S............. 232

LOCAL NUMBER 004 N005W36R03S . . . . . . . . . . . .

LOCAL NUMBER $005 N 003$ W03H02S................... 232

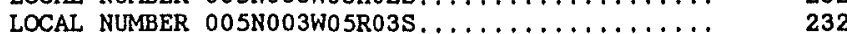

LOCAL NUMBER $005 N 004$ W11P03S.............. 233, 257

LOCAL NUMBER $005 N 004$ W13F02S.............. 233

LOCAL NUMBER $005 N 004$ W25N01S................. 233, 257

LOCAL NUMBER $005 N 004$ W27D08S................. 233

LOCAL NUMBER $005 N 004$ W30K02S.................. 234

LOCAL NUMBER 005N006W12E02S............... 234

LOCAL NUMBER 005N007W24D03S............. 234

LOCAL NUMBER DO6N003W34J03S ............... 234

LOCAL NUMBER 006 N004W30D10S............... 235, 257

LOCAL NUMBER $006 N 005$ W12H01S................ 235, 257

LOCAL NUMBER 006N005W19J02S.................. 235

LOCAL NUMBER 006N006W21J02S............. 235

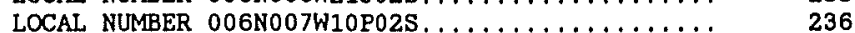

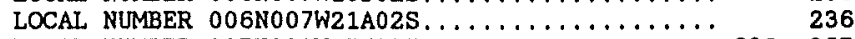

LOCAL NUMBER 007 N004W07K02S................ 236, 257

LOCAL NUMBER 007 N007W10C02S .............. 236

LOCAL NUMBER 008N001W29D02S............. 237

LOCAL NUMBER 008 N003W05J01S................. 237, 257

LOCAL NUMBER 008 N004W13B01S............. 237, 257

LOCAL NUMBER 008 N004W15C01S............... 237

LOCAL NUMBER 008 N004W23Q01S................. 238

LOCAL NUMBER 009 N001W10G06S............ 238, 257

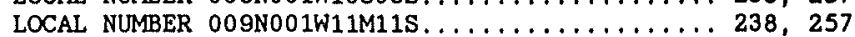

LOCAL NUMBER 009N001W11R02S ................ 238, 257

LOCAL NUMBER 009 N001W13B02S................. 239, 257

LOCAL NUMBER 009 N001W13B03S ................ 239, 257

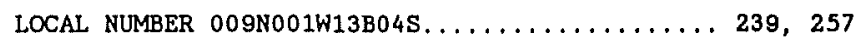

LOCAL NUMBER 009 N001W13H02S.................. 239, 257

LOCAL NUMBER 009 N002W05N07S............... 240, 257

LOCAL NUMBER 009N002W06A04S............... 240

LOCAL NUMBER 009 N003W13R01S.................... 240

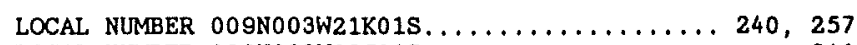

LOCAL NUMBER 009 N003W22J01S................... 240,241

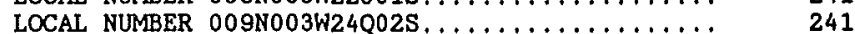

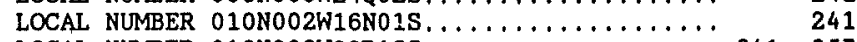

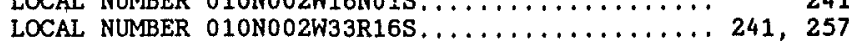


SAN BERNARDINO COUNTY--Continued

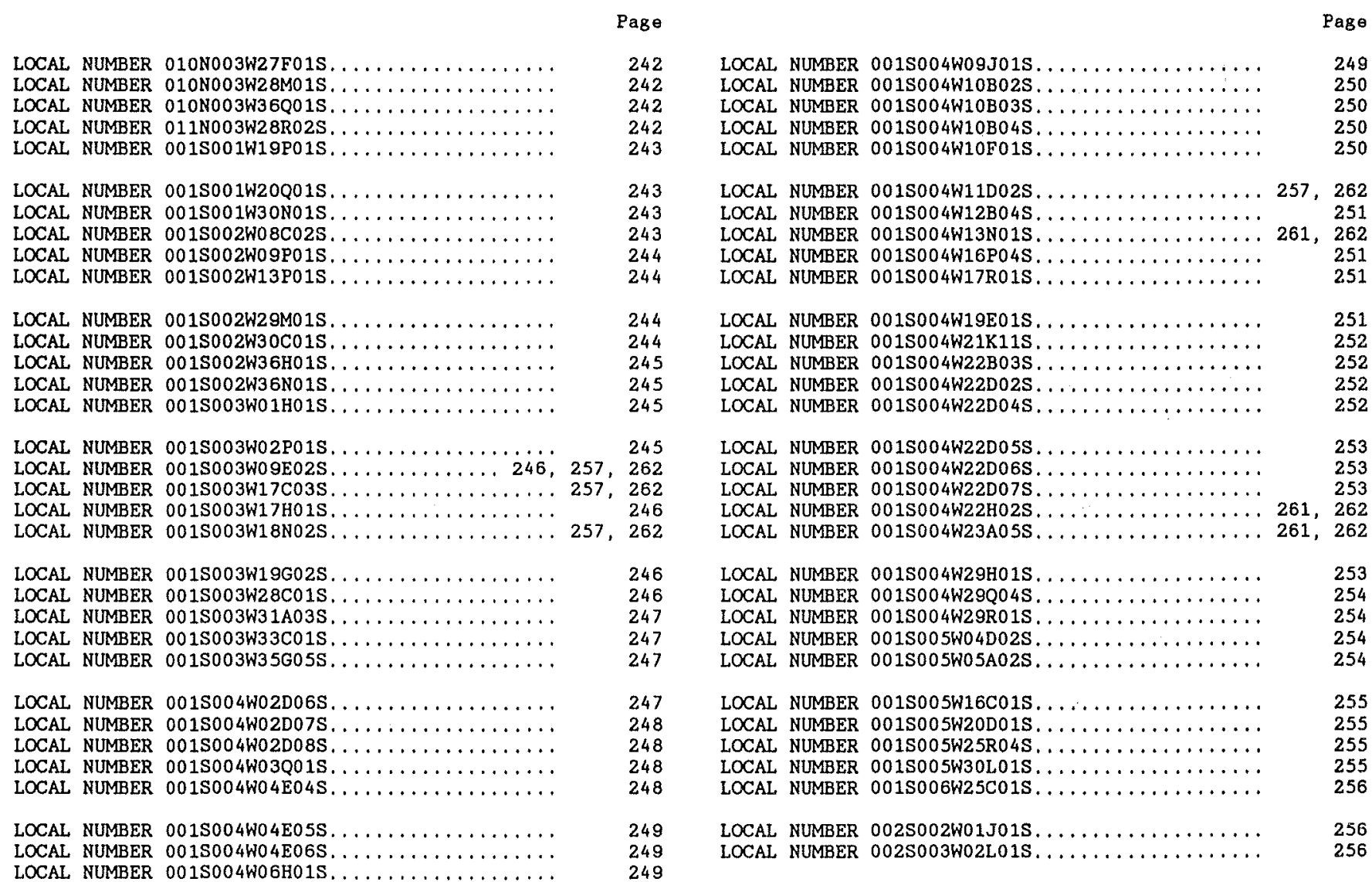




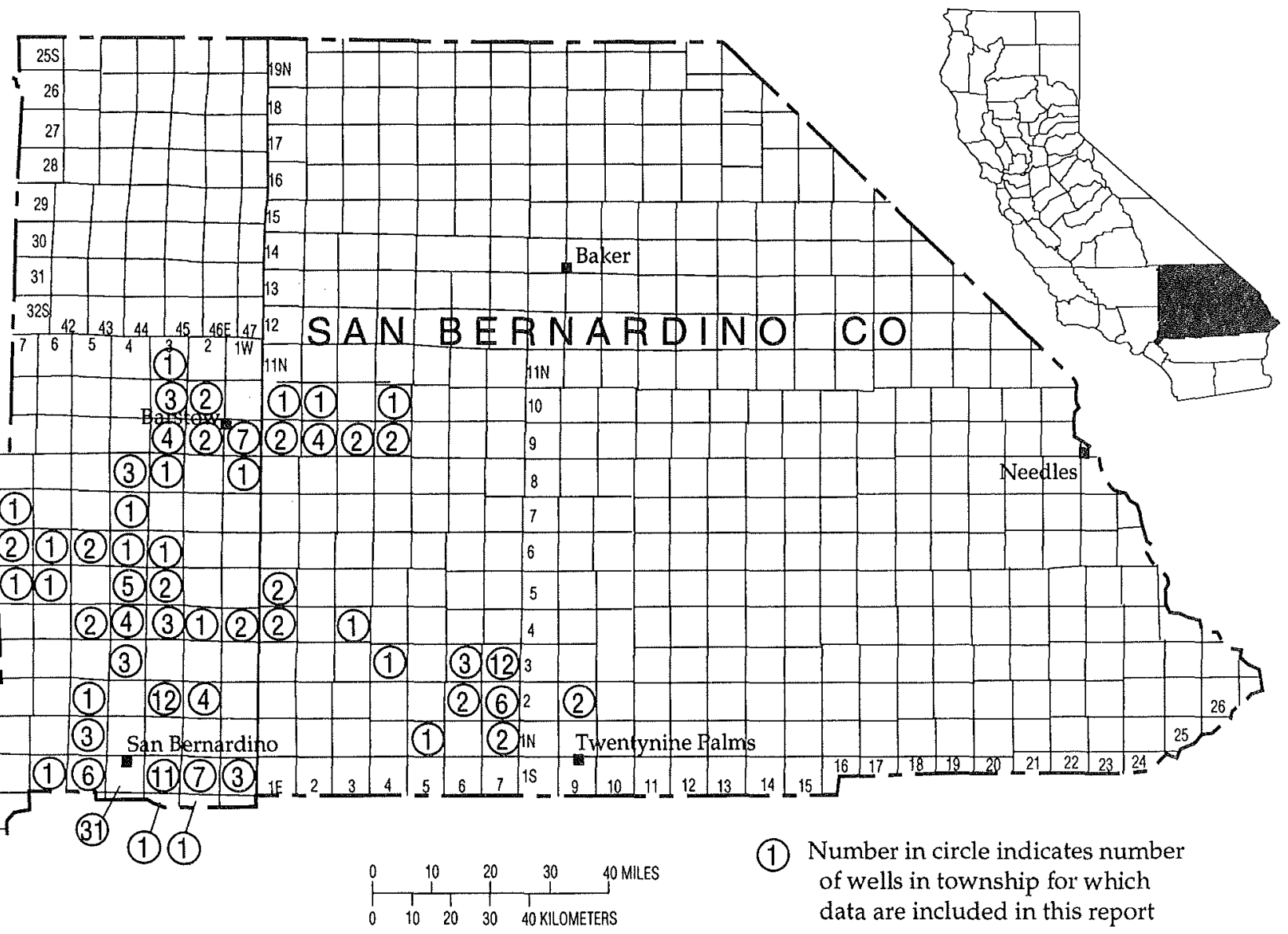

Figure 24. Location of wells in San Bernardino County. 


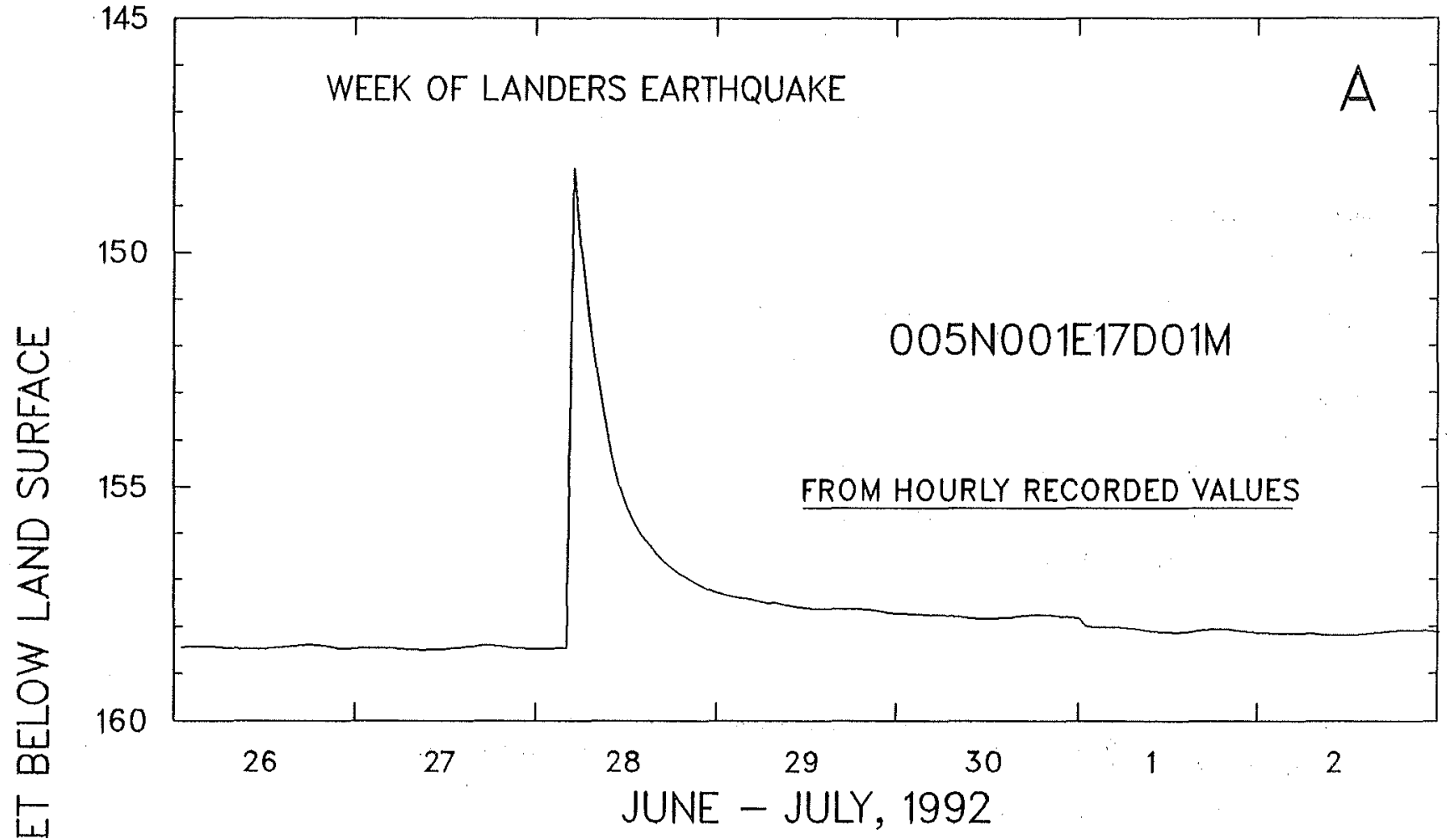

出

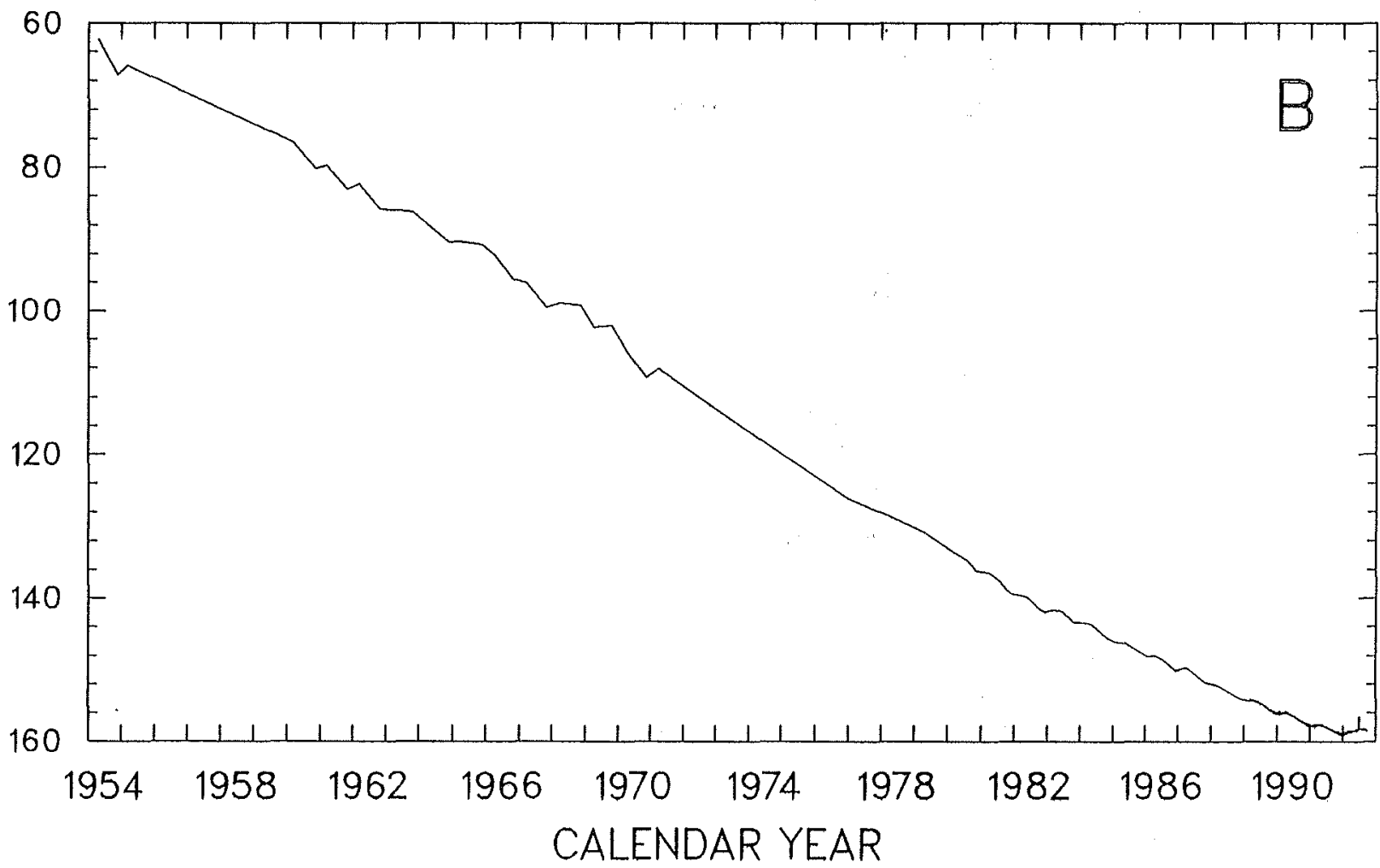

Figure 25. Hydrographs for well in San Bernardino County (005N001E17D01S). 
WELL DESCRIPTIONS AND WATER-LEVEL MEASUREMENTS

WATER YEAR OCTOBER 1991 TO SEPTEMBER 1992

\begin{abstract}
SITE NUMBER 341144116260601
\end{abstract}
LOCAL NUMBER O01N005E02N01S

About 5.5 miles north of Yucca Valley. Drilled unused well in alluvium. Diameter 8 inches, depth unknown. Altitude of land-surface datum 3,520 feet. Water-level records available 1952-53, 1958-71, 1978, 1980 to current year.

WATER LEVELS IN FEET BELOW LAND-SURFACE DATUM

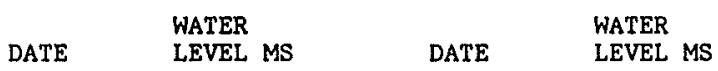
OCT $29,199165.90 \mathrm{~S} \quad \operatorname{MAY} 28,199268.29 \mathrm{~S}$

PERIOD OF RECORD HIGHEST 30.41 APR 14, 1982 LOWEST 80.17 APR 04, 1968

SITE NUMBER 340923116142501

LOCAL NUMBER O01N007E22L02S

About $0.5 \mathrm{mile}$ south of Sunfair, $1.5 \mathrm{miles}$ north of Highway 62 , and 0.35 mile east of Sunfair Road. Drilled domestic water-table well. Diameter 12 inches, depth 392.75 feet, perforated 241-387 feet. Altitude of land-surface datum 2,408 feet. Water-level records available 1963, 1980 to current year.

WATER LEVELS IN FEET BELOW LAND-SURFACE DATUM

$$
\begin{array}{ll} 
& \text { WATER } \\
\text { DATE } & \text { WEVEL MS } \\
\text { DATE } & \text { LEVEL MS }
\end{array}
$$

OCT 29, 1991233.37 SR MAY 27, 1992234.71 SR

$\begin{array}{lllllll}\text { PERIOD OF RECORD } & \text { HIGHEST } & 233.61 & \text { MAR } 25,1981 & \text { LOWEST } & 236.5 & 1963\end{array}$

SITE NUMBER 340945116125001

LOCAL NUMBER OO1N007E23A01S

About 1.9 miles east of Sunfair. Drilled unused water-table well. Diameter 10 inches, depth 368.5 feet, perforated 360-370 feet. Altitude of land-surface datum 2,376 feet. Water-level records available 1969 , 1978 to current year.

WATER LEVELS IN FEET BELOW LAND-SURFACE DATUM

$$
\begin{array}{lll} 
& \text { WATER } & \\
\text { DATE } & \text { WEVEL MS } & \text { DATE } \\
\text { LEVEL MS }
\end{array}
$$

OCT 31, $1991211.33 \mathrm{~S} \quad$ MAY 26, $1992211.50 \mathrm{~S}$

PERIOD OF RECORD HIGHEST 209.96 JAN 09, 1980 LOWEST 211.50 MAY 26, 1992

SITE NUMBER 341622116194601

LOCAL NUMBER O02NOO6E11M01S

On Twentynine Palms Marine Corps Base, along Giant Rock Road. Drilled observation water-table well in alluvium of Tertiary age. Diameter 4 inches, depth 790 feet. Altitude of land-surface datum 2,803 feet. Water-level records available 1986 to current year.

WATER LEVELS IN FEET BELOW LAND-SURFACE DATUM

$$
\begin{array}{ll} 
& \text { WATER } \\
\text { DATE } & \text { LEVEL MS }
\end{array}
$$

AUG 28, $1992484.77 \mathrm{~S}$

PERIOD OF RECORD HIGHEST 482.0 MAR 13, 1986 LOWEST 485.87 OCT 09, 1987 
SITE NUMBER 341556116233401

LOCAL NUMBER 002N006E18B01S

In Landers, southwest of post office. Drilled public-supply well. Diameter 14 inches, depth 310 feet, perforated 187-305 feet. Altitude of land-surface datum 3,087 feet. Water-ievel records available 1963,1980 to current year.

WATER LEVELS IN FEET BELOW LAND-SURFACE DATUM

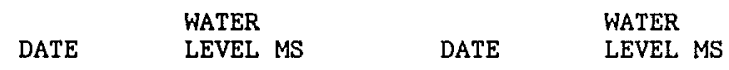

OCT 29, 1991225.78 S $\quad$ MAY 27, 1992223.90 SR

PERIOD OF RECORD HIGHEST 185.60 JUL 19, $1963 \quad$ LOWEST 225.78 OCT 29, 1991

SITE NUMBER 341741116132501

LOCAL NUMBER 002N007E02C01S

On Twentynine Palms Marine Corps Base, about 10.5 miles northwest of Headquarters, Drilled observation watertable well in alluvium. Diameter 10 inches, depth 341.8 feet in 1982 . Altitude of land-surface datum 2,272.11 feet. Water-level records available 1941, 1952-67, 1970, 1973, 1982, 1984, 1987 to current year.

WATER LEVELS IN FEET BELOW LAND-SURFACE DATUM

$$
\begin{array}{lll} 
& \text { WATER } & \\
\text { DATE } & \text { WEVEL MS } & \text { WATER } \\
\text { LEVEL MS }
\end{array}
$$

JUN $04,1992163.74 \mathrm{~S} \quad$ AUG $28,1992129.75 \mathrm{~S}$

PERIOD OF RECORD HIGHEST 25.00 MAR 14, 1952 LOWEST 163.74 JUN 04, 1992

SITE NUMBER 341736116141201

LOCAL NUMBER 002N007E03B01S

On Twentynine Palms Marine Corps Base, about 11 miles northwest of Headquarters. Drilled institutional. water-table well in alluvium. Diameter 12 inches, depth 800 feet, cased to 700 feet. Altitude of land-surface datum $2,355.3$ feet. Water-level records available 1953-68, 1970, 1972-75, 1982, 1984 to current year.

WATER LEVELS IN FEET BELOW LAND-SURFACE DATUM

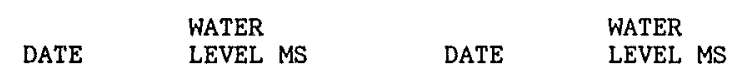

JUN 04, $1992 \quad \mathrm{P} \quad$ AUG 28, $1992 \quad \mathrm{P}$

PERIOD OF RECORD HIGHEST 104.21 MAR 27, 1953 LOWEST $156.63 \quad$ JUN 13,1989

SITE NUMBER 341720116145601

LOCAL NUMBER 002N007E04H01S

On Twentynine Palms Marine Corps Base, about 11.5 miles northwest of Headquarters. Drilled observation water-table well in alluvium. Diameter 10 inches, depth measured 482.4 feet in 1982 . Altitude of land-surface datum 2,442.17 feet. Water-level records avallable 1952-68, 1970, 1973, 1975, 1982 , 1984 to current year.

WATER LEVELS IN FEET BELOW LAND-SURFACE DATUM

$$
\begin{array}{lll} 
& \text { WATER } & \\
\text { DATE } & \text { LEVEL MS } & \text { WATER } \\
& \text { DATE } & \text { LEVEL MS }
\end{array}
$$

JUN $04,1992220.97$ S AUG 28, $1992221.17 \mathrm{~S}$

PERIOD OF RECORD HIGHEST 188.64 MAY $30,1953 \quad$ LOWEST 221.17 AUG 28,1992 
WELL DESCRIPTIONS AND WATER-LEVEL MEASUREMENTS

WATER YEAR OCTOBER 1991 TO SEPTEMBER 1992

SITE NUMBER 341742116160701

LOCAL NUMBER 002N007E05B01S

On Twentynine Palms Marine Corps Base, about 3 miles east of Surprise Spring. Drilled observation water-table well in alluvium of Tertiary age. Diameter 4 inches, depth 405 feet, perforated 385-405 feet. Altitude of land-surface datum $2,586.72$ feet. Water-level records available 1986 to current year.

WATER LEVELS IN FEET BELOW LAND-SURFACE DATUM

$\begin{array}{lll} & \text { WATER } & \\ \text { DATE } & \text { WATER } \\ \text { LEVEL MS } & \text { DATE }\end{array}$
JUN $04,1992355.69 \mathrm{~S}$
AUG $28,1992355.03 \mathrm{~S}$

PERIOD OF RECORD HIGHEST 351.04 JAN 13,1987

LOWEST 356.45 OCT 09,1987

SITE NUMBER 341520116130101

LOCAL NUMBER 002N007E14K01S

On Twentynine Palms Marine Corps Base, about 3 miles south of Surprise Spring. Unused water-table well in alluvium. Dlameter 10 inches, depth 644 feet, perforated $450-525,538-548,550-558$ feet. Altitude of land-surface datum 2,538 feet. Water-level records available 1952-67, 1970, 1973, 1975, 1979-80, 1987, $1989,1992$.

WATER LEVELS IN FEET BELOW LAND-SURFACE DATUM

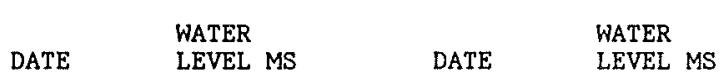

JUN $05,1992342.00 \mathrm{~S} \quad$ AUG $28,1992342.37 \mathrm{~S}$

PERIOD OF RECORD HIGHEST 334.00 JUL 25,1952 LOWEST 357.68 APR 26, 1979

SITE NUMBER 341502116050101

LOCAL NUMBER 002NOO9E19D02S

About 4 miles northwest of the main gate of the U.S. Marine Corps Air Ground Combat Center. Drilled unused well. Diameter 8 inches, depth 220 feet. Altitude of land-surface datum 1,840 feet. Water-levei records available 1989 to current year.

WATER LEVELS IN FEET BELOW LAND-SURFACE DATUM

$$
\begin{array}{lll} 
& \text { WATER } & \\
\text { DATE } & \text { WETER } \\
\text { LEVEL MS } & \text { LEVEL MS }
\end{array}
$$

JUN $05,1992140.71 \mathrm{~S} \quad$ AUG $28,1992140.85 \mathrm{~S}$

PERIOD OF RECORD HIGHEST 140.28 SEP 27,1990 LOWEST 141.79 JUN 13, 1989

SITE NUMBER 341458116051601

LOCAL NUMBER 002N009E19D04S

About 4 miles northwest of the main gate of the U.S. Marine Corps Air Ground Combat Center between Ocotillo Heights housing and the golf course clubhouse. Drilied unused well. Diameter 8 inches, depth 519 feet, perforated 259-479, 499-519 feet. Altitude of land-surface datum 1,860 feet. Water-level records available 1990 to current year.

WATER LEVELS IN FEET BELOW LAND-SURFACE DATUM

$$
\begin{array}{lll} 
& \text { WATER } & \text { WATER } \\
\text { DATE } & \text { LEVEL MS } & \text { DATE }
\end{array}
$$
JUN $05,199288.80$ s
AUG $28,199288.80 \mathrm{~S}$

PERIOD OF RECORD HIGHEST 88.24 JUN 20, 1990 LOWEST 88.80 JUN 05, AUG 28, 1992 
SITE NUMBER 342229116341001

LOCAL NUMBER 003N004E04K02S

About 23 miles east of Lucerne Valley. Drilled unused well. Diameter 4 inches, depth 510 feet, perforated 360-510 feet. Altitude of land-surface datum 2,915 feet. Water-level records avallable 1989 to current year.

WATER LEVELS IN FEET BELOW LAND-SURFACE DATUM

$$
\text { DATE WATER }
$$

JUN $04,1992155.56 \mathrm{~S}$

PERIOD OF RECORD HIGHEST 155.44 APR 30,1991 LOWEST 155.56 JUN 04, 1992

SITE NUMBER 342221116190801

LOCAL NUMBER 003N006E02J01S

On Twentynine Palms Marine Corps Base, about $6 \mathrm{miles}$ southeast of Emerson Dry Lake. Drilled observation water-table well in alluvium of Tertiary age. Diameter 4 inches, depth 335 feet, perforated $315-335$ feet. Altitude of land-surface datum 2,413 feet. Water-level records avatlable 1986 to current year.

WATER LEVELS IN FEET BELOW LAND-SURFACE DATUM

$$
\text { DATE WATER } \quad \text { LEVEL MS }
$$

AUG $28,1992160.37 \mathrm{~S}$

PERIOD OF RECORD HIGHEST 159.49 JUN 12,1986 LOWEST 160.48 MAY 20, 1991

SITE NUMBER 342107116210401

LOCAL NUMBER 003N006E16A01S

On Twentynine Palms Marine Corps Base, about 6 miles south of Emerson Dry Lake. Drilled observation water-table well in alluvium of Tertiary age. Diameter 4 inches, depth 295 feet, perforated 275-295 feet. Altitude of land-surface datum 2,507 feet. Water-level records available 1986 to current year.

WATER LEVELS IN FEET BELOW LAND-SURFACE DATUM

$$
\begin{array}{ll} 
& \text { WATER } \\
\text { DATE } & \text { LEVEL MS }
\end{array}
$$

AUG $28,1992175.89 \mathrm{~S}$

PERIOD OF RECORD HIGHEST 174.64 MAR 13, 1986 LOWEST 175.89 AUG 28, 1992

SITE NUMBER 341925116202501

LOCAL NUMBER 003N006E27B01S

On Twentynine Palms Marine Corps Base, about 5 miles northeast of Landers. Drilled observation water-table well in alluvium of Tertiary age, Diameter 4 inches, depth 415 feet, perforated $395-415$ feet. Altitude of land-surface datum 2,643 feet. Water-level records available 1986 to current year.

WATER LEVELS IN FEET BELOW LAND-SURFACE DATUM

$$
\begin{array}{lll} 
& \text { WATER } & \\
\text { DATE } & \text { LEVEL MS } & \text { WATER } \\
& \text { DATE } & \text { LEVEL MS }
\end{array}
$$

JUN $04,1992309.53 \mathrm{~S} \quad$ AUG $28,1992309.49 \mathrm{~s}$

PERIOD OF RECORD GIGHEST 277.9 MAR 13, 1986 LOWEST $309.53 \quad$ JUN 04,1992 
WELL DESCRIPTIONS AND WATER-LEVEL MEASUREMENTS

WATER YEAR OCTOBER 1991 TO SEPTEMBER 1992

SITE NUMBER 341939116174501

LOCAL NUMBER 003N007E19N01S

On Twentynine Palms Marine Corps Base, about $4.5 \mathrm{miles}$ northwest of Surprise Spring. Drilled observation water-table well in alluvium of Tertiary age. Diameter 4 inches, depth 334 feet, perforated $275-295$ feet. Altitude of land-surface datum $2,488,4$ feet. Water-level records available 1986 to current year.

WATER LEVELS IN FEET BELOW LAND-SURFACE DATUM

$$
\begin{array}{lll} 
& \text { WATER } & \\
\text { DATE } & \text { WEVER MS }
\end{array}
$$

JUN $04,1992240.06 \mathrm{~S} \quad$ AUG $28,1992240.56 \mathrm{~S}$

PERIOD OF RECORD HIGHEST 236.8 MAR 13, 1986 LOWEST 240.56 AUG 28, 1992

SITE NUMBER 342111116174801

LOCAL NUMBER 003N007E20C01S

On Twentynine Palms Marine Corps Base, about $4 \mathrm{miles}$ northwest of Surprise Spring. Drilled observation water-table well in alluvium of Tertiary age. Diameter 2 inches, depth 605 feet, screened 228-244, 601-605 feet. Altitude of land-surface datum $2,444.9$ feet. Water-level records available 1975-76, 1980 to current year.

WATER LEVELS IN FEET BELOW LAND-SURFACE DATUM

$\begin{array}{lll} & \text { WATER } & \text { WATER } \\ \text { DATE } & \text { LEVEL MS } & \text { DATE }\end{array}$

JUN $04,1992198.15 \mathrm{~S} \quad$ AUG $28,1992197.27 \mathrm{~s}$

PERIOD OF RECORD HIGHEST 189.7 APR 02, 1986 LOWEST 198.15 JUN 04, 1992

SITE NUMBER 341952116164601

LOCAL NUMBER 003N007E20M01S

On Twentynine Palms Marine Corps Base, about 3.8 miles northwest of Surprise Spring. Drilled observation water-table well in alluvium of Tertiary age. Diameter 4 inches, depth 295 feet, perforated 275-295 feet. Altitude of land-surface datum 2,457.5 feet. Water-level records available 1986 to current year.

WATER LEVELS IN FEET BELOW LAND-SURFACE DATUM

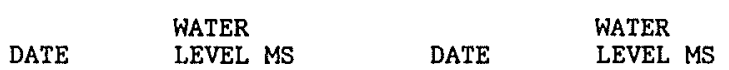

JUN $04,1992210.02 \mathrm{~S} \quad$ AUG $28,1992210.30 \mathrm{~s}$

PERIOD OF RECORD HIGHEST 207.15 JUL 22, 1986 LOWEST 210.30 AUG 28, 1992

SITE NUMBER 341909116134101

LOCAL NUMBER 003N007E27H01S

About 1.7 miles north of Surprise Spring. Drilled test well in alluvium. Diameter 4 inches, depth 587 feet, perforated 472-492, 552-572 feet. Altitude of land-surface datum 2,240 feet. Water-level records available 1987 to current year.

WATER LEVELS IN FEET BELOW LAND-SURFACE DATUM

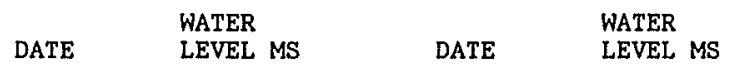

JUN 05, $1992412.07 \mathrm{~S} \quad$ AUG $28,1992412.55 \mathrm{~s}$

PERIOD OF RECORD HIGHEST 408.79 JAN 30, 1987 LOWEST 412.55 AUG 28,1992 
SITE NUMBER 341923116153201

LOCAL NUMBER 003N007E28D02S

On Twentynine Palms Marine Corps Base, supply well 10A, about 2.8 miles northwest of Surprise Spring. Drilled water-table well in alluvium. Diameter 16 inches, depth 600 feet, perforated $390-580$ feet. Altitude of landsurface datum 2,473 feet. Water-level records available 1991 to current year.

WATER LEVELS IN FEET BELOW LAND-SURFACE DATUM

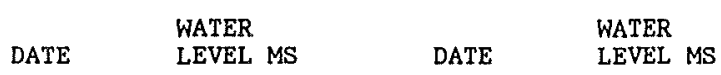

JUN $04,1992235.14$ SR AUG 28, $1992 \quad$ P

PERIOD OF RECORD HIGHEST 230.53 MAY 21, 1991 LOWEST 230.53 MAY 21, 1991

SITE NUMBER 341905116163401

LOCAL NUMBER 003N007E29F01S

On Twentynine Palms Marine Corps Base, supply wel1 11A, about 3.5 miles northwest of Surprise Spring. Drilled water-table well in alluvium. Diameter 16 inches, depth 600 feet, perforated $390-580$ feet. Altitude of land-surface datum 2,510 feet. Water-level records available 1991 to current year.

WATER LEVELS IN FEET BELOW LAND-SURFACE DATUM

$$
\begin{array}{lll} 
& \text { WATER } & \text { WATER } \\
\text { DATE } & \text { LEVEL MS } & \text { DATE }
\end{array}
$$

JUN $04,1992277.52 \mathrm{~S} \quad$ AUG $28,1992278.88 \mathrm{~s}$

PERIOD OF RECORD HIGHEST 271.05 MAY 21, 1991 LOWEST 278.88 AUG 28, 1992

SITE NUMBER 341912116160801

LOCAL NUMBER 003N007E29G01S

On Twentynine Palms Marine Corps Base, about 3 miles northwest of Surprise Spring. Drilled observation water-table well in alluvium of Tertiary age. Diameter 2 inches, depth 348 feet, perforated $312-348$ feet. Altitude of land-surface datum 2,500.6 feet. Water-level records available 1976, 1982,1984 , 1986 to current year.

WATER LEVELS IN FEET BELOW LAND-SURFACE DATUM

$$
\begin{array}{lll} 
& \text { WATER } & \\
\text { DATE } & \text { WATER } \\
\text { LEVEL MS } & \text { DATE } & \text { LEVEL MS }
\end{array}
$$

JUN $04,1992257.33 \mathrm{~S} \quad$ AUG $28,1992257.13 \mathrm{~S}$

PERIOD OF RECORD HIGHEST 248.00 MAR 01, MAR 02, $1976 \quad$ LOWEST 257.33 JUN 04,1992

SITE NUMBER 341843116155201

LOCAL NUMBER 003N007E29R01S

On Twentynine Palms Marine Corps Base, about $2,7 \mathrm{miles}$ northwest of Surprise Spring. Drilled institutional water-table well in alluvium. Diameter 16 inches, depth 600 feet. Altitude of land-surface datum $2,519.9$ feet. Water-level records avallable $1978,1982,1984$ to current year.

WATER LEVELS IN FEET BELOW LAND-SURFACE DATUM

$$
\begin{array}{lll} 
& \text { WATER } & \\
\text { DATE } & \text { LEVEL MS } & \text { WATER } \\
& \text { DATE } & \text { LEVEL MS }
\end{array}
$$

JUN $04,1992280.08 \mathrm{~S} \quad$ AUG $28,1992280.82 \mathrm{~S}$

PERIOD OF RECORD HIGHEST 270.60 AUG 03, 1978 LOWEST 280.94 AUG 22,1991 
WELL DESCRIPTIONS AND WATER-LEVEL MEASUREMENTS

WATER YEAR OCTOBER 1991 TO SEPTEMBER 1992

SITE NUMBER 341826116164501

LOCAL NUMBER O03N007E32DO1S

About 3 miles west of Surprise Spring. Drilled test well in alluvium. Diameter 4 inches, depth 620 feet, perforated 418-438, 498-538, 578-598 feet. Altitude of land-surface datum 2,544 feet. Water-level records available 1987 to current year.

WATER LEVELS IN FEET BELOW LAND-SURFACE DATUM

$\begin{array}{lll} & \text { WATER } & \text { WATER } \\ \text { DATE } & \text { LEVEL MS } & \text { DEVEL MS }\end{array}$

JUN 04, 1992307.15 SS AUG 28, 1992309.05 SS

PERIOD OF RECORD HIGHEST 295.28 OCT 09, 1987 LOWEST 299.72 AUG 22, 1991

\section{SITE NUMBER 341825116164501}

LOCAL NUMBER OO3N007E32DO2S

On Twentynine Palms Marine Corps Base, supply well 12A, about 3.4 miles west of Surprise Spring. Drilled watertable well in alluvium. Diameter 16 inches, depth 570 feet, perforated 390-550 feet. Altitude of land-surface datum 2,544 feet. Water-level records available 1991 to current year.

WATER LEVELS IN FEET BELOW LAND-SURFACE DATUM

$$
\begin{array}{ll}
\text { WATER } & \text { WEVEL MS }
\end{array}
$$

JUN 06,1992

$$
\mathrm{P}
$$

PERIOD OF RECORD HIGHEST 290.05 MAY 21, 1991 LOWEST 290.05 MAY 21, 1991

SITE NUMBER 341833116144201

LOCAL NUMBER 003N007E34D01S

On Twentynine Palms Marine Corps Base, about 1.5 miles northwest of Surprise Spring. Drilled observation watertable well in alluvium of Tertiary age. Diameter 2 inches, depth 405 feet. Altitude of land-surface datum $2,500.9$ feet. Water-level records available 1976, 1986 to current year.

WATER LEVELS IN FEET BELOW LAND-SURFACE DATUM

$$
\begin{array}{lll} 
& \text { WATER } & \text { WATER } \\
\text { DATE } & \text { LEVEL MS }
\end{array}
$$

JUN 04, $1992271.43 \mathrm{~S}$ AUG 28, $1992272.99 \mathrm{~S}$

PERIOD OF RECORD HIGHEST 257.00 MAR 1, 2, 1976 LOWEST 368.85 MAY 21, 1991 
SITE NUMBER 341809116115801

LOCAL NUMBER 003N007E36G01S

On Twentynine Palms Marine Corps base, about 11 miles northwest of Headquarters. Drilled test water-table well in alluvium. Diameter 2 inches, depth measured 385 feet in 1982. Altitude of land-surface datum 2,111 feet. Waterlevel records available 1968, 1970, 1973, 1975, 1982, 1984 to current year.

WATER LEVELS IN FEET BELOW LAND-SURFACE DATUM

$$
\begin{array}{lll} 
& \text { WATER } & \\
\text { DATE } & \text { WEVER MS } & \text { DATE } \\
\text { LEVEL MS }
\end{array}
$$

JUN $05,1992282.88 \mathrm{~S} \quad$ AUG $28,1992283.08 \mathrm{~S}$

PERIOD OF RECORD HIGHEST 279.7 NOV 28,1970 LOWEST 286.09 JUL 22, 1986

SITE NUMBER 342603116501801

LOCAL NUMEER DO4N001E13MO1S

In town of Lucerne Valley about $0.35 \mathrm{mile}$ south of Highway 247 on Dallas Avenue. Drilled domestic water-table well. Diameter unknown, depth 270 feet in 1954. Altitude of land-surface datum 3,014 feet. Water-level records available $1953-71,1980$ to current year.

WATER LEVELS IN FEET BELOW LAND-SURFACE DATUM

$$
\begin{array}{lll} 
& \text { WATER } & \text { WATER } \\
\text { DATE } & \text { DEVEL MS } & \text { DETE }
\end{array}
$$

OCT 29, $1991175.99 \mathrm{~S} \quad$ MAY 28, $1992176.15 \mathrm{~S}$

PERIOD OF RECORD HIGHEST 170.00 JAN 1953 LOWEST 182.00 MAR 26, 1981

\section{SITE NUMBER 342609116541001}

LOCAL NUMBER 004N001E17LO1S

About 5 miles east of Lucerne Valley. Drilled domestic well. Diameter 8 inches, depth 301 feet, perforated 189-210, 230-250, 271-291 feet. Altitude of land-surface datum 2,979 feet. Water-level records available 1987 to current year.

WATER LEVELS IN FEET BELOW LAND-SURFACE DATUM

$$
\text { DATE HATER } \quad \text { LEVEL MS }
$$

JUN 04, $1992166.55 \mathrm{SR}$

PERIOD OF RECORD HIGHEST 149 JUL 28, 1987 LOWEST 154.17 MAY 22, 1990 
WATER LEVELS, SAN BERNARDINO COUNTY--Continued

WELL DESCRIPTIONS AND WATER-LEVEL MEASUREMENTS

WATER YEAR OCTOBER 1991 TO SEPTEMBER 1992

SITE NUMBER 342324116374901

LOCAL NUMBER 004N003E35J01S

About 20 miles east of Lucerne Valley, Drilled unused well. Diameter 4 inches, depth 512 feet, perforated 200-500 feet. Altitude of land-surface datum 2,920 feet. Recorder installed 1989. Water-levei records available 1989 to current year.

Missing record due to malfunction of recording instrument.

WATER LEVELS IN FEET BELOW LAND-SURFACE DATUM (OBSERVATIONS AT 1200 HOURS)

\begin{tabular}{|c|c|c|c|c|c|c|c|c|c|c|c|c|}
\hline DAY & $\propto C T$ & NOV & DEC & JAN & FEB & MAR & APR & MAY & JUN & JUL & AUG & SEP \\
\hline 1 & 136.03 & 136.02 & 136.04 & 136.03 & 136.01 & 136.03 & 136.01 & -- & $\cdots$ & -- & -- & - \\
\hline 2 & 136.04 & 136.03 & 136.01 & 135.99 & 136.04 & 136.01 & 136.04 & $\cdots$ & -- & --- & -- & -- \\
\hline 3 & 136.04 & 136.04 & 136.02 & 136.01 & 136.02 & 136.05 & 136.03 & $\cdots$ & -- & $\cdots$ & 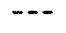 & -- \\
\hline 4 & 136.06 & 136.02 & 136.00 & 136.03 & 136.02 & 136.03 & 136.02 & --- & -- & --- & --- & -- \\
\hline 5 & 136.06 & 136.01 & 136.02 & 135.96 & 136.02 & 136.03 & 136.05 & --- & $\ldots$ & -- & $-\cdots$ & -- \\
\hline 6 & 136.05 & 136.02 & 136.01 & 136.04 & 136.02 & 136.03 & 136.05 & $\cdots$ & -- & -- & $\cdots$ & -- \\
\hline 7 & 136.05 & 136.04 & 136.01 & 136.02 & 136.05 & 136.02 & 136.04 & -- & -- & --- & --- & $-\infty$ \\
\hline 8 & 136.06 & 136.02 & 136.04 & 136.05 & 136.04 & 136.05 & 136.03 & -- & -- & -- & -- & -- \\
\hline 9 & 136.05 & 136.02 & 136.02 & 136.02 & 136.01 & 136.06 & 136.03 & -- & -- & -- & -- & -- \\
\hline 10 & 136.05 & 136.03 & 136.00 & 136.00 & 136.05 & 136.02 & 136.03 & --- & -- & -- & $\ldots$ & -- \\
\hline 11 & 136.03 & 136.04 & 136.04 & 135.97 & 136.05 & 136.02 & 136.03 & $-\cdots$ & -- & --- & --- & $\mathrm{S} 136.16$ \\
\hline 12 & 136.06 & 136.02 & 136.04 & 136.04 & 136.00 & 136.03 & 136.03 & --- & --- & $\cdots$ & $\cdots$ & 136.17 \\
\hline 13 & 136.06 & 135.98 & 136.05 & 136.01 & 136.03 & 136.02 & --- & -- & $\cdots$ & -- & --- & 136.17 \\
\hline 14 & 136.05 & 136.00 & 136.02 & 136.02 & 136.03 & 136.02 & -- & $\cdots$ & $\cdots$ & $\cdots$ & $\cdots$ & 136.17 \\
\hline 15 & 136.04 & 136.05 & 136.01 & 136.03 & 136.02 & 136.02 & $-\cdots$ & -- & --- & -- & -- & 136.18 \\
\hline 16 & 136.05 & 136.04 & 136.01 & 135,98 & 136.04 & 136.02 & $\cdots$ & -- & $\cdots$ & $\cdots$ & $\cdots$ & 136.19 \\
\hline 17 & 136.06 & 136.01 & 136.01 & 136.00 & 136.03 & 136.04 & --- & $\cdots$ & -- & $\cdots$ & $\cdots$ & 136.17 \\
\hline 18 & 136.06 & 136.04 & 136.01 & 136.04 & 136.04 & 136.03 & -- & -- & $\cdots$ & $\cdots$ & $\cdots$ & 136.16 \\
\hline 19 & 136.03 & 136.05 & 136.02 & 136.03 & 136.03 & 136.04 & $\cdots$ & -- & -- & $\ldots$ & $\cdots$ & 136.17 \\
\hline 20 & 136.04 & 136.01 & 136,04 & 136.02 & 136.03 & 136.01 & -- & -- & -- & $\cdots$ & $\cdots$ & 136.17 \\
\hline 21 & 136.03 & 136.02 & 136.01 & 136.04 & 136.05 & 136.05 & -- & -- & $\cdots$ & $\ldots$ & -- & 136.18 \\
\hline 22 & 136.06 & 136.05 & 136.03 & 136.03 & 136.03 & 136.03 & --- & --- & -- & $\cdots$ & -- & 136.17 \\
\hline 23 & 136.06 & 136.03 & 136.02 & 136.02 & 136.03 & 136.05 & $\cdots$ & --- & -- & $\cdots$ & $\cdots$ & 136.17 \\
\hline 24 & 136.05 & 136.00 & 136.02 & 136.01 & 136.03 & 136.03 & $\cdots$ & --- & -- & --- & -- & 136.16 \\
\hline 25 & 136.04 & 136.01 & 136.03 & 135.99 & 136.03 & 136.02 & --- & $\cdots$ & $\cdots$ & $\cdots$ & $\cdots$ & 136.19 \\
\hline 26 & 136.03 & 136.01 & 136.03 & 136.02 & 136.03 & 136.00 & -- & $\ldots$ & $\cdots$ & $\ldots$ & $\cdots$ & 136.19 \\
\hline 27 & 136.05 & 135.99 & 135.99 & 136.05 & 136.03 & 136.03 & $\cdots$ & S136.05 & $\cdots$ & $\cdots$ & $\cdots$ & 136.17 \\
\hline 28 & 136.03 & 136.03 & 136.02 & 136.02 & 136.01 & 136.04 & -- & --- & -- & $\ldots$ & $\cdots$ & 136.17 \\
\hline 29 & 135.99 & 135.99 & 136.00 & 136.02 & 136.00 & 136.02 & $\cdots$ & -- & $\cdots$ & -- & -- & 136.17 \\
\hline 30 & 136.05 & 136.03 & 136.05 & 136.03 & -- & 136.01 & -- & --- & $\cdots$ & -- & -- & 136.18 \\
\hline 31 & 136.02 & $\ldots$ & 136.02 & 136.00 & -- & 136.04 & --- & $\cdots$ & $\cdots$ & $\cdots$ & $\cdots$ & -- \\
\hline
\end{tabular}

PERIOD OF RECORD HIGHEST 135.96 JAN 05, 1992 LOWEST 136.19 SEP 16, 25, 26, 1992

S Manual steel tape measurement. 
WELL DESCRIPTIONS AND WATER-LEVEL MEASUREMENTS

WATER YEAR OCTOBER 1991 TO SEPTEMBER 1992

SITE NUMBER 343153116542301

LOCAL NUMBER 005N001E17D01S

About $6.5 \mathrm{miles}$ north of Lucerne Valley. Drilled unused water-table well in alluvium. Diameter 14 inches, depth

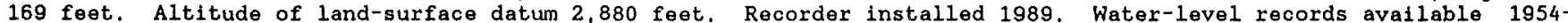
$55,1960-71,1976,1978$ to current year

Missing record due to malfunction of recording instrument.

WATER LEVELS IN FEET BELOW LAND-SURFACE DATUM (OBSERVATIONS AT 1200 HOURS)

\begin{tabular}{|c|c|c|c|c|c|c|c|c|c|c|c|c|}
\hline DAY & $\alpha C T$ & Nov & DEC & JAN & FEB & MAR & APR & MAY & JUN & JUL & AUG & SEP \\
\hline $\begin{array}{l}1 \\
2 \\
3 \\
4 \\
5\end{array}$ & $\begin{array}{l}158.61 \\
158.54 \\
158.56 \\
158.64 \\
158.70\end{array}$ & $\begin{array}{l}158.74 \\
158.77 \\
158.92 \\
158.81 \\
158.69\end{array}$ & $\begin{array}{l}159.17 \\
158.91 \\
159.03 \\
158.82 \\
158.85\end{array}$ & $\begin{array}{l}159.05 \\
158.87 \\
158.77 \\
158.99 \\
158.71\end{array}$ & $\begin{array}{l}158.76 \\
158.94 \\
158.90 \\
158.94 \\
158.82\end{array}$ & $\begin{array}{l}158.62 \\
158.55 \\
158.83 \\
158.80 \\
158.74\end{array}$ & $\begin{array}{l}158.51 \\
158.64 \\
158.59 \\
158.48 \\
158.60\end{array}$ & $\begin{array}{l}--- \\
--- \\
--- \\
-m- \\
---\end{array}$ & $\begin{array}{l}158.37 \\
158.37 \\
158.44 \\
158.37 \\
158.31\end{array}$ & $\begin{array}{l}158.13 \\
158.19 \\
158.15 \\
158.21 \\
158.22\end{array}$ & $\begin{array}{l}158.35 \\
158.28 \\
158.24 \\
158.29 \\
158.29\end{array}$ & $\begin{array}{l}158.35 \\
158.43 \\
158.36 \\
158.40 \\
158.39\end{array}$ \\
\hline $\begin{array}{r}6 \\
7 \\
8 \\
9 \\
10\end{array}$ & $\begin{array}{l}158.69 \\
158.65 \\
158.68 \\
158.70 \\
158.70\end{array}$ & $\begin{array}{l}158.68 \\
158.83 \\
158.77 \\
158.71 \\
158.82\end{array}$ & $\begin{array}{l}158.88 \\
158.75 \\
159.04 \\
159.09 \\
158.81\end{array}$ & $\begin{array}{l}159.03 \\
159.00 \\
159.19 \\
159.06 \\
158.90\end{array}$ & $\begin{array}{l}158.71 \\
158.83 \\
159.01 \\
158.82 \\
158.86\end{array}$ & $\begin{array}{l}158.73 \\
158.58 \\
158.73 \\
158.86 \\
158.82\end{array}$ & $\begin{array}{l}158.66 \\
158.63 \\
158.54 \\
158.52 \\
158.51\end{array}$ & $\begin{array}{l}--- \\
--- \\
--- \\
--- \\
---\end{array}$ & $\begin{array}{l}158.39 \\
158.51 \\
158.50 \\
158.44 \\
158.41\end{array}$ & $\begin{array}{l}158.27 \\
158.34 \\
158.38 \\
158.28 \\
158.20\end{array}$ & $\begin{array}{l}158.31 \\
158.33 \\
158.35 \\
158.35 \\
158.32\end{array}$ & $\begin{array}{l}158.36 \\
158.41 \\
158.38 \\
158.38 \\
158.40\end{array}$ \\
\hline $\begin{array}{l}11 \\
12 \\
13 \\
14 \\
15\end{array}$ & $\begin{array}{l}158.58 \\
158.68 \\
158.76 \\
158.77 \\
158.63\end{array}$ & $\begin{array}{l}158.93 \\
158.91 \\
158.63 \\
158.61 \\
158.95\end{array}$ & $\begin{array}{l}158.91 \\
159.13 \\
159.15 \\
159.02 \\
158.94\end{array}$ & $\begin{array}{l}158.63 \\
159.10 \\
159.10 \\
158.96 \\
159.09\end{array}$ & $\begin{array}{l}159.01 \\
158.70 \\
158.73 \\
158.92 \\
158.72\end{array}$ & $\begin{array}{l}158.68 \\
158.69 \\
158.69 \\
158.65 \\
158.64\end{array}$ & $\begin{array}{r}158.52 \\
158.56 \\
--- \\
--- \\
---\end{array}$ & $\begin{array}{l}-+- \\
--- \\
--- \\
--- \\
---\end{array}$ & $\begin{array}{l}158.47 \\
158.51 \\
158.36 \\
158.35 \\
158.43\end{array}$ & $\begin{array}{l}158.18 \\
158.28 \\
158.34 \\
158.33 \\
158.26\end{array}$ & $\begin{array}{l}158.25 \\
158.29 \\
158.36 \\
158.38 \\
158.29\end{array}$ & $\begin{array}{l}158.41 \\
158.40 \\
158.39 \\
158.39 \\
158.42\end{array}$ \\
\hline $\begin{array}{l}16 \\
17 \\
18 \\
19 \\
20\end{array}$ & $\begin{array}{l}158.64 \\
158.78 \\
158.87 \\
158.67 \\
158.59\end{array}$ & $\begin{array}{l}159.08 \\
158.79 \\
158.93 \\
159.17 \\
158.89\end{array}$ & $\begin{array}{l}158.92 \\
158.84 \\
158.87 \\
158.89 \\
159.17\end{array}$ & $\begin{array}{l}158.85 \\
158.71 \\
158.99 \\
159.07 \\
158.95\end{array}$ & $\begin{array}{l}158.95 \\
158.91 \\
158.90 \\
158.84 \\
158.75\end{array}$ & $\begin{array}{l}158.61 \\
158.67 \\
158.71 \\
158.73 \\
158.53\end{array}$ & $\begin{array}{l}-\cdots \\
--- \\
--- \\
---\end{array}$ & $\begin{array}{l}--- \\
--- \\
--- \\
--- \\
---\end{array}$ & $\begin{array}{l}158.56 \\
158.43 \\
158.43 \\
158.42 \\
158.40\end{array}$ & $\begin{array}{l}158.25 \\
158.31 \\
158.33 \\
158.27 \\
158.17\end{array}$ & $\begin{array}{l}158.25 \\
158.30 \\
158.36 \\
158.33 \\
158.30\end{array}$ & $\begin{array}{l}158.54 \\
158.43 \\
158.37 \\
158.43 \\
158.43\end{array}$ \\
\hline $\begin{array}{l}21 \\
22 \\
23 \\
24 \\
25\end{array}$ & $\begin{array}{l}158.66 \\
158.74 \\
158.79 \\
158.79 \\
158.76\end{array}$ & $\begin{array}{l}158.70 \\
158.99 \\
159.06 \\
158.78 \\
158.78\end{array}$ & $\begin{array}{l}158.95 \\
159.00 \\
158.94 \\
158.88 \\
158.97\end{array}$ & $\begin{array}{l}158.98 \\
159.02 \\
158.99 \\
158.88 \\
158.82\end{array}$ & $\begin{array}{l}158.86 \\
158.77 \\
158.85 \\
158.84 \\
158.79\end{array}$ & $\begin{array}{l}158.61 \\
158.67 \\
158.71 \\
158.76 \\
158.57\end{array}$ & $\begin{array}{l}--- \\
-\cdots \\
--- \\
---\end{array}$ & $\begin{array}{l}--- \\
--- \\
--- \\
--- \\
---\end{array}$ & $\begin{array}{l}158.47 \\
158.56 \\
158.45 \\
158.42 \\
158.47\end{array}$ & $\begin{array}{l}158.24 \\
158.25 \\
158.28 \\
158.34 \\
158.40\end{array}$ & $\begin{array}{l}158.28 \\
158.26 \\
158.32 \\
158.40 \\
158.46\end{array}$ & $\begin{array}{l}158.49 \\
158.52 \\
158.44 \\
158.37 \\
158.45\end{array}$ \\
\hline $\begin{array}{l}26 \\
27 \\
28 \\
29 \\
30 \\
31\end{array}$ & $\begin{array}{l}158.70 \\
158.77 \\
158.90 \\
158.56 \\
159.02 \\
158.88\end{array}$ & $\begin{array}{r}158.82 \\
158.71 \\
158.91 \\
158.62 \\
159.01 \\
-\end{array}$ & $\begin{array}{l}159.04 \\
158.89 \\
158.92 \\
158.89 \\
159.12 \\
159.14\end{array}$ & $\begin{array}{l}158.86 \\
159.08 \\
158.93 \\
158.96 \\
158.94 \\
158.82\end{array}$ & $\begin{array}{r}158.82 \\
158.78 \\
158.71 \\
158.57 \\
\ldots \ldots\end{array}$ & $\begin{array}{l}158.47 \\
158.52 \\
158.73 \\
158.72 \\
158.57 \\
158.59\end{array}$ & $\begin{array}{l}-\ldots- \\
-\cdots- \\
--- \\
--- \\
---\end{array}$ & $\begin{array}{c}- \\
S 158.41 \\
- \\
158.43 \\
158.49 \\
158.51\end{array}$ & $\begin{array}{r}158.48 \\
158.49 \\
155.44 \\
157.62 \\
157.84 \\
\end{array}$ & $\begin{array}{l}158.35 \\
158.29 \\
158.28 \\
158.24 \\
158.31 \\
158.33\end{array}$ & $\begin{array}{l}158.46 \\
158.39 \\
158.31 \\
158.31 \\
158.30 \\
158.31\end{array}$ & $\begin{array}{r}158.59 \\
158.51 \\
158.47 \\
158.50 \\
158.51\end{array}$ \\
\hline
\end{tabular}

PERIOD OF RECORD HIGHEST 62.27 APR 22, 1954 LOWEST 159.19 JAN 08, 1992

$S$ Manual steel tape measurement. 
WELL DESCRIPTIONS AND WATER-LEVEL MEASUREMENTS

WATER YEAR OCTOBER 1991 TO SEPTEMBER 1992

SITE NUMBER 342837116540401

LOCAL NUMBER 005N001E32L01S

About 2.5 miles northeast of Lucerne Valley, $2.25 \mathrm{miles}$ north of Highway 247 on Midway Avenue. Domestic well. Diameter 12 inches, depth 200 feet. Altitude of land-surface datum 2,870 feet. Water-level records available 1980 to current year.

WATER LEVELS IN FEET BELOW LAND-SURFACE DATUM

$\begin{array}{ll}\text { WATER } & \text { WATER } \\ \text { DATE } & \text { DATE }\end{array}$

OCT 29, 1991165.54 SP MAY 27, $1992 \quad$ P

PERIOD OF RECORD HIGHEST 136.88 MAR 19, 1980 LOWEST 168.38 OCT 31,1990

SITE NUMBER 345413116512902

LOCAL NUMBER O09N001E03H05S

About 1.5 miles west of Yermo. Drilled domestic well. Diameter 6 inches, depth 200 feet, perforated 100-200 feet. Altitude of land-surface datum 1,950 feet. Water-level records available 1989 to current year.

WATER LEVELS IN FEET BELOW LAND-SURFACE DATUM

$$
\begin{array}{ll} 
& \text { WATER } \\
\text { DATE } & \text { LEVEL MS }
\end{array}
$$

MAY 27, $1992 \quad P$

PERIOD OF RECORD HIGHEST 105.49 NOV 14,1990 LOWEST 121.44 JUN 01, 1989

SITE NUMBER 345410116544101

LOCAL NUMBER OO9NO01E06HO2S

About 5 miles northeast of Barstow. Drilled domestic woll. Diameter 6 inches, depth 320 feet. Altitude of land-surface datum 2,000 feet. Water-level records available 1988 to current year.

WATER LEVELS IN FEET BELOW LAND-SURFACE DATUM

$$
\text { DATE WATER }
$$

MAY $29,1992129.81 \mathrm{~S}$

PERIOD OF RECORD HIGHEST 124.37 MAY 24, 1990 LOWEST 129.81 MAY 29, 1992

SITE NUMBER 345249116483401

LOCAL NUMBER 009N002E07Q01S

About $1.8 \mathrm{miles}$ south of Yermo. Drilled irrigation well. Diameter 14 inches, depth 360 feet, perforated 100-360 feet. Altitude of land-surface datum 1,931 feet. Water-level records avallable 1987 to current year.

WATER LEVELS IN FEET BELOW LAND-SURFACE DATUM

$\begin{array}{lll} & \text { WATER } & \\ \text { DATE } & \text { LEVEL MS } & \text { DATER } \\ \text { LEVEL MS }\end{array}$

NOV 13, $1991 \quad \mathrm{P} \quad \mathrm{MAY} 28,1992122.60 \mathrm{~S}$

PERIOD OF RECORD HIGHEST 105.17 JUN 01, 1987 LOWEST 122.60 MAY 28,1992 
SITE NUMBER 345334116442801

LOCAL NUMBER OOSNO02E11C03S

About 4 miles east of Yermo and 2 miles south of Interstate 15. Drilled domestic well, Diameter 8 inches, depth 190 feet, perforated 50-190 feet. Altitude of land-surface datum 1,878 feet. Water-level records available 1987 to current year.

WATER LEVELS IN FEET BELOW LAND-SURFACE DATUM

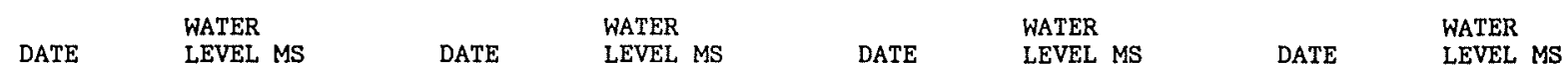

NOV $13,1991123.23 \mathrm{~S} \quad$ NOV $19,1991124.77 \mathrm{~S}$

MAR 10, $1992123.99 \mathrm{~S} \quad$ MAY $28,1992124.76 \mathrm{~S}$

PERIOD OF RECORD HIGHEST 111.61 MAY 20, 1988 LOWEST 124.77 NOV 19, 1991

SITE NUMBER 345206116445201

LOCAL NUMBER OO9NOO2E14NO2S

About 2.5 miles east of Barstow/Daggett airport. Irrigation water-table well. Diameter 12 inches, depth reported 300 feet, depth measured 215.3 feet in 1961. Altitude of land-surface datum 1,886 feet. Water-level records available $1928,1930-35,1937-58,1960-61,1980$ to current year.

WATER LEVELS IN FEET BELOW LAND-SURFACE DATUM

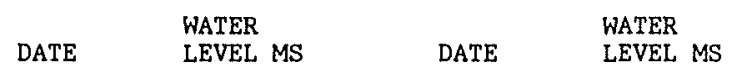

OCT 31, $199182.95 \mathrm{~S} \quad$ MAY 28, $199287.37 \mathrm{~s}$

PERIOD OF RECORD HIGHEST 15.30 NOV 16, 1945 LOWEST 87.37 MAY 28, 1992

SITE NUMBER 345208116424901

LOCAL NUMBER OO9NOO3E18NO1S

About 3 miles north of Newberry Springs. Drilled irrigation well, Diameter 14 inches, depth 400 feet, perforated 140-400 feet. Altitude of land-surface datum 1,863 feet. Water-level records available 1987,1989 to current year.

WATER LEVELS IN FEET BELOW LAND-SURFACE DATUM

$$
\begin{array}{ll} 
& \text { WATER } \\
\text { DATE } & \text { LEVEL MS }
\end{array}
$$

JUN $03,1992 \quad P$

$\begin{array}{lllllll}\text { RERIOD OF RECORD } & \text { HIGHEST } & 107 & \text { JAN 26, } 1987 & \text { LOWEST } & 107 & \text { JAN 26, } 1987\end{array}$

SITE NUMBER 344927116394101

LOCAL NUMBER 009N003E34N01S

In Newberry Springs, east of post office. Drilled domestic well. Diameter 8 inches, depth 99 feet in 1959. Altitude of land-surface datum 1,820 feet. Water-level records avallable 1959, 1964, 1967-71, 1980 to current year.

WATER LEVELS IN FEET BELOW LAND-SURFACE DATUM

$$
\text { DATE WATER } \quad \text { LEVEL MS }
$$

$\operatorname{OCT} 31,199185.12 \mathrm{~s}$

PERIOD OF RECORD HIGHEST 23.10 DEC 17, 1959 LOWEST 85.12 OCT 31,1991 
WELL DESCRIPTIONS AND WATER-LEVEL MEASUREMENTS

WATER YEAR OCTOBER 1991 TO SEPTEMBER 1992

SITE NUMBER 345343117350501

LOCAL NUMBER 009N004E05P02S

About 5 miles southeast of Harvard. Drilled domestic well. Diameter 6 inches, depth 180 feet, perforated 100-180 feet. Altitude of land-surface datum 1,780 feet. Water-level records available 1984, 1987 to current year.

WATER LEVELS IN FEET BELOW LAND-SURFACE DATUM

DATE WATER

JUN $03,199269.32 \mathrm{~s}$

PERIOD OF RECORD HIGHEST 64.44 MAY 22, $1990 \quad$ LOWEST 69.32 JUN 03, 1992

SITE NUMBER 345155116333601

LOCAL NUMBER 009N004E21B02S

About 8 miles northeast of Newberry Springs. Drilled domestic well. Diameter 6.62 inches, depth 160 feet, perforated 60-160 feet. Altitude of land-surface datum 1,783 feet. Water-level records available 1989 to current year.

WATER LEVELS IN FEET BELOW LAND-SURFACE DATUM

DATE $\quad$ WATER

JUN 03, $1992 \quad P$

PERIOD OF RECORD HIGHEST -. LOWEST --

SITE NUMBER 345600116523901

LOCAL NUMBER 010N001E28G03S

About 8 miles northeast of Barstow. Drilled domestic wel1. Diameter 6 inches, depth 227 feet, perforated 127-227 feet. Altitude of land-surface datum 1,985 feet. Water-level records available 1989 to current year.

WATER LEVELS IN FEET BELOW LAND-SURFACE DATUM

$$
\text { DATE WATER }
$$

MAY 29, $1992145.68 \mathrm{~S}$

PERIOD OF RECORD HIGHEST 131.16 MAY 24, 1990 LOWEST 145.68 MAY 29, 1992

SITE NUMBER 345446116485101

LOCAL NUMBER O1ON002E31LO3S

About 0.5 mile north of Yermo, Drilled domestic well. Diameter 6 inches, depth 240 feet, perforated 100-240 feet. Altitude of land-surface datum 1,935 feet. Water-level records available 1988 to current year. WATER LEVELS IN FEET BELOW LAND-SURFACE DATUM

DATE WATER

JUN 02, $1992121.91 \mathrm{~S}$

PERIOD OF RECORD HIGHEST 107.22 MAY 23, 1990 LOWEST 121.91 JUN 02, 1992 
SITE NUMBER 345703116350801

LOCAL NUMBER 010N004E2ODO1S

About 2 miles south of Manix. Drilled public-supply well. Diameter 6 inches, depth 300 feet, perforated 190-300 feet. Altitude of land-surface datum 1,735 feet. Water-level records available 1988 to current year.

WATER LEVELS IN FEET BELOW LAND-SURFACE DATUM

$$
\text { DATE WATER }
$$

Nov 13, $1991 \quad$ P

PERIOD OF RECORD HIGHEST 152.98 MAY 25, 1988 LOWEST 156.84 MAY 24, 1990

\section{SITE NUMBER 340907117133501}

LOCAL NUMBER O01N003W19R02S

In Del Rosa area of San Bernardino. Drilled domestic well in alluvium. Diameter 14 inches, depth 100 feet. Altitude of land-surface datum 1,447 feet. Water-level records available 1944, 1955, 1986 to current year.

WATER LEVELS IN FEET BELOW LAND-SURFACE DATUM

$\begin{array}{lll} & \text { WATER } & \\ \text { DATE } & \text { WETER } \\ \text { LEVE } & \text { LEVEL MS }\end{array}$

NOV 25, $199116.77 \mathrm{~S}$ JUN $17,199213.22 \mathrm{~s}$

PERIOD OF RECORD HIGHEST 11.82 MAY 21, 1986 LOWEST 38.4 JUL 20, 1955

SITE NUMBER 340830117133002

LOCAL NUMBER 001N003W29M02S

In San Bernardino at Patten State Hospital. Drilled unused well. Depth cased 501 feet, depth measured 366 feet in 1992. Altitude of land-surface datum 1,344 feet. Water-level records available 1964, 1992.

WATER LEVELS IN FEET BELOW LAND-SURFACE DATUM

$$
\begin{array}{ll}
\text { WATER } \\
\text { DATE } & \text { LEVEL MS }
\end{array}
$$

AUG $11,1992325.03 \mathrm{~S}$

PERIOD OF RECORD HIGHEST 325.03 AUG 11,1992 LOWEST 366.7 DEC 21,1964

SITE NUMBER 340722117135801

LOCAL NUMBER OO1NO03W31Q02S

In Harlem Springs area of San Bernardino near intersection of Base Line Street and Victoria Avenue. Diameter 12 inches, depth 201 feet. Altitude of land-surface datum 1,120 feet. Water-level records available 1929, $1986-89,1992$.

WATER LEVELS IN FEET BELOW LAND-SURFACE DATUM

$$
\text { DATE WATER } \quad \text { LEVEL MS }
$$

AUG $11,1992124.90 \mathrm{~S}$

PERIOD OF RECORD HIGHEST 44.13 JUN 19, 1986 LOWEST 124.90 AUG 11, 1992 
WELL DESCRIPTIONS AND WATER-LEVEL MEASUREMENTS

WATER YEAR OCTOBER 1991 TO SEPTEMBER 1992

SITE NUMBER 340808117120501

LOCAL NUMBER 001N003W33C01S

In San Bernardino, near Highland and Palm Avenues. Drilled unused well in alluvium. Diameter 24 inches, depth 598 feet, perforated 160-190, 370-412, 441-530 feet. Altitude of land-surface datum 1,424 feet. Water-level records available 1927, 1935-36, 1992 .

WATER LEVELS IN FEET BELOW LAND-SURFACE DATUM

DATE WATER

AUG $12,1992400.7 \quad \mathrm{~V}$

PERIOD OF RECORD HIGHEST 294.0 DEC 18,1927 LOWEST 400.7 AUG 12,1992

SITE NUMBER 340949117181501

LOCAL NUMBER 001N004W21B02S

In San Bernardino on Kendall Drive in Shandin Hills area. Drilled unused well in alluvium. Diameter 14 inches, depth 1,100 feet, perforated 200-350, 415-1,100 feet. Aititude of land-surface datum 1,322,40 feet. Water-level records avallable 1960 to current year.

WATER LEVELS IN FEET BELOW LAND-SURFACE DATUM

$\begin{array}{lll} & \text { WATER } & \text { WATER } \\ \text { DATE } & \text { LEVEL MS }\end{array}$

NOV $25,1991172.32 \mathrm{~S}$ JUN $16,1992179.25 \mathrm{~S}$

PERIOD OF RECORD HIGHEST 75.4 NOV 19, 1981 LOWEST 224.90 MAR 20, 1968

SITE NUMBER 340936117160802

LOCAL NUMBER 001N004W23G02S

In San Bernardino, south of 40 th Street on the levee. Drilled observation well in alluvium. Diameter 2 inches, depth 195 feet, perforated 175-195 feet. Altitude of land-surface datum 1,343.03 feet. Water-level records available 1971, 1986 to current year.

WATER LEVELS IN FEET BELOW LAND-SURFACE DATUM

$\begin{array}{lll} & \text { WATER } & \text { WATER } \\ \text { DATE } & \text { LEVEL MS }\end{array}$

NOV 21,1991 SD JUN $16,1992142.71 \mathrm{~S}$

PERIOD OF RECORD HIGHEST 136.34 FEB 05, 1988 LOWEST 181.35 JUN 20, 1991

SITE NUMBER 340936117160803

LOCAL NUMBER OO1N004W23G03S

In San Bernardino, south of 40 th Street on the levee. Drilled observation well in alluvium. Diameter 2 inches, depth measured 90 feet in 1986, perforated 75-95 feet. Altitude of land-surface datum 1,345.03 feet. Water-level records available 1971,1986 to current year.

WATER LEVELS IN FEET BELOW LAND-SURFACE DATUM

$$
\begin{array}{lll} 
& \text { WATER } & \\
\text { DATE } & \text { WATER } \\
\text { LATE } & \text { LEVEL MS }
\end{array}
$$

NOV 25,1991 SD JUN 16, $1992 \quad$ SD

PERIOD OF RECORD HIGHEST -- LOWEST -- 
WATER LEVELS, SAN BERNARDINO COUNTY--Continued

WELL DESCRIPTIONS AND WATER-LEVEL MEASUREMENTS

WATER YEAR OCTOBER 1991 TO SEPTEMBER 1992

\section{SITE NUMBER 340905117160402}

LOCAL NUMBER 001N004W23Q03S

In San Bernardino, south of 40 th Street on the levee. Drilled observation well in alluvium. Diameter 2 inches, depth 230 feet, perforated 167-230 feet. Altitude of land-surface datum 1,284.39 feet. Water-level records avallable 1971, 1986 to current year.

WATER LEVELS IN FEET BELOW LAND-SURFACE DATUM

$\begin{array}{lll} & \text { WATER } & \\ \text { DATE } & \text { LEVEL MS } & \text { WATER } \\ \text { LEVEL } & \text { LEVEL }\end{array}$

NOV $25,1991 \quad$ D $\quad J U N 16,1992178.89 \mathrm{~S}$

PERIOD OF RECORD HIGHEST 178.51 JUN $23,1986 \quad$ LOWEST 206.87 FEB 05,1988

SITE NUMBER 340905117160403

LOCAL NUMBER 001N004W23Q04S

In San Bernardino, south of 40 th Street on the levee. Drilled observation well in alluvium. Diameter 2 inches, original depth 115 feet, depth measured 108 feet in 1986, perforated 94-115 feet. Altitude of land-surface datum $1,284,39$ feet. Water-level records available 1971,1986 to current year.

WATER LEVELS IN FEET BELOW LAND-SURFACE DATUM

$$
\begin{array}{lll} 
& \text { WATER } & \\
\text { DATE } & \text { LEVEL MS } & \text { WATER } \\
& \text { DATE } & \text { LEVEL MS }
\end{array}
$$

NOV 25, $1991 \quad$ D $\quad$ JUN $16,1992 \quad$ SD

PERIOD OF RECORD HIGHEST -- LOWEST --

SITE NUMBER 340903117155101

LOCAL NUMBER 001N004W23R02S

In San Bernardino, south of 40 th Street on the levee. Drilled observation well in alluvium. Diameter 4 inches, depth 392 feet, perforated 245-392 feet. Altitude of land-surface datum 1, 268 feet. Water-1evel records available $1971-79,1986$ to current year.

WATER LEVELS IN FEET BELOW LAND-SURFACE DATUM

$$
\begin{array}{lll} 
& \text { WATER } & \\
\text { DATE } & \text { LEVEL MS } & \text { WATER } \\
\text { LETE } & \text { LEVEL MS }
\end{array}
$$

NOV 25, $1991 \quad$ D JUN 16, $1992 \quad$ SD

PERIOD OF RECORD HIGHEST 163.35 JUN 23,1986 LOWEST 255.93 DEC 12,1972 JAN 11,1973

SITE NUMBER 340903117155102

LOCAL NUMBER 001N004W23R03S

In San Bernardino, south of 40 th Street on the levee. Drilled observation well in alluvium. Diameter 2 inches, original depth 205 feet, depth measured 174 feet in 1988, perforated 163-205 feet. Altitude of land-surface datum 1,268 feet. Water-level records available 1971 , 1986 to current year.

WATER LEVELS IN FEET BELOW LAND-SURFACE DATUM

$$
\begin{array}{lll} 
& \text { WATER } & \\
\text { DATE } & \text { LEVEL MS } & \text { WATER } \\
& & \text { LEVEL MS }
\end{array}
$$

NOV 25,1991 D JUN $16,1992169.78 \mathrm{~S}$

PERIOD OF RECORD HIGHEST 160.44 JUN 23,1986 LOWEST 172.28 JUN 17,1987 
WELL DESCRIPTIONS AND WATER-LEVEL MEASUREMENTS

WATER YEAR OCTOBER 1991 TO SEPTEMBER 1992

SITE NUMBER 340903117155104

LOCAL NUMBER 001N004W23R05S

In San Bernardino, south of 40 th Street on the levee, Drilled observation well in alluvium. Diameter 2 inches, original depth 72 feet, depth measured 67 feet in 1988, perforated 58-72 feet. Altitude of land-surface datum 1,268 feet. Water-level records available 1971,1986 to current year.

WATER LEVELS IN FEET BELOW LAND-SURFACE DATUM

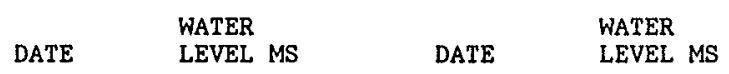

NOV 25,1991 D JUN 16,1992 SD

PERIOD OF RECORD HIGHEST -- LOWEST --

SITE NUMBER 340851117171101

LOCAL NUMBER 001N004W27B01S

In San Bernardino on Mountain View Avenue. Drilled public-supply well in alluvium. Diameter 20 inches, depth 577 feet, perforated 325-553 feet. Altitude of land-surface datum 1,253 feet. Water-level records available 1962 to current year.

WATER LEVELS IN FEET BELOW LAND-SURFACE DATUM

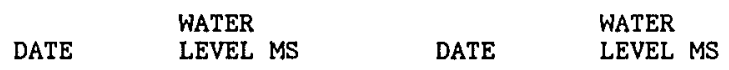
NOV 25, 1991
P JUN $16,1992263.58 \mathrm{~V}$

PERIOD OF RECORD HIGHEST 184.75 DEC 28,1987 LOWEST $296.00 \quad$ NOV 30,1967

SITE NUMBER 340743117162001

LOCAL NUMBER 001N004W35L01S

In San Bernardino about 0.1 mile southwest of intersection of 16 th Street and Crestriew Avenue. Drilled observation well in alluvium. Dianeter 3 inches, depth 256 feet, no perforations. Altitude of land-surface datum $1,130.34$ feet. Water-level records available 1888,1904 to current year.

WATER LEVELS IN FEET BELOW LAND-SURFACE DATUM (READINGS ABOVE LAND SURFACE INDICATED BY "+")

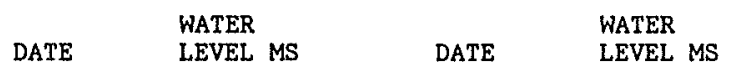
NOV $25,1991177,40 \mathrm{~S}$ JUN $16,1992176.51 \mathrm{~s}$

PERIOD OF RECORD HIGHEST +7.22 MAR 02, 1917 LOWEST 216.14 AUG 31,1967

SITE NUMBER 341209117260701

LOCAL NUMBER O01N005W06F01S

About 6 miles northwest of Rialto in Lytle Creek Wash. Drilled public-supply well. Diameter unknown, depth 113 feet. Altitude of land-surface datum 2,242.4 feet. A " 2 " site status is water level affected by falling water. Water-level records available 1919 to current year.

WATER LEVELS IN FEET BELOW LAND-SURFACE DATUM

$\begin{array}{lll} & \text { WATER } & \\ \text { DATE } & \text { LEVEL MS } & \text { WATER } \\ & \text { LEVEL MS }\end{array}$

NOV $25,1991 \quad \mathrm{P} \quad$ JUN $16,199263.47 \mathrm{VZ}$

PERIOD OF RECORD HIGHEST 19.7 MAY 01, $1980 \quad$ LOWEST 108.7 AUG 15,1923 
STTE NUMBER 341003117232001

LOCAL NUMBER O01N005W15Q01S

About 6 miles northwest of Rialto in Lytle Creek Wash. Drilled unused well in alluvium. Diameter 20 inches, original depth 425 feet, depth measured 387 feet in 1987. Altitude of land-surface datum 1,592.12 feet. Water-level records available 1929-65, 1987 to current year.

WATER LEVELS IN FEET BELOW LAND-SURFACE DATUM

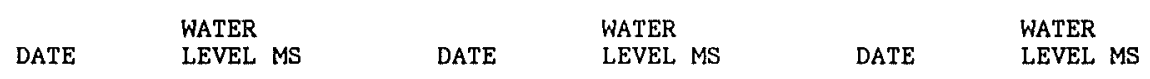

NOV 25, 1991 D JUN 15, $1992 \quad$ SD AUG 11, $1992 \quad$ D

PERIOD OF RECORD HIGHEST 48.7 OC'T 04, 1956 AUG 06, 29, OCT 03, NOV 04, DEC 05, 1957

LOWEST 424.7 NOV 01, DEC 01, 1961 JAN 01, FEB 01, MAR 01, APR 01, AUG 02, 31, 0CT 02, 1962

SITE NUMBER 340218117234601

LOCAL NUMBER 001N005W34M01S

About 2 miles northwest of Rialto. Drilled municipal well. Diameter 20 inches, depth 887 feet, perforated 525-860 feet. Altitude of land-surface datum 1,410 feet. Water-level records available 1986 , 1989 to current year.

WATER LEVELS IN FEET BELOW LAND-SURFACE DATUM

$\begin{array}{lll} & \text { WATER } & \\ \text { DATE } & \text { WEVEL MS } & \text { WATER } \\ & & \text { LEVEL MS }\end{array}$

DEC 05, 1991369.76 SV JUN $15,1992378.76 \mathrm{~S}$

PERIOD OF RECORD HIGHEST 357.88 SEP 21, $1989 \quad$ LOWEST $378.76 \quad$ JUN 15,1992

SITE NUMBER 341249117241101

LOCAL NUMBER 002N005W33J04S

South of Devore in Cajon Canyon Wash. Unused well. Diameter 6 inches, depth 87 feet. Altitude of land-surface datum 2,010 feet. Water-level records available 1986-92.

WATER LEVELS IN FEET BELOW LAND-SURFACE DATUM

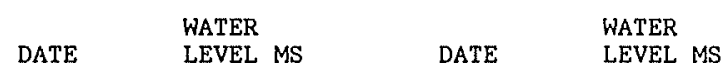

NOV $25,199165.09 \mathrm{~S}$ JUN $15,1992 \quad$ W

PERIOD OF RECORD HIGHEST 64.07 JUN 25, 1986 LOWEST 66.29 NOV 30, 1989

SITE NUMBER 342300117160301

LOCAL NUMBER 003N004W02C01S

About 4 miles southeast of Hesperia. Drilled public-supply well. Diameter 18 inches, depth 700 feet, perforated 420-700 feet. Altitude of land-surface datum 3,200 feet. Water-level records available 1988 to current year.

WATER LEVELS IN FEET BELOW LAND-SURFACE DATUM

DATE WATER

MAY 28, $1992362.63 \mathrm{~S}$

PERIOD OF RECORD HIGHEST 361,22 SEP 27, 1990 LOWEST 362,63 MAY 28, 1992 
WELL DESCRIPTIONS AND WATER-LEVEL MEASUREMENTS

WATER YEAR OCTOBER 1991 TO SEPTEMBER 1992

SITE NUMBER 342055117150201

LOCAL NUMBER 003N004W13F02S

About 5 miles southeast of Hesperia, 0.2 mile northwest of Mojave Forks Reservoir access road. Drilled domestic well. Diameter 8 inches, depth 269 feet, perforated 219-269 feet. Altitude of land-surface datum 3,064 feet. Water-level records available 1988 to current year.

WATER LEVELS IN FEET BELOW LAND-SURFACE DATUM

$\begin{array}{ll}\text { DATE } & \text { WATER } \\ \text { LEVEL MS }\end{array}$

$\operatorname{OCT} 29,1991 \quad P$

PERIOD OF RECORD HIGHEST -- LOWEST --

SITE NUMBER 341823117174501

LOCAL NUMBER 003N004W33H05S

About 1.2 miles east of Silverwood Lake outflow on Highway 173. Drilled domestic well. Diameter 6 inches, depth 105 feet, perforated 63-105 feet. Altitude of land-surface datum 3,210 feet. Water-level records available 1988 to current year. Previously published as well 003N004W33H01S.

WATER LEVELS IN FEET BELOW LAND-SURFACE DATUM

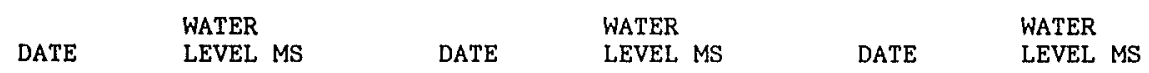

$\begin{array}{llllllll}\text { OCT 29, } 1991 & F & \text { MAR 11, } 1992 & \text { F } & \text { MAY 27, } 1992 & \text { F }\end{array}$

PERIOD OF RECORD HIGHEST -- LOWEST --

SITE NUMBER 342625116571301

LOCAL NUMBER 004N001W14C02S

In town of Lucerne Valley near intersection of Highways 247 and 18 . Drilled unused water-table well. Diameter 10 inches, depth 105 feet, cased to 30 feet. Altitude of land-surface datum 2,950 feet. Water-level records available 1954,1982 to current year.

WATER LEVELS IN FEET BELOW LAND-SURFACE DATUM

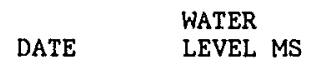

CT 29, $199121.93 \mathrm{~s}$

PERIOD OF RECORD HIGHEST 1.50 FEB $19,1954 \quad$ LOWEST 28.27 OCT 16,1985

SITE NUMBER 342519116591401

LOCAL NUMBER 004N001W21G01S

About 2 miles southwest of town of Lucerne Valley, 0.4 mile west of intersection of Custer Avenue and Sutter Road. Domestic well. Diameter unknown, depth 250 feet. Altitude of land-surface datum 3,121 feet. Water-1eve1 records available 1980 to current year.

WATER LEVELS IN FEET BELOW LAND-SURFACE DATUM

$\begin{array}{lll} & \text { WATER } & \\ \text { DATE } & \text { WEVEL MS } & \text { DATE } \\ \text { LEVEL MS }\end{array}$

OCT 29, 1991 173.90 SR MAY 27, 1992173.90 SR

PERIOD OF RECORD HIGHEST 168.35 DEC 11,1980 LOWEST 183.00 OCT 16,1985 
SITE NUMBER 342618117060701

LOCAL NUMBER 004N002W16E01S

About 7.2 miles southeast of Apple Valley. Drilled unused well. Diameter 8.62 inches, depth 380 feet, perforated 325-375 feet. Altitude of land-surface datum 3,190 feet. Water-1evel records avallable 1989 to current year.

WATER LEVELS IN FEET BELOW LAND-SURFACE DATUM

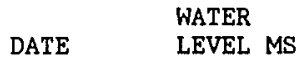

JUN 04, $1992339.48 \mathrm{~s}$

PERIOD OF RECORD HIGHEST 330 NOV 24,1980 LOWEST 339.48 JUN 04, 1992

SITE NUMBER 342630117104501

LOCAL NUMBER 004N003W15C01S

About 3 miles south of Apple Valley, Drilled domestic well. Diameter 8 inches, depth 390 feet, perforated 330-390 feet. Altitude of land-surface datum 3,076 feet. Water-level records available 1975 , 1987 to current year.

WATER LEVELS IN FEET BELOW LAND-SURFACE DATUM

$$
\begin{array}{ll} 
& \text { WATER } \\
\text { DATE } & \text { LEVEL MS }
\end{array}
$$

MAY $27,1992303.25 \mathrm{~S}$

PERIOD OF RECORD HIGHEST 215 JAN 23, 1975 LOWEST 303.25 MAY 27,1992

SITE NUMBER 342512117121801

LOCAL NUMBER 004N003W21M03S

About $5 \mathrm{miles}$ south of Apple Valley. Drilled unused well. Diameter 6 inches, depth 360 feet, perforated $300-320$,

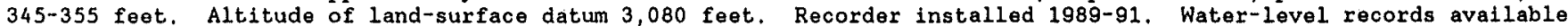
1982, 1989 to current year.

WATER LEVELS IN FEET BELOW LAND-SURFACE DATUM

$$
\begin{array}{ll} 
& \text { WATER } \\
\text { DATE } & \text { LEVEL MS }
\end{array}
$$

MAY 29, 1992278.79 SS

PERIOD OF RECORD HIGHEST 262 DEC 22, $1982 \quad$ LOWEST $285.20 \quad$ APR 30,1991

SITE NUMBER 342436117133801

LOCAL NUMBER 004N003W3OAOGS

About 7 miles south of Hesperia, 0.2 mile east of Mojave River, and 0.4 mile southwest of intersection of Rock Springs and Deep Creek Roeds. Drilled domestic we11. Diameter 8 inches, depth 123 feet, perforated $70-120$ feet. Altitude of land-surface datum 2,894 feet. Water-level records available 1987 to current year.

WATER LEVELS IN FEET BELOW LAND-SURFACE DATUM

$$
\begin{array}{llllll} 
& \text { WATER } & & \text { WATER } & & \text { WATER } \\
\text { DATE } & \text { LEVEL MS } & \text { DATE } & \text { LEVEL MS } & \text { DATE } & \text { LEVEL MS }
\end{array}
$$

OCT 29, $199187.27 \mathrm{~S} \quad$ MAR 11, $1992 \quad 69.94 \mathrm{~s} \quad \mathrm{MAY} 26,1992 \quad 67.50 \quad \mathrm{~V} \quad$ JUN $03,1992 \quad 69.15 \quad \mathrm{~S}$

PERIOD OF RECORD HIGHEST 67.50 MAY 26, 1992 LOWEST 87.27 OCT 29, 1991 
WELL DESCRIPTIONS AND WATER-LEVEL MEASUREMENTS

WATER YEAR OCTOBER 1991 TO SEPTEMBER 1992

SITE NUMBER 342813117143101

LOCAL NUMBER 004N004W01A01S

About 6 miles southeast of Victorville, near intersection of Bear Valley cutoff and Apple Valley Road. Drilled public-supply well. Diameter 14 inches, depth 275 feet, perforated 130-275 feet. Altitude of land-surface datum 2,836 feet. Water-level records available 1984 to current year.

WATER LEVELS IN FEET BELOW LAND-SURFACE DATUM
$\begin{array}{ll} & \text { WATER } \\ \text { DATE LEVEL MS }\end{array}$
DATE WATER
$\begin{array}{ll} & \text { WATER } \\ \text { DATE } & \text { LEVEL MS }\end{array}$
DATE
WATER

CT 29, $199154.59 \mathrm{~S}$

MAR 11, $1992 \quad 53.33 \quad \mathrm{~S}$

MAY 27, $199251.02 \mathrm{~S}$

JUN 03, $199250.72 \mathrm{~s}$

PERIOD OF RECORD HIGHEST 44 OCT 11, $1984 \quad$ LOWEST 55.64 JUN 19, 1991

SITE NUMBER 342740117165501

LOCAL NUMBER 004N004W03Q01S

About 2 miles north of Hesperia. Drilled industrial well. Diameter 6 inches, depth 400 feet, perforated 240-400 feet. Altitude of land-surface datum 3,021 feet. Water-level records avallable 1988 to current year.

WATER LEVELS IN FEET BELOW LAND-SURFACE DATUM

DATE WATER

MAY 28, $1992250.02 \mathrm{~V}$

PERIOD OF RECORD HIGHEST 250.02 MAY 28, 1992 LOWEST 256.18 JUN 18, 1991

SITE NUMBER 342639117194001

LOCAL NUMBER 004N004W08N03S

In Hesperia, about 1.2 miles north of intersection of Main Street and Balsam Road. Drilled public-supply well. Diameter 16 inches, depth 900 feet, perforated 600-900 feet. Altitude of land-surface datum 3,127 feet. Water-1evel records available 1988 to current year. Previously published in WDR CA-88-5 as well 004N004W08N01S.

WATER LEVELS IN FEET BELOW LAND-SURFACE DATUM

$$
\begin{array}{ll} 
& \text { WATER } \\
\text { DATE } & \text { LEVEL MS }
\end{array}
$$

MAY $03,1992437.20 \quad \mathrm{R}$

PERIOD OF RECORD HIGHEST 413 JUN 24, 1981 LOWEST 437.20 MAY 03, 1992

\section{SITE NUMBER 342450117151201}

LOCAL NUMBER 004N004W24P03S

In Hesperia, about $0.1 \mathrm{mile}$ north of the intersection of Main Street and Rock Springs Road. Drilled public-supply well. Diameter 18 inches, depth 605 feet, perforated 245-605 feet. Altitude of land-surface datum 3,045 feet. Water-level records available 1988 to current year. Previously published in WDR CA-88-5 as we11 004N004W24P01S.

WATER LEVELS IN FEET BELOW LAND-SURFACE DATUM

$$
\text { DATE WATER }
$$

MAY 28, $1992197.30 \mathrm{~S}$

PERIOD OF RECORD HIGHEST 180 APR 06, 1982 LOWEST 205.70 SEP 28, 1990 
SITE NUMBER 342743117211701

\section{LOCAL NUMBER 004N005W01K04S}

About 4 miles northwest of Hesperia. Drilled industrial well. Diameter 10.75 inches, depth 807 feet, perforated 465.5-807 feet. Altitude of land-surface datum 3,250 feet. Water-level records available 1986 , 1988 to current year. Previously published in WDR CA-88-5 as well. 004N005W01K01S.

WATER LEVELS IN FEET BELOW LAND-SURFACE DATUM

$$
\text { DATE WATER }
$$

MAY $27,1992454.47 \mathrm{~V}$

PERIOD OF RECORD HIGHEST 429 NOV 07, 1986 LOWEST 454,47 MAY 27, 1992

SITE NUMBER 342301117205001

LOCAL NUMBER 004N005W36R03S

About 4 miles southwest of Hesperia. Drilled public-supply well. Diameter 14 inches, depth 1,015 feet, perforated 795-1,015 feet. Altitude of land-surface datum 3,558 feet. Water-level records available 1984 , 1989 to current year.

WATER LEVELS IN FEET BELOW LAND-SURFACE DATUM

$$
\begin{array}{ll}
\text { WATER } \\
\text { DATE }
\end{array}
$$

MAY 29, $1992741.93 \mathrm{~V}$

PERIOD OF RECORD HIGHEST 735 APR 25, 1984 LOWEST 741.93 MAY 29, 1992

SITE NUMBER 343307117103201

LOCAL NUMBER 005N003W03H02S

About 3 miles north of Apple Valley. Drilled domestic well. Diameter 6 inches, depth 223 feet, perforated 108-129 feet. Altitude of land-surface datum 2,920 feet. Water-level records available 1987 to current year. WATER LEVELS IN FEET BELOW LAND-SURFACE DATUM

$$
\text { DATE WATER }
$$

MAY 27, $1992159.89 \mathrm{~S}$

PERIOD OF RECORD HIGHEST 105 JUL 07, 1987 LOWEST 159.89 MAY 27, 1992

SITE NUMBER 343218117123401

LOCAL NUMBER 005N003W05R03S

About 3 miles northwest of Apple Valley. Drilled domestic well. Diameter 7 inches, depth 150 feet. Altitude of land-surface datum 2,904 feet. Water-level records available 1989 to current year.

WATER LEVELS IN FEET BELOW LAND-SURFACE DATUM

$$
\text { DATE WATER }
$$

JUN $04,1992109.71 \mathrm{~S}$

PERIOD OF RECORD HIGHEST 109.71 JUN 04, 1992 LOWEST 111.57 MAY 21, 1990 
WELL DESCRIPTIONS AND WATER-LEVEL MEASUREMENTS

WATER YEAR OCTOBER 1991 TO SEPTEMBER 1992

SITE NUMBER 343150117151502

LOCAL NUMBER 005N004W11P03S

In Apple Valley. Drilled domestic water-table well. Diameter 8 inches, depth 145 feet. Altitude of land-surface datum 2,788 feet. Water-level records avallable 1978, 1980 to current year.

WATER LEVELS IN FEET BELOW LAND-SURFACE DATUM

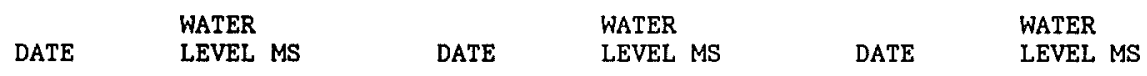

$\begin{array}{llllllllll}\text { NOV 01, } 1991 & 60.02 & \mathrm{~S} & \text { MAY } 11,1992 & 57.42 & \mathrm{~S} & \text { JUN } 03,1992 & 57.24 & \mathrm{~S} \\ \text { MAR 11, } 1992 & 57.42 & \mathrm{~S} & 26 & & 61.48 & \mathrm{~S} & & & \end{array}$

PERIOD OF RECORD HIGHEST 51.27 OCT $19,1983 \quad$ LOWEST 65.47 JUN 18, 1991

SITE NUMBER 343104117150501

LOCAL NUMBER 005N004W13F02S

About 3 miles east of Victorville, 0.6 mile southeast of intersection of Apple Valley Road and Chamber Lane. Drilled public-supply well. Diameter 12 inches, depth 260 feet, perforated $80-260$ feet. Altitude of land-surface datum 2,820 feet. Water-level records available 1988 to current year. Previously published in WDR CA-88-5 as well $005 \mathrm{~N} 004 \mathrm{~W} 13 \mathrm{P} 01 \mathrm{~S}$.

WATER LEVELS IN FEET BELOW LAND-SURFACE DATUM

DATE WATER

MAY 28, $199293.17 \mathrm{~S}$

PERIOD OF RECORD HIGHEST 93.17 MAY 28, 1992 LOWEST 93.17 MAY 28, 1992

SITE NUMBER 342918117153201

LOCAL NUMBER O05N004W25N01S

About 3 miles south of Victorville, 1.2 miles north of Bear Valley Road and about 0.5 mile east of Mojave River. Drilled domestio well. Diameter 14 inches, depth 400 feet, perforated 100-400 feet. Altitude of land-surface datum 2,800 feet. Water-level records available 1988 to current year.

WATER LEVELS IN FEET BELOW LAND-SURFACE DATUM

$\begin{array}{lll} & \text { WATER } & \\ \text { DATE } & \text { WEVEL MS } & \text { DATE } \\ \text { LEVEL MS }\end{array}$

NOV 01, $1991 \quad$ P MAY 26, $1992 \quad 40.95$ SR

PERIOD OF RECORD HIGHEST -- LOWEST --

SITE NUMBER 342953117173101

LOCAL NUMBER OOSNOO4W27DO8S

About 5 miles north of Hesperia. Drilled public-supply well. Diameter 16 inches, depth 520 feet, perforated 290-510 feet. Altitude of land-surface datum 2,929 feet. Water-level records available 1988 to current year. Previously published in WDR CA-88-5 as well 005N004W27D01S.

WATER LEVELS IN FEET BELOW LAND-SURFACE DATUM

$\begin{array}{ll} & \text { WATER } \\ \text { DATE } & \text { LEVEL MS }\end{array}$

MAY 27, 1992122.64 SR

PERIOD OF RECORD HIGHEST 128.48 JUN 19, 1991 LOWEST 128.48 JUN 19, 1991 
SITE NUMBER 342931117201501

LOCAL NUMBER 005N004W30K02S

About 5 miles northwest of Hesperia. Drilled public-supply well. Diameter 16 inches, depth 650 feet, perforated 340-480, 500-640 feet. Altitude of land-surface datum 3,058 feet. Water-level records avallable 1985, 1988 to current year. Previously published in WDR CA-88-5 as well 005N004W30K01S.

WATER LEVELS IN FEET BELOW LAND-SURFACE DATUM

$$
\begin{array}{ll} 
& \text { WATER } \\
\text { DATE } & \text { LEVEL MS }
\end{array}
$$

MAY 27, $1992304,84 \mathrm{~S}$

PERIOD OF RECORD HIGHEST 275 OCT 22,1985 LOWEST 304.84 MAY 27, 1992

SITE NUMBER 343213117275301

LOCAL NUMBER OOSNOOGW12E02S

About 4 miles southwest of Adelanto. Drilled domestic well. Diameter 8 inches, depth 400 feet, perforated 230-500 feet. Altitude of land-surface datum 3,060 feet. Water-level records available 1989 to current year,

WATER LEVELS IN FEET BELOW LAND-SURFACE DATUM

$$
\begin{array}{ll} 
& \text { WATER } \\
\text { DATE } & \text { LEVEL MS }
\end{array}
$$

MAY 29, $1992176.02 \mathrm{~S}$

PERIOD OF RECORD HIGHEST 172.21 MAY 17,1989 LOWEST 178.00 NOV 15,1990

SITE NUMBER 343038117341701

LOCAL NUMBER 005N007W24D03S

North of Pearblossom Highway on Old Phelan Road. Drilled public-supply well. Diameter 14 inches, depth 660 feet. Altitude of land-surface datum 3,347 feet. Water-level records available 1980 to current year.

WATER LEVELS IN FEET BELOW LAND-SURFACE DATUM

$$
\begin{array}{lll} 
& \text { WATER } & \\
\text { DATE } & \text { LEVEL MS } & \text { WATER } \\
& \text { DATE } & \text { LEVEL MS }
\end{array}
$$

$\operatorname{OCT} 30,1991435.74 \mathrm{~s} \quad \operatorname{MAY} 27,1992 \quad 0$

PERIOD OF RECORD HIGHEST 402.09 DEC 01, $1981 \quad$ LOWEST $435.74 \quad$ OCT 30,1991

SITE NUMBER 343341117101601

LOCAL NUMBER 006N003W34J03S

About 4 miles north of Apple Valley. Drilled public-supply well, Diameter 14 inches, depth 305 feet, perforated 175-305 feet. Altitude of land-surface datum 2,975 feet. Water-level records available 1988 to current year. Previously published in WDR CA-88-5 as well 006N003W34J01S.

WATER LEVELS IN FEET BELOW LAND-SURFACE DATUM

$$
\text { DATE HATER }
$$

MAY 29, 1992229.28 SR

PERIOD OF RECORD HIGHEST 227.07 NOV $21,1990 \quad$ LOWEST $228.55 \quad$ MAY 12,1988 
WELL DESCRIPTIONS AND WATER-LEVEL MEASUREMENTS

WATER YEAR OCTOBER 1991 TO SEPTEMBER 1992

SITE NUMBER 343458117204201

LOCAL NUMBER O06N004W30D10S

About 2 miles east of George AFB, 0.6 mile northeast of intersection of Shay and Turner Roads. Drilled domestic we11. Diameter 8 inches, depth 145 feet, perforated 25-145 feet. Altitude of land-surface datum 2,691 feet, Watex-level records available 1988 to current year. Previously published in WDR CA-88-5 as well 006N004W30DO1s.

WATER LEVELS IN FEET BELOW LAND-SURFACE DATUM

$\begin{array}{llll} & \text { WATER } & \text { WATER } & \text { WATER } \\ \text { DATE } & \text { LEVEL MS } & \text { DATE } & \text { LEVEL MS }\end{array}$

$\begin{array}{lllllllll}\text { OCT 30, } 1991 & P & \text { MAR } 10,1992 & 49.70 & \text { SR } & \text { MAY 26, } 1992 & 44.75 \mathrm{~S}\end{array}$

PERIOD OF RECORD HIGHEST 29.04 MAY 18, 1988 LOWEST 44.75 MAY 26, 1992

SITE NUMBER 343723117205501

LOCAL NUMBER 006N005W12H01S

About 1.5 miles north of Oro Grande and 0.2 mile west of National Trails Highway. Drilled irrigation well. Diameter 16 inches, depth 150 feet, perforated 30-150 feet. Altitude of land-surface datum 2,580 feet.

Water-level records available 1976, 1987 to current year.

WATER LEVELS IN FEET BELOW LAND-SURFACE DATUM

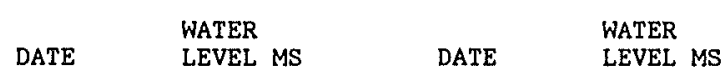

OCT 29, $1991 \quad 15.15 \mathrm{~S} \quad$ MAY 26, $199215.45 \mathrm{~S}$

PERIOD OF RECORD HIGHEST 14.35 MAY 23, 1990 LOWEST 30 FEB 01, 1976

SITE NUMBER 343900117261801

LOCAL NUMBER 006N005W19J02S

About 1.5 miles northwest of Adelanto. Drilled unused water-table well. Diameter 9 inches, depth 1,200 feet. Altitude of land-surface datum 2,838 feet. Water-level records available 1979 to current year.

WATER LEVELS IN FEET BELOW LAND-SURFACE DATUM

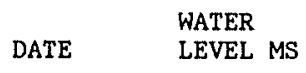

OCT 30, $1991 \quad 0$

PERIOD OF RECORD HIGHEST 78.3 APR $17,27,1979$ OCT 25,1989 LOWEST 80.03 OCT 17,1985

SITE NUMBER 343534117303001

LOCAL NUMBER OO6N006W21J02S

About 8 miles west of Adelanto. Drilled domestic well. Diameter 6 inches, depth 200 feet, perforated 110-140, 160-200 feet. Altitude of land-surface datum 2,886 feet. Water-level records available 1987-89, 1991 to current year. Previously published in WDR CA-88-5 as well 006N006W21J01S. Water levels published in 1989-90 were for wel1 006N006W21J01S.

WATER LEVELS IN FEET BELOW LAND-SURFACE DATUM

$$
\begin{array}{ll}
\text { WATER } & \text { WEVEL MS }
\end{array}
$$

MAY 27, $1992133.03 \mathrm{~s}$

PERIOD OF RECORD HIGHEST 133,03 MAY 27, 1991 MAY 27, 1992 LOWEST 140 JAN 16, 1987 
SITE NUMBER 343712117361701

LOCAL NUMBER O06N007W10P02S

About 1.3 miles north of El Mirage Road on El Mirage Airport Road. Domestic well. Diameter 12 inches 0-260 feet, 10 inches 260-290 feet, 8 inches 290-320 feet, depth 320 feet, perforated $70-85,110-170,210-265,300-320$ feet. Altitude of land-surface datum 2,864 feet. Water-level records available 1962, 1980 to current year.

WATER LEVELS IN FEET BELOW LAND-SURFACE DATUM

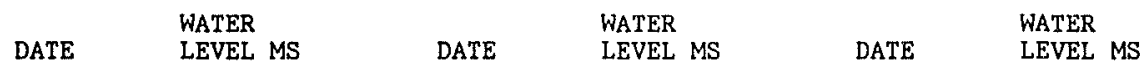

OCT 30, $199137.49 \mathrm{~S} \quad$ MAY 26, $199238.30 \mathrm{~s} \quad$ JUN 23, $199238.60 \mathrm{~s}$

PERIOD OF RECORD HIGLEST 26.52 APR 10, 1980 LOWEST 40.00 JAN 1962

SITE NUMBER 343606117365601

LOCAL NUMBER O06N007W21A02S

About 1 mile east of El Mirage. Drilled domestic well. Diameter 6 inches, depth 200 feet, perforated 80-100, 162-194 feet. Altitude of land-surface datum 2,909 feet. Water-level records available 1989 to current year.

WATER LEVELS IN FEET BELOW LAND-SURFACE DATUM

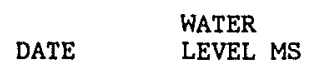

MAY 26, $199271.20 \mathrm{~S}$

PERIOD OF RECORD HIGHEST 70.64 NOV 01, $1990 \quad$ LOWEST 71.69 JUL 26, 1989

SITE NUMBER 344230117200501

LOCAL NUMBER 007N004W07K02S

About 2.6 miles south of Helendale on National Trails Highway. Drilled domestio well. Diameter 6 inches, depth 200 feet, perforated 80-180 feet. Altitude of land-surface datum 2,490 feet. Water-level records available 1988 to current year.

WATER LEVELS IN FEET BELOW LAND-SURFACE DATUM

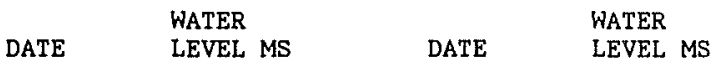

OCT $29,199150.37 \mathrm{~S} \quad \mathrm{MAY} 26,1992 \quad 51.51 \mathrm{SR}$

PERIOD OF RECORD HIGHEST 44.4 JUN 03, 1988 LOWEST 51.61 JUN 20, 1991

SITE NUMBER 344301117361901

LOCAL NUMBER 007N007W10C02S

About 17 miles west of Adelanto. Domestic well. Diameter 6 inches, depth reported 300 feet. Altitude of land-surface datum 3,257 feet. Water-level records available 1989 to current year.

WATER LEVELS IN FEET BELOW LAND-SURFACE DATUM

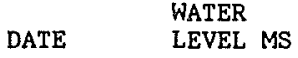

MAY 26, 1992

PERIOD OF RECORD

HIGHES

80.85 NOV 01,1990

LOWEST

82.60 MAY 19, 1989 
WELL DESCRIPTIONS AND WATER-LEVEL MEASUREMENTS

WATER YEAR OCTOBER 1991 TO SEPTEMBER 1992

SITE NUMBER 344535117003701

LOCAL NUMBER 008N001W29D02S

About 10 miles south of Barstow. Drilled unused well. Diameter 6 inches, depth 213 feet, perforated $60-213$ feet. Altitude of land-surface datum 2,850 feet. Water-level records available 1988 to current year. Previously published in WDR CA-88-5 as well 008N001W29D01S.

WATER LEVELS IN FEET BELOW LAND-SURFACE DATUM

$$
\text { DATE WATER } \quad \text { LEVEL MS }
$$

MAY $28,1992 \quad 91.07 \mathrm{~S}$

PERIOD OF RECORD HIGHEST 90.85 JUN 25, 1991 LOWEST 100.75 NOV 14, 1990

SITE NUMBER 344841117125301

LOCAL NUMBER 008N003W05J01S

About 9 miles northeast of Helendale, 2.1 miles southeast of intersection of Hinkley Road and National Trails Highway, adjacent to south bank of Mojave River. Drilled domestic well. Diameter 9 inches, depth 115 feet. Altitude of land-surface datum 2,290 feet. Water-level records available 1987 to current year.

WATER LEVELS IN FEET BELOW LAND-SURFACE DATUM

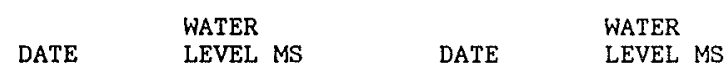
OCT $30,1991 \quad 16.67 \mathrm{~S}$
MAY $26,199211.22 \mathrm{~S}$

PERIOD OF RECORD HIGHEST 6.49 APR 22, 1987 LOWEST 16.67 OCT 30,1991

SITE NUMBER 344726117145501

LOCAL NUMBER 008N004W13B01S

About 15 miles southwest of Barstow, north of National Trails Highway. Drilled commercial well. Diameter 10 inches, depth unknown. Altitude of land-surface datum 2,330 feet. Water-level records available 1988 to current year.

WATER LEVELS IN FEET BELOW LAND-SURFACE DATUM

$\begin{array}{ll}\text { DATE } & \text { WATER } \\ \text { LEVEL MS }\end{array}$

OCT $30,1991 \quad P$

PERIOD OF RECORD HIGHEST _.. LOWEST _.

SITE NUMBER 344714117172001

LOCAL NUMBER 008N004W15C01S

About 6 miles southwest of Hodge. Drilled domestic well. Diameter 8 inches, depth 131 feet, perforated

55-131 feet. Altitude of land-surface datum 2,365 feet. Water-level records available July 1989 to current year.

WATER LEVELS IN FEET BELOW LAND-SURFACE DATUM

$\begin{array}{ll} & \text { WATER } \\ \text { DATE } & \text { LEVEL MS }\end{array}$

NOV 13,1991

$$
\mathrm{p}
$$

PERIOD OF RECORD

HIGHEST 33.90 JUL 14,1989 LOWEST 43.14 OCT 26,1990 
LOCAL NUMBER 008N004W23Q01S

About 4 miles northeast of Helendale. Drilled domestic well. Diameter 6 inches, depth 253 feet, perforated 152-253 feet. Altitude of land-surface datum 2,489 feet. Water-level records available 1987 to current year. WATER LEVELS IN FEET BELOW LAND-SURFACE DATUM

$$
\text { DATE WATER }
$$

MAY $28,1992173.30 \quad \mathrm{RR}$

PERIOD OF RECORD HIGHEST 170.25 SEP 23,1987 LOWEST 181.48 JUN 14,1990

SITE NUMBER 345314116580401

LOCAL NUMBER 009N001W10G06S

About 1.5 miles southeast of Interstate 15 in the Mojave River. Drilled domestic well. Diameter 8 inches, depth 107 feet, perforated 75-105 feet. Altitude of land-surface datum 2, 058 feet. Water-1evel records available 1987 to current year. Previously published in WDR-CA-89-5 as well 009N001W10G05S.

WATER LEVELS IN FEET BELOW LAND-SURFACE DATUM

$$
\begin{array}{lll} 
& \text { WATER } & \\
\text { DATE } & \text { WEVEL MS } & \text { WATER } \\
\text { LATE } & \text { LEVEL MS }
\end{array}
$$

$\operatorname{OCT} 30,199117.51 \mathrm{~S} \quad \operatorname{MAY} 29,199217.82 \mathrm{~s}$

PERIOD OF RECORD HIGHEST 10.76 OCT 28, 1988 LOWEST 17.82 MAY 29, 1992

SITE NUMBER 345254116572404

LOCAL NUMBER DO9N001W11M11S

In Barstow on U.S. Marine Corps Base, west of golf course, Drilled domestic well, Diameter 4 inches, depth 75 feet, perforated 35-75 feet. Altitude of land-surface datum 2,015 feet. Water-level records available 1985 to current year.

WATER LEVELS IN FEET BELOW LAND-SURFACE DATUM

$\begin{array}{lllll} & & \text { WATER } & \text { WATER } \\ \text { DATE } & \text { WATER } & \text { DATE } & \text { LEVEL MS } & \text { DATE }\end{array}$

OCT $30,1991 \quad 11.24 \mathrm{~s} \quad \operatorname{MAR} 11,199211.14 \mathrm{~s} \quad$ MAY $27,199211.16 \mathrm{~s}$

PERIOD OF RECORD HIGHEST 3.40 JUN 19,1985 LOWEST 11.24 OCT 30,1991

SITE NUMBER 345243116563802

LOCAL NUMBER 009N001W11R02S

In Barstow on U.S. Marine Corps Base near golf course. Drilled observation water-table well. Diameter 2 inches, depth 102 feet, 97.90 feet in 1992, well point 100-102 feet. Altitude of land-surface datum $2,032.51$ feet. Water-level records available 1972-73, 1975 to current year.

WATER LEVELS IN FEET BELOW LAND-SURFACE DATUM

$\begin{array}{llllll} & & & & \text { WATER } \\ \text { DATE } & \text { WATER } & \text { WATER } & \text { WATER } \\ \text { LEVEL MS } & \text { DATE } & \text { LEVEL MS } & \text { DATE } & \text { LEVEL MS }\end{array}$

$\begin{array}{llllllllllllll}\text { OCT } 30,1991 & 25.64 & \mathrm{~S} & \text { MAR } 11,1992 & 25.49 & \mathrm{~S} & \text { MAY } 28, & 1992 & 25.37 & \mathrm{~S} & \text { MAY } 29,1992 \quad 57.87\end{array}$

PERIOD OF RECORD HIGHEST 19.02 JUN $17,1983 \quad$ LOWEST 57.87 MAY 29,1992 
WELL DESCRIPTIONS AND WATER-LEVEL MEASUREMENTS

WATER YEAR OCTOBER 1991 TO SEPTEMBER 1992

SITE NUMBER 345228116560001

LOCAL NUMBER 009N001W13B02S

About 4 miles southeast of Barstow and 0.7 mile north of Interstate 40 . Drilled domestic well. Diameter 8 inches, depth 110 feet, perforated 30-110 feet, Altitude of land-surface datum 2,000 feet. Water-level records available 1987 to current year.

WATER LEVELS IN FEET BELOW LAND-SURFACE DATUM

$\begin{array}{lll} & \text { WATER } & \\ \text { DATE } & \text { WETER } \\ \text { LEVEL MS } & \text { LEVEL MS }\end{array}$

OCT $30,199124.72 \mathrm{~S} \quad$ MAY $27,199226.50 \mathrm{~S}$

PERIOD OF RECORD HIGHEST 14.80 MAY 25, 1988 LOWEST 26.50 MAY 27, 1992

SITE NUMBER 345225116555001

LOCAL NUMBER 009N001W13B03S

About 4 miles southeast of Barstow, 0.5 mile northwest of intersection of National Trails Highway and Nebo Street, near bank of Mojave River channel. Drilled observation well in alluvium. Diameter 2 inches, depth 60 feet, perforated 45-50 feet. Altitude of land-surface datum 1,995 feet. Water-level records available 1987 to ourrent year.

WATER LEVELS IN FEET BELOW LAND-SURFACE DATUM

$\begin{array}{lllll} & \text { WATER } & \text { WATER } & \text { WATER } \\ \text { DATE } & \text { LEVEL MS } & \text { DATE } & \text { LEVEL MS } & \text { DATE }\end{array}$

$\operatorname{OCT} 30,1991 \quad 19.76 \mathrm{~s} \quad$ MAR 10, $1992 \quad 21.05 \mathrm{~s} \quad$ MAY 27, $1992 \quad 21.64 \mathrm{~s}$

PERIOD OF RECORD HIGHEST 8.75 MAY 21, 1987 LOWEST 21.64 MAY 27, 1992

SITE NUMBER 345225116555002

LOCAL NUMBER 009N001W13B04S

About 4 miles southeast of Barstow, 0.5 mile northwest of intersection of National Trails Highway and Nebo Street, near bank of Mojave River channel. Drilled observation well in alluvium. Diameter 2 inches, depth 120 feet, perforated 105-110 feet. Altitude of land-surface datum 1,995 feet. Water-level records avallable 1987 to current year.

WATER LEVELS IN FEET BELOW LAND-SURFACE DATUM

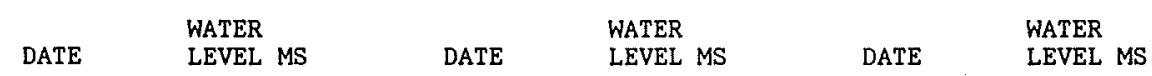
OCT $30,199119.42 \mathrm{~S}$
MAR 10, $1992 \quad 20.72 \quad \mathrm{~S}$
MAY 27, $1992 \quad 21.29 \mathrm{~S}$

PERIOD OF RECORD HIGHEST 8.37 MAY 21, 1987 LOWEST 23.97 JUN 11, 1991

SITE NUMBER 345214116554001

LOCAL NUMBER OO9NOO1W13H02S

About 4 miles southeast of Barstow and 0.5 mile north of Interstate 40 . Drilled domestic well. Diameter 8 inches, depth 160 feet, perforated 65-108 feet. Altitude of land-surface datum 2,000 feet. Water-level records available 1954, 1958, 1960, 1988 to current year.

WATER LEVELS IN FEET BELOW LAND-SURFACE DATUM

$\begin{array}{lllll} & \text { WATER } & & & \text { WATER } \\ \text { DATE } & \text { LEVEL MS } & \text { DATE } & \text { WEVEL MS } & \text { DATE }\end{array}$

OCT 30, $199127.04 \mathrm{~S} \quad \operatorname{MAR} 10,1992 \quad 28.43 \quad \mathrm{~S} \quad \operatorname{MAY} 27,199229.15 \mathrm{~s}$

PERIOD OF RECORD HIGHEST 17.78 MAY 25, 1988 LOWEST 57.39 SEP 23, 1960 
SITE NUMBER 345339117065001

LOCAL NUMBER 009N002W05N07S

About 6 miles west of Barstow, $0.2 \mathrm{mile}$ south of Mojave River, and 0.3 mile northeast of intersection of Lenwood and Agate Roads. Drilled domestic well. Diameter 8 inches, depth 195 feet, perforated 80-195 feet. Altitude of land-surface datum 2,184 feet. Water-level records available 1987 to current year.

WATER LEVELS IN FEET BELOW LAND-SURFACE DATUM

$$
\begin{array}{lll} 
& \text { WATER } & \\
\text { DATE } & \text { WEVEL MS } & \text { DATE } \\
\text { LEVEL MS }
\end{array}
$$

OCT $30,199199.53 \mathrm{~S} \quad$ MAY $27,199298.14 \mathrm{~S}$

PERIOD OF RECORD HIGHEST 65.24 MAY 29, 1987 LOWEST 99.53 OCT 30, 1991

SITE NUMBER 345422117071001

LOCAL NUMBER O09N002W06A04S

About 5 miles west of Barstow. Drilled domestic well. Diameter 8 inches, depth 300 feet, perforated $80-300$ feet. Altitude of land-surface datum 2,181 feet. Water-level records available 1989 to current year.

WATER LEVELS IN FEET BELOW LAND-SURFACE DATUM

$$
\text { DATE WATER }
$$

MAY 28, $1992101.82 \mathrm{SR}$

PERIOD OF RECORD HIGHEST -- LOWEST --

SITE NUMBER 345153117080701

LOCAL NUMBER 009N003W13R01S

About 2 miles southwest of Lenwood. Drilled domestic water-table well in alluvium. Diameter 12 inches, depth 212 feet. Altitude of land-surface datum 2,245 feet. Water-level records available 1954, 1963-71, 1979 to current year.

WATER LEVELS IN FEET BELOW LAND-SURFACE DATUM

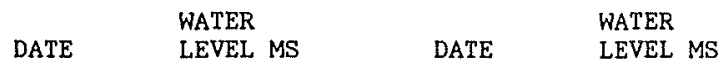

OCT 30, $1991104.89 \mathrm{~S} \quad$ MAY 28, $199298.44 \mathrm{~V}$

PERIOD OF RECORD HIGHEST 60.60 APR 20, 1954 LOWEST 104.89 OCT 30,1991

SITE NUMBER 345119117113601

LOCAL NUMBER 009N003W21K01S

About 5.5 miles south of Hinkley, 2.3 miles northwest of Johnston Corner and National Trails Highway. Drilled domestic well. Diameter 8 inches, depth 150 feet, perforated 50-150 feet. Altitude of land-surface datum 2,246 feet. Water-level records available 1987 to current year.

WATER LEVELS IN FEET BELOW LAND-SURFACE DATUM

$$
\begin{array}{lll} 
& \text { WATER } & \\
\text { DATE } & \text { WEVEL MS } & \text { DATE } \\
\text { LEVEL MS }
\end{array}
$$

NOV $13,199195.68 \mathrm{~S} \quad \mathrm{MAY} 28,1992 \quad 83.15 \mathrm{SP}$

PERIOD OF RECORD HIGHEST 73.02 OCT 26,1988 LOWEST 95.68 NOV 13,1991 
WELL DESCRIPTIONS AND WATER-LEVEL MEASUREMENTS

WATER YEAR OCTOBER 1991 TO SEPTEMBER 1992

\section{SITE NUMBER 345112117101901}

LOCAL NUMBER OOSN003W22J01S

About 10 miles southwest of Barstow. Drilled irrigation well. Diameter 12 inches, depth 205 feet, perforated 70-205 feet. Altitude of land-surface datum 2,240 feet. Water-level records available 1987-91. We11 destroyed.

WATER LEVELS IN FEET BELOW LAND-SURFACE DATUM

$$
\text { DATE WATER } \quad \text { LEVEL MS }
$$

Nov $13,1991 \quad$ W

PERIOD OF RECORD HIGHEST 62 MAR 30, 1987 LOWEST 92.06 MAY 25, 1990

SITE NUMBER 345059117082901

LOCAL NUMBER 009N003W24Q02S

About 8 miles southwest of Barstow. Drilled domestic well, Diameter 8 inches, depth 232 feet, perforated 122-132 feet. Altitude of land-surface datum 2,340 feet. Water-level records available 1987 to current year.

WATER LEVELS IN FEET BELOW LAND-SURFACE DATUM

$$
\text { DATE WATER } \quad \text { LEVEL MS }
$$

MAY 28, 1992199.45

PERIOD OF RECORD HIGHEST 192.93 JUN 13, 1990 LOWEST 199.45 MAY 28, 1992

SITE NUMBER 345703117055101

LOCAL NUMBER 010N002W16N01S

About 6 miles northwest of Barstow. Drilled domestic well. Diameter 6 inches, depth 238 feet, perforated 195-235 feet. Altitude of land-surface datum 2,230 feet. Water-level records available 1978, 1988 to current year.

WATER LEVELS IN FEET BELOW LAND-SURFACE DATUM

$$
\text { DATE } \quad \text { WATER }
$$

MAY 27, $1992132.00 \mathrm{~S}$

PERIOD OF RECORD HIGHEST 125 DEC 16, 1978 LOWEST 132.05 JUN 12, 1991

SITE NUMBER 345426117050801

LOCAL NUMBER 010NO02W33R16S

About 3.5 miles west-northwest of Barstow, $0.3 \mathrm{mile}$ south of Highway 58 . Drilled domestic well. Diameter 8 inches, depth 150 feet, perforated $80-150$ feet. Altitude of land-surface datum 2,159 feet. Water-level records available 1987 to current year. Previously published in WDR CA-88-5 as well 010N002W33R01S.

WATER LEVELS IN FEET BELOW LAND-SURFACE DATUM

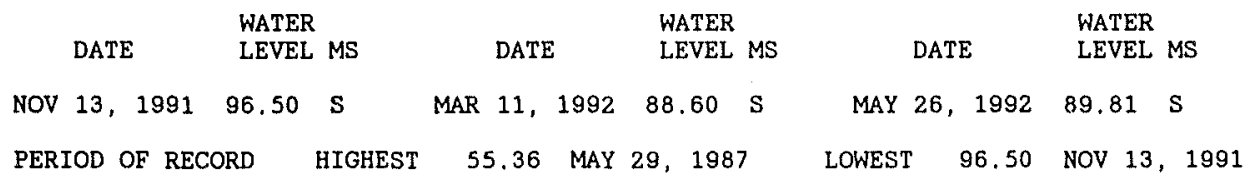


SITE NUMBER 345546117104601

LOCAL NUMBER 010N003W27F01S

About 11 miles west of Barstow. Drilled domestio well. Diameter 6 inches, depth 130 feet, perforated $80-130$ feet. Altitude of land-surface datum 2,169 feet. Water-level records available 1989 to current year.

WATER LEVELS IN FEET BELOW LAND-SURFACE DATUM

$$
\begin{array}{ll} 
& \text { WATER } \\
\text { DATE } & \text { LEVEL MS }
\end{array}
$$

MAY $27,1992 \quad$ P

PERIOD OF RECORD HIGHEST 91.00 MAY 26, 1989 LOWEST $92.85 \quad$ JUN 11,1991

SITE NUMBER 345542117121201

LOCAL NUMBER 010N003W28M01S

About 5 miles north of Hodge. Drilled domestic well. Diameter 6 inches, depth 217 feet, perforated $75-217$ feet. Altitude of land-surface datum 2,176 feet. Water-level records available 1987 to current year.

WATER LEVELS IN FEET BELOW LAND-SURFACE DATUM

$$
\begin{array}{ll} 
& \text { WATER } \\
\text { DATE } & \text { LEVEL MS }
\end{array}
$$

MAY 26, $199266.75 \mathrm{~S}$

PERIOD OF RECORD HIGHEST 66 JUL $21,1987 \quad$ LOWEST 72.57 MAY 21,1990

SITE NUMBER 345428117083201

LOCAL NUMBER 010N003W36Q01S

About $9.4 \mathrm{miles}$ southwest of Barstow. Drilled irrigation well. Diameter 12 inches, depth 254 feet, perforated 103-254 feet. Altitude of land-surface datum 2,188 feet. Water-level records available 1978 , 1989 to current year. WATER LEVELS IN FEET BELOW LAND-SURFACE DATUM

$$
\begin{array}{ll} 
& \text { WATER } \\
\text { DATE } & \text { LEVEL MS }
\end{array}
$$

MAY 27, 1992

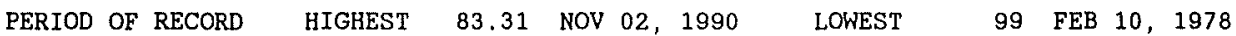

SITE NUMBER 350039117112101

LOCAL NUMBER 011N003W28R02S

About 5 miles north of Hinkley. Drilled unused well. Diameter 12 inches, depth 241.8 feet in 1978 , 222.75 feet in 1990. Altitude of land-surface datum 2,073 feet. Water-level records available 1953-71, 1978, 1980 to current year.

WATER LEVELS IN FEET BELOW LAND-SURFACE DATUM

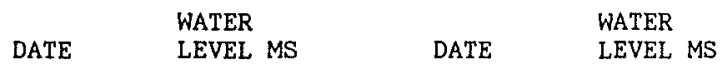

OCT 31, 199159.05 SR MAY 27, $1992 \quad \mathrm{P}$

PERIOD OF RECORD HIGHEST 26,00 JUN $26,1953 \quad$ LOWEST $57,80 \quad$ JUN 11,1991 
WELL DESCRIPTIONS AND WATER-LEVEL MEASUREMENTS

WATER YEAR OCTOBER 1991 TO SEPTEMBER 1992

SITE NUMBER 340348117013001

LOCAL NUMBER 001S001W19P01S

About 2 miles north of Yucaipa. Drilled unused well in alluvium. Diameter 16 inches, depth drilled 495 feet, perforated 49-350 feet. Altitude of land-surface datum 2,940 feet. Water-level records available 1949, 1951 , $1953,1960,1963,1968,1970,1987$ to current year.

WATER LEVELS IN FEET BELOW LAND-SURFACE DATUM

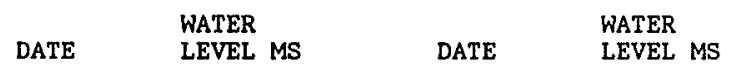

NOV 26, $1991343.63 \mathrm{~S}$ JUN $15,1992343.20 \mathrm{~V}$

PERIOD OF RECORD HIGHEST 337.11 SEP 18, 1989 LOWEST 433.32 OCT 02, 1970

SITE NUMBER 340355117001901

LOCAL NUMBER 001S001W20Q01S

About 2.5 miles northeast of Yucaipa, Domestic well, Diameter 16 inches, depth unknown, Altitude of land-surface datum 3,390 feet. Water-level records available 1955, 1960, 1968, 1986 to current year.

WATER LEVELS IN FEET BELOW LAND-SURFACE DATUM

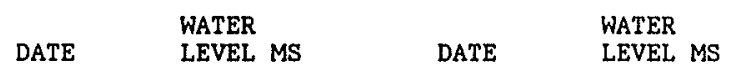

NOV $26,199151.82 \mathrm{~S} \quad$ JUN $15,199249.66 \mathrm{~S}$

PERIOD OF RECORD HIGHEST 45.54 JAN 27, 1986 LOWEST 64.8 MAR 31, 1960

SITE NUMBER 340257117015301

LOCAL NUMBER 001S001W30N01S

About 1 mile north of Yucaipa near intersection of Oak Glen Road and Bryant Street. Drilled observation well in alluvium. Diameter 2 inches, original depth 500 feet, depth measured 457 feet in 1970, perforated 440-460 feet. Altitude of land-surface datum 2,820 feet. Water-level records available 1969-70, 1987 to current year.

WATER LEVELS IN FEET BELOW LAND-SURFACE DATUM

$\begin{array}{lll} & \text { WATER } & \\ \text { DATE } & \text { WATER } \\ \text { LEVEL MS } & \text { DEVEL MS }\end{array}$

NOV 26, $1991377.78 \mathrm{~s} \quad$ JUN $15,1992393.00 \mathrm{~V}$

PERIOD OF RECORD HIGHEST 230,44 JUN 21, $1991 \quad$ LOWEST 454 OCT 15, 1970

SITE NUMBER 340620117065001

LOCAL NUMBER 001S002W08C02S

About 3 miles east of East Highlands, north of Greenspot Road, and west of the Santa Ana River. Drilled unused well. Diameter 24 inches, depth measured 176.1 feet in 1988. Altitude of land-surface datum 1,806.7 feet. Water-level records available 1927-28, 1940,1950-51,1954-67, 1978 to current year.

WATER LEVELS IN FEET BELOW LAND-SURFACE DATUM

$\begin{array}{lll} & \text { WATER } & \\ \text { DATE } & \text { WATER } \\ \text { LEVEL MS } & \text { LEVEL MS }\end{array}$

NOV $26,199175.39 \mathrm{~s} \quad$ JUN $12,1992 \quad 59.29 \mathrm{~s}$

PERIOD OF RECORD HIGHEST 19.7 MAR 15, 1978 LOWEST 149.2 DEC 04, 1959 
SITE NUMBER 340533117053901

LOCAL NUMBER 001 SOO2W09P01S

About 2 miles northeast of Mentone, west of Emerald Road in citrus grove. Diameter 18 inches, depth measured 172.7 feet in 1988, perforated 60-120, 125-152 feet. Altitude of land-surface datum 2,130 feet. Water-1eve1 records available 1925,1986 to current year.

WATER LEVELS IN FEET BELOW LAND-SURFACE DATUM

$\begin{array}{lll} & \text { WATER } & \\ \text { DATE } & \text { WEVEL MS } & \text { WATER } \\ & \text { LEVEL MS }\end{array}$

NOV $26,1991160.16 \mathrm{~S}$ JUN $15,1992144.51 \mathrm{~S}$

PERIOD OF RECORD HIGHEST 80 MAY 21, 1925 LOWEST 160.16 NOV 26, 1991

SITE NUMBER 340443117023501

LOCAL NUMBER 001S002W13P01S

About 2 miles north of Yucaipa at U.S. Forest Service Station on Mill Creek Road, Drilled unused well in alluvium. Diameter 12 inches, depth measured 101.5 feet in 1988, perforated 126-144 feet. Altitude of land-surface datum 2,705 feet. Water-level records available 1954-55, 1985 to current year.

WATER LEVELS IN FEET BELOW LAND-SURFACE DATUM

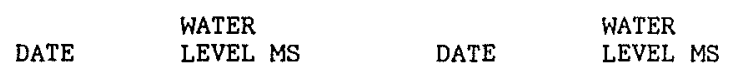

NOV $26,199197.72 \mathrm{~S} \quad$ JUN $15,199291.62 \mathrm{~S}$

PERIOD OF RECORD HIGHEST 73.96 SEP 19, 1985 LOWEST 109.35 APR 27, 1955

SITE NUMBER 340307117070201

LOCAL NUMBER 001S002W29M01S

About 2.5 miles east of Redlands, east of Crafton Avenue on Highland Avenue. Drilled irrigation well in alluvium. Diameter 20 inches, depth drilled 506 feet, perforated 235-490 feet. Altitude of land-surface datum 1,852 feet. Water-level records available 1948, 1950-56, 1962 to current year.

WATER LEVELS IN FEET BELOW LAND-SURFACE DATUM

$$
\begin{array}{lll} 
& \text { WATER } & \text { WATER } \\
\text { DATE } & \text { LEVEL MS }
\end{array}
$$

NOV 26, $1991245.15 \mathrm{~S} \quad$ AUG $12,1992232.76 \mathrm{~S}$

PERIOD OF RECORD HIGHEST 176.4 SEP $18,1980 \quad$ LOWEST 370.5 OCT 27,1950

SITE NUMBER 340346117080001

LOCAL NUMBER 001S002W30C01S

In Redlands at intersection of Colton and Opal Avenues, Drilled irrigation well in alluvium. Diameter 18 inches, depth 285 feet, perforated 134-248 feet. Altitude of land-surface datum 1,649 feet. Water-level records available $1928,1930,1932,1936-92$.

WATER LEVELS IN FEET BELOW LAND-SURFACE DATUM

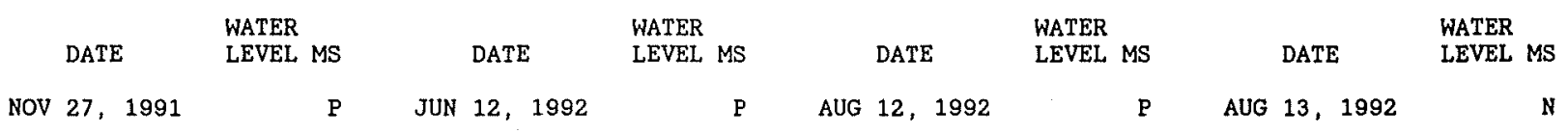

PERIOD OF RECORD HIGHEST 48.0 APR 17, 1948 LOWEST 163.6 OCT 20, 1953 
WELL DESCRIPTIONS AND WATER-LEVEL MEASUREMENTS

WATER YEAR OCTOBER 1991 TO SEPTEMBER 1992

SITE NUMBER 340234117020201

LOCAL NUMBER 001S002W36H01S

In Yucaipa on Bryant Street. Drilled unused well in alluvium. Diameter 12 inches to 422 feet, 10.75 inches 417-517 feet, 8 inches 517-614 feet, original depth 426 feet in 1946, deepened to 614 feet in 1951 . Altitude of land-surface datum 2,770 feet. Water-level records available 1953, 1987-92.

WATER LEVELS IN FEET BELOW LAND-SURFACE DATUM

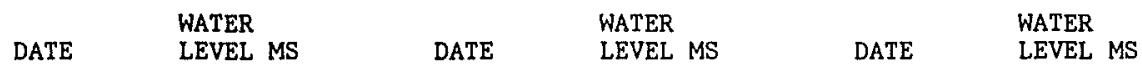

NOV 26, 1991 D JUN 15, 1992 VD AUG 13, 1992 N

PERIOD OF RECORD HIGHEST 311.78 JUN 29, 1988 LOWEST 378 SEP 16, 1953

SITE NUMBER 340215117025701

LOCAL NUMBER 001S002W36N01S

In Yucaipa on Cedar Avenue, Drilled public-supply well. Diameter 15 inches, depth 523 feet. Altitude of land-surface datum 2,559 feet. Water-level records available 1945-60, 1971 to current year.

WATER LEVELS IN FEET BELOW LAND-SURFACE DATUM

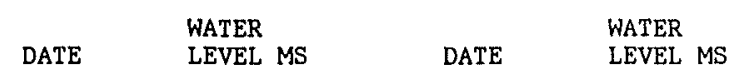

NOV $26,1991205.65 \mathrm{~S}$ JUN $15,1992208.94 \mathrm{~S}$

PERIOD OF RECORD HIGHEST 136.9 APR 03, 1946 LOWEST 294.5 APR 06, 1977

SITE NUMBER 340649117082901

LOCAL NUMBER 001S003W01H01S

East of East Highlands. Drilled irrigation well. Diameter 20 inches, depth 465 feet, perforated 250-308, 326-348, 360-414 feet. Altitude of land-surface datum 1,541.3 feet. Water-level records available 1931-76, 1986 to current year.

WATER LEVELS IN FEET BELOW LAND-SURFACE DATUM

$\begin{array}{ll} & \text { WATER } \\ \text { DATE } & \text { LEVEL MS }\end{array}$

AUG 12, $1992289.15 \mathrm{~S}$

PERIOD OF RECORD HIGHEST 135.2 MAR 24, 1970 LOWEST 417.6 AUG 19, 1965

SITE NUMBER 340637117095201

LOCAL NUMBER 001S003W02P01S

In East Highlands on Greenspot Road. Drilled unused well. Diameter 18 inches, depth 438 feet, perforated 180-418 feet. Altitude of land-surface datum 1,369.4 feet. Water-level records available 1992.

WATER LEVELS IN FEET BELOW LAND-SURFACE DATUM

$$
\text { DATE WATER }
$$

AUG $12,1992184.17 \mathrm{~S}$

PERIOD OF RECORD HIGHEST 184.17 AUG 12, 1992 LOWEST 184.17 AUG 12, 1992 
SITE NUMBER 340605117122401

LOCAL NUMBER 001S003W09E02S

Northwest of Redlands, east of Norton AFB. Drilled industrial well in alluvium. Diameter 14 inches, depth 401 feet, perforated 211-288, 315-320, 355-385 feet. Altitude of land-surface datum 1, 190 feet. Water-1evel records available 1955 to current year.

WATER LEVELS IN FEET BELOW LAND-SURFACE DATUM

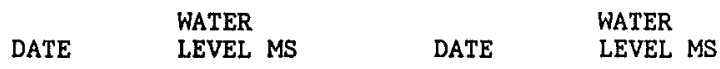

NOV $27,1991159.95$ VR JUN $12,1992154.34$ SS

PERIOD OF RECORD HIGHEST 51.9 OCT $31,1983 \quad$ LOWEST $231.2 \quad$ OCT 27,1965

SITE NUMBER 340506117122901

LOCAL NUMBER 001S003W17H01S

In Redlands, west of Alabama Street. Drilled unused well in alluvium. Diameter 18 inches, depth 220 feet, perforated 97-220 feet. Altitude of land-surface datum 1,222.65 feet. Water-level records available 1926, $1931-64,1986$ to current year.

WATER LEVELS IN FEET BELOW LAND-SURFACE DATUM

$$
\begin{array}{lll} 
& \text { WATER } & \\
\text { DATE } & \text { LEVEL MS } & \text { DATER }
\end{array}
$$

NOV $27,1991167.24 \mathrm{~s}$ JUN $12,1992164.81 \mathrm{~s}$

PERIOD OF RECORD HIGHEST 49.3 APR 28, 1941 LOWEST 204.5 FEB 28, 1963

SITE NUMBER 340426117135401

LOCAL NUMBER 001S003W19G02S

About 1.5 miles northeast of Loma Linda, west of California Street, and north of Lugonia Avenue. Unused well. Diameter 20 inches, depth unknown. Altitude of land-surface datum 1,135 feet. Water-level records available $1931-82,1985$ to current year.

WATER LEVELS IN FEET BELOW LAND-SURFACE DATUM

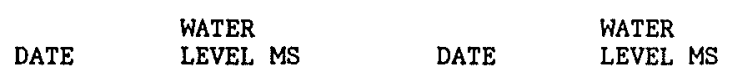

DEC $02,1991126.86$ SS JUN $12,1992122,68$ SS

PERIOD OF RECORD HIGHEST 14.9 MAR 30, 1945 LOWEST 223.3 APR 07,1966

SITE NUMBER 340340117115901

LOCAL NUMBER 001S003W28C01S

In Redlands near intersection of Redlands Boulevard and Tennessee Street. Drilled unused well in alluvium. Diameter 18 inches, depth 309 feet, perforated 80-90, 174-204, 220-309 feet. Altitude of land-surface datum 1,270 feet. Water-level records available 1928, 1936, 1954-55, 1987 to current year.

WATER LEVELS IN FEET BELOW LAND-SURFACE DATUM

$$
\begin{array}{lll} 
& \text { WATER } & \\
\text { DATE } & \text { LEVEL MS } & \text { WATER } \\
\text { LEVEL MS }
\end{array}
$$

NOV $27,1991153.29 \mathrm{~S}$ JUN $12,1992152.54 \mathrm{~s}$

PERIOD OF RECORD HIGHEST 69.7 JUN 09,1928 LOWEST 153.29 NOV 27,1991 
LOCAL NUMBER O01S003W31A03S

In Bryn Mawr area of San Bernardino on Whittier Avenue. Drilled unused well. Diameter 16 inches, depth 472 feet. Altitude of land-surface datum 1,203 feet. Water-level records available 1935-65, 1970-71, 1987 to current year.

WATER LEVELS IN FEET BELOW LAND-SURFACE DATUM

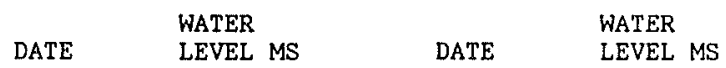

Nov $27,1991146.81 \mathrm{~V}$ JUN $12,1992135.64 \mathrm{~V}$

PERIOD OF RECORD HIGHEST 64.9 MAR 28, 1945 LOWEST 237.8 NOV 24, 1964

SITE NUMBER 340244117120001

LOCAL NUMBER 001S003W33C01S

In Redlands. Unused well. Diameter 10 inches, original depth 462 feet, depth measured 264 feet in 1953 , Altitude of land-surface datum 1,310 feet. Water-level records available 1931-32, 1935-49, 1954, 1975-79, 1986 to current year.

WATER LEVELS IN FEET BELOW LAND-SURFACE DATUM

$$
\begin{array}{lll} 
& \text { WATER } & \\
\text { DATE } & \text { WEVEL MS } & \text { DATE } \\
\text { LEVEL MS }
\end{array}
$$

NOV $27,1991159.79 \mathrm{~S} \quad$ JUN $12,1992146.16 \mathrm{~S}$

PERIOD OF RECORD HIGHEST 73.8 MAR 28, 1945 LOWEST 159.79 NOV 27, 1991

SITE NUMBER 340241117094402

LOCAL NUMBER 001S003W35G05S

In Redlands near intersection of Highland Avenue and Roosevelt Road. Drilled unused well, Diameter 10 inches, depth 570 feet. Altitude of land-surface datum $1,534.8$ feet. Water-level records available 1913, $1921,1925-57$, $1962-65,1969-71,1985$ to current year.

WATER LEVELS IN FEET BELOW LAND-SURFACE DATUM (READINGS ABOVE LAND SURFACE INDICATED. BY "+")

$\begin{array}{lll} & \text { WATER } & \\ \text { DATE } & \text { WEVEL MS } & \text { DATE } \\ \text { LEVEL MS }\end{array}$

NOV $27,199164.52 \mathrm{~S} \quad$ JUN $12,199256.63 \mathrm{~s}$

PERIOD OF RECORD HIGHEST +1.8 JUN 26,1945 LOWEST 163.3 SEP 29, 1956

SITE NUMBER 340707117162706

LOCAL NUMBER 001S004W02D06S

In San Bernardino near Sierra High School. Drilled monitoring well in alluvium. Diameter 2 inches, depth 530 feet, perforated 520-530 feet. One of three multi-level wells in vault at this site. Altitude of land-surface datum 1,077 feet. Water-level records available 1991 to current year.

WATER LEVELS IN FEET BELOW LAND-SURFACE DATUM

$\begin{array}{lllll} & \text { WATER } & \text { WATER } & \text { WATER } \\ \text { DATE } & \text { LEVEL MS } & \text { DATE } & \text { LEVEL MS } & \text { DATE } \\ \text { LEVEL MS }\end{array}$

$\begin{array}{lllllllllll}\text { OCT } 29,1991 & 133.15 & \mathrm{~S} & \text { JAN } 02,1992130.56 & \mathrm{~S} & \text { JUN } 23,1992136.99 & \mathrm{~S} \\ \text { NOV } 19 & & 130.13 & \mathrm{~S} & \text { APR } 08 & 100.96 & \mathrm{~S} & \text { SEP } 17 & 142.65 & \mathrm{~S}\end{array}$

PERIOD OF RECORD HIGHEST 100.96 APR 08, 1992 LOWEST 142.65 SEP 17, 1992 
SITE NUMBER 340707117162707

LOCAL NUMBER 001S004W02D07S

In San Bernardino near Sierra High School. Drilled monitoring well in alluvium. Diameter 6 inches, depth 400 feet, perforated $340-400$ feet. One of three multi-level wells in vault at this site. Altitude of land-surface datum 1,077 feet. Water-level records available 1991 to current year.

WATER LEVELS IN FEET BELOW LAND-SURFACE DATUM

$$
\begin{array}{lllll} 
& \text { WATER } & \text { WATER } & \text { WATER } \\
\text { DATE } & \text { LEVEL MS } & \text { DATE } & \text { LEVEL MS }
\end{array}
$$

OCT 29, $1991126.30 \mathrm{~s} \quad$ JAN 02, $1992120.71 \mathrm{~S} \quad$ JUN 23, $1992132.50 \mathrm{~s}$

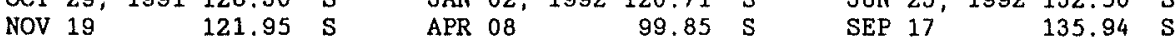

PERIOD OF RECORD HIGHEST 99.85 APR 08, 1992 LOWEST 135.94 SEP 17, 1992

SITE NUMBER 340707117162708

LOCAL NUMBER 001SO04W02D08S

In San Bernardino near Sierra High School, Drilled monitoring well in alluvium. Diameter 2 inches, depth 190 feet, perforated 170-190 feet. One of three multi-level wells in vault at this site. Altitude of land-surface datum 1,077 feet. Water-level records available 1991 to current year.

WATER LEVELS IN FEET BELOW LAND-SURFACE DATUM

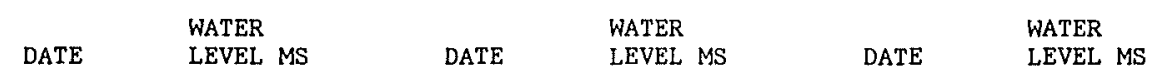

OCT 29, $199184.80 \mathrm{~s} \quad$ JAN 02, $199287.37 \mathrm{~s} \quad$ JUN $23,199290.02 \mathrm{~s}$

\begin{tabular}{|c|}
\hline ov 19 \\
\hline
\end{tabular}

PERIOD OF RECORD HIGHEST 84.80 OCT 29,1991 LOWEST 94.03 SEP 17, 1992

SITE NUMBER 340632117170501

LOCAL NUMBER $001 \mathrm{~S} 004$ W03Q01S

In San Bernardino near intersection of 5 th Street and Sierra Way. Drilled unused well. Diameter 8 inches, depth 200 feet. Altitude of land-surface datum 1,042 feet. Water-level records available 1942 to current year.

WATER LEVELS IN FEET BELOW LAND-SURFACE DATUM (READINGS ABOVE LAND SURFACE INDICATED BY "+")

$\begin{array}{lll} & \text { WATER } & \\ \text { DATE } & \text { WEVEL MS } & \text { WATER } \\ \text { LEVEL MS }\end{array}$

NOV $25,199153.07 \mathrm{~S} \quad$ JUN $15,199255.88 \mathrm{~S}$

PERIOD OF RECORD HIGHEST +23.44 APR 04, 1945 LOWEST 98.0 DEC 10,1968

SITE NUMBER 340655117184004

LOCAL NUMBER 001S004W04E04S

In San Bernardino in Garner Park. Drilled observation well. Diameter 2 inches, depth 176 feet, perforated 162-176 feet. One of four multi-level wells in cement vault at this site. Altitude of land-surface datum 1,120 feet. Water-level records available 1986 to current year.

WATER LEVELS IN FEET BELOW LAND-SURFACE DATUM

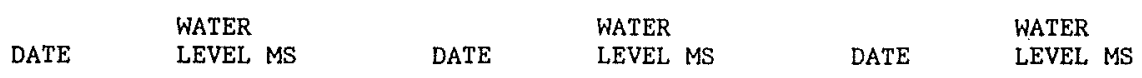

OCT 31, $1991111.72 \mathrm{~S} \quad$ APR 08, $1992113.67 \mathrm{~S} \quad$ SEP 17, $1992120.15 \mathrm{~S}$

JAN $02,1992113.94 \mathrm{~S} \quad$ JUN $23 \quad 116.67 \mathrm{~S}$

PERIOD OF RECORD HIGHEST 37.77 JUN 20, 1986 LOWEST 120.15 SEP 17, 1992 
WELL DESCRIPTIONS AND WATER-LEVEL MEASUREMENTS

WATER YEAR OCTOBER 1991 TO SEPTEMBER 1992

SITE NUMBER 340655117184005

LOCAL NUMBER 001S004W04E05S

In San Bernardino in Garner Park. Drilled observation well. Diameter 2 inches, depth 258 feet, perforated 243-258 feet. One of four multi-level wells in cement vault at this site. Altitude of land-surface datum 1,120 feet. Water-level records available 1986 to current year.

WATER LEVELS IN FEET BELOW LAND-SURFACE DATUM

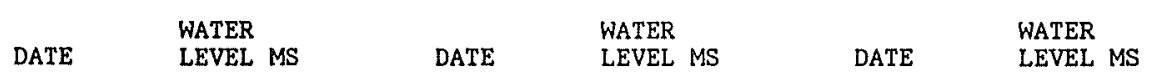

OCT 31, $1991127.43 \mathrm{~S} \quad$ APR 08, $1992117.39 \mathrm{~S} \quad$ SEP 17, $1992133.92 \mathrm{~S}$

JAN 02, $1992125.99 \mathrm{~S}$ JUN 23 127.62 $\mathrm{S}$

PERIOD OF RECORD HIGHEST 40.65 JUN 20, 1986 LOWEST 133.92 SEP 17, 1992

SITE NUMBER 340655117184006

LOCAL NUMBER O01S004W04E06S

In San Bernardino in Garner Park. Drilled observation well. Diameter 2 inches, depth 552 feet, perforated 538-552 feet. One of four multi-level wells in cement vault at this site. Altitude of land-surface datum 1,120 feet. Water-level records available 1986 to current year.

WATER LEVELS IN FEET BELOW LAND-SURFACE DATUM

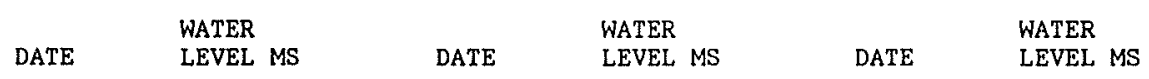

OCT 31, $1991150.27 \mathrm{~S} \quad$ APR 08, $1992127.12 \mathrm{~S} \quad$ SEP 17, $1992154.09 \mathrm{~S}$

JAN 02, $1992150.12 \mathrm{~S} \quad$ JUN $23, r 96.06 \mathrm{~S}$

PERIOD OF RECORD HIGHEST 29.89 JUN 20, 1986 LOWEST 154.09 SEP 17, 1992

SITE NUMBER 340652117195801

LOCAL NUMBER 001S004W06H01S

In San Bernardino in Lytle Creek Wash area, north of Foothill Boulevard. Drilled unused well in alluvium. D1 ameter 16 inches, depth 636 feet, perforated 224-252, 288-346, 378-420, 448-490, 500-510, 604-636 feet. Altitude of land-surface datum 1,160 feet. Water-level records available 1927-58, 1962-77, 1980 to current year.

WATER LEVELS IN FEET BELOW LAND-SURFACE DATUM

$\begin{array}{lll} & \text { WATER } & \\ \text { DATE } & \text { WETER MS }\end{array}$

NOV 27, 1991161.88 SS JUN $17,1992162.46$ SS

PERIOD OF RECORD HIGHEST 19.0 APR 02, 1945 LOWEST 922.1 JUL 07, 1931

SITE NUMBER 340552117175801

LOCAL NUMBER 001S004W09J01S

In San Bernardino near G Street. Drilled unused artesian well. Diameter 16 inches, depth 508 feet, perforated 130-152, 208-250, 276-296, 338-348 feet. Altitude of land-surface datum 1,030 feet. Water-1evel records available 1947 to current year.

WATER LEVELS IN FEET BELOW LAND-SURFACE DATUM

$\begin{array}{lll} & \text { WATER } & \\ \text { DATE } & \text { WEVEL MS } & \text { DATER } \\ \text { LEVEL MS }\end{array}$

NOV 27, $199183.75 \mathrm{~S}$ JUN $17,199288.82 \mathrm{~S}$

PERIOD OF RECORD HIGHEST 0.2 MAR 09, 1951 LOWEST 88.82 JUN 17, 1992 
SITE NUMBER 340615117170902

LOCAL NUMBER 001S004W10B02S

In San Bernardino. Drilled observation well. Diameter 6 inches, depth sounded 740 feet in 1990, original depth 700 feet, perforated $680-700$ feet. One of three multi-level welis in vault at this site. Altitude of land-surface datum 1,015 feet. Water-level records available 1991 to current year.

WATER LEVELS IN FEET BELOW LAND-SURFACE DATUM

$$
\begin{array}{llll} 
& \text { WATER } & \text { WATER } & \text { WATER } \\
\text { DATE } & \text { DEVEL MS } & \text { DATE } & \text { LEVEL MS }
\end{array}
$$

OCT 30, 199180.17 S $\quad$ APR 08, 199247.66 S $\quad$ SEP 17, $1992 \quad 92.36$ S

JAN $02,199270.93 \mathrm{~S} \quad$ JUN $23,1992,89.48 \mathrm{~s}$

PERIOD OF RECORD HIGHEST 47.66 APR 08, 1992 LOWEST 92.36 SEP 17, 1992

SITE NUMBER 340615117170903

LOCAL NUMBER 001S004W10B03S

In San Bernardino. Drilled observation well. Diameter 2 inches, depth sounded 303 feet in 1990, original depth 320 feet, perforated $300-320$ feet. One of three multi-level wells in vault at this site. Altitude of land-surface datum 1,015 feet. Water-level records available 1991 to current year.

WATER LEVELS IN FEET BELOW LAND-SURFACE DATUM

$$
\begin{array}{lllll} 
& \text { WATER } & \text { WATER } & \text { WATER } \\
\text { DATE } & \text { LEVEL MS } & \text { DATE } & \text { LEVEL MS } & \text { DATE }
\end{array}
$$

OCT 30, $199137.96 \mathrm{~S} \quad$ APR 08, $199229.92 \mathrm{~S} \quad$ SEP 17, $199247.29 \mathrm{~S}$

JAN 02, $1992 \quad 38.92 \mathrm{~S} \quad$ JUN $23,1992 \quad 41.44 \mathrm{~S}$

PERIOD OF RECORD HIGHEST 29.92 APR 08, 1992 LOWEST 47.29 SEP 17, 1992

SITE NUMBER 340615117170904

LOCAL NUMBER 001S004W10B04S

In San Bernardino. Drilled observation well. Diameter 2 inches, depth sounded 100 feet in 1990, original depth 120 feet, perforated 100-120 feet. One of three multi-level wells in vault at this site. Altitude of land-surface datum 1,015 feet. Water-level records available 1991 to current year.

WATER LEVELS IN FEET BELOW LAND-SURFACE DATUM

$$
\begin{array}{lllll} 
& & \text { WATER } & \text { WATER } \\
\text { DATE } & \text { WEVEL MS } & \text { DATE } & \text { LEVEL MS } & \text { DATE }
\end{array}
$$

OCT $30,199121.46$ S $\quad$ APR $08,1992 \quad 22.54$ S $\quad$ SEP 17, $1992 \quad 26.96$ S

JAN 02, $199222.99 \mathrm{~S} \quad$ JUN $23 \quad 25.54 \mathrm{~S}$

PERIOD OF RECORD HIGHEST 21.46 OCT 30,1991 LOWEST 26.96 SEP 17, 1992

\section{SITE NUMBER 340608117172601}

LOCAL NUMBER O01S004W1OF01S

In San Bernardino near $D$ and 2nd Streets. Drilled unused artesian well. Diameter 20 inches, depth 752 feet, perforated 680-734 feet. Altitude of land-surface datum 1,029.7 feet. Water-level records available 1920, 1922 , 1926 to current year.

WATER LEVELS IN FEET BELOW LAND-SURFACE DATUM

$$
\begin{array}{lll} 
& \text { WATER } & \\
\text { DATE } & \text { WETER MS }
\end{array}
$$

NOV $25,199136.58 \mathrm{~S} \quad$ JUN $17,199245.61 \mathrm{~S}$

PERIOD OF RECORD HIGHEST 0.2 NOV 21,1983 LOWEST 105.5 OCT 21,1964 
WELL DESCRIPTIONS AND WATER-LEVEL MEASUREMENTS

WATER YEAR OCTOBER 1991 TO SEPTEMBER 1992

SITE NUMBER 340615117145901

LOCAL NUMBER 001S004W12B04S

On Norton AFB. Drilled public-supply well in alluvium. Diameter 10 inches, depth drilled 818 feet. Altitude of land-surface datum 1,087 feet. Water-level records available 1933-40,1943-44, 1946, 1951, 1982-83, 1986 to current year.

WATER LEVELS IN FEET BELOW LAND-SURFACE DATUM (READINGS ABOVE LAND SURFACE INDICATED BY "+")

$\begin{array}{lll} & \text { WATER } & \\ \text { DATE } & \text { WEVEL MS } \\ \text { LATE } & \text { LEVEL MS }\end{array}$

NOV 27, 1991115.64 SR JUN 12, $1992113.34 \mathrm{~S}$

PERIOD OF RECORD HIGHEST T0.6 APR 06, 1934 LOWEST 113.34 JUN 12, 1992

SITE NUMBER 340441117182701

LOCAL NUMBER 001S004W16P04S

In Colton, north of Colton Avenue. Drilled observation well. Diameter 2 inches, original depth 208.5 feet, depth measured 191 feet in 1965, perforated 187.5-208.5 feet. Altitude of land-surface datum 1,014.50 feet.

Water-level records available $1964-70,1987$ to current year.

WATER LEVELS IN FEET BELOW LAND-SURFACE DATUM

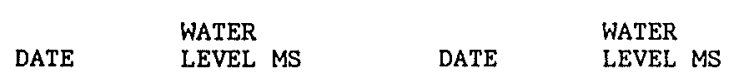

NOV $27,1991114.35 \mathrm{~S}$ JUN $17,1992115.03 \mathrm{~s}$

PERIOD OF RECORD HIGHEST 100,8 OCT 07,1987 LOWEST 146.50 APR 05, 1965

SITE NUMBER 340439117185501

LOCAL NUMBER 001S004W17R01S

In Colton on Olive Street. Drilled observation well. Diameter 2 inches, original depth 209 feet, depth measured 192 feet in 1964, perforated 88-209 feet. Altitude of land-surface datum 1,014.52 feet. Water-level records available $1964-65,1967-70,1987$ to current year.

WATER LEVELS IN FEET BELOW LAND-SURFACE DATUM

$\begin{array}{lll} & \text { WATER } & \\ \text { DATE } & \text { WEVEL MS } & \text { WATE } \\ \end{array}$

NOV 27, $1991116.07 \mathrm{~S}$ JUN 17, $1992108.31 \mathrm{~S}$

PERIOD OF RECORD HIGHEST 83.85 OCT 07, 1987 LOWEST 148.48 JAN 02, 1969

SITE NUMBER 340416117205101

LOCAL NUMBER 001S004W19E01S

In southwest Colton, east of Meridian Avenue, and north of Valley Boulevard. Drilled observation well in alluvium. Diameter 2 inches, original depth 251 feet, measured 222 feet in 1964, perforated $223-244$ feet Altitude of land-surface datum $1,038.9$ feet. Water-level records available 1964, 1967-70, 1977 to current year.

WATER LEVELS IN FEET BELOW LAND-SURFACE DATUM

$\begin{array}{lll} & \text { WATER } & \\ \text { DATE } & \text { LEVEL MS } & \text { WATER }\end{array}$

NOV $27,1991163.15 \mathrm{~S}$ JUN $17,1992162.35 \mathrm{~s}$

PERIOD OF RECORD HIGHEST 148.38 MAY 15, 1986 LOWEST 193.94 JAN 02, 1969 


\section{SITE NUMBER 340413117180501}

LOCAL NUMBER 001S004W21K11S

In Colton, north of Fairway Drive. Unused well. Diameter 8 inches, depth unknown, Altitude of land-surface datum 963 feet. Water-level records available 1967-68, 1972 to current year.

WATER LEVELS IN FEET BELOW LAND-SURFACE DATUM

\begin{tabular}{|c|c|c|}
\hline & WATER & \\
\hline DATE & LEVEL MS & DATE \\
\hline
\end{tabular}

NOV 29, $199141.91 \mathrm{~S} \quad$ JUN $17,1992 \quad 28.73 \mathrm{~S}$

PERIOD OF RECORD HIGHEST 13.1 MAR 20, 1982 LOWEST 98.2 JUL 07, 1977

SITE NUMBER 340432117165701

LOCAL NUMBER 001S004W22B03S

In south San Bernardino, west of Waterman Avenue. Drilled unused well. Diameter 8 inches, depth reported 200 feet. Altitude of land-surface datum 1,001.9 feet. Water-level records available 1933 to current year.

WATER LEVELS IN FEET BELOW LAND-SURFACE DATUM

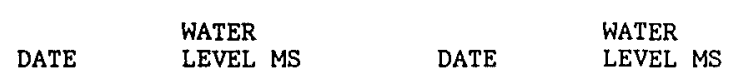

NOV $29,199172.98 \mathrm{~S} \quad$ JUN $17,199241.89 \mathrm{~S}$

PERIOD OF RECORD HIGHEST 0.10 JUN $12,1947 \quad$ LOWEST $107.30 \quad$ DEC 11,1968

SITE NUMBER 340439117173902

LOCAL NUMBER 001S004W22D02S

In San Bernardino, near Orange Show Road and E Street. Drilled observation well. Diameter 6 inches, depth 200 feet, perforated $160-200$ feet. One of five multi-level wells in concrete vault at this site. Altitude of land-surface datum 975.1 feet. Water-level records available 1991 to current year.

WATER LEVELS IN FEET BELOW LAND-SURFACE DATUM

$\begin{array}{llllll} & \text { WATER } & \text { WATER } & & \text { WATER } \\ \text { DATE } & \text { LEVEL MS } & \text { DATE } & \text { LEVEL MS } & \text { DATE } & \text { LEVEL MS }\end{array}$

$\begin{array}{llllllllllllllllll}\text { OCT } 29, & 1991 & 24.28 & \mathrm{~S} & \text { NOV } 15,1991 & 24.34 & \mathrm{~S} & \text { APR } & 08, & 1992 & 9.13 & \mathrm{~S} & \mathrm{JUL} & 01, & 1992 & 20.01 & \mathrm{~S}\end{array}$

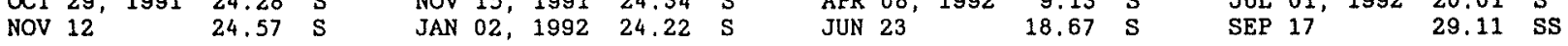

PERIOD OF RECORD HIGHEST 9.13 APR 08, 1992 LOWEST 24.57 NOV 12,1991

SITE NUMBER 340439117173904

LOCAL NUMBER 001S004W22D04S

In San Bernardino, near Orange Show Road and E Street. Drilled observation well. Diameter 2 inches, depth 655 feet, perforated 600-655 feet. One of five multi-level wells in concrete vault at this site. Altitude of land-surface datum 975.1 feet. Water-level records available 1991 to current year.

WATER LEVELS IN FEET BELOW LAND-SURFACE DATUM

\begin{tabular}{|c|c|c|c|c|c|c|c|c|c|c|c|c|}
\hline DATE & $\begin{array}{l}\text { WATER } \\
\text { LEVEL MS }\end{array}$ & & DATE & $\begin{array}{l}\text { WATER } \\
\text { LEVEL }\end{array}$ & MS & & DATE & $\begin{array}{l}\text { WATER } \\
\text { LEVEL }\end{array}$ & MS & & DATE & $\begin{array}{l}\text { WATER } \\
\text { LEVEL }\end{array}$ \\
\hline $\begin{array}{l}29,1991 \\
12\end{array}$ & $\begin{array}{l}29.73 \\
30.84\end{array}$ & $\begin{array}{l}\text { NOV } \\
\text { JAN }\end{array}$ & $\begin{array}{ll}15, & 1991 \\
02, & 1992\end{array}$ & $\begin{array}{l}31.04 \\
34.62\end{array}$ & $\begin{array}{l}S \\
S\end{array}$ & $\begin{array}{l}\text { APR } \\
\text { JUN }\end{array}$ & $\begin{array}{l}08,1992 \\
23\end{array}$ & $\begin{array}{l}21.98 \\
22.81\end{array}$ & $\begin{array}{l}S \\
S\end{array}$ & $\begin{array}{l}\text { JUL } \\
\text { SEP }\end{array}$ & $\begin{array}{l}01,1992 \\
17\end{array}$ & $\begin{array}{l}23.71 \\
34.20\end{array}$ \\
\hline
\end{tabular}

PERIOD OF RECORD HIGHEST 21.98 APR 08,1992 LOWEST 34.62 JAN 02,1992 
WELL DESCRIPTIONS AND WATER-LEVEL MEASUREMENTS

WATER YEAR OCTOBER 1991 TO SEPTEMBER 1992

SITE NUMBER 340439117173905

LOCAL NUMBER 001S004W22D05S

In San Bernardino, near Orange Show Road and E Street. Drilled observation well. Diameter 2 inches, depth measured 524 feet in 1990, original depth 574 feet, perforated 555-574 feet. One of five multi-level wells in concrete vault at this site. Altitude of land-surface datum 975.1 feet. Water-level records available 1991 to current year.

WATER LEVELS IN FEET BELOW LAND-SURFACE DATUM

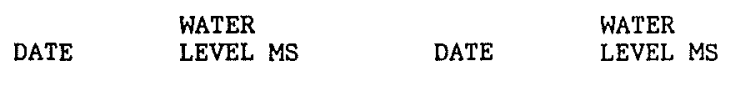

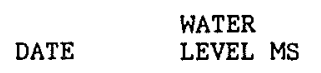

NOV $15,199110.05 \mathrm{~S}$

OCT 29, 1991

NOV 12

$10.38 \mathrm{~s}$

JAN 02, $1992 \quad 10.29 \mathrm{~S}$

PERIOD OF RECORD

HIGHEST

10.05 NOV $12,15,1991$
APR 08, $1992 \quad 10.43 \quad \mathrm{~S}$

JUN $23,199210.43 \mathrm{~S}$

LOWEST 10.54 JUN 23,1992

\section{SITE NUMBER 340439117173906}

\section{LOCAL NUMBER 001S004W22D06S}

In San Bernardino, near Orange Show Road and E Street. Drilled observation well. Diameter 2 inches, depth 398 feet, perforated 388-398 feet. One of five multi-level wells in concrete vault at this site. Altitude of land-surface datum 975.1 feet. Water-level records avallable 1991 to current year.

WATER LEVELS IN FEET BELOW LAND-SURFACE DATUM

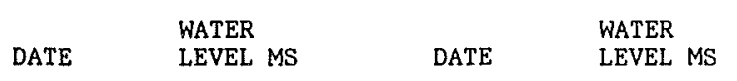

OCT 29, $199125.98 \quad \mathrm{~S}$

NOV 12
NOV $15,199125.70 \mathrm{~s}$

JAN 02, $1992 \quad 26.71 \mathrm{~S}$

\section{DATE}

APR 08, 1992

JUN 23 $\begin{array}{ll} & \text { WATER } \\ \text { DATE } & \text { LEVEL MS }\end{array}$

JUL $01,199210.44 \mathrm{~S}$

PERIOD OF RECORD HIGHEST

13.78 APR 08, 1992

LOWEST

26.71 JAN 02, 1992

SITE NUMBER 340439117173907

LOCAL NUMBER 001S004W22D07S

In San Bernardino, near Orange Show Road and E Street. Drilled observation well. Diameter 6 inches, depth

52 feet, perforated $10-20,35-45$ feet. One of five multi-level wells in concrete vaut at this site. Altitude of land-surface datum 975.1 feet. Water-level records available 1991 to current year.

WATER LEVELS IN FEET BELOW LAND-SURFACE DATUM

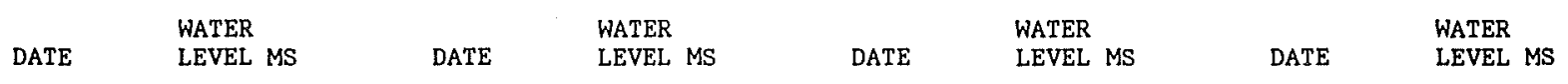

$\begin{array}{lllllllllllllll}\text { OCT } 29,1991 & 18.11 & \mathrm{~S} & \text { NOV } 15,1991 & 19.48 & \mathrm{~S} & \text { APR } & 08,1992 & 8.25 & \mathrm{~S} & \text { JUL } & 01,1992 & 13.07 & \mathrm{~S}\end{array}$

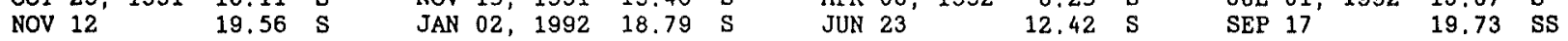

PERIOD OF RECORD HIGHEST 8,25 APR 08, 1992 LOWEST 19.56 NOV 12, 1991

SITE NUMBER 340319117185501

LOCAL NUMBER 001S004W29H01S

In Colton along the Santa Ana River. Drilled public-supply well in alluvium. Diameter 20 inches, depth 345 feet, depth of hole 530 feet, perforated $68-88,108-220$ feet. Altitude of land-surface datum 923.34 feet. Water-level records available 1929 to current year.

WATER LEVELS IN FEET BELOW LAND-SURFACE DATUM

$\begin{array}{lllll} & \text { WATER } & & & \text { WATER } \\ \text { DATE } & \text { LEVEL MS } & \text { DATE } & \text { WATER } & \text { LEVEL MS } \\ \end{array}$

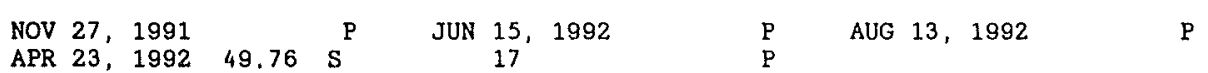

PERIOD OF RECORD HIGHEST 2.2 FEB 05, 1943 LOWEST 150.8 JUN 18, 1965 
SITE NUMBER 340255117191201

LOCAL NUMBER 001S004W29Q04S

In Colton along the Santa Ana River. Drilled unused well in alluvium and channel deposits. Diameter 16 inches, depth 448 feet, perforated 152-438 feet. Altitude of land-surface datum 924.5 feet. Water-level records available 1971 to current year.

WATER LEVELS IN FEET BELOW LAND-SURFACE DATUM

\begin{tabular}{llcccc} 
DATE & $\begin{array}{l}\text { WATER } \\
\text { LEVEL MS }\end{array}$ & DATE & $\begin{array}{l}\text { WATER } \\
\text { LEVEL MS }\end{array}$ & DATE & \multicolumn{2}{l}{ WATER } \\
LEVEL MS
\end{tabular}

NOV 27, $1991 \quad \mathrm{P} \quad$ JUN $15 ; 199252.31 \mathrm{~S} \quad$ AUG $13,1992 \quad \mathrm{P}$

PERIOD OF RECORD HIGHEST 19.43 JUN 20, 1986 LOWEST 83.6 JUN 08, 1977

SITE NUMBER 340307117190301

LOCAL NUMBER 001S004W29R01S

In Colton along the Santa Ana River. Drilled unused well in alluvium and channel deposits. Diameter 20 inches, depth 403 feet, perforated 100-248,290-354 feet. Altitude of land-surface datum 923 feet. Water-1evel records available 1992 .

WATER LEVELS IN FEET BELOW LAND-SURFACE DATUM

$$
\begin{array}{ll}
\text { WATER } \\
\text { DATE } & \text { LEVEL MS }
\end{array}
$$

AUG $13,199258.57 \mathrm{~S}$

PERIOD OF RECORD HIGHEST 58,57 AUG 13, 1992 LOWEST 58.57 AUG 13, 1992

SITE NUMBER 340709117245301

LOCAL NUMBER 001S005W04D02S

In Fontana near intersection of Baseline and Adler Avenues, Drilled public-supply well. Diameter 16 inches, depth 553 feet. Altitude of land-surface datum 1,400 feet. Water-level records available 1931-42, 1958-82, 1986 to current year.

WATER LEVELS IN FEET BELOW LAND-SURFACE DATUM

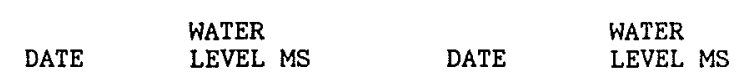

DEC 05, $1991301.87 \mathrm{~S} \quad$ JUN 15, $1992305.30 \mathrm{~S}$

PERIOD OF RECORD HIGHEST 257.78 MAR 12, 1986 LOWEST 305.30 JUN 15, 1992

\section{SITE NUMBER 340716117251001}

LOCAL NUMBER 001S005W05A02S

In Rialto along Baseline Avenue, west of Adler Avenue. Drilled unused well in alluvium. Diameter unknown, depth 543 feet. Altitude of land-surface datum 1,410 feet. Water-level records available $1925-76,1987$ to current year.

WATER LEVELS IN FEET BELOW LAND-SURFACE DATUM

$$
\begin{array}{lll} 
& \text { WATER } & \text { WATER } \\
\text { DATE } & \text { LEVEL MS } & \text { DATE }
\end{array}
$$

DEC 05, $1991290.02 \mathrm{~S} \quad$ JUN $15,1992281.69 \mathrm{~S}$

PERIOD OF RECORD HIGHEST 201.6 FEB 04, 1932 LOWEST 372.3 OCT 03, 1957 
WELL DESCRIPTIONS AND WATER-LEVEL MEASUREMENTS

WATER YEAR OCTOBER 1991 TO SEPTEMBER 1992

SITE NUMBER 340531117243601

LOCAL NUMBER 001S005W16C01S

In Fontana. Drilled unused well in alluvium. Diameter 20 inches, depth 531 feet, perforated 354-386, 390-398, 410-420, 472-490 feet. Altitude of land-surface datum 1,227.3 feet. Water-level records available 1929 to current year.

WATER LEVELS IN FEET BELOW LAND-SURFACE DATUM

$$
\begin{array}{lll} 
& \text { WATER } & \\
\text { DATE } & \text { WEVEL MS } & \text { DATE } \\
\text { LEVEL MS }
\end{array}
$$

DEC 05, $1991401.83 \mathrm{~S} \quad$ JUN $15,1992401.05 \mathrm{~S}$

PERIOD OF RECORD HIGHEST 370.0 APR 20,1929 LOWEST 430.0 OCT 03, 1978

SITE NUMBER 340439117260201

LOCAL NUMBER OO1SO05W2ODO1S

In south Fontana near intersection of Sierra and San Bernardino Avenues. Drilled public-supply well. Diameter 20 inches, depth reported 510 feet. Altitude of land-surface datum 1,157 feet. Water-level records avallable $1931-35,1938-40,1943-49,1953,1956,1959-60,1964,1986$ to current year.

WATER LEVELS IN FEET BELOW LAND-SURFACE DATUM

$$
\begin{array}{lll} 
& \text { WATER } & \\
\text { DATE } & \text { WEVEL MS } \\
\text { LATE } & \text { LEVEL MS }
\end{array}
$$

DEC 05, $1991390.89 \mathrm{~S}$ JUN 16, $1992388.50 \mathrm{~V}$

PERIOD OF RECORD HIGHEST 283.45 FEB 19, 1964 LOWEST 448.3 APR 29, 1944

SITE NUMBER 340255117211001

LOCAL NUMBER 001S005W25R04S

In south Rialio, west of Santa Ana River on Agua Mansa Road. Drilled public-supply well in alluvium. Diameter 20 inches, depth 302 feet, perforated 50-280 feet. Altitude of land-surface datum 880 feet. Water-level records available 1962,1985 to current year.

WATER LEVELS IN FEET BELOW LAND-SURFACE DATUM

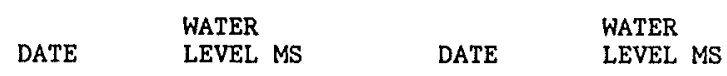

NOV $27,199134.90 \mathrm{~S}$ JUN $17,1992 \quad 22.70 \mathrm{~s}$

PERIOD OF RECORD HIGHEST 16.00 NOV 06, 1985 LOWEST 58.0 JUL 06, 1962

SITE NUMBER 340320117263701

LOCAL NUMBER 001S005W30L01S

In south Fontana near intersection of Santa Ana and Cypress Avenues. Drilled unused well. Diameter 16 inches, depth reported 492 feet. Altitude of land-surface datum 1,049 feet. Water-level records available 1912-51, 1956 to current year.

WATER LEVELS IN FEET BELOW LAND-SURFACE DATUM

$$
\begin{array}{lll} 
& \text { WATER } & \text { WATER } \\
\text { DATE } & \text { LEVEL MS } & \text { DATE }
\end{array}
$$

DEC 05, $1991287.55 \mathrm{~S}$ JUN 15, $1992288.06 \mathrm{~S}$

PERIOD OF RECORD HIGHEST 225.4 OCT 06,1924 LOWEST 311.8 OCT 03,1978 
SITE NUMBER 340346117274201

LOCAL NUMBER 001S006W25C01S

In south Fontana. Drilled unused well. Diameter 16 inches, depth measured 854 feet in 1912 . Altitude of land-surface datum 1,050 feet. Water-level records available 1912, 1914-19, 1953 to current year.

WATER LEVELS IN FEET BELOW LAND-SURFACE DATUM

$$
\begin{array}{lll} 
& \text { WATER } & \\
\text { DATE } & \text { LEVEL MS } & \text { WATER } \\
& \text { DATE } & \text { LEVEL MS }
\end{array}
$$

DEC $05,1991301.30 \mathrm{~s} \quad$ JUN $15,1992301.02 \mathrm{~s}$

PERIOD OF RECORD HIGHEST 237.9 APR $03,1919 \quad$ LOWEST $321.5 \quad$ OCT 03,1978

STTE NUMBER 340124117021301

LOCAL NUMBER 002S002W01J01S

In Yucaipa east of 2nd Street. Drilled observation well. Depth drilled 481 feet, perforated $330-350$ feet. Altitude of land-surface datum 2,600 feet. Water-level records available 1992 .

WATER LEVELS IN FEET BELOW LAND-SURFACE DATUM

$$
\begin{array}{ll} 
& \text { WATER } \\
\text { DATE } & \text { LEVEL MS }
\end{array}
$$

AUG $13,1992314.27 \mathrm{~S}$

PERIOD OF RECORD HIGHEST 314.27 AUG 13,1992 LOWEST 314.27 AUG 13,1992

SITE NUMBER 340133117095401

LOCAL NUMBER 002S003W02L01S

In Redlands Heights on Sunset Drive. Drilled observation well in alluvium. Diameter 2 inches, depth 441 feet, perforated 420-441 feet. Altitude of land-surface datum 1,857,51 feet. Water-1evel records available 1967 , 1987 to current year.

WATER LEVELS IN FEET BELOW LAND-SURFACE DATUM

$$
\begin{array}{lll} 
& \text { WATER } & \\
\text { DATE } & \text { LEVEL MS } & \text { WATER } \\
& \text { LEVEL MS }
\end{array}
$$

NOV $26,1991246.83 \mathrm{~S} \quad$ JUN $12,1992243.62 \mathrm{~S}$

PERIOD OF RECORD HIGHEST 243.62 JUN 12, 1992 LOWEST 273.11 OCT 02,1967 
WATER QUALITY, SAN BERNARDINO COUNTY

INORGANIC DATA

WATER YEAR OCTOBER 1991 TO SEPTEMBER 1992

$\begin{array}{ccc}\text { LOCAL } & & \\ \text { NUMBER } & \text { DATE } & \text { TIME } \\ & & \\ \text { 002N007E03B01S } & 06-04-92 & 1230 \\ \text { 002N007E03E01S } & 06-04-92 & 1330 \\ \text { 003N007E28D02S } & 06-04-92 & 1440 \\ \text { 003N007E32D02S } & 06-04-92 & 1620 \\ \text { 009N002E03E01S } & 05-26-92 & 1520 \\ & & \\ \text { 009N002E07Q01S } & 06-02-92 & 1320 \\ \text { 009N002E11C03S } & 05-28-92 & 1130 \\ \text { 010N004E20D01S } & 05-28-92 & 1320 \\ \text { 001N004W26E02S } & 07-01-92 & 0900 \\ \text { 001N004W34G03S } & 11-26-91 & 1115 \\ & & \\ & 07-30-92 & 1600 \\ \text { 003N004W13F02S } & 10-29-91 & 1005 \\ \text { 003N004W33H05S } & 10-29-91 & 0820 \\ \text { 004N003W30A06S } & 10-29-91 & 1145 \\ \text { 004N004W01A01S } & 10-29-91 & 1405 \\ \text { 005N004W11P03S } & 11-01-91 & 1330 \\ \text { 005N004W25N01S } & 11-01-91 & 0950 \\ \text { 006N004W30D10S } & 10-30-91 & 1630 \\ \text { 006N005W12H01S } & 10-29-91 & 1700 \\ \text { 007N004W07K02S } & 10-29-91 & 1545 \\ & & \\ \text { 008N003W05J01S } & 10-30-91 & 1325 \\ \text { 008N004W13B01S } & 10-30-91 & 1440 \\ \text { 009N001W10G06S } & 10-30-91 & 1005 \\ \text { 009N001W11M11S } & 05-27-92 & 1425 \\ \text { 009N001W11R02S } & 05-29-92 & 0810 \\ & & \\ \text { 009N001W13B02S } & 05-27-92 & 1010 \\ \text { 009N001W13B03S } & 05-27-92 & 1118 \\ \text { 009N001W13B04S } & 05-27-92 & 1200 \\ \text { 009N001W13H02S } & 05-27-92 & 0835 \\ \text { 009N002W05N07S } & 10-30-91 & 1220 \\ \text { 009N003W21K01S } & 05-28-92 & 1645 \\ \text { 010N002W33R16S } & 05-26-92 & 1200 \\ \text { 001S003W09E02S } & 11-25-91 & 1330 \\ & 06-30-92 & 1315 \\ \text { 001S003W17C03S } & 11-25-91 & 1215 \\ & & \\ \text { 001S003W18N02S } & 06-30-92 & 1445 \\ & 12-02-91 & 0915 \\ \text { 001S004W11D02S } & 06-30-92 & 1030 \\ & 11-25-91 & 1015 \\ & 07-30-92 & 1050\end{array}$

$\begin{array}{lllcl}\text { DEPTH } & & & \text { PH } \\ \text { BELOW } & & \text { SPE- } & \text { WATER } & \\ \text { LAND } & \text { DEPTH } & \text { CIFIC } & \text { WHOLE } & \\ \text { SURFACE } & \text { OF } & \text { CON- } & \text { FIELD } & \text { TEMPER- } \\ \text { (WATER } & \text { WELL, } & \text { DUCT- } & \text { (STAND- } & \text { ATURE } \\ \text { LEVEL) } & \text { TOTAL } & \text { ANCE } & \text { ARD } & \text { WATER } \\ \text { (FEET) } & \text { (FEET) } & \text { (US/CM) } & \text { UNITS) } & \text { (DEG C) }\end{array}$

$\begin{array}{llc}\text { HARD- } & & \text { MAGNE- } \\ \text { NESS } & \text { CALCIUM } & \text { SIUM, } \\ \text { TOTAL } & \text { DIS- } & \text { DIS- } \\ \text { (MG/L } & \text { SOLVED } & \text { SOLVED } \\ \text { AS } & \text { (MG/L } & \text { (MG/L } \\ \text { CACO3) } & \text { AS CA) } & \text { AS MG) }\end{array}$

700

$\begin{array}{rll}26 & 10 & 0.26 \\ 18 & 7.1 & 0.10 \\ 34 & 13 & 0.30 \\ 28 & 11 & 0.17 \\ 120 & 39 & 5.5 \\ & & \end{array}$

\section{$\begin{array}{ll}-- & 360\end{array}$}

$124.76 \quad 190$

-- 300

-- 689

$-708$

$\begin{array}{ll}8.8 & 27.0\end{array}$

8.928 .0

$8.5 \quad 27.0$

8.6

26.5

$\begin{array}{lll}289 & 8.6 & 26.5 \\ 447 & 8.0 & 22.0\end{array}$

$--708$

$\begin{array}{r}269 \\ -\quad \mathrm{F} \quad 105 \\ \hline\end{array}$

$87.27 \quad 123$

$54.59 \quad 275$

$7.5 \quad 18.5$

706
435
1390

1390

$\begin{array}{ll}8.1 & 24.0 \\ 8.8 & 25.5\end{array}$

7.4

558
580

7.7

17.0

96

180

19.5

280

32

3.2

590

7.7

19.5

$\begin{array}{ll}7.4 & 15.5 \\ 8.0 & 16.5\end{array}$

$7.4 \quad 14.0$

270

296
315

224

14.0
15.0

95
110

$60.02 \quad 145$

.02
.$- \quad 400$

-. 145

$15.15 \quad 150$

$50.37 \quad 200$

8.5

8.7

20.0

212

183
827

7.6

17.0

19.5

16.0

1170

7.7

19.5

$16.67 \quad 115$

7.9

17.0

1040

7.4

$17.51 \quad 107$

$11.16 \quad 75$

18.0

19.0

19.5

1110

7.2

7.4

21.5

26. $50 \quad 110$

$21.64 \quad 60$

21.29 120

$29.15 \quad 160$

19.0

7.5

1390

1340

1160

19.5

20.5

21.5

7.6

19.0

1310

22.0

$83.15 \quad 150$

$89.81 \quad 150$

-- 401

$\begin{array}{ll}- & 401 \\ -- & 296\end{array}$

18.5

7.4

7.5

7.6

18.5

19.0

259

19.5

296

-. 988

-. 988

- 963

$\begin{array}{ll}7.5 & 20.0 \\ 8.2 & 18.0\end{array}$

$8.3 \quad 21.0$

$8.1 \quad 22.5$

$8.0 \quad 22.5$

130

86

3.8

0.26

12

15

15
5.5
5.6

5.6
4.3

7.8

$42 \quad 13 \quad 2.2$

40

220

80

$340 \quad 110$

1.2

13

15

16

$\begin{array}{lll}270 & 84 & 14\end{array}$

$\begin{array}{lll}440 & 140 & 21\end{array}$

$270 \quad 85$

$360 \quad 110$

$430 \quad 130-25$

$390 \quad 120 \quad 22$

$390 \quad 120 \quad 22$

$\begin{array}{rrr}350 & 110 & 19 \\ 170 & 55 & 8\end{array}$

$\begin{array}{llr}170 & 55 & 8 \\ 280 & 87 & 15\end{array}$

288

314

340

$\begin{array}{rr}350 & 110 \\ 240 & 76 \\ 93 & 29 \\ 93 & 29 \\ 380 & 120 \\ 390 & 120 \\ 89 & 32 \\ 36 & 13 \\ 92 & 32 \\ 90 & 31\end{array}$

19

12 4.9
5.1

19

21

2.3

0.77

2.9

F Flowing. 
WATER YEAR OCTOBER 1991 TO SEPTEMBER 1992

\begin{tabular}{|c|c|c|c|c|c|c|c|c|c|c|}
\hline $\begin{array}{l}\text { LOCAL } \\
\text { NUMBER }\end{array}$ & DATE & $\begin{array}{l}\text { SODIUM, } \\
\text { DIS- } \\
\text { SOLVED } \\
\text { (MG/L } \\
\text { AS NA) }\end{array}$ & $\begin{array}{l}\text { SODIUM } \\
\text { PERCENT }\end{array}$ & $\begin{array}{l}\text { SODIUM } \\
\text { AD- } \\
\text { SORP- } \\
\text { TION } \\
\text { RATIO }\end{array}$ & $\begin{array}{l}\text { POTAS- } \\
\text { SIUM, } \\
\text { DIS- } \\
\text { SOLVED } \\
(M G / L \\
\text { AS K) }\end{array}$ & $\begin{array}{c}\text { CAR- } \\
\text { BONATE } \\
\text { WATER } \\
\text { WH IT } \\
\text { FIELD } \\
\text { MG /L AS } \\
\text { CO3 }\end{array}$ & $\begin{array}{c}\text { BICAR- } \\
\text { BONATE } \\
\text { WATER } \\
\text { WH IT } \\
\text { FIELD } \\
\text { MG/L AS } \\
\text { HCO3 }\end{array}$ & $\begin{array}{c}\text { ALKA- } \\
\text { LINITY } \\
\text { WAT WH } \\
\text { TOT IT } \\
\text { FIELD } \\
\text { MG/L AS } \\
\text { CACO3 }\end{array}$ & $\begin{array}{l}\text { SULFATE } \\
\text { DIS- } \\
\text { SOLVED } \\
\text { (MG/L } \\
\text { AS SO4) }\end{array}$ & $\begin{array}{l}\text { CHLO- } \\
\text { RIDE, } \\
\text { DIS- } \\
\text { SOLVED } \\
\text { (MG/L } \\
\text { AS CL) }\end{array}$ \\
\hline $\begin{array}{l}002 N 007 E 03 B 01 S \\
002 N 007 E 03 E 01 S \\
003 N 007 E 28 D 02 S \\
003 N 007 E 32 D 02 S \\
009 N 002 E 03 E 01 S\end{array}$ & $\begin{array}{l}06-04-92 \\
06-04-92 \\
06-04-92 \\
06-04-92 \\
05-26-92\end{array}$ & $\begin{array}{r}47 \\
52 \\
48 \\
45 \\
49\end{array}$ & $\begin{array}{l}78 \\
85 \\
74 \\
76 \\
47\end{array}$ & $\begin{array}{l}4 \\
5 \\
4 \\
4 \\
2\end{array}$ & $\begin{array}{l}2.1 \\
2.0 \\
3.1 \\
2.3 \\
1.7\end{array}$ & $\begin{array}{l}-- \\
-- \\
-- \\
--\end{array}$ & $\begin{array}{l}-- \\
- \\
- \\
- \\
190\end{array}$ & $\begin{array}{l}1_{66} \\
1_{69} \\
1_{72} \\
1_{66} \\
156\end{array}$ & $\begin{array}{l}33 \\
35 \\
34 \\
34 \\
34\end{array}$ & $\begin{array}{l}22 \\
24 \\
28 \\
24 \\
27\end{array}$ \\
\hline $\begin{array}{l}\text { 009N002E07Q01S } \\
\text { 009N002E11C03S } \\
010 \mathrm{~N} 004 \mathrm{E} 20 \mathrm{D} 01 \mathrm{~S} \\
001 \mathrm{~N} 004 \mathrm{~W} 26 \mathrm{E} 02 \mathrm{~S} \\
001 \mathrm{~N} 004 \mathrm{~W} 34 \mathrm{GO} S\end{array}$ & $\begin{array}{l}06-02-92 \\
05-28-92 \\
05-28-92 \\
07-01-92 \\
11-26-91\end{array}$ & $\begin{array}{r}54 \\
53 \\
300 \\
46 \\
14\end{array}$ & $\begin{array}{l}35 \\
54 \\
98 \\
36 \\
10\end{array}$ & $\begin{array}{r}2 \\
2 \\
43 \\
2 \\
0.4\end{array}$ & $\begin{array}{l}2.5 \\
1.7 \\
3.2 \\
2.3 \\
3.7\end{array}$ & $\begin{array}{l}0 \\
0 \\
0 \\
0\end{array}$ & $\begin{array}{l}174 \\
185 \\
270 \\
176 \\
242\end{array}$ & $\begin{array}{l}142 \\
151 \\
253 \\
144 \\
198\end{array}$ & $\begin{array}{r}85 \\
33 \\
120 \\
91 \\
58\end{array}$ & $\begin{array}{r}71 \\
26 \\
220 \\
31 \\
23\end{array}$ \\
\hline $\begin{array}{l}003 N 004 W 13 F 02 S \\
003 N 004 W 33 H 05 S \\
004 N 003 W 30 A 06 S \\
004 N 004 W 01 A 01 S\end{array}$ & $\begin{array}{l}07-30-92 \\
10-29-91 \\
10-29-91 \\
10-29-91 \\
10-29-91\end{array}$ & $\begin{array}{l}14 \\
26 \\
27 \\
17 \\
17\end{array}$ & $\begin{array}{l}10 \\
37 \\
34 \\
34 \\
22\end{array}$ & $\begin{array}{l}0.4 \\
1 \\
1 \\
0.9 \\
0.7\end{array}$ & $\begin{array}{l}3.5 \\
1.8 \\
1.6 \\
1.6 \\
2.0\end{array}$ & $\begin{array}{l}0 \\
0 \\
0 \\
0 \\
0\end{array}$ & $\begin{array}{r}242 \\
122 \\
189 \\
72 \\
139\end{array}$ & $\begin{array}{r}198 \\
100 \\
155 \\
59 \\
114\end{array}$ & $\begin{array}{l}54 \\
32 \\
4.2 \\
13 \\
27\end{array}$ & $\begin{array}{l}28 \\
14 \\
10 \\
24 \\
13\end{array}$ \\
\hline $\begin{array}{l}005 \mathrm{~N} 004 \mathrm{~W} 11 \mathrm{P} 03 \mathrm{~S} \\
005 \mathrm{~N} 004 \mathrm{~W} 25 \mathrm{~N} 01 \mathrm{~S} \\
006 \mathrm{~N} 004 \mathrm{~W} 30 \mathrm{D} 10 \mathrm{~S} \\
006 \mathrm{~N} 005 \mathrm{~W} 12 \mathrm{H} 01 \mathrm{~S} \\
007 \mathrm{~N} 004 \mathrm{~W} 07 \mathrm{~K} 02 \mathrm{~S}\end{array}$ & $\begin{array}{l}11-01-91 \\
11-01-91 \\
10-30-91 \\
10-29-91 \\
10-29-91\end{array}$ & $\begin{array}{r}31 \\
24 \\
100 \\
87 \\
120\end{array}$ & $\begin{array}{l}61 \\
56 \\
49 \\
42 \\
43\end{array}$ & $\begin{array}{l}2 \\
2 \\
3 \\
2 \\
3\end{array}$ & $\begin{array}{l}1.1 \\
1.0 \\
0.60 \\
2.7 \\
2.9\end{array}$ & $\begin{array}{l}3 \\
5 \\
0 \\
0 \\
0\end{array}$ & $\begin{array}{r}90 \\
85 \\
344 \\
303 \\
259\end{array}$ & $\begin{array}{r}79 \\
78 \\
282 \\
248 \\
212\end{array}$ & $\begin{array}{l}15 \\
8.5 \\
120 \\
130 \\
210\end{array}$ & $\begin{array}{l}9.6 \\
4.9 \\
42 \\
67 \\
110\end{array}$ \\
\hline $\begin{array}{l}\text { 008N003W05J01S } \\
008 \text { N004W13B01S } \\
009 \text { N001W10G06S } \\
009 \text { N001W11M11S } \\
\text { 009N001W11R02S }\end{array}$ & $\begin{array}{l}10-30-91 \\
10-30-91 \\
10-30-91 \\
05-27-92 \\
05-29-92\end{array}$ & $\begin{array}{r}120 \\
160 \\
110 \\
98 \\
160\end{array}$ & $\begin{array}{l}49 \\
44 \\
47 \\
37 \\
45\end{array}$ & $\begin{array}{l}3 \\
3 \\
3 \\
2 \\
3\end{array}$ & $\begin{array}{l}3.1 \\
3.0 \\
3.2 \\
3.3 \\
4.5\end{array}$ & $\begin{array}{l}0 \\
0 \\
0 \\
0 \\
0\end{array}$ & $\begin{array}{l}359 \\
354 \\
244 \\
251 \\
354\end{array}$ & $\begin{array}{l}294 \\
290 \\
200 \\
206 \\
290\end{array}$ & $\begin{array}{l}150 \\
200 \\
210 \\
160 \\
200\end{array}$ & $\begin{array}{r}89 \\
230 \\
74 \\
140 \\
200\end{array}$ \\
\hline $\begin{array}{l}009 N 001 W 13 B 02 S \\
009 N 001 W 13 B 03 S \\
009 N 001 W 13 B 04 S \\
009 N 001 W 13 H 02 S \\
009 N 002 W 05 N 07 S\end{array}$ & $\begin{array}{l}05-27-92 \\
05-27-92 \\
05-27-92 \\
05-27-92 \\
10-30-91\end{array}$ & $\begin{array}{r}150 \\
140 \\
110 \\
76 \\
88\end{array}$ & $\begin{array}{l}45 \\
44 \\
40 \\
48 \\
40\end{array}$ & $\begin{array}{l}3 \\
3 \\
3 \\
3 \\
2\end{array}$ & $\begin{array}{l}3.5 \\
3.3 \\
3.6 \\
2.8 \\
2.7\end{array}$ & $\begin{array}{l}0 \\
0 \\
0 \\
0 \\
0\end{array}$ & $\begin{array}{l}325 \\
348 \\
334 \\
207 \\
167\end{array}$ & $\begin{array}{l}266 \\
285 \\
274 \\
169 \\
137\end{array}$ & $\begin{array}{r}210 \\
200 \\
160 \\
79 \\
150\end{array}$ & $\begin{array}{r}180 \\
160 \\
130 \\
66 \\
120\end{array}$ \\
\hline $\begin{array}{l}009 N 003 W 21 K 01 S \\
010 N 002 W 33 R 16 S \\
001 S 003 W 09 E 02 S\end{array}$ & $\begin{array}{l}05-28-92 \\
05-26-92 \\
11-25-91 \\
06-30-92 \\
11-25-91\end{array}$ & $\begin{array}{r}130 \\
110 \\
14 \\
13 \\
38\end{array}$ & $\begin{array}{l}44 \\
50 \\
24 \\
23 \\
18\end{array}$ & $\begin{array}{l}3 \\
3 \\
0.6 \\
0.6 \\
0.9\end{array}$ & $\begin{array}{l}3.7 \\
3.9 \\
2.7 \\
2.6 \\
5.4\end{array}$ & $\begin{array}{l}0 \\
0 \\
0 \\
0 \\
0\end{array}$ & $\begin{array}{l}209 \\
334 \\
115 \\
118 \\
393\end{array}$ & $\begin{array}{r}171 \\
274 \\
94 \\
96 \\
322\end{array}$ & $\begin{array}{r}260 \\
98 \\
15 \\
16 \\
59\end{array}$ & $\begin{array}{c}150 \\
69 \\
9.5 \\
9.8 \\
53\end{array}$ \\
\hline $001 \mathrm{~S} 003 \mathrm{~W} 18 \mathrm{~N} 02 \mathrm{~S}$ & $\begin{array}{l}06-30-92 \\
12-02-91 \\
06-30-92 \\
11-25-91 \\
07-30-92\end{array}$ & $\begin{array}{l}54 \\
26 \\
54 \\
35 \\
35\end{array}$ & $\begin{array}{l}23 \\
38 \\
76 \\
44 \\
45\end{array}$ & $\begin{array}{l}1 \\
1 \\
4 \\
2 \\
2\end{array}$ & $\begin{array}{l}5.6 \\
1.7 \\
1.7 \\
3.1 \\
2.8\end{array}$ & $\begin{array}{l}0 \\
0 \\
0 \\
0\end{array}$ & $\begin{array}{l}- \\
134 \\
149 \\
139 \\
129\end{array}$ & $\begin{array}{r}1_{301} \\
110 \\
122 \\
114 \\
106\end{array}$ & $\begin{array}{l}57 \\
18 \\
20 \\
41 \\
43\end{array}$ & $\begin{array}{l}59 \\
9.4 \\
7.5 \\
11 \\
14\end{array}$ \\
\hline
\end{tabular}

L Laboratory value. 
INORGANIC DATA

WATER YEAR OCTOBER 1991 TO SEPTEMBER 1992

LOCAL

NUMBER

002N007E03B01S 002N007E03E01S 003 N007E28D02S 003N007E32D02S 009 N002E03E01S

009N002E07Q01S 009N002E11C03S 010 N004E20D01S 001 N004W26E02S $001 N 004 W 34 G 03 S$

$003 N 004 W 13 F 02 S$ 003 N0O4W33H05S 004N003W30A06S 004N0O4W01A01S

$005 N 004$ W11P03S 005N004W25N01S 006N004W3OD10S $006 \mathrm{~N} 005 \mathrm{~W} 12 \mathrm{HO} 1 \mathrm{~S}$ 007 N004W07K02S

008 N003W05J01S $008 \mathrm{~N} 004 \mathrm{~W} 13 \mathrm{BO} 1 \mathrm{~S}$ OOON001W10G06S 009N001W11M11S 0O9N001W11RO2S

009N001W13B02S 009 N001W13B03S $009 N 001 W 13 B 04 S$ $009 N 001 W 13 \mathrm{H} 02 \mathrm{~S}$ 009 N002W05NOTS

009N003W21K01S 010N002W33R16S 001 S003W09E02S

$001 \mathrm{~S} 003 \mathrm{~W} 17 \mathrm{C0} 03 \mathrm{~S}$

0015003 W18N02S

0015004 W11D02S
DATE

\begin{tabular}{|c|c|c|c|}
\hline $\begin{array}{l}06-04-92 \\
06-04-92 \\
06-04-92 \\
06-04-92 \\
05-26-92\end{array}$ & $\begin{array}{l}0.60 \\
0.50 \\
0.40 \\
0.50 \\
0.50\end{array}$ & $\begin{array}{l}18 \\
18 \\
19 \\
19 \\
24\end{array}$ & $\begin{array}{l}-- \\
-- \\
-- \\
-- \\
270\end{array}$ \\
\hline $\begin{array}{l}06-02-92 \\
05-28-92 \\
05-28-92 \\
07-01-92 \\
11-26-91\end{array}$ & $\begin{array}{l}0.50 \\
0.40 \\
5.3 \\
0.80 \\
0.40\end{array}$ & $\begin{array}{l}22 \\
26 \\
60 \\
32 \\
22\end{array}$ & $\begin{array}{l}415 \\
270 \\
860 \\
364 \\
359\end{array}$ \\
\hline $\begin{array}{l}07-30-92 \\
10-29-91 \\
10-29-91 \\
10-29-91 \\
10-29-91\end{array}$ & $\begin{array}{l}0.40 \\
1.0 \\
0.30 \\
0.30 \\
0.20\end{array}$ & $\begin{array}{l}24 \\
27 \\
24 \\
20 \\
25\end{array}$ & $\begin{array}{l}354 \\
184 \\
175 \\
132 \\
204\end{array}$ \\
\hline $\begin{array}{l}11-01-91 \\
11-01-91 \\
10-30-91 \\
10-29-91 \\
10-29-91\end{array}$ & $\begin{array}{l}0.50 \\
0.40 \\
0.70 \\
0.70 \\
0.40\end{array}$ & $\begin{array}{l}21 \\
21 \\
38 \\
28 \\
28\end{array}$ & $\begin{array}{l}126 \\
120 \\
524 \\
573 \\
752\end{array}$ \\
\hline $\begin{array}{l}10-30-91 \\
10-30-91 \\
10-30-91 \\
05-27-92 \\
05-29-92\end{array}$ & $\begin{array}{l}0.70 \\
0.70 \\
0.60 \\
0.50 \\
0.50\end{array}$ & $\begin{array}{l}24 \\
29 \\
23 \\
23 \\
22\end{array}$ & $\begin{array}{l}642 \\
938 \\
618 \\
712 \\
942\end{array}$ \\
\hline $\begin{array}{l}05-27-92 \\
05-27-92 \\
05-27-92 \\
05-27-92 \\
10-30-91\end{array}$ & $\begin{array}{l}0.50 \\
0.50 \\
0.50 \\
0.60 \\
0.50\end{array}$ & $\begin{array}{l}27 \\
27 \\
25 \\
27 \\
29\end{array}$ & $\begin{array}{l}898 \\
856 \\
718 \\
416 \\
584\end{array}$ \\
\hline $\begin{array}{l}05-28-92 \\
05-26-92 \\
11-25-91 \\
06-30-92 \\
11-25-91\end{array}$ & $\begin{array}{l}0.50 \\
0.40 \\
0.30 \\
0.30 \\
0.30\end{array}$ & $\begin{array}{l}26 \\
25 \\
24 \\
26 \\
23\end{array}$ & $\begin{array}{l}816 \\
576 \\
165 \\
157 \\
595\end{array}$ \\
\hline $\begin{array}{l}06-30-92 \\
12-02-91 \\
06-30-92 \\
11-25-91 \\
07-30-92\end{array}$ & $\begin{array}{l}0.30 \\
0.80 \\
1.3 \\
0.60 \\
0.70\end{array}$ & $\begin{array}{l}25 \\
18 \\
17 \\
20 \\
22\end{array}$ & $\begin{array}{l}-- \\
174 \\
207 \\
213 \\
213\end{array}$ \\
\hline
\end{tabular}

$\begin{array}{lcclcccc} & & \text { SOLIDS, } & \text { SOLIDS, } & \text { NITRO- } & \text { NITRO- } & \text { NITRO- } & \text { NITRO- } \\ \text { FLUO- } & \text { SILICA, } & \text { RESIDUE } & \text { SUM OF } & \text { GEN, } & \text { GEN, } & \text { GEN, } & \text { GEN, } \\ \text { RIDE, } & \text { DIS- } & \text { AT 180 } & \text { CONSTI- } & \text { NITRITE } & \text { NO2+NO3 } & \text { AMMONIA } & \text { AMONIA } \\ \text { DIS- } & \text { SOLVED } & \text { DEG, C } & \text { TUENTS, } & \text { DIS- } & \text { DIS- } & \text { DIS- } & \text { DIS- } \\ \text { SOLVED } & \text { (MG/L } & \text { DIS- } & \text { DIS- } & \text { SOLVED } & \text { SOLVED } & \text { SOLVED } & \text { SOLVED } \\ \text { (MG/L } & \text { AS } & \text { SOLVED } & \text { SOLVED } & \text { (MG/L } & \text { (MG/L } & \text { (MG/L } & \text { (MG/L } \\ \text { AS F) } & \text { SIO2) } & (M G / L) & (M G / L) & \text { AS N) } & \text { AS N) } & \text { AS N) } & \text { AS NH4) }\end{array}$

$\begin{array}{rcccl}79 & <0.010 & 1.30 & <0.010 & -- \\ 87 & <0.010 & 1.10 & <0.010 & -- \\ 85 & <0.010 & 1.90 & <0.010 & -- \\ 81 & <0.010 & 1.20 & <0.010 & -- \\ 77 & <0.010 & 0.630 & 0.020 & 0.03 \\ & & & & \\ 06 & <0.010 & 1.80 & <0.010 & -- \\ 84 & <0.010 & 3.90 & 0.020 & 0.03 \\ 48 & <0.010 & <0.050 & 0.170 & 0.22 \\ 65 & <0.010 & 2.70 & 0.040 & 0.05 \\ 74 & <0.010 & 7.50 & <0.010 & -- \\ & & & & \\ 72 & <0.010 & 6.70 & <0.010 & -- \\ 02 & <0.010 & 1.20 & -- & -- \\ 02 & <0.010 & <0.050 & -- & -- \\ 42 & <0.010 & 1.10 & -- & -- \\ 20 & <0.010 & 4.60 & -- & -- \\ & & & & \\ 44 & <0.010 & 0.650 & -- & -- \\ 24 & <0.010 & 0.540 & -- & -- \\ 53 & <0.010 & 0.210 & -- & -- \\ 65 & <0.010 & <0.050 & -- & -- \\ 41 & <0.010 & 3.60 & -- & -- \\ 88 & 0.040 & 5.80 & -- & -- \\ 10 & <0.010 & 11.0 & -- & -- \\ 52 & <0.010 & 2.50 & -- & -- \\ 81 & <0.010 & 0.470 & 0.010 & 0.01 \\ 28 & 0.500 & 2.20 & 0.130 & 0.17 \\ 83 & <0.010 & 2.10 & 0.010 & 0.01 \\ 854 & <0.010 & 2.10 & 0.020 & 0.03 \\ 55 & <0.010 & 7.30 & 0.020 & 0.03 \\ 21 & <0.010 & 0.750 & 0.010 & 0.01 \\ 82 & <0.010 & 1.60 & -- & -- \\ 11 & <0.010 & 1.90 & 0.010 & 0.01 \\ 568 & <0.010 & 2.10 & 0.010 & 0.01 \\ 61 & <0.010 & 1.20 & <0.010 & -- \\ 66 & <0.010 & 1.30 & 0.040 & 0.05 \\ 47 & 0.030 & 8.00 & <0.010 & -- \\ & & & & \\ 51 & <0.010 & 11.0 & 0.020 & .0 .03 \\ 84 & <0.010 & 2.20 & <0.010 & -- \\ 92 & <0.010 & 0.720 & 0.030 & 0.04 \\ 18 & <0.010 & 0.920 & <0.010 & -- \\ 19 & <0.010 & 0.910 & 0.010 & 0.01 \\ & & & & \end{array}$

< Actual value is less than value shown. 
WATER YEAR OCTOBER 1991 TO SEPTEMBER 1992

\begin{tabular}{|c|c|c|c|c|c|c|c|c|c|}
\hline $\begin{array}{l}\text { LOCAL } \\
\text { NUMBER }\end{array}$ & DATE & $\begin{array}{l}\text { NITRO- } \\
\text { GEN, AM- } \\
\text { MONIA + } \\
\text { ORGANIC } \\
\text { DIS. } \\
\text { (MG/L } \\
\text { AS N) }\end{array}$ & $\begin{array}{c}\text { PHOS- } \\
\text { PHORUS } \\
\text { DIS- } \\
\text { SOLVED } \\
\text { (MG/L } \\
\text { AS P) }\end{array}$ & $\begin{array}{l}\text { PHOS- } \\
\text { PHORUS } \\
\text { ORTHO, } \\
\text { DIS- } \\
\text { SOLVED } \\
\text { (MG/L } \\
\text { AS P) }\end{array}$ & $\begin{array}{l}\text { PHOS- } \\
\text { PHATE, } \\
\text { ORTHO, } \\
\text { DIS- } \\
\text { SOLVED } \\
\text { (MG/L } \\
\text { AS PO4) }\end{array}$ & $\begin{array}{l}\text { ARSENIC } \\
\text { DIS- } \\
\text { SOLVED } \\
\text { (UG/L } \\
\text { AS AS) }\end{array}$ & $\begin{array}{l}\text { BORON, } \\
\text { DIS- } \\
\text { SOLVED } \\
\text { (UG/L } \\
\text { AS B) }\end{array}$ & $\begin{array}{l}\text { IRON, } \\
\text { DIS- } \\
\text { SOLVED } \\
\text { (UG/L } \\
\text { AS FE) }\end{array}$ & $\begin{array}{l}\text { MANGA- } \\
\text { NESE, } \\
\text { DIS- } \\
\text { SOLVED } \\
\text { (UG/L } \\
\text { AS MN) }\end{array}$ \\
\hline $\begin{array}{l}\text { 002N007E03B01S } \\
002 \mathrm{~N} 007 \mathrm{E} 03 \mathrm{E} 01 \mathrm{~S} \\
003 \mathrm{~N} 007 \mathrm{E} 28 \mathrm{D} 02 \mathrm{~S} \\
003 \mathrm{~N} 007 \mathrm{E} 32 \mathrm{DO2S} \\
009 \mathrm{~N} 002 \mathrm{E} 03 \mathrm{E} 01 \mathrm{~S}\end{array}$ & $\begin{array}{l}06-04-92 \\
06-04-92 \\
06-04-92 \\
06-04-92 \\
05-26-92\end{array}$ & $\begin{array}{l}<0.20 \\
<0.20 \\
<0.20 \\
<0.20 \\
-.\end{array}$ & $\begin{array}{l}0.030 \\
0.020 \\
0.020 \\
0.020 \\
-.\end{array}$ & $\begin{array}{r}<0.010 \\
<0.010 \\
<0.010 \\
<0.010 \\
0.020\end{array}$ & $\begin{array}{l}-- \\
-- \\
-- \\
0.06\end{array}$ & $\begin{array}{l}-- \\
-- \\
-- \\
-- \\
\end{array}$ & $\begin{array}{r}60 \\
50 \\
50 \\
40 \\
150\end{array}$ & $\begin{array}{r}<3 \\
<3 \\
4 \\
<3 \\
4\end{array}$ & $\begin{array}{l}<1 \\
<1 \\
<1 \\
<1 \\
<1\end{array}$ \\
\hline $\begin{array}{l}\text { 009N002E07Q01S } \\
009 N 002 E 11 \mathrm{CO} 3 \mathrm{~S} \\
010 \mathrm{~N} 004 \mathrm{E} 20 \mathrm{DO} 1 \mathrm{~S} \\
001 \mathrm{~N} 004 \mathrm{~W} 26 \mathrm{E} 02 \mathrm{~S} \\
001 \mathrm{~N} 004 \mathrm{~W} 34 \mathrm{G} 03 \mathrm{~S}\end{array}$ & $\begin{array}{l}06-02-92 \\
05-28-92 \\
05-28-92 \\
07-01-92 \\
11-26-91\end{array}$ & $\begin{array}{l}-- \\
-- \\
<0.20 \\
<0.20\end{array}$ & $\begin{array}{c}-- \\
-- \\
0.020 \\
<0.010\end{array}$ & $\begin{array}{l}0.050 \\
0.040 \\
0.060 \\
0.020 \\
0.010\end{array}$ & $\begin{array}{l}0.15 \\
0.12 \\
0.18 \\
0.06 \\
0.03\end{array}$ & $\begin{array}{r}1 \\
2 \\
--\end{array}$ & $\begin{array}{r}130 \\
140 \\
3200 \\
310 \\
40\end{array}$ & $\begin{array}{r}6 \\
6 \\
14 \\
6 \\
3\end{array}$ & $\begin{aligned}<1 \\
<1 \\
8 \\
<1 \\
<1\end{aligned}$ \\
\hline $\begin{array}{l}003 \mathrm{~N} 004 \mathrm{~W} 13 \mathrm{~F} 02 \mathrm{~S} \\
003 \mathrm{~N} 004 \mathrm{~W} 33 \mathrm{H} 05 \mathrm{~S} \\
004 \mathrm{~N} 003 \mathrm{~W} 30 \mathrm{AO} 6 \mathrm{~S} \\
004 \mathrm{~N} 004 \mathrm{~W} 01 \mathrm{AO} 1 \mathrm{~S}\end{array}$ & $\begin{array}{l}07-30-92 \\
10-29-91 \\
10-29-91 \\
10-29-91 \\
10-29-91\end{array}$ & $\begin{array}{l}<0.20 \\
-- \\
-- \\
-- \\
--\end{array}$ & $\begin{array}{c}<0.010 \\
-- \\
-- \\
-- \\
--\end{array}$ & $\begin{array}{l}0.010 \\
-- \\
-- \\
-- \\
--\end{array}$ & $\begin{array}{l}0.03 \\
-- \\
-- \\
-- \\
--\end{array}$ & $\begin{array}{l}<1 \\
<1 \\
<1 \\
<1\end{array}$ & $\begin{array}{r}40 \\
50 \\
<10 \\
30 \\
20\end{array}$ & $\begin{array}{l}<3 \\
10 \\
38 \\
29 \\
10\end{array}$ & $\begin{array}{r}<1 \\
<1 \\
8 \\
4 \\
2\end{array}$ \\
\hline $\begin{array}{l}005 \mathrm{~N} 004 \mathrm{~W} 11 \mathrm{P} 03 \mathrm{~S} \\
005 \mathrm{~N} 004 \mathrm{~W} 25 \mathrm{NO} 1 \mathrm{~S} \\
006 \mathrm{~N} 004 \mathrm{~W} 30 \mathrm{D} 10 \mathrm{~S} \\
006 \mathrm{~N} 005 \mathrm{~W} 12 \mathrm{HO} 1 \mathrm{~S} \\
007 \mathrm{~N} 004 \mathrm{~W} 07 \mathrm{~K} 02 \mathrm{~S}\end{array}$ & $\begin{array}{l}11-01-91 \\
11-01-91 \\
10-30-91 \\
10-29-91 \\
10-29-91\end{array}$ & $\begin{array}{l}-- \\
-- \\
-- \\
-- \\
--\end{array}$ & $\begin{array}{l}-- \\
-- \\
-- \\
--\end{array}$ & $\begin{array}{l}-- \\
-- \\
-- \\
--\end{array}$ & $\begin{array}{l}-- \\
-- \\
-- \\
--\end{array}$ & $\begin{array}{r}2 \\
6 \\
2 \\
<1 \\
3\end{array}$ & $\begin{array}{r}120 \\
60 \\
140 \\
230 \\
<10\end{array}$ & $\begin{array}{r}37 \\
3 \\
11 \\
4100 \\
12\end{array}$ & $\begin{array}{r}<1 \\
<1 \\
<1 \\
830 \\
<1\end{array}$ \\
\hline $\begin{array}{l}008 \mathrm{~N} 003 \mathrm{~W} 05 \mathrm{~J} 01 \mathrm{~S} \\
008 \mathrm{~N} 004 \mathrm{~W} 13 \mathrm{~B} 01 \mathrm{~S} \\
009 \mathrm{~N} 001 \mathrm{~W} 10 \mathrm{G} 06 \mathrm{~S} \\
009 \mathrm{~N} 001 \mathrm{~W} 11 \mathrm{M} 11 \mathrm{~S} \\
009 \mathrm{~N} 001 \mathrm{~W} 1 \mathrm{R} 02 \mathrm{~S}\end{array}$ & $\begin{array}{l}10-30-91 \\
10-30-91 \\
10-30-91 \\
05-27-92 \\
05-29-92\end{array}$ & $\begin{array}{l}-- \\
-- \\
-- \\
-- \\
--\end{array}$ & $\begin{array}{l}-- \\
-- \\
-- \\
-- \\
--\end{array}$ & $\begin{array}{c}\cdots \\
0.080 \\
<0.010\end{array}$ & $\begin{array}{l}-- \\
-- \\
0.25\end{array}$ & $\begin{array}{r}<1 \\
3 \\
2 \\
2 \\
<1\end{array}$ & $\begin{array}{l}320 \\
350 \\
540 \\
420 \\
990\end{array}$ & $\begin{array}{r}15 \\
4 \\
7 \\
16 \\
940\end{array}$ & $\begin{array}{l}47 \\
<1 \\
66 \\
51 \\
68\end{array}$ \\
\hline $\begin{array}{l}009 \mathrm{~N} 001 \mathrm{~W} 13 \mathrm{~B} 02 \mathrm{~S} \\
009 \mathrm{~N} 001 \mathrm{~W} 13 \mathrm{~B} 03 \mathrm{~S} \\
009 \mathrm{~N} 001 \mathrm{~W} 13 \mathrm{~B} 04 \mathrm{~S} \\
009 \mathrm{~N} 001 \mathrm{~W} 13 \mathrm{H} 02 \mathrm{~S} \\
009 \mathrm{~N} 002 \mathrm{~W} 05 \mathrm{~N} 07 \mathrm{~S}\end{array}$ & $\begin{array}{l}05-27-92 \\
05-27-92 \\
05-27-92 \\
05-27-92 \\
10-30-91\end{array}$ & $\begin{array}{l}-- \\
-- \\
-- \\
--\end{array}$ & $\begin{array}{l}-- \\
-- \\
-- \\
--\end{array}$ & $\begin{array}{r}0.020 \\
0.020 \\
0.050 \\
<0.010 \\
---\end{array}$ & $\begin{array}{l}0.06 \\
0.06 \\
0.15 \\
-- \\
--\end{array}$ & $\begin{array}{l}1 \\
1 \\
2 \\
4 \\
5\end{array}$ & $\begin{array}{l}680 \\
580 \\
410 \\
310 \\
230\end{array}$ & $\begin{array}{r}11 \\
6 \\
3 \\
<3 \\
16\end{array}$ & $\begin{array}{r}<1 \\
2 \\
3 \\
<1 \\
<1\end{array}$ \\
\hline $\begin{array}{l}009 \mathrm{~N} 003 \mathrm{~W} 21 \mathrm{~K} 01 \mathrm{~S} \\
010 \mathrm{~N} 002 \mathrm{~W} 33 \mathrm{R} 16 \mathrm{~S} \\
001 \mathrm{~S} 003 \mathrm{~W} 09 \mathrm{E} 02 \mathrm{~S}\end{array}$ & $\begin{array}{l}05-28-92 \\
05-26-92 \\
11-25-91 \\
06-30-92 \\
11-25-91\end{array}$ & $\begin{array}{l}-- \\
-- \\
<0.20 \\
<0.20 \\
<0.20\end{array}$ & $\begin{array}{r}0.020 \\
0.030 \\
<0.010\end{array}$ & $\begin{array}{r}<0.010 \\
0.020 \\
0.040 \\
0.030 \\
<0.010\end{array}$ & $\begin{array}{l}-- \\
0.06 \\
0.12 \\
0.09 \\
--\end{array}$ & $\begin{array}{r}2 \\
\quad 1 \\
\ldots \\
\cdots \\
\cdots\end{array}$ & $\begin{array}{r}280 \\
240 \\
30 \\
30 \\
240\end{array}$ & $\begin{array}{r}69 \\
7 \\
<3 \\
6 \\
8\end{array}$ & $\begin{array}{r}5 \\
1 \\
<1 \\
<1 \\
12\end{array}$ \\
\hline $001 \mathrm{~S} 004 \mathrm{~W} 11 \mathrm{D} 02 \mathrm{~S}$ & $\begin{array}{l}06-30-92 \\
12-02-91 \\
06-30-92 \\
11-25-91 \\
07-30-92\end{array}$ & $\begin{array}{l}<0.20 \\
2.3 \\
<0.20 \\
<0.20 \\
<0.20\end{array}$ & $\begin{array}{r}<0.010 \\
0.020 \\
0.010 \\
<0.010 \\
<0.010\end{array}$ & $\begin{array}{r}<0.010 \\
<0.010 \\
0.010 \\
0.020 \\
0.020\end{array}$ & $\begin{array}{l}-- \\
-- \\
0.03 \\
0.06 \\
0.06\end{array}$ & $\begin{array}{l}-- \\
-- \\
-- \\
--\end{array}$ & $\begin{array}{r}340 \\
20 \\
50 \\
30 \\
30\end{array}$ & $\begin{array}{r}<3 \\
9 \\
<3 \\
<3 \\
<3\end{array}$ & $\begin{array}{r}7 \\
1 \\
<1 \\
<1 \\
<1\end{array}$ \\
\hline
\end{tabular}

$<$ Actual value is less than value shown. 
LOCAL
NUMBER

$301 \mathrm{~S} 004 \mathrm{~W} 22 \mathrm{HO} 2 \mathrm{~S}$

J01S004W23A05S

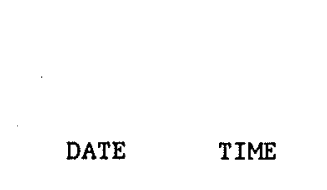

$\begin{array}{ll} & \\ \text { DEPTH } & \text { SPE- } \\ \text { OF } & \text { CON }- \\ \text { WELL, } & \text { DUCT- } \\ \text { TOTAL } & \text { ANCE } \\ \text { (FEET) } & \text { (US/CM) }\end{array}$

PH

WATER

WHOLE

TEMPER-

(STAND- ATURE

ARD

UNITS)

WATER
(DEG C)

(EEET) (US/CM

11-26-91

06-30-92

0945

0815

325
325

$11-25-91$

07-30-92

11-26-91

1315

1020

798
810

810

398

1106
704

7.5
7.8
8.4
8.2
8.0

18.5
19.0

28.0

28.0

18.0

530

8.0

$\begin{array}{cc}\begin{array}{c}\text { SODIUM } \\ \text { AD- }\end{array} & \begin{array}{r}\text { POTAS } \\ \text { SIUM }\end{array} \\ \text { SORP- } & \text { DIS- } \\ \text { TION } & \text { SOLVED } \\ \text { RATIO } & \text { (MG/L } \\ & \text { AS K) } \\ 1 & 4.2 \\ 1 & 4.2 \\ 5 & 1.8 \\ 5 & 1.7 \\ 0.6 & 2.3\end{array}$

CAR-
BONATE
WATER
WH IT
FIELD
MG /L AS
CO3

BICAR-
BONATE
WATER
WH IT
FIELD
MG/L AS
HCO3

ALKA-

LINITY

WAT WH SULFATE

TOT IT DIS-

FIELD SOLVED

MG/L AS (MG/L

$\mathrm{CACO} 3$ AS SO4)

$\begin{array}{llll}49 & 25 & 1 & 4.2 \\ 50 & 24 & 1 & 4.2 \\ 71 & 81 & 5 & 1.8 \\ 69 & 80 & 5 & 1.7 \\ 21 & 16 & 0.6 & 2.3\end{array}$

$\begin{array}{ll}11-26-91 & 49 \\ 06-30-92 & 50 \\ 11-25-91 & 71 \\ 07-30-92 & 69 \\ 11-26-91 & 21\end{array}$

298

283

132

124

$244 \quad 140$

$232 \quad 140$

$116 \quad 46$

10243

$148 \quad 49$

LOCAL,

NUMBER

$001 S 004 W 13 N 01 S$

$0015004 W 22 H 02 S$

$001 S 004 W 23 A 05 S$
CHLO-

RIDE,

DIS-

SOLVED

(MG/L

AS CL)

25
28
28
32
23

FLUO- SILICA,

RIDE, DIS-

DIS- SOLVED

(MG/L AS

AS F)

$\begin{array}{ll}0.50 & 20 \\ 0.50 & 21 \\ 0.90 & 15 \\ 1.0 & 16 \\ 0.40 & 20\end{array}$

SOLIDS, SOLIDS,

RESIDUE SUM OF

AT 180 CONSTI-

DEG, C TUENTS,

DIS- DIS-

SOLVED SOLVED

(MG/L) (MG/L)

468
521
196
268
305

534
545
249
240
344

$<0.010$

$<0.010$

$<0.010$

$<0.010$
NITRO-

GEN,

NITRITE

DIS-

SOLVED

$\mathrm{NO} 2+\mathrm{NO} 3$

DIS-

SOLVED

(MG/L

AS N)

7.50

8.40

0.540

0.520

12.0

NITRO-

GEN,

AMMONIA

DOIS-

(MG/L

AS N)

$<0.010$

0.030

$<0.010$

$<0.010$

$<0,010$
LOCAL
NUMBER

001S004W13N01S

$0015004 W 22 H 02 S$

001S004W23A05S
NITRO- NITRO-

GEN, GEN, AM- PHOS-

AMMONIA MONIA + PHORUS

DIS- ORGANIC DIS

SOLVED

SOLVED DIS.

(MG/L (MG/L

DIS-
SOLVED

(MG/L

AS P)

$-$

$<0.20$

$0.04<0.20$

$--\quad<0.20$

$--\quad<0.20$

$--\quad<0,20$

$<0.010$

0.010

$<0.010$

$<0.010$

$<0.010$
PHOS-

PHORUS

ORTHO,

DIS-

SOLVED

(MG/L

AS P)

0.010

$<0.010$

0.010

$<0.010$

0.010
PHOS-

PHATE,

ORTHO,

DIS-

SOLVED

(MG/L

AS PO4)

BORON,
DIS-
SOLVED
(UG /L
AS B)
150
140
80
90
50

MANGA-

IRON, NESE,

DIS- DIS-

SOLVED SOLVED

(UG/L (UG/L

AS FE) AS MN)

$\begin{array}{lr}0.03 & 150 \\ -- & 140 \\ 0.03 & 80 \\ -- & 90 \\ 0.03 & 50\end{array}$

$\begin{aligned} 3 & <1 \\ <3 & <1 \\ <3 & <1 \\ <3 & <1 \\ 3 & <1\end{aligned}$

< Actual value is less than value shown. 


\begin{tabular}{|c|c|c|c|c|c|c|c|c|c|}
\hline $\begin{array}{l}\text { LOCAL } \\
\text { NUMBER }\end{array}$ & DATE & TIME & $\begin{array}{l}\text { OF } \\
\text { WELL, } \\
\text { TOTAL } \\
\text { (FEET) }\end{array}$ & $\begin{array}{l}\text { BENZENE } \\
\text { TOTAL } \\
\text { (UG/L) }\end{array}$ & $\begin{array}{l}\text { BROMO- } \\
\text { FORM } \\
\text { TOTAL } \\
\text { (UG/L) }\end{array}$ & $\begin{array}{l}\text { CHLO- } \\
\text { RIDE } \\
\text { TOTAL } \\
\text { (UG/L) }\end{array}$ & $\begin{array}{l}\text { CHLORO- } \\
\text { BENZENE } \\
\text { TOTAL } \\
\text { (UG/L) }\end{array}$ & $\begin{array}{l}\text { BROMO- } \\
\text { METHANE } \\
\text { TOTAL } \\
\text { (UG/L) }\end{array}$ & $\begin{array}{l}\text { CHLORO- } \\
\text { ETHANE } \\
\text { TOTAL } \\
\text { (UG/L) }\end{array}$ \\
\hline $\begin{array}{l}\text { 001N004W26E02S } \\
001 N 004 W 34 G 03 S \\
001 S 003 W 09 E 02 S\end{array}$ & $\begin{array}{l}07-01-92 \\
11-26-91 \\
07-30-92 \\
11-25-91 \\
06-30-92\end{array}$ & $\begin{array}{l}0900 \\
1115 \\
1600 \\
1330 \\
1315\end{array}$ & $\begin{array}{l}689 \\
708 \\
708 \\
401 \\
401\end{array}$ & $\begin{array}{l}<0.2 \\
<0.2 \\
<0.2 \\
<0.2 \\
<0.2\end{array}$ & $\begin{array}{l}<0.2 \\
<0.2 \\
<0.2 \\
<0.2 \\
<0.2\end{array}$ & $\begin{array}{l}<0.2 \\
<0.2 \\
<0.2 \\
<0.2 \\
<0.2\end{array}$ & $\begin{array}{l}<0.20 \\
<0.20 \\
<0.20 \\
<0.20 \\
<0.20\end{array}$ & $\begin{array}{l}<0.2 \\
<0.2 \\
<0.2 \\
<0.2 \\
<0.2\end{array}$ & $\begin{array}{l}<0.2 \\
<0.2 \\
<0.2 \\
<0.2 \\
<0.2\end{array}$ \\
\hline $\begin{array}{l}001 \mathrm{~S} 003 \mathrm{~W} 17 \mathrm{C0} 3 \mathrm{~S} \\
001 \mathrm{~S} 003 \mathrm{~W} 18 \mathrm{~N} 02 \mathrm{~S} \\
001 \mathrm{~S} 004 \mathrm{~W} 11 \mathrm{D} 02 \mathrm{~S}\end{array}$ & $\begin{array}{l}11-25-91 \\
06-30-92 \\
12-02-91 \\
06-30-92 \\
11-25-91\end{array}$ & $\begin{array}{l}1215 \\
1445 \\
0915 \\
1030 \\
1015\end{array}$ & $\begin{array}{l}296 \\
296 \\
988 \\
988 \\
963\end{array}$ & $\begin{array}{l}<0.2 \\
<0.2 \\
<0.2 \\
<0.2 \\
<0.2\end{array}$ & $\begin{array}{l}<0.2 \\
<0.2 \\
<0.2 \\
<0.2 \\
<0.2\end{array}$ & $\begin{array}{l}<0.2 \\
<0.2 \\
<0.2 \\
<0.2 \\
<0.2\end{array}$ & $\begin{array}{l}<0.20 \\
<0.20 \\
<0.20 \\
<0.20 \\
<0.20\end{array}$ & $\begin{array}{l}<0.2 \\
<0.2 \\
<0.2 \\
<0.2 \\
<0.2\end{array}$ & $\begin{array}{l}<0.2 \\
<0.2 \\
<0.2 \\
<0.2 \\
<0.2\end{array}$ \\
\hline $001 \mathrm{~S} 004 \mathrm{~W} 13 \mathrm{NO} 1 \mathrm{~S}$ & $\begin{array}{l}07-30-92 \\
11-26-91 \\
06-30-92 \\
11-25-91 \\
07-30-92\end{array}$ & $\begin{array}{l}1050 \\
0945 \\
0815 \\
0930 \\
1315\end{array}$ & $\begin{array}{r}963 \\
325 \\
325 \\
1106 \\
1106\end{array}$ & $\begin{array}{l}<0.2 \\
<0.2 \\
<0.2 \\
<0.2 \\
<0.2\end{array}$ & $\begin{array}{l}<0.2 \\
<0.2 \\
<0.2 \\
<0.2 \\
<0.2\end{array}$ & $\begin{array}{l}<0.2 \\
<0.2 \\
<0.2 \\
<0.2 \\
<0.2\end{array}$ & $\begin{array}{l}<0.20 \\
<0.20 \\
<0.20 \\
<0.20 \\
<0.20\end{array}$ & $\begin{array}{l}<0.2 \\
<0.2 \\
<0.2 \\
<0.2 \\
<0.2\end{array}$ & $\begin{array}{l}<0.2 \\
<0.2 \\
<0.2 \\
<0.2 \\
<0.2\end{array}$ \\
\hline $001 \mathrm{~S} 004 \mathrm{~W} 23 \mathrm{~A} 05 \mathrm{~S}$ & $11-26-91$ & 1020 & 704 & $<0.2$ & $<0.2$ & $<0.2$ & $<0.20$ & $<0.2$ & $<0.2$ \\
\hline
\end{tabular}

\begin{tabular}{|c|c|c|c|c|c|c|c|c|c|}
\hline $\begin{array}{l}\text { LOCAL } \\
\text { NUMBER }\end{array}$ & DATE & $\begin{array}{l}\text { CHLORO- } \\
\text { PROPENE } \\
\text { TOTAL } \\
\text { (UG/L) }\end{array}$ & $\begin{array}{l}\text { CHLORO- } \\
\text { FORM } \\
\text { TOTAL } \\
\text { (UG/L) }\end{array}$ & $\begin{array}{l}\text { BROMO- } \\
\text { METHANE } \\
\text { TOTAL } \\
\text { (UG/L) }\end{array}$ & $\begin{array}{l}\text { FLUORO- } \\
\text { METHANE } \\
\text { TOTAL } \\
\text { (UG/L) }\end{array}$ & $\begin{array}{l}\text { ETHYL- } \\
\text { BENZENE } \\
\text { TOTAL } \\
\text { (UG/L) }\end{array}$ & $\begin{array}{l}\text { METHYL- } \\
\text { BROMIDE } \\
\text { TOTAL } \\
\text { (UG/L) }\end{array}$ & $\begin{array}{l}\text { CHLO- } \\
\text { RIDE } \\
\text { TOTAL } \\
\text { (UG/L) }\end{array}$ & $\begin{array}{l}\text { CHLO- } \\
\text { RIDE } \\
\text { TOTAL } \\
\text { (UG/L) }\end{array}$ \\
\hline $\begin{array}{l}001 \mathrm{~N} 004 \mathrm{~W} 26 \mathrm{E} 02 \mathrm{~S} \\
001 \mathrm{~N} 004 \mathrm{~W} 34 \mathrm{G} 03 \mathrm{~S}\end{array}$ & $\begin{array}{l}07-01-92 \\
11-26-91 \\
07-30-92 \\
11-25-91 \\
06-30-92\end{array}$ & $\begin{array}{l}<0.2 \\
<0.2 \\
<0.2 \\
<0.2 \\
<0.2\end{array}$ & $\begin{array}{l}<0.2 \\
<0.2 \\
<0.2 \\
<0.2 \\
<0.2\end{array}$ & $\begin{array}{l}<0.2 \\
<0.2 \\
<0.2 \\
<0.2 \\
<0.2\end{array}$ & $\begin{array}{r}<0.2 \\
2.1 \\
3.0 \\
<0.2 \\
<0.2\end{array}$ & $\begin{array}{l}<0.2 \\
<0.2 \\
<0.2 \\
<0.2 \\
<0.2\end{array}$ & $\begin{array}{l}<0.2 \\
<0.2 \\
<0.2 \\
<0.2 \\
<0.2\end{array}$ & $\begin{array}{l}<0.2 \\
<0.2 \\
<0.2 \\
<0.2 \\
<0.2\end{array}$ & $\begin{array}{l}<0.2 \\
<0.2 \\
<0.2 \\
<0.2 \\
<0.2\end{array}$ \\
\hline $\begin{array}{l}001 \mathrm{~S} 003 \mathrm{~W} 17 \mathrm{C} 03 \mathrm{~S} \\
001 \mathrm{~S} 003 \mathrm{~W} 18 \mathrm{~N} 02 \mathrm{~S}\end{array}$ & $\begin{array}{l}11-25-91 \\
06-30-92 \\
12-02-91 \\
06-30-92 \\
11-25-91\end{array}$ & $\begin{array}{l}<0.2 \\
<0.2 \\
<0.2 \\
<0.2 \\
<0.2\end{array}$ & $\begin{aligned}<0.2 \\
0.2 \\
<0.2 \\
<0.2 \\
<0.2\end{aligned}$ & $\begin{array}{l}<0.2 \\
<0.2 \\
<0.2 \\
<0.2 \\
<0.2\end{array}$ & $\begin{array}{l}<0.2 \\
<0.2 \\
<0.2 \\
<0.2 \\
<0.2\end{array}$ & $\begin{array}{l}<0.2 \\
<0.2 \\
<0.2 \\
<0.2 \\
<0.2\end{array}$ & $\begin{array}{l}<0.2 \\
<0.2 \\
<0.2 \\
<0.2 \\
<0.2\end{array}$ & $\begin{array}{l}<0.2 \\
<0.2 \\
<0.2 \\
<0.2 \\
<0.2\end{array}$ & $\begin{array}{l}<0.2 \\
<0.2 \\
<0.2 \\
<0.2 \\
<0.2\end{array}$ \\
\hline $001 \mathrm{~S} 004 \mathrm{~W} 13 \mathrm{~N} 01 \mathrm{~S}$ & $\begin{array}{l}07-30-92 \\
11-26-91 \\
06-30-92 \\
11-25-91 \\
07-30-92\end{array}$ & $\begin{array}{l}<0.2 \\
<0.2 \\
<0.2 \\
<0.2 \\
<0.2\end{array}$ & $\begin{array}{l}<0.2 \\
<0.2 \\
<0.2 \\
<0.2 \\
<0.2\end{array}$ & $\begin{array}{l}<0.2 \\
<0.2 \\
<0.2 \\
<0.2 \\
<0.2\end{array}$ & $\begin{array}{l}<0.2 \\
<0.2 \\
<0.2 \\
<0.2 \\
<0.2\end{array}$ & $\begin{array}{l}<0.2 \\
<0.2 \\
<0.2 \\
<0.2 \\
<0.2\end{array}$ & $\begin{array}{l}<0.2 \\
<0.2 \\
<0.2 \\
<0.2 \\
<0.2\end{array}$ & $\begin{array}{l}<0.2 \\
<0.2 \\
<0.2 \\
<0.2 \\
<0.2\end{array}$ & $\begin{array}{l}<0.2 \\
<0.2 \\
<0.2 \\
<0.2 \\
<0.2\end{array}$ \\
\hline $001 \mathrm{~S} 004 \mathrm{~W} 23 \mathrm{~A} 05 \mathrm{~S}$ & $11-26-91$ & $<0.2$ & $<0.2$ & $<0.2$ & $<0.2$ & $<0.2$ & $<0.2$ & $<0.2$ & $<0.2$ \\
\hline
\end{tabular}

$<$ Actual value is less than value shown. 
ORGANIC DATA

WATER YEAR OCTOBER 1991 TO SEPTEMBER 1992

\begin{tabular}{|c|c|c|c|c|c|c|c|c|c|}
\hline $\begin{array}{l}\text { LOCAL } \\
\text { NUMBER }\end{array}$ & DATE & $\begin{array}{l}\text { CHLORO- } \\
\text { THATE } \\
\text { (UG/L) }\end{array}$ & $\begin{array}{l}\text { ETHYL- } \\
\text { ENE } \\
\text { TOTAL } \\
\text { (UG/L) }\end{array}$ & $\begin{array}{l}\text { CHLORO- } \\
\text { ETHANE } \\
\text { TOTAL } \\
\text { (UG/L) }\end{array}$ & $\begin{array}{l}\text { WATER } \\
\text { UNFLTRD } \\
\text { REC } \\
\text { (UG/L) }\end{array}$ & $\begin{array}{l}\text { CHLORO- } \\
\text { PROPENE } \\
\text { TOTAL } \\
\text { (UG/L) }\end{array}$ & $\begin{array}{l}\text { CHLORO- } \\
\text { ETHANE } \\
\text { TOTAL } \\
\text { (UG/L) }\end{array}$ & $\begin{array}{l}\text { CHLORO- } \\
\text { WAT UNF } \\
\text { REC } \\
\text { (UG/L) }\end{array}$ & $\begin{array}{l}\text { WATER } \\
\text { UNFLTRD } \\
\text { REC } \\
\text { (UG/L) }\end{array}$ \\
\hline $\begin{array}{l}001 \mathrm{~N} 004 \mathrm{~W} 26 \mathrm{E} 02 \mathrm{~S} \\
001 \mathrm{~N} 004 \mathrm{~W} 34 \mathrm{G} 03 \mathrm{~S}\end{array}$ & $\begin{array}{l}07-01-92 \\
11-26-91 \\
07-30-92 \\
11-25-91 \\
06-30-92\end{array}$ & $\begin{array}{l}<0.2 \\
<0.2 \\
<0.2 \\
<0.2 \\
<0.2\end{array}$ & $\begin{array}{l}<0.2 \\
<0.2 \\
<0.2 \\
<0.2 \\
<0.2\end{array}$ & $\begin{array}{l}<0.2 \\
<0.2 \\
<0.2 \\
<0.2 \\
<0.2\end{array}$ & $\begin{array}{l}<0.20 \\
<0.20 \\
<0.20 \\
<0.20 \\
<0.20\end{array}$ & $\begin{array}{l}<0.20 \\
<0.20 \\
<0.20 \\
<0.20 \\
<0.20\end{array}$ & $\begin{array}{l}<0.2 \\
<0.2 \\
<0.2 \\
<0.2 \\
<0.2\end{array}$ & $\begin{array}{l}<0.2 \\
<0.2 \\
<0.2 \\
<0.2 \\
<0.2\end{array}$ & $\begin{array}{l}<0.20 \\
<0.20 \\
<0.20 \\
<0.20 \\
<0.20\end{array}$ \\
\hline $\begin{array}{l}0015003 W 18 N 02 S \\
001 S 004 W 11 D 02 S\end{array}$ & $\begin{array}{l}11-25-91 \\
06-30-92 \\
12-02-91 \\
06-30-92 \\
11-25-91\end{array}$ & $\begin{array}{l}<0.2 \\
<0.2 \\
<0.2 \\
<0.2 \\
<0.2\end{array}$ & $\begin{array}{l}<0.2 \\
<0.2 \\
<0.2 \\
<0.2 \\
<0.2\end{array}$ & $\begin{array}{l}<0.2 \\
<0.2 \\
<0.2 \\
<0.2 \\
<0.2\end{array}$ & $\begin{array}{l}<0.20 \\
<0.20 \\
<0.20 \\
<0.20 \\
<0.20\end{array}$ & $\begin{array}{l}<0.20 \\
<0.20 \\
<0.20 \\
<0.20 \\
<0.20\end{array}$ & $\begin{array}{l}<0.2 \\
<0.2 \\
<0.2 \\
<0.2 \\
<0.2\end{array}$ & $\begin{array}{l}<0.2 \\
<0.2 \\
<0.2 \\
<0.2 \\
<0.2\end{array}$ & $\begin{array}{l}<0.20 \\
<0.20 \\
<0.20 \\
<0.20 \\
<0.20\end{array}$ \\
\hline $\begin{array}{l}001 \mathrm{~S} 004 \mathrm{~W} 13 \mathrm{NO} 1 \mathrm{~S} \\
001 \mathrm{~S} 004 \mathrm{~W} 22 \mathrm{HO} 2 \mathrm{~S}\end{array}$ & $\begin{array}{l}07-30-92 \\
11-26-91 \\
06-30-92 \\
11-25-91 \\
07-30-92\end{array}$ & $\begin{array}{l}<0.2 \\
<0.2 \\
<0.2 \\
<0.2 \\
<0.2\end{array}$ & $\begin{array}{l}<0.2 \\
<0.2 \\
<0.2 \\
<0.2 \\
<0.2\end{array}$ & $\begin{array}{l}<0.2 \\
<0.2 \\
<0.2 \\
<0.2 \\
<0.2\end{array}$ & $\begin{array}{l}<0.20 \\
<0.20 \\
<0.20 \\
<0.20 \\
<0.20\end{array}$ & $\begin{array}{l}<0.20 \\
<0.20 \\
<0.20 \\
<0.20 \\
<0.20\end{array}$ & $\begin{array}{l}<0.2 \\
<0.2 \\
<0.2 \\
<0.2 \\
<0.2\end{array}$ & $\begin{array}{l}<0.2 \\
<0.2 \\
<0.2 \\
<0.2 \\
<0.2\end{array}$ & $\begin{array}{l}<0.20 \\
<0.20 \\
<0.20 \\
<0.20 \\
<0.20\end{array}$ \\
\hline $0015004 \mathrm{~W} 23 \mathrm{~A} 05 \mathrm{~S}$ & $11-26-91$ & $<0.2$ & $<0.2$ & $<0.2$ & $<0.20$ & $<0.20$ & $<0.2$ & $<0.2$ & $<0.20$ \\
\hline $\begin{array}{l}\text { LOCAL } \\
\text { NUMBER }\end{array}$ & DATE & $\begin{array}{l}1,2- \\
\text { DIBROMO } \\
\text { ETHANE } \\
\text { WATER } \\
\text { WHOLE } \\
\text { TOTAL } \\
\text { (UG/L) }\end{array}$ & $\begin{array}{c}1,2-D I- \\
\text { CHLORO- } \\
\text { ETHANE } \\
\text { TOTAL } \\
\text { (UG/L) }\end{array}$ & $\begin{array}{c}\text { 1,2-DI- } \\
\text { CHLORO- } \\
\text { PROPANE } \\
\text { TOTAL } \\
\text { (UG/L) }\end{array}$ & $\begin{array}{l}\text { DIBROMO } \\
\text { CHLORO- } \\
\text { PROPANE } \\
\text { WATER } \\
\text { WHOLE } \\
\text { TOT.REC } \\
\text { (UG/L) }\end{array}$ & $\begin{array}{c}1,2- \\
\text { TRANSDI } \\
\text { CHLORO- } \\
\text { ETHENE } \\
\text { TOTAL } \\
\text { (UG/L) }\end{array}$ & $\begin{array}{c}\text { BENZENE } \\
1,4-D I- \\
\text { CHLORO- } \\
\text { WATER } \\
\text { UNFLTRD } \\
\text { REC } \\
\text { (UG/L) }\end{array}$ & $\begin{array}{l}\text { STYRENE } \\
\text { TOTAL } \\
\text { (UG/L) }\end{array}$ & $\begin{array}{l}\text { TETRA- } \\
\text { CHLORO- } \\
\text { ETHYL- } \\
\text { ENE } \\
\text { TOTAL } \\
\text { (UG/L) }\end{array}$ \\
\hline $\begin{array}{l}001 \mathrm{~N} 004 \mathrm{~W} 26 \mathrm{E} 02 \mathrm{~S} \\
001 \mathrm{~N} 004 \mathrm{~W} 34 \mathrm{G} 03 \mathrm{~S}\end{array}$ & $\begin{array}{l}07-01-92 \\
11-26-91 \\
07-30-92 \\
11-25-91 \\
06-30-92\end{array}$ & $\begin{array}{l}<0.0 \\
<0.2 \\
<0.0 \\
<0.2 \\
<0.0\end{array}$ & $\begin{array}{l}<0.2 \\
<0.2 \\
<0.2 \\
<0.2 \\
<0.2\end{array}$ & $\begin{array}{l}<0.2 \\
<0.2 \\
<0.2 \\
<0.2 \\
<0.2\end{array}$ & $\begin{array}{l}<0.0 \\
<0.0 \\
<0.0 \\
<0.0 \\
<0.0\end{array}$ & $\begin{array}{r}<0.2 \\
0.2 \\
0.2 \\
<0.2 \\
<0.2\end{array}$ & $\begin{array}{l}<0.20 \\
<0.20 \\
<0.20 \\
<0.20 \\
<0.20\end{array}$ & $\begin{array}{l}<0.2 \\
<0.2 \\
<0.2 \\
<0.2 \\
<0.2\end{array}$ & $\begin{array}{r}<0.2 \\
2.7 \\
3.3 \\
<0.2 \\
<0.2\end{array}$ \\
\hline $001 \mathrm{~S} 003 \mathrm{~W} 17 \mathrm{C} 03 \mathrm{~S}$ & $\begin{array}{l}11-25-91 \\
06-30-92 \\
12-02-91 \\
06-30-92 \\
11-25-91\end{array}$ & $\begin{array}{l}<0.2 \\
<0.0 \\
<0.2 \\
<0.0 \\
<0.2\end{array}$ & $\begin{array}{l}<0.2 \\
<0.2 \\
<0.2 \\
<0.2 \\
<0.2\end{array}$ & $\begin{array}{l}<0.2 \\
<0.2 \\
<0.2 \\
<0.2 \\
<0.2\end{array}$ & $\begin{array}{l}<0.0 \\
<0.0 \\
<0.0 \\
<0.0 \\
<0.0\end{array}$ & $\begin{array}{l}<0.2 \\
<0.2 \\
<0.2 \\
<0.2 \\
<0.2\end{array}$ & $\begin{array}{l}<0.20 \\
<0.20 \\
<0.20 \\
<0.20 \\
<0.20\end{array}$ & $\begin{array}{l}<0.2 \\
<0.2 \\
<0.2 \\
<0.2 \\
<0.2\end{array}$ & $\begin{array}{l}<0.2 \\
<0.2 \\
<0.2 \\
<0.2 \\
<0.2\end{array}$ \\
\hline $001 \mathrm{~S} 004 \mathrm{~W} 22 \mathrm{H} 02 \mathrm{~S}$ & $\begin{array}{l}07-30-92 \\
11-26-91 \\
06-30-92 \\
11-25-91 \\
07-30-92\end{array}$ & $\begin{array}{l}<0.0 \\
<0.2 \\
<0.0 \\
<0.2 \\
<0.0\end{array}$ & $\begin{array}{l}<0.2 \\
<0.2 \\
<0.2 \\
<0.2 \\
<0.2\end{array}$ & $\begin{array}{r}<0.2 \\
0.4 \\
0.3 \\
<0.2 \\
<0.2\end{array}$ & $\begin{array}{r}<0.0 \\
0.1 \\
0.1 \\
<0.0 \\
<0.0\end{array}$ & $\begin{array}{r}<0.2 \\
0.7 \\
0.5 \\
<0.2 \\
<0.2\end{array}$ & $\begin{array}{l}<0.20 \\
<0.20 \\
<0.20 \\
<0.20 \\
<0.20\end{array}$ & $\begin{array}{l}<0.2 \\
<0.2 \\
<0.2 \\
<0.2 \\
<0.2\end{array}$ & $\begin{array}{l}<0.2 \\
<0.2 \\
<0.2 \\
<0.2 \\
<0.2\end{array}$ \\
\hline $0015004 \mathrm{~W} 23 \mathrm{~A} 05 \mathrm{~S}$ & $11-26-91$ & $<0.2$ & $<0.2$ & $<0.2$ & 0.4 & $<0.2$ & $<0.20$ & $<0,2$ & $<0.2$ \\
\hline
\end{tabular}

< Actual value is less than value shown. 
ORGANIC DATA

WATER YEAR OCTOBER 1991 TO SEPTEMBER 1992

\begin{tabular}{|c|c|c|c|c|c|c|c|c|}
\hline $\begin{array}{l}\text { LOCAL } \\
\text { NUMBER }\end{array}$ & DATE & $\begin{array}{l}\text { TOLUENE } \\
\text { TOTAL } \\
\text { (UG/L) }\end{array}$ & $\begin{array}{l}\text { TRANS- } \\
1,3-D I- \\
\text { CHLORO- } \\
\text { PROPENE } \\
\text { TOTAL } \\
\text { (UG/L) }\end{array}$ & $\begin{array}{c}\text { TRI- } \\
\text { CHLORO- } \\
\text { ETHYL- } \\
\text { ENE } \\
\text { TOTAL } \\
\text { (UG/L) }\end{array}$ & $\begin{array}{l}\text { TRI- } \\
\text { CHLORO- } \\
\text { FLUORO- } \\
\text { METHANE } \\
\text { TOTAL } \\
\text { (UG/L) }\end{array}$ & $\begin{array}{c}2- \\
\text { CHLORO- } \\
\text { ETHYL- } \\
\text { VINYL- } \\
\text { ETHER } \\
\text { TOTAL } \\
\text { (UG/L) }\end{array}$ & $\begin{array}{l}\text { VINYL } \\
\text { CHLO- } \\
\text { RIDE } \\
\text { TOTAL } \\
\text { (UG/L) }\end{array}$ & $\begin{array}{l}\text { XYLENE } \\
\text { WATER } \\
\text { UNFLTRD } \\
\text { REC } \\
\text { (UG/L) }\end{array}$ \\
\hline $\begin{array}{l}001 N 004 W 26 E 02 S \\
001 N 004 W 34 G 03 S \\
001 S 003 W 09 E 02 S\end{array}$ & $\begin{array}{l}07-01-92 \\
11-26-91 \\
07-30-92 \\
11-25-91 \\
06-30-92\end{array}$ & $\begin{array}{l}<0.2 \\
<0.2 \\
<0.2 \\
<0.2 \\
<0.2\end{array}$ & $\begin{array}{l}<0.2 \\
<0,2 \\
<0.2 \\
<0,2 \\
<0,2\end{array}$ & $\begin{array}{r}<0.2 \\
1.7 \\
1.7 \\
<0.2 \\
<0.2\end{array}$ & $\begin{array}{r}<0.2 \\
0.4 \\
0.6 \\
<0.2 \\
<0.2\end{array}$ & $\begin{array}{l}<0.2 \\
<0.2 \\
<0.2 \\
<0.2 \\
<0.2\end{array}$ & $\begin{array}{l}<0.2 \\
<0.2 \\
<0.2 \\
<0.2 \\
<0.2\end{array}$ & $\begin{array}{l}<0.20 \\
<0.20 \\
<0.20 \\
<0.20 \\
<0.20\end{array}$ \\
\hline $\begin{array}{l}0015003 W 17 \mathrm{C03S} \\
0015003 W 18 N 02 \mathrm{~S} \\
0015004 \mathrm{~W} 11 \mathrm{D} 02 \mathrm{~S}\end{array}$ & $\begin{array}{l}11-25-91 \\
06-30-92 \\
12-02-91 \\
06-30-92 \\
11-25-91\end{array}$ & $\begin{array}{l}<0.2 \\
<0.2 \\
<0.2 \\
<0.2 \\
<0.2\end{array}$ & $\begin{array}{l}<0.2 \\
<0.2 \\
<0.2 \\
<0.2 \\
<0.2\end{array}$ & $\begin{array}{l}<0.2 \\
<0.2 \\
<0.2 \\
<0.2 \\
<0.2\end{array}$ & $\begin{array}{l}<0.2 \\
<0.2 \\
<0.2 \\
<0.2 \\
<0.2\end{array}$ & $\begin{array}{l}<0.2 \\
<0.2 \\
<0.2 \\
<0.2 \\
<0.2\end{array}$ & $\begin{array}{l}<0.2 \\
<0.2 \\
<0.2 \\
<0.2 \\
<0.2\end{array}$ & $\begin{array}{l}<0.20 \\
<0.20 \\
<0.20 \\
<0.20 \\
<0.20\end{array}$ \\
\hline $\begin{array}{l}001 \mathrm{~S} 004 \mathrm{~W} 13 \mathrm{~N} 01 \mathrm{~S} \\
001 \mathrm{~S} 004 \mathrm{~W} 22 \mathrm{H} 02 \mathrm{~S}\end{array}$ & $\begin{array}{l}07-30-92 \\
11-26-91 \\
06-30-92 \\
11-25-91 \\
07-30-92\end{array}$ & $\begin{array}{l}<0.2 \\
<0.2 \\
<0.2 \\
<0.2 \\
<0.2\end{array}$ & $\begin{array}{l}<0.2 \\
<0.2 \\
<0.2 \\
<0.2 \\
<0.2\end{array}$ & $\begin{array}{r}<0.2 \\
0.5 \\
0.4 \\
<0.2 \\
<0.2\end{array}$ & $\begin{array}{l}<0.2 \\
<0.2 \\
<0.2 \\
<0.2 \\
<0.2\end{array}$ & $\begin{array}{l}<0.2 \\
<0.2 \\
<0.2 \\
<0.2 \\
<0.2\end{array}$ & $\begin{array}{l}<0.2 \\
<0.2 \\
<0.2 \\
<0.2 \\
<0.2\end{array}$ & $\begin{array}{l}<0.20 \\
<0.20 \\
<0.20 \\
<0.20 \\
<0.20\end{array}$ \\
\hline $001 \mathrm{~S} 004 \mathrm{~W} 23 \mathrm{AO} 5 \mathrm{~S}$ & $11-26-91$ & $<0.2$ & $<0.2$ & $<0.2$ & $<0.2$ & $<0.2$ & $<0,2$ & $<0.20$ \\
\hline
\end{tabular}

< Actual value is less than value shown. 

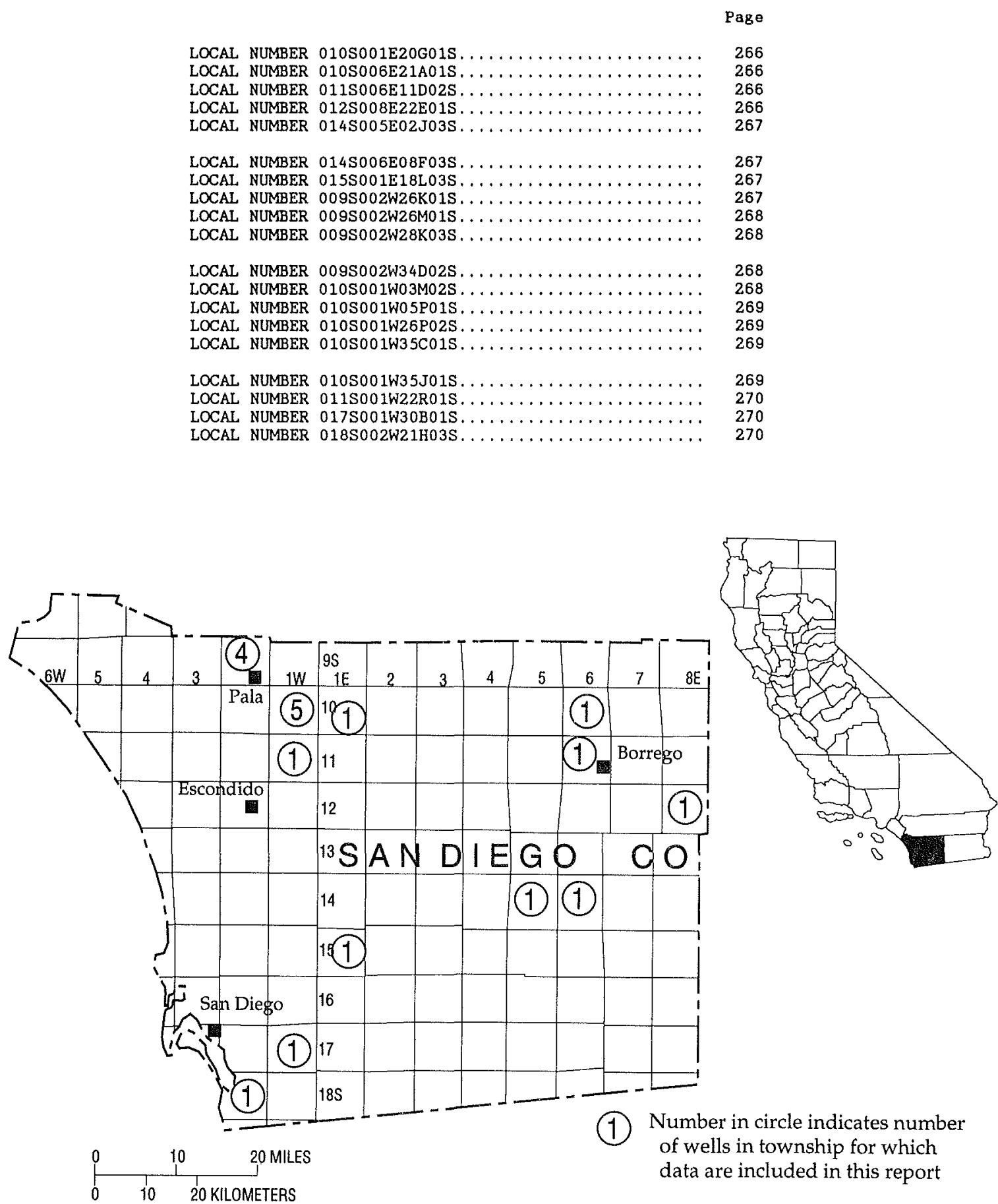

(1) Number in circle indicates number of wells in township for which data are included in this report

Figure 26. Location of wells in San Diego County. 
WELL DESCRIPTIONS AND WATER-LEVEL MEASUREMENTS

WATER YEAR OCTOBER 1991 TO SEPTEMBER 1992

SITE NUMBER 331737116540701

LOCAL NUMBER 010S001E20G01S

About 10 miles west of Lake Henshaw on Highway 76 . Drilled unused water-table well. Diameter 8 inches, depth 70 feet. Altitude of land-surface datum 2,580 feet. Water-level records available 1960, 1967, 1971, 1976-85, 1989 to current year.

WATER LEVELS IN FEET BELOW LAND-SURFACE DATUM

$\begin{array}{lll} & \text { WATER } & \\ \text { DATE } & \text { WETEL MS }\end{array}$

DEC 18, $199123.23 \mathrm{~V} \quad$ MAR $17,1992 \quad 18.70 \mathrm{~S}$

PERIOD OF RECORD HIGHEST 7.25 MAR 31, 1980 LOWEST 37.75 AUG 25, 1977

SITE NUMBER 331800116210001

LOCAL NUMBER 010S006E21A01S

North of Borrego Springs, about $0.1 \mathrm{mile}$ southeast of intersection of Borrego Valley and Henderson Canyon Roads. Drilled unused water-table well in alluvium. Diameter 12 inches, depth 310 feet. Altitude of land-surface datum 640 feet. Recorder installed by Californi a Department of Water Resources in 1952 . Water-level records available 1952-76, 1978,1980 to current year.

WATER LEVELS IN FEET BELOW LAND-SURFACE DATUM

$$
\begin{array}{lll} 
& \text { WATER } & \text { WATER } \\
\text { DATE LEVEL MS DATE LEVEL MS }
\end{array}
$$

MAR 16, $1992191.35 \mathrm{~S} \cdot \operatorname{SEP} 24,1992194.15 \mathrm{~S}$

PERIOD OF RECORD HIGHEST 130.55 JAN 03, $1953 \quad$ LOWEST 194.15 SEP 24, 1992

SITE NUMBER 331432116194602

LOCAL NUMBER 011S006E11D02S

East of Borrego Springs, about 1 mile southeast of intersection of Borrego Valley Road and Palm Canyon Drive. Drilled unused water-table well in alluvium. Diameter 14 inches, depth 218 feet. Altitude of land-surface datum 500 feet. Water-level records available 1953-71, 1978, 1980 to current year.

WATER LEVELS IN FEET BELOW LAND-SURFACE DATUM

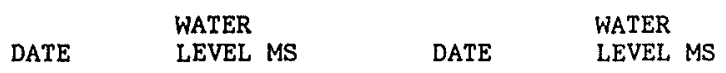

MAR 16, $1992 \quad 52.06 \mathrm{~S} \quad \operatorname{SEP} 24,199254.35 \mathrm{~S}$

PERIOD OF RECORD HIGHEST 17.53 NOV 16, 1953 LOWEST 65.60 AUG 14, 1958

SITE NUMBER 330639116074701

LOCAL NUMBER 012SO08E22E01S

About 2 miles south of Ocotillo Wells, 2.5 miles southeast of intersection of Highway 78 and Split Mountain Road. Drilled unused water-table well in aliuvium. Diameter 16 inches, depth 226 feet. Altitude of land-surface datum 110 feet. Water-level records available 1951, 1953-71, 1978, 1980-85, 1989 to current year.

WATER LEVELS IN FEET BELOW LAND-SURFACE DATUM

\begin{tabular}{|c|c|c|c|c|c|}
\hline & WATER & & WATER & & WATER \\
\hline DATE & LEVEL MS & DATE & LEVEL MS & DATE & LEVEL MS \\
\hline
\end{tabular}

OCT 02, $1991109.21 \mathrm{~s} \quad$ MAR 16, $1992109.18 \mathrm{~S} \quad$ SEP 24, $1992109.34 \mathrm{~S}$

PERIOD OF RECORD HIGHEST 101.83 NOV 10,1954 LOWEST 119.16 NOV 10,1970 
WELL DESCRIPTIONS AND WATER-LEVEL MEASUREMENTS

WATER YEAR OCTOBER 1991 TO SEPTEMBER 1992

\section{SITE NUMBER 325848116260301}

LOCAL NUMBER 014S005E02J03S

About 0.2 mile north of Agua Caliente. Drilled domestic water-table well in alluvium. Diameter 10 inches, depth 181 feet. Altitude of land-surface datum 2,030 feet. Water-level records available 1978, 1980 to current year.

WATER LEVELS IN FEET BELOW LAND-SURFACE DATUM

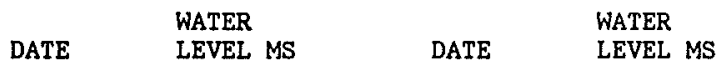

MAR 23, $1992 \quad 47.38 \mathrm{~S}$ SEP 08, $199250.67 \mathrm{~S}$

PERIOD OF RECORD HIGHEST 40.35 MAR 01, 1985 LOWEST 74.10 DEC 27,1978

SITE NUMBER 325808116232801

LOCAL NUMBER 014S006E08F03S

About $1 \mathrm{mile}$ northeast of Troutman Mountain. Unused water-table well. Diameter 8 inches, depth 110 feet. Altitude of land-surface datum 1,645 feet. Water-level records available 1960, 1962, 1964-66, 1968, 1978, 1980 to current year.

WATER LEVELS IN FEET BELOW LAND-SURFACE DATUM

$$
\begin{array}{lll} 
& \text { WATER } & \\
\text { DATE } & \text { WETER MS }
\end{array}
$$

MAR 23, $199261.33 \mathrm{~S} \quad$ SEP 08, $199261.35 \mathrm{~S}$

PERIOD OF RECORD HIGHEST 61.33 MAR 23, 1992 LOWEST 78.20 OCT 01, 1982

SITE NUMBER 325159116551101

LOCAL NUMBER 015S001E18L03S

North of Lakeside, about $0.3 \mathrm{mile}$ northeast of intersection of Mapleview Street and Highway 67 . Drilled unused water-table well in alluvium. Diameter and depth unknown. Altitude of land-surface datum 395 feet.

Water-level records available 1978 to current year.

WATER LEVELS IN FEET BELOW LAND-SURFACE DATUM

$$
\text { DATE WATER } \quad \text { LEVEL MS }
$$

MAR 23, $1992 \quad 20.87 \mathrm{~S}$

PERIOD OF RECORD HIGHEST 15.41 MAR 11, 1983 LOWEST 25.52 SEP 16, 1987

SITE NUMBER 332153117032901

LOCAL NUMBER 009S002W26K01S

On Pala Indian Reservation, about 0.07 mile east of Concord Road. Drilled irrigation water-table well. Diameter 10 inches, depth 173 feet. Altitude of land-surface datum 470 feet. Water-level records available 1967, 1971-72, 1975-84, 1989 to current year.

WATER LEVELS IN FEET BELOW LAND-SURFACE DATUM

$$
\begin{array}{lll} 
& \text { WATER } & \\
\text { DATE } & \text { WEVEL MS } & \text { WATER } \\
& \text { LEVEL MS }
\end{array}
$$

DEC $18,1991 \quad 85.34 \mathrm{~S} \quad$ MAR $17,1992 \quad 81.14 \mathrm{~s}$

PERIOD OF RECORD HIGHEST 59.07 APR 26, 1983 LOWEST 89.64 SEP 28, 1977 
SITE NUMBER 332151117040001

LOCAL NUMBER 009S002W26M01S

On Pala Indian Reservation, north of Highway 76. Drilled irrigation water-table well. Diameter 10 inches, depth 148 feet. Altitude of land-surface datum 425 feet. Water-level records available 1964-73, 1975-85, 1989 to current year.

WATER LEVELS IN FEET BELOW LAND-SURFACE DATUM

$$
\begin{array}{ll}
\text { WATER } \\
\text { LEVEL MS }
\end{array}
$$

MAR 17, $1992 \quad 57.96 \mathrm{SP}$

PERIOD OF RECORD HIGHEST 35.48 MAY 14, 1980 LOWEST 65.60 SEP 27, 1977

SITE NUMBER 332147117052301

LOCAL NUMBER O09S002W28K03S

About 1 mile west of Pala, and 550 feet south of Highway 76. Drilled irrigation water-table well. Diameter and depth unknown. Altitude of land-surface datum 355 feet. Water-level records available 1975-85, 1989 to current year.

WATER LEVELS IN FEET BELOW LAND-SURFACE DATUM

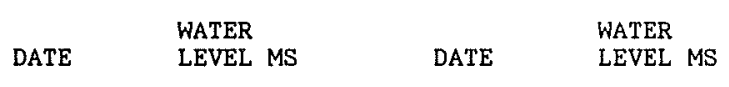

DEC 18, $1991 \quad 18.55 \mathrm{~S} \quad$ MAR $17,199216.85 \mathrm{~S}$

PERIOD OF RECORD HIGHEST 4.47 APR 11, 1979 LOWEST 23.10 NOV 03, 1980

SITE NUMBER 332119117045101

LOCAL NUMBER O095002W34D02S

On Pala Indian Reservation, south of Highway 76. Drilled irrigation water-table well. Diameter 12 inches, depth 50 feet. Altitude of land-surface datum 375 feet. Water-level records available 1975-85, 1989 to current year.

WATER LEVELS IN FEET BELOW LAND-SURFACE DATUM

\begin{tabular}{|c|c|c|c|}
\hline DATE & $\begin{array}{l}\text { WATER } \\
\text { LEVEL MS }\end{array}$ & DATE & $\begin{array}{l}\text { WATER } \\
\text { LEVEL MS }\end{array}$ \\
\hline
\end{tabular}

DEC $18,1991 \quad 19.79 \mathrm{~S} \quad$ MAR $17,1992 \quad 20.25 \mathrm{~S}$

PERIOD OF RECORD HIGHEST 6.49 APR 26, 1983 LOWEST 23.26 DEC 20, 1977

SITE NUMBER 331957116584601

LOCAL NUMBER 010S001W03MO2S

On Pauma Indian Reservation, about 1.3 miles northwest of Highway 76. Drilled domestic well. Diameter 8 inches, depth 400 feet, perforated 100-400 feet. Altitude of land-surface datum 1,110 feet. Water-level records available 1966, 1971-72, 1989 to current year.

WATER LEVELS IN FEET BELOW LAND-SURFACE DATUM

$\begin{array}{lll} & \text { WATER } & \\ \text { DATE } & \text { WETER } \\ \text { LATE } & \text { LEVEL MS }\end{array}$

DEC $18,1991 \quad 72.08 \mathrm{~S} \quad \operatorname{MAR} 17,1992 \quad 70.61 \mathrm{~s}$

PERIOD OF RECORD HIGHEST 69.43 OCT 19,1971 LOWEST 93.43 SEP 29, 1966 
WELL DESCRIPTIONS AND WATER-LEVEL MEASUREMENTS

WATER YEAR OCTOBER 1991 TO SEPTEMBER 1992

SITE NUMBER 331949117003301

LOCAL NUMBER 010S001W05P01S

In Pauma Valley, about $0.5 \mathrm{mile}$ southwest of Highway 76. Drilled irrigation well. Diameter 12 inches $0-178$ feet, 10 inches 178-340 feet, depth 340 feet, perforated 46-66, 133-147, 172-174, 194-215, 230-245, 260-340 feet.

Altitude of land-surface datum 705 feet. Water-level records available 1990 to current year.

WATER LEVELS IN FEET BELOW LAND-SURFACE DATUM

$\begin{array}{lll} & \text { WATER } & \\ \text { DATE } & \text { WATER } \\ \text { LEVEL MS } & \text { DETE }\end{array}$

DEC 18, $199168.40 \mathrm{~S} \quad \operatorname{MAR} 17,1992 \quad 40.85 \mathrm{~S}$

PERIOD OF RECORD HIGHEST 34.34 MAR 28, 1991 LOWEST 68.40 DEC 18, 1991

SITE NUMBER 331626116571401

LOCAL NUMBER 010S001W26P02S

On Rincon Indian Reservation in the San Luis Rey River Valley, about 370 feet west of Highway 6 . Unused wel1. Diameter 12 inches, depth 193 feet. Altitude of land-surface datum 853 feet. Water-level records available 1962 , $1967,1971-72,1978-85,1989$ to current year.

WATER LEVELS IN FEET BELOW LAND-SURFACE DATUM

$$
\begin{array}{lll} 
& \text { WATER } & \\
\text { DATE } & \text { WATER } \\
& \text { LEVEL MS } & \text { LEVEL MS }
\end{array}
$$

DEC 18, $199135.88 \mathrm{~V} \quad$ MAR $17,1992 \quad 35.53 \mathrm{~S}$

PERIOD OF RECORD HIGHEST 9.44 MAY 15, 1980 LOWEST 49.91 MAR 30, 1967

SITE NUMBER 331613116570901

LOCAL NUMBER 010S001W35C01S

On Rincon Indian Reservation, west of Highway 6 and south of section line road. Drilled unused water-table well. Diameter 16 inches, depth 105 feet. Altitude of land-surface datum 860 feet. Water-level records avallable 1938 , $1940,1950,1959-63,1971-73,1975-85,1989$ to current year.

WATER LEVELS IN FEET BELOW LAND-SURFACE DATUM

$$
\begin{array}{lll} 
& \text { WATER } & \\
\text { DATE } & \text { WEVEL MS } & \text { DATE } \\
\text { LEVEL MS }
\end{array}
$$

DEC $18,1991 \quad 35.48 \mathrm{~S} \quad$ MAR $17,1992 \quad 35.15 \mathrm{~S}$

PERIOD OF RECORD HIGHEST 9.16 MAR 31,1980 LOWEST 48.50 JUN 1963

SITE NUMBER 331535116564101

LOCAL NUMBER 010S001W35J01S

In San Luis Rey River Valley, about $0.6 \mathrm{mile}$ southeast of Highway 6 and about 150 feet west of Rincon Indian Reservation. Dug unused water-table well. Diameter 6 feet, depth 42 feet. Altitude of land-surface datum 874 feet. Water-level records available 1923-24,1926, 1929-34, 1940,1959-60,1967, 1971-72, 1976 to current year.

WATER LEVELS IN FEET BELOW LAND-SURFACE DATUM

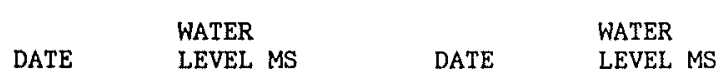

DEC $18,199130.39 \mathrm{~S} \quad$ MAR $17,1992 \quad 22.08 \mathrm{~S}$

PERIOD OF RECORD HIGHEST 4.69 APR 10, 1979 LOWEST 38.38 NOV 19, 1976 
SITE NUMBER 331205116575601

LOCAL NUMBER 011S001W22R01S

On San Pasqual Indian Reservation in Woods Valley, about 0.30 mile west of Lake Wohlford Road. Dug unused water-table well. Diameter 42 inches, depth 27 feet. Altitude of land-surface datum 1,675 feet. Water-level records avallable $1967,1976-85,1989$ to current year.

WATER LEVELS IN FEET BELOW LAND-SURFACE DATUM

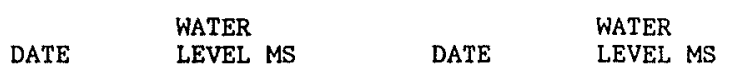

DEC $18,1991 \quad 12.36 \mathrm{~s} \quad$ MAR $17,1992 \quad 4.17 \mathrm{~s}$

PERIOD OF RECORD HIGHEST 2.67 MAR 22, 1979 LOWEST $23.30 \quad$ DEC 20,1977

SITE NUMBER 324005117012001

LOCAL NUMBER 017S001W30B01S

In Sunnyside, about $0.25 \mathrm{mile}$ southeast from corner of Bonita and Central Avenues. Drilled unused well. Diameter 12 inches, depth unknown. Altitude of land-surface datum 85 feet. Water-level records available 1978 to current year.

WATER LEVELS IN FEET BELOW LAND-SURFACE DATUM

$\begin{array}{lll} & \text { WATER } & \\ \text { DATE } & \text { LEVEL MS } & \text { WATER } \\ & & \text { LEVEL MS }\end{array}$

MAR 23, $1992 \quad 9.44 \mathrm{~s} \quad$ SEP 03, $1992 \quad 12.57 \mathrm{~S}$

PERIOD OF RECORD HIGHEST 7.02 MAR 11, $1983 \quad$ LOWEST 13.66 SEP 12,1990

SITE NUMBER 323530117050701

LOCAL NUMBER 018S002W21H03S

East of Imperial Beach about 0.25 mile south of Main Street near Interstate 5 . Drilled irrigation well. Diameter 13 inches, depth unknown. Altitude of land-surface datum 12 feet. Water-1evel records available 1978 to current year.

WATER LEVELS IN FEET BELOW LAND-SURFACE DATUM

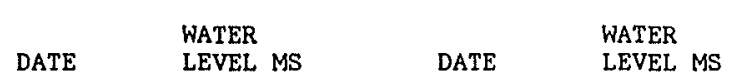

MAR 23, $1992 \quad 7.27 \mathrm{~s} \quad \operatorname{SEP} 03,1992 \quad 8.03 \mathrm{~S}$

PERIOD OF RECORD HIGHEST 7.16 MAR 09, 1988 LOWEST $11.03 \quad$ DEC 07,1978 


\section{SAN FRANCISCO COUNTY}

Page

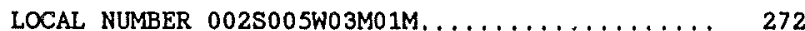

LOCAL NUMBER 002S005W06M01M. . . . . . . . . . . 272

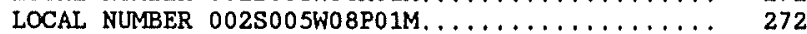

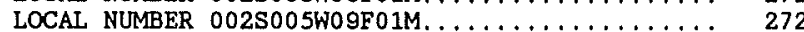

LOCAL NUMBER 002SO05W16E01M............. 273

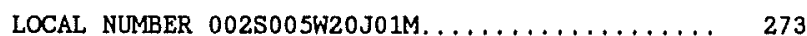

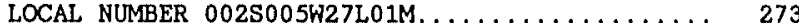

LOCAL NUMBER $002 S 005 W 27 N 01 M \ldots \ldots \ldots \ldots \ldots \ldots \ldots \ldots . \ldots \ldots . \ldots \ldots$

LOCAL NUMBER $0025005 W 33 L 01 \mathrm{M} \ldots \ldots \ldots \ldots \ldots \ldots \ldots \ldots . \ldots \ldots$

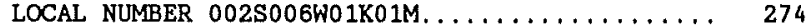

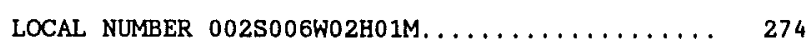

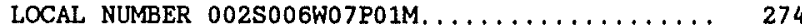

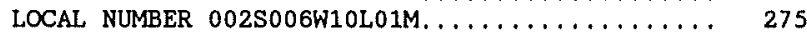

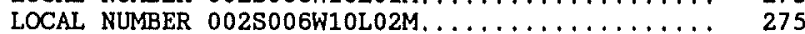

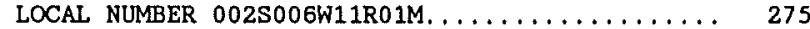

LOCAL NUMBER $002 S 006 W 11 R 02 M \ldots \ldots \ldots \ldots \ldots \ldots \ldots \ldots$

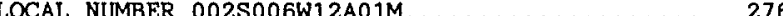

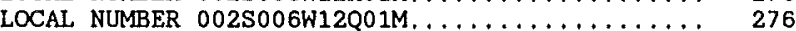

Page

LOCAL NUMBER 002S006W15C01M. . . . . . . . . 276

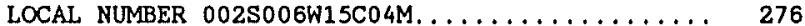

LOCAL NUMBER $0025006 W 15 C 05 M \ldots \ldots \ldots \ldots \ldots \ldots \ldots \ldots . \ldots . \ldots . \ldots \ldots$

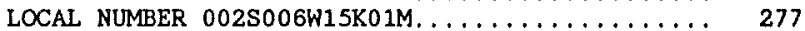

LOCAL NUMBER 002S006W15P01M. . . . . . . . . . . 277

LOCAL NUMBER 002S006W22J01M. . . . . . . . . . . 277

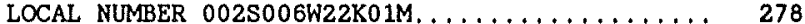

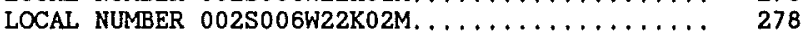

LOCAL NUMBER $0025006 W 23001 M \ldots \ldots \ldots \ldots \ldots \ldots \ldots . \ldots \ldots . \ldots \ldots$

LOCAL NUMBER 002S006W26A01M. . . . . . . . . . 278

LOCAL NUMBER $0025006 W 26 P 01 M \ldots \ldots \ldots \ldots \ldots \ldots \ldots . \ldots \ldots$

LOCAL NUMBER 002S006W27B06M. .............. 279

LOCAL NUMBER 0025006 W27J01M. . . . . . . . . . 279

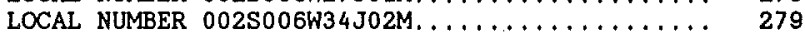

LOCAL NUMBER 002S006W34J03M. . . . . . . . ... 280

LOCAL NUMBER 002S006W35H01M. . . . . . . . . . 280

LOCAL NUMBER 002 S006W35Q01M. . . . . . . . . . . . . 280

LOCAL NUMBER 002 S006W36N01M. . . . . . . . . . 280

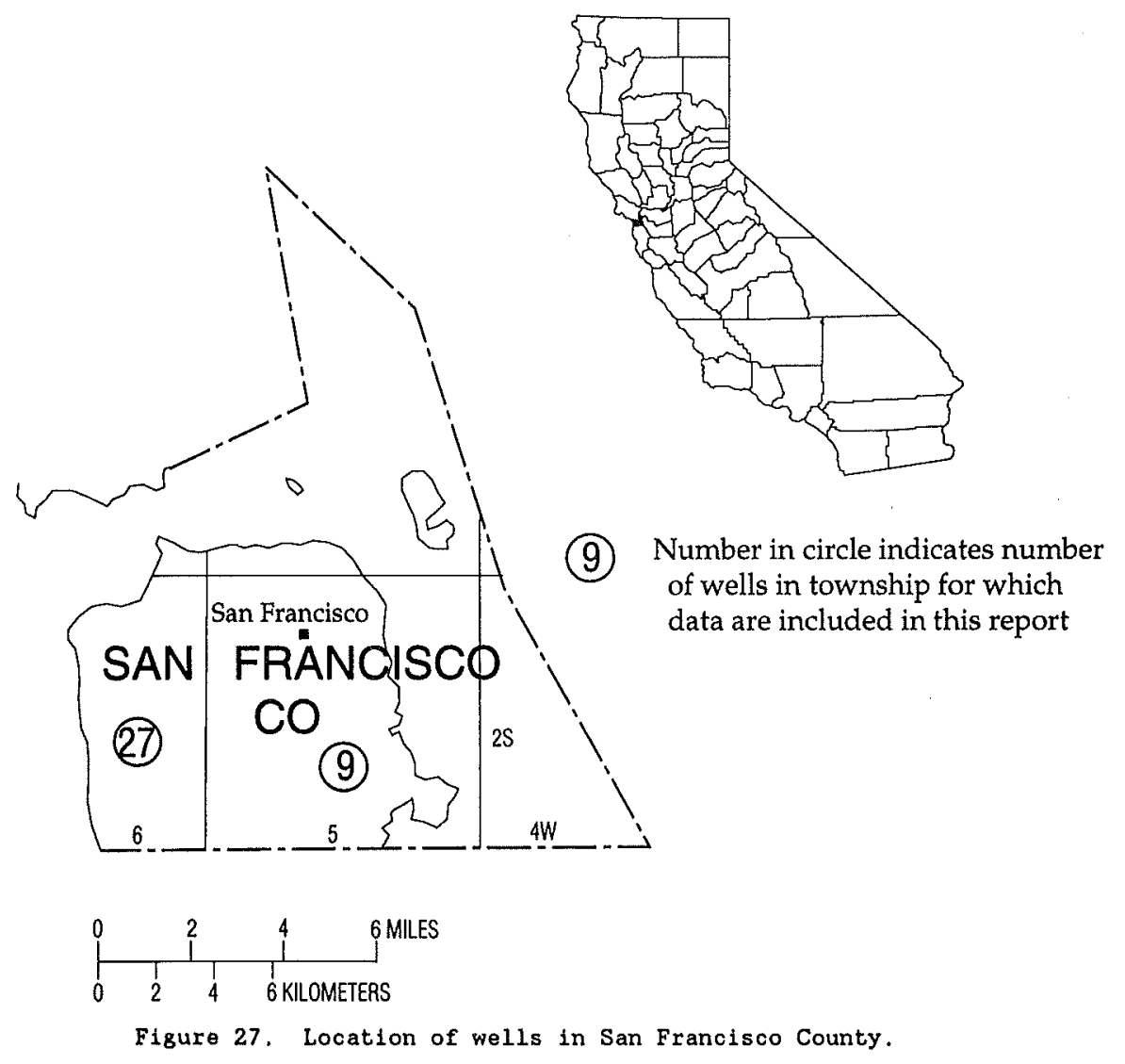


SITE NUMBER 374711122241601

LOCAL NUMBER 002S005W03M01M

In San Francisco on Market Street, east of Stockton Street. Unused well. Diameter 18 inches, depth measured 102.5 feet in 1989. Altitude of land-surface datum 25.55 feet. Water-level records available 1988 to current year.

WATER LEVELS IN FEET BELOW LAND-SURFACE DATUM

\begin{tabular}{llllll} 
& WATER & & & & \multicolumn{2}{c}{ WATER } \\
DATE & LEVEL MS & DATE & LEVEL MS & DATE & LEVEL MS \\
04,1992 & $31.17 \mathrm{~S}$ & JUN 10,1992 & $29.92 \mathrm{~S}$ & SEP 15, 1992 & $28.47 \mathrm{~S}$
\end{tabular}

PERIOD OF RECORD HIGHEST 27.75 MAR 16, 1989 LOWEST 33.65 NOV 06, 1990

SITE NUMBER 374713122274201

LOCAL NUMBER 002S00SWO6MO1M

In San Francisco, north of Lake Street. Irrigation well. Diameter 20 inches, depth measured about 80 feet in 1990. Altitude of land-surface datum 195.13 feet. Water-level records available 1988 to current year.

WATER LEVELS IN FEET BELOW LAND-SURFACE DATUM

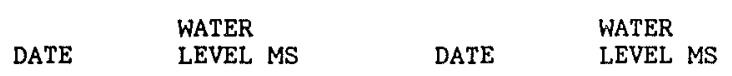

FEB $05,1992 \quad 51.89 \mathrm{~s} \quad$ JUN $11,1992 \quad 57.36 \mathrm{~s}$

PERIOD OF RECORD HIGHEST 44,56 MAR 16, 1989 LOWEST 57.36 JUN 11,1992

SITE NUMBER 374604122260101

LOCAL NUMBER OO2S005W08P01M

In San Francisco on 14 th Street, south of Castro Street. Drilled unused well. Diameter 6 inches, depth 230 feet, perforated 130-210 feet. Altitude of land-surface datum 200.56 feet. Water-level records available 1980 , 1988 to current year.

WATER LEVELS IN FEET BELOW LAND-SURFACE DATUM

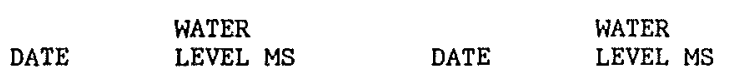

FEB $04,1992 \quad 26.65 \mathrm{~S}$ JUN $10,1992 \quad 26.50 \mathrm{~s}$

PERIOD OF RECORD HIGHEST 25.76 MAR 16, 1989 LOWEST 27.40 FEB 06,1990

SITE NUMBER 374632122251001

LOCAL NUMBER 002S005W09F01M

In San Francisco on Oak Street, at International Center. Unused well. Diameter 16 inches, depth measured 107 feet in 1989. Altitude of land-surface datum 35.23 feet. Water-level records available 1988 to current year.

WATER LEVELS IN FEET BELOW LAND-SURFACE DATUM

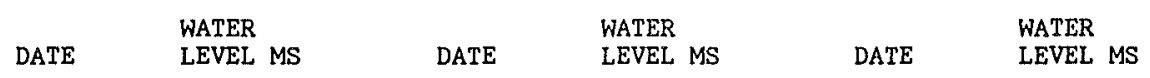

FEB $04,1992 \quad 6.78 \mathrm{~s} \quad$ JUN $10,1992 \quad 6.07 \mathrm{~s} \quad$ SEP $15,1992 \quad 6.08 \mathrm{~s}$

PERIOD OF RECORD HIGHEST 4.00 OCT $19,1988 \quad$ LOWEST $\quad 7.03 \quad$ FEB 05,1991 
WELL DESCRIPTIONS AND WATER-LEVEL MEASUREMENTS

WATER YEAR OCTOBER 1991 TO SEPTEMBER 1992

SITE NUMBER 374541122251201

LOCAL NUMBER O02S005W16E01M

In San Francisco, south of 18th Street on Lexington Street. Commercial well, Diameter 10 inches, depth measured 93.9 feet in 1989. Altitude of land-surface datum 33.32 feet. Water-level records aval lable 1988 to current year.

WATER LEVELS IN FEET BELOW LAND-SURFACE DATUM

$\begin{array}{lllll}\text { DATE } & \text { WATER } & \text { WATER } & \text { WATER } \\ \text { LEVEL MS } & \text { DATE } & \text { LEVEL MS } & \text { DATE } & \text { LEVEL MS }\end{array}$

FEB 04, $1992 \quad 7.43 \mathrm{~S}$ JUN 10,1992 $6.98 \mathrm{~S} \quad$ SEP 15, $1992 \quad 7.26 \mathrm{~S}$

PERIOD OF RECORD HIGHEST 6.98 JUN 10, 1992 LOWEST 7.53 AUG 01, 1990

SITE NUMBER 374437122253501

LOCAL NUMBER 002S005W20JO1M

In San Francisco, west of Church Street, and north of 29 th Street at St. Paul's Church. Institutional well. Diameter 60 inches, depth measured 42 feet in 1989. Altitude of land-surface datum 133.61 feet. Water-level records available 1988 to current year.

WATER LEVELS IN FEET BELOW LAND-SURFACE DATUM

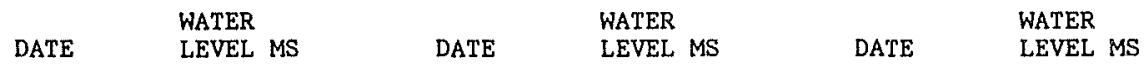

FEB 04, $1992 \quad 18.36 \mathrm{~S}$ JUN 10, $1992 \quad 16.85 \mathrm{~S} \quad$ SEP 15, $199217.48 \mathrm{~S}$

PERIOD OF RECORD HIGHEST 16.74 MAR 16, 1989 LOWEST 23.90 OCT 19,1988

SITE NUMBER 374335122235001

LOCAL NUMBER 002S005W27L01M

In San Francisco on Egbert Street. Commercial well. Diameter 12 inches, depth 100 feet, perforated 23-89 feet. Altitude of land-surface datum 29.78 feet. Water-level records available 1966,1988 to current year.

WATER LEVELS IN FEET BELOW LAND-SURFACE DATUM

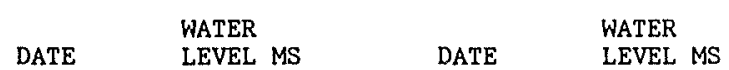

FEB $04,1992 \quad 4.22 \mathrm{~S} \quad \operatorname{SEP} 15,1992 \quad 3.79 \mathrm{~S}$

PERIOD OF RECORD HIGHEST 2.65 MAY 16, 1989 LOWEST 4.36 FEB 05, 1991

SITE NUMBER 374332122241601

LOCAL NUMBER 002S005W27NO1M

In San Francisco on Brussels Street. Domestic well. Diameter 30 inches, depth measured 20 feet in 1990. Altitude of land-surface datum 70.16 feet. Water-level records available 1988 to current year.

WATER LEVELS IN FEET BELOW LAND-SURFACE DATUM

$\begin{array}{ll} & \text { WATER } \\ \text { DATE } & \text { LEVEL MS }\end{array}$

FEB 04, $19929.88 \mathrm{~S}$

PERIOD OF RECORD HIGHEST 7.83 MAR 16, 1989 LOWEST 10.13 NOV 06, 1990 
SITE NUMBER 374247122245701

LOCAL NUMBER 002S005W33L01M

In San Francisco, north of Sunnydale Avenue in Herz playground. Drilled observation well, Diameter 2 inohes, depth 80.75 feet, perforated $65.75-75.75$ feet. Altitude of land-surface datum 120 feet. Water-level records available 1989 to current year.

WATER LEVELS IN FEET BELOW LAND-SURFACE DATUM

$$
\begin{array}{lll} 
& \text { WATER } & \\
\text { DATE } & \text { LEVEL MS } & \text { WATER } \\
& \text { LATE } & \text { LEVEL MS }
\end{array}
$$

FEB 04, $1992 \quad 59.46 \mathrm{~s} \quad \operatorname{SEP} 15,1992 \quad 60.38 \mathrm{~s}$

PERIOD OF RECORD HIGHEST 50,47 JUL $07,1989 \quad$ LOWEST $60.38 \quad$ SEP 15,1992

SITE NUMBER 374715122280801

LOCAL NUMBER 002S006W01K01M

In San Francisco on the Presidio, at Mountain Lake. Unused well. Diameter 14 inches, depth reported 90 feet. Altitude of land-surface datum 136.31 feet. Water-level records available 1988 to current year.

WATER LEVELS IN FEET BELOW LAND-SURFACE DATUM

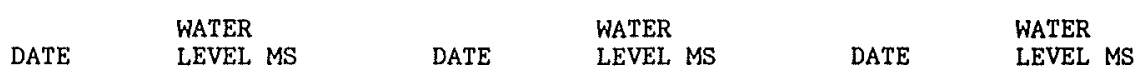

FEB $05,1992 \quad 5.63 \mathrm{~s} \quad$ JUN $10,1992 \quad 15.24 \mathrm{SS} \quad$ SEP $16,1992 \quad 12.66 \mathrm{~s}$

PERIOD OF RECORD HIGHEST 1.56 MAR 20,1989 LOWEST $12.66 \quad$ SEP 16,1992

SITE NUMBER 374725122290101

LOCAL NUMBER 002S006W02H01M

In San Francisco on the Presidio, near Baker Beach. Institutional well. Diameter 20 inches, depth measured 60 feet in 1990. Altitude of land-surface datum 24.46 feet. Water-level records available 1988 to current year. WATER LEVELS IN FEET BELOW LAND-SURFACE DATUM

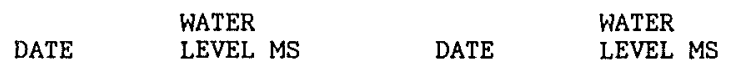

FEB 05, $1992 \quad 5.96 \mathrm{~S} \quad$ JUN $11,1992 \quad 7.25 \mathrm{~S}$

PERIOD OF RECORD HIGHEST 5.90 FEB 06, 1990 LOWEST 7.32 OCT 24,1989

SITE NUMBER 374602122271001

LOCAL NUMBER 002S006W07P01M

In San Francisco in Golden Gate Park. Observation well, Dianeter 2 inches, depth drilled 331 feet, depth cased 249 feet. Altitude of land-surface datum 273.47 feet. Water-level records available 1982 , 1987 to current year. WATER LEVELS IN FEET BELOW LAND-SURFACE DATUM

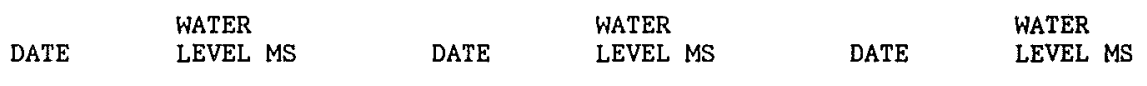

FEB 05, $1992 \quad 58.67 \mathrm{~S} \quad$ JUN $11,1992 \quad 57.74 \mathrm{~S} \quad$ SEP $15,1992 \quad 56.76 \mathrm{~S}$

PERIOD OF RECORD HIGHEST 51.1 AUG $11,18,1982 \quad$ LOWEST 80.20 DEC 10,1987 
WELL DESCRIPTIONS AND WATER-LEVEL MEASUREMENTS

WATER YEAR OCTOBER 1991 TO SEPTEMBER 1992

SITE NUMBER 374616122303001

LOCAL NUMBER 002S006W10LO1M

In San Francisco in Golden Gate Park. Irrigation well. Diameter unknown, depth 156 feet. Altitude of land-surface datum 33.19 feet. Water-level records available 1987 to current year.

WATER LEVELS IN FEET BELOW LAND-SURFACE DATUM

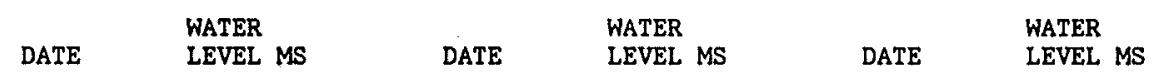

FEB 05, $1992 \quad 20.68 \mathrm{~S}$ JUN 11, $1992 \quad 21.06 \mathrm{~S} \quad$ SEP 15, $1992 \quad 21.00 \mathrm{~s}$

PERIOD OF RECORD HIGHEST 16.22 DEC 10, 1987 LOWEST 22.83 MAY 01, 1990

SITE NUMBER 374616122303002

LOCAL NUMBER 002S006W10L02M

In San Francisco in Golden Gate Park, near Fulton Street. Irrigation well. Diameter unknown, depth 281 feet. Altitude of land-surface datum 44.76 feet. Water-level records available 1987 to current year.

WATER LEVELS IN FEET BELOW LAND-SURFACE DATUM

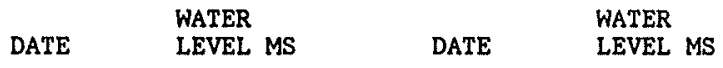

FEB 05, $1992 \quad 31.10 \mathrm{~S} \quad$ JUN $11,1992 \quad 31.45 \mathrm{~S}$

PERIOD OF RECORD HIGHEST 25.85 DEC 10, 1987 LOWEST 33.24 MAY 01, 1990

SITE NUMBER 374605122284801

LOCAL NUMBER 002S006W11R01M

In San Francisco in Golden Gate Park, near Elk Glen Lake. Drilled irrigation well. Diameter 14 inches, depth 360 feet, perforated 170-350 feet. Altitude of land-surface datum 169.12 feet. Water-level records available 1987 to current year.

WATER LEVELS IN FEET BELOW LAND-SURFACE DATUM

$$
\text { DATE WATER } \quad \text { LEVEL MS }
$$

FEB 04, $1992 \quad 50.02 \mathrm{~s}$

PERIOD OF RECORD HIGHEST 43.92 JAN 15, 1988 LOWEST 76.85 FEB 07, 1989

SITE NUMBER 374604122284701

LOCAL NUMBER 002S006W11R02M

In San Francisco in Golden Gate Park, east of Elk Glen Lake. Drilled observation well in Colma Formation. Diameter 2 inches, depth 72 feet, perforated 58-68 feet. Altitude of land-surface datum 173.34 feet. Water-1evel records available 1989 to current year.

WATER LEVELS IN FEET BELOW LAND-SURFACE DATUM

$$
\text { DATE WATER }
$$

FEB $04,1992 \quad 44.35 \mathrm{~S}$

PERIOD OF RECORD HIGHEST 42.33 FEB 06, 1990 LOWEST 46.16 FEB 07, 1991 
SITE NUMBER 374647122274401

LOCAL NUMBER 002S006W12A01M

In San Francisco, west of 5th Avenue at Geary Street, at hospital. Unused well. Diameter 24 inches, depth measured 100 feet in 1990. Altitude of land-surface datum 185.67 feet. Water-ievel records availabie 1988 to current year.

WATER LEVELS IN FEET BELOW LAND-SURFACE DATUM

\begin{tabular}{|c|c|c|c|c|c|}
\hline DATE & $\begin{array}{l}\text { WATER } \\
\text { LEVEL MS }\end{array}$ & DATE & $\begin{array}{l}\text { WATER } \\
\text { LEVEL MS }\end{array}$ & DATE & $\begin{array}{l}\text { WATER } \\
\text { LEVEL MS }\end{array}$ \\
\hline 05,1992 & $39.59 \mathrm{~s}$ & JUN 10,1992 & $39.85 \mathrm{~S}$ & 16,1992 & $40.27 \mathrm{~s}$ \\
\hline
\end{tabular}

PERIOD OF RECORD HIGHEST 33.25 OCT 19, 1988 LOWEST 40.27 SEP 16, 1992

SITE NUMBER 374558122281001

LOCAL NUMBER 002S006W12Q01M

In San Francisco in Golden Gate Park, north of Lincoln Way. Irrigation well. Diameter 12 inches, depth 250 feet. Altitude of land-surface datum 206.99 feet. Water-level records available 1987 to current year.

WATER LEVELS IN FEET BELOW LAND-SURFACE DATUM

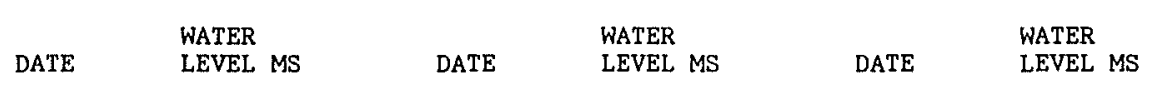

FEB 05, $1992 \quad 43.53 \mathrm{~s}$ JUN $11,199247.54 \mathrm{~S} \quad \operatorname{SEP} 16,199249.09 \mathrm{~S}$

PERIOD OF RECORD HIGHEST 35.22 JAN 15, 1988 LOWEST 78.0 OCT 02, 1987

SITE NUMBER 374544122303001

LOCAL NUMBER 002S006W15C01M

In San Francisco on the Great Highway, north of Irving Street. Observation well, Diameter 4 inches, depth

65 feet. Altitude of land-surface datum 19.77 feet. Water-level records available 1980 to current year.

Measurements 1980-86 available in the files of the San Francisco Clean Water Program.

WATER LEVELS IN FEET BELOW LAND-SURFACE DATUM

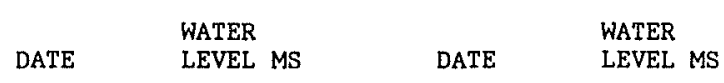

FEB 05, $1992 \quad 9.69 \mathrm{~S} \quad \operatorname{SEP} 16,1992 \quad 10.56 \mathrm{~S}$

PERIOD OF RECORD HIGHEST 8.17 DEC 10, $1987 \quad$ LOWEST 13.09 SEP 18, 1991

SITE NUMBER 374553122302502

LOCAL NUMBER 002S006W15C04M

In San Francisco in Golden Gate Park, near the south windmill on South Drive. Drilled observation well. Diameter 2 inches, depth 140 feet, perforated 118-138 feet. Middle well of three cluster wells. Altitude of land-surface datum 27.35 feet. Water-level records available 1989 to current year.

WATER LEVELS IN FEET BELOW LAND-SURFACE DATUM

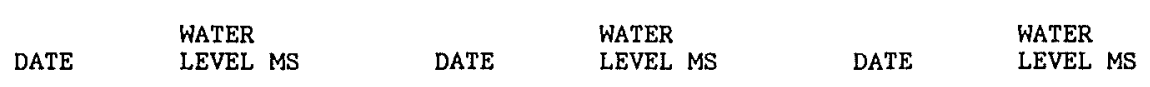

FEB $04,1992 \quad 17.85 \mathrm{~s} \quad$ JUN $11,1992 \quad 16.15 \mathrm{~S} \quad$ SEP $15,1992 \quad 17.96 \mathrm{~s}$

PERIOD OF RECORD HIGHEST 13.11 OCT 16,1989 LOWEST 20.35 JUN 05, 1990 
WELL DESCRIPTIONS AND WATER-LEVEL MEASUREMENTS

WATER YEAR OCTOBER 1991 TO SEPTEMBER 1992

SITE NUMBER 374553122302503

LOCAL NUMBER 002S006W15C05M

In San Francisco in Golden Gate Park, near the south windmill on South Drive. Drilled observation well. Diameter 2 inches, depth 57 feet, perforated 30-50 feet. Shallow well of three cluster wells. Altitude of land-surface datum 27.23 feet. Water-level records available 1989 to current year.

WATER LEVELS IN FEET BELOW LAND-SURFACE DATUM

$\begin{array}{llll} & \text { WATER } & \text { WATER } & \text { WATER } \\ \text { DATE } & \text { DEVEL MS } & \text { DATE } & \text { LEVEL MS }\end{array}$

FEB 04, $1992 \quad 15.40 \mathrm{~S}$ JUN 11, $1992 \quad 15.01 \mathrm{~S} \quad$ SEP $15,1992 \quad 15.74 \mathrm{~S}$

PERIOD OF RECORD HIGHEST 12.03 OCT 16,1989 LOWEST 17.15 JUN 05, 1990

SITE NUMBER 374523122300901

LOCAL NUMBER 002S006W15K01M

In San Francisco on 44 th Avenue, about 40 feet north of Moraga Street. "nused well. Diameter 12 inches, depth 260 feet. Altitude of land-surface datum 59,34 feet. Water-level records available 1988-92.

WATER LEVELS IN FEET BELOW LAND-SURFACE DATUM

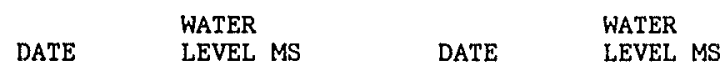

FEB 05, $1992 \quad 46,83 \mathrm{~S} \quad$ SEP $15,1992 \quad \mathrm{~N}$

PERIOD OF RECORD HIGHEST 43.88 APR 20, 1988 LOWEST 47.87 SEP 18, 1991

SITE NUMBER 374516122302701

LOCAL NUMBER 002SO06W15P01M

In San Francisco on Moraga Street. Observation well. Diameter 2 inches, depth 68 feet. Alt1tude of land-surface datum 22.64 feet. Water-level records available 1980 to current year. Measurements 1980-86 available in the files of the San Francisco Clean Water Program.

WATER LEVELS IN FEET BELOW LAND-SURFACE DATUM

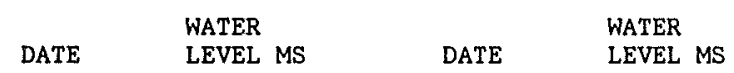

FEB 05, $1992 \quad 13.73 \mathrm{~S} \quad$ SEP $16,1992 \quad 14.85 \mathrm{~S}$

PERIOD OF RECORD HIGHEST 12.00 DEC 10, 1987 LOWEST 15.11 SEP 18, 1991

SITE NUMBER 374436122300501

LOCAL NUMBER O02S006W22J01M

In San Francisco on 44 th Avenue south of Santiago Street. Unused well. Diameter 12 inches, depth 253.3 feet. Altitude of land-surface datum 63.33 feet. Water-level records available 1987-92.

WATER LEVELS IN FEET BELOW LAND-SURFACE DATUM

$\begin{array}{lll} & \text { WATER } & \text { WATER } \\ \text { DATE } & \text { LEVEL MS }\end{array}$
FEB $05,1992 \quad 51.68 \mathrm{~s}$
SEP 15, 1992
N

PERIOD OF RECORD HIGHEST 48.27 DEC 09, $1987 \quad$ LOWEST 52.50 JUL 19, 1988 
SITE NUMBER 374438122301701

LOCAL NUMBER 002S006W22K01M

In San Francisco, northwest of intersection of 47 th Avenue and Santiago Street. Observation well. Diameter 2 inches, depth 61 feet. Altitude of land-surface datum 32.59 feet. Water-level records available 1980 to current year. Measurements 1980-86 available in the files of the San Francisco Clean Water Program.

WATER LEVELS IN FEET BELOW LAND-SURFACE DATUM

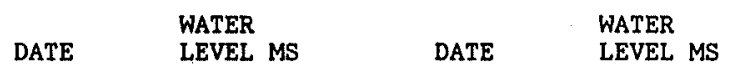

FEB 05, $1992 \quad 20.02 \mathrm{~S} \quad$ SEP 16, $1992 \quad 19.52 \mathrm{~s}$

PERIOD OF RECORD HIGHEST 16.00 FEB 24,1988 LOWEST 20.02 FEB 05,1992

SITE NUMBER 374437122302001

LOCAL, NUMBER 002S006W22K02M

In San Francisco, east of Great Highway on Santiago Street. Observation well. Diameter 2 inches, depth 41 feet. Alt1tude of land-surface datum 21.17 feet. Water-level records available 1980 to current year. Measurements 1980-86 available in the files of the San Francisco Clean Water Program.

WATER LEVELS IN FEET BELOW LAND-SURFACE DATUM

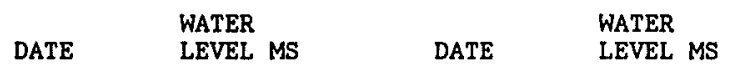

FEB 04, $1992 \quad 13.07 \mathrm{~S} \quad$ SEP $15,1992 \quad 14.24 \mathrm{~S}$

PERIOD OF RECORD HIGHEST 11.70 JAN 15, 1988 LOWEST 15.93 JUL 19,1988

SITE NUMBER 374418122290601

LOCAL NUMBER 002S006W23Q01M

In San Francisco at Edgewood School, near Stern Grove. Irrigation well. Diameter unknown, depth measured 213.8 feet in 1989. Altitude of land-surface datum 157.70 feet. Water-level records available 1988 to current year.

WATER LEVELS IN FEET BELOW LAND-SURFACE DATUM

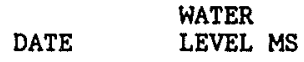

SEP 16, $1992116.23 \mathrm{~V}$

PERIOD OF RECORD HIGHEST 108.32 NOV 15, 1988 LOWEST 116.23 SEP 16, 1992

SITE NUMBER 374411122285001

LOCAL NUMBER O02S006W26A01M

In San Francisco in Stern Grove, Observation well. Diameter 12 inches, depth 268.5 feet, perforated 98,5-228.5 feet. Altitude of land-surface datum 83.90 feet. Water-level records available 1987 to ourrent year. WATER LEVELS IN FEET BELOW LAND-SURFACE DATUM

$\begin{array}{lllll}\text { DATE } & \text { WATER } & \text { WATER } & \text { WATER } \\ \text { LEVEL MS } & \text { DATE } & \text { LEVEL MS } & \text { DATE } & \text { LEVEL MS }\end{array}$
FEB 05,1992
$26.11 \mathrm{~s}$
JUN 11,1992
$27.34 \mathrm{~S}$
SEP 15, 1992
$27.57 \mathrm{~s}$

PERIOD OF RECORD HIGHEST 19.93 APR 20, 1988 LOWEST 27.57 SEP 15, 1992 
WELL DESCRIPTIONS AND WATER-LEVEL MEASUREMENTS

WATER YEAR OCTOBER 1991 TO SEPTEMBER 1992

SITE NUMBER 374323122292001

LOCAL NUMBER 002S006W26P01M

In San Francisco in Harding Park. Irrigation well. Diameter 12 inches $0-144$ feet, 8 inches 144-265 feet, depth 265 feet, perforated 50-265 feet. Altitude of land-surface datum 67.78 feet. Water-level records available 1957,1987 to current year.

WATER LEVELS IN FEET BELOW LAND-SURFACE DATUM

$\begin{array}{llll}\text { WATE } & \text { WATER } & \text { WATER } & \text { WATER } \\ \text { LEVEL MS } & \text { DATE } & \text { LEVEL MS } & \text { DATE }\end{array}$

FEB 04, $1992 \quad 61.13 \mathrm{~s}$ JUN 11, $1992 \quad 62.32 \mathrm{~S} \quad$ SEP 15, $199263.55 \mathrm{~s}$

PERIOD OF RECORD HIGHEST 42 MAY 08, 1957 LOWEST 63.55 SEP 15, 1992

SITE NUMBER 374406122301702

LOCAL NUMBER 002S006W27B06M

In San Francisco at Fleishhacker Zoo. Irrigation well. Diameter 16 inches, depth reported 200 feet. Altitude of land-surface datum 12.62 feet. Water-level records available 1988 to current year.

WATER LEVELS IN FEET BELOW LAND-SURFACE DATUM

DATE WATER

FEB $04,1992 \quad 4.49 \mathrm{~S}$

PERIOD OF RECORD HIGHEST 3.04 FEB 08, $1990 \quad$ LOWEST 5.93 NOV 09, 1988 JUL 06,1989

SITE NUMBER 374346122300501

LOCAL NUMBER O02S006W27J01M

In San Francisco, northwest of Lake Merced. Observation well. Diameter 2 inches, depth 100 feet. Altitude of land-surface datum 48.90 feet. Water-level records available 1980 to current year, Measurements $1980-86$ available in the files of the San Francisco Clean Water Program.

WATER LEVELS IN FEET BELOW LAND-SURFACE DATUM

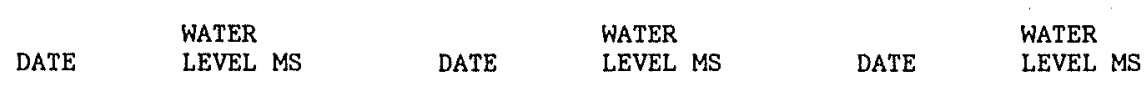

FEB 05, $1992 \quad 39.65 \mathrm{~S} \quad$ JUN 11, $1992 \quad 39.68 \mathrm{~S} \quad$ SEP 13, $1992 \quad 42.51 \mathrm{~S}$

PERIOD OF RECORD HIGHEST 36.03 JUN 14, 1988 LOWEST 42.51 SEP 13, 1992

SITE NUMBER 374246122295402

LOCAL NUMBER 002S006W34J02M

In San Francisco at Fort Funston. Drilled observation well in Merced Formation. Diameter 2 inches, depth 602 feet, perforated $572-592$ feet. Middle well of three cluster wells. Altitude of land-surface datum 190 feet. Water-level records available 1989 to current year.

WATER LEVELS IN FEET BELOW LAND-SURFACE DATUM

$\begin{array}{ll} & \text { WATER } \\ \text { DATE } & \text { LEVEL MS }\end{array}$

JUN $11,1992165.21 \mathrm{~V}$

PERIOD OF RECORD HIGHEST 162,62 MAY 02, 1990 LOWEST 194.10 SEP 20, 1989 


\section{SITE NUMBER 374246122295403}

LOCAL NUMBER 002S006W34J03M

In San Francisco at Fort Funston. Drilled observation well in Merced Formation. Diameter 2 inches, depth 278 feet, perforated 250-270 feet. Shallow well of three cluster wells. Altitude of land-surface datum 190 feet. Water-level records available 1989 to current year.

WATER LEVELS IN FEET BELOW LAND-SURFACE DATUM

$$
\begin{array}{lllll} 
& \text { WATER } & \text { WATER } & & \text { WATER } \\
\text { DATE } & \text { LEVEL MS } & \text { DATE } & \text { LEVEL MS } & \text { DATE }
\end{array}
$$

FEB 05, $1992183.39 \mathrm{~V}$ JUN 11, $1992182.82 \mathrm{~V} \quad \operatorname{SEP} 15,1992183.75 \mathrm{~V}$

PERIOD OF RECORD HIGHEST 178.82 SEP 20, 1989 LOWEST 184.03 JUL 02, 1990

SITE NUMBER 374306122285801

LOCAL NUMBER 002S006W35HO1M

In San Francisco on Higuera Street at San Francisco State College. Drilled observation well. Diameter 4 inches, depth 87 feet. Altitude of land-surface datum 90 feet. Water-level records available $1990-92$.

WATER LEVELS IN FEET BELOW LAND-SURFACE DATUM

$\begin{array}{lllll} & \text { WATER } & \text { WATER } & \text { WATER } \\ \text { DATE } & \text { LEVEL MS } & \text { DATE } & \text { LEVEL MS } & \text { DATE }\end{array}$

FEB $05,1992 \quad 81.58 \mathrm{~S} \quad$ JUN $11,199281.84 \mathrm{~S} \quad$ SEP $16,1992 \quad \mathrm{~N}$

PERIOD OF RECORD HIGHEST 80.18 AUG 01, 1990 LOWEST 82.56 JUL 18, 1990

SITE NUMBER 374230122291101

LOCAL NUMBER 002S006W35Q01M

In San Francisco at the Olympic Country Club. Drilled observation well. Diameter 2 inches, depth 51 feet, perforated 36-46 feet. Altitude of land-surface datum 31.30 feet. Water-level records available 1989 to current year.

WATER LEVELS IN FEET BELOW LAND-SURFACE DATUM

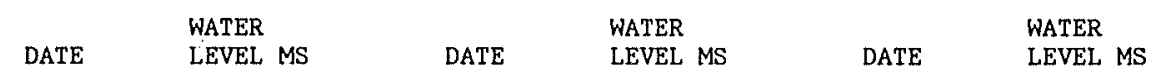

FEB 05, $1992 \quad 32.42 \mathrm{~S} \quad$ JUN 11, $1992 \quad 32.48 \mathrm{~S} \quad$ SEP 15, $1992 \quad 33.07 \mathrm{~S}$

PERIOD OF RECORD HIGHEST 28.63 JUL 07, 1989 LOWEST 33.07 SEP 15, 1992

SITE NUMBER 374235122283601

LOCAL. NUMBER 002SO06W36NO1M

In San Francisco at the San Francisco Golf Club. Drilled irrigation well. Diameter 14 inches, depth 324 feet, perforated 200-310 feet. Altitude of land-surface datum 143.02 feet. Water-level records available 1951 , 1987 to current year.

WATER LEVELS IN FEET BELOW LAND-SURFACE DATUM

$$
\begin{array}{lllll}
\text { DATE } & \text { WATER } & \text { WATER } & \text { WATER } \\
\text { LEVEL MS } & \text { DATE } & \text { LEVEL MS } & \text { DATE } & \text { LEVEL MS }
\end{array}
$$

FEB $04,1992178.42 \mathrm{~V}$ JUN $11,1992181.58 \mathrm{~V} \quad \operatorname{SEP} 15,1992183.03 \mathrm{~V}$

PERIOD OF RECORD HIGHEST 60 APR 24, 1951 LOWEST 183.03 SEP 15, 1992 


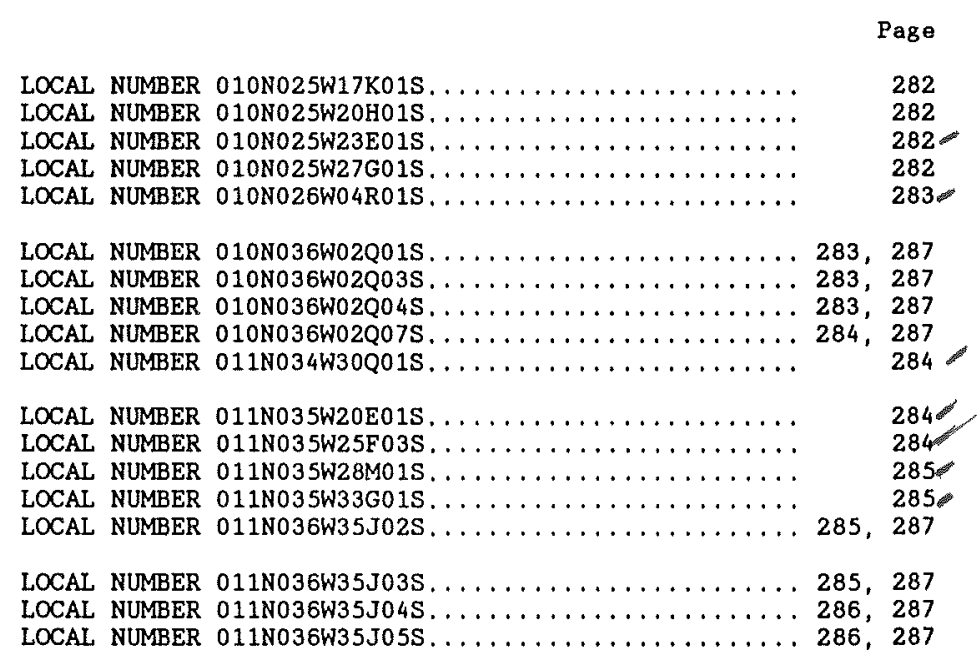

(1) Number in circle indicates number of wells in township for which data are included in this report

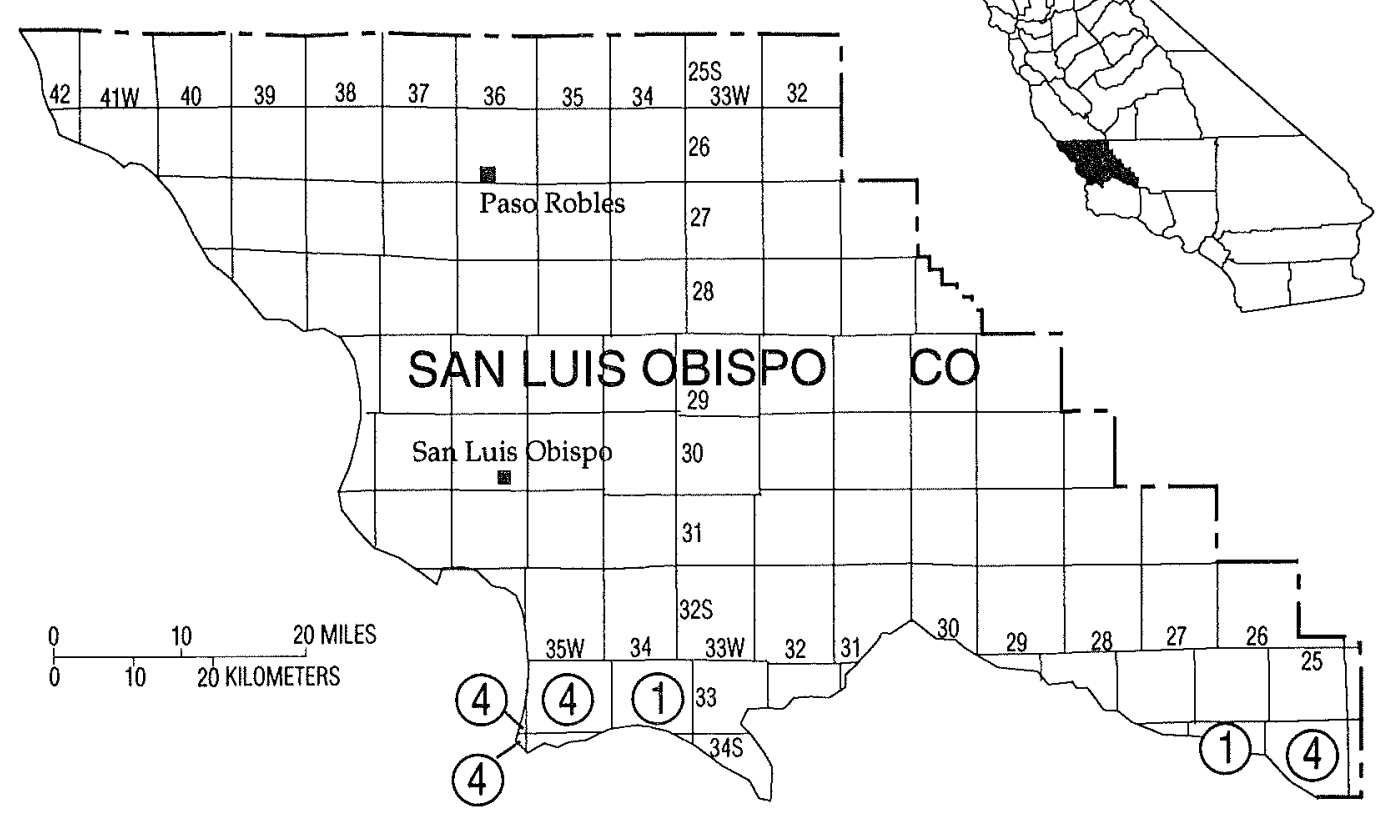

Figure 28, Location of wells in San Luis Obispo County. 
SITE NUMBER 345644119341501

LOCAL NUMBER 010N025W17K01S

East of Cuyama, north of Highway 166. Drilled unused water-table well. Diameter 16 inches, depth measured 203.5 feet in 1990. Altitude of land-surface datum 2, 310 feet. Measurements provided by Santa Barbara County Water Agency beginning in 1982. Water-level records available 1947, 1955, 1966, $1968,1982-92$.

WATER LEVELS IN FEET BELOW LAND-SURFACE DATUM

$\begin{array}{ll} & \text { WATER } \\ \text { DATE } & \text { LEVEL MS }\end{array}$

APR 01, $1992 \quad \mathrm{RD}$

PERIOD OF RECORD HIGHEST 49.10 OCT $23,1947 \quad$ LOWEST $217.74 \quad$ DEC 11,1968

SITE NUMBER 345604119340001

LOCAL NUMBER 010N025W2OHO1S

About 1.4 miles east of Cuyama near Highway 166. Drilled irrigation water-table well. Diameter 10 inches, depth 656 feet in 1946, perforated 108-656 feet. Altitude of land-surface datum 2,335 feet. Measurements provided by Santa Barbara County Water Agency beginning in 1980. Water-level records available 1946-47, 1956, 1961, 1966, 1968,1979 to current year.

WATER LEVELS IN FEET BELOW LAND-SURFACE DATUM

$\begin{array}{ll} & \text { WATER } \\ \text { DATE } & \text { LEVEL MS }\end{array}$

APR $01,1992 \quad 356.61 \mathrm{R}$

PERIOD OF RECORD HIGHEST 59.00 JUL 08, 1946 LOWEST 356,61 APR 01,1992

SITE NUMBER 345612119313001

LOCAL NUMBER 010N025W23E01S

About 5 miles east of Cuyama. Drilled water-table well. Diameter 16 inches, depth 810 feet, perforated 175-810 feet. Altitude of land-surface datum 2,385 feet. Measurements provided by Santa Barbara County Water Agency beginning in 1981. Water-level records available 1947-48, 1953, 1955, 1966 , 1981 to current year.

WATER LEVELS IN FEET BELOW LAND-SURFACE DATUM

$$
\begin{array}{ll} 
& \text { WATER } \\
\text { DATE } & \text { LEVEL MS }
\end{array}
$$

APR 02, $1992 \quad 406.28 \mathrm{R}$

PERIOD OF RECORD HIGHEST 106.10 FEB 10, $1947 \quad$ LOWEST 406.28 APR 02,1992

SITE NUMBER 345512119322101

LOCAL NUMBER 010N025W27G01S

In Cuyama Valley, about 1 mile south of Highway 166 and 1.5 miles west of Highway 33 . Drilled irrigation well. Diameter 16 inches, depth measured 401.5 feet in 1990 , original depth 666 feet, perforated $119-400$, $410-665$ feet. Altitude of land-surface datum 2, 418 feet. Measurements provided by Santa Barbara County Water Agency beginning in 1986. Water-level records available 1986 to current year.

WATER LEVELS IN FEET BELOW LAND-SURFACE DATUM

$\begin{array}{ll} & \text { WATER } \\ \text { DATE } & \text { LEVEL MS }\end{array}$

APR 02,1992

PERIOD OF RECORD HIGHEST 388,40 APR 12, 1986 LOWEST 400.64 APR 09,1991 
WELL DESCRIPTIONS AND WATER-LEVEL MEASUREMENTS

WATER YEAR OCTOBER 1991 TO SEPTEMBER 1992

SITE NUMBER 345822119391801

LOCAL NUMBER 010N026W04R01S

About 3.2 miles northwest of Cuyama. Drilled domestic well, Diameter 16 inches, depth 238 feet, perforated 108-232 feet. Altitude of land-surface datum 2,116 feet. Measurements provided by Santa Barbara County Agency beginning in 1980. Water-level records available 1947, 1955, 1957 to current year.

WATER LEVELS IN FEET BELOW LAND-SURFACE DATUM

$$
\text { DATE WATER }
$$

APR 01, $1992106.34 \mathrm{R}$

PERIOD OF RECORD HIGHEST 18,60 APR 13, 1955 LOWEST 106.34 APR 01, 1992

SITE NUMBER 345823120383901

LOCAL NUMBER 010N036W02Q01S

North of Santa Maria River and west of Highway 101. Observation well. Diameter 2 inches, depth 671 feet, perforated 568-671 feet. Alt1tude of land-surface datum 20 feet. Water-level records available 1977 to current year.

WATER LEVELS IN FEET BELOW LAND-SURFACE DATUM (READINGS ABOVE LAND SURFACE INDICATED BY "+")

$$
\begin{array}{ll} 
& \text { WATER } \\
\text { DATE } & \text { LEVEL MS }
\end{array}
$$

AUG 26, $1992+1.20 \mathrm{~s}$

PERIOD OF RECORD HIGHEST +2.80 AUG 26,1991 LOWEST +1.20 AUG 26,1992

SITE NUMBER 345823120383903

LOCAL NUMBER 010N036W02Q03S

North of Santa Maria River and west of Highway 101. Observation well. Diameter 2 inches, depth 444 feet, perforated 397-444 feet, Altitude of land-surface datum 20 feet. Water-level records available 1977 to current year.

WATER LEVELS IN FEET BELOW LAND-SURFACE DATUM (READINGS ABOVE LAND SURFACE INDICATED BY "+")

$$
\text { DATE WATER } \quad \text { LEVEL MS }
$$

AUG $26,1992 \quad 0.22 \mathrm{~s}$

PERIOD OF RECORD HIGHEST +1.89 MAY 24, 1978 LOWEST 0.22 AUG 26, 1992

SITE NUMBER 345823120383904

LOCAL NUMBER 010N036W02Q04S

North of Santa Maria River and west of Highway 101. Observation well. Diameter 2 inches, depth 378 feet, perforated 291-378 feet. Altitude of land-surface datum 20 feet. Water-level records avallable 1977 to current year.

WATER LEVELS IN FEET BELOW LAND-SURFACE DATUM (READINGS ABOVE LAND SURFACE INDICATED BY "+")

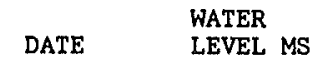

AUG 26, $1992 \quad 0.22 \mathrm{~s}$

PERIOD OF RECORD HIGHEST +1.94 MAY 24, 1978 LOWEST 1.22 OCT 19, 1977 
WELL DESCRIPTIONS AND WATER-LEVEL MEASUREMENTS

WATER YEAR OCTOBER 1991 TO SEPTEMBER 1992

SITE NUMBER 345823120383907

LOCAL NUMBER 010N036W02Q07S

West of Guadalupe. Observation we11. Diameter 2 inches, depth measured 44 feet in 1991 , original depth 47 feet, perforated 18.5-46.5 feet. Altitude of land-surface datum 15.2 feet. Water-level records available 1970 to current year.

WATER LEVELS IN EEET BELOW LAND-SURFACE DATUM

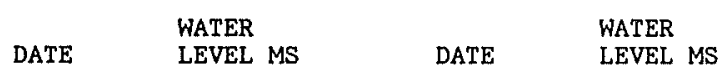

AUG $26,1992 \quad 4.59 \mathrm{~S} \quad$ SEP 09, $1992 \quad 4.75 \mathrm{~s}$

PERIOD OF RECORD HIGHEST 3.76 JUL 24,1990 LOWEST $8.8 \quad$ JUN 03,1970

SITE NUMBER 345950120294501

LOCAL NUMBER 011N034W30Q01S

Northwest of Santa Maria, southeast of intersection of Nipomo-Oso Flaco Lake and Bonita School Roads. Drilled irrigation water-table well. Diameter 16 inches, depth 180 feet in 1923 . Altitude of land-surface datum 148 feet. Measurements provided by Santa Maria Valley Water Conservation District 1958-85, Santa Barbara County Water Agency beginning in 1990. Water-level records available 1930, 1933, 1936, 1938-85, 1990 to current year.

WATER LEVELS IN FEET BELOW LAND-SURFACE DATUM

$$
\begin{array}{ll} 
& \text { WATER } \\
\text { DATE } & \text { LEVEL MS }
\end{array}
$$

MAR 27, $1992103.84 \mathrm{R}$

PERIOD OF RECORD HIGHEST 34.59 MAY $16,1941 \quad$ LOWEST $114.90 \quad$ OCT 01,1966

\section{SITE NUMBER 350107120353201}

LOCAL NUMBER 011N035W20E01S

Northwest of Guadalupe, $1.5 \mathrm{miles}$ northwest of intersection of Oso Flaco Lake Road and Highway 1 . Drilled irrigation well. Diameter 14 inches, depth 525 feet, perforated $150-440$ feet, cemented at 440 feet. Altitude of land-surface datum 48.74 feet. Measurements provided by Santa Maria Valley Water Conservation District to 1985 and Santa Barbara County Water Agency begiming in 1990. Water-level records available $1938-85$, 1990 to current year.

WATER LEVELS IN FEET BELOW LAND-SURFACE DATUM

$$
\begin{array}{ll} 
& \text { WATER } \\
\text { DATE } & \text { LEVEL MS }
\end{array}
$$

MAR 24, $1992 \quad 22,83 \mathrm{R}$

PERIOD OF RECORD HIGHEST 0.01 DEC $30,1943 \quad$ LOWEST $37.10 \quad$ JUL 15,1983

SITE NUMBER 350014120310501

LOCAL NUMBER 011N035W25F03S

Northeast of Guadalupe, near intersection of Bonita School Road and Division Street. Irrigation well.

Diameter and depth unknown. Altitude of land-surface datum 130 feet. Measurements provided by Santa Maria Valley Water Conservation District to 1985, and Santa Barbara County Water Agency beginning in 1990 . Water-level records available $1979-85,1990$ to current year.

WATER LEVELS IN FEET BELOW LAND-SURFACE DATUM

$$
\begin{array}{ll} 
& \text { WATER } \\
\text { DATE } & \text { LEVEL MS }
\end{array}
$$

MAR 24, $199288.14 \mathrm{R}$

PERIOD OF RECORD HIGHEST 47.00 APR 15,1985 LOWEST 88.69 APR 20, 1990 
WELL DESCRIPTIONS AND WATER-LEVEL MEASUREMENTS

WATER YEAR OCTOBER 1991 TO SEPTEMBER 1992

SITE NUMBER 350012120342601

LOCAL NUMBER 011N035W28M01S

About 2 miles north of Guadalupe, southwest of intersection of Oso Flaco Lake Road and Highway 1 . Drilled irrigation wel1. Diameter 16 inches, depth 376 feet, perforated 235-239, 272-276, 300-372 feet. Altitude of land-surface datum 77 feet. Measurements provided by Santa Maria Valley Water Conservation District to 1985, and Santa Barbara County Water Agency beginning in 1990. Water-level records available 1929, 1932-33, 1935, 1938-85, 1990 to current year.

WATER LEVELS IN FEET BELON LAND-SURFACE DATUM

$$
\text { DATE HATER }
$$

MAR 24, $1992 \quad 45.84 \mathrm{R}$

PERIOD OF RECORD HIGHEST 11.09 DEC 30,1943 LOWEST 64.0 JUL 01, 1968

SITE NUMBER 345926120340001

LOCAL NUMBER 011N035W33G01S

North of Guadalupe, east of intersection of Division Street and Highway 1. Drilled irrigation well. Diameter unknown, depth 141 feet. Altitude of land-surface datum 90 feet. Measurements provided by Santa Maria Valley Water Conservation District to 1985, and Santa Barbara County Water Agency beginning in 1990 . Water-level records available $1930,1933-34,1937-85,1990$ to current year.

WATER LEVELS IN FEET BELOW LAND-SURFACE DATUM

$$
\text { DATE WATER }
$$

MAR 27, $199251.68 \mathrm{R}$

PERIOD OF RECORD HIGHEST 16.49 FEB 29, 1944 LOWEST 78.40 OCT 01, 1967

SITE NUMBER 345921120381601

LOCAL, NUMBER 011N036W35J02S

About 2 miles north of Guadalupe, south of dump. Observation well. Diameter 2 inches, depth 615 feet, perforated 527-615 feet. Altitude of land-surface datum 40 feet. Water-level records available 1977 to current year.

WATER LEVELS IN FEET BELOW LAND-SURFACE DATUM (READINGS ABOVE LAND SURFACE INDICATED BY "+")

$$
\begin{array}{ll} 
& \text { WATER } \\
\text { DATE } & \text { LEVEL MS }
\end{array}
$$

AUG $25,1992 \quad 4.56 \mathrm{~S}$

PERIOD OF RECORD HIGHEST +1.90 OCT 02,1980 LOWEST 4.56 AUG 25,1992

SITE NUMBER 345921120381602

LOCAL NUMBER 011N036W35J03S

About 2 miles north of Guadalupe, south of dump. Observation well. Diameter 2 inches, depth 495 feet, perforated 247-495 feet. Altitude of land-surface datum 40 feet. Water-levei records available 1977 to current year.

WATER LEVELS IN FEET BELOW LAND-SURFACE DATUM (READINGS ABOVE LAND SURFACE INDICATED BY "+")

$$
\text { DATE WATER }
$$

AUG $25,1992 \quad 6.20 \mathrm{~S}$

PERIOD OF RECORD HIGHEST t0.72 SEP 19, 1989 LOWEST 6.23 JUL 20,1977 
SITE NUMBER 345921120381603

LOCAL NUMBER 011N036W35J04S

About 2 miles north of Guadalupe, south of dump. Observation well. Diameter 2 inches, depth 228 feet, perforated 175-228 feet. Altitude of land-surface datum 40 feet. Water-level records available 1977 to current year.

WATER LEVELS IN FEET BELOW LAND-SURFACE DATUM (READINGS ABOVE LAND SURFACE INDICATED BY "+")

$$
\begin{array}{lll} 
& \text { WATER } & \\
\text { DATE } & \text { WATER } \\
& \text { LEVEL MS } & \text { LEVEL MS }
\end{array}
$$

AUG $25,1992 \quad 6.40 \mathrm{~s} \quad$ AUG $26,1992 \quad 6.36 \mathrm{~s}$

PERIOD OF RECORD HIGHEST to.47 MAR 24, 1978 LOWEST 6.40 AUG 25, 1992

SITE NUMBER 345921120381604

LOCAL NUMBER 011N036W35J05S

About 2 miles north of Guadalupe, south of dump, Observation well. Diameter 2 inches, depth measured 135 feet in 1991, original depth 138 feet, perforated $74-138$ feet. Altitude of land-surface datum 40 feet. Water-1evel records available 1977 to current year.

WATER LEVELS IN FEET BELOW LAND-SURFACE DATUM (READINGS ABOVE LAND SURFACE INDICATED BY "+")

$$
\begin{array}{lll} 
& \text { WATER } & \\
\text { DATE } & \text { WEVEL MS } & \text { WATER } \\
\text { LEVEL MS }
\end{array}
$$

AUG $25,1992 \quad 5.96 \mathrm{~S} \quad$ AUG $26,1992 \quad 6.12 \mathrm{~s}$

PERIOD OF RECORD HIGHEST +0.58 MAR 24, 1978 LOWEST 6.12 AUG 26, 1992 
INORGANIC DATA

WATER YEAR OCTOBER 1991 TO SEFTEMBER 1992

\begin{tabular}{|c|c|c|c|c|c|c|c|c|c|}
\hline $\begin{array}{l}\text { LOCAL } \\
\text { NUMBER }\end{array}$ & DATE & TIME & $\begin{array}{l}\text { DEPTH } \\
\text { BELOW } \\
\text { LAND } \\
\text { SURFACE } \\
\text { (WATER } \\
\text { LEVEL) } \\
\text { (FEET) }\end{array}$ & $\begin{array}{l}\text { DEPTH } \\
\text { OF } \\
\text { WELL, } \\
\text { TOTAL } \\
\text { (FEET) }\end{array}$ & $\begin{array}{l}\text { SPE- } \\
\text { CIFIC } \\
\text { CON- } \\
\text { DUCT- } \\
\text { ANCE } \\
\text { (US/CM) }\end{array}$ & $\begin{array}{c}\text { PH } \\
\text { WATER } \\
\text { WHOLE } \\
\text { FIELD } \\
\text { (STAND- } \\
\text { ARD } \\
\text { UIIIS) }\end{array}$ & $\begin{array}{l}\text { TEMPER- } \\
\text { ATURE } \\
\text { WATER } \\
\text { (DEG C) }\end{array}$ & $\begin{array}{l}\text { HARD- } \\
\text { NESS } \\
\text { TOTAL } \\
\text { (MG/L } \\
\text { AS } \\
\text { CACO3) }\end{array}$ & $\begin{array}{l}\text { CALCIUM } \\
\text { DIS- } \\
\text { SOLVED } \\
\text { (MG/L } \\
\text { AS CA) }\end{array}$ \\
\hline $\begin{array}{l}010 N 036 W 02 Q 01 S \\
010 N 036 W 02 Q 03 S \\
010 N 036 W 02 Q 04 S \\
010 N 036 W 02 Q 07 S \\
011 N 036 W 35 J 02 S\end{array}$ & $\begin{array}{l}08-26-92 \\
08-26-92 \\
08-26-92 \\
09-11-92 \\
08-25-92\end{array}$ & $\begin{array}{l}1255 \\
1405 \\
1445 \\
1115 \\
1255\end{array}$ & $\begin{array}{r}+1.20 \\
0.22 \\
0.22 \\
-. \\
4.56\end{array}$ & $\begin{array}{r}671 \\
444 \\
378 \\
44 \\
615\end{array}$ & $\begin{array}{r}1110 \\
1010 \\
968 \\
1960 \\
1070\end{array}$ & $\begin{array}{l}7.4 \\
7.5 \\
7.6 \\
7.4 \\
7.6\end{array}$ & $\begin{array}{l}22.0 \\
20.5 \\
20.0 \\
17.0 \\
21.0\end{array}$ & $\begin{array}{l}480 \\
440 \\
430 \\
720 \\
470\end{array}$ & $\begin{array}{r}110 \\
100 \\
97 \\
170 \\
110\end{array}$ \\
\hline $\begin{array}{l}011 \mathrm{~N} 036 \mathrm{~W} 35 \mathrm{~J} 03 \mathrm{~S} \\
011 \mathrm{~N} 036 \mathrm{~W} 35 \mathrm{~J} 04 \mathrm{~S} \\
011 \mathrm{~N} 036 \mathrm{~W} 35 \mathrm{~J} 05 \mathrm{~S}\end{array}$ & $\begin{array}{l}08-25-92 \\
08-26-92 \\
08-26-92\end{array}$ & $\begin{array}{l}1325 \\
0935 \\
1045\end{array}$ & $\begin{array}{l}6.20 \\
6.36 \\
6.12\end{array}$ & $\begin{array}{l}495 \\
228 \\
135\end{array}$ & $\begin{array}{l}1510 \\
1840 \\
1510\end{array}$ & $\begin{array}{l}7.6 \\
7.6 \\
7.5\end{array}$ & $\begin{array}{l}20.0 \\
18.0 \\
18.0\end{array}$ & $\begin{array}{l}670 \\
920 \\
750\end{array}$ & $\begin{array}{l}160 \\
220 \\
190\end{array}$ \\
\hline $\begin{array}{l}\text { LOCAL } \\
\text { NUMBER }\end{array}$ & DATE & $\begin{array}{l}\text { MAGNE- } \\
\text { SIUM, } \\
\text { DIS- } \\
\text { SOLVED } \\
\text { (MG /L } \\
\text { AS MG) }\end{array}$ & $\begin{array}{l}\text { SODIUM, } \\
\text { DIS- } \\
\text { SOLVED } \\
\text { (MG/L } \\
\text { AS NA) }\end{array}$ & $\begin{array}{l}\text { SODIUM } \\
\text { PERCENT }\end{array}$ & $\begin{array}{l}\text { SODIUM } \\
\text { AD- } \\
\text { SORP- } \\
\text { TION } \\
\text { RATIO }\end{array}$ & $\begin{array}{l}\text { POTAS- } \\
\text { SIUM, } \\
\text { DIS- } \\
\text { SOLVED } \\
\text { (MG/L } \\
\text { AS K) }\end{array}$ & $\begin{array}{c}\text { BICAR- } \\
\text { BONATE } \\
\text { WATER } \\
\text { WH IT } \\
\text { FIELD } \\
\text { MG/L AS } \\
\text { HCO3 }\end{array}$ & $\begin{array}{c}\text { ALKA- } \\
\text { LINITY } \\
\text { WAT WH } \\
\text { TOT IT } \\
\text { FIELD } \\
\text { MG/L AS } \\
\text { CACO3 }\end{array}$ & $\begin{array}{l}\text { SULFATE } \\
\text { DIS- } \\
\text { SOLVED } \\
\text { (MG/L } \\
\text { AS SO4) }\end{array}$ \\
\hline $\begin{array}{l}010 N 036 W 02 Q 01 S \\
010 N 036 W 02 Q 03 S \\
010 N 036 W 02 Q 04 S \\
010 N 036 W 02 Q 07 S \\
011 N 036 W 35 J 02 S\end{array}$ & $\begin{array}{l}08-26-92 \\
08-26-92 \\
08-26-92 \\
09-11-92 \\
08-25-92\end{array}$ & $\begin{array}{l}51 \\
47 \\
46 \\
71 \\
48\end{array}$ & $\begin{array}{r}54 \\
50 \\
48 \\
130 \\
57\end{array}$ & $\begin{array}{l}19 \\
20 \\
19 \\
28 \\
21\end{array}$ & $\begin{array}{l}1 \\
1 \\
1 \\
2 \\
1\end{array}$ & $\begin{array}{l}3.3 \\
3.0 \\
2.8 \\
6.0 \\
3.2\end{array}$ & $\begin{array}{l}273 \\
229 \\
254 \\
339 \\
264\end{array}$ & $\begin{array}{l}224 \\
188 \\
208 \\
278 \\
216\end{array}$ & $\begin{array}{l}330 \\
300 \\
280 \\
200 \\
320\end{array}$ \\
\hline $\begin{array}{l}011 \text { N036W35J03S } \\
011 N 036 W 35 J 04 S \\
011 N 036 W 35 J 05 S\end{array}$ & $\begin{array}{l}08-25-92 \\
08-26-92 \\
08-26-92\end{array}$ & $\begin{array}{l}66 \\
90 \\
68\end{array}$ & $\begin{array}{l}83 \\
93 \\
74\end{array}$ & $\begin{array}{l}21 \\
18 \\
17\end{array}$ & $\begin{array}{l}1 \\
1 \\
1\end{array}$ & $\begin{array}{l}3.9 \\
4.5 \\
3.9\end{array}$ & $\begin{array}{l}283 \\
361 \\
315\end{array}$ & $\begin{array}{l}232 \\
296 \\
258\end{array}$ & $\begin{array}{l}520 \\
700 \\
550\end{array}$ \\
\hline $\begin{array}{l}\text { LOCAL } \\
\text { NUMBER }\end{array}$ & DATE & $\begin{array}{l}\text { CHLO- } \\
\text { RIDE; } \\
\text { DIS- } \\
\text { SOLVED } \\
\text { (MG/L } \\
\text { AS CL) }\end{array}$ & $\begin{array}{l}\text { FLUO- } \\
\text { RIDE, } \\
\text { DIS- } \\
\text { SOLVED } \\
\text { (MG/L } \\
\text { AS F) }\end{array}$ & $\begin{array}{l}\text { SILICA, } \\
\text { DIS- } \\
\text { SOLVED } \\
\text { (MG/L } \\
\text { AS } \\
\text { SIO2) }\end{array}$ & $\begin{array}{l}\text { SOLIDS, } \\
\text { RESIDUE } \\
\text { AT } 180 \\
\text { DEG. C } \\
\text { DIS- } \\
\text { SOLVED } \\
(M G / L)\end{array}$ & $\begin{array}{l}\text { SOLIDS, } \\
\text { SUM OF } \\
\text { CONSTI- } \\
\text { TUENTS, } \\
\text { DIS- } \\
\text { SOLVED } \\
\text { (MG/L) }\end{array}$ & $\begin{array}{l}\text { NITRO- } \\
\text { GEN, } \\
\text { NITRITE } \\
\text { DIS- } \\
\text { SOLVED } \\
\text { (MG/L } \\
\text { AS N) }\end{array}$ & $\begin{array}{c}\text { NITRO- } \\
\text { GEN } \\
\text { NO2+NÓ3 } \\
\text { DIS- } \\
\text { SOLVED } \\
\text { (MG/L } \\
\text { AS N) }\end{array}$ & \\
\hline $\begin{array}{l}010 N 036 W 02 Q 01 S \\
010 N 036 W 02 Q 03 S \\
010 N 036 W 02 Q 04 S \\
010 N 036 W 02 Q 07 S \\
011 N 036 W 35 J 02 S\end{array}$ & $\begin{array}{l}08-26-92 \\
08-26-92 \\
08-26-92 \\
09-11-92 \\
08-25-92\end{array}$ & $\begin{array}{r}31 \\
25 \\
25 \\
440 \\
31\end{array}$ & $\begin{array}{l}0.30 \\
0.40 \\
0.20 \\
0.50 \\
0.60\end{array}$ & $\begin{array}{l}43 \\
40 \\
38 \\
1.1 \\
41\end{array}$ & $\begin{array}{r}784 \\
730 \\
690 \\
1210 \\
746\end{array}$ & $\begin{array}{r}759 \\
680 \\
664 \\
1190 \\
743\end{array}$ & $\begin{array}{l}<0.010 \\
<0.010 \\
<0.010 \\
<0.010 \\
<0.010\end{array}$ & $\begin{array}{l}0.490 \\
0.460 \\
0.550 \\
0.080 \\
0.480\end{array}$ & \\
\hline $\begin{array}{l}011 N 036 W 35 J 03 S \\
011 N 036 W 35 J 04 S \\
011 N 036 W 35 J 05 S\end{array}$ & $\begin{array}{l}08-25-92 \\
08-26-92 \\
08-26-92\end{array}$ & $\begin{array}{l}59 \\
77 \\
58\end{array}$ & $\begin{array}{l}0.30 \\
0.60 \\
0.50\end{array}$ & $\begin{array}{l}32 \\
29 \\
29\end{array}$ & $\begin{array}{l}1080 \\
1480 \\
1190\end{array}$ & $\begin{array}{l}1080 \\
1410 \\
1140\end{array}$ & $\begin{array}{r}<0.010 \\
<0.010 \\
0.030\end{array}$ & $\begin{array}{l}4.60 \\
3.90 \\
1.60\end{array}$ & \\
\hline $\begin{array}{l}\text { LOCAL } \\
\text { NUMBER }\end{array}$ & DATE & $\begin{array}{l}\text { NITRO- } \\
\text { GEN, } \\
\text { AMMONIA } \\
\text { DIS- } \\
\text { SOLVED } \\
(M G / L \\
\text { AS N) }\end{array}$ & $\begin{array}{c}\text { NITRO- } \\
\text { GEN, } \\
\text { AMMONIA } \\
\text { DIS- } \\
\text { SOLVED } \\
\text { (MG/L } \\
\text { AS NH4) }\end{array}$ & $\begin{array}{l}\text { PHOS- } \\
\text { PHORUS } \\
\text { ORTHO, } \\
\text { DIS- } \\
\text { SOLVED } \\
\text { (MG /L } \\
\text { AS P) }\end{array}$ & $\begin{array}{c}\text { PHOS- } \\
\text { PHATE, } \\
\text { ORTHO, } \\
\text { DIS- } \\
\text { SOLVED } \\
\text { (MG/L } \\
\text { AS PO4) }\end{array}$ & $\begin{array}{l}\text { BORON, } \\
\text { DIS- } \\
\text { SOLVED } \\
\text { (UG/L } \\
\text { AS B) }\end{array}$ & $\begin{array}{l}\text { IRON, } \\
\text { DIS- } \\
\text { SOLVED } \\
\text { (UG /L } \\
\text { AS FE) }\end{array}$ & $\begin{array}{l}\text { MANGA- } \\
\text { NESE, } \\
\text { DIS- } \\
\text { SOLVED } \\
\text { (UG/L } \\
\text { AS MN) }\end{array}$ & \\
\hline $\begin{array}{l}010 N 036 \mathrm{~W} 02 \mathrm{Q} 01 \mathrm{~S} \\
010 \mathrm{~N} 036 \mathrm{~W} 02 \mathrm{Q} 03 \mathrm{~S} \\
010 \mathrm{~N} 036 \mathrm{~W} 02 \mathrm{Q} 04 \mathrm{~S} \\
010 \mathrm{~N} 036 \mathrm{~W} 02 \mathrm{Q} 07 \mathrm{~S} \\
011 \mathrm{~N} 036 \mathrm{~W} 35 \mathrm{~J} 02 \mathrm{~S}\end{array}$ & $\begin{array}{l}08-26-92 \\
08-26-92 \\
08-26-92 \\
09-11-92 \\
08-25-92\end{array}$ & $\begin{array}{r}<0.010 \\
0.020 \\
<0.010 \\
3.00 \\
0.010\end{array}$ & $\begin{array}{l}\overline{-} \\
0.03 \\
-- \\
3.9 \\
0.01\end{array}$ & $\begin{array}{r}0.020 \\
0.010 \\
0.010 \\
0.020 \\
<0.010\end{array}$ & $\begin{array}{l}0.06 \\
0.03 \\
0.03 \\
0.06 \\
. .\end{array}$ & $\begin{array}{l}140 \\
180 \\
120 \\
170 \\
140\end{array}$ & $\begin{array}{r}15 \\
10 \\
13 \\
1900 \\
<3\end{array}$ & $\begin{array}{r}<1 \\
<1 \\
<1 \\
580 \\
<1\end{array}$ & \\
\hline $\begin{array}{l}011 \mathrm{~N} 036 \mathrm{~W} 35 \mathrm{~J} 03 \mathrm{~S} \\
011 \mathrm{~N} 036 \mathrm{~W} 35 \mathrm{~J} 04 \mathrm{~S} \\
011 \mathrm{~N} 036 \mathrm{~W} 35 \mathrm{~J} 05 \mathrm{~S}\end{array}$ & $\begin{array}{l}08-25-92 \\
08-26-92 \\
08-26-92\end{array}$ & $\begin{array}{r}<0.010 \\
0.010 \\
<0.010\end{array}$ & 0.01 & $\begin{array}{l}0.010 \\
0.020 \\
0.090\end{array}$ & $\begin{array}{l}0.03 \\
0.06 \\
0.28\end{array}$ & $\begin{array}{l}210 \\
240 \\
200\end{array}$ & $\begin{array}{l}30 \\
15 \\
19\end{array}$ & $\begin{array}{l}<1 \\
<1 \\
49\end{array}$ & \\
\hline
\end{tabular}

+ Indicates measurement above land surface.

< Actual value is less than value shown. 

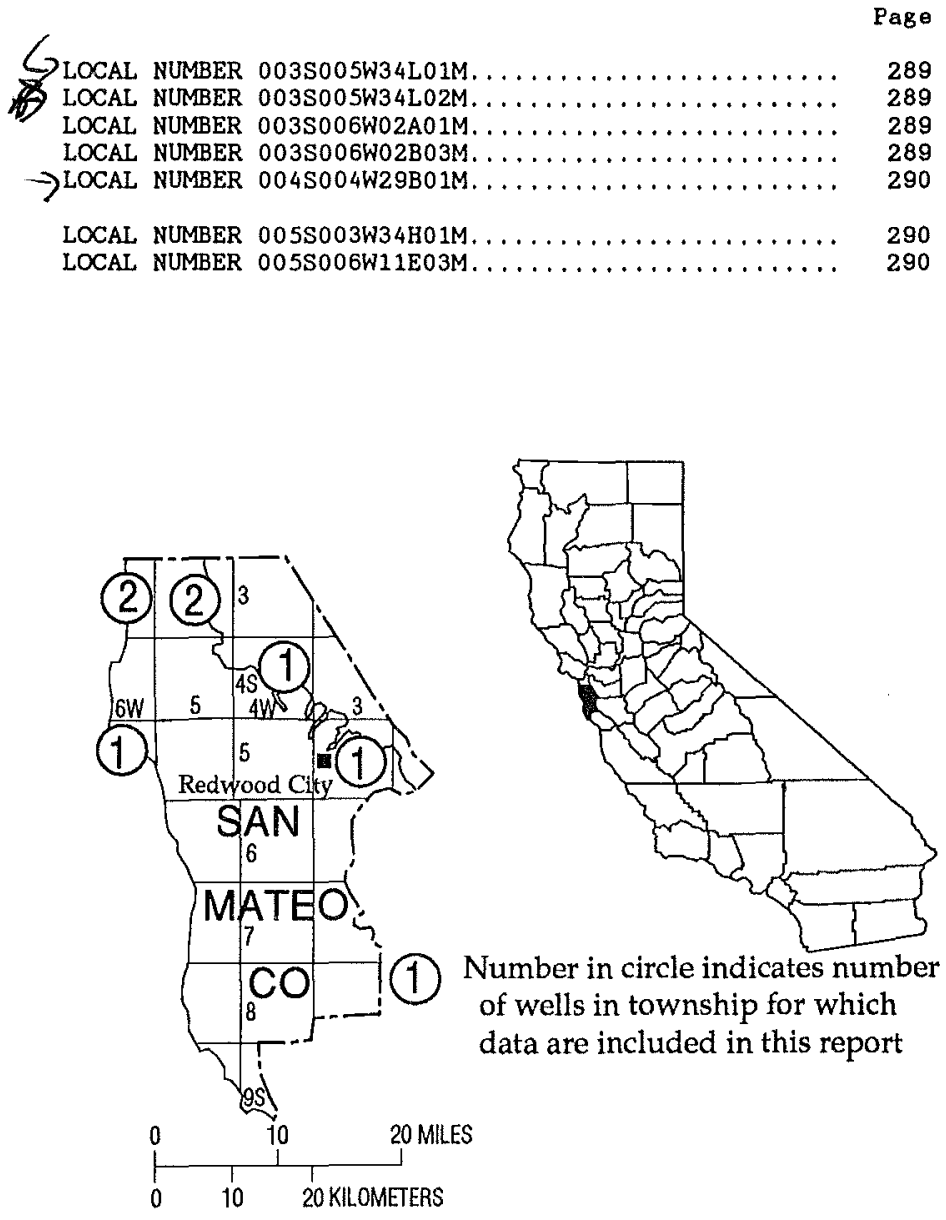

Number in circle indicates number of wells in township for which data are included in this report

Figure 29. Location of wells in San Mateo County. 
WELL DESCRIPTIONS AND WATER-LEVEL MEASUREMENTS

WATER YEAR OCTOBER 1991 TO SEPTEMBER 1992

SITE NUMBER 373741122235201

LOCAL NUMBER 0035005W34L01M

At San Francisco International Airport. Drilled observation well. Diameter 2 inches, depth 144 feet, perforated 116-136 feet. Deep well of two cluster wells. Altitude of land-surface datum 10 feet. Water-level records avallable 1989 to current year.

WATER LEVELS IN FEET BELOW LAND-SURFACE DATUM

$$
\begin{array}{lll} 
& \text { WATER } & \text { WATER } \\
\text { DATE } & \text { DEVEL MS } & \text { LEVEL MS }
\end{array}
$$

FEB $04,1992 \quad 48.67 \mathrm{~S} \quad \operatorname{SEP} 15,199248.97 \mathrm{~V}$

PERIOD OF RECORD HIGHEST 42.28 OCT 27,1989 LOWEST 48.97 SEP 15, 1992

SITE NUMBER 373741122235202

LOCAL NUMBER 0035005W34LO2M

At San Francisco International Airport. Drilled observation well. Diameter 2 inches, depth 35 feet, perforated 20-30 feet. Shallow well of two cluster wells. Altitude of land-surface datum 10 feet. Water-level records available 1989 to current year.

WATER LEVELS IN FEET BELOW LAND-SURFACE DATUM

$\begin{array}{lll} & \text { WATER } & \text { WATER } \\ \text { DATE } & \text { LEVEL MS }\end{array}$

FEB 04, $1992 \quad 5.38 \mathrm{~S} \quad$ SEP $15,1992 \quad 5.93 \mathrm{~S}$

PERIOD OF RECORD HIGHEST 4.72 MAR 06, 1990 LOWEST 6.57 DEC 04, 1990

SITE NUMBER 374228122285301

LOCAL NUMBER 003S006W02A01M

In San Francisco at the San Francisco Golf Club. Drilled observation well. Diameter 2 inches, depth 62 feet, perforated 49-59 feet. Altitude of land-surface datum 40.90 feet. Water-level records available 1989 to current year.

WATER LEVELS IN FEET BELOW LAND-SURFACE DATUM

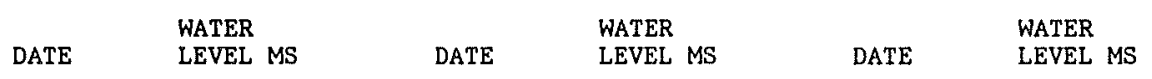

FEB 04, $1992 \quad 41.92 \mathrm{~S}$ JUN 11, $1992 \quad 42.14 \mathrm{~S} \quad$ SEP 15, $199242.46 \mathrm{~S}$

PERIOD OF RECORD HIGHEST 38.00 JUL 07, 1989 LOWEST 42.46 SEP 15,1992

SITE NUMBER 374224122290701

LOCAL, NUMBER 0035006W02B03M

South of Lake Merced, west of Lake Merced Boulevard, Drilled irrigation well in Merced Formation. Diameter 12 inches 0-456 feet, 8 inches 456-524 feet, depth 524 feet, perforated 242-499 feet. Altitude of land-surface datum 61.52 feet. Water-level records available 1982,1988 to current year.

WATER LEVELS IN FEET BELOW LAND-SURFACE DATUM

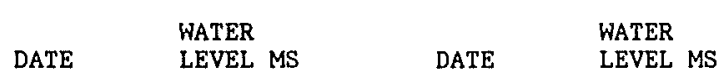

FEB $04,199294.95 \mathrm{~S} \quad \operatorname{SEP} 15,1992107.92 \mathrm{~V}$

PERIOD OF RECORD HIGHEST 90.83 MAR 15, 1989 LOWEST 107.92 SEP 15, 1992 
SITE NUMBER 373338122191301

LOCAL NUMBER 004S004W29B01M

In San Mateo. Drilled irrigation water-table well in alluvium of Quaternary age. Diameter 12 inches, depth 180 feet. Altitude of land-surface datum 32 feet. Water-level records available 1979 to current year.

WATER LEVELS IN FEET BELOW LAND-SURFACE DATUM

$$
\text { DATE WATER } \quad \text { LEVEL MS }
$$

MAY $21,1992 \quad 34.74 \mathrm{SR}$

PERIOD OF RECORD HIGHEST 24.01 APR 18, 1979 LOWEST 33.08 NOV 15, 1990

SITE NUMBER 372722122100501

LOCAL NUMBER 005S003W34H01M

In Menlo Park. Drilled industrial water-table well in alluvium of Quaternary age. Diameter 16 to 8 inches, depth 290 feet, perforated 180-200, 250-270 feet. Altitude of land-surface datum 53 feet. Water-level records available 1977 to current year.

WATER LEVELS IN FEET BELOW LAND-SURFACE DATUM

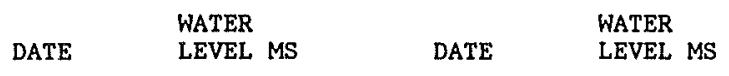

NOV $20,1991 \quad 47.30 \mathrm{~s} \quad \operatorname{MAY} 21,1992 \quad 44.81 \mathrm{~s}$

PERIOD OF RECORD HIGHEST 22.59 APR 20, 1983 LOWEST 54.70 MAY 22, 1990

SITE NUMBER 373045122292801

LOCAL NUMBER O05S006W11E03M

About 5 miles northwest of Half Moon Bay. Drilled unused water-table well in terrace deposits of Pleistocene age. Diameter 12 inches, depth measured 87 feet, original depth 92 feet, perforated 12-88 feet. Altitude of land-surface datum 49 feet. Water-level records available 1979 to current year.

WATER LEVELS IN FEET BELOW LAND-SURFACE DATUM

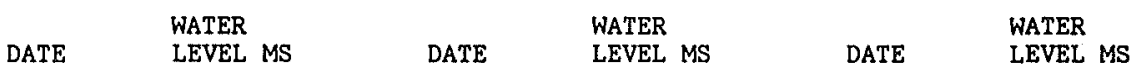

OCT 08, $1991 \quad 38.19 \mathrm{~S}$ NOV $20,1991 \quad 39.34 \mathrm{~S}$ APR 15, $1992 \quad 3.10 \mathrm{~s}$

PERIOD OF RECORD HIGHEST 3.10 APR 15, 1992 LOWEST 45.17 OCT 12, 1989 
SANTA BARBARA COUNTY

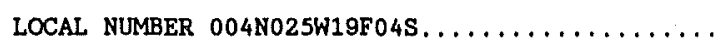

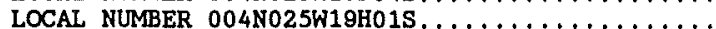

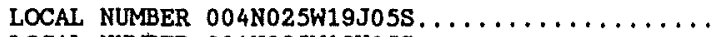

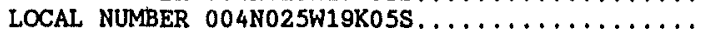

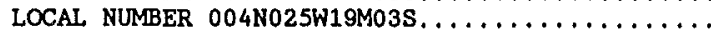

LOCAL NUMBER 004N025W20K03S...............

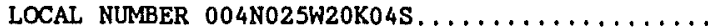

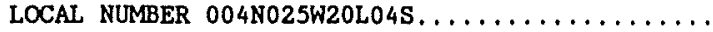

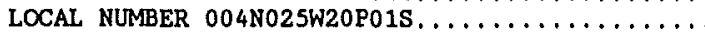

LOCAL NUMBER 004 N025W21N01S $\ldots \ldots \ldots \ldots \ldots$

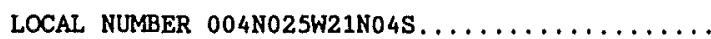

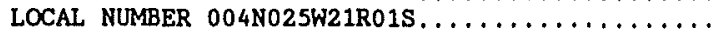

LOCAL NUMBER 004N025W22R03S. . . . . . . . .

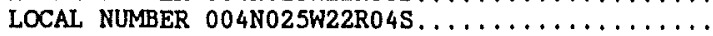

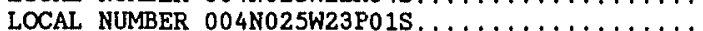

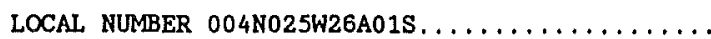

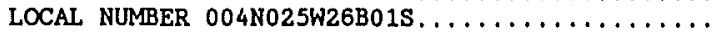

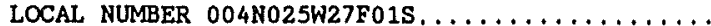

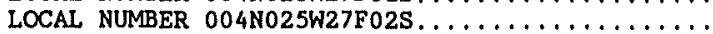

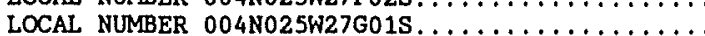

LOCAL NUMBER 004N025W27Q06S..............

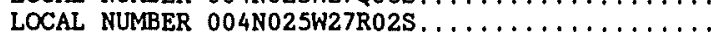

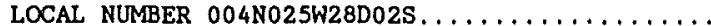

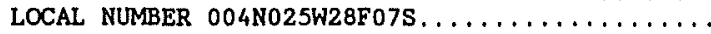

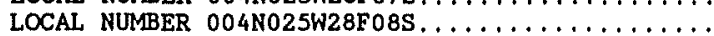

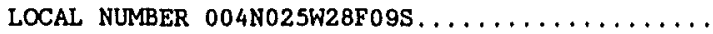

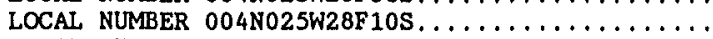

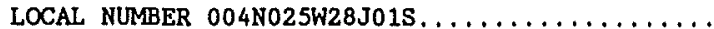

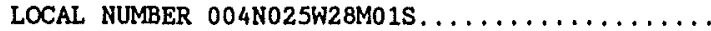

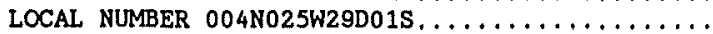

LOCAL NUMBER 004N025W29D07S. . . . . . . . . .

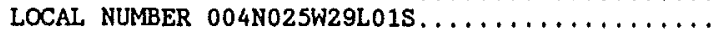

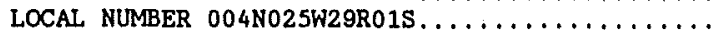

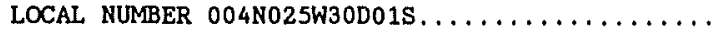

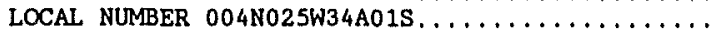

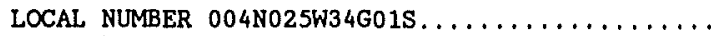

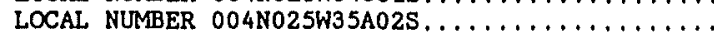

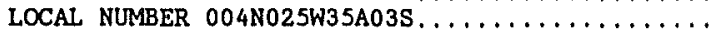

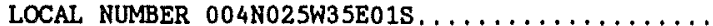

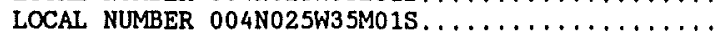

LOCAL NUMBER 004N026W23A02S..............

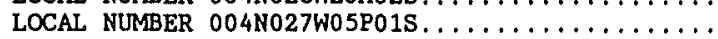

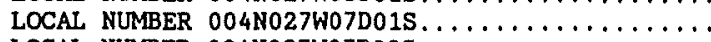

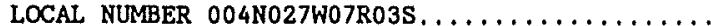

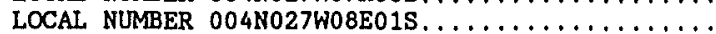

LOCAL NUMBER 004N027W08L03S.

LOCAL NUMBER 004N027W08M05S.................

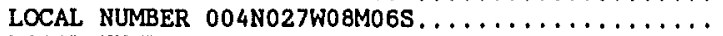

LOCAL NUMBER 004N027W08R02S.

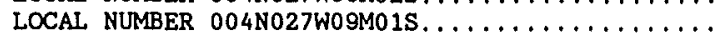

LOCAL NUMBER $004 N 027$ W09Q01S . . . . . . . . . . .

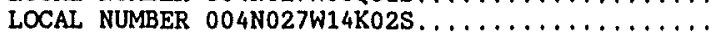

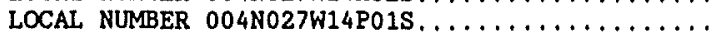

LOCAL NUMBER 004N027W14R01S . . . . . . . . . . .

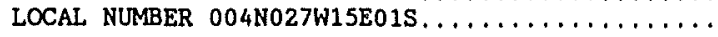

LOCAL NUMBER 004N027W15J02S

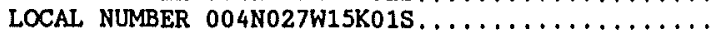

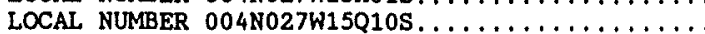

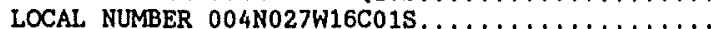

LOCAL NUMBER $004 N 027 W 16 C 025 \ldots \ldots \ldots \ldots \ldots \ldots \ldots$
Page

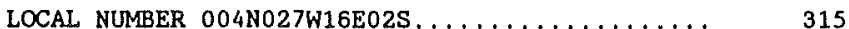

LOCAL NUMBER 004 N027W16R01S............... 315

LOCAL NUMBER 004N027W17J015.............. 315, 402

LOCAL NUMBER 004 N027W17LO2S.................. 316

LOCAL NUMBER 004N027W17L03S.............. 316

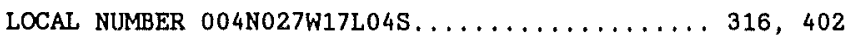

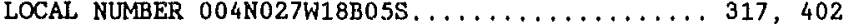

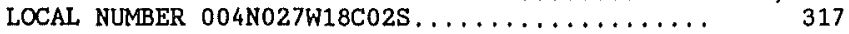

LOCAL NUMBER 004 N027W18C03S................. 317

LOCAL NUMBER 004N027W18Q01S, ............. 318

LOCAL NUMBER 004 N027W18Q0 4 S............. 318

LOCAL NUMBER 004N027W19A01s.............. 318

LOCAL NUMBER 004N027W19A02S.............. 319

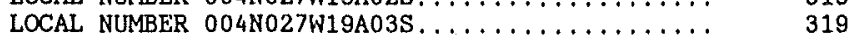

LOCAL NUMBER 004 N027W21B01S............... 319

LOCAL NUMBER 004 N027W21E01S............. 320

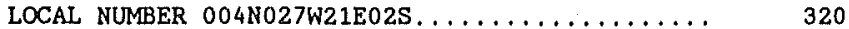

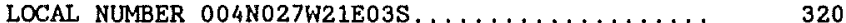

LOCAL NUMBER 004N027W21F01S............... 321

LOCAL NUMBER 004N027W21F02S............. 321, 402

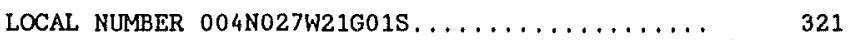

LOCAL NUMBER 004 N027W21G025................. 322, 402

LOCAL NUMBER 004 N027W22A02S.............. 322

LOCAL NUMBER $004 N 027 W 22 A 035 \ldots \ldots \ldots \ldots \ldots \ldots \ldots, 322$

LOCAL NUMBER 004N027W22A04S............... 323, 402

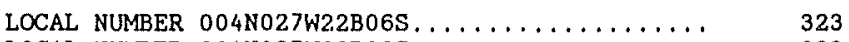

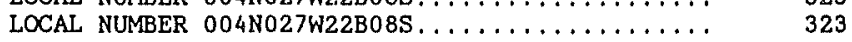

LOCAL NUMBER 004N027W22B09S.............. 323

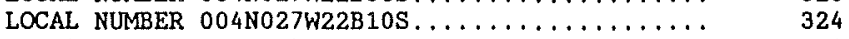

LOCAL NUMBER 004 N027W22B11S...............

LOCAL NUMBER 004 N027W22C01S............. 324

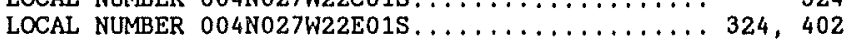

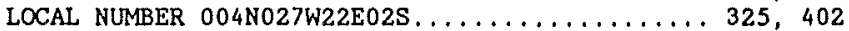

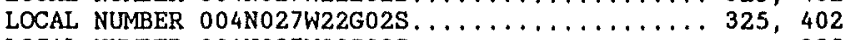

LOCAL NUMBER 004 N027W22G03S................. 325

LOCAL NUMBER $004 N 027 W 22 G 04 S \ldots \ldots \ldots \ldots \ldots \ldots \ldots 326,402$

LOCAL NUMBER 004 N027W22M01S................. 326

LOCAL NUMBER 004 N027W22M02S.............. 326

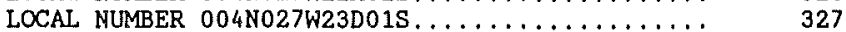

LOCAL NUMBER 004N027W23E05S................ 327, 402

LOCAL NUMBER 004N027W23E06S.............. 327

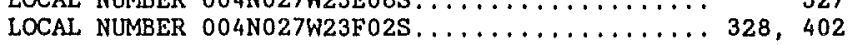

LOCAL NUMBER 004 N027W23F03S............. 328, 402

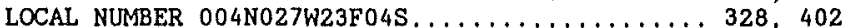

LOCAL NUMBER 004 N027W23F08S............. $329,402,406$

LOCAL NUMBER 004N027W23F09S............. 329, 406

LOCAL NUMBER $004 N 027$ W23H015................. 329

LOCAL NUMBER 004 N027W23H04S................ 330, 406

LOCAL NUMBER 004N028W02P03S............. 330

LOCAL NUMBER 004 N028W12H0 4 S................ 330

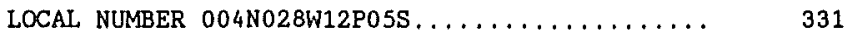

LOCAL NUMBER 004 N028W12R03S.................. 331

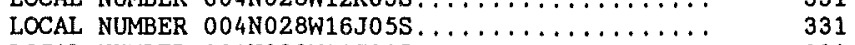

LOCAL NUMBER 004N030W01G01S.............. 331

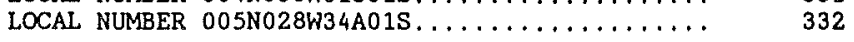

LOCAL NUMBER 005N029W01C01S............. 332

LOCAL NUMBER O05N029W31C015................ 332

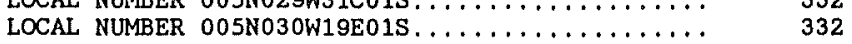

LOCAL NUMBER 005 N030W28R01S................ 333

LOCAL NUMBER 005 N030W3ONO2S .............. 333 
SANTA BARBARA COUNTY--Continued

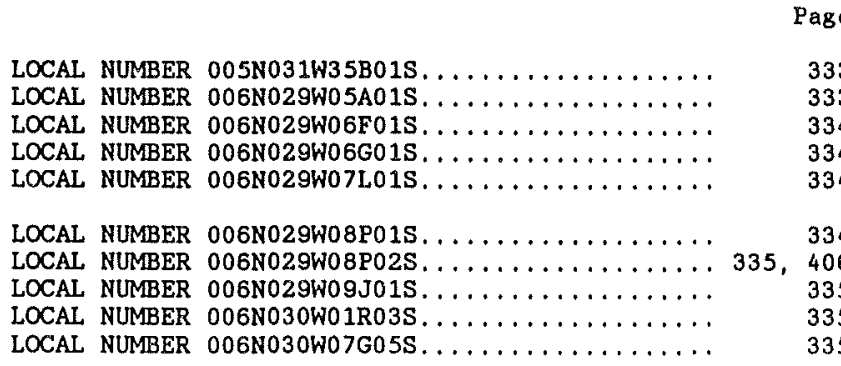

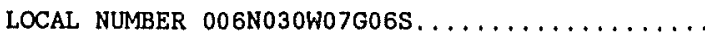

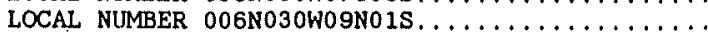

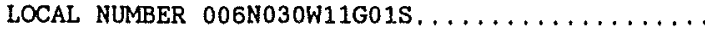

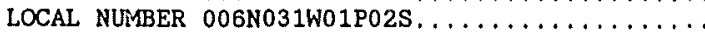

LOCAL NUMBER $006 N 031$ W01P03S . . . . . . . . . .

LOCAL NUMBER 006N031W02K01S. . . . . . . . .

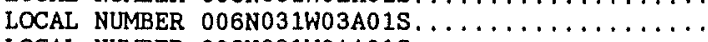

LOCAL NUMBER $006 N 031 W 04 A 015 \ldots \ldots \ldots \ldots \ldots$

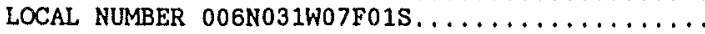

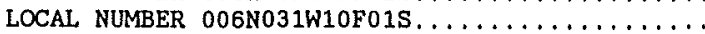

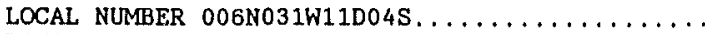

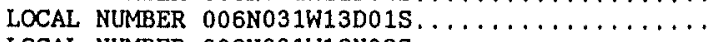

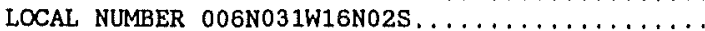

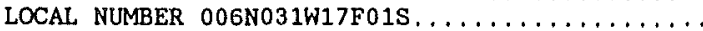

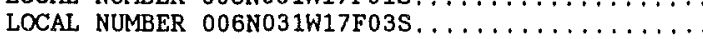

LOCAL NUMBER 006N032W02Q01S. . . . . . . . .

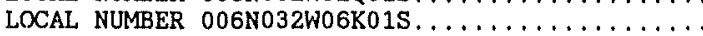

LOCAL NUMBER $006 N 032 W 11 L 035 \ldots \ldots \ldots \ldots \ldots \ldots \ldots$

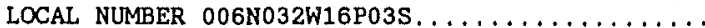

LOCAL NUMBER 006 N032W18H01S . . . . . . . . . .

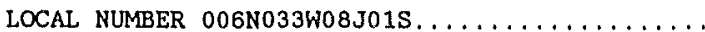

LOCAL NUMBER $006 N 033 W 11 M 015 \ldots \ldots \ldots \ldots \ldots \ldots$

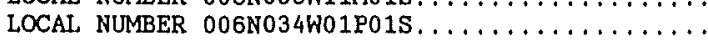

LOCAL NUMBER $006 N 034 W 06 C 045 \ldots \ldots \ldots \ldots \ldots \ldots$

LOCAL NUMBER D07N024W13C02S. . . . . . . . . .

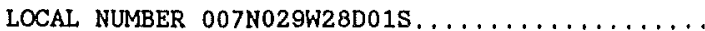

LOCAL NUMBER 007N029W29R01S.............

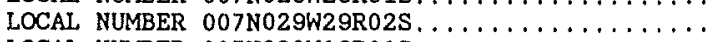

LOCAL NUMBER 007N030W16B01S..............

LOCAL NUMBER 007N030W19H01S.............

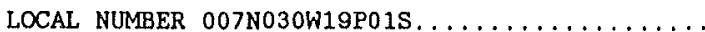

LOCAL NUMBER 007 N030W22E01S. . . . . . . . . .

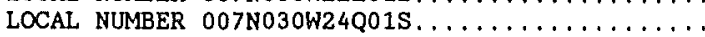

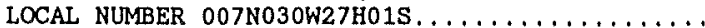

LOCAL NUMBER 007N030W27Q01S . . . . . . . . .

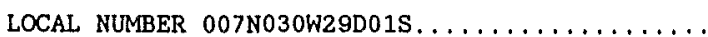

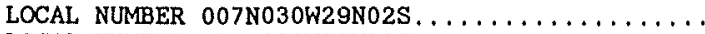

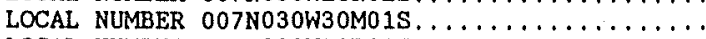

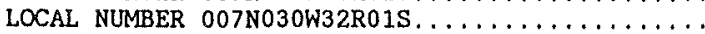

LOCAL NUMBER 007NO3OW33MO1S. . . . . . . . .

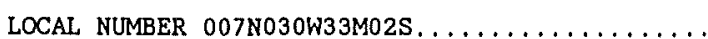

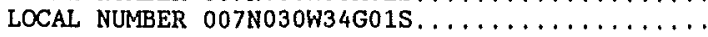

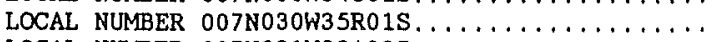

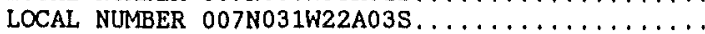

LOCAL NUMBER 007N031W23P01S . . . . . . . . . .

LOCAL NUMBER 007 N031W34M01S . . . . . . . . . .

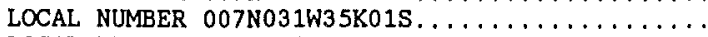

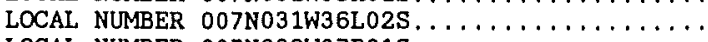

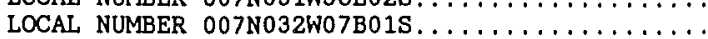

LOCAL NUMBER 007 N032W31M01S . . . . . . . . . .
Page
LOCAL NUMBER 007N033W16G02S............

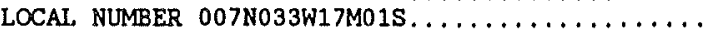
LOCAL NUMBER 007N033W17N02S............

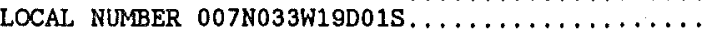
LOCAL NUMBER 007N033W19Q02S . . . . . . . . . .

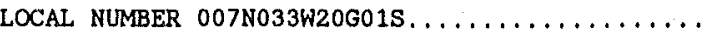

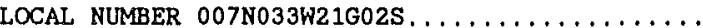

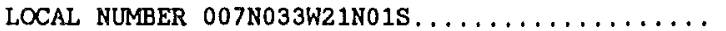
LOCAL NUMBER $007 N 033 W 27 G 015 \ldots \ldots \ldots \ldots \ldots \ldots$ LOCAL NUMBER 007 N033W27J01S . . . . . . . . .

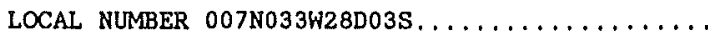
LOCAL NUMBER 007N033W30B02S............. LOCAL NUMBER 007N033W36J01S. . . . . . . . . . LOCAL NUMBER $007 N 033 W 36 J 025 \ldots \ldots \ldots \ldots \ldots \ldots \ldots \ldots$

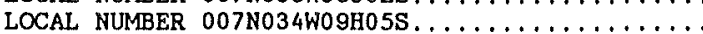

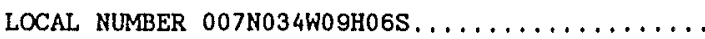

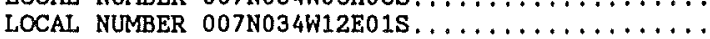

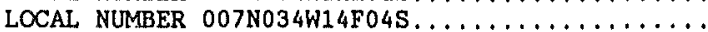
LOCAL NUMBER $007 N 034 W 14 \mathrm{~L} 015 \ldots \ldots \ldots \ldots \ldots \ldots$

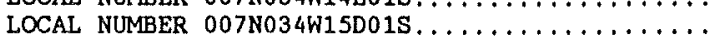

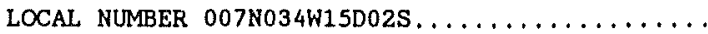

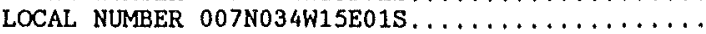

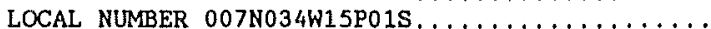
LOCAL NUMBER 007 N034W20K0 4 S . . . . . . . . . LOCAL NUMBER 007 N034W20K06S $\ldots \ldots \ldots \ldots \ldots \ldots$

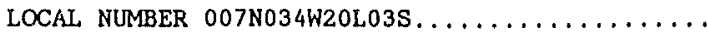

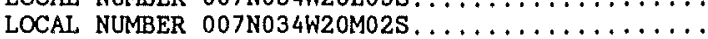

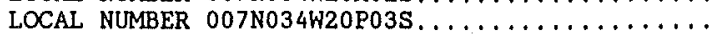

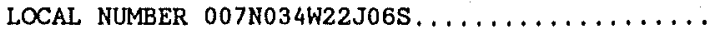
LOCAL NUMBER 007 N034W24N01S .............

LOCAL NUMBER 007 N034W26H03S . . . . . . . . .

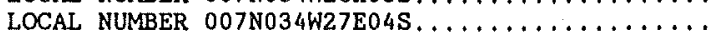

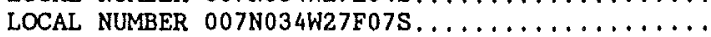

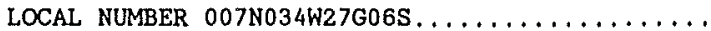

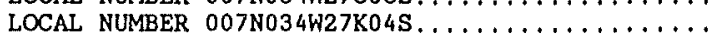

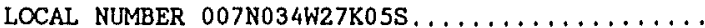

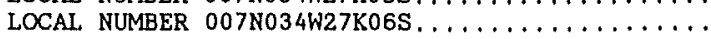

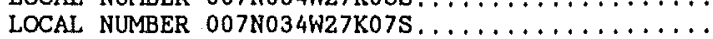

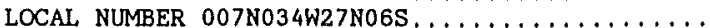

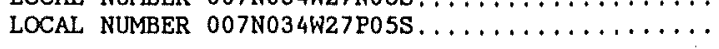

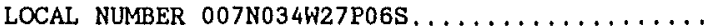

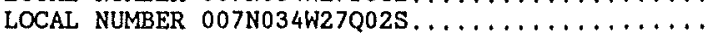

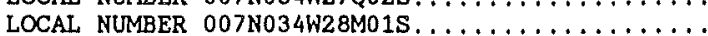

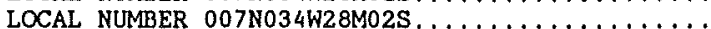
LOCAL NUMBER 007N034W28Q01S. . . . . . . . . .

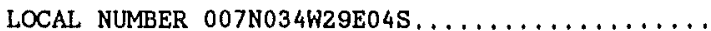
LOCAL NUMBER 007 N034W29F01S. . . . . . . . . . .

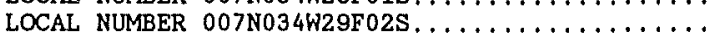

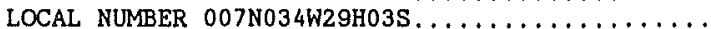

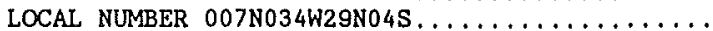

LOCAL NUMBER 007N034W29N05S...

359, 406

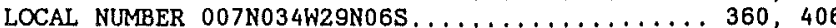

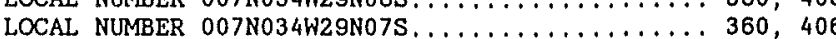

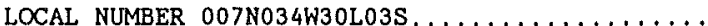
LOCAL NUMBER 007N034W30L10S . . . . . . . . .

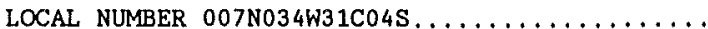

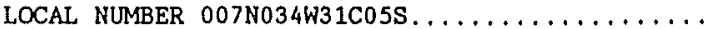
LOCAL NUMBER $007 N 034 \mathrm{~W} 31 \mathrm{R} 02 \mathrm{~A} \ldots \ldots \ldots \ldots \ldots \ldots \ldots \ldots \ldots$

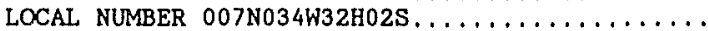
LOCAL NUMBER 007 N034W33E05S . . . . . . . . . 
SANTA BARBARA COUNTY--Continued

Page

LOCAL NUMBER 007N034W34A04S.

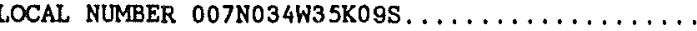

LOCAL NUMBER 007N035W13N02S,

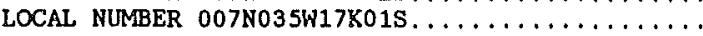

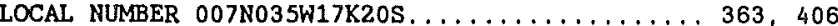

LOCAL NUMBER 007 N035W17K21S............. 363, 406

LOCAL NUMBER 007N035W17M01S.............. 364

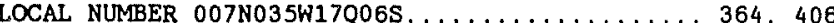

LOCAL NUMBER 007N035W18H01S.............. 364

LOCAL NUMBER 007N035W18J02S.............. 364

LOCAL NUMBER 007N035W21G025.............. 365, 406

LOCAL NUMBER 007N035W22J01S................ 365

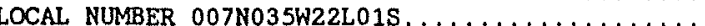

LOCAL NUMBER 007N035W22M01S............. 365

LOCAL NUMBER 007N035W23B02S . . . . . . . . . 366,406

LOCAL NUMBER 007N035W23E02S . . . . . . . . . . . 366

LOCAL NUMBER 007N035W23J05S,............. 366

LOCAL NUMBER $007 N 035$ W23Q02S................. 366, 406

LOCAL NUMBER 007N035W23Q03S............ 367, 406

LOCAL NUMBER $007 N 035 W 23 Q 04 S \ldots \ldots \ldots \ldots \ldots \ldots \ldots 367,406$

LOCAL NUMBER 007N035W24J04S............... 367

LOCAL NUMBER $007 N 035 W 24 K 05 S \ldots \ldots \ldots \ldots \ldots \ldots \ldots, 367$

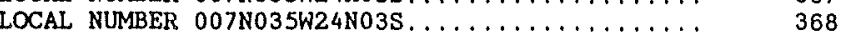

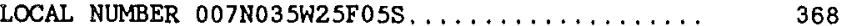

LOCAL NUMBER 007 N035W25F06S............. 368

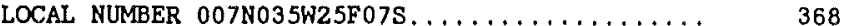

LOCAL NUMBER 007N035W26F04S............. 369

LOCAL NUMBER $007 N 035 W 26 F 05 S \ldots \ldots \ldots \ldots \ldots \ldots \ldots \ldots, 406$

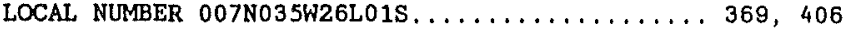

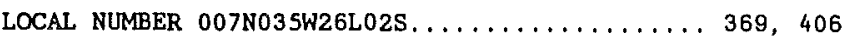

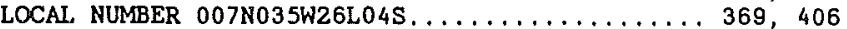

LOCAL NUMBER 007 N035W27F015................... 370

LOCAL NUMBER $007 N 035$ W27H01S................ 370

LOCAL NUMBER 007N035W27P01S............. 370

LOCAL NUMBER 007 N035W30G015............ 370

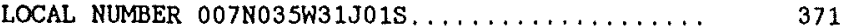

LOCAL NUMBER 007 N035W32N01S.................

LOCAL NUMBER 007 N035W33J01S................. 371

LOCAL NUMBER 007N035W33J02S............ 371, 406

LOCAL NUMBER 007N035W33J03S.............

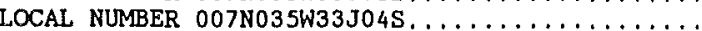

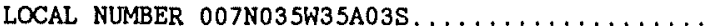

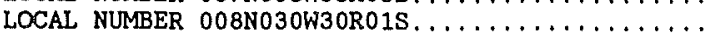

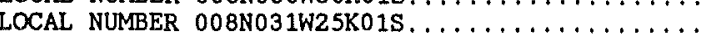

LOCAL NUMBER 008N031W25Q01S. . . . . . . . . .

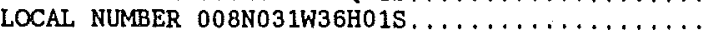

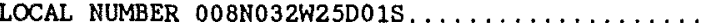

LOCAL NUMBER $008 N 032 W 27 P 025, \ldots \ldots \ldots \ldots \ldots \ldots \ldots \ldots$

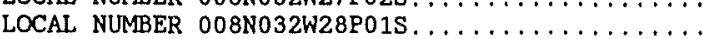

LOCAL NUMBER 008N032W29L02S. . . . . . . . .

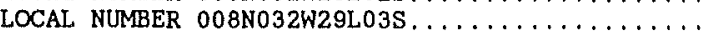

LOCAL NUMBER 008N032W30D01S . . . . . . . . . . .

LOCAL NUMBER $008 N 033 W 19 G 02 S \ldots \ldots \ldots \ldots \ldots \ldots \ldots$

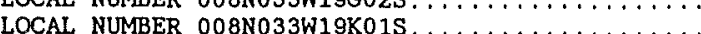

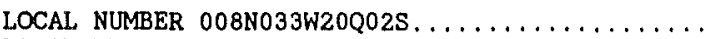

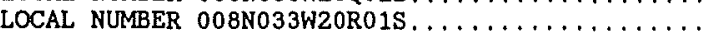

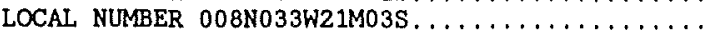

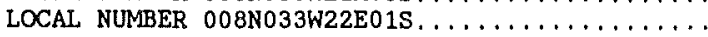

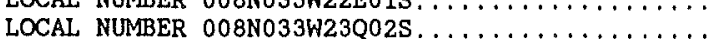

372

406

372

372

372

373

373

373

406

373

374

406

374

406

374

374

375

375

406

375

62
62

3

3

LOCAL NUMBER 008N034W02M01S . .

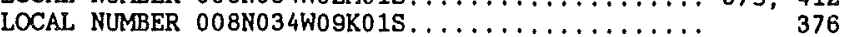

LOCAL NUMBER 008N034W14L01S.............. 376

LOCAL NUMBER 008N034W15F04S .............. 376

LOCAL NUMBER 008N034W16C01S .............. 376

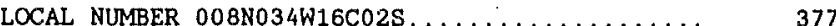

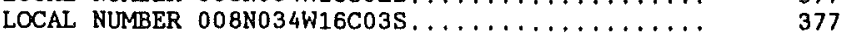

LOCAL NUMBER $008 \mathrm{~N} 034 \mathrm{~W} 16 \mathrm{C} 04 \mathrm{~S} \ldots \ldots \ldots \ldots \ldots \ldots \ldots \ldots . \ldots \ldots$

LOCAL NUMBER DO8N034W16C05S $\ldots \ldots \ldots \ldots \ldots \ldots \ldots$

LOCAL NUMBER 008 N034W16D02S................ 377

LOCAL NUMBER 008N034W16F01S. . . . . . . . . . .

LOCAL NUMBER 008 N034W16G02S.............. 378

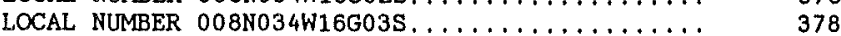

LOCAL NUMBER D08N034W17E01S $\ldots \ldots \ldots \ldots \ldots \ldots \ldots \ldots$

LOCAL NUMBER 008 N034W17G01S................. 379

LOCAL NUMBER 008N034W17H01S.

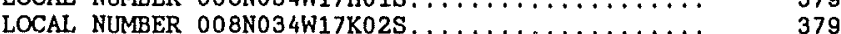

LOCAL NUMBER $008 N 034$ W17Q01S...................

LOCAL NUMBER O08N034W21A01S. . . . .

LOCAL NUMBER 008N034W22F01S............. 380

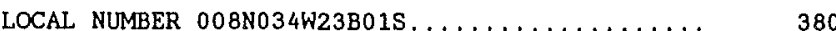

LOCAL NUMBER D08N034W24E01S . . . . . . . . . . . 380

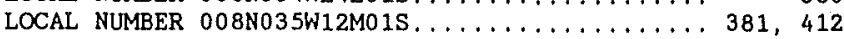

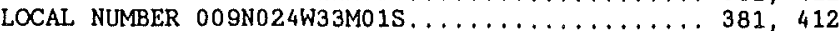

LOCAL NUMBER 009N025W13B01S............. 381

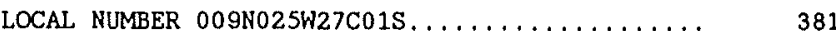

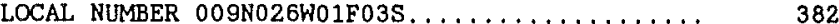

LOCAL NUMBER OOSN032W06D01S.................

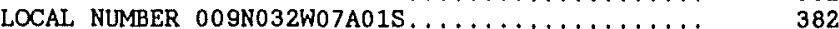

LOCAL NUMBER DOSN032W07Q01S.............. 382

LOCAL NUMBER OOSNO32WOBNO1S.............. 383

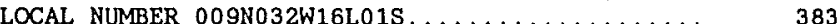

LOCAL NUMBER 009 N032W17G01S................ 383

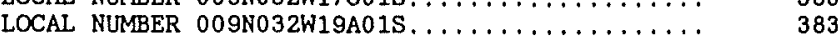

LOCAL NUMBER 009N032W20E01S.............. 384

LOCAL NUMBER 009N032W2ZD01S. . . . . . . . . . . 384

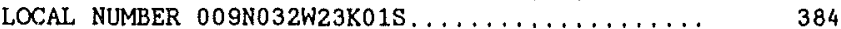

LOCAL NUMBER 009N032W32K01S.............. 384

LOCAL NUMBER OOSN032W33M01S.................

LOCAL NUMBER 009N033W02A07S ............. 385

LOCAL NUMBER 009N033W12C01S............

LOCAL NUMBER 009 N033W24L015. $\ldots \ldots \ldots \ldots \ldots \ldots \ldots . \ldots$

LOCAL NUMBER O09N034W03A02S............. 386

LOCAL NUMBER 009 N034W03F01S................. 386

LOCAL NUMBER 009 N034W06C01S ............. 386

LOCAL NUMBER 009N034W06K02S............ 386

LOCAL NUMBER 009 N034W08H01s.............. 387

LOCAL NUMBER 009 N034W09R01S . . . . . . . . . . . . . . 387

LOCAL NUMBER $009 N 034$ W14D01S................. 387

LOCAL NUMBER 009N034W34P01S.............. 387

LOCAL NUMBER 010N025W29K02S............ 388

LOCAL NUMBER 010 N025W30F01S................. 388

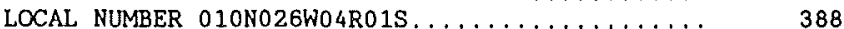

LOCAL NUMBER O1ON026W09R03S................. 412

LOCAL NUMBER O1ON026W15N01S................. 388

LOCAL NUMBER 010 N026W16001S.............. 389

LOCAL NUMBER 010N026W18F01S.............. 389

LOCAL NUMBER 010N026W20M01S................. 389

LOCAL NUMBER 010 N026W21G01S.................. 412

LOCAL, NUMBER 010 N027W11A03S................ 389 


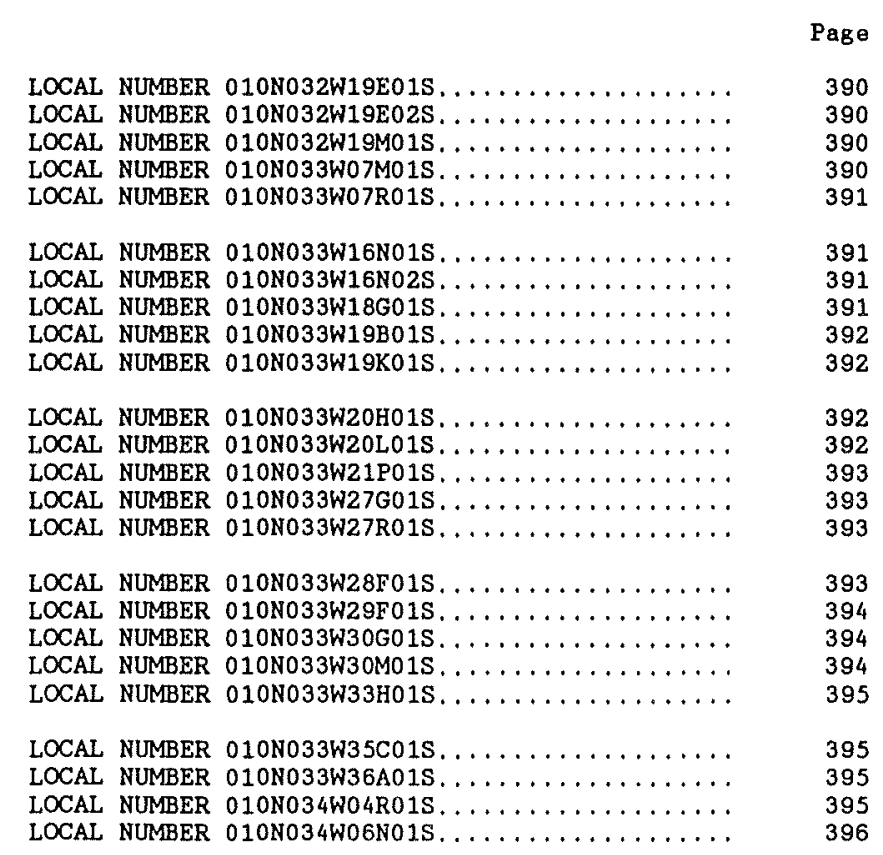

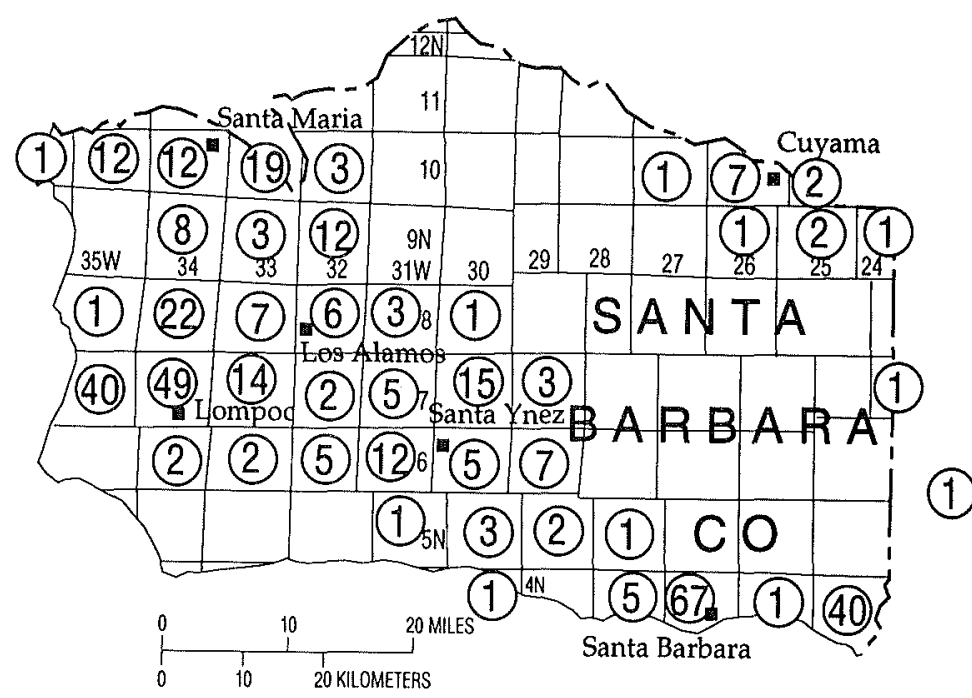

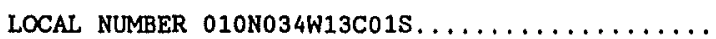
LOCAL NUMBER 010N034W13G015.............

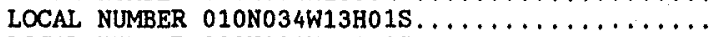

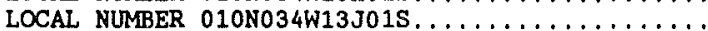

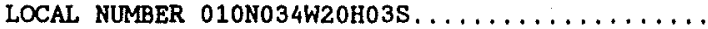

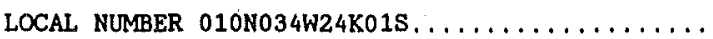

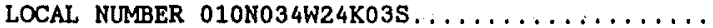
LOCAL NUMBER 010 N034W26H02S.............. 398,

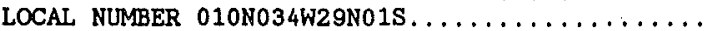
LOCAL NUMBER 010N034W31L02S, . . . . . . . . .

LOCAL NUMBER 010N035W09E05S.

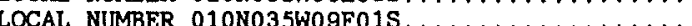

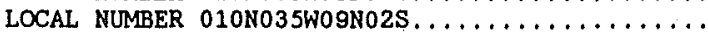

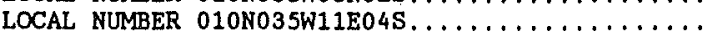

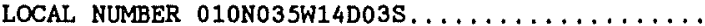

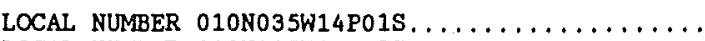

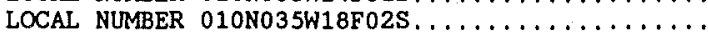

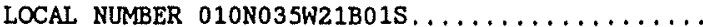

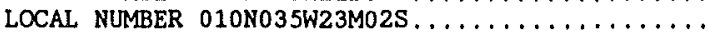

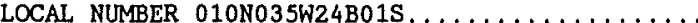

LOCAL NUMBER 010N035W24Q01S. . . . . . . . . . .

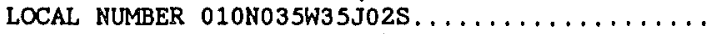

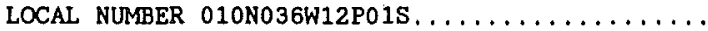

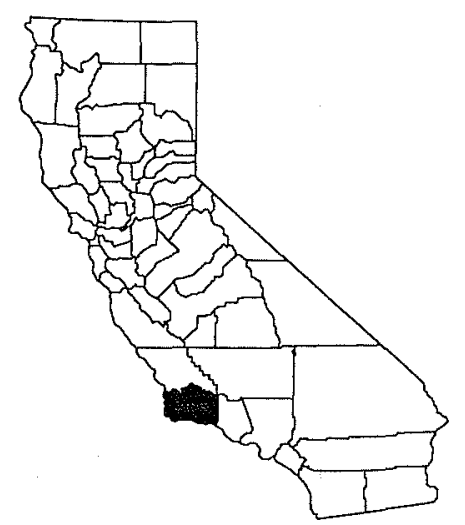

(1) Number in circle indicates number of wells in township for which data are included in this report

Figure 30. Location of wells in Santa Barbara County. 
WELL DESCRIPTIONS AND WATER-LEVEL MEASUREMENTS

WATER YEAR OCTOBER 1991 TO SEPTEMBER 1992

SITE NUMBER 342501119323101

LOCAL NUMBER 004N025W19F04S

In Carpinteria about $1.2 \mathrm{miles}$ east of Nidever Road and north of Foothill Road. Drilled unused water-table well. Diameter 8 inches, depth 250 feet in 1941, depth measured 197.2 feet in 1990. Alt1tude of land-surface datum 106 feet. Measurements provided by Carpinteria County Water District beginning in January 1978 . Water-level records available 1941 to current year.

WATER LEVELS IN FEET BELOW LAND-SURFACE DATUM

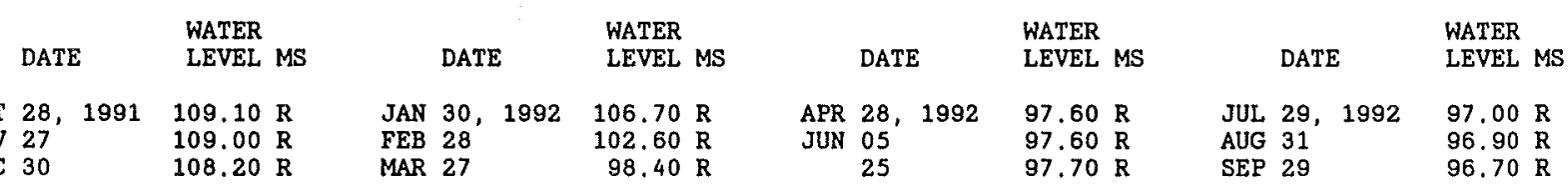

PERIOD OF RECORD HIGHEST 52.93 MAR 22, 1983 LOWEST 123.40 MAR 22, 1950

SITE NUMBER 342503119320101

LOCAL NUMBER 004N025W19H01S

In Carpinteria, east of Cravens Lane, and north of Foothill Road. Drilled unused water-table well. Diameter 10 inches, depth 360 feet in 1949, depth measured 237.6 feet in 1990. Altitude of land-surface datum 120 feet. Measurements provided by Carpinteria County Water District beginning in October 1977. Water-level records avallable 1948-50,1956,1972, 1977 to current year.

WATER LEVELS IN FEET BELOW LAND-SURFACE DATUM

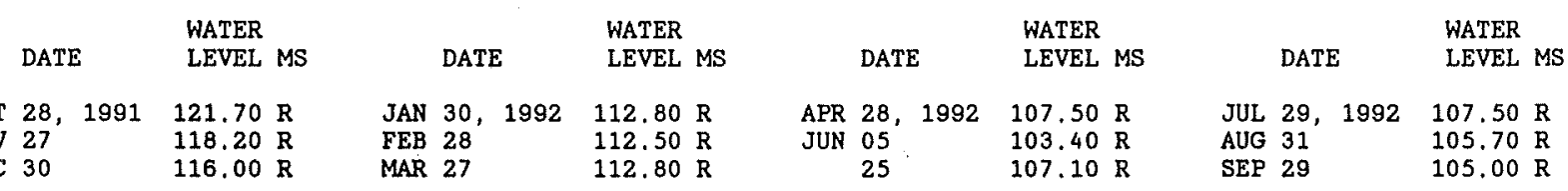

PERIOD OF RECORD HIGHEST 62.74 JUN 16,1983 LOWEST 122.70 OCT 30,1990

SITE NUMBER 342446119315701

LOCAL NUMBER 004N025W19J05S

In Carpinteria, south of Foothill Road, and east of Cravens Lane. Drilled unused well. Diameter 8 inches, depth 100 feet. Altitude of land-surface datum 55 feet. Measurements provided by Carpinteria County Water District beginning in August 1978. Water-level records available 1941-74, 1978 to current year.

WATER LEVELS IN FEET BELOW LAND-SURFACE DATUM

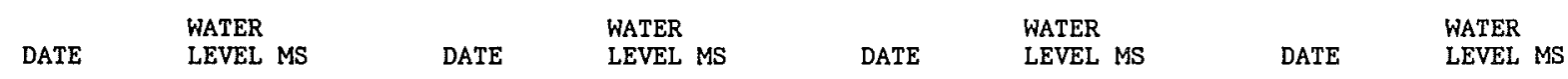

$\begin{array}{lllllllllll}\text { OCT 29, } 1991 & 81.70 \mathrm{R} & \text { JAN 30, } 1992 & 76.30 \mathrm{R} & \text { APR 28, } 1992 & 70.50 \mathrm{R} & \text { JUL } 29,1992 & 69.80 \mathrm{R} \\ \text { NOV 27 } & 80.30 \mathrm{R} & \text { FEB 28 } & 75.30 \mathrm{R} & \text { JUN } 05 & & 68.30 \mathrm{R} & \text { SEP } 01 & 68.20 \mathrm{R} \\ \text { DEC 31 } & 78.30 \mathrm{R} & \text { MAR 30 } & 73.50 \mathrm{R} & & 29 & 72.00 \mathrm{R} & & & \end{array}$

PERIOD OF RECORD HIGHEST 20.67 MAY 17, $1983 \quad$ LOWEST $92.95 \quad$ SEP 25, 1951 
SITE NUMBER 342445119320401

LOCAL NUMBER 004N025W19K05S

In Carpinteria, southeast of intersection of Foothi11 Road and Cravens Lane. Irrigation well. Diameter 4 inches, depth unknown. Altitude of land-surface datum 43 feet. Measurements provided by Carpinteria County Water District beginning in 1989. Water-level records avallable 1946, 1949, 1989 to current year.

WATER LEVELS IN FEET BELOW LAND-SURFACE DATUM

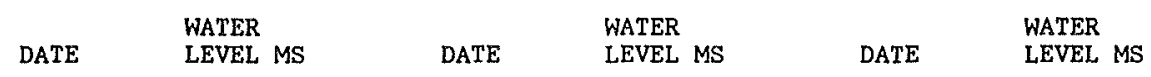

OCT 30, 1991 RP $\quad$ FEB 28, $1992 \quad 55.40 \mathrm{R} \quad$ JUN 25, $1992 \quad$ RP

$\begin{array}{llllllll}\text { NOV } 27 & & 60.50 \mathrm{R} & \text { MAR } 31 & 51.70 \mathrm{R} & \text { AUG } 31 & \text { RP } \\ \text { JAN } 31,1992 & & \text { RP } & \text { JUN } 09 & \text { RP } & \text { SEP } 30 & 47.60 \text { R }\end{array}$

PERIOD OF RECORD HIGHEST 12.00 APR $17,1946 \quad$ LOWEST 65.08 DEC 13,1949

SITE NUMBER 342448119323601

LOCAL NUMBER 004N025W19M03S

In Carpinteria, west of Cravens Lane, and south of Foothill Road. Drilled unused artesian well. Diameter 6 inches, depth reported 204 feet, depth measured 82.5 feet in 1990 . Altitude of land-surface datum 65 feet. Measurements provided by Carpinteria County Water District beginning in October 1977 . Water-level records available $1949-50,1956,1959,1977$ to current year.

WATER LEVELS IN FEET BELOW LAND-SURFACE DATUM

\begin{tabular}{|c|c|c|c|c|c|c|c|c|c|c|}
\hline DATE & $\begin{array}{l}\text { WATER } \\
\text { LEVEL MS }\end{array}$ & & DATE & $\begin{array}{l}\text { WATER } \\
\text { LEVEL MS }\end{array}$ & & DATE & $\begin{array}{l}\text { WATER } \\
\text { LEVEL MS }\end{array}$ & & DATE & $\begin{array}{l}\text { WATER } \\
\text { LEVEL MS }\end{array}$ \\
\hline $\begin{array}{ll}28, & 1991 \\
27 & \\
30 & \end{array}$ & $\begin{array}{l}78.10 \mathrm{R} \\
77.80 \mathrm{R} \\
76.70 \mathrm{R}\end{array}$ & $\begin{array}{l}\text { JAN } \\
\text { FEB } \\
\text { MAR }\end{array}$ & $\begin{array}{l}30,1992 \\
28 \\
27\end{array}$ & $\begin{array}{ll}74.70 & R \\
73.00 & R \\
70.80 & R\end{array}$ & $\begin{array}{l}\text { APR } \\
\text { JUN }\end{array}$ & $\begin{array}{ll}28, & 1992 \\
05 & \\
25 & \end{array}$ & $\begin{array}{ll}69.60 & R \\
68.00 & R \\
68.40 & R\end{array}$ & $\begin{array}{l}\text { JUL } \\
\text { AUG } \\
\text { SEP }\end{array}$ & $\begin{array}{ll}29, & 1992 \\
31 & \\
28 & \end{array}$ & $\begin{array}{ll}67.70 & R \\
67.80 & R \\
67.90 & R\end{array}$ \\
\hline
\end{tabular}

PERIOD OF RECORD HIGHEST 29.66 MAR 22,1983 LOWEST 88.28 DEC 14,1949

\section{SITE NUMBER 342440119310601}

LOCAL NUMBER 004N025W20K03S

In Carpinteria, west of Carpinteria High School. Drilled unused water-table well. Diameter 10 inches, depth 408 feet in 1949. Altitude of land-surface datum 45 feet. Measurements provided by Carpinteria County Water District beginning in October 1977. Water-level records avallable 1949-50, 1956, 1959, 1977 to current year.

WATER LEVELS IN FEET BELOW LAND-SURFACE DATUM

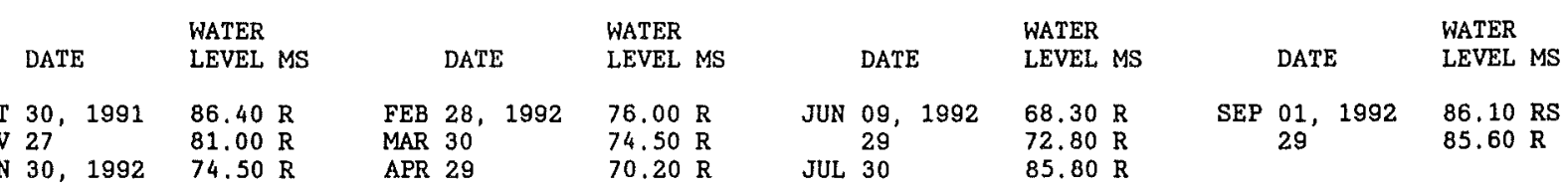

PERIOD OF RECORD HIGHEST 0.32 JUN 17,1983 LOWEST 95.00 JAN 29, 1991 
WELL DESCRIPTIONS AND WATER-LEVEL MEASUREMENTS

WATER YEAR OCTOBER 1991 TO SEPTEMBER 1992

SITE NUMBER 342438119310601

LOCAL NUMBER 004N025W20K04S

In Carpinteria, west of Carpinteria High School. Drilled public-supply well. Diameter 16 inches $0-353$ feet, 14 inches 353-903 feet, depth 903 feet, depth of hole 1,988 feet, screened 355-375, 395-415, 615-645, 705-725, 745-765, 825-885 feet. Altitude of land-surface datum 42.3 feet. Measurements provided by Carpinteria County Water District. Water-level records available 1989 to current year.

WATER LEVELS IN FEET BELOW LAND-SURFACE DATUM

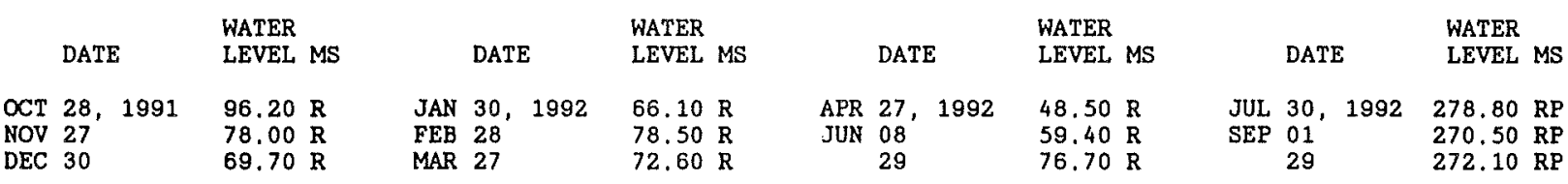

PERIOD OF RECORD HIGHEST 48.50 APR 27, 1992 LOWEST 295.71 FEB 26, 1991

SITE NUMBER 342451119313001

LOCAL NUMBER 004N025W2OL04S

In Carpinteria about 60 feet east of Santa Monica Creek and north of Foothill Road. Drilled unused water-table well. Diameter 10 inches, original depth 264 feet, depth measured 257.3 feet in 1990, perforated 62-254 feet. Altitude of land-surface datum 111 feet. Measurements provided by Carpinteria County Water District beginning in February 1977. Water-level records available 1947, 1949 to current year.

WATER LEVELS IN FEET BELOW LAND-SURFACE DATUM

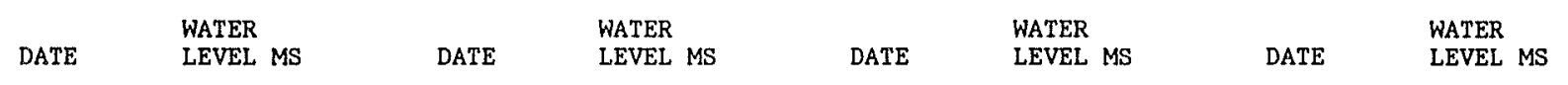

$\begin{array}{llllll}\text { OCT } 28,1991 & 147.20 \mathrm{R} & \text { JAN } 30,1992 & 138.50 \mathrm{R} \\ \text { NOV } 27 & & 146.10 \mathrm{R} & \text { FEB } 28 & & 137.90 \mathrm{R} \\ \text { DEC 30 } & 141.20 \mathrm{R} & \text { MAR } 26 & & 135.70 \mathrm{R}\end{array}$

$\begin{array}{lll}\text { APR } & 28,1992 & 133.10 \mathrm{R} \\ \text { JUN } & 05 & 128.00 \mathrm{R} \\ & 25 & 130.30 \mathrm{R}\end{array}$

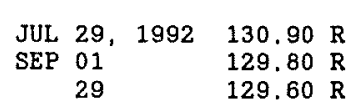

PERIOD OF RECORD HIGHEST 71.04 JUN 15, 1983 LOWEST 153.17 SEP 25, 1951

SITE NUMBER 342432119312101

LOCAL NUMBER 004N025W20PO1S

In Carpinteria. Drilled commercial well. Diameter 6 inches, depth reported 220 feet. Altitude of land-surface datum 54 feet. Measurements provided by Carpinteria County Water District beginning in 1990. Water-1evel records available 1941, 1945-46,1949-50,1954,1956,1959, 1990 to current year.

WATER LEVELS IN FEET BELOW LAND-SURFACE DATUM

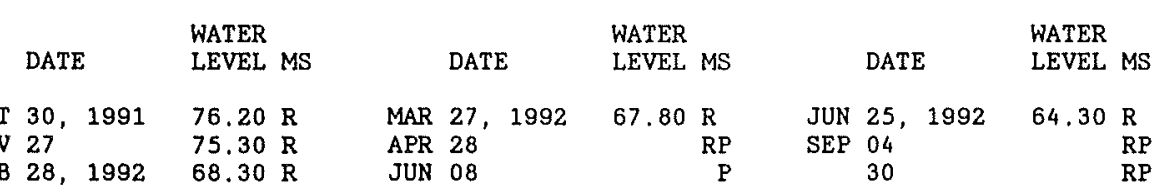

PERIOD OF RECORD HIGHEST 35.75 MAR 19, 1945 LOWEST 117.70 NOV 10, 1954 
WELL DESCRIPTIONS AND WATER-LEVEL MEASUREMENTS

WATER YEAR OCTOBER 1991 TO SEPTEMBER 1992

\section{SITE NUMBER 342428119304201}

LOCAL NUMBER 004N025W21N01S

In Carpinteria about 100 feet north of Foothill Road and 700 feet east of Linden Avenue. Drilled unused well. Diameter 12 and 6 inches, depth 405 feet. Altitude of land-surface datum 41 feet. Measurements provided by Carpinteria County Water District beginning in 1982, Water-level records available 1936, 1938, 1941-42, 1945-46, $1949-50,1959,1982$ to current year.

WATER LEVELS IN FEET BELOW LAND-SURFACE DATUM (READINGS ABOVE LAND SURFACE INDICATED BY "+")

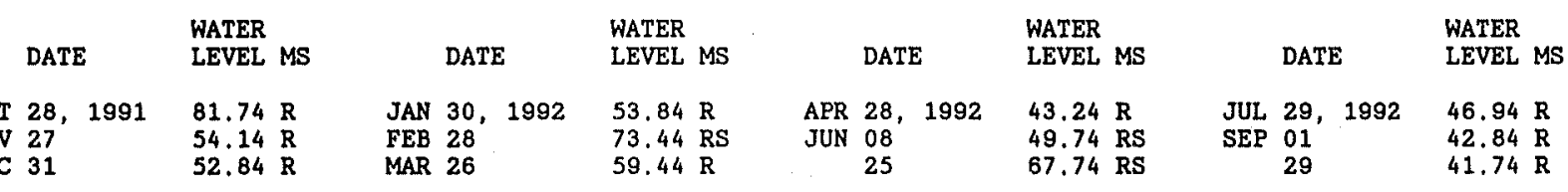

PERIOD OF RECORD HIGHEST +0.29 JUL 21, 1982 LOWEST 81.74 OCT 28,1991

\section{SITE NUMBER 342425119303302}

LOCAL NUMBER 004N025W21N04S

In Carpinteria. Drilled unused well. Diameter 10 inches, depth 406 feet. Altitude of land-surface datum 51 feet. Measurements provided by Carpinteria County Water District beginning in 1982. Water-level records available 1949, 1982 to current year.

WATER LEVELS IN FEET BELOW LAND-SURFACE DATUM

\begin{tabular}{|c|c|c|c|c|c|c|c|c|c|c|c|}
\hline DATE & $\begin{array}{l}\text { WATER } \\
\text { LEVEL MS }\end{array}$ & & DATE & $\begin{array}{l}\text { WATER } \\
\text { LEVEL MS }\end{array}$ & & DATE & & $\begin{array}{l}\text { WATER } \\
\text { LEVEL MS }\end{array}$ & & DATE & $\begin{array}{l}\text { WATER } \\
\text { LEVEL MS }\end{array}$ \\
\hline $\begin{array}{ll}30, & 1991 \\
27 & \\
30, & 1992\end{array}$ & $\begin{array}{ll}92.20 & R \\
70,20 & R \\
72.00 & R\end{array}$ & $\begin{array}{l}\text { FEB } \\
\text { MAR } \\
\text { APR }\end{array}$ & $\begin{array}{ll}28, & 1992 \\
30 & \\
28 & \end{array}$ & $\begin{array}{r}104.00 \mathrm{RS} \\
66.30 \mathrm{R} \\
53.30 \mathrm{R}\end{array}$ & $\begin{array}{l}\text { JUN } \\
\text { JUL }\end{array}$ & $\begin{array}{l}09 \\
29 \\
30\end{array}$ & 1992 & $\begin{array}{l}86.50 \mathrm{RS} \\
95.20 \mathrm{RS} \\
60.40 \mathrm{R}\end{array}$ & SEP & $\begin{array}{l}01, \quad 1992 \\
29\end{array}$ & $\begin{array}{l}55.20 \mathrm{R} \\
54.30 \mathrm{R}\end{array}$ \\
\hline ) & & & 0.22 & 17,1982 & LOWES & & 92.20 & Ост 30, & & & \\
\hline
\end{tabular}

SITE NUMBER 342427119294601

LOCAL NUMBER 004N025W21R01S

Northeast of Carpinteria. Drilled unused water-table well. Diameter 12 inches, depth 434 feet, depth of hole 468 feet, perforated $82-90,120-150,170-176,239-240,289-304,314-318,340-341,356-386,412-416$ feet. Alt1tude of land-surface datum 108 feet. Measurements provided by Carpinteria County Water District beginning in 1978. Water-level records avallable 1941 to current year.

WATER LEVELS IN FEET BELOW LAND-SURFACE DATUM

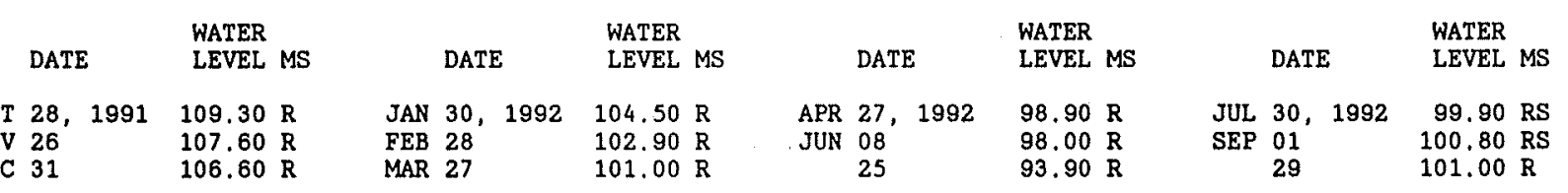

PERIOD OF RECORD HIGHEST 30.28 FEB 17, 1984 LOWEST 126.08 NOV 26, 1951 
WATER LEVELS, SANTA BARBARA COUNTY--Continued

WELL DESCRIPTIONS AND WATER-LEVEL MEASUREMENTS

WATER YEAR OCTOBER 1991 TO SEPTEMBER 1992

SITE NUMBER 342430119284901

LOCAL NUMBER 004N025W22R03S

East of Carpinteria, west of Lillingston Canyon Road. Irrigation well. Diameter 10 inches, depth 264 feet, perforated 96-264 feet. Altitude of land-surface datum 212 feet. Measurements provided by Carpinteria County Water District beginning in 1978. Water-level records available 1948-49, 1954, 1956, 1978 to current year.

WATER LEVELS IN FEET BELOW LAND-SURFACE DATUM

\begin{tabular}{|c|c|c|c|c|c|c|c|c|c|}
\hline DATE & $\begin{array}{l}\text { WATER } \\
\text { LEVEL MS }\end{array}$ & DATE & $\begin{array}{l}\text { WATER } \\
\text { LEVEL MS }\end{array}$ & & DATE & $\begin{array}{l}\text { WATER } \\
\text { LEVEL }\end{array}$ & MS & DATE & $\begin{array}{l}\text { WATER } \\
\text { LEVEL MS }\end{array}$ \\
\hline $\begin{array}{ll}28, & 1991 \\
26 & \\
02, & 1992 \\
29 & \end{array}$ & $\begin{array}{rl} & R P \\
& R P \\
69.20 & R \\
59.80 & R\end{array}$ & $\begin{array}{lll}\text { JAN } & 30, & 1992 \\
\text { FEB } & 28 & \\
\text { MAR } & 30 & \\
\text { APR } & 28 & \end{array}$ & $\begin{array}{ll}54.50 & R \\
45.60 & R \\
35.90 & R \\
& R P\end{array}$ & $\begin{array}{l}\text { JUN } \\
\text { JUL } \\
\text { SEP }\end{array}$ & $\begin{array}{ll}08, & 1992 \\
25 & \\
30 & \\
01 & \end{array}$ & $46.00 \begin{array}{l}R \\
R \\
R \\
R\end{array}$ & $\begin{array}{l}\text { RP } \\
\text { RP } \\
\text { RS } \\
\text { RP }\end{array}$ & SEP 29, 1992 & $57.30 \mathrm{RS}$ \\
\hline
\end{tabular}

PERIOD OF RECORD HIGHEST 11.98 APR 19, 1983 LOWEST 162.90 NOV 10, 1954

SITE NUMBER 342430119284701

LOCAL NUMBER 004N025W22R04S

East of Carpinteria, east of Lillingston Canyon Road. Drilled domestic water-table well. Diameter 10 inches, depth reported 504 feet, perforated 192-504 feet. Altitude of land-surface datum 218 feet. Measurements provided by Carpinteria County Water District beginning in 1981. Water-level records available 1949, 1950, 1956,1981 to current year.

WATER LEVELS IN FEET BELOW LAND-SURFACE DATUM

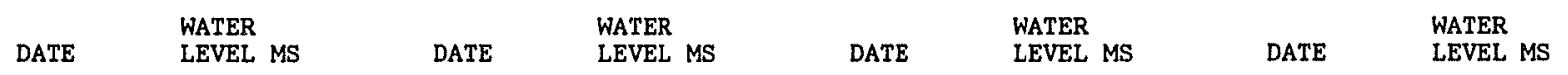

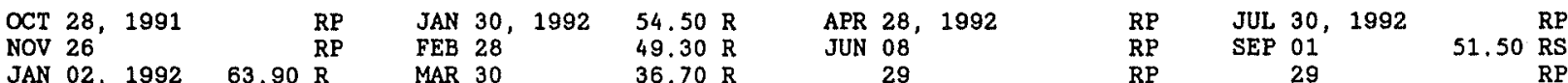

JAN $02,199263,90 \mathrm{R}$

LOWEST 97.43 NOV 24,1950

SITE NUMBER 342427119281701

LOCAL NUMBER 004N025W23P01S

East of Carpinteria. Drilled unused well. Diameter 10 inches, depth reported 465 feet in 1944 . Altitude of land-surface datum 480 feet. Measurements provided by Carpinteria County Water District beginning in 1977. Water-level records available 1945-46, 1948-50,1954-55, 1959, 1977 to current year.

WATER LEVELS IN FEET BELOW LAND-SURFACE DÁTUM

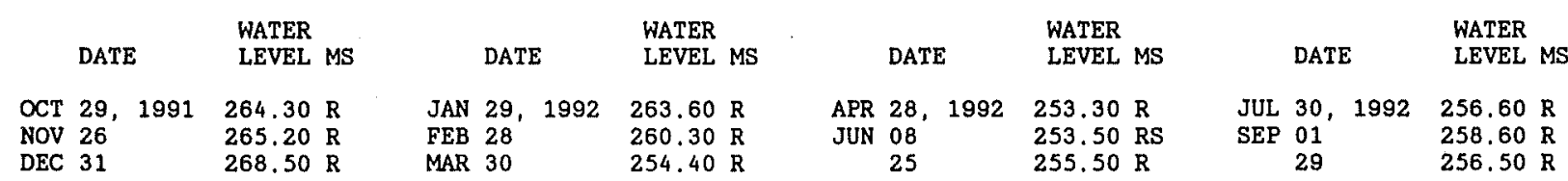

PERIOD OF RECORD HIGHEST 204.85 JAN 17, 1984 LOWEST 341.80 DEC 14, 1949 
LOCAL NUMBER 004N025W26A01S

East of Carpinteria, north of Shepherd Road, and west of Highway 150. Drilled unused well. Diameter 10 inches, depth 480 feet, perforated 228-480 feet. Altitude of land-surface datum 420 feet. Measurements provided by Carpinteria County Water District beginning in 1978. Water-level records available 1941, 1944 to current year.

WATER LEVELS IN FEET BELOW LAND-SURFACE DATUM

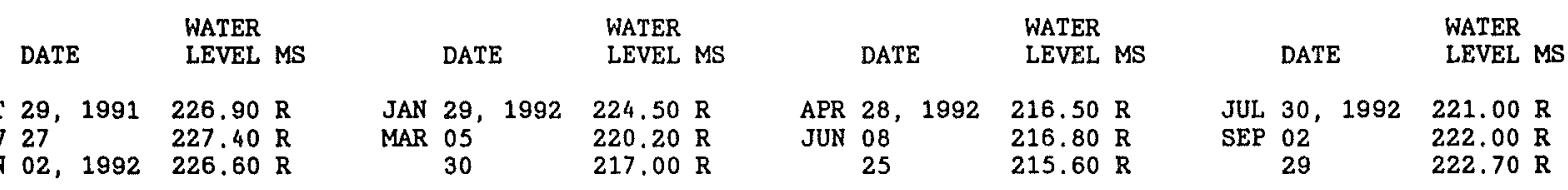

PERIOD OF RECORD HIGHEST 174.44 JAN 17, 1984 LOWEST 387.00 NOV 28, 1955

SITE NUMBER 342409119275001

LOCAL NUMBER 004N025W26B01S

East of Carpinteria, south of Gobernador Road. Drilled unused well. Diameter 14 inches, depth 552 feet, perforated 240-552 feet. Altitude of land-surface datum 455 feet. Measurements provided by Carpinteria County Water District beginning in 1987. Water-level records available 1944-46, 1987 to current year.

WATER LEVELS IN FEET BELOW LAND-SURFACE DATUM

$\begin{array}{lllll} & \text { WATER } & \text { WATER } & \text { WATER } \\ \text { DATE } & \text { LEVEL MS } & \text { DATE } & \text { LEVEL MS } & \text { DATE }\end{array}$

$\begin{array}{llllll}\text { NOV 27, } 1991 & \text { RP } & \text { JUN 25, } 1992 & \text { RP } & \text { SEP 02, } 1992 & \text { RP } \\ \text { JUN 08, 1992 } & \text { RP } & \text { JUL 30 } & \text { RP } & & \end{array}$

SEP 021,1992200

PERIOD OF RECORD HIGHEST 109.21 JUL 25, 1945 LOWEST 260.35 DEC 16, 1944

SITE NUMBER 342407119291801

LOCAL NUMBER 004N025W27F01S

East of Carpinteria on Casitas Pass Road. Drilled irrigation well. Diameter 12 inches, depth drilled 543 feet. Altitude of land-surface datum 121 feet. Measurements provided by Carpinteria County Water District beginning in 1990. Water-level records available $1934,1937-38,1941,1950,1990$ to current year.

WATER LEVELS IN FEET BELOW LAND-SURFACE DATUM

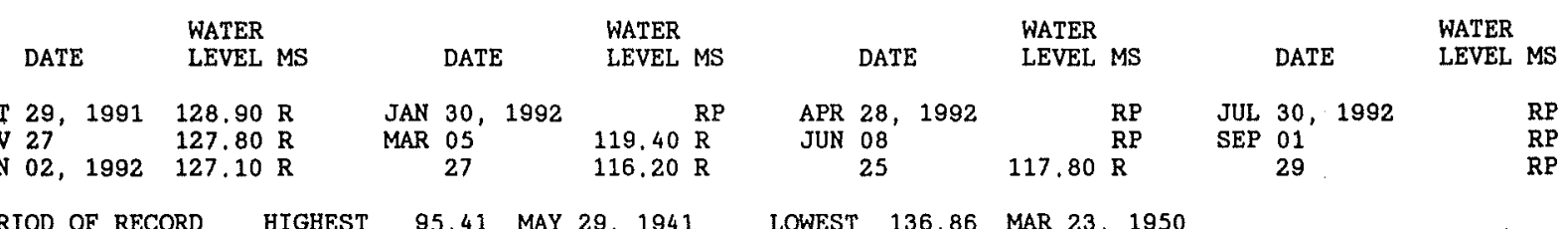

PERIOD OF RECORD HIGHEST 95.41 MAY 29, 1941 LOWEST 136.86 MAR 23, 1950 
WELL DESCRIPTIONS AND WATER-LEVEL MEASUREMENTS

WATER YEAR OCTOBER 1991 TO SEPTEMBER 1992

\begin{abstract}
SITE NUMBER 342404119291201
\end{abstract}
LOCAL NUMBER 004N025W27F02S

In Carpinteria, south of Lillingston Canyon Road, and west of Casitas Pass Road. Drilled public-supply well. Diameter 10 inches to 451 feet, 14 inches to 825 feet, depth 825 feet, perforated $455-801$ feet. Altitude of land-surface datum 133 feet. Measurements provided by Carpinteria County Water District beginning in 1977. Water-level records available 1975, 1977 to current year.

WATER LEVELS IN FEET BELOW LAND-SURFACE DATUM

\begin{tabular}{|c|c|c|c|c|c|c|c|c|c|c|c|}
\hline DATE & $\begin{array}{l}\text { WATER } \\
\text { LEVEL }\end{array}$ & MS & & DATE & $\begin{array}{l}\text { WATER } \\
\text { LEVEL MS }\end{array}$ & & DATE & $\begin{array}{l}\text { WATER } \\
\text { LEVEL MS }\end{array}$ & & DATE & $\begin{array}{l}\text { WATER } \\
\text { LEVEL MS }\end{array}$ \\
\hline $\begin{array}{ll}29, & 1991 \\
26 & \\
02, & 1992\end{array}$ & 155.67 & $\begin{array}{l}R \\
R P \\
R P\end{array}$ & $\begin{array}{l}\text { JAN } \\
\text { MAR }\end{array}$ & $\begin{array}{ll}30, & 1992 \\
05 & \\
30 & \end{array}$ & $\begin{array}{l}142.37 \mathrm{RS} \\
136.77 \mathrm{R} \\
134.67 \mathrm{R}\end{array}$ & $\begin{array}{l}\text { APR } \\
\text { JUN }\end{array}$ & $\begin{array}{ll}27, & 1992 \\
03 & \\
25 & \end{array}$ & $\begin{array}{rl}125.97 & \mathrm{R} \\
& \mathrm{RP} \\
126.57 & \mathrm{R}\end{array}$ & $\begin{array}{l}\text { JUL } \\
\text { SEP }\end{array}$ & $\begin{array}{ll}30, & 1992 \\
01 & \\
29 & \end{array}$ & $\mathrm{RI}$ \\
\hline
\end{tabular}

PERIOD OF RECORD HIGHEST 31.57 MAR 14, 1984 LOWEST 155.67 OCT 29, 1991

\title{
SITE NUMBER 342400119285401
}

LOCAL NUMBER 004N025W27G01S

In Carpinteria, north of Casitas Pass Road, and south of Gobernador Creek. Drilled irrigation well. Diameter unknown, depth reported 600 feet. Altitude of land-surface datum 174 feet. Measurements provided by Carpinteria County Water District beginning in 1977. Water-level records available 1941, 1949, 1977 to current year.

WATER LEVELS IN FEET BELOW LAND-SURFACE DATUM

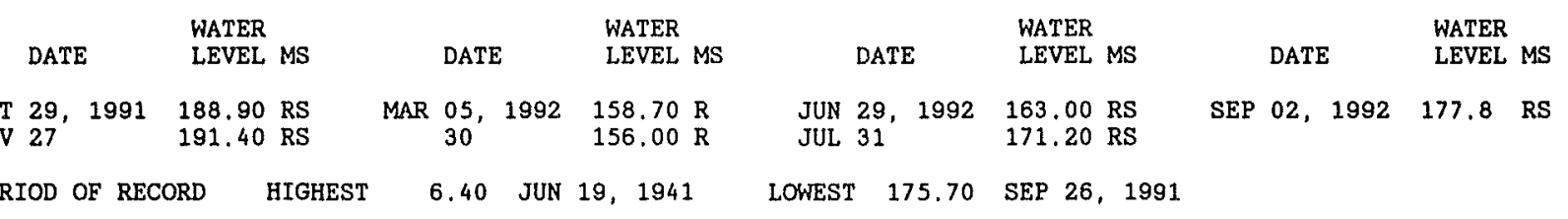

SITE NUMBER 342341119290801

LOCAL NUMBER 004N025W27Q06S

East of Carpinteria, south of Casitas Pass Road. Drilled irrigation well. Diameter 10 inches, depth 580 feet, perforated 100-580 feet. Altitude of land-surface datum 130 feet. Measurements provided by Carpinteria County Water District beginning in 1989. Water-level records available 1949, 1989 to current year.

WATER LEVELS IN FEET BELOW LAND-SURFACE DATUM

\begin{tabular}{|c|c|c|c|c|c|c|c|c|c|c|}
\hline DATE & $\begin{array}{l}\text { WATER } \\
\text { LEVEL MS }\end{array}$ & & DATE & $\begin{array}{l}\text { WATER } \\
\text { LEVEL MS }\end{array}$ & & DATE & $\begin{array}{l}\text { WATER } \\
\text { LEVEL MS }\end{array}$ & & DATE & $\begin{array}{l}\text { WATER } \\
\text { LEVEL MS }\end{array}$ \\
\hline $\begin{array}{ll}29, & 1991 \\
27 & \\
02, & 1992\end{array}$ & $\begin{array}{rl}140.20 & R \\
& R P \\
140.60 & R\end{array}$ & $\begin{array}{l}\text { JAN } \\
\text { MAR }\end{array}$ & $\begin{array}{ll}30, & 1992 \\
05 & \\
30 & \end{array}$ & $\begin{array}{l}136.90 \mathrm{R} \\
132.10 \mathrm{R} \\
129.50 \mathrm{R}\end{array}$ & $\begin{array}{l}\text { APR } \\
\text { JUN }\end{array}$ & $\begin{array}{ll}28, & 1992 \\
08 & \\
25 & \end{array}$ & $130.50 \stackrel{R}{R P}$ & $\begin{array}{l}\text { JUL } \\
\text { SEP }\end{array}$ & $\begin{array}{ll}30, & 1992 \\
01 & \\
29 & \end{array}$ & $\begin{array}{l}\text { RE } \\
\text { RE } \\
\text { RF }\end{array}$ \\
\hline
\end{tabular}

PERIOD OF RECORD HIGHEST 108.50 JUL 28,1989 LOWEST 140.60 JAN 02, 1992 
SITE NUMBER 342339119284301

LOCAL NUMBER 004N025W27R02S

In Carpinteria. Drilled irrigation well. Diameter 12 inches, depth 421 feet, perforated 295-310, 350-378, 392-420 feet. Altitude of land-surface datum 132 feet. Measurements provided by Carpinteria County Water District beginning in 1977. Water-level records available 1941 to current year.

WATER LEVELS IN FEET BELOW LAND-SURFACE DATUM

\begin{tabular}{|c|c|c|c|c|c|c|c|c|c|c|c|}
\hline DATE & $\begin{array}{l}\text { WATER } \\
\text { LEVEL MS }\end{array}$ & & DATE & $\begin{array}{l}\text { WATER } \\
\text { LEVEL MS }\end{array}$ & & DATE & $\begin{array}{l}\text { WATER } \\
\text { LEVEL }\end{array}$ & MS & & DATE & $\begin{array}{l}\text { WATER } \\
\text { LEVEL MS }\end{array}$ \\
\hline $\begin{array}{ll}30, & 1991 \\
26 & \\
02, & 1992\end{array}$ & $\begin{array}{l}130.30 \mathrm{R} \\
131.10 \mathrm{R} \\
129.30 \mathrm{R}\end{array}$ & $\begin{array}{l}\text { JAN } \\
\text { MAR }\end{array}$ & $\begin{array}{ll}30, & 1992 \\
05 & \\
30 & \end{array}$ & $\begin{array}{l}128.10 \mathrm{R} \\
125.60 \mathrm{R} \\
123.50 \mathrm{R}\end{array}$ & $\begin{array}{l}\text { APR } \\
\text { JUN }\end{array}$ & $\begin{array}{ll}28, & 1992 \\
08 & \\
25 & \end{array}$ & 129.40 & $\begin{array}{l}\mathbf{R P} \\
\mathbf{R} \\
\mathbf{R P}\end{array}$ & $\begin{array}{l}\text { JUL } \\
\text { SEP }\end{array}$ & $\begin{array}{ll}30, & 1992 \\
01 & \\
29 & \end{array}$ & $\begin{array}{r}125.70 \mathrm{R} \\
131.30 \mathrm{R} \\
\mathrm{R}\end{array}$ \\
\hline
\end{tabular}

PERIOD OF RECORD HIGHEST 32.70 JAN 18, 1984 LOWEST 182.23 SEP 25, 1951

SITE NUMBER 342422119303701

LOCAL NUMBER 004N025W28D02S

In Carpinteria in EI Carro Park, south of Foothill Road. Drilled irrigation well. Diameter 16 inches 0-284 feet, 14 inches 284-1,214 feet, depth' drilled 2,706 feet, perforated 284-324, 854-914, 1,094-1, 194 feet. Altitude of land-surface datum 60 feet. Measurements provided by Carpinteria County Water District beginning in 1990 . Water-level records available 1990 to current year.

WATER LEVELS IN FEET BELOW LAND-SURFACE DATUM

\begin{tabular}{|c|c|c|c|c|c|c|c|}
\hline DATE & $\begin{array}{l}\text { WATER } \\
\text { LEVEL MS }\end{array}$ & DATE & $\begin{array}{l}\text { WATER } \\
\text { LEVEL MS }\end{array}$ & DATE & $\begin{array}{l}\text { WATER } \\
\text { LEVEL MS }\end{array}$ & DATE & $\begin{array}{l}\text { WATER } \\
\text { LEVEL MS }\end{array}$ \\
\hline $\begin{array}{ll}31, & 1991 \\
27 & \\
31 & \end{array}$ & $\begin{array}{l}99.40 \mathrm{R} \\
75.60 \mathrm{R} \\
72.10 \mathrm{R}\end{array}$ & $\begin{array}{lll}\text { JAN } & 30, & 1992 \\
\text { FEB } 28 & \\
\text { MAR } 27 & \end{array}$ & $\begin{array}{rl}77.50 & \mathrm{R} \\
& \mathrm{RP} \\
79.70 & \mathrm{R}\end{array}$ & $\begin{array}{lll}\text { APR } 27, & 1992 \\
\text { JUN } 08 & \\
25 & \end{array}$ & $53.90 \begin{array}{r}\mathrm{R} \\
\mathrm{RP} \\
\mathrm{RP}\end{array}$ & $\begin{array}{lll}\text { JUL } & 30, & 1992 \\
\text { SEP } & 01 & \\
& 29 & \end{array}$ & $\begin{array}{l}63.80 \\
58.70 \\
58.30\end{array}$ \\
\hline
\end{tabular}

PERIOD OF RECORD HIGHEST 53.90 APR 27, 1992 LOWEST 225.36 FEB 26, 1991

SITE NUMBER 342409119302101

LOCAL NUMBER 004N025W28F07S

In Carpinteria on corner of Casitas Pass Road and Shemara Street. Drilled public-supply artesian well. Diameter 16 inches $0-290$ feet, 10 inches $290-1,245$ feet, depth 1,245 feet, depth of hole 1,271 feet, perforated $310-350$, 400-420, 890-930, 1,140-1,180,1,210-1,230 feet. Altitude of land-surface datum 63 feet. Measurements provided by Carpinteria County Water District. Water-level records available 1976 to current year.

WATER LEVELS IN FEET BELOW LAND-SURFACE DATUM

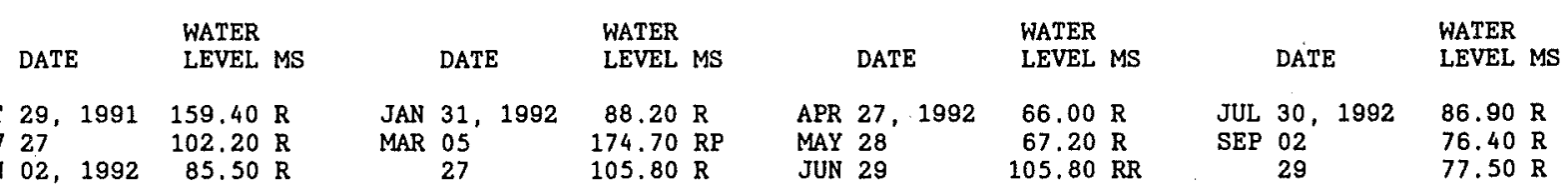

PERIOD OF RECORD HIGHEST 8.40 JAN 19, 1981 LOWEST 159.40 OCT 29, 1991 
WELL DESCRIPTIONS AND WATER-LEVEL MEASUREMENTS

WATER YEAR OCTOBER 1991 TO SEPTEMBER 1992

SITE NUMBER 342407119302201

LOCAL NUMBER 004N025W28F08S

In Carpinteria, south of intersection of Casitas Pass Road and Shemara Street. Drilled observation artesian well. Diameter 2 inches, depth 300 feet. Altitude of land-surface datum 62 feet. Measurements provided by Carpinteria County Water District. Water-level records available 1977-1992.

WATER LEVELS IN FEET BELOW LAND-SURFACE DATUM

\begin{tabular}{|c|c|c|c|c|c|}
\hline ATE & $\begin{array}{l}\text { WATER } \\
\text { LEVEL MS }\end{array}$ & DATE & $\begin{array}{l}\text { WATER } \\
\text { LEVEL MS }\end{array}$ & DATE & $\begin{array}{l}\text { WATER } \\
\text { LEVEL MS }\end{array}$ \\
\hline
\end{tabular}

OCT 30, $1991 \quad 70.20 \mathrm{R}$ NOV $27,1991 \quad 70.80 \mathrm{R} \quad$ JAN 31, $1992 \quad 71.70 \mathrm{R}$

PERIOD OF RECORD HIGHEST 1.00 FEB 18, MAR 17, APR 20, MAY 15, 1981 LOWEST 78.91 FEB 03, 1986

SITE NUMBER 342407119302202

LOCAL NUMBER O04N025W28FO9S

In Carpinteria, south of intersection of Casitas Pass Road and Shemara Street. Drilled observation artesian well. Diameter 2 inches, depth 900 feet. Altitude of land-surface datum 62 feet. Measurements provided by Caprinteria County Water District. Water-level records avallable 1977-1992.

WATER LEVELS IN FEET BELOW LAND-SURFACE DATUM

$\begin{array}{lllll}\text { DATE } & \text { WATER } & \text { WATER } & & \text { WATER } \\ \text { LEVEL MS } & \text { DATE } & \text { LEVEL MS } & \text { DATE } & \text { LEVEL MS }\end{array}$

OCT 30, $199181.10 \mathrm{R}$ NOV 27, $1991 \quad 82.20 \mathrm{R} \quad$ JAN 31, $1992 \quad 82.80 \mathrm{R}$

PERIOD OF RECORD HIGHEST 7.43 MAY 15, 1981 LOWEST 82.80 JAN 31, 1992

SITE NUMBER 342407119302203

LOCAL NUMBER 004N025W28F10S

In Carpinteria, south of intersection of Casitas Pass Road and Shemara Street. Drilled observation artesian well. Diameter 2 inches, depth 1,140 feet. Altitude of land-surface datum 62 feet. Measurements provided by Carpinteria County Water District. Water-level records available 1977-1992.

WATER LEVELS IN FEET BELOW LAND-SURFACE DATUM

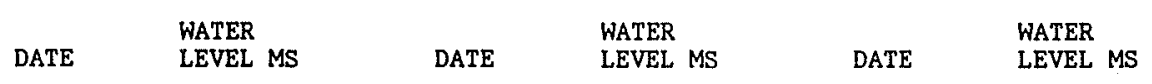

OCT 30, $1991 \quad 41.10 \mathrm{R}$ NOV 27, $1991 \quad 41.70 \mathrm{R} \quad$ JAN 31, $1992 \quad 42.60 \mathrm{R}$

PERIOD OF RECORD HIGHEST 1.78 MAR 17, 1982 LOWEST 42.60 JAN 31,1992

SITE NUMBER 342357119295501

LOCAL NUMBER 004N025W28J01S

East of Carpinteria. Drilled irrigation well. Diameter 12 inches, depth 175 feet. Altitude of land-surface datum 89 feet. Measurements provided by Carpinteria County Water District beginning in 1977. Water-level records available 1919,1930,1937-38,1940-50, 1952 to current year.

WATER LEVELS IN FEET BELOW LAND-SURFACE DATUM

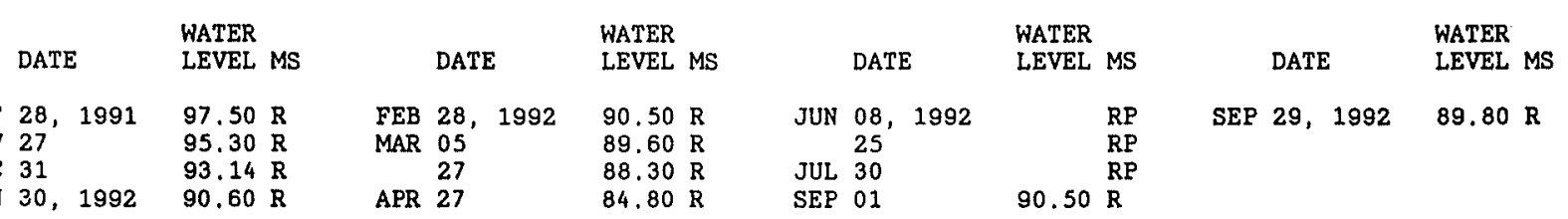

PERIOD OF RECORD HIGHEST 14.44 JAN 16, 1984 LOWEST 138.20 OCT 24,1960 


\section{SITE NUMBER 342354119303001}

LOCAL NUMBER 004N025W28M01S

In Carpinteria about 1,700 feet northeast of Highway 101 and 200 feet northwest of Casitas Pass Road. Drilled observation artesian well. Diameter 2 inches, original depth 151.7 feet, depth measured 48.53 feet in 1990. Altitude of land-surface datum 53 feet. Measurements provided by Carpinteria County Water District beginning in 1978. Water-level records available 1941 to current year.

WATER LEVELS IN FEET BELOW LAND-SURFACE DATUM (READINGS ABOVE LAND SURFACE INDICATED BY "+")

$\begin{array}{llllll} & \text { WATER } & & & \text { WATER } \\ \text { DATE } & \text { LEVEL MS } & \text { DATE } & \text { WATER } & \text { DATER } & \text { LS } \\ \text { LEVEL MS }\end{array}$

OCT 30, 1991

NOV 27

DEC 31

\begin{abstract}
JAN 31, 1992
FEB 28
\end{abstract}

MAR 30
LEVEL MS

RO
RO
O

$\begin{array}{lll}\text { APR } & 28,1992 \\ \text { JUN } & 09\end{array}$

29
$46.08 \mathrm{R}$

RO
RO

JUL 31,1992

SEP 02
30
RO

RO

PERIOD OF RECORD HIGHEST to.75 MAR 25, $1986 \quad$ LOWEST $95.61 \quad$ OCT 31,1951

\section{SITE NUMBER 342418119314601}

LOCAL NUMBER 004N025W29D01S

In Carpinteria about 1 mile north of Highway 101 on west side of Santa Monica Road. Drilled unused artesian well. Diameter 12 inches, original depth 147 feet, depth measured 105.45 feet in 1990 . Altitude of land-surface datum 17 feet. Measurements provided by Carpinteria County Water District beginning in February 1977 . Water-level records available 1938,1941 to current year.

WATER LEVELS IN FEET BELOW LAND-SURFACE DATUM (READINGS ABOVE LAND SURFACE INDICATED BY "+")

\begin{tabular}{|c|c|c|c|c|c|c|c|}
\hline & WATER & & WATER & & WATER & & WATER \\
\hline DATE & LEVEL MS & DATE & LEVEL MS & DATE & LEVEL MS & DATE & LEVEL MS \\
\hline
\end{tabular}

\begin{tabular}{|c|c|c|c|c|c|c|c|c|c|c|c|c|}
\hline $\begin{array}{ll}\text { OCT } & 28, \\
\text { NOV } & 27 \\
\text { JAN } & 30,\end{array}$ & 1991 & $\begin{array}{l}44.15 \mathrm{R} \\
41.85 \mathrm{R} \\
37.25 \mathrm{RS}\end{array}$ & $\begin{array}{ll}\text { FEB } & 28, \\
\text { MAR } & 27 \\
\text { APR } & 28\end{array}$ & 1992 & $\begin{array}{l}37.05 \mathrm{RS} \\
36.05 \mathrm{R} \\
31.35 \mathrm{R}\end{array}$ & $\begin{array}{l}\text { JUN } \\
\text { JUL }\end{array}$ & $\begin{array}{l}05 \\
25 \\
29\end{array}$ & 1992 & $\begin{array}{l}29.25 \mathrm{R} \\
32.55 \mathrm{RS} \\
31.05 \mathrm{R}\end{array}$ & $\begin{array}{l}\text { AUG } 31 \text {, } \\
\text { SEP } 28\end{array}$ & 1992 & $\begin{array}{l}28.85 \mathrm{R} \\
28.05 \mathrm{R}\end{array}$ \\
\hline
\end{tabular}

PERIOD OF RECORD HIGHEST +1.00 OCT 28,1969 MAR 26, 1970 LOWEST 57.28 SEP 25,1951

SITE NUMBER 342420119313801

LOCAL NUMBER 004N025W29D07S

In Carpinteria about $0.04 \mathrm{mile}$ north of Via Real and $0.1 \mathrm{mile}$ east of Santa Monica Road. Drilled public-supply artesian well. Diameter 14.5 inches, depth 950 feet, perforated 215-950 feet. Altitude of land-surface datum 25 feet. Measurements provided by Carpinteria County Water District. Water-level records available 1977 to current year.

WATER LEVELS IN FEET BELOW LAND-SURFACE DATUM (READINGS ABOVE LAND SURFACE INDICATED BY "+")

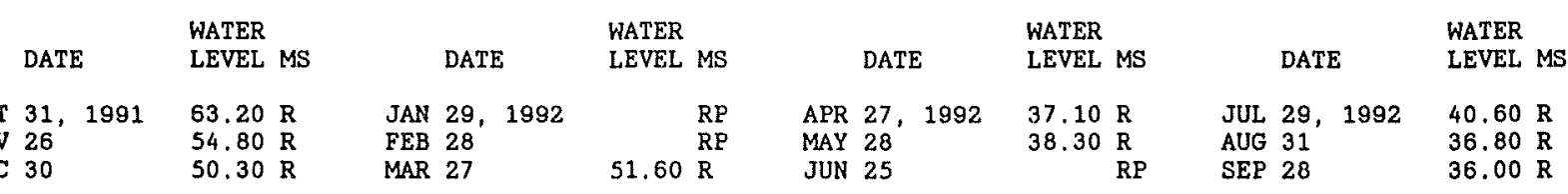

PERIOD OF RECORD HIGHEST +2.45 NOV $20,1979 \quad$ LOWEST 72.50 AUG 27,1991 
WELL DESCRIPTIONS AND WATER-LEVEL MEASUREMENTS

WATER YEAR OCTOBER 1991 TO SEPTEMBER 1992

\section{SITE NUMBER 342349119312801}

LOCAL NUMBER 004N025W29L01S

In Carpinteria near intersection of Holly Avenue and 3rd Street. Drilled unused artesian well. Diameter 2 inches, original depth 110 feet. Depth measured 106.1 feet in 1990 . Altitude of land-surface datum 18 feet. Measurements provided by Carpinteria County Water District beginning in January 1978. Water-level records available 1946, 1950-71, 1977 to current year.

WATER LEVELS IN FEET BELOW LAND-SURFACE DATUM (READINGS ABOVE LAND SURFACE INDICATED BY "+")

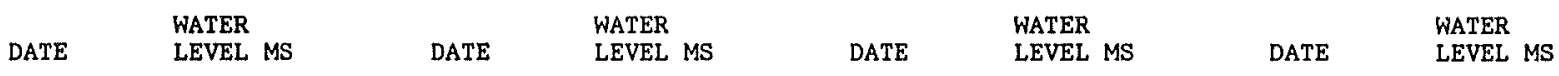

$\begin{array}{llllllllll}\text { OCT 28, } 1991 & 44.50 \mathrm{R} & \text { JAN 29, } 1992 & 32.20 \mathrm{R} & \text { APR 27, } 1992 & 15.90 \mathrm{R} & \text { JUL 29, } 1992 & 18.70 \mathrm{R} \\ \text { NOV 26 } & 31.40 \mathrm{R} & \text { FEB 28 } & 37.40 \mathrm{R} & \text { MAY 28 } & 17.60 \mathrm{R} & \text { AUG } 31 & 15.40 \mathrm{R} \\ \text { DEC 30 } & 27.90 \mathrm{R} & \text { MAR 26 } & 30.40 \mathrm{R} & \text { JUN } 25 & 33.90 \mathrm{R} & \text { SEP 28 } & 14.50 \mathrm{R}\end{array}$

PERIOD OF RECORD HIGHEST +1.60 OCT 28,1969 LOWEST 51.24 SEP 25, 1951

SITE NUMBER 342343119305201

LOCAL NUMBER 004N025W29R01S

In Carpinteria at Carpinteria Union Jr. High School, south of tennis court. Drilled unused artesian well. Diameter 10 inches, original depth 176 feet, depth measured 163.12 feet in 1990. Altitude of land-surface datum 32 feet. Measurements provided by Carpinteria County Water District beginning in January 1978. Water-level records available 1941 to current year.

WATER LEVELS IN FEET BELOW LAND-SURFACE DATUM (READINGS ABOVE LAND SURFACE INDICATED BY "+")

$\begin{array}{llllll} & \text { WATER } & \text { WATER } & \text { WATER } & \text { WATER } \\ \text { DATE } & \text { LEVEL MS } & \text { DATE } & \text { LEVEL MS } & \text { DATE } & \text { LEVEL MS }\end{array}$

$\begin{array}{lllllllllll}\text { OCT 29, } 1991 & 31.02 \mathrm{R} & \text { JAN 29, 1992 } & 23,52 \mathrm{R} & \text { APR 27, } 1992 & 20.02 \mathrm{R} & \text { JUL } 29,1992 & 23.52 \mathrm{R} \\ \text { NOV 26 } & 27.22 \mathrm{R} & \text { MAR } 05 & 24.42 \mathrm{R} & \text { JUN } 04 & 20.02 \mathrm{R} & \text { SEP } 01 & 21.82 \mathrm{R} \\ \text { DEC 30 } & 25.52 \mathrm{R} & & 30 & 24.12 \mathrm{R} & & 29 & 24.32 \mathrm{R} & & 29 & 21.67 \mathrm{R}\end{array}$

PERIOD OF RECORD HIGHEST to.28 AUG 15, 1985 LOWEST 73.90 SEP 21, 1961

SITE NUMBER 342419119323701

LOCAL NUMBER 004N025W30D01S

West of Carpinteria. Drilled unused artesian well. Diameter 10 inches, original depth 210 feet, depth measured 59.7 feet in 1990. Altitude of land-surface datum 7 feet. Measurements provided by Carpinteria County Water District beginning in January 1978. Water-level records available 1941, 1945, 1947-66, 1969-71, 1977 to current year.

WATER LEVELS IN FEET BELOW LAND-SURFACE DATUM (READINGS ABOVE LAND SURFACE INDICATED BY "+")

$\begin{array}{llllll} & \text { WATER } & \text { WATER } & \text { WATER } & \text { WATER } \\ \text { DATE } & \text { LEVEL MS } & \text { DATE } & \text { LEVEL MS } & \text { DATE } & \text { LEVEL MS }\end{array}$

$\begin{array}{llllllllrr}\text { OCT 28, } 1991 & 20.00 \mathrm{R} & \text { JAN 30, } 1992 & 15.80 \mathrm{R} & \text { APR 28, } 1992 & 12.50 \mathrm{R} & \text { JUL } 29,1992 & 11.20 \mathrm{R} \\ \text { NOV 27 } & 19.30 \mathrm{R} & \text { FEB 28 } & 15.60 \mathrm{R} & \text { JUN } 05 & 10.97 \mathrm{R} & \text { AUG } 31 & 10.80 \mathrm{R} \\ \text { DEC 30 } & 17.80 \mathrm{R} & \text { MAR 27 } & 14.60 \mathrm{R} & & 25 & 11.35 \mathrm{R} & \text { SEP 28 } & 9.82 \mathrm{R}\end{array}$


SITE NUMBER 342319119284901

LOCAL NUMBER 004N025W34A01S

About 2 miles east of Carpinteria. Drilled irrigation well. Diameter 10 inches, depth reported 250 feet, Altitude of land-surface datum 242 feet. Measurements provided by Carpinteria County Water District beginning in 1991. Water-level records available 1941, 1947, 1991 to current year.

WATER LEVELS IN FEET BELOW LAND-SURFACE DATUM

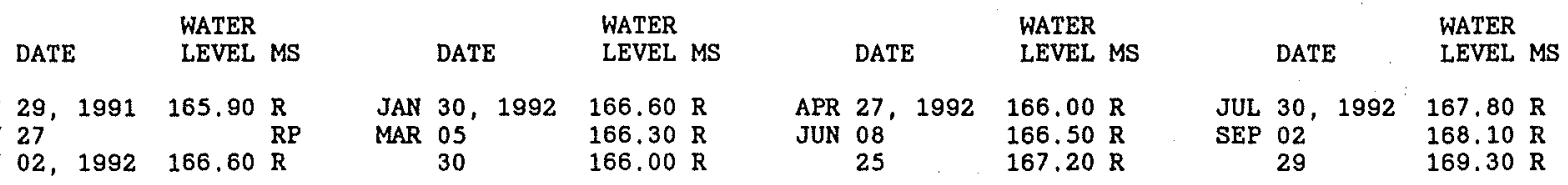

PERIOD OF RECORD HIGHEST 165.35 MAY 07, 1991 LOWEST 175.38 MAR 07, 1947

SITE NUMBER 342317119285601

LOCAL NUMBER 004N025W34G01S

In Carpinteria. Drilled observation well. Diameter 6 inches, depth 278 feet, perforated 25-45, 218-278 feet. Altitude of land-surface datum 188 feet. Measurements provided by Carpinteria County Water District. Water-1evel records available 1990 to current year.

WATER LEVELS IN FEET BELOW LAND-SURFACE DATUM

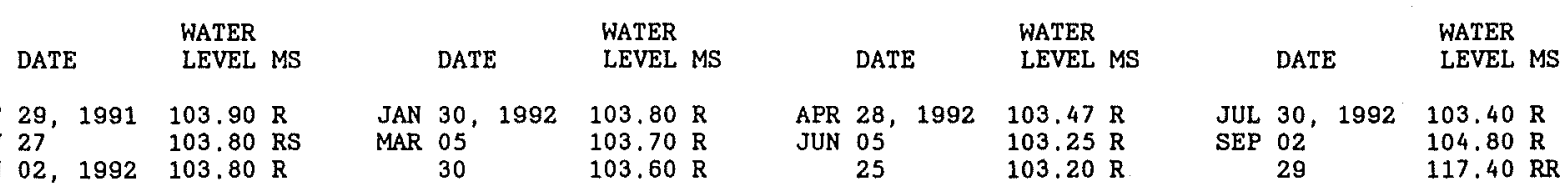

PERIOD OF RECORD HIGHEST 103.20 JUN 25, 1992 LOWEST 104.80 SEP 02, 1992

SITE NUMBER 342323119274601

LOCAL NUMBER 004N025W35A02S

About 3 miles east of Carpinteria. Drilled unused water-table well. Diameter 12 inches, depth measured 203.4 feet in 1990, original depth 403 feet, perforated 55-179, 231-401 feet. Altitude of land-surface datum 147 feet. Measurements provided by Carpinteria County Water District beginning in 1979. Water-level records available 1941, $1945,1949-50,1955-56,1979$ to current year.

WATER LEVELS IN FEET BELOW LAND-SURFACE DATUM

\begin{tabular}{|c|c|c|c|c|c|c|c|}
\hline DATE & $\begin{array}{l}\text { WATER } \\
\text { LEVEL MS }\end{array}$ & & DATE & $\begin{array}{l}\text { WATER } \\
\text { LEVEL MS }\end{array}$ & & DATE & $\begin{array}{l}\text { WATER } \\
\text { LEVEL MS }\end{array}$ \\
\hline $\begin{array}{ll}29, & 1991 \\
27 & \\
29, & 1992\end{array}$ & $\begin{array}{l}15.64 \mathrm{RS} \\
15.80 \mathrm{RS} \\
15.30 \mathrm{R}\end{array}$ & $\begin{array}{l}\text { MAR } \\
\text { JUN }\end{array}$ & $\begin{array}{ll}31, & 1992 \\
08 & \\
29 & \end{array}$ & $\begin{array}{l}13.48 \mathrm{R} \\
14.60 \mathrm{RS} \\
15.20 \mathrm{R}\end{array}$ & $\begin{array}{l}\text { JUL } \\
\text { SEP }\end{array}$ & $\begin{array}{ll}30, & 1992 \\
02 & \\
29 & \end{array}$ & $\begin{array}{l}13.90 \mathrm{R} \\
14.70 \mathrm{RS} \\
16.80 \mathrm{R}\end{array}$ \\
\hline
\end{tabular}

PERIOD OF RECORD HIGHEST 11.09 FEB 20, 1980 LOWEST 85.76 DEC 13, 1949 
East of Carpinteria, east of Rincon Creek Road, and south of Rincon Creek bridge. Drilled unused well. Diameter 10 inches, depth 145.8 feet in 1941. Altitude of land-surface datum 147 feet. Measurements provided by Carpinteria County Water District beginning in 1978. Water-level records available 1941, 1945-46, 1949-50, 1955-56, 1959 to current year.

WATER LEVELS IN FEET BELOW LAND-SURFACE DATUM

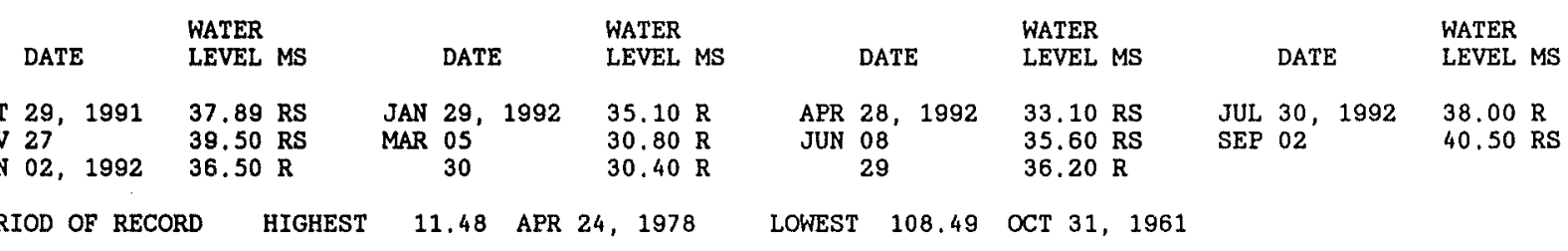

\section{SITE NUMBER 342315119282901}

LOCAL NUMBER 004N025W35E01S

About 2 miles southeast of Carpinteria. Drilled irrigation well, Diameter 10 inches, depth 420 feet, perforated 260-385 feet. Altitude of land-surface datum 249 feet. Measurements provided by Carpinteria County Water District beginning in 1977. Water-level records available 1939, 1941, 1949, 1956, 1977 to current year.

WATER LEVELS IN FEET BELOW LAND-SURFACE DATUM

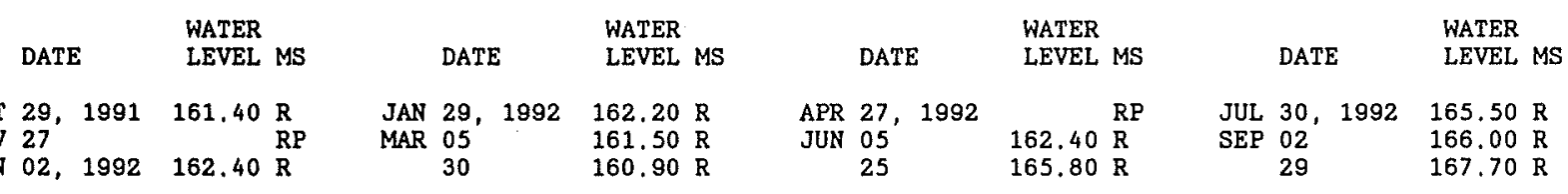

PERIOD OF RECORD HIGHEST 160.75 MAR 24, 1989 LOWEST $210.80 \quad$ DEC 19,1977

SITE NUMBER 342300119281901

LOCAL NUMBER 004N025W35MO1S

East of Carpinteria, east of Rincon Hill Road. Drilled irrigation well. Diameter 12 inches, depth reported 115 feet. Altitude of land-surface datum 85 feet. Measurements provided by Carpinteria County Water District

beginning in 1990. Water-level records available 1941, 1943-44, 1949-50,1955-56, 1959, 1990 to current year.

WATER LEVELS IN FEET BELOW LAND-SURFACE DATUM

\begin{tabular}{|c|c|c|c|c|c|c|c|}
\hline DATE & $\begin{array}{l}\text { WATER } \\
\text { LEVEL MS }\end{array}$ & DATE & $\begin{array}{l}\text { WATER } \\
\text { LEVEL MS }\end{array}$ & DATE & $\begin{array}{l}\text { WATER } \\
\text { LEVEL MS }\end{array}$ & DATE & WATER \\
\hline $\begin{array}{l}27,1991 \\
31,1992 \\
05\end{array}$ & 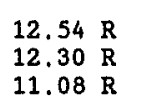 & $\begin{array}{ll}\text { MAR } 30,1992 \\
\text { APR } 28 \\
\text { JUN } 05\end{array}$ & $\begin{array}{rl}10.92 & \mathrm{R} \\
& \mathrm{RP} \\
11.55 & \mathrm{RS}\end{array}$ & $\begin{array}{lll}\text { JUN } & 29,1992 \\
\text { JUL } & 30 & \\
\text { SEP } & 02\end{array}$ & $\begin{array}{rl}R P \\
R P \\
13.00 & R\end{array}$ & SEP 29, 1992 & $12.50 \mathrm{R}$ \\
\hline
\end{tabular}

PERIOD OF RECORD HIGHEST 8.37 MAR 02, 1944 LOWEST 37.63 APR 23, 1956 
SITE NUMBER 342512119335701

LOCAL NUMBER 004N026W23A02S

West of Santa Barbara Polo and Racquet Club at end of Serpolla Drive. Drilled unused water-table well. Diameter 10 inches, depth 330 feet. Altitude of land-surface datum 63 feet. Measurements provided by Carpinteria County Water District beginning in 1978. Water-level records available 1941, 1947-74, 1978 to current year.

WATER LEVELS IN FEET BELOW LAND-SURFACE DATUM

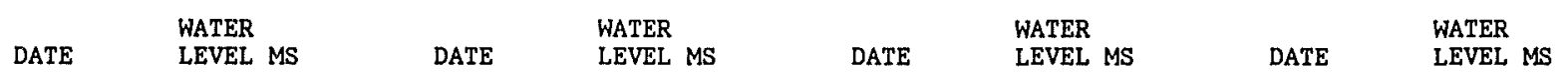

OCT 28, 1991

$62.60 \mathrm{R}$

JAN 30, 1992

LEVEL MS

LEVEL MS

DATE

$59.30 \mathrm{R}$

NOV 27

$63.50 \mathrm{R}$

FEB 28

$54.90 \mathrm{R}$

APR 28, 1992

$57.70 \mathrm{R}$

$54.90 \mathrm{R}$

JUL 29, 1992

AUG 31

$57.50 \mathrm{R}$

$55.80 \mathrm{R}$

25

$57.20 \mathrm{R}$

SEP 29

$61.25 \mathrm{R}$

PERIOD OF RECORD HIGHEST 40.20 JUN 16, 1983 LOWEST 79.91 AUG 31,1953

SITE NUMBER 342705119435801

LOCAL NUMBER 004N027W05P01S

In Santa Barbara about 700 feet north of Foothill Road in San Roque channel. Observatton well. Diameter 5 inches, depth 200 feet, cased to 160 feet, perforated 60-160 feet. Altitude of land-surface datum 305 feet. Reported measurements provided by city of Santa Barbara. Water-level records available 1984 to current year.

WATER LEVELS IN FEET BELOW LAND-SURFACE DATUM

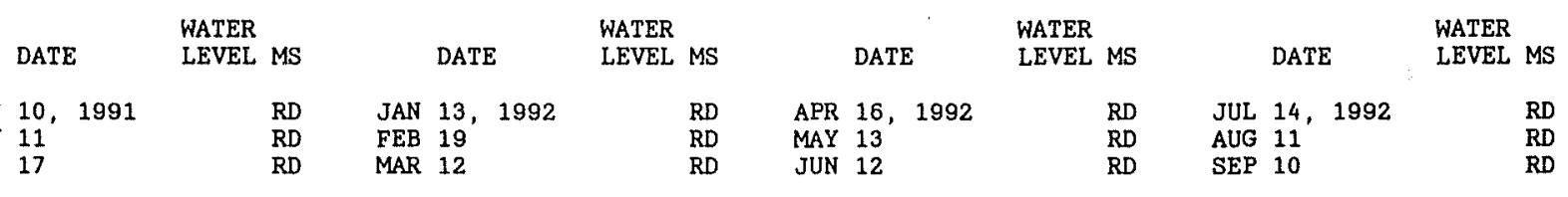

PERIOD OF RECORD HIGHEST 100.55 JUL 07, 1986 LOWEST 137.93 DEC 04, 1985

SITE NUMBER 342647119451701

LOCAL NUMBER 004N027W07D01S

In Santa Barbara about 15 feet south of Via Diego, at Via Rosa. Drilled production well. Diameter 16 inches, depth 490 feet, perforated 215-310, 330-480 feet. Altitude of land-surface datum 180 feet. Reported measurements provided by city of Santa Barbara. Water-level records available 1985 to current year.

WATER LEVELS IN FEET BELOW LAND-SURFACE DATUM

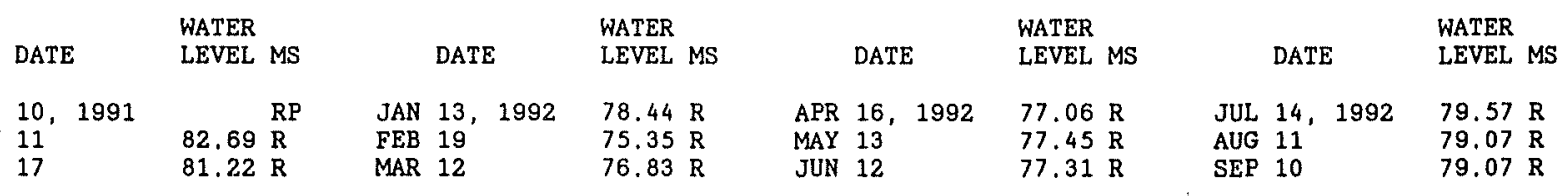

PERIOD OF RECORD HIGHEST 52.88 FEB 07, 1986 LOWEST 95.15 OCT 14, 1988 
WELL DESCRIPTIONS AND WATER-LEVEL MEASUREMENTS

WATER YEAR OCTOBER 1991 TO SEPTEMBER 1992

SITE NUMBER 342608119444001

LOCAL NUMBER 004N027W07R03S

In Santa Barbara, 100 feet south of YMCA Building, and 270 feet west of Hitchcock Street. Drilled observation well, diameter 10 inches, depth 510 feet, perforated 165-500 feet. Altitude of land-surface datum 185 feet. Reported measurements provided by city of Santa Barbara. Water-level records available 1983 to current year.

WATER LEVELS IN FEET BELOW LAND-SURFACE DATUM

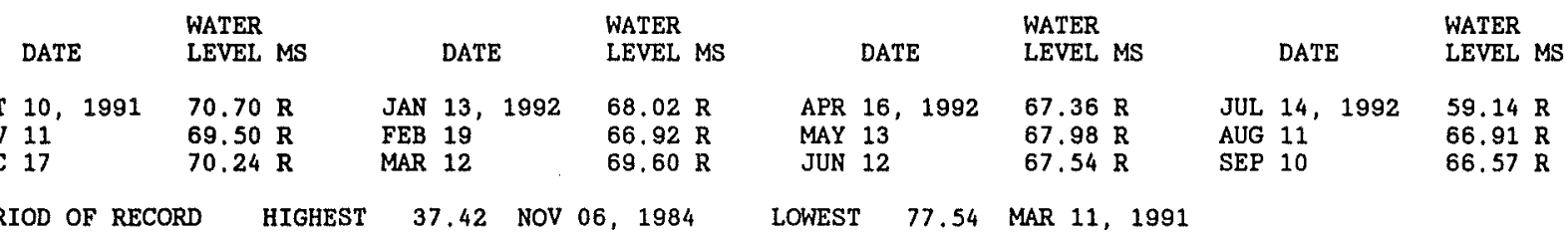

SITE NUMBER 342637119440901

LOCAL NUMBER 004N027W08E01S

In Santa Barbara in Triangular Park, about $0.4 \mathrm{mile}$ north of Chuparosa Road. Drilled production well, diameter 114 inches, depth 580 feet, perforated 52-580 feet. Altitude of land-surface datum 251 feet. Reported measurements provided by city of Santa Barbara. Water-level records available 1948, 1976 , 1984 to current year.

WATER LEVELS IN FEET BELOW LAND-SURFACE DATUM

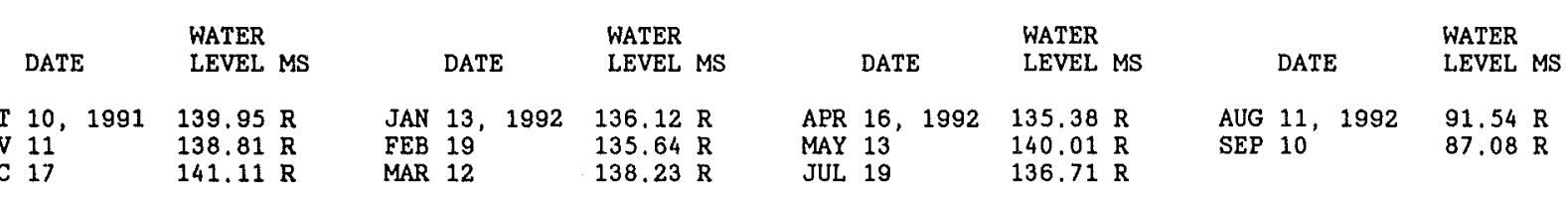

PERIOD OF RECORD HIGHEST 72.00 JAN 07, 1986 LOWEST 150.38 MAR 11, 1991

\section{SITE NUMBER 342624119435701}

LOCAL NUMBER 004N027W08L03S

In Santa Barbara, southeast of State Street and Los Positas Road. Drilled public-supply well. Diameter 14 inches 0-260 feet, 12 inches 260-610 feet, depth 610 feet, perforated $260-370,390-470,490-510,540-550,590-600$ feet. Altitude of land-surface datum 230 feet. Water-level records available 1983,1985 to current year.

WATER LEVELS IN FEET BELOW LAND-SURFACE DATUM

\begin{tabular}{|c|c|c|c|c|c|c|c|}
\hline DATE & $\begin{array}{l}\text { WATER } \\
\text { LEVEL MS }\end{array}$ & DATE & $\begin{array}{l}\text { WATER } \\
\text { LEVEL MS }\end{array}$ & DATE & $\begin{array}{l}\text { WATER } \\
\text { LEVEL MS }\end{array}$ & DATE & $\begin{array}{l}\text { WATER } \\
\text { LEVEL MS }\end{array}$ \\
\hline $\begin{array}{l}10,1991 \\
11 \\
17\end{array}$ & $\begin{array}{l}85.78 \mathrm{R} \\
83.69 \mathrm{R} \\
93.50 \mathrm{R}\end{array}$ & $\begin{array}{lll}\text { JAN } & 13,1992 \\
\text { FEB } & 19 & \\
\text { MAR } & 12 & \end{array}$ & 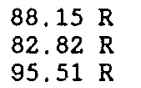 & $\begin{array}{lll}\text { APR } & 16, & 1992 \\
\text { MAY } & 13 & \\
\text { JUN } & 12 & \end{array}$ & 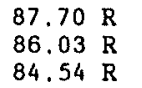 & $\begin{array}{lll}\text { JUL } & 14,1992 \\
\text { AUG } & 11 & \\
\text { SEP } & 10 & \end{array}$ & 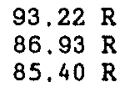 \\
\hline
\end{tabular}

PERIOD OF RECORD HIGHEST 38.21 AUG 14, 1985 LOWEST 133.50 JUN 05, 1987 
SITE NUMBER 342630119442301

LOCAL NUMBER 004N027W08M05S

In Santa Barbara at intersection of San Roque Creek and San Remo Drive. Drilled observation well. Diameter 5 inches 0-60 feet, 4 inches 60-680 feet, depth 680 feet, perforated $620-660$ feet. Altitude of land-surface datum 217 feet. Recorder installed in 1987. Water-level records available 1987 to current year.

Missing record due to malfunction of recording instrument.

WATER LEVELS IN FEET BELOW LAND-SURFACE DATUM (OBSERVATIONS AT 1200 HOURS)

\begin{tabular}{|c|c|c|c|c|c|c|c|c|c|c|c|c|}
\hline DAY & OCT & NOV & DEC & JAN & FEB & MAR & APR & MAY & JUN & JUL & AUG & SEF \\
\hline $\begin{array}{l}1 \\
2 \\
3 \\
4 \\
5\end{array}$ & $\begin{array}{l}99.10 \\
99.15 \\
99.25 \\
99.30 \\
99.39\end{array}$ & $\begin{array}{l}98.17 \\
98.09 \\
98.16 \\
98.21 \\
98.21\end{array}$ & $\begin{array}{r}97.30 \\
97.36 \\
97.44 \\
100.26 \\
99.97\end{array}$ & $\begin{array}{l}96.04 \\
95.98 \\
95.83 \\
95.81 \\
95.57\end{array}$ & $\begin{array}{l}94.36 \\
94.36 \\
94.32 \\
94.30 \\
94.27\end{array}$ & $\begin{array}{l}93.44 \\
93.44 \\
93.52 \\
93.52 \\
93.50\end{array}$ & $\begin{array}{l}94.01 \\
93.99 \\
93.99 \\
93.99 \\
94.03\end{array}$ & $\begin{array}{l}94.74 \\
94.76 \\
94.82 \\
94.83 \\
94.83\end{array}$ & $\begin{array}{l}94.43 \\
94.43 \\
94.41 \\
94.41 \\
94.32\end{array}$ & $\begin{array}{l}95.24 \\
95.26 \\
95.30 \\
95.30 \\
95.27\end{array}$ & $\begin{array}{l}94.97 \\
94.70 \\
94.47 \\
94.37 \\
94.21\end{array}$ & $\begin{array}{l}93.65 \\
93.54 \\
93.43 \\
93.31 \\
93.26\end{array}$ \\
\hline $\begin{array}{r}6 \\
7 \\
8 \\
9 \\
10\end{array}$ & $\begin{array}{l}99.47 \\
99.53 \\
99.57 \\
99.59 \\
99.62\end{array}$ & $\begin{array}{l}98.06 \\
97.94 \\
97.88 \\
97.88 \\
97.87\end{array}$ & $\begin{array}{r}99.30 \\
98.82 \\
98.23 \\
97.97 \\
100.86\end{array}$ & $\begin{array}{l}95.55 \\
95.40 \\
95.39 \\
95.34 \\
95.25\end{array}$ & $\begin{array}{l}94.16 \\
94.01 \\
94.00 \\
93.90 \\
93.80\end{array}$ & $\begin{array}{l}93.50 \\
93.42 \\
93.35 \\
93.34 \\
97.95\end{array}$ & $\begin{array}{l}94.03 \\
94.11 \\
94.11 \\
94.13 \\
94.13\end{array}$ & $\begin{array}{l}94.83 \\
94.79 \\
94.70 \\
94.64 \\
94.64\end{array}$ & $\begin{array}{r}94.28 \\
94.28 \\
94.28 \\
\ldots \\
\ldots-\end{array}$ & $\begin{array}{r}95.21 \\
\text { s100.00 } \\
100.17 \\
598.57 \\
98.15\end{array}$ & $\begin{array}{l}94.09 \\
93.92 \\
93.77 \\
93.62 \\
93.49\end{array}$ & $\begin{array}{l}93.22 \\
93.17 \\
93.16 \\
93.12 \\
93.12\end{array}$ \\
\hline $\begin{array}{l}11 \\
12 \\
13 \\
14 \\
15\end{array}$ & $\begin{array}{l}99.62 \\
99.66 \\
99.76 \\
99.80 \\
99.80\end{array}$ & $\begin{array}{l}97.81 \\
97.80 \\
97.78 \\
97.65 \\
97.61\end{array}$ & $\begin{array}{r}100.01 \\
99.01 \\
98.40 \\
98.02 \\
97.78\end{array}$ & $\begin{array}{l}95.09 \\
95.02 \\
94.99 \\
94.94 \\
94.92\end{array}$ & $\begin{array}{l}93.72 \\
93.49 \\
93.45 \\
93.45 \\
93.39\end{array}$ & $\begin{array}{l}98.19 \\
96.76 \\
95.96 \\
95.36 \\
94.95\end{array}$ & $\begin{array}{l}94.13 \\
94.13 \\
94.13 \\
94.15 \\
94.17\end{array}$ & $\begin{array}{l}94.66 \\
94.67 \\
94.63 \\
94.60 \\
94.49\end{array}$ & $\begin{array}{r}--- \\
--- \\
--- \\
94.46\end{array}$ & $\begin{array}{l}97.46 \\
96.94 \\
96.55 \\
96.22 \\
95.98\end{array}$ & $\begin{array}{l}93.49 \\
93.40 \\
93.30 \\
93.30 \\
93.27\end{array}$ & $\begin{array}{l}93.29 \\
93.52 \\
93.63 \\
93.79 \\
93.86\end{array}$ \\
\hline $\begin{array}{l}16 \\
17 \\
18 \\
19 \\
20\end{array}$ & $\begin{array}{l}99.74 \\
99.67 \\
99.50 \\
99.41 \\
99.29\end{array}$ & $\begin{array}{l}97.63 \\
97.62 \\
97.62 \\
97.64 \\
97.64\end{array}$ & $\begin{array}{l}97.57 \\
97.41 \\
97.27 \\
97.19 \\
97.09\end{array}$ & $\begin{array}{l}94.89 \\
94.76 \\
94.65 \\
94.65 \\
94.62\end{array}$ & $\begin{array}{l}93.39 \\
93.39 \\
93.39 \\
93.39 \\
93.32\end{array}$ & $\begin{array}{l}94.73 \\
94.54 \\
94.48 \\
94.37 \\
94.30\end{array}$ & $\begin{array}{l}94.19 \\
94.19 \\
94.22 \\
94.31 \\
94.35\end{array}$ & $\begin{array}{l}94.55 \\
94.57 \\
94.61 \\
94.63 \\
94.60\end{array}$ & $\begin{array}{l}94.46 \\
94.48 \\
94.47 \\
94.45 \\
94.47\end{array}$ & $\begin{array}{l}95.88 \\
95.74 \\
95.73 \\
95.68 \\
95.63\end{array}$ & $\begin{array}{l}93.28 \\
93.25 \\
93.25 \\
93.25 \\
93.22\end{array}$ & $\begin{array}{l}93.89 \\
93.93 \\
93.94 \\
94.02 \\
94.07\end{array}$ \\
\hline $\begin{array}{l}21 \\
22 \\
23 \\
24 \\
25\end{array}$ & $\begin{array}{l}99.22 \\
99.08 \\
99.00 \\
98.91 \\
98.86\end{array}$ & $\begin{array}{l}97.68 \\
97.58 \\
97.62 \\
97.64 \\
97.57\end{array}$ & $\begin{array}{l}97.06 \\
97.00 \\
96.92 \\
96.86 \\
96.76\end{array}$ & $\begin{array}{l}94.62 \\
94.62 \\
94.62 \\
94.52 \\
94.52\end{array}$ & $\begin{array}{l}93.32 \\
93.29 \\
93.21 \\
93.22 \\
93.18\end{array}$ & $\begin{array}{l}94.18 \\
94.08 \\
94.06 \\
94.06 \\
94.06\end{array}$ & $\begin{array}{l}94.37 \\
94.46 \\
94.46 \\
94.46 \\
94.55\end{array}$ & $\begin{array}{l}94.59 \\
94.57 \\
94.63 \\
94.67 \\
94.60\end{array}$ & $\begin{array}{l}94.53 \\
94.56 \\
94.67 \\
94.73 \\
94.78\end{array}$ & $\begin{array}{l}95.63 \\
95.60 \\
95.57 \\
95.57 \\
95.56\end{array}$ & $\begin{array}{l}93.11 \\
93.19 \\
93.44 \\
93.79 \\
94.14\end{array}$ & $\begin{array}{l}94.09 \\
94.19 \\
94.20 \\
94.20 \\
94.19\end{array}$ \\
\hline $\begin{array}{l}26 \\
27 \\
28 \\
29 \\
30 \\
31\end{array}$ & $\begin{array}{l}98.81 \\
98.61 \\
98.53 \\
98.38 \\
98.31 \\
98.24\end{array}$ & $\begin{array}{r}97.57 \\
97.57 \\
97.44 \\
97.29 \\
97.28 \\
---\end{array}$ & $\begin{array}{l}96.72 \\
96.57 \\
96.47 \\
96.21 \\
96.16 \\
96.15\end{array}$ & $\begin{array}{l}94.49 \\
94.49 \\
94.47 \\
94.47 \\
94.44 \\
94.36\end{array}$ & $\begin{array}{r}93.17 \\
93.24 \\
93.38 \\
93.38 \\
\ldots\end{array}$ & $\begin{array}{l}94.03 \\
93.99 \\
93.99 \\
94.01 \\
94.01 \\
94.01\end{array}$ & $\begin{array}{r}94.69 \\
94.72 \\
94.75 \\
94.77 \\
94.77 \\
-.-\end{array}$ & $\begin{array}{l}94.46 \\
94.37 \\
94.37 \\
94.37 \\
94.43 \\
94.43\end{array}$ & $\begin{array}{r}94.83 \\
94.92 \\
95.15 \\
95.14 \\
95.17 \\
-\end{array}$ & $\begin{array}{l}95.49 \\
95.46 \\
95.46 \\
95.41 \\
95.35 \\
95.16\end{array}$ & $\begin{array}{l}94.36 \\
94.37 \\
94.12 \\
93.94 \\
93.84 \\
93.69\end{array}$ & $\begin{array}{r}94.19 \\
94.27 \\
94.29 \\
94.35 \\
94.36 \\
-\end{array}$ \\
\hline
\end{tabular}

PERIOD OF RECORD HIGHEST 93.11 AUG 21, $1992 \quad$ LOWEST $123.74 \quad$ FEB 23, 1991

S Manual steel tape measurement.

\section{SITE NUMBER 342630119442302}

LOCAL NUMBER 004N027W08MO6S

In Santa Barbara at intersection of San Roque Creek and San Remo Drive. Drilled observation well. D1ameter 2 inches, depth 280 feet, perforated 260-280 feet. Altitude of land-surface datum 217 feet. Water-level records available 1987 to current year.

WATER LEVELS IN FEET BELOW LAND-SURFACE DATUM

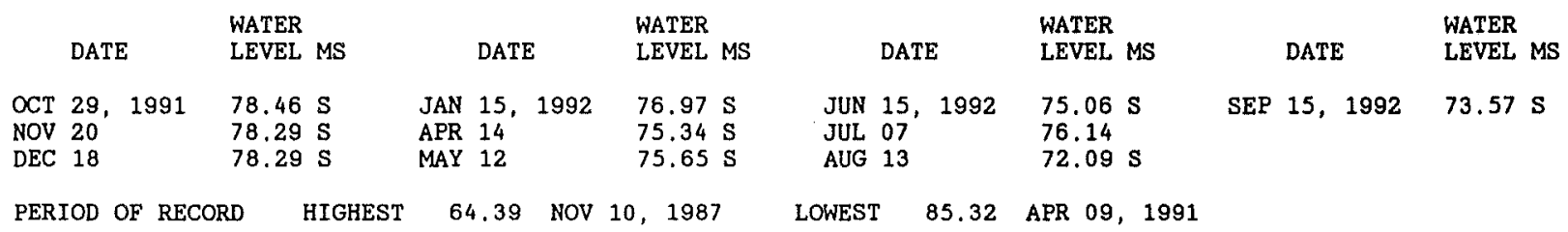


WELL DESCRIPTIONS AND WATER-LEVEL MEASUREMENTS

WATER YEAR OCTOBER 1991 TO SEPTEMBER 1992

\begin{abstract}
SITE NUMBER 342618119432501
\end{abstract}
LOCAL NUMBER O04N027W08R02S

In Santa Barbara at intersection of Alamar Avenue and State Street. Drilled observation water-table well. Diameter 6 inches, depth 205 feet, depth of hole 265 feet, perforated 155-205 feet. Altitude of land-surface datum 215 feet. Reported measurements provided by city of Santa Barbara. Water-level records available 1982 to current year.

WATER LEVELS IN FEET BELOW LAND-SURFACE DATUM

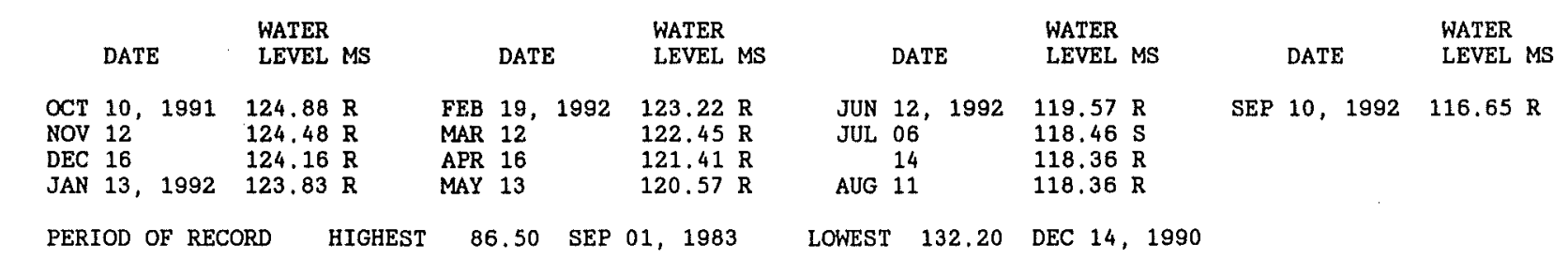

\title{
SITE NUMBER 342611119432701
}

LOCAL NUMBER 004N027W09M01S

In Santa Barbara along Mission Creek, north of State Street. Drilled observation water-table well. Diameter 6 inches, depth 120 feet, depth of hole 180 feet, perforated 30-110 feet. Altitude of land-surface datum 240 feet. Reported measurements provided by city of Santa Barbara. Water-level records available 1981 to current year.

WATER LEVELS IN FEET BELOW LAND-SURFACE DATUM

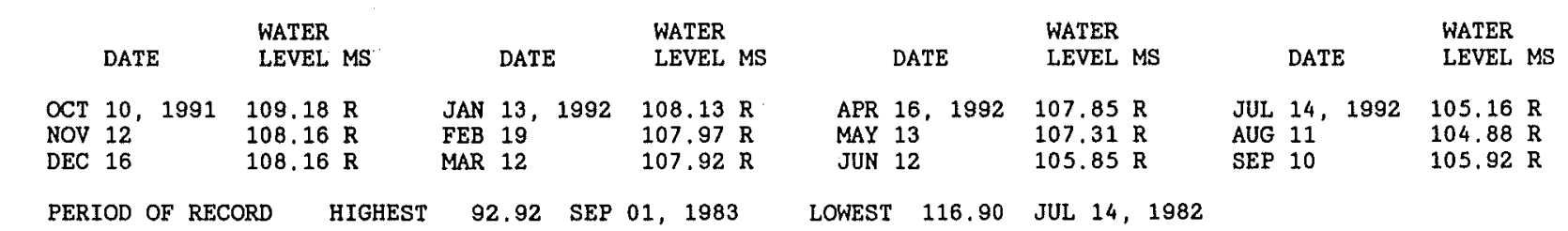

\section{SITE NUMBER 342618119423701}

LOCAL NUMBER 004N027W09Q01S

In Santa Barbara at Plaza Rubio, near Mission Park. Drilled observation well. Diameter 2 inches, depth 240 feet. Altitude of land-surface datum 306 feet. Reported measurements provided by city of Santa Barbara. Water-1eve1 records available $1984-86,1988$ to current year.

WATER LEVELS IN FEET BELOW LAND-SURFACE DATUM

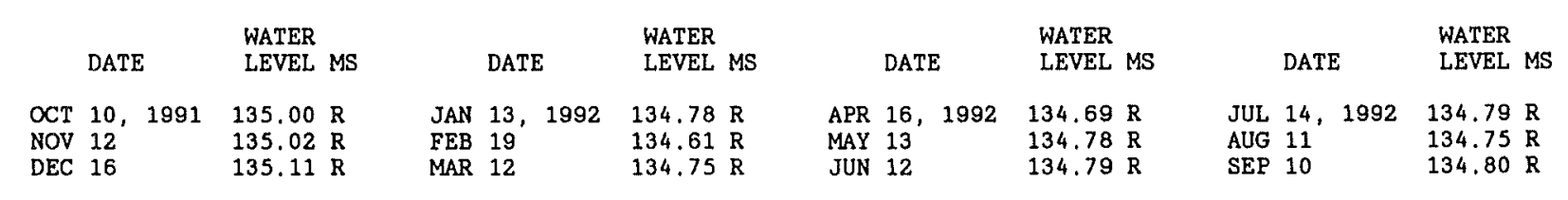

PERIOD OF RECORD HIGHEST 129,80 JUN 06, AUG 14, 1985 LOWEST 137.33 MAR 30,1984 
SITE NUMBER 342534119404301

LOCAL NUMBER 004N027W14K02S

In Santa Barbara, north of Voluntario Street in school parking lot. Drilled observation water-table well in Santa Barbara Formation of Pleistocene age. Diameter 8 inches, depth 380 feet, depth of hole 450 feet, perforated 260-380 feet. Altitude of land-surface datum 42.3 feet. Reported measurements provided by city of Santa Barbara. Water-level records avallable 1979 to current year.

WATER LEVELS IN FEET BELOW LAND-SURFACE DATUM

\begin{tabular}{|c|c|c|c|c|c|c|c|}
\hline DATE & $\begin{array}{l}\text { WATER } \\
\text { LEVEL MS }\end{array}$ & DATE & $\begin{array}{l}\text { WATER } \\
\text { LEVEL MS }\end{array}$ & DATE & $\begin{array}{l}\text { WATER } \\
\text { LEVEL MS }\end{array}$ & DATE & $\begin{array}{l}\text { WATER } \\
\text { LEVEL MS }\end{array}$ \\
\hline $\begin{array}{l}10,1991 \\
12 \\
16\end{array}$ & $\begin{array}{l}32.85 \mathrm{R} \\
32.21 \mathrm{R} \\
32.65 \mathrm{R}\end{array}$ & $\begin{array}{lll}\text { JAN } & 13,1992 \\
\text { FEB } & 19 & \\
\text { MAR } & 12 & \end{array}$ & $\begin{array}{ll}31.63 & R \\
30.32 & R \\
29.69 & R\end{array}$ & $\begin{array}{lll}\text { APR } & 16,1992 \\
\text { MAY } & 13 & \\
\text { JUN } & 15 & \end{array}$ & 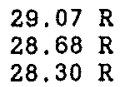 & $\begin{array}{lll}\text { JUL } & 14,1992 \\
\text { AUG } & 11 & \\
\text { SEP } & 10 & \end{array}$ & 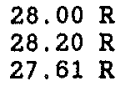 \\
\hline
\end{tabular}

PERIOD OF RECORD HIGHEST 25.95 MAY 08, 1989 LOWEST 67.31 OCT 09, 1989

SITE NUMBER 342524119405201

LOCAL NUMBER 004N027W14P01S

In Santa Barbara, southwest of Milpas Street, and north of Highway 101 . Drilled unused water-table well. Diameter 14 inches, depth 783 feet, depth of hole 950 feet, perforated 181-783 feet. Altitude of land-surface datum 18 feet. Measurements provided by city of Santa Barbara beginning July 1976. Water-level records available $1950-55,1957,1968,1976$ to current year.

WATER LEVELS IN FEET BELOW LAND-SURFACE DATUM

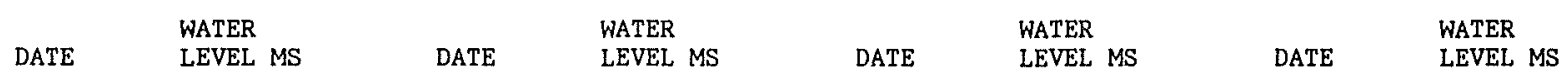

$\begin{array}{lllllllllll}\text { OCT 10, } 1991 & 14.98 \mathrm{R} & \text { JAN 13, } 1992 & 11.68 \mathrm{R} & \text { MAY 13, } 1992 & 7.73 \mathrm{R} & \text { AUG } 11,1992 & 6.10 \mathrm{R} \\ \text { NOV 12 } & 13.67 \mathrm{R} & \text { FEB 19 } & 9.93 \mathrm{R} & \text { JUN } 12 & & 7.48 \mathrm{R} & \text { SEP } 10 & \\ \text { DEC } 16 & 13.46 \mathrm{R} & \text { MAR } 12 & & 9.70 \mathrm{R} & \text { JUL } 14 & & 6.51 \mathrm{R} & & & \end{array}$

M.70 12

PERIOD OF RECORD HIGHEST

4.21 MAY 08, 1989

LOWEST $\quad 88.80$ JUL 23,1968

SITE NUMBER 342523119402001

LOCAL NUMBER 004N027W14R01S

In Santa Barbara about 0.3 mile northeast of Milpas Street and 0.1 mile southeast of Carpinteria Street. Drilled unused water-table well. Diameter 13 inches, depth 700 feet, perforated 107-635 feet. Altitude of land-surface datum 27.84 feet. Measurements provided by city of Santa Barbara beginning June 1976. Water-level records available 1948-55, 1968, 1976 to current year.

WATER LEVELS IN FEET BELOW LAND-SURFACE DATUM

\begin{tabular}{|c|c|c|c|c|c|c|c|}
\hline DATE & $\begin{array}{l}\text { WATER } \\
\text { LEVEL MS }\end{array}$ & DATE & $\begin{array}{l}\text { WATER } \\
\text { LEVEL MS }\end{array}$ & DATE & $\begin{array}{l}\text { WATER } \\
\text { LEVEL MS }\end{array}$ & DATE & $\begin{array}{l}\text { WATER } \\
\text { LEVEL MS }\end{array}$ \\
\hline $\begin{array}{ll}10, & 1991 \\
16 & \\
13, & 1992\end{array}$ & $\begin{array}{r}19.92 \mathrm{R} \\
19.64 \mathrm{R} \\
8.60 \mathrm{R}\end{array}$ & $\begin{array}{lll}\text { FEB } & 19, & 1992 \\
\text { MAR } & 12 & \\
\text { APR } & 16 & \end{array}$ & 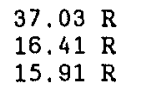 & $\begin{array}{lll}\text { MAY } & 13,1992 \\
\text { JUN } & 12 & \\
\text { JUL } 14 & \end{array}$ & 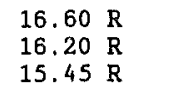 & $\begin{array}{l}\text { AUG } 11,1992 \\
\text { SEP } 10\end{array}$ & $\begin{array}{l}15.37 \mathrm{R} \\
14.32 \mathrm{R}\end{array}$ \\
\hline
\end{tabular}


In Santa Barbara near Alameda Plaza. Unused well. Diameter 8 inches, depth unknown. Altitude of land-surface datum 145 feet. Measurements provided by city of Santa Barbara beginning May 1976. Water-level records available 1976 to current year.

WATER LEVELS IN FEET BELOW LAND-SURFACE DATUM

$$
\begin{array}{lll} 
& \text { WATER } & \\
\text { DATE } & \text { WETER } \\
\text { LEVEL MS } & \text { LEVEL MS }
\end{array}
$$

OCT $10,1991 \quad 110.82 \mathrm{R}$

NOV $12 \quad 109.67 \mathrm{R}$

DEC $16 \quad 108.77 \mathrm{R}$

$\begin{array}{lll}\text { JAN } & 13,1992 & 108.49 \mathrm{R} \\ \text { FEB } & 19 & 107.32 \mathrm{R} \\ \text { MAR } & 12 & 106.80 \mathrm{R}\end{array}$

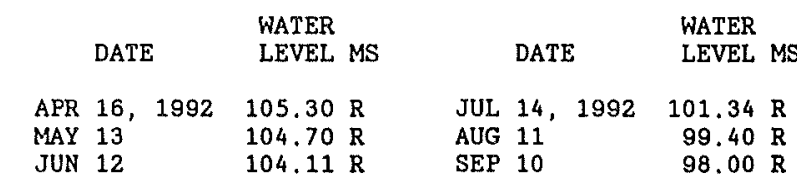

PERIOD OF RECORD HIGHEST 91.30 APR 08, 1976 LOWEST 130.08 OCT 11, 1990

\section{SITE NUMBER 342530119410701}

LOCAL NUMBER 004N027W15J02S

In Santa Barbara, northeast of intersection of Ortega and Salsipuedes Streets. Drilled public-supply water-table wel1. Diameter 14 inches 0-298 feet, 12.75 inches 298-579 feet, depth 579 feet, depth of hole 661 feet, perforated 198-218, 266-276, 363-383, 430-440,478-488,515-535, 539-579 feet. Altitude of land-surface datum 12.31 feet. Measurements provided by city of Santa Barbara beginning June 1976. Water-level records available 1975 to current year.

WATER LEVELS IN FEET BELOW LAND-SURFACE DATUM (READINGS ABOVE LAND SURFACE INDICATED BY "+")

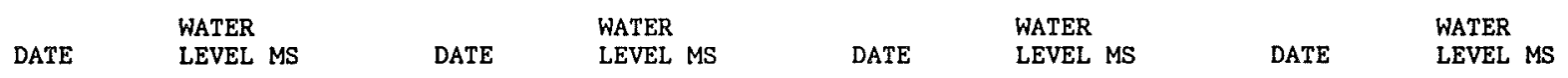

\begin{tabular}{|c|c|c|c|c|c|c|c|c|}
\hline $\begin{array}{lll}\text { OCT } & 10,1991 \\
\text { NOV } & 12 & \\
\text { DEC } & 16\end{array}$ & $\begin{array}{l}5.72 \mathrm{R} \\
4.12 \mathrm{R} \\
4.22 \mathrm{R}\end{array}$ & $\begin{array}{lll}\text { JAN } & 13,1992 \\
\text { FEB } & 19 & \\
\text { MAR } & 12 & \end{array}$ & $\begin{array}{l}2.49 \\
0.74\end{array}$ & $\begin{array}{ll}\text { APR } & 16, \\
\text { MAY } & 13 \\
\text { JUN } & 12\end{array}$ & 1992 & +0.76 & $\begin{array}{l}R \\
R F \\
R F\end{array}$ & $\begin{array}{ll}\text { JUL } & 14,1992 \\
\text { AUG } & 11 \\
\text { SEP } & 10\end{array}$ \\
\hline
\end{tabular}

PERIOD OF RECORD HIGHEST to.76 APR 16, 1992 LOWEST 76.00 OCT 14,1981

\section{SITE NUMBER 342538119413401}

\section{LOCAL NUMBER 004N027W15K01S}

In Santa Barbara near intersection of Salsipuedes and Canon Perdido Streets. Drilled observation water-table well in Santa Barbara Formation of Pleistocene age. Diameter 8 inches, depth 464 feet, depth of hole 500 feet, perforated 280-464 feet. Altitude of land-surface datum 18.9 feet. Reported measurements provided by city of Santa Barbara. Water-level records available 1979 to current year.

WATER LEVELS IN FEET BELOW LAND-SURFACE DATUM (READINGS ABOVE LAND SURFACE INDICATED BY "+")

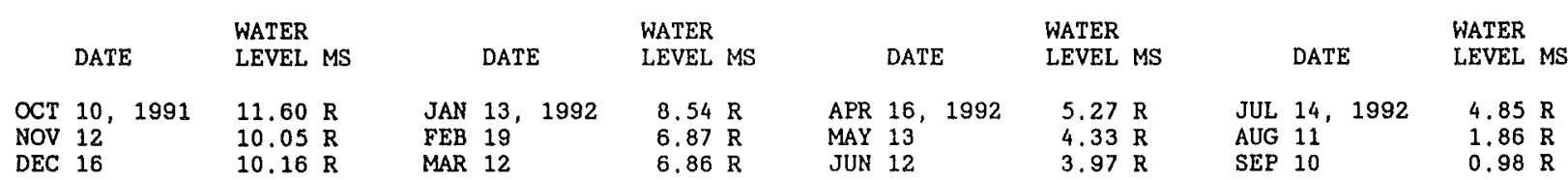

PERIOD OF RECORD HIGHEST 0.98 SEP 10, 1992 LOWEST 77.77 NOV 13, 1979


SITE NUMBER 342523119412901

LOCAL NUMBER 004N027W15Q10S

In Santa Barbara, southeast of intersection of Ortega and Vine Streets. Drilled public-supply well in alluvium. Diameter 14 inches, depth 675 feet, perforated 195-205, 240-260, 380-400, 415-425, 495-505, 585-605, 615-655 feet. Altitude of land-surface datum 11 feet. Measurements provided by city of Santa Barbara beginning 1982. Water-level records available 1982 to current year.

WATER LEVELS IN FEET BELOW LAND-SURFACE DATUM

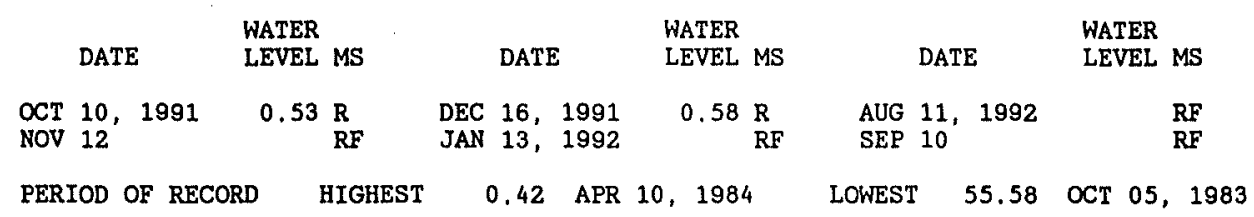

\section{SITE NUMBER 342603119430401}

LOCAL NUMBER 004N027W16C01S

In Santa Barbara on State Street, near Pueblo Street. Drilled observation water-table well. Diameter 6 inches, depth 330 feet, perforated 290-330 feet. Altitude of land-surface datum 210 feet. Reported measurements provided by city of Santa Barbara. Previously published measurements for November 1985-December 1989 were for well 16C2. Water-level records available 1981 to current year.

WATER LEVELS IN FEET BELOW LAND-SURFACE DATUM

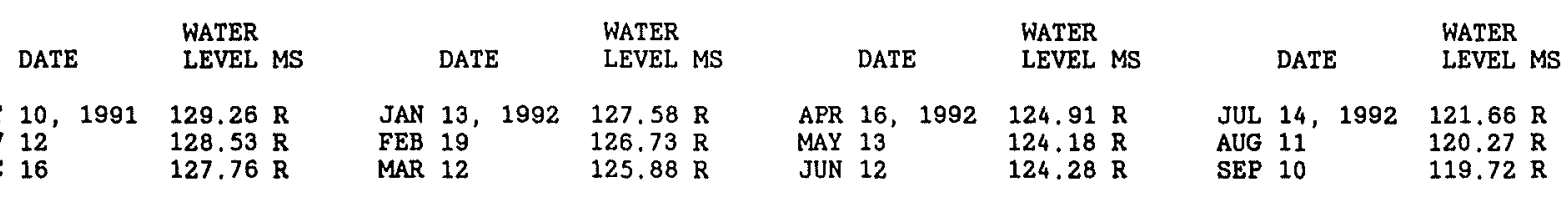

PERIOD OF RECORD HIGHEST 109.33 APR 10, 1984 LOWEST 143.14 NOV 12, 1990

SITE NUMBER 342603119430402

LOCAL NUMBER 004N027W16CO2S

In Santa Barbara on State Street, near Pueblo Street. Drilled observation water-table well. Diameter 2 inches, depth 230 feet, perforated 190-230 feet. Altitude of land-surface datum 210 feet. Reported measurements provided by city of Santa Barbara. Previously published measurements for November 1985-December 1989 were for well 16C1. Water-level records avallable 1981 to current year.

WATER LEVELS IN FEET BELOW LAND-SURFACE DATUM

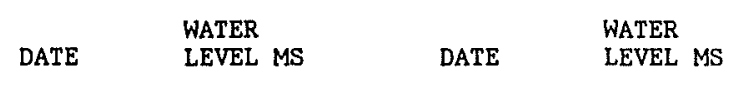

OCT $10,1991 \quad 132.17 \mathrm{R}$

NOV $12 \quad 131.67 \mathrm{R}$

DEC $16 \quad 133.00 \mathrm{R}$ $\begin{array}{llll}\text { JAN } & 13,1992 & 131.07 \mathrm{R} \\ \text { FEB } & 19 & & 130.44 \mathrm{R}\end{array}$

MAR $12 \quad 129.74 \mathrm{R}$

PERIOD OF RECORD HIGHEST 113.54 MAY 03, 1984

DATE WATER

APR 16, 1992

MAY 13

JUN 12
$127,82 \mathrm{R}$

LOWEST 145.52 NOV 12,1990

$\begin{array}{ll} & \text { WATER } \\ \text { DATE } & \text { LEVEL MS } \\ \text { JUL } 14,1992 & 127.03 \mathrm{R} \\ \text { AUG 11 } & 126.13 \mathrm{R} \\ \text { SEF } 10 & 125.67 \mathrm{R}\end{array}$


WELL DESCRIPTIONS AND WATER-LEVEL MEASUREMENTS

WATER YEAR OCTOBER 1991 TO SEPTEMBER 1992

SITE NUMBER 342543119431101

LOCAL NUMBER 004N027W16E02S

In Santa Barbare near the intersection of Castillo and Padre Streets. Drilled public-supply well. Diameter 12 inches, depth 403 feet, perforated 203-223, 263-283, 303-383 feet. Altitude of land-surface datum 125 feet. Reported measurements provided by city of Santa Barbara. Water-level records available 1985 to current year.

WATER LEVELS IN FEET BELOW LAND-SURFACE DATUM

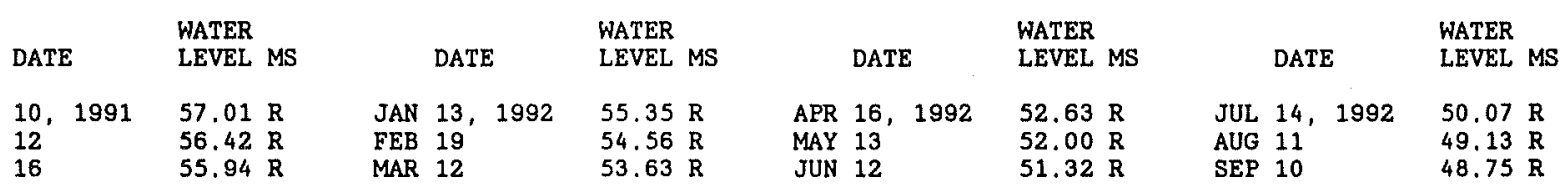

PERIOD OF RECORD HIGHEST 44.20 JUN 06, 1985 LOWEST 69.65 OCT 11, 1990

SITE NUMBER 342521119422401

LOCAL NUMBER 004N027W16R01S

In Santa Barbara near intersection of Victoria and De La Vina Streets. Drilled observation water-table well in Santa Barbara Formation of Pleistocene age. Diameter 6 inches, depth 625 feet, depth of hole 720 feet, perforated 545-625 feet. Altitude of land-surface datum 84.8 feet. Reported measurements provided by city of Santa Barbara. Water-level records available 1979 to current year.

WATER LEVELS IN FEET BELOW LAND-SURFACE DATUM

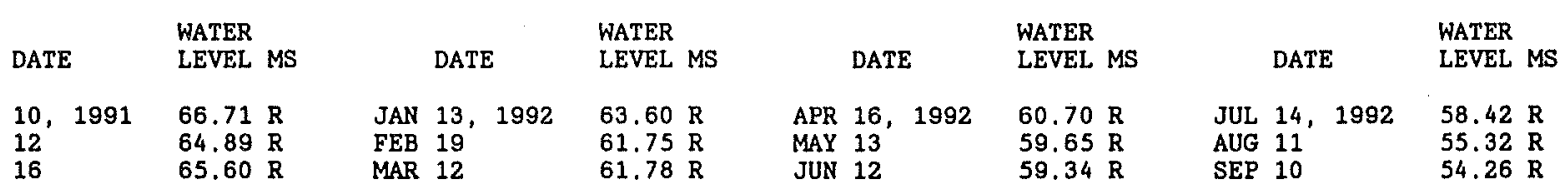

$\begin{array}{llll}\mathrm{DEC} 16 & 65.60 \mathrm{R} & \text { MAR } 12 & 61.78 \mathrm{R}\end{array}$

PERIOD OF RECORD HIGHEST 54.13 MAY 08, 1989 LOWEST 142.54 OCT 11, 1990

SITE NUMBER 342541119433501

LOCAL NUMBER 004N027W17J01S

In Santa Barbara on bank of Mission Creek, west of Junipero Street. Drilled observation water-table well in Santa Barbara Formation of Pleistocene age. Dianeter 8 inches, depth 320 feet, depth of hole 385 feet, perforated 190-320 feet. Altitude of land-surface datum 138.8 feet. Reported measurements provided by city of Santa Barbara. Water-level records avatlable 1979 to current year.

WATER LEVELS IN FEET BELOW LAND-SURFACE DATUM

\begin{tabular}{|c|c|c|c|c|c|c|c|c|c|c|}
\hline$i$ & DATE & $\begin{array}{l}\text { WATER } \\
\text { LEVEL MS }\end{array}$ & & DATE & $\begin{array}{l}\text { WATER } \\
\text { LEVEL MS }\end{array}$ & DATE & $\begin{array}{l}\text { WATER } \\
\text { LEVEL MS }\end{array}$ & & DATE & $\begin{array}{l}\text { WATER } \\
\text { LEVEL MS }\end{array}$ \\
\hline $\begin{array}{l}\text { OCT } \\
\text { NOV } \\
\text { DEC } \\
\text { JAN }\end{array}$ & $\begin{array}{ll}10, & 1991 \\
12 & \\
16 & \\
13, & 1992\end{array}$ & $\begin{array}{ll}56.62 & \mathrm{R} \\
56.19 & \mathrm{R} \\
55.80 & \mathrm{R} \\
55.33 & \mathrm{R}\end{array}$ & $\begin{array}{l}\text { FEB } \\
\text { MAR } \\
\text { APR } \\
\text { MAY }\end{array}$ & $\begin{array}{ll}19, & 1992 \\
12 & \\
16 & \\
13 & \end{array}$ & $\begin{array}{ll}55.02 & \mathrm{R} \\
54.01 & \mathrm{R} \\
53.19 & \mathrm{R} \\
52.71 & \mathrm{R}\end{array}$ & $\begin{array}{lll}\text { JUN } & 12,1992 \\
\text { JUL } & 09 \\
& 14 \\
\text { AUG } & 11\end{array}$ & $\begin{array}{l}52.13 \mathrm{R} \\
51.75 \mathrm{~S} \\
51.68 \mathrm{R} \\
50.79 \mathrm{R}\end{array}$ & SEP & 10,1992 & $50.41 \mathrm{R}$ \\
\hline
\end{tabular}

PERIOD OF RECORD HIGHEST 35.90 MAY 03, 1985 LOWEST 69.94 NOV 12,1990 
WELL DESCRIPTIONS AND WATER-LEVEL MEASUREMENTS

WATER YEAR OCTOBER 1991 TO SEPTEMBER 1992

SITE NUMBER 342533119435501

LOCAL NUMBER 004N027W17L02S

In Santa Barbara in the 2500 block of Modoc Road. Drilled observation well. Diameter 2 inches, depth 300 feet, perforated 260-300 feet. Deepest of three cluster wells. Altitude of land-surface datum 138.27 feet. Reported measurements provided by city of Santa Barbara. Water-level records available 1989 to current year.

WATER LEVELS IN FEET BELOW LAND-SURFACE DATUM

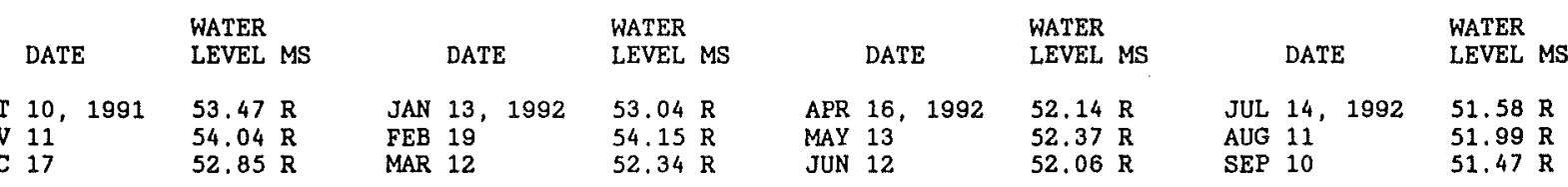

PERIOD OF RECORD HIGHEST 44.46 MAR 06, 1989 LOWEST 57.42 NOV 12, 1990

\section{SITE NUMBER 342533119435502}

LOCAL NUMBER 004N027W17LO3S

In Santa Barbara in the 2500 block of Modoc Road. Drilled observation well. Diameter 2 inches, depth 220 feet, perforated 190-220 feet. Middle depth of three cluster wells. Altitude of land-surface datum 138.27 feet. Reported measurements provided by city of Santa Barbara beginning in 1989. Water-level records available 1989 to current year.

WATER LEVELS IN FEET BELOW LAND-SURFACE DATUM

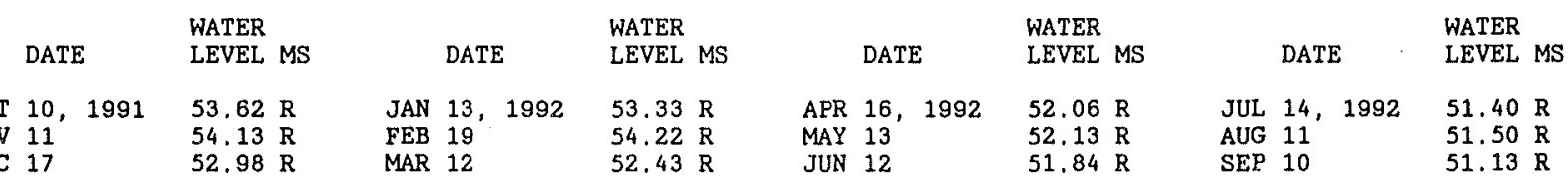

PERIOD OF RECORD HIGHEST 44.39 MAR 06, 1989 LOWEST 58.14 NOV 12, 1990

SITE NUMBER 342533119435503

LOCAL NUMBER 004N027W17L04S

In Santa Barbara in the 2500 block of Modoc Road. Drilled observation well. Diameter 2 inches, depth 140.35 feet in 1992, perforated 100-140 feet. Shallowest of three cluster wells. Altitude of land-surface datum 138.27 feet. Reported measurements provided by city of Santa Barbara. Water level records available 1989 to current year.

WATER LEVELS IN FEET BELOW LAND-SURFACE DATUM

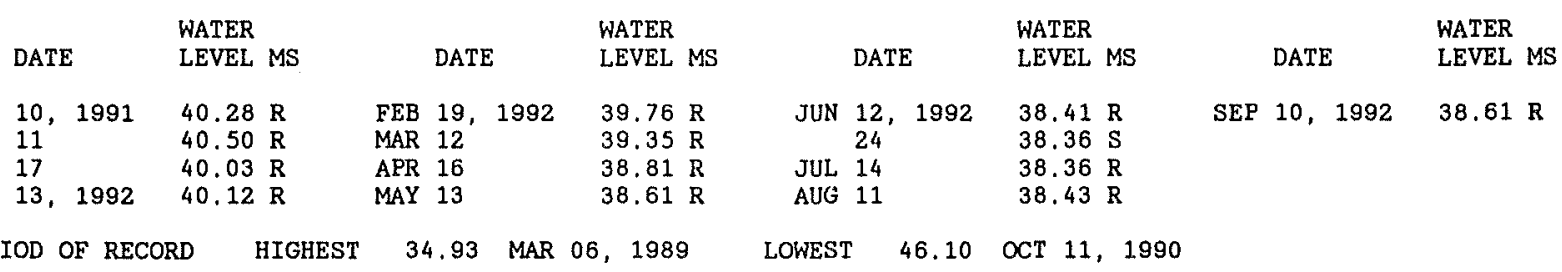


WATER LEVELS, SANTA BARBARA COUNTY--Continued

WELL DESCRIPTIONS AND WATER-LEVEL MEASUREMENTS

WATER YEAR OCTOBER 1991 TO SEPTEMBER 1992

SITE NUMBER 342606119445201

LOCAL NUMBER 004N027W18B05S

In Santa Barbara along Calle Real, near Arroyo Burro Creek. Drilled observation well, Diameter 6 inches, depth 85 feet, perforated 65-85 feet. Altitude of land-surface datum 166.44 feet. Reported measurements provided by olty of Santa Barbara. Water-level records avallable 1989 to current year.

WATER LEVELS IN FEET BELOW LAND-SURFACE DATUM

\begin{tabular}{|c|c|c|c|c|c|c|c|}
\hline DATE & $\begin{array}{l}\text { WATER } \\
\text { LEVEL MS }\end{array}$ & DATE & $\begin{array}{l}\text { WATER } \\
\text { LEVEL MS }\end{array}$ & DATE & $\begin{array}{l}\text { WATER } \\
\text { LEVEL MS }\end{array}$ & DATE & $\begin{array}{l}\text { WATER } \\
\text { LEVEL MS }\end{array}$ \\
\hline $\begin{array}{ll}10, & 1991 \\
11 & \\
17 & \end{array}$ & 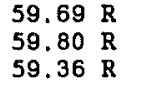 & $\begin{array}{lll}\text { FEB } & 19, & 1992 \\
\text { MAR } & 12 & \\
\text { APR } & 16 & \end{array}$ & 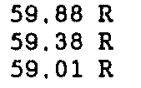 & $\begin{array}{lll}\text { MAY } & 13, & 1992 \\
\text { JUN } & 12 & \\
\text { JUL } & 06 & \end{array}$ & $\begin{array}{l}58.78 \mathrm{R} \\
58.65 \mathrm{R} \\
58.61 \mathrm{~S}\end{array}$ & $\begin{array}{lll}\text { JUL } & 14,1992 \\
\text { AUG } & 11 & \\
\text { SEP } & 10 & \end{array}$ & $\begin{array}{ll}58.66 & R \\
58.71 & R \\
58.75 & R\end{array}$ \\
\hline
\end{tabular}

SITE NUMBER 342600119445201

LOCAL NUMBER 004N027W18C02S

In Santa Barbara, south of Modoc Road between La Cumbre and Las Positas Roads. Drilled unused well. Diameter 4 inches, depth 88 feet, perforated 78-88 feet. Altitude of land-surface datum 159.69 feet. Measurements provided by city of Santa Barbara beginning in 1987 . Water-level records avallable 1987 to current year.

WATER LEVELS IN FEET BELOW LAND-SURFACE DATUM

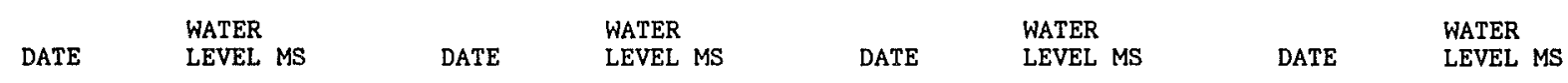

$\begin{array}{lllllllllll}\text { OCT } 10,1991 & 54.97 \mathrm{R} & \text { JAN 13, } 1992 & 54.67 \mathrm{R} & \text { APR } 16,1992 & 52.16 \mathrm{R} & \text { JUL } 14,1992 & 52.70 \mathrm{R} \\ \text { NOV } 11 & & 55.21 \mathrm{R} & \text { FEB } 19 & & 53.78 \mathrm{R} & \text { MAY } 13 & 51.71 \mathrm{R} & \text { AUG } 11 & 53.28 \mathrm{R} \\ \text { DEC } 17 & 55.24 \mathrm{R} & \text { MAR } 12 & & 53.02 \mathrm{R} & \text { JUN } 12 & 51.93 \mathrm{R} & \text { SEP } 10 & 53.79 \mathrm{R}\end{array}$

PERIOD OF RECORD HIGHEST 44.56 JUL 06, $1988 \quad$ LOWEST 55.24 DEC 17, 1991

SITE NUMBER 342600119445202

LOCAL NUMBER 004N027W18C03S

In Santa Barbara, south of Modoc Road between La Cumbre and Las Positas Roads. Drilled observation well. Diameter 2 inches, depth 257 feet, perforated 237-257 feet. Altitude of land-surface datum 159.77 feet. Measurements provided by city of Santa Barbara beginning in 1987. Water-level records available 1987 to current year.

WATER LEVELS IN FEET BELOW LAND-SURFACE DATUM

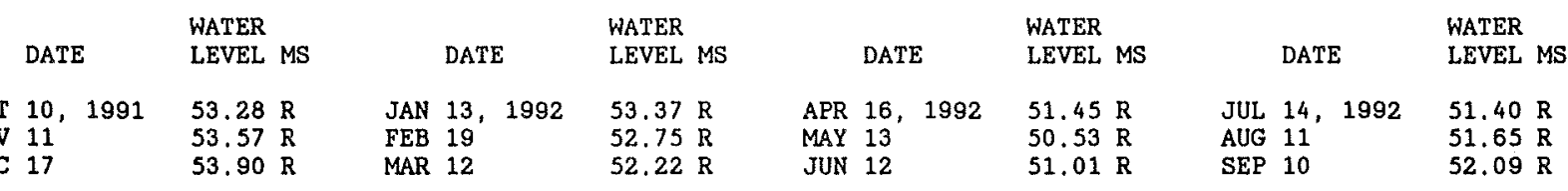

PERIOD OF RECORD HIGHEST 43.66 JUL 06,1988 LOWEST 53.90 DEC 17,1991 
SITE NUMBER 342525119444701

LOCAL NUMBER 004N027W18Q01S

In Santa Barbara, east of Modoc Road, and north of Palermo Road. Drilled observation well. Diameter and depth unknown. Altitude of land-surface datum 110 feet. Reported measurements provided by city of Santa Barbara. Water-level records available 1983 to current year.

WATER LEVELS IN FEET BELOW LAND-SURFACE DATUM

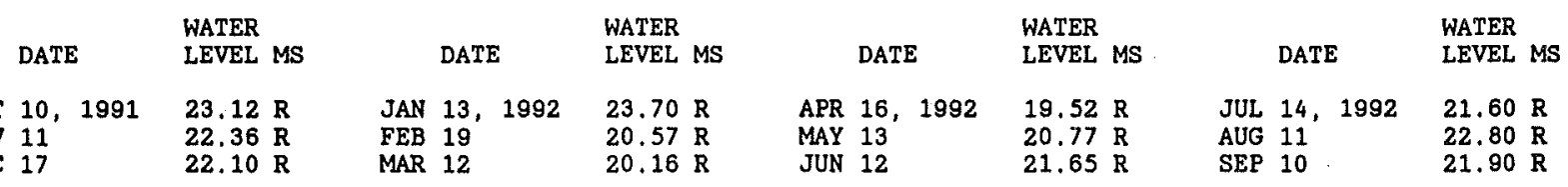

PERIOD OF RECORD HIGHEST 11.48 NOV 12, 1986 LOWEST 37.70 JAN 09, 1990

SITE NUMBER 342520119445501

LOCAL NUMBER 004N027W18Q04S

In Santa Barbara about 120 feet south-southwest of Torino Drive crossing over Arroyo Burro. Public-supply well. Diameter 8 inches, depth 240 feet, perforated 90-230 feet. Altitude of land-surface datum 105 feet. Reported measurements provided by city of Santa Barbara. Water-level records available 1984 to current year.

WATER LEVELS IN FEET BELOW LAND-SURFACE DATUM

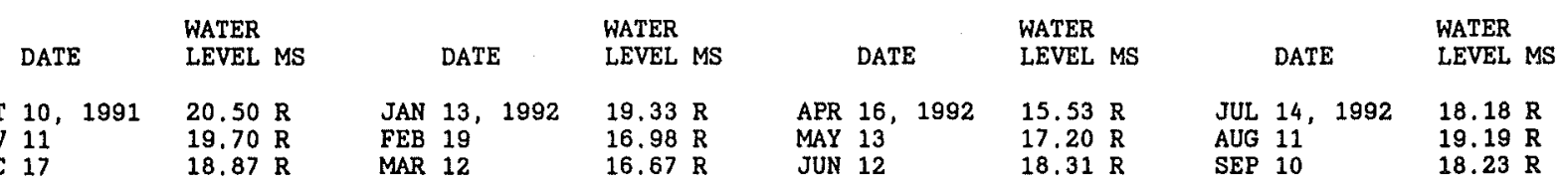

PERIOD OF RECORD HIGHEST 7.87 OCT 13, 1986 LOWEST 22.63 MAR 11,1991

SITE NUMBER 342512119443701

LOCAL NUMBER 004N027W19A01S

In Santa Barbara at the Hillside House. Drilled observation well. Diameter 2 inches, depth 360 feet, perforated 320-360 feet. Deepest of three oluster wells. Altitude of land-surface datum 86.08 feet. Reported measurements provided by city of Santa Barbara. Water-level records available 1989 to current year.

WATER LEVELS IN FEET BELOW LAND-SURFACE DATUM

\begin{tabular}{|c|c|c|c|c|c|c|c|}
\hline DATE & $\begin{array}{l}\text { WATER } \\
\text { LEVEL MS }\end{array}$ & DATE & $\begin{array}{l}\text { WATER } \\
\text { LEVEL MS }\end{array}$ & DATE & $\begin{array}{l}\text { WATER } \\
\text { LEVEL MS }\end{array}$ & DATE & $\begin{array}{l}\text { WATER } \\
\text { LEVEL MS }\end{array}$ \\
\hline $\begin{array}{l}10,1991 \\
11 \\
17\end{array}$ & $\begin{array}{l}2.30 \mathrm{R} \\
2.43 \mathrm{R} \\
1.58 \mathrm{R}\end{array}$ & $\begin{array}{ll}\text { JAN } & 13, \\
\text { FEB } & 19 \\
\text { MAR } & 12\end{array}$ & $\begin{array}{ll}2.36 & R \\
2.37 & R \\
2.23 & R\end{array}$ & $\begin{array}{lll}\text { APR } & 16,1992 \\
\text { MAY } & 13 & \\
\text { JUN } & 12 & \end{array}$ & $\begin{array}{ll}2.16 & \mathrm{R} \\
2.37 & \mathrm{R} \\
2.41 & \mathrm{R}\end{array}$ & $\begin{array}{ll}\text { JUL } & 14,1992 \\
\text { AUG } & 11 \\
\text { SEP } & 10\end{array}$ & $\begin{array}{l}2.34 \mathrm{R} \\
2.44 \mathrm{R} \\
2.39 \mathrm{R}\end{array}$ \\
\hline
\end{tabular}

PERIOD OF RECORD HIGHEST 0.16 JUN 05, 1989 LOWEST 3.79 FEB 12, 1991 
In Santa Barbara at the Hillside House. Drilled observation well. Diameter 2 inches, depth 180 feet, perforated 160-180 feet. Middle depth of three cluster wells. Altitude of land-surface datum 86.08 feet. Measurements provided by city of Santa Barbara beginning in 1989. Water-level records avallable 1989 to current year.

WATER LEVELS IN FEET BELOW LAND-SURFACE DATUM

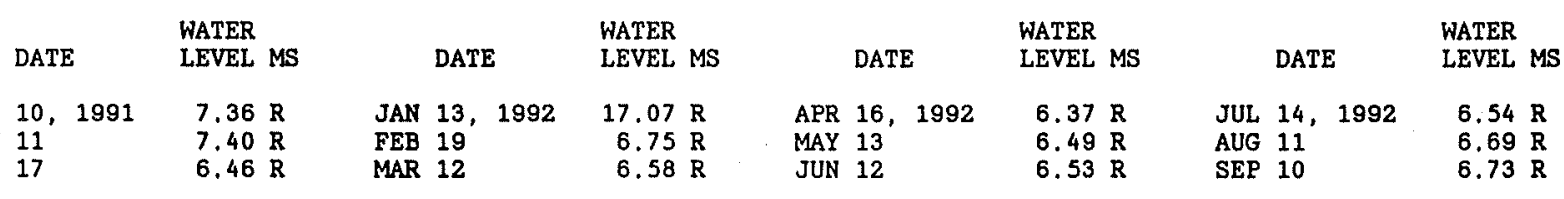

PERIOD OF RECORD HIGHEST 6.32 SEP 17, $1991 \quad$ LOWEST 17.07 JAN 13, 1992

SITE NUMBER 342512119443703

LOCAL NUMBER 004N027W19A03S

In Santa Barbara at the Hillside House. Drilled observation well. Diameter 2 inches, depth 110 feet, 105.6 feet in 1992, perforated 70-110 feet. Shallowest of three cluster wells. Altitude of land-surface datum 86.08 feet. Reported measurements provided by city of Santa Barbara. Water-level records available 1989 to current year.

WATER LEVELS IN FEET BELOW LAND-SURFACE DATUM

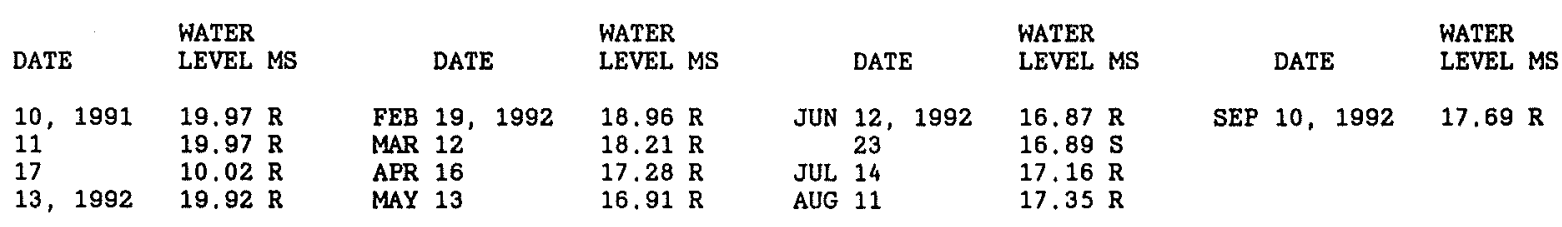

PERIOD OF RECORD HIGHEST 10.02 DEC 17, 1991 LOWEST 24.27 OCT 11, 1990

SITE NUMBER 342506119423801

LOCAL NUMBER 004N027W21B01S

In Santa Barbara near Wilson School. Unused artesian we11 in Santa Barbara Formation. Diameter 16 inches, depth 454 feet, perforated 145-350 feet. Altitude of land-surface datum 68 feet. Measurements provided by city of Santa Barbara beginning May 1976. Recorder installed 1979-84. Water-level records avallable 1931, 1948-50, 1956 to current year.

WATER LEVELS IN FEET BELOW LAND-SURFACE DATUM

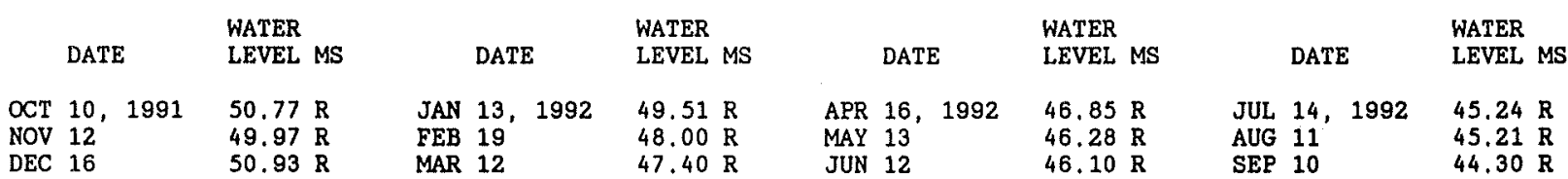

MAR $12 \quad 47.40 \mathrm{R}$

PERIOD OF RECORD

HIGHEST

37.00 JAN 23,1948

LOWEST 94.84 SEP 14,1948 
SITE NUMBER 342502119431401

LOCAL NUMBER 004N027W21E01S

In Santa Barbara. Drilled observation well. Diameter 2 inches, depth 410 feet, perforated 250-290 feet. Altitude of land-surface datum 90 feet. Reported measurements provided by city of Santa Barbara. Water-level records avallable 1989 to current year.

WATER LEVELS IN FEET BELOW LAND-SURFACE DATUM

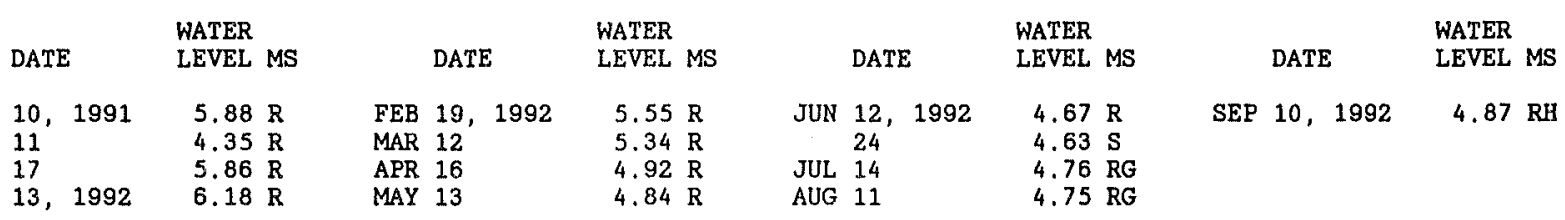

JAN $13,1992 \quad 6.18$

MAY $13 \quad 4.84 \mathrm{R}$

PERIOD OF RECORD

HIGHEST

3.42 MAR 06, 1989

LOWEST

9.22 MAR 11, 1991

SITE NUMBER 342502119431402

LOCAL NUMBER 004N027W21E02S

In Santa Barbara. Drilled observation well. Diameter 2 inches, depth 200 feet, perforated 180-200 feet. Altitude of land-surface datum 90 feet. Reported measurements provided by city of Santa Barbara. Water-level records available 1989 to current year.

WATER LEVELS IN FEET BELOW LAND-SURFACE DATUM (READINGS ABOVE LAND SURFACE INDICATED BY "+")

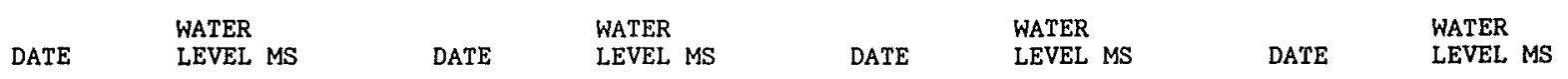

\begin{tabular}{|c|c|c|c|c|c|c|c|c|c|c|c|c|c|c|c|c|}
\hline $\begin{array}{l}\text { OCT } \\
\text { NOV } \\
\text { DEC }\end{array}$ & $\begin{array}{l}10 \\
11 \\
17\end{array}$ & 1991 & $\begin{array}{ll}1.86 & R \\
2.37 & R \\
1.30 & R\end{array}$ & $\begin{array}{l}\text { JAN } \\
\text { FEB } \\
\text { MAR }\end{array}$ & $\begin{array}{l}13 \\
19 \\
12\end{array}$ & 1992 & $\begin{array}{r}1.12 \mathrm{R} \\
+.14 \mathrm{R} \\
+.84 \mathrm{R}\end{array}$ & $\begin{array}{l}\text { APR } \\
\text { MAY } \\
\text { JUN }\end{array}$ & $\begin{array}{l}16 \\
13 \\
12\end{array}$ & 1992 & $\begin{array}{l}+2.82 \\
+3.20\end{array}$ & $\begin{array}{l}\mathrm{R} \\
\mathrm{R} \\
\mathrm{RF}\end{array}$ & $\begin{array}{l}\text { JUL } \\
\text { AUG } \\
\text { SEP }\end{array}$ & $\begin{array}{l}14, \\
11 \\
10\end{array}$ & 1992 & $\begin{array}{r}+3.15 \mathrm{RF} \\
+.73 \mathrm{RH} \\
+.23 \mathrm{RE}\end{array}$ \\
\hline
\end{tabular}

PERIOD OF RECORD HIGHEST +3.20 MAY 13, 1992 LOWEST 5.74 DEC 14, 1990

\section{SITE NUMBER 342502119431403}

LOCAL NUMBER 004N027W21E03S

In Santa Barbara near intersection of Robins and Valerio. Drilled observation well. Diameter 2 inches, depth

85.5 feet, perforated 52.5-82.5 feet. Altitude of land-surface datum 90 feet. Reported measurements provided by city of Santa Barbara. Water-level records available 1989 to current year.

WATER LEVELS IN FEET BELOW LAND-SURFACE DATUM (READINGS ABOVE LAND SURFACE INDICATED BY "+")

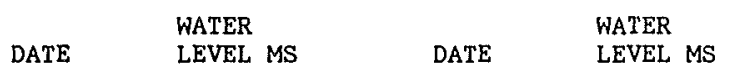

OCT 10,1991

NOV 11

DEC 17

$$
\begin{aligned}
& 1.79 \mathrm{R} \\
& 1.69 \mathrm{R} \\
& 1.13 \mathrm{R}
\end{aligned}
$$

$$
\begin{array}{r}
.41 \mathrm{R} \\
+.14 \mathrm{R}
\end{array}
$$$$
+1.24 \mathrm{R}
$$

DATE

APR 16, 1992

MAY 13

JUN 12
WATER

LEVEL MS

$+2.19 \mathrm{R}$

$+4.00 \mathrm{R}$

$\mathrm{RF}$
DATE

WATER

LEVEL MS

PERIOD OF RECORD HIGHEST +4.00 MAY 13, 1992 LOWEST 5.09 DEC 14, 1990

JUL 14,1992
AUG 11
SEP 10

$+3.88 \mathrm{RF}$ $+1.50 \mathrm{RH}$ $+1.04 \mathrm{RE}$ 
WELI. DESCRIPTIONS AND WATER-LEVEL MEASUREMENTS

WATER YEAR OCTOBER 1991 TO SEPTEMBER 1992

\begin{abstract}
SITE NUMBER 342456119425201
\end{abstract}
LOCAL NUMBER 004N027W21F01S

In Santa Barbara. Drilled observation well. Diameter 2 inches, depth 150 feet, perforated 140-150 feet. Altitude of land-surface datum 80 feet. Reported measurements provided by city of Santa Barbara. Water-level records available 1991 to current year.

WATER LEVELS IN FEET BELOW LAND-SURFACE DATUM

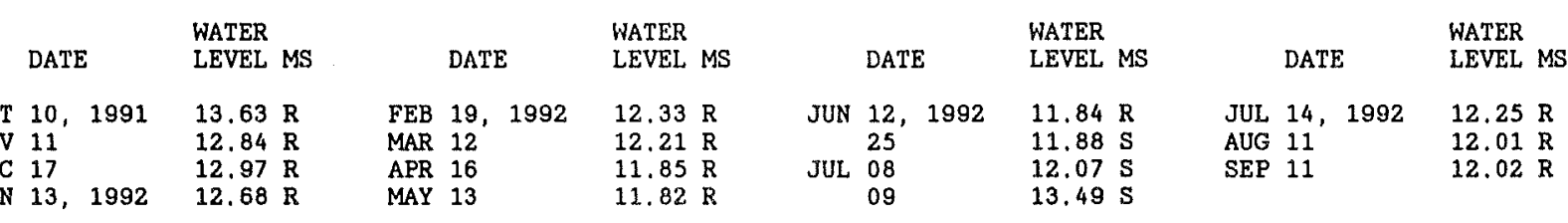

PERIOD OF RECORD HIGHEST 11.82 MAY 13, 1992 LOWEST 13.63 OCT 10, 1991

SITE NUMBER 342456119425202

LOCAL NUMBER 004N027W21F02S

In Santa Barbara. Drilled observation well. Diameter 2 inches, depth 80 feet, perforated $70-80$ feet. Altitude of land-surface datum 80 feet. Reported measurements provided by city of Santa Barbara, Water-1evel records available 1991 to current year.

WATER LEVELS IN FEET BELOW LAND-SURFACE DATUM

$\begin{array}{llllll} & \text { WATER } & & & \text { WATER } & \text { WATER } \\ \text { DATE } & \text { LEVEL MS } & \text { DATE } & \text { WEVEL MS } & \text { DATE } & \text { LEVEL MS }\end{array}$

$\begin{array}{llllllllll}\text { OCT } 10,1991 & 9.39 \mathrm{R} & \text { FEB } 19,1992 & 7.73 \mathrm{R} & \text { JUN } 12,1992 & 7.44 \mathrm{R} & \text { AUG } 11,1992 & 7.89 \mathrm{R} \\ \text { NOV } 11 & 9.55 \mathrm{R} & \text { MAR } 12 & & 7.34 \mathrm{R} & & 25 & 7.53 \mathrm{~S} & \text { SEP } 10 & \\ \text { DEC 17 } & 9.62 \mathrm{R} & \text { APR } 16 & & 7.09 \mathrm{R} & \text { JUL } 08 & 7.62 \mathrm{~S} & & \\ \text { JAN 13, } 1992 & 9.16 \mathrm{R} & \text { MAY } 13 & 7.21 \mathrm{R} & & 14 & 7.67 \mathrm{R} & & \end{array}$

PERIOD OF RECORD HIGHEST 7.09 APR 16, 1992 LOWEST 9.62 DEC 17, 1991

SITE NUMBER 342452119424001

LOCAL NUMBER 004N027W21G01S

In Santa Barbara. Drilled observation we11. Diameter 4 inches, depth measured 109.7 feet in 1992, original depth unknown, perforated 100-110 feet. Altitude of land-surface datum 66 feet. Reported measurements provided by city of Santa Barbara. Well recovers slowly to static water level after pumping. Water levels with a " $Z$ " status represent this condition. Water-level records avallable 1991 to current year.

WATER LEVELS IN FEET BELOW LAND-SURFACE DATUM

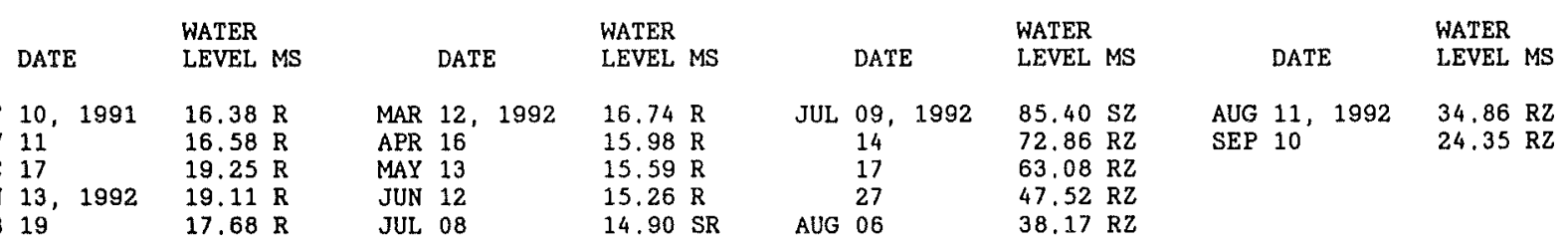

PERIOD OF RECORD HIGHEST 15.26 JUN 12, 1992 LOWEST 31.30 AUG 06, 1991 
WELL DESCRIPTIONS AND WATER-LEVEL MEASUREMENTS

WATER YEAR OCTOBER 1991 TO SEPTEMBER 1992

SITE NUMBER 342452119424002

LOCAL NUMBER 004N027W21G02S

In Santa Barbara. Drilled observation well. Diameter 2 inches, depth measured 38.6 feet in 1992 , perforated 28-38 feet. Altitude of land-surface datum 66 feet. Reported measurements provided by city of Santa Barbara. Water-level records available 1991 to current year.

WATER LEVELS IN FEET BELOW LAND-SURFACE DATUM

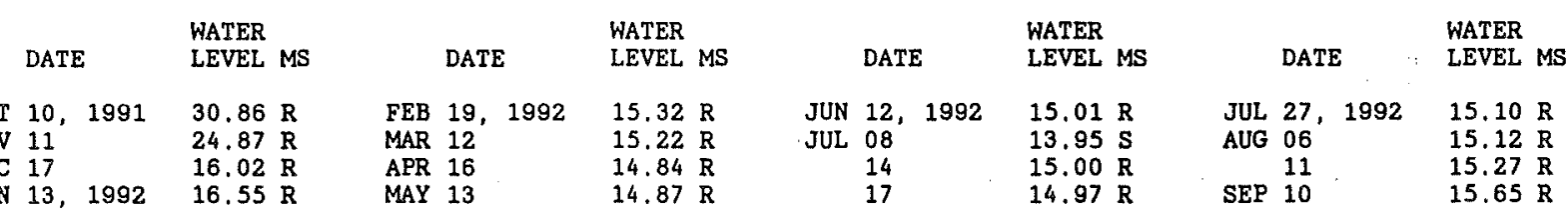

JAN $13,1992 \quad 16.55 \mathrm{R} \quad$ MAY $13 \quad 14.87 \mathrm{R}$

PERIOD OF RECORD HIGHEST 13.95 JUL 08, 1992 LOWEST 30.86 OCT 10, 1991

SITE NUMBER 342506119412201

LOCAL NUMBER 004N027W22A02S

In Santa Barbara on east Palm Avenue. Drilled observation well. Diameter 2 inches, depth measured 279.35 feet in 1992, perforated 270-290 feet. Shallowest of three cluster welis, Altitude of land-surface datum 6.52 feet. Reported measurements provided by city of Santa Barbara. Water-level records available 1987 to current year.

WATER LEVELS IN FEET BELOW LAND-SURFACE DATUM (READINGS ABOVE LAND SURFACE INDICATED BY "+")

$$
\begin{array}{lllll} 
& \text { WATER } & \text { WATER } & \text { WATER } \\
\text { DATE } & \text { LEVEL MS } & \text { DATE } & \text { LEVEL MS } & \text { DATE } \\
\text { LEVEL MS }
\end{array}
$$

MAY 13, 1992 RF JUN 25, $1992+9.20 \mathrm{R} \quad$ AUG $11,1992+8.31 \mathrm{RF}$

$\begin{array}{llllll}\text { JUN } 12 & \text { RF } & \text { JUL } 14 & +5.96 R F & \text { SEP } 10 & +9.82 \\ \text { RF }\end{array}$

PERIOD OF RECORD HIGHEST +9.20 JUN 25, 1992 LOWEST 58.30 SEP 13, 1990

\section{SITE NUMBER 342506119412202}

LOCAL NUMBER OO4N027W22A03S

In Santa Barbara on east Palm Avenue. Drilled observation well. Diameter 2 inches, depth measured 518.55 feet in 1992, original depth 290 feet, perforated 505-525 feet. Middle depth of three cluster wells. Altitude of land-surface datum 6.52 feet. Reported measurements provided by city of Santa Barbara. Water-level records available 1987 to current year.

WATER LEVELS IN FEET BELOW LAND-SURFACE DATUM (READINGS ABOVE LAND SURFACE INDICATED BY "+")

$$
\begin{array}{lllll} 
& & \text { WATER } & \text { WATER } \\
\text { DATE } & \text { WEVEL MS } & \text { DATE } & \text { LEVEL MS } & \text { DATE }
\end{array}
$$

MAY 13, 1992 RF JUN 25, $1992+10,65 \mathrm{R}$ AUG $11,1992+11.48 \mathrm{RF}$

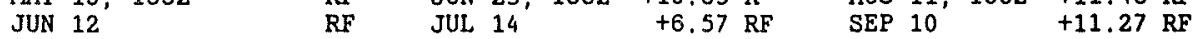

PERIOD OF RECORD HIGHEST +10.7 FEB 06, 1989 LOWEST 45.49 SEP 13, 1990 
WELL DESCRIPTIONS AND WATER-LEVEL MEASUREMENTS

WATER YEAR OCTOBER 1991 TO SEPTEMBER 1992

SITE NUMBER 342506119412203

LOCAL NUMBER 004N027W22A04S

In Santa Barbara on east Palm Avenue. Drilled observation well. Diameter 5 inches $0-90$ feet, 4 inches 90-740 feet, depth 740 feet, perforated 692-732 feet. Deepest of three cluster wells, Altitude of land-surface datum 6.52 feet. Measurements provided by city of Santa Barbara beginning in 1987 . Water-level records available 1987 to current year.

WATER LEVELS IN FEET BELOW LAND-SURFACE DATUM (READINGS ABOVE LAND SURFACE INDICATED BY "+")

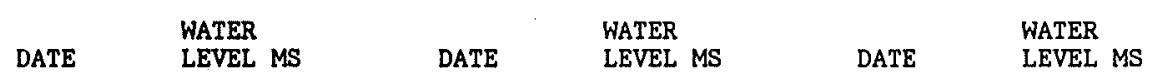

$\begin{array}{llllll}\text { MAY 13, } 1992 & \text { RF } & \text { JUN 25, } 1992 & \text { F } & \text { AUG 11, } 1992+14.16 \text { RF } \\ \text { JUN 12 } & \text { RF } & \text { JUL 14 } & 0.94 \text { RG } & \text { SEP } 10 & \\ +15.00 & \text { RF }\end{array}$

PERIOD OF RECORD HIGHEST +15.00 SEP 10, 1992 LOWEST 88.63 SEP 13, 1990

SITE NUMBER 342510119413801

LOCAL NUMBER 004N027W22B06S

In Santa Barbara, near Cota Street and Vera Cruz Avenue. Drilled public-supply water-table well. Diameter 14 inches, depth reported 700 feet, perforated $210-240,415-435,465-480,560-580,600-620,640-670$ feet. Altitude of land-surface datum 35 feet. Reported measurements provided by city of Santa Barbara. Water-level records available 1976, 1982 to current year.

WATER LEVELS IN FEET BELOW LAND-SURFACE DATUM (READINGS ABOVE LAND SURFACE INDICATED BY "+")

$\begin{array}{lllllll} & \text { WATER } & \text { WATER } & & \text { WATER } & \text { WATER } \\ \text { DATE } & \text { LEVEL MS } & \text { DATE } & \text { LEVEL MS } & \text { DATE } & \text { LEVEL MS } & \text { DATE }\end{array}$

\begin{tabular}{|c|c|c|c|c|c|c|c|c|c|c|}
\hline $\begin{array}{ll}\text { OCT } & 10, \\
\text { NOV } & 12 \\
\text { DEC } & 16\end{array}$ & 1991 & $\begin{array}{l}8.78 \mathrm{R} \\
6.46 \mathrm{R} \\
7.32 \mathrm{R}\end{array}$ & $\begin{array}{ll}\text { JAN } & 13, \\
\text { FEB } & 19 \\
\text { MAR } & 12\end{array}$ & 1992 & $\begin{array}{rl}5.52 & R \\
3.95 \mathrm{R} \\
\mathrm{R}\end{array}$ & $\begin{array}{cc}\text { APR } & 1 \\
\text { MAY } & 1 \\
\text { JUN } & 1\end{array}$ & $\begin{array}{l}16,1992 \\
13 \\
12\end{array}$ & $\begin{array}{l}3.80 \\
4.66 \\
2.08\end{array}$ & $\begin{array}{l}\mathrm{R} \\
\mathrm{R} \\
\mathrm{R}\end{array}$ & $\begin{array}{lll}\text { JUL } & 14,1992 \\
\text { AUG } & 11 & \\
\text { SEP } & 10 & \end{array}$ \\
\hline
\end{tabular}

PERIOD OF RECORD HIGHEST $+1.30 \quad$ SEP $10,1992 \quad$ LOWEST 79.87 JUL 07,1986

SITE NUMBER 342508119413702

LOCAL NUMBER 004N027W22B08S

In Santa Barbara in Vera Cruz Park. Drilled observation well. Diameter 2 inches, depth 780 feet, perforated 760-780 feet. Altitude of land-surface datum 17.37 feet. Reported measurements provided by city of Santa Barbara. Water-level records available 1992

WATER LEVELS IN FEET BELOW LAND-SURFACE DATUM (READINGS ABOVE LAND SURFACE INDICATED BY "+")

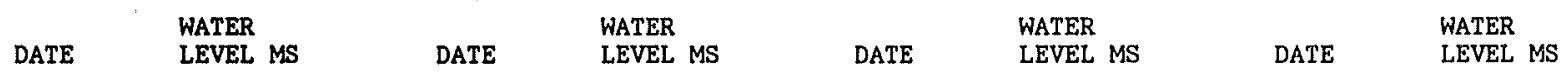
JUN 23, 1992
0.71 SG
JUL $14,1992+0.30 \mathrm{RF}$
AUG 11, $1992+0.76 \mathrm{RF}$
SEP 10,1992
$+1.95 \mathrm{RF}$

PERIOD OF RECORD HIGHEST -- LOWEST --

SITE NUMBER 342508119413703

LOCAL NUMBER 004N027W22B09S

In Santa Barbara in Vera Cruz Park. Drilled observation well. Diameter 2 inches, depth 669.3 feet, perforated 650-670 feet. Altitude of land-surface datum 17.37 feet. Reported measurements provided by city of Santa Barbara. Water-level records available 1992 .

WATER LEVELS IN FEET BELOW LAND-SURFACE DATUM (READINGS ABOVE LAND SURFACE INDICATED BY "+")

\begin{tabular}{|c|c|c|c|c|c|c|c|}
\hline & WATER & & WATER & & WATER & & WATER \\
\hline DATE & LEVEL MS & DATE & LEVEL MS & DATE & LEVEL MS & DATE & LEVEL MS \\
\hline 23,1992 & +1.47 & JUL 14,1992 & $12.67 \mathrm{R}$ & AUG 11,1992 & $+2.39 \mathrm{RF}$ & SEP 10,1992 & $+3.37 \mathrm{RF}$ \\
\hline
\end{tabular}

PERIOD OF RECORD HIGHEST 12.67 JUL 14,1992 LOWEST 12.67 JUL 14,1992 
SITE NUMBER 342508119413704

LOCAL NUMBER 004N027W22B1OS

In Santa Barbara in Vera Cruz Park. Drilled observation well. Diameter 2 inches, depth 440.9 feet, perforated 420-440 feet. Altitude of land-surface datum 17.37 feet. Reported measurements provided by city of Santa Barbara. Water-level records available 1992 .

WATER LEVELS IN FEET BELOW LAND-SURFACE DATUM (READINGS ABOVE LAND SURFACE INDICATED BY "+")

\begin{tabular}{|c|c|c|c|c|c|c|c|c|}
\hline DATE & $\begin{array}{l}\text { WATER } \\
\text { LEVEL MS }\end{array}$ & DATE & $\begin{array}{l}\text { WATER } \\
\text { LEVEL MS }\end{array}$ & DATE & $\begin{array}{l}\text { WATER } \\
\text { LEVEL MS }\end{array}$ & & DATE & $\begin{array}{l}\text { WATER } \\
\text { LEVEL MS }\end{array}$ \\
\hline JUN 23,1992 & +1.21 & JUL 14,1992 & $0.99 \mathrm{RG}$ & AUG 11,1992 & $+1.81 \mathrm{RF}$ & SEP & 10,1992 & $+2.66 \mathrm{RF}$ \\
\hline
\end{tabular}

SITE NUMBER 342508119413705

LOCAL NUMBER 004N027W22B11S

In Santa Barbara in Vera Cruz Park. Drilled observation well. Diameter 2 inches, depth 220.5 feet, perforated 200-220 feet. Altitude of land-surface datum 17.37 feet. Reported measurements provided by city of Santa Barbara. Water-level records available 1992.

WATER LEVELS IN FEET BELOW LAND-SURFACE DATUM (READINGS ABOVE LAND SURFACE INDICATED BY "+")

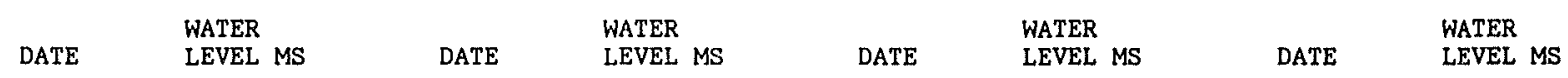

JUN 23, $1992 \quad 1.51 \mathrm{SG}$ JUL 14, $1992 \quad 5.91 \mathrm{RG} \quad$ AUG 11, $1992 \quad 1.99 \mathrm{R} \quad$ SEP $10,1992 \quad+0.30 \mathrm{R}$

PERIOD OF RECORD HIGHEST to.30 SEP 10, 1992 LOWEST 1.99 AUG 11, 1992

SITE NUMBER 342513119414901

LOCAL NUMBER 004N027W22C01S

In Santa Barbara, south of Anacapa and De La Guerra Streets in parking lot of City Hall. Drilled public-supply well. Diameter 14 inches 0-300 feet, 12 inches 300-635 feet, depth 635 feet, perforated 180-215, 300-315, 430-440, 510-520, 535-565, 580-615 feet. Altitude of land-surface datum 40 feet. Reported measurements provided by city of Santa Barbara. Water-level records available 1976, 1982 to current year.

WATER LEVELS IN FEET BELOW LAND-SURFACE DATUM

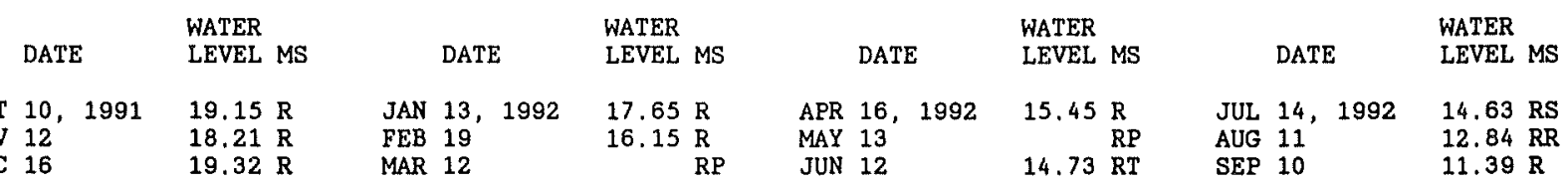

PERIOD OF RECORD HIGHEST 10.53 MAR 07, 1989 LOWEST 111.00 APR 03, 1985

SITE NUMBER 342501119421001

LOCAL NUMBER 004N027W22E01S

In Santa Barbara. Drilled observation well. Diameter 2 inches, depth 120 feet, perforated 110-120 feet. Altitude of land-surface datum 40 feet. Reported measurements provided by city of Santa Barbara. Water-level records available 1991 to current year.

WATER LEVELS IN FEET BELOW LAND-SURFACE DATUM

\begin{tabular}{|c|c|c|c|c|c|c|c|}
\hline DATE & $\begin{array}{l}\text { WATER } \\
\text { LEVEL MS }\end{array}$ & DATE & $\begin{array}{l}\text { WATER } \\
\text { LEVEL MS }\end{array}$ & DATE & $\begin{array}{l}\text { WATER } \\
\text { LEVEL MS }\end{array}$ & DATE & $\begin{array}{l}\text { WATER } \\
\text { LEVEL MS }\end{array}$ \\
\hline $\begin{array}{ll}10, & 1991 \\
11 & \\
17 & \\
13, & 1992\end{array}$ & $\begin{array}{ll}29.21 & R \\
29.05 & R \\
28.95 & R \\
28.98 & R\end{array}$ & $\begin{array}{lll}\text { FEB } & 19, & 1992 \\
\text { MAR } & 12 & \\
\text { APR } & 16 & \\
\text { JUN } & 12 & \end{array}$ & $\begin{array}{ll}28.17 & R \\
27.66 & R \\
27.07 & R \\
26.57 & R\end{array}$ & $\begin{array}{lll}\text { JUN } & 26, & 1992 \\
\text { JUL } & 08 & \\
& 14 & \\
\text { AUG } & 11 & \end{array}$ & $\begin{array}{l}26.12 \mathrm{~S} \\
26.05 \mathrm{~S} \\
26.37 \mathrm{R} \\
25.26 \mathrm{R}\end{array}$ & SEP 10,1992 & $26.07 \mathrm{R}$ \\
\hline
\end{tabular}

PERIOD OF RECORD HIGHEST 25.26 AUG 11, 1992 LOWEST 31.78 AUG 07, 1991 
WELL DESCRIPTIONS AND WATER-LEVEL MEASUREMENTS

WATER YEAR OCTOBER 1991 TO SEPTEMBER 1992

SITE NUMBER 342501119421002

LOCAL NUMBER 004N027W22E02S

In Santa Barbara. Drilled observation well. Diameter 2 inches, depth 70 feet, perforated 60-70 feet. Altitude of land-surface datum 40 feet. Reported measurements provided by city of Santa Barbara. Water-level records available 1991 to current year.

WATER LEVELS IN FEET BELOW LAND-SURFACE DATUM

\begin{tabular}{|c|c|c|c|c|c|c|c|c|c|c|c|c|}
\hline DATE & $\begin{array}{l}\text { WATER } \\
\text { LEVEL MS }\end{array}$ & & DATE & $\begin{array}{l}\text { WATER } \\
\text { LEVEL MS }\end{array}$ & & DATE & & $\begin{array}{l}\text { WATER } \\
\text { LEVEL MS }\end{array}$ & & DATE & & $\begin{array}{l}\text { WATER } \\
\text { LEVEL MS }\end{array}$ \\
\hline $\begin{array}{ll}10, & 1991 \\
11 & \\
17 & \\
13, & 1992\end{array}$ & 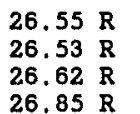 & $\begin{array}{l}\text { FEB } \\
\text { MAR } \\
\text { APR } \\
\text { JUN }\end{array}$ & $\begin{array}{ll}19, & 1992 \\
12 & \\
16 & \\
12 & \end{array}$ & $\begin{array}{ll}25.36 & R \\
24.54 & R \\
23.80 & R \\
23.39 & R\end{array}$ & $\begin{array}{l}\text { JUN } \\
\text { JUL } \\
\text { AUG }\end{array}$ & $\begin{array}{l}26 \\
08 \\
14 \\
11\end{array}$ & 1992 & $\begin{array}{ll}23.10 & \mathrm{~S} \\
23.04 & \mathrm{~S} \\
23.36 & \mathrm{R} \\
23.41 & \mathrm{R}\end{array}$ & SEP & 10 & 1992 & $23.50 \mathrm{R}$ \\
\hline
\end{tabular}

PERIOD OF RECORD HIGHEST 23,04 JUL 08, 1992 LOWEST 27.79 AUG 07, 1991

SITE NUMBER 342455119412401

LOCAL NUMBER O04N027W22G02S

In Santa Barbara near intersection of Gutierrez Strest and Motor Way. Drilled observation well. Diameter 2 inches, depth 200 feet, depth of hole 740 feet, perforated 180-200 feet. Altitude of land-surface datum 20.24 feet. Reported measurements provided by city of Santa Barbara. Water-level records available 1987 to current year.

WATER LEVELS IN FEET BELOW LAND-SURFACE DATUM

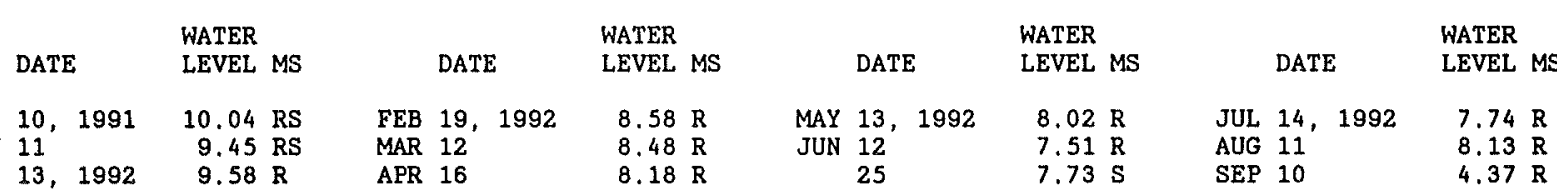

JAN $13,1992 \quad 9.58 \mathrm{R} \quad$ APR $16 \quad 8.18 \mathrm{R}$

PERIOD OF RECORD HIGHEST 4.37 SEP 10, 1992 LOWEST 32.53 SEP 12, 1990

SITE NUMBER 342455119412402

LOCAL NUMBER 004N027W22G03S

In Santa Barbara near intersection of Gutierrez Street and Motor Way. Drilled observation well. Diameter 2 inches, depth 420 feet, depth of hole 740 feet, perforated $400-420$ feet. Altitude of land-surface datum 20.24 feet. Reported measurements provided by city of Santa Barbara. Water-level records available 1987 to current year.

WATER LEVELS IN FEET BELOW LAND-SURFACE DATUM

\begin{tabular}{|c|c|c|c|c|c|c|c|}
\hline DATE & $\begin{array}{l}\text { WATER } \\
\text { LEVEL MS }\end{array}$ & DATE & $\begin{array}{l}\text { WATER } \\
\text { LEVEL MS }\end{array}$ & DATE & $\begin{array}{l}\text { WATER } \\
\text { LEVEL MS }\end{array}$ & DATE & $\begin{array}{l}\text { WATER } \\
\text { LEVEL MS }\end{array}$ \\
\hline $\begin{array}{ll}10, & 1991 \\
11 & \\
13, & 1992\end{array}$ & 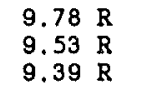 & $\begin{array}{lll}\text { FEB } & 19, & 1992 \\
\text { MAR } & 12 & \\
\text { APR } & 16\end{array}$ & $\begin{array}{ll}8.07 & \mathrm{R} \\
7.86 & \mathrm{R} \\
7.65 & \mathrm{R}\end{array}$ & $\begin{array}{lll}\text { MAY } & 13, & 1992 \\
\text { JUN } & 12 & \\
\text { JUL } & 14\end{array}$ & $\begin{array}{l}7.66 \mathrm{R} \\
7.56 \mathrm{R} \\
7.07 \mathrm{R}\end{array}$ & $\begin{array}{l}\text { AUG } 11,1992 \\
\text { SEP } 10\end{array}$ & $\begin{array}{l}7.47 \mathrm{R} \\
6.52 \mathrm{R}\end{array}$ \\
\hline
\end{tabular}

PERIOD OF RECORD HIGHEST 5.77 DEC 14, 1988 LOWEST 44.62 SEP 12, 1990 
SITE NUMBER 342455119412403

LOCAL NUMBER 004N027W22G04S

In Santa Barbara near intersection of Gutierrez Street and Motor Way. Drilled observation well. Diameter 5 inches, depth 690 feet, perforated 650-690 feet. Altitude of land-surface datum 20.24 feet. Reported measurements provided by city of Santa Barbara. Water-level records available 1987 to current year.

WATER LEVELS IN FEET BELOW LAND-SURFACE DATUM

\begin{tabular}{|c|c|c|c|c|c|c|c|}
\hline DATE & $\begin{array}{l}\text { WATER } \\
\text { LEVEL MS }\end{array}$ & DATE & $\begin{array}{l}\text { WATER } \\
\text { LEVEL MS }\end{array}$ & DATE & $\begin{array}{l}\text { WATER } \\
\text { LEVEL MS }\end{array}$ & DATE & $\begin{array}{l}\text { WATER } \\
\text { LEVEL MS }\end{array}$ \\
\hline $\begin{array}{ll}10, & 1991 \\
11 & \\
13, & 1992\end{array}$ & $\begin{array}{l}9.74 \mathrm{R} \\
7.99 \mathrm{R} \\
7.18 \mathrm{R}\end{array}$ & $\begin{array}{lll}\text { FEB } & 19, & 1992 \\
\text { MAR } & 12 & \\
\text { APR } & 16 & \end{array}$ & $\begin{array}{l}5.19 \mathrm{R} \\
6.19 \mathrm{R} \\
4.23 \mathrm{R}\end{array}$ & $\begin{array}{lll}\text { MAY } & 13,1992 \\
\text { JUN } & 12 & \\
\text { JUL } & 07 & \end{array}$ & $\begin{array}{l}3.39 \mathrm{R} \\
3.22 \mathrm{R} \\
1.82 \mathrm{~S}\end{array}$ & $\begin{array}{lll}\text { JUL } & 14,1992 \\
\text { AUG } & 11 \\
\text { SEP } & 10\end{array}$ & $\begin{array}{ll}1.69 & R \\
0.96 & R \\
& R F\end{array}$ \\
\hline
\end{tabular}

PERIOD OF RECORD HIGHEST 0.59 NOV 10,1988 LOWEST 98.74 SEP 12, 1990

SITE NUMBER 342444119421801

LOCAL NUMBER 004N027W22M01S

In Santa Barbara. Drilled observation well. Diameter 2 inches, depth 120 feet, perforated 100-110 feet. Altitude of land-surface datum 53 feet. Reported measurements provided by city of Santa Barbara. Water-level records available 1991 to current year.

WATER LEVELS IN FEET BELOW LAND-SURFACE DATUM

\begin{tabular}{|c|c|c|c|c|c|c|c|}
\hline DATE & $\begin{array}{l}\text { WATER } \\
\text { LEVEL MS }\end{array}$ & DATE & $\begin{array}{l}\text { WATER } \\
\text { LEVEL MS }\end{array}$ & DATE & $\begin{array}{l}\text { WATER } \\
\text { LEVEL MS }\end{array}$ & DATE & $\begin{array}{l}\text { WATER } \\
\text { LEVEL MS }\end{array}$ \\
\hline $\begin{array}{l}10,1991 \\
11 \\
17\end{array}$ & 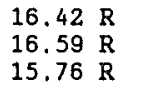 & $\begin{array}{lll}\text { JAN } & 13,1992 \\
\text { FEB } & 19 & \\
\text { MAR } & 12 & \end{array}$ & 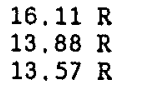 & $\begin{array}{lll}\text { APR } & 16, & 1992 \\
\text { MAY } & 13 & \\
\text { JUN } & 12 & \end{array}$ & $\begin{array}{l}12.92 \mathrm{R} \\
13.21 \mathrm{R} \\
13.50 \mathrm{R}\end{array}$ & $\begin{array}{lll}\text { JUL } & 14, & 1992 \\
\text { AUG } & 11 \\
\text { SEP } & 10\end{array}$ & $\begin{array}{l}13.74 \mathrm{R} \\
14.02 \mathrm{R} \\
14.37 \mathrm{R}\end{array}$ \\
\hline
\end{tabular}

PERIOD OF RECORD HIGHEST 12.92 APR 16, 1992 LOWEST 16.59 NOV 11, 1991

SITE NUMBER 342444119421802

LOCAL NUMBER 004N027W22MO2S

In Santa Barbara. Drilled observation well. Diameter 2 inches, depth 65.7 feet, perforated 55-65 feet. Altitude of land-surface datum 53 feet. Reported measurements provided by city of Santa Barbara. Water-level records available 1991 to current year.

WATER LEVELS IN FEET BELOW LAND-SURFACE DATUM

\begin{tabular}{|c|c|c|c|c|c|c|c|}
\hline DATE & $\begin{array}{l}\text { WATER } \\
\text { LEVEL MS }\end{array}$ & DATE & $\begin{array}{l}\text { WATER } \\
\text { LEVEL MS }\end{array}$ & DATE & $\begin{array}{l}\text { WATER } \\
\text { LEVEL MS }\end{array}$ & DATE & $\begin{array}{l}\text { WATER } \\
\text { LEVEL MS }\end{array}$ \\
\hline $\begin{array}{ll}10, & 1991 \\
11 & \\
17 & \end{array}$ & $\begin{array}{l}16.01 \mathrm{R} \\
16.31 \mathrm{R} \\
15.58 \mathrm{R}\end{array}$ & $\begin{array}{lll}\text { JAN } & 13,1992 \\
\text { FEB } & 19 & \\
\text { MAR } & 12 & \end{array}$ & $\begin{array}{l}15.87 \mathrm{R} \\
13.53 \mathrm{R} \\
13.29 \mathrm{R}\end{array}$ & $\begin{array}{lll}\text { APR } & 16,1992 \\
\text { MAY } & 14 \\
\text { JUN } & 12\end{array}$ & $\begin{array}{l}12.75 \mathrm{R} \\
13.04 \mathrm{R} \\
13.22 \mathrm{R}\end{array}$ & $\begin{array}{lll}\text { JUL } & 14,1992 \\
\text { AUG } & 11 \\
\text { SEP } & 10\end{array}$ & $\begin{array}{l}13.55 \mathrm{R} \\
13.82 \mathrm{R} \\
14.24 \mathrm{R}\end{array}$ \\
\hline
\end{tabular}

$\begin{array}{lllll}\text { DEC } 17 & 15.58 \mathrm{R} & \text { MAR } 12 & 13.29 \mathrm{R}\end{array}$

PERIOD OF RECORD HIGHEST 12.75 ARR 16, 1992 LOWEST 16.31 NOV 11, 1991 
WELL DESCRIPTIONS AND WATER-LEVEL MEASUREMENTS

WATER YEAR OCTOBER 1991 TO SEPTEMBER 1992

SITE NUMBER 342507119411101

LOCAL NUMBER 004N027W23D01S

In Santa Barbare, south of Highway 101 near sewage treatment plant. Drilled observation water-table well in Santa Barbara Formation of Pleistocene age. Diameter 8 inches, depth 380 feet, depth of hole 400 feet, perforated 240-380 feet. Altitude of land-surface datum 12 feet. Reported measurements provided by city of Santa Barbara. Water-level records available 1979 to current year.

WATER LEVELS IN FEET BELOW LAND-SURFACE DATUM (READINGS ABOVE LAND SURFACE INDICATED BY "+")

$\begin{array}{llllll} & \text { WATER } & & & \text { WATER } & \text { WATER } \\ \text { DATE } & \text { LEVEL MS } & \text { DATE } & \text { LEVEL MS } & \text { DATE } & \text { LEVEL MS }\end{array}$

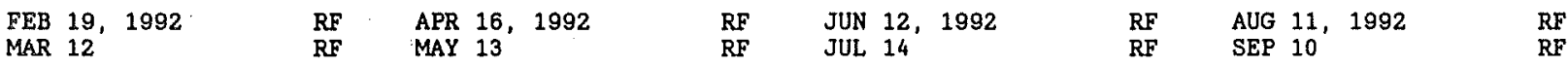

PERIOD OF RECORD HIGHEST +1.50 FEB 21, 1983 LOWEST 35.98 SEP 04, 1979

\section{SITE NUMBER 342501119410501}

LOCAL NUMBER 004N027W23E05S

In Santa Barbara on west side of sewage-treatment plant. Drilled observation well. Diameter 5 inches $0-100$ feet, 4.5 inches 100-714 feet, depth 760 feet, screened 674-714 feet. Altitude of land-surface datum 11.3 feet.

Reported measurements provided by city of Santa Barbara, Water-level records available 1986 to current year.

WATER LEVELS IN FEET BELOW LAND-SURFACE DATUM (READINGS ABOVE LAND SURFACE INDICATED BY "+")

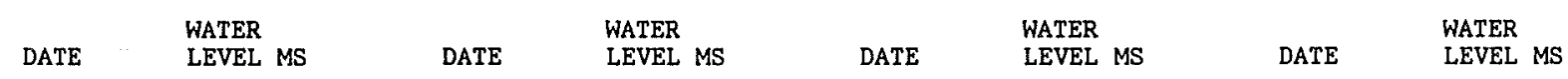

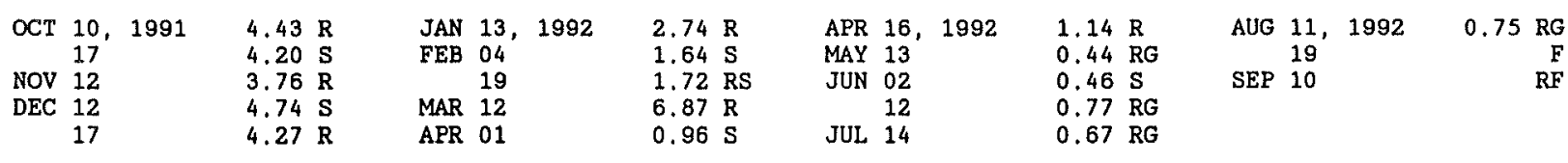

PERIOD OF RECORD HIGHEST +2.04 FEB 02, 1989 LOWEST 37.87 OCT 17,1990

SITE NUMBER 342501119410502

LOCAL NUMBER 004N027W23E06S

In Santa Barbara on west side of sewage-treatment plant. Drilled observation well. Diameter 2 inches, depth 495 feet, perforated 475-495 feet. Altitude of land-surface datum 11.31 feet. Used as saltwater intrusion monitor. Reported measurements provided by city of Santa Barbara. Water-level records available 1986 to current year.

WATER LEVELS IN FEET BELOW LAND-SURFACE. DATUM

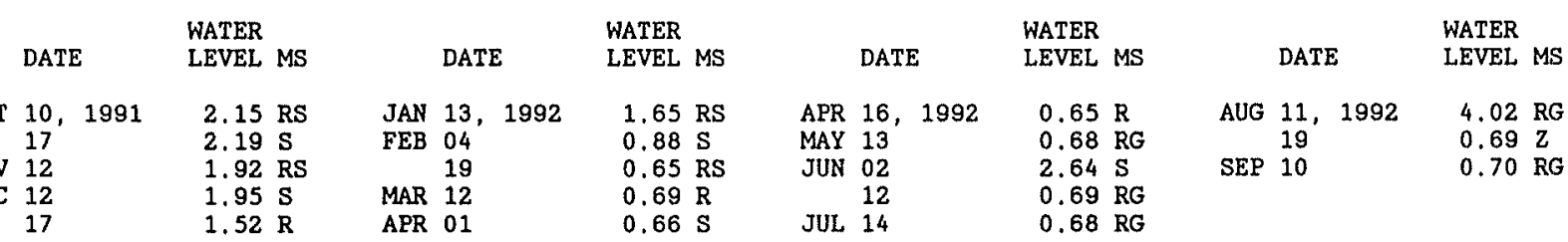

PERIOD OF RECORD HIGHEST 0.09 JUL 19,1988 LOWEST 35.38 OCT 17,1990 
In city of Santa Barbara Public Works parking lot, about 50 feet north of Cabrillo Boulevard, and $0.5 \mathrm{mtle}$ west of Cabrillo Park. Drilled observation well. Diameter 2 inches, depth 380 feet, perforated $360-380$ feet. Altitude of land-surface datum 6.5 feet. Used as saltwater intrusion monitor. Reported measurements provided by city of Santa Barbara. Water-level records available 1986 to current year.

WATER LEVELS IN FEET BELOW LAND-SURFACE DATUM (READINGS ABOVE LAND SURFACE INDICATED BY "+")

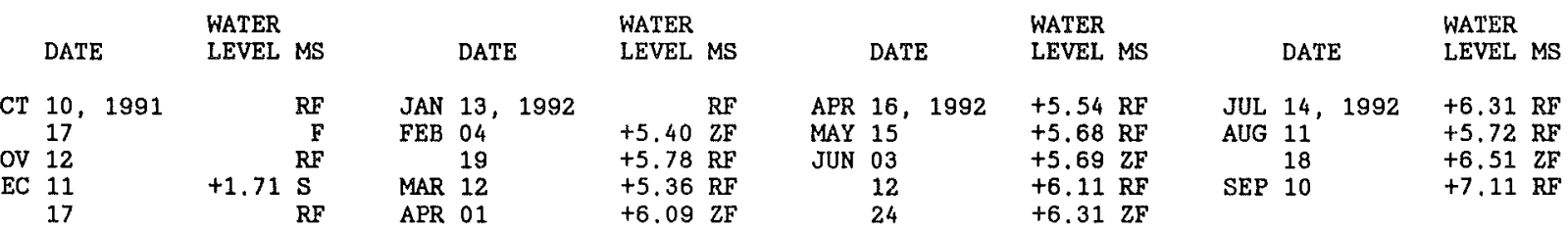

PERIOD OF RECORD HIGHEST +7.11 SEP 10, 1992 LOWEST 44.35 SEP 14, 1986

SITE NUMBER 342455119405603

LOCAL NUMBER 004N027W23F03S

In city of Santa Barbara Public Works parking lot about 50 feet north of Cabrillo Boulevard, and $0.5 \mathrm{mile}$ west of Cabrillo Park. Drilled observation well. Diameter 2 inches, depth 550 feet, perforated $530-550$ feet. Altitude of land-surface datum 6.8 feet. Used as saltwater intrusion monitor. Reported measurements provided by city of Santa Barbara. Water-level records available 1986 to current year.

WATER LEVELS IN FEET BELOW LAND-SURFACE DATUM (READINGS ABOVE LAND SURFACE INDICATED BY "+")

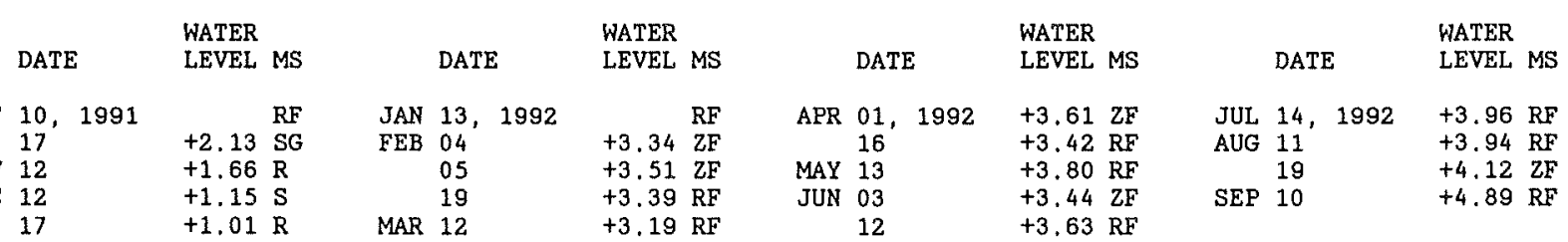

PERIOD OF RECORD HIGKEST +4.89 SEP $10,1992 \quad$ LOWEST 25.78 OCT 17,1990

SITE NUMBER 342455119405604

LOCAL NUMBER 004N027W23F04S

In ctty of Santa Barbara Public Works parking lot about 50 feet north of Cabrillo Boulevard, and 0.5 mile west of Cabrillo Park. Drilled observation well. Diameter 4.5 inches, depth 802 feet, perforated $762-802$ feet. Altitude of land-surface datum 6.8 feet. Reported measurements provided by city of Santa Barbara. Water-level records available 1986 to current year.

WATER LEVELS IN FEET BELOW LAND-SURFACE DATUM

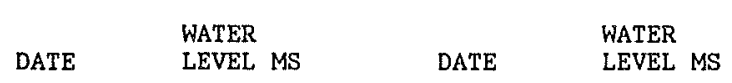

OCT 10,1991

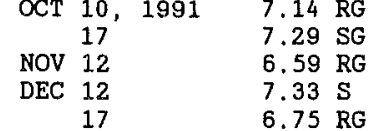

JAN 13,1992

FEB 04

$\begin{array}{ll}19 \\ M A R & 12\end{array}$

$\begin{array}{ll}\text { MAR } & 12 \\ \text { APR } & 01\end{array}$
LEVEL MS

$5.73 \mathrm{RG}$

5.00 SG

$4.87 \mathrm{RG}$

5.14 RG

$4.41 \mathrm{~S}$

PERIOD OF RECORD HIGHEST

1.77 FEB 16,1989

WATER

LEVEL MS

4.65 RG

4.27 RG

$4.36 \mathrm{~S}$

5.31 RG

3. $82 \mathrm{RG}$
WATER

DATE LEVEL MS

JUL 14

LOWEST 36.76 SEP 12,1990 
WELL DESCRIPTIONS AND WATER-LEVEL MEASUREMENTS

WATER YEAR OCTOBER 1991 TO SEPTEMBER 1992

SITE NUMBER 342452119405504

LOCAL NUMBER 004N027W23F08S

In Santa Barbara, about 300 feet south of Cabrillo Boulevard. Drilled observation well. Diameter 2 inches, depth 430 feet, perforated 410-430 feet. Altitude of land-surface datum 9.56 feet. Used as saltwater intrusion monitor. Reported measurements provided by city of Santa Barbara. Water-level records available 1991 to current year.

WATER LEVELS IN FEET BELOW LAND-SURFACE DATUM

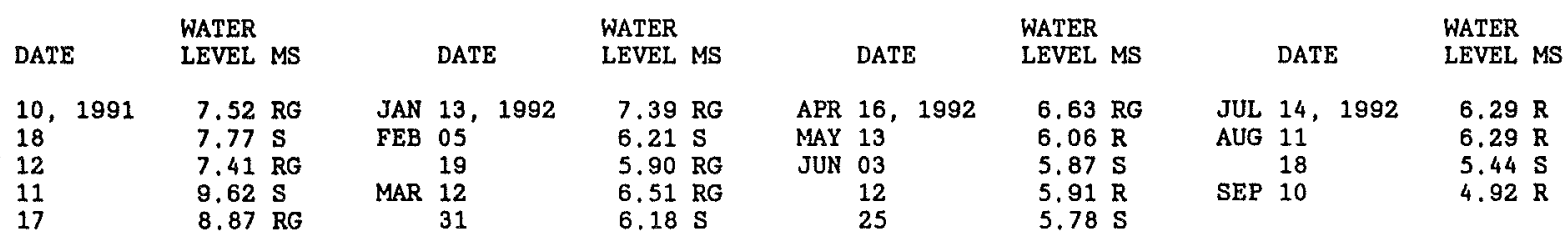

PERIOD OF RECORD HIGHEST 4.92 SEP 10,1992 LOWEST 17.38 JAN 31,1991

SITE NUMBER 342452119405505

LOCAL NUMBER 004N027W23F09S

In Santa Barbara, about 300 feet south of Cabrillo Boulevard, Drilled observation well. Diameter 2 inches, depth 195 feet, perforated 175-195 feet. Altitude of land-surface datum 9.56 feet. Used as saltwater intrusion monitor. Reported measurements provided by oity of Santa Barbara. Water-level records available 1991 to current year.

WATER LEVELS IN FEET BELOW LAND-SURFACE DATUM

\begin{tabular}{|c|c|c|c|c|c|c|}
\hline DATE & $\begin{array}{l}\text { WATER } \\
\text { LEVEL MS }\end{array}$ & DATE & $\begin{array}{l}\text { WATER } \\
\text { LEVEL MS }\end{array}$ & DATE & $\begin{array}{l}\text { WATER } \\
\text { LEVEL MS }\end{array}$ & $\begin{array}{l}\text { WATER } \\
\text { LEVEL MS }\end{array}$ \\
\hline
\end{tabular}

$\begin{array}{llllll}\text { OCT } 10,1991 & 3.90 \mathrm{R} & \text { JAN } 13,1992 & 2.94 \mathrm{RG} \\ 18 & 3.33 \mathrm{~S} & \text { FEB } 05 & 2.18 \mathrm{~S} \\ \text { NOV } 12 & 3.26 \mathrm{R} & & 19 & 1.73 \mathrm{RG} \\ \text { DEC } 11 & 3.13 \mathrm{~S} & \text { MAR } 12 & 2.46 \mathrm{RG} \\ 17 & 3.50 \mathrm{R} & 31 & 1.96 \mathrm{~S}\end{array}$

$\begin{array}{lll}\text { APR } & 16,1992 & 2.40 \mathrm{RG} \\ \text { MAY } & 13 & 1.90 \mathrm{R} \\ \text { JUN } & 03 & 2.48 \mathrm{~S} \\ 12 & 2.25 \mathrm{R} \\ 25 & 2.44 \mathrm{~S}\end{array}$

$\begin{array}{llll}\text { JUL } 14,1992 & 2.50 \mathrm{R} \\ \text { AUG } 11 & & 2.37 \mathrm{R} \\ & 18 & 2.19 \mathrm{~S} \\ \text { SEP } 10 & & 1.90 \mathrm{R}\end{array}$

PERIOD OF RECORD HIGHEST

1.73 FEB 19, 1992 LOWEST 7.24 JAN 31, 1991

SITE NUMBER 342459119402601

LOCAL NUMBER 004N027W23H01S

In Santa Barbara, northeast of Cabrillo Boulevard and Punta Gorda. Diameter 2 inches, depth 781 feet, depth of hole 845 feet, screened 746,5-751.5, 756.7-761.7, 766,9-771.9, 777.5-781 feet. Altitude of land-surface datum 7 feet. Reported measurements provided by city of Santa Barbara beginning January 1980 . Water-level records available 1978 to current year.

WATER LEVELS IN FEET BELOW LAND-SURFACE DATUM (READINGS ABOVE LAND SURFACE INDICATED BY "+")

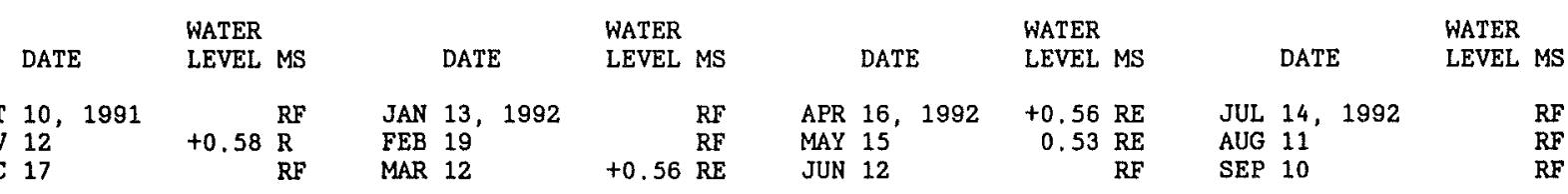

PERIOD OF RECORD HIGHEST +0.75 MAR 01, $1984 \quad$ LOWEST 25.53 JUL 23, 1980 
HATER YEAR OCTOBER 1991 TO SEPTEMBER 1992

SITE NUMBER 342459119402604

LOCAL NUMBER 004N027W23H04S

In Santa Barbara, northeast of Cabrillo Boulevard and Punta Gorda. Diameter 2 inches, depth measured 93.6 feet in 1992, original depth 95 feet, depth of hole 635 feet, screened 75.5-91 feet. Altitude of land-surface datum 7 feet. Reported measurements provided by city of Santa Barbara. Water-level records available 1978 to current year.

WATER LEVELS IN FEET BELOW LAND-SURFACE DATUM (READINGS ABOVE LAND SURFACE INDICATED BY "+")

\begin{tabular}{|c|c|c|c|c|c|c|c|c|c|c|c|}
\hline DATE & $\begin{array}{l}\text { WATER } \\
\text { LEVEL MS }\end{array}$ & & DATE & $\begin{array}{l}\text { WATER } \\
\text { LEVEL MS }\end{array}$ & & DATE & $\begin{array}{l}\text { WATER } \\
\text { LEVEL MS }\end{array}$ & & DATE & & $\begin{array}{l}\text { WATER } \\
\text { LEVEL }\end{array}$ \\
\hline $\begin{array}{ll}10, & 1991 \\
12 & \\
17 & \\
13, & 1992\end{array}$ & $\begin{array}{rl}1.42 & R \\
+0.11 & R \\
0.00 & R \\
& R F\end{array}$ & $\begin{array}{l}\text { FEB } \\
\text { MAR } \\
\text { APR } \\
\text { MAY }\end{array}$ & $\begin{array}{ll}19, & 1992 \\
12 & \\
16 & \\
13 & \end{array}$ & $\begin{array}{rl}0.99 & R \\
+0.81 & R E \\
0.70 & R E \\
5.63 & R E\end{array}$ & $\begin{array}{l}\text { JUN } \\
\text { JUL } \\
\text { AUG }\end{array}$ & $\begin{array}{ll}12, & 1992 \\
22 & \\
14 & \\
11 & \end{array}$ & $\begin{array}{ll}1.01 & R G \\
1.20 & \mathrm{~S} \\
0.87 & \mathrm{R} \\
0.83 & \mathrm{R}\end{array}$ & SEP & 10 & 1992 & 0.80 \\
\hline
\end{tabular}

PERIOD OF RECORD HIGHEST to.90 JUN 24, 1987 LOWEST 10.51 OCT 09, 1989

SITE NUMBER 342709119471401

LOCAL NUMBER 004N028W02P03S

In Goleta, north of Cathedral Oaks Road at east end of Tuckers Grove. Drilled irrigation well. Diameter 8 inches, depth measured 271.4 feet, perforated 40-297 feet. Alt1tude of land-surface datum 180 feet. Water-level records available 1946 , 1951-52, 1967 to current year.

WATER LEVELS IN FEET BELOW LAND-SURFACE DATUM

$$
\text { DATE WATER }
$$

MAR 05, $1992 \quad 28.18 \mathrm{R}$

PERIOD OF RECORD HIGHEST 23.88 APR $12,1984 \quad$ LOWEST 73.38 OCT 10,1968

SITE NUMBER 342639119452801

LOCAL NUMBER 004N028W12H04S

In Santa Barbara about 110 feet south of La Colina Road and 60 feet west of Pesetas Lane in northeast corner of Santa Barbara Medical Foundation parking lot, Diameter 8 inches, depth 290 feet, perforated 200-250, 260-280 feet. Altitude of land-surface datum 172 feet. Reported measurements provided by city of Santa Barbara. Water-level records available $1977,1978,1984$ to current year.

WATER LEVELS IN FEET BELOW LAND-SURFACE DATUM

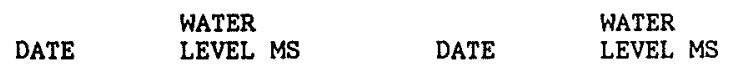

OCT 10, 1991

NOV 11

DEC 17

$80.28 \mathrm{R}$

$72.11 \mathrm{R}$

$70.94 \mathrm{R}$
JAN 13, 1992

FEB 19

MAR 12

\section{$67.89 \mathrm{R}$}

$64.07 \mathrm{R}$

$65.44 \mathrm{R}$

\begin{tabular}{ll}
\multicolumn{2}{c}{ DATE } \\
APR 16,1992 \\
MAY 13 \\
JUN 12
\end{tabular}

WATER

LEVEL MS

$67.56 \mathrm{R}$

$68.12 \mathrm{R}$

$68.50 \mathrm{R}$

\section{DATE}

JUL 19, 1992

AUG 11

SEP 10
WATER

LEVEL MS

$69.99 \mathrm{R}$

$70.27 \mathrm{R}$

$70.81 \mathrm{R}$

PERIOD OF RECORD HIGHEST 35.37 NOV 15, 1984 LOWEST 90.76 AUG 08, 1990 
WATER LEVELS, SANTA BARBARA COUNTY--Continued

WELL DESCRIPTIONS AND WATER-LEVEL MEASUREMENTS

WATER YEAR OCTOBER 1991 TO SEPTEMBER 1992

SITE NUMBER 342617119455801

LOCAL NUMBER 004N028W12P0SS

In Santa Barbara in Hope Ranch area. Diameter 16 inches, depth 355 feet. Altitude of land-surface datum 105 feet. Measurements provided by Goleta Water District and the City of Santa Barbara beginning in 1971 . Water-level records avallable 1956, 1959 to current year.

WATER LEVELS IN FEET BELOW LAND-SURFACE DATUM

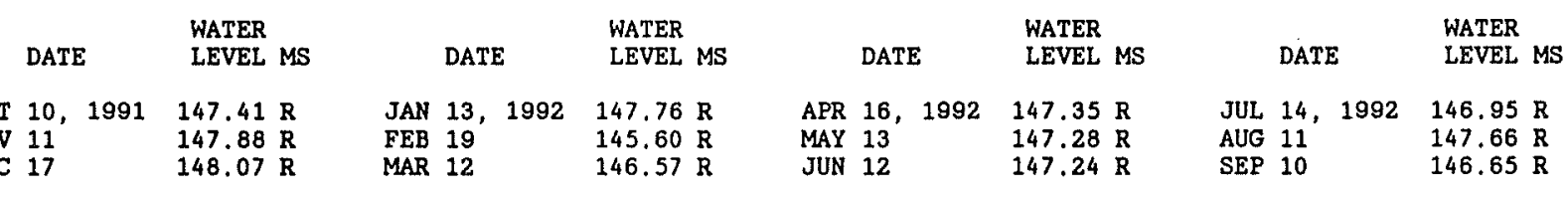

PERIOD OF RECORD HIGHEST 115.18 APR 02, 1979 LOWEST 207.78 OCT 29, 1970

SITE NUMBER 342623119453301

LOCAL NUMBER 004N028W12R03S

In Goleta, south of Hollister Avenue, and west of San Marcos Pass Road. Drilled unused well. Diameter 10 inches 0-215 feet, 6 inches 215-380 feet, depth 396 feet, cased to 380 feet, perforated 142-212, 215-370 feet.

Altitude of land-surface datum 135 feet. Measurements provided by city of Santa Barbara beginning in 1985 .

Water-level records available 1985 to current year.

WATER LEVELS IN FEET BELOW LAND-SURFACE DATUM

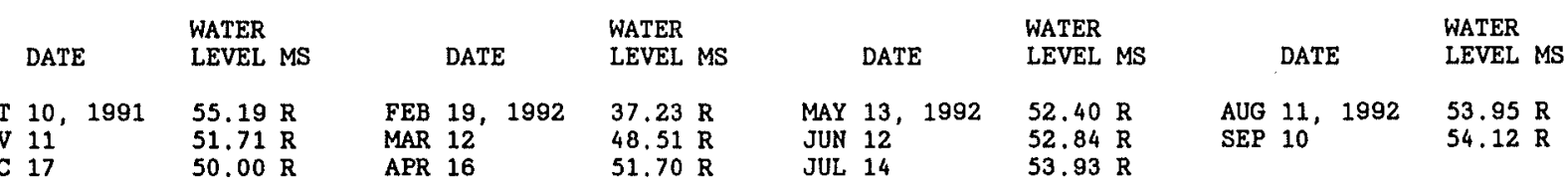

PERIOD OF RECORD HIGHEST 37.23 FEB 19, 1992 LOWEST 66.33 SEP 12, 1990

SITE NUMBER 342539119483504

LOCAL NUMBER 004N028W16J05S

In Goleta, south of Hollister Avenue and east of More Road. Drilled observation well. Diameter 1.25 inches, depth drilled 62 feet, well point 18.2-20.2 feet. Altitude of land-surface datum 25 feet. Water-level records available 1962 to current year.

WATER LEVELS IN FEET BELOW LAND-SURFACE DATUM

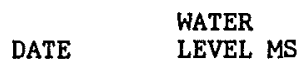

MAR $05,1992 \quad 11.22 \mathrm{R}$

PERIOD OF RECORD HIGHEST 3.00 DEC 01, 13, 1962 LOWEST 11.89 MAR 11, 1991

SITE NUMBER 342732119583101

LOCAL NUMBER 004N030W01G01S

About 13 miles east of Gaviota, north of Las Varas Ranch Headquarters. Irrigation well. Diameter 12 inches, depth 350 feet. Altitude of land-surface datum 180 feet. Measurements provided by Santa Barbara County Water Agency beginning in 1980. Water-level records avallable 1970 to current year.

WATER LEVELS IN FEET BELOW LAND-SURFACE DATUM

$$
\text { DATE WATER }
$$

MAR 04, $1992 \quad 291.75 \mathrm{R}$

PERIOD OF RECORD HIGHEST 89,80 MAR 25, 1971 LOWEST 291.75 MAR 04,1992 


\section{SITE NUMBER 342844119474401}

LOCAL NUMBER 005N028W34A01S

North of Goleta on Old San Marcos Pass Road. Drilled domestic well. Diameter 5 inches, depth 780 feet, perforated 620-760 feet. Altitude of land-surface datum 977 feet. Measurements provided by Santa Barbara County Water Agency beginning in 1987. Water-level records available 1987 to current year.

WATER LEVELS IN FEET BELOW LAND-SURFACE DATUM

$$
\begin{array}{ll}
\text { WATER } & \text { WEVEL MS }
\end{array}
$$

MAR 05, $1992 \quad$ RO

PERIOD OF RECORD HIGHEST 404.80 MAR 29, 1991 LOWEST 411 MAR 21, 1989

SITE NUMBER 343251119522201

LOCAL NUMBER 005N029W01C01S

East of Lake Cachuma, north of San Marcos Pass Road at San Marcos Ranch. Drilled unused well. Diameter 16 inches, depth measured 34.4 feet in 1990. Altitude of land-surface datum 790 feet. Measurements provided by Santa Barbara County Water Agency beginning in 1980. Water-level records available 1950, 1977 to current year.

WATER LEVELS IN FEET BELOW LAND-SURFACE DATUM

$$
\text { DATE WATER }
$$

MAR 11, $1992 \quad 5.05 \mathrm{R}$

PERIOD OF RECORD HIGHEST 4.05 MAR 27, 1978 LOWEST 15.12 NOV 28, 1977

SITE NUMBER 342838119573501

LOCAL NUMBER 005N029W31C01S

About 14 miles east of Gaviota, about 1.75 miles north of Highway 101 in Las Varas Canyon. Drilled unused well. Diameter 10 inches $0-209$ feet, 8 inches 0-1,510 feet, depth 1,510 feet, perforated 290-320, 400-420, 470-510, 550-600, 620-1,510 feet. Altitude of land-surface datum 400 feet. Measurements provided by Santa Barbara County Water Agency beginning in 1980. Water-level records available 1962 to current year.

WATER LEVELS IN FEET BELOW LAND-SURFACE DATUM

$$
\text { DATE WATER }
$$

MAR 04, $1992 \quad 119.17 \mathrm{R}$

PERIOD OF RECORD HIGHEST 33.42 APR 04, 1983 LOWEST 126.59 MAR 11, 1991

SITE NUMBER 343008120035801

LOCAL NUMBER O05N030W19E01S

About 2.5 miles north of Highway 101 in Canada del Refugio. Drilled irrigation well. Diameter 12 inches 0-43 feet, 8 inches 43-254 feet, 6 inches 254-397 feet, depth 505 feet, perforated 205-347 feet. Altitude of land-surface datum 330 feet. Measurements provided by Santa Barbara County Water Agency beginning in 1980. Water-level records available 1962 to current year.

WATER LEVELS IN FEET BELOW LAND-SURFACE DATUM (READINGS ABOVE LAND SURFACE INDICATED BY "+")

$$
\begin{array}{ll}
\text { WATER } \\
\text { DATE }
\end{array}
$$

MAR 09, $1992 \quad 28.89 \mathrm{R}$

PERIOD OF RECORD HIGHEST t0.82 MAR 31, 1983 LOWEST 39.60 MAR 27, 1990 
WELL DESCRIPTIONS AND WATER-LEVEL MEASUREMENTS

WATER YEAR OCTOBER 1991 TO SEPTEMBER 1992

SITE NUMBER 342845120010701

LOCAL NUMBER O05N030W28R01S

About 1.2 miles north of Highway 101 and El Capitan Beach State Park in Canada del Capitan. Domestic well.

Diameter 8 inches, depth 198 feet. Altitude of land-surface datum 350 feet. Measurements provided by Santa Barbara County Water Agency beginning in 1980. Water-level records available 1963-75, 1977 to current year.

WATER LEVELS IN FEET BELOW LAND-SURFACE DATUM

$$
\text { DATE WATER }
$$

MAR 06, $1992 \quad 51.59 \mathrm{R}$

PERIOD OF RECORD HIGHEST 18.30 MAR 31, 1970 LOWEST 74.82 MAR 21, 1990

SITE NUMBER 342850120040002

LOCAL NUMBER OOSNO3OW3ONO2S

About 1 mile north of Refugio Beach State Park in Canada del Refugio. Irrigation well. Diameter 10 inches, depth 93 feet. Altitude of land-surface datum 85 feet. Measurements provided by Santa Barbara County Water Agency beginning in 1980. Water-level records available 1962-76, 1978 to current year.

WATER LEVELS IN FEET BELOW LAND-SURFACE DATUM

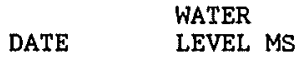

MAR 09, $199213.19 \mathrm{R}$

PERIOD OF RECORD HIGHEST 11.87 MAR 24, 1986 LOWEST 36.46 JUL 27,1964

SITE NUMBER 342845120053801

LOCAL NUMBER 005N031W35B01S

About 1.2 miles north of Highway 101 in Tajiguas Canyon. Drilled irrigation well. Diameter 12 inches, depth 76 feet. Altitude of land-surface datum 80 feet. Measurements provided by Santa Barbara County Water Agency beginning in 1980. Water-level records available 1962 to current year.

WATER LEVELS IN FEET BELOW LAND-SURFACE DATUM

DATE HATER $\quad$ LEVEL MS

MAR 09, $1992 \quad 9.77 \mathrm{R}$

PERIOD OF RECORD HIGHEST 5.49 MAY 01, 1979 LOWEST 13.75 APR 15, 1977

SITE NUMBER 343755119570901

LOCAL NUMBER O06N029W05A01S

About 3 miles north of Lake Cachuma. Stock well. Diameter unknown, depth 93 feet. Altitude of land-surface datum 1,190 feet. Measurements provided by Santa Barbara County Water Agency beginning in 1980. Water-level records available 1964, 1966-1973, 1975 to current year.

WATER LEVELS IN FEET BELOW LAND-SURFACE DATUM (READINGS ABOVE LAND SURFACE INDICATED BY "+")

$$
\begin{array}{ll}
\text { WATER } \\
\text { DATE }
\end{array}
$$

MAR 17, $1992 \quad 9.75 \mathrm{R}$

PERIOD OF RECORD HIGHEST t0.01 APR 01, 1983 LOWEST 23.95 OCT 19, 1967 
SITE NUMBER 343746119583101

LOCAL NUMBER OOGNO29W06F01S

In Happy Canyon about $0.8 \mathrm{mile}$ northeast of Happy Canyon Creok bridge. Drilled 1rrigation well. Diameter 10 inches, depth 72 feet, perforated 35-53 feet. Altitude of land-surface datum 840 feet. Measurements provided by Santa Barbara County Water Agency beginning in 1980. Water-level records available 1952, 1954-59, 1963-64, $1967-74,1976$ to current year.

WATER LEVELS IN FEET BELOW LAND-SURFACE DATUM

$$
\text { DATE NATER } \quad \text { LEVEL MS }
$$

MAR 17, $199213.54 \mathrm{R}$

PERIOD OF RECORD HIGHEST 7.69 MAR 24, 1969 LOWEST 33.68 OCT 11, 1955

SITE NUMBER 343746119582201

LOCAL NUMBER 006N029W06G01S

In Happy Canyon about 1 mile northeast of Happy Canyon Creek bridge. Drilled unused well. Diameter 10 inches, depth 102 feet, perforated 57-96 feet. Altitude of land-surface datum 875 feet. Measurements provided by Santa Barbara County Water Agency beginning in 1980. Water-level records available 1954, 1957-58, 1966 to current year.

WATER LEVELS IN FEET BELOW LAND-SURFACE DATUM

$$
\begin{array}{ll} 
& \text { WATER } \\
\text { DATE } & \text { LEVEL MS }
\end{array}
$$

MAR 17, $199250.20 \mathrm{R}$

PERIOD OF RECORD HIGHEST 27.99 MAR 24, 1969 LOWEST 55.00 AUG 10, 1954

SITE NUMBER 343646119583001

LOCAL NUMBER 006N029W07L01S

Northeast of Cachuma Village. Unused well. Diameter 8 inches, depth 440 feet. Altitude of land-surface datum 868 feet. Measurements provided by Senta Barbara County Water Agency beginning in 1980. Water-level records available $1960,1963-76,1978$ to current year.

WATER LEVELS IN FEET BELOW LAND-SURFACE DATUM

$$
\text { DATE WATER }
$$

MAR 17, $1992 \quad 207.01 \mathrm{R}$

PERIOD OF RECORD HIGHEST 193.58 ARR 13, 1984 LOWEST 225.85 OCT 14, 1969

SITE NUMBER 343632119573301

LOCAL NUMBER O06N029W08P01S

Northeast of Cachuma Village. Drilled domestic well. Diameter 10 inches, depth 252 feet, perforated $210-237$ feet. Altitude of land-surface datum 910 feet. Measurements provided by Santa Barbara County Water Agency beginning in 1980. Water-level records available 1934, 1964, 1967 to current year.

WATER LEVELS IN FEET BELOW LAND-SURFACE DATUM

$$
\text { DATE WATER }
$$

MAR 17, $1992 \quad 222.67 \mathrm{R}$

PERIOD OF RECORD HIGHEST 198.00 OCT 06, 1934 LOWEST 247.95 MAR 24, 1967 
WELL DESCRIPTIONS AND WATER-LEVEL MEASUREMENTS

WATER YEAR OCTOBER 1991 TO SEPTEMBER 1992

SITE NUMBER 343632119573302

LOCAL NUMBER 006N029W08P02S

Northeast of Cachuma Village. Domest,ic well. Diameter 12 inches, depth unknown. Altitude of land-surface datum 910 feet. Measurements provided by Santa Barbara County Water Agency beginning in 1980. Water-level records available 1966 to current year.

WATER LEVELS IN FEET BELOW LAND-SURFACE DATUM

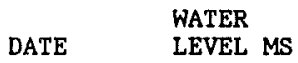

MAR 17, $1992223.72 \mathrm{R}$

PERIOD OF RECORD HIGHEST 216.63 MAR 17, 1988 LOWEST 238.03 MAR 25, 1970

SITE NUMBER 343636119555701

LOCAL NUMBER 006N029W09J01S

North of Lake Cachuma on Janeway Ranch. Domestic well. Diameter 8 inches, depth 200 feet. Altitude of land-surface datum 803 feet. Measurements provided by Santa Barbara County Water Agency beginning in 1980. Water-level records available 1963-1964, 1966-73, 1975 to current year.

WATER LEVELS IN FEET BELOW LAND-SURFACE DATUM

$$
\text { DATE WATER }
$$

MAR 17, $1992 \quad 12.10 \mathrm{R}$

PERIOD OF RECORD HIGHEST 10.58 MAR 27, 1981 LOWEST 28.13 APR 01, 1983

SITE NUMBER 343718119592001

LOCAL NUMBER 006N030W01R03S

In Happy Canyon about 250 feet south of Happy Canyon Road. Drilled unused well. Diameter 12 inches, depth 340 feet, perforated 28-110 feet. Altitude of land-surface datum 760 feet. Measurements provided by Santa Barbara County Water Agency beginning in 1980. Water-level records available 1949, 1954-59, 1963-64, 1966 to current year.

WATER LEVELS IN FEET BELOW LAND-SURFACE DATUM

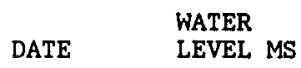

MAR 17, $1992 \quad 19.00 \mathrm{R}$

PERIOD OF RECORD HIGHEST 8,48 ARR 01, 1983 LOWEST 31.26 MAR 15, 1971

SITE NUMBER 343651120043401

LOCAL NUMBER 006N030W07G05S

In Santa Ynez, west of Santa Barbara Avenue extension. Public-supply well. Diameter unknown, depth 158 feet. Altitude of land-surface datum 600 feet. Measurements provided by Santa Barbara County Water Agency beginning in 1981. Water-level records available 1962-79, 1981, 1983 to current year.

WATER LEVELS IN FEET BELOW LAND-SURFACE DATUM

$$
\text { DATE WATER }
$$

MAR 12, $1992 \quad 88.31 \mathrm{R}$

PERIOD OF RECORD HIGHEST 41,64 JUN 28, 1962 LOWEST 92.73 MAR 27, 1991 
SITE NUMBER 343651120043402

LOCAL NUMBER 006N030W07G06S

In Santa Ynez, west of Santa Barbara Avenue extension. Drilled public-supply well. Diameter 16 inches, depth 564 feet, perforated $305-326,331-355,395-410$ feet. Altitude of land-surface datum 600 feet. Measurements provided by Santa Barbara County Water Agency beginning in 1980. Water-level records available 1962-77, 1979 to current year.

WATER LEVELS IN FEET BELOW LAND-SURFACE DATUM

$\begin{array}{ll} & \text { WATER } \\ \text { DATE } & \text { LEVEL MS }\end{array}$

MAR 12, $1992 \quad 84.11 \mathrm{R}$

PERIOD OF RECORD HIGHEST 40.06 APR 22, 1963 LOWEST 89.39 MAR 27, 1991

SITE NUMBER 343627120030801

LOCAL NUMBER 006N030W09N01S

East of Santa Ynez, south of Highway 150. Drilled stock well. Diameter 8 inches, depth 160 feet. Altitude of land-surface datum 660.01 feet. Measurements provided by Santa Barbara County Water Agency beginning in 1980 . Water-level records available 1941 to current year.

WATER LEVELS IN FEET BELOW LAND-SURFACE DATUM

$$
\begin{array}{ll} 
& \text { WATER } \\
\text { DATE } & \text { LEVEL MS }
\end{array}
$$

MAR 12, $1992 \quad 58.37 \mathrm{R}$

PERIOD OF RECORD HIGHEST 24.06 JUL 27,1960 LOWEST 58.37 MAR 12,1992

SITE NUMBER 343649120001801

LOCAL NUMBER 006N030W11G01S

East of Santa Ynez, west of Alisos Avenue in Happy Canyon. Irrigation well. Diameter 15 inches, depth measured 120 feet in 1991 . Altitude of land-surface datum 680 feet. Measurements provided by Santa Barbara County Water Agency beginning in 1980. Water-level records available 1943, 1974, 1976, 1978 to current year.

WATER LEVELS IN FEET BELOW LAND-SURFACE DATUM

$$
\text { DATE WATER }
$$

$\operatorname{MAR} 17,199243.14 \mathrm{R}$

PERIOD OF RECORD HIGHEST 20.00 NOV 01, 1943 LOWEST 49.50 MAR 26, 1990

SITE NUMBER 343727120055801

LOCAL NUMBER O06N031W01P02S

In Santa Ynez, north of Santa Ynez Union High School, and west of Refugio Road. Irrigation well. Diameter unknown, depth reported 350 feet. Altitude of land-surface datum 620 feet. Measurements provided by Santa Barbara County Water Agency beginning in 1980. Water-level records available 1956-58, 1966 to current year.

WATER LEVELS IN FEET BELOW LAND-SURFACE DATUM

$$
\begin{array}{ll} 
& \text { WATER } \\
\text { DATE } & \text { LEVEL MS }
\end{array}
$$

MAR $12,1992 \quad 85.30 \mathrm{R}$

PERIOD OF RECORD HIGHEST 40.90 MAR 12, 1958 LOWEST 85.30 MAR 12, 1992 
WELL DESCRIPTIONS AND WATER-LEVEL MEASUREMENTS

WATER YEAR OCTOBER 1991 TO SEPTEMBER 1992

SITE NUMBER 343728120055101

LOCAL NUMBER 006N031W01P03S

In Santa Ynez, north of Santa Ynez Union High School, and west of Refugio Road. Drilled public-supply well. Diameter 16 inches, depth 508 feet, depth of hole 1,006 feet, perforated 195-360, 410-490 feet. Altitude of land-surface datum 640 feet. Measurements provided by Santa Barbara County Water Agency beginning in 1980. Water-level records avallable 1967 to current year.

WATER LEVELS IN FEET BELOW LAND-SURFACE DATUM

$$
\text { DATE HATER }
$$

MAR 12, $1992115.32 \mathrm{R}$

PERIOD OF RECORD HIGHEST 79.76 MAR 27, 1969 LOWEST 115.32 MAR 12, 1992

SITE NUMBER 343741120064801

LOCAL NUMBER 006N031W02K01S

In Ballard about 2,850 feet south of Base Line Avenue and 700 feet east of Alamo Pintado Road. Drilled irrigation well. Diameter 10 inches, depth 71.3 feet. Altitude of land-surface datum 627 feet. Measurements provided by Santa Barbara County Water Agency beginning in 1980. Water-level records available 1942, 1947 to current year.

WATER LEVELS IN FEET BELOW LAND-SURFACE DATUM

$$
\begin{array}{ll}
\text { DATE } & \text { WATER } \\
\text { LEVEL MS }
\end{array}
$$

MAR 16, $1992 \quad 29.24 \mathrm{R}$

PERIOD OF RECORD HIGHEST 18.02 APR 05, $1983 \quad$ LOWEST 58.90 OCT 11,1968

SITE NUMBER 343759120072901

LOCAL NUMBER O06N031W03A01S

About $0.25 \mathrm{mile}$ west of Ballard and $0.5 \mathrm{mile}$ west of Alamo Pintado Road. Unused well. Diameter 12 inches, depth 196 feet. Altitude of land-surface datum 760 feet. Measurements provided by Santa Barbara County Water Agency beginning in 1980. Water-level records available 1963-64, 1966 to current year.

WATER LEVELS IN FEET BELOW LAND-SURFACE DATUM

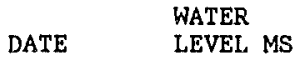

MAR 16, $1992159.73 \mathrm{R}$

PERIOD OF RECORD HIGHEST 148.65 SEP 14, 1971 LOWEST 168.49 APR 19, 1984

SITE NUMBER 343800120083001

LOCAL NUMBER O06N031W04A01S

About 3 miles north of Solvang in Ballard Canyon. Domestic well. Diameter 7 inches, depth unknown. Altitude of land-surface datum 615 feet. Measurements provided by Santa Barbara County Water Agency beginning in 1980. Water-level records available 1956-58, 1963-64, 1966 to current year.

WATER LEVELS IN FEET BELOW LAND-SURFACE DATUM

$$
\begin{array}{ll} 
& \text { WATER } \\
\text { DATE } & \text { LEVEL MS }
\end{array}
$$

MAR 13, $199294.00 \mathrm{R}$

PERIOD OF RECORD HIGHEST 80.00 NOV 01, 1956 LOWEST 94.52 APR 17, 1987 
SITE NUMBER 343655120111201

LOCAL NUMBER O06N031W07F01S

In Buellton. Drilled 1rrigation well. Diameter 10 inches, depth 633 feet in 1985, original depth 700 feet, perforated 250-300, 390-650,670-700 feet. Altitude of land-surface datun 385 feet. Measurements provided by Santa Barbara County Water Agency beginning in 1984. Water-level records available 1984 to current year.

WATER LEVELS IN FEET BELOW LAND-SURFACE DATUM

$$
\text { DATE WATER } \quad \text { LEVEL MS }
$$

MAR 13, $1992 \quad 72.36 \mathrm{R}$

PERIOD OF RECORD HIGHEST 66.36 MAR 21, 1985 LOWEST 73.05 MAR 27, 1991

SITE NUMBER 343656120080601

LOCAL NUMBER 006N031W10F01S

About 1.5 miles north of Highway 150 in Solvang and 100 feet west of Adobe Canyon Road. Drilled irrigation well. Diameter 8 inches, depth 265 feet. Altitude of land-surface datum 540 feet. Measurements provided by Santa Barbara County Water Agency beginning in 1980. Water-level records available 1966 to current year.

WATER LEVELS IN FEET BELOW LAND-SURFACE DATUM

$$
\text { DATE WATER }
$$

MAR $24,199267,14 \mathrm{R}$

PERIOD OF RECORD HIGHEST 62.59 MAR 22, 1985 LOWEST 75.00 OCT 01, 1966

\section{SITE NUMBER 343705120071001}

LOCAL NUMBER O06N031W11D04S

In Solvang on north bank of Alamo Pintado Creek. Drilled irrigation well. Diameter 12 inches, depth 452 feet, perforated 93-102, 131-147, 197-205, 227-447 feet. Altitude of land-surface datum 558.51 feet. Measurements provided by Santa Barbara County Water Agency beginning in 1980. Water-level records available. 1955-59, 1963-64, 1966 to current year.

WATER LEVELS IN FEET BELOW LAND-SURFACE DATUM

$$
\text { DATE WATER }
$$

MAR 13, $1992 \quad 28,48 \mathrm{R}$

PERIOD OF RECORD HIGHEST 23.40 APR 06, 1983 LOWEST 67.19 OCT 31, 1957

SITE NUMBER 343623120061201

LOCAL NUMBER O06N031W13D01S

In Santa Ynez about 150 feet south of Highway 150. Drilled domestic well. Diameter 10 inches, depth measured 152 feet in 1991. Altitude of land-surface datum 608 feet. Measurements provided by Santa Barbara County Water Agency beginning in 1980. Water-level records available 1941 to current year.

WATER LEVELS IN FEET BELOW LAND-SURFACE DATUM

$$
\text { DATE WATER }
$$

MAR 13, $1992 \quad 112.62 \mathrm{R}$

PERIOD OF RECORD HIGHEST 102.58 MAR 09, 1942 LOWEST 132.10 OCT 23, 1961 
WELL DESCRIPTIONS AND WATER-LEVEL MEASUREMENTS

WATER YEAR OCTOBER 1991 TO SEPTEMBER 1992

SITE NUMBER 343535120091101

LOCAL NUMBER O06NO31W16NO2S

About 2.5 miles east of Buellton. Diameter 16 inches, depth 47 feet. Altitude of land-surface datum 366.29 feet. Measurements provided by Santa Barbara County Water Agency beginning in 1980 . Water-level records available $1941-42,1949$ to current year.

WATER LEVELS IN FEET BELOW LAND-SURFACE DATUM

DATE WATER

MAR 13, $1992 \quad 25.00 \mathrm{R}$

PERIOD OF RECORD HIGHEST 6.48 APR 06, 1942 LOWEST 35.92 NOV 24, 1961

SITE NUMBER 343609120101201

LOCAL NUMBER 006N031W17FO1S

About 1.5 miles east of Buellton. Dus domestic well. Diameter 12 inches, depth 43 feet, 40 feet measured in 1990. Altitude of land-surface datum 362.9 feet. Measurements provided by city of Santa Barbara 1931-41, and Santa Barbara County Water Agency beginning in 1980. Water-level records available 1931 to current year.

WATER LEVELS IN FEET BELOW LAND-SURFACE DATUM

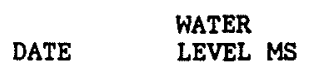

MAR 13, $199238.34 \mathrm{R}$

PERIOD OF RECORD HIGHEST 14.80 APR 09, 1941 LOWEST 38.45 SEP 28, 1964

SITE NUMBER 343608120101001

LOCAL NUMBER O06N031W17F03S

About 1 mile northwest of Solvang, south of Highway 150. Irrigation well. Diameter 14 inches, depth measured 70.8 feet in 1992. Altitude of land-surface datum 360 feet. Measurements provided by Santa Barbaxa County Water Agency beginning in 1987. Water-level records available 1987 to current year.

WATER LEVELS IN FEET BELOW LAND-SURFACE DATUM

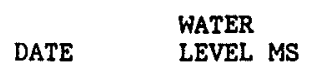

MAR $13,1992 \quad 38.84 \mathrm{R}$

PERIOD OF RECORD HIGHEST 38.84 MAR 13, 1992 LOWEST 43.07 MAR 27, 1990

SITE NUMBER 343719120124901

LOCAL NUMBER 006N032W02Q01S

About 1.3 miles northwest of Buellton, 100 feet north of Highway 150. Drilled domestic well. Diameter 8 inches, depth 110 feet, perforated 50-76 feet. Altitude of land-surface datum 359.46 feet. Measurements provided by Santa Barbara County Water Agency beginning 1980. Water-level records available 1949 to current year.

WATER LEVELS IN FEET BELOW LAND-SURFACE DATUM

DATE WATER

MAR 13, $1992 \quad 58.74 \mathrm{R}$

PERIOD OF RECORD HIGHEST 55.33 APR 01, 1969 LOWEST 63.79 MAR 23, 1991 
SITE NUMBER 343739120171301

LOCAL NUMBER O06N032W06K01S

About 6 miles northwest of Buellton, west of Highway 150. Drilled domestic well. Diameter 12 inches, depth 74 feet. Altitude of land-surface datum 383.55 feet. Measurements provided by city of Santa Barbara in $1932-34$, and Santa Barbara County Water Agency beginning in 1980. Water-level records available $1932-34$, 1941 to current year.

WATER LEVELS IN FEET BELOW LAND-SURFACE DATUM

$\begin{array}{ll} & \text { WATER } \\ \text { DATE } & \text { LEVEL MS }\end{array}$

MAR 13, $1992 \quad 37.74 \mathrm{R}$

PERIOD OF RECORD HIGHEST 10.50 JUL 09,1932 LOWEST 39.55 MAR 23,1991

SITE NUMBER 343646120131801

LOCAL NUMBER O06NO32W11LO3S

About 1.6 miles west of Buellton, 2,400 feet north of Santa Rosa Road. Dug domestic well. Diameter 12 inches, depth 42 feet in 1949 , perforated $20-42$ feet. Altitude of land-surface datum 302.38 feet. Measurements provided by Santa Barbara County Water Agency beginning in 1980. Water-1evel records available 1949-55, 1970-1972, 1976 to current year.

WATER LEVELS IN FEET BELOW LAND-SURFACE DATUM

$$
\begin{array}{ll} 
& \text { WATER } \\
\text { DATE } & \text { LEVEL MS }
\end{array}
$$

MAR 13, $1992 \quad 7.90 \mathrm{R}$

PERIOD OF RECORD HIGHEST 5.13 APR $25,1978 \quad$ LOWEST $15.00 \quad$ OCT 27,1955

SITE NUMBER 343544120151801

LOCAL NUMBER 006N032W16P03S

About 4.5 miles west of Bueliton on Santa Rosa Road. Drilled irribation well. Diameter 16 inches, depth 70 feet. Altitude of land surface datum 293.18 feet. Measurements provided by Santa Barbara County Water Agency beginning in 1980. Water-level records available 1941 to current year.

WATER LEVELS IN FEET BELOW LAND-SURFACE DATUM

$$
\begin{array}{ll} 
& \text { WATER } \\
\text { DATE } & \text { LEVEL MS }
\end{array}
$$

MAR 13, $1992 \quad 45.67 \mathrm{R}$

PERIOD OF RECORD HIGHEST 41.82 FEB 24, 1943 LOWEST 53.66 NOV 27,1961

SITE NUMBER 343613120164501

LOCAL NUMBER 006N032W18H01S

About 6 miles west of Buellton on Santa Rosa Road, Drilled observation well. Diameter 8 inches, depth 50 feet. Altitude of land-surface datum 267.09 feet. Measurements provided by city of Santa Barbara in 1932-34, 1936-42, and Santa Barbara County Water Agency beginning in 1980. Water-level records available 1932-34, 1936-42, 1949 to current year.

WATER LEVELS IN FEET BELOW LAND-SURFACE DATUM

$\begin{array}{ll} & \text { WATER } \\ \text { DATE } & \text { LEVEL MS }\end{array}$

MAR 13, $1992 \quad 34.13 \mathrm{R}$

PERIOD OF RECORD HIGHEST 27.90 MAR 07, 1941 LOWEST 42.79 SEP 27,1961 
WELL DESCRIPTIONS AND WATER-LEVEL MEASUREMENTS

WATER YEAR OCTOBER 1991 TO SEPTEMBER 1992

\section{SITE NUMBER 343643120220001}

LOCAL NUMBER 006N033W08J01S

East of Lompoc. Drilled domestic well. Diameter 10 inches, original depth 61.6 feet, depth measured 46 feet in 1990. Altitude of land-surface datum 200,57 feet. Measurements provided by the U.S. Bureau of Reclamation and Santa Barbara County Water Agency. Water-level records available 1941-42, 1949 to current year.

WATER LEVELS IN FEET BELOW LAND-SURFACE DATUM

$$
\text { DATE WATER }
$$

MAR 13, $1992 \quad 39.74 \mathrm{R}$

PERIOD OF RECORD HIGHEST 33.6 MAR 28, 1983 LOWEST 52.84 OCT 24, 1961

SITE NUMBER 343647120194601

LOCAL NUMBER 006N033W11MO1S

East of Lompoc. Drilled irrigation well. Diameter 16 inches, depth 65 feet, perforated 4-30, 56-63 feet. Altitude of land-surface datum 203.82 feet. Measurements provided by U.S. Bureau of Reclamation and Santa Barbara County Water Agency beginning in 1971. Water-level records available 1947, 1949 to current year.

WATER LEVELS IN FEET BELOW LAND-SURFACE DATUM

$$
\text { DATE WATER }
$$
MAR 13, 1992
$9.10 \mathrm{R}$

PERIOD OF RECORD HIGHEST

4.29 FEB 27, 1950

LOWEST $18.13 \quad$ SEP 27,1961

SITE NUMBER 343737120245901

LOCAL NUMBER 006N034W01P01S

East of Lompoc. Drilled irrigation well. Diameter 16 inches, depth 164 feet, perforated 54-72, 127-162 feet. Altitude of land-surface datum 150.36 feet. Measurements provided by Santa Barbara County Water Agency beginning in 1980. Water-level records available 1948-1975, 1978 to current year.

WATER LEVELS IN FEET BELOW LAND-SURFACE DATUM

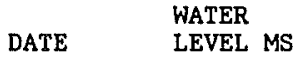

MAR 11, 1992 RO

PERIOD OF RECORD HIGHEST 34.47 MAR 22, 1967 LOWEST 46.86 SEP 27, 1961

SITE NUMBER 343815120300602

LOCAL NUMBER O06N034W06C04S

West of Lompoc and south of the intersection of Ocean Avenue and San Pasqual Canyon Road. Drilled domestic well. Diameter 6 inches, depth 112 feet, perforated 77-112 feet. Altitude of land-surface datum 103 feet. Measurements provided by Santa Barbara County Water Agency beginning in 1990. Water-level records available 1990 to current year.

WATER LEVELS IN FEET BELOW LAND-SURFACE DATUM

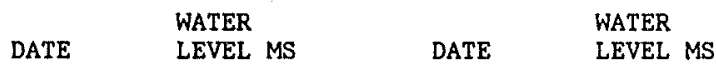

MAR 06, $1992 \quad 67.19 \mathrm{R} \quad$ SEP 02, $1992 \quad 74.19 \mathrm{~S}$

PERIOD OF RECORD HIGHEST 67.19 MAR 06, 1992 LOWEST 82.23 AUG 23, 1990 
SITE NUMBER 344224119223901

LOCAL NUMBER O07N024W13C02S

At Apache School, in Cuyama River flood plain. Drilled observation well. Diameter 8 inches, depth 165 feet Altitude of land-surface datum 3,435 feet. Measurements provided by Santa Barbara County Water Agency beginning in 1980. Water-level records available 1950 to current year.

WATER LEVELS IN FEET BELOW LAND-SURFACE DATUM

$$
\text { DATE WATER }
$$

APR 02, $1992 \quad$ RO

PERIOD OF RECORD HIGHEST 17.98 APR 10, 1979 LOWEST 44.23 DEC 27, 1951

SITE NUMBER 343946119563701

LOCAL NUMBER O07N029W28D01S

About 9 miles northeast of Santa Ynez, about 125 feet west of Happy Canyon Road. Unused well. Diameter 12 inches, depth 73.8 feet. Altitude of land-surface datum 1,130 feet. Measurements provided by Santa Barbara County Water Agency beginning in 1980. Water-level records available 1941-43, 1954-59, 1963-64, 1966-1992. Wel1 destroyed.

WATER LEVELS IN FEET BELOW LAND-SURFACE DATUM

$$
\text { DATE WATER } \quad \text { LEVEL MS }
$$

MAR 17, $1992 \quad$ W

PERIOD OF RECORD HIGHEST 6.05 APR 26, 1978 LOWEST 60.53 MAR 26, 1990

SITE NUMBER 343900119570201

LOCAL NUMBER OO7NO29W29RO1S

About 8 miles northeast of Santa Ynez in Happy Canyon. Irrigation well. Diameter 10 inches, depth measured 128.2 feet in 1991. Altitude of land-surface datum 1,050 feet. Measurements provided by Santa Barbara County Water Agency beginning in 1980. Water-level records available 1956, 1964, 1966-74, 1978 to current year.

WATER LEVELS IN FEET BELOW LAND-SURFACE DATUM

$$
\text { DATE WATER }
$$

MAR $18,1992 \quad 42.17 \mathrm{R}$

PERIOD OF RECORD HIGHEST 20.65 MAR 24, 1969 LOWEST 91.86 MAR 27, 1990

SITE NUMBER 343900119570301

LOCAL NUMBER 007NO29W29R02S

About $8 \mathrm{miles}$ northeast of Santa Ynez in Happy Canyon. Irrigation well. Diameter 8 inches, depth measured 158 feet in 1991. Altitude of land-surface datum 1,050 feet. Measurements provided by Santa Barbara County Water Agency beginning in 1980. Water-level records available 1964, 1966-76, 1978 to current year.

WATER LEVELS IN FEET BELOW LAND-SURFACE DATUM

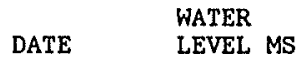

MAR 18, $1992 \quad 41.26 \mathrm{R}$

PERIOD OF RECORD HIGHEST 18.15 APR 04, 1983 LOWEST 96.93 OCT 17, 1964 
WELL DESCRIPTIONS AND WATER-LEVEL MEASUREMENTS

WATER YEAR OCTOBER 1991 TO SEPTEMBER 1992

SITE NUMBER 344127120023301

LOCAL NUMBER 007N030W16B01S

Northeast of Los Olivos. Drilled irrigation well. Diameter 14 inches, depth 150 feet. Altitude of land-surface datum 1,077 feet. Measurements provided by Santa Barbara County Water Agency beginning in 1980 . Water-1eve1 records available 1950, 1954-59, 1963-64, 1976-72, 1975, 1978 to current year.

WATER LEVELS IN FEET BELOW LAND-SURFACE DATUM

$$
\text { DATE WATER } \quad \text { LEVEL MS }
$$

MAR 17, $1992 \quad 32.09 \mathrm{R}$

PERIOD OF RECORD HIGHEST 8.48 APR 04, 1983 LOWEST 36.84 MAR 31, 1991

SITE NUMBER 344028120041801

LOCAL NUMBER OO7NO3OW19H01S

About $2.3 \mathrm{mlles}$ east of Los Olivos and $1.8 \mathrm{miles}$ north of Roblar Avenue. Stock well. Diameter 8 inches, depth unknown. Altitude of land-surface datum 1, 120 feet. Measurements provided by Santa Barbara County Water Agency beginning in 1980. Water-level records avallable 1954-58, 1963-64, 1966 to current year.

WATER LEVELS IN FEET BELOW LAND-SURFACE DATUM

$$
\begin{array}{ll} 
& \text { WATER } \\
\text { DATE } & \text { LEVEL MS }
\end{array}
$$

MAR 18, $1992177.73 \mathrm{R}$

PERIOD OF RECORD HIGHEST 171.69 APR 20, 1984 MAR 25, 1985 LOWEST 199.89 APR 08, 1977

SITE NUMBER 344005120045501

LOCAL NUMBER 007N030W19P01S

About 1.6 miles east of Los Olivos and 1.2 miles north of Roblair Avenue. Stock well. Diameter 8 inches, depth measured 103 feet in 1991. Altitude of land-surface datum 920 feet. Measurements provided by Santa Barbara County Water Agency beginning in 1980. Water-level records available 1954-59, 1964, 1966-75, 1978 to current year.

WATER LEVELS IN FEET BELOW LAND-SURFACE DATUM

$$
\text { DATE WATER }
$$

MAR 18, $199274.91 \mathrm{R}$

PERIOD OF RECORD HIGHEST 67.18 APR 05, 1983 LOWEST 85.80 APR 27, 1978

SITE NUMBER 344023120015101

LOCAL NUMBER OO7N03OW22E01S

About 4 miles east of Los Olivos in Santa Agueda Creek Canyon. Drilled domestic well. Diameter 12 inches, depth 229 feet. Altitude of land-surface datum 920 feet. Measurements provided by Santa Barbara County Water Agency beginning in 1980. Water-level records available 1948, 1954-59, 1963-64, 1966-73, 1975 to current year.

WATER LEVELS IN FEET BELOW LAND-SURFACE DATUM

$$
\text { DATE WATER }
$$

MAR 17, $1992 \quad 5.79 \mathrm{R}$

PERIOD OF RECORD HIGHEST 1.28 MAR 16, 1971 LOWEST 11.19 APR 20, 1964 
SITE NUMBER 343956119592401

LOCAL NUMBER 007N030W24Q01S

About 7 miles east of Los Olivos in Marre Canyon. Stock well. Diameter 8 inches, depth measured 185 feet in 1991. Altitude of land-surface datum 1,190 feet. Measurements provided by Santa Barbara County Water Agency beginning in 1980. Water-level records available 1966 to current year.

WATER LEVELS IN FEET BELOW LAND-SURFACE DATUM

$$
\text { DATE WATER }
$$

MAR 17, $199253.29 \mathrm{R}$

PERIOD OF RECORD HIGHEST 37.05 OCT 14, 1970 LOWEST 62.12 MAR 27, 1990

SITE NUMBER 343935120010801

LOCAL NUMBER 007N03OW27H01S

About 5 miles east of Los Olivos in Corrales Canyon. Drilled irrigation well. Diameter 10 inches, depth 188 feet, perforated 145-171 feet. Altitude of land-surface datum 852 feet. Measurements provided by Santa Barbara County Water Agency beginning in 1980. Water-level records available 1939, 1942, 1954-59, 1963, 1966, 1968 to current year.

WATER LEVELS IN FEET BELOW LAND-SURFACE DATUM

$$
\text { DATE WATER }
$$

MAR 17, $1992 \quad 5.33 \mathrm{R}$

PERIOD OF RECORD HIGHEST 4.28 APR 15, 1980 LOWEST 22.60 OCT 11, 1966

SITE NUMBER 343900120013401

LOCAL NUMBER 007N030W27Q01S

About 5 miles east of Los Olivos, about 1 mile north of Base Line Avenue. Drilled stock well. Diameter 8 inches, depth measured 91.4 feet in 1991. Altitude of land-surface datum 789 feet. Measurements provided by Santa Barbara County Water Agency beginning in 1980. Water-level records available 1942, 1945, 1950, 1954-59, 1963-64, 1966 to current year.

WATER LEVELS IN FEET BELOW LAND-SURFACE DATUM

$$
\text { DATE WATER }
$$

MAR 17, $1992 \quad 23.09 \mathrm{R}$

PERIOD OF RECORD HIGHEST 8.55 APR 04, 1983 LOWEST 47.16 MAR 23, 1990

SITE NUMBER 343946120035801

LOCAL NUMBER 007N03OW29D01S

About $3 \mathrm{miles}$ east of Los Olivos, about $1 \mathrm{mile}$ north of Roblar Avenue. Stock well. Diameter 6 inches, depth 290 feet. Altitude of land-surface datum 910 feet. Measurements provided by Santa Barbara County Water ABency beginning in 1980. Water-level records available 1941, 1957-59, 1963-64, 1966-72, 1976, 1978 to current year.

WATER LEVELS IN FEET BELOW LAND-SURFACE DATUM

$$
\text { DATE WATER }
$$

MAR 18, $1992 \quad 25.05 \mathrm{R}$

PERIOD OF RECORD HIGHEST 15.57 APR 05,1983 LOWEST 110.60 OCT 16,1968 
WELL DESCRIPTIONS AND WATER-LEVEL MEASUREMENTS

WATER YEAR OCTOBER 1991 TO SEPTEMBER 1992

\section{SITE NUMBER 343903120040701}

LOCAL NUMBER OO7N03OW29N02S

About 2 miles east of Los Olivos, about 360 feet north of Roblar Avenue. Drilled irrigation well. Diameter unknown, depth 485 feet. Altitude of land-surface datum 820 feet. Measurements provided by Santa Barbara County Water Agency beginning in 1980. Water-level records avallable 1954-59, 1963-64, 1966-72, 1974 to current year.

WATER LEVELS IN FEET BELOW LAND-SURFACE DATUM

$$
\text { DATE HATER }
$$

MAR $18,1992 \quad 305.49 \mathrm{R}$

PERIOD OF RECORD HIGHEST 230.00 MAR 01, $1954 \quad$ LOWEST 305.49 MAR 18, 1992

SITE NUMBER 343921120051601

LOCAL NUMBER OO7NO3OW3OMO1S

About 1 mile east of Los Olivos, about 2,000 feet north of Roblar Avenue. Drilled irrigation well. Diameter 12 inches, depth 200 feet. Altitude of land-surface datum 795 feet. Measurements provided by Santa Barbara County Water Agency beginning in 1980. Water-level records available 1939, 1956-59, 1966-74, 1976 , 1978 to current year.

WATER LEVELS IN FEET BELOW LAND-SURFACE DATUM

$$
\begin{array}{ll}
\text { WATER } \\
\text { DATE }
\end{array}
$$

MAR 18, $1992159.32 \mathrm{R}$

PERIOD OF RECORD HIGHEST $102.00 \quad 1939$ LOWEST 184.74 APR 27, 1978

SITE NUMBER 343812120031701

LOCAL NUMBER OOTNO3OW32RO1S

About 3 miles east of Ballard and 225 feet north of Base Line Avenue. Domestic well. Diameter 10 inches, depth measured 186 feet in 1991. Altitude of land-surface datum 701 feet. Measurements provided by Santa Barbara County Agency beginning in 1980. Water-level records available 1942-43, 1945, 1950, 1952-59, 1977 to current year.

WATER LEVELS IN FEET BELOW LAND-SURFACE DATUM

$$
\text { DATE HATER } \quad \text { LEVEL MS }
$$

MAR 18, $1992 \quad$ RD

PERIOD OF RECORD HIGHEST 82.15 APR 10, 1945 LOWEST 191.09 JUN 12,1978

SITE NUMBER 343834120030001

LOCAL NUMBER 007N030W33M02S

About 3.5 miles east of Ballard, north of Base Line Avenue on Mora Avenue. Drilled unused well. Diameter 10 inches, depth 318 feet, perforated 165-173, 178-183, 196-199, 211-216, 221-241, 254-292, 299-318 feet. Altitude of land-surface datum 746 feet. Measurements provided by Santa Barbara County Water Agency beginning in 1980 . Water-level records available 1953-72, 1974-75, 1978 to current year.

WATER LEVELS IN FEET BELOW LAND-SURFACE DATUM

$$
\begin{array}{ll} 
& \text { WATER } \\
\text { DATE } & \text { LEVEL MS }
\end{array}
$$

MAR 18， $1992 \quad 227.25 \mathrm{R}$

PERIOD OF RECORD HIGHEST 158.00 FEB 13, 1953 LOWEST 227.25 MAR 18, 1992 
SITE NUMBER 343809120000601

LOCAL NUMBER OO7NO3OW35RO1S

About 6 miles east of Ballard in Marre Canyon. Drilled stock well. Diameter 10 inches, depth 300 feet, perforated 201-203, 223-242, 264-268, 283-288 feet. Altitude of land-surface datum 880 feet. Measurements provided by Sante Barbara County Water Agency beginning in 1980. Water-level records available 1952, 1956-59, $1963-64,1966-72,1974-76,1978$ to current year.

WATER LEVELS IN FEET BELOW LAND-SURFACE DATUM

$$
\text { DATE WATER }
$$

MAR $17,1992 \quad 232.56 \mathrm{R}$

PERIOD OF RECORD HIGHEST 186.49 MAR 20, 1985 LOWEST 236.73 ARR 26, 1978

\section{SITE NUMBER 344044120072801}

LOCAL NUMEER O07N031W22A03S

Northwest of Los Olivos on Foxen Canyon Road. Irrigation well. Diameter 9 inches, depth 224 feet, perforated 95-105, 110-135, 140-150,182-188, 210-218 feet. Altitude of land-surface datum 865 feet. Measurements provided by Santa Barbara County Water Agency beginning in 1980. Water-level records available 1958-59, 1963-64, 1966 to current year.

WATER LEVELS IN FEET BELOW LAND-SURFACE DATUM

$$
\text { DATE WATER } \quad \text { LEVEL MS }
$$

MAR 16, $1992 \quad 70.23 \mathrm{R}$

PERIOD OF RECORD HIGHEST 31.62 APR 20, 1984 LOWEST 77.98 MAR 31, 1991

SITE NUMBER 344002120070001

LOCAL NUMBER 007N031W23P01S

In Los Olivos about 850 feet west of Grand Avenue and 450 feet east of Alamo Pintado Creek. Drilled irrigation well. Diameter 8 inches, depth 141.4 feet. Altitude of land-surface datum 822 feet. Measurements provided by Santa Barbara County Water Agency beginning in 1980. Water-level records available 1942 to current year.

WATER LEVELS IN FEET BELOW LAND-SURFACE DATUM

$\begin{array}{ll} & \text { WATER } \\ \text { DATE } & \text { LEVEL MS }\end{array}$

MAR 16, $1992 \quad 58.04 \mathrm{R}$

PERIOD OF RECORD HIGHEST 8.00 JAN 01, 1942 LOWEST 105.08 AUG 22, 1966

SITE NUMBER 343824120081801

LOCAL NUMBER 007N031W34M01S

About 3.8 miles north of Solvang in Ballard Canyon. Stock well. Diameter 8 inches, depth unknown. Altitude of land-surface datum 650 feet. Measurements provided by Santa Barbara County Water Agency beginning in 1980.

Water-level records available 1966-75, 1977 to current year.

WATER LEVELS IN FEET BELOW LAND-SURFACE DATUM

DATE WATER

MAR 13, $1992146,16 \mathrm{R}$

PERIOD OF RECORD HIGHEST 126.88 APR 03, 1967 LOWEST 146.16 MAR 13,1992 
WELL DESCRIPTIONS AND WATER-LEVEL MEASUREMENTS

WATER YEAR OCTOBER 1991 TO SEPTEMBER 1992

\begin{abstract}
SITE NUMBER 343827120064601
\end{abstract}
LOCAL NUMBER O07N031W35K01S

In Ballard, north of Baseline Avenue and 550 feet east of Alamo-Pintado Road. Irrigation well.. Diameter unknown, depth 149,4 feet. Altitude of land-surface datum 683 feet. Measurements provided by Santa Barbara County Water Agency beginning in 1980. Water-level records available 1942-43, 1945, 1957-59, 1964, 1967-72, 1974 to current year.

WATER LEVELS IN FEET BELOW LAND-SURFACE DATUM

$$
\text { DATE WATER }
$$

MAR 16, $199240.99 \mathrm{R}$

PERIOD OF RECORD HIGHEST 20.47 APR 05, 1983 LOWEST 100.54 OCT 23, 1967

SITE NUMBER 343831120055001

LOCAL NUMBER 007N031W36LO2S

East of Ballard, north of Base Line Avenue and 50 feet west of Refuglo Road. Drilled domestic well. Diameter 12 inches, depth 230 feet. Altitude of land-surface datum 721 feet. Measurements provided by Santa Barbara County Water Agency beginning in 1980. Water-level records available 1942 to current year.

WATER LEVELS IN FEET BELOW LAND-SURFACE DATUM

$$
\text { DATE WATER } \quad \text { LEVEL MS }
$$

MAR 16, $199260.57 \mathrm{R}$

PERIOD OF RECORD HIGHEST 16.54 APR 07, 1943 LOWEST 128,20 OCT 14,1968

SITE NUMBER 344215120170001

LOCAL NUMBER 007N032W07B01S

About 2 miles south of Los Alamos in Drum Canyon. Stock well. Diameter 16 inches, depth 77 feet. Altitude of land-surface datum 1,030 feet. Measurements provided by Santa Barbara County Water Agency beginning in 1980 . Water-level records available $1941,1945,1954-59,1966$ to current year.

WATER LEVELS IN FEET BELOW LAND-SURFACE DATUM

$$
\text { DATE WATER }
$$

MAR $13,1992 \quad 46.37 \mathrm{R}$

PERIOD OF RECORD HIGHEST 5.01 APR 06, 1983 LOWEST 58.84 OCT 21, 1969

SITE NUMBER 343821120173601

LOCAL NUMBER 007N032W31M01S

About 7 miles northwest of Buellton, north of Highway 150 . Irrigation well. Diameter 12 inches, depth measured 136.9 feet in 1991, 137.5 feet in 1992. Altitude of land-surface datum 450 feet. Measurements provided by Santa Barbara County Water Agency beginning in 1980. Water-level records ava11able 1954-59, 1966-75, 1977 to current year.

WATER LEVELS IN FEET BELOW LAND-SURFACE DATUM

$$
\begin{array}{ll} 
& \text { WATER } \\
\text { DATE } & \text { LEVEL MS }
\end{array}
$$

MAR 13, $199269.04 \mathrm{R}$

PERIOD OF RECORD HIGHEST 51.95 MAR 26, 1985 LOWEST 73.05 SEP 20, 1971 
SITE NUMBER 344116120212101

LOCAL NUMBER OO7N033W16G02S

In Santa Rita Valley about 1.7 miles northeast of Tularosa Road and Highway 246. Drilled unused well. Diameter 6 inches, depth 410 feet. Altitude of land-surface datum 520 feet. Reported measurements provided by Santa Barbara County Water Agency. Water-level records available 1987, 1990 to current year.

WATER LEVELS IN FEET BELOW LAND-SURFACE DATUM

$$
\begin{array}{ll} 
& \text { WATER } \\
\text { DATE } & \text { LEVEL MS }
\end{array}
$$

MAR 10, $1992367.89 \mathrm{R}$

PERIOD OF RECORD HIGHEST 366.82 APR 06, 1990 LOWEST 367.89 MAR 10, 1992

SITE NUMBER 344100120224901

LOCAL NUMBER 007N033W17M01S

In Lompoc Valley about 2 miles north of Cebada Canyon Road and Highway 246. Drilled unused well. Diameter 8 inches, depth 290 feet. Altitude of land-surface datum 360 feet. Reported measurements provided by Santa Barbara County Water Agency. Water-level records available 1987, 1990 to current year.

WATER LEVELS IN FEET BELOW LAND-SURFACE DATUM

$$
\begin{array}{lll} 
& \text { WATER } & \\
\text { DATE } & \text { WATER } \\
\text { LEVEL MS } & \text { LEVEL MS }
\end{array}
$$

MAR 10, $1992 \quad 264.58 \mathrm{R} \quad$ SEP $01,1992 \quad 271.42 \mathrm{R}$

PERIOD OF RECORD HIGHEST 263.75 MAR 21, 1991 LOWEST 274.30 AUG 27, 1991

SITE NUMBER 344051120224901

LOCAL NUMBER OO7N033W17N02S

Northeast of Lompoc in Cebada Canyon. Irrigation well. Diameter 8 inches, depth unknown. Altitude of land-surface datum 360 feet. Measurements provided by Santa Barbara County Water Agency beginning in 1980. Water-level records available 1966 to current year.

WATER LEVELS IN FEET BELOW LAND-SURFACE DATUM

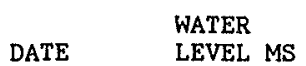

MAR 10, $1992 \quad 286.24 \mathrm{R}$

PERIOD OF RECORD HIGHEST 262.98 MAR 30, 1967 LOWEST 301.15 APR 02, 1981

SITE NUMBER 344035120235901

LOCAL NUMBER 007N033W19D01S

Northeast of Lompoc in Cebada Canyon. Drilled irrigation well. Diameter 14 inches, depth 760 feet, cased to 552 feet, perforated 228-264 feet. Altitude of land-surface datum 270 feet. Measurements provided by Santa Barbara County Water Agency beginning in 1980. Water-level records available 1958, 1966 to current year.

WATER LEVELS IN FEET BELOW LAND-SURFACE DATUM

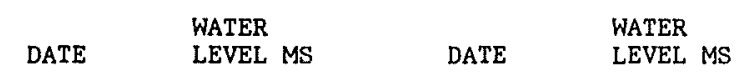

MAR 10, $1992 \quad 199.75 \mathrm{R} \quad$ SEP 01, $1992 \quad 201.15 \mathrm{SR}$

PERIOD OF RECORD HIGHEST 186.30 MAR 30, 1967 LOWEST 202.38 OCT 19, 1970 
WELL DESCRIPTIONS AND WATER-LEVEL MEASUREMENTS

WATER YEAR OCTOBER 1991 TO SEPTEMBER 1992

SITE NUMBER 344025120221601

LOCAL NUMBER 007N033W20G01S

About 6 miles east of Bueliton, 0.7 mile north of Highway 246 . Diameter 6 inches; depth measured 385 feet in 1991 , original depth 460 feet, perforated 220-320, 360-460 feet. Altitude of land-surface datum 400 feet. Reported measurements provided by Santa Barbara County Water Agency. Water-level records ava1lable 1977, 1987, 1990 to current year.

WATER LEVELS IN FEET BELOW LAND-SURFACE DATUM

$\begin{array}{lllll} & & \text { WATER } & \text { WATER } \\ \text { DATE } & \text { WEVEL MS } & \text { DATE } & \text { LEVEL MS } & \text { DATE } \\ \text { LEVEL MS }\end{array}$

MAR 10, $1992 \quad 314.14 \mathrm{R} \quad$ MAR 22, $1992 \quad 314.14 \mathrm{R}$

SEP 01, $1992 \quad 316.19 \mathrm{R}$

PERIOD OF RECORD HIGHEST 304.41 OCT 17,1977 LOWEST 318.15 AUG 04, 1987

SITE NUMBER 344025120211501

LOCAL NUMBER 007N033W21G02S

In Santa Rita Valley about 1.5 miles northeast of Tularosa Road and Highway 246 . Drilled irrigation well.

Diameter 6 inches, depth unknown. Altitude of land-surface datum 430 feet. Water-level records available 1987 , 1990 to current year.

WATER LEVELS IN FEET BELOW LAND-SURFACE DATUM

$\begin{array}{lll} & \text { WATER } & \\ \text { DATE } & \text { WEVEL MS } & \text { DATE } \\ \text { LEVEL MS }\end{array}$

MAR 10, $1992 \quad 313.22 \mathrm{R} \quad \mathrm{SEP} 01,1992 \quad 313.80 \mathrm{R}$

PERIOD OF RECORD HIGHEST 310.09 AUG 04, 06, 1987 LOWEST 313.80 SEP 01, 1992

SITE NUMBER 343956120214001

LOCAL NUMBER 007N033W21N01S

About 5 miles northeast of Lompoc. Unused well. Diameter 14 inches, depth unknown. Altitude of land-surface datum 360 feet. Measurements provided by Santa Barbara County Water Agency beginning in 1980. Water-level records available 1967-71, 1973-76, 1978 to current year.

WATER LEVELS IN FEET BELOW LAND-SURFACE DATUM

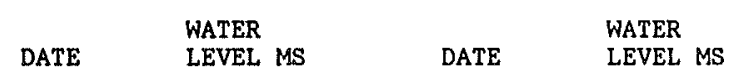

MAR 10, $1992 \quad 296.34 \mathrm{R} \quad$ SEP 01, $1992 \quad 298.73 \mathrm{R}$

PERIOD OF RECORD HIGHEST 278.35 MAR 26, 1969 LOWEST 302.08 SEP 08, 1991

SITE NUMBER 343926120201001

LOCAL NUMBER 007N033W27G01S

About $8 \mathrm{miles}$ east of Lompoc, $0.5 \mathrm{mile}$ south of Highway 150. Drilled irrigation well. Diameter 14 inches, depth 735 feet, perforated $435-735$ feet. Altitude of land-surface datum 432 feet. Measurements provided by Santa Barbara County Water Agency beginning in 1980. Water-level records available 1967, 1969-71, 1973 to current year.

WATER LEVELS IN FEET BELOW LAND-SURFACE DATUM

DATE WATER

MAR $10,1992 \quad 359.86 \mathrm{R}$

PERIOD OF RECORD HIGHEST 347.77 APR $11,1974 \quad$ LOWEST 374.08 DEC 27,1977 
SITE NUMBER 343923120200101

LOCAL NUMBER 007N033W27J01S

About $8 \mathrm{miles}$ east of Lompoc, $0.5 \mathrm{mile}$ south of Highway 150 . Dug stock well. Diameter 20 inches, depth 52.5 feet. Altitude of land-surface datum 458.2 feet. Measurements provided by Santa Barbara County Water Agency beginning in 1980. Water-level records available 1941, 1945, 1955-58, 1966-74, 1976 to current year.

WATER LEVELS IN FEET BELOW LAND-SURFACE DATUM

$$
\text { DATE WATER MS }
$$

MAR 10, $199219.56 \mathrm{R}$

PERIOD OF RECORD HIGHEST 17.11 APR 06, 1983 LOWEST 35.05 NOV 08, 1957

SITE NUMBER 343946120215301

LOCAL NUMBER 007N033W28D03S

About 6 miles east of Lompoc, south of Highway 246. Drilled irrigation well. Diameter 14 inches, depth 600 feet, perforated 440-590 feet. Altitude of land-surface datum 360 feet. Measurements provided by Santa Barbara County Water Agency beginning in 1988. Water-level records avallable 1971, 1973, 1988 to ourrent year.

WATER LEVELS IN FEET BELOW LAND-SURFACE DATUM

$$
\text { DATE WATER } \quad \text { LEVEL MS }
$$

MAR 10, $1992292.56 \mathrm{R}$

PERIOD OF RECORD HIGHEST 278.00 NOV 24, 1971 LOWEST 297.48 AUG 23, 1990

SITE NUMBER 343949120232901

LOCAL NUMBER 007N033W30B02S

About 3 miles northeast of Lompoc on Highway 246. Drilled domestic well. Diameter 6 inches, depth 240 feet, perforated 200-210 feet. Altitude of land-surface datum 230 feet. Measurements provided by Santa Barbara County Water Agency beginning in 1991. Water-level records available 1981, 1991 to current year.

WATER LEVELS IN FEET BELOW LAND-SURFACE DATUM

$$
\text { DATE WATER }
$$

MAR 10, $1992175.31 \mathrm{R}$

PERIOD OF RECORD HIGHEST 175.30 MAR 21, $1991 \quad$ LOWEST 176 JUL 1981

SITE NUMBER 343824120175201

LOCAL NUMBER 007N033W36J01S

About 10 miles east of Lompoc and northwest of intersection of Drum Canyon Road and Highway 150 . Drilled irrigation well. Diameter 8 inches, depth measured 157 feet in 1990 , original depth 190 feet, perforated 125-190 feet. Altitude of land-surface datum 495 feet. Measurements provided by Santa Barbara County Water Agency beginning in 1980. Water-level records available 1941, 1945-46, 1948, 1954-59, 1966 to current year.

WATER LEVELS IN FEET BELOW LAND-SURFACE DATUM

$$
\text { DATE WATER } \quad \text { LEVEL MS }
$$

MAR 13, $1992 \quad$ RD

PERIOD OF RECORD HIGHEST 114.10 OCT 22, 1946 LOWEST 155.15 MAR 20, 1989 
WELL DESCRIPTIONS AND WATER-LEVEL MEASUREMENTS

WATER YEAR OCTOBER 1991 TO SEPTEMBER 1992

SITE NUMBER 343825120174601

LOCAL NUMBER OO7N033W36J02S

About 10 miles east of Lompoc and northwest of intersection of Drum Canyon Road and Highway 150 . Unused well. Diameter 8 inches, depth 97.5 feet. Altitude of land-surface datum 478 feet. Measurements provided by Santa Barbara County Water Agency beginning in 1980. Water-level records available 1941, 1954, 1957,1966 to current yoar.

WATER LEVELS IN FEET BELOW LAND-SURFACE DATUM

DATE WATER

MAR 13, $199262.72 \mathrm{R}$

PERIOD OF RECORD HIGHEST 51.00 APR 23, 1984 LOWEST 69.77 NOV 03, 1954

SITE NUMBER 344221120273501

LOCAL NUMBER 007N034WOGH05S

About 3 miles north of Lompoc, northeast of Lompoc-Casmalia Road. Drilled unused well. Diameter 14 inches, depth 853 feet, perforated 653-853 feet. Altitude of land-surface datum 275 feet. Measurements provided by Santa Barbara County Water Agency beginning in 1980. Water-level records avallable 1960-76, 1978 to current year.

WATER LEVELS IN FEET BELOW LAND-SURFACE DATUM

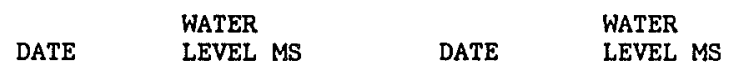

MAR 10, $1992 \quad 254.22 \mathrm{R} \quad$ SEP 02, $1992 \quad 254.09 \mathrm{R}$

PERIOD OF RECORD HIGHEST 218.90 MAR 28, 1962 LOWEST 284.70 MAR 03, 1966

SITE NUMBER 344221120273502

LOCAL NUMBER OOTN034WOGH06S

About 3 miles north of Lompoc, north of intersection of Lompoc-Casmalia Road and Cabrillo Highway. Industrial well. Diameter 16 inches, depth 810 feet, perforated $594-810$ feet. Altitude of land-surface datum 275 feet. Measurements provided by Santa Barbara County Water Agency beginning in 1980. Water-level records available 1966 to current year.

WATER LEVELS IN FEET BELOW LAND-SURFACE DATUM

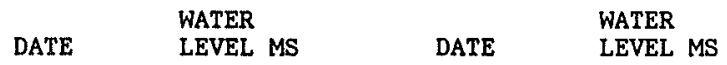
MAR $10,1992 \quad 254.92 \mathrm{R}$
SEP $02,1992 \quad 255.72 R$

PERIOD OF RECORD HIGHEST 241.60 MAR 23, 1976 LOWEST $255.72 \quad$ SEP 02,1992

SITE NUMBER 344219120250601

LOCAL NUMBER 007N034W12E01S

About 4 miles north-northeast of Lompoc, east of Highway 1. Drilled observation well. Diameter 8 inches 0-278 feet, 6 inches 278-385 feet, depth 377 feet, orisinal depth 385 feet, perforated $345-385$ feet. Altitude of land-surface datum 385.83 feet. Measurements provided by Santa Barbara County Water Agency beginning in 1980. Water-level records avallable 1949-50,1956, 1959, 1961-66, 1970 to current year.

WATER LEVELS IN FEET BELOW LAND-SURFACE DATUM

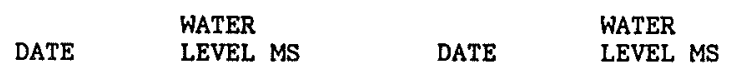

MAR 06, $1992 \quad 323.69 \mathrm{R} \quad \mathrm{SEP} 02,1992 \quad 324.04 \mathrm{R}$

PERIOD OF RECORD HIGHEST 301.00 JUN 19,1949 LOWEST 324.04 SEP 02, 1992 
WATER LEVELS, SANTA BARBARA COUNTY--Continued

WELL DESCRIPTIONS AND WATER-LEVEL MEASUREMENTS

WATER YEAR OCTOBER 1991 TO SEPTEMBER 1992

SITE NUMBER 344126120255201

LOCAL NUMBER 007N034W14F04S

About 3 miles north of Lompoc. Drilled public-supply well. Diameter 16 inches, depth 540 feet, perforated

280-530 feet. Altitude of land-surface datum 272 feet. Measurements provided by Santa Barbara County Water Agency beginning in 1989. Water-level records available 1982, 1989 to current year.

WATER LEVELS IN FEET BELOW LAND-SURFACE DATUM

$\begin{array}{lll} & \text { WATER } & \\ \text { DATE } & \text { LEVEL MS } & \text { WATER } \\ & \text { LEVEL MS }\end{array}$

MAR 09, $1992 \quad 225.57 \mathrm{R} \quad \mathrm{SEP} 02,1992 \quad 227.63 \mathrm{R}$

PERIOD OF RECORD HIGHEST 210 AUG 26, 1982 LOWEST 227.63 SEP 02,1992

SITE NUMBER 344117120255001

LOCAL, NUMBER 007N034W14L01S

North of Lompoc on Burton Mesa Road, Observation well. Diameter 6 inches, depth 550 feet, perforated

200-550 feet. Altitude of land-surface datum 250 feet. Measurements provided by Santa Barbara County Water Agency beginning in 1981. Water-level records available 1981 to current year.

WATER LEVELS IN FEET BELOW LAND-SURFACE DATUM

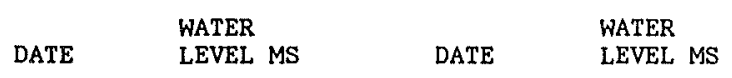

MAR 09, $1992 \quad 213.82 \mathrm{R} \quad \operatorname{SEP} 02,1992 \quad 215.90 \mathrm{R}$

PERIOD OF RECORD HIGHEST 205.85 APR 25, 1984 LOWEST $215.90 \quad$ SEP 02,1992

SITE NUMBER 344140120272301

LOCAL NUMBER 007N034W15D01S

About $3 \mathrm{miles}$ north of Lompoc on Lompoc-Casmalia Road. Drilled public-supply well. Diameter 24 to 16 inohes, depth 790 feet, perforated 450-790 feet. Altitude of land-surface datum 180 feet. Measurements provided by Santa Barbara County Water Agency beginning in 1980. Water-level records available 1960-74, 1978 to current year.

WATER LEVELS IN FEET BELOW LAND-SURFACE DATUM

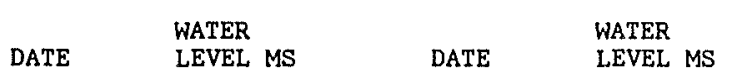

MAR 10, $1992129.14 \mathrm{R} \quad \operatorname{SEP} 02,1992 \quad 132.31 \mathrm{R}$

PERIOD OF RECORD HIGHEST 111.22 JUL 27, 1960 LOWEST 150,00 JAN 28, 1960

SITE NUMBER 344140120272302

LOCAL NUMBER 007N034W15D02S

About 3 miles north of Lompoc on Lompoc-Casmalia Road. Drilled unused well. Diameter 16 inches, depth 690 feet, perforated 486-666 feet. Altitude of land-surface datum 180 feet. Measurements provided by Santa Barbara County Water Agency beginning in 1980. Water-level records available 1970, 1977 to current year.

WATER LEVELS IN FEET BELOW LAND-SURFACE DATUM

$\begin{array}{lll} & \text { WATER } & \\ \text { DATE } & \text { LEVEL MS } & \text { WATER } \\ & \text { LEVEL MS }\end{array}$

MAR 10, $1992 \quad 128.44 \mathrm{R} \quad$ SEP 02, $1992 \quad 131.42 \mathrm{R}$

PERIOD OF RECORD HIGHEST 123.54 OCT 20,1970 LOWEST 131.44 AUG 24,1990 
WELL DESCRIPTIONS AND WATER-LEVEL MEASUREMENTS

WATER YEAR OCTOBER 1991 TO SEPTEMBER 1992

SITE NUMBER 344134120272201

LOCAL NUMBER 007N034W15E01S

Solxtheast of Vandenberg Village on Casmalia Road. Drilled public-supply well. Diameter 18 inches, depth 749 feet, perforated 511-725 feet. Atlitude of land-surface datum 180 feet. Measurements provided by Santa Barbara County Water Agency beginning in 1980. Water-level records available 1964, 1968 to current year.

WATER LEVELS IN FEET BELOW LAND-SURFACE DATUM

$$
\text { DATE WATER }
$$

MAR 10, $1992130.65 \mathrm{R}$

PERIOD OF RECORD HIGHEST 122.06 MAR 07, 1968 LOWEST 136.30 APR 10,1974

SITE NUMBER 344100120270401

LOCAL NUMBER 007N034W15P01S

South of Mission Hills about 0.25 mile northeast of intersection of Highway 1 and Lompoc-Casmalia Road. Drilled stock well. Dlameter 14 inches, depth 924 feet, perforated 200-440,800-920 feet. Altitude of land-surface datum 300 feet. Measurements provided by Santa Barbara County Water Agency beginning in 1980. Water-level records ava1lable $1963,1969,1974$ to current year.

WATER LEVELS IN FEET BELOW LAND-SURFACE DATUM

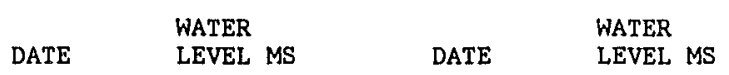

MAR 09, $1992 \quad 253.22 \mathrm{R}$ SEP 02, $1992 \quad 253.46 \mathrm{R}$

PERIOD OF RECORD HIGHEST 225.00 NOV 1963 LOWEST 277.13 MAR 23, 1976

SITE NUMBER 344017120285502

LOCAL NUMBER 007N034W20K04S

On Vandenberg AFB, south of Federal Corrections Institute. Drilled public-supply water-table well. Diameter 18 inches, original depth 186 feet, depth measured 144.5 feet in 1966, perforated 90-174 feet. Altitude of land-surface datum 75 feet. Water-level records available 1941-42, 1955, 1966-77, 1979 to current year.

WATER LEVELS IN FEET BELOW LAND-SURFACE DATUM

$$
\begin{array}{ll}
\text { DATER } & \text { WETEL MS }
\end{array}
$$

SEP 03, 1992

$$
\mathrm{P}
$$

PERIOD OF RECORD HIGHEST 15.70 OCT 18,1942 LOWEST 37.00 APR 04, 1974

SITE NUMBER 344023120292802

LOCAL NUMBER 007N034W20M02S

On Vandenberg AFB, southwest of Federal Corrections Institute. Drilled public-supply water-table well. Diameter 18 inches, original depth 192 feet, perforated 77-93, 101-177 feet. Altitude of land-surface datum 70 feet. Water-level records available 1941-42, 1955-56, 1966-73, 1975-78, 1980 to current year.

WATER LEVELS IN FEET BELOW LAND-SURFACE DATUM

$$
\text { DATE WATER }
$$

SEP 03, 1992

FERIOD OF RECORD

$$
\text { o }
$$$$
\text { HIGHEST } 18.40 \text { APR 01, } 1942 \text { LOWEST } 52.56 \text { AUG } 30,1989
$$ 
SITE NUMBER 344016120290101

LOCAL NUMBER 007N034W20P03S

North of Lompoc at the Federal Corrections Institute. Drilled observation well in Careaga Sand of Pliocene age. Diameter 6 inches, depth 320 feet, perforated 260-320 feet. Altitude of land-surface datum 71 feet. Reported measurements provided by Santa Barbara County Water Agency. Water-level records avallable 1987 , 1990 to current year.

WATER LEVELS IN FEET BELOW LAND-SURFACE DATUM

$$
\begin{array}{lll} 
& \text { WATER } & \\
\text { DATE } & \text { WEVEL MS } & \text { WATER } \\
& \text { LEVEL MS }
\end{array}
$$

MAR 06, $1992 \quad 25.87 \mathrm{R} \quad$ SEP 03, $1992 \quad 33.86 \mathrm{R}$

PERIOD OF RECORD HIGHEST 25.87 MAR 06, 1992 LOWEST 41.62 AUG 28,1991

SITE NUMBER 344033120263404

LOCAL NUMBER 007N034W22J06S

West of La Purisima Mission along Lompoc-Casmalla Road. Drilled irrigation well. Diameter unknown, depth 135 feet. Altitude of land-surface datum 97 feet. Measurements provided by Santa Barbara County Water Agency beginning in 1980. Water-level records available 1949-54, 1957-58, 1961 to current year.

WATER LEVELS IN FEET BELOW LAND-SURFACE DATUM

$$
\begin{array}{ll} 
& \text { WATER } \\
\text { DATE } & \text { LEVEL MS }
\end{array}
$$

MAR 09, $1992 \quad 45.77 \mathrm{R}$

PERIOD OF RECORD HIGHEST 27.77 NOV 23,1949 LOWEST 55.34 APR 16,1973

SITE NUMBER 344010120251601

LOCAL NUMBER 007N034W24N01S

Southeast of La Purisima Mission along Lompoc-Casmalia Road. Drilled irrigation well. Diameter 16 inches, depth 183 feet. Altitude of land-surface datum 130.4 feet. Measurements provided by city of Santa Barbara 1930-35, and Santa Barbara County Water Agency beginning in 1980. Water-level records available 1924, 1930-35, 1949, 1953 to current yoar.

WATER LEVELS IN FEET BELOW LAND-SURFACE DATUM

$$
\begin{array}{lll} 
& \text { WATER } & \\
\text { DATE } & \text { LEVEL MS } & \text { WATER }
\end{array}
$$

MAR 09, $1992 \quad 77.33 \mathrm{R} \quad$ SEP 01, $1992 \quad 80.04 \mathrm{R}$

PERIOD OF RECORD HIGHEST 38.00 JUL 1924 LOWEST $80.04 \quad$ SEP 01,1992

SITE NUMBER 343943120252201

LOCAL NUMBER 007N034W26H03S

About $1.5 \mathrm{miles}$ northeast of Lompoc. Drilled unused well. Diameter 16 inches, depth drilled 123 feet, depth measured 43 in 1966, cleaned out to 83 feet in 1967, depth measured 66.9 feet in 1991. Altitude of land-surface datum 112.92 feet. Records provided by Santa Barbara County Water Agency beginning in 1980, other reported measurements provided by U.S. Bureau of Reclamation. Water-level records available 1950 to current year.

WATER LEVELS IN FEET BELOW LAND-SURFACE DATUM

$$
\begin{array}{ll} 
& \text { WATER } \\
\text { DATE } & \text { LEVEL MS }
\end{array}
$$

MAR 09, $1992 \quad 61.70 \mathrm{R}$

PERIOD OF RECORD HIGHEST 40.13 MAR 09, 1950 LOWEST 63.07 MAR 21, 1991 
WELL DESCRIPTIONS AND WATER-LEVEL MEASUREMENTS

WATER YEAR OCTOBER 1991 TO SEPTEMBER 1992

SITE NUMBER 343939120271801

LOCAL NUMBER 007N034W27E04S

In Lompoc Valley, about 300 feet northeast of $H$ Street and Central Avenue. Drilled observation well in alluvium. Diameter 2 inches, depth 58.5 feet, perforated 48.5-58.5 feet. Altitude of land-surface datum 89 feet. Reported measurements provided by city of Lompoc. Water-level records available 1987 to current year.

WATER LEVELS IN FEET BELOW LAND-SURFACE DATUM

$$
\begin{array}{lll} 
& \text { WATER } & \\
\text { DATE } & \text { WEVEL MS } \\
& \text { DATE } & \text { LEVEL MS }
\end{array}
$$

FEB $20,1992 \quad 42.75 \mathrm{R}$ AUG $12,1992 \quad 41.58 \mathrm{R}$

PERIOD OF RECORD HIGHEST 39.90 OCT 30,1987 LOWEST 52.74 AUG 23, 1990

SITE NUMBER 343949120265301

LOCAL NUMBER 007N034W27F07S

In Lompoc, northeast of intersection of Central Avenue and H Street. Drilled observation well. Diameter 6 inches, depth 200 feet, perforated 90-190 feet. Altitude of land-surface datum 90 feet. Reported measurements provided by city of Lompoc. Water-level records available 1991 to current year.

WATER LEVELS IN FEET BELOW LAND-SURFACE DATUM

$\begin{array}{lll} & \text { WATER } & \\ \text { DATE } & \text { WEVEL MS } & \text { DATER } \\ \text { LEVEL MS }\end{array}$
FEB 28, 1992
$45.15 \mathrm{~V}$
SEP 02, 1992
$46.55 \mathrm{R}$

PERIOD OF RECORD HIGHEST 45.15 FEB 28, 1992 LOWEST 46.55 SEP 02, 1992

SITE NUMBER 343949120264901

LOCAL NUMBER 007N034W27G06S

In Lompoc, northeast of intersection of North A Street and Canfield Drive. Drilled irrigation well. Diameter 12 inches, depth reported 160 feet, perforated 120-160 feet. Altitude of land-surface datum 90 feet. Measurements provided by Santa Barbara County Water Agency. Water-level records available 1989 to current year.

WATER LEVELS IN FEET BELOW LAND-SURFACE DATUM

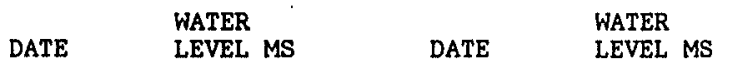

MAR 09, $199238.33 \mathrm{R} \quad$ SEP 01, $1992 \quad 40.47 \mathrm{R}$

PERIOD OF RECORD HIGHEST 38.33 MAR 09, 1992 LOWEST 54.80 AUG 23, 1990

SITE NUMBER 343938120264101

LOCAL NUMBER 007N034W27K04S

In Lompoc Valley, about $0.7 \mathrm{mile}$ east of $\mathrm{H}$ Street and A Street. Augered observation well in alluvium. Diameter 2 inches, depth 62.1 feet, perforated $57.1-62.1$ feet. Altitude of land-surface datum $102.35 \mathrm{ft}$. Reported measurements provided by city of Lompoc. Water-level records available 1987 to current year.

WATER LEVELS IN FEET BELOW LAND-SURFACE DATUM

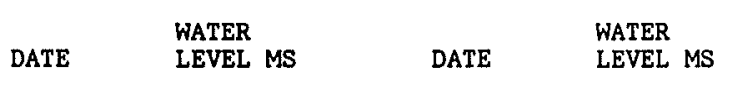
FEB 20, $1992 \quad 56.62 \mathrm{R}$
AUG $12,1992 \quad 54.32 \mathrm{R}$

PERIOD OF RECORD HIGHEST 46.42 MAY 07, 1987 LOWEST 58.4 JAN 26, FEB 02, 09, 1989 
SITE NUMBER 343938120264102

LOCAL NUMBER 007N034W27K05S

In Lompoc Valley, about $0.7 \mathrm{mile}$ east of $\mathrm{H}$ Street and A Street. Augered observation well in alluvium. Diameter 2 inches, depth 97.3 feet, perforated 92.3-97.3 feet. Altitude of land-surface datum 102.59 feet. Reported measurements provided by city of Lompoc. Water-level records avallable 1987 to current year.

WATER LEVELS IN FEET BELOW LAND-SURFACE DATUM

$$
\begin{array}{lll} 
& \text { WATER } & \\
\text { DATE } & \text { WEVEL MS } & \text { DATE }
\end{array}
$$

FEB 20, $199261.98 \mathrm{R}$ AUG $12,1992 \quad 55.34 \mathrm{R}$

PERIOD OF RECORD HIGHEST 44.28 MAR 18, 1987 LOWEST 78.04 FEB 13, 1991

\section{SITE NUMBER 343938120264001}

LOCAL NUMBER 007N034W27K06S

In Lompoc Valley about $0.7 \mathrm{mile}$ east of A Street and Central Avenue. Drilled observation well in Careaga Sand of Pliocene Age. Diameter 16 inches, 0-22 feet, 6.63 inches, 22-383 feet, depth 383 feet, depth of hole 1,025 feet, perforated 270-350 feet. Altitude of land-surface datum 98.63 feet. Reported measurements provided by city of Lompoc. Water-level records available 1987 to current year.

WATER LEVELS IN FEET BELOW LAND-SURFACE DATUM

$\begin{array}{lllll} & & \text { WATER } & \text { WATER } \\ \text { DATE } & \text { LEVEL MS } & \text { DATE } & \text { LEVEL MS } & \text { DATE }\end{array}$

FEB 19, $1992 \quad 50.29 \mathrm{R} \quad$ AUG $11,1992 \quad 51.34 \mathrm{R} \quad$ SEP 03, $1992 \quad 51.57 \mathrm{RS}$

PERIOD OF RECORD HIGHEST 47.4 MAY 03, 1988 LOWEST 62.70 AUG 23, 1990

SITE NUMBER 343938120264002

LOCAL NUMBER 007N034W27K07S

In Lompoc Valley, about $0.7 \mathrm{mile}$ east of A Street and Central Avenue. Drilled public supply well in alluvium. Diameter 30 inches 0-50 feet, 16 inches 50-175 feet, depth 175 feet, perforated 95-165 feet. Altitude of

land-surface datum 101.78 feet. Reported measurements provided by city of Lompoc. Water-level records available 1988 to current year.

WATER LEVELS IN FEET BELOW LAND-SURFACE DATUM

$\begin{array}{lll} & \text { WATER } & \\ \text { DATE } & \text { WEVEL MS } & \text { WATER } \\ \end{array}$

FEB 19, $1992 \quad 53.75 \mathrm{R}$ AUG 11, $1992 \quad 52.67 \mathrm{R}$

PERIOD OF RECORD HIGHEST 50.8 MAY 18, 1988 LOWEST 70.88 FEB 23, 1991

SITE NUMBER 343920120271401

LOCAL NUMBER 007N034W27N06S

In Lompoc about $0.4 \mathrm{mile}$ southeast of $\mathrm{H}$ Street and Central Avenue. Augered observation well in alluvium. Diameter 2 inches, depth 95.5 feet, perforated 85.5-95.5 feet. Altitude of land-surface datum 79 feet. Reported measurements provided by city of Lompoc. Water-level records available 1987 to current year.

WATER LEVELS IN FEET BELOW LAND-SURFACE DATUM

\begin{tabular}{|c|c|c|c|c|c|}
\hline DATE & $\begin{array}{l}\text { WATER } \\
\text { LEVEL MS }\end{array}$ & DATE & $\begin{array}{l}\text { WATER } \\
\text { LEVEL MS }\end{array}$ & DATE & $\begin{array}{l}\text { WATER } \\
\text { LEVEL MS }\end{array}$ \\
\hline
\end{tabular}

FEB 19, $1992 \quad 43.97 \mathrm{R} \quad$ AUG $11,1992 \quad 43.75 \mathrm{R} \quad$ SEP 02, $1992 \quad 47.88 \mathrm{RS}$

PERIOD OF RECORD HIGHEST 41.44 APR $14,1988 \quad$ LOWEST 52.17 AUG 28,1991 
In Lompoc. Drilled public-supply well. Diameter 14 inches, depth 172 feet, perforated 100-172 feet. Altitude of land-surface datum 92.35 feet. Records provided by U.S. Bureau of Reclamation and city of Lompoc beginning in 1972. Water-level records available 1965, 1968, 1972 to current year.

WATER LEVELS IN FEET BELOW LAND-SURFACE DATUM

$$
\text { DATE WATER }
$$

SEP 02, $1992 \quad 56.13 \mathrm{R}$

PERIOD OF RECORD HIGHEST 34.2 JUN 26,1980 LOWEST 68.20 AUG 15,1972 JAN 22,1974

SITE NUMBER 343919120270801

LOCAL NUMBER 007N034W27P06S

In Lompoc Valley, about $0.3 \mathrm{mile}$ northeast of $\mathrm{H}$ Street and D Street. Augered observation well. Diameter 2 inches, depth 56.5 feet, perforated 51-56 feet. Altitude of land-surface datum 80 feet. Reported measurements provided by city of Lompoc. Water-level records avallable 1987 to current year.

WATER LEVELS IN FEET BELOW LAND-SURFACE DATUM

$\begin{array}{lll} & \text { WATER } & \text { WATER } \\ \text { DATE } & \text { LEVEL MS } & \text { DATE }\end{array}$
FEB 20, $1992 \quad 46.17 \mathrm{R}$
AUG 12, 1992
$46.18 \mathrm{R}$

PERIOD OF RECORD HIGHEST

42.4 DEC 09, 1987

LOWEST 54.09 AUG 21, 1991

SITE NUMBER 343924120264401

LOCAL NUMBER 007N034W27Q02S

In Lompoc about $0.3 \mathrm{mile}$ southeast of A Street and Central Avenue, Drilled municipal well in alluvium. Diameter 14 inches, depth 185 feet, perforated 95-175 feet. Altitude of land-surface datum 98.97 feet. Reported measurements provided by city of Lompoc. Water-level records available 1990 to current year.

WATER LEVELS IN FEET BELOW LAND-SURFACE DATUM

$\begin{array}{lll} & \text { WATER } & \\ \text { DATE } & \text { WEVEL MS } & \text { DATE } \\ \text { LEVEL MS }\end{array}$

FEB 20, $1992 \quad 56.48 \mathrm{R} \quad$ AUG $11,1992 \quad 55.77 \mathrm{R}$

PERIOD OF RECORD HIGHEST 55.77 AUG 11, 1992 LOWEST 75.58 AUG 24, 1990

SITE NUMBER 343938120282401

LOCAL NUMBER 007N034W28M01S

In Lompoc Valley, about 100 feet southeast of Central Avenue and V Street. Drilled observation well in alluvium. Diameter 2 inches, depth 72 feet, perforated 62-72 feet. Altitude of land-surface datum 78 feet. Reported measurements provided by city of Lompoc. Water-level records available 1987 to current year.

WATER LEVELS IN FEET BELOW LAND-SURFACE DATUM

$$
\begin{array}{lll} 
& \text { WATER } & \text { WATER } \\
\text { DATE } & \text { DEVEL MS } & \text { DATE } \\
\text { LEVEL MS }
\end{array}
$$

FEB $20,1992 \quad 34.94 \mathrm{R} \quad$ SEP 02, $1992 \quad 39.43 \mathrm{R}$

PERIOD OF RECORD HIGHEST 32.2 JAN 28, 1988 LOWEST 46.95 AUG 23, 1990 
STTE NUMBER 343938120282402

LOCAL NUMBER 007N034W28M02S

In Lompoc Valley, about 100 feet southeast of Central Avenue and V Street, Drilled observation well in alluvium. Diameter 2 inches, depth 162.3 feet, perforated 142.3-162.3 feet. Altitude of land-surface datum 78 feet. Reported measurements provided by city of Lompoc. Water-level records available 1987 to ourrent year.

WATER LEVELS IN FEET BELOW LAND-SURFACE DATUM

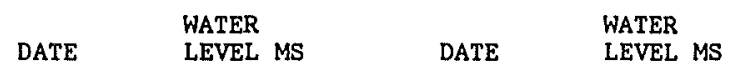

FEB 20, $1992 \quad 34.25 \mathrm{R} \quad \mathrm{SEP} 02,1992 \quad 40.45 \mathrm{R}$

PERIOD OF RECORD HIGHEST 32,9 JAN 20, 28, MAR 03, 1988 LOWEST 49.64 AUG 23, 1990

SITE NUMBER 343924120274101

LOCAL NUMBER 007N034W28Q01S

In Lompoc Valley, about $0.18 \mathrm{mile}$ southeast of 0 Street and Barton Street. Drilled observation well in alluvium, Diameter 2 inches, depth 67.1 feet, perforated $57.1-67.1$ feet. Altitude of land-surface datum 70 feet. Reported measurements provided by city of Lompoc. Water-level records available 1987-88, 1990 to current year.

WATER LEVELS IN FEET BELOW LAND-SURFACE DATUM

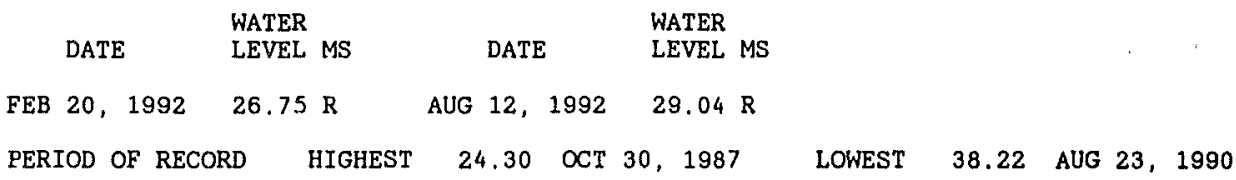

SITE NUMBER 343948120292002

LOCAL NUMBER 007N034W29E04S

About 2.5 miles northwest of Lompoc. Drilled irrigation well. Diameter 16 inches, depth 176 feet. Altitude of land-surface datum 67.71 feet. Measurements provided by Santa Barbara County Water Agency beginning in 1980 . Water-level records available 1945-76, 1978 to current year.

WATER LEVELS IN FEET BELOW LAND-SURFACE DATUM

$$
\begin{array}{ll} 
& \text { WATER } \\
\text { DATE } & \text { LEVEL MS }
\end{array}
$$

MAR 06, $1992 \quad 27.76 \mathrm{R}$

PERIOD OF RECORD HIGHEST 18.36 MAR 31,1958 LOWEST $48,14 \quad$ JUL 24,1963

SITE NUMBER 343944120290101

LOCAL NUMBER 007N034W29F01S

In Lompoc Valley, about $0.7 \mathrm{mile}$ northwest of Central Avenue and V Street. Drilled observation well in alluvium. Diameter 2 inches, depth of well 164 feet, depth of hole 180 feet, perforated 144-164 feet. Altitude of land-surface datum 62.67 feet. Reported measurements provided by city of Lompoc. Water-1evel records avallable 1987 to current year.

WATER LEVELS IN FEET BELOW LAND-SURFACE DATUM

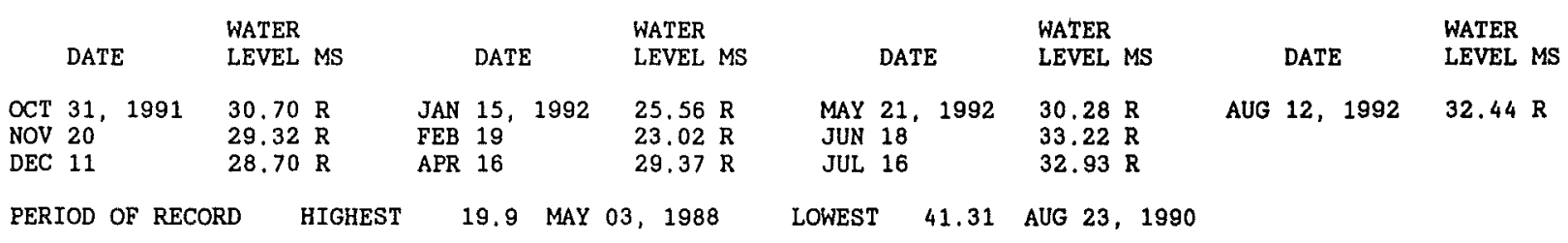


WELI DESCRIPTIONS AND WATER-LEVEL MEASUREMENTS

WATER YEAR OCTOBER 1991 TO SEPTEMBER 1992

SITE NUMBER 343944120290102

LOCAL NUMBER 007N034W29F02S

In Lompoc Valley, about $0.7 \mathrm{mile}$ northwest of Central Avenue and $\mathrm{V}$ street. Drilled observation well in alluvium. Diameter 2 inches, depth 60.6 feet, perforated 40.6-60.6 feet. Altitude of land-surface datum 62.68 feet. Reported measurements provided by city of Lompoc. Water-level records available 1987 to current year.

WATER LEVELS IN FEET BELOW LAND-SURFACE DATUM

\begin{tabular}{|c|c|c|c|c|c|c|c|}
\hline DATE & $\begin{array}{l}\text { WATER } \\
\text { LEVEL MS }\end{array}$ & DATE & $\begin{array}{l}\text { WATER } \\
\text { LEVEL MS }\end{array}$ & DATE & $\begin{array}{l}\text { WATER } \\
\text { LEVEL MS }\end{array}$ & DATE & $\begin{array}{l}\text { WATER } \\
\text { LEVEL MS }\end{array}$ \\
\hline $\begin{array}{ll}31, & 1991 \\
20 & \\
11 & \end{array}$ & 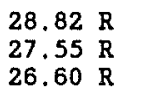 & $\begin{array}{lll}\text { JAN } & 15, & 1992 \\
\text { FEB } & 19 & \\
\text { APR } & 16\end{array}$ & 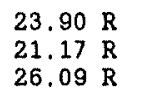 & $\begin{array}{lll}\text { MAY } & 21, & 1992 \\
\text { JUN } & 18 & \\
\text { JUL } 16 & \end{array}$ & $\begin{array}{l}27.47 \mathrm{R} \\
30.08 \mathrm{R} \\
29.81 \mathrm{R}\end{array}$ & AUG 12,1992 & $29.39 \mathrm{R}$ \\
\hline
\end{tabular}

PERIOD OF RECORD HIGHEST 20.6 DEC 20, 1987 JAN 20, 28, 1988 LOWEST 33.82 AUG 23,1990

SITE NUMBER 343944120283201

LOCAL NUMBER 007N034W29H03S

In Lompoc Valley about $0.25 \mathrm{mile}$ northwest of Central Avenue and $\mathrm{v}$ Street. Drilled observation well in alluvium. Diameter 2 inches, depth 75.2 feet, perforated $65.2-75.2$ feet. Altitude of land-surface datum 71.17 feet. Reported measurements provided by city of Lompoc. Water-level records available 1987 to current year.

WATER LEVELS IN FEET BELOW LAND-SURFACE DATUM

\begin{tabular}{|c|c|c|c|c|c|c|c|}
\hline DATE & $\begin{array}{l}\text { WATER } \\
\text { LEVEL MS }\end{array}$ & DATE & $\begin{array}{l}\text { WATER } \\
\text { LEVEL MS }\end{array}$ & DATE & $\begin{array}{l}\text { WATER } \\
\text { LEVEL MS }\end{array}$ & DATE & $\begin{array}{l}\text { WATER } \\
\text { LEVEL MS }\end{array}$ \\
\hline $\begin{array}{ll}31, & 1991 \\
20 & \\
11 & \end{array}$ & $\begin{array}{ll}37.02 & R \\
36.56 & R \\
35.25 & R\end{array}$ & $\begin{array}{lll}\text { JAN } & 15, & 1992 \\
\text { FEB } & 19 & \\
\text { APR } & 16 & \end{array}$ & $\begin{array}{l}32.59 \mathrm{R} \\
29.83 \mathrm{R} \\
32.60 \mathrm{R}\end{array}$ & $\begin{array}{lll}\text { MAY } & 21, & 1992 \\
\text { JUN } & 18 & \\
\text { JUL } & 16\end{array}$ & $\begin{array}{l}33.29 \mathrm{R} \\
35.63 \mathrm{R} \\
35.88 \mathrm{R}\end{array}$ & AUG 12,1992 & $35.54 \mathrm{R}$ \\
\hline
\end{tabular}

SITE NUMBER 343926120292402

LOCAL NUMBER 007N034W29N04S

In Lompoc Valley about $0.6 \mathrm{mile}$ southeast of Central and Floradale Avenues. Augered observation well in alluvium. Diameter 2 inches, depth 60 feet, perforated 55-60 feet. Altitude of land-surface datum 67.34 feet. Water-level records available 1987 to current year.

WATER LEVELS IN FEET BELOW LAND-SURFACE DATUM

$\begin{array}{lll} & \text { WATER } & \\ \text { DATE } & \text { WEVEL MS } & \text { WATER }\end{array}$

MAR 09, $1992 \quad 28.90 \mathrm{R} \quad \operatorname{SEP} 01,1992 \quad 32.34 \mathrm{R}$

PERIOD OF RECORD HIGHEST 25.63 MAR 12, 1987 LOWEST 36.21 AUG 24, 1990

SITE NUMBER 343926120292403

LOCAL NUMBER 007N034W29N05S

In Lompoc Valley about $0.6 \mathrm{mile}$ southeast of Central and Floradale Avenues. Augered observation well in alluvium. Diameter 2 inches, depth 95 feet, perforated 90-95 feet. Altitude of land-surface datum 67.36 feet. Reported measurements provided by Santa Barbara County Water Agency. Water-level records available 1987 to current year.

WATER LEVELS IN FEET BELOW LAND-SURFACE DATUM

$\begin{array}{ll} & \text { WATER } \\ \text { DATE } & \text { LEVEL MS }\end{array}$

SEP 01, $1992 \quad 37,98 \mathrm{R}$

PERIOD OF RECORD HIGHEST 23.08 MAR 09, 1987 LOWEST 46.54 AUG 24, 1990 
SITE NUMBER 343926120293001

LOCAL NUMBER 007N034W29N06S

In Lompoc Valley about $0.35 \mathrm{mile}$ southeast of Central and Floradale Avenues. Drilled observation well in alluvium. Diameter 2 inches, depth of well 160 feet, depth of hole 600 feet, perforated 140-160 feet. Altitude of land-surface datum 66.70 feet. Reported measurements provided by Santa Barbara County Water Agency. Water-level records available 1987 to current year.

WATER LEVELS IN FEET BELOW LAND-SURFACE DATUM

$\begin{array}{lll} & \text { WATER } & \\ \text { DATE } & \text { WEVEL MS }\end{array}$

MAR 06, $1992 \quad 28.40 \mathrm{R} \quad$ SEP 01, $1992 \quad 39.01 \mathrm{R}$

PERIOD OF RECORD HIGHEST 23.6 MAY 03, 1988 LOWEST 48.66 AUG 23, 1990

SITE NUMBER 343926120293002

LOCAL NUMBER 007N034W29N07S

In Lompoc Valley about $0.35 \mathrm{mile}$ southeast of Central and Floradale Avenues. Drilled observation well in alluvium. Diameter 4.5 inches, depth 420 feet, depth of hole 600 feet, perforated 320-360, 380-400 feet. Altitude of land-surface datum 66.70 feet. Reported measurements provided by Santa Barbara County Water Agency. Water-level records available 1987 to current year.

WATER LEVELS IN FEET BELOW LAND-SURFACE DATUM

\begin{tabular}{|c|c|c|}
\hline DATE & $\begin{array}{l}\text { WATER } \\
\text { LEVEL MS }\end{array}$ & DATE \\
\hline
\end{tabular}

MAR 06, $1992 \quad 28.48 \mathrm{R} \quad$ SEP $01,1992 \quad 37.13 \mathrm{R}$

PERIOD OF RECORD HIGHEST 26.13 MAR 13, 1987 LOWEST 45.27 AUG 23, 1990

SITE NUMBER 343941120300102

LOCAL. NUMBER 007N034W30L03S

West of Lompoc, south of Central Avenue and east of Artesia School. Drilled observation well. Diameter 2 inches, depth 31.8 feet in 1947. Altitude of land-surface datum 58.79 feet. Measurements provided by Santa Barbara County Water Agency beginning in 1980. Water-level records available 1945 to current year.

WATER LEVELS IN FEET BELOW LAND-SURFACE DATUM

$$
\text { DATE WATER }
$$

MAR 06, 1992

PERIOD OF RECORD HIGHEST 13.74 APR 08, 1983 LOWEST 29.52 MAR 12, 1991

SITE NUMBER 343941120300106

LOCAL NUMBER 007N034W30L10S

About 2 miles west of Lompoc. Drilled irrigation well. Diameter 15 inches, depth 176 feet, perforated 136-176 feet. Alt1tude of land-surface datum 59 feet. Measurements provided by Santa Barbara County Water Agency beginning in 1991 . Water-level records available 1991 to current year.

WATER LEVELS IN FEET BELOW LAND-SURFACE DATUM

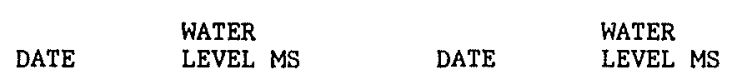

MAR 06, $1992 \quad 23.49 \mathrm{R} \quad \mathrm{SEP} 02,1992 \quad 40.15 \mathrm{R}$

PERIOD OF RECORD HIGHEST 23.49 MAR 06, 1992 LOWEST 40.15 SEP 02, 1992 
WELL DESCRIPTIONS AND WATER-LEVEL MEASUREMENTS

WATER YEAR OCTOBER 1991 TO SEPTEMBER 1992

SITE NUMBER 343901120300303

LOCAL NUMBER 007N034W31C04S

About 2.25 miles west of Lompoc. Observation well. Diameter 1.25 inches, depth cased 61.4 feet, depth measured 60.0 feet in 1991, well point at 61.4 feet. Altitude of land-surface datum 64.6 feet. Measurements provided by Santa Barbara County Water Agency beginning in 1980. Water-level records available 1962 to current year.

WATER LEVELS IN FEET BELOW LAND-SURFACE DATUM

DATE WATER

MAR 06, $1992 \quad 24.19 \mathrm{R}$

PERIOD OF RECORD HIGHEST 13.28 APR 08, 1983 LOWEST 25.10 MAR 22, 1991

SITE NUMBER 343903120300301

LOCAL NUMBER 007N034W31C05S

About 2 miles west of Lompoc. Drilled irrigation well. Diameter 16 inches, depth 270 feet, perforated 100-120, 145-265 feet. Altitude of land-surface datum 64 feet. Measurements provided by Santa Barbara County Water Agency. Water-level records available 1990 to current year.

WATER LEVELS IN FEET BELOW LAND-SURFACE DATUM

DATE $\quad$ WATER

MAR 06, $1992 \quad 24.23 R$

PERIOD OF RECORD HIGHEST 24.23 MAR 06, 1992 LOWEST 41.41 APR 13, 1990

SITE NUMBER 343828120293201

LOCAL NUMBER 007N034W31R02S

In Lompoc Valley along Floradale Avenue. Drilled domestic well. Diameter 6 inches, depth measured 87.5 feet in 1991, perforated 29-44 feet. Altitude of land-surface datum 70.35 feet. Reported measurements provided by Santa Barbara County Water Agency. Water-level records available 1941-42, 1950-59, 1972, 1990 to current year.

WATER LEVELS IN FEET BELOW LAND-SURFACE DATUM

$\begin{array}{lll} & \text { WATER } & \\ \text { DATE } & \text { WEVEL MS } & \text { DATE } \\ \text { LEVEL MS }\end{array}$

MAR 06, $1992 \quad 34.92 \mathrm{R} \quad$ SEP 03, $1992 \quad 42.92 \mathrm{R}$

PERIOD OF RECORD HIGHEST 7.00 APR 01, 1941 LOWEST 52.32 APR 18, 1990

SITE NUMBER 343901120284201

LOCAL NUMBER 007N034W32H02S

In Lompoc, south of Central Avenue and east of Bailey Avenue. Drilled irrigation well. Diameter 16 inches, depth reported 220 feet, perforated 50-220 feet. Altitude of land-surface datum 78 feet. Measurements provided by Santa Barbara County Water Agency. Water-level records available 1986 to current year.

WATER LEVELS IN FEET BELOW LAND-SURFACE DATUM

DATE WATER

MAR 06, $1992 \quad 38.65 \mathrm{R}$

PERIOD OF RECORD HIGHEST 32.62 APR 01, 1986 LOWEST 47.01 APR 19, 1990 
In Lompoc Valley, about 30 feet southeast of $V$ Street and College Avenue. Drilled observation well in alluvium. Diameter 2 inches, depth 56.5 feet, perforated $46.5-56.5$ feet. Altitude of land-surface datum 82 feet. Reported measurements provided by city of Lompoc. Water-level records available 1987-88, 1990 to current year.

WATER LEVELS IN FEET BELOW LAND-SURFACE DATUM

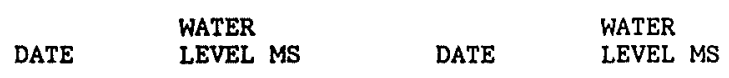

FEB 20, $1992 \quad 47.02 \mathrm{R} \quad$ AUG $12,1992 \quad 45.69 \mathrm{R}$

PERIOD OF RECORD HIGHEST 37.78 JAN 06, 1988 LOWEST 47.40 AUG 21,1991

SITE NUMBER 343901120262202

LOCAL NUMBER 007N034W34A04S

In Lompoc about $0.9 \mathrm{mile}$ southeast of A Street and Central Avenue. Drilled municipal well in alluvium. Diameter 14 inches, depth 170 feet, perforated 150-170 feet. Reported measurements provided by city of Lompoc. Water-level records available 1982 to current year.

WATER LEVELS IN FEET BELOW LAND-SURFACE DATUM

$\begin{array}{lll} & \text { WATER } & \\ \text { DATE } & \text { LEVEL MS } & \text { WATER } \\ & \text { DATE } & \text { LEVEL MS }\end{array}$

FEB 20, $1992 \quad 49.46 \mathrm{R} \quad$ AUG $11,1992 \quad 52.85 \mathrm{R}$

PERIOD OF RECORD HIGHEST 49.46 FEB 20,1992 LOWEST 76.86 AUG 24,1990

SITE NUMBER 343911120264001

LOCAL NUMBER 007N034W34B01S

In Lompoc. Drilled public-supply water-table well. Diameter 14 inches, depth 195 feet, perforated 96-192 feet. Altitude of land-surface datum 101.52 feet. Records beginning in 1972 provided by U.S. Bureau of Reclamation and and city of Lompoo. Water-level records available 1965, 1972-88, 1990 to current year.

WATER LEVELS IN FEET BELOW LAND-SURFACE DATUM

$\begin{array}{lll} & \text { WATER } & \\ \text { DATE } & \text { LEVEL MS } & \text { WATER } \\ & \text { LEVEL MS }\end{array}$

FEB $19,1992 \quad 55.43 \mathrm{R} \quad$ SEP $02,1992 \quad 57.20 \mathrm{R}$

PERIOD OF RECORD HIGHEST 40.70 APR 23, 1975 FEB 22, $1979 \quad$ LOWEST 77.29 AUG 23, 1990

SITE NUMBER 343840120254701

LOCAL NUMBER 007N034W35K09S

In Lompoc. Drilled irrigation well. Diameter 16 inches, depth 128 feet, perforated 52-80, $112-124$ feet. Records in 1960-69 and reported measurements 1970-79 provided by U.S. Bureau of Reclamation; measurements provided by Santa Barbara County Water Agency beginning in 1980. Water-level records available 1950 , 1952 to current year.

WATER LEVELS IN FEET BELOW LAND-SURFACE DATUM

$$
\begin{array}{ll} 
& \text { WATER } \\
\text { DATE } & \text { LEVEL MS }
\end{array}
$$

MAR 09, $1992 \quad 21.95 \mathrm{R}$

PERIOD OF RECORD HIGHEST 18.4 MAR 28, APR 26, 1983 LOWEST 43.28 MAR 07, 1991 
WELI DESCRIPTIONS AND WATER-LEVEL MEASUREMENTS

WATER YEAR OCTOBER 1991 TO SEPTEMBER 1992

SITE NUMBER 344109120312401

LOCAL NUMBER O07N035W13N02S

On Vandenberg AFB, west of Federal Corrections Institute. Drilled observation well. Diameter 4 inches, depth measured 40.9 feet in 1991, original depth 44 feet, perforated 22-44 feet. Altitude of land-surface datum 35 feet. Reported measurements provided by Barbara County Water Agency. Water-level records available 1980-85, 1990 to current year.

WATER LEVELS IN FEET BELOW LAND-SURFACE DATUM

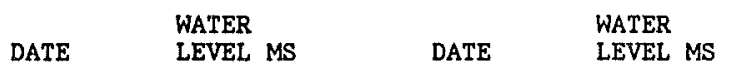

MAR 04, $1992 \quad 19.22 \mathrm{R} \quad$ SEP 03, $1992 \quad 22.96 \mathrm{R}$

PERIOD OF RECORD HIGHEST 3.95 APR 06, 1983 LOWEST 30.15 MAR 08, 1991

SITE NUMBER 344116120351401

LOCAL NUMBER 007N035W17K01S

On Vandenberg AFB, west of Ocean Beach Park at Surf, south of lagoon. Drilled unused water-table well. Diameter 8 inohes, original depth 190 feet, depth measured 186.7 feet in 1966, perforated 175-185 feet. Altitude of land-surface datum 10 feet. Water-level records available 1966 to current year.

WATER LEVELS IN FEET BELOW LAND-SURFACE DATUM

DATE WATER

AUG $31,1992 \quad 3.02 \mathrm{~s}$

PERIOD OF RECORD HIGHEST 0.02 APR 05, 1967 LOWEST 4.75 APR 18, 1991

SITE NUMBER 344112120351001

LOCAL NUMBER O07N035W17K20S

On Vandenberg AFB, east of Ocean Beach Park at Surf, south of lagoon. Diameter 6 inches, depth 126 feet, perforated 96-116 feet. Altitude of land-surface datum 24 feet. Water-level records available 1982 to current year.

WATER LEVELS IN FEET BELOW LAND-SURFACE DATUM

DATE WATER

AUG $31,199216.09 \mathrm{~s}$

PERIOD OF RECORD HIGHEST 15.21 NOV 20,1984 LOWEST 18.67 APR 18, 1991

SITE NUMBER 344117120350601

LOCAL NUMBER 007N035W17K21S

In Lompoc Valley on Vandenberg AFB about 2.3 miles northwest of Ocean Avenue and Renwick Avenue. Drilled observation well in alluvium. Diameter 2 inches, depth 175 feet, depth measured 171.3 feet in 1991 , perforated 155-175 feet. Altitude of land-surface datum 9 feet. Water-level records available 1987 to current year.

WATER LEVELS IN FEET BELOW LAND-SURFACE DATUM

$\begin{array}{lll} & \text { WATER } & \\ \text { DATE } & \text { WATER } \\ \text { LEVEL MS } & \text { DATE } & \text { LEVEL MS }\end{array}$

MAR 04, $1992 \quad 3.41 \mathrm{R} \quad$ AUG $31,1992 \quad 2.28 \mathrm{~S}$

PERIOD OF RECORD HIGHEST 1.0 APR 27, MAY 3, 13, 18, 28, JUN 3, 9, 1988 LOWEST 5.63 SEP 05,1990 
SITE NUMBER 344114120353501

LOCAL NUMBER OO7N035W17M01S

East of Ocean Beach Park at Surf. Drilled observation well. Diameter 8 inches, depth measured 158.75 feet 1n 1991, perforated 115-120 feet. Altitude of land-surface datum 9.74 feet. Measurements provided by Santa Barbara County Water Agency beginning in 1980. Water-level records available 1964 to current year.

WATER LEVELS IN FEET BELOW LAND-SURFACE DATUM

$\begin{array}{lll} & \text { WATER } & \\ \text { DATE } & \text { WEVEL MS } \\ \text { DATE } & \text { LEVEL MS }\end{array}$

MAR 04, $1992 \quad 3.82 \mathrm{R} \quad$ SEP 03, $1.992 \quad 2.96 \mathrm{R}$

PERIOD OF RECORD HIGHEST 1.68 JAN 26, 1968 LOWEST 5.40 AUG 07,1978

SITE NUMBER 344110120351201

LOCAL NUMBER 007N035W17Q06S

On Vandenberg AFB, west of Ocean Beach Park at Surf. Diameter 6 inches, depth 87 feet, perforated 57-77 feet. Altitude of land-surface datum 20 feet. Water-level records available ig82 to current year.

WATER LEVELS IN FEET BELOW LAND-SURFACE DATUM

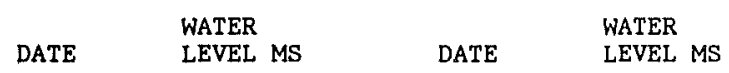

MAR $04,1992 \quad 11.02 \mathrm{R} \quad$ AUG $31,1992 \quad 10.90 \mathrm{~S}$

PERIOD OF RECORD HIGHEST 9.57 NOV $20,1984 \quad$ LOWEST $12.50 \quad$ OCT 31,1985

SITE NUMBER 344135120355201

LOCAL NUMBER 007N035W18H01S

On Vandenberg AFB, north of Ocean Beach Park at Surf and lagoon. Drilled observation well. Diameter 8 inches, depth 111.5 feet in 1966, perforated 83-88 feet. Altitude of land-surface datum 5.87 feet. Measurements provided by Santa Barbara County Water Agency beginning in 1980. Water-level records available 1963-76, 1978 to current year.

WATER LEVELS IN FEET BELOW LAND-SURFACE DATUM

$$
\begin{array}{ll} 
& \text { WATER } \\
\text { DATE } & \text { LEVEL MS }
\end{array}
$$

MAR 04, $1992 \quad 1.69 \mathrm{R}$

PERIOD OF RECORD HIGHEST 0.44 APR $07,1981 \quad$ LOWEST $\quad 2.60$ APR 05,1967

SITE NUMBER 344118120355902

LOCAL NUMBER 007N035W18J02S

Near south exit road at Ocean Beach Park. Drilled observation well. Diameter 8 inches, depth 121.7 feet in 1966 , perforated 100-105 feet. Altitude of land-surface datum 7.38 feet. Measurements provided by Santa Barbara County Water Agency beginning in 1980. Water-level records available 1963 to current year.

WATER LEVELS IN FEET BELOW LAND-SURFACE DATUM

$$
\begin{array}{ll} 
& \text { WATER } \\
\text { DATE } & \text { LEVEL MS }
\end{array}
$$

MAR 04, $1992 \quad 2.45 \mathrm{R}$

PERIOD OF RECORD HIGHEST 2,34 DEC $29,1964 \quad$ LOWEST 4.85 MAR 26, 1969 
WELL DESCRIPTIONS AND WATER-LEVEL MEASUREMENTS

WATER YEAR OCTOBER 1991 TO SEPTEMBER 1992

SITE NUMBER 344041120341101

LOCAL NUMBER 007N035W21G02S

On Vandenberg AFB. Drilled observation well. Diameter 6 inches, depth 180 feet, perforated 150-170 feet. Altitude of land-surface datum 20 feet. Water-level records available 1982 to current year.

WATER LEVELS IN FEET BELOW LAND-SURFACE DATUM

DATE WATER

SEP $02,1992 \quad 12.53 \mathrm{~S}$

PERIOD OF RECORD HIGHEST 10.14 MAY 25, 1983 LOWEST 14.21 JUL 25, 1990

SITE NUMBER 344021120324101

LOCAL NUMBER 007N035W22J01S

On Vandenberg AFB about 1 mile west of Main Gate, at Point Arguello. Drilled irrigation well. Diameter 16 inches, depth 182 feet, perforated 133-180 feet. Altitude of land-surface datum 31.76 feet. Measurements provided by city of Santa Barbara 1930-35 and Santa Barbara County Water Agency beginning in 1980. Water-level records available $1930-35,1941-42,1945-76,1978$ to current year.

WATER LEVELS IN FEET BELOW LAND-SURFACE DATUM

$$
\begin{array}{lll} 
& \text { WATER } & \\
\text { DATE } & \text { WETER } \\
\text { LEVE } & \text { DATE } & \text { LEVEL MS }
\end{array}
$$

MAR 04, $1992 \quad 11.97 \mathrm{R} \quad$ SEP 03, $1992 \quad 22.96 \mathrm{R}$

PERIOD OF RECORD HIGHEST 6.01 APR 08, 1958 LOWEST 25.93 AUG 27, 1991

SITE NUMBER 344025120331301

LOCAL NUMBER 007N035W22L01S

On Vandenberg AFB about 3 miles southeast of Surf, and $0.5 \mathrm{mile}$ north of Highway 246 on Renwick Avenue. Drilled irrigation water-table well. Diameter 12 inches, depth 219 feet, perforated $172-189$ feet. Alt1tude of land-surface datum 30 feet. Water-level records available 1934-35, 1953, 1960, 1966 to current year.

WATER LEVELS IN FEET BELOW LAND-SURFACE DATUM

DATE WATER $\quad$ LEVEL MS

SEP 03, $1992 \quad 0$

PERIOD OF RECORD HIGHEST 10.70 APR 05, 1967 LOWEST 24.80 SEP 19, 1935

SITE NUMBER 344025120333401

LOCAL NUMBER 007N035W22M01S

On Vandenberg AFB about 3 miles southeast of Surf, and west of Renwick Avenue, Drilled irrigation water-table well. Diameter 12 inches, depth 180 feet. Altitude of land-surface datum 29 feet. Water-level records available 1933, 1935, 1947 to current year.

WATER LEVELS IN FEET BELOW LAND-SURFACE DATUM

WATER

DATE LEVEL MS

SEP $03,199213.80 \mathrm{~s}$

PERIOD OF RECORD HIGHEST 2.87 MAR 27, 1952 LOWEST 20.20 SEP 19, 1935 
SITE NUMBER 344048120320201

LOCAL NUMBER 007N035W23BO2S

About 4 miles west of Surf, southeast of intersection of 13 th and Terra Street. Diameter 6 inches, depth 80 feet, perforated 20-60 feet. Altitude of land-surface datum 30 feet. Reported measurements provided by Santa Barbara County Water Agency beginning in 1990. Water-level records avallable 1979 to current year.

WATER LEVELS IN FEET BELOW LAND-SURFACE DATUM

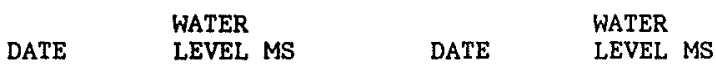

MAR 04, $1992 \quad 16.76 \mathrm{R} \quad$ SEP 02, $1992 \quad 18.81 \mathrm{R}$

PERIOD OF RECORD HIGHEST 16.76 MAR 04, 1992 LOWEST 21.66 AUG 23, 1990

SITE NUMBER 344043120322402

LOCAL NUMBER 007N035W23E02S

About 4 miles east of Surf. Drilled irrigation well. Diameter 16 inches, depth 212 feet, perforated $170-190$ feet. Altitude of land-surface datum 36.11 feet. Measurements provided by city of Santa Barbara $1930-35$ and Santa Barbara County Water Agenoy beginning in 1980. Water-level records available 1930-35, 1937-38, 1941-76, 1978 to current year.

WATER LEVELS IN FEET BELOW LAND-SURFACE DATUM

$\begin{array}{lll} & \text { WATER } & \\ \text { DATE } & \text { LEVEL MS } & \text { WATER } \\ & \text { LEVEL MS }\end{array}$

MAR $04,1992 \quad 14.54 \mathrm{R} \quad \mathrm{SEP} 03,1992 \quad 21.69 \mathrm{R}$

PERIOD OF RECORD HIGHEST 8.03 SEP 28, 1954 LOWEST 65,00 APR 28, MAY 12, 19, 26, 1943

SITE NUMBER 344025120313701

LOCAL NUMBER 007N035W23J05S

About 3 miles northwest of Lompoc, about $0.8 \mathrm{mile}$ north of intersection of Artesia and Central Avenues. Unused well. Diameter 16 inches, depth measured 142.5 feet in 1991. Altitude of land-surface datum 43 feet.

Measurements provided by Santa Barbara County Water Agency beginning in 1980 . Water-level records available $1966-72,1974-75,1978,1980$ to current year.

WATER LEVELS IN FEET BELOW LAND-SURFACE DATUM

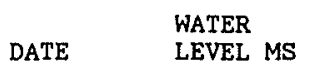

MAR $04,199216.34 \mathrm{R}$

PERIOD OF RECORD HIGHEST 12.76 APR 25, 1978 LOWEST 32.79 APR 13,1990

SITE NUMBER 344009120320402

LOCAL NUMBER 007N035W23Q02S

In Lompoc Valley about $0.6 \mathrm{mile}$ northeast of Central and Union Sugar Avenues. Augered observation well in alluvium, Diameter 2 inches, depth 51 feet, perforated 46-51 feet. Altitude of land-surface datum 37.22 feet. Reported measurements provided by Santa Barbara County Water Agency. Water-1evel records available 1987 to current year.

WATER LEVELS IN FEET BELOW LAND-SURFACE DATUM

$$
\begin{array}{ll} 
& \text { WATER } \\
\text { DATE } & \text { LEVEL MS }
\end{array}
$$

MAR 04, $1992 \quad 11.02 \mathrm{R}$

PERIOD OF RECORD HIGHEST 10.31 JUN $24,1987 \quad$ LOWEST 18,8 OCT 28,1988 
WELL DESCRIPTIONS AND WATER-LEVEL MEASUREMENTS

WATER YEAR OCTOBER 1991 TO SEPTEMBER 1992

SITE NUMBER 344009120320403

LOCAL NUMBER 007N035W23Q03S

In Lompoc Valley about $0.6 \mathrm{mile}$ northeast of Central and Union Sugar Avenues. Augered observation well in alluvium. Diameter 2 inches, depth 82.5 feet, perforated 77.5-82.5 feet, Aititude of land-surface datum. 37.11 feet. Reported measurements provided by Santa Barbara County Water Agency. Water-level records available 1987 to current year.

WATER LEVELS IN FEET BELOW LAND-SURFACE DATUM

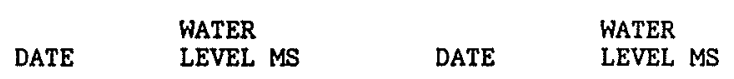

MAR 04, $1992 \quad 13.24 \mathrm{R} \quad$ SEP 01, $1992 \quad 30.19 \mathrm{R}$

PERIOD OF RECORD HIGHEST 12.40 JAN 05, 20, $1988 \quad$ LOWEST 37.0 SEP 02, 1988

SITE NUMBER 344008120320901

LOCAL NUMBER 007 N035W23Q04S

In Lompoc Valley about $0.5 \mathrm{mile}$ north of Central and Union Sugar Avenues. Drilled observation well in alluvium. Diameter 4.5 inches, depth 190 feet, depth of hole 440 feet, perforated $160-180$ feet. Altitude of land-surface datum 37.32 feet. Reported measurements provided by Santa Barbara County Water Agency. Digital recorder October 1987 to October 1988. Water-level records available 1987 to current year.

WATER LEVELS IN FEET BELOW LAND-SURFACE DATUM

$$
\begin{array}{lll} 
& \text { WATER } & \\
\text { DATE } & \text { WEVEL MS } & \text { DATE } \\
\text { LEVEL MS }
\end{array}
$$

MAR 04, $1992 \quad 13.14 \mathrm{R} \quad \mathrm{SEP} 01,1992 \quad 30.62 \mathrm{R}$

PERIOD OF RECORD HIGHEST 13.14 MAR 04, 1992 LOWEST 38.75 AUG 22, 1990

SITE NUMBER 344021120303504

LOCAL NUMBER D07N035W24J04S

About 3 miles northwest of Lompoc. Drilled unused well. Diameter 8 inches, depth 171 feet, perforated 165-170 feet. Alt1tude of land-surface datum 52 feet. Measurements provided by Santa Barbara County Water Agency beginning in 1980. Water-level records available 1961, 1974 to current year.

WATER LEVELS IN FEET BELOW LAND-SURFACE DATUM

$\begin{array}{lll} & \text { WATER } & \text { WATER } \\ \text { DATE LEVEL MS } & \text { DATE }\end{array}$

MAR 04, $1992 \quad 25.95 \mathrm{R} \quad$ SEP 02, $1992 \quad 39.70 \mathrm{R}$

PERIOD OF RECORD HIGHEST 24.77 APR 05, 1982 LOWEST 52.00 JAN 01, 1961

SITE NUMBER 344029120310305

LOCAL NUMBER O07N035W24K05S

About 2 miles west of Lompoc, about $0.9 \mathrm{mile}$ north of Central Avenue, and 75 feet east of De Wolf Avenue.

Irrigation well. Diameter 14 inches, depth 174 feet, perforated 130-135, 140-170 feet. Altitude of land-surface datum 51 feet. Measurements provided by Santa Barbara County Water Agency beginning in 1980. Water-level records available 1973-74, 1978 to current year.

WATER LEVELS IN FEET BELOW LAND-SURFACE DATUM

$$
\begin{array}{lll} 
& \text { WATER } & \\
\text { DATE } & \text { WEVEL MS } & \text { WATER }
\end{array}
$$

MAR 04, $1992 \quad 19.36 \mathrm{R} \quad \operatorname{SEP} 02,1992 \quad 29.48 \mathrm{R}$

PERIOD OF RECORD HIGHEST 18.62 APR 25, 1978 LOWEST 34.45 AUG 23, 1990 
SITE NUMBER 344046120321401

LOCAL NUMBER O07N035W24N03S

West of Lompoc on Artesia Avenue, north of Central Avenue. Drilled irrigation well. Diameter 12 inches, depth 185 feet, perforated 145-185 feet. Measurements provided by Santa Barbara County Water Agency beginning tn 1990. Water-level records available 1990 to current year.

WATER LEVELS IN FEET BELOW LAND-SURFACE DATUM

$$
\begin{array}{ll} 
& \text { WATER } \\
\text { DATE } & \text { LEVEL MS }
\end{array}
$$

MAR $04,1992 \quad 12.89 \mathrm{R}$

PERIOD OF RECORD HIGHEST 12.89 MAR 04, 1992 LOWEST 28.05 ARR 13, 1990

SITE NUMBER 343947120310701

LOCAL NUMBER 007N035W25F05S

About $2.5 \mathrm{miles}$ northwest of Lompoc, about $0.25 \mathrm{mile}$ west of De Wolf Avenue, and 500 feet north of Central Avenue. Drilled irrigation well. Diameter 12 inches, depth 184 feet, perforated 145-175 feet. Altitude of land-surface datum 47 feet. Measurements provided by Santa Barbara County Water Agency beginning in 1983 . Water-level records available 1939-41, 1945-48,1950,1952-55, 1957-73, 1983 to current year.

WATER LEVELS IN FEET BELOW LAND-SURFACE DATUM

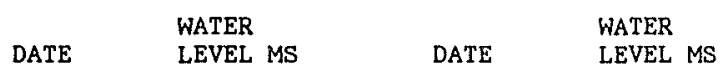

MAR 04, $1992 \quad 17.26 \mathrm{R} \quad \mathrm{SEP} 03,1992 \quad 30.33 \mathrm{R}$

PERIOD OF RECORD HIGHEST 5.72 MAR 27, 1941 LOWEST 40.11 AUG 23, 1990

SITE NUMBER 343947120310703

LOCAL NUMBER 007N035W25F06S

About 2.5 miles northwest of Lompoc, north of Central Avenue, and west of De Wolf Avenue, Drilled observation we11. Diameter 2 inches, depth 19.4 feet in 1991 . Altitude of land-surface datum 47 feet. Measurements provided by Santa Barbara County Water Agency beginning in 1980 . Water-level records available 1945 to current year.

WATER LEVELS IN FEET BELOW LAND-SURFACE DATUM

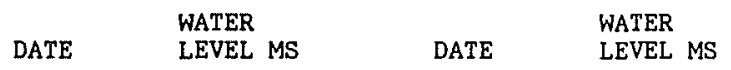

MAR 04, $1992 \quad 13.00 \mathrm{R} \quad$ SEP 03, $1992 \quad 14.91 \mathrm{R}$

PERIOD OF RECORD HIGHEST 3.84 APR 04,1969 LOWEST $16,18 \quad$ OCT 28,1964

SITE NUMBER 343947120310702

LOCAL NUMBER 007N035W25F07S

About 2.5 miles northwest of Lompoc, north of Central Avenue, and west of De Wolf Avenue. Drilled observation well. Diameter 1.25 inches, depth 29.5 feet, sand point 29.5 feet. Altitude of land-surface datum 46.9 feet. Measurements provided by Santa Barbara County Water Agency beginning in 1980 . Water-level records available 1962 to current year.

WATER LEVELS IN FEET BELOW LAND-SURFACE DATUM

$\begin{array}{lll} & \text { WATER } & \\ \text { DATE } & \text { LEVEL MS } & \text { WATER } \\ & \text { DATE } & \text { LEVEL MS }\end{array}$

MAR 04, $1992 \quad 14.33 \mathrm{R} \quad$ SEP 03, $1992 \quad 13.88 \mathrm{R}$

PERIOD OF RECORD HIGHEST 5.70 APR 25, 1978 LOWEST 19.96 FEB 04,1964 
WELL DESCRIPTIONS AND WATER-LEVEL MEASUREMENTS

WATER YEAR OCTOBER 1991 TO SEPTEMBER 1992

SITE NUMBER 343948120320901

LOCAL NUMBER 007N035W26F04S

About 3.5 miles northwest of Lompoc, north of Central Avenue, and west of Union Avenue. Drilled 1 rrigation well. Diameter 16 inches, depth 190 feet in 1952. Altitude of land-surface datum 35 feet. Measurements provided by Santa Barbara County Water Agency beginning in 1980. Water-level records avallable 1970-77, 1979 to current year.

WATER LEVELS IN FEET BELOW LAND-SURFACE DATUM

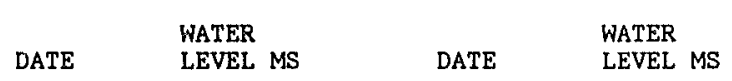
MAR 04, $1992 \quad 11.82 \mathrm{R}$
SEP 03, 1992
$41.38 \mathrm{RS}$

PERIOD OF RECORD HIGHEST 10.43 APR 02, 1982 LOWEST 33.74 AUG 24, 1990

SITE NUMBER 343929120321001

LOCAL NUMBER 007N035W26L01S

In Lompoc Valley about $0.25 \mathrm{mile}$ south of Central and Union Sugar Avenues. Augered observation well in alluvium. Diameter 2 inches, depth 22.5 feet, perforated 17.5-22.5 feet. Altitude of land-surface datum 36.09 feet. Reported measurements provided by Santa Barbara County Water Agency. Water-level records available $1987-88,1990$ to current year.

WATER LEVELS IN FEET BELOW LAND-SURFACE DATUM

$\begin{array}{lll} & \text { WATER } & \text { WATER } \\ \text { DATE } & \text { LEVEL MS } & \text { DATE } \\ \text { LEVEL MS }\end{array}$

MAR 04, $1992 \quad 5.59 \mathrm{R} \quad$ SEP 02, $1992 \quad 7.55 \mathrm{R}$

PERIOD OF RECORD HIGHEST 5.11 MAR 08, 1991 LOWEST 7.55 SEP 02, 1992

SITE NUMBER 343929120321002

LOCAL NUMBER 007N035W26L02S

In Lompoc Valley about $0.25 \mathrm{mile}$ south of Central and Union Sugar Avenues. Augered observation well in alluvium. Diameter 2 inches, depth 82 feet, perforated 77-82 feet. Altitude of land-surface datum 35.77 feet. Reported measurements provided by Santa Barbara County Water Agency. Water-level records available 1987-88, 1990 to current year.

WATER LEVELS IN FEET BELOW LAND-SURFACE DATUM

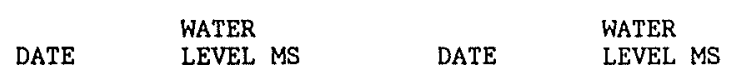

MAR 04, $1992 \quad 5.25 \mathrm{R} \quad \mathrm{SEP} 02,1992 \quad 12.74 \mathrm{R}$

PERIOD OF RECORD HIGHEST 3.45 MAR 18, 1987 LOWEST 17.73 AUG 20, 1990

SITE NUMBER 343929120321004

LOCAL NUMBER 007N035W26L04S

In Lompoc Valley about $0.25 \mathrm{mile}$ south of Central and Union Sugar Avenues. Drilled observation well in Careaga Sand of Pliocene age. Diameter 4.5 inches, depth 299 feet, depth of hole 340 feet, perforated

259-299 feet. Altitude of land-surface datum 36 feet. Reported measurements provided by Santa Barbara County Water Agency. Water-level records available 1987 to current year.

WATER LEVELS IN FEET BELOW LAND-SURFACE DATUM

$\begin{array}{lll} & \text { WATER } & \\ \text { DATE } & \text { WATER } \\ \text { LEVEL MS } & \text { DEVEL MS }\end{array}$

MAR 04, $1992 \quad 6.18 \mathrm{R} \quad$ SEP 02, $1992 \quad 21.17 \mathrm{R}$

PERIOD OF RECORD HIGHEST 6.18 MAR 04, 1992 LOWEST 29.55 AUG 22, 1990 
SITE NUMBER 343952120332001

LOCAL NUMBER 007N035W27F01S

On south Vandenberg AFB about $0.45 \mathrm{mile}$ east of Arguello Boulevard and 50 feet north of Clark Street. Drilled unused water-table well. Diameter 12 inches, depth 123 feet. Altitude of land-surface datum 27.63 feet. Measurements prior to 1942 provided by city of Santa Barbara. Water-level records available 1930-32, $1934,1942,1953$ to current year.

WATER LEVELS IN FEET BELOW LAND-SURFACE DATUM

$$
\text { DATE WATER }
$$

SEP $03,199214.89 \mathrm{~S}$

PERIOD OF RECORD HIGHEST 2.52 APR 21, 1958 LOWEST 28.28 AUG 16, 1990

SITE NUMBER 343948120330101

LOCAL NUMBER 007N035W27H01S

On south Vandenberg AFB about 200 feet south of Highway 246. Drilled irrigation water-table well. Diameter 16 inches, depth 142 feet, perforated 80-124 feet. Altitude of land-surface datum 27 feet. Measurements provided by city of Santa Barbara 1935-42. Water-level records available 1935-42, 1953-59, 1966 to current year.

WATER LEVELS IN FEET BELOW LAND-SURFACE DATUM

$$
\text { DATE WATER }
$$

SEP $03,1992 \quad 15.24 \mathrm{~S}$

PERIOD OF RECORD HIGHEST 1.50 APR 02, 1941 LOWEST 30.60 AUG 16, 1990

SITE NUMBER 343923120332501

LOCAL NUMBER 007N035W27P01S

On south Vandenberg AFB, south of Highway 246. Drilled unused water-table well. Diameter 6 inches, depth 677 feet, perforated 562-577 feet. Altitude of land-surface datum 260 feet. Water-level records available $1963-69,1971$ to current year.

WATER LEVELS IN FEET BELOW LAND-SURFACE DATUM

$$
\text { DATE WATER }
$$

SEP $03,1992 \quad 228.20 \mathrm{~S}$

PERIOD OF RECORD HIGHEST 222.24 APR 24, 1963 LOWEST 235.20 APR 10, 1979

SITE NUMBER 343944120361901

LOCAL NUMBER 007N035W30G01S

On south Vandenberg AFB about 1.5 miles south of Surf and 0.1 mile south of road to Launch Complex A. Drilled observation water-table well. Diameter 8 inches, depth 277 feet, perforated 115-275 feet. Altitude of land-surface datum 130 feet. Water-level records available 1958-61, 1963 to current year.

WATER LEVELS IN FEET BELOW LAND-SURFACE DATUM

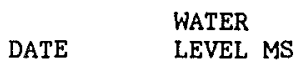

SEP $10,199297.59 \mathrm{~S}$

PERIOD OF RECORD HIGHEST 95.82 MAR 13, 1985 LOWEST 98.34 JUL 31, 1958 
WELL DESCRIPTIONS AND WATER-LEVEL MEASUREMENTS

WATER YEAR OCTOBER 1991 TO SEPTEMBER 1992

SITE NUMBER 343841120355201

LOCAL NUMBER 007N035W31J01S

On Vandenberg AFB in Bear Creek area. Diameter 6 inches, depth 625 feet, perforated 571-591 feet. Altitude of land-surface datum 160 feet. Water-level records avallable 1963-69, 1971 to current year.

WATER LEVELS IN FEET BELOW LAND-SURFACE DATUM

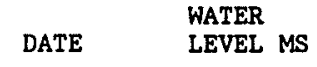

SEP $10,1992 \quad 51.39 \mathrm{~S}$

PERIOD OF RECORD HIGHEST 48.18 OCT 30,1963 LOWEST 66.49 JAN 04, 1963

SITE NUMBER 343831120354301

LOCAL NUMBER O07N035W32N01S

On south Vandenberg AFB in Bear Creek area. Drilled unused water-table well. Diameter 8 inches, original depth 300 feet, depth measured 224.6 feet in 1966, perforated 10-210 feet. Altitude of land-surface datum 175 feet. Water-level records available 1958-69, 1971 to current year.

WATER LEVELS IN FEET BELOW LAND-SURFACE DATUM

$$
\begin{array}{ll}
\text { WATE } & \text { WATER } \\
\text { LEVEL MS }
\end{array}
$$

SEP $10,1992 \quad 4.36 \mathrm{~S}$

PERIOD OF RECORD HIGHEST 1.94 MAY 02, $1988 \quad$ LOWEST 9.47 JUN 25, 1958

SITE NUMBER 343846120334801

LOCAL NUMBER O07N035W33J01S

On south Vandenberg AFB in Lompoc Canyon. Drilled unused water-table well. Diameter 8 inches, depth measured 186 feet in 1966, original depth 380 feet, perforated 113-155, 173-244 feet. Altitude of land-surface datum 177 feet. Water-level records available 1958-69, 1971 to current year.

WATER LEVELS IN FEET BELOW LAND-SURFACE DATUM

$$
\text { DATE WATER }
$$

SEP $03,1992123.10 \mathrm{~S}$

PERIOD OF RECORD HIGHEST 109.89 NOV 25, 1958 LOWEST 127.29 JUN 05, 1967

SITE NUMBER 343846120334802

LOCAL NUMBER 007N035W33J02S

On south Vandenberg AFB about $1.8 \mathrm{miles}$ south in Lompoc Canyon. Drilled public-supply water-table well. Diameter 14 inches, depth measured 460 feet, original depth 530 feet, perforated 170-210, 375-465 feet, Altitude of land-surface datum 177 feet. Water-level records available 1958-69, 1971 to current year.

WATER LEVELS IN FEET BELOW LAND-SURFACE DATUM

$$
\text { DATE WATER }
$$

SEP 03, $1992128.11 \mathrm{~s}$

PERIOD OF RECORD HIGHEST 112.94 OCT 29, 1958 LOWEST 151.00 SEP 28, 1965 
SITE NUMBER 343837120335201

LOCAL NUMBER 007N035W33J03S

On south Vandenberg AFB in Lompoc Canyon. Drilled public-supply water-table well. Diameter 14 inches, depth 660 feet, perforated $96-160,428-460$ feet. Altitude of land-surface datum 220 feet. Water-level records available 1960-69, 1971 to current year.

WATER LEVELS IN FEET BELOW LAND-SURFACE DATUM

$$
\begin{array}{ll} 
& \text { WATER } \\
\text { DATE } & \text { LEVEL MS }
\end{array}
$$

SEP $03,1992134.36 \mathrm{~S}$

PERIOD OF RECORD HIGHEST 127.92 MAR 13, 1985 LOWEST 164.74 OCT 28, 1964

SITE NUMBER 343859120314003

LOCAL NUMBER 007N035W35A03S

About 3 miles west of Lompoc at intersection of Artesia Avenue and Highway 246. Drilled irrigation well. Diameter 14 inches, depth 100 feet, perforated $78-92$ feet. Altitude of land-surface datum 45.68 feet. Measurements provided by Santa Barbara County Water Agency beginning in 1980. Water-level records available 1945, 1947-75, 1977 to current year.

WATER LEVELS IN FEET BELOW LAND-SURFACE DATUM

$$
\begin{array}{ll} 
& \text { WATER } \\
\text { DATE } & \text { LEVEL MS }
\end{array}
$$

MAR $04,199216.79 \mathrm{R}$

PERIOD OF RECORD HIGHEST 5.41 APR 29, 1958 LOWEST 25.81 JUL 27, 1950

SITE NUMBER 344420120041701

LOCAL NUMBER 008N030W3OR01S

About 2 miles east of Los Olivos. Public-supply well. Diameter 12 inches, depth 271 feet in 1964 . Altitude of land-surface datum 1255 feet. Measurements provided by Santa Barbara County Water Agency beginning in 1989. Water-level records available 1964,1989 to current year.

WATER LEVELS IN FEET BELOW LAND-SURFACE DATUM

$$
\text { DATE WATER }
$$

MAR 18, $1992 \quad 11.64 \mathrm{R}$

PERIOD OF RECORD HIGHEST 10.53 APR 02, 1991 LOWEST 20.77 MAR 23, 1990

SITE NUMBER 344430120054001

LOCAL NUMBER 008N031W25K01S

About 6 miles north of Los Olivos in Sycamore Canyon. Drilled irrigation well. Diameter 8 inches, depth reported 180 feet in 1964. Altitude of land-surface datum 1,275 feet. Measurements provided by Santa Barbara County Water Agency beginning in 1986. Water-level records available 1986 to current year.

WATER LEVELS IN FEET BELOW LAND-SURFACE DATUM

$$
\begin{array}{ll} 
& \text { WATER } \\
\text { DATE } & \text { LEVEL MS }
\end{array}
$$

MAR 24, $1992 \quad 94.75 \mathrm{R}$

PERIOD OF RECORD HIGHEST 89.70 APR 02, 1991 LOWEST 101.05 APR 15, 1987 
WELL DESCRIPTIONS AND WATER-LEVEL MEASUREMENTS

WATER YEAR OCTOBER 1991 TO SEPTEMBER 1992

SITE NUMBER 344418120053101

LOCAL NUMBER O08N031W2SQ01S

About 5 miles north of Los Olivos in Sycamore Canyon. Stock well. Diameter 12 inches, depth 190 feet. Altitude of land-surface datum 1,220 feet. Measurements provided by Santa Barbara County Water Agency beginning in 1980. Water-level records available 1967-73, 1975 76, 1978 to current year.

WATER LEVELS IN FEET BELOW LAND-SURFACE DATUM

$$
\text { DATE WATER }
$$

MAR 24, $1992 \quad 45.23 \mathrm{R}$

PERIOD OF RECORD HIGHEST 15.49 APR 05, 1983 LOWEST 49.44 MAR 18, 1988 MAR 23, 1990

SITE NUMBER 344354120051501

LOCAL NUMBER 008N031W36H01S

About 5 miles north of Los Olivos. Unused well. Diameter 8 inches, depth measured 73 feet in 1991. Altitude of land-surface datum 1,180 feet. Measurements provided by Santa Barbara County Water Agency beginning in 1989. Water-level records available 1989 to current year.

WATER LEVELS IN FEET BELOW LAND-SURFACE DATUM

$$
\begin{array}{ll} 
& \text { WATER } \\
\text { DATE } & \text { LEVEL MS }
\end{array}
$$

MAR 18, $199210.21 \mathrm{R}$

PERIOD OF RECORD HIGHEST 10.21 MAR 18, 1992 LOWEST 45.70 MAR 22, 1990

SITE NUMBER 344457120122101

LOCAL NUMBER 008N032W25D01S

About 4 miles east of Los Alamos, 2.4 miles north of Highway 101 on Aliso Canyon Road. Drilled irrigation water-table well. Diameter 12.75 inches, depth 881 feet, perforated 268-700 feet. Altitude of land-surface datum 763 feet. Water-level records available 1977, 1979 to current year.

WATER LEVELS IN FEET BELOW LAND-SURFACE DATUM

DATE WATER

SEP $10,1992 \quad 81.76 \mathrm{~S}$

PERIOD OF RECORD HIGHEST 43.83 APR 22, 1977 LOWEST 87.01 AUG 15, 1990

SITE NUMBER 344417120151001

LOCAL NUMBER 008N032W28P01S

About 1.5 miles east of Los Alamos. Irrigation well. Diameter 12 inches, depth 235 feet, perforated 67-79, 81-101 feet. Altitude of land-surface datum 616 feet. Measurements provided by Santa Barbara County Water Agency beginning in 1983. Water-level records available 1943,1955, 1958, 1978, 1983 to current year.

WATER LEVELS IN FEET BELOW LAND-SURFACE DATUM

DATE WATER

SEP $10,199276.47 \mathrm{~S}$

PERIOD OF RECORD HIGHEST 23.12 DEC 02, 1943 LOWEST 80.53 JUN 21, 1989 
WATER YEAR OCTOBER 1991 TO SEPTEMBER 1992

SITE NUMBER 344437120161401

LOCAL NUMBER O08NO32W29LO2S

North of Los Alamos near Highway 101. Drilled well. Diameter 9 inches, depth unknown. Altitude of land-surface datum 590 feet. Measurements provided by Santa Barbara County Water Ageney beginning in 1980. Water-1evel records available 1977 to current year.

WATER LEVELS IN FEET BELOW LAND-SURFACE DATUM

DATE WATER $\quad$ LEVEL MS

MAR 16, $1992 \quad 42.94 \mathrm{R}$

RERIOD OF RECORD HIGHEST 13.89 APR 14, 1983 LOWEST 45.86 APR 05, 1991

SITE NUMBER 344457120174001

LOCAL NUMBER O08N032W30D01S

In Los Alamos, north of Highway 135 , and 0.3 mile west of Bell Street. Drilled unused water-table well in sand and Bravel. Diameter 16 inches, depth 899 feet, perforated 265-355, 378-409, 463-523, 667-895 feet. Altitude of land-surface datum 540 feet. Recorder installed December 1977 to October 1982. Water-level records available 1977 to current year.

WATER LEVELS IN FEET BELOW LAND-SURFACE DATUM

$\begin{array}{ll} & \text { WATER } \\ \text { DATE } & \text { LEVEL MS }\end{array}$

SEP $09,199279.05 \mathrm{~S}$

PERIOD OF RECORD HIGHEST 23.18 APR 30,1978 LOWEST 91.06 AUG 15, 1991

SITE NUMBER 344530120231601

LOCAL, NUMBER 008N033W19K01S

About 6 miles west of Los Alamos. Drilled irrigation well. Diameter 12 inches, depth 300 feet. Altitude of land-surface datum 368 feet. Water-level records available 1952, 1958, 1978,1983 to current year.

WATER LEVELS IN FEET BELOW LAND-SURFACE DATUM

$$
\begin{array}{ll}
\text { WATER } \\
\text { DATE }
\end{array}
$$

SEP 09, $1992 \quad 75.38 \mathrm{~S}$

PERIOD OF RECORD HIGHEST 45.58 JUN 17,1958 LOWEST 75.38 SEP 09, 1992

SITE NUMBER 344518120221002

LOCAL NUMBER OOBNO33W20Q02S

About 5.5 miles west of Los Alamos on Highway 135 and 200 feet west of Bachelor Road. Drilled water-table well. Diameter 16 inches, depth 260 feet. Altitude of land-surface datum 408 feet. Measurements provided by Santa

Barbara County Water Agency beginning in 1980. Water-level records avallable 1958, 1967-71, 1973 to current year.

WATER LEVELS IN FEET BELOW LAND-SURFACE DATUM

$$
\begin{array}{ll} 
& \text { WATER } \\
\text { DATE } & \text { LEVEL MS }
\end{array}
$$

MAR 16， $199234.83 \mathrm{R}$

PERIOD OF RECORD HIGHEST 7.37 JAN 16,1958 LOWEST 46.34 AUG 29, 1968 
WELL DESCRIPTIONS AND WATER-LEVEL MEASUREMENTS

WATER YEAR OCTOBER 1991 TO SEPTEMBER 1992

SITE NUMBER 344517120220701

LOCAL NUMBER 008N033W20R01S

About 5.2 miles west of Los Alamos and 50 feet south of Los Alamos Road. Drilled water-table well. Diameter 10 inches, depth 75 feet. Altitude of land-surface datum 410 feet. Measurements provided by Santa Barbara County Water Agency beginning in 1980. Water-level records available 1943 to current year.

WATER LEVELS IN FEET BELOW LAND-SURFACE DATUM

$$
\text { DATE WATER }
$$

MAR 16, $1992 \quad 44.45 \mathrm{R}$

PERIOD OF RECORD HIGHEST 21.20 JAN 30, 1947 LOWEST 51,42 APR 20,' 1990

SITE NUMBER 344517120214301

LOCAL NUMBER 008N033W21M03S

About 4.5 miles west of Los Alamos on Highway 135. Drilled irrigation water-table well. Diameter 14 inches 0-207 feet, 12 inches 207-447 feet, depth 447 feet, perforated 348-447 feet. Altitude of land-surface datum 400 feet. Water-level records available 1966, 1968, 1980 to current year.

WATER LEVELS IN FEET BELOW LAND-SURFACE DATUM (READINGS ABOVE LAND SURFACE INDICATED BY "+")

WATER

DATE LEVEL MS

SEP 09, $1992 \quad 54.04 \mathrm{~S}$

PERIOD OF RECORD HIGHEST +1.39 JAN 19, 1968 LOWEST 58.18 MAR 13, 1990

SITE NUMBER 344516120190201

LOCAL NUMBER 008N033W23Q02S

About $3 \mathrm{miles}$ west of Los Alamos, north of Highway 135. Drilled irrigation water-table well. Diameter 14 inches, depth 311 feet, cased to 220 feet, perforated 52-220 feet. Altitude of land-surface datum 495 feet.

Water-level records available $1955,1978,1980$ to current year.

WATER LEVELS IN FEET BELOW LAND-SURFACE DATUM

$$
\text { DATE WATER }
$$

SEP $09,1992 \quad 21.09 \mathrm{~S}$

PERIOD OF RECORD HIGHEST 6.90 APR 21, 1955 LOWEST 44.53 SEP 25, 1985

SITE NUMBER 344802120255901

LOCAL NUMBER 008N034W02M01S

About 5 miles south of Orcutt, 0.5 mile east of intersection of Cabrillo Highway and Vandenbers. Road. Irrigation well. Diameter and depth unknown. Altitude of land-surface datum 420 feet. Water-level records available 1977 to current year.

WATER LEVELS IN FEET BELOW LAND-SURFACE DATUM

$$
\text { DATE WATER }
$$

SEP 09, $1992129,14 \mathrm{~S}$

PERIOD OF RECORD HIGHEST 83,52 JAN 18, 1978 LOWEST 131.99 AUG 14, 1991 
SITE NUMBER 344712120273901

LOCAL NUMBER 008N034W09K01S

On Vandenberg AFB about 1,7 miles northeast of intersection of Vandenberg and San Antonio Roads. Drilled unused water-table well. Diameter 6 inches, depth 620 feet, perforated 120-320, 380-580 feet. Altitude of land-surface datum 425 feet. Water-level records available 1978 to current year.

WATER LEVELS IN FEET BELOW LAND-SURFACE DATUM

$$
\text { DATE WATER }
$$

SEP $10,1992103.09 \mathrm{~S}$

PERIOD OF RECORD HIGHEST 82.75 MAR 22, 1979 LOWEST 103.09 SEP 10, 1992

SITE NUMBER 344624120253901

LOCAL NUMBER OOBN034W14LO1S

About 6 miles south of Orcutt, 80 feet west of Highway 1, and 85 feet north of San Antonio Road. Drilled observation well. Diameter 4 inches, depth 593 feet, perforated 320-440,500-560 feet. Altitude of

land-surface datum 330 feet. Recorder installed 1980-86. Water-level records available 1980 to current year.

WATER LEVELS IN FEET BELOW LAND-SURFACE DATUM

$$
\text { DATE WATER } \quad \text { LEVEL MS }
$$

SEP $09,199240.94 \mathrm{~S}$

PERIOD OF RECORD HIGHEST 17,59 MAR 19, 1981 LOWEST 40.94 SEP 09, 1992

SITE NUMBER 344628120264203

LOCAL NUMBER 008N034W15F04S

On Vandenberg AFB at east end of Barka Slough. Drilled observation water-table well in Paso Robles Formation of Pleistocene age. Diameter 6 inches, depth 475 feet, perforated $375-435$ feet. Altitude of land-surface datum 310 feet. Water-level records avallable 1981 to current year.

WATER LEVELS IN FEET BELOW LAND-SURFACE DATUM

$$
\text { DATE WATER } \quad \text { LEVEL MS }
$$

SEP $10,1992 \quad 29.24 \mathrm{~S}$

PERIOD OF RECORD HIGHEST 10.05 FEB 24, 1984 LOWEST 29.24 SEP 10, 1992

SITE NUMBER 344640120274401

LOCAL NUMBER 008 N034W16C01S

On Vandenberg AFB, south of Vandenberg Road. Drilled observation well. Diameter 2 inches, depth measured 86.25 feet, screened 85-89 feet. Altitude of land-surface datum 340 feet. Water-level records available 1970 to current year.

WATER LEVELS IN FEET BELOW LAND-SURFACE DATUM

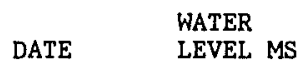

SEP 09, $199283.18 \mathrm{~S}$

PERIOD OF RECORD HIGHEST 39.92 FEB 05,1970 LOWEST 83.18 SEP 09, 1992 
WELL DESCRIPTIONS AND WATER-LEVEL MEASUREMENTS

WATER YEAR OCTOBER 1991 TO SEPTEMBER 1992

SITE NUMBER 344640120274402

LOCAL NUMBER 008N034W16C02S

On Vandenberg AFB, south of Vandenberg Road. Drilled observation well. Dianeter 2 inches, depth measured 169.43 feet in 1970, screened 168-172 feet. Altitude of land-surface datum 340 feet. Water-level records avallable 1970 to current year.

WATER LEVELS IN FEET BELOW LAND-SURFACE DATUM

DATE WATER

SEP 09, $1992 \quad 70.70 \mathrm{~s}$

PERIOD OF RECORD HIGHEST 33.91 FEB 05, 1970 LOWEST $70.70 \quad$ SEP 09, 1992

SITE NUMBER 344640120274403

LOCAL NUMBER 008N034W16C03S

On Vandenberg AFB, south of Vandenberg Road. Drilled observation well. Diameter 2 inches, depth measured 317.68 feet in 1970, screened $320-324$ feet. Altitude of land-surface datum 340 feet. Water-level records available 1970 to current year.

WATER LEVELS IN FEET BELOW LAND-SURFACE DATUM

$$
\text { DATE WATER }
$$

SEP $09,1992 \quad 57.02 \mathrm{~s}$

PERIOD OF RECORD HIGHEST 27.05 AUG 26, 1971 LOWEST 57.02 SEP 09, 1992

SITE NUMBER 344640120274404

LOCAL NUMBER 008N034W16C04S

On Vandenberg AFB, south of Vandenberg Road. Drilled observation well. Dlameter 2 inches, depth 560 feet, screened 556-560 feet. Altitude of land-surface datum 340 feet. Water-level records available 1970 to current year.

WATER LEVELS IN FEET BELOW LAND-SURFACE DATUM

$$
\text { DATE WATER }
$$

SEP $09,1992 \quad 59.87 \mathrm{~S}$

PERIOD OF RECORD HIGHEST 21.06 FEB 05, $1970 \quad$ LOWEST 59.87 SEP 09, 1992

SITE NUMBER 344638120280701

LOCAL NUMBER O08N034W16D02S

On Vandenberg AFB in Barka Slough area. Drilled observation water-table well. Diameter 2 inches, depth 62 feet, well point 60.5-62 feet. Altitude of land-surface datum 280 feet. Water-level records available $1976-1992$.

WATER LEVELS IN FEET BELOW LAND-SURFACE DATUM (READINGS ABOVE LAND SURFACE INDICATED BY "+")

DATE WATER $\quad$ LEVEL MS

SEP 10, $1992 \quad 0$

PERIOD OF RECORD HIGHEST +2.66 APR 26, 1977 LOWEST 20.78 AUG 29, 1989 
SITE NUMBER 344636120274201

LOCAL NUMBER 008N034W16F01S

On Vandenberg AFB in Barka Slough area. Drilled observation water-table well. Diameter 2 inches, depth 59 feet, well point 57.5-59 feet. Altitude of land-surface datum 290 feet. Water-level records available 1976 to current year.

WATER LEVELS IN FEET BELOW LAND-SURFACE DATUM (READINGS ABOVE LAND SURFACE INDICATED BY "+")

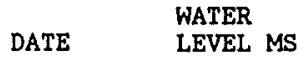

SEP $10,1992 \quad 30.87 \mathrm{~S}$

PERIOD OF RECORD HIGHEST +0.99 JAN 22, 1979 LOWEST 30.87 SEP 10, 1992

SITE NUMBER 344628120273201

LOCAL NUMBER 008N034W16G02S

On Vandenberg AFB in Barka Slough area, Drilled public-supply water-table well in Paso Robles Formation. Diameter 14 inches, depth 700 feet, perforated 52-680 feet. Altitude of land-surface datum 306 feet. Water-level records available 1964, 1966-69, 1971 to current year.

WATER LEVELS IN FEET BELOW LAND-SURFACE DATUM

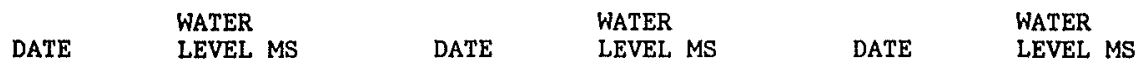

OCT 08, $199152.94 \mathrm{~S} \quad$ DEC 03, $199150.25 \mathrm{~S} \quad$ FEB $10,1992 \quad 49.23 \mathrm{~S}$

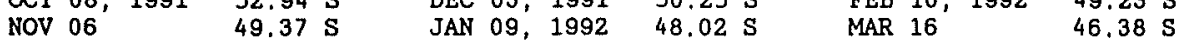

PERIOD OF RECORD HIGHEST 14.67 DEC 01, 1966 LOWEST $52.94 \quad$ OCT 08, 1991

SITE NUMBER 344626120272901

LOCAL NUMBER 008N034W16G03S

On Vandenberg AFB in Barka Slough area. Drilled observation water-table well. Diameter 2 inches, depth 58 feet, well point 54,5-56 feet. Altitude of land-surface datum 290 feet. Water-level records available 1976 to current year.

WATER LEVELS IN FEET BELOW LAND-SURFACE DATUM

$$
\text { DATE WATER }
$$

SEP $10,1992 \quad 35.30 \mathrm{~S}$

PERIOD OF RECORD HIGHEST 10.08 APR 29, 1976 LOWEST 35.30 SEP 10,1992

SITE NUMBER 344630120290101

LOCAL NUMBER DOBN034W17E01S

On Vandenberg AFB in Barka Slough area. Drilled observation water-table well. Diameter 2 inches, depth 87 feet, well point $87.5-89$ feet. Altitude of land-surface datum 270 feet. Water-level records available 1976 to current year.

WATER LEVELS IN FEET BELOW LAND-SURFACE DATUM

$$
\begin{array}{ll}
\text { WATER } \\
\text { DATE }
\end{array}
$$

SEP 09, $1992 \quad 12.07 \mathrm{~S}$

PERIOD OF RECORD HIGHEST 5.26 JUN 27, 1983 LOWEST 12.07 SEP 09, 1992 
WELL DESCRIPTIONS AND WATER-LEVEL MEASUREMENTS

WATER YEAR OCTOBER 1991 TO SEPTEMBER 1992

SITE NUMBER 3446371.20282901

LOCAL NUMBER 008N034W17G01S

On Vandenberg AFB in Barka Slough area. Drilled observation water-table well. Diameter 2 inches, depth 61 feet, well point 59.5-61 ceet. Altitude of land-surface datum 260 feet. Water-level records available 1976 to current year.

WATER LEVELS IN FEET BELOW LAND-SURFACE DATUM (READINGS ABOVE LAND SURFACE INDICATED BY "+")

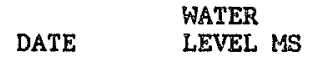

SEP $10,1992 \quad 0.34 \mathrm{~S}$

PERIOD OF RECORD HIGHEST +9.01 SEP $26,1978 \quad$ LOWEST $0.34 \quad$ SEP 10,1992

SITE NUMBER 344633120281901

LOCAL NUMBER 008N034W17H01S

On Vandenberg AFB in Barka Slough area. Drilled observation water-table well. Diameter 2 inches, depth 61 feet, well point 59.5-61 feet. Altitude of land-surface datum 260 feet. Water-level records available 1976 to current year.

WATER LEVELS IN FEET BELOW LAND-SURFACE DATUM (READINGS ABOVE LAND SURFACE INDICATED BY "+")

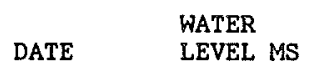

SEP $10,1992 \quad 5.90 \mathrm{~S}$

PERIOD OF RECORD HIGHEST +7.54 FEB 25,1983 LOWEST 5.90 SEP 10,1992

SITE NUMBER 344618120283201

LOCAL NUMBER 008N034W17K02S

On Vandenberg AFB in Barka Slough area. Drilled observation water-table well. Diameter 2 inches, depth 60 feet, well point 58.5-60 feet. Altitude of land-surface datum 260 feet. Water-level records available 1976 to current year.

WATER LEVELS IN FEET BELOW LAND-SURFACE DATUM (READINGS ABOVE LAND SURFACE INDICATED BY "+")

$$
\text { DATE WATER }
$$

SEP 09, $1992+0.23 \mathrm{~S}$

PERIOD OF RECORD HIGHEST +9.55 FEB 25,1983 LOWEST 4.00 JUL 02, 1987

SITE NUMBER 344611120283001

LOCAL NUMBER 008N034W17Q01S

On Vandenberg AFB in Barka Slough area. Drilled observation water-table well. Diameter 2 inches, depth 47.5 feet, well point 46-47.5 feet. Altitude of land-surface datum 270 feet. Water-level records available 1976 to current year.

WATER LEVELS IN FEET BELOW LAND-SURFACE DATUM (READINGS ABOVE LAND SURFACE INDICATED BY "+")

$$
\begin{array}{ll}
\text { DATE } & \text { WATER } \\
\text { LEVEL MS }
\end{array}
$$

SEP 09, $1992+0.23 \mathrm{~S}$

PERIOD OF RECORD HIGHEST $+8,55$ APR 26,1983 LOWEST +0,23 SEP 09, 1992 
SITE NUMBER 344550120273901

LOCAL NUMBER 008N034W21A01S

On Vandenberg AFB in Barka Slough area. Drilled observation water-table well in Careaga Sand of Pliocene age. Diameter 6 inches, depth 271 feet, perforated 71-271 feet. Altitude of land-surface datum 300 feet. Water-1evel records available 1977 to current year.

WATER LEVELS IN FEET BELOW LAND-SURFACE DATUM

$$
\text { DATE WATER }
$$

SEP $09,1992 \quad 17.43 \mathrm{~s}$

PERIOD OF RECORD HIGHEST 2.10 APR 25, 1978 LOWEST 17.43 SEP 09, 1992

SITE NUMBER 344539120263902

LOCAL NUMBER 008N034W22F01S

About 7 miles south of Orcutt, 1.25 miles west of Highways 135 and 1, south of San Antonio Road. Drilled unused water-table well. Diameter 8 inches, depth 269 feet, perforated 105-115, 168-173 feet. Altitude of land-surface datum 310 feet. Water-level records available 1967, 1971, 1980 to current year.

WATER LEVELS IN FEET BELOW LAND-SURFACE DATUM

$$
\begin{array}{ll} 
& \text { WATER } \\
\text { DATE } & \text { LEVEL MS }
\end{array}
$$

SEP 09, $199249.93 \mathrm{~S}$

PERIOD OF RECORD HIGHEST 26.00 AUG 28, 1967 LOWEST 49.93 SEP 09, 1992

SITE NUMBER 344546120252901

LOCAL NUMBER 008N034W23B01S

About 8.3 miles west of Los Alamos. Drilled water-table well. Diameter 12 inches, depth 150 feet. Altitude of land-surface datum 315 feet. Measurements provided by Santa Barbara County Water Agency beginning in 1980 . Water-level records available 1943 to current year.

WATER LEVELS IN FEET BELOW LAND-SURFACE DATUM

$$
\text { DATE WATER }
$$

MAR 16, $1992 \quad 25.42 \mathrm{R}$

PERIOD OF RECORD HIGHEST 12.57 MAR 01, 1945 LOWEST 36.36 MAR 08, 1972

SITE NUMBER 344530120245201

LOCAL NUMBER 008N034W24E01S

South of Orcutt, southeast of intersection of Highways 1 and 135. Drilled irrigation well. Diameter 16 inches, depth 580 feet, perforated 310-570 feet. Altitude of land-surface datum 350 feet. Water-level records available 1977, 1980 to current year.

WATER LEVELS IN FEET BELOW LAND-SURFACE DATUM

$$
\begin{array}{ll} 
& \text { WATER } \\
\text { DATE } & \text { LEVEL MS }
\end{array}
$$

SEP 09, $199265.14 \mathrm{~S}$

PERIOD OF RECORD HIGHEST 50.42 APR 26,1983 LOWEST 71.70 SEP 27,1983 
WELL DESCRIPTIONS AND WATER-LEVEL MEASUREMENTS

WATER YEAR OCTOBER 1991 TO SEPTEMBER 1992

SITE NUMBER 344650120312001

LOCAI, NUMBER 008N035W12M01S

On Vandenberg AFB along San Antonio Creek, 2 miles west of Barka Slough. Drilled observation water-table well. Diameter 6 inches, depth 120 feet, perforated 60-100 feet. Altitude of land-surface datum 145 feet.

Water-level records available 1978 to current year.

WATER LEVELS IN FEET BELOW LAND-SURFACE DATUM

$$
\begin{array}{ll} 
& \text { WATER } \\
\text { DATE } & \text { LEVEL MS }
\end{array}
$$

SEP 02, $1992 \quad 15.43 \mathrm{~S}$

PERIOD OF RECORD HIGHEST 6.35 ARR 08, 1983 LOWEST 16.48 AUG 15, 1990

SITE NUMBER 344910119270501

LOCAL NUMBER O09N024W33M01S

About $1 \mathrm{mile}$ southeast of Ventucopa, $0.5 \mathrm{mile}$ east of Highway 33, and north of Quatal Canyon Road. Drilled observation well. Diameter 13 inches, depth 233 feet. Altitude of land-surface datum 3,049 feet. Measurements provided by Santa Barbara County Water Agency beginning in 1980. Water-level records available 1950-76, 1978 to current year.

WATER LEVELS IN FEET BELOW LAND-SURFACE DATUM

$$
\text { DATE WATER }
$$

APR 02, $1992 \quad 178.45 \mathrm{RR}$

PERIOD OF RECORD HIGHEST 126.64 MAY 11, 1984 LOWEST 207.54 APR 06, 1976

SITE NUMBER 345206119294701

LOCAL NUMBER DO9N025W13B01S

About 3 miles north of Ventucopa. Drilled domestic well. Diameter 10 inches, depth 119 feet, deepened to 175 feet In 1966, perforated 120-175 feet. Altitude of land-surface datum 2,700 feet. Measurements provided by Santa Barbara County Water Agency beginning in 1980. Water-level records available 1946, 1952-76, 1978 to current year.

WATER LEVELS IN FEET BELOW LAND-SURFACE DATUM

$$
\text { DATE WATER }
$$

APR 02, $1992 \quad$ RO

PERIOD OF RECORD HIGHEST 84.47 APR 22, 1983 LOWEST 123.35 DEC 27, 1960

SITE NUMBER 345023119322601

LOCAL NUMBER 009N025W27C01S

In Santa Barbara Canyon, southwest of Cuyama River. Drilled stock well. Diameter 6 inches, depth 95 feet. Altitude of land-surface datum 2,820 feet. Measurements provided by Santa Barbara County Water Agency beginning in 1980. Water-level records available 1941-43, 1966, 1977 to current year.

WATER LEVELS IN FEET BELOW LAND-SURFACE DATUM

$$
\text { DATE WATER }
$$

APR 02, $1992 \quad 77.50 \mathrm{R}$

PERIOD OF RECORD HIGHEST 33.69 APR 22, 1983 LOWEST 83.85 DEC 27, 1977 
LOCAL NUMBER OOSNO26W01F03S

About $3 \mathrm{miles}$ south of Cuyama, $0.6 \mathrm{mile}$ south of Foothill Road. Drilled domestic water-table well. Diameter 6 inches, depth 500 feet. Altitude of land-surface datum 2,604.5 feet. Measurements provided by Santa Barbara County Water Agency beginning in 1983. Water-level records available 1983 to current year.

WATER LEVELS IN FEET BELOW LAND-SURFACE DATUM

$$
\begin{array}{ll} 
& \text { WATER } \\
\text { DATE } & \text { LEVEL MS }
\end{array}
$$

APR 01, $1992302.29 \mathrm{R}$

PERIOD OF RECORD HIGHEST 293,78 APR 23, 1983 LOWEST 302.29 APR 01,1992

SITE NUMBER 345323120173801

LOCAL NUMBER 009N032W06D01S

About 1 mile east of Garey, south of Santa Maria Mesa Road. Irrigation well. Diameter and depth unknown. Altitude of land-surface datum 435 feet. Measurements provided by Santa Barbara County Water Agency beginning tn 1980 . Water-level records available 1966-1975, 1977 to current year.

WATER LEVELS IN FEET BELOW LAND-SURFACE DATUM

$$
\begin{array}{ll} 
& \text { WATER } \\
\text { DATE } & \text { LEVEL MS }
\end{array}
$$

MAR 30, $1992120.82 \mathrm{R}$

PERIOD OF RECORD HIGHEST 60.72 APR 07,1969 LOWEST 131,65 APR 07,1991

SITE NUMBER 345238120164701

LOCAL NUMBER 009N032W07A01S

About 1.5 miles southeast of Garey, south of Santa Maria Mesa Road. Drilled irrigation well. Diameter 16 Inches, depth 452 feet, perforated 107-115, 154-189, 235-238, 282-290,410-416, 428-436 feet. Altitude of land-surface datum 490 feet. Measurements provided by Santa Barbara County Water Agency beginning in 1980 . Water-level records avallable 1944 , 1966 to current year.

WATER LEVELS IN FEET BELOW LAND-SURFACE DATUM

$$
\begin{array}{ll} 
& \text { WATER } \\
\text { DATE } & \text { LEVEL MS }
\end{array}
$$

MAR 30, $1992173.78 \mathrm{R}$

PERIOD OF RECORD HIGHEST $96 \quad$ NOV $18,1944 \quad$ LOWEST 176.49 APR 07,1991

SITE NUMBER 345155120170001

LOCAL NUMBER 009N032W07Q01S

About $0.5 \mathrm{mile}$ east of Sisquoc. Drilled domestic well. Diameter 10 inches, depth unknown. Altitude of land-surface datum 421 feet. Measurements provided by Santa Barbara County Water Agency beginning in 1980 . Water-level records available 1935, 1940-42,1947, 1950,1966-75, 1977 to current year.

WATER LEVELS IN FEET BELOW LAND-SURFACE DATUM

$$
\begin{array}{ll} 
& \text { HATER } \\
\text { DATE } & \text { LEVEL MS }
\end{array}
$$

MAR 30, $1992 \quad 125.29 \mathrm{R}$

PERIOD OF RECORD HIGHEST 19.00 AUG 01,1941 LOWEST 125.29 MAR 30, 1992 
WELL DESCRIPTIONS AND WATER-LEVEL MEASUREMENTS

WATER YEAR OCTOBER 1991 TO SEPTEMBER 1992

SITE NUMBER 345152120162801

LOCAL NUMBER OO9N032W08N01S

About $0.9 \mathrm{mile}$ east of Sisquoc. Domestic well. Diameter 8 inches, depth measured 103.5 feet in 1991, Altitude of land-surface datum 420 feet. Measurements provided by Santa Barbara County Water Agency beginning in 1980. Water-level records available 1958, 1966-74, 1977 to current year.

WATER LEVELS IN FEET BELOW LAND-SURFACE DATUM

$$
\text { DATE WATER }
$$

MAR 30, $1992101.79 \mathrm{R}$

PERIOD OF RECORD HIGHEST 17.80 APR 07, 1969 LOWEST 101.79 MAR 30, 1992

SITE NUMBER 345116120150601

LOCAL NUMBER O09N032W16L01S

About 2 miles southwest of Sisquoc, $0.3 \mathrm{mile}$ north of $\mathrm{Sisquoc}$ Road, and $0.4 \mathrm{mile}$ east of Tepusquet Canyon Road, Irrigation well. Diameter 16 inches, depth measured 120 feet in 1992, perforated 36-95 feet. Altitude of land-surface datum 468 feet. Measurements provided by Santa Barbara County Water Agency beginning in

1980. Water-level records available 1929, 1938, 1942, 1945, 1947, 1950, 1953, 1958-59, 1966 to current year.

WATER LEVELS IN FEET BELOW LAND-SURFACE DATUM

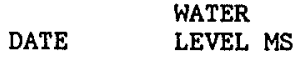

MAR 30, $199245.49 \mathrm{R}$

PERIOD OF RECORD HIGHEST 9.75 MAY 20, 1938 LOWEST 80.61 APR 08, 1991

SITE NUMBER 345129120160301

LOCAL NUMBER 009N032W17G01S

About 2 miles east of Sisquoc on Foxen Canyon Road. Drilled domestic well. Diameter 6 inches, original depth 107 feet, depth measured 72 feet in 1990 . Altitude of land-surface datum 447 feet. Measurements provided by Santa Barbara County Water Agency beginning in 1980. Water-level records available 1941 to current year.

WATER LEVELS IN FEET BELOW LAND-SURFACE DATUM

$$
\text { DATE WATER }
$$

MAR 30, $1992 \quad$ RD

PERIOD OF RECORD HIGHEST 11.22 APR 05, 1943 LOWEST 86.59 OCT 26, 1960

SITE NUMBER 345056120163901

LOCAL NUMBER OO9N032W19A01S

About $1 \mathrm{mile}$ southeast of Sisquoc in Long Canyon. Domestic well. Diameter 13 inches, depth 357.77 feet in 1961 , deepened and cemented at 550 feet in 1964, perforated 500-530, 548-550 feet. Altitude of land-surface datum 727.6 feet. Measurements provided by Santa Barbara County Water Agency beginning in 1980 . Water-1evel records available 1961, 1964, 1966-71, 1973-76, 1978 to current year.

WATER LEVELS IN FEET BELOW LAND-SURFACE DATUM

$$
\text { DATE WATER } \quad \text { LEVEL MS }
$$

MAR 31, $1992 \quad 381.60 \mathrm{R}$

PERIOD OF RECORD HIGHEST 350.59 APR 06, 1970 LOWEST 432.40 APR 07, 1969 
SITE NUMBER 345035120162901

LOCAL NUMBER O09N032W20E01S

About 1.5 miles southeast of Sisquoc in Long Canyon. Unused well. Diameter 6 inches, depth unknown. Altitude of land-surface datum 638 feet. Measurements provided by Santa Barbara County Water Agency beginning in 1980. Water-level records avallable 1964, 1966-77, 1979 to current year,

WATER LEVELS IN FEET BELOW LAND-SURFACE DATUM

$$
\begin{array}{ll}
\text { WATER } \\
\text { DATE }
\end{array}
$$

MAR 31, $1992266.76 \mathrm{R}$

PERIOD OF RECORD HIGHEST 246.90 MAR 17, 1971 LOWEST 283.35 APR 26, 1990

SITE NUMBER 345053120141501

LOCAL NUMBER O09N032W22D01S

About $3.5 \mathrm{miles}$ southeast of Sisquoc, $0.2 \mathrm{mile}$ east of Foxen Canyon Road. Drilled irrigation well. Diameter 14 inches, depth measured 203 feet in 1992. Altitude of land-surface datum 495 feet. Measurements provided by by Santa Barbara County Water Agency beginning 1980. Water-level records available 1950 , 1958 , 1961 to current year.

WATER LEVELS IN FEET BELOW LAND-SURFACE DATUM

$$
\begin{array}{ll} 
& \text { WATER } \\
\text { DATE } & \text { LEVEL MS }
\end{array}
$$

$\operatorname{MAR} 30,1992 \quad 24.07 \mathrm{R}$

PERIOD OF RECORD HIGHEST 4.26 APR 10,1979 LOWEST 44.88 JAN 05,1978

SITE NUMBER 345035120123501

LOCAL NUMBER 009N032W23K01S

About 5 miles southeast of Sisquoc, east of Foxen Canyon Road. Drilled irrigation well. Diameter 16 inches, depth 75 feet, perforated 34-55 feet. Altitude of land-surface datun 532 feet. Measurements provided by Santa Barbaxa County Water Agency beginning in 1980. Water-level records available 1940, 1943, 1950-52, 1958, 1960-75, 1978 to current year.

WATER LEVELS IN FEET BELOW LAND-SURFACE DATUM

$$
\begin{array}{ll} 
& \text { WATER } \\
\text { DATE } & \text { LEVEL MS }
\end{array}
$$

MAR $30,199213,77 \mathrm{R}$

$\begin{array}{lllllllll}\text { PERIOD OF RECORD HIGHEST } & 5.90 & \text { APR } & 27, & 1978 & \text { LOWEST } & 33 & \text { SEP } & 19,1940\end{array}$

SITE NUMBER 344840120155701

LOCAL NUMBER 009N032W32K01S

About 4 miles south of Sisquoc in Cat Canyon. Domestic well. Diameter 8 inches, depth unknown. Altitude of land-surface datum 720 feet. Measurements provided by Santa Barbara County Water Agency beginning in 1980. Water-level records available 1961, 1966 to current year.

WATER LEVELS IN FEET BELOW LAND-SURFACE DATUM

$$
\begin{array}{ll} 
& \text { WATER } \\
\text { DATE } & \text { LEVEL MS }
\end{array}
$$

MAR 30, $1992 \quad 44.69 \mathrm{R}$

PERIOD OF RECORD HIGHEST 13.80 MAR 28, $1974 \quad$ LOWEST $58.24 \quad$ OCT 17,1968 
WELL DESCRIPTIONS AND WATER-LEVEL MEASUREMENTS

WATER YEAR OCTOBER 1991 TO SEPTEMBER 1992

SITE NUMBER 344835120152701

LOCAL NUMBER 009N032W33M01S

About 4.5 miles southeast of Sisquoc in Cat Canyon. Stock well. Diameter 10 inches, depth 89 feet in 1991 , 99 feet in 1967 . Altitude of land-surface datum 745 feet. Measurements provided by Santa Barbara County Water Agency beginning in 1980. Water-level records available 1961, 1966 to current year.

WATER LEVELS IN FEET BELOW LAND-SURFACE DATUM

$$
\text { DATE HATER }
$$

MAR 30, $1992 \quad 59.91 \mathrm{R}$

PERIOD OF RECORD GIGHEST 53.77 MAR 28, $1974 \quad$ LOWEST 73.20 MAR 25, 1976

SITE NUMBER 345325120184201

L.OCAL NUMBER OO9N033W02A07S

East of Santa Maria, north of intersection of Wicks and Andrew Avenues. Drilled irrigation well. Diameter 14 inches, depth 512 feet, perforated 125-507 feet. Altitude of land-surface datum 377 feet. Measurements provided by Santa Barbara County Water Agency. Watex-level records available 1990 to current year.

WATER LEVELS IN FEET BELOW LAND-SURFACE DATUM

$$
\text { DATE WATER }
$$

MAR 30, $1992 \quad 122.18 \mathrm{R}$

PERIOD OF RECORD HIGHEST 122.18 MAR 30, 1992 LOWEST 125.16 APR 07, 1991

SITE NUMBER 345233120181001

LOCAL NUMBER 009N033W12C01S

About 1.2 miles southeast of Garey. Ixrigation well. Diameter unknown, depth unknown. Altitude of land-surface datum 399 feet. Measurements provided by Santa Barbara County Water Agency beginning in 1980 . Water-level records available 1948, 1950, 1966-75, 1977 to current year.

WATER LEVELS IN FEET BELOW LAND-SURFACE DATUM

$$
\text { DATE WATER }
$$

MAR 30, $1992 \quad 157.57 \mathrm{R}$

PERIOD OF RECORD HIGHEST 59.50 MAR 09, 1948 LOWEST 157.57 MAR 30,1992

SITE NUMBER 345024120181801

LOCAL NUMBER 009N033W24L01S

About 1.5 miles south of Sisquoc, north of intersection of Palmer and Cat Canyon Roads. Drilled public-supply well. Diameter 12 inches, depth 622 feet, perforated 432-600 feet. Altitude of 1 and-surface datum 531 feet. Measurements provided by Santa Barbara County Water Agency beginning in 1980 . Water-level records available $1951-57,1961-62,1964,1970-71,1973-75,1977$ to current year.

WATER LEVELS IN FEET BELOW LAND-SURFACE DATUM

$$
\begin{array}{ll} 
& \text { WATER } \\
\text { DATE } & \text { LEVEL MS }
\end{array}
$$

MAR 30, $1992 \quad 206,82 \mathrm{R}$

PERIOD OF RECORD HYGHEST 157.28 JUL 01, 1955 LOWEST 218.00 APR 01,1961 
SITE NUMBER 345340120261801

LOCAL NUMBER OO9N034W03A02S

East of Santa Marla airport, about $0.2 \mathrm{mile}$ west of Broadway Avenue, and 50 feet south of Lakeview Road. Drilled public-supply well. Diameter 16 inches, depth 350 feet, perforated 247-251, 258-271, 284-331 feet. Altitude of land-surface datum 270 feet. Measurements provided by Santa Barbara County Water Agency beginning in 1980 . Water-level records avallable 1967-75, 1977 to current year.

WATER LEVELS IN FEET BELOW LAND-SURFACE DATUM

$$
\text { DATE WATER }
$$

MAR 27, $1992224.14 \mathrm{R}$

PERIOD OF RECORD HIGHEST 177.13 APR 09, 1986 LOWEST 235.00 OCT 08, 1968

SITE NUMBER 345324120263801

LOCAL NUMBER 009N034W03F01S

East of Santa Maria alrport, about $0.5 \mathrm{mile}$ west of Orcutt Road, and $0.35 \mathrm{mile}$ south of Lakeview Road. Drilled public-supply well. Diameter 16 inches, depth 461 feet, perforated $321-327,345-358,368-376,382-392$,

419-449 feet. Altitude of land-surface datum 265 feet. Measurements provided by Santa Barbara County Water Agency beginning in 1980. Water-level records available 1942, 1966-67, 1969-72, 1974, 1977 to current year.

WATER LEVELS IN FEET BELOW LAND-SURFACE DATUM

$$
\text { DATE WATER }
$$

MAR 27, $1992 \quad 231.00 \mathrm{R}$

PERIOD OF RECORD HIGHEST 150.00 AUG 01, 1942 LOWEST 250,50 APR 05, 1977

SITE NUMBER 345330120300801

LOCAL NUMBER 009N034W06C01S

About 3 miles west of Santa Maria. Domestic well. Diameter 10 inches, depth 300 feet. Altitude of land-surface datum 132 feet. Measurements provided by Santa Barbara County Water Agency beginning in 1980. Water-1evel records avallable 1966-75, 1977 to current year.

WATER LEVELS IN FEET BELOW LAND-SURFACE DATUM

$$
\text { DATE WATER } \quad \text { LEVEL MS }
$$

MAR 27, $199290.82 \mathrm{R}$

PERIOD OF RECORD HIGHEST 45.98 ARR 21, 1987 LOWEST 111.00 AUG 01, 1966

SITE NUMBER 345300120294101

LOCAL NUMBER OOMN034W06KO2S

About 1.5 miles southwest of Santa Maria airport, about 970 feet north of junction of Orcutt-Casmalia Road, and 50 feet west of Casmalia Highway. Drilled industrial well. Diameter 12 inches, depth 139 feet. Altitude os land-surface datum 161 feet. Measurements provided by Santa Barbara County Water Agency beginning in 1980. Water-level records available 1942, 1950 to current year.

WATER LEVELS IN FEET BELOW LAND-SURFACE DATUM

$$
\text { DATE WATER }
$$

MAR 26, $199288.86 \mathrm{R}$

PERIOD OF RECORD HIGHEST 59.00 MAR 01, 1942 LOWEST 99.67 OCT 08, 1968


WELL DESCRIPTIONS AND WATER-LEVEL MEASUREMENTS

WATER YEAR OCTOBER 1991 TO SEPTEMBER 1992

SITE NUMBER 345225120283101

LOCAL NUMBER 009N034W08H01S

About $1 \mathrm{mile}$ west of Orcutt, 100 feet south of Highway 1. Drilled industrial well. Diameter 12 inches, depth 200 feet. Altitude of land-surface datum 222 feet. Measurements provided by Santa Barbara County Water Agency beginning in 1980 . Water-level records available 1955 to current year.

WATER LEVELS IN FEET BELOW LAND-SURFACE DATUM

$$
\text { DATE WATER }
$$

MAR 27, $1992 \quad 140.00 \mathrm{R}$

PERIOD OF RECORD HIGHEST 119.73 MAR 25, 1958 LOWEST 156.20 JUL 27, 1959

\section{SITE NUMBER 345205120271801}

LOCAL NUMBER OO9N034WO9R01S

In Orcutt about $0.1 \mathrm{mile}$ west of Blosser Road and $0.3 \mathrm{mile}$ north of Highway 1. Drilled industrial well. Diameter 12 inches, depth 510 feet, perforated 363-382, 418-458 feet. Altitude of land-surface datum 275 feet. Measurements provided by Santa Barbara County Water Agency beginning in 1980 . Water-level records available 1956, 1967 to current year.

WATER LEVELS IN FEET BELOW LAND-SURFACE DATUM

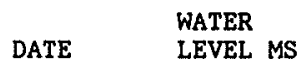

MAR 27, $1992 \quad 211.30 R$

PERIOD OF RECORD HIGHEST 168.85 APR 08, 1986 LOWEST 224.89 DEC 27, 1977

SITE NUMBER 345144120260201

LOCAL NUMBER 009N034W14D01S

In Orcutt. Unused well. Diameter 8 inches, depth 447 feet in 1945. Altitude of land-surface datum 390 feet. Measurements provided by Santa Barbara County Water Agency beginning in 1989 . Water-level records available 1989 to current year.

WATER LEVELS IN FEET BELOW LAND-SURFACE DATUM

$$
\text { DATE WATER }
$$

MAR 27, $1992 \quad$ RD

PERIOD OF RECORD HIGHEST 319.00 MAR 29, 1989 LOWEST 328.38 APR 22, 1990

\section{SITE NUMBER 344836120265401}

LOCAL NUMBER OO9N034W34P01S

On Vandenberg AFB about 4 miles south of Orcutt. Diameter 6 inches, depth 240 feet, perforated 100-220 feet. Altitude of land-surface datum 425 feet. Measurements provided by Santa Barbara County Water Agency beginning in 1980. Water-level records available 1979 to current year.

WATER LEVELS IN FEET BELOW LAND-SURFACE DATUM

$$
\begin{array}{ll}
\text { WATER } \\
\text { DATE LEVEL MS }
\end{array}
$$

SEP 09, $199269.06 \mathrm{R}$

PERIOD OF RECORD HIGHEST 46.60 OCT 24,1979 LOWEST 69.06 SEP 09,1992 
SITE NUMBER 345500119343201

LOCAL NUMBER 010N025W29K02S

About $6.5 \mathrm{miles}$ ast of New Cuyama. Irrigation water-table well. Diameter 14 inches, depth 296 feet, depth of hole 450 feet, perforated 120-296 feet. Altitude of land-surface datum 2,357 feet. Measurements provided by Santa Barbara County Water Agency beginning in 1980. Water-level records available 1966, 1968 , 1977 to current year.

WATER LEVELS IN FEET BELOW LAND-SURFACE DATUM

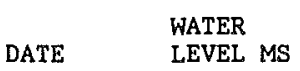

APR $01,1992 \quad 368.09 \mathrm{R}$

PERIOD OF RECORD HIGHEST 121.20 DEC 11,1968 LOWEST 368.09 APR 01,1992

SITE NUMBER 345512119354101

LOCAL NUMBER 010N025W30F01S

About $1.5 \mathrm{miles}$ southwest of Cuyama and $0.9 \mathrm{mile}$ south of Highway 166 on Kirschenmann Road. Drilled unused well. Diameter 16 inches, depth 212 feet in 1970, original depth 376 feet, perforated 124-160, $170-187$, 196-202, 229-232, 241-250, 265-268, 274-313, 332-370 feet. Altitude of land-surface datum 2, 320 feet. Measurements provided by Santa Barbara County Water Agency beginning in 1980. Water-level records ava1lable 1941-56, 1966, 1968, 1970 to current year.

WATER LEVELS IN FEET BELOW LAND-SURFACE DATUM

$$
\begin{array}{ll} 
& \text { WATER } \\
\text { DATE } & \text { LEVEL MS }
\end{array}
$$

APR 01, $1992172.75 \mathrm{R}$

PERIOD OF RECORD HIGHEST 47.36 APR 24, 1945 LOWEST 172.75 APR 01 , 1992

SITE NUMBER 345822119391801

LOCAL NUMBER 010N026W04R01S

About 3.2 miles northwest of Cuyama. Drilled domestic we11. Diameter 16 inches, depth 238 feet, perforated 108-232 feet. Altitude of land-surface datum 2,116 feet. Measurements provided by Santa Barbara County Agency beginning in 1980. Water-level records available 1947, 1955, 1957 to current year.

WATER LEVELS IN FEET BELOW LAND-SURFACE DATUM

$$
\begin{array}{ll} 
& \text { WATER } \\
\text { DATE } & \text { LEVEL MS }
\end{array}
$$

APR $01,1992106.34 \mathrm{R}$

PERIOD OF RECORD HIGHEST 18.60 APR 13, 1955 LOWEST 106.34 APR 01,1992

SITE NUMBER 345631119391501

LOCAL NUMBER 010N026W15N01S

About 1 mile east of New Cuyama, north of Highway 166. Drilled unused well. Diameter 14 inches, depth 550 feet, perforated 94-106, 118-124, 142-154, 172-190, 196-226, 238-244, 262-274, 286-346, 358-370, 382-388, 406-418, 430-484, 502-544 feet. Altitude of land-surface datum 2,210 feet. Measurements March 24,1970 to April 27,1977 previously published as well 010N026W16Q01S. Measurements provided by Santa Barbara County Water Agency beginning in 1980. Water-level records available 1948, 1955, 1958, 1966, 1968, 1970 to current year.

WATER LEVELS IN FEET BELOW LAND-SURFACE DATUM

$$
\begin{array}{ll} 
& \text { WATER } \\
\text { DATE } & \text { LEVEL MS }
\end{array}
$$

APR $01,1992190.18 \mathrm{R}$

PERIOD OF RECORD HIGHEST 25,00 NOV 1948 LOWEST 190.18 APR 01,1992 
WELL DESCRIPTIONS AND WATER-LEVEL MEASUREMENTS

WATER YEAR OCTOBER 1991 TO SEPTEMBER 1992

SITE NUMBER 345637119394701

LOCAL NUMBER 010N026W16Q01S

About 1 mile east of New Cuyama. Drilled unused well. Diameter 14 inches, depth 646 feet, perforated 166-238, 262-286, 310-382, 412-526, 544-550, 568-574, 592-640 feet. Altitude of land-surface datum 2,205 feet. Measurements provided by Santa Barbara County Water Agency beginning in 1991. Water-level records avallable $1950-51,1954-69,1977,1991$ to current year.

WATER LEVELS IN FEET BELOW LAND-SURFACE DATUM

DATE WATER

APR 01, $1992169.84 \mathrm{R}$

PERIOD OF RECORD HIGHEST 33.77 JAN 26, 1954 LOWEST 169.84 APR 01, 1992

SITE NUMBER 345709119415501

LOCAL NUMBER 010N026W18F01S

About 1 mile west of New Cuyama. Drilled irrigation well. Diameter 14 inches, depth reported 240 feet in 1941. Altitude of land-surface datum 2,090 feet. Measurements provided by Santa Barbara County Water Agency beginning in 1990. Water-level records avallable 1941-47, 1949, 1966, 1968, 1990 to current year.

WATER LEVELS IN FEET BELOW LAND-SURFACE DATUM

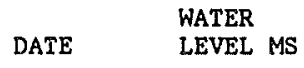

MAR 31, $1992 \quad 99.66 \mathrm{R}$

PERIOD OF RECORD HIGHEST 43.12 AUG 04, 1941 LOWEST 99.89 APR 08, 1991

SITE NUMBER 345603119411901

LOCAL NUMBER 010N026W20M01S

South of New Cuyama. Diameter 16 inches, depth 790 feet, perforated 350-790 feet. Altitude of land-surface datum 2,165 feet. Measurements provided by Santa Barbara County Water Agency beginning in 1981. Water-level records available 1981 to current year.

WATER LEVELS IN FEET BELOW LAND-SURFACE DATUM

$$
\text { DATE WATER } \quad \text { LEVEL MS }
$$

MAR $31,1992 \quad 51.36 \mathrm{R}$

PERIOD OF RECORD HIGHEST 51.36 MAR 31, 1992 LOWEST 88.12 APR 16, 1982

SITE NUMBER 345807119433501

LOCAL NUMBER O1ON027W11A03S

About 3 miles northwest of New Cuyama. Unused well. Diameter 12 inches, depth 115 feet. Altitude of land-surface datum 1,978 feet. Measurements provided by Santa Barbara County Water Agency beginning in 1980. Water-leve1 records available 1955-60, 1966 to current year.

WATER LEVELS IN FEET BELOW LAND-SURFACE DATUM

$$
\text { DATE HATER }
$$

APR 01, $1992 \quad 64.23 \mathrm{R}$

PERIOD OF RECORD HIGHEST 21.85 FEB 27, 1959 LOWEST 77.74 AUG 25, 1976 
SITE NUMBER 345555120172701

LOCAL NUMBER 010N032W19E01S

About 3 miles south of Twitche11 Dam. Drilled irrigation well. Diameter 18 inches, depth measured 74.9 feet in 1991. Altitude of land-surface datum 380 feet. Measurements provided by Santa Barbara County Water Agenoy beginning in 1980. Water-level records available 1967 to current year.

WATER LEVELS IN FEET BELOW LAND-SURFACE DATUM

$$
\text { DATE WATER }
$$

MAR 29, $1992 \quad 10,46 \mathrm{R}$

PERIOD OF RECORD HIGHEST 6.35 MAR 18, 1968 LOWEST 18.20 APR 07,1991

SITE NUMBER 345554120172701

LOCAL NUMBER 010N032W19E02S

In Cuyama River Valley about 3 miles north of Santa Maria Mesa Road. Drilled unused well. Diameter 18 inches, depth 78 feet, perforated $35-72$ feet. Altitude of land-surface datum 380 feet. Measurements provided by Santa Barbara County Water Agency beginning in 1980. Water-level records available 1963 , 1966 to current year.

WATER LEVELS IN FEET BELOW LAND-SURFACE DATUM

DATE $\quad$ WATER

MAR 29, $199210.19 \mathrm{R}$

PERIOD OF RECORD HIGHEST 7.72 NOV 06, 1967 LOWEST 17.73 APR 07,1991

SITE NUMBER 345541120173001

LOCAL. NUMBER 010N032W19M01S

In Cuyama River Valley about $2.5 \mathrm{miles}$ north of Santa Maria Mesa Road. Drilled irrigation well. Diameter 18 inches, depth measured 53.3 feet in 1992 . Altitude of land-surface datum 380 feet. Measurements provided by Santa Barbara County Water Agency beginning in 1980. Water-level records available 1958, $1967-74$, 1976 to current year.

WATER LEVELS IN FEET BELOW LAND-SURFACE DATUM

$$
\begin{array}{ll}
\text { WATER } \\
\text { DATE } & \text { LEVEL MS }
\end{array}
$$

MAR 29, $1992 \quad 8.06 \mathrm{R}$

PERIOD OF RECORD HIGHEST 5.70 NOV 06, 1967 LOWEST 14.22 APR 07,1991

SITE NUMBER 345725120235701

LOCAL NUMBER 010N033W07M01S

East of Santa Maria, about 0.3 mile north of Main Street. Drilled irrigation well. Diameter 16 inches, depth 253 feet, perforated 130-146, 186-214, 218-226, 229-238 feet. Altitude of land-surface datum 255 feet.

Measurements provided by Santa Barbara County Water Agency beginning in 1980 . Water-1evel records available 1940, $1942,1955,1958-59,1967-75,1978$ to current year.

WATER LEVELS IN FEET BELOW LAND-SURFACE DATUM

$$
\begin{array}{ll}
\text { DATE } & \text { WATER } \\
\text { LEVEL MS }
\end{array}
$$

MAR 28, 1992119.42 RS

PERIOD OF RECORD HIGHEST 58.49 APR 11,1979 LOWEST 146.99 APR 24, 1990 
WELL DESCRIPTIONS AND WATER-LEVEL MEASUREMENTS

WATER YEAR OCTOBER 1991 TO SEPTEMBER 1992

SITE NUMBER 345710120230801

LOCAL NUMBER 010N033W07R01S

In Santa Maria about 35 feet north of Main Street and $1.3 \mathrm{miles}$ east of Suey Road. Drilled unused well. Diameter 16 Inches, depth 210 feet. Altitude of land-surface datum 270 feet. Measurements provided by Santa Barbara County Water Agency beginning in 1980. Water-level records available 1928, 1934, 1936, 1942, 1947, 1955-74, 1978 to current year.

WATER LEVELS IN FEET BELOW LAND-SURFACE DATUM

$$
\begin{array}{ll} 
& \text { WATER } \\
\text { DATE } & \text { LEVEL MS }
\end{array}
$$

MAR 28, $1992100.99 \mathrm{R}$

PERIOD OF RECORD HIGHEST 38.05 APR 11, 1979 LOWEST 139.68 NOV 19, 1964

SITE NUMBER 345627120214201

LOCAL, NUMBER 010N033W16N01S

About 1.1 miles northwest of Gates, and 0.6 mile east of Philbric Road. Drilled irrigation well. Diametex 16 inches, depth measured 161 feet in 1991. Altitude of land-surface datum 292 feet. Measurements provided by Santa Barbara County Water Agency beginning in 1980. Water-level records available 1946, 1955,1968 to current year.

WATER LEVELS IN FEET BELOW LAND-SURFACE DATUM

$$
\text { DATE WATER }
$$

MAR 29, $199263.19 \mathrm{RT}$

PERIOD OF RECORD HIGHEST 13.73 APR 11, 1979 LOWEST 123.90 APR 06, 1991

SITE NUMBER 345627120214401

LOCAL NUMBER O1ON033W16N02S

About 1.1 miles northwest of Gates, and 0.6 mile east of Philbric Road. Drilled irxigation well. Diameter

14 inches, depth 240 feet. Altitude of land-surface datum 292 feet. Measurements provided by Santa Barbara County Water Agency beginning in 1980. Water-level records available 1952, 1955, 1958-59, 1967-75, 1977 to current year.

WATER LEVELS IN FEET BELOW LAND-SURFACE DATUM

$$
\begin{array}{ll}
\text { DATE } & \text { WETER } \\
\text { LEVEL MS }
\end{array}
$$

MAR 29, 199267.22 RR

PERIOD OF RECORD HIGHEST 12.61 APR 11, 1979 LOWEST 125.71 APR 06, 1991

SITE NUMBER 345645120231101

LOCAL NUMBER 010N033W18G01S

In Santa Maria, 0.75 mile east of intersection of Rosemary and Jones Roads. Drilled irrigation well. Diameter 16 inches, depth 436 feet, perforated 132-142, 288-320, 336-340, 408-422 feet, cemented at 424 feet. Alt1tude of land-surface datum 273 feet. Measurements provided by Santa Maria Valley Water Conservation District to 1985, and Santa Barbara County Water Agency beginning in 1990. Water-level records available 1939-85, 1990 to current year.

WATER LEVELS IN FEET BELOW LAND-SURFACE DATUM

$$
\text { DATE WATER }
$$

MAR 27, $1992112.23 \mathrm{R}$

PERIOD OF RECORD HIGHEST 54.50 OCT 15,1980 LOWEST 135.10 OCT 01,1966 
SITE NUMBER 345616120231001

LOCAL NUMBER 010N033W19B01S

In Santa Maria, $0.7 \mathrm{mile}$ east of Rosemary and Stowell Roads. Drilled irrigation well. Diameter 16 inches, depth 307 feet, perforated 92-97, 116-125, 190-215, 238-248 feet. Altitude of land-surface datum 275 feet.

Measurements provided by Santa Maria Valley Water Conservation District to 1985 and Santa Barbara County Water

Agency beginning in 1990. Water-level records available 1927, 1929-85, 1990 to current year.

WATER LEVELS IN FEET BELOW LAND-SURFACE DATUM

$$
\text { DATE WATER }
$$

MAR 28, $1992163.50 \mathrm{R}$

PERIOD OF RECORD HIGHEST 61.50 JUL 10,1980 LOWEST 191.30 AUG 30,1965

SITE NUMBER 345538120231101

LOCAL NUMBER 010N033W19K01S

Southeast of Santa Maria, about 0.25 mile north of Betteravia Road. Drilled irrigation well. Diameter unknown, original depth 292 feet, deepened to 423 feet in 1957 , perforated 262-295, 305-417 feet. Altitude of land-surface datum 280 feet. Measurements provided by Santa Barbara County Water Agency beginning in 1980 . Water-level records available 1950, 1953, 1957, 1966-72, 1974-75, 1977 to current year.

WATER LEVELS IN FEET BELOW LAND-SURFACE DATUM

$$
\text { DATE WATER }
$$

MAR 28, $1992191.50 \mathrm{R}$

PERIOD OF RECORD HIGHEST 106.01 APR 02, 1985 LOWEST 191.50 MAR 28, 1992

SITE NUMBER 345552120220001

LOCAL NUMBER O1ON033W2OHO1S

About $0.7 \mathrm{mile}$ west of Gates, and $0.5 \mathrm{mile}$ east of Philbric Road. Drilled irrigation well. Diameter 16 inches, depth 251 feet, perforated 100-106, 126-132, 161-175 feet. Altitude of land-surface datum 300 feet. Reported measurements provided by San Joaquin Power Division of Pacific Gas and Electric Company, J.B. Lippincott

Engineering offices prior to 1980 , and by Santa Barbara County Water Agency beginning in 1980. Water-level records avallable $1929-40,1942,1950,1955,1958$ to current year.

WATER LEVELS IN FEET BELOW LAND-SURFACE DATUM

$$
\begin{array}{ll}
\text { WATE } & \text { WETER } \\
\text { LEVEL MS }
\end{array}
$$

MAR 28, $199299.36 \mathrm{R}$

PERIOD OF RECORD HIGHEST 51.10 APR 29, 1980 LOWEST 145.17 APR 06, 1991

SITE NUMBER 345547120222301

LOCAL NUMBER 010N033W20LO1S

About $1 \mathrm{mile}$ west of Gates, and 30 feet west of Philbric Road. Irrigation well. Diameter 16 inches, depth 300 feet, perforated 95-105, 114-130, 148-160,264-278 feet. Altitude of land-surface datum 294 feet.

Measurements provided by Santa Barbara County Water Agency beginning in 1980 . Water-level records available 1937 , $1942,1950,1958$ to current year.

WATER LEVELS IN FEET BELOW LAND-SURFACE DATUM

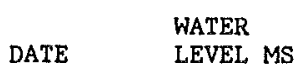

MAR 28, $1992144.02 \mathrm{R}$

PERIOD OF RECORD HIGHEST 63.04 JAN 22, 1970 LOWEST $172,30 \quad$ JUN 27,1961 
WELL DESCRIPTIONS AND WATER-LEVEL MEASUREMENTS

WATER YEAR OCTOBER 1991 TO SEPTEMBER 1992

\section{SITE NUMBER 345534120212001}

LOCAL NUMBER 010N033W21P01S

East of Santa Maria, north of Sisquoc Road. Domestic well. Diameter 16 inches, depth 284 feet, perforated 100-237, 255-272 feet. Altitude of land-surface datum 314 feet. Measurements provided by Santa Maria Valley Water Conservation District to 1985 and Santa Barbara County Water Agency beginning in 1990. Water-level records available 1934-36, 1938, 1942, 1947-48, 1950,1952, 1955, 1958-59, 1981-85, 1990 to current year.

WATER LEVELS IN FEET BELOW LAND-SURFACE DATUM

$$
\text { DATE WATER }
$$

MAR 29, $1992 \quad 97.50 \mathrm{RP}$

PERIOD OF RECORD HIGHEST 46.30 APR 15, 1984 LOWEST 144.80 APR 06, 1991

SITE NUMBER 345458120200601

LOCAL NUMBER 010N033W27G01S

Southeast of Gates. Drilled domestic well. Diameter 16 inches, depth 272 feet, perforated 140-180, 240-260 feet. Altitude of land-surface datum 338 feet. Measurements provided by Santa Maria Valley Water Conservation District to 1985 and Santa Barbara County Water Agency beginning in 1990. Water-level records ava1lable 1929-33, 1936, $1938-85,1990$ to current year.

WATER LEVELS IN FEET BELOW LAND-SURFACE DATUM

DATE $\quad$ WATER

MAR 29, $199289.23 \mathrm{R}$

PERIOD OF RECORD HIGHEST 26.00 JUL 01, 1938 LOWEST 130.76 APR 06, 1991

SITE NUMBER 345433120194401

LOCAL NUMBER O1ON033W27R01S

About 1.9 miles southeast of Gates. Drilled irrigation well. Diameter 16 inches, depth measured 138 feet in 1990 , original depth 255 feet, perforated 130-224 feet. Altitude of land-surface datum 352 feet. Measurements provided by Santa Barbara County Water Agency beginning in 1980. Water-level records available 1926, 1937-39, $1942-43,1947,1949-50,1955,1958-59,1966$ to current year.

WATER LEVELS IN FEET BELOW LAND-SURFACE DATUM

$\begin{array}{ll} & \text { WATER } \\ \text { DATE } & \text { LEVEL MS }\end{array}$

MAR 31, $1992 \quad 115.68 \mathrm{RS}$

PERIOD OF RECORD HIGHEST 35.50 MAY 21, 1943 LOWEST 120.50 AUG 09, 1950

SITE NUMBER 345500120211901

LOCAL NUMBER 010N033W28F01S

About 0.6 mile south of Gates. Irrigation well. Diameter 16 inches, depth 450 feet, perforated 149-154, 170-175, 196-216, 238-248, 291-311, 320-326, 342-348 feet. Altitude of land-surface datum 316 feet. Measurements provided by Santa Barbara County Water Agency beginning in 1980. Water-level records available 1933, 1936-37, 1942, $1947,1949-50,1955,1958,1966-76,1978$ to current year.

WATER LEVELS IN FEET BELOW LAND-SURFACE DATUM

$$
\text { DATE WATER }
$$

MAR 29, $1992198.50 \mathrm{R}$

PERIOD OF RECORD HIGHEST 72.50 JAN 29, 1942 LOWEST 198.50 MAR 29, 1992 
SITE NUMBER 345459120222301

LOCAL NUMBER 010N033W29F01S

About 1.2 miles southwest of Gates. Irrigation well. Diameter and depth unknown. Altitude of land-surface datum 315 feet. Measurements provided by Santa Barbara County Water Agency beginning in 1980, Water-level records available 1958-59, 1964, 1966 to current year.

WATER LEVELS IN FEET BELOW LAND-SURFACE DATUM

$$
\text { DATE WATER }
$$

MAR 28, $1992 \quad 220.42 \mathrm{R}$

PERIOD OF RECORD HIGHEST 137.03 AFR 02, 1985 LOWEST 220.42 MAR 28, 1992

SITE NUMBER 345459120232301

LOCAL NUMBER 010N033W30G01S

Southeast of Santa Maria, west of the intersection of Prell and Telephone Roads. Drilled domestic well. Diameter 16 inches, depth 676 feet, perforated 325-370, 397-432, 454-486, 505-512, 529-561, 575-585, 612-662 feet. Altitude of land-surface datum 320 feet. Measurements provided by Santa Maria Valley Water Conservation District to 1985 and Santa Barbara County Water Agency beginning in 1990. Water-level records available 1948, 1951-85, 1990 to current year.

WATER LEVELS IN FEET BELOW LAND-SURFACE DATUM

$$
\begin{array}{ll} 
& \text { WAT'ER } \\
\text { DATE } & \text { LEVEL MS }
\end{array}
$$

MAR 27, $1992 \quad 244.15 \mathrm{R}$

PERIOD OF RECORD HIGHEST 155.00 DEC 20, 1948 LOWEST 244.15 MAR 27, 1992

SITE NUMBER 345457120233801

LOCAL NUMBER O1ON033W30M01S

Southeast of Santa Maria, west of the intersection of Prell and Telephone Roads. Drilled industrial well. Diameter 16 inches, depth 500 feet, perforated 190-210, 218-244, 268-286, 310-315, 327-342, 385-418, 450-485 feet. Altitude of land-surface datum 310 feet. Measurements provided by Santa Maria Valley Water Conservation District to 1985 and Santa Barbara County Water Agency beginning in 1990. Water-level records avallable 1937, 1951-85, 1990 to current year.

WATER LEVELS IN FEET BELOW LAND-SURFACE DATUM

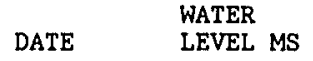

MAR 28, $1992246.88 \mathrm{R}$

PERIOD OF RECORD HIGHEST 166.00 APR 24, 1937 LOWEST 246.88 MAR 28, 1992 
WELL DESCRIPTIONS AND WATER-LEVEL MEASUREMENTS

WATER YEAR OCTOBER 1991 TO SEPTEMBER 1992

SITE NUMBER 345406120204901

LOCAL NUMBER 010N033W33H01S

About 2 miles south of Gates in Bradley Canyon. Drilled domestic well. Diameter 16 inches, depth measured 248 feet in 1990, original depth 290 feet, perforated 204-232, 245-250, 270-280 feet. Alt1tude of land-surface datum 402 feet. Measurements provided by Santa Barbara County Water Agency beginning in 1980 . Water-1evel records available 1929, 1947 to current year.

WATER LEVELS IN FEET BELOW LAND-SURFACE DATUM

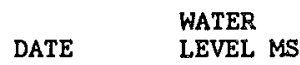

MAR 29, 1992

$$
\text { RP }
$$

PERIOD OF RECORD HIGHEST 179.50 JAN 29, 1947 LOWEST 264.30 SEP 28, 1965

SITE NUMBER 345426120191501

LOCAL NUMBER 010N033W35C01S

About 1 mile north of Garey. Drilled irrigation well. Diameter 20 inches, depth 159.5 feet in 1992 . Altitude of land-surface datum 348 feet. Measurements provided by Santa Barbara County Water Agency beginning in 1980.

Water-level records available 1947, 1955, 1958-59, 1966 to current year.

WATER LEVELS IN FEET BELOW LAND-SURFACE DATUM

$\begin{array}{ll} & \text { WATER } \\ \text { DATE } & \text { LEVEL MS }\end{array}$

MAR 29, $199244.69 \mathrm{R}$

PERIOD OF RECORD HIGHEST 3.55 MAR 19, 1968 LOWEST 92.91 APR 06, 1991

SITE NUMBER 345423120175001

LOCAL NUMBER 010N033W36A01S

About $1 \mathrm{mile}$ north of Garey. Drilled irrigation water-table well in alluvium. Diameter 16 inches, depth 162 feet, perforated 30-76 feet. Altitude of land-surface datum 372 feet. Measurements provided by Santa Barbara County Water Agency beginning in 1980. Water-level records available 1939-42, 1951-53, 1958-59, 1966-75, 1978 to current year.

WATER LEVELS IN FEET BELOW LAND-SURFACE DATUM

DATE WATER

MAR 29, $1992 \quad 18.74 \mathrm{R}$

PERIOD OF RECORD HIGHEST 7.00 JAN 01, 1942 LOWEST 37.96 APR 07, 1991

SITE NUMBER 345807120270901

LOCAL NUMBER 010N034W04R01S

In Santa Maria, northwest of the intersection of Donovan and Blosser Roads. Drilled irrigation well. Diameter 16 inches, depth 186 feet, perforated 90-108, 133-174 feet, cemented at 182 feet. Altitude of land-surface datum 1,921 feet. Measurements provided by Santa Maria Valley Water Conservation District to 1985 and Santa Barbara County Water Agency beginning in 1990. Water-level records available 1934-35, 1939, 1942-85, 1990 to current year.

WATER LEVELS IN FEET BELOW LAND-SURFACE DATUM

$$
\text { DATE WATER }
$$

MAR 27, $1992140.39 \mathrm{R}$

PERIOD OF RECORD HIGHEST 71.00 APR 15, 1984 LOWEST 152.20 MAR 21, 1967 
SITE NUMBER 345818120300601

LOCAL NUMBER 010N034W06N01S

West of Santa Maria. Drilled irrigation well. Diameter 16 inches, depth 190 feet in 1924 . Altitude of land-surface datum 152 feet. Measurements provided by Santa Maria Valley Water Conservation District to 1985 and Santa Barbara County Water Agency beginning in 1990. Water-level records available 1930, 1934 , $1936-85$, 1990 to current year.

WATER LEVELS IN FEET BELOW LAND-SURFACE DATUM

$$
\begin{array}{ll} 
& \text { WATER } \\
\text { DATE } & \text { LEVEL MS }
\end{array}
$$

MAR 24, $1992109.06 \mathrm{R}$

PERIOD OF RECORD HIGHEST 48.40 APR 01, 1943 LOWEST 159.30 APR 05,1978

SITE NUMBER 345657120242901

LOCAL NUMBER 010N034W13C01S

In Santa Maria, $0.25 \mathrm{mile}$ south of east Main Street, Drilled irrigation well. Diameter 16 inches, depth 209 feet, perforated 125-175, 182-204 feet. Altitude of land-surface datum 249 feet. Measurements provided by Santa Barbara County Water Agency beginning in 1980. Water-level records available 1927, 1929-53, 1959, 1967 to current year.

WATER LEVELS IN FEET BELOW LAND-SURFACE DATUM

$$
\begin{array}{ll} 
& \text { WATER } \\
\text { DATE } & \text { LEVEL MS }
\end{array}
$$

MAR 28, $1992 \quad 147.89 \mathrm{R}$

PERIOD OF RECORD HIGHEST 86.30 MAY $10,1984 \quad$ LOWEST 190.80 OCT 08,1953

SITE NUMBER 345644120241801

LOCAL NUMBER 010N034W13G01S

In Santa Maria, $0.5 \mathrm{mile}$ south of east Main Street on Jones Road, Drilled irrigation well. Diameter 16 inches, depth 450 feet, perforated 136-160,165-170,344-360,363-373,390-395. Altitude of land-surface datum

253 feet. Measurements provided by Santa Barbara County Water Agency beginning in 1980 . Water-leve1 records available 1933-53, 1959, 1967 to current year. Water level and depth data published for 1990 was in error and deleted from data base.

WATER LEVELS IN FEET BELOW LAND-SURFACE DATUM

$$
\begin{array}{ll} 
& \text { WATER } \\
\text { DATE } & \text { LEVEL MS }
\end{array}
$$

MAR 28, $1992148.63 \mathrm{R}$

PERIOD OF RECORD HIGHEST 89.50 APR $01,1970 \quad$ LOWEST 161.20 APR 05,1991

SITE NUMBER 345644120235801

LOCAL NUMBER 010N034W13H01S

East of Santa Maria near the intersection of Jones Street and Rosemary Road. Drilled irrigation well. Diameter 14 inches, depth 515 feet, perforated 98-224, 264-388, 429-514 feet. Altitude of land-surface datum 257 feet. Measurements provided by Santa Barbara County Water Agency beginning in 1990 . Water-level records available 1990 to current year.

WATER LEVELS IN FEET BELOW LAND-SURFACE DATUM

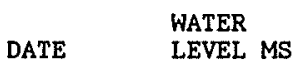

$\operatorname{MAR} 28,1992131.33 \mathrm{R}$

PERIOD OF RECORD HIGHEST 131.33 MAR 28, 1992 LOWEST 150.18 APR 24, 1990 
WELL DESCRIPTIONS AND WATER-LEVEL MEASUREMENTS

WATER YEAR OCTOBER 1991 TO SEPTEMBER 1992

SITE NUMBER 345635120235901

LOCAL NUMBER 010N034W13J01S

East of Santa Maria, about 0.3 mile north of intersection of Rosemary and Stowell Roads. Drilled irrigation well. Diameter 16 inches, depth 308 feet. Altitude of land-surface datum 260 feet. Measurements provided by Santa Barbara County Water Agency beginning in 1980. Water-level records avallable 1947-52, 1955, 1959, 1967-75, 1977-78, 1980 to current year. Water-level and depth data published for 1990 was in error and deleted from data base.

WATER LEVELS IN FEET BELOW LAND-SURFACE DATUM

$\begin{array}{ll}\text { WATER } \\ \text { DATE } & \text { LEVEL MS }\end{array}$

MAR 28, $1992137.03 \mathrm{R}$

PERIOD OF RECORD HIGHEST 85.74 MAY 11,1984 LOWEST 188.65 APR 05, 1991

SITE NUMBER 345604120282202

LOCAL NUMBER 010N034W20H03S

In Santa Maria, south of west Main Street, and $1 \mathrm{mile}$ west of Blosser Road. Drilled unused well. Diameter 16 inches, depth 252 feet, perforated 134-172, 182-234 feet. Altitude of land-surface datum 182 feet.

Measurements provided by Santa Maria Valley Water Conservation District to 1985 and Santa Barbara County Water Agency beginning in 1990. Water-level records available 1957, 1967-75, 1978-85, 1990 to current year.

WATER LEVELS IN FEET BELOW LAND-SURFACE DATUM

$\begin{array}{ll} & \text { WATER } \\ \text { DATE } & \text { LEVEL MS }\end{array}$

MAR 27, $1992130.18 \mathrm{R}$

PERIOD OF RECORD HIGHEST 87.50 APR 15,1985 LOWEST 143.62 NOV 02, 1967

SITE NUMBER 345548120242202

LOCAL NUMBER 010N034W24K01S

East of Highway 101 and south of Battles Road. Drilled unused well in alluvium. Diameter 16 inches, depth 714 feet, perforated 650-657, 692-710 feet. Altitude of land-surface datum 254 feet. Measurements provided by Santa Maria Valley Water Conservation Water District to 1985 and Santa Barbara County Water Agency beginning in 1990. Water-level records available 1941, 1972-85, 1990 to current year.

WATER LEVELS IN FEET BELOW LAND-SURFACE DATUM

DATE WATER

MAR 28, $1992184.97 \mathrm{R}$

PERIOD OF RECORD HIGHEST 75.32 DEC 30,1941 LOWEST 215.50 JUL 01,1972

SITE NUMBER 345548120242201

LOCAL NUMBER 010N034W24KO3S

In Santa Maria about 0.7 mile southeast of the intersection of Stowell Road and Highway 101. Drilled industrial well. Diameter 16 inches, depth 792 feet, perforated 708-778 feet. Altitude of land-surface datum 254 feet. Measurements provided by Santa Maria Valley Water Conservation District to 1985 and Santa Barbara County Water Agency beginning in 1990. Water-level records available 1947-85, 1990 to current year.

WATER LEVELS IN FEET BELOW LAND-SURFACE DATUM

$$
\text { DATE WATER }
$$

MAR 28, $1992179.20 \mathrm{R}$

PERIOD OF RECORD HIGHEST 71.00 JAN 22, 1947 LOWEST 188.00 OCT 01, 1966 
SITE NUMBER 345459120250301

LOCAL NUMBER 010N034W26H02S

In Santa Maria, southwest of intersection of Prell and Bradley Roads. Irrigation well. Diameter and depth unknown. Altitude of land-surface datum 260 feet. Measurements provided by Santa Barbara County Water Agency beginning in 1980. Water-level records available 1957-59, 1966-75, 1978, 1980 to current year.

WATER LEVELS IN FEET BELOW LAND-SURFACE DATUM

$$
\text { DATE WATER }
$$

MAR 27, $1992 \quad 209.47 \mathrm{R}$

PERIOD OF RECORD HIGHEST 153.47 APR 03, 1985 LOWEST 212.00 MAR 22, 1967

SITE NUMBER 345358120295401

LOCAL NUMBER 010N034W31LO2S

About $1 \mathrm{mile}$ west of Santa Maria airport, 1.4 miles south of Betteravia Road, and 0.5 mile west of Mahoney Road. Drilled domestic well. Diameter 8 inches, depth 140 feet, perforated $120-140$ feet. Altitude of land-surface datum 175 feet. Measurements provided by Santa Barbara County Water Agency beginning in 1980. Water-level records avallable 1955, 1966-75, 1977 to current year.

WATER LEVELS IN FEET BELOW LAND-SURFACE DATUM

$$
\text { DATE WATER }
$$

MAR 27, $1992 \quad 120.62 \mathrm{R}$

PERIOD OF RECORD HIGHEST 91.27 APR 08, 1986 LOWEST 139.22 OCT 08, 1968

\section{SITE NUMBER 345750120343001}

LOCAL NUMBER 010N035W09E05S

In Guadalupe, southeast of the intersection of 5th and Tognazzini Streets. Drilled irrigation well.

Diameter 12.75 inches, depth drilled 908 feet, perforated $698-848$ feet. Altitude of land-surface datum 80 feet. Measurements provided by Santa Maria Valley Water Conservation District to 1985 and Santa Barbara County Water Agency beginning in 1990. Water-level records available 1974, 1979-85, 1990 to current year.

WATER LEVELS IN FEET BELOW LAND-SURFACE DATUM

$$
\begin{array}{ll} 
& \text { WATER } \\
\text { DATE } & \text { LEVEL MS }
\end{array}
$$

MAR 24, $1992 \quad 52.77 \mathrm{R}$

PERIOD OF RECORD HIGHEST 25.00 JAN 15, $1984 \quad$ LOWEST 52.77 MAR 24, 1992

SITE NUMBER 345751120340001

LOCAL NUMBER O10N035WO9F01S

In Guadalupe, east of Obispo Street. Drilled irrigation well. Diameter 12 inches, depth 400 feet, perforated 228-240 feet. Altitude of land-surface datum 88 feet. Measurements provided by Santa Barbara County Water Agency beginning in 1980. Water-level records available 1930, 1933, 1935-36, 1938-74, 1976 to current year.

WATER LEVELS IN FEET BELOW LAND-SURFACE DATUM

$$
\begin{array}{ll} 
& \text { WATER } \\
\text { DATE } & \text { LEVEL MS }
\end{array}
$$

MAR 24, $1992 \quad 51.74 \mathrm{R}$

PERIOD OF RECORD HIGHEST 13.74 FEB 29, $1944 \quad$ LOWEST 74.24 JUL 24,1964 
WELL DESCRIPTIONS AND WATER-LEVEL MEASUREMENTS

WATER YEAR OCTOBER 1991 TO SEPTEMBER 1992

SITE NUMBER 345725120342503

LOCAL NUMBER 010N035W09N02S

In Guadalupe, southwest of the intersection of West Main Street and Highway 1. Drilled irrigation well. Diameter 16 inches, depth 464 feet in 1924 . Altitude of land-surface datum 85.87 feet. Measurements provided by Santa Barbara County Water Agency beginning in 1986. Water-level records available 1942, 1950, 1955, 1958,1986 to current year.

WATER LEVELS IN FEET BELOW LAND-SURFACE DATUM

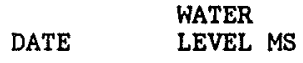

MAR 24, $199250.09 \mathrm{R}$

PERIOD OF RECORD HIGHEST 16.73 FEB 10, 1942 LOWEST 54.97 APR 20, 1990

SITE NUMBER 345748120321901

LOCAL NUMBER 010N035W11E04S

About 1 mile east of Guadalupe. Drilled irrigation well. Diameter 14 inches, depth 430 feet, perforated 230-430 feet. Altitude of land-surface datum 118 feet. Measurements provided by Santa Barbara County Water Agency beginning in 1986 . Water-level records avallable 1986 to current year.

WATER LEVELS IN FEET BELOW LAND-SURFACE DATUM

$$
\text { DATE WATER }
$$

MAR 24, $199278.19 \mathrm{R}$

PERIOD OF RECORD HIGHEST 45.3 APR 07, 1986 LOWEST 80.54 APR 20, 1990

SITE NUMBER 345624120320901

LOCAL. NUMBER 010N035W14P01S

About 2 miles southeast of Guadalupe. Drilled irrigation well. Diameter 16 inches, depth 320 feet, perforated 96-103, 129-132, 151-158, 180-193, 280-310 feet. Altitude of land-surface datum 122 feet. Measurements provided by Santa Barbara County Water Agency beginning in 1983. Water-level records available 1983 to current year.

HATER LEVELS IN FEET BELOW LAND-SURFACE DATUM

$$
\begin{array}{ll} 
& \text { WATER } \\
\text { DATE } & \text { LEVEL MS }
\end{array}
$$

MAR 26, $1992 \quad 76.92 \mathrm{R}$

PERIOD OF RECORD HIGHEST 41.72 APR 07, 1986 LOWEST 76.92 MAR 26, 1992

SITE NUMBER 345659120362002

LOCAL NUMBER 010N035W18F02S

About 1 mile southwest of Guadalupe, about $0.8 \mathrm{mile}$ south of Main Street, and $1.8 \mathrm{miles}$ west of Highway 1 . Drilled irrigation well. Diameter 14 inches, depth 360 feet, perforated 143-180, 236-251 feet. Altitude of 1and-surface datum 49 feet. Measurements provided by Santa Barbara County Water Agency beginning in 1980 . Water-level records available 1968 to current year.

WATER LEVELS IN FEET BELOW LAND-SURFACE DATUM

$$
\begin{array}{ll} 
& \text { WATER } \\
\text { DATE } & \text { LEVEL MS }
\end{array}
$$

$\operatorname{MAR} 24,199219,49 \mathrm{R}$

PERIOD OF RECORD HIGHEST 3.38 APR 08, 1986 LOWEST 29.13 OCT 07,1968 
STTE NUMBER 345621120340101

LOCAL NUMBER 010N035W21B01S

About 1.5 miles southwest of Guadalupe. Drilled irrigation well. Diameter 16 inches, depth 310 feet, perforated 102-118, 134-136, 145-175, 246-248, 251-300 feet. Altitude of land-surface datum 94 feet. Measurements provided by Santa Maria Valley Water Conservation District to 1985 and Santa Barbara County Water Agency beginning in 1990. Water-level records available 1936, 1938-85, 1990 to current year.

WATER LEVELS IN FEET BELOW LAND-SURFACE DATUM

$$
\text { DATE WATER }
$$

MAR 26, $1992 \quad 47.33 \mathrm{R}$

PERIOD OF RECORD HIGHEST 7.85 FEB 29, $1944 \quad$ LOWEST $73.45 \quad$ FEB 23,1972

SITE NUMBER 345544120322501

LOCAL NUMBER 010N035W23M02S

About 3 miles southeast of Guadalupe. Drilled irrigation well. Diameter 16 inches, depth 180 feet. Altitude of land-surface datum 125 feet. Measurements provided by Santa Barbara County Water Agency beginning in 1980.

Water-level records available 1966 to current year.

WATER LEVELS IN FEET BELOW LAND-SURFACE DATUM

$$
\begin{array}{ll} 
& \text { WATER } \\
\text { DATE } & \text { LEVEL MS }
\end{array}
$$

MAR 26, $1992 \quad 71.84 \mathrm{R}$

PERIOD OF RECORD HIGHEST 38.83 APR 07, 1986 LOWEST $98.00 \quad$ SEP 15, 1966

SITE NUMBER 345620120305201

LOCAL NUMBER 010N035W24B01S

Southeast of Guadalupe near intersection of Brown and Ray Roads. Drilled irrigation well. Diameter 16 inches, depth 290 feet, perforated 122-153, 169-175, 178-288 feet. Altitude of land-surface datum 144 feet. Measurements provided by Santa Maria Valley Water Conservation District to 1985 and Santa Barbara County Water Agency beginning in 1990. Water-level records available 1928, 1932-85, 1990 to current year.

WATER LEVELS IN FEET BELOW LAND-SURFACE DATUM

$$
\text { DATE WATER }
$$

MAR 26, $1992100.17 \mathrm{R}$

PERIOD OF RECORD HIGHEST 40.00 NOV 1928 LOWEST 127.83 OCT 19,1966

SITE NUMBER 345538120304801

LOCAL NUMBER O1ON035W24Q01S

About 3.5 miles southeast of Guadalupe, 0.6 mile north of Betteravia Road and 0.1 mile west of Sinton Road. Stock well. Diameter 12 inches, depth 310 feet. Altitude of land-surface datum 162 feet. Measurements provided by Santa Barbara County Water Agency beginning in 1980 . Water-1eve1 records available 1958 , 1970 to current year.

WATER LEVELS IN FEET BELOW LAND-SURFACE DATUM

$$
\begin{array}{ll} 
& \text { WATER } \\
\text { DATE } & \text { LEVEL MS }
\end{array}
$$

MAR 26, $1992116.18 \mathrm{R}$

PERIOD OF RECORD HIGHEST 77.66 APR 08, 1986 LOWEST 123.20 APR 09,1973 
WELL DESCRIPTIONS AND WATER-LEVEL MEASUREMENTS

WATER YEAR OCTOBER 1991 TO SEPTEMBER 1992

SITE NUMBER 345406120313501

LOCAL NUMBER 010N035W35JO2S

About 4.5 miles southeast of Guadalupe on Highway 1. Drilled irrigation well. Diameter 14 inches, depth 760 feet, perforated $580-660,730-750$ feet. Altitude of land-surface datum 110 feet. Measurements provided by Santa Barbara County Water Agency beginning in 1980. Water-level records available 1977 to current year.

WATER LEVELS IN FEET BELOW LAND-SURFACE DATUM

DATE HATER

MAR 26, $199266.46 \mathrm{R}$

PERIOD OF RECORD HIGHEST 12.29 APR 01, 1985 LOWEST 66.46 MAR 26, 1992

SITE NUMBER 345724120374101

LOCAL NUMBER 010N036W12P01S

About 3 miles west of Guadalupe, and 2.8 miles northeast of Mussel Point. Drilled domestic well. Diameter 4 inches, depth 172 feet. Altitude of land-surface datum 29 feet. Measurements provided by Santa Barbara County Water Agency beginning in 1980. Water-level records available 1942, 1961 to current year.

WATER LEVELS IN FEET BELOW LAND-SURFACE DATUM (READINGS ABOVE LAND SURFACE INDICATED BY "+")

$$
\text { DATE WATER }
$$

MAR 24, $1992 \quad 1.85 \mathrm{R}$

PERIOD OF RECORD HIGHEST +2.78 FEB 27, 1962 LOWEST 7.50 OCT 09, 1968 
WATER YEAR OCTOBER 1991 TO SEPTEMBER 1992

\begin{tabular}{|c|c|c|c|c|c|c|c|c|c|}
\hline $\begin{array}{l}\text { LOCAL } \\
\text { NUMBER }\end{array}$ & DATE & TIME & $\begin{array}{l}\text { DEPTH } \\
\text { BELOW } \\
\text { LAND } \\
\text { SURFACE } \\
\text { (WATER } \\
\text { LEVEL) } \\
\text { (FEET) }\end{array}$ & $\begin{array}{l}\text { DEPTH } \\
\text { OF } \\
\text { WELL, } \\
\text { TOTAL } \\
\text { (FEET) }\end{array}$ & $\begin{array}{l}\text { SPE- } \\
\text { CIFIC } \\
\text { CON- } \\
\text { DUCT- } \\
\text { ANCE } \\
\text { (US/CM) }\end{array}$ & $\begin{array}{c}\text { PH } \\
\text { WATER } \\
\text { WHOLE } \\
\text { FIELD } \\
\text { (STAND- } \\
\text { ARD } \\
\text { UNITS) }\end{array}$ & $\begin{array}{l}\text { TEMPER- } \\
\text { ATURE } \\
\text { WATER } \\
\text { (DEG C) }\end{array}$ & $\begin{array}{l}\text { HARD- } \\
\text { NESS } \\
\text { TOTAL } \\
\text { (MG /L } \\
\text { AS } \\
\text { CACO3) }\end{array}$ & $\begin{array}{l}\text { CALCIUM } \\
\text { DIS- } \\
\text { SOLVED } \\
\text { (MG/L } \\
\text { AS CA) }\end{array}$ \\
\hline $\begin{array}{l}004 \mathrm{~N} 027 \mathrm{~W} 07 \mathrm{D0} 1 \mathrm{~S} \\
004 \mathrm{~N} 027 \mathrm{~W} 08 \mathrm{M} 05 \mathrm{~S} \\
004 \mathrm{~N} 027 \mathrm{~W} 08 \mathrm{MO} 06 \mathrm{~S} \\
004 \mathrm{~N} 027 \mathrm{~W} 08 \mathrm{R} 02 \mathrm{~S} \\
004 \mathrm{~N} 027 \mathrm{~W} 17 \mathrm{~J} 01 \mathrm{~S}\end{array}$ & $\begin{array}{l}07-09-92 \\
07-09-92 \\
07-07-92 \\
07-06-92 \\
07-09-92\end{array}$ & $\begin{array}{l}1505 \\
1310 \\
1040 \\
1430 \\
1000\end{array}$ & $\begin{array}{r}98.57 \\
76.14 \\
118.46 \\
51.75\end{array}$ & $\begin{array}{l}490 \\
680 \\
280 \\
205 \\
320\end{array}$ & $\begin{array}{r}1590 \\
1160 \\
681 \\
1210 \\
860\end{array}$ & $\begin{array}{l}7.2 \\
8.2 \\
6.6 \\
6.8 \\
8.2\end{array}$ & $\begin{array}{l}21.5 \\
23.0 \\
19.5 \\
19.0 \\
20.5\end{array}$ & $\begin{array}{l}610 \\
490 \\
260 \\
570 \\
370\end{array}$ & $\begin{array}{r}140 \\
130 \\
68 \\
160 \\
100\end{array}$ \\
\hline $\begin{array}{l}004 \mathrm{~N} 027 \mathrm{~W} 17 \mathrm{LO} 4 \mathrm{~S} \\
004 \mathrm{~N} 027 \mathrm{~W} 18 \mathrm{~B} 05 \mathrm{~S} \\
004 \mathrm{~N} 027 \mathrm{~W} 21 \mathrm{~F} 02 \mathrm{~S} \\
004 \mathrm{~N} 027 \mathrm{~W} 21 \mathrm{G} 02 \mathrm{~S} \\
004 \mathrm{~N} 027 \mathrm{~W} 22 \mathrm{~A} 04 \mathrm{~S}\end{array}$ & $\begin{array}{l}06-24-92 \\
07-06-92 \\
07-08-92 \\
07-08-92 \\
06-25-92\end{array}$ & $\begin{array}{l}0900 \\
1700 \\
1420 \\
1640 \\
1630\end{array}$ & $\begin{array}{r}38.36 \\
58.61 \\
7.62 \\
13.95 \\
F\end{array}$ & $\begin{array}{r}140 \\
85 \\
80 \\
38 \\
740\end{array}$ & $\begin{array}{r}1030 \\
1470 \\
1390 \\
2070 \\
748\end{array}$ & $\begin{array}{l}7.0 \\
7.3 \\
7.8 \\
7.7 \\
7.6\end{array}$ & $\begin{array}{l}20.5 \\
23.5 \\
20.0 \\
22.0 \\
22.0\end{array}$ & $\begin{array}{r}300 \\
640 \\
87 \\
-- \\
290\end{array}$ & $\begin{array}{c}88 \\
160 \\
20 \\
-- \\
80\end{array}$ \\
\hline $\begin{array}{l}004 \mathrm{~N} 027 \mathrm{~W} 22 \mathrm{E} 01 \mathrm{~S} \\
004 \mathrm{~N} 027 \mathrm{~W} 22 \mathrm{E} 02 \mathrm{~S} \\
004 \mathrm{~N} 027 \mathrm{~W} 22 \mathrm{G} 02 \mathrm{~S} \\
004 \mathrm{~N} 027 \mathrm{~W} 22 \mathrm{G} 04 \mathrm{~S} \\
004 \mathrm{~N} 027 \mathrm{~W} 23 \mathrm{E} 05 \mathrm{~S}\end{array}$ & $\begin{array}{l}07-08-92 \\
07-08-92 \\
06-25-92 \\
07-07-92 \\
10-17-91\end{array}$ & $\begin{array}{l}0935 \\
1130 \\
0900 \\
1735 \\
1025\end{array}$ & $\begin{array}{r}26.05 \\
23.04 \\
7.73 \\
1.82 \\
4.20\end{array}$ & $\begin{array}{r}120 \\
70 \\
200 \\
690 \\
760\end{array}$ & $\begin{array}{r}815 \\
953 \\
847 \\
755 \\
9140\end{array}$ & $\begin{array}{l}7.3 \\
7.2 \\
6.7 \\
7.6 \\
6.4\end{array}$ & $\begin{array}{l}19.0 \\
20.0 \\
20.5 \\
24.0 \\
22.5\end{array}$ & $\begin{array}{r}280 \\
400 \\
320 \\
300 \\
--\end{array}$ & $\begin{array}{r}66 \\
100 \\
82 \\
85 \\
--\end{array}$ \\
\hline & $\begin{array}{l}12-12-91 \\
02-04-92 \\
04-01-92 \\
06-02-92 \\
08-19-92\end{array}$ & $\begin{array}{l}1030 \\
1130 \\
0950 \\
1125 \\
1530\end{array}$ & $\begin{array}{r}4.74 \\
1.64 \\
0.96 \\
0.46 \\
F\end{array}$ & $\begin{array}{l}760 \\
760 \\
760 \\
760 \\
760\end{array}$ & $\begin{array}{r}9300 \\
9310 \\
9490 \\
10400 \\
10600\end{array}$ & $\begin{array}{l}6.5 \\
6.5 \\
6.4 \\
6.5 \\
6.5\end{array}$ & $\begin{array}{l}22.5 \\
22.5 \\
22.5 \\
22.5 \\
23.0\end{array}$ & $\begin{array}{l}-- \\
-- \\
-- \\
--\end{array}$ & $\begin{array}{l}-- \\
-- \\
-- \\
--\end{array}$ \\
\hline $004 \mathrm{~N} 027 \mathrm{~W} 23 \mathrm{~F} 02 \mathrm{~S}$ & $\begin{array}{l}10-17-91 \\
12-11-91 \\
02-04-92 \\
04-01-92 \\
06-03-92\end{array}$ & $\begin{array}{l}1200 \\
1100 \\
1355 \\
1145 \\
1000\end{array}$ & $\begin{aligned} & F \\
&+1.71+5.40 \\
&+6.09 \\
&+5.69\end{aligned}$ & $\begin{array}{l}380 \\
380 \\
380 \\
380 \\
380\end{array}$ & $\begin{array}{l}602 \\
611 \\
618 \\
599 \\
593\end{array}$ & $\begin{array}{l}6.9 \\
7.0 \\
6.9 \\
6.9 \\
6.9\end{array}$ & $\begin{array}{l}20.5 \\
20.5 \\
20.5 \\
20.5 \\
20.5\end{array}$ & $\begin{array}{l}-- \\
-- \\
-- \\
--\end{array}$ & $\begin{array}{l}-- \\
-- \\
-- \\
-- \\
--\end{array}$ \\
\hline & $\begin{array}{l}06-24-92 \\
08-18-92\end{array}$ & $\begin{array}{l}1430 \\
1505\end{array}$ & $\begin{array}{l}+6.31 \\
+6.51\end{array}$ & $\begin{array}{l}380 \\
380\end{array}$ & $\begin{array}{l}606 \\
611\end{array}$ & $\begin{array}{l}6.8 \\
7.0\end{array}$ & $\begin{array}{l}20.0 \\
20.0\end{array}$ & 240 & $\begin{array}{c}64 \\
--\end{array}$ \\
\hline 004N027W23F03S & $\begin{array}{l}10-17-91 \\
02-05-92 \\
06-03-92\end{array}$ & $\begin{array}{l}1445 \\
1015 \\
0930\end{array}$ & $\begin{array}{l}+2.13 \\
+3.51 \\
+3.44\end{array}$ & $\begin{array}{l}550 \\
550 \\
550\end{array}$ & $\begin{array}{r}960 \\
1260 \\
1040\end{array}$ & $\begin{array}{l}6.9 \\
7.0 \\
7.0\end{array}$ & $\begin{array}{l}21.5 \\
21.0 \\
21.0\end{array}$ & $\begin{array}{l}-- \\
-- \\
--\end{array}$ & $\begin{array}{l}-- \\
--\end{array}$ \\
\hline $004 \mathrm{NO} 27 \mathrm{~W} 23 \mathrm{~F} 04 \mathrm{~S}$ & $\begin{array}{l}10-17-91 \\
12-12-91 \\
02-04-92 \\
04-01-92 \\
06-02-92\end{array}$ & $\begin{array}{l}1250 \\
1250 \\
1450 \\
1230 \\
1400\end{array}$ & $\begin{array}{l}7.29 \\
7.33 \\
5.00 \\
4.41 \\
4.36\end{array}$ & $\begin{array}{l}802 \\
802 \\
802 \\
802 \\
802\end{array}$ & $\begin{array}{l}30500 \\
30500 \\
30900 \\
32300 \\
32600\end{array}$ & $\begin{array}{l}6.5 \\
6.6 \\
6.6 \\
6.4 \\
6.6\end{array}$ & $\begin{array}{l}23.0 \\
23.0 \\
22.5 \\
22.5 \\
22.5\end{array}$ & $\begin{array}{l}-- \\
-- \\
-- \\
--\end{array}$ & $\begin{array}{l}-- \\
-- \\
-- \\
--\end{array}$ \\
\hline $004 \mathrm{~N} 027 \mathrm{~W} 23 \mathrm{~F} 08 \mathrm{~S}$ & $\begin{array}{l}08-19-92 \\
10-18-91 \\
12-11-91 \\
02-05-92 \\
03-31-92\end{array}$ & $\begin{array}{l}1200 \\
1030 \\
1405 \\
1235 \\
1245\end{array}$ & $\begin{array}{l}3.58 \\
7.77 \\
9.62 \\
6.21 \\
6.18\end{array}$ & $\begin{array}{l}802 \\
430 \\
430 \\
430 \\
430\end{array}$ & $\begin{array}{l}32400 \\
43100 \\
43000 \\
43500 \\
43800\end{array}$ & $\begin{array}{l}6.6 \\
6.4 \\
6.4 \\
6.3 \\
6.2\end{array}$ & $\begin{array}{l}23.0 \\
20.5 \\
20.5 \\
20.5 \\
20.5\end{array}$ & $\begin{array}{l}-- \\
-- \\
--\end{array}$ & $\begin{array}{l}-- \\
-- \\
-- \\
-- \\
--\end{array}$ \\
\hline
\end{tabular}

F Flowing

+ Indicates measurement above land surface. 
INORGANIC DATA

WATER YEAR OCTOBER 1991 TO SEPTEMBER 1992

LOCAL

NUMBER

004N027W07D01S $004 \mathrm{~N} 027$ W08M05S $004 N 027$ WOBM06S 004N027W08R02S 004N027W17J01S

004N027W17L04S 004 N027W18B05S 004 N027W21F02S $004 \mathrm{N027W21G02S}$ $004 \mathrm{~N} 027 \mathrm{~W} 22 \mathrm{~A} 04 \mathrm{~S}$

$004 \mathrm{~N} 027 \mathrm{~W} 22 \mathrm{E} 01 \mathrm{~S}$ 004N027W22E02S $004 \mathrm{N027W22G02S}$ $004 \mathrm{~N} 027 \mathrm{~W} 22 \mathrm{G} 04 \mathrm{~S}$ 004N027W23E05S

$004 N 027 W 23 F 03 S$

$004 \mathrm{~N} 027 \mathrm{~W} 23 \mathrm{~F} 04 \mathrm{~S}$

004N027W23F08S

\begin{tabular}{|c|c|c|c|c|c|c|c|c|}
\hline DATE & $\begin{array}{l}\text { MAGNE- } \\
\text { SIUM, } \\
\text { DIS- } \\
\text { SOLVED } \\
\text { (MG/L } \\
\text { AS MG) }\end{array}$ & $\begin{array}{l}\text { SODIUM, } \\
\text { DIS- } \\
\text { SOLVED } \\
\text { (MG/L } \\
\text { AS NA) }\end{array}$ & $\begin{array}{l}\text { SODIUM } \\
\text { PERCENT }\end{array}$ & $\begin{array}{l}\text { SODIUM } \\
\text { AD- } \\
\text { SORP- } \\
\text { TION } \\
\text { RATIO }\end{array}$ & $\begin{array}{l}\text { POTAS- } \\
\text { SIUM, } \\
\text { DIS- } \\
\text { SOLVED } \\
\text { (MG/L } \\
\text { AS K) }\end{array}$ & $\begin{array}{c}\text { BICAR- } \\
\text { BONATE } \\
\text { WATER } \\
\text { WH IT } \\
\text { FIELD } \\
\text { (MG/L AS } \\
\text { HCO3) }\end{array}$ & $\begin{array}{l}\text { ALKA- } \\
\text { LINITY } \\
\text { WAT WH } \\
\text { TOT FET } \\
\text { FIELD } \\
\text { (MG /L AS } \\
\text { CACO3) }\end{array}$ & $\begin{array}{c}\text { SULFA } \\
\text { DIS- } \\
\text { SOLV } \\
\text { (MG } \\
\text { AS S }\end{array}$ \\
\hline $\begin{array}{l}07-09-92 \\
07-09-92 \\
07-07-92 \\
07-06-92 \\
07-09-92\end{array}$ & $\begin{array}{l}62 \\
39 \\
23 \\
41 \\
29\end{array}$ & $\begin{array}{r}120 \\
80 \\
50 \\
53 \\
46\end{array}$ & $\begin{array}{l}30 \\
26 \\
29 \\
17 \\
21\end{array}$ & $\begin{array}{l}2 \\
2 \\
1 \\
1 \\
1\end{array}$ & $\begin{array}{l}1.7 \\
2.3 \\
1.0 \\
1.1 \\
1.2\end{array}$ & $\begin{array}{l}395 \\
393 \\
229 \\
293 \\
293\end{array}$ & $\begin{array}{l}-\overline{318} \\
-- \\
-- \\
--\end{array}$ & $\begin{array}{l}260 \\
270 \\
130 \\
320 \\
140\end{array}$ \\
\hline $\begin{array}{l}06-24-92 \\
07-06-92 \\
07-08-92 \\
07-08-92 \\
06-25-92\end{array}$ & $\begin{array}{c}20 \\
59 \\
9.1 \\
-- \\
21\end{array}$ & $\begin{array}{c}120 \\
100 \\
270 \\
-- \\
55\end{array}$ & $\begin{array}{r}46 \\
25 \\
86 \\
-\quad \\
29\end{array}$ & $\begin{array}{r}3 \\
2 \\
13 \\
-- \\
1\end{array}$ & $\begin{array}{l}1.4 \\
2.8 \\
6.4 \\
-. \\
1.5\end{array}$ & $\begin{array}{l}405 \\
539 \\
534 \\
564 \\
325\end{array}$ & $\begin{array}{c}330 \\
439 \\
-- \\
460 \\
264\end{array}$ & $\begin{array}{r}190 \\
270 \\
22 \\
-- \\
130\end{array}$ \\
\hline $\begin{array}{l}07-08-92 \\
07-08-92 \\
06-25-92 \\
07-07-92 \\
10-17-91\end{array}$ & $\begin{array}{l}29 \\
36 \\
28 \\
22 \\
--\end{array}$ & $\begin{array}{c}56 \\
58 \\
53 \\
54 \\
--\end{array}$ & $\begin{array}{r}30 \\
24 \\
26 \\
28 \\
--\end{array}$ & $\begin{array}{r}1 \\
1 \\
1 \\
1 \\
--\end{array}$ & $\begin{array}{l}1.6 \\
1.4 \\
1.4 \\
1.5 \\
--\end{array}$ & $\begin{array}{l}244 \\
311 \\
259 \\
351 \\
--\end{array}$ & $\begin{array}{l}-- \\
254 \\
210 \\
-- \\
--\end{array}$ & $\begin{array}{r}110 \\
150 \\
120 \\
110 \\
--\end{array}$ \\
\hline $\begin{array}{l}12-12-91 \\
02-04-92 \\
04-01-92 \\
06-02-92 \\
08-19-92\end{array}$ & $\begin{array}{l}-- \\
-- \\
-- \\
--\end{array}$ & $\begin{array}{l}-- \\
-- \\
-- \\
--\end{array}$ & $\begin{array}{l}-- \\
-- \\
-- \\
--\end{array}$ & $\begin{array}{l}-- \\
-- \\
-- \\
-- \\
--\end{array}$ & $\begin{array}{l}-- \\
-- \\
-- \\
--\end{array}$ & $\begin{array}{l}-- \\
-- \\
-- \\
--\end{array}$ & $\begin{array}{l}-- \\
-- \\
--\end{array}$ & $\begin{array}{l}-- \\
-- \\
=\end{array}$ \\
\hline $\begin{array}{l}10-17-91 \\
12-11-91 \\
02-04-92 \\
04-01-92 \\
06-03-92\end{array}$ & $\begin{array}{l}-- \\
-- \\
-- \\
--\end{array}$ & $\begin{array}{l}-- \\
-- \\
-- \\
-- \\
--\end{array}$ & $\begin{array}{l}-- \\
-- \\
-- \\
--\end{array}$ & $\begin{array}{l}-- \\
-- \\
-- \\
--\end{array}$ & $\begin{array}{l}-- \\
-- \\
-- \\
--\end{array}$ & $\begin{array}{l}-- \\
-- \\
-- \\
--\end{array}$ & $\begin{array}{l}-- \\
-- \\
-- \\
--\end{array}$ & $\begin{array}{l}-- \\
-- \\
--\end{array}$ \\
\hline $\begin{array}{l}06-24-92 \\
08-18-92 \\
10-17-91 \\
02-05-92 \\
06-03-92\end{array}$ & $\begin{array}{l}19 \\
-- \\
-- \\
--\end{array}$ & $\begin{array}{c}38 \\
-- \\
-- \\
-- \\
--\end{array}$ & $\begin{array}{l}26 \\
-- \\
-- \\
--\end{array}$ & $\begin{array}{l}1 \\
-- \\
-- \\
--\end{array}$ & $\begin{array}{l}1.2 \\
-- \\
-- \\
-- \\
--\end{array}$ & $\begin{array}{l}235 \\
-- \\
-- \\
-- \\
--\end{array}$ & $\begin{array}{l}191 \\
-- \\
-- \\
--\end{array}$ & $\begin{array}{c}100 \\
-- \\
-- \\
--\end{array}$ \\
\hline $\begin{array}{l}10-17-91 \\
12-12-91 \\
02-04-92 \\
04-01-92 \\
06-02-92\end{array}$ & $\begin{array}{l}-- \\
-- \\
-- \\
--\end{array}$ & $\begin{array}{l}-- \\
-- \\
-- \\
--\end{array}$ & $\begin{array}{l}-- \\
-- \\
-- \\
--\end{array}$ & $\begin{array}{l}-- \\
-- \\
-- \\
--\end{array}$ & $\begin{array}{l}-- \\
-- \\
-- \\
--\end{array}$ & $\begin{array}{l}-- \\
-- \\
-- \\
--\end{array}$ & $\begin{array}{l}-- \\
-- \\
-- \\
--\end{array}$ & $\begin{array}{l}-- \\
-- \\
-- \\
--\end{array}$ \\
\hline $\begin{array}{l}08-19-92 \\
10-18-91 \\
12-11-91 \\
02-05-92 \\
03-31-92\end{array}$ & $\begin{array}{l}-- \\
-- \\
-- \\
--\end{array}$ & $\begin{array}{l}-- \\
-- \\
--\end{array}$ & $\begin{array}{l}-- \\
-- \\
-- \\
-- \\
--\end{array}$ & $\begin{array}{l}-- \\
-- \\
-- \\
-- \\
--\end{array}$ & $\begin{array}{l}-- \\
-- \\
-- \\
--\end{array}$ & $\begin{array}{l}-- \\
-- \\
-- \\
--\end{array}$ & $\begin{array}{l}-- \\
-- \\
-- \\
--\end{array}$ & $\begin{array}{l}-- \\
-- \\
-- \\
--\end{array}$ \\
\hline
\end{tabular}




\begin{tabular}{|c|c|c|c|c|c|c|c|c|c|}
\hline $\begin{array}{l}\text { LOCAL } \\
\text { NUMBER }\end{array}$ & DATE & $\begin{array}{l}\text { CHLO- } \\
\text { RIDE, } \\
\text { DIS- } \\
\text { SOLVED } \\
\text { (MG/L } \\
\text { AS CL) }\end{array}$ & $\begin{array}{l}\text { FLUO- } \\
\text { RIDE, } \\
\text { DIS- } \\
\text { SOLVED } \\
\text { (MG/L } \\
\text { AS F) }\end{array}$ & $\begin{array}{l}\text { SILICA, } \\
\text { DIS- } \\
\text { SOLVED } \\
\text { (MG/L } \\
\text { AS } \\
\text { SIO2) }\end{array}$ & $\begin{array}{l}\text { SOLIDS, } \\
\text { RESIDUE } \\
\text { AT } 180 \\
\text { DEG. C } \\
\text { DIS- } \\
\text { SOLVED } \\
\text { (MG/L) }\end{array}$ & $\begin{array}{c}\text { SOLIDS, } \\
\text { SUM OF } \\
\text { CONSTI- } \\
\text { TUENTS, } \\
\text { DIS- } \\
\text { SOLVED } \\
\text { (MG/L) }\end{array}$ & $\begin{array}{c}\text { NITRO- } \\
\text { GEN, } \\
\text { NITRATE } \\
\text { DIS- } \\
\text { SOLVED } \\
\text { (MG/L } \\
\text { AS NO3) }\end{array}$ & $\begin{array}{c}\text { NITRO- } \\
\text { GEN, } \\
\text { NITRATE } \\
\text { DIS- } \\
\text { SOLVED } \\
\text { (MG/L } \\
\text { AS N) }\end{array}$ & $\begin{array}{c}\text { NITRO- } \\
\text { GEN, } \\
\text { NITRITE } \\
\text { DIS- } \\
\text { SOLVED } \\
\text { (MG/L } \\
\text { AS N) }\end{array}$ \\
\hline $\begin{array}{l}004 \text { N027W07D01S } \\
004 \mathrm{~N} 027 \text { W08M05S } \\
004 \mathrm{~N} 027 \text { W08M06S } \\
004 \mathrm{~N} 027 \text { W08R02S } \\
004 \mathrm{~N} 027 \text { W } 17 \mathrm{~J} 01 \mathrm{~S}\end{array}$ & $\begin{array}{l}07-09-92 \\
07-09-92 \\
07-07-92 \\
07-06-92 \\
07-09-92\end{array}$ & $\begin{array}{r}190 \\
53 \\
35 \\
73 \\
71\end{array}$ & $\begin{array}{l}0.50 \\
0.60 \\
0.30 \\
0.30 \\
0.40\end{array}$ & $\begin{array}{l}26 \\
30 \\
42 \\
32 \\
35\end{array}$ & $\begin{array}{r}1070 \\
754 \\
445 \\
860 \\
554\end{array}$ & $\begin{array}{r}1010 \\
800 \\
467 \\
838 \\
568\end{array}$ & $\begin{array}{l}20 \\
-- \\
-- \\
--\end{array}$ & $\begin{array}{l}4.47 \\
-- \\
-- \\
-- \\
--\end{array}$ & $\begin{array}{r}0.030 \\
<0.010 \\
<0.010 \\
<0.010 \\
<0.010\end{array}$ \\
\hline $\begin{array}{l}004 \mathrm{~N} 027 \mathrm{~W} 17 \mathrm{~L} 04 \mathrm{~S} \\
004 \mathrm{~N} 027 \mathrm{~W} 18 \mathrm{~B} 05 \mathrm{~S} \\
004 \mathrm{~N} 027 \mathrm{~W} 21 \mathrm{~F} 02 \mathrm{~S} \\
004 \mathrm{~N} 027 \mathrm{~W} 21 \mathrm{G} 02 \mathrm{~S} \\
004 \mathrm{~N} 027 \mathrm{~W} 22 \mathrm{~A} 04 \mathrm{~S}\end{array}$ & $\begin{array}{l}06-24-92 \\
07-06-92 \\
07-08-92 \\
07-08-92 \\
06-25-92\end{array}$ & $\begin{array}{r}47 \\
94 \\
190 \\
-- \\
29\end{array}$ & $\begin{array}{l}0.40 \\
0.50 \\
1.3 \\
0.30\end{array}$ & $\begin{array}{l}38 \\
22 \\
16 \\
-- \\
30\end{array}$ & $\begin{array}{l}-- \\
988 \\
764 \\
-- \\
--\end{array}$ & $\begin{array}{r}705 \\
990 \\
806 \\
-- \\
507\end{array}$ & $\begin{array}{l}-- \\
16 \\
-- \\
--\end{array}$ & $\begin{array}{l}3.57 \\
-- \\
-- \\
--\end{array}$ & $\begin{array}{r}<0.010 \\
0.030 \\
<0.010 \\
<0.010 \\
<0.010\end{array}$ \\
\hline $\begin{array}{l}004 \mathrm{~N} 027 \mathrm{~W} 22 \mathrm{E} 01 \mathrm{~S} \\
004 \mathrm{~N} 027 \mathrm{~W} 22 \mathrm{E} 02 \mathrm{~S} \\
004 \mathrm{~N} 027 \mathrm{~W} 22 \mathrm{G} 02 \mathrm{~S} \\
004 \mathrm{~N} 027 \mathrm{~W} 22 \mathrm{G} 04 \mathrm{~S} \\
004 \mathrm{~N} 027 \mathrm{~W} 23 \mathrm{E} 05 \mathrm{~S}\end{array}$ & $\begin{array}{l}07-08-92 \\
07-08-92 \\
06-25-92 \\
07-07-92 \\
10-17-91\end{array}$ & $\begin{array}{r}68 \\
65 \\
74 \\
27 \\
3100\end{array}$ & $\begin{array}{l}0.60 \\
0.50 \\
0.50 \\
0.20\end{array}$ & $\begin{array}{l}35 \\
27 \\
37 \\
30 \\
--\end{array}$ & $\begin{array}{c}508 \\
588 \\
-- \\
-468 \\
--\end{array}$ & $\begin{array}{l}526 \\
605 \\
564 \\
503 \\
-\cdots\end{array}$ & $\begin{array}{c}-- \\
13 \\
-- \\
--\end{array}$ & $\begin{array}{l}-- \\
2.87 \\
-- \\
-- \\
--\end{array}$ & $\begin{array}{r}<0.010 \\
0.030 \\
<0.010 \\
<0.010 \\
. .-\end{array}$ \\
\hline & $\begin{array}{l}12-12-91 \\
02-04-92 \\
04-01-92 \\
06-02-92 \\
08-19-92\end{array}$ & $\begin{array}{l}3500 \\
3400 \\
2900 \\
3600 \\
3400\end{array}$ & $\begin{array}{l}-- \\
-- \\
-- \\
--\end{array}$ & $\begin{array}{l}-- \\
-- \\
-- \\
-- \\
--\end{array}$ & $\begin{array}{l}-- \\
-- \\
--\end{array}$ & $\begin{array}{l}-- \\
-- \\
-- \\
--\end{array}$ & $\begin{array}{l}-- \\
-- \\
-- \\
--\end{array}$ & $\begin{array}{l}-- \\
-- \\
-- \\
--\end{array}$ & $\begin{array}{l}-- \\
-- \\
-- \\
--\end{array}$ \\
\hline 004N027W23F02S & $\begin{array}{l}10-17-91 \\
12-11-91 \\
02-04-92 \\
04-01-92 \\
06-03-92\end{array}$ & $\begin{array}{l}22 \\
26 \\
26 \\
24 \\
20\end{array}$ & $\begin{array}{l}-- \\
-- \\
-- \\
--\end{array}$ & $\begin{array}{l}-- \\
-- \\
-- \\
--\end{array}$ & $\begin{array}{l}-- \\
-- \\
-- \\
--\end{array}$ & $\begin{array}{l}-- \\
- \\
-- \\
--\end{array}$ & $\begin{array}{l}-- \\
-- \\
-- \\
--\end{array}$ & $\begin{array}{l}-- \\
-- \\
-- \\
--\end{array}$ & $\begin{array}{l}-- \\
-- \\
-- \\
--\end{array}$ \\
\hline & $\begin{array}{l}06-24-92 \\
08-18-92\end{array}$ & $\begin{array}{l}26 \\
21\end{array}$ & 0.40 & 38 & $\begin{array}{l}-- \\
--\end{array}$ & 403 & $\begin{array}{l}-- \\
--\end{array}$ & $=-$ & $<0.010$ \\
\hline 004N027W23F03S & $\begin{array}{l}10-17-91 \\
02-05-92 \\
06-03-92\end{array}$ & $\begin{array}{l}110 \\
210 \\
150\end{array}$ & $\begin{array}{l}-- \\
-- \\
--\end{array}$ & $\begin{array}{l}-- \\
--\end{array}$ & $\begin{array}{l}-- \\
-- \\
--\end{array}$ & $\begin{array}{l}-- \\
-- \\
--\end{array}$ & $\begin{array}{l}-- \\
-- \\
--\end{array}$ & $\begin{array}{l}-- \\
-- \\
--\end{array}$ & $\begin{array}{l}-- \\
--\end{array}$ \\
\hline $004 N 027 W 23 F 04 S$ & $\begin{array}{l}10-17-91 \\
12-12-91 \\
02-04-92 \\
04-01-92 \\
06-02-92\end{array}$ & $\begin{array}{l}12000 \\
-- \\
11000 \\
11000 \\
12000\end{array}$ & $\begin{array}{l}-- \\
-- \\
-- \\
--\end{array}$ & $\begin{array}{l}-- \\
-- \\
-- \\
--\end{array}$ & $\begin{array}{l}-- \\
-- \\
-- \\
--\end{array}$ & $\begin{array}{l}-- \\
-- \\
-- \\
--\end{array}$ & $\begin{array}{l}-- \\
-- \\
-- \\
--\end{array}$ & $\begin{array}{l}-- \\
-- \\
-- \\
--\end{array}$ & $\begin{array}{l}-- \\
-- \\
-- \\
--\end{array}$ \\
\hline 004N027W23F08S & $\begin{array}{l}08-19-92 \\
10-18-91 \\
12-11-91 \\
02-05-92 \\
03-31-92\end{array}$ & $\begin{array}{l}12000 \\
18000 \\
17000 \\
16000 \\
16000\end{array}$ & $\begin{array}{l}-- \\
-- \\
-- \\
--\end{array}$ & $\begin{array}{l}-- \\
-- \\
-- \\
--\end{array}$ & $\begin{array}{l}-- \\
-- \\
-- \\
--\end{array}$ & $\begin{array}{l}-- \\
-- \\
-- \\
--\end{array}$ & $\begin{array}{l}-- \\
-- \\
-- \\
--\end{array}$ & $\begin{array}{l}-- \\
-- \\
-- \\
--\end{array}$ & $\begin{array}{l}-- \\
-- \\
-- \\
--\end{array}$ \\
\hline
\end{tabular}


NITRO- NITROGEN

$\mathrm{NO} 2+\mathrm{NO} 3$

GEN,

DIS-

AMMONIA

DIS-

PHATE, PHORUS

SOLVED SOLVED

$\begin{array}{ll}\text { (MG } / L & \text { (MG/L } \\ \text { AS N) } & \text { AS N })\end{array}$

DIS-

DIS-

AS $\mathrm{PO}$ ) AS P)

$07-09-92$

$07-09-92$

4.50

0.010

0.360

$<0.010$

0.12

0.03

0.040

$07-07-92$

1.10

$<0.010$

0.31

07-06-92

2. 90

0.030

0.34
0.28

0.010

0.100

07-09-92

$<0.010$

06-24-92

$07-06-92$

$<0.050$

0.020

$07-08-92$

3.60

0.010

$<0.050$

0.220

$07-08-92$

$<0.050$

0.060

0.09

06-25-92

$<0.050$

0.070

0.09

5.5

0.15

0.28

0.110

0.090

BORON

DIS-

SOLVED

(UG/L

AS B)

0.030

0.030

1.80

07-08-92

8.70

0.020

07-08-92

2.90

06-25-92

9. 20

$<0.010$

07-07-92

$<0.050$

0.77

0.050

0.090

190
190

30

70

0.250

0.89

0.28

0.290

10-17-91

--

--

0.40

0.090

0.130

70
240

2800

70

$-$

--

90

190
60

50

$--$

$12-12-91$
$02-04-92$

$04-01-92$

06-02-92

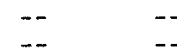

08-19-92

$--$

--

$--$

--

$-$

004N027W23F02S

004N027W23F03S

$10-17-91$ $12-11-91$ $02-04-92$ 04-01-92

$-$

--

$-$

$--$

$-$

$-$

--

$-$

06-03-92

$<0.050 \quad 0.020$

$06-24-92$

$08-18-92$
$10-17-91$

$02-05-92$

$06-03-92$

$-$

004N027W23F04S

10-17-91

12-12-91

$02-04-92$

$04-01-92$

06-02-92

08-19-92

$10-18-91$

$12-11-91$

$02-05-92$

03-31-92

-
-
-
-
-
-
-
-
-
-
-
-
-

$--$

$-$

$--$

--

--
--
--

$\begin{array}{ll}-- & - \\ -- & --\end{array}$

$-$

0.28

0.090

--

--

$-$

--

$-$

--

--

--

$--\quad-$

$--\quad-$

$--$

$-$

< Actual value is less than value shown. 


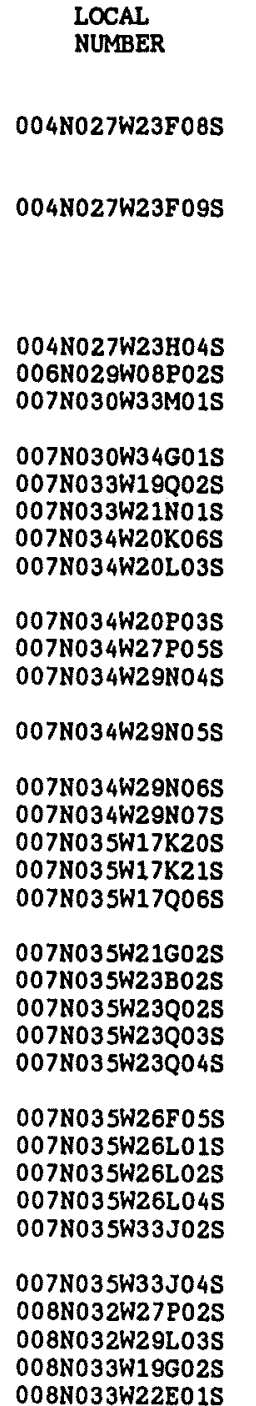

DEPTH
BELOW
LAND
SURFACE
(WATER
LEVEL)
(FEET)

$\begin{array}{ll}06-03-92 & 1145 \\ 06-25-92 & 1600 \\ 08-18-92 & 1300 \\ 12-11-91 & 1230 \\ 03-31-92 & 1415 \\ 06-25-92 & 1400 \\ 08-18-92 & 1415 \\ 06-22-92 & 1505 \\ 07-01-92 & 0945 \\ 07-01-92 & 1200 \\ 07-01-92 & 1115 \\ 09-04-92 & 1000 \\ 07-01-92 & 0745 \\ 09-03-92 & 1120 \\ 09-03-92 & 1025 \\ 09-04-92 & 0900 \\ 06-30-92 & 1410 \\ 08-27-92 & 0900 \\ 09-01-92 & 0945 \\ 09-01-92 & 1600 \\ & \\ 09-01-92 & 1050 \\ 09-01-92 & 1235 \\ 08-31-92 & 1735 \\ 08-31-92 & 1525 \\ 08-31-92 & 1600 \\ & \\ 09-02-92 & 1045 \\ 09-02-92 & 0900 \\ 09-01-92 & 1540 \\ 09-01-92 & 1605 \\ 09-01-92 & 1445 \\ & \\ 06-30-92 & 1315 \\ 09-02-92 & 1355 \\ 09-02-92 & 1315 \\ 09-02-92 & 1230 \\ 09-03-92 & 1230 \\ & \\ 09-03-92 & 1245 \\ 09-11-92 & 0900 \\ 09-08-92 & 1505 \\ 09-08-92 & 1400 \\ 09-08-92 & 1250 \\ & \end{array}$

5.87

5.78

5.44

3.13

1.96

2.44

2.19

1.20

$-$

$--$

$--$

$--$

$--$

$-$

33.86

$--$

32.25

32.34

37.98

39.01

37.13

16.09

2.28

10.90

12.53

18.81

15.94

30.19

30.62$$
--
$$

7.55

12.74

21.17

128.11

$\begin{array}{cc}-- & 462 \\ -- & --- \\ 98.69 & 490 \\ -- & 109 \\ -- & 150\end{array}$

195

195

95

340

900

210

-.-

320

172
PH

$\begin{array}{cccc}\text { WATER } & & \text { HARD- } \\ \text { WHOLE } & & \text { NESS } & \text { CALCIUM } \\ \text { FIELD } & \text { TEMPER- } & \text { TOTAL } & \text { DIS- } \\ \text { (STAND- } & \text { ATURE } & \text { (MG/L } & \text { SOLVED } \\ \text { ARD } & \text { WATER } & \text { AS } & \text { (MG/L } \\ \text { UNITS) } & \text { (DEG C) } & \text { CACO3) } & \text { AS CA) }\end{array}$

(FEET) (US/CM)

\section{$430 \quad 45300$}

$430 \quad 42900$

$430 \quad 46100$

$195 \quad 1120$

1120
836

195745

690
1280

1280

770

835

624
753

1290

1360

1580

1930

4100

3650

3100

2540

1500

5220

6350

11300

\section{4}

6.5

6.5

6.8

6.7

6.8

7.0

7.4

7.8

7.7

20.5
21.0

20.0

19.5

20.0

20.5

19.5

20.5

22.0

20.5

$-$

5900

--

--

$8.0 \quad 19.5$

$6.8 \quad 23.0$

$7.0 \quad 20.5$

$7.6 \quad 23.0$

$7.7 \quad 23.0$

$7.9 \quad 24.0$

$7.4 \quad 17.0$

$7.1 \quad 17.5$

$7.6 \quad 16.0$

$7.4 \quad 17.5$

120

510

290

360

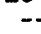

130

46

29

420

180

180

490

37
46

45

140

$440 \quad 130$

$460 \quad 86$

$940 \quad 210$

$2400 \quad 390$

$2400 \quad 400$

$1500 \quad 310$

$\begin{array}{llll}7.2 & 17.5 & 1700 \quad 360\end{array}$

$\begin{array}{rrrr}7.2 & 19.0 & 610 & 160\end{array}$

$\begin{array}{llll}7.3 & 18.5 & 1200 & 210\end{array}$

$\begin{array}{llll}7.3 & 19.5 & 1700 & 290\end{array}$

$\begin{array}{llll}7.6 & 22.0 & 2000 & 270\end{array}$

$\begin{array}{llll}7.5 & 18.5 & 1300 \quad 270\end{array}$

$\begin{array}{llrr}7.8 & 16.0 & 230 & 54\end{array}$

$\begin{array}{llll}7.8 & 16.5 & 2100 & 300\end{array}$

$\begin{array}{llll}7.3 & 17.0 & 1500 & 330\end{array}$

$\begin{array}{llll}7.4 & 18.0 & 1400 \quad 310\end{array}$

3090

2950

7.4

7.4

7.3

7.7

7.4

18.

18.5

18.0

20.0

$1300 \quad 270$

1050

18.0

$430 \quad 83$

$380 \quad 88$

$7.7 \quad 19.5$

1140

.1 21.5

$6.8 \quad 18.0$

$7.6 \quad 19.5$

20.5
$400 \quad 110$

40

$180 \quad 43$

$520 \quad 110$

$570 \quad 160$ 
INORGANIC DATA

WATER YEAR OCTOBER 1991 TO SEPTEMBER 1992

LOCAL

NUMBER

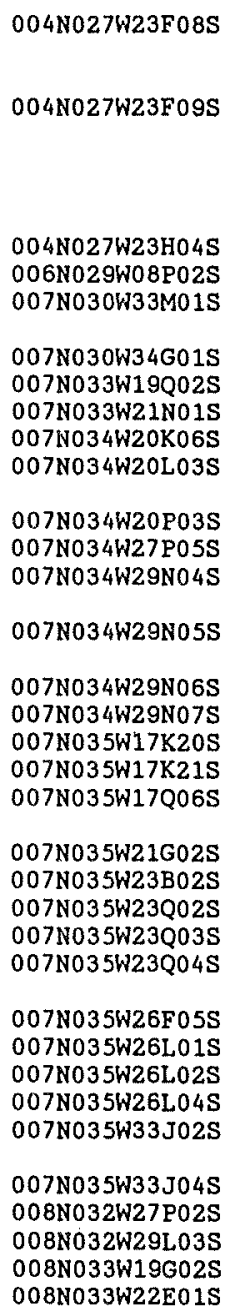

MAGNE-

SIUM, SODIUM,

DIS-

SOLVED SOLVED

(MG/L (MG/L

AS MG) AS NA) PERCENT

DATE

$\begin{array}{lccc}06-03-92 & -- & -- & -- \\ 06-25-92 & 1000 & 8900 & -7 \\ 08-18-92 & -- & -- & -- \\ 12-11-91 & -- & -- & -- \\ 03-31-92 & -- & -- & \\ & & & 66 \\ 06-25-92 & 12 & 110 & -- \\ 08-18-92 & -- & -- & 26 \\ 06-22-92 & 44 & 81 & 15 \\ 07-01-92 & 42 & 23 & 12 \\ 07-01-92 & 71 & 24 & \\ 07-01-92 & 79 & 29 & 13\end{array}$

07-01-92

$09-04-92$

$07-01-92$

$09-03-92$

$09-03-92$

79
16

16
17

29

55
73

85

09-04-92 $60 \quad 120$

06-30-92 100

$08-27-92 \quad 350$

09-01-92 350

09-01-92 170

120

250

270

09-01-92 190

09-01-92 52

$08-31-92 \quad 170$

08-31-92 240

08-31-92 330

$$
140
$$

160

110

740

920

2100

09-02-92 $140 \quad 200$

09-02-92 $24 \quad 160$

$09-01-92 \quad 340 \quad 730$

$09-01-92 \quad 160 \quad 200$

09-01-92 $150 \quad 220$

06-30-92 $140 \quad 190$

$09-02-92 \quad 240 \quad 400$

$09-02-92 \quad 53$

$09-02-92 \quad 38$

$09-03-92$

17

$09-03-92$

31

$09-11-92$

$09-08-92$

$09-08-92$

09-08-92

31
17
59
41

13
39
46
27
35
35
22
18
19
17
17
28
56
54
68

$\begin{array}{ccccl} & & \text { BICAR- } & \text { ALKA- } & \\ \text { SODIUM } & \text { POTAS- } & \text { BONATE } & \text { LINITY } & \\ \text { AD- } & \text { SIUM, } & \text { WATER } & \text { WAT WH } & \text { SULFATE } \\ \text { SORP- } & \text { DIS- } & \text { WH IT } & \text { TOT FET } & \text { DIS- } \\ \text { TION } & \text { SOLVED } & \text { FIELD } & \text { FIELD } & \text { SOLVED } \\ \text { RATIO } & (M G / L & (M G / L \text { AS } & (\text { MG /L AS } & \text { (MG /L } \\ & \text { AS K) } & \text { HCO3) } & \text { CACO3) } & \text { AS SO4) }\end{array}$

$\begin{array}{ccc}-- & -- & - \\ 50 & 29 & - \\ -- & -- & - \\ -- & -- & - \\ - & -- & -\end{array}$

114

114

$92 \quad 2400$

$--$

$\begin{array}{lll}- & - & -\end{array}$

$\begin{array}{lllll}4 & 1.4 & 195 & 160 & 97\end{array}$

$2 \quad \begin{array}{llll}1.5 & 381 & 310 & 160\end{array}$

$\begin{array}{lllll}0.6 & 1.5 & 381 & 307 & 8.4\end{array}$

$\begin{array}{lllll}0.6 & 1.1 & 488 & 394 & 36\end{array}$

$\begin{array}{rrr}488 & 394 & 36 \\ -\quad 112 & 49\end{array}$

$86 \quad 71 \quad 72$

$400 \quad 326.170$

$\begin{array}{llll}4.1 & 383 & 309 & 150\end{array}$

$11, \quad--\quad 130 \quad 330$

$\begin{array}{rrrr}5.4 & 556 & 448 & 620 \\ 5.0 & -- & 404 & 2200\end{array}$

$3.1 \quad--\quad 506 \quad 2000$

$\begin{array}{llll}4.8 & -- & 462 & 990\end{array}$

$6.3 \quad--\quad 441 \quad 1200$

$\begin{array}{llll}4.4 & - & 354 & 310\end{array}$

$32 \quad 544 \quad 438 \quad 420$

$19 \quad--\quad 508 \quad 750$

$\begin{array}{llll}60 & 644 & 522 & 990\end{array}$

$\begin{array}{llllll}26 & 2 & 11 & 650 & 529 & 580\end{array}$

$\begin{array}{llll}1.9 & 417 & 339 & 170\end{array}$

$5.3 \quad--\quad 402 \quad 2300$

$5.7 \quad-3 \quad 300 \quad 1000$

$12 \quad \ldots \quad 404 \quad 990$

$\begin{array}{llll}7.0 & 513 & 416 \quad 760\end{array}$

$0.90 \quad--\quad 420 \quad 1300$

$2.8 \quad--\quad 278 \quad 140$

$\begin{array}{rrrr}18 & - & 250 & 120 \\ 3.0 & 107 & 87 & 37\end{array}$

$\begin{array}{llll}5.1 & 234 & 190 & 120\end{array}$

$2.6 \quad 146 \quad 120 \quad 51$

$\begin{array}{rrrr}2.8 & 103 & 85 & 87\end{array}$

$\begin{array}{llll}4.0 & 515 & 418 & 260\end{array}$

$\begin{array}{llll}3.7 & 342 & 278 & 120\end{array}$ 


\begin{tabular}{|c|c|c|c|c|c|c|c|c|c|}
\hline $\begin{array}{l}\text { LOCAL } \\
\text { NUMBER }\end{array}$ & DATE & $\begin{array}{l}\text { CHLO- } \\
\text { RIDE, } \\
\text { DIS- } \\
\text { SOLVED } \\
\text { (MG/L } \\
\text { AS CL) }\end{array}$ & $\begin{array}{l}\text { FLUO- } \\
\text { RIDE, } \\
\text { DIS- } \\
\text { SOLVED } \\
\text { (MG/L } \\
\text { AS F) }\end{array}$ & $\begin{array}{l}\text { SILICA, } \\
\text { DIS- } \\
\text { SOLVED } \\
\text { (MG/L } \\
\text { AS } \\
\text { SIO2) }\end{array}$ & $\begin{array}{l}\text { SOLIDS, } \\
\text { RESIDUE } \\
\text { AT } 180 \\
\text { DEG. C } \\
\text { DIS- } \\
\text { SOLVED } \\
(\mathrm{MG} / \mathrm{L})\end{array}$ & $\begin{array}{c}\text { SOLIDS, } \\
\text { SUM OF } \\
\text { CONSTI- } \\
\text { TUENTS, } \\
\text { DIS- } \\
\text { SOLVED } \\
\text { (MG/L) }\end{array}$ & $\begin{array}{c}\text { NITRO- } \\
\text { GEN, } \\
\text { NITRATE } \\
\text { DIS- } \\
\text { SOLVED } \\
\text { (MG /L } \\
\text { AS NO3) }\end{array}$ & $\begin{array}{c}\text { NITRO- } \\
\text { GEN, } \\
\text { NITRATE } \\
\text { DIS- } \\
\text { SOLVED } \\
\text { (MG/L } \\
\text { AS N) }\end{array}$ & $\begin{array}{c}\text { NITRO- } \\
\text { GEN, } \\
\text { NITRITE } \\
\text { DIS- } \\
\text { SOLVED } \\
\text { (MG/L } \\
\text { AS N) }\end{array}$ \\
\hline $004 \mathrm{~N} 027 \mathrm{~W} 23 \mathrm{~F} 08 \mathrm{~S}$ & $\begin{array}{l}06-03-92 \\
06-25-92 \\
08-18-92\end{array}$ & $\begin{array}{l}17000 \\
17000 \\
17000\end{array}$ & $\begin{array}{l}-- \\
2.2 \\
--\end{array}$ & 30 & $\begin{array}{l}-- \\
--\end{array}$ & $\begin{array}{l}-- \\
30100 \\
--\end{array}$ & $\begin{array}{l}-- \\
-- \\
--\end{array}$ & $=$ & $<0.010$ \\
\hline $004 \mathrm{~N} 027 W 23 F 09 \mathrm{~S}$ & $\begin{array}{l}12-11-91 \\
03-31-92\end{array}$ & $\begin{array}{r}170 \\
92\end{array}$ & -- & -- & -- & $\begin{array}{l}-- \\
--\end{array}$ & -- & -- & -- \\
\hline & $\begin{array}{l}06-25-92 \\
08-18-92\end{array}$ & $\begin{array}{l}71 \\
46\end{array}$ & $\begin{array}{l}0.80 \\
--\end{array}$ & 43 & -- & $\begin{array}{l}467 \\
--\end{array}$ & -- & -- & $<0.010$ \\
\hline $\begin{array}{l}004 \mathrm{~N} 027 \mathrm{~W} 23 \mathrm{H} 04 \mathrm{~S} \\
006 \mathrm{~N} 029 \mathrm{~W} 08 \mathrm{PO} 2 \mathrm{~S} \\
007 \mathrm{~N} 030 \mathrm{~W} 33 \mathrm{MO} 1 \mathrm{~S}\end{array}$ & $\begin{array}{l}06-22-92 \\
07-01-92 \\
07-01-92\end{array}$ & $\begin{array}{r}170 \\
32 \\
44\end{array}$ & $\begin{array}{l}0.30 \\
0.30 \\
0.20\end{array}$ & $\begin{array}{l}32 \\
39 \\
41\end{array}$ & $\begin{array}{l}-- \\
356 \\
443\end{array}$ & $\begin{array}{l}812 \\
387 \\
473\end{array}$ & $\begin{array}{l}4.3 \\
-- \\
--\end{array}$ & $\begin{array}{l}0.970 \\
-- \\
--\end{array}$ & $\begin{array}{r}0.030 \\
<0.010 \\
<0.010\end{array}$ \\
\hline $\begin{array}{l}007 N 030 W 34 G 01 S \\
007 N 033 W 19 Q 02 S \\
007 N 033 W 21 N 01 S \\
007 N 034 W 20 K 06 S \\
007 N 034 W 20 L 03 S\end{array}$ & $\begin{array}{l}07-01-92 \\
09-04-92 \\
07-01-92 \\
09-03-92 \\
09-03-92\end{array}$ & $\begin{array}{r}37 \\
100 \\
150 \\
170 \\
160\end{array}$ & $\begin{array}{l}0.20 \\
0.40 \\
0.20 \\
0.20 \\
0.20\end{array}$ & $\begin{array}{l}40 \\
58 \\
51 \\
48 \\
49\end{array}$ & $\begin{array}{l}499 \\
-- \\
495 \\
820 \\
848\end{array}$ & $\begin{array}{l}507 \\
396 \\
469 \\
850 \\
821\end{array}$ & $\begin{array}{l}-- \\
-- \\
-- \\
--\end{array}$ & $\begin{array}{l}-- \\
-- \\
-- \\
--\end{array}$ & $\begin{array}{l}<0.010 \\
<0.010 \\
<0.010 \\
<0.010\end{array}$ \\
\hline $\begin{array}{l}007 \mathrm{~N} 034 \mathrm{~W} 20 \mathrm{PO} 3 \mathrm{~S} \\
007 \mathrm{~N} 034 \mathrm{~W} 27 \mathrm{P0} 5 \mathrm{~S} \\
007 \mathrm{~N} 034 \mathrm{~W} 29 \mathrm{~N} 04 \mathrm{~S}\end{array}$ & $\begin{array}{l}09-04-92 \\
06-30-92 \\
08-27-92 \\
09-01-92 \\
09-01-92\end{array}$ & $\begin{array}{l}200 \\
130 \\
280 \\
410 \\
260\end{array}$ & $\begin{array}{l}0.10 \\
0.40 \\
0.20 \\
0.50 \\
0.40\end{array}$ & $\begin{array}{l}17 \\
36 \\
26 \\
25 \\
26\end{array}$ & $\begin{array}{l}-- \\
1560 \\
-- \\
--\end{array}$ & $\begin{array}{r}903 \\
1500 \\
3750 \\
3770 \\
2180\end{array}$ & $\begin{array}{l}-- \\
-- \\
-- \\
1.7 \\
--\end{array}$ & $\begin{array}{l}-- \\
-- \\
-- \\
0.390 \\
--\end{array}$ & $\begin{array}{r}<-- \\
<0.010 \\
<0.010 \\
0.020 \\
<0.010\end{array}$ \\
\hline $\begin{array}{l}007 N 034 W 29 N 06 S \\
007 N 034 W 29 N 07 S \\
007 N 035 W 17 K 20 S \\
007 N 035 W 17 K 21 S \\
007 N 035 W 17 Q 06 S\end{array}$ & $\begin{array}{l}09-01-92 \\
09-01-92 \\
08-31-92 \\
08-31-92 \\
08-31-92\end{array}$ & $\begin{array}{r}250 \\
110 \\
1500 \\
1900 \\
3500\end{array}$ & $\begin{array}{l}0.30 \\
0.20 \\
0.30 \\
0.40 \\
2.6\end{array}$ & $\begin{array}{l}36 \\
36 \\
34 \\
36 \\
34\end{array}$ & $\begin{array}{l}-- \\
-- \\
3570 \\
-- \\
8140\end{array}$ & $\begin{array}{r}2480 \\
995 \\
3380 \\
4470 \\
7610\end{array}$ & $\begin{array}{l}-- \\
-- \\
-- \\
--\end{array}$ & $\begin{array}{l}-- \\
-- \\
-- \\
--\end{array}$ & $\begin{array}{c}-- \\
<0.010 \\
<0.010\end{array}$ \\
\hline $\begin{array}{l}007 \mathrm{~N} 035 \mathrm{~W} 21 \mathrm{GO} 2 \mathrm{~S} \\
007 \mathrm{~N} 035 \mathrm{~W} 23 \mathrm{~B} 02 \mathrm{~S} \\
007 \mathrm{~N} 035 \mathrm{~W} 23 \mathrm{Q} 02 \mathrm{~S} \\
007 \mathrm{~N} 035 \mathrm{~W} 23 \mathrm{Q} 03 \mathrm{~S} \\
007 \mathrm{~N} 035 \mathrm{~W} 23 \mathrm{Q} 04 \mathrm{~S}\end{array}$ & $\begin{array}{l}09-02-92 \\
09-02-92 \\
09-01-92 \\
09-01-92 \\
09-01-92\end{array}$ & $\begin{array}{l}430 \\
180 \\
630 \\
520 \\
400\end{array}$ & $\begin{array}{l}0.30 \\
0.50 \\
0.80 \\
0.30 \\
0.40\end{array}$ & $\begin{array}{l}38 \\
26 \\
20 \\
26 \\
39\end{array}$ & $\begin{array}{l}2020 \\
696 \\
-- \\
-- \\
--\end{array}$ & $\begin{array}{r}1990 \\
823 \\
4810 \\
2430 \\
2370\end{array}$ & $\begin{array}{c}-- \\
240 \\
-- \\
--\end{array}$ & $\begin{array}{r}-- \\
-- \\
53.5 \\
-- \\
--\end{array}$ & $\begin{array}{r}<0.010 \\
<0.010 \\
0.500 \\
<0.010 \\
<0.010\end{array}$ \\
\hline $\begin{array}{l}007 N 035 W 26 F 05 S \\
007 N 035 W 26 L 01 S \\
007 N 035 W 26 L 02 S \\
007 N 035 W 26 L 04 S \\
007 N 035 W 33 J 02 S\end{array}$ & $\begin{array}{l}06-30-92 \\
09-02-92 \\
09-02-92 \\
09-02-92 \\
09-03-92\end{array}$ & $\begin{array}{l}340 \\
390 \\
170 \\
130 \\
150\end{array}$ & $\begin{array}{r}0.30 \\
0.70 \\
0.10 \\
<0.10 \\
0.30\end{array}$ & $\begin{array}{l}39 \\
25 \\
37 \\
51 \\
33\end{array}$ & $\begin{array}{l}2230 \\
-- \\
-- \\
-- \\
396\end{array}$ & $\begin{array}{r}2000 \\
2970 \\
746 \\
658 \\
413\end{array}$ & $\begin{array}{c}-- \\
160 \\
-- \\
--\end{array}$ & $\begin{array}{r}-- \\
36.6 \\
-- \\
--\end{array}$ & $\begin{array}{r}<0.010 \\
0.390 \\
<0.010 \\
-- \\
<0.010\end{array}$ \\
\hline $\begin{array}{l}007 N 035 W 33 J 04 S \\
008 N 032 W 27 P 02 S \\
008 N 032 W 29 L 03 S \\
008 N 033 W 19 G 02 S \\
008 N 033 W 22 E 01 S\end{array}$ & $\begin{array}{l}09-03-92 \\
09-11-92 \\
09-08-92 \\
09-08-92 \\
09-08-92\end{array}$ & $\begin{array}{r}190 \\
73 \\
64 \\
140 \\
200\end{array}$ & $\begin{array}{l}0.20 \\
0.20 \\
0.20 \\
0.20 \\
0.20\end{array}$ & $\begin{array}{l}50 \\
39 \\
53 \\
40 \\
49\end{array}$ & $\begin{array}{l}684 \\
313 \\
337 \\
988 \\
882\end{array}$ & $\begin{array}{r}691 \\
346 \\
371 \\
1030 \\
912\end{array}$ & $\begin{array}{l}-- \\
-- \\
-- \\
--\end{array}$ & $\begin{array}{l}-- \\
-- \\
-- \\
--\end{array}$ & $\begin{array}{l}<0.010 \\
<0.010 \\
<0.010 \\
<0.010 \\
<0.010\end{array}$ \\
\hline
\end{tabular}


INORGANIC DATA

WATER YEAR OCTOBER 1991 TO SEPTEMBER 1992

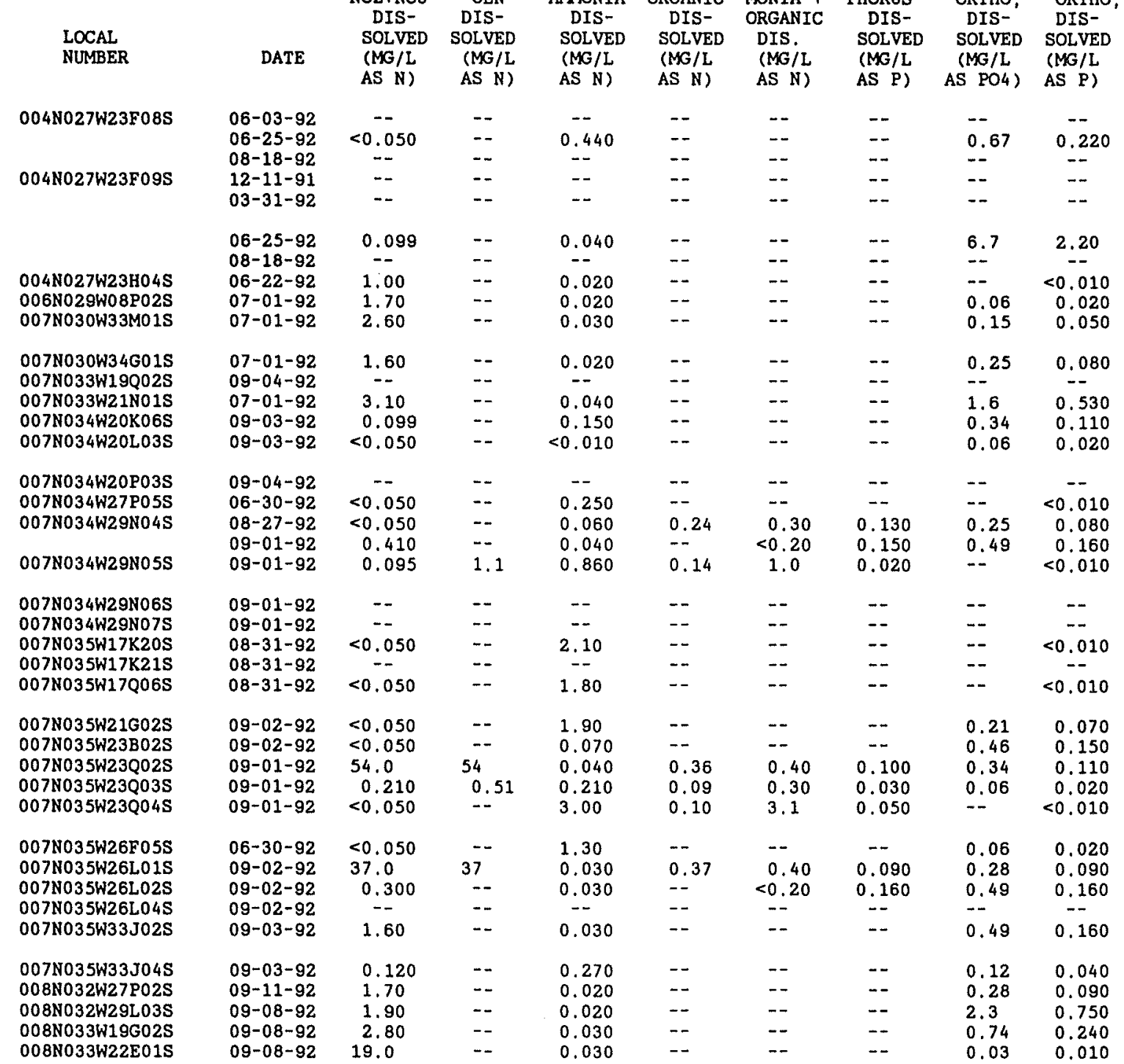

< Actual value is less than value shown. 
LOCAL

NUMBER

004N027W23F08S

004N027W23F09S

004N027W23H04S 006N029W08P02S 007N030W33M01S

$007 N 030$ W34G01S 007N033W19Q02S 007N033W21N01S 007N034W20K06S 007N034W2OLO3S

007N034W20P03S $007 N 034 W 27 \mathrm{P0} 5 \mathrm{~S}$ $007 N 034 W 29 N 04 S$

007N034W29N05S

007N034W29N06S $007 N 034 W 29 N 07 S$ $007 N 035 W 17 K 20 S$ 007 N035W17K21S 007N035W17Q06S

007 N035W21G02S $007 N 035 W 23 B 02 S$ $007 N 035 W 23002 S$ 007N035W23003S $007 N 035 W 23 Q 045$

007N035W26F05S 007N035W26L01S 007N035W26LO2S 007 N035W26L04S 007N035W33J02S

007N035W33J04S 008N032W27 P02S 008 N032W29L03S 008 N033W19G02S 008N033W22E01S

\section{DATE}

$06-03-92$
$06-25-92$
$08-18-92$
$12-11-91$

03-31-92

06-25-92

08-18-92

$06-22-92$

07-01-92

07-01-92

$07-01-92$

09-04-92

07-01-92

09-03-92

09-03-92

09-04-92

06-30-92

08-27-92

09-01-92

09-01-92

09-01-92

09-01-92

08-31-92

08-31-92

08-31-92

09-02-92

$09-02-92$

09-01-92

09-01-92

09-01-92

06-30-92

$09-02-92$

09-02-92

09-02-92

09-03-92

09-03-92

09-11-92

09-08-92

09-08-92

08-08-92

$\begin{array}{lc}\text { BORON, } & \text { CADMIUM } \\ \text { DIS- } & \text { DIS- } \\ \text { SOLVED } & \text { SOLVED } \\ \text { (UG/L } & \text { (UG/L } \\ \text { AS B) } & \text { AS CD) }\end{array}$

CHROSOLVED SOLVED SOLVED SOLVED SOLVED SOLVED (UG/L (UG/L (UG/L (UG/L (UG/L (UG/L AS (R) AS (O) AS CU) AS FE) AS PB) AS MN)

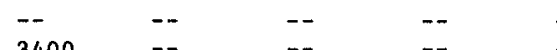

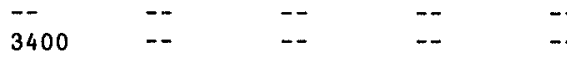

$\begin{array}{lllll}-- & -- & -- & - & - \\ -2 & -2 & - & -\end{array}$

$\begin{array}{lll}-- & -- & --\end{array}$

$--$

$--$

$\begin{aligned} 60 & - \\ -- & - \\ 160 & - \\ 50 & -- \\ 100 & --\end{aligned}$

$--$

$-$

$--$

--
--

--
--
--
--

$--$

130
70

70

180

$--$

$-$

$--$

270

$--$

$--$

$--$

410
560

1100

1200

550

700
490

510

400
1800

$--$

$--$

$--$

--

$-$

$--\quad--$

$\begin{array}{llll}- & - & - & -\end{array}$

$\begin{array}{lll}-2 & -1 \\ -- & - & -\end{array}$

$--$

$--$

$--$

340
280

290
2600

470
590

$--$

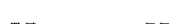

$--$

$--$

$-$

380
1100

1100

120
140

140
70

$-$

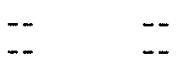

$--$

$-$

$\begin{array}{llll}-- & - & - & - \\ -- & -- & -- & -\end{array}$

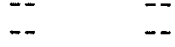

$<1.0$

10

$-$

$--$

60
120

90

440

$--$

$\begin{array}{ll}-- & - \\ -- & - \\ -\infty & -\end{array}$

--
--
--

$\begin{array}{ccc}-- & -- & - \\ -- & -- & 3000 \\ -- & -- & -- \\ -- & -- & --\end{array}$

$280 \quad--\quad 81$

$360 \quad--\quad 1300$

$<3 \quad--\quad<1$

$\begin{array}{rrr}3 & -- & <1 \\ 1100 & -- & 80 \\ 39 & -- & 2\end{array}$

$\begin{array}{rrr}260 & -- & --\end{array}$

$72 \quad--\quad 150$

$1600 \quad \cdots \quad 900$

$\begin{array}{rrr}30 & -- & 2400 \\ 10 & -- & 520\end{array}$

$760 \quad-\quad 2300$

$5500 \quad--\quad 1800$

59 -. $\quad 77$

$\begin{array}{rll}6500 & -- & -- \\ 6100 & -- & 2100\end{array}$

$20 \quad \ldots \quad \ldots$

$2900 \quad--\quad--$

$\begin{array}{rrr}380 & -- & - \\ 10 & - & 440\end{array}$

$780 \quad--\quad 800$

$2700 \quad--\quad 520$

$2400 \quad--\quad 1200$

$10 \quad--\quad 1900$

$\begin{array}{rrr}10 & -- & 1700 \\ 7 & - & 32\end{array}$

$140 \quad-1.0 \quad \ldots<\ldots<\ldots$

< Actual value is less than value shown. 
INORGANIC DATA

WATER YEAR OCTOBER 1991 TO SEPTEMBER 1992

LOCAL

NUMBER

004 N027W23F08S

004 N027W23F09S

$004 \mathrm{~N} 027 \mathrm{~W} 23 \mathrm{H} 04 \mathrm{~S}$ 006 N029W08P02S 007 N030W33M015

007 N030W34G01S $007 \mathrm{~N} 033 \mathrm{~W} 19 \mathrm{Q} 02 \mathrm{~S}$ 007 N033W21N01S 007 N034W20K06S 007N034W20L03S

007 N034W20P03S $007 N 034 \mathrm{~W} 27 \mathrm{PO} 5 \mathrm{~S}$ 007 N034W29N04S

007 N034W29N05S

007N034W29N06S 007 N034W29N07S 007 N035W17K20S 007 N035W17K21S $007 N 035 W 170065$

$007 \mathrm{~N} 035 \mathrm{~W} 21 \mathrm{G} 02 \mathrm{~S}$ 007 N035W23B02S $007 N 035 W 230025$ $007 N 035 \mathrm{~W} 23 \mathrm{Q} 03 \mathrm{~S}$ 007 N035W23004S

007N035W26F05S 007N035W26L.01S $007 \mathrm{~N} 035 \mathrm{~W} 26 \mathrm{~L} 02 \mathrm{~S}$ 007 N035W26L04S 007 N035W33J02S

007 N035W33J04S 008 N032W27P02S $008 \mathrm{~N} 032 \mathrm{~W} 2 \mathrm{LL} 03 \mathrm{~S}$ 008 N033W19G02S 008 N033W22E01S

\begin{tabular}{|c|c|c|c|c|c|c|c|c|}
\hline DATE & $\begin{array}{c}\text { MERCURY } \\
\text { DIS- } \\
\text { SOLVED } \\
\text { (UG/L } \\
\text { AS HG) }\end{array}$ & $\begin{array}{l}\text { MOLYB- } \\
\text { DENUM, } \\
\text { DIS- } \\
\text { SOLVED } \\
\text { (UG/L } \\
\text { AS MO) }\end{array}$ & $\begin{array}{l}\text { NICKEL, } \\
\text { DIS- } \\
\text { SOLVED } \\
\text { (UG/L } \\
\text { AS NI) }\end{array}$ & $\begin{array}{c}\text { STRON- } \\
\text { TIUM, } \\
\text { DIS- } \\
\text { SOLVED } \\
\text { (UG/L } \\
\text { AS SR) }\end{array}$ & $\begin{array}{l}\text { VANA- } \\
\text { DIUM, } \\
\text { DIS- } \\
\text { SOLVED } \\
\text { (UG/L } \\
\text { AS Y) }\end{array}$ & $\begin{array}{l}\text { ZINC, } \\
\text { DIS- } \\
\text { SOLVED } \\
\text { (UG/L } \\
\text { AS ZN) }\end{array}$ & $\begin{array}{c}\text { LITHIUM } \\
\text { DIS- } \\
\text { SOLVED } \\
\text { (UG/L } \\
\text { AS LI) }\end{array}$ & $\begin{array}{l}\text { SELE- } \\
\text { NIUM, } \\
\text { DIS- } \\
\text { SOLVED } \\
\text { (UG/L } \\
\text { AS SE) }\end{array}$ \\
\hline
\end{tabular}

\begin{tabular}{|c|c|c|c|c|c|c|c|c|}
\hline $06-03-92$ & $\cdots$ & -- & - & -- & -- & -- & -- & -- \\
\hline $06-25-92$ & -- & - & -- & -- & -- & -- & -- & -- \\
\hline $08-18-92$ & -- & -- & -- & -- & -- & -- & -- & -. \\
\hline $12-11-91$ & -- & - & -- & -- & - & $\ldots$ & -- & -- \\
\hline $03-31-92$ & -- & -- & -- & -- & -- & -- & -- & -- \\
\hline $06-25-92$ & -- & -- & -- & -- & -- & $=$ & -- & -- \\
\hline $08-18-92$ & -- & -- & -- & -- & -- & $-\infty$ & -- & - \\
\hline $06-22-92$ & -- & -- & -- & -- & - & -- & -- & -- \\
\hline $07-01-92$ & -- & -- & -- & -- & - & -- & -- & -- \\
\hline $07-01-92$ & -- & -- & -- & -- & -- & -- & -- & -- \\
\hline $07-01-92$ & $\cdots$ & -- & -- & -- & -- & -- & -- & -- \\
\hline $09-04-92$ & -- & -- & -- & -- & -- & -- & -- & - \\
\hline $07-01-92$ & -- & -- & -- & -- & -- & -- & -- & -- \\
\hline $09-03-92$ & $\cdots$ & -- & - & -- & -- & -- & -- & -- \\
\hline $09-03-92$ & -- & $\cdots$ & -- & -- & -- & -- & -- & -- \\
\hline $09-04-92$ & - & -- & -- & -- & - & -- & - & -- \\
\hline $06-30-92$ & -- & -- & - & - & -- & -- & $-\cdots$ & -- \\
\hline $08-27-92$ & -- & -- & -- & -- & -- & -- & -- & -- \\
\hline $09-01-92$ & $\cdots$ & -- & -- & -- & -- & -- & -- & -- \\
\hline $09-01-92$ & -- & -- & -- & -- & -- & $=-$ & -- & -- \\
\hline $09-01-92$ & -. & -- & -. & -- & -- & -- & -- & -- \\
\hline $09-01-92$ & -- & -- & - & -- & -- & -- & -- & - \\
\hline $08-31-92$ & -- & - & -- & -- & -- & -- & -- & - \\
\hline $08-31-92$ & -- & -- & -- & -- & -- & -- & -- & - \\
\hline $08-31-92$ & - & -- & -- & -- & -- & -- & -- & -- \\
\hline $09-02-92$ & -- & -- & -- & -- & - - & -- & -- & -. \\
\hline $09-02-92$ & - & -- & -- & -- & - & - & -- & -- \\
\hline $09-01-92$ & -- & - & -- & -- & -- & -- & -- & -- \\
\hline $09-01-92$ & -- & -- & -- & -- & $\cdots$ & -- & -. & -- \\
\hline $09-01-92$ & -- & -- & -- & -- & -- & -- & -- & -- \\
\hline $06-30-92$ & -- & -- & -. & -- & -. & -- & $\ldots$ & -- \\
\hline $09-02-92$ & - & -- & -- & -- & -- & -- & -. & -- \\
\hline $09-02-92$ & -- & -- & -- & -- & - & -- & - & -- \\
\hline $09-02-92$ & -- & - & -- & -- & -- & -- & -. & -- \\
\hline $09-03-92$ & $<0.1$ & 9 & 1 & 100 & 4 & 6 & 7 & 3 \\
\hline $09-03-92$ & -- & -- & -- & -- & -. & -- & -- & -. \\
\hline $09-11-92$ & -- & -- & -- & -- & -- & -- &.- & -- \\
\hline $09-08-92$ & -- & -- & -- & -- & -- & -- & -- & -. \\
\hline $09-08-92$ & $<0.1$ & 41 & 2 & 650 & 6 & 28 & 85 & 20 \\
\hline $09-08-92$ & -- & -- & -- & -- & - & -- & -- & -- \\
\hline
\end{tabular}

< Actual value is less than value shown. 
INORGANIC DATA

WATER YEAR OCTOBER 1991 TO SEPTEMBER 1992

\begin{tabular}{|c|c|c|c|c|c|c|c|c|c|c|}
\hline $\begin{array}{l}\text { LOCAL } \\
\text { NUMBER }\end{array}$ & DATE & TIME & $\begin{array}{l}\text { DEPTH } \\
\text { BELOW } \\
\text { LAND } \\
\text { SURFACE } \\
\text { (WATER } \\
\text { LEVEL) } \\
\text { (FEET) }\end{array}$ & $\begin{array}{l}\text { DEPTH } \\
\text { OF } \\
\text { WELL, } \\
\text { TOTAL } \\
\text { (FEET) }\end{array}$ & $\begin{array}{l}\text { SPE- } \\
\text { CIFIC } \\
\text { CON- } \\
\text { DUCT- } \\
\text { ANCE } \\
\text { (US/CM) }\end{array}$ & $\begin{array}{l}\text { PH } \\
\text { WATER } \\
\text { WHOLE } \\
\text { FIELD } \\
\text { (STAND- } \\
\text { ARD } \\
\text { UNITS) }\end{array}$ & $\begin{array}{l}\text { TEMPER- } \\
\text { ATURE } \\
\text { WATER } \\
\text { (DEG C) }\end{array}$ & $\begin{array}{l}\text { HARD- } \\
\text { NESS } \\
\text { TOTAL } \\
\text { (MG/L } \\
\text { AS } \\
\text { CACO3) }\end{array}$ & $\begin{array}{l}\text { CALCIUM } \\
\text { DIS- } \\
\text { SOLVED } \\
\text { (MG/L } \\
\text { AS CA) }\end{array}$ & $\begin{array}{l}\text { MAGNE- } \\
\text { SIUM, } \\
\text { DIS- } \\
\text { SOLVED } \\
\text { (MG/L } \\
\text { AS MG) }\end{array}$ \\
\hline $\begin{array}{l}\text { 008N034W02M01S } \\
008 N 034 W 16 C 05 S \\
008 N 035 W 12 \mathrm{M} 01 \mathrm{~S} \\
009 \mathrm{~N} 024 \mathrm{~W} 3 \mathrm{MM} 01 \mathrm{~S} \\
010 \mathrm{~N} 026 \mathrm{~W} 09 \mathrm{R} 03 \mathrm{~S}\end{array}$ & $\begin{array}{l}09-09-92 \\
09-09-92 \\
09-02-92 \\
06-29-92 \\
07-02-92\end{array}$ & $\begin{array}{l}0900 \\
1050 \\
1605 \\
1025 \\
0920\end{array}$ & $\begin{array}{c}129.14 \\
-- \\
15.43 \\
-- \\
--\end{array}$ & $\begin{array}{l}-- \\
334 \\
100 \\
233 \\
648\end{array}$ & $\begin{array}{r}822 \\
713 \\
5650 \\
1010 \\
2000\end{array}$ & $\begin{array}{l}7.4 \\
7.4 \\
7.1 \\
7.9 \\
7.5\end{array}$ & $\begin{array}{l}21.0 \\
20.5 \\
18.5 \\
21.0 \\
19.0\end{array}$ & $\begin{array}{r}220 \\
210 \\
720 \\
160 \\
1100\end{array}$ & $\begin{array}{r}64 \\
63 \\
150 \\
52 \\
270\end{array}$ & $\begin{array}{l}15 \\
12 \\
83 \\
7.4 \\
93\end{array}$ \\
\hline $\begin{array}{l}010 N 026 \mathrm{~W} 21 \mathrm{G} 01 \mathrm{~S} \\
010 \mathrm{~N} 034 \mathrm{~W} 26 \mathrm{H} 02 \mathrm{~S} \\
010 \mathrm{~N} 034 \mathrm{~W} 29 \mathrm{~N} 01 \mathrm{~S} \\
010 \mathrm{~N} 035 \mathrm{~W} 14 \mathrm{D} 03 \mathrm{~S}\end{array}$ & $\begin{array}{l}06-29-92 \\
08-27-92 \\
06-30-92 \\
06-30-92\end{array}$ & $\begin{array}{l}1205 \\
1325 \\
0950 \\
1125\end{array}$ & $m-$ & $\begin{array}{r}372 \\
-- \\
112 \\
--\end{array}$ & $\begin{array}{r}1740 \\
1540 \\
872 \\
1890\end{array}$ & $\begin{array}{l}7.6 \\
7.6 \\
7.7 \\
7.5\end{array}$ & $\begin{array}{l}24.5 \\
18.0 \\
19.0 \\
18.0\end{array}$ & $\begin{array}{l}790 \\
640 \\
390 \\
800\end{array}$ & $\begin{array}{r}220 \\
140 \\
89 \\
190\end{array}$ & $\begin{array}{l}58 \\
70 \\
41 \\
80\end{array}$ \\
\hline
\end{tabular}

\section{LOCAL \\ NUMBER}

008N034W02M01S $008 \mathrm{~N} 034 \mathrm{~W} 16 \mathrm{C} 05 \mathrm{~S}$ $008 \mathrm{~N} 035 \mathrm{~W} 12 \mathrm{M} 01 \mathrm{~S}$ 009 N024W33M01S 010NO26W09R03S

$010 \mathrm{~N} 026 \mathrm{~W} 21 \mathrm{G} 01 \mathrm{~S}$ $010 \mathrm{NO} 34 \mathrm{~W} 26 \mathrm{HO} 02 \mathrm{~S}$ 010 N034W29N01S 010N035W14D03S
LOCAL

NUMBER

008N034W02M01S $008 N 034 \mathrm{~W} 16 \mathrm{C} 05 \mathrm{~S}$ 008N035W12M01S 009N024W33M01S 010N026W09R03S

010 N026W21G01S $010 \mathrm{~N} 034 \mathrm{~W} 26 \mathrm{H} 02 \mathrm{~S}$ 010N034W29N01S 010 N035W14D03S
BICAR- ALKA-

\begin{tabular}{cccccll} 
& \multicolumn{1}{c}{ BICAR- } & ALKA- & & & \\
SODIUM & POTAS- & BONATE & LINITY & & CHLO- & FLUO- \\
AD- & SIUM, & WATER & WAT WH & SULFATE & RIDE, & RIDE, \\
SORP- & DIS- & WH IT & TOT FET & DIS- & DIS- & DIS- \\
TION & SOLVED & FIELD & FIELD & SOLVED & SOLVED & SOLVED \\
RATIO & $(M G / L$ & $(M G / L A S$ & $(M G / L$ AS & (MG/L & (MG/L & (MG/L \\
& AS K) & HCO3) & CACO3) & AS SO4) & AS CL) & AS F)
\end{tabular}

$\begin{array}{rr}09-09-92 & 88 \\ 09-09-92 & 60 \\ 09-02-92 & 1100 \\ 06-29-92 & 150 \\ 07-02-92 & 96 \\ & \\ 06-29-92 & 120 \\ 08-27-92 & 87 \\ 06-30-92 & 44 \\ 06-30-92 & 120\end{array}$

$\begin{array}{rr}46 & 3 \\ 38 & 2 \\ 76 & 18 \\ 67 & 5 \\ 16 & 1 \\ & \\ 25 & 2 \\ 23 & 1 \\ 20 & 1 \\ 24 & 2\end{array}$

185
205
1680
268
190
160
283
--
327

$\begin{array}{rc}150 & 92 \\ 166 & 76 \\ 1350 & -- \\ 217 & 170 \\ 153 & 1100 \\ & \\ 130 & 840 \\ 228 & 380 \\ 205 & 270 \\ 264 & 490\end{array}$

120

0.30

0.20

0.30

0.80
0.50

2.1

1300

1300
80
23

$\begin{array}{cc}5.2 & 160 \\ 3.4 & 283 \\ 2.6 & -- \\ 4.3 & 327\end{array}$

14
110

0.20

0.40

0.20

0.30

SOLIDS, SOLIDS, NITRO- NITRO- NITRO- NITRO- NITRO- PHOS-

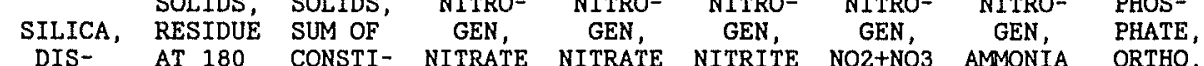
DIS- AT 180 CONSTI- NITRATE NITRATE NITRITE NO2+NO3 AMMONIA ORTHO,

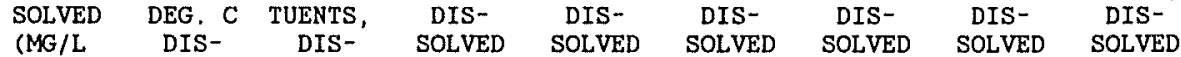
AS SOLVED SOLVED (MG/L (MG/L (MG/L (MG/L) (MG/L (MG/L SIO2) (MG/L) (MG/L) AS NO3) AS N) AS N) AS N) AS N) AS PO4)

\begin{tabular}{|c|c|c|c|c|c|c|c|c|c|}
\hline $\begin{array}{l}09-09-92 \\
09-09-92 \\
09-02-92 \\
06-29-92 \\
07-02-92\end{array}$ & $\begin{array}{l}52 \\
44 \\
33 \\
26 \\
22\end{array}$ & $\begin{array}{r}516 \\
438 \\
3330 \\
629 \\
1860\end{array}$ & $\begin{array}{r}528 \\
446 \\
-- \\
622 \\
1710\end{array}$ & $\begin{array}{l}-- \\
-- \\
-- \\
-- \\
11\end{array}$ & $\begin{array}{l}-- \\
-- \\
-- \\
-- \\
2.59\end{array}$ & $\begin{array}{r}<0.010 \\
<0.010 \\
<0.010 \\
<0.010 \\
0.010\end{array}$ & $\begin{array}{c}<0.050 \\
<0.050 \\
<0.050 \\
0.270 \\
2.60\end{array}$ & $\begin{array}{l}0.290 \\
0.280 \\
1.30 \\
0.030 \\
0.060\end{array}$ & $\begin{array}{l}0.77 \\
1.3 \\
1.8 \\
0.09 \\
--\end{array}$ \\
\hline $\begin{array}{l}06-29-92 \\
08-27-92 \\
06-30-92 \\
06-30-92\end{array}$ & $\begin{array}{l}37 \\
28 \\
37 \\
29\end{array}$ & $\begin{array}{r}1470 \\
1030 \\
633 \\
1400\end{array}$ & $\begin{array}{r}1370 \\
1020 \\
631 \\
1290\end{array}$ & $\begin{array}{l}1.0 \\
-- \\
-- \\
--\end{array}$ & $\begin{array}{l}0.230 \\
-- \\
-- \\
--\end{array}$ & $\begin{array}{r}0.010 \\
<0.010 \\
<0.010 \\
<0.010\end{array}$ & $\begin{aligned} 0.240 \\
15.0 \\
0.540 \\
13.0\end{aligned}$ & $\begin{array}{r}0.330 \\
<0.010 \\
0.030 \\
0.020\end{array}$ & $\begin{array}{l}-- \\
0.06 \\
-- \\
--\end{array}$ \\
\hline
\end{tabular}

< Actual value is less than value shown. 
INORGANIC DATA

WATER YEAR OCTOBER 1991 TO SEPTEMBER 1992

LOCAL

NUMBER

008 N034W02M01S

$008 \mathrm{~N} 034 \mathrm{~W} 16 \mathrm{CO} 5 \mathrm{~S}$

008 N035W12M01S

009 N024W33M01S

010N026W09R03S

$010 \mathrm{NO} 26 \mathrm{~W} 21 \mathrm{GO} 1 \mathrm{~S}$

$010 \mathrm{NO} 34$ W26HO2S

010N034W29N01S

010N035W14D03S

LOCAL

NUMBER

008N034W02M01S

$008 \mathrm{~N} 034 \mathrm{~W} 16 \mathrm{CO} 5 \mathrm{~S}$

$008 \mathrm{~N} 035 \mathrm{~W} 12 \mathrm{M} 01 \mathrm{~S}$

009 N024W33M01S

010N026W09R03S

$010 \mathrm{~N} 026 \mathrm{~W} 21 \mathrm{GO} 1 \mathrm{~S}$

010N034W26H02S

$010 N 034$ W29N01S

010 N035W14D03S
PHOS-

PHORUS

ORTHO,

DIS-

SOLVED

(MG/L

AS P)

09-09-92

$09-09-92$

09-02-92

06-29-92

$07-02-92$

06-29-92

$08-27-92$

$06-30-92$

$06-30-92$

\subsection{0}

0.440

0.590

0.030

$<0.010$

BORON, CADMIUM

DIS-

DIS-

CHRO-

MIUM, COBALT,

DIS-

(UG/L

SOLVED SOLVED

(UG/L (UG/L

AS $(R)$ AS (O)

AS B)

$<0.010$

0.020

$<0.010$

$<0.010$

170
120
9100
370
230

200
190
120
280

--

$<1.0$

$-\infty$

$--$

$--$

$--$

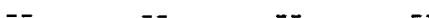

$-$

--

-

\begin{abstract}
A
\end{abstract}

--
--
--
--
--
--
--

$\begin{array}{lll}\text { IRON, } & \text { LEAD, } & \text { MANGA- } \\ \text { NESE, } \\ \text { DIS- } & \text { DIS- } & \text { DIS- } \\ \text { SOLVED } & \text { SOLVED } & \text { SOLVED } \\ \text { (UG/L } & \text { (UG/L } & \text { (UG/L } \\ \text { AS FE) } & \text { AS PB) } & \text { AS MN) }\end{array}$

$\begin{array}{ll}-- & -- \\ -<1 & -- \\ -- & -- \\ -- & 3 \\ -- & <10 \\ -- & 100 \\ -- & <1 \\ -- & 1 \\ -- & <1\end{array}$

$<$ Actual value is less than value shown.

\begin{tabular}{|c|c|c|c|c|c|c|c|c|}
\hline $\begin{array}{l}09-09-92 \\
09-09-92\end{array}$ & $\begin{array}{l}-- \\
<0.1\end{array}$ & 110 & $<1$ & -- & -- & $<3$ & -- & -- \\
\hline $09-02-92$ & -- & $-\infty$ & -- & -- & -- & -- & -- & -- \\
\hline $06-29-92$ & -- & -- & -- & - & -- & -- & -- & -- \\
\hline $07-02-92$ & -- & -- & -- & -- & -- & -- & -- & -- \\
\hline $06-29-92$ & -- & -- & -- & -- & - & -- & -- & -- \\
\hline $08-27-92$ & -- & -- & -- & $\cdots$ & -- & -- & -- & -- \\
\hline $06-30-92$ & -- & -- & -- & -- & -- & -- & -- & -- \\
\hline $06-30-92$ & -- & -- & -- & -- & -- & -- & -- & -- \\
\hline
\end{tabular}



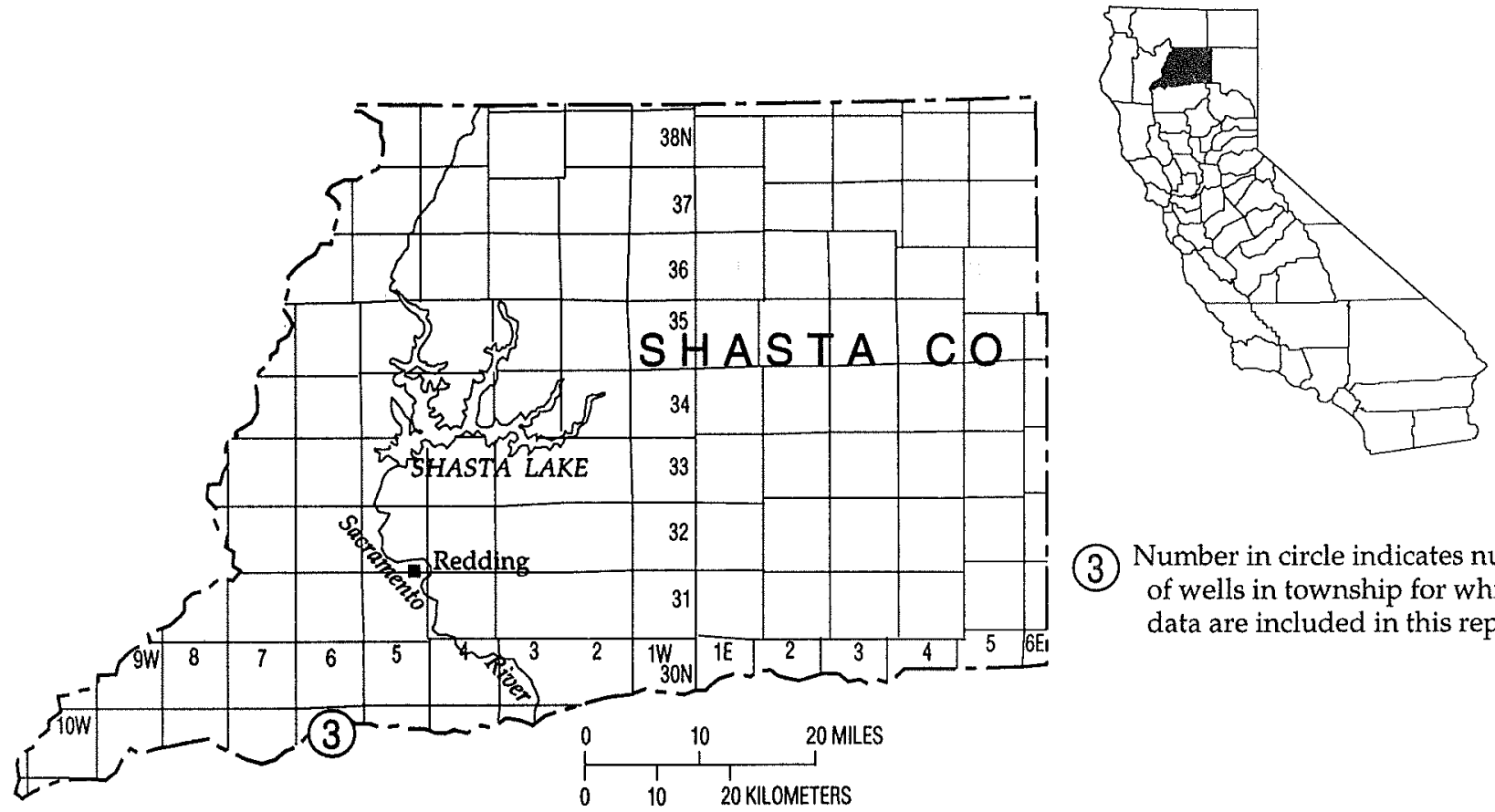

Figure 31. Location of wells in Shasta County.

(3) Number in circle indicates number of wells in township for which data are included in this report 
WATER LEVELS, SHASTA COUNTY

WELL DESCRIPTIONS AND WATER-LEVEL MEASUREMENTS

WATER YEAR OCTOBER 1991 TO SEPTEMBER 1992

SITE NUMBER 402259122295601

LOCAL NUMBER 029N006W12E01M

About 11.6 miles west of Cottonwood. Drilled observation well in Tehama Formation of Pliocene age. Diameter 6 inches, depth 80 feet, perforated $65-75$ feet. Altitude of land-surface datum 520 feet. Water-level records available 1984-85, 1988 to current year.

WATER LEVELS IN FEET BELOW LAND-SURFACE DATUM (READINGS ABOVE LAND SURFACE INDICATED BY "+")

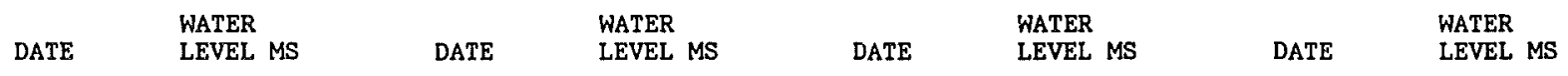

$\begin{array}{lllllllll}\text { OCT 08, 1991 } & \text { F } & \text { JAN 13, } 1992 & \text { F } & \text { APR 10, 1992 } & \text { F } & \text { JUL 07, } 1992 \\ \text { NOV 04 } & \text { F } & \text { FEB 06 } & \text { F } & \text { MAY 11 } & \text { F } & \text { AUG 06 } & \text { F } \\ \text { DEC 13 } & \text { F } & \text { MAR 04 } & \text { JUN 02 } & \text { F } & \text { SEP 01 } & \text { F }\end{array}$

PERIOD OF RECORD HIGHEST +4.53 JUL 21,1989 LOWEST +1.15 JUN 23,1984

SITE NUMBER 402259122295602

LOCAL NUMBER O29N006W12E02M

About 11.6 miles west of Cottonwood. Drilled observation well in alluvium. Diameter 6 inches, depth 40 feet, perforated 25-35 feet. Altitude of land-surface datum 520 feet. Water-level records available 1984-85, 1988 to current year.

WATER LEVELS IN FEET BELOW LAND-SURFACE DATUM

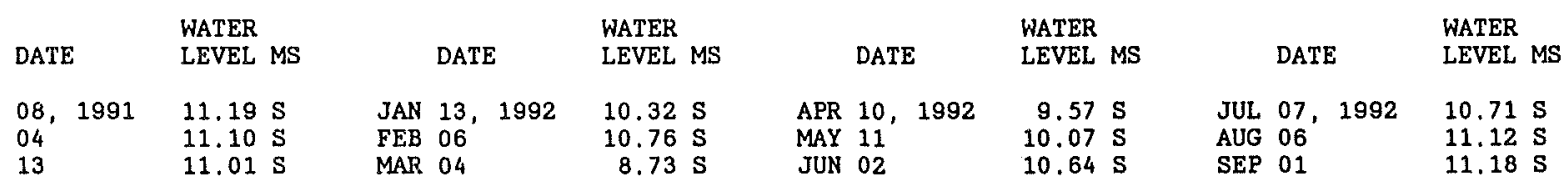

PERIOD OF RECORD HIGHEST 7.47 MAR 20, 1989 LOWEST 11.37 SEP 06, 1991

SITE NUMBER 402300122295601

LOCAL NUMBER 029NO06W12E03M

About 11.6 miles west of Cottonwood. Drilled observation well in alluvium. Diameter 6 inches, depth 40 feet, perforated 25-35 feet. Altitude of land-surface datum 520 feet. Water-level records available 1984-85, 1988 to current year.

WATER LEVELS IN FEET BELOW LAND-SURFACE DATUM

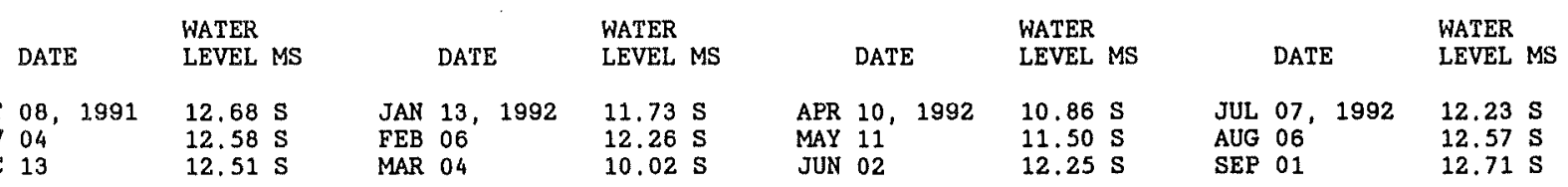

PERIOD OF RECORD HIGHEST 9.17 MAR 20, 1989 LOWEST 12.83 SEP 06, 1991 [B 


\section{SUTTER COUNTY}
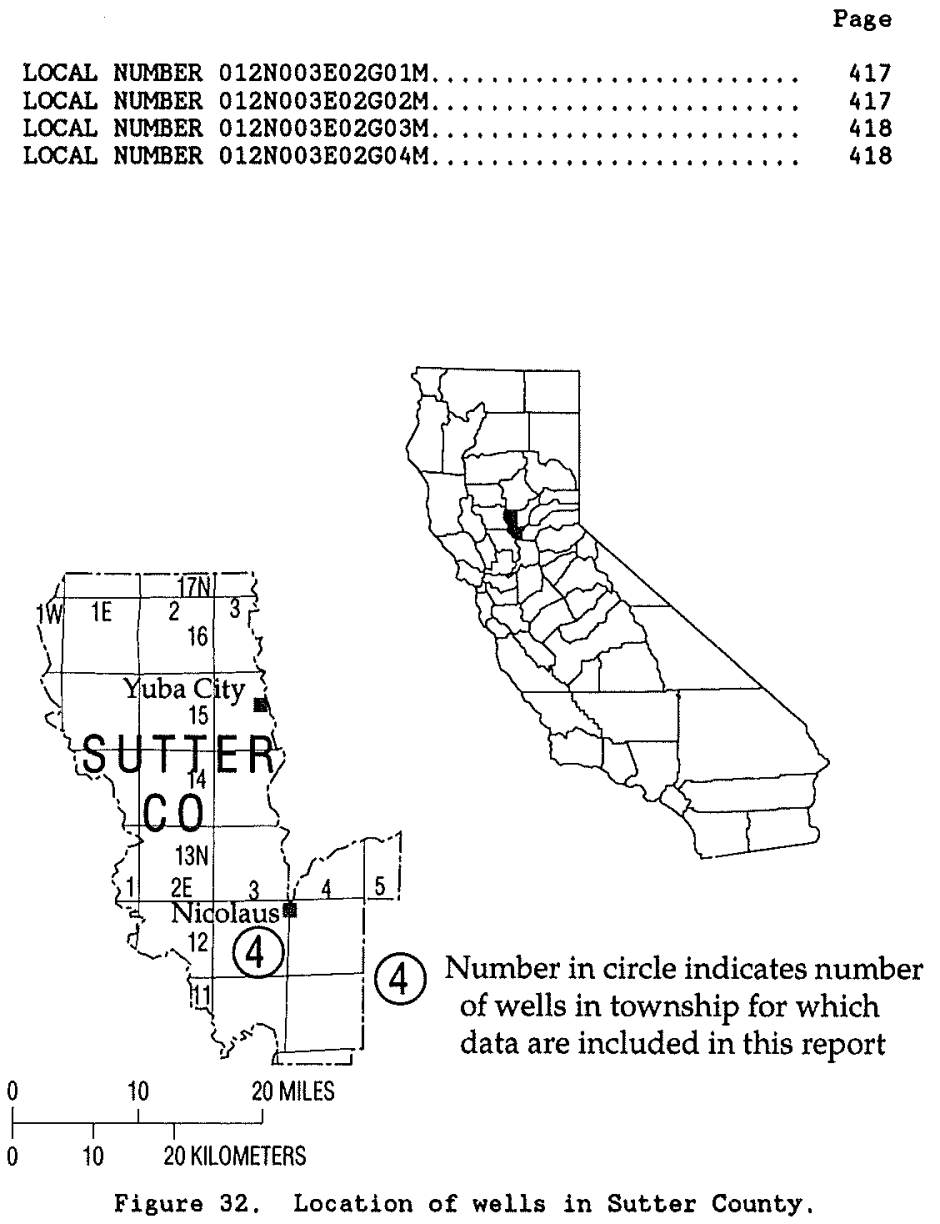
WELI DESCRIPTIONS AND WATER-LEVEL MEASUREMENTS

WATER YEAR OCTOBER 1991 TO SEPTEMBER 1992

SITE NUMBER 385501121361901

LOCAL NUMBER 012N003E02G01M

About 1.7 miles northwest of Nicolaus. Drilled observation water-table well in alluvium. Diameter 3 inches, depth 1,081 feet, screened 1,066-1,071 feet. Alt1tude of land-surface datum 32.54 feet. Recorder installed 1980 to current year. Water-level records avallable 1980 to current year.

WATER LEVELS IN FEET BELOW LAND-SURFACE DATUM

$\begin{array}{lllllll}\text { DATE } & \begin{array}{l}\text { WATER } \\ \text { LEVEL MS }\end{array} & \text { DATE } & \begin{array}{l}\text { WATER } \\ \text { LEVEL MS }\end{array} & \text { DATE } & \text { WATER } \\ \text { LEVEL MS }\end{array}$

PERIOD OF RECORD HIGHEST 11.50 APR $18,1987 \quad$ LOWEST 33.88 SEP 09, 1981

SITE NUMBER 385501121361902

LOCAL NUMBER 012N003E02G02M

About 1.7 miles northwest of Nicolaus. Drilled observation water-table well in alluvium. Diameter 3 inches, depth 721 feet, screened 706-711 feet. Altitude of land-surface datum 32.54 feet. Recorder installed 1980 to current year. Water-level records available 1980 to current year.

Missing record due to malfunction of recording instrument.

WATER LEVELS IN FEET BELOW LAND-SURFACE DATUM (OBSERVATIONS AT 1200 HOURS)

\begin{tabular}{|c|c|c|c|c|c|c|c|c|c|c|c|c|}
\hline DAY & $\mathrm{OCT}$ & NOV & DEC & JAN & FEB & MAR & APR & MAY & JUN & JUL & AUG & SEP \\
\hline $\begin{array}{l}1 \\
2 \\
3 \\
4 \\
5\end{array}$ & $\begin{array}{r}--- \\
18.43 \\
18.36 \\
18.31\end{array}$ & $\begin{array}{l}16.87 \\
16.85 \\
16.85 \\
16.64 \\
16.58\end{array}$ & $\begin{array}{l}15.87 \\
15.82 \\
15.79 \\
15.74 \\
15.66\end{array}$ & $\begin{array}{l}14.87 \\
14.83 \\
14.78 \\
14.78 \\
14.66\end{array}$ & $\begin{array}{l}14.13 \\
14.08 \\
14.06 \\
13.97 \\
13.91\end{array}$ & $\begin{array}{l}12.97 \\
12.96 \\
12.88 \\
12.78 \\
12.75\end{array}$ & $\begin{array}{r}12.21 \\
12.20 \\
\ldots \\
\ldots \\
\ldots\end{array}$ & $\begin{array}{l}12.66 \\
12.69 \\
12.72 \\
12.76 \\
12.76\end{array}$ & $\begin{array}{l}16.28 \\
16.34 \\
16.41 \\
16.47 \\
16.51\end{array}$ & $\begin{array}{l}18.72 \\
18.82 \\
18.85 \\
18.90 \\
18.96\end{array}$ & $\begin{array}{l}20.48 \\
20.55 \\
20.60 \\
20.67 \\
20.77\end{array}$ & $\begin{array}{l}22.00 \\
22.06 \\
22.09 \\
22.16 \\
22.17\end{array}$ \\
\hline $\begin{array}{r}6 \\
7 \\
8 \\
9 \\
10\end{array}$ & $\begin{array}{l}18.25 \\
18.20 \\
18.10 \\
18.05 \\
17.99\end{array}$ & $\begin{array}{l}16.52 \\
16.50 \\
16.45 \\
16.42 \\
16.39\end{array}$ & $\begin{array}{l}15.66 \\
15.67 \\
15.58 \\
15.56 \\
15.60\end{array}$ & $\begin{array}{l}14.68 \\
14.70 \\
14.69 \\
14.67 \\
14.66\end{array}$ & $\begin{array}{l}13.91 \\
13.87 \\
13.77 \\
13.77 \\
13.64\end{array}$ & $\begin{array}{l}12.69 \\
12.71 \\
12.72 \\
12.69 \\
12.64\end{array}$ & $\begin{array}{l}--- \\
--- \\
--- \\
---\end{array}$ & $\begin{array}{l}12.86 \\
12.94 \\
12.98 \\
13.03 \\
13.12\end{array}$ & $\begin{array}{l}16.57 \\
16.72 \\
16.80 \\
16.89 \\
16.97\end{array}$ & $\begin{array}{l}19.02 \\
19.04 \\
19.06 \\
19.08 \\
19.12\end{array}$ & $\begin{array}{l}20.82 \\
20.88 \\
20.92 \\
20.94 \\
21.03\end{array}$ & $\begin{array}{l}22.18 \\
22.19 \\
22.20 \\
22.18 \\
22.15\end{array}$ \\
\hline $\begin{array}{l}11 \\
12 \\
13 \\
14 \\
15\end{array}$ & $\begin{array}{l}17.87 \\
17.84 \\
17.81 \\
17.74 \\
17.65\end{array}$ & $\begin{array}{l}16.30 \\
16.24 \\
16.23 \\
16.30 \\
16.19\end{array}$ & $\begin{array}{l}15.62 \\
15.60 \\
15.55 \\
15.51 \\
15.43\end{array}$ & $\begin{array}{l}14.66 \\
14.66 \\
14.65 \\
14.64 \\
14.52\end{array}$ & $\begin{array}{l}13.59 \\
13.54 \\
13.43 \\
13.41 \\
13.41\end{array}$ & $\begin{array}{l}12.61 \\
12.56 \\
12.51 \\
12.48 \\
12.47\end{array}$ & $\begin{array}{r}--- \\
-\cdots- \\
12.24 \\
12.26\end{array}$ & $\begin{array}{l}13.30 \\
13.42 \\
13.53 \\
13.64 \\
13.77\end{array}$ & $\begin{array}{l}17.12 \\
17.25 \\
17.32 \\
17.35 \\
17.41\end{array}$ & $\begin{array}{l}19.18 \\
19.25 \\
19.31 \\
19.37 \\
19.41\end{array}$ & $\begin{array}{l}21.06 \\
21.11 \\
21.20 \\
21.27 \\
21.30\end{array}$ & $\begin{array}{l}22.13 \\
22.06 \\
21.98 \\
21.87 \\
21.81\end{array}$ \\
\hline $\begin{array}{l}16 \\
17 \\
18 \\
19 \\
20\end{array}$ & $\begin{array}{l}17.58 \\
17.53 \\
17.53 \\
17.44 \\
17.32\end{array}$ & $\begin{array}{l}16.19 \\
16.19 \\
16.17 \\
16.13 \\
16.11\end{array}$ & $\begin{array}{l}15.39 \\
15.41 \\
15.40 \\
15.38 \\
15.35\end{array}$ & $\begin{array}{l}14.48 \\
14.47 \\
14.47 \\
14.49 \\
14.49\end{array}$ & $\begin{array}{l}13.39 \\
13.32 \\
13.29 \\
13.29 \\
13.27\end{array}$ & $\begin{array}{l}12.42 \\
12.38 \\
12.29 \\
12.24 \\
12.25\end{array}$ & $\begin{array}{l}12.27 \\
12.30 \\
12.30 \\
12.30 \\
12.31\end{array}$ & $\begin{array}{l}13.90 \\
14.06 \\
14.25 \\
14.41 \\
14.59\end{array}$ & $\begin{array}{r}17.47 \\
17.50 \\
17.59 \\
17.63 \\
17.69\end{array}$ & $\begin{array}{l}19.47 \\
19.47 \\
19.51 \\
19.51 \\
19.52\end{array}$ & $\begin{array}{l}21.30 \\
21.32 \\
21.44 \\
21.52 \\
21.55\end{array}$ & $\begin{array}{l}21.75 \\
21.66 \\
21.58 \\
21.49 \\
21.37\end{array}$ \\
\hline $\begin{array}{l}21 \\
22 \\
23 \\
24 \\
25\end{array}$ & $\begin{array}{l}17.24 \\
17.22 \\
17.23 \\
17.20 \\
17.19\end{array}$ & $\begin{array}{l}16.12 \\
16.08 \\
16.04 \\
15.96 \\
15.93\end{array}$ & $\begin{array}{l}15.35 \\
15.25 \\
15.22 \\
15.18 \\
15.10\end{array}$ & $\begin{array}{l}14.49 \\
14.48 \\
14.43 \\
14.39 \\
14.39\end{array}$ & $\begin{array}{l}13.22 \\
13.21 \\
13.21 \\
13.21 \\
13.22\end{array}$ & $\begin{array}{l}12.23 \\
12.26 \\
12.23 \\
12.20 \\
12.18\end{array}$ & $\begin{array}{l}12.31 \\
12.38 \\
12.43 \\
12.45 \\
12.42\end{array}$ & $\begin{array}{l}14.77 \\
14.96 \\
15.14 \\
15.30 \\
15.47\end{array}$ & $\begin{array}{r}17.80 \\
- \\
18.11 \\
18.21\end{array}$ & $\begin{array}{l}19.60 \\
19.60 \\
19.73 \\
19.81 \\
19.87\end{array}$ & $\begin{array}{l}21.58 \\
21.62 \\
21.64 \\
21.70 \\
21.78\end{array}$ & $\begin{array}{l}21.27 \\
21.18 \\
21.11 \\
21.01 \\
20.91\end{array}$ \\
\hline $\begin{array}{l}26 \\
27 \\
28 \\
29 \\
30 \\
31\end{array}$ & $\begin{array}{l}17.02 \\
17.06 \\
17.04 \\
16.97 \\
16.97 \\
16.97\end{array}$ & $\begin{array}{r}15.93 \\
15.87 \\
15.88 \\
15.88 \\
15.88 \\
\end{array}$ & $\begin{array}{l}15.05 \\
14.91 \\
14.93 \\
14.95 \\
14.95 \\
14.94\end{array}$ & $\begin{array}{l}14.33 \\
14.32 \\
14.28 \\
14.20 \\
14.15 \\
14.15\end{array}$ & $\begin{array}{r}13.22 \\
13.10 \\
13.03 \\
12.98 \\
\ldots \\
\ldots\end{array}$ & $\begin{array}{l}12.19 \\
12.22 \\
12.23 \\
12.23 \\
12.21 \\
12.20\end{array}$ & $\begin{array}{r}12.44 \\
12.52 \\
12.55 \\
12.58 \\
12.62 \\
\end{array}$ & $\begin{array}{l}15.61 \\
15.74 \\
15.91 \\
15.99 \\
16.10 \\
16.17\end{array}$ & $\begin{array}{r}18.30 \\
18.33 \\
18.40 \\
18.59 \\
-\end{array}$ & $\begin{array}{l}19.90 \\
19.93 \\
20.04 \\
20.21 \\
20.31 \\
20.33\end{array}$ & $\begin{array}{l}21.82 \\
21.87 \\
21.88 \\
21.92 \\
21.98 \\
22.00\end{array}$ & $\begin{array}{r}20.79 \\
20.68 \\
20.58 \\
20.46 \\
20.39 \\
.\end{array}$ \\
\hline
\end{tabular}


WELL DESCRIPTIONS AND WATER-LEVEL MEASUREMENTS

WATER YEAR OCTOBER 1991 TO SEPTEMBER 1992

SITE NUMBER 385501121361903

LOCAL NUMBER 012N003E02G03M

About 1.7 miles northwest of Nicolaus. Drilled observation water-table well in alluvium. Diameter 3 inches, depth 321 feet, screened 306-311 feet. Altitude of land-surface datum 32.54 feet. Recorder installed 1980 to current year. Water-level records available 1980 to current year.

Missing record due to malfunction of recording instrument.

WATER LEVELS IN FEET BELOW LAND-SURFACE DATUM (OBSERVATIONS AT 1200 HOURS)

\begin{tabular}{|c|c|c|c|c|c|c|c|c|c|c|c|c|}
\hline DAY & OC'T & NOV & DEC & JAN & FEB & MAR & APR & MAY & JUN & JUL & AUG & SEP \\
\hline 1 & --- & -- & -- & -- & 11.04 & 8.54 & 8.63 & --- & 11.05 & -- & --- & 13.48 \\
\hline 2 & 511.78 & -- & -- & -- & 11.04 & 8.59 & 8.72 & -- & --- & -- & $-\cdots$ & 13.64 \\
\hline 3 & - & -- & -- & --- & 11.07 & 8.51 & -- & --- & -- & $\cdots$ & --- & $\ldots$ \\
\hline 4 & -- & -- & --- & --- & 11.02 & 8.55 & -- & --- & -- & --- & --- & -- \\
\hline 5 & -- & $\cdots$ & -- & --- & 11.02 & 8.35 & --- & -- & --- & --- & -- & --- \\
\hline 6 & --- & --- & -- & -- & 11.08 & 8.29 & -- & --- & -- & -- & --- & --- \\
\hline 7 & --- & -- & -- & --- & 10.94 & 8.35 & $-\infty$ & -- & -- & --- & --- & --- \\
\hline 8 & -- & -- & -- & --- & 10.98 & 8.36 & -- & 10.56 & -- & -- & -- & -- \\
\hline 9 & $\cdots$ & --- & -- & -- & 11.06 & 8.28 & --- & 10.45 & -- & 514.40 & $\cdots$ & $\cdots$ \\
\hline 10 & -- & -- & -- & -- & 10.85 & 8.21 & $\cdots$ & 10.47 & -- & $-\cdots$ & -- & 13.34 \\
\hline 11 & --- & --- & -- & -- & 10.77 & 8.22 & $-\cdots$ & 10.58 & $\cdots$ & -- & --- & 13.32 \\
\hline 12 & -- & -- & -- & -- & 10.57 & 8.22 & $\cdots$ & 10.64 & --- & -- & $\cdots$ & 13.25 \\
\hline 13 & -- & --- & --- & --- & 10.37 & 8.21 & $\cdots$ & 10.81 & $\cdots$ & --- & $\cdots$ & 13.12 \\
\hline 14 & $\cdots$ & -- & --- & -- & 10.23 & 8.21 & $\cdots$ & 11.01 & $\cdots$ & $\ldots$ & -- & 13.02 \\
\hline 15 & --- & $\mathrm{S} 12.15$ & -- & --- & 10.06 & 8.22 & -- & 11.20 & -- & -- & --- & 13.02 \\
\hline 16 & --- & --- & --- & --- & 9.81 & 8.17 & --- & 11.30 & --- & --- & --- & 13.01 \\
\hline 17 & --- & -- & $\cdots$ & $\cdots$ & 9.59 & 8.10 & $\cdots$ & 11.47 & -- & $\mathrm{S} 12.50$ & -- & 12.95 \\
\hline 18 & --- &.-- & -- & -- & 9.45 & 7.97 & -- & 11.65 & -- & -- & --- & 12.64 \\
\hline 19 & -- & --- & --- & --- & 9.36 & 8.03 & --- & 11.64 & $\cdots$ & $\cdots$ & --- & 12.50 \\
\hline 20 & --- & -- & -- & --- & 9.18 & 8.09 & --- & 11.58 & --- & --- & --- & 12.41 \\
\hline 21 & -- & -- & -- & -- & 8.92 & 8.19 & -- & 11.41 & -- & -- & --- & 12.34 \\
\hline 22 & -- & $\cdots$ & --- & --- & 8.78 & 8.23 & --- & 11.24 & $\cdots$ & -- & $\cdots$ & 12.30 \\
\hline 23 & -- & $\cdots$ & $\cdots$ & -- & 8.66 & 8.16 & -- & 11.12 & -.- & $\cdots$ & $\cdots$ & 12.21 \\
\hline 24 & $\cdots$ & $\cdots$ & $\cdots$ & $\mathrm{S} 11.26$ & 8.64 & 8,15 & -- & 11.02 & --- & --- & --- & 12.17 \\
\hline 25 & -- & -- & -- & 11.24 & 8.61 & 8.10 & -- & 10.90 & $-\infty$ & -- & S13.10 & 12.14 \\
\hline 26 & $-\infty$ & $-\infty$ & $\cdots$ & 11.25 & 8.56 & 8.31 & $\cdots$ & 10.92 & $-\cdots$ & $\cdots$ & 13.17 & 12.36 \\
\hline 27 & -- & $\cdots$ & -- & 11.20 & 8.38 & 8.41 & -- & 10.82 & $\cdots$ & --- & 13.16 & 12.39 \\
\hline 28 & -- & --- & $\ldots$ & 11.15 & 8.38 & 8.42 & --- & 10.82 & $\cdots$ & $\cdots$ & 13.12 & 12.32 \\
\hline 29 & -- & $-\cdots$ & -- & 11.01 & 8.36 & 8.44 & --- & 10.89 & $\cdots$ & -- & 13.11 & 12.24 \\
\hline 30 & $\cdots$ & --- & $\cdots$ & 11.05 & -- & 8.48 & $\rightarrow-$ & 10.96 & $\cdots$ & -- & 13.23 & 12.30 \\
\hline 31 & $\cdots$ & --- & --- & 11.12 & -- & 8.60 & $\cdots$ & 11.03 & -- & --- & 13.34 & $-\ldots$ \\
\hline
\end{tabular}

PERIOD OF RECORD HIGHEST 0.96 MAR 17, 1983 LOWEST 14.40 JUL 09, 1992

S Manual steel tape measurement.

SITE NUMBER 385501121361904

LOCAL NUMBER 012N003E02G04M

About 1.7 miles northwest of Nicolaus. Drilled domestic well in alluvium. Diameter 8 inches, depth 54 feet. Altitude of land-surface datum 32.54 feet. Water-level records available July 1985 to current year.

WATER LEVELS IN FEET BELOW LAND-SURFACE DATUM

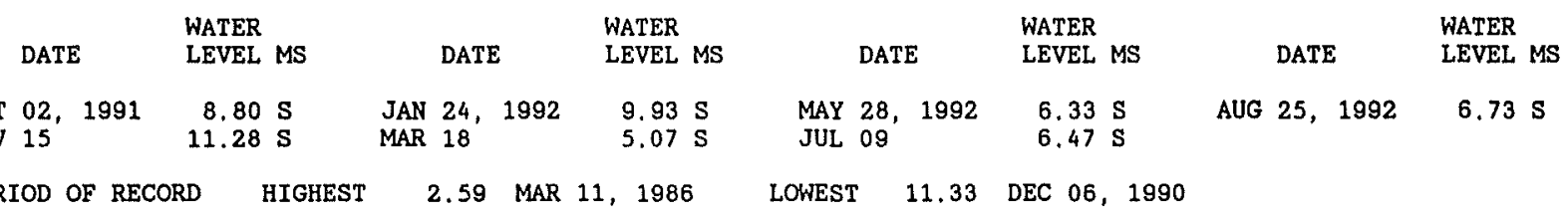




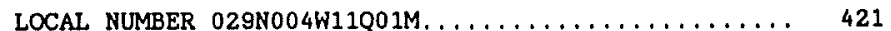

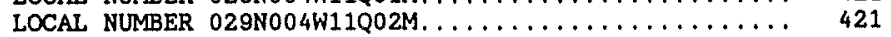

LOCAL NUMBER 029N004W14B01M. ............... 421

LOCAL NUMBER 029N004W17B01M.

LOCAL NUMBER 029N004W17B02M............... 422

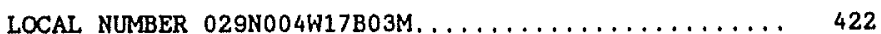

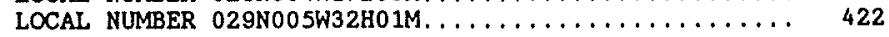

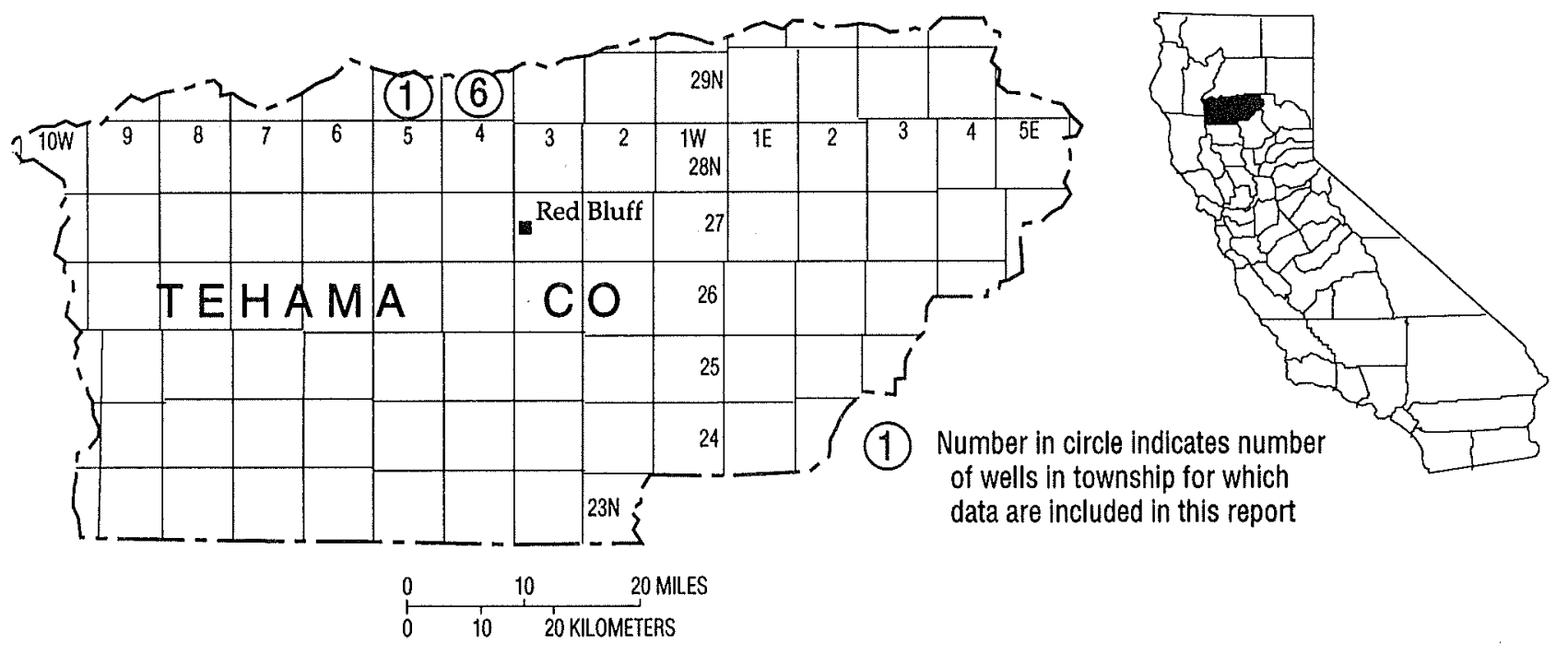

Figure 33. Location of wells in Tehama County. 


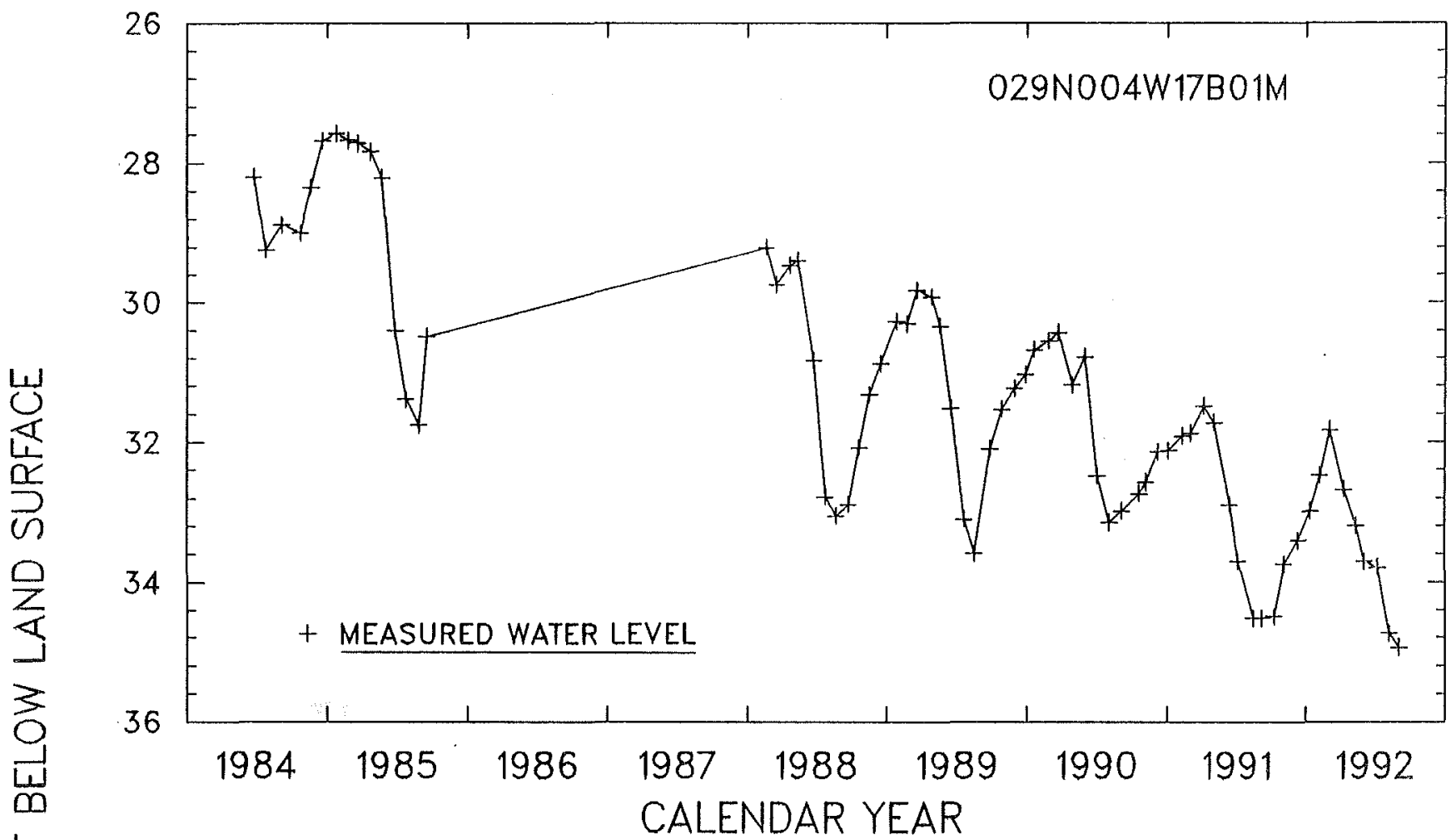

넌

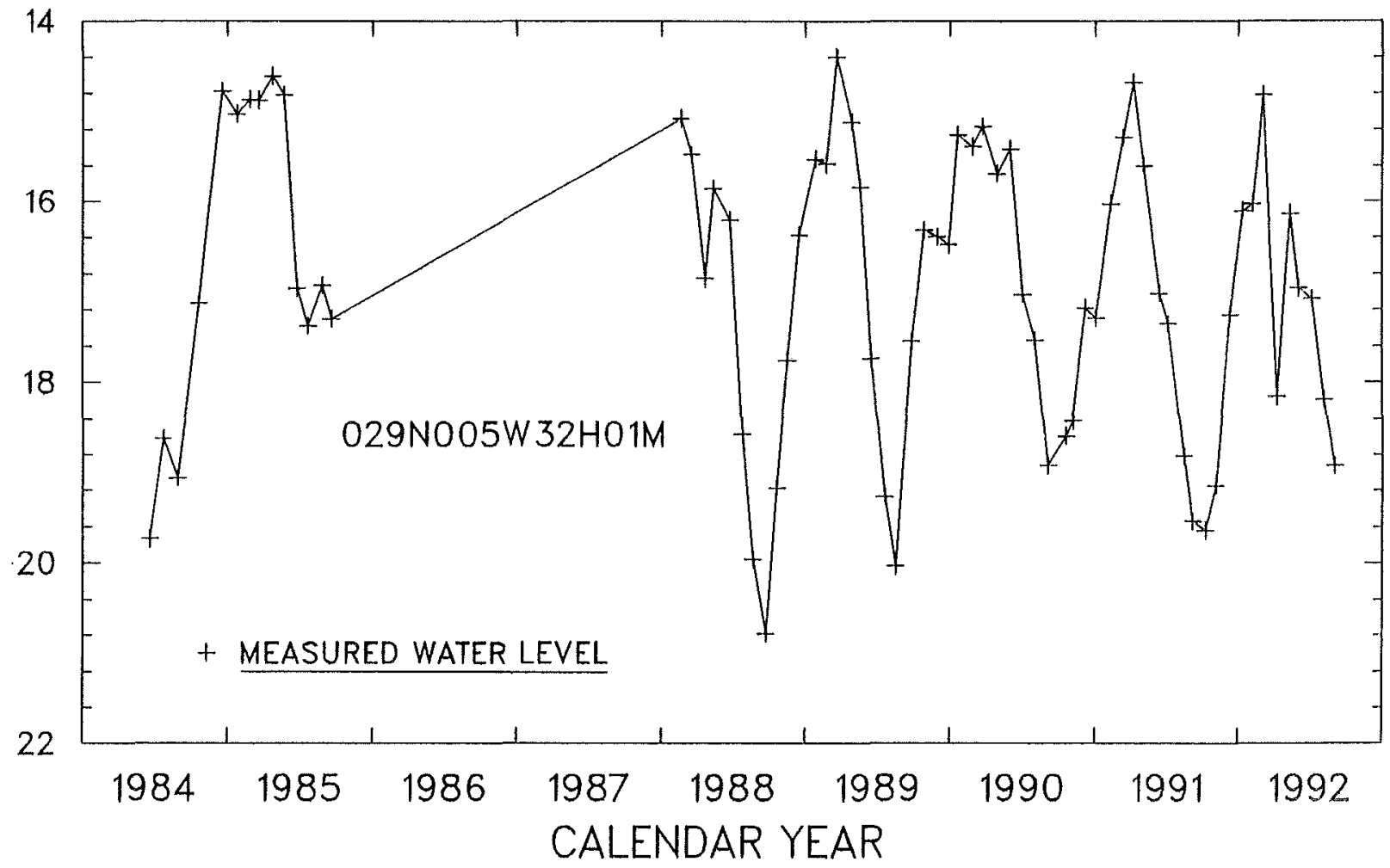

Figure 34. Hydrographs for wells in Tehama County. (029N004W17B01M and 029N005W32H01M) 
WELL DESCRIPTIONS AND WATER-LEVEL MEASUREMENTS

WATER YEAR OCTOBER 1991 TO SEPTEMBER 1992

SITE NUMBER 402230122165101

LOCAL NUMBER 029N004W11Q01M

About 0.7 mile south of Cottonwood. Drilled observation well in Tehama Formation of Pliocene age. Diameter 6 inches, depth 93 feet, perforated 78-88 feet. Altitude of land-surface datum of 400 feet. Water-1evel records avallable $1984-85,1988$ to current year.

WATER LEVELS IN FEET BELOW LAND-SURFACE DATUM

\begin{tabular}{|c|c|c|c|c|c|c|c|}
\hline DATE & $\begin{array}{l}\text { WATER } \\
\text { LEVEL MS }\end{array}$ & DATE & $\begin{array}{l}\text { WATER } \\
\text { LEVEL MS }\end{array}$ & DATE & $\begin{array}{l}\text { WATER } \\
\text { LEVEL MS }\end{array}$ & DATE & $\begin{array}{l}\text { WATER } \\
\text { LEVEL MS }\end{array}$ \\
\hline $\begin{array}{ll}08, & 1991 \\
04 & \\
13 & \end{array}$ & $\begin{array}{r}9.38 \mathrm{~S} \\
9.24 \mathrm{~S} \\
10.05 \mathrm{~S}\end{array}$ & $\begin{array}{lll}\text { JAN } & 13, & 1992 \\
\text { FEB } & 06 & \\
\text { MAR } & 04\end{array}$ & $\begin{array}{l}9.27 \mathrm{~S} \\
9.52 \mathrm{~S} \\
7.69 \mathrm{~S}\end{array}$ & $\begin{array}{lll}\text { APR } & 10,1992 \\
\text { MAY } & 11 & \\
\text { JUN } & 02 & \end{array}$ & $\begin{array}{l}9.20 \mathrm{~S} \\
8.88 \mathrm{~S} \\
9.11 \mathrm{~S}\end{array}$ & $\begin{array}{lll}\text { JUL } & 07, & 1992 \\
\text { AUG } & 06 & \\
\text { SEP } & 01\end{array}$ & $\begin{array}{l}9.08 \mathrm{~s} \\
9.68 \mathrm{~s} \\
9.60 \mathrm{~s}\end{array}$ \\
\hline
\end{tabular}

PERIOD OF RECORD HIGHEST 5.92 DEC 18, 1984 LOWEST 10.05 DEC 13, 1991

SITE NUMBER 402230122165201

LOCAL NUMBER O29N004W11Q02M

About 0.7 mile south of Cottonwood. Drilled observation well in alluvium. Diameter 6 inches, depth 45 feet, perforated 30-40 feet. Altitude of land-surface datum $400 \mathrm{ft}$. Water-level records available 1984-85, 1988 to current year.

WATER LEVELS IN FEET BELOW LAND-SURFACE DATUM

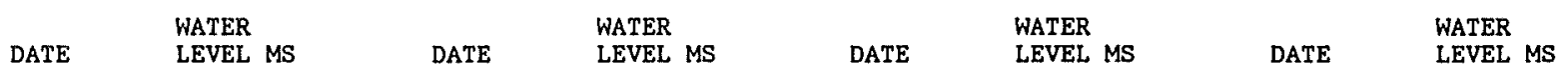

$\begin{array}{lllllllllll}\text { OCT 08, } 1991 & 9.03 \mathrm{~S} & \text { JAN 13, } 1992 & 9.20 \mathrm{~S} & \text { APR 10,1992 } & 8.56 \mathrm{~S} & \text { JUL } 07,1992 & 8.92 \mathrm{~S} \\ \text { NOV } 04 & 9.12 \mathrm{~S} & \text { FEB 06 } & 9.51 \mathrm{~S} & \text { MAY } 11 & 8.63 \mathrm{~S} & \text { AUG } 06 & 9.33 \mathrm{~S} \\ \text { DEC 13 } & 9.95 \mathrm{~S} & \text { MAR 04 } & 7.68 \mathrm{~S} & \text { JUN } 02 & 8.88 \mathrm{~S} & \text { SEP 01 } & 9.34 \mathrm{~S}\end{array}$

PERIOD OF RECORD HIGHEST 5.89 DEC 18, 1984 LOWEST 9.95 DEC 13, 1991

SITE NUMBER 402229122165201

LOCAL NUMBER 029N004W14B01M

About 0.7 mile south of Cottonwood. Drilled observation well in alluvium. Diameter 6 inches, depth 47 feet, perforated 32-42 feet. Altitude of land-surface datum 400 feet. Water-level records available $1984-85$, 1988 to current year.

WATER LEVELS IN FEET BELOW LAND-SURFACE DATUM

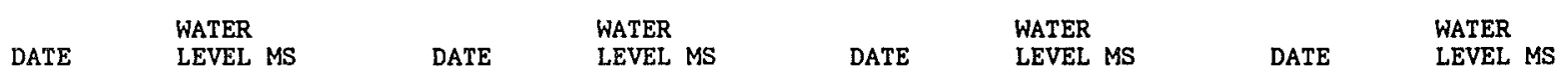

$\begin{array}{lllllllllll}\text { OCT 08, } 1991 & 8.78 \mathrm{~S} & \text { JAN 13, } 1992 & 8.97 \mathrm{~S} & \text { APR } 10,1992 & 8.37 \mathrm{~S} & \text { JUL } 07,1992 & 8.70 \mathrm{~S} \\ \text { NOV 04 } & 8.91 \mathrm{~S} & \text { FEB 06 } & 9.27 \mathrm{~S} & \text { MAY 11 } & 8.43 \mathrm{~S} & \text { AUG } 06 & 9.04 \mathrm{~S} \\ \text { DEC 13 } & 9.70 \mathrm{~S} & \text { MAR 04 } & 7.52 \mathrm{~S} & \text { JUN 02 } & 8.68 \mathrm{~S} & \text { SEP 01 } & 9.00 \mathrm{~S}\end{array}$

PERIOD OF RECORD HIGHEST 5.57 DEC 18, 1984 LOWEST 9.70 DEC 13, 1991

SITE NUMBER 402225122201201

LOCAL NUMBER 029N004W17B01M

About 3.2 miles southwest of Cottonwood. Drilled observation well in Tehama Formation of Pliocene age. Diameter 6 inches, depth 160 feet, perforated 150-160 feet. Altitude of land-surface datum 440 feet. Water-level records available $1984-85,1988$ to current year.

WATER LEVELS IN FEET BELOW LAND-SURFACE DATUM

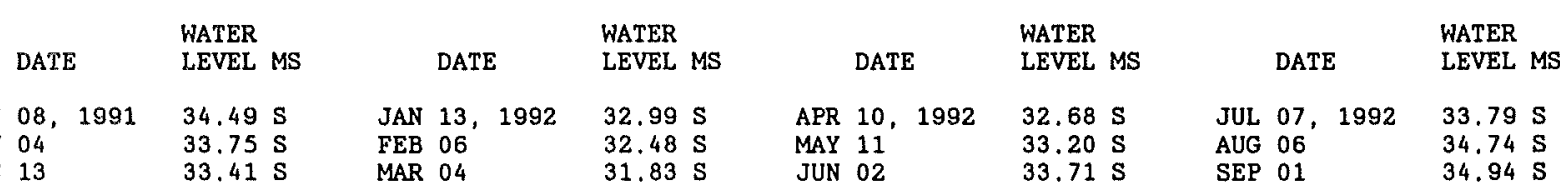

PERIOD OF RECORD HIGHEST 27.58 JAN 25, 1985 LOWEST 34.94 SEP 01,1992 
SITE NUMBER 402225122201202

LOCAL NUMBER 029N004W17BO2M

About 3.2 miles southwest of Cottonwood. Drilled observation well in alluvium. Diameter 6 inches, depth 80 feet, perforated 70-80 feet. Altitude of land-surface datum 440 feet. Water-level records available 1984-85, 1988 to current year.

WATER LEVELS IN FEET BELOW LAND-SURFACE DATUM

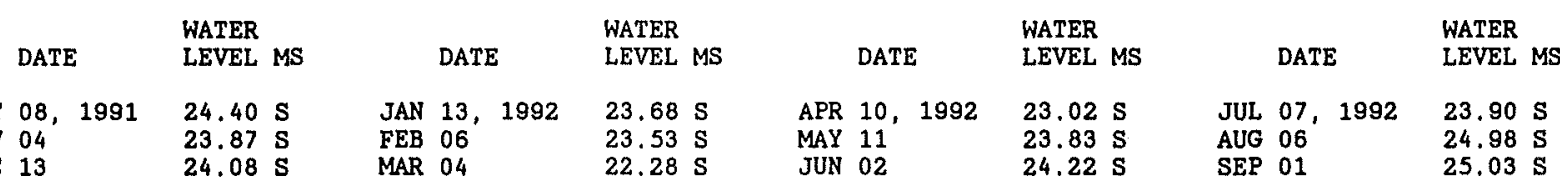

PERIOD OF RECORD HIGHEST 14,59 JUN 25,1984 LOWEST 26.83 FEB 25, 1985

SITE NUMBER 402224122201001

LOCAL NUMBER 029N004W17B03M

About 3.2 miles southwest of Cottonwood. Drilled observation well in alluvium. Diameter 6 inches, depth 80 feet, perforated 70-80 feet. Altitude of land-surface datum 440 feet. Water-level records available 1984-85, 1988 to current year.

WATER LEVELS IN FEET BELOW LAND-SURFACE DATUM

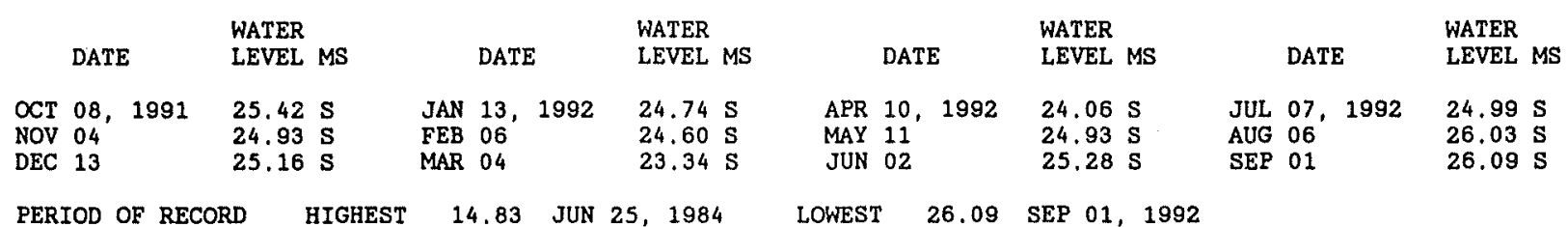

PERIOD OF RECORD HIGHEST 14.83 JUN 25,1984 LOWEST 26.09 SEP 01, 1992

SITE NUMBER 401932122263301

LOCAL NUMBER 029N005W32H01M

About 9.8 miles southwest of Cottonwood. Drilled observation well in Tehama Formation of Pliocene age. Dianeter 6 inches, depth 105 feet, perforated 90-100 feet. Altitude of land-surface datum 510 feet. Water-level records available 1984-85, 1988 to current year.

WATER LEVELS IN FEET BELOW LAND-SURFACE DATUM

\begin{tabular}{|c|c|c|c|c|c|c|c|}
\hline DATE & $\begin{array}{l}\text { WATER } \\
\text { LEVEL MS }\end{array}$ & DATE & $\begin{array}{l}\text { WATER } \\
\text { LEVEL MS }\end{array}$ & DATE & $\begin{array}{l}\text { WATER } \\
\text { LEVEL MS }\end{array}$ & DATE & $\begin{array}{l}\text { WATER } \\
\text { LEVEL MS }\end{array}$ \\
\hline $\begin{array}{l}08,1991 \\
04 \\
13\end{array}$ & $\begin{array}{l}19.64 \mathrm{~S} \\
19.15 \mathrm{~S} \\
17.26 \mathrm{~S}\end{array}$ & $\begin{array}{lll}\text { JAN } & 13,1992 \\
\text { FEB } & 06 & \\
\text { MAR } & 04\end{array}$ & $\begin{array}{l}16.11 \mathrm{~S} \\
16.03 \mathrm{~S} \\
14.82 \mathrm{~S}\end{array}$ & $\begin{array}{lll}\text { APR } & 10,1992 \\
\text { MAY } & 11 & \\
\text { JUN } & 02 & \end{array}$ & $\begin{array}{l}18.16 \mathrm{~S} \\
16.14 \mathrm{~S} \\
16.96 \mathrm{~S}\end{array}$ & $\begin{array}{lll}\text { JUL } & 07, & 1992 \\
\text { AUG } & 06 & \\
\text { SEP } & 01\end{array}$ & $\begin{array}{l}17.08 \mathrm{~S} \\
18.19 \mathrm{~s} \\
18.92 \mathrm{~s}\end{array}$ \\
\hline
\end{tabular}

PERIOD OF RECORD HIGHEST 14,40 MAR 20, $1989 \quad$ LOWEST 20.78 SEP 23,1988




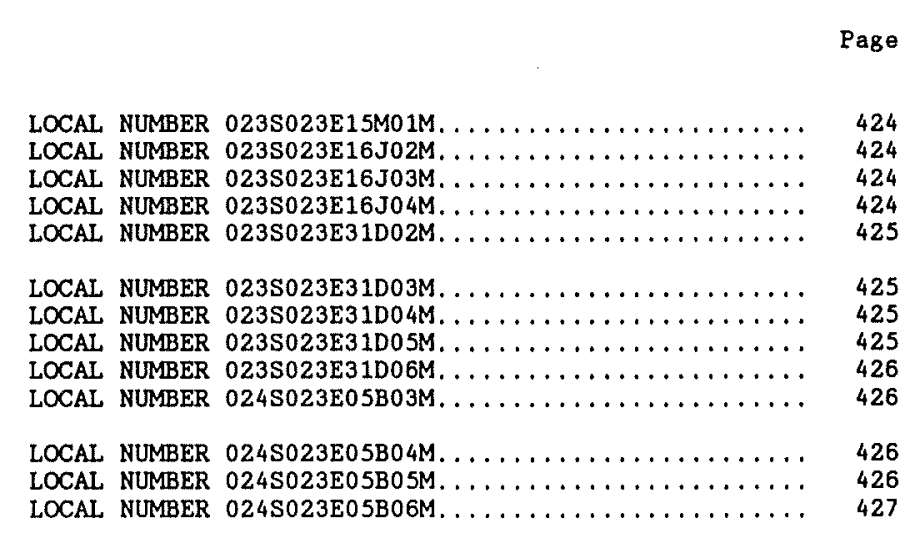

(4) Number in circle indicates number of wells in township for which data are included in this report
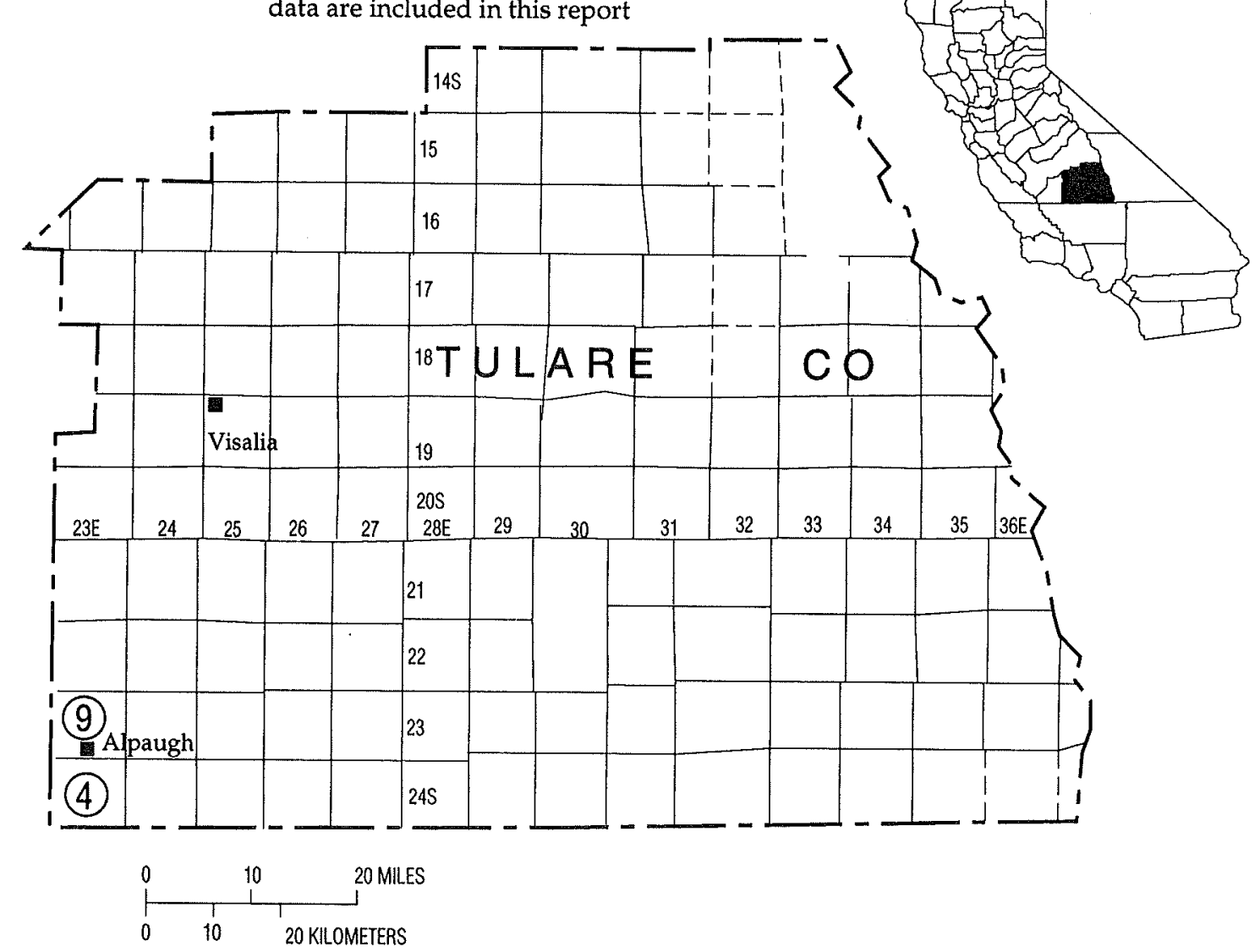

Figure 35. Location of wells in Tulare County. 
SITE NUMBER 355530119285201

LOCAL NUMBER 023S023E15M01M

About 2 miles north of Alpaugh. Drilled observation well. Diameter 2 inches, depth 20 feet, perforated

10-20 feet. Altitude of land-surface datum 200 feet. Water-level records available 1989 to current year.

WATER LEVELS IN FEET BELOW LAND-SURFACE DATUM

\begin{tabular}{|c|c|c|c|c|c|c|c|}
\hline DATE & $\begin{array}{l}\text { WATER } \\
\text { LEVEL MS }\end{array}$ & DATE & $\begin{array}{l}\text { WATER } \\
\text { LEVEL MS }\end{array}$ & DATE & $\begin{array}{l}\text { WATER } \\
\text { LEVEL MS }\end{array}$ & DATE & $\begin{array}{l}\text { WATER } \\
\text { LEVEL MS }\end{array}$ \\
\hline
\end{tabular}

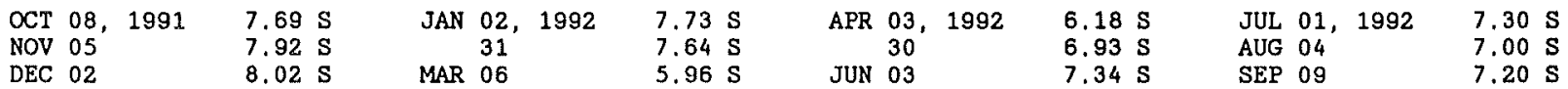

DEC 02

PERIOD OF RECORD

HIGHEST

5.96 MAR 06, 1992

LOWEST

8.02 DEC 02, 1991

SITE NUMBER 355526119285502

LOCAL NUMBER 023S023E16J02M

About 2 miles north of Alpaugh. Drilled observation well. Diameter 2 inches, depth 54 feet, perforated

39-49 feet. Altitude of land-surface datum 200 feet. Water-level records available 1991 to current year.

WATER LEVELS IN FEET BELOW LAND-SURFACE DATUM

\begin{tabular}{llllll} 
& WATER & & WATER & \multicolumn{2}{c}{ WATER } \\
DATE & LEVEL MS & DATE & LEVEL MS & DATE & LEVEL MS
\end{tabular}

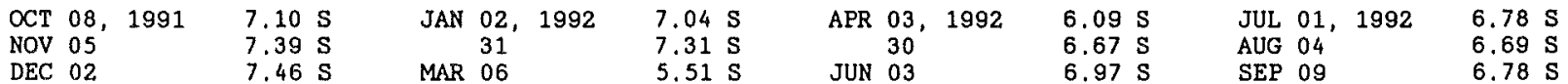

DEC 02

MAR $06 \quad 5.51$

PERIOD OF RECORD HIGHEST

5.44 JUL 02,1991

LOWEST $\quad 7.46 \quad$ DEC 02, 1981

SITE NUMBER 355526119285503

LOCAL NUMBER 023S023E16J03M

About 2 miles north of Alpaugh. Drilled observation well. Diameter 2 inches, depth 99 feet, perforated 81-91 feet. Altitude of land-surface datum 200 feet. Water-level records available 1991 to current year.

WATER LEVELS IN FEET BELOW LAND-SURFACE DATUM

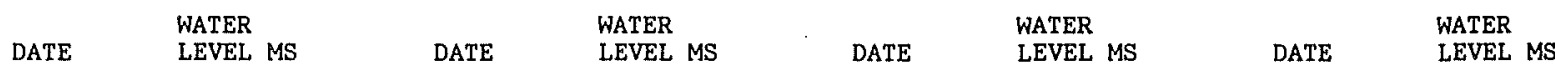

OCT $08,1991 \quad 11.68 \mathrm{~s}$

DEC 02

$\begin{array}{llll}\text { JAN } 02,1992 & 12.21 \mathrm{~S} \\ & 31 & & 12.16 \mathrm{~S} \\ \text { MAR } 06 & & 11.81 \mathrm{~S}\end{array}$

APR 03, 1992

DATE

LEVEL MS

$12.16 \mathrm{~S} \quad \mathrm{MAR} 06$

JUN 03

$11.57 \mathrm{~S}$
$11.62 \mathrm{~S}$

JUL 01, 1992

$12.12 \mathrm{~s}$

HIGHEST

11.44 AUG 30,1991

LOWEST 12.24 SEP 09, 1992

SITE NUMBER 355526119285504

LOCAL NUMBER 0235023E16J04M

About 2 miles north of Alpaugh. Drilled observation well. Diameter 2 inches, depth 201 feet, perforated 186-196 feet. Altitude of land-surface datum 200 feet. Water-level records avallable 1991 to current year. WATER LEVELS IN FEET BELOW LAND-SURFACE DATUM

\begin{tabular}{|c|c|c|c|c|c|c|c|c|c|c|}
\hline DATE & $\begin{array}{l}\text { WATER } \\
\text { LEVEL MS }\end{array}$ & & DATE & $\begin{array}{l}\text { WATER } \\
\text { LEVEL MS }\end{array}$ & & DATE & $\begin{array}{l}\text { WATER } \\
\text { LEVEL MS }\end{array}$ & & DATE & $\begin{array}{l}\text { WATER } \\
\text { LEVEL }\end{array}$ \\
\hline $\begin{array}{l}08, \quad 1991 \\
05 \\
02\end{array}$ & $\begin{array}{l}58.29 \mathrm{~S} \\
58.68 \mathrm{~S} \\
58.75 \mathrm{~S}\end{array}$ & $\begin{array}{l}\text { JAN } \\
\text { MAR }\end{array}$ & $\begin{array}{l}02 \\
31 \\
06\end{array}$ & $\begin{array}{l}58.49 \mathrm{~S} \\
58.03 \mathrm{~S} \\
57.15 \mathrm{~S}\end{array}$ & $\begin{array}{l}\text { APR } \\
\text { JUN }\end{array}$ & $\begin{array}{ll}03, & 1992 \\
30 & \\
03 & \end{array}$ & $\begin{array}{l}57.09 \mathrm{~S} \\
57.04 \mathrm{~S} \\
57.79 \mathrm{~S}\end{array}$ & $\begin{array}{l}\text { JUL } \\
\text { AUG } \\
\text { SEP }\end{array}$ & $\begin{array}{ll}01, & 1992 \\
04 & \\
09 & \end{array}$ & $\begin{array}{l}58.00 \\
58.88 \\
59.62\end{array}$ \\
\hline
\end{tabular}

PERIOD OF RECORD HIGHEST 56.30 JUL 02,1991 LOWEST 59.62 SEP 09,1992 
WATER LEVELS, TULARE COUNTY--Continued

WELL DESCRIPTIONS AND WATER-LEVEL MEASUREMENTS

WATER YEAR OCTOBER 1991 TO SEPTEMBER 1992

SITE NUMBER 355317119320301

LOCAL NUMBER 023S023E31D02M

About 4 miles west of Alpaugh. Drilled observation well. Diameter 2 inches, depth 20 feet, perforated 10-20 feet. Altitude of land-surface datum 199 feet. Water-level records available 1991 to current year.

WATER LEVEIS IN FEET BELOW LAND-SURFACE DATUM

\begin{tabular}{|c|c|c|c|c|c|c|c|}
\hline DATE & $\begin{array}{l}\text { WATER } \\
\text { LEVEL MS }\end{array}$ & DATE & $\begin{array}{l}\text { WATER } \\
\text { LEVEL MS }\end{array}$ & DATE & $\begin{array}{l}\text { WATER } \\
\text { LEVEL MS }\end{array}$ & DATE & $\begin{array}{l}\text { WATER } \\
\text { LEVEL MS }\end{array}$ \\
\hline
\end{tabular}

$\begin{array}{llllllllll}\text { OCT 08, } 1991 & 7.49 \mathrm{~S} & \text { JAN 02, } 1992 & 8.19 \mathrm{~S} & \text { APR 03, } 1992 & 6.54 \mathrm{~S} & \text { JUL } 01,1992 & 6.83 \mathrm{~S} \\ \text { NOV 05 } & 7.83 \mathrm{~S} & 31 & 8.10 \mathrm{~S} & & 30 & 6.47 \mathrm{~S} & \text { AUG } 04 & 6.69 \mathrm{~S} \\ \text { DEC 02 } & 8.09 \mathrm{~S} & \text { MAR } 06 & 7.03 \mathrm{~S} & \text { JUN } 03 & 6.67 \mathrm{~S} & \text { SEP } 09 & 6.64 \mathrm{~S}\end{array}$

PERIOD OF RECORD HIGHEST 6.13 JUL 02, $1991 \quad$ LOWEST 8.19 JAN 02, 1992

SITE NUMBER 355317119320002

LOCAL NUMBER 023S023E31D03M

About 4 miles west of Alpaugh. Drilled observation well. Diameter 2 inches, depth 58 feet, perforated $43-53$ feet. Altitude of land-surface datum 199 feet. Water-level records available 1991 to current year.

WATER LEVELS IN FEET BELOW LAND-SURFACE DATUM

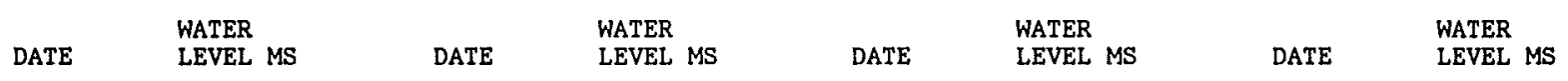

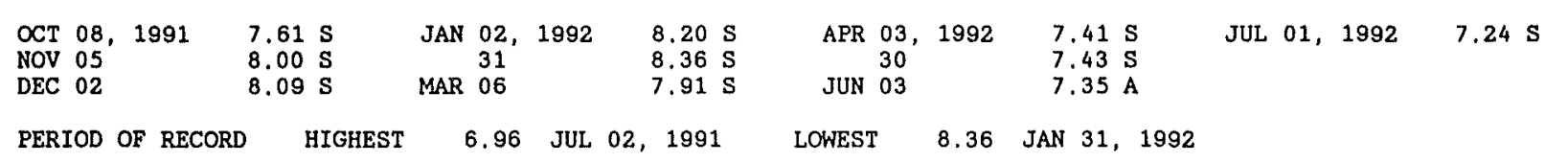

SITE NUMBER 355317119320003

LOCAL NUMBER 0235023E31D04M

About 4 miles west of Alpaugh. Drilled observation well. Diameter 2 inches, depth 98 feet, perforated 78-83 feet. Altitude of land-surface datum 199 feet. Water-level records available 1991 to current year.

WATER LEVELS IN FEET BELOW LAND-SURFACE DATUM

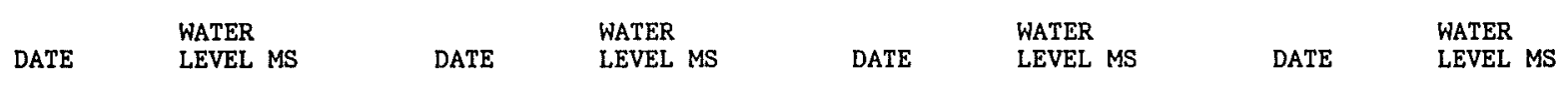

$\begin{array}{llllllllll}\text { OCT 08, } 1991 & 7.87 \mathrm{~S} & \text { JAN 02, } 1992 & 8.42 \mathrm{~S} & \text { APR } 03,1992 & 7.73 \mathrm{~S} & \text { JUL } 01,1992 & 7.53 \mathrm{~S} \\ \text { NOV 05 } & 8.18 \mathrm{~S} & 31 & 8.58 \mathrm{~S} & 30 & 7.76 \mathrm{~S} & \text { AUG } 04 & 7.49 \mathrm{~S} \\ \text { DEC 02 } & 8.34 \mathrm{~S} & \text { MAR 06 } & 8.29 \mathrm{~S} & \text { JUN 03 } & 7.64 \mathrm{~S} & \text { SEP } 09 & 7.48 \mathrm{~S}\end{array}$

PERIOD OF RECORD HIGHEST 7.48 SEP 09, $1992 \quad$ LOWEST 8.58 JAN 31,1992

SITE NUMBER 355317119320004

LOCAL NUMBER 023S023E31D05M

About 4 miles west of Alpaugh. Drilled observation well. Diameter 2 inches, depth 200 feet, perforated 185-195 feet. Altitude of land-surface datum 199 feet. Water-level records available 1991 to current year.

WATER LEVELS IN FEET BELOW LAND-SURFACE DATUM

\begin{tabular}{|c|c|c|c|c|c|c|c|c|c|}
\hline DATE & $\begin{array}{l}\text { WATER } \\
\text { LEVEL MS }\end{array}$ & & DATE & $\begin{array}{l}\text { WATER } \\
\text { LEVEL MS }\end{array}$ & & DATE & $\begin{array}{l}\text { WATER } \\
\text { LEVEL MS }\end{array}$ & DATE & $\begin{array}{l}\text { WATER } \\
\text { LEVEL MS }\end{array}$ \\
\hline $\begin{array}{ll}08,1991 \\
05 & \\
02 & \end{array}$ & $\begin{array}{l}36.63 \mathrm{~s} \\
37.05 \mathrm{~S} \\
38.17 \mathrm{~S}\end{array}$ & $\begin{array}{l}\text { JAN } \\
\text { MAR }\end{array}$ & $\begin{array}{ll}02, & 1992 \\
31 & \\
06 & \end{array}$ & $\begin{array}{l}37.18 \mathrm{~s} \\
37.15 \mathrm{~S} \\
37.18 \mathrm{~S}\end{array}$ & $\begin{array}{l}\text { APR } \\
\text { JUN }\end{array}$ & $\begin{array}{ll}03,1992 \\
30 \\
03\end{array}$ & $\begin{array}{l}37.18 \mathrm{~S} \\
37.28 \mathrm{~S} \\
37.24 \mathrm{~S}\end{array}$ & $\begin{array}{lll}\text { JUL } & 01,1992 \\
\text { AUG } & 04 & \\
\text { SEP } & 09\end{array}$ & $\begin{array}{l}37.34 \mathrm{~S} \\
37.55 \mathrm{~S} \\
37.67 \mathrm{~S}\end{array}$ \\
\hline
\end{tabular}

PERIOD OF RECORD HIGHEST 36.20 JUL 02, $1991 \quad$ LOWEST 38.17 DEC 02, 1991 
WELL DESCRIPTIONS AND WATER-LEVEL MEASUREMENTS

WATER YEAR OCTOBER 1991 TO SEPTEMBER 1992

\section{SITE NUMBER 355317119320005}

LOCAL NUMBER 023S023E31D06M

About 4 miles west of Alpaugh. Drilled observation well. Diameter 2 inches, depth 20 feet, perforated 10-20 feet. Altitude of land-surface datum 199 feet, Water-level records available 1992.

WATER LEVELS IN FEET BELOW LAND-SURFACE DATUM

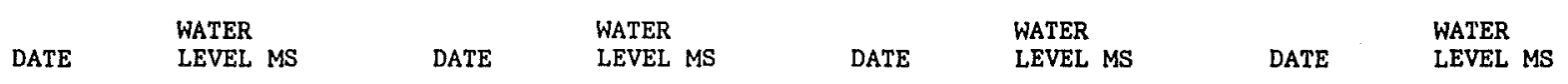

$\begin{array}{llllllllll}\text { MAR 06, } 1992 & 5.77 \mathrm{~S} & \text { APR } 30,1992 & 6.13 \mathrm{~S} & \text { JUL 01, } 1992 & 6.35 \mathrm{~S} & \text { SEP } 09,1992 & 5.99 \mathrm{~S} \\ \text { APR 03 } & 6.24 \mathrm{~S} & \text { JUN 03 } & 6.63 \mathrm{~S} & \text { AUG 04 } & 6.01 \mathrm{~S} & & \end{array}$

PERIOD OF RECORD HIGHEST 5.77 MAR 06, 1992 LOWEST 6.63 JUN 03, 1992

SITE NUMBER 355225119301601

LOCAL NUMBER 024S023E05B03M

About 1.5 miles southwest of Alpaugh. Drilled observation well. Diameter 2 inches, depth 20 feet, screened 10-20 feet. Altitude of land-surface datum 210 feet. Water-level records avallable 1990 to current year.

WATER LEVELS IN FEET BELOW LAND-SURFACE DATUM

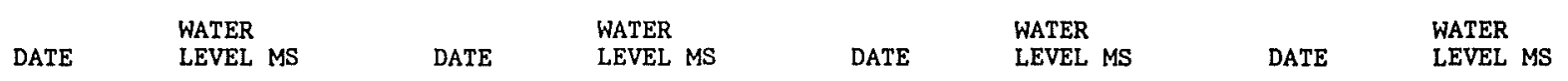

$\begin{array}{lrrrrrrrrrr}\text { OCT 08, } 1991 & 8.78 \mathrm{~S} & \text { JAN 02, } 1992 & 10.44 \mathrm{~S} & \text { APR } 03,1992 & 7.24 \mathrm{~S} & \text { JUL } 01,1992 & 7.47 \mathrm{~S} \\ \text { NOV 05 } & 10.80 \mathrm{R} & 31 & 9.48 \mathrm{~S} & & 30 & 6.99 \mathrm{SS} & \text { AUG } 04 & 7.89 \mathrm{~S} \\ \text { DEC 02 } & 10.20 \mathrm{~S} & \text { MAR 06 } & 6.79 \mathrm{~S} & \text { JUN } 03 & 7.35 \mathrm{~S} & \text { SEP } 09 & 9.21 \mathrm{~S}\end{array}$

PERIOD OF RECORD HIGHEST 6.79 JUL 02, 1991 MAR 06, 1992 LOWEST 10.80 NOV 05, 1991

SITE NUMBER 355225119301602

LOCAL NUMBER 024S023E05B04M

About $1.5 \mathrm{miles}$ southwest of Alpaugh. Drilled observation well. Diameter 2 inches, depth 68 feet, screened 53-63 feet. Altitude of land-surface datum 210 feet. Water-level records available 1990 to current year.

WATER LEVELS IN FEET BELOW LAND-SURFACE DATUM

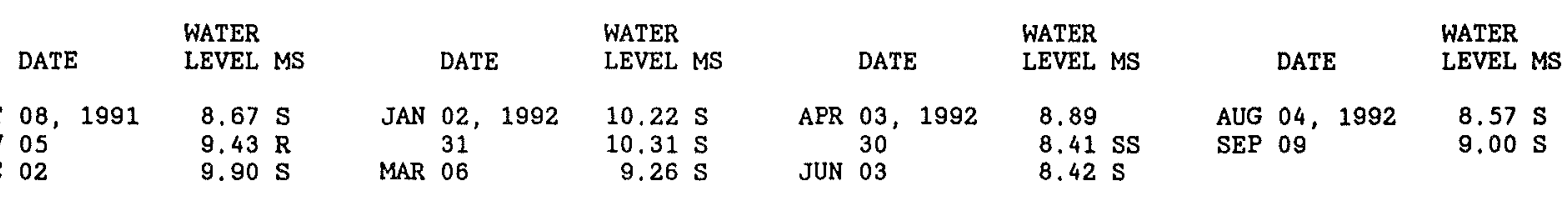

PERIOD OF RECORD HIGHEST 7.93 JUL 24, 1991 LOWEST 10.36 JAN 31, 1991

SITE NUMBER 355225119301603

LOCAL NUMBER 024S023E05B05M

About 1.5 miles southwest of Alpaugh. Drilled observation well. Diameter 2 inches, depth 100 feet, screened 90-100 feet. Altitude of land-surface datum 210 feet. Water-level records available 1990 to current year.

WATER LEVELS IN FEET BELOW LAND-SURFACE DATUM

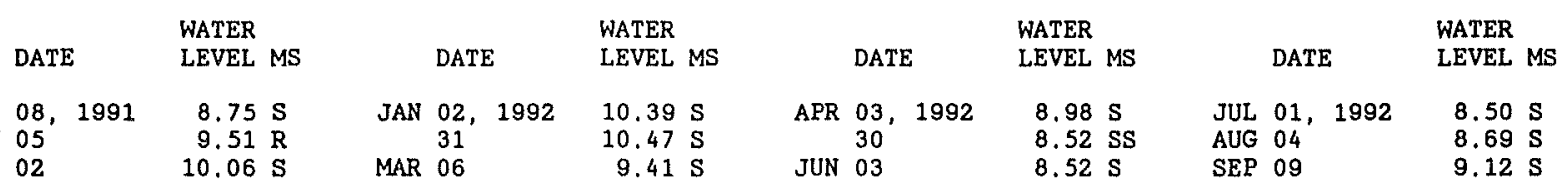

PERIOD OF RECORD HIGHEST 8.01 JUL 24,1991 LOWEST 10.48 JAN 31, 1991 
WELL DESCRIPTIONS AND WATER-LEVEL MEASUREMENTS

WATER YEAR OCTOBER 1991 TO SEPTEMBER 1992

SITE NUMBER 355225119301604

LOCAL NUMBER 024S023E05B06M

About 1.5 miles southwest of Alpaugh. Drilled observation well. Diameter 3 inches, depth 190 feet, screened 175-185 feet. Altitude of land-surface datum 210 feet. Water-level records available 1990 to current year.

WATER LEVELS IN FEET BELOW LAND-SURFACE DATUM

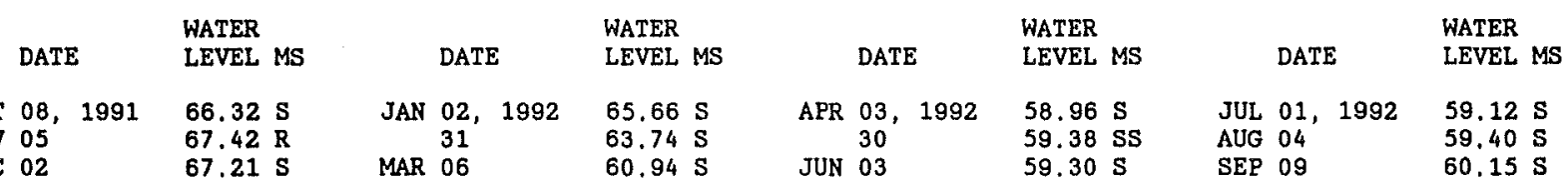

PERIOD OF RECORD HIGHEST 58.96 APR 03, 1992 LOWEST 67.42 NOV 05, 1991 
(2) Number in circle indicates number of wells in township for which data are included in this report
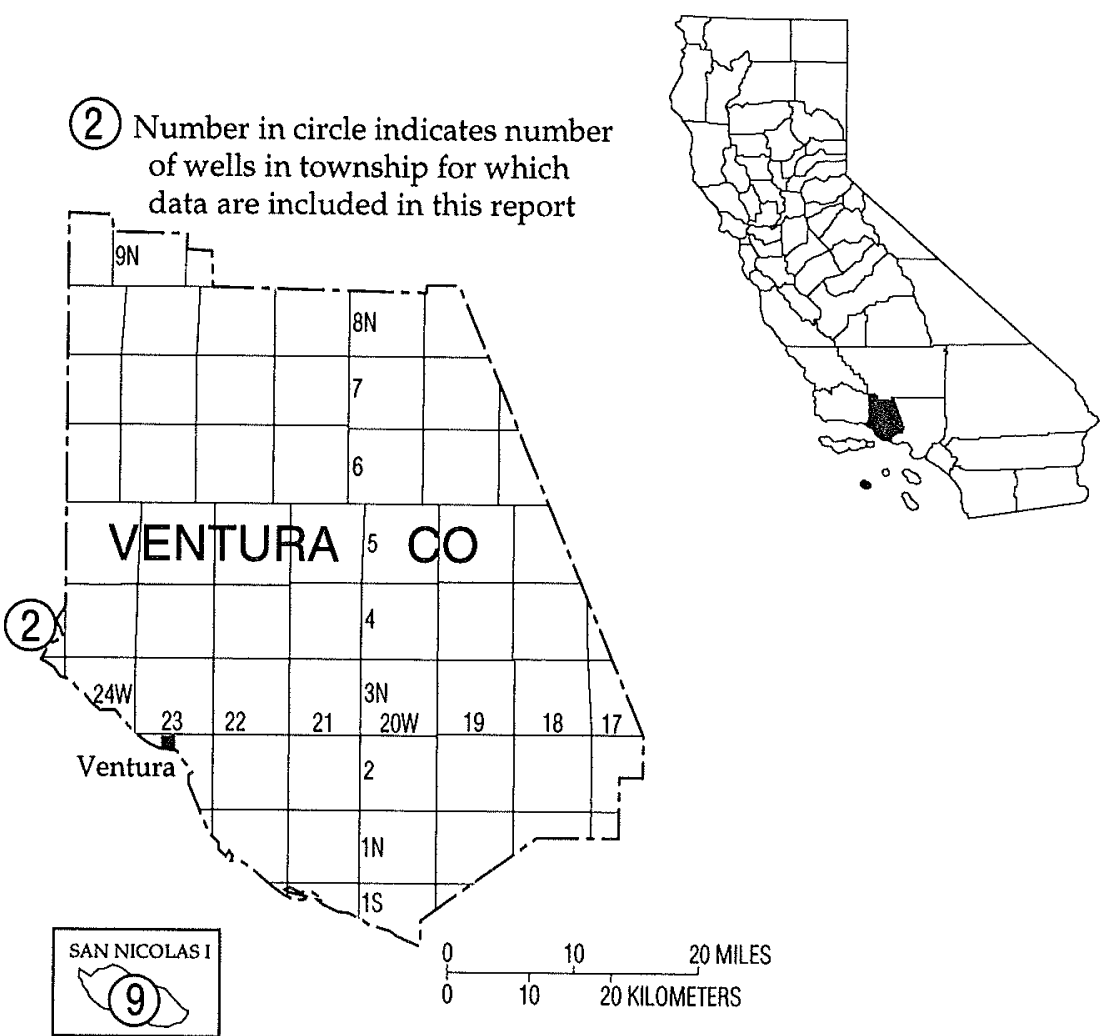

Figure 36, Location of wells in Ventura County. 
WELL DESCRIPTIONS AND WATER-LEVEL MEASUREMENTS

WATER YEAR OCTOBER 1991 TO SEPTEMBER 1992

SITE NUMBER 342323119274601

LOCAL, NUMBER 004N025W35A02S

About 3 miles east of Carpinteria. Drilled unused water-table well. Diameter 12 inches, depth measured 203.4 feet in 1990, original depth 403 feet, perforated 55-179, 231-401 feet. Altitude of land-surface datum 147 feet.

Measurements provided by Carpinteria County Water District beginning in 1979. Water-level records available 1941, $1945,1949-50$, 1955-56, 1979 to current year.

WATER LEVELS IN FEET BELOW LAND-SURFACE DATUM

$\begin{array}{llll}\text { WATER } & & \text { WATER } & \text { WATER } \\ \text { DATE } & \text { DEVEL MS } & \text { DATE } & \text { LEVEL MS }\end{array}$

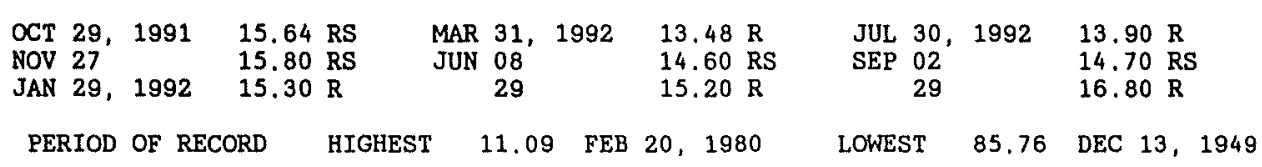

SITE NUMBER 342321119274601

LOCAL NUMBER O04N025W35A03S

East of Carpinteria, east of Rincon Creek Road, and south of Rincon Creek bridge. Drilled unused well. Diameter 10 inches, depth 145.8 feet in 1941. Altitude of land-surface datum 147 feet. Measurements provided by Carpinteria County Water District beginning in 1978. Water-level records available 1941, 1945-46, 1949-50, $1955-56,1959$ to current year.

WATER LEVELS IN FEET BELOW LAND-SURFACE DATUM

\begin{tabular}{lllllllll}
\multicolumn{1}{c}{$\begin{array}{l}\text { WATER } \\
\text { DATE }\end{array}$} & LEVEL MS & DATE & $\begin{array}{l}\text { WATER } \\
\text { LEVEL MS }\end{array}$ & DATE & WATER & LEVEL MS & WATE & WATER \\
LEVEL MS
\end{tabular}




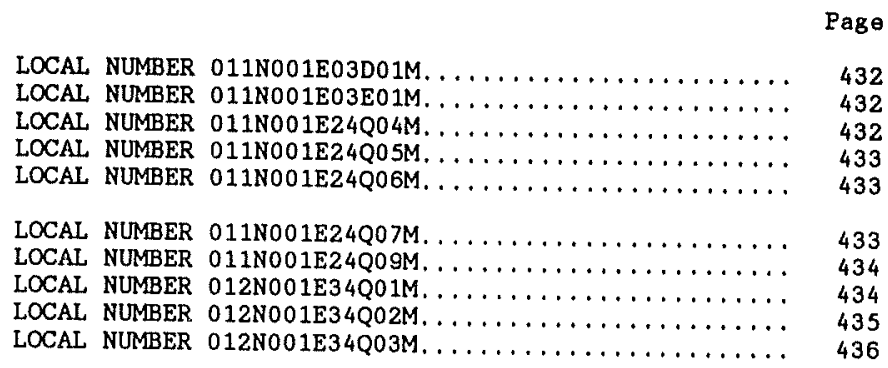

(3) Number in circle indicates number of wells in township for which data are included in this report

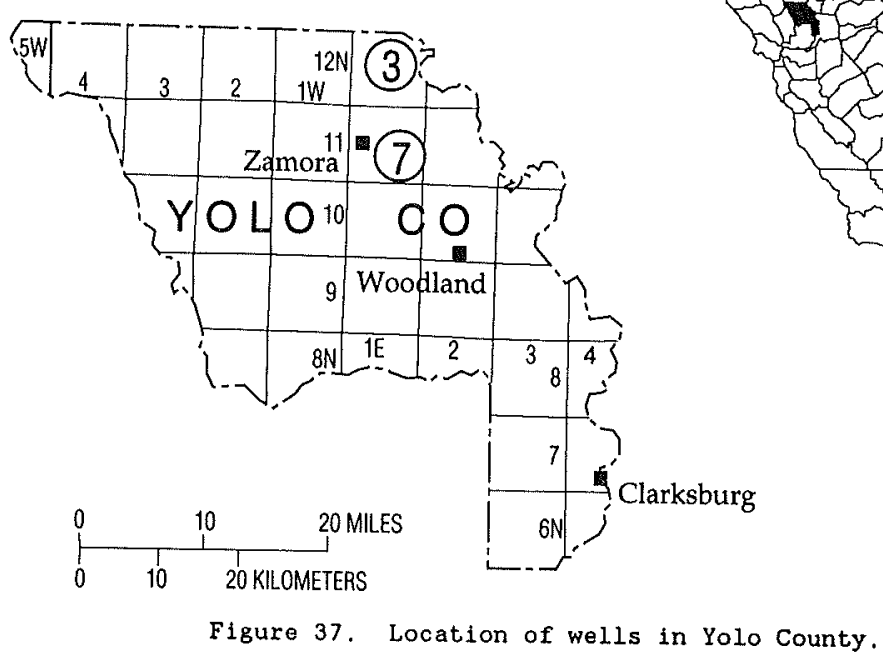




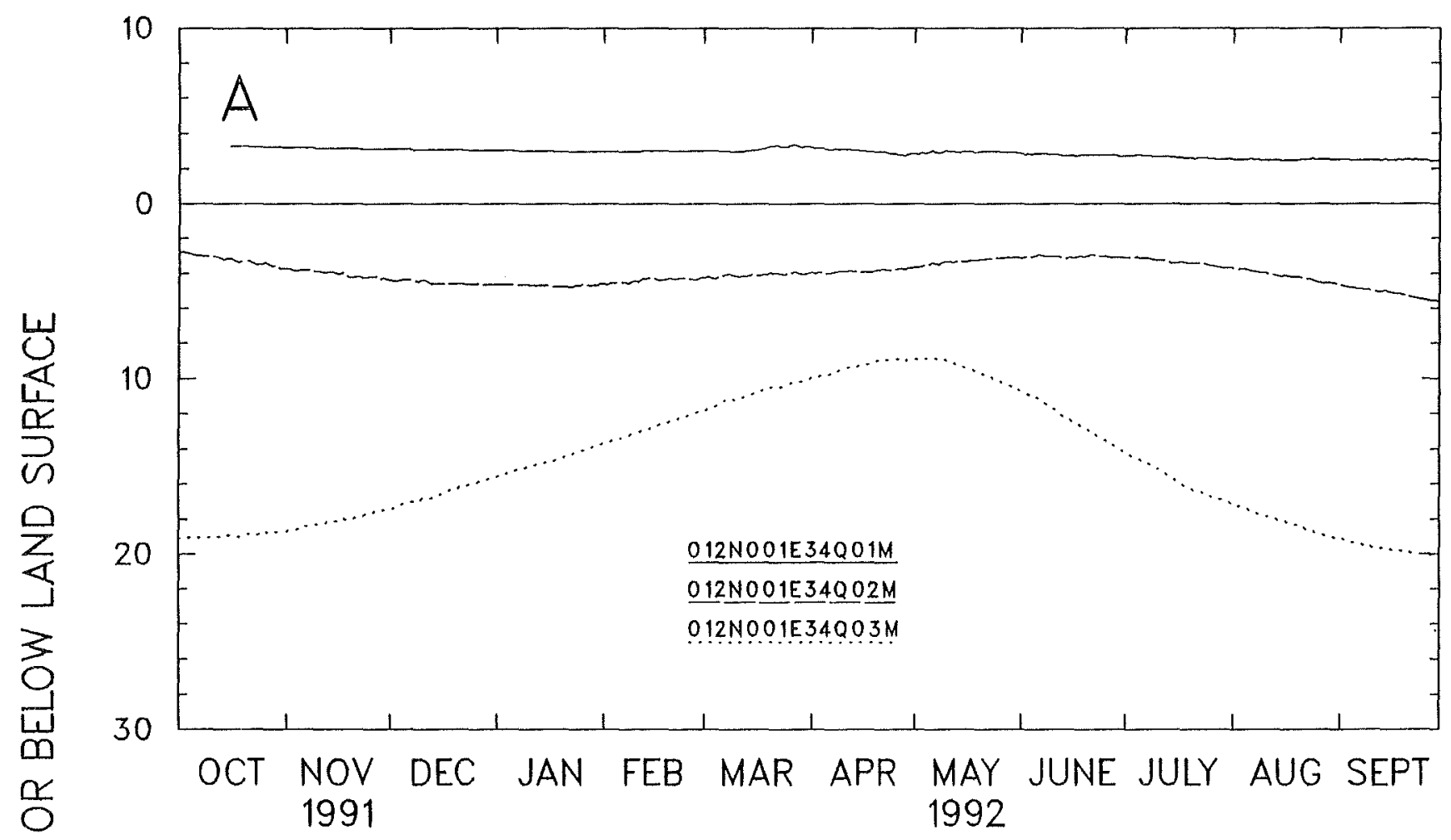

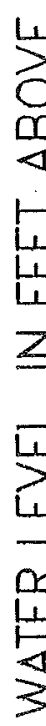

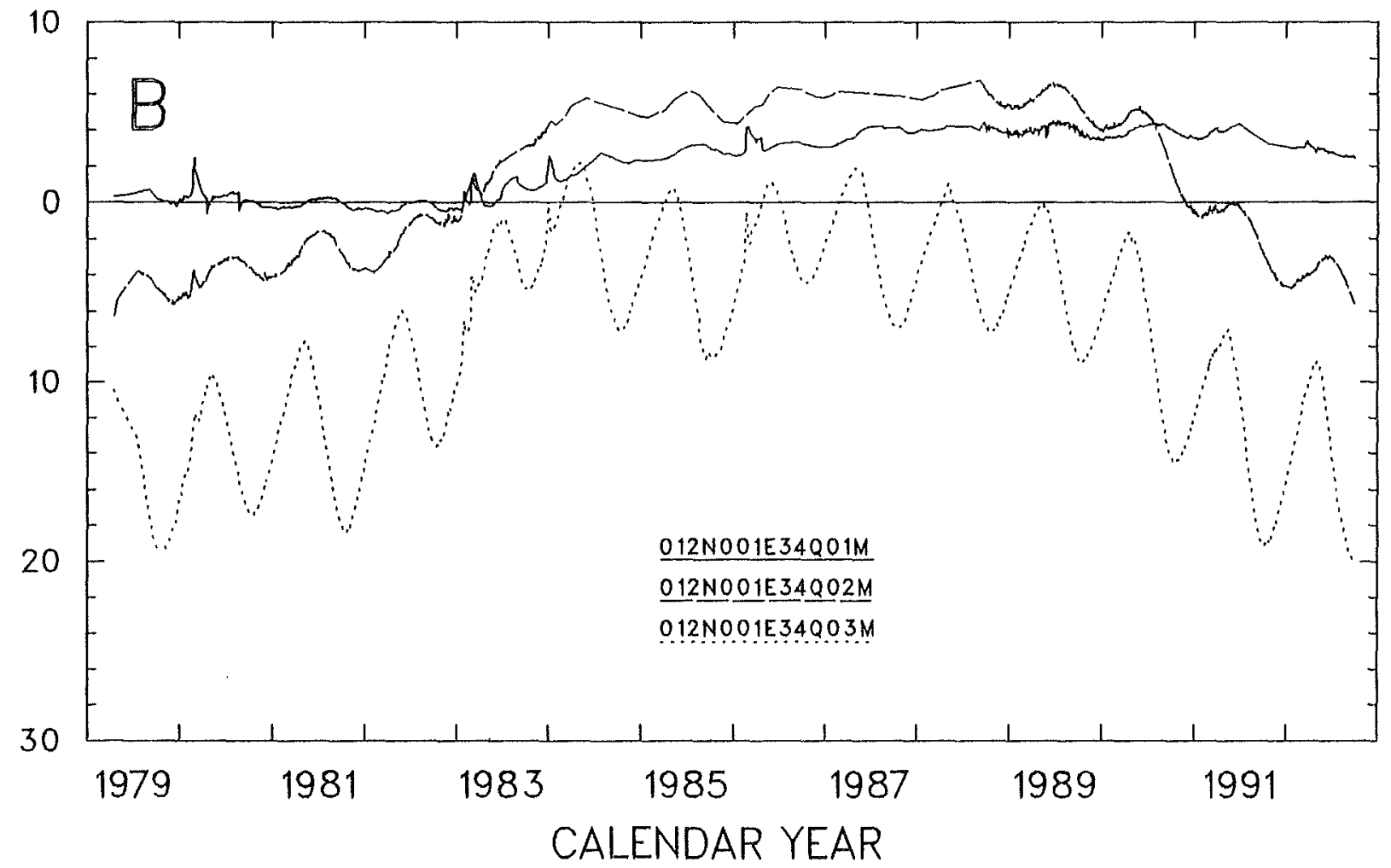

Figure 38. Hydrographs for wells in Yolo County. (012N001E34Q01M, 012N001E34Q02M, and 012N001E34Q03M) 
WELL DESCRIPTIONS AND WATER-LEVEL MEASUREMENTS

WATER YEAR OCTOBER 1991 TO SEPTEMBER 1992

SITE NUMBER 385009121512401

LOCAL NUMBER 011N001E03D01M

About $2.9 \mathrm{miles}$ northeast of Zamora. Unused water-table well in alluvium. Diameter 10 inches, depth 500 feet. Altitude of land-surface datum 30.16 feet. Water-level records available 1979 to current year.

WATER LEVELS IN FEET BELOW LAND-SURFACE DATUM

\begin{tabular}{llllll} 
& WATER & \multicolumn{2}{c}{ WATER } & & \multicolumn{2}{c}{ WATER } \\
DATE & LEVEL MS & DATE & LEVEL MS & DATE & LEVEL MS \\
16,1991 & $42.02 \mathrm{SS}$ & JAN 13,1992 & $18.41 \mathrm{~s}$ & AUG 06, 1992 & $53.12 \mathrm{~S}$ \\
14 & $26.37 \mathrm{~S}$ & MAR 04 & $14.39 \mathrm{~S}$ & &
\end{tabular}

PERIOD OF RECORD HIGHEST 4.85 MAR 02, $1984 \quad$ LOWEST 55.29 AUG 02,1991

SITE NUMBER 384951121512401

LOCAL NUMBER 011N001E03E01M

About $2.7 \mathrm{miles}$ northeast of Zamora. Domestic water-table well in alluvium. Diameter 8 inches, depth 260 feet, perforated 220-260 feet. Altitude of land-surface datum 33.13 feet. Water-1evel records avałlable 1979 to current year.

WATER LEVELS IN FEET BELOW LAND-SURFACE DATUM

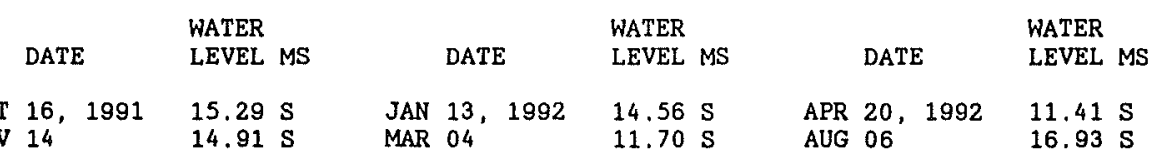

PERIOD OF RECORD HIGHEST 2.15 FEB 28, $1980 \quad$ LOWEST 16.93 AUG 06,1992

SITE NUMBER 384646121483801

LOCAL NUMBER 011N001E24Q04M

About $7 \mathrm{miles}$ north of Woodland, $2 \mathrm{miles}$ west of Highway 113. Drilled observation well in alluvium. Diameter 2 inches, depth 809 feet, perforated 784-789 feet. Deepest of four cluster wells. Altitude of land-surface datum 42.14 feet (1988). Data logger with pressure transducer June 1988 to current year. Water-level records available 1987 to current year.

WATER LEVELS IN FEET BELOW LAND-SURFACE DATUM

\begin{tabular}{|c|c|c|c|c|c|c|c|c|c|c|c|}
\hline DATE & $\begin{array}{l}\text { WATER } \\
\text { LEVEL MS }\end{array}$ & & DATE & $\begin{array}{l}\text { WATER } \\
\text { LEVEL MS }\end{array}$ & & DATE & $\begin{array}{l}\text { WATER } \\
\text { LEVEL MS }\end{array}$ & & DATY & & $\begin{array}{l}\text { WATER } \\
\text { LEVEL }\end{array}$ \\
\hline $\begin{array}{ll}16, & 1991 \\
14 & \\
19 & \end{array}$ & $\begin{array}{l}59.82 \mathrm{~s} \\
53.83 \\
52.98 \mathrm{~S}\end{array}$ & $\begin{array}{l}\text { DEC } \\
\text { JAN }\end{array}$ & $\begin{array}{ll}17, & 1991 \\
13, & 1992 \\
17 & \end{array}$ & $\begin{array}{l}47.85 \mathrm{~S} \\
43.75 \mathrm{~S} \\
43.16 \mathrm{~S}\end{array}$ & $\begin{array}{l}\text { FEB } \\
\text { MAR } \\
\text { APR }\end{array}$ & $\begin{array}{ll}14, & 1992 \\
18 & \\
20 & \end{array}$ & $\begin{array}{l}39.65 \mathrm{~S} \\
36.39 \mathrm{~S} \\
34.41 \mathrm{~S}\end{array}$ & $\begin{array}{l}\text { MAY } \\
\text { JUL } \\
\text { AUG }\end{array}$ & $\begin{array}{l}14, \\
23 \\
25\end{array}$ & 1992 & $\begin{array}{l}37.92 \\
62.77 \\
64.97\end{array}$ \\
\hline
\end{tabular}

PERIOD OF RECORD HIGHEST 24.73 MAR 18,1988 LOWEST $65.74 \quad$ SEP 12,1991 
WELL DESCRIPTIONS AND WATER-LEVEL MEASUREMENTS

WATER YEAR OCTOBER 1991 TO SEPTEMBER 1992

SITE NUMBER 384646121483802

LOCAL NUMBER 011N001E24Q05M

About 7 miles north of Woodland, 2 miles west of Highway 113 . Drilled observation well in alluvium. Diameter 2 inches, depth 608 feet, perforated 583-588 feet. Second deepest of four cluster wells. Altitude of land-surface datum 42.14 feet (1988). Data logger with pressure transducer June 1988 to current year. Water-level records available 1987 to current year.

WATER LEVELS IN FEET BELOW LAND-SURFACE DATUM

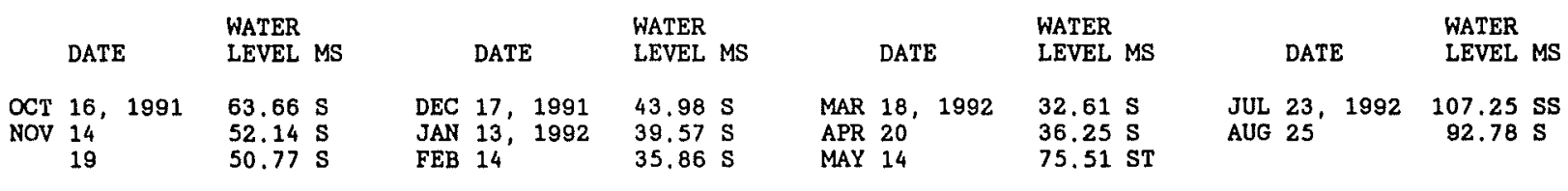

PERIOD OF RECORD HIGHEST 22.10 MAR 08, 1988 LOWEST 107.17 AUG 02, 1991

\section{SITE NUMBER 384646121483803}

LOCAL NUMBER O11N001E24Q06M

About 7 miles north of Woodland, 2 miles west of Highway 113. Drilled observation well in alluvium. Diameter 2 inches, depth 407 feet, perforated 382-387 feet. Second shallowest of four cluster wells. Altitude of land-surface datum 42.14 feet (1988). Data logger with pressure transducer June 1988 to current year. Water-level records available 1987 to current year.

WATER LEVELS IN FEET BELOW LAND-SURFACE DATUM

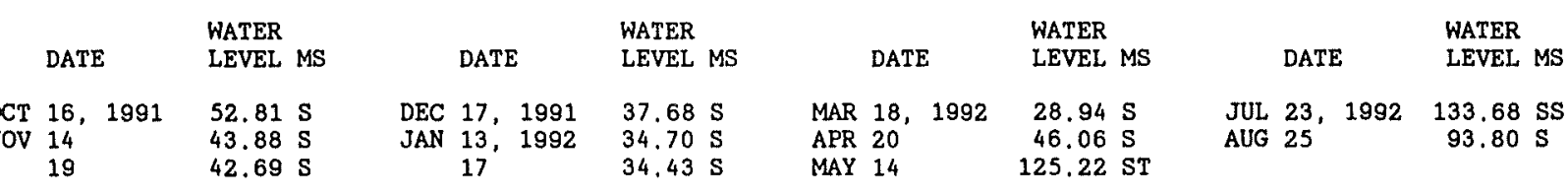

PERIOD OF RECORD HIGHEST 20.08 MAR 08, 1988 LOWEST 138.62 JUL 07, 1989

\section{SITE NUMBER 384646121483804}

LOCAL NUMBER O11N001E24Q07M

About 7 miles north of Woodland, 2 miles west of Highway 113. Drilled observation water-table well in alluvium. Diameter 2 inches, depth 220 feet, perforated 180-200 feet. Shallowest of four cluster wells. Altitude of land-surface datum 42.14 feet (1988). Data logger with pressure transducer June 1988 to current year. Water-level records avallable 1987 to current year.

WATER LEVELS IN FEET BELOW LAND-SURFACE DATUM

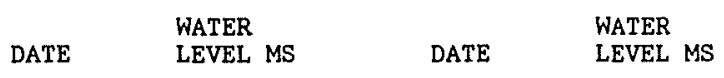

OCT 16,1991

NOV 14

DEC 17

$49.53 \mathrm{~S}$

$42.06 \mathrm{~S}$

$36.52 \mathrm{~S}$

$\begin{array}{lll}\text { JAN } 13,1992 & 33.92 \mathrm{~S} \\ \text { FEB } 14 & & 31.71 \mathrm{~S} \\ \text { MAR } 18 & 28.49 \mathrm{~S}\end{array}$

PERIOD OF RECORD HIGHEST 21.31 FEB 27, 1989 LOWEST 121.84 JUN 13, 1990

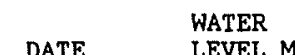

AUG $25,1992 \quad 83,73 \mathrm{~s}$ 
SITE NUMBER 384645121483801

LOCAL NUMBER O11N001E24Q09M

About 7 miles north of Woodland, 2 miles west of Highway 113. Drilled observation water-table well in alluvium. Diameter 2 inches, depth 215 feet, perforated 189-194 feet. Altitude of land-surface datum 41.9 feet. (1990). Water-level records available 1990 to current year.

WATER LEVELS IN FEET BELOW LAND-SURFACE DATUM

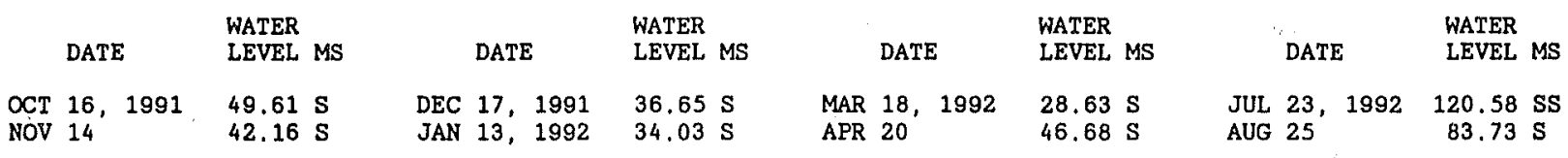

PERIOD OF RECORD HIGHEST 28.63 MAR 18, 1992 LOWEST 113.10 AUG 02,1991

SITE NUMBER 385020121503601

LOCAL NUMBER 012N001E34Q01M

About 4 miles northeast of Zamora, Drilled observation water-table well in alluvium, Diameter 2 inches, depth 2,125 feet, screened 2,120-2,125 feet. Deepest of three cluster wells. Altitude of land-surface datum 24,27 feet. Recorder installed 1979 to current year. Water-level records available 1979 to current year.

Missing record due to malfunction of recording instrument.

WATER LEVELS IN FEET BELOW LAND-SURFACE DATUM (READINGS ABOVE LAND SURFACE INDICATED BY "+") (OBSERVATIONS AT 1200 HOURS)

\begin{tabular}{|c|c|c|c|c|c|c|c|c|c|c|c|c|}
\hline DAY & OCT & NOV & DEC & JAN & FEB & MAR & APR & MAY & JUN & JUL & AUG & SEP \\
\hline $\begin{array}{l}1 \\
2 \\
3 \\
4 \\
5\end{array}$ & $\begin{array}{l}--- \\
--- \\
--- \\
--- \\
---\end{array}$ & $\begin{array}{l}--- \\
--- \\
--- \\
--- \\
---\end{array}$ & $\begin{array}{l}--- \\
--- \\
--- \\
--- \\
---\end{array}$ & $\begin{array}{l}--\infty \\
--- \\
--- \\
--- \\
---\end{array}$ & $\begin{array}{l}--- \\
--- \\
--- \\
---\end{array}$ & $\begin{array}{r}--- \\
--- \\
S+3.02 \\
\\
---\end{array}$ & $\begin{array}{l}+3.21 \\
+3.18 \\
+3.17 \\
+3.17 \\
+3.11\end{array}$ & $\begin{array}{l}+2.85 \\
+2.82 \\
+2.87 \\
+2.90 \\
+2.94\end{array}$ & $\begin{array}{l}+2.83 \\
+2.82 \\
+2.83 \\
+2.82 \\
+2.83\end{array}$ & $\begin{array}{l}+2.72 \\
+2.73 \\
+2.73 \\
+2.75 \\
+2.75\end{array}$ & $\begin{array}{l}+2.54 \\
+2.52 \\
+2.51 \\
+2.51 \\
+2.52\end{array}$ & $\begin{array}{l}+2.52 \\
+2.52 \\
+2.51 \\
+2.49 \\
+2.50\end{array}$ \\
\hline $\begin{array}{r}6 \\
7 \\
8 \\
9 \\
10\end{array}$ & $\begin{array}{l}--- \\
--- \\
--- \\
--- \\
---\end{array}$ & $\begin{array}{l}--- \\
--- \\
--- \\
--- \\
---\end{array}$ & $\begin{array}{l}--- \\
--- \\
--- \\
--- \\
---\end{array}$ & $\begin{array}{l}--\infty \\
--- \\
--\infty \\
--\infty \\
--\infty\end{array}$ & $\begin{array}{l}-\cdots \\
--- \\
-\cdots \\
---\end{array}$ & $\begin{array}{l}-m- \\
--- \\
--- \\
---\end{array}$ & $\begin{array}{l}+3.08 \\
+3.09 \\
+3.11 \\
+3.08 \\
+3.11\end{array}$ & $\begin{array}{l}+2.99 \\
+2.94 \\
+2.88 \\
+2.94 \\
+2.99\end{array}$ & $\begin{array}{l}+2.85 \\
+2.84 \\
+2.83 \\
+2.81 \\
+2.81\end{array}$ & $\begin{array}{l}+2.73 \\
+2.73 \\
+2.71 \\
+2.70 \\
+2.68\end{array}$ & $\begin{array}{l}+2.56 \\
+2.52 \\
+2.51 \\
+2.49 \\
+2.49\end{array}$ & $\begin{array}{l}+2.48 \\
+2.49 \\
+2.50 \\
+2.50 \\
+2.49\end{array}$ \\
\hline $\begin{array}{l}11 \\
12 \\
13 \\
14 \\
15\end{array}$ & $\begin{array}{l}--- \\
--- \\
--- \\
--- \\
---\end{array}$ & $\begin{array}{r}--- \\
--- \\
-\cdots \\
S+3.17 \\
---\end{array}$ & $\begin{array}{l}--- \\
--- \\
-\cdots \\
--- \\
---\end{array}$ & $S+3.01$ & $\begin{array}{l}--- \\
--- \\
--- \\
--- \\
---\end{array}$ & $\begin{array}{r}+2.97 \\
+3.02 \\
+3.01 \\
+3.04\end{array}$ & $\begin{array}{l}+3.08 \\
+3.08 \\
+3.03 \\
+3.04 \\
+3.01\end{array}$ & $\begin{array}{l}+2.98 \\
+2.95 \\
+2.94 \\
+2.92 \\
+2.96\end{array}$ & $\begin{array}{l}+2.81 \\
+2.78 \\
+2.72 \\
+2.71 \\
+2.72\end{array}$ & $\begin{array}{l}+2.68 \\
+2.68 \\
+2.67 \\
+2.65 \\
+2.64\end{array}$ & $\begin{array}{l}+2.50 \\
+2.49 \\
+2.50 \\
+2.49 \\
+2.47\end{array}$ & $\begin{array}{l}+2.50 \\
+2.49 \\
+2.50 \\
+2.51 \\
+2.50\end{array}$ \\
\hline $\begin{array}{l}16 \\
17 \\
18 \\
19 \\
20\end{array}$ & $\begin{array}{r}S+3.27 \\
-- \\
-- \\
--\end{array}$ & $\begin{array}{l}--- \\
--- \\
--- \\
--- \\
---\end{array}$ & $\begin{array}{l}--- \\
--- \\
--- \\
--- \\
---\end{array}$ & $\begin{array}{l}--- \\
--- \\
--- \\
--- \\
---\end{array}$ & $\begin{array}{l}--- \\
--- \\
--- \\
---\end{array}$ & $\begin{array}{l}+3.05 \\
+3.07 \\
+3.13 \\
+3.18 \\
+3.27\end{array}$ & $\begin{array}{r}+2.99 \\
+2.98 \\
+2.96 \\
+2.96 \\
---\end{array}$ & $\begin{array}{l}+2.95 \\
+2.96 \\
+2.94 \\
+2.93 \\
+2.97\end{array}$ & $\begin{array}{l}+2.74 \\
+2.73 \\
+2.77 \\
+2.78 \\
+2.77\end{array}$ & $\begin{array}{l}+2.62 \\
+2.63 \\
+2.62 \\
+2.60 \\
+2.59\end{array}$ & $\begin{array}{l}+2.48 \\
+2.48 \\
+2.49 \\
+2.49 \\
+2.49\end{array}$ & $\begin{array}{l}+2.50 \\
+2.50 \\
+2.51 \\
+2.50 \\
+2.51\end{array}$ \\
\hline $\begin{array}{l}21 \\
22 \\
23 \\
24 \\
25\end{array}$ & $\begin{array}{l}--- \\
--- \\
--- \\
--- \\
---\end{array}$ & $\begin{array}{l}--- \\
--- \\
--- \\
--- \\
---\end{array}$ & $\begin{array}{l}--- \\
--- \\
--- \\
---\end{array}$ & $\begin{array}{r}S+2.98 \\
--- \\
--- \\
-- \\
---\end{array}$ & $\begin{array}{l}--- \\
--- \\
--- \\
--- \\
-\cdots\end{array}$ & $\begin{array}{l}+3.29 \\
+3.32 \\
+3.24 \\
+3.22 \\
+3.26\end{array}$ & $\begin{array}{r}--- \\
+2.97 \\
+2.88 \\
+2.83 \\
+2.83\end{array}$ & $\begin{array}{l}+2.96 \\
+2.96 \\
+2.96 \\
+2.95 \\
+2.95\end{array}$ & $\begin{array}{l}+2.78 \\
+2.78 \\
+2.76 \\
+2.75 \\
+2.75\end{array}$ & $\begin{array}{l}+2.58 \\
+2.59 \\
+2.59 \\
+2.59 \\
+2.57\end{array}$ & $\begin{array}{l}+2.52 \\
+2.52 \\
+2.54 \\
+2.56 \\
+2.53\end{array}$ & $\begin{array}{l}+2.51 \\
+2.50 \\
+2.52 \\
+2.49 \\
+2.50\end{array}$ \\
\hline $\begin{array}{l}26 \\
27 \\
28 \\
29 \\
30 \\
31\end{array}$ & $\begin{array}{l}--- \\
--- \\
--- \\
--- \\
--- \\
---\end{array}$ & $\begin{array}{l}--- \\
--- \\
--- \\
--- \\
--- \\
---\end{array}$ & $\begin{array}{l}--- \\
--- \\
--- \\
--- \\
--- \\
-\cdots\end{array}$ & $\begin{array}{l}--- \\
--- \\
--- \\
--- \\
--- \\
---\end{array}$ & $\begin{array}{l}--- \\
--- \\
--- \\
--- \\
--- \\
---\end{array}$ & $\begin{array}{l}+3.33 \\
+3.36 \\
+3.27 \\
+3.21 \\
+3.22 \\
+3.23\end{array}$ & $\begin{array}{r}+2.77 \\
+2.76 \\
+2.72 \\
+2.79 \\
+2.85 \\
---\end{array}$ & $\begin{array}{l}+2.94 \\
+2.91 \\
+2.92 \\
+2.93 \\
+2.91 \\
+2.88\end{array}$ & $\begin{array}{r}+2.76 \\
+2.75 \\
+2.75 \\
+2.69 \\
+2.70 \\
---\end{array}$ & $\begin{array}{l}+2.55 \\
+2.54 \\
+2.54 \\
+2.53 \\
+2.54 \\
+2.55\end{array}$ & $\begin{array}{l}+2.53 \\
+2.51 \\
+2.51 \\
+2.53 \\
+2.52 \\
+2.51\end{array}$ & $\begin{array}{r}+2.48 \\
+2.46 \\
+2.46 \\
+2.45 \\
+2.45 \\
---\end{array}$ \\
\hline
\end{tabular}

PERIOD OF RECORD HIGHEST $+4.43 \quad$ SEP 19, $1988 \quad$ LOWEST 0.57 APR 05, 1982

S Manual steol tape measurement. 
WATER LEVELS, YOLO COUNTY--Continued

WELL DESCRIPTIONS AND WATER-LEVEL MEASUREMENTS

WATER YEAR OCTOBER 1991 TO SEPTEMBER 1992

SITE NUMBER 385020121503602

LOCAL NUMBER 012N001E34Q02M

About 4 miles northeast of Zamora. Drilled observation water-table well in alluvium. Diameter 3 inches, depth 1,401 feet, screened 1,396-1,401 feet. Middle of three cluster wells. Altitude of land-surface datum 24.27 feet. Recorder installed 1979 to current year. Water-level records avallable 1979 to current year.

Missing record due to malfunction of recording instrument.

WATER LEVELS IN FEET BELOW LAND-SURFACE DATUM (READINGS ABOVE LAND SURFACE INDICATED BY "+") (OBSERVATIONS AT 1200 HOURS)

\begin{tabular}{|c|c|c|c|c|c|c|c|c|c|c|c|c|}
\hline DAY & $O C T$ & NOV & DEC & JAN & FEB & MAR & APR & MAY & JUN & JUL & AUG & SEP \\
\hline $\begin{array}{l}1 \\
2 \\
3 \\
4 \\
5\end{array}$ & $\begin{array}{l}2.81 \\
2.78 \\
2.81 \\
2.86 \\
2.93\end{array}$ & $\begin{array}{l}3.75 \\
3.78 \\
3.85 \\
3.87 \\
3.84\end{array}$ & $\begin{array}{l}4.42 \\
4.44 \\
4.45 \\
4.40 \\
4.39\end{array}$ & $\begin{array}{l}--- \\
--- \\
--- \\
--\end{array}$ & $\begin{array}{l}4.63 \\
4.66 \\
4.59 \\
4.59 \\
4.58\end{array}$ & $\begin{array}{l}4.26 \\
4.22 \\
4.31 \\
4.34 \\
4.24\end{array}$ & $\begin{array}{l}3.98 \\
4.01 \\
3.99 \\
3.99 \\
4.03\end{array}$ & $\begin{array}{l}3.67 \\
3.67 \\
3.62 \\
3.57 \\
3.49\end{array}$ & $\begin{array}{l}3.15 \\
3.12 \\
3.08 \\
3.07 \\
3.02\end{array}$ & $\begin{array}{l}3.13 \\
3.15 \\
3.13 \\
3.07 \\
3.14\end{array}$ & $\begin{array}{l}3.73 \\
3.77 \\
3.76 \\
3.76 \\
3.78\end{array}$ & $\begin{array}{l}4.66 \\
4.72 \\
4.77 \\
4.80 \\
4.82\end{array}$ \\
\hline $\begin{array}{r}6 \\
7 \\
8 \\
9 \\
10\end{array}$ & $\begin{array}{l}2.97 \\
2.98 \\
3.01 \\
3.04 \\
3.05\end{array}$ & $\begin{array}{l}3.81 \\
3.84 \\
3.84 \\
3.88 \\
3.88\end{array}$ & $\begin{array}{l}4.37 \\
4.38 \\
4.43 \\
4.51 \\
4.41\end{array}$ & $\begin{array}{l}--- \\
--- \\
--- \\
--- \\
---\end{array}$ & $\begin{array}{l}4.54 \\
4.55 \\
4.62 \\
4.50 \\
4.47\end{array}$ & $\begin{array}{r}4.17 \\
4.09 \\
4.07 \\
4.15 \\
-.-\end{array}$ & $\begin{array}{l}4.03 \\
3.99 \\
3.94 \\
3.91 \\
3.90\end{array}$ & $\begin{array}{l}3.41 \\
3.45 \\
3.50 \\
3.38 \\
3.33\end{array}$ & $\begin{array}{l}2.95 \\
2.97 \\
3.02 \\
3.04 \\
3.02\end{array}$ & $\begin{array}{l}3.16 \\
3.16 \\
3.20 \\
3.24 \\
3.27\end{array}$ & $\begin{array}{l}3.87 \\
3.89 \\
3.95 \\
3.97 \\
3.99\end{array}$ & $\begin{array}{l}4.87 \\
4.89 \\
4.93 \\
4.93 \\
4.97\end{array}$ \\
\hline $\begin{array}{l}11 \\
12 \\
13 \\
14 \\
15\end{array}$ & $\begin{array}{l}3.04 \\
3.08 \\
3.16 \\
3.21 \\
3.18\end{array}$ & $\begin{array}{l}3.95 \\
3.99 \\
3.92 \\
3.93 \\
4.01\end{array}$ & $\begin{array}{l}4.43 \\
4.57 \\
4.60 \\
4.62 \\
4.61\end{array}$ & s4.69 & $\begin{array}{l}4.49 \\
4.34 \\
4.35 \\
4.31 \\
4.26\end{array}$ & $\begin{array}{r}54.22 \\
4.17 \\
4.16 \\
4.13 \\
4.11\end{array}$ & $\begin{array}{l}3.89 \\
3.86 \\
3.90 \\
3.91 \\
3.91\end{array}$ & $\begin{array}{l}3.33 \\
3.38 \\
3.36 \\
3.34 \\
3.31\end{array}$ & $\begin{array}{l}3.03 \\
3.08 \\
3.15 \\
3.12 \\
3.06\end{array}$ & $\begin{array}{l}3.28 \\
3.26 \\
3.34 \\
3.37 \\
3.39\end{array}$ & $\begin{array}{l}4.00 \\
4.02 \\
4.08 \\
4.14 \\
4.17\end{array}$ & $\begin{array}{l}5.01 \\
5.05 \\
5.04 \\
5.02 \\
5.07\end{array}$ \\
\hline $\begin{array}{l}16 \\
17 \\
18 \\
19 \\
20\end{array}$ & $\begin{array}{l}3.18 \\
3.27 \\
3.39 \\
3.36 \\
3.30\end{array}$ & $\begin{array}{l}4.07 \\
3.99 \\
4.17 \\
4.26 \\
4.24\end{array}$ & $\begin{array}{c}4.60 \\
- \\
- \\
--\end{array}$ & $\begin{array}{l}--- \\
--- \\
--- \\
--\end{array}$ & $\begin{array}{l}4.33 \\
4.39 \\
4.39 \\
4.34 \\
4.31\end{array}$ & $\begin{array}{l}4.10 \\
4.10 \\
4.05 \\
4.05 \\
3.99\end{array}$ & $\begin{array}{r}3.89 \\
3.91 \\
3.89 \\
3.87 \\
\text { s3.81 }\end{array}$ & $\begin{array}{l}3.30 \\
3.25 \\
3.27 \\
3.28 \\
3.22\end{array}$ & $\begin{array}{l}3.10 \\
3.08 \\
3.02 \\
3.05 \\
3.03\end{array}$ & $\begin{array}{l}3.37 \\
3.33 \\
3.38 \\
3.44 \\
3.44\end{array}$ & $\begin{array}{l}4.15 \\
4.17 \\
4.20 \\
4.23 \\
4.23\end{array}$ & $\begin{array}{l}5.14 \\
5.18 \\
5.18 \\
5.21 \\
5.25\end{array}$ \\
\hline $\begin{array}{l}21 \\
22 \\
23 \\
24 \\
25\end{array}$ & $\begin{array}{l}3.31 \\
3.39 \\
3.50 \\
3.52 \\
3.51\end{array}$ & $\begin{array}{l}4.21 \\
4.23 \\
4.31 \\
4.28 \\
4.26\end{array}$ & $\begin{array}{l}--- \\
--- \\
-\cdots \\
--- \\
---\end{array}$ & $\begin{array}{r}54.74 \\
4.77 \\
4.76 \\
4.70 \\
4.69\end{array}$ & $\begin{array}{l}4.33 \\
4.35 \\
4.30 \\
4.30 \\
4.31\end{array}$ & $\begin{array}{l}4.01 \\
4.01 \\
4.07 \\
4.10 \\
4.02\end{array}$ & $\begin{array}{l}--- \\
3.78 \\
3.80 \\
3.81 \\
3.79\end{array}$ & $\begin{array}{l}3.23 \\
3.20 \\
3.18 \\
3.18 \\
3.15\end{array}$ & $\begin{array}{l}2.96 \\
2.96 \\
3.04 \\
3.02 \\
3.03\end{array}$ & $\begin{array}{l}3.43 \\
3.42 \\
3.46 \\
3.50 \\
3.53\end{array}$ & $\begin{array}{l}4.25 \\
4.33 \\
4.34 \\
4.38 \\
4.50\end{array}$ & $\begin{array}{l}5.30 \\
5.36 \\
5.42 \\
5.46 \\
5.48\end{array}$ \\
\hline $\begin{array}{l}26 \\
27 \\
28 \\
29 \\
30 \\
31\end{array}$ & $\begin{array}{l}3.41 \\
3.60 \\
3.64 \\
3.61 \\
3.73 \\
3.76\end{array}$ & $\begin{array}{r}4.24 \\
4.27 \\
4.32 \\
4.31 \\
4.37 \\
. .-\end{array}$ & $\overline{-}$ & $\begin{array}{l}4.65 \\
4.69 \\
4.75 \\
4.70 \\
4.71 \\
4.61\end{array}$ & $\begin{array}{r}4.35 \\
4.38 \\
4.37 \\
4.27 \\
\ldots \ldots\end{array}$ & $\begin{array}{l}3.99 \\
3.92 \\
4.01 \\
4.06 \\
4.04 \\
4.01\end{array}$ & $\begin{array}{r}3.74 \\
3.73 \\
3.78 \\
3.72 \\
3.68 \\
-\end{array}$ & $\begin{array}{l}3.14 \\
3.14 \\
3.10 \\
3.09 \\
3.10 \\
3.12\end{array}$ & $\begin{array}{r}3.06 \\
3.09 \\
3.09 \\
3.10 \\
3.12 \\
-\end{array}$ & $\begin{array}{l}3.60 \\
3.63 \\
3.66 \\
3.68 \\
3.68 \\
3.68\end{array}$ & $\begin{array}{l}4.55 \\
4.59 \\
4.58 \\
4.59 \\
4.63 \\
4.64\end{array}$ & $\begin{array}{r}5.52 \\
5.57 \\
5.58 \\
5.57 \\
5.64 \\
---\end{array}$ \\
\hline
\end{tabular}

PERIOD OF RECORD HIGHEST +6.74 SEP 06, 1988 LOWEST 6.31 APR 18, 1979

S Manual steel tape measurement. 
WELL DESCRIPTIONS AND WATER-LEVEL MEASUREMENTS

WATER YEAR OCTOBER 1991 TO SEPTEMBER 1992

SITE NUMBER 385020121503603

LOCAL NUMBER 012N001E34Q03M

About 4 miles northeast of Zamora, Drilled observation water-table well in alluvium. Diameter 3 inches, depth 947 feet, screened 942-947 feet. Shallowest of three cluster wolls. Altitude of land-surface datum 24.27 feet. Recorder installed 1979 to current year. Water-level records avałlable 1979 to current year.

Missing record due to malfunction of recording instrument.

WATER LEVELS IN FEET BELOW LAND-SURFACE DATUM (READINGS ABOVE LAND SURFACE INDICATED BY "+") (OBSERVATIONS AT 1200 HOURS)

\begin{tabular}{|c|c|c|c|c|c|c|c|c|c|c|c|c|}
\hline DAY & OCT & NOV & DEC & JAN & FEB & MAR & APR & MAY & JUN & JUL & AUG & SEP \\
\hline $\begin{array}{l}1 \\
2 \\
3 \\
4 \\
5\end{array}$ & $\begin{array}{l}19.10 \\
19.07 \\
19.05 \\
19.05 \\
19.09\end{array}$ & $\begin{array}{l}18.68 \\
18.64 \\
18.63 \\
18.59 \\
18.51\end{array}$ & $\begin{array}{l}17.44 \\
17.38 \\
17.31 \\
17.21 \\
17.13\end{array}$ & $\begin{array}{l}-\cdots \\
--- \\
\cdots- \\
---\end{array}$ & $\begin{array}{l}--- \\
--- \\
--- \\
---\end{array}$ & $\begin{array}{r}\ldots- \\
\cdots 11.61 \\
\cdots\end{array}$ & $\begin{array}{l}9.95 \\
9.93 \\
9.87 \\
9.83 \\
9.80\end{array}$ & $\begin{array}{l}8.90 \\
8.91 \\
8.87 \\
8.85 \\
8.83\end{array}$ & $\begin{array}{l}10.73 \\
10.82 \\
10.90 \\
10.99 \\
11.06\end{array}$ & $\begin{array}{l}14.23 \\
14.35 \\
14.42 \\
14.47 \\
14.61\end{array}$ & $\begin{array}{l}17.16 \\
17.23 \\
17.26 \\
17.31 \\
17.41\end{array}$ & $\begin{array}{l}19.10 \\
19.17 \\
19.24 \\
19.30 \\
19.32\end{array}$ \\
\hline $\begin{array}{r}6 \\
7 \\
8 \\
9 \\
10\end{array}$ & $\begin{array}{l}19.09 \\
19.06 \\
19.05 \\
19.05 \\
19.05\end{array}$ & $\begin{array}{l}18.44 \\
18.40 \\
18.36 \\
18.33 \\
18.27\end{array}$ & $\begin{array}{l}17.06 \\
17.02 \\
16.99 \\
16.99 \\
16.85\end{array}$ & $\begin{array}{l}--- \\
--- \\
--- \\
---\end{array}$ & $\begin{array}{l}--- \\
-- \\
--- \\
---\end{array}$ & $\begin{array}{l}11.24 \\
11.24 \\
11.20 \\
11.24\end{array}$ & $\begin{array}{l}9.75 \\
9.67 \\
9.60 \\
9.52 \\
9.46\end{array}$ & $\begin{array}{l}8.81 \\
8.87 \\
8.94 \\
8.94 \\
8.95\end{array}$ & $\begin{array}{l}11.13 \\
11.27 \\
11.43 \\
11.58 \\
11.68\end{array}$ & $\begin{array}{l}14.68 \\
14.76 \\
14.90 \\
14.98 \\
15.11\end{array}$ & $\begin{array}{l}17.55 \\
17.61 \\
17.69 \\
17.74 \\
17.81\end{array}$ & $\begin{array}{l}19.38 \\
19.42 \\
19.48 \\
19.50 \\
19.57\end{array}$ \\
\hline $\begin{array}{l}11 \\
12 \\
13 \\
14 \\
15\end{array}$ & $\begin{array}{l}19.00 \\
19.01 \\
19.02 \\
19.03 \\
18.96\end{array}$ & $\begin{array}{l}18.26 \\
18.26 \\
18.16 \\
18.12 \\
18.12\end{array}$ & $\begin{array}{l}16.82 \\
16.83 \\
16.81 \\
16.71 \\
16.63\end{array}$ & S14.85 & $\begin{array}{l}--- \\
--- \\
--- \\
---\end{array}$ & $\begin{array}{l}11.16 \\
11.07 \\
11.00 \\
10.93 \\
10.87\end{array}$ & $\begin{array}{l}9.41 \\
9.34 \\
9.32 \\
9.29 \\
9.25\end{array}$ & $\begin{array}{l}9.00 \\
9.12 \\
9.20 \\
9.27 \\
9.31\end{array}$ & $\begin{array}{l}11.80 \\
11.98 \\
12.17 \\
12.28 \\
12.35\end{array}$ & $\begin{array}{l}15.22 \\
15.32 \\
15.48 \\
15.62 \\
15.76\end{array}$ & $\begin{array}{l}17.87 \\
17.93 \\
18.00 \\
18.07 \\
18.12\end{array}$ & $\begin{array}{l}19.61 \\
19.66 \\
19.65 \\
19.64 \\
19.69\end{array}$ \\
\hline $\begin{array}{l}16 \\
17 \\
18 \\
19 \\
20\end{array}$ & $\begin{array}{l}18.92 \\
18.97 \\
18.02 \\
18.98 \\
18.89\end{array}$ & $\begin{array}{l}18.12 \\
17.97 \\
18.05 \\
18.05 \\
17.99\end{array}$ & $\begin{array}{r}16.56 \\
16.45 \\
--- \\
-- \\
---\end{array}$ & $\begin{array}{r}-.7 \\
\text { S14. } \\
-.- \\
--\end{array}$ & $\begin{array}{l}--- \\
--- \\
--- \\
---\end{array}$ & $\begin{array}{l}10.79 \\
10.73 \\
10.64 \\
10.60 \\
10.50\end{array}$ & $\begin{array}{r}9.21 \\
9.17 \\
9.15 \\
9.08 \\
\text { s8.99 }\end{array}$ & $\begin{array}{l}9.38 \\
9.44 \\
9.52 \\
9.60 \\
9.68\end{array}$ & $\begin{array}{l}12.47 \\
12.57 \\
12.67 \\
12.77 \\
12.90\end{array}$ & $\begin{array}{l}15.88 \\
15.99 \\
16.12 \\
16.24 \\
16.31\end{array}$ & $\begin{array}{l}18.14 \\
18.20 \\
18.28 \\
18.32 \\
18.35\end{array}$ & $\begin{array}{l}19.75 \\
19.79 \\
19.80 \\
19.82 \\
19.85\end{array}$ \\
\hline $\begin{array}{l}21 \\
22 \\
23 \\
24 \\
25\end{array}$ & $\begin{array}{l}18.85 \\
18.86 \\
18.90 \\
18.87 \\
18.83\end{array}$ & $\begin{array}{l}17.91 \\
17.87 \\
17.86 \\
17.77 \\
17.70\end{array}$ & $\begin{array}{l}--- \\
--- \\
--- \\
--\end{array}$ & $\begin{array}{r}\text { S14. } 41 \\
\cdots \\
-\cdots \\
-\cdots\end{array}$ & $\begin{array}{l}--- \\
-- \\
-- \\
-- \\
-\cdots\end{array}$ & $\begin{array}{l}10.49 \\
10.48 \\
10.49 \\
10.46 \\
10.34\end{array}$ & $\begin{array}{l}-.- \\
8.95 \\
8.95 \\
8.96 \\
8.92\end{array}$ & $\begin{array}{r}9.77 \\
9.83 \\
9.93 \\
10.02 \\
10.10\end{array}$ & $\begin{array}{l}13.00 \\
13.10 \\
13.30 \\
13.41 \\
13.50\end{array}$ & $\begin{array}{l}16.38 \\
16.42 \\
16.49 \\
16.59 \\
16.66\end{array}$ & $\begin{array}{l}18.41 \\
18.56 \\
18.61 \\
18.67 \\
18.79\end{array}$ & $\begin{array}{l}19.89 \\
19.91 \\
19.97 \\
19.99 \\
20.01\end{array}$ \\
\hline $\begin{array}{l}26 \\
27 \\
28 \\
29 \\
30 \\
31\end{array}$ & $\begin{array}{l}18.69 \\
18.80 \\
18.79 \\
18.71 \\
18.75 \\
18.74\end{array}$ & $\begin{array}{r}17.61 \\
17.58 \\
17.56 \\
17.49 \\
17.47 \\
.\end{array}$ & $\begin{array}{l}--- \\
-- \\
-- \\
--- \\
--\end{array}$ & 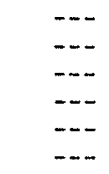 & $\begin{array}{l}--- \\
--- \\
--- \\
--- \\
---\end{array}$ & $\begin{array}{l}10.26 \\
10.15 \\
10.17 \\
10.15 \\
10.09 \\
10.02\end{array}$ & $\begin{array}{l}8.89 \\
8.90 \\
8.98 \\
8.95 \\
8.90 \\
-.-\end{array}$ & $\begin{array}{l}10.19 \\
10.29 \\
10.34 \\
10.43 \\
10.53 \\
10.65\end{array}$ & $\begin{array}{r}13.63 \\
13.75 \\
13.84 \\
13.97 \\
14.11 \\
.-5\end{array}$ & $\begin{array}{l}16.75 \\
16.81 \\
16.86 \\
16.89 \\
16.98 \\
17.06\end{array}$ & $\begin{array}{l}18.86 \\
18.91 \\
18.93 \\
18.97 \\
19.03 \\
19.07\end{array}$ & $\begin{array}{l}20.04 \\
20.06 \\
20.08 \\
20.06 \\
20.08 \\
\end{array}$ \\
\hline
\end{tabular}

PERIOD OF RECORD HIGHEST +2.16 MAY 3-5, 1984 LOWEST 20.08 SEP 28, 30, 1992

S Manual steel tape measurement. 
ACCESS TO WATSTORE DATA.

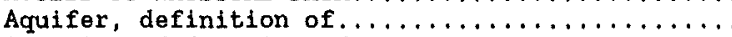

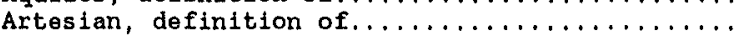

Calaveras County, index map to

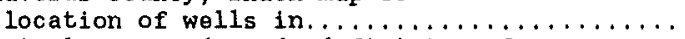

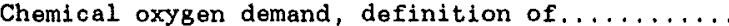

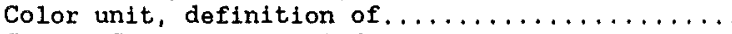

Contra Costa County, index map to

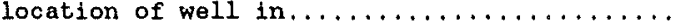

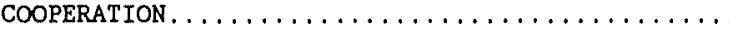

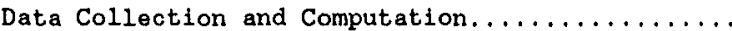

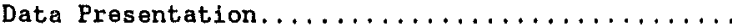

DEFINITION OF TERMS . . . . . . . . . . . . . .

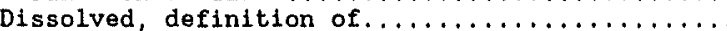

Dissolved-solids concentration,

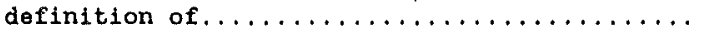

EXPLANATION OF THE RECORDS ..............

Fresno County, index map to location

of wells in....................... 24

Glenn County, hydrographs for wells in........ 38 Glenn County, index map to location

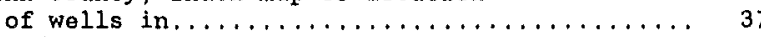

Ground-water levels, records of.............. 3

Ground-water levels and quality records by

county, index to:

Contra Costa County ...................... 22

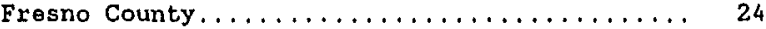

Glenn County....................... 37

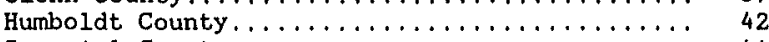

Imperial County..................... 44

Kern County........................ 55

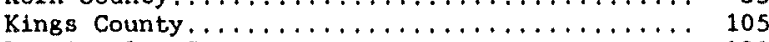

Los Angeles County...................... 108

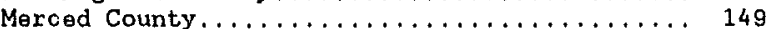

Mono County........................ 170

Monterey County..................... 185

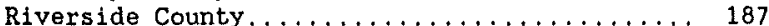

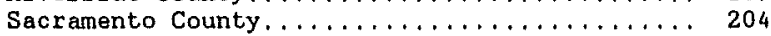

San Bernardino County.................... 207

San Diego County...................... 265

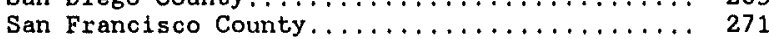

San Luis Obispo County................. 281

San Mateo County....................... 288

Santa Barbara County.................... 291

Shasta County........................... 414

Sutter County..................... 416

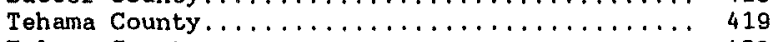

Tulare County........................ 423

Ventura County........................ 428

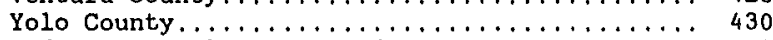

Ground-water quality, records of...............

GROUND-WATER RECORDS $\ldots \ldots \ldots \ldots$

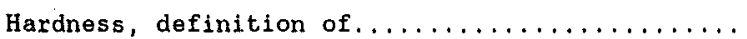
Humboldt County, index map to location

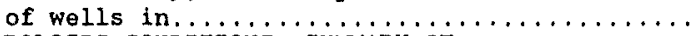

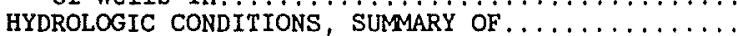

Imperial County, index map to location

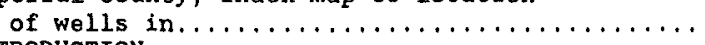

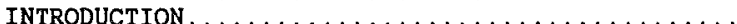

Kern County, hydrographs for wells in.........

Kern County, Index map to location

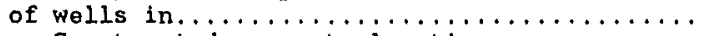

Kings County, index map to location

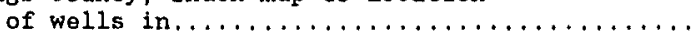

Land-surface datum, definition of........... GP0 /85-833/9909g
Page

Latitude-Longitude System................

Local Well Numbers...................... 3

Los Angeles County, hydrographs for weils in.... 110

Los Angeles County, index map to location of wells in....................... 109

Merced County, hydrographs for wells in........ 150

Merced County, index map to location of wells in....................... 149

Methylene blue active substance.

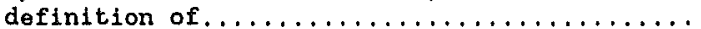

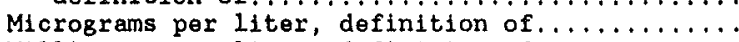

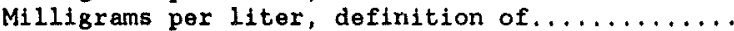

Mono County, index map to location

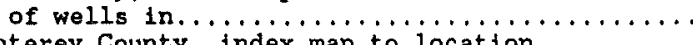

Monterey County, index map to location

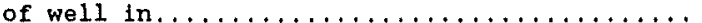

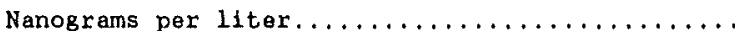

Pesticides, definition of $\ldots \ldots \ldots \ldots \ldots \ldots \ldots$

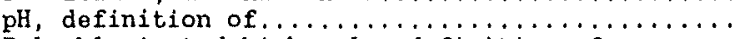
Polychlorinated biphenyls, definition of........ PUBLICATIONS ON TECHNIQUES OF WATERRESOURCES INVESTIGATIONS.

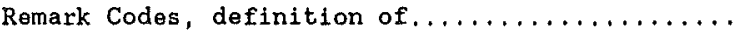
Riverside County, index map to location

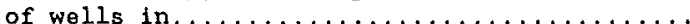

Sacramento County, hydrographs for well in..... Sacramento County, Index map to location

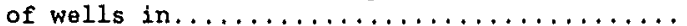

San Bernardino County, hydrographs

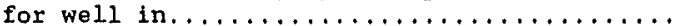

San Bernardino County, index map to location of wells in........................ 209

San Diego County, index map to location

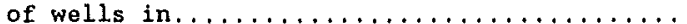

San Francisco County, index map to location

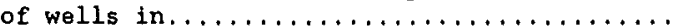

San Luis Obispo County, index map to location

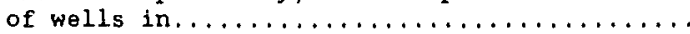

San Mateo County, index map to location

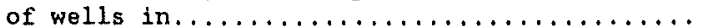

Santa Barbara County, index map to location

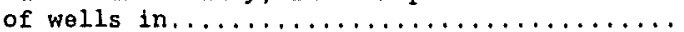

Shasta County, index map to location

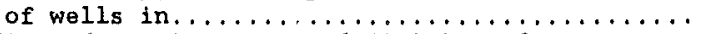

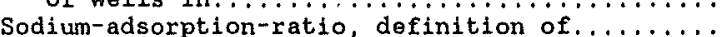

Specific conductance, definition of.............

Sutter County, index map to location

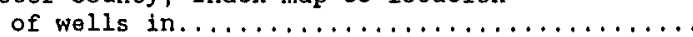

Tehama County, hydrographs for wells in........

Tehama County, index map to location

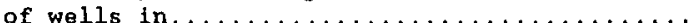

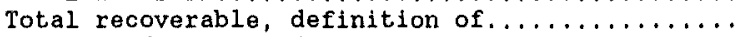

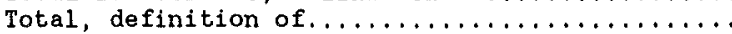

Tulare County, index map to location

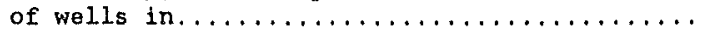

Ventura County, index map to location

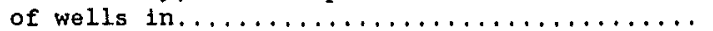

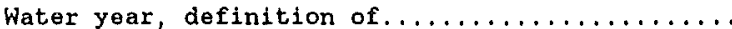

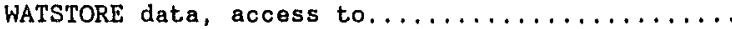

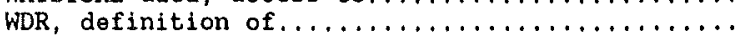

Well numbering system.................

WSP, definition of $\ldots \ldots \ldots \ldots \ldots \ldots \ldots \ldots \ldots \ldots \ldots$

Yolo County, hydrographs for wells in.........

Yolo County, index map to location

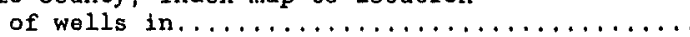




\section{FACTORS FOR CONVERTING INCH-POUND UNITS TO INTERNATIONAL SYSTEM UNITS (SI)}

The following factors may be used to convert the inch-pound units published herein to the International System of Units (SI).

Multiply inch-pound units

inches (in)
feet (ft)
miles (mi)

acres

square miles $\left(\mathrm{mi}^{2}\right)$

gallons (gal)

million gallons

cfs-days

acre-feet (acre-ft) cubic feet $\left(\mathrm{ft}^{3}\right)$

By

\section{Length}

$2.54 \times 10$ $2.54 \times 10^{-2}$

$3.048 \times 10^{-1}$

$1.609 \times 10^{\circ}$

Area

$4.047 \times 10^{3}$

$4.047 \times 10^{-1}$

$4.047 \times 10^{-3}$

$2.590 \times 10^{0}$

Volume

$3.785 \times 10^{0}$

$3.785 \times 10^{0}$

$3.785 \times 10^{-3}$

$3.785 \times 10^{3}$

$3.785 \times 10^{-3}$

$2.832 \times 10^{1}$

$2.832 \times 10^{-2}$

$2.447 \times 10^{3}$

$2.447 \times 10^{-3}$

$1.233 \times 10^{3}$

$1.233 \times 10^{-3}$

$1.233 \times 10^{6}$

Flow

cubic feet per second $\left(\mathrm{ft}^{3} / \mathrm{s}\right)$

gallons per minute (gal/min)

million gallons per day

tons (short)
$2.832 \times 10^{1}$

$2.832 \times 10^{1}$

$2.832 \times 10^{-2}$

$6.309 \times 10^{-2}$

$6.309 \times 10^{-2}$

$6.309 \times 10^{-5}$

$4.381 \times 10^{1}$

$4.381 \times 10^{-2}$

Mass

$9.072 \times 10^{-1}$
To obtain SI units millimeters (mm)

meters (m)

meters (m)

kilometers $(\mathrm{km})$

square meters $\left(\mathrm{m}^{2}\right)$

square hectometers $\left(\mathrm{hm}^{2}\right)$

square kilometers $\left(\mathrm{km}^{2}\right)$

square kilometers $\left(\mathrm{km}^{2}\right)$

\section{liters (L)}

cubic decimeters $\left(\mathrm{dm}^{3}\right)$

cubic meters $\left(\mathrm{m}^{3}\right)$

cubic meters $\left(\mathrm{m}^{3}\right)$

cubic hectometers $\left(\mathrm{hm}^{3}\right)$

cubic decimeters $\left(\mathrm{dm}^{3}\right)$

cubic meters $\left(\mathrm{m}^{3}\right)$

cubic meters $\left(\mathrm{m}^{3}\right)$

cubic hectometers $\left(\mathrm{hm}^{3}\right)$

cubic meters $\left(\mathrm{m}^{3}\right)$

cubic hectometers $\left(\mathrm{hm}^{3}\right)$

cubic kilometers $\left(\mathrm{km}^{3}\right)$

\section{liters per second $(\mathrm{L} / \mathrm{s})$}

cubic decimeters per second $\left(\mathrm{dm}^{3} / \mathrm{s}\right)$

cubic meters per second $\left(\mathrm{m}^{3} / \mathrm{s}\right)$

liters per second $(\mathrm{L} / \mathrm{s})$

cubic decimeters per second $\left(\mathrm{dm}^{3} / \mathrm{s}\right)$

cubic meters per second $\left(\mathrm{m}^{3} / \mathrm{s}\right)$

cubic decimeters per second $\left(\mathrm{dm}^{3} / \mathrm{s}\right)$

cubic meters per second $\left(\mathrm{m}^{3} / \mathrm{s}\right)$

megagrams (Mg) or metric tons 
U.S. DEPARTMENT OF THE INTERIOR

U.S. Geological Survey, Room W-2233

2800 Cottage Way, Federal Building

Sacramento, CA 95825

OFFICIAL BUSINESS

PENALTY FOR PRIVATE USE $\$ 300$

SPECIAL 4TH CLASS BOOK RATE 